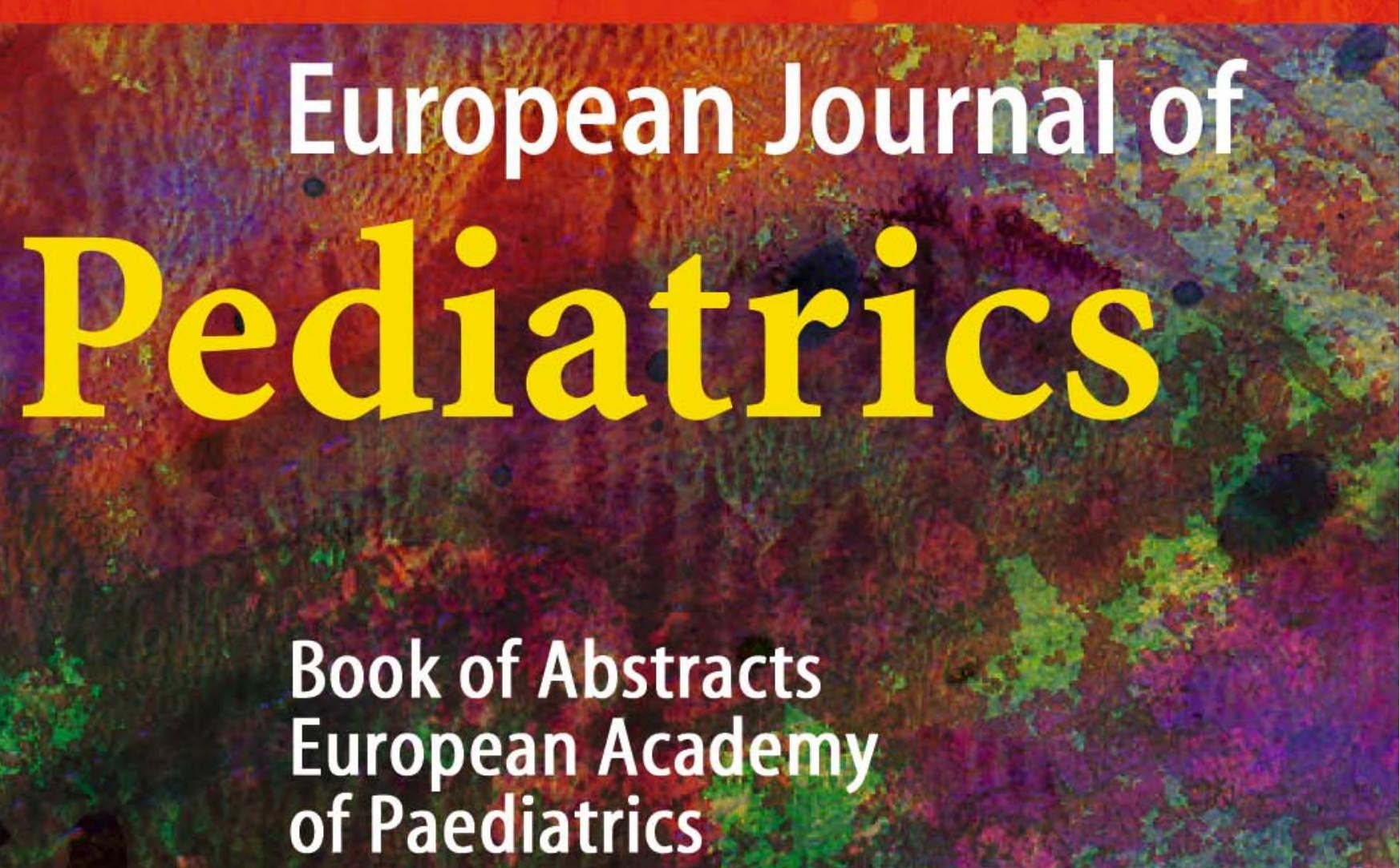

Barcelona, Spain, October 7-10, 2006

- Free color illustrationst

Online First Immediately Online

springerlink.com

Submit manuscripts:

8 http://www.editorialmanager.com/ejpe

2) Springer 


\section{EUROPEAN ACADEMY OF PAEDIATRICS}

Barcelona, Spain, 0ctober 7-10, 2006

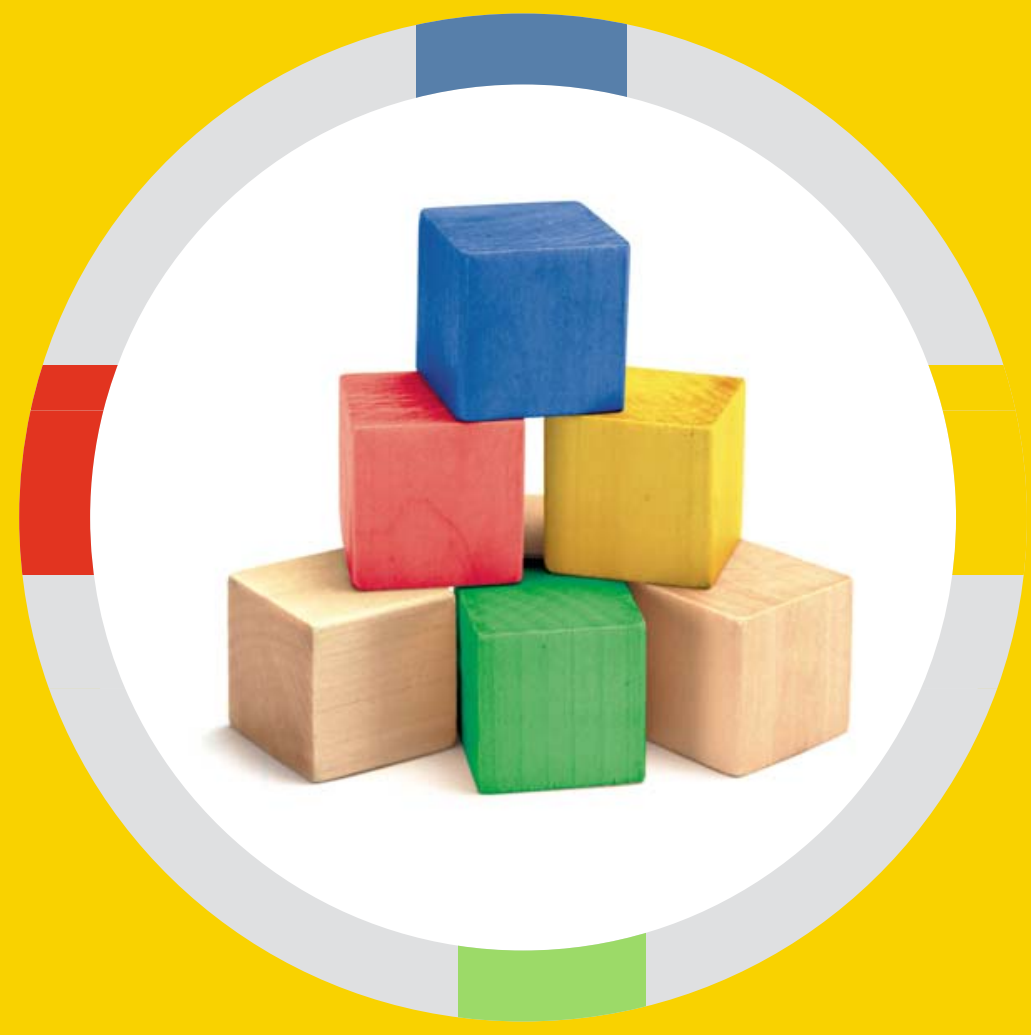

도

E

$=$

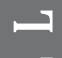

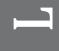

다

E

$\varangle$

थ

थ

थ

[다

$\propto$

0

2

0

$\circlearrowright$

도

$\propto$

ค

1

es

$E$

$\mho$

$\leftarrow$

$\Leftrightarrow$

E

A B S T R A C T S

es

$\oplus$

$\leftarrow$

Pre Congress Satellite Symposium 
BODY COMPOSITION IN EARLY GROWTH: LESSONS FROM DOMESTIC ANIMALS

\section{J E Harding}

Liggins Institute, University of Auckland, New Zealand

The effects of changes in nutrition on body composition of the growing animal depend on the timing, as well as severity, duration and nature, of the nutritional insult. In sheep, both maternal undernutrition around the time of conception and a high plane of nutrition in a young mother still growing herself result in impaired maternal metabolic adaptation to pregnancy, and hence greater substrate distribution to maternal at the expense of conceptus tissue accretion. Maternal nutrition in early pregnancy also determines subsequent growth and body composition of the offspring, with mild-moderate maternal undernutrition resulting in offspring of normal body weight at birth but with increased weight of fat depots thereafter. Undernutrition in late pregnancy commonly reduces offspring birthweight, and this in turn affects later growth and body composition. After birth, growth of different tissues is strongly influenced by both prior and current nutritional experiences. Lambs of lower birthweight take longer to start to gain weight after birth. They also require a higher feed intake to attain a given weight gain, and more of that weight is gained as fat and less as muscle compared with lambs of higher birthweight. However for a given birthweight, slower postnatal weight gain is generally associated with a proportionately greater muscle growth. Rate of growth also determines other aspects of body composition. Muscle fibre number, which limits final muscle size, is generally determined before birth, and indeed before the last trimester of pregnancy in many species. Lambs growing rapidly either before or after birth tend to have proportionately larger livers, whereas chronically slow growth is associated with reduced liver size relative to body weight. Similar patterns are seen in the lymphoreticular tissues such as spleen and thymus. It seems likely that these variations in body composition will have important functional consequences for later health and wellbeing.

\section{EARLY GROWTH AND BODY COMPOSITION OF ADOLESCENTS}

L A Moreno ${ }^{1}$, I Labayen ${ }^{2}$

${ }^{1}$ E.U. Ciencias de la Salud, Universidad de Zaragoza, Zaragoza, Spain, ${ }^{2}$ Department of Nutrition and Food Science, University of The Basque Country, Vitoria, Spain

Background and Aims: There are critical periods during childhood that influence the development of obesity, including gestation and early infancy. Size at birth and early postnatal growth are determinants of adult height and body mass index. The aim of this study was to assess if birth weight has any effect on body composition and/or fat distribution in adolescents.

Methods: Body composition was assessed by both skinfold thickness measurements and dual energy X-ray absorptiometry (DXA) in 234 adolescents born at term (140 females and 94 males), now aged 13-18 y and living in the city of Zaragoza (AVENA-Zaragoza cohort). Relative fat distribution was estimated with the ratio of the subscapular to triceps skinfolds $(\mathrm{S} / \mathrm{T})$. Birth weight and gestational age were assessed by means of a questionnaire, considering the paediatric neonatal records.

Results: Birth weight was inversely associated with $\mathrm{S} / \mathrm{T}$ ratio $(\mathrm{P}<0.05)$ in males and directly associated with bone mass $(\mathrm{P}<0.01)$ and fat free mass $(\mathrm{P}<0.05)$ in females. This association was independent of age, Tanner stage, gestational age, socioeconomic status, physical activity and height.

Conclusions: In adolescents born at term, genetic and/or intrauterine environmental factors that influence foetal growth and are reflected in birth weight have long term consequences on bone and muscle mass, measured by DXA, in females and in subcutaneous fat distribution in males.

\section{PROGRAMMING OF OBESITY AND INSULIN RESISTANCE: THE EFFECT OF EARLY CATCH UP GROWTH - LESSONS FROM ANIMAL MODELS}

\section{Remacle, B Reusens}

Institute of Life Science, University of Louvain, Louvain-la-Neuve, Belgium

Background and Aims: A body of evidence now suggests that poor intrauterine milieu elicited by maternal nutritional disturbance or placental insufficiency may programme susceptibility in the fetus to later develop chronic degenerative disease such as diabetes, obesity, hypertension and cardio-vascular disease. The compatibility of early postnatal nutrition with fetal experience will also be decisive for the rate of occurrence of degenerative disease.

Methods: The understanding of mechanisms underlying the programming of the metabolic syndrome is now progressing thanks to animal studies in which the fetal and neonatal environment has been manipulated.

Results: Similarly to data from epidemiological studies in humans, obesity in rodent models arises principally in offspring submitted to malnutrition in early stages of gestation and which presented an early catch-up growth. Four different pathways for programming obesity may be proposed: 1) programming central circuits in the hypothalamic area controlling feeding and metabolism, 2) modification of energy expenditure, 3) generation of a hormonal context favouring adipose tissue development, 4) modification of the pools of fat cell precursors which should lead to hypertrophic / hyperplastic obesity. These pathways and their respective importance according to the time-window of exposure and the type of malnutrition are now subject to intense study. Studies on the progressive appearance of insulin resistance in rodents which had been growthretarded in utero revealed an increased sensitivity of target tissues to insulin in young adults, followed by insulin resistance in older age. The insulin resistance was associated with changes in expression of key components of the insulin-signalling cascade in muscle and adipocytes. The changes in expression of such molecular markers have been detected also in muscle 
and fat biopsies of men with a low birth weight. Possible molecular mechanisms involved in nutritional programming are just starting to emerge, such as early definitive changes in the organelle central to energy utilisation, i.e. mitochondria, or epigenetic changes in nuclear DNA.

Conclusions: Animal models provide efficient tools to reveal the time windows of nutritional experience in early life favouring later obesity, as well as to decipher progressively physiological and molecular mechanisms involved in such metabolic programming. 


\section{EUROPEAN ACADEMY OF PAEDIATRICS}

Barcelona, Spain, 0ctober 7-10, 2006

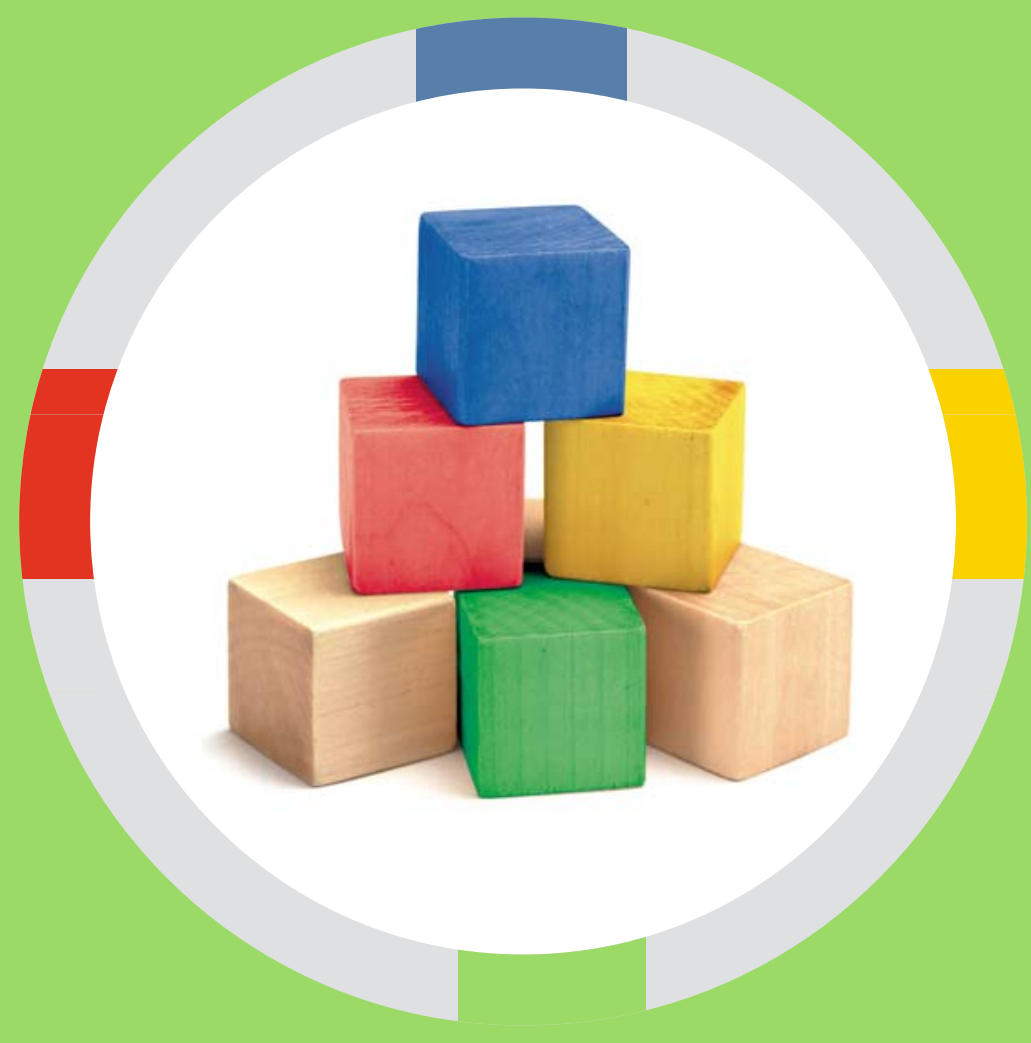

$$
\begin{aligned}
& \text { es } \\
& \text { z } \\
& 0 \\
& \text { E } \\
& \varangle \\
& E \\
& \text { z } \\
& \text { [디 } \\
& \text { es } \\
& \text { ㄸ } \\
& \propto \\
& \text { e }
\end{aligned}
$$

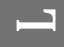

$$
\begin{aligned}
& \varangle \\
& \propto \\
& 0 \\
& \text { I } \\
& \text { es } \\
& \text { E } \\
& 0 \\
& \varangle \\
& \propto \\
& \text { E } \\
& \text { es } \\
& \infty \\
& \varangle
\end{aligned}
$$

A B S T R A C T S
ORAL PRESENTATIONS 
HUMAN GENOME: IMPLICATIONS FOR CLINICAL PRACTICE

\section{J-J Cassiman}

Center for Human Genetics, University of Leuven, Belgium

The complete and detailed sequence of a representative sample of human DNA is almost fully known, except for a few stretches of hard to sequence DNA. This historic achievement has already generated a few surprises. The number of genes (about 25,000) hidden in the 3 billion nucleotides that make up the human genome is much smaller than initially expected and is only slightly higher than in flies, while the total protein coding sequence is only about $3 \%$ of the genome. The OMIM register at www3.ncbi.nlm.nih.gov/Omim/ lists more than 12,000 entries for monogenic or Mendelian diseases, including 60 mitochondrial diseases. More than 7,000 of these have been already mapped to a particular chromosomal region, while another 2,000 have a definite gene locus. The remaining entries are only phenotypic descriptions or other entries. The molecular basis of these diseases is rapidly being uncovered. In some genes one or a few defects are responsible for a particular phenotype; in others, mutations can be scattered all over the gene e.g. the more than 1000 different mutations in the CFTR gene in cystic fibrosis. The majority of our normal traits and diseases are inherited in a multifactorial fashion. This means that a series of genes (usually tens of genes) are involved, due to either normal variation in their sequence or real dysfunctional mutations. In addition, environmental factors modulate the way in which these genes influence the final phenotype. Traits inherited in this fashion are mainly quantitative (measurable) traits such as height, weight, IQ etc. and show a normal (Gaussian) distribution in the population. Lamarck, who in the 19th century claimed that acquired traits can be inherited, has taken a little revenge in recent years. Some examples have been found of how nutritional factors can condition DNA and affect future generations. As our understanding of the human genome increases, it has become increasingly clear that our knowledge is at present largely insufficient to attempt to explain all the phenotypic variability observed between individuals affected by the same disease. It is only gradually that we will unravel all the genetic and environmental factors responsible for this variability. Nevertheless, many families can already be helped by genetic testing in order to identify with precision what the nature of the disease is and what the recurrence risks are. Even fairly precise prediction of the risk to develop a disease in adult life (presymptomatic diagnosis) or of the susceptibility to develop serious multifactorial diseases, such as hereditary breast or colorectal cancer, is available. Nevertheless we have to guarantee our clients that these tests are done with the best quality available and in the context of appropriate genetic counseling.
GENES AND ENVIRONMENTAL INFLUENCE IN ASTHMA AND ATOPY

\section{R Nickel}

Department of Pediatric Pneumology and Immunology, Charité, Berlin, Germany

Background and Aims: Despite a strong genetic basis for asthma and atopy, no genetic marker(s) unambiguously associated with atopy-related traits has yet been identified. Variants in $>100$ candidate genes were associated with atopy-related traits. However, replication (at least twice) was reported for less than 30 genes. Discrepant results may partly be attributed type 1 and type 2 errors due to small sample sizes or no correction for multiple tests. Also, different ethnicities of study populations have to be considered. There is growing evidence that gene-by-gene- and gene-byenvironment interactions strongly influence disease susceptibility and that single variant association studies are not sufficient to identify the genetic basis for atopic disorders. With regard to the development of asthma and atopy, environmental factors (e.g. factors associated with living on a farm, allergen exposure, infections) appear to be particularly important very early in life. Methods: Therefore, many gene-by-environmentinteraction studies have been performed in pediatric populations and birth cohorts.

Results: Strong evidence for interactions has been presented for genes involved in LPS (lipopolysaccharide) binding and signalling: One of the most extensively examined candidate gene for atopy is CD14, coding for a receptor for LPS. A CD14 promoter variant was associated with both, low and high total serum $\operatorname{IgE}$ levels (tIgE), other studies showed no effect on tIgE. Recent studies that focused on CD14 genotypes and endotoxin exposure suggest strong gene-by-environment interactions with the CD14 variant being either protective or a risk factor for asthma or increased $\operatorname{tgE}$ dependent on low or high endotoxin exposure. Toll like receptor (TLR) genes have also been investigated in asthma and atopy. TLRs are crucial for recognition of microbial pattern associated molecules. A TLR2 variant was associated with a decreased risk for asthma, but this effect was exclusively seen in children living on a farm, also implying a gene-by-environment interaction. Environmental tobacco smoke (ETS) exposure has been intensely investigated in gene-by-environment interaction studies. An example is studies of an IL13 variant within a large German birth cohort. Significant associations with tIgE and a common promoter variant were observed at any age from 1 to 7 years. ETS exposure in utero and/or infancy resulted in even higher tIgE in children homozygous for the variant, but with no effect on $\operatorname{tgE}$ in the remaining children.

Conclusions: ETS-, endotoxin- and allergen exposure are important environmental factors with regard to asthma and atopy. Exposure can be assessed and quantified in standardized procedures. Other factors that 
may contribute to the increased prevalence of atopy (and therefore of interest for gene-by-environment interaction studies) are harder to assess in a standardized fashion (e.g. diet, infections, medication, stress), which is an ongoing effort of current birth cohort studies in our division. Taken together, genetic studies of asthma and atopy not only require large carefully characterized, ethnically homogeneous study populations, but also data on environmental exposure such as endotoxin, ETS and allergens. Birth cohorts are particularly valuable for gene-by-environment interaction studies since exposure can be assessed in early infancy, a most vulnerable period of life with regard to the development of asthma and atopy.

\section{CLINICAL IMPLICATIONS OF SYSTEMS BIOLOGY AND HIGH-THROUGHPUT TECHNOLOGY IN PEDIATRICS}

\section{Benson}

Department of Pediatrics, Queen Silvia Children's Hospital, Gothenburg, Sweden

Background and Aims: Complex diseases like diabetes, allergy and cancer depend on multiple interacting genes. It is probably not possible to find a key gene in such diseases, a "silver bullet" that can be used for diagnostic or therapeutic purposes. New technologies, like DNA microarrays allow simultaneous analysis of expression of all human genes. We have applied these technologies and systems biology to allergic rhinitis. The clinical aim is to identify markers for treatment response. It is possible that similar principles are generally applicable to complex diseases.

Methods: DNA microarrays, clique-based graph algorithms, pathway analysis, real-time PCR, immunohistochemistry, flow cytometry, SNP analysis

Results: Getting a functional understanding of the large datasets generated by DNA microarray studies of complex diseases is a significant challenge. One such study may identify hundreds or even thousands of genes that differ in expression between patients and controls. While this may be seen as discouraging it does reflect the complexity of the diseases that we study. Moreover, if we can get a grip on this complexity this may be used for clinical purposes, for example to find combinations of markers for individualized therapy. Systems biology is an emerging discipline that aims to understand how complex systems relate to biological effects. One important theoretical framework is network-based analysis. In the context of DNA microarray studies, genes that differ in expression between patients and controls are organized in gene interaction networks. Such networks can be analyzed in a top-down fashion. The idea is the same as a microscopic examination. You start with a low magnification to identify general features of the sample and then proceed to detailed studies of individual cells. A gene interaction network can be analyzed to find sub-networks or pathways with distinct functions, such as cell proliferation or apoptosis. These sub-networks can be further dissected to find upstream genes with putative key regulatory functions. Typically, several sub-networks or pathways are involved in complex diseases. However, a top-down analysis may result in identification of combinations of key genes that can be used for clinical purposes, such as to find markers for personalized medication. In this presentation examples from our own research in allergic disease will be given, as well as from oncological research. In the latter case customized DNA microarrays are tried to find gene expression signatures for personalized medication in breast cancer.

Conclusions: High-throughput studies and systems biology may contribute to understanding of complex diseases. A clinical implication may be identification of markers for personalized medication.

\section{WRITING FOR SUCCESS: OR EVERYTHING I WANTED TO KNOW ABOUT GETTING PUBLISHED BUT WAS AFRAID TO ASK!}

\section{J W Albarran}

\section{University of the West of England, Bristol, UK}

Background and Aims: Nurses, whether as academics or practitioners are increasingly required to disseminate the findings of research or share ideas and examples of best practice through publication. Lack of confidence, time and familiarity with different journal house-styles are often cited as barriers. These issues will be considered. Moreover, writing informatively and in an accessible format is not easy, but it can be learned through following certain principles, commitment, time and enthusiasm [1-2]. Aim: The purpose of this workshop is for delegates to understand how to develop a successful strategy that will lead to publication in a peer-reviewed journal.

Methods: The workshop will address the following areas:

1) Choosing a topic area

2) Decisions about choosing journal (impact factors and dissemination)

3) Preparing the manuscript (conventions for research and non-research papers)

4) Multiple publications from research projects

5) Authorship and co-authorship

6) Dealing with reviewers comments

Results: The workshop will be based on a power-point presentation but delegates will be expected to participate through open discussion and questions to explore a wide range of other related issues.

Conclusions: The conclusion will summarise the seven key stages on preparing a paper for publication including the importance of considering international readers.

References: Murray R (2005) Writing for academic journals. Open University Press, Maidenhead Albarran JW and Scholes J (2005) How to get published: Seven easy steps, Nursing in Critical Care, 10 (2): 72-77.

\section{THE ROLE OF A HOSPITAL PLAY SPECIALIST IN ENSURING THE DEVELOPMENT OF LONG-TERM VENTILATED CHILDREN ON AN INTENSIVE CARE UNIT}

\author{
L Bassett, C M Pierce
}


Paediatric and Neonatal Intensive Care Unit, Great Ormond Street Hospital, London, UK

Background and Aims: Play therapy has been part of the intensive care service in our hospital since 1996. Hospital Play Specialists (HPS) work as part of the multidisciplinary and psychosocial team within the intensive care unit. We reviewed our experience of play therapy in long-term patients whilst on intensive care.

Methods: We performed a review of long-term ventilated patients on our intensive care unit, between 2002-2006 in which we routinely devised 'play programmes'. The average stay was 12 months. Reason for contact and outcome were reviewed.

Results: The HPS assessed the child's needs and devised appropriate programmes for them, which fit a daily routine. The programme ensured appropriate stimulation as well as establishing periods of rest and sleep, whilst still appreciating the child's medical and nursing input. The programmes changed to fit the child's development and looked at the psychosocial needs of the child and the family whilst requiring intensive care. Examples are given.

Conclusions: A play specialist can become an integral part of the intensive care unit working alongside the medical and nursing staff to improve the long-term patient's development. The HPS teaches nurses and parents to play within the intensive care environment. The HPS works with the long-term patient, their family and the intensive care staff to overcome issues such as behaviour, eating, and communication problems. The Play Specialist meets the patient's needs through play; they provide fun and stimulation trying to provide some normality in an otherwise stressful and frightening environment.

\section{DOES HUMOUR DELIVERED THROUGH CLOWNING BENEFIT CHILDREN IN HOSPITAL? THE PERCEPTIONS OF THE THEODORA CHILDREN'S TRUST SPECIAL CLOWNS}

\author{
E A Glasper ${ }^{1,2}$, G Prudhoe ${ }^{1}$ \\ ${ }^{1}$ University of Southampton, ${ }^{2}$ Southampton University \\ Hospitals Trust, Southampton, UK
}

Background and Aims: The use of humour as a therapy is receiving increasing attention from health care professionals. This study discusses the views of The English Theodora Children's Trust Clown Doctors of what works best and least well during a clown encounter with a sick child in hospital.

Methods: Nominal group technique (NGT). Sample size $=12$.

Results: What works best when a clown doctor visits a sick child?

JUNIOR CLOWNS

1. Spontaneity

2. To empower the child

3. Listening, watching and then responding

4. Distraction

5. Child's consent

SENIOR CLOWNS

1. Being $100 \%$ present
2. Sensitivity

3. Feeling and giving happiness

4. Play and the laughter (2 joint items)

5. Changing atmosphere: What works least well when a clown doctor visits a sick child?

JUNIOR CLOWNS

1. Insensitivity

2. Talking down to the child

3. Ego

4. Competing with another clown doctor for attention

5. Disrespect of hospital procedure and staff

SENIOR CLOWNS

1. Being Insensitive

2. Being on an ego trip

3. Not connecting and relatives or staff saying the child hates clowns when in fact they themselves do

4. Bad communication, not having enough time, working with no feeling and ignoring the child's feelings 5. Not judging the situation, when a child is diagnosed as the terminally ill/dying, lack of information on the child's status and not feeling at your best

Conclusions: This report of the perceptions of Clown Doctors has shown that there are a number of elements which enhance a clown doctor encounter with a sick child.

\section{CYSTIC FIBROSIS AND GENES: A BRIEF HISTORY, BUT A LOT OF NEWS}

\section{S Cunningham}

Department of Respiratory and Sleep Medicine, Royal Hospital for Sick Children, Edinburgh, UK

Cystic Fibrosis, first described in 1938, was quickly recognised, like so many other conditions as being of autosomal recessive inheritance. There was understandable great excitement surrounding the discovery in 1989 of DeltaF508, the most common gene mutation for cystic fibrosis. As a recessive single gene defect disease the possibility of replacing the defective gene was a stimulating prospect. Seventeen years later, whilst other single gene defects have been corrected, a solution to the cystic fibrosis gene defect remains unresolved. The reasons for this lack of resolution are frustrating to patients and clinicians, but have opened novel and fruitful research directions. What can be so complex about this single gene defect? Firstly, there isn't just one gene defect, there are no more than 1000 genes identified associated with cystic fibrosis, falling into five functional classes. Secondly, we have become increasingly aware that the interplay of ancillary 'modifier' genes play an important but as yet poorly quantified effect on the principle gene defect. Thirdly, as with other diseases, the complex evolving phenotype of cystic fibrosis, incorporates the interplay of genotype and environment, together with the possible role of epigenetics (heritable changes in gene function that occur without a change in the DNA sequence). Together these factors add a substantial complexity to what was considered a simple model of disease. Into this environment, the next generation of pulmonary trials for correction of the cystic fibrosis gene defect are now being planned. The first pulmonary trials were successful (in that gene transfer occurred), but not 
enough and side effects too many. Important lessons were learnt, and following a hiatus to reform the whole infrastructure of the gene delivery systems further trials are now planned. There are expected additional problems in children, not least of which is how will we know if gene transfer works. Yet children are considered the linchpin to the success of such studies (repairing the defect before major pulmonary injury has occurred) and research is underway to ensure that children should not be disenfranchised from this major biotechnological advance.

\section{BETAMETHASONE EFFECTS ON PERINATAL PULMONARY DEVELOPMENT IN AN ESCHERICHIA COLI EXPERIMENTAL MODEL OF INTRA UTERINE INFECTION IN THE RABBIT}

N Joram ${ }^{1,3}$, E Launay ${ }^{1,3}$, J C Roze ${ }^{3}$, M L Franco-Montoya ${ }^{2}$, J Bourbon ${ }^{2}$, C Gras-Le Guen ${ }^{1,3}$

${ }^{1}$ Laboratoire d'Antibiologie, CHU Nantes, France

${ }^{2}$ Laboratoire INSERM U651, Créteil, France

${ }^{3}$ Departement de Perinatologie, CHU Nantes, France

Background and Aims: Chorioamnionitis is known to play a central role in its pathogenesis of bronchopulmonary dysplasia. In clinical practice, glucocorticoids are used before a premature birth for their role in pulmonary maturation, decreasing the risk of morbidity and mortality. However, some animal studies suggest that they may also have adverse effects on alveolar development. In this context, we hypothesized that glucorticoids could major alveolarization impairment caused by chorioamnionitis.

Methods: We used a model of Escherichia coli chorioamnionitis in rabbits. Pregnant females $(n=20)$ receiving an intrauterine inoculation with $103 \mathrm{E}$. Coli on day 25 of gestation received antibiotics and either one or two doses of betamethasone $(0,1 \mathrm{mg} / \mathrm{kg} / \mathrm{dose})$ or saline serum, 6 hours after inoculation. Surviving foetuses were studied at first day after normal delivery. Alveolar development was compared between the three groups performing morphometric analysis $(n=31)$. Inflammatory process was analyzed measuring cellularity in amniotic fluid and bronchoalveolar lavage fluid.

Results: With one or two doses of bethamethasone versus saline serum, a statistically higher mortality rate $(p<0,001)$ was found and birth weight and lung volume were significantly decreased $(p<0,05)$. With two doses of glucocorticoids versus one dose and versus saline serum, specific alveolar area, intersticium volume, elastin fibers length and blood vessels volume were decreased $(p<0,05)$, and alveolar airspace volume was increased $(p<0,05)$. No significant difference was found in inflammatory process.

Conclusions: Glucocorticoids major significantly infectious-induced alveolar impairment in this experimental model of evolutive antenatal infection, which confirms their serious potential long term adverse effects on the preterm lung.
STRUCTURAL LUNG ABNORMALITIES IN BRONCHOPULMONARY DYSPLASIA (BPD): A NEW SCORING SYSTEM FOR LUNG-CT SCAN EVALUATION

J E H Bunt ${ }^{1}$, V Casotti $^{2}$, L Rubin $^{3}$, R Failo $^{3}$, M H Lequin ${ }^{4}$, W Hop ${ }^{5}$, H A Tiddens ${ }^{6}$

${ }^{1}$ Department of Paediatrics-Neonatology, Erasmus MC-Sophia, Rotterdam, The Netherlands, ${ }^{2}$ Department of Paediatrics, Policlinico G.B. Rossi, Verona, Italy ${ }^{3}$ Department of Radiology, Policlinico G.B. Rossi, Verona, Italy, ${ }^{4}$ Department of Radiology, Erasmus MCSophia, Rotterdam, The Netherlands, ${ }^{5}$ Department of Biostatistics, Erasmus MC, Rotterdam, The Netherlands ${ }^{6}$ Department of Paediatrics-Pulmonology, Erasmus MCSophia, Rotterdam, The Netherlands

Background and Aims: Little is known about the structural substrate causing pulmonary abnormalities in patients with BPD. Aims: First: To develop a scoring system to standardize the description of lung-CT scans in BPD. Second: To study long term structural changes on CT scans in BPD in relation to lung function and neonatal risk factors.

Methods: Retrospective analysis of lung-CT scans in BPD. Patients received a lung-CT scan when indicated by the paediatric pulmonologist. CT scans were scored for: decreased density/hypovascularity (0-3 points); linear opacities (0-3 points); bullae (0-3 points); air trapping ( $0-1$ point); consolidations ( $0-3$ points); bronchiectasis ( $0-1$ point), and airway wall thickening (0-1 point). The maximum score could be $85(=100 \%)$. Three independent readers scored all scans to determine the reproducibility.

Results: 37 BPD patients were identified. Age at CT was 2 months to 17 years. Mean CT score was $27 \%$ (range 4-53\%). Inter- and intra-observer agreement, assessed by the intra-class correlation coefficient, was good (ICC from 0.830 to 0.933 ). The mean CT score correlated positively with the mean alveolar pressure during mechanical ventilation at 24 hours after birth $(\mathrm{R}=0.588, \mathrm{p}<0.008)$, and the days of $\mathrm{O} 2$ need postnatally $(\mathrm{R}=0.530, \mathrm{p}<0.002)$. The mean $\mathrm{CT}$ score correlated negatively with the FEV1 $(\mathrm{R}=-0.612$, $\mathrm{p}<0.002$ ).

Conclusions: CT scans in BPD show structural abnormalities and can be scored in a standardized reproducable way. The CT score correlated with classic neonatal risk factors for BPD and lung function at later age. A prospective study on CT scans in BPD should be performed in the new era of neonatal care.

\section{IMPACT OF ASTHMA ON CHILDREN'S PHYSICAL ACTIVITY, EMOTIONAL WELLBEING AND RISK OF OBESITY}

C Glazebrook $^{1}$, A McPherson ${ }^{2}$, C Ramsay ${ }^{1}$, R Newbould ${ }^{1}$, I MacDonald ${ }^{3}$, A Smyth ${ }^{4}$

${ }^{1}$ School of Community Health Sciences, University of Nottingham, UK, ${ }^{2}$ School of Nursing, University of Nottingham, UK, ${ }^{3}$ School of Biomedical Sciences, University of Nottingham, UK, ${ }^{4}$ Division of Child Health, University of Nottingham, UK 
Background and Aims: Exercise is a frequently cited trigger for asthma symptoms in childhood. Any barrier to physical activity has implications for weight management and emotional wellbeing. This study aims to investigate the impact of asthma on children's customary activity, body mass index (BMI) and mental health.

Methods: A controlled, cross-sectional study of children aged 7-14 attending hospital outpatient clinics, either for asthma $(n=56)$, or for ENT or dermatological conditions $(\mathrm{n}=61)$. Measures were BMI and International Task Force classification of obesity. Parent-rated Strengths and Difficulties Questionnaire (SDQ) assessed children's mental health and child-rated Physical Activity Questionnaire (PAQ) assessed total sedentary and physical activities in previous 24 hours.

Results: The groups were well matched for demographic variables. The asthma group had higher BMI $(\mathrm{p}=0.008)$ and $21.4 \%$ were obese compared to $6.6 \%$ in the non-asthma group (OR 3.89, 95\% CI 1.1712.88). Children with asthma reported fewer physical activities $(p=0.002)$ but comparable levels of sedentary activities. Obese children were less active $(\mathrm{p}=0.008)$ but regression analysis showed asthma was the strongest predictor of lower activity scores, followed by younger age (adjusted $\mathrm{r} 2=0.104$ ). The asthma group had higher levels of emotional difficulties $(\mathrm{p}=0.05)$ and, within this group, PAQ scores negatively correlated with SDQ scores indicating that more active children had better mental health $(\mathrm{p}=0.009)$. More parents $(60.7 \%)$ and children $(66.1 \%)$ in the asthma group identified the child's health as a barrier to exercise compared to the non-asthma group $(\mathrm{p}<0.001)$.

Conclusions: Interventions to promote physical activity in children with asthma may reduce the risk of obesity and improve mental health.

\section{EFFECTS OF INDOMETHACIN AND IBUPROFEN ON ENDOTHELINS IN THE DEVELOPING RAT LUNG}

\author{
H D Modanlou ${ }^{1}, \mathrm{~K}$ D Beharry ${ }^{1}, \mathrm{~J} \mathrm{Hasan}^{1,2}$, \\ J V Aranda ${ }^{3}$ \\ ${ }^{I}$ Department of Pediatrics, University of California \\ Irvine, Irvine, CA, USA, ${ }^{2}$ Department of Pediatrics, \\ Miller Children's Hospital, Long Beach, CA, USA \\ ${ }^{3}$ Pediatric Pharmacology Research Unit Network, \\ Children's Hospital of Michigan, Detroit, MI, USA
}

Background and Aims: Indomethacin (IN) is used for PDA closure, but its use is associated with changes in pulmonary vascular tone. These effects may be due to endothelin-1 (ET-1). We compared the effects of early postnatal IN and ibuprofen (IB) on ET-1 and big ET-1 levels in the rat lungs.

Methods: Newborn rats ( $\mathrm{n}=15$ /group) received IP injections at birth (P1), P2, and P3 of either saline (Sal); $0.2 \mathrm{mg} / \mathrm{kg} \mathrm{IN}$ on P1 followed by $0.1 \mathrm{mg} / \mathrm{kg}$ on $\mathrm{P} 2$ and $\mathrm{P} 3 ; 1.0 \mathrm{mg} / \mathrm{kg} \mathrm{IN}$ on P1 followed by $0.5 \mathrm{mg} / \mathrm{kg}$ on P2 and $\mathrm{P} 3 ; 10 \mathrm{mg} / \mathrm{kg} \mathrm{IB}$ on $\mathrm{P} 1$ followed by $5 \mathrm{mg} / \mathrm{kg}$ on P2 and P3; and $50 \mathrm{mg} / \mathrm{kg}$ IB on P1 followed by $25 \mathrm{mg} / \mathrm{kg}$ on P2 and P3. At birth, P14 and P21, lung ET-1 and big ET-1 levels were examined.
Results: ET-1 levels were higher than big ET-1 levels and remained unchanged from birth through P21 in the controls. In contrast, big ET-1 levels increased at P14 compared to birth $(\mathrm{p}<0.05)$ and P21 $(\mathrm{p}<0.05)$. Low-dose IN increased ET-1 levels compared to Sal $(\mathrm{p}<0.01)$, low-dose IB $(p<0.001)$, high-dose IB $(p<0.001)$, and high-dose IN $(p<0.001)$. Big ET-1 levels were lower in all treatment groups $(\mathrm{p}<0.01)$.

Conclusions: Suppression of big ET-1 with IN and IB suggest an interaction between COX and endothelin. Changes in pulmonary vascular tone with therapeutic doses of IN may be due to increased production of ET-1. IB does not have similar effects on pulmonary ET-1 levels.

\section{LASER CO2 ARYEPIGLOTTOPLASTY IN INFANTS WITH SEVERE LARYNGOMALACIA: EFFECTS ON BREATHING PATTERN}

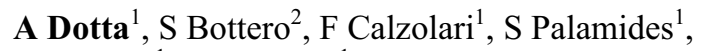 \\ $\mathrm{C}$ Corchia $^{1}$, M Orzalesi ${ }^{1}$ \\ ${ }^{l}$ Department of Medical and Surgical Neonatology, \\ Bambino Gesù Children's Hospital, Rome, Italy \\ ${ }^{2}$ Otorinolaryngoiatry Unit, Bambino Gesù Children's \\ Hospital, Rome, Italy
}

Background and Aims: Laryngomalacia is the most common cause of stridor in infants. Current treatment in severe forms is aryepiglottoplasty. Little is known about its effects on respiratory function. The aim was to evaluate changes in breathing pattern after aryepiglottoplasty in infants with severe laryngomalacia. Methods: Twenty infants were studied before and 4-6 weeks after laser $\mathrm{CO} 2$ aryepiglottoplasty. Laryngomalacia was diagnosed clinically and by laryngo-tracheal endoscopy. Breathing pattern was studied with an ultrasonic flow-meter measuring: Tidal Volume (Vt), Respiratory Rate (RR), Time to peak expiratory flow/expiratory time ratio (tPTEF/Te, an index of lower airways patency), Mean Expiratory/Mean Inspiratory Flow ratio (MEF/MIF, an index of upper airways patency). The patients underwent laser $\mathrm{CO} 2$ ariepiglottoplasty and following the procedure were kept intubated for $24 \mathrm{hrs}$. The comparison between pre (T1) and post aryepiglottoplasty (T2) test was done by the Student's t-test for paired values.

Results: Values are expressed as means \pm SD. Infants were studied at $2.9 \pm 1.7$ (T1) and $4.5 \pm 1.8$ (T2) months of age. Before surgery the breathing pattern was characterized by low tidal volumes and high $\mathrm{tPTEF} / \mathrm{Te}$ and MEF/MIF ratios. After surgery these variables improved significantly ( $\mathrm{T} 1$ vs $\mathrm{T} 2$ : $\mathrm{Vt}=5.5 \pm 1.7$ vs 8.0 $\mathrm{ml} / \mathrm{kg}, \quad \mathrm{p}<0.001 ; \quad \mathrm{tPTEF} / \mathrm{te}=0.34 \pm 0.11$ vs $0.23 \pm 0.11$, $\mathrm{p}<0.005 ; \quad \mathrm{MEF} / \mathrm{MIF}=1.6 \pm 0.8 \quad$ vs $\quad 0.9 \pm 0.2, \quad \mathrm{p}<0.001)$ reaching age specific normal values. The surgical procedure was well tolerated in all infants and no perioperative or long term complications were observed.

Conclusions: Laser $\mathrm{CO} 2$ aryepiglottoplasty in infancy is safe and effective and significantly improves pulmonary function at least in the short term. 


\section{SERUM LEVELS OF SEVEN CYTOKINES IN PRETERM VENTILATED NEWBORNS: CORRELATION WITH OLD AND NEW FORMS OF BRONCHOPULMONARY-DYSPLASIA}

M Tana ${ }^{1}$, S Boccacci $^{1}$, V Vendettuoli ${ }^{1}$, G Giuratrabocchetta ${ }^{1}$, A De Cunto ${ }^{1}$, C Tirone ${ }^{1}$, E Capoluongo $^{2}$, C Santonocito ${ }^{2}$, F Vendittelli $^{2}$, P Concolino ${ }^{2}$, M P De Carolis ${ }^{1}$, G Vento ${ }^{1}$, F Ameglio ${ }^{2}$, C Romagnoli

${ }^{I}$ Department of Pediatrics, Division of Neonatology, Catholic University of The Sacred Heart, Rome, Italy ${ }^{2}$ Department of Biochemistry and Clinical Biochemistry, Catholic University of The Sacred Heart, Rome, Italy

Background and Aims: Besides old classification of Chronic Lung Disease (CLD) - O2-dependency at 36 weeks of postmenstrual age-, recently a new definition for CLD has been proposed: New BronchopulmonaryDysplasia (BPD), which uses total duration of $\mathrm{O} 2$ supplementation and positive pressure requirements to delineate three degrees of severity: mild, moderate and severe, according to the respiratory status at 36 weeks post-menstrual age. Objective of this report is to analyse the balance of serum pro-inflammatory and profibrotic/angiogenic cytokine concentrations in relation to CLD or New BPD definition.

Methods: Thirty-one preterm neonates with a gestational age of 24 to 29 weeks, previously enrolled in a randomized clinical trial to compare the effects of high-frequency oscillatory ventilation versus intermittent mandatory ventilation in terms of pulmonary mechanics and lung cytokines, were actually studied to evaluate their serum cytokine concentration. At days 1, 3 and 5 after birth, serum samples were collected until extubation to detect the levels of three pro-inflammatory cytokines (IL-6, IL-8, MCP-1) plus four profibrotic/angiogenic cytokines (IL-10, TGF- $\beta 1$, VEGF, PDGF-BB), and correlation was made with old CLD or New BPD development.

Results: Ventilation treatments were homogeneously distributed between the groups and did not interfere with the results presented here. Old CLD development, mainly corresponding to the moderate/severe forms of New BPD, was associated with increased proinflammatory and profibrotic/angiogenic cytokines, while mild forms of New BPD were only characterized by increases of pro-fibrotic/angiogenic cytokines.

Conclusions: These data suggest a different balance of two pathogenic mechanisms in different phases of the disease.

\section{TNF-ALPHA-308 AND TGF-BETA1-800 POLYMORPHISMS AND RISK OF BRONCHOPULMONARY DYSPLASIA IN PRETERM NEWBORNS}

A De Cunto ${ }^{1}, \mathrm{~S}_{\text {Boccacci }}{ }^{1}, \mathrm{~V}$ Vendettuoli ${ }^{1}$, G Giuratrabocchetta ${ }^{1}$, M Tana $^{1}, \mathrm{C}_{\text {Tirone }}{ }^{1}$, E Capoluongo $^{2}$, C Santonocito ${ }^{2}$, P Concolino $^{2}$, F Vendittelli ${ }^{2}$, M P De Carolis ${ }^{1}$, G Vento ${ }^{1}$, C Romagnoli ${ }^{1}$

${ }^{I}$ Division of Neonatology, Department of Pediatrics, Università Cattolica S. Cuore, Rome, Italy
${ }^{2}$ Departments of Biochemistry and Clinical Biochemistry, Università Cattolica S. Cuore, Rome, Italy

Background and Aims: TNF-alpha -308 (G/A) has been associated with increased levels of TNF-alpha, involved in the BPD development. TGF-beta1-800 (G/A) is in linkage disequilibrium with TGF-beta1-509, associated with increased production of TGF-beta1, a marker of fibrotic response in BPD.

Methods: PCR analyses were performed with DNA extracted from whole blood samples on 55 newborns with GA $<32$ wks. 27 newborns with BPD (GA=26.7+/$1.8 \mathrm{wks} ; \mathrm{BW}=821+/-223 \mathrm{~g})$ and 28 controls $(\mathrm{GA}=29.5+/-$ 1.7wks; BW=1274+/-358g p $<0.0001$ ) were studied.

Results: The AA and GA TNF-alpha-308 and the GA TGF-beta1-800 genotypes occurred more frequently in infants with $\operatorname{BPD}(p=0.58$ and $p=0.54$ respectively). Subdividing newborns according to their genotype, duration of mechanical ventilation and O2-therapy was longer in the group TNF-alpha-308 AA/GA and TGFbeta1-800 GA respect to GG groups (Tab 1-2).

Conclusions: TNF-alpha-308 and TGF-beta1-800 polymorphisms may play a role in susceptibility to VILI.

Tab 1
\begin{tabular}{|l|l|l|l|}
\hline \multicolumn{1}{|c|}{ TNF $-\alpha$} & GG & $\begin{array}{l}\text { AA/GA } \\
\text { rF } 32\end{array}$ & P \\
\hline GA (wks) & $282 \pm 2.1$ & $28 \pm 2.3$ & 0.81 \\
BW(g) & $1066 \pm 279$ & $1041 \pm 434$ & 0.80 \\
Mecharical Vertilation (h) & $46(0-873)$ & $119(0-1176)$ & 0.14 \\
Oxygen therapy (h) & $142(9-3900)$ & $435(0-2528)$ & 0.74 \\
Survival (\%) & $22(96)$ & $30(94)$ & 1 \\
\hline
\end{tabular}

Tab 2
\begin{tabular}{|l|ll|l|}
\hline TGF- $\beta 1$ & GG & GA & P \\
& rF41 & rF 14 & \\
\hline GA (wks) & $28 \pm 2.2$ & $28.4 \pm 2.4$ & 0.51 \\
BW (gx) & $1059 \pm 359$ & $1029 \pm 431$ & 0.79 \\
Mecharical Vertilation (h) & $79(0-1176)$ & $136(2-873)$ & 0.33 \\
Oxygen therapy (h) & $2070-3960)$ & $459(16-2720)$ & 0.25 \\
Survival (\%) & $39(95)$ & $13(93)$ & 1 \\
\hline
\end{tabular}
Median vahes (ranze) or n(\%)

\section{THE ALPHA-DEFENSINS IN BALF OF PRETERM INFANTS STUDIED WITH PROTEOMIC APPROACH}

S Boccacci ${ }^{1}$, V Vendettuoli ${ }^{1}$, M Tana ${ }^{1}$, A De Cunto ${ }^{1}$, G Giuratrabocchetta $^{1}, \mathrm{C}$ Tirone $^{1}$, E Capoluongo ${ }^{2}$, R Inzitari ${ }^{2}$, M Castagnola ${ }^{2}$, M P De Carolis ${ }^{1}, \mathrm{G}$ Vento ${ }^{1}$, C Romagnoli ${ }^{1}$

${ }^{I}$ Department of Pediatrics, Division of Neonatology, Catholic University of Sacred Heart, Rome, Italy ${ }^{2}$ Department of Biochemistry and Clinical Biochemistry, Catholic University of Sacred Heart, Rome, Italy

Background and Aims: There is no evidence about the production of neutrophilic alpha-defensins (HNP 1-4) in preterms BALF and their role as anti-inflammatory markers.

Methods: We collected 31 BALF samples in 2nd and 3rd day of life from 21 ventilated neonates with RDS and $\mathrm{GA}<35$ weeks. In 10 patients a second sample was collected in 5th or 7th day. The mass spectrometry ESIION TRAP plus HPLC have been used for qualitative and semiquantitative analysis (Massa Area with EIC) of the alpha-defensins. 
Results: The studied population was divided in 2 groups: one of 8 neonates (GA: 27,9+/-4,1 weeks; BW: $1192+/-760 \mathrm{~g}$ ) with pneumonia in the first week of life and one of 13 controls (GA: 28,5+/-3,2 weeks; BW: $1190+/-599 \mathrm{~g})$. The alpha-defensins's sum and the number of PMN have been found significantly higher in the pneumonia group (Fig.1). Especially in the pneumonia group, exists a positive significant correlation between the absolute number of PMN in BALF and the levels corresponding to semiquantitative sum of defensins (Fig.2).

Conclusions: These data confirm the prevalent neutrophilic origin of alpha-defensins and the capacity of response of immune system during infections in preterm infants.

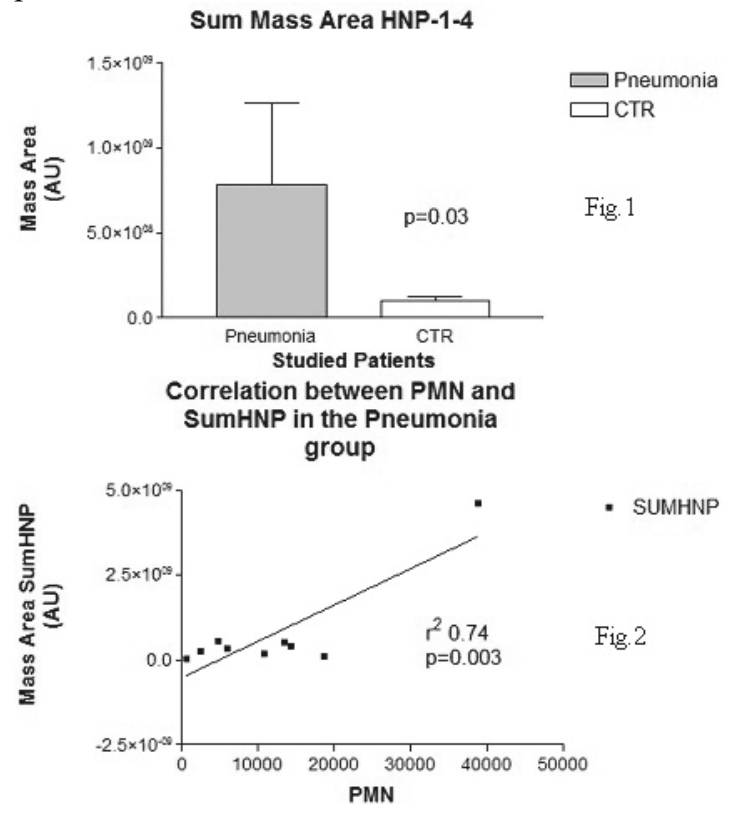

\section{RESEARCH IN MEDICINES FOR CHILDREN: THE NEW REGULATION AND THE ROLE OF THE EMEA}

\section{Brasseur}

University Children's Hospital Queen Fabiola, Brussels, Belgium and Chair of CHMP/Paediatric Expert Group at the European Medicines Agency (EMEA), London, $U K$

The European Union has since June 2006 a Regulation on 'Better Medicines for Children'. Currently the provisions of the text await final implementation: the new system should be operational in Q2 of 2007 at the latest. The purposes are to facilitate and increase the development of medicines for children, promote a high quality of research, consolidate the ethical approach of clinical trials. The system hits inevitably new products at first submission or if claiming a new indication, a new route of administration, a new dosage or a new pharmaceutical form. The system is optional for unpatented drugs. Those still patented get a 6 months extension of their protection, the others a 10 years paediatric registration if they comply with the paediatric investigational plan (PIP). The PIP intents to assure availability of data and an age appropriate formulation. PIP means a research and development programme intended to obtain a paediatric indication. PIP will specify the timing and measures proposed to assess the quality, safety and efficacy in all subsets of the paediatric populations possibly concerned. The scope of the PIP includes the formulation(s), the preclinical safety, the clinical efficacy, the pharmaco-vigilance and any appropriate risk management plan. The timing of the PIP should be precised. In specific circumstances a PIP will be waived (likelihood of inefficacy, safety concerns, conditions occurring in adults only, or some ages groups might be waived [neonates...]). The PIP will be assessed by a Paediatric Committee (PECO) where each member state will be represented, as will be healthcare professionals and patients/parents (groups). The PECO will assess the proposed PIP and an agreement (or deferral/waiver) should be reached before the submission of the adult (part of the) dossier. If no discussion of PECO is included in a new application, the adult dossier cannot be validated. The PECO will also define compliance criteria that the company should fulfil before it can be decided that the requirements of the PIP have been met. This compliance will condition the 6 months extension, whatever the final results of the studies conducted. A failed PIP can thus lead to the incentives, provided this 'negative' information is incorporated in the product information. In order to facilitate the conduct of the PIP the European Medicines Agency (EMEA) has set a number of measures: guidance on quality assessments of paediatric formulations, guidance on preclinical toxicity studies in juvenile animals, proposals on PK-PD studies and some reflection on the paediatric specificity of pharmacovigilance and risk minimisation.

\section{A BLINDED RANDOMIZED CLINICAL TRIAL OF CLONIDINE IN COMBINATION WITH DILUTED TINCTURE OF OPIUM (DTO) VERSUS DTO ALONE FOR NEONATAL ABSTINENCE SYNDROME (NAS)}

A G Agthe ${ }^{1,2}, \mathrm{~K}$ B Mathias ${ }^{3}$, C W Hendrix ${ }^{2}$, L Jansson ${ }^{2,4}$, M Yaster', T R Roark ${ }^{2}$, E B Gauda ${ }^{2,3,4}$

${ }^{1}$ University of Maryland Medical Center, Baltimore, MD, USA, ${ }^{2} J$ ohns Hopkins Medical Institution, Baltimore, MD, USA, ${ }^{3}$ Mt. Washington Pediatric Hospital, Baltimore, MD, USA ${ }^{4}$ Johns Hopkins Bayview Medical Center, Baltimore, MD, USA

Background and Aims: Opioids are the current standard for treating moderate and severe NAS. Clonidine is used widely as a non-opioid alternative for managing opioid withdrawal in adults. To determine whether clonidine in combination with tapering doses of DTO will significantly reduce the length of therapy and the amount of opioids used in infants with NAS.

Methods: 80 Infants with history of maternal methadone or heroin use during pregnancy, having 2 consecutive modified Finnegan Scores (MFS) $\geq 9$ were randomized to receive clonidine (Duraclon, $1 \mathrm{mcg} / \mathrm{kg}$ $\mathrm{q} 4 \mathrm{~h})$ or placebo. Excluded infants were $<35$ weeks gestational age, $<5$ th $\%$ birthweight, breastfeeding. DTO dose was increased in 6 steps from $0.2 \mathrm{ml} \mathrm{q} 4 \mathrm{~h}$ up to $0.9 \mathrm{ml} \mathrm{q} 3 \mathrm{~h}$ if 2 consecutive MFS $\geq 9$. Infants with 2 consecutive MFS $\geq 9$ on $0.9 \mathrm{ml} \mathrm{q} 3 \mathrm{~h}$ were classified as 
treatment failure. Infants were monitored for hypotension, bradycardia, and oxygen desaturations.

Results: 40 infants were assigned to two groups, that were similar with regard to maternal and infant demographic factors. The median length of therapy was $23 \%$ shorter for infants in the clonidine group compared to placebo ( $11.5 \pm 1.8$ vs. $14.9 \pm 1.2$ days, logrank, $\mathrm{p}<0.05)$. The maximum daily dose of DTO tended to be less $(2.4 \pm 2.4 \mathrm{ml}$ vs. $3.4 \pm 2.7 \mathrm{ml})(\mathrm{P}=0.10)$ in the clonidine group. The placebo group had more seizures (3 vs. 0) and a higher rate of treatment failure (5 vs. 0 ). Other adverse events (hypertension, hypotension, bradycardia, desaturations) were not significantly different.

Conclusions: Clonidine in combination with DTO is safe and more efficacious than DTO alone for the treatment of moderate to severe NAS. Supported by NIDA\#IR21DAO16288.

\section{ELECTRON MICROSCOPY FINDINGS OF RENAL GLOMERULI AND TUBULES IN RAT PUPS TREATED WITH INDOMETHACIN AND IBUPROFEN}
A Kent ${ }^{1,5}, \mathrm{~L} \mathrm{Maxwell}^{2}, \mathrm{M} \mathrm{Koina}^{2}, \mathrm{M} \mathrm{C} \mathrm{Falk}{ }^{3,5}$, D Willenborg ${ }^{4,5}, \mathrm{~J}$ E Dahlstrom ${ }^{2,5}$
${ }^{1}$ Dept of Neonatology, The Canberra Hospital, ${ }^{2}$ Dept of Anatomical Pathology, ${ }^{3}$ Dept of Renal Medicine, The Canberra Hospital, Woden, ${ }^{4}$ Neurosciences Research Unit, Australian National University, ${ }^{5}$ Dept of
Paediatrics, Australian National University Medical School, Canberra, Australia

Background and Aims: Indomethacin and ibuprofen are drugs that are commonly used to treat a patent ductus arteriosus in premature infants. These drugs are known to cause acute renal failure in up to $25 \%$ of infants treated. Infants are usually treated between 24-28 weeks gestation at a time when glomerulogenesis is still occurring. To determine whether there are renal glomerular and tubular changes found on electron microscopy (EM) following administration of indomethacin and ibuprofen to rat pups.

Methods: Rat pups were administered indomethacin $0.1 \mathrm{mg} / \mathrm{kg}$ or $0.2 \mathrm{mg} / \mathrm{kg}$ or ibuprofen $5 \mathrm{mg} / \mathrm{kg}$ or 10 $\mathrm{mg} / \mathrm{kg}$ intraperitoneally for 5 days from day 1 of life (equivalent to 24-26 weeks gestation in the human). Control groups consisted of no drug administration and normal saline administered intraperitoneally. The pups were sacrificed using carbon dioxide at 14 days of age, equivalent to term in the human. The systemic circulation was perfused with heparinized saline, papaverine, normal saline and fixed with $2.5 \%$ glutaraldehyde in $0.1 \mathrm{~mol} / \mathrm{L}$ phosphate buffer. Three sections were taken for EM examination.

Results: Six pups were included in each group. There were no glomerular or tubular anomalies in either the control or the saline group. Mitochondrial changes were seen in the tubules in both the indomethacin and ibuprofen treated animals. Changes to the foot processes and basement membrane of the glomeruli were seen in both the indomethacin and ibuprofen treated groups.

Conclusions: Indomethacin and ibuprofen affect both glomeruli and tubules in the developing kidney.

\section{ADENOSINE FOR THE MANAGEMENT OF PERSISTENT PULMONARY HYPERTENSION OF THE NEWBORN?}

T R Thiruchelvam, A J Petros, C M Pierce

Paediatric Intensive Care Unit, Great Ormond Street Hospital, London, UK

Background and Aims: Persistent pulmonary hypertension of the newborn (PPHN) causes significant morbidity and mortality. Nitric oxide (iNO) reduces the need for ECMO in these infants. Adenosine is a potent pulmonary vasodilator that has also been used in PPHN. We reviewed our experience of adenosine in this population.

Methods: Retrospective review of case notes of neonates admitted to our intensive care between Jan 2001 - Jan 2006 with clinical and echocardiographic evidence of PPHN. Clinical demographics and therapy were recorded.

Results: 192 cases of PPHN were admitted. All received iNO, 26 were given adenosine $(50 \mu \mathrm{g} / \mathrm{kg} / \mathrm{min})$. The median age of the whole group was $1 \mathrm{~d}(1-2)$ and the adenosine subset $1 \mathrm{~d}(1-2)$ : weights were $3.4 \mathrm{~kg}(3.0-3.7)$ and $3.3 \mathrm{~kg}(3.3-3.37)$ respectively. Oxygenation index (OI) was 54 (31.8-62) for the adenosine subset $(n=26)$ compared to $30(16.4-46.8)$ for the whole group $(n=192)$. Adenosine recipients referred for ECMO $(n=10)$ had an OI of 65 (57-80) with no deaths vs. those not given adenosine and referred to ECMO $(n=39), 48.5$ (27-62) $(\mathrm{p}=0.06)$, with 8 deaths $(\mathrm{p}=0.18$ Fisher's exact $)$. Those not referred to ECMO $(n=16)$ had an OI of 42.5 (28.5-54) and 3 deaths vs. those who did not get adenosine and not referred to ECMO $(n=127), 24$ (14-40) $(\mathrm{p}=0.014)$ with 15 deaths $(\mathrm{p}=0.42)$.

Conclusions: The group receiving adenosine had a higher OI before needing ECMO, without a higher mortality rate. Those not needing ECMO also had a higher OI. Adenosine reduced the need for ECMO. We need a randomised controlled trial to elucidate the role of adenosine in PPHN.

\section{USE AND MISUSE OF CORTICOSTEROIDS IN THE PEDIATRIC INTENSIVE CARE UNIT}

\section{Mul, J J Verhoeven, K F M Joosten}

Department of Pediatric Intensive Care, Erasmus MCSophia, Rotterdam, The Netherlands

Background and Aims: Corticosteroid (CS) treatment is used for several indications in the pediatric intensive care unit (PICU). However, consensus on which CS to use and/or its dose is frequently lacking. Given the well known side-effects of CS on several metabolic and endocrine pathways careful use of CS should be considered.

Methods: Retrospective analysis of the use of CS in our PICU during a random 11-months period in 2005. Indications, type of CS and dosing values were collected.

Results: In 109 of 520 PICU admissions (21\%) CS were used. Indications are summarised in the table. Sixty-one percent of patients were male, mean age was 4.0 yrs (SD 4.8). Median cumulative dosage $(\mathrm{mg} / \mathrm{kg})$ during 
admission was 3,2 for prednisone, 1,1 for dexamethasone and 5,8 for hydrocortisone. For all CS, a wide range in cumulative dose was observed, mainly skewed to the higher values: ranges were 0,5-11,2 $\mathrm{mg} / \mathrm{kg}$ for prednison, $0,2-7,7 \mathrm{mg} / \mathrm{kg}$ for dexamethasone and $1,4-30,4 \mathrm{mg} / \mathrm{kg}$ for hydrocortisone.

Conclusions: In $21 \%$ of PICU admissions, CS were used in various doses; the choice of CS for specific indications were not uniform. Cumulative CS doses differ considerably within identical indications, and reach values with higher risk for occurrence of side-effects in metabolic or endocrine pathways.

\begin{tabular}{|c|c|c|c|c|}
\hline Diagnasis/indication & $\begin{array}{l}\text { Total } \\
(n=109)\end{array}$ & $\begin{array}{l}\text { Prechisone } \\
(n=45)\end{array}$ & $\begin{array}{l}\text { Dexamethasone } \\
(n=48)\end{array}$ & $\begin{array}{l}\text { Hydrocortisone } \\
(n=17)\end{array}$ \\
\hline $\begin{array}{l}\text { Respiratory } \\
\text { insufficiency / asthma }\end{array}$ & 36 & 22 & 14 & 0 \\
\hline Sepsis & 12 & 3 & 4 & 5 \\
\hline Upper respiratory tract & 30 & 8 & 17 & 5 \\
\hline Oncology & 8 & 2 & 3 & 3 \\
\hline Other & 23 & 9 & 10 & 4 \\
\hline
\end{tabular}

\section{GOOD CLINICAL OUTCOME AFTER ACUTE POISONINGS IN CHILDREN, A TWO-YEAR PROSPECTIVE STUDY}

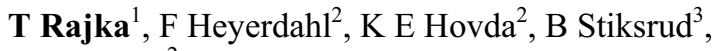
D Jacobsen ${ }^{2}$

${ }^{I}$ Department of Paediatric Intensive Care, Ullevaal University Hospital, Oslo, Norway, ${ }^{2}$ Department of Acute Medicine, Ullevaal University Hospital, Oslo, Norway, ${ }^{3}$ Oslo Walk-In Casualty Center, Oslo, Norway

Background and Aims: The incidence and types of acute poisonings in children changes over time. This study was designed to evaluate the clinical course and outcome of poisonings among children in Oslo, and findings were compared to a similar study from 1980 .

Methods: Prospective observational study including all children $<16$ years of age with a main diagnosis of acute poisoning admitted to hospital or the Central outpatient clinic in Oslo during two years.

Results: 234 episodes (226 children) of acute poisoning were included at outpatient clinic (97) or hospital (137). Annual incidence was $1.2 \%$, significantly lower than in 1980 ( $\mathrm{p}<0.001)$. Toxic agents were pharmaceuticals $(42 \%)$, domestic products $(30 \%)$ and plants or mushrooms $(16 \%)$ in younger children; older children ingested ethanol (51\%) and tablets (47\%). No active treatment was needed in $85 \%$ (outpatient clinic) and $26 \%$ (hospital). As in 1980, charcoal (40\%), gastric lavage $(18 \%)$, and emetics $(10 \%)$ were the most common hospital treatment, though in reversed order. Antidotes were only given to hospitalized children $(15 \%) .9 \%$ of all children were comatose. Complications were seen in $17 \%$, no differences regarding age, gender or place of treatment. For details about older vs. younger children, see table 1. All children survived without sequelae.

Conclusions: Clinical outcome after acute poisonings in children was good. Incidence was reduced since 1980. Older children present with more severe poisonings than younger.

\begin{tabular}{lcc} 
Table 1 & \multicolumn{1}{c}{ - Years } & $>$ 8 Years \\
\hline Female / Male & $57 / 84$ & $62 / 31$ \\
Coma on admission & $2(1 \%)$ & $20(22 \%)$ \\
Outpatient clinic/ Hospital & $50 / \mathbf{9 1}$ & $47 / \mathbf{4 6}$ \\
$>1$ day in hospital & $15(16 \%)$ & $15(33 \%)$ \\
ICU & $3(3 \%)$ & $13(28 \%)$ \\
Treated with antidote & $5(6 \%)$ & $15(33 \%)$ \\
Treatment (> observation and antidote) & $65(71 \%)$ & $34(74 \%)$ \\
Complications & $14(15 \%)$ & $10(22 \%)$
\end{tabular}

\section{ORAL VERSUS INTRANASAL MIDAZOLAM PREMEDICATION FOR INFANTS DURING ECHOCARDIOGRAPHIC STUDY}

\author{
S V Yildirim ${ }^{1}$, B E Guc ${ }^{2}, \mathrm{~N} \mathrm{Bozdogan}^{3}$, E Cekilmez ${ }^{2}$, \\ $\mathrm{T} \mathrm{Cemil}^{2}, \mathrm{~K}$ Tokel $^{4}$ \\ ${ }^{1}$ Department of Pediatric Cardiology, Baskent \\ University, Adana Teaching and Medical Research \\ Center, Adana, Turkey, ${ }^{2}$ Department of Pediatrics, \\ Baskent University, Adana Teaching and Medical \\ Research Center, Adana, Turkey, ${ }^{3}$ Department of \\ Anaesthesiology, Baskent University, Adana Teaching \\ and Medical Research Center, Adana, Turkey \\ ${ }^{4}$ Department of Pediatric Cardiology, Baskent \\ University, Faculty of Medicine, Adana, Turkey
}

Background and Aims: Movement and anxiety during echocardiographic study may reduce the reliability and compromise the quality of echocardiographic images; sedation is essential component for the procedure especially in infants. Purpose of this study: To evaluate the acceptance and effectiveness of intranasal vs. oral midazolam for infants during the transtorasic echocardiography.

Methods: A randomized, prospective blinded placebo controlled study. Seventy-four infants, between the ages of 6 months to 3 years, presenting for elective echocardiographic study were divided 3 groups. Oral midazolam group (group 1) received $0.4 \mathrm{mg}-\mathrm{kg}-1$ of injectable midazolam mixed with an equal volume of strawberry fruit, Intranasal midazolam group (group 2) received $0.2 \mathrm{mg}-\mathrm{kg}-1$, and placebo group (group 3) received oral strawberry fruit or intranasal serum physiologic. A blinded echocardiographer assessed the sedation and echocardiographer's satisfaction scores for each child, and score for the easiness of administration was recorded by the nurse.

Results: Intranasal route was more willingly accepted by infants than oral route $(\mathrm{P}<0.001)$. There was not significant differences between the group 1 and group 2 for the effect of sedation $(\mathrm{P}=0.952)$, but a very significant differences between the groups of 1 and 3,2 and 3 for the echocardiographer's satisfaction $(\mathrm{P}<0.001$ and $\mathrm{P}<0.005$, respectively).

Conclusions: Although there was not difference of sedation score between oral and nasal route, intranasal midazolam was well accepted than oral midazolam by infants, and proved that under the effect of midazolam, echocardiography was studied easily and more reliable compared the control group. 
DOES MATERNAL ALLOPURINOL (ALLO) ADMINISTRATION DURING PERINATAL HYPOXIC-ISCHEMIA (P-HI) TRANSFER THE PLACENTA IN SHEEP AND HUMANS?

M J N L Benders ${ }^{1}$, H L Torrance ${ }^{1}$, J B Derks ${ }^{1}$, M Oudijk ${ }^{1}$, C M Rademaker ${ }^{2}$, F Groenendaal ${ }^{1}$, D A Giussani ${ }^{3}, \mathrm{G} \mathrm{H}$ Visser ${ }^{1}, \mathrm{~F}$ van $\mathrm{Bel}^{1}$

${ }^{I}$ Department of Perinatology, University Medical Center Utrecht, Wilhelmina Children's Hospital, Utrecht, The Netherlands, ${ }^{2}$ Department of Pharmacy, University Medical Center Utrecht, Wilhelmina Children's Hospital, Utrecht, The Netherlands ${ }^{3}$ Department of Physiology, University of Cambridge, $U K$

Background and Aims: ALLO inhibits xanthine oxidase (XO) and thereby prevents formation of toxic superoxide-radicals during $\mathrm{P}-\mathrm{HI}$ and early reperfusion. Postnatal ALLO does not seem neuroprotective, probably because treatment is started too late to prevent the XO-surge. Antenatal ALLO-administration to mothers during P-HI may provide neuroprotection. This study evaluated placental passage after ALLOadministration to mothers during P-HI through measurement of ALLO and oxypurinol (OXY) concentrations in mothers and hypoxic fetuses. In a previous study therapeutic levels $(>4 \mathrm{mg} / \mathrm{L})$ were reached in newborns after oral ALLO-administration of $600 \mathrm{mg}$ to women in uncomplicated labour (Boda 1999).

Methods: Pregnant ewes at 0.8 of gestation received a single IV ALLO-dose of $900 \mathrm{mg}$ during intermittent umbilical cord clamping. Women in labour with GA $>36$ wks and signs of fetal distress received a single ALLO-dose of $500 \mathrm{mg}$ IV or $600 \mathrm{mg}$ orally. ALLO and OXY concentrations were measured in maternal and umbilical cord (UC) blood.

Results: Therapeutic levels were reached in the ewe and sheep fetus. In humans iv-administration caused therapeutic levels in the mother, whereas UC levels were subtherapeutical. After oral administration levels were extremely low.

Conclusions: During P-HI high dosed ALLO passes the sheep placenta. However, iv-ALLO-administration in humans during P-HI leads to suboptimal fetal levels (unlike in fetuses without P-HI), probably because of a compromised placental transfer. We conclude therapeutic UC levels may be reached by increasing the iv-dose and oral administration is not indicated in case of P-HI.

\begin{tabular}{|c|c|c|c|c|c|c|}
\hline \multicolumn{7}{|c|}{ ALLO and $O X Y$ concentration $(\mathrm{mg} / \mathrm{l})$ mean $\leq S D$ at $\pm 2 \mathrm{~h}$ post-infusion } \\
\hline & \multirow{2}{*}{\multicolumn{2}{|c|}{\begin{tabular}{|c|} 
SHEEP \\
$\mathrm{IV}(\mathrm{n}=6)$ \\
at $\pm 2 \mathrm{~h}$ post-infusion
\end{tabular}}} & \multicolumn{4}{|c|}{ HUMANS } \\
\hline & & & \multicolumn{2}{|c|}{$\begin{array}{c}\text { IV }(n=16) \\
\text { at } 22 h \text { post-infusion }\end{array}$} & \multicolumn{2}{|c|}{$\begin{array}{c}\text { ORAL }(n=2) \\
\text { at : } 1 \mathrm{~h} \text { post administration }\end{array}$} \\
\hline & Ewe & UC & Mother & UC & Mother & UC \\
\hline LLO & $5.1 \pm 5.5$ & $3.8 \div 1.1$ & $3.8 \pm 2.5$ & $1.6 \div 1.7$ & $0.7 \div 0.9$ & $1.5: 2.1$ \\
\hline OXY & $10.0 \pm 3.5$ & $1.0=0.3$ & $3.6 \pm 1.3$ & $1.9 \pm 1.2$ & $0.6 \div 0.8$ & $1.2: 1.6$ \\
\hline
\end{tabular}

\section{EFFECT OF PROPOFOL ON SEIZURE LIKE PHENOMENA AND EEG ACTIVITY IN CHILDREN WITH EPILEPSY VS. MENTAL RETARDATION}

L Gortner ${ }^{1,2}$, S Meyer ${ }^{1}$, M G Shamdeen ${ }^{2}$, B Kegel ${ }^{2}$, T Mencke ${ }^{3}$, S Gottschling ${ }^{2}$, U Grundmann ${ }^{3}$
${ }^{1}$ Department of Pediatrics and Pediatric Intensive Care Medicine, University Hospital of Saarland, Homburg, Germany, ${ }^{2}$ Section of Neuropediatrics, University Hospital of Saarland, Homburg, Germany, ${ }^{3}$ Department of Anesthesiology, University Hospital of Saarland, Homburg, Germany

Background and Aims: Propofol (PROP) is widely used for sedation in children. Until today, there is an ongoing debate whether PROP exhibits pro- or anticonvulsant effects, and whether it should be used in patients with epilepsy. This prospective study was done to assess seizure like phenomena (SLP) and EEG changes in children with epilepsy receiving PROP sedation for MRI studies, and to compare these data to non-epileptic children.

Methods: We prospectively assessed the occurrence of SLP and the effects of PROP on the EEG in 25 children with epilepsy and 25 children with mental retardation undergoing sedation for MRI studies.

Results: In both groups, no child demonstrated SLP of epileptic origin during and after PROP sedation. Immediately after stopping PROP, characteristic EEG changes in the epilepsy group consisted of increased beta wave activity (23/25 children), and suppression of pre-existing theta rhythms (11/16 children). In addition, 16 of 18 children with epilepsy and documented EEG seizure activity demonstrated suppression of spike-wave patterns after PROP sedation. In all 25 children with mental retardation an increase in beta wave activity was seen. Suppression of theta rhythms occurred in 11 of 12 children at the end of the MRI study. In no child of either group, a primary occurrence, or an increase in spike-wave patterns was seen following PROP administration. The occurrence of beta wave activity (mental retardation and epilepsy group) and suppression of spike-wave patterns (epilepsy group) were transient, and disappeared after 4 hours.

Conclusions: This study demonstrates characteristic, time-dependent EEG patterns induced by PROP in children with epilepsy and mental retardation. Our data support the concept of PROP being a sedative-hypnotic agent with anti-convulsant properties.

\section{CONCEPTS OF CARDIAC DEVELOPMENT}

\section{A F M Moorman, V M Christoffels}

Department of Anatomy and Embryology, Academic Medical Centre, University of Amsterdam, The Netherlands

In this presentation we discuss the formation of the synchronously contracting chambered heart from a peristaltically contracting linear heart tube. It is proposed that members of the T-box family of transcription factors play a crucial role in the formation of the building plan of the heart. Tbx 5 confers craniocaudal polarity to the heart tube, whereas $\mathrm{Tbx} 2$ and Tbx 3 prevent the cardiac inflow tract, atrioventricular region, outflow tract, as well as the cardiac inner curvatures from chamber differentiation. With the exception of the outflow tract that becomes incorporated into the ventricles, these regions contribute to the cardiac conduction system. Tbx1 specifically is involved 
in the formation of the outflow tract, whereas Tbx18 is involved in the formation of the systemic venous tributaries. One of the most fascinating aspects in the formation of the heart is the very early development of the electrical patterning as can be registered by the ECG, which is the registration of the rhythmic waves of depolarising activity over the cardiac muscle. In the mature heart the conduction system is responsible for the rhythmic excitations and contractions. However, in chicken embryos a sinusoidal type of ECG can already be derived from the linear heart tube stages at about two days of development, and less than one day later when chamber formation has just been initiated, an adult type of ECG can be monitored. The question can thus justifiably be asked whether the presence of an adult type of ECG in these early embryonic hearts implies the presence of a conduction system. Generally, the conduction system is defined as the system of specialized myocardial tissues responsible for initiation and propagation of the sinus impulse. If we accept this functional definition, then the early embryonic heart has a conduction system, which is already in place, because there is an adult type of ECG. On the other hand, if we would apply strict anatomical and histological definitions, the embryonic heart undeniably lacks a conduction system. This field of tension concerning the recognition of the conduction system using physiological versus anatomical / histological criteria has led to many controversies in the field.. In this contribution we discuss the specification of the embryonic areas that will become chamber and those areas that will not form chambers and participate in the formation of the distinct components of the conduction system. Another intriguing aspect is how during evolution the cardiac components of the pulmonary circulation evolved. Recently, a novel cardiac field, the so-called secondary heart-forming field, has been described. This field is localized centrally from the classic cardiac crescent on the embryonic disc. We discuss the view that the so-called second heart-forming field contributes to the cardiac components of the evolutionary new pulmonary circulation, being the right ventricle and outflow tract as well as the mediastinal myocardium, which gives rise to the atrial septum and the dorsal wall of the left atrium. Both types of myocardium are derived from the mesenchyme that is localized centrally from the classic cardiac crescent.

\section{CARDIAC OUTPUT MEASUREMENT USING A MODIFIED CARBON DIOXIDE FICK METHOD: A VALIDATION STUDY IN VENTILATED LAMBS}

W P de Boode ${ }^{1}$, J G van der Hoeven ${ }^{2}$, O Daniëls ${ }^{3}$, J C W Hopman ${ }^{4}$, K D Liem ${ }^{1}$

${ }^{l}$ Department of Neonatology, Radboud University Nijmegen Medical Centre, Nijmegen, The Netherlands ${ }^{2}$ Department of Intensive Care, Radboud University Nijmegen Medical Centre, Nijmegen, The Netherlands

${ }^{3}$ Department of Pediatric Cardiology, Radboud University Nijmegen Medical Centre, Nijmegen, The Netherlands, ${ }^{4}$ Department of Clinical Physics, Radboud University Nijmegen Medical Centre, Nijmegen, The Netherlands
Background and Aims: Cardiac output can be measured using a modified carbon dioxide Fick (mCO2F) method. We performed a validation study comparing mCO2-F method derived cardiac output (QmCO2F) with measured pulmonary blood flow by an ultrasonic flowprobe (Qufp).

Methods: The study was approved by the Ethical Committee on Animal Research and performed in 7 random-bred newborn lambs $(2.9-6.4 \mathrm{~kg})$. The $\mathrm{mCO} 2-$ F method is based on the principle, that in a steady state carbon dioxide production equals pulmonary carbon dioxide exchange. Cardiac output was manipulated by creating hemorrhagic hypotension. Bias, defined as QmCO2F minus Qufp, was calculated for each measurement. For every venous sampling site mean bias with standard deviation (sd), 95\% confidence intervals and limits of agreement (LOA) were calculated. LOA are defined as mean bias $\pm 1.96 \mathrm{sd}$.

Results: The table shows the mean bias in cardiac output in $1 / \mathrm{min}$ for the three different venous sample sites (right atrium, superior vena cava and inferior vena cava).

Conclusions: Measurement of cardiac output with the $\mathrm{mCO} 2-\mathrm{F}$ method is reliable in newborn lambs and can be used for trend monitoring. Venous blood sampling from the right atrium, superior vena cava or inferior vena cava can be used for this measurement. The $\mathrm{mCO} 2-\mathrm{F}$ method is a promising technique that can be used to measure cardiac output in critically ill newborns.

\begin{tabular}{|c|c|c|c|c|}
\hline \multicolumn{5}{|c|}{ Bias in cardiac output in I/min $\left(\mathbf{Q}^{\mathrm{mCO2F}}-\mathbf{Q}^{\text {ufp }}\right)$} \\
\hline $\begin{array}{c}\text { Sampling } \\
\text { site }\end{array}$ & $\begin{array}{c}\text { Mean } \\
\text { Bias }\end{array}$ & $95 \%-C l$ & SD & LOA \\
\hline RA & -0.082 & $\begin{array}{c}-0.130 \text { to } \\
-0.035\end{array}$ & 0.123 & $\begin{array}{c}-0.323 \text { to } \\
0.159\end{array}$ \\
\hline SVC & -0.085 & $\begin{array}{c}-0.138 \text { to } \\
-0.032\end{array}$ & 0.125 & $\begin{array}{c}-0.330 \text { to } \\
0.160\end{array}$ \\
\hline IVC & -0.183 & $\begin{array}{c}-0.227 \text { to } \\
-0.139\end{array}$ & 0.116 & $\begin{array}{c}-0.410 \text { to } \\
0.044\end{array}$ \\
\hline
\end{tabular}

\section{THE USE OF PRO B-TYPE NATRIURETIC PEPTIDE AS A MEASURE OF LEFT VENTRICULAR FUNCTION IN PRETERM NEONATES}

A F El-Khuffash ${ }^{1}, \mathrm{M} \mathrm{Ambruso}^{2}, \mathrm{M} \mathrm{Culliton}^{2}, \mathrm{~J} \mathrm{Loyta}^{3}$, K Walsh ${ }^{4}$, E J Molloy ${ }^{1}$

${ }^{1}$ Department of Neonatology, National Maternity Hospital, Dublin, Ireland, ${ }^{2}$ Department of Biochemistry, National Maternity Hospital, Dublin, Ireland

${ }^{3}$ Department of Biostatistics, University College Dublin, Ireland, ${ }^{4}$ Department of Paediatric Cardiology, Our Lady's Hospital for Sick Children, Dublin, Ireland

Background and Aims: Brain natriuretic peptide (BNP) and Pro brain natriuretic peptide (Pro-BNP) are useful markers of left ventricular function in adults and are widely used to diagnose cardiac failure. Their use is yet to be explored in neonates. Adequate left ventricular function is vital to maintain sufficient systemic perfusion in adults and possibly in preterm neonates. We hypothesize that Pro-BNP can be a useful marker of left ventricular function in neonates.

Methods: Preterm neonates less than $1500 \mathrm{~g}$ and less than 30 weeks were enrolled in the prospective blinded study. Echocardiographic measurement of Stroke 
volume (SV) and left ventricular output (LVO) were carried out on days 1, 3, and five of life. Pro-BNP levels were measured at the same time as echo measurement.

Results: Fifteen neonates [gestational age Mean (SD) 27 weeks (1.8), mean weight 1064g (213)] had 44 paired echocardiography and pro-BNP determinations. ProBNP ranged from 94 to $35353 \mathrm{pmol} / \mathrm{L} .18$ of 44 echocardiograms showed PDA. In 7 neonates (Group A in table) average SV and LVO were significantly lower than in the other 8 (Group B). Pro-BNP had a statistically significant inverse relationship with both SV and LVO.

Conclusions: Pro-BNP is a useful indicator of left ventricular function in neonates. It may have a role in distinguishing heart from respiratory disease, aid in the decision of starting ionotropes and monitoring response to treatment. It may also have a role in assessing systemic perfusion as an adjunct to blood pressure monitoring.

\begin{tabular}{|l|c|c|c|}
\hline & $\begin{array}{c}\text { Group A } \\
\mathbf{n}=\mathbf{7}\end{array}$ & $\begin{array}{c}\text { Group B } \\
\mathbf{n = 8}\end{array}$ & p value \\
\hline Stroke Volume (m/s) & 1.14 & 1.98 & 0.00001 \\
\hline LV output (mis/kgimin) & 117 & 252 & 0.0007 \\
\hline Pro-BNP & 11273 & 2594 & 0.001 \\
\hline
\end{tabular}

\section{TRANSPULMONARY THERMODILUTION CARDIAC OUTPUT MEASUREMENT VERSUS ULTRASOUND FLOWPROBE IN A NEWBORN LAMB MODEL}

\author{
J Lemson ${ }^{1}$, W P de Boode ${ }^{2}$, J G van der Hoeven ${ }^{1}$ \\ ${ }^{l}$ Department of Intensive Care Medicine, Radboud \\ University Nijmegen Medical Centre, Nijmegen, The \\ Netherlands, ${ }^{2}$ Department of Neonatology, Radboud \\ University Nijmegen Medical Centre, Nijmegen, The \\ Netherlands
}

Background and Aims: Cardiac output (CO) measurement in critically ill children is cumbersome, but may improve haemodynamic management. Transpulmonary thermodilution (TPTD) technique has been used but validation in small children is limited. This is the first study to compare TPTD with ultrasound flow probes in a paediatric animal model.

Methods: We prospectively studied 9 mechanically ventilated and sedated lambs with a median weight of $6.5 \mathrm{~kg}(4.7-12.5 \mathrm{~kg})$ under various circulatory conditions. TPTD measurements were performed with ice-cold saline using the PiCCOplus system (Pulsion Medical Systems). The mean of 4 consecutive measurements with a normal TPTD curve were compared to a simultaneous registration of an ultrasound perivascular flowprobe around the pulmonary artery (Transonic Systems Inc.).

Results: A total of 65 simultaneous measurements fulfilled the quality control criteria and were analysed. Mean $\mathrm{CO}$ was $1.8 \mathrm{l} / \mathrm{min}$ (range $0.72-3.6 \mathrm{l} / \mathrm{min}$ ). The figure depicts the Bland Altman analysis for the two measurement techniques. Bias is $0.3 \mathrm{l} / \mathrm{min}$ with a precision of $0.3 \mathrm{l} / \mathrm{min}$. The correlation coefficient (pearson) is $0.96(\mathrm{p}<0.0001)$.

Conclusions: In this paediatric animal model transpulmonary thermodilution provides an accurate measurement of cardiac output, therefore it can be useful as a monitoring tool in children with haemodynamic instability.

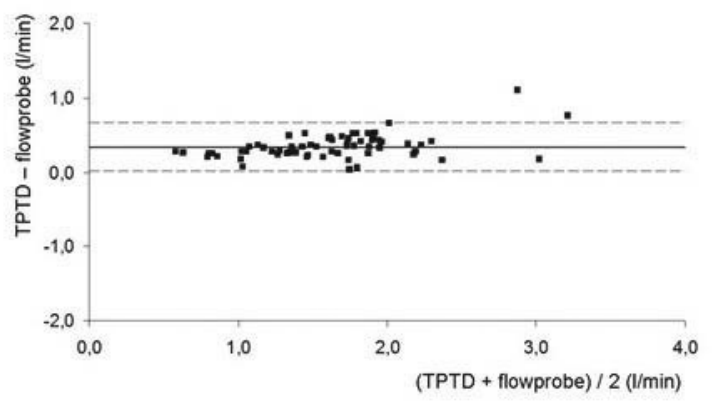

\section{FETAL GROWTH CHARACTERISTICS AND LEFT CARDIAC STRUCTURE IN EARLY INFANCY. THE GENERATION R STUDY}

J J M Geelhoed ${ }^{1,2}$, V W V Jaddoe ${ }^{1,2,3}$, L M Van Osch-Gevers ${ }^{3}$, A Hofman ${ }^{2}$, W A Helbing ${ }^{3}$, J C M Witteman ${ }^{2}$

${ }^{I}$ The Generation R Study Group, Erasmus Medical Center, Rotterdam, The Netherlands, ${ }^{2}$ Department of Epidemiology and Biostatistics, Erasmus Medical Center, Rotterdam, The Netherlands, ${ }^{3}$ Department of Pediatrics, Erasmus Medical Center, Rotterdam, The Netherlands

Background and Aims: Left ventricular hypertrophy may be partly originated in early life. The aim of this study was to examine the associations of fetal growth characteristics in the second and third trimester of pregnancy and at birth with left cardiac structure in early infancy.

Methods: This study was embedded in the Generation R Study, a prospective population-based cohort study from fetal life until adulthood. Fetal growth characteristics were assessed by ultrasounds in each trimester in pregnant women. Echocardiographic follow-up studies were performed in a subgroup of 791 infants aged 6 weeks and 6 months.

Results: We did not find associations of second trimester growth characteristics with postnatal cardiac structures. Third trimester abdominal circumference was positively associated with postnatal left ventricular mass and aortic root diameter. For each SD increase in third trimester abdominal circumference, left ventricular mass increased by $0.33(95 \%$ CI $0.059,0.61)$ grams and aortic root diameter increased by 0.13 (95\% CI: $0.03,0.22)$ $\mathrm{mm}$. Birth weight was associated with aortic root diameter leading to an increase of 0.21 ( $95 \%$ CI: 0.07 , $0.34) \mathrm{mm}$ per SD increase in birth weight. These associations were adjusted for age, gender, weight and length. The analyses focused on left cardiac structure at the age of 6 months are currently undertaken and will be presented at the congress.

Conclusions: Fetal growth retardation in second half of pregnancy is associated with persistent smaller left ventricular mass and aortic root diameter in early infancy. Follow-up studies are needed to examine if these cardiac changes will persist throughout childhood. 


\section{LUNG ANTIOXIDANT ENZYMES ARE REGULATED BY INCREASED PULMONARY BLOOD FLOW IN THE NEONATAL LAMB}

S M Black ${ }^{1}$, A Grobe $^{2}$, P Oishi ${ }^{3}$, A Azakie $^{4}$, $\mathrm{J}_{\mathrm{R}}$ Fineman ${ }^{3}$

${ }^{I}$ Vascular Biology Center, The Medical College of Georgia, Augusta, GA, USA, ${ }^{2}$ Biomedical and

Pharmaceutical Sciences, The University of Montana, Missoula, MT, USA, ${ }^{3}$ Department of Pediatrics, UC San Francisco, San Francisco, CA, USA, ${ }^{4}$ Department of Surgery, UC San Francisco, San Francisco, CA, USA

Background and Aims: Our previous studies have indicated that an increase in oxidative stress is involved in the development of pulmonary hypertension in a lamb model of increased pulmonary blood flow (Shunts). However, it is not clear whether this is due, at least in part, to alterations in lung antioxidant enzymes. Thus, the goal of this study was to determine whether the expression and activity of lung antioxidant enzymes are altered during the development of pulmonary hypertension in Shunt lambs.

Methods: Eighteen fetal lambs underwent in utero placement of an aortopulmonary vascular graft. Two-, four, and eight- weeks following spontaneous delivery, peripheral lung tissue was collected. Expression of catalase, SODI and SOD2 were evaluated by Western blotting. Catalase and SOD activities were also determined. A $\mathrm{P}<0.05$ was considered statistically significant.

Results: Catalase expression and activity were significantly decreased in 2-week Shunt compared to Controls but this decrease was not maintained at 4- and 8 -weeks. No change in SOD1 expression were observed in Shunts compared to Controls at any age group but SOD2 expression was found to be decreased at 2-weeks of age in Shunts. However, no change in total SOD activity was observed between Shunt and Controls at any age group

Conclusions: Lung antioxidant enzyme expression and activites are altered in lambs with increased pulmonary blood flow and these differences may play a role in the development of pulmonary hypertension.

\section{OPTIMAL SETTINGS DURING TVI RECORDING AND OFF LINE STRAIN AND STRAIN-RATE ANALYSIS IN TERM NEONATES}

E Nestaas $^{1,2}$, A Støylen ${ }^{3,4}$, L Sandvik ${ }^{5}$, L Brunvand ${ }^{1}$, D Fugelseth ${ }^{1}$

${ }^{I}$ Department of Paediatrics, Ullevål University Hospital, Oslo, Norway, ${ }^{2}$ Department of Paediatrics, Hospital of Vestfold, Tønsberg, Norway, ${ }^{3}$ Department of Cardiology, St. Olavs Hospital, Trondheim, Norway ${ }^{4}$ Department of Circulation and Medical Imaging, Faculty of Medicine, Norwegian University of Science and Technology, Trondheim, Norway, ${ }^{5}$ Centre for Clinical Research, Ullevål University Hospital, Oslo, Norway

Background and Aims: Strain and strain-rate (SR) measurements from tissue velocity images (TVI) are noisy. A novel method for noise estimation, based on the measured variation between two consecutive heart beats, was used for assessing the optimal settings during TVI recording and deformation analysis in term neonates.

Methods: The strain and SR beat to beat variation (BBV) were assessed in 8 good-quality TVI for each of the following probe and frame rate (FR) settings (Vivid 7, GE Vingmed, range $+/-16 \mathrm{~cm} / \mathrm{sec}$ ); $5 \mathrm{~S}$ probe default FR, 10S default FR and 10S low FR. Two segments per wall were analysed using 48 different combinations of region of interest (ROI) size and strain length (SL). Results: Both BBVs were lower in the $5 \mathrm{~S}$ than in both the $10 \mathrm{~S}$ series $(\mathrm{p}<0.05)$. Both BBVs decreased with increased SL in each series $(\mathrm{p}<0.05)$. Except for the $10 \mathrm{~S}$ default FR strain BBV $(p=0.086)$ both BBVs decreased with increased ROI length $(\mathrm{p}<0.05)$. Of the combination of ROI length and SL eligible for two-segment analyses, the lowest BBVs in all series were found using ROI length $1 \mathrm{~mm}$ and SL $10 \mathrm{~mm}$. The optimal ROI width was smaller using the $10 \mathrm{~S}$ probe $(1 \mathrm{~mm})$ than the $5 \mathrm{~S}$ probe (3mm).

Conclusions: The BBVs can be used to assess the optimal settings during TVI recording and analysis. The BBVs were lower using the $5 \mathrm{~S}$ probe than the $10 \mathrm{~S}$ probe. In two-segment analysis, the optimal ROI length was $1 \mathrm{~mm}$ and SL was $10 \mathrm{~mm}$, and the optimal ROI width was $1 \mathrm{~mm}$ using the $10 \mathrm{~S}$ probe and $3 \mathrm{~mm}$ using the $5 \mathrm{~S}$ probe.

\section{NEONATAL RESPIRATORY ECMO IN THE UK: WHERE ARE WE 10 YEARS AFTER UK ECMO TRIAL?}

\author{
A Karimova ${ }^{1}, \mathrm{~J} \mathrm{Cassidy}^{2}, \mathrm{C} \mathrm{Davis}^{3}, \mathrm{H}^{\mathrm{H}}$ Pandya ${ }^{4}$, \\ K Harrington ${ }^{1}$, W Beierlein ${ }^{1}$, A P Goldman ${ }^{1}$

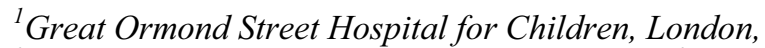 \\ ${ }^{2}$ Freeman Hospital, Newcastle upon Tyne, UK, ${ }^{3}$ The \\ Royal Hospital for Sick Children, Glasgow, UK, \\ ${ }^{4}$ Glenfield Hospital, Leicester, UK
}

Background and Aims: After the UK neonatal ECMO trial (1993-1995), a national ECMO service was established (1996). ELSO reports worldwide decline in neonatal ECMO. This paper reviews neonatal respiratory ECMO in the UK focusing on last 10 years.

Methods: Retrospective review: patient demographics; illness severity; ECMO indication, characteristics and outcome.

Results: 804 neonates were supported between 19892005. Cases/year and diagnoses are shown in the graph. Pre-ECMO treatment changed (1993-2005): iNO 0\% to $92 \%$; HFOV $0 \%$ to $61 \%$ and surfactant from 5 to $63 \%$. No change occurred in the patient age, length of ventilation and oxygenation index pre-ECMO. The use of V-V ECMO increased from $40 \%$ (1995) to $69 \%$ (2005). Overall survival to discharge was $79 \%$; MAS 96\%, PPHN 84\%, HMD 90\%, sepsis 60\%, CDH 55\% and others $24 \%$.

Conclusions: Overall and diagnosis specific survival is comparable in the UK to worldwide. More babies are supported for MAS and less with $\mathrm{CDH}$ in the UK compared with ELSO. It is unclear why, despite having a centralized national ECMO service, the frequency of neonatal ECMO has not consistently fallen in the UK. 


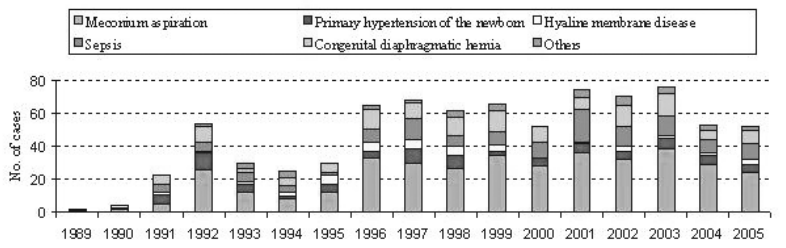

\section{CHANGING DEMOGRAPHICS IN NEONATAL EXTRACORPOREAL MEMBRAN \\ OXYGENATION (ECMO) - NEW RESULTS FROM THE GERMAN ECMO CENTER MANNHEIM}

F Loersch, S T Hien, S Demirakca, C H v. Buch, H Wirth, V Varnholt, T H Schaible

NICU, University Childrens Hospital, Mannheim, Germany

Background and Aims: ECMO is a standard treatment in the algorithm of neonatal respiratory failure who can provide oxygenation and may reduce ventilator induced injury. Furthermore ECMO helps to avoid high doses of inotropic medications and may help to overcome pulmonary hypertension. Over the last 20 years marked changes have occurred by new technical developments and indications for neonatal ECMO therapy. We analysed the changes in our ECMO center over this time period.

Methods: From 1987-2006, 281 neonates were treated with ECMO in Mannheim. From 1987-1991, 33 patients, from 1992-1996, 68 and from 1997-2001, 85 patients were treated with ECMO. In the last period from 2002-2006, 95 patients put on either VV or VAECMO.

Results: The over all survival of this 281 neonates were $72,2 \%$ (mean; Period 1-4: 60\%/64\%/76\%/77\%). The mean maximum ventilation pressure was $36,1 \mathrm{mbar}$ $(45,4 / 42,6 / 35,6 / 30,4)$ the average ECMO time was $167 \mathrm{~h}$ $(83 \mathrm{~h} / 109 \mathrm{~h} / 162 \mathrm{~h} / 223 \mathrm{~h})$. The Oxygenation Index (OI) was calculated of 51,9 before ECMO $(69,5 / 58,2 / 62,5 / 36,0)$, NO was used in $31 \%$ $(0 \% / 25 \% / 38 \% / 41 \%)$ and HFOV by $22 \%$ of the patients $(84 \% / 28 \% / 9 \% 76 \%)$. The indication for ECMO was in $22 \%$ MAS $(12 \% / 32 \% / 31 \% / 12 \%)$ in $50 \% \quad \mathrm{CDH}$ $(27 \% / 27 \% / 47 \% 778 \%)$ and in $28 \%$ others $(61 \% / 41 \% / 22 \% / 10 \%)$.

Conclusions: The survival after ECMO was increased in this period. ECMO was started with lower values of positive inspiratory peak pressures, and therefore lower oxygenation index. The time under ECMO was longer. The spectrum has totally changed to the main patient group of $\mathrm{CDH}$. Although advances in mechanical ventilation have been made the necessity for ECMO remains a tool to override several neonatal lung failure.

\section{BRONCHIOLITIS IN EUROPE - A COMPARATIVE SURVEY}

\author{
A Seiz \\ Department of Pediatric Emergency, University \\ Hospital, Saint Etienne, France
}

Background and Aims: Bronchiolitis is a common cause of illness and an increasingly important cause of hospitalisation. It remains a major concern for paediatricians and typically affects infants younger than two years, principally during winter months. Bronchiolitis is a self-limited illness in most previously healthy infants and resolves frequently without complications. However, severely affected infants are at increased risk for complications. Assessment of disease severity is very important in the management of bronchiolitis. Neither for assessment of severity nor for hospitalisation nor for treatment recommendations are official criteria in place. Although there is a lack of well established criteria a number of guidelines have been published.

Methods: In this comparative survey appropriate guidelines were taken from the European French and German speaking paediatric scholarly societies (Austria, Belgium, France, Germany, and Switzerland). These guidelines were evaluated to address the issue of consistency with evidence from large randomised trials, meta-analyses, and systematic reviews.

Results: In case of bronchiolitis we have consistent rates in Europe concerning mortality, morbidity, days of hospitalisation, need of intensive care, and duration of mechanical ventilation. Despite of this fact clinical practice and therapeutically recommendations varies widely. There are remarkable differences in indications for hospitalisation and treatment proposals.

Conclusions: In the aggregate, evidence from large randomised trials, meta-analyses, and systematic reviews should become generally accepted. They should enter standardised the official recommendations of paediatric scholarly societies in Europe. This could render epidemiological research in this field easier which promotes the comparability and quality rating at those points where an agreement is not yet achieved.

\section{PENTAVALENT (HUMAN-BOVINE) REASSORTANT ROTAVIRUS VACCINE (PRV) REDUCED HOSPITALIZATIONS AND EMERGENCY DEPARTMENT (ED) VISITS FOR ROTAVIRUS (RV) ACUTE GASTROENTERITIS (AGE): ASSESSING THE ROBUSTNESS OF THE RESULTS}

R Itzler ${ }^{1}, \mathrm{~T}$ Vesikari ${ }^{2}$, D Matson ${ }^{3}, \mathrm{M} \mathrm{Coia}^{1}, \mathrm{~J} \mathrm{Cook}^{1}$, $\mathrm{G}^{\text {Davies }}{ }^{1}$, M DiNubile ${ }^{1}$, P Heaton ${ }^{1}, \mathrm{~J} \mathrm{Heyse}^{1}$, G Koch $^{4}$

${ }^{1}$ Merck Research Labs, West Point, PA, USA

${ }^{2}$ University of Tampere, Finland, ${ }^{3}$ Eastern Virginia Medical School, Norfolk, VA, USA, ${ }^{4}$ University of North Carolina, Chapel Hill, NC, USA

Background and Aims: Between 2001-2004, 70,301 healthy infants 6-12 weeks old were enrolled from 11 countries in the Rotavirus Efficacy and Safety Trial (REST) to receive 3 oral doses of PRV expressing human serotypes G1-4 and P[8] or placebo. Active surveillance for hospitalizations and ED visits for AGE was conducted 7,14 , and 42 days after each dose and every 6 weeks thereafter for up to two years. Three doses of PRV reduced the rate of G1-4 RV-associated hospitalizations/ED visits by $94.5 \%$ [95\% CI: 91.2, 
96.6] (N Engl J Med 2006; 354:23-33). We assessed the rate reductions in all subjects receiving $\geq 1$ dose.

Methods: The case definition for RV AGE was vomiting and/or $\geq 3$ loose stools within 24 hours and RV detection in stool by EIA. Serotypes were identified by PCR. Analyses were performed using Poisson regression.

Results: Mean length of follow-up after the first dose was 314.5 days in PRV recipients and 314.4 days in placebo recipients. Rate reduction for hospitalizations/ED visits due to G1-4 RV AGE occurring after the first dose was $91.9 \%$ [95\% CI: 88.0, 94.5] in PRV recipients. Rate reductions for the 5 most commonly observed serotypes are shown in the table.

Conclusions: PRV consistently decreased hospitalizations/ED visits for RV AGE caused by each of the 5 most common serotypes observed in the large international REST.

\begin{tabular}{|c|c|c|cc|}
\hline $\begin{array}{c}\text { VP7 } \\
\text { Serotype }\end{array}$ & $\begin{array}{c}\text { Number of Cases } \\
\text { PRV } \\
\mathrm{N}=34,035\end{array}$ & $\begin{array}{c}\text { Phacebo } \\
\text { N=34,003 }\end{array}$ & $\begin{array}{c}\text { Efficacy } \\
\text { Estimate } \\
(\%)\end{array}$ & $\begin{array}{c}\mathbf{9 5} \% \\
\text { Confidence } \\
\text { Interv al }\end{array}$ \\
\hline $\mathrm{G} 1$ & 32 & 414 & 92.3 & $88.2,95.0$ \\
$\mathrm{G} 2$ & 1 & 12 & 91.7 & $34,7,99.0$ \\
$\mathrm{G} 3$ & 3 & 20 & 85.1 & $49.6,95.6$ \\
$\mathrm{G} 4$ & 2 & 20 & 90.1 & $57.2,97.7$ \\
$\mathrm{G} 9$ & 2 & 25 & 92.1 & $66.1,98.2$ \\
\hline
\end{tabular}

\section{RESULTS FROM THE REVEAL STUDY - PEDIATRIC ROTAVIRUS GASTROENTERITIS: AGE DISTRIBUTION AND SEASONAL TRENDS AMONG 2846 CHILDREN PRESENTING WITH SYMPTOMS OF ACUTE GASTROENTERITIS IN SEVEN EUROPEAN COUNTRIES}

\author{
F A Allaert ${ }^{9}$, C Giaquinto $^{1}$, L Gothefors ${ }^{2}$, C Huelsse ${ }^{4}$,

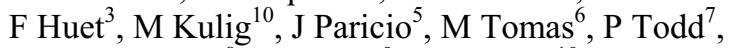 \\ P Van Damme $^{8}$, N Vernet $^{9}, M$ Watson ${ }^{10}$ \\ ${ }^{I}$ Department of Pediatrics, University of Padova, Italy, \\ ${ }^{2}$ Barn-Och Ungdomskliniken, Norrlands \\ Universitetssjukhus, Umeà, Sweden, ${ }^{3}$ Department of \\ Pediatrics, University Hospital, Dijon, France \\ ${ }^{4}$ Landesgesundheitsamt, Rostock, Germany \\ ${ }^{5}$ Department of Pediatrics, Hospital Marina Alta, \\ Denia, Spain, ${ }^{6}$ Department of Pediatrics, Hospital \\ Francesc de Borja, Gandia, Spain, ${ }^{7}$ Arrowe Park \\ Hospital, Wirral, UK, ${ }^{8}$ Center for the Evaluation of \\ Vaccination, University of Antwerp, Belgium \\ ${ }^{9}$ Department of Epidemiology, McGill University and \\ Cenbiotech, Dijon, France, ${ }^{10}$ Sanofi Pasteur MSD, \\ Lyon, France
}

Background and Aims: Paediatric Rotavirus Gastroenteritis (PRG) affects the majority of children during the first years of life and causes a considerable medical and economic burden of disease (BOD). In order to quantify this BOD and the beneficial impact of preventative vaccination, further understanding of seasonal and age distribution of PRG is required.

Methods: A prospective, observational, study was conducted in seven coutries (Belgium, France, Germany, Italy, Spain, Sweden, United Kingdom). Children up to 5 years of age with physician-diagnosed acute gastroenteritis (AGE) were included. They were recruited from 12 hospitals, 16 emergency departments and 139 paediatricians (FP/GP) between Oct 2004 and Sept 2005. Rotavirus was determined by ELISA testing. Results: Among 2846 children with AGE, 1102 had PRG. For the percentage of PRG among AGE see table1. More than $94 \%$ of those presenting with PRG were aged $>=6$ months. The temporal distribution of PRG varied between the winter and summer months, with a peak between January and March (57\% to $64 \%$ of all AGE cases).

Conclusions: More than $94 \%$ of PRG presented after the age of 6 months with the highest proportion of PRG seen in hospitals. During the winter months, the majority of AGE cases are due to PRG. Vaccination of infants against rotavirus infection should significantly reduce the annual and winter burden of AGE during early childhood.

\begin{tabular}{|c|c|c|c|c|c|c|c}
\hline $\begin{array}{c}\text { Proportion of PRG } \\
\text { among AGE cases (\%) } \\
\text { in Hospitals }\end{array}$ & $\begin{array}{c}\text { Antwerp } \\
\text { (Gelgium) }\end{array}$ & $\begin{array}{c}\text { Dijon } \\
\text { (France) }\end{array}$ & $\begin{array}{c}\text { Rostock } \\
\text { (Germany) }\end{array}$ & $\begin{array}{c}\text { Padowa } \\
\text { (Italy) }\end{array}$ & $\begin{array}{c}\text { Gandial } \\
\text { Denia } \\
\text { (Spain) }\end{array}$ & $\begin{array}{c}\text { Umea } \\
\text { (Sweden) }\end{array}$ & $\begin{array}{c}\text { Wiral } \\
\text { (UK) } \\
68\end{array}$ \\
\hline in Emergency roomsiGP/FP & 41 & 36 & 65 & 69 & 53 & 62 & 61 \\
\hline
\end{tabular}

\section{hMPV INFECTIONS ARE NOT GENERALLY MILDER THAN RSV INFECTIONS}

O Schildgen ${ }^{1}$, A Wilkesmann ${ }^{2}$, A M Eis-Hübinger ${ }^{2}$, T Geikoswski ${ }^{2}$, T Glatzel ${ }^{2}$, M J Lentze ${ }^{1}$, U Bode $^{1}$, A Simon ${ }^{1}$

${ }^{\text {I}}$ Childrens Hospital, Medical Center, University of Bonn, Germany, ${ }^{2}$ Institute for Medical Microbiology, Immunology and Parasitology, Department of Virology, University of Bonn, Germany

Background and Aims: Human Metapneumovirus (hMPV) is a worldwide distributed pathogen in pediatric viral respiratory tract infection (VRTI). Earlier publications suggest milder illness severity in hMPVcompared to Respiratory Syncytial Virus (RSV)infection.

Methods: 637 nasopharyngeal aspirates from pediatric patients were tested by hMPV-PCR in two consecutive seasons. Risk factors, clinical, and laboratory items were analyzed. The hMPV-infected patients were compared with hMPV-negative, RSV-infected patients by matched pair analysis.

Results: hMPV was detected in $17.9 \%$ of all NPA. In total, $88 \mathrm{hMPV}$-infected patients with complete dataset were considered. More than one half of all hMPV patients were older than 12 months. $45.5 \%$ had at least one risk factor for severe courses of the VRTI; $27.3 \%$ were prematurely born infants, $15.9 \%$ with a birth weight $<1500 \mathrm{~g}$. Among hMPV-infected patients 39 $(44.3 \%)$ were coinfected with other respiratory viruses (RSV in 29.5\%). Coinfection did not result in higher illness severity. On matched pair analysis (hMPV+/RSV- vs. hMPV-/RSV+) the epidemiological and clinical features of hMPV-infection were similar to those of RSV infection although somewhat higher proportions of patients with hypoxemia on admission (33 vs. $21 \%$ ) and of intensive care treatment (20.8 vs. $10.4 \%$ ) were observed in the hMPV group than in the RSV group. The hMPV patients more often showed lobar infiltrates in radiologically chest examination. In $60 \%$ of all hMPV-infections, the attending physicians prescribed antimicrobial chemotherapy. 
Conclusions: The earlier assumption that hMPV infections are milder than RSV infections does not apply in hospitalized patients (Wilkesmann et al., Eur. J. Ped., in press). The first two authors contributed equally to this work.

\section{POST NEONATAL TETANUS - PREVENTABLE BUT STILL PREVALENT AMONG RURAL POPULATION OF INDIA}

P H Shah ${ }^{1}$, S M Nimbalkar ${ }^{1}$, S N Vani ${ }^{1}$, A A Kapoor ${ }^{1}$, A P Shah ${ }^{2}$

${ }^{I}$ Department of Paediatrics, Pramukhswami Medical College and Hospital, Karamsad, Gujarat, India

${ }^{2}$ Department of Microbiology, Government Medical College and New Civil Hospital, Surat, Gujarat, India

Background and Aims: Tetanus is a neurological disorder with spasticity, muscle spasms and autonomic disturbances caused by neurotoxin 'tetanospasmin' produced by clostridium tetani. Tetanus was first described in Egypt over 3000 years ago and was prevalent throughout the ancient world. Very few reviews of tetanus in children are available in India and other countries. Our aim was to study the mode of infection, incubation period, period of onset, duration of spasms, complications, vaccination status and outcome.

Methods: Diagnosis of tetanus was made on clinical grounds. Cases of tetanus between 1 month and 16 years of age admitted between August 1999 to December 2002 were evaluated retrospectively. Severity was graded on the basis of Patel and Joag criteria.

Results: Overall incidence was $1.09 \%$. Out of 40 cases $(\mathrm{M}: \mathrm{F}=1: 1), 27$ were discharged, 9 were deaths and 4 had DAMA (Discharge Against Medical Advise). Traumatic tetanus $(42.5 \%)$ was more common than otogenic (37.5\%). The mortality was seen among severe cases (9 of 10). Severe attack and poor prognosis has been associated with short incubation period and period of onset. Thrombophlebitis is the most common complication encountered followed by lower respiratory tract infection. Autonomic imbalances continue to be difficult to treat and associated with high mortality.

Conclusions: Post neonatal tetanus continues to be a major cause of mortality and morbidity among rural population in India. An incomplete or poor immunization status in combination with CSOM and a high chance of injury to the feet and subsequent contamination with tetanus bacilli puts the rural poor at a high risk.

\section{DEVELOPMENT OF AN ANTIVIRAL FOR RESPIRATORY SYNCYTIAL VIRUS (RSV) UTILIZING RNA INTERFERENCE (RNAI)}

J P DeVincenzo ${ }^{1}, \mathrm{R}_{\text {Alvarez }}{ }^{2}, \mathrm{R}$ Tripp ${ }^{3}, \mathrm{~J} \mathrm{Cehelsky}^{2}$, $\mathrm{P}$ Hadwiger $^{2}$, R Pandey ${ }^{2}, \mathrm{P} \mathrm{Ge}^{2}$, R Constein ${ }^{2}$, S Elbashir ${ }^{2}, \mathrm{M} \mathrm{John}^{2}$, S Shanmugan ${ }^{2}$, R Meyers ${ }^{2}$

${ }^{I}$ Department of Pediatrics, University of Tennessee, Memphis, TN, USA, ${ }^{2}$ Alnylam Pharmaceuticals, Cambridge, MA, USA, ${ }^{3}$ University of Georgia, Athens, GA, USA
Background and Aims: RSV is the leading cause of hospitalisation of infants and is an important pathogen of the elderly and the immunosuppressed. Existing preventions and treatments are limited. RNAi utilizes existing ubiquitous cellular proteins to catalyze targeted mRNA degradation in a sequence-specific manner. Aims: To evaluate small inhibiting RNAs (siRNA) as potential antivirals and to test their safety in humans.

Methods: The RSV genome was screened bioinformatically for consensus sequences across RSV strains while avoiding human homology by BLAST. Resulting siRNAs were screened invitro by plaque reduction. Promising candidates targeting RSV P, N and $\mathrm{L}$ mRNA were screened invivo using BALB/c mouse models utilizing mismatched siRNA controls. ALNRSV01 (N target) was further evaluated by formal toxicology studies. Single and multiple rising doses of ALN-RSV01 nasal spray were evaluated in healthy adults in a double-blind, placebo-(saline)-controlled phase 1 trial.

Results: Given $4 \mathrm{hr}$ before or $24 \mathrm{hr}$ after infection, a single dose $(2 \mathrm{mg} / \mathrm{kg})$ of selected $\mathrm{P}, \mathrm{N}$ or L targeted siRNAs reduced lung virus by 4 or $3 \log \mathrm{pfu} / \mathrm{g}$ respectively and prevented disease (weight loss). Mismatch siRNA controls showed no viral reduction. No toxicity was observed in rats and monkeys exposed IV or to 4 doses via intranasal aspiration (NOEL $>48 \mathrm{mg} / \mathrm{kg}$ in rats; $>24 \mathrm{mg} / \mathrm{kg}$ in monkeys). 65 adults receiving $1.5-150 \mathrm{mg}$ of ALN-RSV01 exhibited similar rates and types of adverse events (AEs) as did placebo recipients. All AEs were mild.

Conclusions: RNAi may offer a new strategy for RSV treatment. ALN-RSV01 appears safe and well tolerated when given intranasally and should undergo further clinical evaluation. (funding: Alnylam Pharmaceuticals).

\section{EFFECTS OF INITIATION OF CONTINUOUS RENAL REPLACEMENT THERAPY ON HAEMODYNAMICS IN CRITICALLY ILL CHILDREN}

\section{J Brierley, M Richardson, M Peters}

Paediatric Intensive Care Unit, Great Ormond Street Hospital, London, UK

Background and Aims: There are no studies analysing the haemodynamic effects of initiating continuous venovenous haemofiltration (CVVH) in critically ill children. Haemodynamic instability is often met by countering 'filter-related decreased systemic vascular resistance (SVR)' with vasoconstrictors. Newer noninvasive haemodynamic techniques may allow more physiological management.

Methods: Prospective observational study of 9 critically ill children (1-9 years) to define haemodynamic responses to commencing CVVH. Heart rate (HR), mean blood pressure (MABP), central venous pressure (CVP), and central venous oxygen saturation ( $\mathrm{ScvO} 2 \%)$ were directly measured at 15 minutely intervals for an hour together with cardiac index (CI), SVR index (SVRi), stroke volume index (SVi) by non-invasive continuous wave Doppler ultrasound device. (USCOM) No changes in inotrope or volume administration occurred during the study. Results: CVVH induced an 
initial significant decrease in the volume-related haemodynamic parameters SVi and CVP. Subsequently a significant increase in SVRi and decrease in CI were seen. ScvO2 remained unchanged.

Conclusions: Initiating CVVH in critically ill children induces hypovolemia leading too a systemic cardiovascular response of vasoconstriction and overall decrease in cardiac output. This may represent haemodilution with lowering of effective inotropic concentration through increased volume of distribution, or there may be a direct negative inotropic effect of initiating CVVH. Physiological based therapy would consist of increasing inotropes, rather than vasoconstrictors.

\begin{tabular}{|c|c|c|c|c|c|}
\hline & Pre CVifH & $15 \mathrm{nins}$ & $30 \mathrm{mins}$ & $45 \mathrm{~m}$ ins & $60 \mathrm{mins}$ \\
\hline H R (beat $\min ^{-1}$ ) & $26(14)$ & $145(14)$ & 125 i12; & $104(12)$ & 105 (11) \\
\hline$c \mid\left(1 / m\right.$ in $\left.\cdot m^{-3}\right)$ & $3.0(0.6)$ & $2.5(0.5)$ & 2.4 (3.6) & $2.4(0.4)$ & $2.2(0.5) \times \pi$ \\
\hline svi(mlmir $\left.m^{-2}\right)$ & $23.8(5.3)$ & $17.2(4.2)$ & $19.2(5.1)^{*}$ & $23.1(4.2)$ & $21(4.2)$ \\
\hline c VF $(m m H g)$ & $\cdot 2.6(1.3)$ & $11 .<(1.5)$ & $9.5(1.7 ; *$ & $8.8(1 . \overline{7})$ & $6.3(1.3)$ \\
\hline MABP $(m \mathrm{mH}$ gi & $61(4.6)$ & $59 . \varepsilon(3.6)$ & $62.3(4.5)$ & $63.3(3.4)$ & $E 0.1(3.3)$ \\
\hline SVRi (dy n.s.cm.m ${ }^{-2}$, & $540(293)$ & $585(277)$ & $658(310)$ & $750(256)$ & $744(296) \times \pi$ \\
\hline$S_{\mathrm{cuv}} C_{2}(\varangle)$ & ;6.5(3.5) & | $4 . \in(4.7)$ & $73.5(5.1)$ & $74.5(5.5)$ & $74.9(5.0)$ \\
\hline
\end{tabular}

\section{RAPID ORAL REHYDRATION THERAPY IN THE MANAGEMENT OF CHILDHOOD GASTROENTERITIS}

\author{
C D Aarts-Tesselaar ${ }^{1}$, A A Vaessen-Verberne ${ }^{2}$

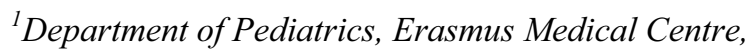 \\ Sophia's Childrens Hospital, Rotterdam, The \\ Netherlands, ${ }^{2}$ Department of Pediatrics, Amphia \\ Hospital, Breda, The Netherlands
}

Background and Aims: Dehydration due to gastroenteritis is a serious health problem in younger children and accounts for a substantial number of admissions at paediatric wards. Despite recent guidelines part of these children are rehydrated with intravenous fluids and hospitalisation stay is prolonged. Our objective is to evaluate whether rehydration with oral rehydration solution during a four-hour period in the hospital is safe and efficient in children with acute gastroenteritis. Primary outcome is the success rate, that is the percentage of children who are successfully rehydrated and sent home after rehydration. Secondary outcomes are duration of diarrhea and/or vomiting and reassessment at the emergency department or requirement of hospitalisation.

Methods: A total of 102 patients (6 months - 6 years) admitted with dehydration due to acute gastroenteritis were enrolled in our study. Exclusion criteria included invagination, ileus, short bowel syndrome, volvulus, diabetes mellitus, HUS and underlying severe parenteral infection. Depending on the severity of dehydration, patients were rehydrated according to ESPGHAN guidelines with 50 to $100 \mathrm{ml} / \mathrm{kg}$ ORS over four hours, followed by continuation of the usual diet.

Results: 97 Patients were rehydrated following our study design. In $80,4 \%$ this was successful and in $19,6 \%$ the hospitalisation had to be prolonged. Biochemical laboratory results and stool cultures were not predictors of failure and had no extra value in the management.

Conclusions: Our results provide support for successful rehydration in four hours with ORS ${ }^{\circledR}$ and for reduction in the need of intravenous therapy and hospitalisation.
After correcting dehydration, early refeeding warrants lasting hydration.

\section{EMOTIONAL/BEHAVIORAL PROBLEMS AND SOCIAL AND ACADEMIC COMPETENCE IN VLBW ADOLESCENTS}

L B Dahl ${ }^{2}$, P I Kaaresen ${ }^{1}$, J Tunby ${ }^{1}$, B H Handegård ${ }^{2}$, $\mathrm{S} \mathrm{Kvernmo}^{2}$, J A Rønning ${ }^{2}$

${ }^{l}$ Department of Pediatrics, University Hospital of North Norway, Tromsø, Norway, ${ }^{2}$ Institute for Clinical Medicine, University of Tromsø, Tromsø, Norway

Background and Aims: Emotional and behavioral problems and reduced social and academic competence are common in VLBW survivors. Studies often conclude from parent-report (CBCL) alone. Youth selfreport (YSR) is uncommon.

Methods: Of 265 VLBW preterms born 1978 - 1989, 174 survived to adolescence (65.7\%). Those 13 to 18 years were addressed. 156 families were eligible, 99 completed CBCL (63.5\%), and 82 completed YSR (52.6\%). CBCL and YSR provide problem and competence scores. Parents of VLBW adolescents were compared to parents of normative adolescents, VLBW adolescents were compared to normative adolescents, VLBW adolescents and their parents were compared in pairs. T-tests and Intraclass correlation were used.

Results: Parents of VLBW adolescents reported more $(\mathrm{p}<0.01)$ total, social and attention problems, and less total and school competence in males, and more internalizing, social and attention problems, and less total and school competence in females. VLBW adolescents males reported less $(\mathrm{p}<0.01)$ total, internalizing, externalizing, and attention problems, and VLBW adolescents females reported less total, externalizing, social, and attention problems, compared to controls. Both gender reported significantly higher activity competence than controls. Female adolescents, in contrast to males, reported significant higher problem scores compared to their parents. Moderate to high correlations were found, except for externalizing behavior in females.

Conclusions: According to parents significant proportions of VLBW adolescents experience more behavioral problems, and less competence than normative adolescents. According to VLBW adolescents they experience less behavioral problems and equal or higher competence than normative controls. Female VLBW adolescents have behavioral problems, in particular externalizing problems, unrecognized by parents.

\section{NEONATAL HYDROCORTISONE TREATMENT: NEURODEVELOPMENTAL OUTCOME AND MRI AT SCHOOL AGE IN PRETERM BORN CHILDREN}

K J Rademaker ${ }^{1}$, C S P M Uiterwaal ${ }^{2}$, F Groenendaal ${ }^{1}$, M M A T Uniken Venema ${ }^{3}, \mathrm{~F}$ van $\mathrm{Bel}^{1}$, F J Beek ${ }^{4}$, I C van Haastert ${ }^{1}$, D E Grobbee ${ }^{2}$, L S De Vries ${ }^{1}$

${ }^{1}$ Department of Neonatology, Wilhelmina Children's

Hospital, University Medical Center Utrecht, The 
Netherlands, ${ }^{2} J u l i u s$ Center for Health Sciences and Primary Care, Utrecht, The Netherlands, ${ }^{3}$ Department of Pediatric Psychology, Wilhelmina Children's Hospital, University Medical Center Utrecht, The Netherlands, ${ }^{4}$ Department of Pediatric Radiology, Wilhelmina Children's Hospital, University Medical Center Utrecht, The Netherlands

Background and Aims: Dexamethasone improves ventilatory status in BPD, but has a negative impact on long-term neurodevelopment. Another corticosteroid with fewer side effects would be welcomed: hydrocortisone may offer such alternative. We investigated neurodevelopment and presence of brain lesions at school age in preterm infants, who had been prescribed hydrocortisone for BPD.

Methods: 226 preterm born children ( $\leq 32$ weeks or birth weight $\leq 1500$ grams) participated in a cohort study with 8 years follow-up. They performed five subtests of the WISC-R, the Visual Motor Integration (VMI) and a memory test. Motor function was assessed using the Movement ABC. Brain lesions were documented by MRI. Sixty-two children who received hydrocortisone for BPD $(5 \mathrm{mg} / \mathrm{kg}$ tapered over a minimum period of 22 days, median duration 27.5 days) were compared with 164 controls.

Results: Hydrocortisone-treated infants were younger, lighter and sicker than their non-steroid counterparts. To deal with group differences, adjustment for gestational age, birth weight, gender, need for mechanical ventilation and small-for-gestational-age was performed. Adjusted mean IQ, VMI and memory results were the same in the hydrocortisone and the non-steroid group (99 vs 101, p:0.62; 97 vs 99, p:0.49, 7.9 vs $7.5, \mathrm{p}: 0.42$ respectively). The incidence of $\mathrm{CP}$ in both groups was equal $(11 \%$ vs $7 \%$, p:0.97. The Total Impairment Score in non-CP children was 5.5 in both groups. Occurrence of brain lesions on MRI and mean midsagittal corpus callosum area were the same.

Conclusions: Hydrocortisone in the mentioned dose, administered in the neonatal period for BPD, does not appear to have long-term effects on neurodevelopment.

\section{FUNCTIONAL OUTCOMES AND PARTICIPATION IN YOUNG ADULTHOOD FOR VERY PRETERM AND VERY-LOW-BIRTH- WEIGHT INFANTS: THE DUTCH POPS-STUDY AT 19 YEARS OF AGE}

E T M Hille ${ }^{1,2}$, N Weisglas-Kuperus ${ }^{1}$, J B van Goudoever ${ }^{1}$, G W Jacobusse ${ }^{2}$, M H Ens-Dokkum ${ }^{3}, \mathrm{~L}$ de $\mathrm{Groot}^{4}, \mathrm{~J} \mathrm{M} \mathrm{Wit}^{5}$, W B Geven 6 , J H Kok ${ }^{7}, \mathrm{M} J \mathrm{~K}$ de Kleine ${ }^{8}$, L A A Kollée ${ }^{9}$, A L M Mulder ${ }^{10}$, H L M Van Straaten ${ }^{11}$, $\mathrm{L} S$ de Vries ${ }^{12}$, M M van Weissenbruch ${ }^{4}$, S P Verloove-Vanhorick ${ }^{2,5}$

${ }^{I}$ Department of Neonatology, Erasmus MC Sophia Children's Hospital, Rotterdam, The Netherlands ${ }^{2}$ Department of Prevention and Health, TNO Quality of Life, Leiden, The Netherlands, ${ }^{3}$ Royal Effatha Guyot Group, Zoetermeer, The Netherlands, ${ }^{4}$ Department of Neonatology, VU Medical Center, Amsterdam, The Netherlands, ${ }^{5}$ Department of Pediatrics, Leiden University Medical Center, Leiden, The Netherlands
${ }^{6}$ Department of Neonatology, UMCG Beatrix Children's Hospital, Groningen, The Netherlands, ${ }^{7}$ Department of Neonatology, AMC Emma Children's Hospital, Amsterdam, The Netherlands, ${ }^{8}$ Department of Pediatrics, Máxima Medical Center, Veldhoven, The Netherlands, ${ }^{9}$ Department of Pediatrics, UMC St Radboud, Nijmegen, The Netherlands, ${ }^{10}$ Department of Neonatology, University Hospital Maastricht, Maastricht, The Netherlands, ${ }^{11}$ Department of Pediatrics/Neonatology, Isala Clinics, Zwolle, The Netherlands, ${ }^{12}$ Department of Neonatology, Wilhelmina Children's Hospital, University Medical Center Utrecht, The Netherlands

Background and Aims: Young adults born very preterm or with a very-low-birth-weight remain at risk for physical and neurodevelopmental problems and lower academic achievement scores. Data however are scarce, hospital-based, mostly done in small populations and need further confirmation.

Methods: Infants born below 32 weeks of gestation and/or with a birth weight less than 1,500 gram in the Netherlands in 1983 (POPS study), were re-examined at age 19. Outcomes were adjusted for nonrespondents using multiple imputation and categorized into none, mild, moderate and severe problems.

Results: Of 959 surviving young adults, 705 (74\%) were assessed and/or completed the questionnaires. Moderate or severe problems were present in $4.3 \%$ for cognition, $1.8 \%$ for hearing, $1.9 \%$ for vision, and $8.1 \%$ for neuromotor functioning. Using the Health Utility Index and the London Handicap Scale, we found 2.0\% and $4.5 \%$, respectively, of the young adults to have three or more affected areas in activities and participation. Special education or lesser level was completed by $24 \%$, and $7.6 \%$ neither had a paid job nor did they follow any education. Overall, $31.7 \%$ suffered from one or more moderate or severe problems in the assessed areas.

Conclusions: $12.6 \%$ young adults born very preterm and/or with a very-low-birth-weight had moderate or severe problems in cognitive or neurosensory functioning. Nearly all young adults were satisfied with their activities and participation in society. Compared with the general Dutch population twice as many young adults born very preterm and/or with a very-low-birthweight were poorly educated and three times as many were neither employed nor in school at age 19.

\section{MOTOR SKILLS AND VISION, PERCEPTION AND PROPRIOCEPTION IN LOW BIRTH WEIGHT ADOLESCENTS}

K A I Evensen ${ }^{1}$, S Lindqvist ${ }^{1}$, S Kulseng ${ }^{2}$, M Indredavik $^{3}$, A M Brubakk ${ }^{1}$, T Vik ${ }^{4}$

${ }^{I}$ Department of Laboratory Medicine, Children's and Women's Health, Faculty of Medicine, Norwegian University of Science and Technology, Trondheim, Norway, ${ }^{2}$ Department of Paediatrics, St. Olavs University Hospital, Trondheim, Norway, ${ }^{3}$ Department of Neuroscience, Faculty of Medicine, Norwegian University of Science and Technology, Trondheim, Norway, ${ }^{4}$ Department of Public Health and General Practice, Faculty of Medicine, Norwegian University of Science and Technology, Trondheim, Norway 
Background and Aims: Motor problems have been frequently reported in low birth weight populations, and may be related to visual, perceptive or proprioceptive deficits. The aim of this study was to examine the association between motor skills and vision, perception and proprioception in adolescents with low birth weight without cerebral palsy (CP).

Methods: Fifty-one very low birth weight adolescents (VLBW: birth weight $\leq 1500 \mathrm{~g}$ ), 57 term small for gestational age (SGA: birth weight $<10$ th centile) and 77 term control adolescents (birth weight $\geq 10$ th centile) without CP were examined by the age of 14 . We performed regression analyses with the total score of the Movement Assessement Battery for Children (Movement $\mathrm{ABC}$ ) as the outcome variable and the Visuo-Motor Integration test (VMI), the Motor Coordination and Visual Perception supplementary tasks, tests of proprioceptive abilities, visual acuity, contrast sensitivity, strabismus and stereopsis as independent variables.

Results: Backward stepwise regression suggested that motor problems in the VLBW group were significantly explained by poor contrast sensitivity, poor stereopsis and a low score on the Motor Coordination task. In the SGA group motor problems were explained by a low score on the Visual Perception task, whereas poor proprioception was associated with motor problems in the control group.

Conclusions: The results suggest that visual deficits are important factors in explaining motor problems in VLBW and SGA adolescents, whereas visual functions in adolescents with normal birth weight do not seem to limit their motor skills.

\section{SENSORIMOTOR OUTCOME AT 2 YEARS IN EXTREMELY PRETERM INFANTS. THE NORWEGIAN EXTREME PREMATURITY STUDY}

T Markestad ${ }^{1}$, R Støen ${ }^{2}$, A Rønnestad ${ }^{3}$, J Skranes ${ }^{4}$, P I Kaaresen ${ }^{5}$, I Elgen ${ }^{1}$, T Farstad ${ }^{6}$, S Retterdal ${ }^{7}$, I E Silberg $^{8}$, I Fagerli ${ }^{9}$, I E Engelund ${ }^{10}$, L M Irgens ${ }^{10}$

${ }^{l}$ Department of Pediatrics, Haukeland University Hospital, Bergen, Norway, ${ }^{2}$ Department of Pediatrics, St. Olav University Hospital, Trondheim, Norway ${ }^{3}$ Department of Pediatrics, Rikshospitalet University Hospital, Oslo, Norway, ${ }^{4}$ Department of Pediatrics, Ullevål University Hospital, Oslo, Norway, ${ }^{5}$ Department of Pediatrics, University Hospital of Northern Norway, Tromsø, Norway, ${ }^{6}$ Department of Pediatrics, Akershus University Hospital, Lørenskog, Norway, ${ }^{7}$ Department of Pediatrics, Stavanger University Hospital, Stavanger, Norway, ${ }^{8}$ Department of Pediatrics, Atfold Central Hospital, Fredrikstad, Norway, ${ }^{9}$ Department of Pediatrics, Nordland Central Hospital, Bodø, Norway,

${ }^{10}$ Medical Birth Registry of Norway, Locus of RegistryBased Epidemiology, Norway

Background and Aims: Perinatal information was obtained on all but two infants with gestational age (GA) <28 weeks born in Norway in 1999-2000. Sensorimotor outcome was assessed at 2 years.

Methods: All pediatric departments in Norway participated. The children had a neurodevelopmental examination, and predefined outcome data were registered.

Results: 364 of 464 neonates (79\%) were resuscitated, $290(80 \%)$ were discharged. Two died later and 2 were not traced, leaving 286 (99\%) for follow-up. 34 (12\%) had sensorimotor handicaps. 3 of 4 blind had grade 5 or 6 ROP before discharge. 15 of 26 with CP (58\%) had major ultrasound (CUS) pathology as neonates while 11 had normal CUS or grade 1-2 hemorrhage. 19 of 34 with major CUS findings had no CP or hydrocephalus.

Conclusions: Survival and intact survival decreased with decreasing GA. For the survivors the handicap rate increased with decreasing GA except for a low rate at 23 weeks, probably because of a low threshold for stopping treatment. CUS had only modest predictive value.

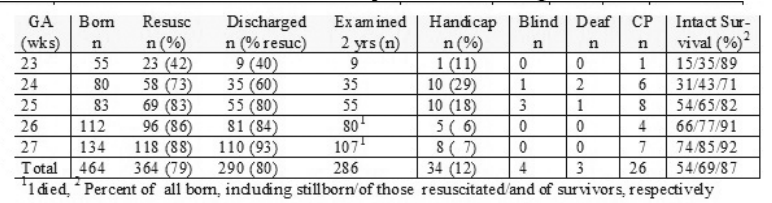

\section{NEURODEVELOPMENTAL OUTCOME OF EXTREMELY LOW BIRTH-WEIGHT (ELBW) INFANTS RANDOMIZED TO HIGH OR LOW BLOOD TRANSFUSION THRESHOLDS (PINTOS)}

R K Whyte ${ }^{1}$, H Kirpalani ${ }^{2}$, E V Asztalos ${ }^{3}$, C Andersen ${ }^{4}$, M A Blajchman ${ }^{2}$, N Heddle ${ }^{2}$, R S Roberts ${ }^{2}$ and The Pintos Study Group 5

${ }^{1}$ Dalhousie University, Halifax, NS, ${ }^{2}$ McMaster University, Hamilton, ON, ${ }^{3}$ University of Toronto, Toronto, ON, Canada, ${ }^{4}$ Mercy Hospital for Women, Melbourne, Australia, ${ }^{5}$ Halifax, NS, Montreal, $P Q$, Kingston, ON, Toronto, ON, Hamilton, ON, Edmonton, AB, Canada, Albany, NY, Brooklyn, NY, USA, Melbourne, Australia

Background and Aims: ELBW infants frequently receive red blood cell transfusions. We previously conducted a multicentre trial (PINT) to compare rates of death or major morbidity at hospital discharge of transfusion at low vs high hemoglobin transfusion thresholds (PAS 2004: 55, LB15); we showed no significant differences in outcomes. In our present study we determined whether or not there were later differences in death or neurodevelopmental outcome at eighteen months corrected age.

Methods: ELBW infants $<48$ hrs of age were randomized to an algorithm of low or high transfusion thresholds, max Hgb $135 \mathrm{~g} / \mathrm{l}$, min $75 \mathrm{~g} / \mathrm{l}$, differing by $10-20 \mathrm{~g} / \mathrm{L}$ depending on age and respiratory support, and maintained until discharge. Infants were followed up for death or neurodevelopmental outcomes at 18 months corrected age.

Results: We randomized 451 infants from 10 NICUs. The groups were well matched for prognostic factors and baseline hemoglobin level. Median (IQR) birthweight was 769 (670-887) g and gestational age 26 (2527) weeks. Groups differed in mean hemoglobin level by approximately $10 \mathrm{~g} / \mathrm{l}$ from weeks one through twelve. 317 of 338 survivors were available for follow up. There were no significant differences in primary (table) or in growth or secondary developmental outcomes. 
Conclusions: Maintaining the hemoglobin of ELBW infants at low rather than high levels with this algorithm does not result in significant differences in survival or neurodevelopmental outcome at 18 months corrected age.

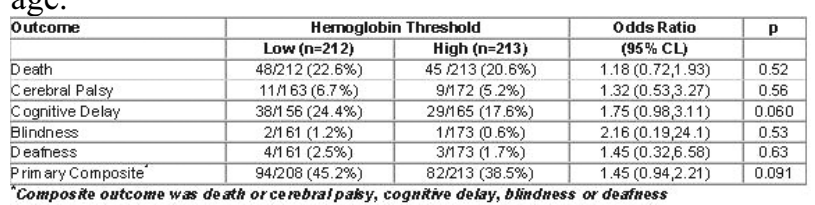

CLINICAL PREDICTORS OF OUTCOME IN A MULTICENTERED RANDOMIZED CONTROLLED TRIAL OF WHOLE BODY HYPOTHERMIA FOR NEONATAL HYPOXICISCHEMIC ENCEPHALOPATHY

\section{S Shankaran}

Hypothermia Subcommittee, NICHD Neonatal Research Network, Bethesda, MD, USA

Background and Aims: Whole body hypothermia reduced the frequency of death or moderate/severe disabilities in neonates with hypoxic-ischemic encephalopathy (HIE). N Engl J Med 2005:353:157484. Predictors of outcome of whole body cooling have not been reported to date. To evaluate a) associations between variables at $<6$ hours of age with outcome at 18 months.

Methods: Variables examined included pre- and perinatal and neonatal variables. Odds ratios and 99\% confidence intervals $(\mathrm{OR}, 99 \% \mathrm{CI})$ were calculated.

Results: The primary outcome was associated with time to spontaneous respiration $>10$ min $5.6(1.4-23.2)$, cord $\mathrm{pH}$ per 0.1 decrease $1.6(1.1-2.1)$, base deficit per 1.0 increase $1.1(1.0-1.3)$, and severe alteration in either level of consciousness 4.7 (1.4 - 15.7), spontaneous activity 5.5 (1.6 -18.7), primitive reflexes $4.1(1.2-14.1)$ or autonomic system 4.4 (1.2 - 16.2). Among control group infants, low Apgar scores at 5 mins 4.8 (1.2 19.8) and 10 mins $6.1(1.6-23.1),>10$ mins to spontaneous respiration $10.1(2.6-38.8)$ and severe alteration in level of consciousness 8.9 (2.2 - 36.6), spontaneous activity $6.0(1.6-22.8)$ or primitive reflexes 6.6 (1.9 - 22.6) were correlated to outcome. Perinatal variables, seizures, out-born birth and time to cooling did not impact outcome.

Conclusions: Predictors of outcome for infants undergoing whole body hypothermia for neonatal HIE include increasing neonatal acidosis, prolonged time to spontaneous respiration, and components of the neurological exam demonstrating encephalopathy. Maternal variables were not predictive. These findings will assist in planning future trials of hypothermia for HIE.

\section{PSYCHOLOGICAL FOLLOW-UP AFTER PREMATURE BIRTH: MATERNAL} EVALUATION 7 YEARS AFTER DISCHARGE

S Denizot $^{1}$, V Lequen ${ }^{1}$, G Caillaux-Varin ${ }^{1}$, V Bureau ${ }^{2}$, E Lemauff ${ }^{2}$, J C Rozé ${ }^{1}$

${ }^{1}$ Service de Néonatologie et Réanimation Néonatale, Hopital Mère Enfant, CHU de Nantes, France
${ }^{2}$ Réseau Grandir Ensemble en Pays de Loire, Hopital Mère Enfant, CHU de Nantes, France

Background and Aims: Premature birth is stressful and associated with more long-term stress. To determine, 7 years after discharge, the maternal psychological consequences of an hospitalisation of a premature infant less than $32 \mathrm{GA}$.

Methods: 66 mothers (mean age 30 years) corresponding to 82 infants (mean gestational age $30+/$ 2.5 weeks, mean birth weight $1435+/-417$ grams) from the EPIPAGE cohort in Pays de Loire were eligible and answered a phone semi structured interview 7 years after discharge (mean neonatal hospitalisation stay 55 +/- 28 days). We analysed all answer taking neonatal characteristics and 5 years follow-up of infant into account.

Results: Remember of neonatal stress remain painful 7 years after discharge. Young maternal age, caesarean section, maternal hospitalisation in USI, and neonatal death of a twin are associated with a difficult emotional feeling. Strictly medical information as CRIB score $(p=$ $0.001)$ and ultrasound neurological abnormalities $(\mathrm{p}=$ 0.003 ) are significantly associated with an increase psychological stress. Emotional reactivation of this stress is still observed 7 years after discharge whatever the presence or not of poor 5 years-outcome. Maternal satisfaction of care givers is better for high risk infants than infants of birth weight $>1500 \mathrm{~g}(\mathrm{p}=0.041)$.

Conclusions: There is practical implications to support parents precociously during hospitalisation and to continue the long-term psychological follow-up. It remains most important for care givers to enhance the quality of medical information even in low risk situation.

\section{MORAL DILEMMAS IN ETHICAL DECISION MAKING FOR NURSES: ROLES AND MODELS}

J E De Vos, A Van Den Hoogen

Department of Neonatology, WKZ, UMC Utrecht, The Netherlands

Background and Aims: Nurses roles in ethical decision making and how nurses are defining an opinion in moral dilemmas is an issue in neonatology and pediatrics. Moral dilemmas arise in discussions on whether or not to start or to continue a treatment for infants in an intensive care unit.

Methods: Two qualitative studies were done. The results were analysed by the method of Maso (1994). Data generation took place by means of semi-structured interviews with nurses working in the NICU of the Wilhelmina Kinderziekenhuis (University Medical Centre in Utrecht) and by literature study. The moral model used was the so called 'Model of Nijmegen' for moral reasoning. The theories on justice and care perspective of Kohlberg, 1984 and Gilligan, 1985 make up the study's theoretical framework.

Results: Nurses mainly act as representatives for the children. They are supporting, assisting and caring. In their moral reasoning nurses especially focus attention on the child. In their considerations the following aspects play a role: the present situation, the future 
situation, and the child's environment (parents). The study's further explores and explains the aspect 'the child's pain and suffering' and 'looking at the child' as a part of the specific know-how of nurses.

Conclusions: Nurses need to emphasize more on their own role and strenghten that role. Integration and participation of nurses in moral discussions is therefore open to improvement. The possibility to discuss emotional aspects is warranted.

\section{PARENTAL PRESENCE DURING RESUSCITATION}

\section{J M Latour}

\section{PICU, Erasmus MC - Sophia Children's Hospital, Rotterdam, The Netherlands}

Background and Aims: The current resuscitation guidelines are supportive to parental presence during cardiopulmonary resuscitation (CPR). However, the subject remains a controversial issue. The aim is to determine the pros and cons of the healthcare professionals' view of witnessed CPR, to discuss the parental empowerment and the impact on parents, and to assess the implication of the current CPR guidelines regarding parental presence during resuscitation.

Methods: A literature search on parental presence during CPR was performed. Particular on the issues of the professionals' views and attitudes, the impact on family and parents and the available position statements of organisations.

Results: Social and cultural differences are diverse and may influence the parents' view on witnessed CPR. However, there is a trend that parents do not like to leave their child. Those parents who have witnessed CPR, would wish to stay again. Parental presence during resuscitation seems to have no long-term adverse effects. Among professionals, controversies remain on this subject. Arguments against parental presence range from traumatic experience of parents to disturbance of professional performance. However, there is a tendency to give parents the choice to stay. Most articles conclude with the words 'allow, support, encourage, give the opportunity' to present the view on parental witnessed resuscitation. Guidelines are therefore necessary to state the departments' vision and practice. An increasing tendency is observed towards the development of official position statements. Supposing that the current data to support parental presence during CPR is insufficient, the latest CPR guidelines provide a positive advice towards parental presence.

Conclusions: Although still controversial in many countries, there is a strong and supportive trend towards parental presence during resuscitation. More evidence becomes available on attitudes and the effects on parents and staff. It is recommended to create guidelines on the principles of parental presence during resuscitation.

\section{HONESTY WITH MERCY}

\section{E Knight}

Evelina Children's Hospital, London, UK
Background and Aims: Honesty can be considered to exist along a continuum ranging from dishonesty to honesty. Every person has their own individual place on this continuum, dictated by their individual backgrounds and experiences. It is hoped to encourage bedside intensive-care nurses to identify where they are on this continuum and to actively participate in helping render patients and their families more "ready" for receiving and coping with bad news.

Methods: The dishonesty/honesty continuum is identified. With examples from the author's own nursing experiences, her movement along this continuum is illustrated giving rise to her current belief in honesty with mercy in nurse relationships with patients and their families. This illustration focuses on three aspects of honesty: truth-telling, timing and lying. Also, it highlights the unique position intensive-care nurses have at the bedside. They often have unique knowledge of the patients' and families' wishes to know information and the amounts that will be manageable by them. Similarly, often they can create, or be presented with, unique opportunities to help vulnerable patients and their families become receptive to disclosure of information.

Results: Ideally, practitioners present will: a) reflect on, and justify, where they are on the dishonesty/honesty continuum; and b) identify ways they can enhance their use of their unique positions in helping patients and families receive bad news.

Conclusions: Bedside nurses can help render patients and their families more "ready" for bad news and should actively take on responsibility for this.

\section{CHALLENGING THE THEORETICAL BASIS OF THE PHILOSOPHY OF 'PARTNERSHIP WITH PARENTS'}

I Coyne, J A Conlon

School of Nursing, Dublin City University, Ireland

Background and Aims: Parent participation is a key issue in children's health care, both in Ireland and internationally. Although nurses are expected to enter into partnership with families in the provision of care, research on parental participation from the UK, Sweden, Australia and from the USA indicates that parental participation is a complex, multidimensional and problematic concept that nurses find difficult to understand and apply to practice. This research focuses on developing an understanding of parents and childrens experiences of consultation and involvement in care during periods of hospitalization.

Methods: Previous research in this area has been descriptive therefore a grounded theory approach was used to develop a theoretical perspective on parent participation in order to explain the underlying process. The data collected contributed to the theoretical framework which Dr. Imelda Coyne developed in her $\mathrm{PhD}$, which enhanced the applicability and generalisability of the theory across many paediatric settings.

Results: Results indicate that there is a concerning lack of understanding about the parents role during a childs hospital admission. Over reliance on parents to 
participate, a lack of clarity of the concept 'Partnership' and factors outside the nurses control are also causing undue distress.

Conclusions: Parents involvement should be voluntary and not necessary. A number of factors need to be addressed in order to ensure this, namely documentation, staffing levels and a greater understanding of the concepts particiaption and partnership.

\section{INVESTIGATION OF CHILDREN'S EXPERIENCES OF INVOLVEMENT AND CONSULTATION IN THEIR HEALTH CARE WITHIN THE HOSPITAL SETTING IN IRELAND}

\section{T Coyne, E Hayes, P Gallagher \\ School of Nursing, Dublin City University, Ireland}

Background and Aims: The past decade has seen the importance of children's rights to participation and consultation established both nationally and internationally. Yet the evidence suggests that children's participation may be more rhetoric than reality. There is limited empirical data on hospitalised children's participation in consultations and decision-making in Ireland. Hence this paper reports on children's experiences of involvement and consultation in their health care in hospital. Ensuring that the views of children are taken into account in matters affecting them is consistent with the vision of the Irish National Children's Strategy, which is "an Ireland where children are respected as young citizens with a valued contribution to make, and a voice of their own" (2000, p4). Aims: (a) To describe children's experiences of consultation in the healthcare setting. (b) To identify the factors that enhance or hinder children's involvement in consultation and the decision making process. (c) To explore strategies that will facilitate children's participation in their own health care decisions.

Methods: The data were collected through focus groups, single interviews, and questionnaires. Data were obtained from children $(n=55)$ aged from 7 to 18 years from ten paediatric wards in three hospitals in Ireland.

Results: The findings revealed that the children wanted to participate in consultations and decisions about their care, but had varying experiences of involvement. The children generally experienced problems in having their voices heard and appeared to occupy a marginalised position in healthcare interactions.

Conclusions: The findings will be presented and reviewed in light of existing theoretical frameworks and published research.

\section{DILEMMA, AN ORGANISATION SUPPORTING PARENTS OF SERIOUSLY ILL OR HANDICAPPED CHILDREN DURING THE PROCESS OF DECISION MAKING}

\author{
N E C Visée ${ }^{1}$, M J K De Kleine ${ }^{1,2}$ \\ ${ }^{I}$ Dilemma Foundation, Utrecht, The Netherlands \\ ${ }^{2}$ Department of Neonatology, Máxima Medical Centre, \\ Veldhoven, The Netherlands
}

Background and Aims: End-of-life decisions precede death in two third of the deaths of children below one year of age and in an unknown percentage of children that do not die. Although many of these decisions are taken in good cooperation between parents and doctors, both the process of decision making and the decisions themselves can create an enormous burden for the parents. During the process of decision making, caregivers frequently disagree, leaving the parents in even greater distress. In the Netherlands, termination of life in newborns with hopeless and unbearable suffering is considered a criminal offence, even if it takes place on explicit request of the parents.

Methods: Dilemma is a foundation that supports parents that have questions about life and death of their seriously ill or handicapped child, either before or after birth. Support is provided in the broadest sense, varying from offering an opportunity to talk, to helping in finding professionals who can mediate or take over the existing treatment. Dilemma runs a telephone number, situated at the desk of the Dutch Paediatric Association. The foundation's backbone is a number of volunteers and a network of independent professional experts.

Results: During the last 10 years some 30 persons contacted Dilemma each year. Of all questions, 30\% regarded information, $50 \%$ counseling and $20 \%$ explicitly requested ending of treatment or ending of life. The content of the questions regarded medical facts like prognosis of the disease, risks of operations, possibilities of other treatments; legal questions like rights of parents, rights of doctors to continue or end treatment and the procedure of a law suit. Counseling was sometimes very practical like helping to arrange a burial of a very young fetus after abortion or providing for a second opinion. In all cases the counseling included the need for conversation, trust and support. The requests for life ending measures lead to extended discussions about future perspective of the child, benefit of treatment, meaning of life and the amount of suffering a human may endure. In turmoil of feelings, anxiety, agony, lost of trust, guilt, love and resignation alternate. Controversies between parents and doctors have been arisen on continuing or withholding treatment, parents' request for purposely ending the life of their child, insufficient communication, counseling or support and confusion about the medical treatment.

Conclusions: We conclude that there's a consistent and urgent need for consultation and information with parents who are involved in decisions regarding the (end-of-life in) sick child. Dilemma provides support in the broadest sense. In our opinion, the information about the support that parents seek and the results of our interventions can be of great value to health care providers who seek to improve the decision making process in sick newborns. 
PNEUMOCOCCAL CONJUGATE VACCINE: IMPACT OF PREVENAR IN NORTH AMERICA

\section{J D Kellner}

Alberta, Canada

Please refer to page 119a.

\section{THE MANAGEMENT OF FEBRILE CHILDREN IN THE PNEUMOCOCCAL CONJUGATE VACCINE ERA}

\section{Isaacman}

\section{Wyeth Pharmaceuticals, Collegeville, PA, USA}

The heptavalent pneumococcal conjugate vaccine (Prevenar) has lead to a marked decrease in the incidence of invasive pneumococcal disease, and significant changes in the epidemiology of occult bacteremia. This talk will review the clinical implications of reductions in invasive pneumococcal disease as they affect the practicing clinician. Particular emphasis will be dedicated to the impact of reductions of pneumococcal bacteremia and meningitis on the management of fever without source in the child 3-36 months of age.

\section{CHANGES IN THE EPIDEMIOLOGY OF INVASIVE PNEUMOCOCCAL DISEASE IN SPAIN}

\section{F Gimenez}

Servicio de Pediatría, Hospital Torrecardenas, Almería, Spain

Objective: To describe the global situation of pneumococcal disease and also the impact of 7-valent pneumococcal conjugate vaccine (7vPn-CRM) in Spain $5 y$ after its commercialization.

Methods: We describe status disease going through all Spanish data published and accessible. We have to take into account an important issue: IPD including meningitis is not a notifiable disease, therefore is difficult to obtain pre-commercialization data to compare with postcommercialization data. It is also important to note that nowadays vaccine coverage is unknown.

Results: In a study conducted in Navarre and Basque Country, by the second year after 7vPn-CRM introduction, comparing with pre-vacunal period (19982001) the incidence of IPD decreased by $69.2 \%, 42.0 \%$ and $39.0 \%$ in children $<1 \mathrm{y},<2 \mathrm{y}$, and $<5 \mathrm{y}$ respectively. Focused on Navarre data until 2005 we do not observe this decrease. The explanation is that the data collection methodology in Emergency Room Units has substantially varied, increasing the number of blood cultures performed, so adjusting IPD rates by blood cultures you could obtain more reliable data on changes of incidence (Fig.1).
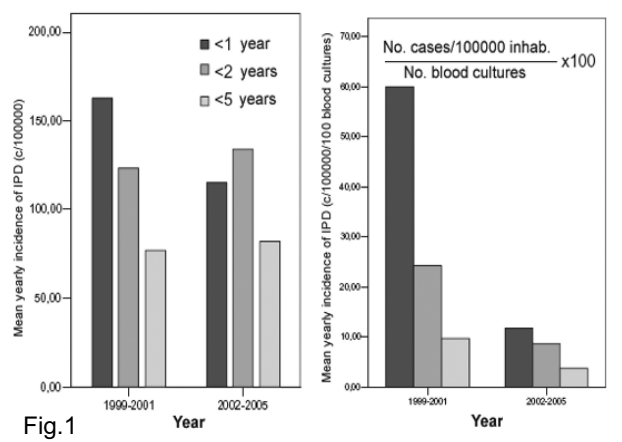

In Madrid based on the data from hospital discharges obtained by the Basic Minimal Data Set, a progressive decrease on the incidence of IPD is detected from 1999 onwards, mainly due to a decrease in pneumococcal pneumonias. This data along time are among the scarce data published. The decrease of the incidence affects all age groups in Madrid, being the most significant decrease in children $<2 \mathrm{y}$ and $<5 \mathrm{y} ; 30.7 \%$ and $28.9 \%$ respectively In the Basque Country and as reported in its Epidemiology Official Report 2003, the incidence of IPD considerably decreased in 2002: $73.8 \%$ in children $<5 y$. The currently available data in this Region at the end of 2005 , collected in public and private hospitals, indicate that the reduction in 2005 with respect to 2000 in children $<5 \mathrm{y},<2 \mathrm{y}$ and $<1 \mathrm{y}$ are: $55.1 \%, 65.6 \%$, and $72.8 \%$ respectively.

An increase in incidence and severity of parapneumonic pleural empyemas has been observed and described in the international literature for years before the beginning of the implementation of the 7vPn-CRM. In Spain there are few data about pneumonia or empyema, according to the data published it cannot be inferred that bacteremic pneumococcal pneumonia has increased or that empyemas are due to Streptococcus pneumoniae. This situation has been described in countries such as UK, which has not implemented 7vPn-CRM. There is no study in the literature that can relate the increase of pneumococcal pneumonia complicated with empyema with the implementation of $7 \mathrm{vPn}-\mathrm{CRM}$.

Conclusions: A decrease of the incidence of invasive pneumococcal disease (IPD) has occurred in Spain, although to a lesser degree than that recorded in countries such as the USA, which have greater vaccine coverage. In long-term surveillance studies is necessary adjusting the results by methodologies changes (blood cultures, PCR...). The disease burden must continue to be monitored and analyzed according to the characteristics of the epidemiological vigilance methods developed in each Region. The $\mathrm{BoH}$ must establish a pneumococcal surveillance system in Spain to control the pneumococcal disease at all ages. To conclude, the Health Authorities should introduce 7vPn-CRM in the National Immunization Programme in order to be able to control more effectively severe childhood pneumococcal disease, as is done in other surrounding countries. 
HONORARY LECTURE: THE IMPACT OF PRENATAL AND POSTNATAL STEROIDS AND SURFACTANT REPLACEMENT THERAPY ON THE OUTCOME OF PRE-TERM INFANTS

\section{E Shinwell}

\section{Professor of Neonatal Paediatrics, Department of Neonatology, Kaplan Medical Center, Rehovot and Hebrew University, Jerusalem, Israel}

Background and Aims: Bengt Robertson is a pioneer of research in lung disease of premature infants. His work has revolutionized the management and outcome of Respiratory Distress Syndrome (RDS) and Bronchopulmonary Dysplasia (BPD). In his honor, the ESPR established this year a Young Investigator's Award to stimulate future leaders in the field. This lecture will trace the development of the major interventions in the field - surfactant replacement and pre- and postnatal steroid therapy. The current state of the art will be reviewed, together with emphasis on the most recent controversial issues of the day.

Methods: Data will be presented from specific studies, meta-analyses and systematic review of each of three issues and their interactions (surfactant, prenatal and postnatal steroids)

Results: Surfactant therapy significantly reduces the severity of RDS and its associated mortality. Current unresolved issues to be discussed include the role of surfactant therapy in Meconium Aspiration Syndrome and Adult-type RDS, the use of surfactant at 23-25 weeks of gestation and the definition of the requirements for an effective synthetic surfactant preparation based on phospholipids combined with peptides that simulate the biophysical function of SP-B and/or SP-C. Antenatal steroid therapy significantly reduces the incidence of RDS, Intraventricular Hemorrhage and mortality. Current unresolved issues that will be discussed include the possibly inadequate effect of current recommended doses in multiple pregnancies (Do we need a higher dose?) and comparison of the effect of different steroid preparations on White Matter Injury in the premature neonate. Postnatal steroid therapy significantly reduces the severity of Bronchopulmonary Dysplasia. However, early postnatal steroid therapy is associated with increased risk for Cerebral Palsy and neurodevelopmental delay. Current issues that will be discussed include the effects of reduced steroid usage at a population level and the identification of a specific high-risk group in whom the benefits of therapy may outweigh the attendant risks.

Conclusions: The prevention and management of respiratory distress in premature infants has progressed by leaps and bounds, thanks to pioneering investigators such as Bengt Robertson and others. However, the current controversies leave much work for future Young Investigators.

\section{ALLERGY AND THE GUT}

A Nieto
Paediatric Allergy Unit, Childrens Hospital La Fe,
Valencia, Spain

Background and Aims: The gut is the way through which the greatest number of substances, and the highest quantities of them come into the body. Many of these substances have high allergenic properties. At the same time considerable number of substances that reach the gut, have enzymatic activity. Thus it is possible that these substances are able to induce injuries in the intestinal mucosa, so disrupting the intestinal barrier, and permitting the pass of allergens into the circulation. Furthermore, there are many evidences that suggest that Allergy is a systemic disease, with a local expression. Thus, and considering the allergic child as a whole, it is convenient to consider the gut, not as a isolated entity, but more properly as an organ interconnected with others which constitute the integral Mucosal Immune System (MIS). The interactions between different organs involved in the MIS are critical in the development of localized symptoms. In consequence, from an allergologic point of view, the gut should be observed: A) As a barrier element, that blocks the pass to the systemic circulation of substances able to develop systemic or remote localized allergic symptoms. B) As a crucial element for the development of immunologic tolerance during the first days/weeks of life. C) As a direct target organ for digestive allergic sensitizations. D) As an indirect target organ for allergic sensitizations acquired through other ways. In the case of predominant digestive symptoms, diagnostic difficulties are remarkable, because of: The similarity of digestive allergic symptoms with other gastro-enterologic diseases. The existence of different immunologic mechanisms involved in the development of food hypersensitivity phenomena. The possibility that the hypersensitivity phenomenon be locally restricted, without a detectable systemic reflection. This can happens in the earlier stages of sensitization.

Conclusions: Anyway, considering the growing prevalence of allergic disease in industrialized countries, it is conceivable that digestive allergy is following the same trend. In consequence, paediatricians should be aware about this possibility in cases or recurrent or persistent gastrointestinal symptoms, particularly when the child has concomitant allergic manifestations in other organs.

\section{ORAL TOLERANCE: WHERE ARE WE?}

A Muraro

Department of Pediatrics, University of Padua, Italy

Abstract not available at the time of printing.

\section{SUCCESSFUL THERAPY OF UNSPECIFIC PROLONGED DIARRHOEA IN INFANTS AND TODDLERS WITH THE PROBIOTIC E. COLI NISSLE 1917}

\author{
J H Henker ${ }^{1}$, B M Blokhin ${ }^{2}$, Y K Bolbot ${ }^{3}$, \\ V G Maydannik $^{4}$, C W Wolff ${ }^{5}$, J S Schulze ${ }^{5}$ \\ ${ }^{1}$ Universitätsklinikum Carl Gustav Carus Der TU \\ Dresden, Germany, ${ }^{2}$ Russian State Medical University, \\ Moscow, Russia, ${ }^{3}$ Dnepropetrovsk State Medical \\ Academy, Dnepropetrovsk, Ukraine, ${ }^{4}$ National Medical
}


University O.O. Bogomolets, Kyiv, Ukraine

${ }^{5}$ Ardeypharm, Herdecke, Germany

Background and Aims: So far no causal therapy exists for infants and toddlers with prolonged diarrhoea. Therefore in a confirmatory, randomized, double-blind clinical trial the efficacy of the probiotic bacterial strain E. coli Nissle $1917(\mathrm{EcN})$ as compared to a placebo was tested.

Methods: 151 children (aged 1 to 47 months, Ø 25) with unspecific prolonged diarrhoea $(>3$ watery or loose stools without blood in 24 hours of a diarrhoeal episode, which has been persisting for more than 4 consecutive but less than 14 days) were randomized to receive either $\mathrm{EcN}$ suspension $(\mathrm{n}=75)$ or placebo $(\mathrm{n}=76)$. All children were dehydrated to a medium extent ( $5-10 \%$ loss of body weight) and received 1 to 3 $\mathrm{ml}$ verum or placebo orally per day depending on the age $(1 \mathrm{ml}$ verum contained 10E8 viable EcN bacteria) for 21 days. At study commencement, rehydration (ORL according to WHO) was performed once.

Results: Already on day 7 more patients in the $\mathrm{EcN}$ (78.7\%) than in the placebo group (59.2\%) showed a reduction of stool frequency to less than 3 watery or loose stools in 24 hours over a period of at least 4 consecutive days. The response rate increased continuously as was measured on days $14(\mathrm{EcN} 93.3 \%$, placebo $65.8 \%)$ and $21(\mathrm{EcN} 98.7 \%$, placebo $71.1 \%)$. The two-sample test of rates following a group sequential test design showed statistically significant superiority for $\mathrm{EcN}$ on both days $14(\mathrm{p}=0.0017)$ and 21 $(\mathrm{p}<0.0001)$.

Conclusions: Prolonged diarrhoea can be successfully treated with the probiotic $\mathrm{EcN}$.

\section{THE NATURAL HISTORY OF COW'S MILK PROTEIN SENSITISATION IN INFANTILE ALLERGIC COLITIS - AN ASSOCIATION WITH MULTIPLE FOOD ALLERGY?}

S L Chuang ${ }^{1}$, P Hayes ${ }^{3}$, J Falconer ${ }^{3}$, M Haddad $^{3}$, T T MacDonald ${ }^{2}$, J M Fell ${ }^{3}$

${ }^{I}$ Department of Neonatology, Chelsea and Westminster Hospital, London, UK, ${ }^{2}$ Division of Infection,

Inflammation, and Repair, University of Southampton School of Medicine, Southampton, UK, ${ }^{3}$ Department of Paediatric Gastroenterology, Chelsea and Westminster Hospital, London, UK

Background and Aims: To document the clinical outcome and evidence of persisting dietary sensitization, in particular, cow's milk protein (CMP), in infants with allergic colitis (AC).

Methods: We carried out a prospective study with comprehensive immunological, endoscopic, clinical evaluation and follow up for assessment of in vivo/in vitro evidence of CMP-sensitivity. $12 \mathrm{AC}$ were diagnosed, median onset at 6 weeks (2 days-7 months). Clinical CMP-sensitivity was defined by resolution of bloody diarrhoea on CMP exclusion (with maternal dairy exclusion or amino-acid formula) and subsequent rechallenge (open in breast-fed, double-blind challenge in amino-acid formula fed). Clinical reactions to sequential dietary antigen introduction during weaning (soya/wheat/egg etc.) were documented.

Results: 10/12 had CMP-sensitivity, 5/10 showed positive Th1/Th2 blood mononuclear responses (IFN- $\gamma$, IL-4, IL-5 cytokine production by ELISPOT to casein and/or beta-lactoglobulin), 3/10 with Th2 lamina propria CMP responses. 9/10 CMP-sensitive-AC were followed up to median $23 \mathrm{~m}(8-34 \mathrm{~m})$. One infant was lost to follow-up before CMP rechallenge. CMP was tolerated in 3 cases by $12 \mathrm{~m}, 1$ case by $18 \mathrm{~m}$ and 2 cases by $24 \mathrm{~m} .3$ cases remained CMP-sensitive at 21,23 and $34 \mathrm{~m}$. Multiple food allergy ( $\geq 2$ antigens) with gastrointestinal and/or cutaneous manifestations, both immediate and delayed-onset, were observed in $6 \mathrm{CMP}$-sensitive cases (soya, $\mathrm{n}=5$; eggs, $\mathrm{n}=3$; others, $\mathrm{n}=2$; wheat, $\mathrm{n}=0$ ). Unrestricted diet was achieved in just 4/8 CMPsensitive-AC in the follow-up.

Conclusions: In this group of AC with early CMPsensitisation, clinical resolution and dietary tolerance were variable and often delayed. Although this may reflect a selective population, it highlights the importance of longer term follow-up despite CMP tolerance by $1 \mathrm{yr}$.

\section{DECREASED HEALTH RELATED QUALITY OF LIFE IN OBESE ADOLESCENTS}

M de Beer ${ }^{1}$, G H Hofsteenge ${ }^{2}$, J W Twisk ${ }^{3}$, H A Delemarre-van de Waal ${ }^{1}$, R J Gemke ${ }^{1}$

${ }^{1}$ Department of Pediatrics, ${ }^{2}$ Department of Dietetics, ${ }^{3}$ Department of Clinical Epidemiology and Biostatistics, $V U$ University Medical Center, Amsterdam, The Netherlands

Background and Aims: Health-related-quality-of-life (HRQoL) is an important indicator of chronic illness, complementary to clinical variables. Although overweight and obesity affect HRQoL, very few studies have assessed this in children and adolescents. In this study we compared HRQoL of obese adolescents with normal weight controls and we explored the relation of Body Mass Index (BMI) with HRQoL.

Methods: Cross-sectional study in 31 adolescents (12-18 yrs), referred for obesity, compared with 62 age and sex matched controls. HRQoL was assessed using the PedsQL and Child Health Questionnaire (CHQ). The main outcome measure was difference in reported HRQoL between the 2 groups and the association of BMI with HRQoL in both groups.

Results: Mean BMI (SD) in patients vs controls was $34.9(8.8)$ vs $19.5(2.2) \mathrm{kg} / \mathrm{m} 2$.* In obese adolescents, lower HRQoL in 3 PedsQL and 3 CHQ scales were found.* Variance in HRQoL scales that was explained by obesity ranged from $8 \%$ to $17 \%$. BMI z-score was inversely correlated with 5 PedsQL and 6 CHQ scales* while the percentage of scale variance explained by BMI z-score ranged from $5 \%$ to $23 \%$. ${ }^{*}$ p. $<0.05$.

Conclusions: HRQoL in obese adolescents is significantly poorer than in normal weight controls and is partially explained by obesity-related comorbidity. Overall HRQoL was inversely associated with BMI. When confirmed in other studies (change in) HRQoL will become an important indicator of impact of obesity and effect of interventions, complimentary to clinical variables. 


\section{EFFECT OF DOMPERIDONE ON \\ GASTROESOPHAGEAL REFLUX IN \\ NEWBORNS ASSESSED BY COMBINED \\ INTRALUMINAL IMPEDANCE AND PH MONITORING}

F Cresi, C Marinaccio, F Savino, L Silvestro, $\mathrm{R}$ Miniero

Department of Pediatrics, University of Turin, Italy

Background and Aims: Gastroesophageal reflux (GER) is common in the neonate. If conservative procedures fail to relieve it, the use of a prokinetic agents is justified. Domperidone has been shown to increase LES pressure, to improve esophageal peristalsis and gastric emptying in children. Aim of this study was to evaluate effect of domperidone on gastroesophageal reflux in newborns assessed by combined intraluminal impedance (MII) and $\mathrm{pH}$ monitoring.

Methods: We performed a paired case control study in 26 formula fed newborns with GERD symptoms referred to our Neonatal Care Unit. The patients were consecutively enrolled and alternatively assigned to the case or control group. MII and $\mathrm{pH}$ monitoring were followed for $24 \mathrm{hr}$. Patients in the treatment group received domperidone $(0.2 \mathrm{mg} / \mathrm{Kg}$ dose $\mathrm{q} 8 \mathrm{hr})$. Simultaneous MII and $\mathrm{pH}$ monitoring identified reflux episodes and illustrated their duration, height and $\mathrm{pH}$. Cases were matched to the control group. They were paired by gestational age and weight. Differences were evaluated with the t-test for paired values with $\mathrm{p}<0.05$ as the significance cut-off. Written consent was obtained from the parents.

Results: Twenty-six (13:13) neonates were studied for a total of 613,3 hours. The mean reflux duration was lower for cases than controls $(15,0 \pm 11,6$ vs $21,44 \pm 11,3$ $\mathrm{sec})$, the reflux frequency was higher for cases than controls $(4,3 \pm 1,6$ vs $3,0 \pm 2,5$ episodes/hr), There were no differences refluxes $\mathrm{pH}$ and height between the two groups.

Conclusions: Efficacy of domperidone in GER newborns needs to be reconsidered, since prokinetics could amplify the gastroesophageal dismotility related to immaturity of gastro-esophageal motor function in these patients.

\section{INHIBITORY EFFECT THE HERBAL FORMULATION OF MATRICARIA RECUTITA, FOENICULUM VULGARE MELISSA OFFICINALIS ON UPPER GASTROINTESTINAL TRANSIT IN MICE IN VIVO}

\author{
R Capasso $^{2}$, F Savino ${ }^{1}$, F Capasso ${ }^{2}$ \\ ${ }^{I}$ Department of Pediatrics, University of Turin, Italy, \\ ${ }^{2}$ Department of Experimental Pharmacology, University \\ of Naples, Italy
}

Background and Aims: Clinical evidence suggests that a phytotherapeutic formulation containing Matricaria recutita flowers extract, Foeniculum vulgare fruit extract and Melissa officinalis aerial parts (ColiMil@) is effective in the treatment of breastfed colicky infants (1). We have now investigated the effect of different dosages of herbal constituents on upper gastrointestinal transit in mice in vivo to enhance our acknowledgement on its supposed dose-dependent effect.

Methods: We have used 16 groups of 10 Male ICR mice (20-22 g) for each experimental group. The mice received orally $0.1 \mathrm{ml} / 10 \mathrm{~g}$ mouse of a black marker ( $10 \%$ charcoal suspension in 5\% gum arabic) $1 \mathrm{~h}$ before oral administration of phytotherapeutic formulation (0.1-0.4 $\mathrm{ml} /$ mouse), Matricariae recutita (0.22-0.89 $\mathrm{mg} /$ mouse), Foeniculum vulgare (2.05-8.21 mg/mouse) or Melissa officinalis (1.61-6.46 mg/mouse). After 20 min the mice were killed by asphyxiation with $\mathrm{CO} 2$ and the gastrointestinal tract removed. The distance travelled by the marker was measured and expressed as a percentage of the total length of the small intestine from pylorus to caecum.

Results: The phytotherapeutic formulation dosedependently delayed upper gastrointestinal motility [ $(\%$ inhibition) $0.1 \mathrm{ml} /$ mouse: $15 \% \pm 6,0.2 \mathrm{ml} /$ mouse: $26 \%$ $\pm 5 ; 0.4 \mathrm{ml} /$ mouse: $34 \% \pm 4](\mathrm{P}<0.05)$. Among the herbal components, Matricariae recutita and Melissa officinalis, but not Foeniculum vulgare, reduced motility.

Conclusions: It is concluded that the phytotherapeutic formulation reduced upper gastrointestinal motility in mice with a dose-dependent effect. These experimental data may be important to better understand the observation that a phytotherapeutic formulation improves colic in breastfed infants. Reference: 1) Savino F. et al Phytother. Res. 2005;19:335-340.

\section{LUNG DEVELOPMENT AND INFLAMMATION}

\section{A H Jobe \\ Professor of Neonatal Paediatrics, Department of Pulmonary Biology, Cincinnati Children's Hospital Medical Center, Cincinnati, OH, USA}

Background and Aims: Inflammation is central to virtually all lung injury syndromes in the newborn and adult. I will focus on antenatal and early postnatal inflammatory effects on the preterm lung, as the effects are directly relevant to bronchopulmonary dysplasia (BPD). The chorioamnionitis frequently associated with preterm delivery has two disparate effects on the fetal lung - an injury similar to mild BPD with alveolar simplification and microvascular injury and induced lung maturation. The lung maturation results in less clinical RDS but many of these infants progress to BPD, perhaps because they have augmented inflammatory responses to postnatal pro-inflammatory stimuli such as resuscitation, lung stretch, and oxygen exposure.

Methods: We have used fetal sheep models to demonstrate that intra-amniotic pro-inflammatory mediators such as E. coli endotoxin, IL-1, or Ureaplasma organisms cause chorioamnionitis and lung inflammation. Following the fetal exposures to intraamniotic mediators, the fetal lungs are assessed for inflammation (inflammatory cells, cytokine expression, vascular injury), and lung maturation (gas exchange, lung gas volumes, surfactant).

Results: The lung maturation requires the recruitment of inflammatory cells to the fetal lung (demonstrated by blocking CD-18 or IL-1 receptors). Lung inflammation is mild, even with repetitive exposures to the mediators. 
The inflammatory exposure also induces maturation of monocytes to alveolar macrophages and modulates systemic and lung innate immune responses. Ventilation of the preterm sheep lung with tidal volumes of 15 $\mathrm{ml} / \mathrm{kg}$ also initiates recruitment of monocytes and granulocytes and induces pro-inflammatory cytokine expression. Subsequent ventilation even after surfactant treatment further increases the inflammatory response. Any mechanical ventilation of the preterm lung likely further promotes inflammation and injury, as does oxygen exposure.

Conclusions: The combination of antenatal and/or resuscitation induced injury and modulation of innate inflammatory responses in the developing lung contribute to the development of BPD.

\section{SURFACTANTS TODAY AND TOMORROW}

\section{H L Halliday}

Royal Maternity Hospital and Honorary Professor of Child Health, Queens University Belfast, Northern Ireland

Background and Aims: Surfactant treatment is now about 25 years old and it has undoubtedly had a major impact on neonatal care and outcomes. The aim was to search for recent clinical trials of surfactant treatment and report their results.

Methods: Recent was defined as published between 2000 and 2005. PubMed was searched on 31st December 2005 using the following terms: surfactant treatment, neonate and clinical trials, with limits of years 2000 to 2005 and age newborn to 1 month. Randomised clinical trials (RCTs) and systematic reviews of RCTs were given priority and studies in children, adults and animals were excluded.

Results: Of the 175 papers found in the search about half were directly related to some aspect of surfactant treatment. Just over one-half of these were either RCTs or systematic reviews. Three of the 34 RCTs of surfactant treatment were excluded as they involved children or animals. Twenty-nine RCTs studied preterm babies with RDS and 2 RCTs term neonates with meconium aspiration. The median sample sizes were 92 and 42 respectively. Eighteen of the RCTs for RDS compared 2 or more surfactant preparations, with the most frequently used being Curosurf and Survanta but altogether 11 different surfactants were studied. Twelve systematic reviews wewre found and these demonstrated the superiority of delivery room surfactant over selective use later in the neonatal unit in babies less than 30 weeks' gestation, natural over synthetic surfactants and the absence of significant long-term developmental problems. Of the non-randomised trials those reporting novel delivery methods, the failure to use evidencebased guidelines and the clear benefit of surfactant treatment in babies of less than 25 weeks were the most interesting.

Conclusions: Surfactant remains one of the most effective and safest treatments in neonatal medicine. Prophylactic natural surfactant is the most evidencebased treatment for babies of less than 30 weeks. Of the newer synthetic surfactants, only Surfaxin has been compared with currently used surfactants and systematic reviews may be needed to determine its role in the treatment of RDS. Improvement in outcome of babies less than 25 weeks has been due to a number of interventions including prenatal steroids, prenatal antibiotics and postnatal surfactant. RCTs of surfactant treatment continue to be published remarkably frequently.

\section{LONG TERM RESPIRATORY OUTCOME FOLLOWING PRE-TERM DELIVERY}

\section{J Stocks}

Professor of Neonatal Paediatrics, Respiratory Physiology, UCL, Institute of Child Health, London, UK

Background and Aims: Advances in neonatal care over the last three decades have resulted in increased survival of extremely preterm infants. However, the prevalence of pulmonary sequelae has not declined as might have been hoped or expected from improved management, thereby increasing the burden of respiratory health care delivery.

Methods: The residual respiratory problems of preterm, low birth weight infants following discharge from neonatal intensive care often persist into childhood with significant respiratory morbidity, including frequent cough and wheeze. Even in the absence of such symptoms, there may be sub-clinical changes associated with airway obstruction, hyperinflation, increased airway reactivity and exercise limitation. These problems may improve with age and somatic growth in some individuals, but remain persistent in others. Although those who required a high level of intensive care or who developed chronic lung disease of infancy/ bronchopulmonary dysplasia, are more likely to be affected beyond infancy, these adverse effects have also been observed in children born prematurely but without significant neonatal disease. Improved ongoing surveillance of individuals born preterm is essential if we are to understand the mechanisms and long term impact of such early life insults.

Results: Despite a vast literature regarding respiratory outcomes of preterm delivery during infancy (see recent series on Lung Function in Infants with CLDI, in Pediatric Pulmonology: 2005-2006), relatively little is known about respiratory health of these subjects during later childhood and adulthood, particularly in those with severe neonatal disease in whom survival was so poor prior to the 1980s. If research into the structural and functional aspects of lung growth and development following preterm delivery is to have any impact on subsequent clinical practice, it is essential to develop more objective physiological definitions of CLDI and to discriminate between what we may be able to influence (i.e. choice of therapeutic modalities) from that which we cannot (such as effects of low birth weight and sex). Consequently, research in this field is challenged by the need to separate the independent effects of a) genetic predisposition, b) preterm delivery per se, c) intrauterine growth retardation d) neonatal respiratory disorders and the related range of treatment strategies and e) impact of early environmental insults (including exposure to tobacco products and infections) on subsequent lung growth and development (see also Stocks J. Pulmonary 
function tests in infants and young children. In Chernick $\mathrm{V}$, Boat TF, Wilmott RW, Bush A, eds. Kendig's disorders of the respiratory tract in children, Philadelphia, USA: Elsevier, 2006).

Conclusions: Well designed multidisciplinary studies are required to follow up graduates of modern neonatal care, as their outcome may be very different to that of cohorts recruited thirty, or even ten, years ago. We need to learn what will happen to the aging lung in these subjects and whether they are at increased risk of acquiring early-onset respiratory failure or chronic obstructive pulmonary disease, in order to plan appropriate services and inform future neonatal care.

\section{A NEW APPROACH TO ACCELERATION OF FETAL LUNG MATURATION BY PIOGLITAZONE}

F Guthmann, B Rüstow

Department of Neonatology, Charite-CCM, Berlin, Germany

Background and Aims: The use of antenatal glucocorticoids is one of the most important reasons for the decreasing mortality of premature infants. However, even with doses scaled to match those used in clinical treatment of humans, side effects occurred in animal brains. Thus, by studying a new approach we aim to induce fetal lung maturation either instead of steroids or supplemental to steroids.

Methods: We determined the expression of i) SP-B which appears to be the most important apoprotein of the surfactant with regard to the development of a respiratory-distress syndrome, ii) SP-C, which increases surfactant spreading and stability, and iii) $\mathrm{ABCA} 3$, a transporter protein in the membrane of lamellar bodies whose expression is inducible by corticosteroids in fetal rat. ABCA3 is expressed in the rat in a developmentspecific manner, and is detected earliest at day 20.5, i.e. shortly before birth.

Results: We found that the PPARgamma ligand pioglitazone, administered antenatally, induces the expression of SP-A, B and C 3.6-, 38- and 6,5-fold, respectively. ABCA3, hardly detectable at day 19.5 , was also drastically increased by pioglitazone as judged by immunfluorescence. Increased ABCA3 is associated with enhanced surfactant lipid synthesis. Incorporation of $[3 \mathrm{H}]$ palmitate into diacylglycerol or into phosphatidylcholine increased around 5-fold in lung homogenates of pioglitazone-treated rats compared to controls.

Conclusions: We follow from the enhanced phosphatidylcholine synthesis, the increased expression of the surfactant apoproteins and $\mathrm{ABCA} 3$, a transport protein being important for the synthesis of surfactant that the antenatal administration of pioglitazone can induce the biochemical maturation of the lung.

\section{LUNG FUNCTION IN HETEROZYGOTE CARRIERS OF ABCA3 MUTATIONS}

O Danhaive ${ }^{1}$, A Dotta $^{1}$, A Turchetta ${ }^{2}$, D Peca ${ }^{3}$, F Savignoni $^{1}$, F Morini $^{4}$, C Corchia $^{1}$
${ }^{1}$ Division of Neonatal Intensive Care, ${ }^{2}$ Division of Sport Medicine and Pulmonary Function, ${ }^{3}$ Laboratory of Neonatal Biology, ${ }^{4}$ Division of Neonatal Surgery, Bambino Gesu Children's Hospital, Rome, Italy

Background and Aims: ABCA3 is a type II pneumocyte phospholipid carrier essential for surfactant synthesis. ABCA3 deficiency causes lethal neonatal RDS and pediatric interstitial lung disease. Most cases carry ABCA3 mutations on both alleles. We investigated relatives of $\mathrm{ABCA} 3$-deficient infants for determining whether heterozygosis leads to subclinical respiratory impairment.

Methods: Genotype was determined by sequencing of PCR-amplified genomic DNA in neonates with suspected genetic surfactant deficiency and relatives. Airway patency was measured by FEV1 in all, except neonates in whom $\mathrm{TPTEF} / \mathrm{Te}$ was used; functional lung volumes were measured by RV/TLC, except in neonates in whom Vt was used. Alveolar exchange was measured by carbon monoxide diffusion capacity (KCO) in subjects $>5$ years, and estimated by $\mathrm{PaO} 2 / \mathrm{FiO} 2$ ratio $<5$ years.

Results: Case 1 carried an $\mathrm{ABCA} 3$ homozygote frameshift mutation on exon 23 (3997del AG) leading to complete ABCA3 deficiency. Case 2 carried biallelic heterozygote missense mutations on exons 6/7. Both died in neonatal period. Case 3, who presented moderate interstial disease from birth, carried a heterozygote missense mutation on exon 8. No $\mathrm{SpB}$ and $\mathrm{SpC}$ mutations were found. 10 heterozygotes were studied, including case 3. All showed normal airway resistances. Lung volumes were normal except case 3 (Vt 5.1cc). Alterations of $\mathrm{KCO}$ were found in relatives carrying C439T and C643T (66.7 vs 95.6\% of predictive value), suggestive of subclinical interstitial disease. $\mathrm{PaO} 2 / \mathrm{FiO} 2$ in case 3 was 115 (normal: >200), suggesting moderate/severe interstitial disease.

Conclusions: Certain heterozygote $\mathrm{ABCA} 3$ mutations may lead to subclinical interstitial lung disease in children and adults, and may be associated with more severe pediatric interstitial lung disease.

\section{HUMAN PULMONARY NITRIC OXIDE SYNTHASES (NOS) AND NITROTYROSINE: FINDINGS DURING NEONATAL DEVELOPMENT AND CHRONIC LUNG DISEASE (CLD)}

M J Sheffield ${ }^{1}$, D W Thibeault ${ }^{2}$, S Mabry ${ }^{2}$, W E Truog ${ }^{2}$

${ }^{1}$ Division of Neonatology, Department of Women and Children, McKay Dee Hospital, Intermountain Health Care, Ogden, UT, USA, ${ }^{2}$ Section of Neonatology, Department of Pediatrics, The Children's Mercy Hospitals and Clinics, University of Missouri-Kansas City School of Medicine, Kansas City, MO, USA

Background and Aims: Nitric Oxide (NO) is a mediator of pulmonary transition to postnatal life. Prematurity and CLD of prematurity may alter ontogeny of NOS expression or function of the NO pathways. NOS presence and NO production in fetal lungs have not been correlated. One key pathway of NO 
metabolism results in nitrotyrosine, a stable, measurable marker of NO production. We compared, by semiquantitative immunohistochemistry (IHC), NOS isoforms and nitrotyrosine at different pulmonary airway and vascular levels in neonates at different gestations and severities of CLD.

Methods: Postmortem lung blocks were compared using IHC, with antibodies to each NOS isoform and nitrotyrosine. Blinded observers evaluated airway and vascular trees for staining intensity ( $0-3$ scale). Controls, postconceptional age 22-42 week infants who died in $<48$ hours, were compared to gestational-matched infants with varying CLD severity.

Results: All three NOS isoforms stained in airway epithelium (bronchus to alveolus) in control and CLD groups. Abundance or distribution of NOS-3 airway staining did not correlate with gestation or CLD severity. In the vasculature, intense NOS-3, moderate NOS-2, and inconsistent NOS-1 staining was found. Nitrotyrosine stained in the pulmonary tree. Compared to controls where nitrotyrosine staining was minimal, regardless of gestation, infants with severe CLD revealed $>4$ fold increase $(p<0.001)$.

Conclusions: All three NOS isoforms and nitrotyrosine are detectable by IHC early in lung development. NOS ontogeny shows no significant changes in abundance or distribution with advancing gestational age or with CLD. However, Nitrotyrosine, a marker of NO production, is significantly increased in severe CLD airways, which may have clinical implications.

\section{RESPIRATORY COMPLICATIONS IN CHILDREN WITH NEUROMUSCULAR DISORDERS}

\section{B Fauroux}

\section{AP-HP, Hopital Armand Trousseau, Department of Pediatric Pulmonary, Research Unit INSERM U719, Universite Pierre et Marie Curie - Paris 6, France}

Background and Aims: In patients with neuromuscular diseases, the development of respiratory muscle weakness represents the major determinant of the respiratory morbidity and mortality. As such, the measurement of lung volumes have been demonstrated to be an useful marker of the severity of the respiratory disease in patients with Duchenne muscular dystrophy (DMD) (1). Routine evaluation of forced vital capacity (FVC), maximal inspiratory and expiratory pressure and peak cough flow is suggested early in the course of the disease by the American Thoracic Society (2). However, noninvasive volitional tests of lung and respiratory muscle function can be misleading because these tests require aptitude, cooperation, motivation and coordination, which explains that reliable measures may be difficult to obtain in young children.

Methods: In order to evaluate inspiratory and expiratory muscle strength in children with various neuromuscular diseases, 41 patients, aged 2 to 18 years, with DMD $(\mathrm{n}=20)$, spinal amyotrophy $(\mathrm{n}=8)$, and congenital myopathy $(n=13)$, were asked to perform a sniff and a cough maneuver, as well as a FVC maneuver. In addition, diaphragmatic strength was assessed by a non- volitional test, i.e the magnetic stimulation of the phrenic nerves (TwPdi) (3).

Results: All the patients were able to perform the sniff and the cough maneuver, whereas only 25 (61\%) patients were able to perform FVC. Sniff transdiaphragmatic pressure decreased with age in patients with DMD whereas it increased with age in patients with spinal amyotrophy and congenital myopathy. Magnetic stimulation of the phrenic nerves was obtained in all patients and in the three groups of patients, a significant correlation was observed between the volitional sniff maneuver and the non-volitional test of diaphragmatic strength, i.e. TwPdi. Also, FVC correlated with sniff transdiaphragmatic pressure and cough gastric pressure. In patients with DMD, minute ventilation and lung volumes, assessed by functional residual capacity and FVC, decreased with age. Thus, in DMD patients, the progression of respiratory muscle weakness with age translates into the development of alveolar hypoventilation. Indeed, the patients with DMD who required non-invasive positive pressure ventilation (NPPV) had significantly weaker inspiratory and expiratory muscles and a lower FVC than those who did not need NPPV. Respiratory muscle output, as reflected by the oesophageal (PTPes) and the diaphragmatic pressure-time products (PTPdi), which represents the work of breathing, was also calculated. In all patients, the PTPes and PTPdi were 2 to 3 times normal values, whatever the diagnosis and age of the patients. This increase in respiratory muscle output may be explained by the microatelectasis, the 'stiffening' and the deformity of the chest and contributes to the ventilatory imbalance. Conclusion: In conclusion, the natural history of respiratory muscle weakness depends on the type of neuromuscular disease. But in these patients, alveolar hypoventilation develops as a consequence of the respiratory muscle weakness. The moderate increase in respiratory load may contribute further to the ventilatory imbalance. References: 1. Smith PEM, Calverley PMA, Edwards RHT, Evans GA, Campbell EJM. Practical problems in the respiratory care of patients with muscular dystrophy. N Engl J Med 1987;316:11971203. 2. Respiratory care of the patient with Duchenne muscular dystrophy. ATS Consensus Conference. Am J Respir Crit Care Med 2004;170:456-465. 3. Nicot F, Hart N, Forin V, Boulé M, Clement A, Polkey MI, Lofaso F, Fauroux B. Respiratory muscle testing: A valuable tool for children with neuromuscular disorders. Am J Respir Crit Care Med 2006; Epub ahead of print.

\section{PHOX2B GENE AND THE INTIMATE RELATIONSHIP WITH DYSAUTONOMY}

\section{Gaultier}

Department of Physiology, Hôpital Robert Debré, Paris, France

Background and Aims: Congenital central hypoventilation syndrome, Hirschsprung disease, and neuroblastomas can occur in combination, and familial cases have been reported in all three conditions. This suggests variable expression of a single genetic abnormality as the common cause to these neural crest disorders. Because the PHOX2B gene is pivotal in the 
development of most relays of the autonomic nervous system, including all autonomic neural crest derivatives, it was considered a candidate gene for the above conditions.

Methods: Molecular genetic analyses were performed in children with CCHS, Hirschsprung disease, and neuroblastomas.

Results: Recent studies have shown that: (i) PHOX2B is the main disease-causing gene for congenital central hypoventilation syndrome, an autosomal dominant disorder. Most of the mutations consisted in alanine expansions within the polyalanine stretch of PHOX2B exon. (ii) PHOX2B is the first gene for which germline mutations have been demonstrated to predispose to neuroblastoma. (iii) Hirschsprung disease was associated with an intronic single-nucleotide polymorphism of the PHOX2B gene in a case-control study.

Conclusions: To clarify the variable clinical expression of the autonomic nervous system dysfunction observed in paediatric disorders with dysautonomy, international databases of clinical symptoms and molecular test results should be established. Furthermore, the development of genetic mouse models should help to improve our understanding of the molecular mechanisms underlying neural crest disorders.

\section{COGNITION AND ANXIETY-RELATED BEHAVIOR, SYNAPTOPHYSIN- AND MAP2- IMMUNOREACTIVITY IN THE ADULT RAT TREATED WITH A SINGLE COURSE OF ANTENATAL BETAMETHASONE}

M Bruschettini ${ }^{1,2,3,5}$, D L A van den Hove ${ }^{1,2,5}$, S Timmers ${ }^{1}$, M Welling ${ }^{1}$, J Prickaerts ${ }^{1}$, D Gazzolo ${ }^{3,4}$, C E Blanco ${ }^{2}$, H W M Steinbusch ${ }^{1,5}$

${ }^{I}$ Department of Psychiatry and Neuropsychology, Division of Neuroscience, University of Maastricht, The Netherlands, ${ }^{2}$ Department of Pediatrics, Research Institute Growth and Development (GROW), Faculty of Medicine, University of Maastricht, The Netherlands, ${ }^{3}$ G. Gaslini Children's Hospital, University of Genoa, Italy, ${ }^{4}$ Maternal Fetal and Neonatal Health, $G$ Garibaldi Hospital, Catania, Italy, ${ }^{5}$ European Graduate School of Neuroscience (EURON), University of Maastricht, The Netherlands

Background and Aims: To investigate the effects of a single course of antenatal betamethasone on cognitionand anxiety-related behavior and synaptophysin- and microtubule-associated protein 2 (MAP2)- immunoreactivity in the adult rat hippocampus.

Methods: On day 20 of gestation pregnant rats were injected with either 1) $170 \mu \mathrm{g} \mathrm{kg}-1$ body weight of betamethasone ('clinically-equivalent dose', equivalent to $12 \mathrm{mg}$ twice, 24 hours apart), 2) Half this dose or 3) Vehicle. Cognition- and anxiety- related behavior of the offspring was analyzed at an age of 5 months using the Morris water maze, object recognition task and open field test. Subsequently, synaptophysin- and MAP2immunoreactivity were measured in the hippocampus.

Results: We report no detrimental effects of antenatal betamethasone on cognition- and anxiety-related behavior and synaptophysin-immunoreactivity in the adult rat. On the other hand, MAP2-immunoreactivity was decreased by betamethasone in males. The lower dose had less influence in terms of fetal growth restriction.

Conclusions: Our findings suggest a permanent impairment in the hippocampus following a single course of antenatal betamethasone. Further research might elucidate whether the betamethasone effect on hippocampal neurons persists later in life and could affect the aging process increasing the risk for neuropathology of the adult. Moreover, lowering the dose appears to reduce the steroid effect on fetal growth restriction - known to be associated with an increased risk of disease in adulthood. (Acknowledgements: DVDV and JP are supported by the EU Framework 6 Integrated Project NEWMOOD, LSHM-CT-2004503474)

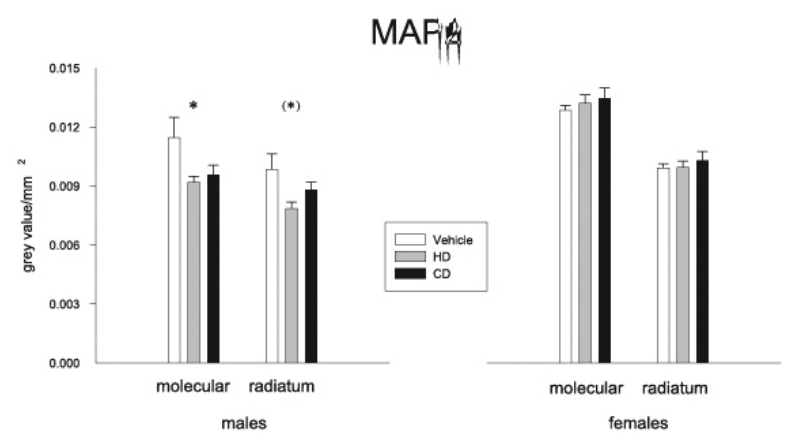

\section{NEONATAL ORAL IMITATION IN PATIENTS WITH SEVERE MOTOR DYSFUNCTION}

T Go ${ }^{1}$, M Yamaoka ${ }^{2}$ E Shimokawa ${ }^{2}$, M Hoshide $^{2}$, H Kobe $^{2}$, H Shinoda ${ }^{2}$, K Uchida ${ }^{2}$, A Hagiwara ${ }^{2}$, M Sato $^{2}$, Y Konishi ${ }^{1}$

${ }^{\prime}$ Department of Infants' Brain and Cognitive Development, Tokyo Women's Medical University, Tokyo, Japan, ${ }^{2}$ Department of Rehabilitation, Saitama Medical Center for the Disabled, Saitama, Japan

Background and Aims: Babies have been shown to reproduce mouth opening and closing in response to an adult model within a few hours after birth. This neonatal oral imitation is observed only in humans and great apes such as chimpanzees, indicating that this oral function is innate and mostly specific in humans. In this study, we investigated the possibility whether patients with severe motor dysfunction demonstrated similar imitation seen in neonate.

Methods: Fourteen patients were included in this study. They were rarely able to vocalize or move their extremities voluntarily. First, a therapist sang a favorite song of each patient for five minutes. Then, the therapist sang a part of it repeatedly with mouse widely and slowly open while making eye contact with the patient. Patients were evaluated as being able to perform oral imitation when they opened their mouth repeatedly in response to the therapists' oral movement.

Results: Seven patients $(50 \%)$ showed oral imitation. Three patients did not move their mouth and four were difficult to be evaluated.

Conclusions: Half of patients with severe motor dysfunction demonstrated similar oral imitation observed in neonate, supporting the subcortical control hypothesis of neonatal imitation. On the other hand, 
singing favorite songs was necessary to induce it, suggesting the involvement of higher brain function. Imitation is considered to precede theory of mind and provide an innate foundation for social cognition even in primitive neonatal imitation. Even patients with severe motor dysfunction were shown to communicate with others by oral imitation.

\section{ISOLATED LANGUAGE REGRESSION IS STRONGLY ASSOCIATED WITH ELECTROGRAPHIC STATUS EPILEPTICUS IN SLEEP}

O Ekinci, E Trevathan, N Reed, U Isik, L L Thio, J Zempel, M Wong, C Gurnett, S Arnold

Division of Pediatric and Developmental Neurology, Washington University in St. Louis School of Medicine, St. Louis Children's Hospital, St. Louis, MO, USA

Background and Aims: We determined the factors associated with electrographic status epilepticus in sleep (ESES) and sleep-activated epileptiform abnormalities (SAEA) among children with autism and/or regression referred for EEG-video studies.

Methods: Data on all children who were referred for 24- hour EEG-video monitoring from 1995-2005 with a question of possible ESES or SAEA were entered into a database for analysis. Senior pediatric neurologists evaluated all children at the time of admission to determine whether children met diagnostic criteria for autism or an autistic spectrum disorder (ASD), or if they had isolated language regression (language regression without autistic features).

Results: 121 children (64\% male) were referred for EEG-video monitoring to evaluate for possible SAEA or ESES. 56 of $121(46 \%)$ children had language, autistic, and/or cognitive/behavioral regression. 66 children had clinical features of autism; 13 children experienced global autistic regression. Of the 33 children with isolated language regression, $19(58 \%)$ had SAEA and $11(33 \%)$ had ESES. Only 1 child with ESES did not have isolated language regression. No child with a diagnosis of global autistic regression had ESES. Isolated language regression was strongly associated with SAEA (odds ratio $=3.07 ; 95 \% \mathrm{CI}=1.34,7.00$ ) and with ESES (odds ratio $=43.5,95 \% \mathrm{CI}=5.33,355.1$ ). Autistic features were not associated with ESES (odds ratio $=0.06,95 \% \mathrm{CI}=0.01,0.49$ ) and or SAEA (odds ratio $=0.31,95 \% \mathrm{CI}=0.14,0.67)$.

Conclusions: Isolated language regression, not autism, was strongly associated with SAEA and ESES.

\section{PYRIDOXINE DEPENDENT SEIZURES (PDS) IN THE NETHERLANDS: STUDY OF AMINO- ADIPINE-SEMI-ALDEHYDE (ASAA) AND PIPECOLIC ACID (PA)}

L A Bok ${ }^{1}$, J V Been $^{2}$, M A Willemsen
C Jakobs

${ }^{I}$ Department of Paediatrics, Máxima Medical Centre, Veldhoven, The Netherlands, ${ }^{2}$ Department of

Paediatrics, Maastricht University Hospital, Maastricht, The Netherlands, ${ }^{3}$ Department of Paediatric Neurology,
Radboud University Medical Centre, Nijmegen, The Netherlands, ${ }^{4}$ Department of Clinical Chemistry, VU University Medical Centre, Amsterdam, The Netherlands

Background and Aims: PDS is a rare, autosomal recessively inherited disorder. Recently PDS was shown to result from AASDH (antiquitin) deficiency, elevated levels of both AASA and PA, that can be used to confirm the diagnosis.

Methods: We reevaluated the recently reported series of Dutch PDS patients (Arch Dis Child 90:1293-1296) by measuring AASA and PA in urine and plasma of all patients $(n=13)$. Because of the ongoing epidemiologic study newly diagnosed patients could be added $(n=6)$.

Results: AASA was elevated in urine and plasma and PA in plasma in most suspected PDS patients (Table). In all patients with definite PDS and in most with probable or possible PDS according to the criteria published by Baxter, the diagnosis of PDS could be confirmed. The birth incidence of 'metabolic proven' PDS in the Netherlands was calculated to be 1:212,000.

Conclusions: This report is the first nationwide population-based study on metabolic confirmed PDS. This study shows that most patients were diagnosed correctly using the Baxter criteria. Metabolic screening of the L-lysine metabolism provides a tool to discriminate between patients with 'classical PDS' and other types of neonatal seizures including pyridoxine responsive seizures.

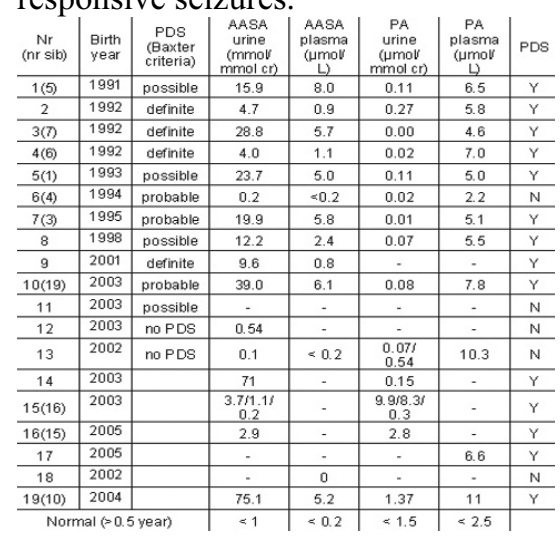

\section{BRAIN DIFFERENTIAL GENE EXPRESSION FOLLOWING 72 HOURS OF GRADED HYPEROXIA IN NEONATAL RATS}

D Torbati ${ }^{1}$, B R Totapally ${ }^{1}, \mathrm{~J}_{\text {Osborne }}{ }^{2}, \mathrm{~L}_{\text {van }} \mathrm{Zyl}^{3}$, S Kalomiris ${ }^{1}$, A Raszynski ${ }^{1}$

${ }^{1}$ Division of Critical Care Medicine, Miami Children's Hospital, Miami, FL, USA, ${ }^{2}$ Department of Statistics, North Carolina State University, Raleigh, NC, USA ${ }^{3}$ ArrayXpress, Raleigh, NC, USA

Background and Aims: Hyperoxia and Hyperoxemiainduced oxidative stress may modulate the expression of specific genes in various organs. Whereas the effects of hyperoxia and hyperoxemia on the developing lungs and retina have been well documented, their effects on developing brain have not been systematically studied. Therefore, we examined, in the present study, differential gene expression in whole brain of neonatal rats exposed to 3 days of normobaric hyperoxia before 
the onset of the clinical signs of pulmonary oxygen toxicity.

Methods: Sixteen newborn male Sprague Dawley rats were randomly divided into an hyperoxia-treated group (72 h, consisting of $98 \%, 60 \%$ and $40 \% \mathrm{O} 2$ breathing at days $1-3$, respectively), or controls $(21 \%$ O2) $(n=8$ in each group). After 72 hours, the pups were euthanized and their brain was rapidly removed and analyzed for differential gene expression, using 9984 oligonucleotide rat array. Samples from both groups were assigned to single slides for the purpose of direct (low variance) comparison. Bonferroni correction was used to control the experimental Type I error at alpha level of 0.05 .

Results: Seventy-two hours of graded exposure to hyperoxia was not associated with statistically significant changes in differential gene expression in the whole brain of newborn rats, as compared to controls.

Conclusions: Seventy-two hours of graded hyperoxia, in the absence of the clinical signs of pulmonary oxygen toxicity, does not affect the whole brain differential gene expression in neonatal rats. Whether hyperoxia in the newborn rats can modify the genomic profile of individual neuroanatomical structures requires further investigation.

\section{END-OF-LIFE DECISIONS IN NEONATES AND INFANTS IN BELGIUM AND THE NETHERLANDS}

L Deliens ${ }^{1}$, A M Vrakking ${ }^{2}$

${ }^{I}$ End-of-Life Care Research Group, Vrije Universiteit Brussel, Brussels, Belgium, ${ }^{2}$ Department of Public Health, Erasmus University Medical Center, Rotterdam, The Netherlands

Background and Aims: Recently, in both Belgium (Flanders) and the Netherlands, studies have been done to get insight in the practice of end-of-life decisionmaking for neonates and infants. We compared the results to gain insight in similarities and differences between these countries.

Methods: Questionnaires were sent to physicians who reported the death of a child who died under the age of one (Belgium: $\mathrm{n}=292$, response $87 \%$; Netherlands: $\mathrm{n}=249$, response $84 \%$ ). The questionnaires included structured questions about whether death had been preceded by end-of-life decisions, and about the decision-making process.

Results: In both countries, in about $25 \%$ of all deaths a life-sustaining treatment was withheld. In Belgium a life-sustaining treatment was less often withdrawn than in the Netherlands $(32 \%, 50 \%$ respectively). In both countries, in about $40 \%$ pain or other symptoms were alleviated taking into account that death might be hastened. Drugs were administered with the explicit intention of hastening death in similar percentages of all deaths (Belgium: 7\%; Netherlands: 9\%), but Dutch physicians more often estimated the shortening of life more than one week than Belgian physicians. In the Netherlands, the decision was more often than in Belgium discussed with parents $(96 \%, 81 \%$ respectively), and with colleague-physicians $(94 \%, 80 \%$ respectively). In Belgium, the decision was discussed with nurses in $32 \%$, and in the Netherlands in $27 \%$.
Conclusions: End-of-life decision-making in severely ill neonates and infants seems to be rather similar in Belgium and the Netherlands. Differences are that Dutch physicians more often withdraw life-sustaining treatment and decide to administer drugs with the intention of hastening death in an earlier stage.

\section{END OF LIFE DECISIONS IN CRITICALLY ILL INFANTS; DIVERSITY WITHIN EUROPE}

\section{P J J Sauer} Department of Pediatrics, University Medical Center
Groningen, The Netherlands

Within Europe a variety in opinions exists among neonatologists and intensivists concerning the question when to start and when to stop or continue life-saving treatment in a critically ill infant. In some countries physicians feel they have the obligation to discuss these issues with parents and, ultimately, to make decisions while in other countries physicians are convinced that these decisions are not part of the medical domain. Quality of life aspects are taken into account in the decision-making process in some countries while in other countries the future quality of life can never be an argument in these decision-making processes. Next to the future quality of life, the presence of suffering can be an aspect in the decision-making process. In some countries physicians are convinced that all suffering can always be sufficiently and effectively treated with medication. In The Netherlands it is accepted that it is not possible to effectively alleviate suffering in all patients with medication and that active ending the life in some infants can be the only way to alleviate suffering. In these extreme situations actively ending the life of an infant is considered to be acceptable in The Netherlands, while in other countries it is considered to be murder. Are opinions regarding these ethical questions indeed very different between countries, and if so, do we know why these differences exist? Or, on the other hand, is it mainly the way we discuss these inssues. In my opinion differences in opinion exist. However, they are not as extreme as they might like. Secondly, physicians are influenced by the cultural and religious environment, and also by the legal system. An open discussion regarding these issues is very important within Europe. Not that one opinion is superior over the other, but these discussions can help physicians in different countries to make their view and thereby the decision making more clear and explicit. Discussing these issues therefore is not telling what is wrong and what is right, but will help to make proper decisions. Ethics is not the science that makes decisions, but the science that helps to assistent by formulating the proper questions in the decision-making process.

\section{CARE AT THE END-OF-LIFE IN THE PAEDIATRIC INTENSIVE CARE UNIT}

\author{
C Pal ${ }^{1}, \mathrm{P} \mathrm{Habibi}^{2}$
}

${ }^{1}$ Paediatric Intensive Care Unit, St Mary's Hospital NHS Trust, London, ${ }^{2}$ Imperial College School of Medicine, London, UK 
Background and Aims: Many deaths on a Paediatric Intensive Care Unit (PICU) can be anticipated. This gives an opportunity to share decisions with parents, and manage a child's end of life care accordingly. We present a review of deaths on a PICU over a 30 month period and three cases to illustrate differences in end of life care. Methods: The 'mode' of death of all children who died in 2003-2006 was categorised: 1. brain stem death, 2. unsuccessful attempts at resuscitation, 3. withdrawal/limitation of therapy. In the latter, the circumstances leading to this decision were noted along with the process, timing and place of therapy withdrawal. Results: There were 53 deaths $(6 \%)$ out of 883 admissions. In 29 cases (55\%), children died following withdrawal or limitation of therapy. Sixteen deaths $(30 \%)$ followed unsuccessful attempts of full resuscitation. Eight children (15\%) died from brain stem death. In the majority of cases, medical staff initiated discussions regarding withdrawal of therapy, usually several discussions took place. Therapy was withdrawn with family present in all cases. Conclusions: The majority of deaths on PICU follow withdrawal or limitation of therapy and can therefore be anticipated. End of life care is discussed with families, so their wishes can be taken into account when managing children dying on PICU. This is a very emotive and challenging area for intensive care, and raises many complex ethical and operational issues. The quality of care at the end of life should be investigated by examining the family's perceptions of such care.

\section{WITH CARE: TEACHING COMPASSIONATE WITHDRAWAL OF LIFE SUPPORT IN THE NICU. WHAT DO PARENTS WANT?}

C Williams ${ }^{1}$, J Cairnie ${ }^{1}$, L Lohfeld ${ }^{2}$, C Patey ${ }^{1}$, V Fines ${ }^{1}$, K Schwarzer ${ }^{1}$, H Kirpalani ${ }^{1}$

\section{${ }^{I}$ McMaster University Health Sciences Centre \\ ${ }^{2}$ McMaster University, Hamilton, ON, Canada}

Background and Aims: Geographic variations in physician practices of withdrawal of life sustaining treatment (WLST) are reported. However, practical aspects of decision making, the withdrawal process and how to support bereaved parents are poorly described. Moreover, parents' perspectives are infrequently discussed. Our purpose was to generate key elements of importance from parents who had experienced WLST in the NICU. Methods: Semi-structured interviews were conducted with parents of infants who underwent WLST within the last 3 years in a tertiary NICU. Parents were asked to describe the events surrounding discussions of decision making, WLST, and follow-up care. Interviews continued to saturation, then were independently coded and reduced. Results: Seven interviews generated 139 items important to parents, organized into 6 themes: Communication e.g. not to be given false hope; not to pass value judgments. Quality of care e.g. continuity of the care plan; that everything possible has been done. Quality of life e.g. balance suffering in life against long term outcome. Decision making e.g. parents feel supported whatever their decision; parents feel the right decision was made. WLST e.g. parents be guided through the steps of WLST. Post-bereavement e.g. retaining a sense of their child's identity; availability of doctors to answer questions. Conclusions: A working set of items important in the delivery of compassionate WLST has been generated based on informed parents' experiences. We are now using these key items to develop a learning package for teaching WLST in the NICU. (This project was funded by a resident award from Physician Services Incorporated, Toronto).

\section{END OF LIFE DECISIONS IN NEWBORNS AND CHILDREN: EXPERIENCES OF A LOCAL REVIEW COMMITTEE}

M A van der Hoeven ${ }^{1}$, D van Waardenburg ${ }^{1}$, C E Blanco ${ }^{1}, \mathrm{~N}$ de Bijl ${ }^{2}$, G de Wert ${ }^{3}$, G Widdershoven ${ }^{4}$

${ }^{I}$ Department of Paediatric Medicine, University Hospital Maastricht, ${ }^{2}$ Department of Health Law, Faculty of Health Sciences, University Maastricht, ${ }^{3}$ Faculty of Medicine, Section Health Ethics and Philosophy, University Maastricht, ${ }^{4}$ Care and Public Health Research Institute, University Maastricht, The Netherlands

Background and Aims: In a third level university hospital a review committee on End-of-life care in Children in the Academic Hospital Maastricht (RECAM) was formed to review medical decisionmaking surrounding the deaths of children younger than 16 years old. Methods: A set of criteria was formulated: the establishment of diagnosis and prognosis of the illness, hopeless and unbearable suffering, communication with the parents, the consultation with other physicians, and the performance of the end of life care. Results: The RECAM reviewed 48 cases, in which in 40 cases a medical end-of-life decision was made. In all cases, the medical decision was repeatedly discussed with the parents. Other physicians were consulted. In $22 \%$ of the reported cases current suffering was deemed unbearable, whereas in all cases unbearable suffering in the future was deemed. In $42 \%$ a decision was made to continue or increase the pain medication without the intention to advance death. In $23 \%$ medication was given with the intention to advance death. In $10 \%$ the RECAM concluded that the doctor's stated intentions did not correspond to the medication given. Conclusions: We conclude that all end-of-life decisions were judged to be in accordance with criteria of due care. We recommend that all cases with an intentional shortening of life should be the object of ethical and legal evaluation by a national review committee.

\section{SHOULD NEONATES OR INFANTS BE DENIED THE RIGHT TO LIFE: THE LEGAL IMPLICATIONS OF LIFE-THREATENING DECISIONS IN ENGLAND AND WALES}

\section{R Stanley}

Faculty of Health and Social Care Sciences, Kingston University and St. George's University of London, Kingston-upon-Thames, Surrey, UK

Background and Aims: Children too young and immature to be competent depend on their parents to 
fulfil their rights to good health and medical care. The advances made by medical technology nevertheless sometimes confront parents and doctors alike with difficult dilemmas over what is the appropriate decision to make. Does a child, for example, have a right to life, at whatever the cost? In questions like this the medical profession wields considerable power and it is the doctors who may in reality decide how best to fulfil a child's rights. In the event of a dispute between parents and doctors, the courts may be asked to intervene. At that point, medical paternalism may be replaced by judicial paternalism, with the child's own perspectives slipping into second place. This aim of this analysis of the neonates' right to life (or death) deals with the extent to which children's right to life can be endangered by decisions relating to their. Specifically this discussion will address 3 objectives: (i) The limits to parents' powers and the relevance of physical or mental handicap (ii) A child's right to life and parents' objections to lifesaving treatment. (iii) Withholding treatment from desperately ill babies.

Methods: This discussion brings together the analytic methodologies of jurisprudence and philosophy. As legal philosophy is nothing else that general philosophy applied to law, it uses the same methodology as philosophy in general. Legal theory, for its part, lays an emphasis on plural and interdisciplinary approaches, in addition to the more traditional analytical methodology. Results: The law continues to cause difficulties for doctors and parents who may be unable to determine in advance what the child's quality of life will be if medical intervention continues. Doctors have an unenviable task when managing the treatment of these babies. Although there have been calls for legislation to clarify the law on neonaticide, the enormity of the moral and legal problems facing an intending legislator makes the prospect of legislation remote. There are obviously no right or wrong answers, but it is certainly unrealistic to claim that every child has a right to life; some children even have the right to die.

Conclusion: Doctors increasingly influence the extent to which children's rights are protected when decisions are made regarding treatment. Although they have the expertise to protect children's medical rights, they are not necessarily in the best position to advise over rights, such as a child's right to bodily integrity, to be consulted, and treated with respect. But whilst doctors are not always the best judges of what is in the child's best interests, nor are the parents, who not surprisingly may find it impossible to be objective over the decisions to be made. The courts too may lose sight of their duty to protect the child's own rights from being infringed by decisions which appear to be in the best interests but which may be more to do with those of her carers. Children's rights should not be lost somewhere in the middle, between parental, medical and judicial paternalism.

\section{CARDIOVASCULAR RISK FACTORS AT AGE 30 FOLLOWING PRETERM BIRTH}

\author{
S R Dalziel ${ }^{1,2,3}$, V Parag ${ }^{3}$, A Rodgers ${ }^{3}$, J E Harding ${ }^{2}$ \\ ${ }^{I}$ Great Ormond Street Hospital, London, UK, ${ }^{2}$ Liggins \\ Institute, The University of Auckland, New Zealand
}

${ }^{3}$ Clinical Trials Research Unit, The University of Auckland, New Zealand

Background and Aims: Recent epidemiological evidence has shown increased rates of cardiovascular mortality and associated risk factors in those born small. However scarce information exists concerning cardiovascular risk factors in adulthood following preterm birth, or distinguishing the relative contributions of length of gestation and fetal growth to small size at birth.

Methods: Prospective follow-up of 458 thirty-year olds whose mothers took part in a randomised controlled trial of antenatal betamethasone for the prevention of neonatal respiratory distress syndrome (147 born at term, 311 born preterm). Follow-up assessments included anthropometry, blood pressure, blood lipids, early morning cortisol levels and $75 \mathrm{~g}$ oral glucose tolerance test.

Results: Gestational age at birth, preterm birth, and birth weight $\mathrm{z}$ score, were not associated with serum cholesterol, triglyceride or cortisol at age 30 . However a low gestational age at birth, and preterm birth, were associated with increased systolic blood pressure ( $\mathrm{p}$ value for preterm birth $=0.009$ ) and insulin resistance at age 30 ( $\mathrm{p}$ value for preterm birth=0.006). Birth weight, adjusted for gestational age, was not associated with these outcomes.

Conclusions: Adults who were born moderately preterm have increased blood pressure and insulin resistance at 30 years of age. Preterm birth rather than poor fetal growth is the major determinant of this. As both the incidence of preterm birth and survival amongst those born preterm are increasing, this group may contribute an increasing proportion of overall cardiovascular disease burden in developed countries.

\section{RISK STRATIFICATION AND EFFECTIVENESS OF INTRAVENOUS IMMUNOGLOBULIN PLUS PREDONISOLONE AS THE INITIAL TREATMENT OF KAWASAKI DISEASE}

K Ikeda ${ }^{1}$, T Kobayashi ${ }^{2}$, Y Inoue ${ }^{2}$, Y Okada ${ }^{2}$, T Tomomasa ${ }^{2}$, M Shinohara ${ }^{2}$, T Kobayashi ${ }^{1}$, $\mathrm{K}$ Takeuchi $^{3}$, A Morikawa $^{2}$

${ }^{I}$ Department of Cardiology, Gunma Children's Medical Center, Shibukawa, Gunma, Japan, ${ }^{2}$ Department of Pediatrics and Developmental Medicine, Gunma University Graduate School of Medicine, Maebashi, Gunma, Japan, ${ }^{3}$ Department of Health and Welfare, Takasaki University of Health and Welfare, Takasaki, Gunma, Japan

Background and Aims: We reported that the combination of intravenous immunoglobulin (IVIG) plus predonisolone as the initial treatment of Kawasaki disease (KD) proved more effective in preventing coronary artery abnormalities than IVIG alone. However, the routine use of corticosteroids may demand careful study because they can be associated with potential adverse reactions. This study aimed to demonstrate the effectiveness of IVIG plus predonisolone by risk stratification. 
Methods: $178 \mathrm{KD}$ patients were randomly assigned to either an IVIG group $(\mathrm{n}=88)$ or an IVIG plus predonisolone (IVIG+PSL) group $(\mathrm{n}=90)$. KD patients were stratified into high or low risk groups by a risk score developed to predict IVIG unresponsiveness from a multiple logistic regression analysis using our institutions 750 consecutive KD patients.

Results: 42 patients in the IVIG group and 45 in the IVIG+PSL group were classified into the high risk group. In the low risk group, clinical outcomes were similar between the two groups (initial treatment failure, $4.3 \%$ vs. $0 \%$ : recurrence $0 \%$ vs. $2.2 \%$ : coronary artery abnormalities, $2.2 \%$ vs. $0 \%$, respectively). In the high risk group, the incidence of initial treatment failure and coronary artery abnormalities was more frequent in the IVIG group (initial treatment failure $33.3 \%$ vs. $11.1 \%$, $\mathrm{P}=0.025$ : coronary artery abnormalities $21.4 \%$ vs. $4.4 \%$, $\mathrm{P}=0.023$, respectively).

Conclusions: IVIG+PSL improved coronary and clinical outcomes in the high risk group. If $\mathrm{KD}$ patients are considered at high risk using our risk score, they should be treated with IVIG+PSL as the initial treatment.

\section{THE SIGNIFICANCE OF BIRTH BEFORE 28 WEEKS OF GESTATION FOR NEONATAL MORTALITY AND MORBIDITY. A NATIONAL POPULATION-BASED STUDY}

H Wold ${ }^{1}$, K Sommerfelt $^{1}$, H Reigstad $^{1}$, A Rønnestad ${ }^{2}$, $\mathrm{S}$ Medbø $\varnothing^{3}, \mathrm{~K}_{\text {Danielsen }}{ }^{4}$, S Spangen ${ }^{5}$, P A Tølløfsrud ${ }^{6}$,

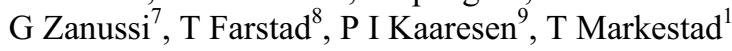

${ }^{l}$ Department of Pediatrics, Haukeland University Hospital, Bergen, Norway, ${ }^{2}$ Department of Pediatrics, Rikshospitalet University Hospital, Oslo, Norway ${ }^{3}$ Department of Pediatrics, Ullevål University Hospital, Oslo, Norway, ${ }^{4}$ Department of Pediatrics, Sørlandet Hospital, Kristiansand, Norway, ${ }^{5}$ Department of Pediatrics, Ålesund Hospital, Alesund, Norway

${ }^{6}$ Department of Pediatrics, Buskerud Hospital, Drammen, Norway, ${ }^{7}$ Department of Pediatrics, Stavanger University Hospital, Stavanger, Norway ${ }^{8}$ Department of Pediatrics, Akershus University Hospital, Lørenskog, Norway, ${ }^{9}$ Department of Pediatrics, University Hospital of Northern Norway, Tromsø, Norway

Background and Aims: To evaluate the effect of being small for gestational age (SGA), defined as birth weight less than the fifth percentile for gestational age, on neonatal mortality and morbidity.

Methods: In this study, prospective data was collected on all births of 22 to 27 completed weeks of gestation, or a birth weight of 500 to $999 \mathrm{~g}$ born during the years, 1999 and 2000 in Norway. Of 636 births, 174 infants were stillborn or died in the delivery room, and data from the remaining 462 were included in the study. Of these, 86 died in the NICU, 87 (19 percent) infants had birth weight $<5 \mathrm{p}$ (SGA), and 372 (81 percent) infants had birth weight $>=5 \mathrm{p}$ (AGA). Mortality and presence of selected neonatal morbidity such as severe cerebral haemorrhage, necrotizing enterocolitis (NEC), periventricular leukomalaci, retinopathy of prematurity, chronic lung disease (CLD- defined as need of oxygen at 36 weeks), persistent ductus arteriosus and total days on ventilator/CPAP were compared between the two groups.

Results: The SGA infants had an almost fourfold mortality, a tripled risk of NEC and a doubled risk of CLD. Similarly, there was an increase in the mean number of total days on ventilator/CPAP support. There were no differences between the SGA-group and the AGA-group in frequency of severe cerebral haemorrhage, retinopathy of prematurity, periventricular leukomalaci, or persistent ductus arteriosus. All analyzes were adjusted for gestational age.

Conclusions: We found indications of increased neonatal mortality and morbidity associated with being born before 28 weeks of gestation and being small-forgestational age.

\section{SILDENAFIL PREVENTS REBOUND PULMONARY HYPERTENSION (PHT) AFTER DISCONTINUATION OF INHALED NITRIC OXIDE (NO) IN CHILDREN: A RANDOMISED, DOUBLE-BLIND PLACEBO-CONTROLLED STUDY}

P Namachivayam ${ }^{1}$, D J Penny ${ }^{2}$, U Thelien ${ }^{1}$, S Cooper ${ }^{1}$, $\mathrm{R} \mathrm{Taib}^{3}$, C Stocker ${ }^{4}$, W Butt ${ }^{1}$, L S Shekerdemian ${ }^{4}$

${ }^{1}$ Intensive Care Unit, Royal Childrens Hospital, Melbourne, Australia, ${ }^{2}$ Department of Cardiology and The Australian and New Zealand Childrens Heart Research Centre, Royal Childrens Hospital, Melbourne, Australia, ${ }^{3}$ Department of Cardiology, Royal Childrens Hospital, Melbourne, Australia, ${ }^{4}$ Intensive Care Unit and The Australian and New Zealand Childrens Heart Research Centre, Royal Childrens Hospital, Melbourne, Australia

Background and Aims: NO reduces PVR, improves oxygenation without altering systemic haemodynamics. Rebound PHT upon withdrawal of NO causes desaturation, hypotension and major instability. Sildenafil, a pulmonary vasodilator increases intrinsic cGMP levels by preventing its breakdown Aim is to establish whether sildenafil has a role in the preventing rebound PHT

Methods: Infants ventilated and receiving NO at 5 parts per million ( $\mathrm{ppm}$ ) or more were eligible. 30 infants were randomised to enteral sildenafil $(0.35 \mathrm{mg} / \mathrm{kg})$ or placebo. NO weaned by 1 ppm every half hour and study drug given when NO was weaned to $2 \mathrm{ppm}$. Arterial pressure, CVP, HR, O2 saturations, ABG and PAP were recorded. Data expressed as means (SD) or median (ranges)

Results: 15 patients randomised to each group. Median age (yrs) $0.47(0.13-1.31)$ for sildenafil and $0.28(0.1-$ 0.81 ) in placebo group. Baseline PAP was 36.5 (12.9) $\mathrm{mmHg}$ in sildenafil group, 30.8 (9.0) $\mathrm{mmHg}$ for placebo. Sildenafil did not cause hypotension, vasodilation or worsening oxygenation. $4(28 \%)$ in placebo group suffered rebound PHT needing recommencement of NO (none in sildenafil group). Mean PAP (\%) between baseline and 1 hour after stopping NO is shown in fig. Rebound was associated with increase in ventilation time

Conclusions: Sildenafil is safe and effective for preventing rebound PHT and also reduced 
hemodynamic incidence of rebound and ventilation time

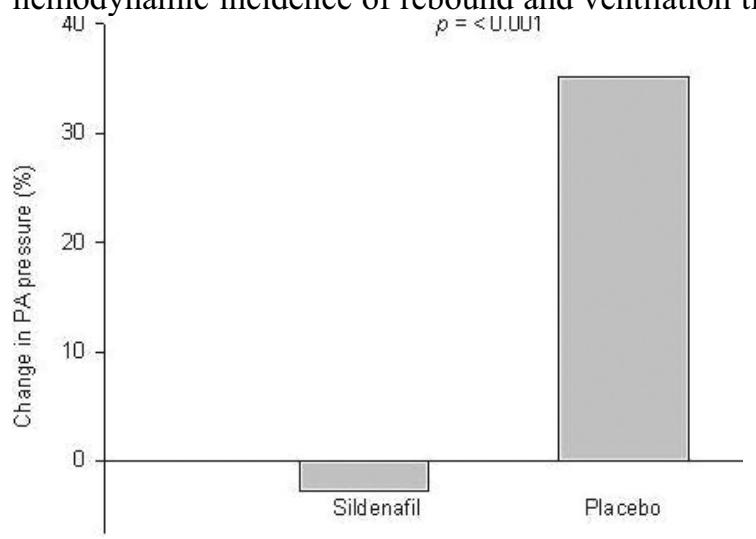

\section{CORRELATION OF GESTATIONAL AGE WITH EXPRESSION OF EPITHELIAL SODIUM CHANNEL (ENAC) IN AIRWAY EPITHELIUM OF NEWBORN INFANTS}

\author{
C Janér, O Helve, O Pitkänen, S Andersson
}

Hospital for Children and Adolescents, Helsinki, Finland

Background and Aims: The fetal lung is filled with liquid that is removed perinatally. Animal experimentation has shown that epithelial sodium channel $(\mathrm{ENaC})$ is rate limiting for clearance of this liquid. In the newborn mouse knock-out of the alpha subunit of the ENaC gene results in early death due to respiratory distress. Human infants that die of respiratory distress shortly after birth have been shown to have a high fluid content of the lungs. Delayed fluid absorption because of low expression of ENaC may thus play a role in the pathogenesis of respiratory distress in the preterm human infant. We therefore studied whether in the early postnatal period a relationship exists between airway expression of subunits of $\mathrm{ENaC}$ and gestational age in newborn infants.

Methods: From 74 newborn infants with gestational age of 26-42 weeks scrape samples from nasal epithelium were taken within 1-6 hours after birth. The expression of alpha, beta and gamma subunit of $\mathrm{ENaC}$ was quantified using RT-PCR.

Results: A significant correlation existed between the expression of alpha $\mathrm{ENaC}$ and gestational age at 1-6 hours after birth $(\mathrm{n}=74, \mathrm{r}=0.393, \mathrm{p}=0.0006)$ and at $22-28$ hours after birth $(n=65, r=0.294, p=0.0175$. The expression of beta $\mathrm{ENaC}$ correlated with gestational age at 1-6 hours after birth $(\mathrm{n}=72, \mathrm{r}=0.2717, \mathrm{p}=0.020)$. In contrast, there was no significant correlation between gamma $\mathrm{ENaC}$ and gestational age.

Conclusions: Insufficient expression of subunits of $\mathrm{ENaC}$ in airway epithelium during the early postnatal period may contribute to the pathogenesis of respiratory distress in the preterm infant.

\section{TRANSPORT OF NEWBORNS WITH ACUTE LUNG FAILURE IN THE PRE-ECMO SITUATION AND ON ECMO}

M Schindler, S Dahlmann, I Berlett, F Loersch

University Childrens Hospital, Mannheim, Germany
Background and Aims: Although ventilation and postpartal management of sick newborns has been optimized, ECMO remains a therapeutic option in the neonatal intensive care of acute lung failure. The ECMO-center Mannheim has performed about 300 ECMO cases since 1987, and currently performs about 25 ECMOs per year. 50 infants per year are evaluated for the option of ECMO-therapy. Half of them are born at the center and the others are transported to the ECMO-center. Some children are too ill for conventional transport, in which case iNO, HFOV or mobile ECMO is necessary.

Results: In the last 5 years we have transported about 50 newborns to our ECMO-center, more than $70 \%$ of them needing iNO or HFOV. 5 children were transported under transport ECMO. Last year we started to installe a special Transport-ECMO unit on a Transport system, which can be used in a standard ambulance or by helicopter. On this transportation unit we use a rollerpump, a ventilator, a heating pump and 12 perfusion pumps. Energy is generated by a UPSU. During the transport the energy comes from the vehicle or helicopter. All 5 newborns transported with this unit have survived and could later have been discharged. The cannulas are placed either by surgeon from the referring hospital or by a surgeon accompanying our transport team.

Conclusions: Special trained transport teams and special equipment are necessary for transporting critically ill children. Transport under ECMO is feasible but remains a high-risk modality that should not replace the prenatal and early postnatal transport to an ECMO center.

\section{NEW TECHNOLOGY IN BRAIN IMAGING IN THE NEONATE}

\section{P S Hüppi}

\section{Department of Pediatrics, University of Geneva, Geneva, Switzerland}

Despite marked improvements in perinatal practice, perinatal brain injury remains one of the most common complications causing chronic handicapping conditions. In recent years remarkable experimental advances have elucidated many of the cellular and vascular mechanisms of perinatal brain damage showing a correlation between the nature of the injury and the maturation of the brain. Advanced Magnetic Resonance Imaging (MRI) techniques have recently provided us with new data on fine structural alteration of the brain in these high risk newborns and have helped us to understand the brains structural alterations and their consequences for later brain development. MRI provides unequaled sensitivity as compared to $\mathrm{CT}$ or ultrasound for evaluating developmental changes and the pathological processes in the perinatal brain. Diffusion tensor imaging (DTI), a recent MR modality which assesses water diffusion in biological tissues at a microstructural level, has revealed a powerful technique to explore the structural basis of normal brain development. In fact, the tissue organization can be probed non-invasively, and the age-related changes of diffusion parameters (mean diffusivity, anisotropy) 
reveal important maturational processes, such as white matter myelination. Early detection of brain injury is crucial for interventions aimed at preventing and reversing ongoing injury. DTI can show early changes at the cellular level that are not detectable by any other imaging modality. Magnetic resonance spectroscopy (MRS) further allows us to some extent to study the underlying metabolic mechanisms that define the pathophysiologic events that take place in perinatal brain injury. Understanding early human brain connectivity is of great clinical importance, as many neurological and neurobehavioral disorders have their origin in early structural and functional cerebral organization. With the development of 3D fiber tractography, based on DTI, the maturation of white matter connectivity can be followed throughout infant development into adulthood with the potential to study correlations between abnormalities on DTI and ultimate neurologic/cognitive outcome. The non-invasive technique of functional MRI can be considered as the leading technique for functional exploration of the infant's brain with localisation of functional brain activation upon sensory, motor and cognitive tasks. Advanced MRI in the newborn brain has allowed to study the structural correlate of early brain development and plasticity in the developing organism.

\section{COULD POWER DOPPLER (PD) BE A DIAGNOSTIC TOOL FOR EARLY} INTERVENTION IN MIDDLE CEREBRAL ARTERY INFARCTION (MCAI) IN NEONATES?

\author{
M C Bravo ${ }^{1}$, A Pellicer ${ }^{1}$, L Dumandzic ${ }^{1}$, R Madero ${ }^{2}$, \\ $\mathrm{J}^{\text {Quero }}{ }^{1}, \mathrm{~F}$ Cabañas ${ }^{1}$ \\ ${ }^{1}$ Department of Neonatology, La Paz University \\ Hospital, Madrid, Spain, ${ }^{2}$ Division of Statistics, La Paz \\ University Hospital, Madrid, Spain
}

Background and Aims: Although lesion topography in MCAI appears crucial regarding outcome, the earlier the identification of events leading to structural brain damage the greater the possibility of intervention. To define the abnormalities of PD in patients with MCAI and whether altered PD patters could anticipate the diagnosis.

Methods: Combined cerebral ultrasound (CUS): To evaluate changes in parenchymal echogenicity and PD studies and to assess for symmetry of flow and to measure blood flow velocity (BFV) -.

Results: 29 infants (BW $2.9 \pm 0.7 \mathrm{~kg}, \mathrm{GA} 38 \pm 3 \mathrm{wk}$ ), who underwent CUS-PD studies as part of routine work up of patients with seizures $(\mathrm{n}=21)$ or risk factors for brain injury $(\mathrm{n}=8)$ had diagnosis of MCAI $(65 \%$ on 1 st CUS; mean time between symptoms and CUS diagnosis $34 \pm 30 \mathrm{hs}$ ). PD showed asymmetric flow (decreased or absent) in $90 \%$ of cases (mean time between symptoms and PD $21 \pm 19 \mathrm{hs}$ ). Abnormal PD preceded abnormal CUS (early-APD) in 5 infants (mean time $54 \pm 48 \mathrm{hs}$ ). PD showed higher systolic, diastolic and temporal mean $\mathrm{BFV}$ in the non-affected as compared with the affected side $(\mathrm{p}<.01)$. Comparisons between early-APD and the remaining infants yielded lower systolic BFV and lower resistance index $(\mathrm{p}=.048)$ in the affected side in the former, but no differences on PD measurements in the non-affected side between groups.

Conclusions: MCAI is clearly defined by CUS-PD. Studies performed soon after onset of symptoms could depict the earliest events evolving into definite structural damage. Studies are needed to address whether this could represent a therapeutic window.

\section{VENTRICULOMEGALY IN ELGA INFANTS - A 3D MRI STUDY}

S Horsch $^{1}$, J Bengtsson ${ }^{2}$, A Nordell ${ }^{2}, \mathrm{Z} \mathrm{Nagy}^{2}$, B Hallberg ${ }^{1}$, H Lagercrantz $^{1}$, B Nordell ${ }^{2}$, U Ådén ${ }^{1}$, M Blennow ${ }^{3}$

${ }^{I}$ Neonatal Clinics, Karolinska University Hospital, Stockholm, Sweden, ${ }^{2}$ Department of Medical Physics, Karolinska University Hospital, Stockholm, Sweden ${ }^{3}$ Department of CLINTEC, Karolinska University Hospital, Stockholm, Sweden

Background and Aims: Cerebral ventriculomegaly at term age is an important predictor of adverse neurological outcome in preterms. It is associated to intraventricular haemorrhages (IVH) and periventricular leukomalcia (PVL), but is also observed in preterms without IVH/ PVL and then representing white matter loss and global cerebral atrophy. 3D MRI provides a valid assessment of ventricle volumes. To identify risk factors for the development of ventriculomegaly we correlated ventricular volumes at term age to perinatal data in a population based cohort of extremely low gestational age (ELGA) infants.

Methods: 34 ELGA infants born in the Stockholm region were included (mean gestational age $25+3$ weeks (SD 9 days); mean birth weight of $773 \mathrm{~g}$ (SD 162g)). Infants were scanned at term equivalent age on a $1.5 \mathrm{~T}$ magnetic resonance system. A T1-weighted 3D-SpoiledGradient-Echo Sequence was used to perform volumetric measurements. Volume of the lateral ventricles was measured applying a software based on a semi-automatic Gradient Vector Flow Active Contour model.

Results: As expected IVH II-IV $(\mathrm{p}=0.041)$ and PVL $(\mathrm{p}=0.000)$ but also postnatal steroids $(\mathrm{p}=0.017)$ were identified as risk factors. In the linear regression analysis gestational age $(\mathrm{p}=0.033)$, days on ventilator $(\mathrm{p}=0.006)$ and cumulative dose of postnatal steroids $(\mathrm{p}=0.041)$ correlated to the ventricular volume at term age.

Conclusions: Ventriculomegaly can be precisely quantified by 3D MRI. Our study underlines that besides IVH and PVL, pulmonary morbidity (including its therapy (respirator, postnatal steroids)) is an important factor in the pathogenesis of ventriculomegaly at term age.

\section{THE ACCURACY OF A-EEG IN BOTH DETECTING POST-ASPHYXIAL NEONATAL SEIZURES AND CLASSIFICATION OF BACKGROUND ACTIVITY}

R Swarte $^{1}$, J Perumpillichira ${ }^{2}$, G Visser ${ }^{2}$, P Govaert ${ }^{1}$ 
${ }^{1}$ Department of Neonatology, Erasmus MC, Sophia's Childrens Hospital, Rotterdam, The Netherlands

${ }^{2}$ Department of Clinical Neurophysiology, Erasmus MC, Sophia's Childrens Hospital, Rotterdam, The

Netherlands

Background and Aims: Amplitude-integrated EEG (aEEG, CFM) is widely used in NICU's. We want to assess the accuracy of a-EEG in both neonatal seizure detection and classification of background activity.

Methods: 14 Full term neonates with asphyxia had seizures recorded on multi-channel EEG. Eight-hoursEEG segments were scored for onset, location, amplitude and frequency of electrographic seizures by a neurophysiologist. A-EEG with single-channel EEG (C3-4) were scored independently at paper speeds of 6 $\mathrm{cm} /$ hour and $30 \mathrm{~cm} /$ hour for seizures and amplitude of the background activity by a neurophysiologist and a neonatologist.

Results: 412 Seizures (mean 29 per patient, range 3144) were detected on EEG. At $6 \mathrm{~cm} / \mathrm{hour}$ and $30 \mathrm{~cm} / \mathrm{hr}$, a-EEG showed a median seizure detection rate of respectively $66 \%$ (mean 53,6 range $0-100$ ) and $72,9 \%$ $(58,2,0-100)$. The inter-rater agreement was $80 \%$. The seizures missed by a-EEG were $<50 \mu \mathrm{V}$ in amplitude, involved temporo-occipital or frontal brain regions, or contained excess of EEG artefacts. In 3 patients all seizures were missed. Classification of voltage of background activity by a-EEG was similar in all patients.

Conclusions: A-EEG detects slightly more than half the seizures in asphyxiated neonates. Some seizures started focally but generalized in the next hours resulting in detection on a-EEG. However, in low-voltage background activity all seizures were missed, which make a multi-channel EEG mandatory. A-EEG appeared to be a reliable tool in assessing the background EEG activity that can be of considerable help in prognostication after birth asphyxia.

\section{COMPARISON OF DOPPLER MEASUREMENTS WITH PHASE-CONTRAST (PC) MR ANGIOGRAPHY (MRA) MEASUREMENTS OF CEREBRAL PERFUSION IN NEWBORNS}

M J N L Benders ${ }^{1}$, J Hendrikse ${ }^{2}$, L S de Vries ${ }^{1}$, $\mathrm{F}$ van $\mathrm{Bel}^{1}, \mathrm{~F}$ Groenendaal ${ }^{1}$

${ }^{1}$ Department of Neonatology, University Medical Center Utrecht, Wilhelmina Children's Hospital, Utrecht, The Netherlands, ${ }^{2}$ Department of Radiology, University Medical Center Utrecht, The Netherlands

Background and Aims: Cerebral blood flow (CBF) alterations are important in the pathogenesis of neonatal ischemic brain damage. Estimations of CBF changes in newborns are mostly based on Doppler velocities in the basilar (BA) and internal carotid (ICA) arteries. Also with PC-MRA, blood flow can be obtained, but there is limited experience with this technique in newborns. Aim is to compare these two methods.

Methods: We compared BA and left/right-ICA's velocities obtained by Doppler velocities with twodimensional PC-MRA measured velocities in 18 newborns. Two-D-PC slice was positioned at the skull base level to measure BA and ICA's volume flow. Immediately thereafter Doppler measurements of these blood vessels were performed. Angle-corrected-timeaveraged flow-velocities were measured over 3-5 cardiac cycles.

Results: Newborns varied in gestational age (range 26$41 \mathrm{wks}$ ), weight (range 1470-5858 g) and age (0-103 days). A significant correlation coefficient was found between PC-MRA and Doppler velocities in BA and ICA's (see figure).

Conclusions: Two dimensional PC-MRA showed a significant positive correlation with Doppler measured cerebral velocities. This may open new possibilities for estimations of cerebral blood flow in ill neonates.
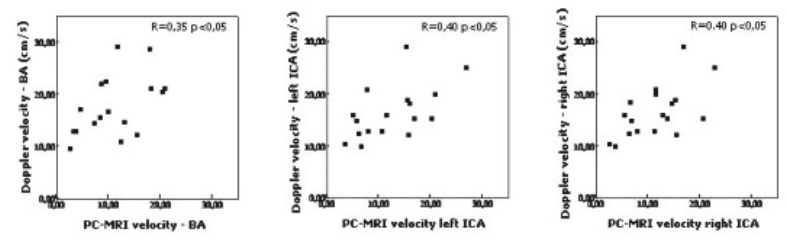

\section{ACCELERATED DEVELOPMENT IN THE VISUAL AREAS OF PRETERM INFANTS: A VOXEL-BASED MORPHOMETRY STUDY ON DIFFUSION TENSOR MR IMAGING (DTI)}

M J Miranda ${ }^{1}$, M Gimenez ${ }^{2}$, A P Born ${ }^{1}, \mathrm{Z} \mathrm{Nagy}^{3}$, $\mathrm{J} \mathrm{Terry}^{4}$, R Egill ${ }^{1}$

${ }^{l}$ Danish Research Centre for Magnetic Resonance, Copenhagen University Hospital, Hvidovre, Denmark ${ }^{2}$ Department of Psychiatry and Psychobiology, University of Barcelona, Spain, ${ }^{3}$ Department of Woman and Child Health, MR Unit, Karolinska Institute, Stockholm, Sweden, ${ }^{4}$ Laboratory of Cognitive Imaging (LOCI), La Jolla, CA, USA

Background and Aims: Premature birth has been related to delayed brain maturation. However, some studies suggest that the extrauterine environment stimulates development, and delayed maturation may therefore be associated with concomitant risk factors, rather than preterm birth itself. The possibility of more rapid maturation of certain brain areas has not yet been demonstrated. 1) To compare brain maturation in a sample of preterm neonates and a control group, and 2) to test the effect of postnatal age (PNA) in brain maturation.

Methods: 27 healthy preterm neonates (mean GA $31 \mathrm{w}$ ) and 10 full-term newborns (mean GA 39w) were included. Both groups were MR scanned at termequivalent ages. Diffusion weighted EPI images were acquired on a Siemens Magneton Trio 3T scanner (b-value $1000 \mathrm{~mm} 2 / \mathrm{s}, 6$ directions). Fractional anisotropy (FA) and apparent diffusion coefficient (ADC) images were calculated, and analysed using VBM technique: images were spatially normalised to a study specific template, and SPM2 tools were used for statistical analysis. We compared FA and ADC images using the two t-test comparison (control > preterm; preterm > control).

Results: When compared with the full-term group, preterm infants at term age showed increased FA in areas related to visual function. The t-test group comparison between preterm and controls showed FA 
increase in the preterm group at the medial temporal region, level of the sagittal stratum (SS). Correlation analysis demonstrated a positive relationship between FA values and PNA in the preterm group in the same areas. ADC showed no differences.

Conclusions: This VBM approach to FA images provides new insight into quantification of WM regional brain maturation. The differences observed in the SS in preterm infants compared to controls suggest that visualrelated brain areas (i.e. face perception) may be stimulated by early experience. Our findings lead to new investigations evaluating adaptive mechanisms of the developing brain at early stages. Sponsored by Mats Blennow.

\section{ACTIVATION OF TEMPORAL CORTEX IN 6-MONTH OLD INFANTS AFTER VISUAL EXPOSURE TO THEIR MOTHER'S FACE AND TO AN UNKNOWN FACE. A NEAR INFRARED SPECTROSCOPY STUDY}

\section{J Carlsson $^{1}$, M Bruschettini ${ }^{1,2}$, M Bartocci ${ }^{1}$, H Lagercrantz ${ }^{1}$ \\ ${ }^{\prime}$ Neonatal Research Unit, Astrid Lindgren's Children's Hospital, Karolinska Institutet, Stockholm, Sweden \\ ${ }^{2}$ G. Gaslini Children's Hospital, University of Genoa, Italy}

Background and Aims: One characteristic that makes human brain so unique is its ability at recognizing. Thus far little is known about neural mechanisms underling the capacity to discriminate objects/faces in human infants, mainly because there are few non-invasive techniques available. In this study we aimed to investigate, using a non-invasive technique such as nearinfrared spectroscopy (NIRS), cortical activation-related haemodynamic changes in response to visual stimulation in 6-month old infants after exposure to a known and an unknown face.

Methods: Thirteen infants have been monitored. We have used 3 different types of visual stimuli: a grey background (GB), the photo of the mother face (MF) and the photo of an unknown person (UF). These images have been presented to the child, on a PC screen, in the following order: GB - MF - GB - UF, keeping the environment around the child as quite as possible. Changes in regional oxy [HbO2], deoxy-[HbH] and total hemoglobin $[\mathrm{Hb}$ tot] have been measured by NIRS in both temporal and occipital areas.

Results: In the temporal area, [Hb tot] increased following the presentation of both mother's face $(+129 \%, \mathrm{P}<0.001)$ and unknown face $(+46 \%, \mathrm{P}<0.001)$. No significant changes were observed during GB. In the occipital area, different kinds of pattern have been observed.

Conclusions: The increase in [Hb tot] observed in the temporal area suggest haemodynamic and metabolic changes coupled to cortical activation in response to face presentation. A non invasive technique such as NIRS can be used to assess and follow the emergence and the development of image processing in infants.

\section{HIGH RESOLUTION VISUALISATION OF PH CHANGES IN MOUSE NEONATAL HYPOXIA ISCHEMIA}

G S Kendall, N J Robertson, D Peebles, G Raivich

Department of Obstetrics and Gynaecology, Centre for Perinatal Brain Protection and Repair, University College London, UK

Background and Aims: Infants with neonatal encephalopathy and experimental hypoxia-ischemia (HI) models show rebound brain intracellular alkalosis, this $\mathrm{pH}$ shift is known to correlate with neurodevelopmental outcome. Here we present an enhancement of neutral red spectrophotometry allowing regional $\mathrm{pH}$ measurements and creation of pseudocolour pH maps.

Methods: P7 mice underwent left carotid occlusion and 90 minutes hypoxia. Following neutral red injection, animals were sacrificed and their brains snap frozen. The cut surface of the brain was captured using a camera with narrow bandwidth filters. $\mathrm{pH}$ was calculated using the $540 \mathrm{~nm} / 470 \mathrm{~nm}$ absorbance ratios and a standard curve of brain homogenates at known $\mathrm{pH}$.

Results: HI resulted in a profound acidosis on the operated side; the moderate effects seen on the contralateral side suggests perfusion steal. Reperfusion resulted in recovery to normal $\mathrm{pH}$ followed by an alkalotic shift at 6 hours (Figure 1).

Conclusions: These techniques can be used to further study the effects of $\mathrm{pH}$ on histopathological outcome following neonatal $\mathrm{HI}$.

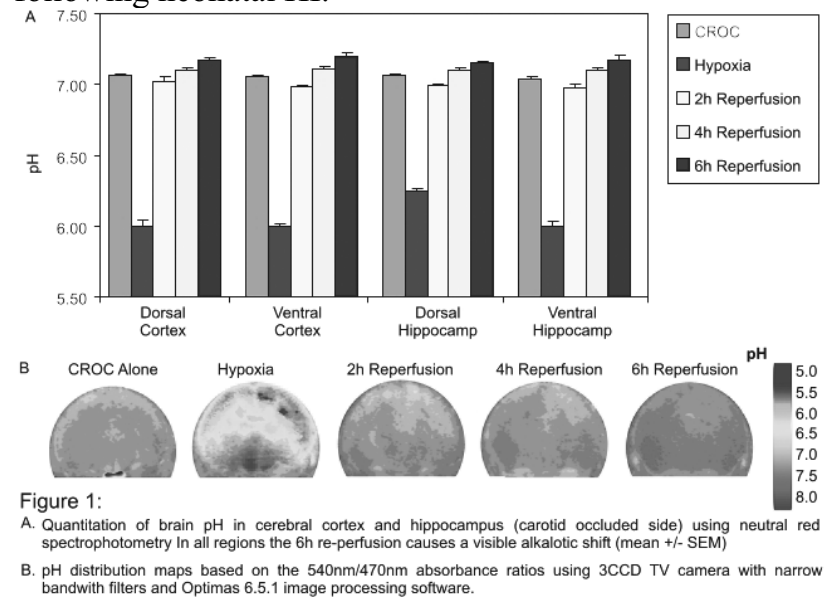

\section{METABOLIC MAPPING: MULTIPLE-VOXEL MRS AND LACTATE PATTERN AFTER SEVERE BIRTH ASPHYXIA}

B Hallberg ${ }^{1}$, L Douglas ${ }^{2}$, S Horsch ${ }^{1}, \mathrm{~K}_{\text {Leisdottir }}{ }^{1}$, M Mosskin $^{3}$, B Nordell ${ }^{2}$, H Lagercrantz ${ }^{1}$, M Blennow ${ }^{1}$

${ }^{I}$ Neonatal Programme, Karolinska Institutet, Karolinska University Hospital, Stockholm, Sweden, ${ }^{2}$ Medical Physics and MRI Unit, Karolinska Institutet, Karolinska University Hospital, Stockholm, Sweden, ${ }^{3}$ Department of Neuroradiology, Karolinska Institutet, Karolinska University Hospital, Stockholm, Sweden

Background and Aims: Several MR techniques are used to evaluate the degree of injury after hypoxic- 
ischemic insults in the newborn infant. Studies using single-voxel based techniques to obtain MR spectra have shown a good correlation between metabolic alterations and outcome. Objective: To develop and evaluate a multiple-voxel based MR-spectroscopy method and correlating metabolic information to the pattern of injury on conventional MRI and outcome.

Methods: Ten full term infants with severe birth asphyxia were included, (mean bw $3382 \mathrm{~g}$, Sarnat score $\mathrm{I}=(1), \mathrm{II}=(8), \mathrm{III}=(1))$. MRI examination on a $1.5 \mathrm{~T}$ MRI system was done at a median age of 7 days. MRspectroscopic imaging was used for each patient 2DMRS images were produced for choline, creatine, Naa, lactate and corresponding ratios using two different echo times, $\mathrm{TE}=136 \mathrm{~ms}, \mathrm{TE}=272 \mathrm{~ms}$, MRSI voxel volume 0.6 $1.1 \mathrm{~cm} 3$. A bicubic interpolation was done of the $24 \times 24$ pixel images to $256 \times 256$ pixel metabolite images with the anatomical contour superimposed. MR images were scored by three independent observers using a predefined scoring system (Barkovich) defining the pattern of injury; Watershed, Basalganglia/Thalamus predominant or normal. MRS injury pattern were scored.

Results: MRI pattern (WS $=6 \mathrm{BG}=4 \mathrm{~N}=0$ ) MRSI pattern $(\mathrm{WS}=6 \mathrm{BG}=4)$. In 3 infants with normal outcome the scorings from MRI and MRSI lactate and lactate/Choline were congruent. (WS=2 $\quad \mathrm{BG}=1$ ) Additional information about severity and the extent of the affected areas were obtained in all infants with neurological sequelae $(n=7)$.

Conclusions: Metabolic mapping using MRSI is possible and gives additional valuable clinical information on brain dammage after birth asphyxia. Table
Measuied

\begin{tabular}{|c|c|c|c|c|c|}
\hline & Pre CVVH & $15 \mathrm{mins}$ & $30 \mathrm{mins}$ & $45 \mathrm{~m}$ ins & $60 \mathrm{mins}$ \\
\hline HR (beat. $\min ^{-1}$ ) & $126(14)$ & $145(14)$ & $125(12)$ & $104(12)$ & 105 (11) \\
\hline $\operatorname{cl}\left(1 \cdot \mathrm{m}\right.$ in $\left.\cdot \mathrm{m}^{-3}\right)$ & $3.0(0.6)$ & $2.5(0.5)$ & $2.4(0.6)$ & $2.4(0.4)$ & $2.2(0.5)^{\pi \times}$ \\
\hline Svi $\left(m+m_{i n} \cdot m^{-2}\right)$ & $23.8(5.3)$ & $17.2(4.2)$ & $19.2(5.1)^{*}$ & $23.1(4.2)$ & $21(4.2)$ \\
\hline$C V P(m m H g)$ & $12.6(1.6)$ & $11.4(1.5)$ & $9.5(1.7)^{x}$ & $8.8(1.7)$ & $9.3(1.3)$ \\
\hline MABP $(m m H g)$ & $61(4.6)$ & 59.5 (3.6) & $62.3(4.5)$ & $63.3(3.4)$ & $60.1(3.3)$ \\
\hline SVR i(dyn.s.cm.m $\left.{ }^{-2}\right)$ & $540(299)$ & $585(277)$ & $658(310)$ & $750(256)$ & $744(296)^{\times x}$ \\
\hline $\mathrm{Sev}_{2}(\%)$ & $76.5(3.6)$ & $74.6(4.7)$ & $73.5(5.1)$ & $74.5(5.5)$ & $74.9(5.0)$ \\
\hline
\end{tabular}

\section{D ECHOCARDIOGRAPHY IN CONGENITAL HEART DISEASES}

\section{P Acar}

Department of Paediatric Cardiology, Hôpital des Enfants, Toulouse, France

Background and Aims: The introduction of real time three-dimensional (3D) echocardiography has led to its use in everyday clinical practice.

Methods: The 3D matrix probe enables the instantaneous acquisition of transthoracic volumes. Several modes of 3D are available: 3D volume, biplan and 3D color Doppler.

Results: Real time 3D echocardiography gave a more accurate description of various congenital heart diseases as well as valvulopathy, shunt and aorta pathology. Fetal 3D echocardiography is available. Quantitative measurement of ventricular volumes could be obtained by $3 \mathrm{D}$ echocardiography.
Conclusion: The facility of utilisation of the matrix probe should lead to routine usage of 3D echocardiography as with 2D and Doppler method. Its values should be decisive in many congenital cardiac lesions requiring surgery or interventional catheterisation.

\section{UPDATED RESULTS OF THE FETO TASK GROUP AS A PRELUDE TO SET-UP OF CLINICAL TRIALS}

A Debeer ${ }^{1}, \mathrm{~J} \mathrm{Jani}^{4}, \mathrm{~K}^{4}$ Allegaert ${ }^{1}, \mathrm{E}$ Gratacos ${ }^{3}$, $\mathrm{S} \mathrm{Salcedo}^{3}, \mathrm{~A} \mathrm{Greenough}^{4}, \mathrm{~K}$ H Nicolaides ${ }^{4}, \mathrm{~J}$ Deprest $^{2}$

${ }^{l}$ Department of Pediatrics/Neonatology, University Hospital Gasthuisberg, Leuven, Belgium, ${ }^{2}$ Department of Obstetrics and Gynaecology, University Hospital Gasthuisberg, Leuven, Belgium, ${ }^{3}$ Department of Obstetrics and Gynaecology, Hospital Clinic, Barcelona, Spain, ${ }^{4}$ Department of Obstetrics and Gynaecology, King's College Hospital, London, UK

Background and Aims: To review the effect of fetoscopic endoluminal tracheal occlusion (FETO) at 26-29 $\mathrm{w}$ on fetuses with severe congenital diaphragmatic hernia $(\mathrm{CDH})$ and define a strategy for future clinical trials.

Methods: Eligibility for FETO was single fetus with severe $\mathrm{CDH}$ (lung-to-head ratio $(\mathrm{LHR})<1$ and intrathoracic liver) without associated problems. FETO was at 26-29 wks gestational age (GA). Outcome measure was postnatal pulmonary outcome i.e. survival without, or death due to pulmonary hypoplasia and/or hypertension.

Results: 43 left and 10 right-sided $\mathrm{CDH}$ cases had a median LHR $=0.7$ prior to FETO (range: 0.5-0.9). Median operation time of 10 min (range: 3-60). Amniorrhexis occurred in $26 \%$ at $<32 \mathrm{wks}$, in the last half it was $16 \%$. Median GA at delivery was 35 wks (range 27-41). Airway patency was restored either perinatally $(\mathrm{n}=18)$ or antenatally at $34 \mathrm{wks}(\mathrm{n}=35)$. Early neonatal survival resp. at discharge were $60 \%(32 / 53)$ and $51 \%(27 / 53)$. Regression analysis showed that LHR prior to FETO is predicting survival, increasing from $13 \%$ for $\mathrm{LHR}<0.6 \quad(\mathrm{n}=8)$ to $52 \%$ for LHR of $0.6 \leq$ LHR $<0.8(n=25)$ and $65 \%$ for $0.8 \leq$ LHR $<1 \quad(n=20)$. Learning curve is suggested by improving PPROM rates and higher GA at delivery.

Conclusions: Survival after FETO is dependent on preoperative LHR and we suggest to report results likewise. When LHR $<0.6$ survival rate is $13 \%$, therefore pulmonary response to very early FETO $(<24 \mathrm{w})$ should be studied. Survival in fetuses with $\mathrm{CDH}$ and $0.6 \leq \mathrm{LHR}<1.0$ is $58 \%$, and in the more common left sided group, we suggest randomisation against optimal postnatal care.

\section{RESUSCITATION WITH $100 \%$ O2 CAUSES AUGMENTED CEREBRAL NECROSIS}

J H Andresen ${ }^{1}$, R Solberg ${ }^{1}$, B H Munkeby ${ }^{1}$, E M Løberg ${ }^{3}$, B Stray Pedersen ${ }^{2}$, O D Saugstad ${ }^{1}$

${ }^{I}$ Department of Pediatric Research, National Hospital, University of Oslo, Norway, ${ }^{2}$ Department of Obstetrics 
and Gynecology, National Hospital, University of Oslo, Norway, ${ }^{3}$ Department of Pathology, Ullevål University Hospital, Oslo, Norway

Background and Aims: Several studies have been undertaken on resuscitation with $100 \%$ oxygen versus room air. It has been shown that resuscitation with $100 \%$ oxygen has detrimental effects. We wish to investigate the effects of resuscitation with $100 \%$ oxygen on cerebral damage after hypoxic/ischemic injury compared to room air.

Methods: Two groups of newborn piglets (12-36 hours) were studied. They were randomized to either hypoxia or normoxia. The hypoxic group was randomized to be resuscitated with either $100 \%$ or $21 \%$ oxygen. Global hypoxia was induced by ventilation with $8 \%$ oxygen in $\mathrm{N} 2$ until Base Excess $<-20 \mathrm{mmol} / \mathrm{L}$ or mean arterial blood pressure $=15 \mathrm{mmHg}$. After hypoxia the animals were observed for $150 \mathrm{~min}$ and then euthanized, the brains removed and the left hemisphere and cerebellum fixated in $10 \%$ buffered formalin. They were stained with hematoxylin and eosin (HE) and immunhistochemistry performed with antiMicrotubule-associated protein 2 (MAP-2) antibodies, MAP-2 being a sensitive marker for ischemic cerebral damage. The HE-stainings and the immunhistochemistry were evaluated, blinded, by a pathologist.

Results: The sections from cerebellum presented increased numbers of necrotic purkinjecells in the $100 \%$ oxygen group (75\%), compared to the $21 \%$ group $(50 \%)$. Preliminary data from striatum present significantly more damage in the $100 \%$ group.

Conclusions: $100 \%$ oxygen appears to cause increased necrosis in cerebellum and striatum in newborn piglets.

\section{DEVELOPMENTAL CARE FOR PRETERM NEONATES: A SURVEY OF PRACTICES IN EUROPEAN NEONATAL UNITS}

M Cuttini ${ }^{1}$, A Maraschini ${ }^{1}$, G Greisen ${ }^{2}$, D Haumont ${ }^{3}$, C Pallàs ${ }^{4}$, V Pierrat ${ }^{5}$, B J Smith ${ }^{6}$, I Warren ${ }^{7}$, B Westrup ${ }^{8}$, J Sizun ${ }^{9}$

\section{${ }^{1}$ Unit of Epidemiology, Paediatric Hospital Bambino Gesù, Rome, Italy, ${ }^{2}$ Department of Neonatology, Rigshospitalet, Copenhagen, Denmark, ${ }^{3}$ Neonatal Unit, St Pierre University Hospital, Brussels, Belgium ${ }^{4}$ Neonatal Unit, Hospital 12 de Octubre, Madrid, Spain ${ }^{5}$ Centre Hospitalier Jeanne de Flandre, Lille, France ${ }^{6}$ Department of Neonatology, Erasmus MC, Rotterdam, The Netherlands, ' Winnicott Baby Unit, St. Mary's Hospital, London, UK, ${ }^{8}$ Neonatal Programme, Karolinska Institute, Stockholm, Sweden, ${ }^{9}$ Department of Paediatrics, Centre Hospitaliere de Brest, France}

Background and Aims: Interventions of "developmental care" are becoming popular in neonatal units to reduce infants' distress and promote well-being. This study, supported by the European Science Foundation, estimates the frequency and patterns of implementation in different European countries.

Methods: 279 neonatal units (response rate 78\%) in eight countries (Belgium, Denmark, France, Italy, Netherlands, UK, Spain and Sweden) answered a structured questionnaire. Over $2 / 3$ of units were 3 rd level; median number of yearly $<1500$ gr. admissions was 57 (from 23 in Sweden to 154 in Netherlands).

Results: Control of lighting was more prevalent than noise control (moderate lighting $24 \mathrm{~h}$ in $59 \%$ of Units; quiet times during the day in $40 \%$ ). Most units used nesting (73 to $100 \%$ ), regular variation of infant's position (54 to $86 \%$ ), and pacifier for comfort (69 to $100 \%)$. Strategies of pain management included written guidelines in $58 \%$ of units $(44 \%$ in Italy to $86 \%$ in Netherlands) and a reference person in $48 \%(15 \%$ in Italy to over $85 \%$ in Netherlands and Sweden). Parents were allowed to visit around the clock in $63 \%$ of units $(1 / 3$ in Italy and Spain to $100 \%$ in Denmark and Sweden), and were offered "Kangaroo care" in 74\% (from $35 \%$ in Spain to $100 \%$ in Sweden and Netherlands). Formal programs such as Neonatal Infant Developmental Care and Assessment Program (NIDCAP) were mostly applied in Denmark, Sweden and Belgium.

Conclusions: Most units routinely apply measures of developmental care; such measures, however, are most often and consistently applied in Sweden and Denmark, and less often in Italy and Spain.

\section{NEONATAL OUTCOME IN MONOCHORIONIC TWINS WITH TWIN-TO-TWIN TRANSFUSION SYNDROME MANAGED BY INTRAUTERINE LASER THERAPY AND DICHORIONIC TWINS: A CASE CONTROL STUDY}

R Lenclen ${ }^{1}$, G Ciarlo ${ }^{1}$, A Paupe ${ }^{1}, \mathrm{~S}$ Couderc

Background and Aims: Monochorionic (MC) pregnancies complicated by twin-to-twin transfusion syndrome (TTTS) are at increased risk for prematurity as well as perinatal mortality and morbidity. Although perinatal outcome in TTTS has improved after laser therapy, neonatal mortality and morbidity remain high. The respective role of prematurity and of fatal disease are ill-defined. The objective of this study was to compare neonatal outcomes between TTTS infants and dichorionic (DC) twins, and to evaluate the impact of laser therapy.

Methods: This was a case-control retrospective study carried out at our center between 2000-2005. Neonatal outcome was assessed in 97 TTTS infants treated in utero with laser ( $\mathrm{n}=56$ pregnancies). Control group consisted of 97 DC twins (56 DC pregnancies) delivered between 24-34 weeks of gestation and matched for gestational age at birth with the cases.

Results: Gestational age at delivery was similar in both groups (median=30;0, IQR 28-34wks), as well as birth weight. Neonatal outcome in TTTS infants was not significantly different from those of DC infants: mortality $(11.3 \%$ vs $12.3 \%)$, severe cerebral lesions (17.9\% vs $12.9 \%)$. The incidence of adverse neonatal outcome (death or severe cerebral lesion) was not different within groups $(21 \%$ vs $18 \%)$. Logistic regression analysis demonstrated that gestational age 
was the only significant factor associated with adverse neonatal outcome; IUGR, intrauterine death and chorionicity failed to show any significant effect.

Conclusions: Neonatal mortality and morbidity rates of TTTS infants treated by laser were not different from those of DC infants matched for gestational age. Neonatal outcome was strongly related to prematurity.

\section{ENTERAL GLUTAMINE SUPPLEMENTATION IN THE NEONATAL PERIOD IN VLBW INFANTS: EFFECT ON INCIDENCE OF ALLERGIC AND INFECTIOUS DISEASES IN THE FIRST YEAR OF LIFE}

\section{A van den Berg ${ }^{1}$, A van Zwol ${ }^{1}, \mathrm{H}^{\mathrm{A}} \mathrm{Moll}^{2}$, W P F Fetter ${ }^{1}$, R M van Elburg ${ }^{1}$ \\ ${ }^{I}$ Department of Pediatrics, Subdivision of Neonatology, $V U$ University Medical Center, Amsterdam, The Netherlands, ${ }^{2}$ Department of Pediatrics, Erasmus Medical Center, Sophia Childrens Hospital, Rotterdam, The Netherlands}

Background and Aims: In a previous, randomised controlled trial of enteral glutamine supplementation in very low birth weight (VLBW) infants the incidence of infectious morbidity was decreased in the glutaminesupplemented group. We hypothesize that glutamineenriched enteral nutrition in the neonatal period may have consequences for the immune response to microorganisms and allergic antigens later in life and the incidence of allergic and infectious disease.

Methods: The parents of 89/102 surviving infants who participated in the initial study (13 infants died) were asked to complete a questionnaire on infectious and allergic disease during the first year of life. The questionnaire was adapted from two validated questionnaires, used in the PIAMA Study and the Generation R Study respectively. Logistic regression was used for data analysis. Corrections were made for possible confounding factors, as smoking at home, childcare attendance, siblings, infection during neonatal period and parental allergic disease.

Results: 77/89 (86\%) questionnaires were completed. The mean corrected age of the infants was $54.8 \pm 5$ weeks. There was less atopic dermatitis in the glutamine-supplemented group (5 versus $20 \%$ ) was found: Odds ratio (OR) $0.13 \quad(95 \%$ CI $0.02-0.96$; $\mathrm{P}=0.05$ ), and a trend towards less bronchial hyperreactivity OR $0.36,(95 \%$ CI $0.1-1.25 ; \mathrm{P}=0.11)$. The incidence of infections in the first year of life was not different in the glutamine-supplemented and control group.

Conclusions: Glutamine-enriched enteral nutrition in VLBW infants in the neonatal period may contribute to less allergic disease in the first year of life. Glutamineenriched enteral nutrition had no effect on the incidence of infectious disease during the first year of life.

\section{THE EFFECT OF GLYCAEMIA ON THE TOI IN VERY LOW BIRTH WEIGHT INFANTS}

J L S Vanderhaegen, G Naulaers, S Vanhaesebrouck, C Vanhole, H Devlieger, P Casaer
Neonatal Intensive Care Unit, University Hospital UZ Gasthuisberg, Leuven, Belgium

Background and Aims: The cerebral TOI (tissue oxygenation index) reflects the cerebral oxygenation. In this study we analyzed the effect of changes in glycaemia on the TOI, as measured by NIRS, in neonates.

Methods: Inclusion criteria were postmenstrual age less than 32 weeks and weight less than 1500 grams. In all patients at least 4 hours of continuous monitoring of TOI with NIRS (NIRO 300, Hamamatsu), glycaemia (Medtronic MiniMed CGMS System Gold), mean arterial blood pressure (MABP), saturation and if possible also $\mathrm{TCO} 2$ was performed during the first days of life. The means of TOI, glycaemia, tPCO2 and MABP were calculated. Multiple regression analysis was performed correcting for saturation, MABP and if possible tPCO2.

Results: 50 Measurements in 15 infants are analysed. In a subgroup of 16 measurements, also the $\mathrm{tPCO} 2$ was continuously monitored. In 29 of the 50 measurements (58\%), a significant positive correlation was found between the TOI and glycaemia after correction for saturation and MABP. In the subgroup additional correction for $\mathrm{tPCO} 2$ influenced the level of significance in only 3 of the 16 measurements. None of the patients suffered from severe hypoglycaemia.

Conclusions: Our results indicate that changes in glycaemia within the normal ranges and even after correction for MABP, saturation and $\mathrm{PCO} 2$, had a direct effect on the cerebral oxygenation in neonates during the first days of life. In studies regarding cerebral oxygenation, glycaemia levels should be included.

\section{PREGNANCY COMPLICATIONS AND OUTCOME IN INFANTS $<32$ WEEKS GESTATION: THE ACTION STUDY}

\author{
L Gagliardi ${ }^{1}$, M Da Frè̀ ${ }^{2}$ E Buiatti ${ }^{2}$, V Carnielli ${ }^{3}$, \\ $\mathrm{C}_{\text {Corchia }}^{4}$, D Di Lallo ${ }^{5}$, F Macagno ${ }^{6}$, S Miniaci ${ }^{7}$, \\ M Cuttini $^{4}$ \\ ${ }^{1}$ Versilia Hospital, Versilia, Italy, ${ }^{2}$ Regional Health \\ Authority, Florence, Italy, ${ }^{3} G$ Salesi Hospital, Ancona, \\ Italy, ${ }^{4}$ Pediatric Hospital Bambino Gesù, Rome, Italy \\ ${ }^{5}$ Agency for Public Health, Rome, Italy, ${ }^{6}$ Udine \\ Hospital, Udine, Italy, ${ }^{7}$ Pugliese-Ciaccio Hospital, \\ Catanzaro, Italy
}

Background and Aims: To study the effect of pregnancy complications leading to preterm birth on outcome of very preterm infants.

Methods: A cohort of NICU admitted infants $<32$ weeks, born in 2003-2004 in 6 Italian regions (ACTION study) was prospectively studied. Pregnancy complications and cause of preterm birth were grouped as follows: preterm labour (PTL: including prelabour rupture of membranes, infection); hypertensive disorders (HD: hypertension, preeclampsia, eclampsia, HELLP); Intra Uterine Growth Restriction (IUGR); placental haemorrhage; malformations. Outcomes were in-hospital mortality, and ultrasound-diagnosed white matter damage (WMD: periventricular leucomalacia 
and/or severe intraventricular haemorrhage) among survivors.

Results: 2953 infants (mean birthweight 1158 g, mean GA 28+3) were analysed; in-hospital mortality was $17.4 \%$; WMD in survivors was $8.3 \%$. PTL frequency was $52.1 \%$, HD $20.9 \%$, IUGR $14.2 \%$, haemorrhage $9.9 \%$; malformations $1.6 \%$. PTL was more frequent in extremely preterms (22-27 weeks), while HD in intermediate preterm (28-31): GA differences could confound the association with outcome. In a multivariate logistic model, after controlling for GA and others factors influencing outcome (antenatal steroids, sex, illness severity (CRIB), twin birth, cesarean section, region), malformations and IUGR were independent risk factors for mortality $(\mathrm{OR}=12.0,95 \% \mathrm{CI} 5.7-25.2$, and 1.6, CI 1.1-2.5 respectively), while HD tended to be protective $(\mathrm{OR}=0.68$, CI $0.45-1.02)$. Using the same model, HD was protective against WMD in survivors $(\mathrm{OR}=0.55$, CI 0.32-0.95).

Conclusions: Different pregnancy complications and the cause of preterm delivery were differently associated with mortality and WMD. This points to different pathways leading to preterm birth and subsequent damage. Overall, HD seems to be protective against adverse outcomes in very preterm infants.

\section{NEW DIAGNOSTIC TECHNIQUES IN RESPIRATORY MEDICINE BEYOND LUNG FUNCTION: INVESTIGATION AND MANAGEMENT OF ASTHMA}

\section{E Baraldi}

\section{Department of Paediatrics, University of Padova, Italy}

The inflammatory state of asthmatic airways may correlate weakly with clinical symptoms and lung function parameters, and that is why the development of non-invasive methods for inflammatory monitoring is very important in asthma. Exhaled nitric oxide (FENO) measurement FENO has been studied extensively, validated as a non-invasive marker of eosinophilic airway inflammation in asthma, and standardized for use in clinical practice. Guidelines for standardized FENO measurement have been published jointly by ATS and ERS and normal reference values with the recommended technique are available for children 4-17 years old. The single breath online method (SBOL) is the gold standard for FENO measurement in cooperating children. The child inhales NO-free air to near-total lung capacity, and then exhales at a constant flow of 50 $\mathrm{ml} / \mathrm{sec}$ for at least 4 seconds. On-line FENO measurement during spontaneous breathing has been applied in children aged 2-5 years old, with the exhalation flow manually adjusted to $50 \mathrm{ml} / \mathrm{sec}$ by changing the exhalation resistance. The method is not well standardized, however, and still requires some passive co-operation. FENO may have a role as a diagnostic tool. Avital et al. showed that FENO can differentiate asthmatic young children from nonasthmatic cases with chronic cough. In addition, in children with recurrent wheezing, raised FENO values may be useful in identifying patients most likely to respond to inhaled corticosteroid (ICS). FENO measurement is also useful in asthma follow-up. In children, FENO levels have been demonstrated to predict asthma relapse after discontinuing steroids and to predict the failure of attempts to reduce ICS dosages. Exhaled breath condensate (EBC) Exhaled breath condensate, obtained by cooling exhaled air, contains water vapor and micro-droplets, the composition of which is believed to reflect that of the airway lining fluid. EBC collection is totally non-invasive and easy to perform even in children. Recommendations have been recently published on how to collect EBC. The drawbacks of EBC analysis lie in that the concentrations of mediators in EBC are low, the anatomical sources of the compounds measured in EBC are not well defined and most of the measurements are not done in real time. A large number of mediators have been measured in EBC. Markers of oxidative stress- as hydrogen peroxide (H2O2), isoprostanes, aldehydes and nitrotyrosine- are increased in asthmatic subjects, while glutathione (a protective lung antioxidant) is reduced. Beside, inflammatory metabolites, such as cysteinyl leukotrienes (Cys-LTs), have been found increased in allergic asthmatic children, despite corticosteroid treatment. Also, a reduced EBC $\mathrm{pH}$ has been reported in asthmatic children, suggesting that a dysregulation in airway $\mathrm{pH}$ homeostasis may have a role in asthma pathogenesis. Conclusion Exhaled nitric oxide has been established as a marker of eosinophilic airway inflammation in asthma and recent studies have included FENO measurement in treatment algorithms. Exhaled breath condensate is a non-invasive technique for sampling the airways, which has the potential to offer new insight on lung disease pathobiology.

\section{IMPACT OF CHANGING PRACTICE WITH THE USE OF CPAP}

T del Moral, N Claure, S Vanbuskirk, E Bancalari

Department of Pediatrics, University of Miami, FL, USA

Background and Aims: Early Continuous Positive Airway Pressure may reduce lung damage by decreasing the need for mechanical ventilation and it is increasingly used as an alternative strategy for ELBW infants with RDS. The objective of this study was to evaluate changes in the use of CPAP and the impact of these changes on the use of surfactant, mechanical ventilation, oxygen therapy and incidence of BPD.

Methods: The study included 606 neonates with BW between $500-1000 \mathrm{~g}$ born at the University of Miami/Jackson Memorial Hospital during two periods of time (1998 to 2000 and 2002 to 2004). Outcome variables were compared between the two periods by $\chi^{2}$. All differences reported were statistically significant $(\mathrm{p}<0.05)$.

Results: During the second time period there was an increased proportion of infants never intubated $(5 \%$ vs $15 \%)$, decrease use of surfactant ( $66 \%$ vs $58 \%)$, and a decreased mechanical ventilation use (intubated at 24 hours $84 \%$ vs $71 \%$ ) and duration (intubated at 7 days $66 \%$ vs $51 \%$ ). These changes were not accompanied by a lower incidence of BPD. In fact, during the later period a larger proportion of infants required oxygen early during their course $(\mathrm{O} 2>21 \%$ at 7 days $39 \%$ vs 
$48 \%)$ and the incidence of BPD was higher ( $29 \%$ vs $39 \%)$.

Conclusions: The reduced use and duration of mechanical ventilation was not associated with improved respiratory outcome. The increased use of supplemental $\mathrm{O} 2$ in infants with non-invasive respiratory support could explain the higher incidence of BPD in the second time period.

\section{ADDITIVE EFFECTS OF KL4 AND POLYMERS FOR PREVENTION OF SURFACTANT INHIBITION}

\section{K W Lu, H W Taeusch}

\section{Department of Pediatrics, University of California San} Francisco School of Medicine, San Francisco, CA, USA

Background and Aims: Inactivation of pulmonary surfactant is caused by acute lung injuries and interferes with the effectiveness of surfactant replacement therapy in these diseases. Surfactant proteins, such as SP-B, and various ionic and nonionic polymers, such as hyaluronan (HA) or polyethylene glycol (PEG), can reduce inhibition of adsorption caused by serum. Our aim was to understand the interaction between an SP-B analogue and polymers in preventing serum inhibition of surfactant.

Methods: We used currently available surfactants, surfactant lipids, or surfactant lipids with either 0.4 or $0.8 \mathrm{mg} / \mathrm{mL}$ of KL4, a synthetic analogue of SP-B (Discovery Laboratories, Inc, USA). Mixtures (1.25 $\mathrm{mg} / \mathrm{mL}$ ) were studied in a pulsating bubble surfactometer that measures surface activity. HA 250 $\mathrm{kDa}, 0.25 \% \mathrm{w} / \mathrm{v}$, or PEG $10 \mathrm{kDa}, 5 \% \mathrm{w} / \mathrm{v}$, were added to some mixtures.

Results: As shown in the table, KL4-surfactant 0.8 $\mathrm{mg} / \mathrm{mL}$ (shaded row) required at least twice the amount of serum to inhibit activity compared with lipids alone, KL4-surfactant $0.4 \mathrm{mg} / \mathrm{mL}$, and versus historical data with beractant, calfactant, or poractant alfa ( $\mathrm{Lu}$ et al. Pediatr Res. 2005;57:237).

Conclusions: Adding KL4 to surfactant lipids reduces serum inhibition in a concentration-dependent manner. Effects of PEG or HA are most evident in the presence of either concentration of KL4. These results indicate additive effects of KL4 and polymers in reducing serum inhibition. Supported by Discovery Labs.

Minimum Surface Tension $(\mathrm{mN} / \mathrm{m})$ in the Presence of Serum

\begin{tabular}{|c|c|c|c|c|}
\hline & \begin{tabular}{|c|}
$\begin{array}{c}\text { Surfactant:Serum } \\
\text { Ratio }^{*}\end{array}$ \\
\end{tabular} & No Polymer ${ }^{b}$ & $+\mathrm{HA}^{\mathrm{b}}$ & $+\mathrm{PEG}^{\mathrm{b}}$ \\
\hline Lipids alone & \begin{tabular}{|l|}
200 \\
\end{tabular} & $29 \pm 1.5$ & $8 \pm 2.5^{\top}$ & $23 \pm 2$ \\
\hline Lipids+0.4 mg/mL KL & 200 & $14 \pm 1$ & $6 \pm 2$ & $5 \pm 0.5$ \\
\hline Lipids $+0.8 \mathrm{mg} / \mathrm{mL} \mathrm{KL}_{4}$ & 50 & $25 \pm 2$ & $4 \pm 2^{\circ}$ & $3 \pm 0.5^{\circ}$ \\
\hline
\end{tabular}

${ }^{a} 1.25 \mathrm{mg}-\mathrm{TPL} / \mathrm{mL}$ surfactant to serum ratio (v:v); ${ }^{b}$ mean \pm SEM. ${ }^{\circ} P<.05$ vs no polymer

\section{IS DECREASING PRESSURE OR INCREASING TIME OFF THE BETTER STRATEGY IN WEANING VLBW INFANTS FROM NASAL CPAP?}

S D Singh ${ }^{1}$, L Bowe $^{1}$, P Clarke $^{1}$, K Glover $^{2}$, A Pasquill ${ }^{3}$, M J Robinson ${ }^{1}$, J Smith ${ }^{4}$

${ }^{1}$ NICU, Hope Hospital, Salford, UK, ${ }^{2}$ NICU, Royal Albert Edward Infirmary, Wigan, UK, ${ }^{3}$ NICU, Royal Bolton Hospital, Bolton, UK, ${ }^{4}$ NICU, Panorama Gardens Hospital, Cape Town, South Africa
Background and Aims: Nasal CPAP (nCPAP) is currently used after extubation from mechanical ventilation. There is, however, uncertainty regarding the best approach to nCPAP weaning. Objective: To compare the effectiveness of pressure reduction with alternating periods of nCPAP with nasal cannula low flow oxygen ('time off') in weaning very low birthweight (VLBW) infants from nCPAP.

Methods: Infants $<1500 \mathrm{~g}$, and stable in $<0.3 \mathrm{FIO} 2$ on nCPAP were randomised to a weaning strategy of either gradual reduction of nCPAP 'pressure', or increasing duration of 'time off' nCPAP. Infants were considered successfully weaned if stable off nCPAP for 7 days.

Results: Median (range) birth weights and gestations were: 'pressure' group, 940g (614 - 1400g) and $27(24-$ 32) wks; 'time off' group, 1080g (520 - 1496g) and 28 (24 - 31) wks. Median PMA at randomisation 29 (28 - 31) wks for both groups. Of 112 eligible infants, $36(65 \%)$ in the 'pressure' and $21(37 \%)$ in the 'time off' arms successfully weaned $(\mathrm{p}=0.006)$ [95\% CI 0.10 $0.46]$. Infants allocated to 'pressure' arm weaned faster and spent fewer days on nCPAP. Median (range) duration of weaning via pressure reduction was $1.5(0.9$ - 50.5) days vs. $8.9(9.0$ - 33.3) days in the 'time off' group. ( $\mathrm{p}=<0.001$ ) Median total time spent on NCPAP was proportionately shorter in the ' pressure' group [6.0 $(2.1-60.0)$ days vs. $13.2(10.0-46.0)](\mathrm{p}=0.001)$.

Conclusions: For VLBW infants, gradual reduction in nCPAP pressure may facilitate more rapid respiratory weaning compared with periods of increasing time spent off nCPAP.

\section{NASAL CONTINUOUS POSITIVE AIRWAY PRESSURE WEANING IN PRETERM INFANTS}

A Soe, J Hodgkinson, B Jani, D A Ducker

Oliver Fisher Neonatal Unit, Medway Maritime Hospital, Gillingham, Kent, UK

Background and Aims: During weaning process from Nasal Continuous Positive Airway Pressure (NCPAP) in preterm infants, it is unclear whether weaning by pressure or time of CPAP is more effective. There are no published data available comparing the two methods. Methods: A prospective study was designed to compare the effects of the two different types of weaning, using infant flow driver. Preterm infants (gestation 24-31 weeks) with respiratory distress syndrome or immature lungs were randomly allocated to Time weaning or Pressure weaning and weaned over 6 days. The data were analysed according to stratification depending on the gestational age.

Results: 98 Infants (24-27 weeks, $\mathrm{n}=44$ and 28-31 weeks, $n=54)$ were randomised to Pressure $(n=49)$ and Time $(\mathrm{n}=49)$ weaning. In the $24-27$ weeks group, 18 $(82 \%)$ in the pressure arm and $12(55 \%)$ in the time arm were weaned successfully $(\mathrm{p}<0.05)$. Total CPAP days were 10 and 15 respectively $(p<0.05)$. At 0,1 and 6 day, there were no differences in the $\mathrm{pH}, \mathrm{PCO} 2, \mathrm{FiO} 2$; number of apnoeas and bradycardias or feeding tolerance between the two arms. There was a trend of less hospital days and less oxygen dependency at 36 weeks in the pressure arm. In the 28-31 weeks gestation group all infants except one in the time arm were 
weaned successfully and there was no difference in the other outcome data.

Conclusions: In infants of gestation 28 weeks onwards, NCPAP weaning can be successful by either method; however, in infants of 24-27 weeks gestation pressure weaning may prove to be more appropriate.

\section{ENDOTOXIN INDUCED CHORIOAMNIONITIS RESULTS IN MATURATION OF ALVEOLAR MACROPHAGES BY TRANSCRIPTION FACTOR PU.1 IN PRETERM SHEEP}

\author{
B W Kramer ${ }^{1,4}$, T J Moss ${ }^{2}$, I Nitsos ${ }^{2}$, J P Newnham ${ }^{2}$, \\ C P Speer ${ }^{1}$, A H Jobe \\ ${ }^{1}$ University Children's Hospital, Wuerzburg, Germany \\ ${ }^{2}$ University of Western Australia, Perth, Australia \\ ${ }^{3}$ Cincinnati Children's Hospital, Cincinatti, OH, USA \\ ${ }^{4}$ University Hospital, Maastricht, The Netherlands
}

Background and Aims: Chorioamnionitis is frequently associated with preterm birth and inflammation in fetal lungs. The fetal lung is immune naive and blood derived monocytes differentiate into alveolar macrophages expressing transcription factor PU.1 late in gestation. Fetal inflammatory responses are minimal to tumor necrosis factor (TNF)-alpha in sheep. We hypothesized that chorioamnionitis induced maturational changes in the monocytes of the fetal lung.

Methods: Time-mated ewes with singletons received $10 \mathrm{mg}$ endotoxin or saline by intra-amniotic injection $2 \mathrm{~d}$ or $7 \mathrm{~d}$ before delivery at $124 \mathrm{~d}$ gestational age $(\mathrm{n}=7$ per group, term 150d). Monocytes/macrophages were isolated with Percoll gradient from the right lung. Monocytes/macrophages were analyzed and cultured with recombinant ovine TNF-alpha (50ng/ml, endotoxin-free).

Results: Macrophages were identified by size and MHCII expression with $98 \pm 12$ mean fluorescent units in controls and $305 \pm 41$ after $7 \mathrm{~d}$. The percentage of macrophages increased to $90 \pm 4 \%$ after $7 \mathrm{~d}$ versus $12 \pm 7 \%$ in controls $(\mathrm{p}<0.05)$. Macrophages isolated after $2 \mathrm{~d}$ of chorioamnionitis produced $28 \pm 5 \mu \mathrm{mol}$ hydrogen peroxide/106 macrophages and $97 \pm 13 \mu \mathrm{mol} / 106$ after $7 \mathrm{~d}$ when stimulated with TNF-alpha $(\mathrm{p}<0.05$ vs same cells with media only and controls $(11 \pm 5 \mu \mathrm{mol} / 106)$ which did not respond to TNF-alpha. Few alveolar macrophages from controls were positive for PU.1 $(8 \pm 3 \%)$ but $55 \pm 10 \%$ after $7 \mathrm{~d}(\mathrm{p}<0.05)$.

Conclusions: Pulmonary inflammation recruited monocytes into the fetal lung which differentiated into macrophages by expressing PU.1. In vitro responses to TNF-alpha were induced within $2 \mathrm{~d}$ of chorioamnionitis. Chorioamnionitis modulates the function of fetal lung monocytes consistent with maturation which may affect subsequent immune responses after preterm birth. Funded by: NIH HL-65397 and HD-12714.

\section{CHARACTERISATION OF AN ANATOMICALLY CORRECT UPPER AIRWAY MODEL IN A 32 WEEKS GESTATIONAL AGE PREMATURE INFANT}

S Minocchieri ${ }^{1}$, J Burren ${ }^{1}$, M A Bachmann ${ }^{1}$, P S Huppi $^{3}$, R Schindel ${ }^{4}$, J H Wildhaber ${ }^{2}$, M Nelle $^{1}$
${ }^{1}$ Division of Neonatology, University Children's Hospital, Bern, Switzerland, ${ }^{2}$ Division of Respiratory Medicine, University Children's Hospital, Zurich, Switzerland, ${ }^{3}$ Child Development Unit, Department of Paediatrics, University Hospital, Geneva, Switzerland, ${ }^{4}$ Institute for Rapid Product Development, University of Applied Sciences, St. Gallen, Switzerland

Background and Aims: Effective airway passage of aerosols in respiratory therapy of neonates is crucial for a positive outcome. Therefore, devices aimed at delivering aerosols to the lungs such as inhalers and nebulisers must be studied thoroughly. For this purpose we constructed an anatomically correct model of the upper airways in a 32 weeks gestational age premature infant. This study describes the construction process, validation and characterization of this airway model.

Methods: Three-dimensional reconstruction of a threeplanar MRI scan was done using Magics(C) software (Materialise $^{\mathrm{TM}}$, Munich, Germany) followed by selection of an optimal air-mucosa threshold. Model reconstruction was achieved using the generated data and Selective Laser Sintering Technique. Subsequently, validation was realized between the original data and a CT scan of our model by comparison of airway volume and anatomical reference points.

Results: The construction process proved to have a high accuracy. The airway volumes of the initial MRI and the control CT scan showed to be $1277 \mathrm{~mm} 3$ and $1265 \mathrm{~mm} 3$, respectively. The final relative deviation in volume was 0.94 percent.

Conclusions: We constructed an anatomically correct model of the upper airways of a 32 week-old premature infant with high accuracy. This model is suitable for further in vitro measurements such as lung dose measurements of aerosols.

\section{CARDIOVASCULAR MONITORING USING TRANSPULMONARY THERMODILUTION VIA FEMORAL ARTERY (QTD FEM) DURING TOTAL LIQUID VENTILATION (TLV)}

H Walti ${ }^{1,2}$, R Robert $^{3}$, J Lebon ${ }^{2}$, O Lesur ${ }^{2}$, J P Praud ${ }^{1,2}$, P Micheau $^{3}$

${ }^{I}$ Department of Paediatrics, Faculty of Medicine, Sherbrooke, QC, Canada, ${ }^{2}$ Department of Physiology, Faculty of Medicine, Sherbrooke, QC, Canada, ${ }^{3}$ Department of Mechanical Engineering, Faculty of Engineering, Sherbrooke, QC, Canada

Background and Aims: Accuracy of QTD FEM, a relatively non-invasive method applicable in a clinical setting, to monitor cardiovascular parameters has not yet been described during TLV. This study evaluates cardiovascular influence of TLV using QTD FEM in a controlled animal experimental trial.

Methods: 16 anesthetised-paralysed healthy newbornlambs instrumented for monitoring of: 1-Pulmonary artery (PAP), central venous pressure (CVP) via SwanGanz catheter; 2-Cardiac index (CI), arterial blood pressure (AP), indexed stroke volume (SVI), systemic vascular resistance (SVRI), intra-thoracic blood-volume (ITBI), extra-vascular lung-water (EVLWI) using QTD fem (PC-8000-PICCO-monitor, Pulsion-Medical- 
System, Ge). Following baseline recording, animals were divided in 2 groups: $\mathrm{GV}=8$, who remained $\mathrm{GV}$ at: $\mathrm{Fr}=40 / \mathrm{min}, \quad \mathrm{PiP}=15 \mathrm{~cm} . \quad \mathrm{H} 2 \mathrm{O}, \quad \mathrm{Peep}=5 \mathrm{~cm} . \mathrm{H} 2 \mathrm{O}$, $\mathrm{FiO} 2=1.0 ; \mathrm{TLV}=8$, whose lungs were filled with $30 \mathrm{ml} / \mathrm{kg}$ PFOB (F2-chemicals, UK) and then ventilated with volume-controlled-pressure-limited liquid ventilator at $\mathrm{Fr}=3-4 / \mathrm{min}, \quad \mathrm{Vt}=25 \mathrm{ml} / \mathrm{kg}, \quad \mathrm{I} / \mathrm{E}=1: 3$, $\mathrm{FiO}=1.0$. Gas exchanges, Lactate/Pyruvate ratio $(\mathrm{L} / \mathrm{P})$ and cardiovascular parameters were recorded at $30 \mathrm{~min}$ intervals during a $120 \mathrm{~min}$ period.

Results: As compared to GV group overall and to baseline GV, TLV animals demonstrated an increase in $\mathrm{PaCO} 2$, and a decrease in $\mathrm{PaO} 2$, but gas exchanges were maintained in the targeted range. A decrease in $\mathrm{pHa}$ was also observed, with no difference in $\mathrm{L} / \mathrm{P}$ however. AP was decreased but maintained in the targeted range. There was no change in PAP or CVP. CI, SVI and ITBI were increased, and SVRI was decreased. Moreover, a $30 \mathrm{ml} / \mathrm{kg}$ increase in EVLWI was demonstrated.

Conclusions: Due to the high thermal conductibility of PFOB, accuracy of QTD FEM during TLV is questionable. Nonetheless, this method could be considered to monitor liquid FRC during TLV.

\section{IMPROVING CPAP DELIVERY USING HELIUM- OXYGEN GAS MIXTURES}

\author{
M Chowdhury ${ }^{1}$, P Dixon $^{2}, \mathrm{P} \mathrm{Habibi}^{1}$ \\ ${ }^{I}$ Department of Paediatrics, Imperial College of \\ Science, Technology and Medicine, London, UK \\ ${ }^{2}$ Viasys Healthcare, London, UK
}

Background and Aims: Continuous positive airways pressure (CPAP) is an established technique for managing acute respiratory failure. Helium-oxygen gas mixtures (heliox), due to its physical properties, may enhance the physiological benefits of CPAP if used as the driving gas. The aim of this study was to assess if heliox can be safely and effectively used as the driving gas through an infant flow driver, one of the commonest CPAP devices in paediatrics.

Methods: The ASL5000 Servo Lung simulator was used to compare heliox/oxygen mixtures versus air/oxygen mixtures as the driving gases of the infant flow driver. Studies were undertaken at 4 different CPAP settings across varying tidal volumes and respiratory rates. In each case pressure, flow and volume changes were measured by the lung simulator. Blender accuracy was noted as well as noise emission.

Results: 1) Up to $6 \mathrm{cmH} 20 \mathrm{CPAP}$ pressure was achievable using heliox. 2) The work of breathing (WOB) was reduced by up to $60 \%$ when using heliox. 3) Maximum gas blender discrepancy using heliox was $10 \%$. 4) CPAP pressure recorded was accurate at all flow rates up to $15 \mathrm{~L} / \mathrm{min}$. 5) Noise emission when using heliox is no more than $1.3 \mathrm{~dB}$ greater than with conventional gases. 6) Heliox-CPAP consumes greater quantities of gas at all CPAP pressure settings.

Conclusions: Heliox/oxygen gas mixtures can be effectively used to deliver CPAP by the Infant Flow Driver. We recommend the following guidelines: 1) Titrate $\mathrm{FiO} 2$ administration using the LCD display. 2) Titrate CPAP pressure based on the actual CPAP pressure displayed.
IMPLICATIONS OF DISCLOSURE OF POSITIVE HIV STATUS FOR MENTAL HEALTH IN ZAMBIAN ADOLESCENTS

N Campain ${ }^{1}$, A Menon ${ }^{2}$, M Ngoma $^{3}$, C Glazebrook $^{1}$

${ }^{1}$ School of Community Health Sciences, University of Nottingham, UK, ${ }^{2}$ Department of Psychology, University of Zambia, Lusaka, Zambia

${ }^{3}$ Department of Paediatrics, University Teaching Hospital, Lusaka, Zambia, ${ }^{4}$ School of Community

Health Sciences, University of Nottingham, UK

Background and Aims: Little research has been conducted in developing countries concerning the psychological wellbeing of HIV+ve children. This study aims to investigate the psychological impact of disclosure of HIV status in Zambian adolescents.

Methods: A cross-sectional survey of HIV+ve Zambian adolescents, 11-15 years, recruited from the Lusaka region. Mental health was rated using the youth-report Strengths and Difficulties Questionnaire (SDQY). Carers completed the parent version of the SDQ. Audiotaped semi-structured interviews were conducted. Transcriptions were subjected to content analysis to determine participants' willingness to engage in a peer support programme.

Results: Participants had high rates of mental health problems and were more likely to score in the abnormal range of the SDQY compared to UK norms (OR 2.1, CI 1.4-3.1), with particular difficulties in relation to peer problems (OR 7.1, CI 4.9, 10.2). The majority of participants (58.3\%) were unaware of their HIV status. Most $(73 \%)$ were receiving antiretroviral therapy and treatment was associated with increased rates of disclosure $(p<0.01)$. Only $42.5 \%$ of children identified themselves as having a health problem and they had higher total SDQY scores $(\mathrm{p}=0.04)$. ANOVA with SDQY total scores as the dependent variable found a main effect of health $(\mathrm{p}=0.05)$, but no effect of disclosure. The covariate age was significant $(\mathrm{p}=0.03)$, with older children having fewer psychological problems. Three children said they would not wish to participate in a peer support programme, but $65 \%$ gave clearly positive responses.

Conclusions: Awareness of positive HIV status was not associated with poorer mental health and disclosure could facilitate provision of peer support.

\section{DEVELOPMENT OF LENTICULOSTRIATE VASCULOPATHY IN INFANTS WITH POSTNATALLY ACQUIRED CMV INFECTION}

M A Verboon-Maciolek ${ }^{1}$, C Koopman-Esseboom ${ }^{1}$, A M van Loon ${ }^{2}$, L S De Vries ${ }^{1}$

${ }^{1}$ Department of Neonatology, University Medical Center, Wilhelmina Children's Hospital, Utrecht, The Netherlands, ${ }^{2}$ Department of Virology, Eijkman Winkler Institute for Microbiology, Infectious Diseases and Inflammation, University Medical Center, Utrecht, The Netherlands

Background and Aims: Lenticulostriate vasculopathy (LSV) (branched echodensities in basal ganglia) has been frequently reported in newborns with different 
disorders. We assessed how often LSV present at term age was due to postnatally acquired CMV infection.

Methods: All infants $<32$ weeks of gestation born between April 2003 and December 2006 and treated in our NICU were studied. Cranial ultrasonography (CUS) was performed several times during the first week after birth and once a week till discharge. The first follow-up visit at term included CUS. In infants with LSV at term CMV culture or CMV PCR in urine was performed. Congenital infection was excluded by a negative CMV PCR or CMV IgM on the Guthrie card. Birth weight and gestational age of infants with CMV infection and without CMV infection were statistically analysed.

Results: Nineteen (4\%) of a total of 475 infants $<32$ weeks assessed at term on follow-up visit developed LSV. LSV was not seen during the first weeks after birth but was present at term age. In $5(26 \%)$ infants the diagnosis of postnatally acquired CMV infection was confirmed. Infants with CMV infection were younger and smaller than infants without infection but there was no statistical difference found $(27 \mathrm{wks}$ and $29 \mathrm{wks}$, respectively, $\mathrm{p}=0.35,950 \mathrm{~g}$ and $1200 \mathrm{~g}$, respectively, $\mathrm{p}=0.08$ ). All CMV positive infants had received breast milk.

Conclusions: LSV can develop weeks after birth in small preterm infants and can be diagnosed by CUS at term. In infants receiving breast milk and developing LSV weeks after birth, postnatally acquired CMV infection should be investigated.

\section{ORBITAL CELLULITIS, A REVIEW OF 17 PATIENTS IN UK}

\author{
K K Z Aabideen ${ }^{2}$, V Munshi ${ }^{1}$, V B Kumar ${ }^{2}$, F Dean ${ }^{1}$ \\ ${ }^{I}$ Department of Ophthalmology, University of Coventry \\ and Warwickshire, Coventry, UK, ${ }^{2}$ Department of \\ Neonatology, University of Coventry and Warwickshire, \\ Coventry, UK
}

Background and Aims: Orbital Cellulitis is a potential serious condition in children. This study is to evaluate its epidemiology, causative organism, clinical presentations, and effectiveness of treatments.

Methods: A retrospective review of all children admitted with diagnosis of Orbital Cellulitis between 1996 to 2004 at University Hospital of Coventry and Warwickshire.

Results: During this period, 17 cases of Orbital Cellulitis were analysed. Of these 11 were, males and 6 were females. All patients were documented as had Hib immunisation. Age of patients ranged from 10 months to 13 years. Out of 17,11 had restriction of eye movement 4 had altered vision, 4 had proptosis and 3 had Chemosis. Out of 17 blood, eye swab and nose swab cultures obtained in 13 patients and results described in table 1. All patients were treated with antibiotic combinations described in table 2. All patients were cured without any complications and sequelae.

Conclusions: With present era of antibiotics, Orbital Cellulitis can be treated completely without any sequelae. After the introduction of Hib vaccine, $\mathrm{H}$ influenza is no longer a significant pathogen in orbital cellulitis.

Table 1 Result of microbiological studies 17 patients
\begin{tabular}{|l|l|l|ll|l|l|}
\hline Source & done & $\begin{array}{l}\text { Positive } \\
\text { culture }\end{array}$ & & \multicolumn{4}{|c|}{ Organism } \\
\cline { 3 - 6 } Blood & 13 & 0 & 0 & 0 & 0 & 0 \\
\hline Eye swab & 13 & 4 & 1 & 1 & 0 & \\
\hline $\begin{array}{l}\text { Nose } \\
\text { swab }\end{array}$ & 13 & 3 & 1 & 0 & 1 & 0 \\
\hline
\end{tabular}

Table 5 Frequency of antibiotic used in patients with Orbital cellulitis \begin{tabular}{|l|l|}
\hline Antibiotic combinations & Number \\
\hline AUGMENTIN & 2 \\
\hline CEFTRIAXONE & 1 \\
\hline CEFTRIAXONE \& CLINDAMYCIN & 8 \\
\hline Other 3 antibiotics & 6 \\
\hline
\end{tabular}

\section{INTERLEUKIN-6 (IL-6) AND LIPOPOLYSACCHARIDE-BINDING PROTEIN (LBP) IN ACUTE APPENDICITIS (AA) IN CHILDREN}

M Derganc ${ }^{1}$, M Groselj-Grenc ${ }^{1}$, Z Dolenc-Strazar ${ }^{2}$, S Hojker ${ }^{3}$, S Repse $^{4}$

${ }^{1}$ Department of Paediatric Surgery and Intensive Care, University Medical Centre, Ljubljana, Slovenia

${ }^{2}$ Medical Faculty, Institute of Pathology, Ljubljana, Slovenia, ${ }^{3}$ Department of Nuclear Medicine, University Medical Centre, Ljubljana, Slovenia, ${ }^{4}$ Department of Abdominal Surgery, University Medical Centre, Ljubljana, Slovenia

Background and Aims: To evaluate the diagnostic accuracy of IL-6 and LBP in children with AA and compare it with the diagnostic accuracy of CRP and WBC count.

Methods: Eighty-two children admitted because of suspected AA classified into two groups: Group 1 (49 children who underwent surgery for AA); and group 2 (33 children with no surgery with diagnosis of nonspecific abdominal pain or sonographic mesenteric lymphadenitis). WBC count and serum CRP, IL-6 and LBP were measured on admission.

Results: The results are shown in the table. Optimum diagnostic cutoff value, area under the ROC curve (AUC), sensitivity, specificity and positive (PPV) and negative (NPV) predictive values (percentages) of WBC count, CRP, IL-6, and LBP for AA.

Conclusions: Only IL-6 showed medium, while other laboratory markers low diagnostic accuracy for AA in children. The new laboratory markers, therefore, do not significantly improve the diagnosis of AA.

\begin{tabular}{|l|c|c|c|c|c|c|}
\hline & Cutoff & AUC & Sen sitivity & Specificity & PPV & NPV \\
\hline WBC & $11.6 \times 10^{9} / \mathrm{L}$ & 0.684 & 73.5 & 65.6 & 63.1 & 75.6 \\
\hline CRP & $9.0 \mathrm{mg} / \mathrm{L}$ & 0.637 & 73.9 & 54.5 & 56.5 & 72.4 \\
\hline ㅍ-6 & $5.4 \mathrm{ng} / \mathrm{L}$ & 0.776 & 73.5 & 69.7 & 65.9 & 76.7 \\
\hline LBP & $20.1 \mathrm{mg} / \mathrm{L}$ & 0.635 & 83.7 & 51.5 & 58.0 & 79.8 \\
\hline
\end{tabular}

\section{SEXUAL BEHAVIORS AMONG GREEK ADOLESCENTS}

A Zervoudakis ${ }^{2}$, G Michas $^{3}$, A Papadopoulou ${ }^{2}$, F Michas ${ }^{4}$, C Tsoumakas ${ }^{1}$, P Georgiopoulou ${ }^{2}$

${ }^{1}$ Faculty of Nursing, University of Athens, Greece, ${ }^{2}$ Department of Pediatrics, KAT General Hospital, Athens, Greece, ${ }^{3}$ Medical School, University of Athens, Greece, ${ }^{4}$ Medical School, University of Patra, Greece 
Background and Aims: Adolescents comprise a separate pediatric group with special needs. Aim of the study was to determine basic knowledge of the risks of sex and sexual attitudes among Greek adolescents

Methods: 413 adolescents (159 boys and 216 girls), answered a specific questionnaire. Data analysis was made with SPSS 8.0.

Results: Knowledge of the risks of sex declared having $87 \%$ of the boys and $84 \%$ of the girls. $91 \%$ of the boys and $92 \%$ of the girls stated that they knew how to protect themselves. The main sources of knowledge were reported to be: friends (boys $50 \%$, girls $57 \%$ ), parents (boys 32\%, girls 14\%), media (boys 17\%, girls $9 \%$ ), books (boys $7 \%$, girls $10 \%$ ), school (boys $4 \%$, girls $12 \%$ ). Sexually active were $47 \%$ of the boys and $31 \%$ of the girls, but of them only $63 \%$ of the boys and $74 \%$ of the girls reported using any form of protection. Boys used exclusively condom, while $70 \%$ of the girls used condom, $8 \%$ pill and $10 \%$ a combination of both. Only one unwanted pregnancy was reported, and no sexually transmitted diseases.......!

Conclusions: Approximately half of the boys and one third of the girls under 18 years reported been sexually active. Despite the fact that they reported knowledge of the risks, a significant percentage (37\% boys and $26 \%$ girls) practiced no form of protection and near zero consequences (one wanders). It is evident that an even more intense and systematic form of information to this group of young people is desperately required.

\section{SKULL FRACTURES AND NON-ACCIDENTAL INJURY: THE DONCASTER EXPERIENCE}

\author{
H F Ayyash ${ }^{1}$, S Soman ${ }^{1}$, R Cocker ${ }^{1}$, A F Sabboubeh ${ }^{2}$ \\ ${ }^{I}$ Department of Paediatrics, Doncaster Royal Infirmary, \\ Doncaster, South Yorkshire, UK, ${ }^{2}$ Department of \\ Orthopaedics, Doncaster Royal Infirmary, Doncaster, \\ South Yorkshire, UK
}

Background and Aims: To find out the number of children who suffered abuse and any signs leading to identification of non- accidental injury (NAI).

Methods: The case notes of children aged less than 3 years with fractured skull between January 1995 and December 2004 were evaluated. Several variables, including age, sex, clinical presentation, mechanism of injury, fracture type and site, height of fall, and claimed aetiology associated with injury, were recorded.

Results: A total of 36 children ( $64 \%$ boys $36 \%$ girls) were identified. Twenty three (64\%) children were less than 1 year old. $53 \%$ sustained injury from less than one meter height. $64 \%$ had parietal skull fracture and the other $36 \%$ had either occipital, frontal and or mixed fractures. $61 \%$ suffered accidental injury, $22 \%$ suspected of NAI and $17 \%$ were proven to have suffered NAI. 70\% occured after drop from carrier's arms or fall from a height. $36 \%$ had head scan and 3 children had sub/extradural haematoma. Eleven children (31\%) had Skeletal survey and only one was abnormal.

Conclusions: Children and mostly boys who are less than 1 year old and fall from less than one meter height, with parietal or mixed fractures, were likely or proven to have sustained injury from abuse. The two way communication between health care professionals and social services has resulted in more cases of child abuse been identified.

\section{DOWN SYNDROME IN THE NETHERLANDS: PREVALENCE NEONATAL CHARACTERISTICS AND FIRST YEAR MORTALITY}

M E Weijerman ${ }^{1}$, A Vonk Noordergraaf ${ }^{1}$, J P v Wouwe ${ }^{2}$, C J M Broers ${ }^{1}$, A M Van Furth ${ }^{1}$, R J B J Gemke ${ }^{1}$

${ }^{I}$ Department of Paediatrics and Infectious Diseases, VU Medical Centre, Amsterdam, The Netherlands, ${ }^{2}$ TNO Quality of Life, Leiden, The Netherlands

Background and Aims: In the Netherlands children with Down syndrome (DS) are registered as a part of the DPSU (Dutch Paediatric Surveillance Unit) since 2003. The aim of this registration was to enclose the exact data of incidence at birth, duration and complications of pregnancy, the clinical symptoms prompting the diagnoses and associated infant morbidity and mortality. Methods: Dutch paediatricians have reported newly diagnosed live born children with DS to the DPSU from January 1st - December 31st 2003. A questionnaire collecting demographic and medical data was completed and was compared to the normal population.

Results: All children with trisomy 21 (182) were analysed; translocations (7) and mozaicism (3) were excluded. The prevalence of DS in the Netherlands in 2003 was 16 per 10.000 live born babies (total prevalence DS, live and stillborn, was 26.8 per 10.000 births). Median age at diagnosis was 1.8 and 10.2 days in hospital vs home born children. Neonatal and infant mortality were $1.65 \%$ and $4 \%$, respectively. Characteristics of Down syndrome children vs the population were: mother's age (33.6 vs 31 yrs), gestational age ( 38 vs 39 wks), breast-feeding ( $51 \%$ vs. $78 \%$ )*, birth weight (đ3119 vs 3525 gr; ㅇ 2901 vs 3389 gr)*. $\left({ }^{*} \mathrm{p}<0.05\right)$.

Conclusions: This study highlights the characteristics of DS in the Netherlands. The DS prevalence in the Netherlands was high. Mortality is higher than in controls but lower than studies on DS in the recent literature. This finding underscores the necessity of assessment of long term morbidity.

\section{WEIGHT CONTROL IN FINNISH AND GREEK ADOLESCENTS BY WEIGHT STATUS AND CORRECTNESS OF THEIR OPINION OF WEIGHT}

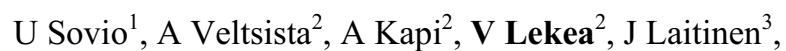
M R Järvelin ${ }^{1,4}$, C Bakoula ${ }^{2}$

${ }^{1}$ Department of Epidemiology and Public Health, Imperial College London, UK, ${ }^{2}$ First Department of Pediatrics, Aghia Sofia Children's Hospital, University of Athens, Greece, ${ }^{3}$ Oulu Regional Institute of Occupational Health, Oulu, Finland, ${ }^{4}$ Department of Public Health and General Practice, University of Oulu, Finland 
Background and Aims: Weight controlling behaviour was compared in Finnish and Greek adolescents by gender, weight status and correctness of their opinion of weight.

Methods: The data comprises Northern Finland 1985/6 birth cohort at age 15-16 and Greek 1983 birth cohort at age 18. The populations were followed up in 2001 using similar methods. Self-reported BMI was classified using IOTF cut-off points. Those who saw themselves more overweight than they were, were classified to "overestimated opinion" class, those less to "underestimated" and the rest to "realistic". Weight controlling methods including dieting/fasting, heavy exercise, vomiting and medication were pooled for the analysis.

Results: $50 \%$ of Finnish girls and 33\% of boys, and $56 \%$ of Greek girls and $47 \%$ of boys controlled their weight, mostly exercising and dieting. Overall, Greek adolescents controlled their weight $24 \%$ (95\% CI $19 \%$, $30 \%$ ) more often than Finns. Normal weight adolescents who saw themselves as overweight (i.e. 23\% of Finnish girls, $6 \%$ of Finnish boys, $22 \%$ of Greek girls and $8 \%$ of Greek boys) controlled their weight as often as overweight and obese adolescents, and twice as often as normal weight adolescents who saw their weight realistically. In Finnish overweight adolescents there was a positive trend between opinion of weight and prevalence of weight controlling. This trend was not seen in Greek overweight adolescents.

Conclusions: Overestimated opinion of weight is associated with weight control behaviour that can be harmful. This behaviour is more common in girls than boys, and more common in Greece than in Finland.

\section{PREVENTING ANAEMIA OF PREMATURITY: COMBINATION OF DELAYED CORD CLAMPING (DCC), EARLY IRON AND RESTRICTED TRANSFUSION REGIME IN VERY LOW BIRTH WEIGHT INFANTS (VLBW)}

H Rabe, J R Fernandez Alvarez, E Day, C Basu, C Lawn, P Amess

Department of Neonatology, Brighton and Sussex University Hospitals, Brighton, UK

Background and Aims: VLBW have a $80 \%$ probability of receiving blood transfusions for anaemia of prematurity. Studies have investigated the preventative effect of DCC, high dose iron or costly recombinant erythropoietin (rh-EPO). We investigated the effect of a preventative treatment regime of combining DCC of 30 seconds with early start of protein and oral feeds, early oral iron $(6 \mathrm{mg} / \mathrm{kg}$ from day $7-10$ of life, if milk feeds $60 \mathrm{ml} / \mathrm{kg} /$ day) and a restrictive transfusion schedule (WHYTE 2004) introduced for VLBW $<32$ weeks gestation (GA), <1500g birth weight (BW).

Methods: Data on blood transfusions in VLBW during the first six weeks of life were collected before the start of the new combination (Period I) and compared with data of consecutively born VLBW after the introduction of the treatment regime (Period II). The day of life, when milk feeds and oral iron were achieved, was recorded. Statistical analysis with Wilcoxon signed ranks test.
Results: VLBW in Period I ( $\mathrm{n}=18$, mean BW $955 \mathrm{~g}$ SD197) received a median of 4 transfusions [0.75:9] compared to Period II ( $\mathrm{n}=22$ mean BW 950g SD200) of 1.5 transfusions [0.75;5] $(\mathrm{p}=0.028)$. Milk feeds of $60 \mathrm{ml} / \mathrm{kgBW} / \mathrm{d}$ were achieved on median day $9[7 ; 13.5]$ and iron was introduced on median day 14 [11:21].

Conclusions: This combination, which has not been described before, reduced the number of blood transfusion by half. It was feasible to introduce oral iron early, but this needs reinforcement so that infants do not get missed out. Combined with rh-EPO it might even be more effective.

\section{COMBINED THERAPY WITH PIMECROLIMUS CREAM 1\% AND PROBIOTIC IN CHILDREN WITH ATOPIC DERMATITIS}

\author{
M L Aryayev, V N Kukushkin
}

Department of Pediatrics and Neonatology, Odessa State Medical University, Odessa, Ukraine

Background and Aims: Pimecrolimus cream 1\% and probiotics have been recently developed for the treatment of atopic dermatitis (AD). The aims of the study were to evaluate the efficacy and safety of combined therapy - pimecrolimus (Elidel ${ }^{\circledR}$ ) and probiotic/prebiotic Lactobacterin plus ${ }^{\circledR}$ (p.Lactobacillus and lactulose; Ukraine).

Methods: This was a 6-week parallel group study. Patients in the age of 3-11 years with mild and moderate $\mathrm{AD}$ were randomly assigned to pimecrolimus treatment group I $(n=30)$ and to pimecrolimus with Lactobacterin plus ${ }^{\circledR}$ group II $(n=30)$. Efficacy of treatment was evaluated using Eczema Area and Severity Index (EASI), Investigator's Global Assessment (IGA) score, pruritus severity score.

Results: EASI and IGA scores were similar between groups at baseline. The median EASI for group I decreased during the study from 10,6 to $4,7(55,7 \%)$, for group II - from 9,8 to 3,6 (63,3\%). Treatment success, defined as IGA score 0-1, was achieved in 21 patients in group I (70,0\%) compared with 25 patients in group II $(83,3 \% ; \mathrm{p}<0,05)$. There were more patients who reported their pruritus to be absent till the study end in group II $(79,8 \%$ and $89,3 \%$ respectively; $\mathrm{p}<0,05)$. During 6 months after the study the exacerbation of $\mathrm{AD}$ was not noticed in $50,1 \%$ patients of group I and in $73,4 \%$ patients of group II $(\mathrm{p}<0,01)$. Conclusions: Long-term management with pimecrolimus cream $1 \%$ improves the symptoms and signs of $\mathrm{AD}$ and enhances the quality of life. The additional administration of probiotic/prebiotic positively influences the number of flares, the intensity of pruritus and prevents early exacerbation of $\mathrm{AD}$.

\section{RANDOMIZED CONTROLLED TRIAL ON THE EFFECTIVENESS OF EARLY RECOMBINANT ERYTHROPOIETIN ADMINISTERED TO PRETERM INFANTS BY CONTINUOUS INTRAVENOUS VERSUS SUBCUTANEOUS ROUTE}

L Maggio, A Scorrano, F Cota, C Romagnoli, A A Zuppa 


\section{NICU, Catholic University, Rome, Italy}

Background and Aims: In very premature infants discomfort, breaks of the skin, and leaking from the injection site could be associated to the subcutaneous injection of recombinant erythropoietin (Epo). We hypothesized that adding Epo to the intravenous solution and administering it as a constant infusion (24h) would result in an erythropoietic effect similar to that seen with subcutaneous administration. Methods: Infants with BW $<1500$ and GA $<32$ weeks were randomly assigned at 72 hours of life to receive Epo (300 units $/ \mathrm{kg}$, 3 times a week until 36 complete wks PMA or discharge), either subcutaneously (ESC) or added to intravenous fluids (EIV). Results: Sixty infants (30 EIV, BW $1120 \pm 280$ gm, GA 29.0 2.3 wks; 30 ESC, BW $1190 \pm 280$ gm, GA $29 \pm 2.1 \mathrm{wks}$ ) met the criteria for randomization. The 2 groups did not differ at study entry with respect to the infants' characteristics, hematologic values, and iron metabolism. EIV and ESC infants received a similar number of EPO administrations (19.2 $\pm 8.8 v \mathrm{vs} 19.4 \pm 17.7)$ and a similar cumulative iron dose $(322 \pm 169 \mathrm{vs} 301 \pm 145$ $\mathrm{mg}$ ). No differences were found between the 2 groups in phlebotomy losses $(27.6 \pm 12.7$ vs $26.2 \pm 13.8 \mathrm{ml})$, incidence of transfusions ( 40.0 vs $36.7 \%$ ), number of transfusion per infant $(1.0 \pm 1.8$ vs $0.8 \pm 1.9)$ and cumulative transfusion volume ( $44.2 \pm 23.8$ vs $53.3 \pm 32.3$ $\mathrm{ml})$. At the end of the study there were no differences in the reticulocyte count $(6.3 \pm 2.3$ vs $6.1 \pm 1.9 \%)$, transferrin saturation $(35 \pm 12 \mathrm{vs} 33 \pm 20 \%)$ and ferritin concentrations $(131 \pm 152$ vs $114 \pm 148 \mathrm{ng} / \mathrm{ml})$. Conclusions: In preterm infants continuous intravenous administration of Epo resulted in similar effectiveness as subcutaneous dosing.

\section{MATURATION OF THE IMMUNE SYSTEM IN HEALTHY INFANTS: THE GENERATION R STUDY}

\author{
L Duijts $^{1,2}$, J A M Labout ${ }^{1,2}$, L E Bakker-Jonges ${ }^{3}$, \\ H Hooijkaas $^{3}$, V W V Jaddoe ${ }^{1,2,4}$, A Hofman ${ }^{4}$, \\ J J M van Dongen ${ }^{3}$, H A Moll ${ }^{1,2}$ \\ ${ }^{I}$ The Generation R Study Group, ${ }^{2}$ Dept of Paediatrics, \\ ${ }^{3}$ Dept of Immunology, ${ }^{4}$ Dept of Epidemiology and \\ Biostatistics, Erasmus MC - Sophia Children's Hospital, \\ Rotterdam, The Netherlands
}

Background and Aims: Differences in maturation of the immune system in infancy may be related to infectious diseases. The aims of this study were to examine the maturation of the immune system in healthy infants and its association with prenatal determinants.

Methods: This study was embedded in the Generation R Study, a population-based prospective cohort study from fetal life until adulthood. Blood samples for measurements of absolute numbers of T-cells, T-helper cells $(\mathrm{CD} 4+)$, T-cytotoxic cells $(\mathrm{CD} 8+)$, B-cells and NK-cells were collected in a subsample at birth $(\mathrm{n}=$ $981)$ and at the age of 6 months $(n=382)$. The measurements are assessed using 6-color flow cytometry.

Results: Preliminary results in the first 206 (birth) and 160 (age 6 months) infants demonstrated that the mean difference of absolute numbers of T-cells, T-helper cells, T-cytotoxic cells and B-cells was 1,74 (standard error (SE) 0,14) x 109/L, 1,26 (SE 0,11) 109/L, 0,43 (SE $0.04) 109 / \mathrm{L}$ and $1,06(\mathrm{SE} 0,07) \times 109 / \mathrm{L}$, respectively (figure 1). Mean difference of absolute numbers of NKcells was $-0,60(\mathrm{SE} 0,05) \times 109 / \mathrm{L}$. Analyses of the associations with prenatal determinants (maternal infections, birth weight, gestational age) are currently undertaken and will be presented additionally.

Conclusions: These preliminary results showed an increase of the absolute number of T-cells, T-helper cells, T-cytotoxic cells and B-cells and a decrease of NK-cells from birth to the age of 6 months.

Fig. 1. Absolute numbers of bymphocyte subsets

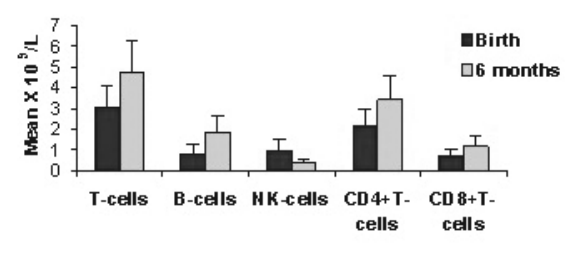

\section{ADIPONECTIN LEVELS IN PATIENTS WITH LYMPHOMAS}

A Varan ${ }^{1}, \mathrm{Y} \mathrm{Koksal}^{1}$, A Alikasifoglu ${ }^{2}$, G Hascelik $^{3}$, M Buyukpamukcu ${ }^{1}$

${ }^{1}$ Department of Pediatric Oncology, Hacettepe University, Faculty of Medicine, Ankara, Turkey ${ }^{2}$ Department of Pediatric Endocrinology, Hacettepe University, Faculty of Medicine, Ankara, Turkey ${ }^{3}$ Department of Biochemistry, Hacettepe University, Faculty of Medicine, Ankara, Turkey

Background and Aims: Adiponectin secreted from adipocytes is an antidiabetic, antiatherogenic, and antiinflammatory hormon. We aimed to investigate the serum adiponectin levels in patients with lymphoma at diagnosis and follow-up.

Methods: 23 newly diagnosed patients with lymphoma were included in this study. Serum adiponectin levels were measured at diagnosis and during the following cycles of chemotherapy. Normal adiponectin level ranged between 0.5 and $30 \mu \mathrm{g} / \mathrm{ml}$. Body mass index (BMI), erythrocyte sedimentation rate, LDH and CRP levels were also detected. Mean and median values were used for analysis. Mann-Whitney U and Wilcoxon rank tests were used to compare for two groups.

Results: There were 11 patients with Hodgkin's lymphoma (HL) and 12 with non-Hodgkin's lymphoma (NHL). The age range was between 3 and 17 with a median of 8 years. Male/female ratio was $15 / 8$. Median adiponectin levels were $15.2 \mu \mathrm{g} / \mathrm{ml}$ and $18.1 \mu \mathrm{g} / \mathrm{ml}$ at diagnosis and follow-up, respectively. 7 patients had increased adiponectin levels (over $30 \mu \mathrm{g} / \mathrm{ml}$ ). The diagnoses of these patients were HL in 5 and NHL in 2 patients. Five of them retained higher levels at the remission. There was no significant correlation between adiponectin levels and BMI and LDH.

Conclusions: Adiponectin levels were normal in most of the patients with lymphoma. This was the first report that evaluates adiponectin levels in children with lymphoma. 


\section{HIGH RISK NEUROBLASTOMA: A SINGLE CENTER EXPERIENCE}

R Gil Gómez, A Herrero Hernández, O Escobosa Sánchez, C Gómez Robles, T Acha García, A Jurado Ortíz

\section{Hospital Materno-Infantil Carlos Haya, Málaga, Spain}

Background and Aims: Children with high risk neuroblastoma (HRNB) represent the largest neuroblastoma subgroup. In spite of standard treatment the 5 years event-free survival rates are $>30 \%$. Our aim is to determine overall survival (Kaplan-Meier) and to evaluate prognostic factors related to the survival (logrank test).

Methods: Retrospective study of 24 HRNB from January 1981-March 2006. Eligibility criteria: Children $>1$ year with stage 4 , or stage 2 and 3 with MYCN amplification. 17/24 males. 22/24 abdominal (14/22 suprarenal) and 2 thoracic. Positive MYCN: 7/14. 19/24 died (3 perioperatory), 4/24 alive with disease, 1/24 alive without disease. Prognostic factors are LDH (group 1 normal $(\mathrm{N})$ levels, group $2<\mathrm{x} 4 \mathrm{~N}$, group 3 $>\mathrm{x} 4 \mathrm{~N}$ ), ferritin (normal, $<\mathrm{x} 4 \mathrm{~N},>\mathrm{x} 4 \mathrm{~N}$ ), enolase (normal, $<x 20 \mathrm{~N},>x 20 \mathrm{~N})$, positive catecholamines, positive MYCN, histology (group 1 neuroblastoma and group 2 ganglioneuroblastoma) and metastasis location (group 1 CNS, skeleton, pleural and lung; group 2 others).

Results: Mean age at diagnosis: $54 \pm 46$ months. Overall mean survival: 33 months (17-49), mean follow up 26 months $(0-120)$. High LDH levels involved significant worse prognosis $(\mathrm{p}=0.01)$. Ferritin and enolase levels, positive catecholamines and metastasis location at diagnosis were not significant. MYCN positive patients had 4 months of mean survival $(\mathrm{p}<0.05)$. Intermediate significance $(\mathrm{p}=0.06)$ was found from group 1 histology. Conclusions: Overall mean survival similar to others pilot studies. Patients with high level of LDH at diagnosis and positive MYCN had a significant poor outcome. No conclusive significant results found for the other prognostic factors, although neuroblastoma is likely to produce a worse outcome than ganglioneuroblastoma.

\section{A MULTICENTER PROSPECTIVE RANDOMIZED TRIAL OF CORTICOSTEROIDS IN PRIMARY THERAPY FOR KAWASAKI DISEASE: CLINICAL COURSE AND CORONARY ARTERY OUTCOME}

Y Inoue ${ }^{1}$, Y Okada $^{1}$, M Shinohara $^{2}$, T Kobayashi ${ }^{1}$, T Kobayashi ${ }^{2}$, T Tomomasa $^{1}, \mathrm{~K}_{\text {Takeuchi }}{ }^{3}$, A Morikawa $^{1}$

${ }^{I}$ Department of Pediatrics and Developmental Medicine, Gunma University Graduate School of Medicine, Maebashi, Gunma, Japan, ${ }^{2}$ Department of Cardiology, Gunma Children's Medical Center, Hokkitsu, Gunma, Japan, ${ }^{3}$ Department of Health Care and Social Welfare, Takasaki University of Health and Welfare, Takasaki, Gunma, Japan

Background and Aims: The role of corticosteroids in initial treatment of Kawasaki disease (KD) is not established.
Methods: Between September 2000 and March 2005 we randomly assigned $178 \mathrm{KD}$ patients from 12 hospitals to either an intravenous immunoglobulin (IVIG) group ( $\mathrm{n}=88 ; 1 \mathrm{~g} / \mathrm{kg}$ for 2 consecutive days) or an IVIG-plus-corticosteroids (IVIG+PSL) group ( $\mathrm{n}=90$ ). The primary end point was coronary artery abnormality (CAA) before a 1-month echocardiographic assessment. Secondary end points included duration of fever, time need for normalization of serum C-reactive protein (CRP), and initial treatment failure requiring additional therapy. Analyses were based on intention to treat.

Results: Baseline characteristics of groups were similar. Fewer IVIG+PSL patients had CAA before 1 month than IVIG patients $(2.2 \%$ vs. $11.4 \%, \mathrm{P}=0.017)$. The duration of fever was shorter $(\mathrm{P}<0.001)$ and CRP decreased more rapidly in the IVIG+PSL group than the IVIG group $(\mathrm{P}=0.001)$. Moreover, initial treatment failure was less frequent (5.6\% vs. $18.2 \%, \mathrm{P}=0.010)$ in the IVIG+PSL group. All patients assigned to the IVIG+PSL group completed treatment without major side effects.

Conclusions: A combination of corticosteroids and IVIG improved clinical course and coronary artery outcome without causing untoward effects in children with acute KD.

\section{MONOCYTE CHEMOATTRACTANT PROTEIN- 1 PLASMA LEVELS ARE ASSOCIATED WITH CLINICAL SEVERITY IN ACUTE PYELONEPHRITIS IN INFANTS}

E Demetriou ${ }^{1}$, A Margeli ${ }^{2}$, F Haliotis ${ }^{3}$, M Sourani ${ }^{3}$, L Zachariadou $^{4}$, A Kariyannis ${ }^{2}$, A Konstantopoulos ${ }^{1}$, I Papassotiriou $^{2}$

${ }^{1}$ Second Department of Paediatrics, Athens University Medical School, Athens, Greece, ${ }^{2}$ Department of Clinical Biochemistry, Aghia Sophia Children's Hospital, Athens, Greece, ${ }^{3}$ Second Department of Paediatrics, Aghia Sophia Children's Hospital, Athens, Greece, ${ }^{4}$ Department of Clinical Microbiology, Aghia Sophia Children's Hospital, Athens, Greece

Background and Aims: MCP-1 is involved in the initiation and progression of tubulointerstitial damage. We studied the role of MCP-1 in the pathogenesis of progression of acute pyelonephritis in febrile infants.

Methods: Twenty children were included in the study. Initial DMSA scan of all patients revealed renal lesions in 12. Follow-up scans performed six months later in all patients showed reversible lesions in 3 and renal scars in 9. MCUG performed in all patients revealed significant vesicureteral reflux (Grade III) in 5/20 and mild reflux (Grade II) in 2/20. MCP-1 plasma levels on admission and 3 days after initiation of treatment were measured.

Results: MCP-1 was increased in all patients at diagnosis of the urinary tract infection $(\mathrm{p}<0.001)$. We compared the values of MCP-1 in patients with normal DMSA and in patients with renal lesions and we observed that MCP-1 either remained high or further increased in patients abnormal DMSA. MCP-1 was not 'affected' by the initiation of treatment in this group of patients and especially in those patients who ended up with renal scarring. Similar results were found in the group of patients with the severe vesicureteral reflux. 
MCP-1 also decreased after treatment in the group of patients with initially normal DMSA, as it also decreased in the small group of patients with the reversible lesions.

Conclusions: Apparently, MCP-1 is more than just a chemoattractant. Its role as a direct elicitor of an inflammatory response may lead to conclusions with more clinical aspects regarding the prognosis of an acute renal damage in humans.

\section{DEVELOPING A MULTI PROFESSIONAL, MULTI SITE CLINICAL NETWORK TO ENHANCE THE ACCESS TO ACUTE SERVICES FOR CHILDREN AND YOUNG PEOPLE WITH LONG TERM VENTILATOR NEEDS}

\section{M Way}

\section{PICU, St Georges Healthcare NHS Trust, London, UK}

Background and Aims: In recent years a number of government and local reports have highlighted the challenges of providing high quality services that meet the needs of technology dependent children. This is particularly pertinent to the care of children and young people who are dependant on long-term ventilation, as their care may become problematic when it needs to be provided across the geographical and professional boundaries of different care settings. The purpose of this project was to develop a multidisciplinary clinical network between three different but complementary care settings that provided acute and community services to local children with LTV needs. Our aim was to insure the provision of timely, proactive admission to paediatric intensive/high dependency care for these children when they became acutely unwell.

\section{Methods:}

-A network between the three sites was established.

-The networked established the key issues in providing a seamless admission pathway for this group of children and young adults through the three centres were identified over a 5 -month period.

-A collaborative three-centre action plan was then developed in light of the key issues.

Results: Key Issues identified for the DGH

-DGH staff had anxiety about caring for these technology dependant children because of the lack of knowledge and expertise e.g. care of the ventilated child.

-The DGH had difficulties in providing staff with the appropriate knowledge and skill to accommodate admission of acutely unwell technology dependant children.

-There were both resource and operational issues that prevented transfer of the sick technology dependant child to another facility better able to manage the technology aspects of care. Key issues identified for the Children's Trust.

-The Children's Trust staff perceived they needed improved immediate access to acute services and nursing/medical expertise and facilities.

-They felt the current provision for updating knowledge and skill regarding the care of the ventilator dependent child needed to be improved, for example, with regard to: cardio-respiratory assessment, update on recognising the deteriorating child.

-They had a lack of access to support regarding equipment and ventilator troubleshooting.

-They felt there was a need for regular multidisciplinary assessment of this group of children in order to more proactively treat and manage any acute illness. In a hope too not only insure timely access to HDU/PICU facilities but also to reduce the need for this access.

Conclusions: As a result of these key issues we have:-

-Established a monthly outreach clinic at the Children's Trust led by the PICU consultant and myself.

-Started to facilitate educational packages across the three sites in relation to high dependency issues.

-Begun to develop shared clinical and patient transfer guidelines.

-Developed closer working relationships with all three centres.

-Began some initial planning to evaluate the effectiveness of the outreach clinic and its impact on the quality of care.

\section{CURRENT PRACTICE AND MANAGEMENT OF CVVH - SETTING THE STANDARD IN PAEDIATRIC INTENSIVE CARE}

\section{J White}

PICU/Forest Ward, Evelina Children's Hospital, Guy's and St Thomas NHS Trust, London, UK

Inconsistencies in practice and differing levels of experience dictate that clear standards and guidelines are available for staff when caring for children who require CVVH. These standards and guidelines provide essential support and guidance to both nursing and medical staff whilst caring for children who require this complex intervention. In the early 1990 's, a core group of nurses experienced in $\mathrm{CVVH}$, were established. This group continue to lead and manage this challenging service. They are responsible for formulating and guiding practice, and provide support within the multidisciplinary team. Achievements of the group to date include:

- Development of a resource booklet? formulation of a competency document .

- Implementation of an education plan.

The booklet includes guidelines based on current best evidence. It provides information on all aspects of CVVH and is utilized by both nurses and doctors when managing the care of children on CVVH. The competency document allows 'experts' in the core group to assess nurses caring for children who require and receive $\mathrm{CVVH}$, ensuring competencies are met and standards maintained. Specific CVVH Study Day's and yearly updates form part of the PICU Education Plan to ensure nurses are given time to develop their knowledge and skills in this challenging area. The use of reflection is encouraged throughout this process. Annual National CVVH meetings also help to ensure knowledge and experiences are shared throughout the United Kingdom and provide the opportunity for staff to discuss their experiences and best practice. The initial aims of the group have been achieved and we continue to review practice whilst evaluating the effectiveness of care 
delivered. The core group and Lead Consultant continue to work collaboratively to meet the educational needs of the nursing and medical staff whilst caring for children who require renal replacement therapy in a safe and consistent environment.

\section{TECHNOLOGICALLY DEPENDENT CHILD AND INFANT AT HOME}

\section{Johansson}

PICU, The Queen Silvia Children's Hospital, Gothenburg, Sweden

Advances in the care of the critically ill child have reduced mortality but have also introduced a new group of patients in our intensive care units. These patients have a constant need of high technology equipment to stay alive and the treatment will unfortunately at times involve prolonged support for weeks, months, years or even permanently. The required treatment will differ from oxygen inhalation to ventilator support, extensive medication to intravenous infusions, peritoneal dialysis or renal replacement therapy etc. Appropriate care is a challenge as life in hospital is an unsuitable environment for these children as well as an inappropriate use of hospital funding and resources. A transition of care has to take place and the pathway is important for the outcome. To shift the responsibility from hospital professionals toward home care services lies mainly on the neonatologist, paediatrician or health care coordinator. It is important to have a strategy and a wellprepared schedule prior to discharge. Many problems with technical equipment, home environment, staff etc will arise during the process and needs to be taken care of in a professional way. We also need to address emotional, economical and moral issues related to caring for a technology- dependent child at home. By using research, case reports and reflections from parents I will try to enlighten the physiological, psychological as well as the social problems we are facing during our efforts to give a good quality care to the child and family in the home environment.

\section{FIRST EXPERIENCES WITH INTRAHOSPITAL TRANSFER OF PRETERM INFANTS AND SICK NEWBORNS ON A OMNIBED AS TRANSPORT INKUBATOR (MONITORING, VENTILATOR) WITHOUT CHANGING THE UNIT}

\section{Schindler, I Berlett, S Dahlmann, F Loersch \\ University Children's Hospital, Mannheim, Germany}

Background and Aims: In the last few years in our hospital we have to transfer sick newborns from delivery room to NICU in a transport incubator. These transports resulting in significant thermal stress, respiratory and hemodynamic instability. We have developed a novel device to improve thermoneutral environment during this early critical phase, which incorporates an overhead warmer and an incubator. The purpose of this study was to compare the impact of using this device on admission thermal stability.
Methods: 35 newborns were included in this cohort study. Twentyone infants with birth weights of less then $1500 \mathrm{~g}$ and fourteen sick children $(\mathrm{CDH}, \mathrm{n}=12$, CCAM $n=2$ ) were transported in a Giraffe OmniBed from delivery room to the NICU. After delivery, the babies were resuscitated in the labor and delivery area using the device in the open bed, radiant warmer mode and then transported in closed bed, incubator mode to the NICU (a distance of approximately $500 \mathrm{~m}$ ).

Results: All initial resuscitation procedures were performed without any problems using the overhead mode. The average rectal temperature measured immediately upon admission to the NICU for all babies was $36,7 \mathrm{C}$ (median, range 36,2-37,2 C). The average temperature was $0,4 \mathrm{C}$ higher than in the control group of 30 babies transported with a conventional transport incubator.

Conclusions: Using this novel hybrid device for resuscitation in the delivery room and for transfer to the NICU resulted in improved thermal stability. This device allows to initiate and maintain mechanical ventilation without any disruption and more aspects of the minimal handling started earlier.

\section{CAN A PALLIATIVE CARE SERVICE PROVIDE AN ACCEPTABLE ALTERNATIVE TO INTENSIVE CARE FOR CHILDREN FACING INEVITABLE DEATH?}

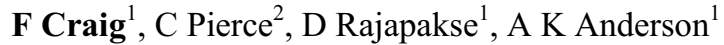 \\ ${ }^{1}$ Department of Palliative Care, Great Ormond Street \\ Hospital, London, UK, ${ }^{2}$ Department of Paediatric \\ Intensive Care, Great Ormond Street Hospital, London, \\ $U K$
}

Background and Aims: Both palliative care (PC) and intensive care (IC) provide support to children and families facing potential or inevitable death. However the focus of IC is to initiate active treatments, whereas the philosophy of PC is to enable children to achieve an optimal quality of life despite no expectation of cure. The purpose of this study was to determine if families would choose a PC approach over IC when their child faces inevitable death.

Methods: Referrals to a PC team were monitored over a 3 -year period. The children referred were assessed in 2 groups: those referred to $\mathrm{PC}$ before an IC admission and those referred whilst on IC. Place of death was recorded for each child.

Results: 203 children were referred to PC, 55 from IC and 148 from non-IC sources. 125 non-IC referrals died, only $2(1.6 \%)$ chose to go to IC at the end-of-life. 54 $(43 \%)$ of them died at home or in a hospice and the remainder died in hospital. Of the 55 children referred from IC, 43 were ventilated at the time of referral, 38 died. Only 18 (42\%) chose to stay on IC. Nine chose to go home or to a hospice and 6 transferred to a hospital nearer to home.

Conclusions: Children and families facing inevitable death are less likely to choose an IC admission when supported by a PC service. Families already on IC will often choose continuing PC support move out of IC to alternative places under PC. 
CRITICALLY ILL CHILDREN WITH A PROLONGED STAY ON THE PICU: THE NEED FOR INTERDISCIPLINARY AND MULTIDISCIPLINARY ROUNDS

P Van Het Einde ${ }^{1}, \mathrm{~S} \mathrm{Naghib}^{1,2}$, R Groenewegen ${ }^{2}$, T Tibboel $^{2}$, K F M Joosten $^{1}$

${ }^{1}$ Department of Pediatrics, Erasmus MC-Sophia Children's Hospital, Rotterdam, The Netherlands ${ }^{2}$ Department of Pediatric Surgery, Erasmus MCChildren's Hospital, Rotterdam, The Netherlands

Background and Aims: There is a group of critically ill children with a prolonged stay on the PICU ( $>28$ days). They take a lot of PICU resource utilisation. The daily care of these children needs special attention of the nursing staff because the difficulties of dealing with an uncertain prognosis and increasing demands of the children and parents. Objective: Investigating the number of children with a prolonged stay in the last 3 years (2003-2005) in context with the weekly support of the medical and nursing staff.

Methods: We looked if and when a primary nurse was appointed to the children, the number of regular conversations with the parents by doctors together with nurses, the number of interdisciplinary and multidisciplinary patient rounds.

Results: In a 3-year period 120 children (equal gender) with a prolonged stay were admitted. Diagnoses were mainly children with (multiple) congenital malformations and tracheal abnormalities. The mean stay of these children was 85 days (range 28-484). Nine children were admitted more than once. $25 \%$ of the children died. Regular conferences with all parents took place once/twice a week. In $19 \%$ of the children interdisciplinary rounds were done and in $12 \%$ multidisciplinary rounds were done. In $68 \%$ a primary nurse was appointed but in the majority these nurses were appointed half way admission.

Conclusions: There is no well-structured protocol for children who have a prolonged stay on the ICU concerning interdisciplinary and multidisciplinary rounds. There is need for the early introduction of a coordinating primary nurse in the overall support of these children.

\section{CO-BEDDING PREMATURE BORN MULTIPLES: AN EVIDENCE-BASED APPROACH}

\section{J M Wielenga}

IC Neonatology, Emma Children's Hospital/Academic Medical Center, Amsterdam, The Netherlands

Background and Aims: After the introduction of developmental care in 2003, professionals as well as parents wished to nurse premature born multiples in one incubator. Co-bedding can be seen as the continuation of the proximity and interaction experienced during the intrauterine period of multiple-gestation infants after birth. It seems reasonably to assume that multiplegestation infants are born with expectations based on their earlier intrauterine experiences. The transition of multiple-gestation to the extra uterine life can be simplified through stimuli generated by the continuous uninterrupted physical contact. The aim of this project was to study literature and collect information on cobedding, mainly on the adverse effects of co-bedding. The information collected was used to compose a bestevidence guideline for the new standard of care: cobedding premature born multiples.

Methods: The study was carried out in the Emma Children s Hospital / Academic Medical Center in Amsterdam, the Netherlands. We used the method of Critical Appraised Topic (CAT) with the PICO (patientintervention-comparison-outcome) system formulated as: Are multiple borns admitted to the NICU (P) who are co-bedded (I) instead of single-bedded (C) exposed to complication risks or physiological instability $(\mathrm{O})$ ? Literature search was performed within PubMed, Cinahl, Embase and the Cochrane Library.

Results: Literature search resulted in 22 hits until December 2003: 17 comments and background articles and 5 scientific papers. From the latter, 3 were selected based on the strength of the designs of the study; one randomised, one prospective cohort and one comparative descriptive design. All studies had small populations, respectively 37- 22-155 infants and cobedding periods varied from 24 hours to 5 days. On complications and physiological instability, was reported in 2 out of 3 studies. No adverse effects or increased infection rates were found. Disruptions of cobedding were never necessary. Physiological stability of co-bedded infants was the same as single-bedded, at some points significant improvement was found, mainly on growth and apnoeas (frequency as well as duration). Co-bedding procedures were described in detail in 2 out of 3 studies.

Conclusions: Based on the findings we made a guideline and to introduced co-bedding in two steps. We started co-bedding with respiratory stable infants after an evaluation we introduced co-bedding to infants on CPAP as well. We still monitor each co-bedding session.

\section{PROCALCITONIN AS A MARKER FOR THE EARLY DIAGNOSIS OF NEONATAL INFECTION}

N Joram ${ }^{1}$, B Isidor ${ }^{1}$, V Loubersac ${ }^{2}$, S Denizot ${ }^{1}$, G Caillaux ${ }^{1}$, N Winer ${ }^{3}$, J C Roze ${ }^{1}$, C Gras-Le Guen ${ }^{1}$

${ }^{1}$ Département de Périnatologie, CHU Nantes, France,

${ }^{2}$ Laboratoire de Biochimie, CHU Nantes, France,

${ }^{3}$ Service de Gynecologie-Obstétrique, Hopital Mère Enfant, CHU Nantes, France

Background and Aims: Materno-foetal and nosocomial infections are frequent causes of morbidity and mortality in neonatal intensive care units (NICU). Their prognosis are closely related to early diagnosis and treatment but clinical signs are very poorly specific nor sensitive. In this context, paediatricians frequently initiate early antibiotherapy, exposing non infected newborns to unnecessary treatment. The aim of our work was to evaluate the value of procalcitonin (PCT) as an early and specific marker of perinatal bacterial sepsis. 
Methods: Two different studies were conducted. In 197 neonates with suspected materno-foetal infection, serum procalcitonin and C-Reactive Protein concentrations were measured simultaneously in umbilical cord blood and in maternal blood. According to clinical and bacteriological course, three groups were defined: group $1=$ infected $(\mathrm{n}=16)$; group $2=$ colonized $(\mathrm{n}=20)$; group 3 $=$ non infected and non colonized In 176 preterm neonates (mean 29,8). Hospitalised in neonatal intensive care units more than $48 \mathrm{~h}$ and with a clinical suspicion of nosocomial infection, PCT was determined immediatly and 24 hours later. Two groups were defined: infected and non infected.

Results: In the population with suspected maternofoetal infection, a statistically higher serum procalcitonin concentration was found in the infected group versus controls and colonized $(\mathrm{p}<0.05)$. According to the the ROC curves, we chose a cut-off point of $0,5 \mathrm{ng} / \mathrm{ml}$ for PCT and $5 \mathrm{mg} / 1$ for CRP. A PCT value greater or equal to $0.5 \mathrm{ng} / \mathrm{ml}$ was observed in $14 / 16$ infected newborns and in $2 / 181$ non infected newborns. The CRP dosage was $>5 \mathrm{mg} / 1$ in $8 / 16$ infected newborns. Sensitivity, specificity, and positive and negative predictive values were respectively $87.5 \%$ (95\% confidence interval 71 to 100 ), $98.7 \%$ (97.7 to 99.7 ), $87.5 \%$ (71 to 100 ), and 98.7\% (97.7 to 99.7) for PCT, and 50\% (25 to 75), $97 \%$ (94 to 100), 67\% (40 to 94 ), and $94 \%$ (81 to 100) for CRP. The positive and negative likelihood ratios were respectively 67.3 and 0.13 for PCT and 16.7 and 0.51 for CRP. Maternal PCT serum level was negative in all cases. In the population with suspected nosocomial infection, 45 cases were defined as infected, 31 infections were proved by bacteriological cultures. Most of the proven infections were caused by coagulase negative staphylocoque $(51,6 \%)$ and staphylocoque aureus $(22,5 \%)$. With the cut off value of $0,5 \mathrm{ng} / \mathrm{ml}$, the sensitivity for diagnosis of bacterial infection was $91,3 \%$, specificity $93,9 \%$, positive predictive value $84 \%$, negative predictive value $96 \%$. Positive and negative likelihood ratios was respectively 14.9 and 0,09 .

Conclusions: Although maternal PCT dosage does not seem to be informative, serum procalcitonin in cord blood seems to be a useful and early marker of antenatal infection. Furthermore, PCT would be an interesting marker of nosocomial infection. This could improve the outcome of perinatal infection allowing early treatment as well as reducing the number of unnecessary antibiotherapies.

\section{PROCALCITONIN IN PAEDIATRIC EMERGENCY DEPARTMENTS FOR THE EARLY DIAGNOSIS OF BACTERIAL INFECTION IN FEBRILE INFANTS}

\section{A Fernandez Lopez}

Spain

Background and Aims: Procalcitonin (PCT) is a potentially useful marker in pediatric Emergency Departments (ED). The basic objectives of this study were to assess the diagnostic performance of PCT for distinguishing between viral and bacterial infections and for the early detection of invasive bacterial infections in febrile children between 1 and 36 months old comparing it with C-reactive protein (CRP) and to evaluate the utility of a qualitative rapid test for PCT in ED. Methods: Prospective, observational and multicenter study that included 445 children who were treated for fever in pediatric ED. Quantitative and qualitative plasma values of PCT and CRP were correlated with the final diagnosis. To obtain the qualitative level of PCT the BRAHMS PCT-Q rapid test was used.

Results: Mean PCT and CRP values in viral infections were $0.26 \mathrm{ng} / \mathrm{ml}$ and $15.5 \mathrm{mg} / \mathrm{l}$, respectively. The area under the curve obtained for PCT in distinguishing between viral and bacterial infections was 0.82 (sensitivity, 65.5\%; specificity, 94.3\%; optimum cutoff, $0.53 \mathrm{ng} / \mathrm{ml}$ ), whereas for CRP it was 0.78 (sensitivity, $63.5 \%$; specificity, $84.2 \%$; optimum cutoff, $27.5 \mathrm{mg} / \mathrm{l}$ ). PCT and CRP values in invasive infections (PCT, 24.3 $\mathrm{ng} / \mathrm{ml}$; CRP $96.5 \mathrm{mg} / \mathrm{l}$ ) were significantly higher than those for noninvasive infections (PCT, $0.32 \mathrm{ng} / \mathrm{ml}$; CRP, $23.4 \mathrm{mg} / \mathrm{l})$. The area under the curve for PCT was 0.95 (sensitivity, 91.3\%; specificity, 93.5\%; optimum cutoff, $0.59 \mathrm{ng} / \mathrm{ml})$, significantly higher $(\mathrm{P}<0.001)$ than that obtained for CRP (0.81). The optimum cutoff value for CRP was $>27.5 \mathrm{mg} / \mathrm{l}$ with sensitivity and specificity of 78 and $75 \%$, respectively. In infants in whom the evolution of fever was $<12 \mathrm{~h}$ ( $\left.\mathrm{n}_{-} 104\right)$, the diagnostic performance of PCT was also greater than that of CRP (area under the curve, 0.93 for PCT and 0.69 for CRP; P $<0.001)$. A good correlation between the quantitative values for PCT and the PCT-Q test was obtained in $87 \%$ of cases (kappa index, 0.8). The sensitivity of the PCT$\mathrm{Q}$ test (cutoff $>0.5 \mathrm{ng} / \mathrm{ml}$ ) for detecting invasive infections and differentiating them from noninvasive infections was $90.6 \%$, with a specificity of $83.6 \%$. Conclusions: PCT offers better specificity than CRP for differentiating between the viral and bacterial etiology of the fever with similar sensitivity. PCT offers better sensibility and specificity than CRP to differentiate between invasive and noninvasive infection. PCT is confirmed as an excellent marker in detecting invasive infections in ED and can even make early detection possible of invasive infections if the evolution of the fever is $<12 \mathrm{~h}$. The PCT-Q test has a good correlation with the quantitative values of the marker.

\section{DISTINGUISHING BETWEEN BACTERIAL AND ASEPTIC MENINGITIS IN THE EMERGENCY DEPARTMENT}

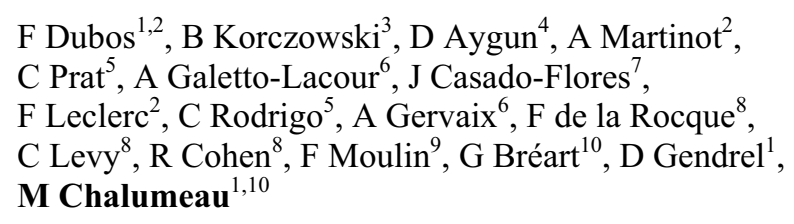

${ }^{1}$ Clinical Epidemiology Unit, Department of Pediatrics, Saint Vincent-de-Paul Hospital, Paris, France, ${ }^{2}$ Jeanne de Flandre Hospital, CHU Lille, France

${ }^{3}$ Regional Hospital, Rzeszów, Poland, ${ }^{4}$ Medical Faculty of Firat University, Elazig, Turkey, ${ }^{5}$ Hospital Universitari Germans Trias I Pujol, Badalona, Spain ${ }^{6}$ HUG Hôpital des Enfants, Geneva, Switzerland ${ }^{7}$ Hospital Universitario del Nino Jesus, Madrid, Spain ${ }^{8}$ Bacterial Meningitis French Surveillance Network, Saint-Maur-des-Faussés, France, ${ }^{9}$ Department of Emergency Medicine, Saint-Vincent-de-Paul Hospital, Paris, France, ${ }^{10}$ INSERM U149, France 
Background and Aims: Distinguishing between bacterial meningitis (BM) and aseptic meningitis (AM) in the emergency department (ED) is sometimes difficult, but could be very helpful to avoid unnecessary hospitalisations and antibiotic treatments. Five clinical decision rules combining clinical and biological parameters available at the time of admission were proposed in the literatture. We herein report a research program aiming at identifying the best rule and at improving it, and we discuss future research issues.

Methods: Data from a retrospective single-center study $(\mathrm{n}=166$ patients, 20 with $\mathrm{BM})$, a secondary analysis of the ACTIV/GPIP French prospective registry of BM in children $(\mathrm{n}=890)$, and a retrospective multicenter European cohort study ( 6 centers in 5 countries, $\mathrm{n}=198$, 96 with BM) were used (1) to compare existing rules and identify the "best" one, (2) to validate the "best" rule on a large population of children with BM, (3) to identify the best biological predictor of $\mathrm{BM}$ (serum procalcitonin -PCT- at the time of admission), (4) to validate this predictor, and (5) to combine this new predictor in a new clinical decision rule.

Results: In the single-center study, the best rule (defined [Dubos, Arch Dis Child, 2006] as the one yielding $100 \%$ sensitivity for BM, the highest specificity $-60 \%$ - and the greatest simplicity for a bedside application) was the Nigrovic rule [Nigrovic, Pediatrics, 2002]: start antibiotics in case of seizure, blood neutrophil count $\geq 10,000 / \mathrm{mm} 3$, positive CSF Gramstaining, CSF protein $\geq 80 \mathrm{mg} / \mathrm{dL}$, or CSF neutrophil count $\geq 1,000 / \mathrm{mm} 3$. However, this rule only achieved $99.3 \%$ sensitivity (95\% CI: 98.8-99.9) on a large population of patients with BM. Both in the singlecenter [Dubos, J Pediatr, 2006] and the multicenter European studies, PCT had the best area under the ROC curve $(0.98,95 \%$ CI: $0.95-0.99)$, statistically different (p $<0.05)$ from the other biologic markers. With a 0.5 $\mathrm{ng} / \mathrm{mL}$ threshold, PCT had $99 \%$ sensitivity (95\% CI: $94-$ $100)$ and $83 \%$ specificity (95\% CI: $74-90)$. Incorporating PCT in a new rule called Meningitest (start antibiotics in case of seizure, purpura, toxic appearance, procalcitonin $\geq 0.5 \mathrm{ng} / \mathrm{mL}$, positive CSF Gram-staining, CSF protein $\geq 50 \mathrm{mg} / \mathrm{dL}$ ) had $100 \%$ sensitivity and $56 \%$ specificity on the population of the single-center study.

Conclusions: To distinguish between bacterial and aseptic meningitis in children in the ED: (1) the Nigrovic rule is a very sensitive and validated tool but do not achieve $100 \%$ sensitivity, (2) PCT is now validated as the best biological predictor, (3) a PCTbased decision rule (the Meningitest) is a promizing tool that needs further (ongoing) validation. Funding: unrestricted educational grants were received from DRC (CRC 03154), FERCMHP, the Fondation Bayer Santé, and from Brahms (the manufacturer of PCT).

\section{PREDICTION OF VESICO-URETHRAL REFLUX AFTER A FIRST FEBRILE URINARY TRACT INFECTION}

S Leroy ${ }^{1,2}$, C Romanello ${ }^{3}$, A Galetto-Lacour ${ }^{4}$, V Smolkin ${ }^{5}$, B Korczowski $^{6}$, C Rodrigo $^{7}$, D Tuerlinckx $^{8}$,

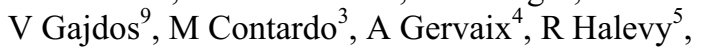

B Duhl ${ }^{6}, \mathrm{C} \mathrm{Prat}^{7}, \mathrm{~T}$ Vander Borght ${ }^{8}, \mathrm{~L}_{\text {Foix-L'Hélias }}{ }^{\text {, }}$ G Bréart $^{2}$, D Gendrel ${ }^{1}$, M Chalumeau ${ }^{1}$

${ }^{1}$ Clinical Epidemiological Unit, Department of Pediatrics, Saint-Vincent-de-Paul Hospital, Paris, France, ${ }^{2}$ INSERM U149, Paris, France, ${ }^{3}$ University of Udine, Italy, ${ }^{4}$ University Hospital of Geneva, Switzerland, ${ }^{5} \mathrm{Ha}^{\prime}$ Emek Medical Center, Afula, Israel, ${ }^{6}$ Regional Hospital N 2, University of Rzeszow, Rzeszow, Poland, ${ }^{7}$ Germans Trias I Pujol Hospital, Badalona, Spain, ${ }^{8}$ UCL Mont-Godinne, Yvoir, Belgium ${ }^{9}$ Antoine-Béclère Hospital, Clamart, France

Background and Aims: Febrile urinary tract infection (FUTI) reveals vesicoureteral reflux (VUR) in $20-40 \%$ of children. Voiding cystourethrogram (VCUG) is then recommended systematically, but is painful, expensive, exposes to radiation and is a posteriori normal in $60-80 \%$ of cases. Then, selective approaches for VCUG are needed. We herein report various strategies proposed to achieve this goal, and discuss future research issues. Methods: A retrospective single-center cohort study ( $\mathrm{n}=136$, including $25 \%$ patients with VUR), and a secondary analysis of 8 prospective European cohort studies ( $\mathrm{n}=398$, including $25 \%$ patients with VUR) were performed, and included consecutive patients aged 1 month to 4 years with a first FUTI. The data were used to evaluate the reproducibility of the clinical decision rule proposed by Oostenbrink et al [Acta Pediatr, 2000] aiming at predicting VUR using a risk score combining clinical (family history of uropathology, male gender, young age), biological (elevated C-reactive protein) and radiological (urinary tract dilation on renal ultrasound) variables. Then, we identified and validated a new predictor of VUR: a high serum procalcitonin (PCT) level at the time of diagnosis. Univariate and multivariate analyses were performed.

Results: The rule proposed by Oostenbrink et al yielded $100 \%$ sensitivity and 3\% specificity for all-grade VUR (vs $100 \%$ sensitivity and $17 \%$ specificity in the original study), and $93 \%$ sensitivity and $13 \%$ specificity for grade $\geq 3$ VUR (vs 100\% sensitivity and 38\% specificity in the original study). Some methodological weaknesses (variables selection and cotation) may explain this lack of reproducibility [Leroy, Arch Dis Child, 2006]. Both in the single-center [Leroy, Pediatrics, 2005] and the multicenter European study [Leroy, in revision], the median value of PCT was significantly higher in children with vs without VUR: 1.6 vs $0.7 \mathrm{ng} / \mathrm{mL}$ (p $<0.001)$. After dichotomisation around a $0.5 \mathrm{ng} / \mathrm{mL}$ threshold, there was a significant association between VUR and high PCT [OR=2.3, 95\% CI 1.3-3.9, $\mathrm{p}=0.001]$. After logistic-regression adjustment for all potential confounders (young age, male gender, positive family history for uropathy, C-reactive protein, urinary tract dilatation on renal ultrasonography, urine technique collection - sterile bags vs suprapubic aspiration or transurethral catheterization), the association remained significant (OR: $2.5 ; 95 \%$ CI: 1.4-4.4; $\mathrm{p}=0.001$ ). The strength of the relationship increased with the grade of reflux VUR grade $(\mathrm{p}<0.001)$. The sensitivity of PCT was $75 \%$ (95\% CI: $66-83)$ for all-grade VUR and $100 \%$ (95\% CI: $81-100$ ) for grade 4 or 5 VUR, both with $43 \%$ specificity (95\% CI: 37-48). 
Conclusions: The potential contribution of the rule proposed by Oostenbrink et al seems to be modest. PCT is a strong, independent and validated predictor for VUR. It could be used to identify patients with low risk for VUR to avoid unnecessary VCUG, alone or combined with other predictors (that need to be identified and validated) in a new clinical decision rule. Then, impact studies with cluster randomization will be needed to evaluate the real impact of this rule in day-today practice. Funding: unrestricted educational grants were received from AJP, FRM, PHRC AOM 05110 and Brahms (the manufacturer of PCT).

\section{GENETICS IN SEPSIS}

\section{J A Hazelzet}

\section{PICU, Erasmus MC, Rotterdam, The Netherlands}

Background and Aims: Sepsis is a polygenic and complex syndrome that is initiated by infection and is characterized by a host response which consist of a systemic inflammatory response. This host response can be quite different between individuals. Genetic polymorphisms in this immune response to infection can partly explain these differences and have been shown to be associated with clinical outcomes. Functional and association studies involving genetic polymorphisms in essential genes. These include specific and aspecific recognition of pathogen associated microbial patterns, signaling receptors like Toll-like receptors, primary and secondary inflammatory proteins like cytokines and chemokines, and coagulation and fibrinolysis factors These polymorphisms have provided important insights into the mechanisms involved in the pathogenesis of sepsis-induced organ dysfunction. The advancement of high-throughput single nucleotide polymorphism (SNP) genotyping will provide valuable information on the interaction of multiple allelic variants and clinical outcome. More precise categorization of patients based on genetic background is likely to lead to individualized targeted treatment. A specific example of pediatric sepsis is the meningococcal sepsis. The clinical presentation of infections caused by Neisseria meningitidis is highly diverse. Some patients develop meningitis, and others present with sepsis or even septic shock. Genetic differences might explain this diversity. Genetic polymorphisms among components of the host response have been shown to be involved in the susceptibility, severity, and outcome of meningococcal disease.

Methods: Literature search and own findings.

Results: An overview will be given of the most recently found polymorphism and their possible impact in the host response.

Conclusions: Future therapeutic trials as well as actual treatment regimens for patients with sepsis are likely to be designed to target specific genotypes and associated cellular responses, maximizing clinical response and patient safety.

\section{IMMUNE DEPRESSION IN POST SEPSIS}

\section{J A Carcillo}

Department of Critical Care Medicine and Pediatrics, University of Pittsburgh, Pittsburgh, PA, USA
Background and Aims: Nosocomial sepsis is a leading cause of morbidity and mortality in critically ill neonates and children. This lecture will describe the elements of CRitical Illness Stress-induced Immune Suppression (CRISIS) as a cause of nosocomial sepsis.

Methods: Immune phenotyping and immune modulation findings from our center, as well as an evidenced based review of basic and clinical literature on this topic, will be presented.

Results: Nosocomial sepsis and mortality risk is predicted by the presence of neutropenia, absolute lymphocyte count $<1,000$, and monocyte HLA-DR expression $<30 \%$ or whole blood TNF response to LPS $<200 \mathrm{pg} / \mathrm{mL}$. Stress or medication induced lymphocyte apoptosis leads to monocyte deactivation (immunoparalysis). Monocyte deactivation leads to the inability of adherent PMNs to apoptose (ARDS). These conditions can be effectively reversed with nutritional and medical strategies.

Conclusions: The study and implementation of immunephenotype-directed therapies are warranted to address the epidemic of nosocomial sepsis in newborns and children.

\section{HEMODYNAMIC CHANGES DURING HEMOFILTRATION IN MENINGOCOCCAL SEPTICAEMIA}

\section{P Maheshwari, R Chhabra, M Clement, C DeMunter}

PICU, St Mary's Hospital, London, UK

Background and Aims: There is good theoretical background for the treatment of severe sepsis with haemofiltration. The aim was to review the cardiovascular effects of veno-venous haemofiltration in severe meningococcal septic shock.

Methods: All patients with severe meningococcal sepsis who were hemofiltered in our paediatric intensive care unit were included in the study. Parameters including inotropic requirement, metabolic acidosis, fluid requirement and blood lactate were recorded 12 hours before starting hemofiltration, at the time of starting, 6 , $12,24,48,72$ and 96 hours after initiating hemofiltration.

Results: 27 patients from 6 months to 16 years of age (median 5.5 years) with increasing fluid and inotropic requirements, anuria, worsening lactic acidemia and base deficits were hemofiltered within 48 hours of admission. Three patients died of fulminant cardiovascular shock within the first 12 hours of their admission and all within 4 hours of initiating haemofiltration. They were not included in the following analysis. The mean PRISM score among the 24 survivors was 64.7. At the time of initiation of haemofiltration, patients were on a mean adrenaline infusion of $2.35 \mathrm{mcg} / \mathrm{Kg} / \mathrm{min}$ and a mean noradrenaline infusion of $0.95 \mathrm{mcg} / \mathrm{Kg} / \mathrm{min}$. There was a rapid and significant improvement in cardiovascular status after a mean of 6 hours of initiation of haemofiltration: Blood pressure stabilised and fluid requirements decreased. Base deficit improved, blood lactic acid levels and doses 
of adrenaline and noradrenaline all rapidly reduced ( $\mathrm{p}$ value $<0.0001$ ) by ANOVA.

Conclusions: The initiation of haemofiltration in cases of severe meningococcal septicaemia was associated with rapid improvements in cardiovascular status.

\section{THE INFLUENCE OF ETOMIDATE ON ADRENAL FUNCTION IN CHILDREN WITH MENINGOCOCCAL SEPSIS}

M den Brinker ${ }^{1}$, A C Hokken-Koelega ${ }^{3}$, J A Hazelzet ${ }^{2}$, F H De Jong ${ }^{4}$, W C Hop ${ }^{5}$, K F Joosten ${ }^{2}$

${ }^{I}$ Department of Pediatrics, ${ }^{2}$ Department of Pediatrics, Division of Paediatric Intensive Care, ${ }^{3}$ Department of Pediatrics, Division of Endocrinology, ${ }^{4}$ Department of Internal Medicine, ${ }^{5}$ Department of Epidemiology and Biostatistics, Erasmus MC, Rotterdam, The Netherlands

Background and Aims: To investigate the influence of one single bolus of etomidate used for intubation on adrenal function in children with meningococcal sepsis.

Methods: Retrospective study of 60 children admitted between 1997 and 2004 to the PICU with meningococcal sepsis, not treated with steroids. Adrenal hormone concentrations were determined as soon as possible after PICU admission, 12 and 24 hours thereafter. To assess disease severity, PRISM score and selected laboratory parameters were determined.

Results: On admission, before blood was drawn, 23 children had been intubated with etomidate, 8 without etomidate and 29 were not intubated. Children who were intubated had significantly higher disease severity parameters, whereas none of these parameters significantly differed between children who were intubated with or without etomidate. Children who received etomidate had significantly lower ratios of cortisol over ACTH and lower ratios of cortisol over 11deoxycortisol than those who did not receive etomidate (figure). Arterial glucose levels were significantly lower in children who were intubated with etomidate than in children who were not intubated. Eight children died, 7 of them had received etomidate. Within $24 \mathrm{~h}$ cortisol/ACTH ratios increased significantly in children who had received etomidate, but not in children who had not receive etomidate, resulting in comparable cortisol/ACTH ratios $24 \mathrm{~h}$ after admission.

Conclusions: Our data imply that even one single bolus of etomidate negatively influences adrenal function and thereby might increase risk of death.

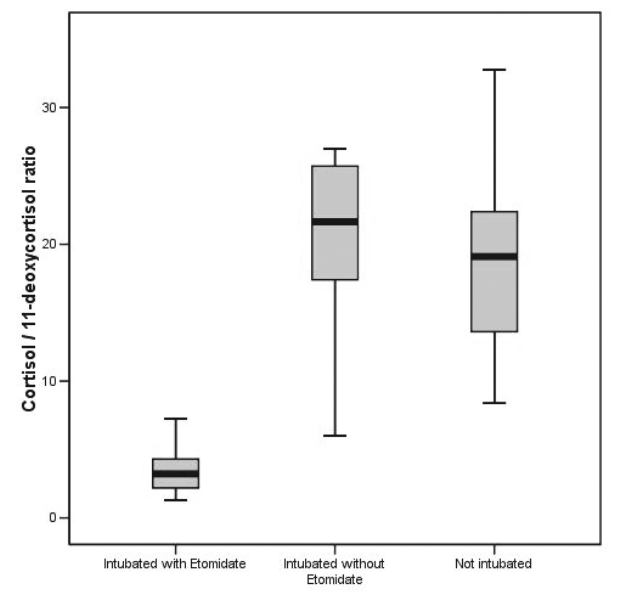

LIPOPOLYSACCHARIDE-BINDING PROTEIN IN CRITICALLY ILL CHILDREN WITH SUSPECTED INFECTION: COMPARISON WITH LIPOPOLYSACCHARIDE, SOLUBLE CD14, PROCALCITONIN, AND C-REACTIVE PROTEIN
M Pavcnik-Arnol ${ }^{1}$, S Hojker ${ }^{2}$, M Derganc ${ }^{1}$

${ }^{1}$ Department of Paediatric Surgery and Intensive Care,

${ }^{2}$ Department of Nuclear Medicine, University Medical Centre, Ljubljana, Slovenia

Background and Aims: To evaluate markers of infection in critically ill children, comparing lipopolysaccharide binding protein (LBP) with lipopolysaccharide (LPS), soluble CD14 (sCD14), procalcitonin (PCT) and C-reactive protein (CRP).

Methods: Prospective observational study in the level III multidisciplinary paediatric intensive care unit. Forty-nine children with systemic inflammatory response syndrome (SIRS) and suspected infection were classified into two groups: SIRS - sepsis $(\mathrm{n}=30)$ and SIRS - no sepsis $(\mathrm{n}=19)$. The median age was 2.4 years. The disease severity was high (mean PRISM III score $=16.5$ ). Serum concentrations of LBP, LPS, sCD14, PCT and CRP were measured on two consecutive days. Area under the receiver operating characteristic (ROC) curve (AUC), sensitivity, specificity and predictive values were evaluated. Correlations between markers of infection were examined.

Results: Serum LBP was significantly higher in children with SIRS - sepsis than in children with noninfectious SIRS at both time points (day $0 \mathrm{p}<0.0001$, day $1 \mathrm{p}=0.003$ ). AUC on the day of suspected infection was 0.82 for LBP, 0.72 for LPS, 0.53 for $\mathrm{sCD} 14,0.76$ for PCT, and 0.69 for CRP. Positive correlation was found between LBP and CRP, and between CRP and PCT concentrations in children with SIRS - sepsis. PCT and $\mathrm{SCD} 14$ concentrations were significantly higher in children with a high PRISM III score.

Conclusions: In critically ill children LBP on the first day of suspected infection is a better marker of sepsis than LPS, sCD14, PCT and CRP. LBP is correlated with CRP in children with sepsis.

\section{VIRAL LARYNGOTRACHEITIS (CROUP SYNDROME) IS SIGNIFICANTLY ASSOCIATED WITH SEVERE SEPSIS AND SEPTIC SHOCK}

\author{
R M Basciani ${ }^{1,2}$, D C Bachmann ${ }^{1}$, I Bolt ${ }^{1}$, \\ B P Wagner ${ }^{1}$ \\ ${ }^{1}$ Department of Paediatrics, University of Berne \\ (Inselspital), ${ }^{2}$ Department of Anaesthesiology, \\ University of Berne (Inselspital), Switzerland
}

Background and Aims: Croup syndrome is a common diagnosis in paediatrics with a benign, self-limited course. Only patients with inspiratory stridor at rest and respiratory distress are hospitalized and about 1\% require intubation. However, bacterial tracheitis, pneumonia, and sepsis can complicate croup syndrome and significantly increase morbidity and mortality. Beside the "classical" respiratory problems there is little awareness about these potentially life-threatening 
complications among paediatricians. This study aims to provide data about the incidence and prognosis of sepsis in croup-patients. Methods: In this observational single centre study all children admitted to our 16-beds, tertiary level paediatric intensive care unit (PICU) due to severe croup syndrome were retrieved from the prospective database. Results: Over a 14 year period, 63 patients with 65 episodes of croup syndromes required mechanical ventilation for an average duration of $4.2 \mathrm{~d}$ (range $0.1-23.8 \mathrm{~d}$ ). 10 patients $(15 \%)$ fulfilled the criteria of severe sepsis or septic shock. In $80 \%$ bacterial cultures of tracheal aspirates were positive. 5 children survived after $9.6 \mathrm{~d}$ (2-14.3) of mechanical ventilation; in this group circulatory failure was present in $80 \%$. The other 5 patients died after a ventilation period of $0.8 \mathrm{~d}$ $(0.1-2.7)$. Main cause of death were persistent hypoxic respiratory failure and brain death after pre-hospital cardiac arrest. Conclusions: Risk for severe sepsis or septic shock in patients with mechanical ventilation due to croup induced respiratory failure is $15 \%$ with a mortality of $50 \%$. Croup-patients with significant respiratory complications should carefully be monitored for sepsis-syndromes with additional organ failures.

\section{CLINICAL SPECTRUM OF SEVERE IMPORTED MALARIA IN CHILDREN}

P Tissieres, V Haas, L Chevret, S Essouri, C Perrot, D Devictor, P Durand

\section{Multidisciplinary Pediatric Intensive Care Unit, Hôpital de Bicêtre, Le Kremlin-Bicêtre, France}

Background and Aims: To characterize clinical spectrum of severe imported malaria in children living in non-endemic region.

Methods: Retrospective study of all children admitted in a single tertiary pediatric ICU over a 15 years period. Severe imported malaria was considered if severe malaria met 2001 WHO criteria and occurred in children after returning from a travel in endemic region.

Results: Twenty-eight children with a mean age of 6.0 \pm 4.2 years-old were identified. In all but one with Plasmodium ovale, Plasmodium falciparum was found. On admission, the most frequently encountered symptoms were fever (96\%), abdominal symptoms (89\%) and apathy (57\%). Seizures occurred in 32\%, including three patients with status epilepticus. $29 \%$ of the patients displayed a dehydration $>10 \%$. Nine patients were mechanically ventilated, seven for a comatos state and two for an ARDS. Two patients presented a hypovolemic shock. Neuromalaria was diagnosed in 11 patients (39\%). Four patients (14\%) had a renal failure requiring renal replacement, two a liver failure and three a disseminated intravascular coagulation. Nine patients $(32 \%)$ displayed one or two failing organs and four patients (14\%), three or more organs. No children died and no children had neurological sequel.

Conclusions: This is the largest reported series of severe imported malaria in children. Clinical spectrum of severe imported malaria in children living in nonendemic region significantly differs from the one in endemic region and classically described in the literature.

\section{MECHANISMS OF VASCULAR DEGENERATION IN RETINOPATHY OF PREMATURITY: O2 AND CO2}

S Chemtob, E Kermorvan, F Sennlaub, D Checchin, M Beauchamp, S Brault, C Quiniou, M Sirinyan, P Hardy, M Balazy

Departments of Pediatrics, Ophthalmology and Pharmacology, Hôpital Ste. Justine, Research Centre, Montreal, QC, Canada

Retinopathy of prematurity (ROP) is characterized by a phase of arrest in vascular development associated with vasoobliteration, which is followed by an ischemic phase resulting in neovascularization. Major factors involved in vascular degeneration include a suppression in VEGF expression combined with a rise in reactive oxygen species and corresponding peroxidation products. On one hand, ischemia-induced retinal (rather than preretinal) neovascularization can be considered beneficial in salvaging the tissue. Yet, despite production of angiogenic factors and nitric oxide within ischemic tissues, compensatory ingrowth of new vessels fails to provide adequate vascularization. Thus, we hypothesized that local factors counter efficient revascularization via nitrosative stress related pathways. Along with hypoxia, ischemia also produces a concomitant elevation of $\mathrm{CO} 2$. In the developing subject, hypercapnia has been associated with the development of ROP in humans and confirmed experimentally. However, the significance of hypercapnia, particularly on neovascularization, has been relatively overlooked compared to the numerous studies on hypoxia. We investigated the mechanisms by which hypercapnia impair vascular development. We found that acute and prolonged $\mathrm{CO} 2$ exposures inhibit developmental neovascularization of the retinal vasculature, as well as revascularization of the ischemic retina. This was associated with increased eNOS, generation of highly reactive nitrogen species (including peroxynitrite) and downregulation of the pro-survival and angiogenic PGE2 receptor, EP3; VEGF and its receptors were not affected. NOS inhibitors as well as peroxynitrite decomposition catalyst FeTPPS prevented hypercapnia-induced vasoattenuation. Furthermore, we illustrate that in vitro $\mathrm{CO} 2$ results in a NO-dependent microvascular endothelial cell (EC) protein nitration and cell death. Mediators of nitrosative stress remain for the most part unknown. Trans-arachidonic acids (TAAs) are recently described major products of $\mathrm{NO}^{\prime}$ radicalmediated isomerization of arachidonic acid, and their formation is enhanced by high $\mathrm{CO} 2$ levels; we explored if and how TAAs reproduce biological effects of nitrosative stress. TAAs are generated in our model of retinal microangiopathy in vivo in a NO-dependent manner. TAAs induced a selective time- and concentration-dependent cell death of ECs in vitro, and resulted in retinal microvascular degeneration ex and in vivo. These effects are mediated by an upregulation of the anti-angiogenic factor thrombospondin-1 (TSP-1), independently of classic arachidonic acid metabolism; accordingly, TAA-induced vasoobliteration was prevented by selective neutralizing antibodies to TSP-1 as well as to its receptor CD-36. In summary, our findings uncover previously undescribed mechanisms of 
hypercapnia-induced cytotoxicity, specifically as it pertains to nitrosative stress in microvascular injury, and provide insight into therapeutic avenues in the management of disorders involving nitrosative stress such as ischemic retinopathies and possibly encephalopathies commonly encountered in the immature subject. Finally, the dogma regarding safety of hypercapnia may need to be revisited.

\section{PREVENTION OF RETINOPATHY OF PREMATURITY}

A Sola, A Schenkman, L Skolinck, M R Rogido

Mid Atlantic Neonatology Associates, Morristown Memorial Hospital, Morristown, NJ, USA

Background and Aims: Oxygen was discovered more than 200 years ago and it has been administered to more infants in the world than any other neonatal treatment. However, we still do not fully know how much is wise to give or how much infants actually need in relation to variations in illness and gestational and postnatal age. "Too much oxygen" damages the retina and now we know that it increases risk for serious illnesses like cancer. In many places, and even in the recent literature, there is a debate about using $21 \% \mathrm{O} 2$ versus $100 \%$ or 'pure' oxygen during resuscitation. This practice cannot be a correct practice for many infants, since it excludes or limits a more reasonable practice, a practice based on using an 'adequate' dose of oxygen (anywhere from $21 \%$ to $100 \%$ ), assessing each infant's needs with an accurate pulse oximeter, targeting for an "acceptable" $\mathrm{SpO} 2$ and using a blender to be able to administer the needed oxygen dose for that target. Measuring $\mathrm{FiO} 2$ and $\mathrm{O} 2$ saturation accurately with a state of the art monitor which incorporates new technologies can today be done routinely from the time of birth in preterm infants treated through out the world. ROP is the most common cause of blindness but many cases can be prevented by practicing differently.

Methods: I will review in some detail how much we know and do not know about oxygenation, alveolar gas equation, normal $\mathrm{PaO} 2$ and $\mathrm{O} 2$ saturation when breathing room air and when breathing oxygen. I will also review pulse oximetry in neonatal medicine, including the most important aspects of $\mathrm{SpO} 2$ and the significant differences in saturation monitors in the market. I will review our peer reviewed publications in relation to prevention of ROP and oxygenation and some related publications by other investigators.

Results: Many $\mathrm{SpO} 2$ monitors have high rates of false alarms, are not accurate in eliminating noise, have 'holding periods' and are more sensitive to light and motion artifacts, not functioning well when clinicians need them the most. In addition, some monitors in the market read $1.5 \%$ - 4\% higher or lower than other monitors, even in very stable conditions. Masimo SET technology is the one with greater and most refined accuracy to resolve most artifacts and to increase sensitivity at low blood flow, pulse amplitude and blood oxygenation values, ensuring better clinical care. More than 90 manuscripts in peer reviewed journals are evidence of the better sensitivity and specificity of this technology. I will present data from our publications and from unpublished work about how the practice of avoiding hyperoxia (i.e.: $\mathrm{SpO} 2>95 \%$ when breathing O2) has been associated with markedly reduced rates of ROP and also with less severe ROP. Avoiding hyperoxia may also be beneficial for conditions like BPD, periventricular leukomalacia, infection and cancer and to avoid oxidative stress which influences apoptosis and cell growth.

Conclusions: Some cases of ROP are associated to inadequate practices and can be prevented by using adequate $\mathrm{SpO} 2$ technology and by changing those practices.

\section{EFFECT OF ACUTE HYPOXIA AND REOXYGENATION WITH ROOM AIR OR DIFFERENT OXYGEN CONCENTRATIONS ON URINARY MARKERS OF OXIDATIVE STRESS}

\author{
R Solberg ${ }^{1}$, Y Andresen ${ }^{1}$, R Escrig ${ }^{2}$, I Izquierdo ${ }^{2}$, \\ P Saenz $^{2}$, M A Asensi ${ }^{3}$, J Sastre ${ }^{3}$, M Vento ${ }^{2}$, \\ O D Saugstad ${ }^{1}$ \\ ${ }^{1}$ Department of Pediatric Research, Rikshospitalet, \\ Oslo, Norway, ${ }^{2}$ Servicio de Neonatologia, Hospital La \\ $\mathrm{Fe}$, Valencia, Spain, ${ }^{3}$ Departamento de Fisiologia, \\ Universidad de Valencia, Spain
}

Background and Aims: Oxidative stress associated with reoxygenation may be responsible for short and long-term pathological consequences. Our aim was to study the effect of acute hypoxia and reoxygenation with room air or $40 \%, 60 \%$ or $100 \%$ oxygen upon urinary markers of oxidative stress, which could be a useful non-invasive tool for monitoring patients in the clinical setting.

Methods: Piglets were asphyxiated following a standardized model, and resuscitated with $21 \%, 40 \%$, $60 \%$ or $100 \%$ oxygen. Once recovered and stabilized, piglets were sacrificed. Urine samples were collected. 8oxo-DHG and 2DG as well as ortotyrosine and phenylalanine were determined by mass spectrometry. Results: Quotient of 8 -oxo-DHG/2DG as well as otyrosine/phenylalanine ratios were significantly higher in piglets resuscitated with high oxygen concentrations (DHG/DG: RAR 5.23 vs OXR100:18.5 microm/L; $<<$ 0.01 and TYR/PHE: RAR 19.07 vs OXR100: 148.7 microm $/ \mathrm{L} ; \mathrm{p}<0.001)$. Moreover there was a significant correlation between the oxygen concentration used at resuscitation and TYR/PHE ratio.

Conclusions: Acute hypoxia during asphyxia and the use of high oxygen concentrations during resuscitation cause oxidation of DNA and PHENYLALANINE. These markers can be easily determined in urine and may be useful as clinical tools in the follow-up of these patients. TYR/PHE is especially sensible.

\section{VASCULAR ENDOTHELIAL GROWTH FACTOR GENE POLYMORPHISM AND SERUM CONCENTRATION AS RISK FACTORS OF RETINOPATHY OF PREMATURITY}

P Kwinta ${ }^{1}$, M Bik-Multanowski ${ }^{1}$, Z Mitkowska ${ }^{1}$, T Tomasik ${ }^{1}$, J Kobylarz ${ }^{2}$, J J Pietrzyk ${ }^{1}$ 
${ }^{I}$ Department of Pediatrics, Jagiellonian University, Cracow, Poland, ${ }^{2}$ Department of Ophthalmology, Jagiellonian University, Cracow, Poland

Background and Aims: Recent experimental studies suggest that vascular endothelial growth factor (VEGF) can play an important role in the development of retinopathy of prematurity (ROP). The aim of the study was analysis of: (1) correlation between serum concentration of VEGF during the first month of life and the risk of ROP (2) association of T (-460C) and G $(+405 \mathrm{C})$ polymorphisms of VEGF gene with ROP.

Methods: 128 newborns with mean birthweight 1073g were included into prospective study. Concentrations of VEGF were measured by Elisa assay in the 2nd, 3rd and 4th weeks of life. Molecular studies of VEGF polymorphisms were performed with PCR-RFLP and RG-PCR. The infants were divided into 2 groups: A) no ROP or ROP not requiring treatment $(n=92)$ and 2) ROP requiring lasertherapy $(\mathrm{n}=36)$.

Results: The serum VEGF concentrations were similar in both groups. The frequency of VEGF $-460 \mathrm{TT}$ allele was higher in the treated group ( 35 vs $24 \%$; $=0,1$ ). Moreover, the VEGF serum concentrations were higher in the TT carriers than TC or CC infants ( 898 vs 688 and $684 \mathrm{pg} / \mathrm{ml} ; \mathrm{p}=0.02)$. The frequency of $+405 \mathrm{CC}$ allele was also higher ( 14 vs $5 \%$; $=0.07)$ in the treated group, but $\mathrm{G}(+405 \mathrm{C})$ polymorphism did not influence VEGF serum concentration. VEGF $-460 \mathrm{TT} /+405 \mathrm{CC}$ haplotype was more prevalent in the treated group (21 vs $2 \%$; $\mathrm{p}=0.016$ ).

Conclusions: $-460 \mathrm{TT} /+405 \mathrm{CC}$ allele carrier state can be associated with the risk of progressive ROP and the VEGF serum concentration is higher in the -460TT carriers. (Study partially supported by State Committee for Scientific Research No 3PO5E02424).

\section{LEVELS OF (SPO2) BETWEEN 85\% AND 93\% ARE ASSOCIATED WITH NORMOXEMIA IN NEWBORNS (NB) RECEIVING OXYGEN THERAPY $(\mathrm{FiO2}>21 \%)$ IN THE NEONATAL INTENSIVE CARE UNIT (NICU)}

\author{
A Castillo $^{1}, \mathrm{H}_{\text {Baquero }}{ }^{3}, \mathrm{~F} \mathrm{Neira}^{3}$, R Deulofeut ${ }^{1}$, \\ A Sola ${ }^{1}$ \\ ${ }^{1}$ Department of Neonatology, Emory University, \\ Atlanta, GA, USA, ${ }^{2}$ Mid Atlantic Neonatology, \\ Morristown, NJ, USA, ${ }^{3}$ Universidad del Norte, \\ Barranquilla, Colombia
}

Background and Aims: NB breathing room air (RA) have normal $\mathrm{SpO} 2$ of $95-100 \%$ but keeping $\mathrm{SpO} 2$ $95-100 \%$ in $\mathrm{NB}$ breathing $\mathrm{FiO} 2>21 \%$ can cause hyperoxia. In 2003, we described that $\mathrm{SpO} 285-95 \%$ is associated with less morbidity. There is concern that these $\mathrm{SpO} 2$ levels may cause persistent or intermittent hypoxemia. Objective: to evaluate $\mathrm{PaO} 2$ at different $\mathrm{SpO} 2$ levels in NB with arterial catheters.

Methods: Prospective comparison of $\mathrm{PaO} 2$ and $\mathrm{SpO} 2$ in stable NB in 4 NICU's of 2 countries. $\mathrm{PaO} 2$ obtained for clinical indications; simultaneous $\mathrm{SpO} 2$ was recorded at the time of the arterial gas. Comparisons were made only in stable $\mathrm{NB}$ if the $\mathrm{SpO} 2$ changed $<1 \%$ before, during and after the collection of the sample. Statistics: Chi square, Fishers, bivariate and multivariate analysis.

Results: $310 \mathrm{SpO} 2$ values in $70 \mathrm{NB} ; 30 \%$ of the samples from NB in RA. GA and BW: $26.5 \pm 2.2 \mathrm{w}$ and $900 \pm 280$ g. NB in RA: median $\mathrm{SpO} 2$ 96\% (88-100\%); with $\mathrm{SpO} 285-93 \%, \mathrm{PaO} 2$ was $51.9 \pm 8 \mathrm{mmHg}$, median of 53 (39-65). When $\mathrm{SpO} 2>94 \%, \quad \mathrm{PaO} 2$ was $65.1 \pm 17 \mathrm{mmHg}$, median 64 (39-113). NB breathing $\mathrm{FiO} 2>21 \%$ : A) With $\mathrm{SpO} 2 \quad 85-93 \%, \mathrm{PaO} 2$ was $52.3 \pm 9 \mathrm{mmHg}$, median 53 (29-83); $\mathrm{PaO} 2$ of $40-80 \mathrm{mmHg}$ in $>90 \%$. B) With $\mathrm{SpO} 2>94 \%, \quad \mathrm{PaO} 2$ was $71.9 \pm 25 \mathrm{mmHg}$, median $63(38-164)(\mathrm{p} \leq 0.001 \mathrm{vs}$ infants with $\mathrm{SpO} 285-93 \%) ; 19 \%$ of the $\mathrm{PaO} 2$ values were $>85 \mathrm{mmHg}$.

Conclusions: NB breathing FiO2>21\%: I) Normoxemia is more frequent with $\mathrm{SpO} 285-93 \%$ than with $\mathrm{SpO} 2>94 \%$; II) $\mathrm{SpO} 285-93 \%$ avoids abnormally high $\mathrm{PaO} 2$ and is infrequently associated with low $\mathrm{PaO} 2$; III) $\mathrm{SpO} 2>94 \%$ is associated with hyperoxia, which may be of risk in some $\mathrm{NB}$ receiving $\mathrm{FiO} 2>21 \%$.

\section{GLUTATHIONE REDOX STATUS AT BIRTH PREDICTS OXYGEN NEEDS IN THE EXTREMELY PRETERM INFANTS}

\author{
R Escrig ${ }^{1}$, I Izquierdo ${ }^{1}$, P Saenz ${ }^{1}$, J Sastre ${ }^{2}$, J Vina ${ }^{2}$, \\ M Vento \\ ${ }^{1}$ Servicio de Neonatologia, Hospital La Fe, Valencia, \\ Spain, ${ }^{2}$ Departamento de Fisiologia, Universidad de \\ Valencia, Spain
}

Background and Aims: Antioxidant enyzme expression matures in the last weeks of gestation. Thus, extremely preterm infants are prone to reactive oxygen derived lung injury. However, prenatal corticosteroids (PCTS) improve postnatal adaptation in these infants. We hypothesize that PCTS favor glutathione cycle enzyme maturation and expression thus reducing alveolar damage.

Methods: Premature infants $<28$ wks gestation receiving (CORT; $\mathrm{n}=15)$ and not receiving NOCORT; $\mathrm{n}=12$ ) antenatal glucocorticoids were enrolled. In cord blood samples and venous blood at $7 \mathrm{~d}$ postnatally total erythrocyte GSH and GSSG, as well as GPx and GReductase activities were determined by methods previously described (Vento M el al JPediatr 2003). Supplemental oxygen given was registered along the study.

Results: CORT group had significantly higher GSH/GSSG ratio at birth $(\mathrm{p}<0.01)$ and 1 week thereafter $(p<0.05)$ as compared to NOCORT group. Moreover, GPx and GReductase activities where also higher in the CORT group at birth $(p<0.05)$ and one week thereafter $(p<0.05)$ than in the NOCORT group. The oxygen needs for the CORT group was significantly lower than in the NOCORT group at 7 days of life $(\mathrm{p}<0.05)$.

Conclusions: Prenatal corticosteroids activate glutathione redox cycle improving postnatal adaptation of extremely preterm infants. 


\section{INCIDENCE AND SEVERITY OF \\ RETINOPATHY OF PREMATURITY (ROP) IS \\ REDUCED IN EXTREMELY LOW \\ GESTATIONAL AGE (GA) NEWBORNS \\ (ELGANS) TREATED WITH RECOMBINANT HUMAN CU/ZN SUPEROXIDE DISMUTASE (rhSOD)}

R B Parad ${ }^{1,2,4}$ E Allred ${ }^{2,3,4}$, J Davis $^{5,6}$ N American rhSOD Study Group ${ }^{6}$

\section{${ }^{I}$ Brigham and Women's Hospital, Boston, MA, USA ${ }^{2}$ Department of Pediatrics, Harvard Medical School, Boston, MA, USA, ${ }^{3}$ Harvard School of Public Health, Boston, MA, USA, ${ }^{4}$ Childrens Hospital, Boston, MA, USA, ${ }^{5}$ Department of Pediatrics, Winthrop University Hospital, Mineola, NY, USA, ${ }^{6}$ SUNY Stony Brook \\ Medical School, Stony Brook, NY, USA}

Background and Aims: ROP is a two-phase disease in which effects of hyperoxia, hypoxia and reactive $\mathrm{O} 2$ species (ROS) lead to abnormal retinal vascularization, particularly in ELGANS. We hypothesized that the highest risk ELGANS $(<25 \mathrm{wks})$ might benefit most (largest effect size) from rhSOD reduction of ROS. Effects on the smallest ELGANS may have been obscured by the total cohort analysis performed in the last rhSOD (Ferring) trial (for the prevention of bronchopulmonary dysplasia (BPD) in infants under 1250g (Pediatrics, 111:469, 2003)).

Methods: In that trial, 302 infants were randomized to intratracheal (IT) rhSOD or placebo at birth and every $48 \mathrm{~h}$ while intubated.

Results: IT administration was associated with prolonged, increased serum (and theoretically intraocular) levels. More rhSOD doses were given with decreasing GA (longer intubation). ROP risk increased with decreasing GA. Analysis of the entire cohort revealed no significant differences in the incidence $(64 \%$ v. $60 \%)$ or the severity [worse than stage $2(>\mathrm{S} 2)]$ of ROP in placebo $\mathrm{v}$. rhSOD treated infants. When a more detailed sub-group analysis (Fisher's exact test) was performed on 72 infants $<26 \mathrm{wk}, 66 \%$ of rhSOD treated infants $(\mathrm{n}=38)$ developed ROP compared to $85 \%$ in controls $(\mathrm{n}=34)(\mathrm{P}=0.06)$. For infants $<25 \mathrm{wk}(\mathrm{n}=24)$, $40 \%$ of rhSOD and $85 \%$ of controls developed any ROP $(\mathrm{P}=0.03)(>50 \%$ incidence reduction $)$, and fewer rhSOD treated infants had ROP $>\mathrm{S} 2 \quad(10 \%)$ compared to controls $(36 \%)(\mathrm{P}=0.17)$.

Conclusions: This post-hoc analysis suggests rhSOD treatment may reduce the incidence and severity of ROP in the most premature infants (more doses with longer intubattion). This has important implications in trials of rhSOD to prevent BPD in high-risk ELGANs.

\section{MICROCIRCULATORY ANALYSIS USING SIDESTREAM DARK FIELD (SDF) IMAGING IN SEPSIS}

\section{Ince}

Department of Physiology, Academic Medical Center, University of Amsterdam, Amsterdam, The Netherlands

Sublingual OPS (orthogonal polarization spectral) imaging has revealed the central role of the microcirculation in the pathophysiology, outcome and treatment of sepsis by its ability to visualise the microcirculation in great detail under clinical conditions. Of particular importance in this context has been the response of the smallest microvessels, the capillaries. OPS imaging illuminates the tissues with polarized green light and measures the reflected light from the tissue surface after filtering out the polarized portion of the reflected light. A clinical scoring system was developed to quantify images in septic patients (1). However OPS imaging was found to lacks sensitivity to study in detail capillary kinetics and morphology. We recently introduced SDF (Sidestream Dark Field) imaging as a new way of clinical observation of the microcirculation. In this modality a light guide imaging the microcirculation is surrounded by light emitting diodes (LED) of a wavelength $(530 \mathrm{~nm})$ absorbed by the hemoglobin of erythrocytes so that they can be clearly observed as flowing cells. Covered by a disposable cap the probe is placed on tissue surfaces. The light from the concentrically placed LED's at the tip of the probe directly penetrate deep into the tissue illuminating the microcirculation. By not being in direct optical contact with the sensing central core of the probe no direct surface reflections interfere with the image of the microcirculation and remarkable clear images can be observed. The deeper sublingual arterioles as well as the glcocalyx lining of the endothelial cells can also be clearly observed. Improved image quality allows better computer automatic analysis of the images and the low energy requirement of SDF imaging further enhances its utility by allowing battery and/or portable computer operation. It is expected that SDF imaging will provide an improved imaging modality of the microcirculation in sepsis especially in pediatriatic patients 1 . Boerma EC, Mathura KR, van der Voort PHJ, Spronk PE, Can Ince (2005) Quantifying bedside-derived imaging of microcirculatory abnormalities in septic patients: a prospective validation study. Critical Care 9:R601-R606 2. Ince $C$ (2005) The microcirculation is the motor of sepsis. Critical Care 9 (suppl. 4): S13-S19 Declared interest: C. Ince is CSO of MicroVisionMedical which is an AMC based company commercializing SDF imaging.

\section{UNRAVELING THE REACTIONS OF NITRIC OXIDE, NITRITE AND HEMOGLOBIN IN HUMAN PHYSIOLOGY AND THERAPEUTICS}

\section{T Gladwin}

\section{Department of Critical Care Medicine, National Heart Lung and Blood Institute, Bethesda, MD, USA}

Nitric oxide (NO) plays a fundamental role in maintaining normal vasomotor tone. Recent data implicate a critical function for hemoglobin and the erythrocyte in regulating the activity of NO in the vascular compartment. Intravascular hemolysis releases hemoglobin from the red blood cell into plasma (plasma cell-free plasma Hemoglobin), which is then able to scavenge endothelial derived NO 600-fold faster than erythrocytic hemoglobin, thereby disrupting NO homoestasis. This may lead to vasoconstriction, decreased blood flow, platelet activation, increased 
endothelin-1 expression (ET-1), and end-organ injury, and thus suggesting a novel mechanism of disease for hereditary and acquired hemolytic conditions such as sickle cell disease and cardiopulmonary bypass. In addition to providing an $\mathrm{NO}$ scavenging role in the physiological regulation of NO-dependent vasodilation, hemoglobin and the erythrocyte may deliver NO as the hemoglobin deoxygenates. While this process has previously been ascribed to S-nitrosated hemoglobin, recent data from our laboratories suggest that deoxygenated hemoglobin reduces nitrite to NO and vasodilates the human circulation along the physiological oxygen gradient. This newly described role of hemoglobin as a nitrite reductase is discussed in the context of blood flow regulation, oxygen sensing, and nitrite-based therapeutics. Reiter CD, Wang X, Tanos-Santos J, Hogg N, Cannon RO, Schechter AN, and Gladwin MT. Cell free hemoglobin limits NO bioavailability in sickle cell disease. Nature Medicine 2002; 8:1383-1389. Cosby K, Partovi KS, Crawford JH, Patel RP, Reiter C, Martyr S, Yang BK, Waclawiw MA, Zalos G, Xu X, Huang KT, Shields H, Kim-Shapiro DB, Schechter A, Cannon RO, and Gladwin MT. Nitrite reduction to nitric oxide by deoxyhemoglobin vasodilates the human circulation. Nature Medicine 2003; 9:1498-1505. Gladwin MT, Sachdev V, Jison M, Plehn JF, Minter K, Brown B, Coles WA, Nichols JS, Ernst I, Hunter LA, Blackwelder W, Schechter AN, Rodgers GP, Castro O, and Ognibene FP. Pulmonary Hypertension as a Risk Factor for Death in Patients with Sickle Cell Disease. New England Journal of Medicine 2004; 350:886-895.

\section{PRECISION OF MEASUREMENT OF CEREBRAL TISSUE OXYGENATION INDEX USING NEAR-INFRARED SPECTROSCOPY IN NEWBORN INFANTS}

\author{
L C Soerensen ${ }^{1}$, G Greisen ${ }^{2}$ \\ ${ }^{I}$ Department of Pediatrics, Copenhagen University \\ Hospital, Hvidovre, Denmark, ${ }^{2}$ Department of \\ Neonatology, National University Hospital; \\ Rigshospitalet, Copenhagen, Denmark
}

Background and Aims: Quantification of the cerebral oxygenation in intensive care is relevant. Spatially resolved near-infrared spectroscopy is suitable, but accuracy is insufficient (1). Our aim was to quantify the precision of the cerebral Tissue Oxygenation Index (c-TOI) doing repeated measurements to see if imprecision could explain the lack of accuracy.

Methods: Twenty-five mature and 37 premature infants were examined using the NIRO300 oximeter (Hamamatsu). The handheld optode was re-sited in different parieto-temporal positions three to nine times within 20 minutes. One-way ANOVA was used to estimate variability.

Results: The mean age at study was $19 \pm 6$ hours. GA was $39.7 \pm 1.3$ and $28.2 \pm 2.6$ weeks and arterial oxygen saturation $96 \pm 2.5 \%$ and $96 \pm 2.8 \%$, respectively. $5 / 37$ of the premature infants were mechanically ventilated. Mean c-TOI was $74.7 \pm 7.8 \%$ and $74.6 \pm 8.5 \%$. The within-infant variation in the two groups was $5.8 \%$ versus $5.2 \%$ (NS). The between-infants variation was $5.2 \%$ versus $6.9 \%(\mathrm{p}<0.005)$.

Conclusions: The 5-6\% precision of c-TOI was not good taking the population standard deviation of 5-7\% into consideration and corresponds almost exactly to the limits of agreement of $+/-12 \%$ in reference (1). The mean c-TOI was similar in the two groups, but the between-infant variation in the premature group was higher. This could reflect greater heterogeneity. Since cTOI is partly venous $(2 / 3)$ and partly arteriel (1/3), an arterial oxygen saturation of $96 \%$ and a c-TOI of $75 \%$ corresponds to a venous saturation of $65 \%$, which is normal. This work has been supported by the Ludvig og Sara Elsass Foundation. 1. Nagdyman N et al. Intensive Care Med 2005; 31: 846-850.

\section{ROLE OF REACTIVE OXYGEN SPECIES IN KV CHANNEL INHIBITION AND VASOCONSTRICTION INDUCED BY TP RECEPTOR ACTIVATION IN RAT PULMONARY ARTERIES}

A L Cogolludo ${ }^{1,2}$, G Frazziano ${ }^{2}$, L Cobeño ${ }^{2}$, L Moreno ${ }^{2}$, F Lodi $^{2}$, J Tamargo ${ }^{2}$, F Perez-Vizcaino ${ }^{2}$, E Villamor ${ }^{1}$

${ }^{1}$ Department of Pediatrics, University Hospital Maastricht, Research Institute Growth and Development (GROW), Maastricht, The Netherlands, ${ }^{2}$ Department of Pharmacology, School of Medicine, Universidad

Complutnese, Madrid, Spain

Background and Aims: Voltage-gated potassium channels (Kv) and thromboxane A2 (TXA2) have been involved in several forms of human and experimental pulmonary hypertension. We have reported that the TXA2 analog U46619, via activation of TP receptors and $\mathrm{PKCz}$, inhibited $\mathrm{Kv}$ currents in rat pulmonary artery (PA) smooth muscle cells, increased cytosolic calcium and induced a contractile response in isolated rat and piglet PA. Herein, we have analyzed the role of reactive oxygen species in the signalling pathway.

Methods: U46619-evoked changes in contractility, dichlorofluorescein fluorescence (an indicator of intracellular hydrogen peroxide), and Kv currents (using the whole-cell configuration of the patch clamp technique) were analyzed in adult rat PA.

Results: In rat PA, U46619 increased dichlorofluorescein fluorescence and this effect was prevented by the NADPH oxidase inhibitor apocynin and by polyethylenglycol-catalase (PEG-catalase, a membrane permeable form of catalase). U46619 inhibited $\mathrm{Kv}$ currents in native PASMC and these effects were strongly inhibited by apocynin. The contractile responses to U46619 in isolated pulmonary arteries were inhibited by PEG-catalase and the NADPH oxidase inhibitors DPI and apocynin. The membrane permeable hydroperoxide t-butyl hydroperoxide also inhibited $\mathrm{Kv}$ currents and induced a contractile response in PA.

Conclusions: Activation of NADPH oxidase and the subsequent production of hydrogen peroxide are involved in the Kv channel inhibition and the contractile response induced by TP receptor activation in rat PA. 


\section{MICROVASCULAR FLOW IS COMPROMISED EARLY IN NEONATAL INFECTION}

I Alba-Alejandre $^{1}, \mathrm{~S} \mathrm{Hiedl}^{1}$, F Christ ${ }^{2}$, O Genzel-Boroviczeny ${ }^{1}$

${ }^{I}$ Division of Neonatology, University Children's Hospital, Ludwig-Maximilians University, Munich, Germany, ${ }^{2}$ Department of Anaesthesiology, Klinikum Grosshadern, Ludwig-Maximilians University, Munich, Germany

Background and Aims: The early diagnosis of neonatal infection remains a challenge. Recent studies using Optical Polarizated Spectral (OPS) imaging techniques, have reported microcirculatory alterations in adult patients with severe sepsis and septic shock. The objective of this study was to compare microcirculatory flow and vascular density between healthy $(\mathrm{H})$ and sick newborns (I) with neonatal infection.

Methods: We obtained prospectively OPS images from the ear conch of 45 infants on their 1st, 2nd and 3rd day of life. Infection was defined as IL- $6>50 \mathrm{pg} / \mathrm{mL}$ and $\mathrm{CRP}>0,5 \mathrm{mg} / \mathrm{dL}$. The OPS sequences were analyzed off-line, blinded to clinical status of the infant, using a semiquantitative assessment of flow based on the work of De Backer and Boerma.

Results: In the 14 infants of group I the percentage of vessels with impaired flow was significantly elevated (mean I [95\%CI]: 34\% [20-47] vs. H: 9\% [6-13] $\mathrm{p}<0,0001)$ whereas the trend to lower vascular density did not reach statistical significance (I: 6,1 [4,67 7,51]vs. H: 6,5 [5,12- 7,88] vessels/mm). Most episodes of infection were not severe (geometrical mean of max. CRP :1,4 [95\%CI: 0,92- 2,2] mg/dL ; IL-6: 87,1 [16,8$450,1] \mathrm{pg} / \mathrm{mL}$ ). Birth weight an gestational age did not differ between the groups.

Conclusions: Even in infants with mild to moderate signs of infection, microcirculatory flow is impaired in a large proportion of vessels early in the course of disease. Since these changes can be easily recognized at the external ear, an on-line evaluation of microcirculatory flow could be an important screening tool for infection in the future.

\section{NEONATES BORN TO MOTHERS WITH PRE- ECLAMPSIA (PE) EXHIBIT ALTERED MICROVASCULAR FUNCTION}

\author{
M J Stark ${ }^{1}$, V L Clifton ${ }^{1}$, I M R Wright ${ }^{1,2}$ \\ ${ }^{I}$ Mother and Babies Research Centre, Hunter Medical \\ Research Institute, University of Newcastle, NSW, \\ Australia, ${ }^{2}$ Kaleidoscope Neonatal Intensive Care Unit, \\ John Hunter Children's Hospital, Newcastle, NSW, \\ Australia
}

Background and Aims: There is abnormal peripheral microvascular function in Pre-eclampsia (PE). We have shown that fetal sex alters maternal microvascular function. It is known that males dominate the poor outcomes in PE (20). We hypothesised that neonatal microvascular function would be altered following PE and be further influenced by sex.

Methods: Peripheral microvascular responses were examined using Laser Doppler flowmetry. Term control infants $(n=31)$ and infants of PE mothers $(n=33)$ where studied at 6 hours and days 1, 3, and 5-7. Baseline perfusion and vasodilatory response to local thermal stimulation and post-occlusive reperfusion were measured.

Results: Baseline blood flow significantly increased from 6 hours to 7 days of age in the PE group (KruskalWallis ANOVA, $\mathrm{P}<0.01$ ). No temporal change was seen in the controls. Within the PE group, male infants where significantly more vasodilated than females at 6 hours (median 13.5 vs $5.9 \mathrm{PU}, \mathrm{P}=0.0289$ ) but not at 72 hours of age. No sex-specific difference was observed in the controls. No significant differences in vasodilatory response to dynamic testing were observed in either group at any time point or by sex.

Conclusions: Following PE infants vasodilate, but not following normotensive pregnancy. The mechanisms of microvascular dysfunction in PE are poorly understood but circulating vasoconstictors play an important role. These may also effect the fetal circulation. The observed differences in microvascular function may be a release phenomenon. In keeping with our previous work we have demonstrated sex significantly influences microvascular function. This could explain the excess of male morbidity and mortality following PE.

\section{FIRST ASSESSMENT OF THE MICROCIRCULATION IN CRITICALLY ILL CHILDREN BY ORTHOGONAL POLARISATION SPECTRAL (OPS) IMAGING}

\author{
P C Top ${ }^{1}$, J E Van Velzen ${ }^{1}$, K R Mathura ${ }^{2}$, C Ince ${ }^{2}$, \\ D Tibboel $^{1}$ \\ ${ }^{I}$ Department of Pediatric Surgical Intensive Care, \\ Erasmus MC-Sophia, Rotterdam, ${ }^{2}$ Department of \\ Physiology, AMC, Amsterdam, The Netherlands
}

Background and Aims: A prominent feature of sepsis is dysfunction of the microcirculation, with impaired perfusion and regional tissue oxygenation. Orthogonal Polarisation Spectral imaging (OPS imaging) is a noninvasive technique that has recently been developed for the study of the microcirculation of mucous membranes and the surface of solid organs. In adults this technique has been proven valuable to predict organ failure and mortality. The aim was to evaluate the feasibility of OPS imaging in children and to determine baseline data of the microcirculation and to evaluate microcirculation in critically ill children.

Methods: The microvascular network of the oral mucosa was studied. The effect of temperature, blood pressure, hemoglobin concentration and $\mathrm{pH}$ were studied. Baseline data were determined.

Results: We found a significant negative correlation between age and the FCD. There were no significant relationships between the FCD and temperature, blood pressure and $\mathrm{pH}$. Furthermore we evaluated the microcirculation of critically ill children and the effect of ECMO on the microcirculation.

Conclusions: Microcirculatory parameters of the oral mucosa can be measured in absolute values in children by OPS imaging. We have successfully determined baseline data of the oral microcirculation, which could serve as reference values for critically ill children. The 
method has been proven useful and is a promising research tool.

\section{HOW BEST CAN WE CARE FOR THE DYING INFANT OR CHILD AND THEIR FAMILY?}

\author{
F Lynch ${ }^{1,2}$, A Van den Hoogen ${ }^{3}$ \\ ${ }^{1}$ Paedatric Intensive Care Unit, Evelina Children's \\ Hospital, London, United Kngdom, ${ }^{2}$ President of \\ Scientific Committee ESPNIC Nursing, ${ }^{3}$ Department of \\ Neonatology, Wilhelmina Childrens Hospital, Utrecht, \\ The Netherlands
}

Background and Aims: This interactive session aims to discuss and explore the roles and responsibilities of the PICU and NICU nurse in caring for the dying child or infant and their family. This important aspect of nursing care challenges even the most experienced nurse. We will share our experiences and generate ideas about best practice through facilitation of audience participation.

Methods: The experience of caring for the dying infant or child in the critical care setting is one which challenges the multidisciplinary team greatly. At a time of immense emotional distress, it is essential that the nurse provides timely, compassionate and therapeutic care for the patient and their family. Insensitive, unhelpful and misguided care can have a lasting negative impact on the grieving process for the family. It is therefore essential that we provide the best care we can to support the family during this difficult time.

Results: The presenters will share with you their individual experiences and knowledge of caring for the dying infant and child. The session will:

- Share the practical issues around caring for the dying patient and their family.

- Explore the complex communication issues with the patient, parents, siblings, family members and members of the multidisciplinary team.

- Discuss the after care required for family members.

- Explore how we can best support and develop other nurses and members of the multidisciplinary team in providing best practice.

- Explore the legislative, spiritual, cultural, professional and ethical issues around providing care in the UK and The Netherlands.

Conclusions: The last section of this session will concentrate on facilitating discussion and debate from the European audience. Both presenters will collate the generated ideas of good practice during the session and conclude the session with a summary of best practice.

\section{PARENTAL CHOICE IN PLACE OF DEATH: GUIDELINES FOR TERMINAL EXTUBATION OUTSIDE PICU}

\section{A P Anderson, F Craig}

Great Ormond Street Hospital, London, UK

Background and Aims: To develop guidelines for terminal extubation outside PICU.

Methods: Retrospective case review of four children whose families have requested terminal extubation to occur outside PICU. Key aspects of care were identified along with difficulties encountered. Based on this experience comprehensive guidelines were developed.

Results: Four families requested for terminal extubation to occur outside PICU. One family chose to be at home and two families chose to go to a hospice local to their homes. The fourth family were unable to be accommodated in their request due to difficulties with local service provision. In all cases, local services requested PICU staff to perform the terminal extubation. Aspects of care highlighted when considering terminal extubation outside PICU were divided into five key areas:

1. Availability of PICU resources for transfer. 2. Planning for anticipated symptoms including medication and education of families and local staff; particularly if death is not imminent on extubation. 3. Availability and experience of local medical and nursing services.

4. The training needs, supervision and clinical competence of PICU staff to provide terminal extubation outside normal working environment.

5. Making provision for care and place of care after death.

Conclusions: Extubation outside PICU is a feasible option and it is hoped that with the guidelines developed, more families will be able to benefit from this option. When a child's death is inevitable, the focus of hope changes, the how, when and where becomes important.

\section{END-OF-LIFE CARE IN PEDIATRIC INTENSIVE CARE: A PRIORITY FOR PEDIATRIC NURSES}

\author{
H Pereth ${ }^{1}$, F Martens ${ }^{1}$, D Danschutter ${ }^{1}$, J Ramet ${ }^{2}$, \\ L Deliens ${ }^{3}$, J Bilsen $^{3}$, S Hachimi-Idrissi ${ }^{1}$ \\ ${ }^{I}$ Pediatric Intensive Care Unit, Vrije Universiteit
} Brussel, Brussels, Belgium, ${ }^{2}$ Department of Pediatrics, Koningin Paola Ziekenhuis, Antwerp, Belgium, ${ }^{3}$ Faculty of Medicine and Pharmacy Medical Sociology, Vrije Universiteit Brussel, Brussels, Belgium

Background and Aims: Care of children at the end of life involves ethical dilemmas and difficult decisions. Empirical research on these decisions focuses predominantly on the physicians' role. Little is known about the role of the pediatric nurses. This paper reports the findings of a study that evaluates the attitude of nurses regarding end-of-life decisions in children. The study also reports their involvement in the decisionmaking process and their participation in the accomplishment of the decision.

Methods: Anonymous questionnaire was send to nurses working in 5 different pediatric intensive care settings, in Belgium.

Results: $63 \%$ of the nurses completed their questionnaire. $84 \%$ of them were confronted at least once with end-of-life decision the last 2 years and $73 \%$ wanted to be involved in making the decision of end-oflife. $66 \%$ of the nurses played an active role in the preparation as well as the accomplishment of the decision. $60 \%$ of the nurses assisted the physician in the accomplishment of the decision, $50 \%$ carried out the decision themselves in the presence of the physician. Almost the majority of the respondent nurses (92\%) 
state that in certain situations it would be preferable not further to treat the child, $6 \%$ of the respondents would never participate in any type of end-of-life decision in children.

Conclusions: The majority of the nurses were confronted with end-of-life decisions in children. $66 \%$ played an active role in the accomplishment of the decision, but only $50 \%$ of them were involved in the decision-making process.

\section{A PARENT TO PARENT SUPPORT PROGRAM FOR MOTHERS OF PRETERM INFANTS IN THE NICU: A RANDOMIZED TRIAL}

T del Moral ${ }^{1}$, L Falcon ${ }^{1}$, A Rodriguez ${ }^{1}$, A Bernacet ${ }^{1}$, J Wilkinson $^{2}$, S Fajardo- Hiriart ${ }^{1}$, R Echeverri ${ }^{1}$

${ }^{I}$ Department of Pediatrics, University of Miami, FL, USA, ${ }^{2}$ Department of Epidemiology and Public Health, University of Miami, FL, USA

Background and Aims: An individual peer support network could be an effective intervention to help parents deal with the stress of having a baby in the NICU. This study aims to assess the stress of parents while their infants are in the NICU and to evaluate the effectiveness, in alleviating stress, of a parent to parent support program.

Methods: Mothers of infants with a birth weight $<1500$ g were randomized to a control or an intervention group. Those in the intervention group were paired with volunteer parents who had previously had a premature infant. These volunteers were trained according to the Parent Partners Listener Training Program (George Washington University). Both groups answered the NICU Family Needs Inventory (NFNI) and the Parental Stressor Scale (PSS) during the first and sixth weeks after admission to NICU. Outcome data were analyzed in an intention to treat basis using T-test.

Results: The PSS scores were significantly higher after six weeks when compared with base line $(2.9+0.9$ vs $2.4+0.7, p=0.006)$. There were not statistically significant differences in PSS $(3.0+0.8$ vs $2.7+0.9)$ or NFNI scores $(2.9+1.1$ vs $2.6+1.1)$ between the control and intervention groups after six weeks.

Conclusions: These results demonstrated that the stress level of mothers of premature infants increases after the infant have been in the NICU for a few weeks. The parent support program was not effective in decreasing the level of parental stress during this initial period of maternal adaptation to the NICU. Studies to measure the long-term effects of this intervention are needed.

\section{PAEDIATRIC ROTAVIRUS \\ GASTROENTERITIS: TIME FOR VACCINATION!}

\section{F Gimenez-Sanchez}

Advisory Committee of Vaccines, Spanish Association of Pediatrics, Paediatric Infectious Diseases,

Torrecardenas Hospital, Almería, Spain

Please refer to page $119 \mathrm{a}$.
RotaTeq ${ }^{\circledR:}$ ENSURE SUCCESSFUL VACCINATION

\section{P Heaton}

Merck Research Laboratories, USA

Please refer to page 119a.

THINKING FORWARD WITH MMRV VACCINE

M Knuf

Germany

Abstract not available at the time of printing.

\section{ProQuad ${ }^{\circledR}$ : MEETING WITH CHALLENGE OF NEW MEASLES, MUMPS, RUBELLA AND VARICELLA VACCINE}

\section{B Kuter}

USA

Abstract not available at the time of printing.

\section{INFECTIOUS DISEASES AND HOST RESPONSE}

\section{R de Groot}

The Netherlands

Abstract not available at the time of printing.

\section{MUCOSAL IMMUNOLOGY: NEONATAL ADAPTATION OF THE MAJOR FIRST LINE DEFENCE SYSTEM}

\section{P Brandtzaeg}

Laboratory for Immunohistochemistry and Immunopathology (LIIPAT), Institute of Pathology, University of Oslo, Rikshospitalet University Hospital, Oslo, Norway

The vast majority of immunological challenges confronting the body make contact with mucosal surfaces, including infectious agents and foreign proteins. To maintain homeostasis in the extensive and vulnerable mucosae, they are protected by specialized mechanisms of immune protection. Numerous genes are involved in the regulation of both innate (natural) and adaptive (acquired) immunity, with a variety of modifications introduced over million of years. Twolayered adaptive mucosal immunity: In the process of evolution, the mucosal immune system has generated two layers of non-inflammatory defence: (i) immune exclusion performed by secretory IgA antibodies to modulate or inhibit surface colonization of microorganisms and dampen penetration of potentially dangerous soluble factors; and (ii) suppressive mechanisms to avoid local and peripheral hypersensitivity to innocuous antigens, particularly food proteins and components of commensal bacteria. When induced via the gut, the latter phenomenon is called 'oral 
tolerance', which appears be a rather robust adaptive immune function in view of the fact that large amounts of food proteins pass through the gut, while overt and persistent food allergy is not so common. Neonatal immune adaptation: The neonatal period is particularly critical, both with regard to infections and priming for allergic disease. This is so because the mucosal barrier function and the immunoregulatory network are poorly developed for a variable period after birth, and successful neonatal immune adaptation to exogenous stimuli is crucial to health. Notably, mucosal homeostasis depends on appropriate microbial colonization as well as adequate timing and dose of foreign food proteins when first introduced in the diet. Dendritic cells are 'decision makers' in the immune system when they perform their antigen-presenting function, thus linking innate and adaptive immunity by sensing the exogenous impact (e.g. conserved microbial molecular patterns) on the mucosa. A balanced indigenous microbiota is apparently required to drive the normal development of both mucosa-associated lymphoid tissue and the secretory IgA system as well as mucosally induced tolerance mechanisms such as the generation of regulatory $\mathrm{T}$ cells. Moreover, the properties of dendritic cells also appear to be influenced by dietary factors such as vitamin A. Other dietary factors including lipids, particularly the polyunsaturated n-3 fatty acids in fish oil, can likewise in several ways have a beneficial effect on the developing immunophenotype of the infant. The same is true for breast milk, which provides both immunoregulatory factors and secretory antibodies reinforcing the infant's mucosal barrier.

\section{MECHANISM OF ALLERGIC SENSITIZATION: THE HYGIENE HYPOTHESIS}

\section{E Isolauri}

Department of Paediatrics, University of Turku, Finland

Background and Aims: The hygiene hypothesis conceives the increase in atopic disease to be related to reduced exposure to microbes early in life.

Methods: The Nutrition, Allergy, Mucosal Immunology and Intestinal Microbiota (NAMI) research group has evaluated the host-microbe interaction in performing studies on:

1. The Association: Mode of delivery-gut microbiotarisk of atopic disease.

2. Clinical Intervention: Probiotics in nutritional management/ risk reduction of atopic disease.

3. Mechanisms: Probiotic / microbiota effects / Pathogenesis of disease.

Results: The microbial provocation is originally obtained from the mother. How the microbiota is originated, depends on genetics, mode of delivery, early feeding strategies and the hygienic conditions. The NAMI research group has provided evidence for an extended hygiene hypothesis. This unifying theory that early and constant microbial stimulus from the gut microbiota may outweigh that of occasional infections provides an explanation for the simultaneous increase of autoimmunity and inflammatory bowel disease (TH1 mediated) and allergic diseases ( $\mathrm{TH} 2$ mediated).
Specific strains of the healthy gut microbiota (probiotics) were shown to counter allergy by generation of anti-inflammatory interleukin-10 and transforming growth factor-beta produced by Tr1- and TH3 cells, respectively, which have a vital role both in suppression of TH2-induced allergic inflammation, induction of oral tolerance, and IgA production, an indispensable component of the mucosal immune defence. The probiotic effects in atopic disease have been attributed to restoration to normal of increased intestinal permeability and unbalanced gut microbiota, improvement of the intestine's immunological barrier functions, and reduced generation of proinflammatory cytokines characteristic of local and systemic allergic inflammation. Gut microbiota provide again maturational signals for the gut-associated lymphoid tissue, particularly for the IgA plasma cells, conferring the first line of host immunological defence. Probiotics have also been show to exert distinct effects on antigen transport, depending on the food matrix, for example the quality of protein in the diet. In a rodent model mucosal transport of degraded macromolecules has been found to be stimulated when Lactobacillus GG is administered together with unhydrolyzed protein, but reduced when administered with hydrolyzed protein. Such protein may thus stimulate the humoral immunity in the gut, but also affect on the induction of oral tolerance, as antigen degradation is an indispensable component in the acquisition of mucosal tolerance. Moreover, polyunsaturated fatty acids have been shown to affect the growth and adhesion of probiotics, and the protective effect of probiotics appear to evolve in joint action with the dietary intake of particular nutrients reducing the risk of allergic disease. These advances have prompted enthusiasm in the scientific community and food industry and have fuelled research activities currently focusing firstly on identification of specific strains with anti-allergenic potential, and secondly on the question how food matrix and dietary content interact with the most efficacious probiotic strains.

Conclusions: Taken together, probiotic effects evidently act cooperatively with other nutritional compounds. In the future, the properties of specific probiotics, and their combinations, in an optimal food matrix, might be exploited in the development of specific prophylactic and therapeutic interventions.

\section{CHANGE IN NURSING PRACTICE STARTS WITH YOU!}

\section{W De Groot-Bolluijt}

GrootBolwerk, Maasdijk, The Netherlands

Background and Aims: Nursing is an innovative and dynamic profession. Since the early days of Florence Nightingale, nursing practice has changed dramatically in order to provide better care and improve the outcome of the patient's illness. The latest development in nursing is the change towards evidence based practice. Knowing the newest developments does not seem to be sufficient for making a change effective. Providing knowledge by education, training and symposia does often not have the required results. Change Management gives realization in different aspects necessary for 
implementing an innovation. The aim of this workshop is to present the Change Work Model to obtain practical skills for a successful implementation of innovations.

Methods: The Change Work Model is based on concepts of various theories of change management and learning styles. In addition, unit culture and innovation techniques are also incorporated in this model. The assumptions of the concepts within the Change Work Model are based on the available literature and are connected within a framework in the set up of the Change Work Model. The model is based on the assumption that before implementing an innovation, nurses have to know the different aspects of change. How they experience there own role and learning styles, the culture of there working place and what they expect from innovation. After the explanation of the theory, the participants will work with checklists based on the different aspects with examples from daily practice. The Change Work Model will lead nurses to become aware of their own role and learning styles, recognise the impact of the organization's culture and to identify recommendations to implement change.

Results: With the Change Work Model different aspects will be important for innovation towards the daily practice. Changing can not only be done through projects in the entire organisation. The workshop sustains working with changes on an individual level and makes the own role of the nurse clear. It also offers tools to work with in a team. As a result, nurses obtain a better understanding of the mechanics and processes involved in innovation. They can use the different aspects of change and this will help them to work on innovation and successful change. Consequently the patient will benefit from any quality improvement in nursing practice.

Conclusions: The Change Work Model guides nurses through a systematic process of change to implement innovations into practice. This model is based on a framework of change management theory, research and clinical experience. The Model is a practical guidance for the individual nurses and applicable in every unit regardless the unit culture. In the rapid changing world of PICU and NICU, nurses need to develop their practice and account for their roles in changing practice. The infants and children on the NICU and PICU will benefit from our attitude. Change in nursing practice starts with you!

\section{A PARENT TELEPHONE SUPPORT NETWORK FOR THE INTENSIVE CARE UNIT}

\author{
A M Scales ${ }^{1}, \mathrm{P}$ L Shroff ${ }^{1}, \mathrm{C}$ M Pierce ${ }^{2}$ \\ ${ }^{\text {I}}$ Paediatric Intensive Care Unit, Great Ormond Street \\ Hospital, London, UK, ${ }^{2}$ Paediatric and Neonatal \\ Intensive Care Unit, Great Ormond Street Hospital, \\ London, UK
}

Background and Aims: The need for family support in intensive care unit is well documented (1). Parents have always offered uniquely understanding support in shared situations. They have an altruistic desire to help others or' give back' into the setting which has had enormous impact on their family. In partnership with professionals, parents can use this compassion and experience, if trained and supported properly, to help other families. In line with government Patient and Public Involvement Strategies and feedback from our intensive care family group a Parent Telephone Support Network (PTSN) was developed and supported by hospital trust management. The aim of the service is to provide unique support for anyone directly affected by an admission to intensive care. Using this to complement the multidisciplinary psychosocial support already offered, thus enhancing the wider support mechanisms available for families.

Methods: Fifteen parents/carers participated in initial training sessions consisting of simulated phone calls, useful skills and helpful strategies to provide confidence in providing this support.

Results: There are three PTSN sessions a week when callers can be connected to a supporter. Supervision and support is provided by the Family Liaison Sisters. Initial feedback from users and supporters is excellent.

Conclusions: Future aims are to expand the service by increasing the number of sessions that are offered. 1. Wheeler, H Nursing in Critical Care 2005 Vol 10 (2) p.56-63, The importance of parental support when caring for the acutely ill child. Acknowledgements: Kolbe, A, Waddington, H, Simmons, J.

\section{PARENT STRESS AND CONCERNS FOR CHILDREN AFTER CARDIAC SURGERY}

\author{
L S Franck ${ }^{1,2}$, A McQuillan ${ }^{2}$, N Power ${ }^{1,2}$, \\ A Goldman ${ }^{1,2}$ \\ ${ }^{1}$ Institute of Child Health, London, UK, ${ }^{2}$ Great Ormond \\ Street Hospital, London, UK
}

Background and Aims: The hospitalisation of a child in intensive care is known to be a stressful experience for parents that may affect family functioning. However, little is known about the time course and the relationships between parental stress and the child's actual or expected recovery. This research project was conducted to investigate pre- and post-operative parental stress and to examine the influencing factors during the postoperative period.

Methods: An exploratory, descriptive study was undertaken, using triangulation of methods, to explore parental stress, expectations and perceptions regarding their child's recovery from cardiac surgery. Structured interviews were conducted with the parents of 117 children pre-operatively and on days 1, 3, 5, 8 and 15 postoperatively (whilst child still in hospital). Parents independently completed a validated parent stressor scales.

Results: Parent stress remained high throughout their child's hospitalisation, regardless of severity of illness. The main themes of parents' expressed concerns were: Parental expectations/perceptions versus actual illness; Changing perceptions over time; and dominance of parental concerns for their child over their own concerns. There were few differences between mothers' and fathers' concerns. Parents whose children had no previous surgery voiced more concerns regarding pain and discomfort, whereas parents of children who had undergone previous surgery were more concerned with complications and physical issues. Parents born outside 
the UK whose first language was not English had higher stress levels and more concerns.

Conclusions: These findings indicate the negative impact of children's surgery and intensive care hospitalisation on parents and identify specific areas for interventions to improve parental support and coping.

\section{THE FETAL ORIGINS OF INSULIN RESISTANCE AND THE METABOLIC SYNDROME}

\section{Lévy-Marchal \\ INSERM U690, Robert Debré Hospital, Paris, France}

Over the past fifteen years, a large body of studies has evidenced the relation between SGA (small for gestational age) and the increased risk of insulinresistance, type 2 diabetes and cardiovascular diseases later in life. The first reports focused on elderly and in populations where the prevalence of diabetes is already high. The association with insulin resistance and the metabolic syndrome was later extended to young adults and to prepubertal children. Our research programme is based on a large community-based cohort where all subjects were carefully selected on birth data and gathers 734 SGA subjects (birth weight $<10$ th percentile) and 886 AGA (adapted for gestational age) subjects $(25<\mathrm{BW}<75$ perc.) born 1971-85. In these adults born SGA, we have reported a number of malfunctions of the adipose tissue persisting after puberty (22 yr. old) suggesting an abnormal adipose tissue which could participate to insulin-resistance and the metabolic syndrome : - They show an excess of fat mass, preferentially abdominal, without obesity - Insulin action on lipolysis is reduced and FFA release is not suppressed under physiological insulin concentrations An excessive lipolysis in response to catecholamines was observed by microdialysis of the abdominal adipose tissue - Leptin levels show an impaired regulation during catch-up and are low in adults - Adiponectin levels are low and the and insulin-sensitizing action is impaired. - Pro12Ala of PPAR actually enhances IR in the SGA individuals whereas it shows a insulinsensitizing effect in AGA's, attesting for a real interaction with the fetal environment. and this observation has been replicated in two independent cohorts At the same time the different features of MS (hypertension, dyslipidemia, impaired glucose tolerance) were manifested in these SGA subjects and were clustering around IR. However, the phenotype was mild meaning that the differences between the subjects born SGA and their pairs born adapted for gestational age (AGA) were indeed significant for all features but of low magnitude. As a matter of fact, the proportion of disorders of glucose tolerance was low but twice as high in SGA in comparison to AGA (3.8\% vs $1.6 \%$ ) and that of MS six fold higher ( $2.3 \%$ vs $0.4 \%)$. We hypothesize that the adipose tissue, which is a major target of fetal growth restriction, is a key-tissue responsible for the metabolic complications and that the process extends beyond the perinatal period and continues to evolve in adults.

\section{CONTROLLED TRIAL OF EARLY LOW-DOSE HYDROCORTISONE TREATMENT IN PREVENTION OF BRONCHOPULMONARY DYSPLASIA IN PRETERM INFANTS: A TWO- YEAR FOLLOW-UP}

O M Peltoniemi, A Lano, K Nikolajev, M A Kari, M Hallman

Department of Pediatrics, University of Oulu, Finland

Background and Aims: Dexamethasone treatment is associated with increased the risk of cerebral palsy (CP) among VLBW infants. Early hydrocortisone (HC) treatment may decrease the incidence of BPD, but no follow up studies have been published so far. Here we present the first preliminary results.

Methods: 51 infants enrolled in a blinded, controlled trial evaluating the effectiveness of postnatal early $\mathrm{HC}$ treatment in prevention of BPD were reviewed. According to present results the infants who had low cortisol values before the supplementation had less severe respiratory course and lower incidence of BPD; however, the infant with high endogenous cortisol had no benefit (J Pediatrics 2005). Here we report the results of physical growth and neurodevelopmental outcome at corrected age of two years.

Results: The growth characteristics were similar in both groups. The mean IQ evaluated by Griffiths Developmental Scale was $94.8 \pm 21.2$ in BM and $99.2 \pm$ 12.4 in placebo groups $(\mathrm{P}=0.45)$. The mean IQ was 97.3 \pm 6.8 in $\mathrm{BM}$ and $95.9 \pm 14.9$ in placebo group among infants with low cortisol values (stimulated cortisol < $270 \mathrm{nmol} / \mathrm{l})$ after birth $(\mathrm{P}=0.95)$. The mean IQ was 91.6 \pm 26.9 in $\mathrm{BM}$ and $100.1 \pm 12.6$ in placebo group among infant with high cortisol values after birth $(\mathrm{P}=0.82)$. Conclusions: Early low dose $\mathrm{HC}$ had no detectable effect on the growth or neurologic outcome at 2 years of adjusted age. The sample size was not calculated to find a difference. We propose that in subsequent trials studying the efficacy and safety of HC supplementation, there is a need to analyze endogenous cortisol levels. $\mathrm{HC}$ or placebo (HC $2.0 \mathrm{mg} / \mathrm{kg}$ i.v. divided into three doses for 2 days, $1.5 \mathrm{mg} / \mathrm{kg}$ i.v. at 8 -hour intervals for 2 days and $0.75 \mathrm{mg} / \mathrm{kg}$ i.v. divided into two doses for 6 days.

\section{INTRAVENOUS ENDOTOXIN RESULTS IN PULMONARY INFLAMMATION AND LUNG REMODELING IN PRETERM FETAL SHEEP}

\author{
B W Kramer ${ }^{1,2}$, C P Speer ${ }^{2}$, A B Coumans ${ }^{2}$, \\ T H Hasaart ${ }^{2}$, Y Garnier ${ }^{2}$ \\ ${ }^{1}$ University Children's Hospital, Wuerzburg, Germany \\ ${ }^{2}$ University Hospital, Maastricht, The Netherlands
}

Background and Aims: Chorioamnionitis and fetal systemic inflammation are associated with a reduction in respiratory distress syndrome but an increase in bronchopulmonary dysplasia (BPD). BPD has been associated with an impaired lung development marked by a rearrangement of elastin deposition. We hypothesized that elastin was part of the structural changes induced by inflammation after intravenous E.coli endotoxin injection into fetal sheep. 
Methods: Fetal sheep were chronically instrumented at $107 \mathrm{~d}$ gestational age and delivered at $117 \mathrm{~d}$ (term $147 \mathrm{~d}$ ). The control group $(\mathrm{n}=6)$ received saline injection and the study group $(n=7) 100 \mathrm{ng}$ endotoxin intravenously. Lung tissues were stained for nuclear factor (NF)kappaB for proinflammatory gene transcription, for surfactant protein B (SP-B) for maturation and for elastin for structural remodeling. Staining intensity was analyzed in a four step semi-quantative scale.

Results: NF-kappaB was detected in the nucleus of endothelial cells after endotoxin (median 3; interquartile range $2.7-3.5 ; \mathrm{p}<0.05$ versus $1 ; 0.4-1.6$; in controls). SP-B was detected in alveolar type II cells in the endotoxin group $(3 ; 2.3-3.6 ; \mathrm{p}<0.05$ versus $1 ; 0.6-2.2$ in controls). Elastin was stained around pulmonary blood vessels in both control and endotoxin group. Elastin fibers were detected in alveolar crests after intravenous endotoxin but in not the control group.

Conclusions: Intravenous injection of endotoxin caused inflammation and maturation in the fetal lungs. Elastin fiber deposition in the fetal lung indicated fetal lung injury and remodeling. These early, strictly intrauterinely induced changes may be a considerate predisposing step to the postnatal development of BPD.

\section{INCREASED ARTERIAL BLOOD PRESSURE AND ARTERIAL STIFFNESS IN YOUNG ADULTS WHO WERE BORN PREMATURE}

C Grosse $^{1}$, L Tauzin ${ }^{1}$, P Rossi ${ }^{2}$, M Tsimaratos ${ }^{3}$, U Simeoni $^{1}$

${ }^{I}$ Division of Neonatology, Assistance Publique, Hopitaux de Marseille and Faculte de Medecine, Universite de la Mediterranee, Marseille, France ${ }^{2}$ Division of Internal Medicine, Assistance Publique, Hopitaux de Marseille and Faculte de Medecine, Universite de la Mediterranee, Marseille, France ${ }^{3}$ Pediatric Nephrology Unit, Assistance Publique, Hopitaux de Marseille and Faculte de Medecine, Universite de la Mediterranee, Marseille, France

Background and Aims: Recent studies have shown that low birth weight infants are at risk of increased arterial blood pressure (ABP) in adulthood. Previous works from our centre and others suggest that $\mathrm{ABP}$ and arterial stiffness (AS) are increased in adolescents who were born preterm. Study aims were to characterize AS and $\mathrm{ABP}$ in young adults who were born preterm with an appropriate birth weight for gestational age compared to a control group born at term with an appropriate birth weight.

Methods: Systemic ABP was measured with an automated oscillometric device. AS was estimated by pulse wave velocity (PWV) between carotid and radial arteries. Increased PWV was considered to correspond to increased AS.

Results: 32 adults were studied at a mean (SD) age of 20.8 (1.5) years. Gestational age of adults who were born preterm was 32.6 (2.2) gestation weeks. They had a significantly higher systolic ( $122 \pm 13$ vs. $112 \pm 8 \mathrm{mmHg}$, $\mathrm{p}<0.01)$, mean $(89 \pm 5$ vs. $85 \pm 4 \mathrm{mmHg}, \mathrm{p}<0.05)$ and diastolic blood pressure $(69 \pm 4$ vs. $65 \pm 4 \mathrm{mmHg} p<0.05)$, and a significantly increased pulse pressure $(53 \pm 11$ vs.
$46 \pm 7 \mathrm{mmHg}, \mathrm{p}<0.05)$ and PWV $(7 \pm 0.8$ vs. $6.4 \pm 0.8 \mathrm{~m} / \mathrm{s}$, $\mathrm{p}<0.05)$.

Conclusions: Young adults whose low birth weight is due to preterm birth have increased ABP and AS. It can be speculated that preterm birth is involved in the early programming of arterial disease in adulthood through increased AS.

\section{METHYLATION PROFILES OF IGF2, H19 AND PEG1/MEST GENES: EPIGENETICS AND FETAL ORIGINS OF ADULT DISEASES}

C Buffat $^{1}$, H Jammes ${ }^{2}$, J M Dupont ${ }^{2}$, L Fayol ${ }^{1}$, M Gamerre ${ }^{3}$, J Tost ${ }^{4}$, I G Gut ${ }^{4}$, D Vaiman ${ }^{2}$, U Simeoni ${ }^{1}$

${ }^{1}$ Université de la Méditerranée, Faculté de Médecine and Service de Néonatologie, Hôpital de la Conception, Marseille, France, ${ }^{2}$ INSERM U709, Hôpital Cochin, Paris, France, ${ }^{3}$ Service de Gynécologie-Obstétrique, Hôpital de la Conception, Marseille, France, ${ }^{4}$ Centre National de Génotypage, Evry, France

Background and Aims: Low birth weight is associated with increased risk of cardiovascular disease, hypertension and type 2 diabetes in adulthood. Mechanisms are incompletely known. DNA methylation may be a common link underlying the association between adverse fetal growth and elevated risk of later chronic diseases. We aimed at determining the methylation profiles of IGF2, H19 (epigenetically deregulated domains in Beckwith-Wiedeman syndrome) and PEG1/MEST DMRs (Differentially Methylated Regions) in placental tissues of pregnancies complicated by intrauterine growth restriction (IUGR) and uncomplicated pregnancies.

Methods: Placental villi samples and maternal peripheral blood lymphocytes were obtained from IUGR pregnancies and controls ( $\mathrm{n}=17, \mathrm{n}=30$, respectively), after informed consent. Protocols for the study were approved by the institutional Ethics Committee. Bisulfite conversion and pyrosequencing technology were used to characterize individual specific methylation profiles in the IGF2, H19 and PEG1/MEST DMRs.

Results: In H19-IGF2 domain, specific methylation profiles were similar in maternal bloods cells and placental villi samples. However, average methylation in this cluster appeared decreased in placenta villi samples of IUGR pregnancies versus controls at full term gestation. Similar results were observed on some CpG dinucleotide for PEG1/MEST DMR.

Conclusions: Differential methylation profiles affect IGF2, H19 and PEG1/MEST genes in IUGR pregnancies. Early nutritional status may influence the regulation of imprinted genes. DNA methylation modifications may induce persistent dysregulation of gene expression and be involved in the pathogenic process underlying early programming of later diseases in low birth weight infants. 


\section{ALTERED HYPOXIC RESPONSE IN NEWBORN MICE WITH TARGETED DELETION OF THE TACHYKININ 1 GENE}

J Berner ${ }^{1}$, Y Shvarev ${ }^{1,3}$, H Lagercrantz ${ }^{1}$, A Bilkei-Gorzo $^{2}$, T Hökfelt ${ }^{1}$, R Wickström ${ }^{1}$

${ }^{I}$ Department of Woman and Child Health, Karolinska Institutet, Stockholm, Sweden, ${ }^{2}$ Department of Molecular Neurobiology, University Hospital Bonn, Germany, ${ }^{3}$ Neurogenomic Laboratory, Institute of Cytology and Genetics, Siberian Division of the Russian Academy of Sciences, Novosibirsk, Russia

Background and Aims: In this study, we used mice, with targeted deletion of the tachykinin 1 (TAC1 -/-) gene, lacking neurokinin A (NKA) and substance $\mathrm{P}$ (SP). Especially SP is known to be involved in respiratory rhythm and pattern generating mechanisms. Methods: We employed in vivo and in vitro approaches to study hypoxic response and changes in chemosensitivity in knock-out mice vs. wild type (WT). In vivo, the effects of intermittent hypoxia (IH) and moderate hypercapnia, were studied using whole-body flow plethysmoraphy at postnatal days 2-3 and 8-10. In vitro, anoxic responses, as well as the effects of hypocapnic and hypercapnic conditions were investigated in brainstem-spinal cord preparation at postnatal day 2 .

Results: In vivo, an attenuated hypoxic response to $\mathrm{IH}$ in knock-out animals (postnatal day 8-10) was observed. Moreover, sigh activity was also considerably affected during $\mathrm{IH}$ in both age groups, demonstrating significantly stronger increase in knock-out mice. As with in vivo, there were no differences between TAC1 /- and WT mice in in vitro anoxic frequency responses. However, in TAC1 -/- mice, the burst amplitude reduction during the anoxic response was significantly more resilient and the recovery period, when the burst amplitude was restored after the initial part of anoxic response, was significantly shorter as compared to WT mice. Conclusions: Thus, our data suggest that deficiency in SP and NKA, considerably modify the respiratory rhythm and pattern generating networks in mice and is particularly affecting the respiratory behaviour during hypoxia. This data contributes to the understanding of disorders such as SIDS.

\section{EARLY SYSTEMIC HYPOTENSION (ESH) AND VASOPRESSOR SUPPORT (VPS) IN LOW BIRTH WEIGHT INFANTS (LBWI): IMPACT ON NEURODEVELOPMENT}

\author{
M C Bravo ${ }^{1}$, F Cabañas ${ }^{1}$, S Salas ${ }^{1}$, R Madero ${ }^{2}$, \\ $\mathrm{J}^{\text {Quero }}{ }^{1}$, A Pellicer ${ }^{1}$

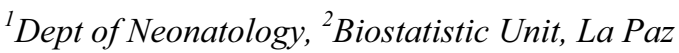 \\ University Hospital, Madrid, Spain
}

Background and Aims: Cerebral circulation is pressure-passive in LBWI with ESH who receive VPS (Pellicer et al, Pediatrics 2005). VPS is controversial as was associated with severe periventricular hemorrhage $(\mathrm{PVH})$. There is no prospective information on VPS and morbidity. Objectives: Prospective study to evaluate the influence of ESH and VPS on neurodevelopment.
Methods: LBWI with ESH ( $<24 \mathrm{~h}$ of life) (group S) were assigned randomly to receive dopamine (2.5-10 $\mu \mathrm{g} / \mathrm{kg} / \mathrm{min})$ or epinephrine $(0.125-0.5 \mu \mathrm{g} / \mathrm{kg} / \mathrm{min})$ in stepwise manner until target blood pressure was attained (treatment success, subgroup TS). Controls (C) were patients without ESH who had parental consent. Outcome measures: cerebral ultrasound (CUS) up to 40 wk, structured neurological evaluation (every 3 months) and neurodevelopmental test at 2-3 years.

Results: 130 patients were included $(\mathrm{S}=60, \mathrm{TS}=38$; $\mathrm{C}=71$ ). 16 infants died; follow-up rate was $88 \%$. Group $\mathrm{S}$ had lower birth weight and GA than group $\mathrm{C}(\mathrm{p}<.001)$. Groups did not differ with respect to initial CUSs; however, final CUSs revealed higher rates of severe $\mathrm{PVH}$ in group $\mathrm{S}(\mathrm{p}<.05)$, and higher rates of normal CUS in group $C(p<.01)$. Only the latter remained when subgroup TS and group $\mathrm{C}$ were compared $(\mathrm{p}<.01)$. Multivariate analysis showed no association between final CUS and VPS. No differences were found on neurological exams or adverse outcome (death or cerebral palsy or severe neurodevelopmental delay) between groups.

Conclusions: Cautious use of VPS to treat ESH in LBWI appears safe. Appropriate study designs should address whether bringing systemic blood pressure to a normal range will improve outcome.

\section{THE IMPACT OF INTRAUTERINE GROWTH, PREMATURITY AND ENVIRONMENT ON COGNITIVE FUNCTION IN VERY PRETERM AND VERY LOW BIRTHWEIGHT INFANTS IN YOUNG ADULTHOOD}

N Weisglas-Kuperus s.4 $^{1,4}$ E T M Hille ${ }^{1,2}$,
H J Duivenvoorden ${ }^{3}$ J B van Goudoever,
S P Verloove-Vanhorick

${ }^{I}$ Dept of Pediatrics, Erasmus MC-Sophia Children's Hospital, Rotterdam, ${ }^{2} T N O$, Quality of Life, Leiden ${ }^{3}$ Dept of Medical Psychology and Psychotherapy, NIHES, Rotterdam, The Netherlands, ${ }^{4}$ On Behalf of The Dutch POPS-19 Collaborative Study Group

Background and Aims: Infants born very preterm or with low very low birthweight are at increased risk of cognitive deficits. Follow-up data into young adulthood however are scarce. We examined the impact of prematurity, intrauterine growth, and environment on cognitive function in young adults born very preterm or with very low birthweight. Methods: A cohort of 562 $(59 \%)$ infants born in the Netherlands in 1983 (POPS study) before 32 weeks of gestation and/or with birthweight less than 1,500 gram had cognitive assessment at age 19 years. Results: The cohort's mean IQ was 100.2 (SD15.0) and did not differ from that in the Dutch norm sample. Adjusted for the other factors, children with a handicap at age 5 (8.8\% of participants) scored 17.0 mean IQ points lower at age 19 years. Participants with high-educated parents scored 11.4 mean IQ points higher than those with low-educated parents. One SD more in birthweight resulted in 2.5 and one week in gestational age in 1.2 mean IQ points more. Males scored 4.0 mean IQ points higher than females and participants born of younger mothers scored 3.0 
mean IQ points lower than those born of mothers aged 25-30 years. Conclusions: Intrauterine growth, prematurity, environment and neonatal complications are of predictive value for later cognitive function. Infants at high biological risk can compensate for their cognitive impairment in a stimulating environment and the good cognitive outcome at young adulthood in these survivors from the early neonatal intensive care era seems mainly due to socioeconomic advantages of the Dutch society, representative for most Western societies.

\section{INTERLEUKIN-1BETA, INTERLEUKIN-18 AND INTERFERON-GAMMA EXPRESSION IN THE CEREBROSPINAL FLUID OF PRETERM INFANTS WITH POSTHEMORRHAGIC HYDROCEPHALUS - MARKERS OF WHITE MATTER DAMAGE?}

\author{
A Heep ${ }^{1}, \mathrm{~T} \mathrm{Schmitz}^{2}$, F Groenendaal ${ }^{3}, \mathrm{P} \mathrm{Bartmann}^{1}$, \\ M Obladen $^{2}$, U Felderhoff-Mueser ${ }^{2}$ \\ ${ }^{I}$ Dept of Neonatology, Friedrich Wilhelms University of \\ Bonn, ${ }^{2}$ Dept of Neonatology, Campus Virchow \\ Klinikum, Charité - Universitätsmedizin Berlin, \\ Germany ${ }^{3}$ Dept of Neonatology, Wilhelmina Children's \\ Hospital, University Medical Center, Utrecht, The \\ Netherlands
}

Background and Aims: To investigate the impact of perinatal brain injury associated with cystic white matter damage (WMD) on the developing preterm brain, we analyzed biomarkers specific for inflammatory responses in the cerebrospinal fluid (CSF) of preterm infants who develop post hemorrhagic hydrocephalus (PHHC).

Methods: 22 preterm infants with PHHC undergoing serial reservoir puncture were included in the study. Patients were grouped according to the diagnosis of signs of cystic WMD on ultrasound at day 28 postnatal age (pna). Group A: 9 infants with PHHC without signs of cystic WMD (median gestational age $27+5$ weeks). Group B: 13 infants with PHHC and cystic WMD (median gestational age $27+3$ weeks). Samples were analyzed at first ventriculostomy (median day 26 pna). 16 age-matched preterm infants with lumbar puncture for the exclusion of meningitis served as controls. CSF concentrations of IL-1beta, IL-18 and IFN-gamma were analysed using ELISA.

Results: Table 1.

Conclusions: IL-1beta and IL-18 seem to be involved in a chronic inflammatory process in patients with PHHC. IFN-gamma levels in the CSF may specifically distinguish the inflammatory process taking place in WMD from that in PHHC and serve as a clinical marker of cystic WMD.

Table 1: levels of proinflammatory cytokines in the different groups
(median; range)
\begin{tabular}{|c|c|c|c|}
\hline & Group A & Group B & Controls \\
\hline IL-1 beta $(\mathrm{pg} / \mathrm{ml})$ & ${ }^{\circ} 13.9(1.1-39.6)$ & ${ }^{*} 4.4(1-245.4)$ & $1(1-3.5)$ \\
\hline IL-18 $(\mathrm{pg} / \mathrm{ml})$ & ${ }^{9} 97.8(12.5-247.6)$ & $41(12.5-153.3)$ & $12.5(12.5-106.7)$ \\
\hline IFN-gamma (pg/ml) & $8(8-23.4)$ & ${ }^{\circ} 21.4(8-899)$ & $10.3(8-81.6)$ \\
\hline
\end{tabular}
${ }^{*} \mathrm{p}<0.001 ;{ }^{\circ}<<0.02 ;{ }^{8} p<0.005$ compared to controls (Mann-Whitney U test)

NEWLY EMERGING VIRAL INFECTIONS

\section{A D M E Osterhaus}

Department of Virology, Erasmus MC, Rotterdam, The Netherlands

In the past century, pandemic outbreaks of influenza and AIDS have cost the lives of tens of millions of people. These events were all caused by multiple introductions of animal viruses - influenza A viruses and SIV of birds and non-human primates respectively - into the human population. Besides these introductions causing major pandemics in humans, a large number of other virus infections have spilled over from animal reservoirs to humans or other susceptible species, resulting in considerable morbidity and mortality as 'virgin soil' epidemics. The most recent examples in humans are the introduction of SARS coronavirus and influenza A viruses (H5N1 and H7N7) from the animal world, which caused global concern about their potential to be at the origin of new pandemics. Over the last decades there seems to be a dramatic increase in the emergence or reemergence of virus threats in humans and animals worldwide. A long list of exotic names like Ebola, Lassa, Rift-Valley, Crimea-Congo, Hendra, Nipah and West-Nile is the illustration of names of just some of the places associated with the origin of viruses that crossed the species boundary to humans, with dramatic consequences in the last ten years alone. Similarly, recent mass mortalities among wild aquatic and terrestrial mammals caused by previously known and newly discovered morbiliviruses, as well as outbreaks of hog cholera, foot-and-mouth disease and fowl plague among domestic animals, highlight this trend. Although improved detection and surveillance techniques, as well as increased media attention may have contributed to our perception of an increase in the incidence of outbreaks of virus infections, it is becoming more and more clear that major changes in our modern society increasingly create new opportunities for virus infections to emerge: a complex mix of changes in social environments, medical and agricultural technologies and ecosystems continues to create new niches for viruses to cross species boundaries and to rapidly adapt to new species. In combating this global threat, we should make optimal use of the new tools provided by the unprecedented advances made in the research areas of virology, molecular biology, immunology, epidemiology, genomics and bioinformatics. Serious investment in these areas in the future will not only be highly cost-effective but will also save many lives of humans and animals. In addition, better collaboration and coordination between all the stakeholders is urgently needed, to establish early warning systems and effective preparedness plans.

\section{UROGENITAL SYMPTOMS DURING PREGNANCY AND BIRTH WEIGHT: THE GENERATION R STUDY}

\author{
L Duijts $^{1,2}$, V W V Jaddoe ${ }^{1,2,3}$, A Hofman ${ }^{1,3}$, \\ H A Moll ${ }^{1,2}$ \\ ${ }^{I}$ The Generation R Study Group, Erasmus MC - \\ Sophia's Children Hospital, Rotterdam, The Netherlands
}


${ }^{2}$ Department of Paediatrics, Erasmus MC - Sophia's Children Hospital, Rotterdam, The Netherlands ${ }^{3}$ Department of Epidemiology and Biostatistics, Erasmus MC - Sophia's Children Hospital, Rotterdam, The Netherlands

Background and Aims: Physician-diagnosed urogenital tract infections in pregnancy are associated with low birth weight and premature birth. The aims of this study were to assess the association of subjective urogenital complaints in different trimesters of pregnancy with birth weight and gestational age.

Methods: This study was embedded in the Generation R Study, a population-based prospective cohort study from fetal life until adulthood in Rotterdam, The Netherlands (2002-2006). Urogenital complaints were assessed by questionnaires in each trimester in pregnant women. Complaints were categorized in mild urethral complaints, mild vaginal complaints and more severe complaints (bleeding after sexual intercourse, nonpainful ulceration, enlarged lymph nodes in the groin). Birth outcomes were obtained from midwife and hospital registries. The present analysis was based on 6,680 prenatally included women with complete records.

Results: In the first trimester mild urethral and more severe complaints were associated with a decrease in birth weight in the offspring of $-33(95 \%$ confidence interval (CI): $-70,-3)$ and -66 (95\% CI:-107,-24) grams respectively, after adjustment for gestational age, ethnicity and education of the mother. We did not find any associations for mild vaginal complaints. Having four or more complaints was associated with a decrease in birth weight of -53 (95\% CI:-96,-11) grams. For urethral complaints, similar associations were found in the second and third trimester. There was no effect on gestational age.

Conclusions: These findings suggest that having urethral or more severe complaints during pregnancy seems to have an adverse effect on birth weight in the offspring.

\section{INCIDENCE AND NON-NUTRITIONAL RISK FACTORS FOR NECROTIZING ENTEROCOLITIS (NEC) IN VERY LOW BIRTHWEIGHT INFANTS (VLBWI)}

L Gagliardi ${ }^{1}$, M De Curtis ${ }^{2}$, R Bellù ${ }^{3}$

${ }^{I}$ Division of Pediatrics and Neonatology, Ospedale Versilia, and Italian Neonatal Network, Lido di Camaiore, Italy, ${ }^{2}$ University La Sapienza, Rome, Italy ${ }^{3}$ Division of Neonatology, Ospedale A Manzoni, Lecco, and Italian Neonatal Network, Lecco, Italy

Background and Aims: To estimate incidence and nonnutritional risk factors for NEC.

Methods: Analysis of a cohort of 2135 VLBWI infants, born in Lombardy (Italy) in 1999-2002 and admitted to 14 Intensive Care Units, participating to the Network Neonatale Lombardo. Mean GA was 29.3 weeks; mean birthweight was 1101 g. Definitions of the VermontOxford network, including NEC definition, were used. Logistic models (adjusting for GA, congenital anomalies, birthweight $<10$ centile, twinning, 1-minute
Apgar, sex, type of delivery, location of birth) were used to estimate and test the association of factors with NEC. Results: NEC incidence was $3.2 \%$ overall $(2.0 \%$ 'medical' NEC, $1.2 \%$ 'surgical'); frequency was higher at low GA: $10.1 \%$ at $23-26$ weeks vs $2.6 \%$ at $27-29$. A wide variation was observed among different units (from 0 to 7\%). Risk factors for NEC were late-onset sepsis (Odds Ratio: 6.05; 95\% CI: 3.18-11.50) and patent ductus arteriosus (OR: 2.05; CI: 1.10-3.82), while mechanical ventilation failed to reach statistical significance (OR: 2.47; CI: 0.95-6.41, $\mathrm{P}=0.062$ ). Use of surfactant (OR: 0.42; CI: 0.19-0.91) and umbilical venous catheter (OR: 0.37; CI: $0.18-0.75$ ) were negatively associated to NEC. Neonates with NEC experienced a 3 -fold increase in mortality $(33.3 \%$ vs 10.3 , without difference between medical and surgical NEC), and had a longer length-of-stay in the hospital (average increase 13 days, adjusted for confounders and procedures).

Conclusions: Many non-nutritional factors influence NEC incidence. NEC frequency varies among neonatal units, perhaps reflecting different nutritional and nonnutritional policies. Our data confirm that NEC has an impact on mortality and length-of-stay in VLBWI.

\section{MITOCHONDRIAL HAPLOTYPE H: BODY TEMPERATURE AND SEPSIS IN VERY LOW BIRTH WEIGHT INFANTS}

W Göpel ${ }^{1}$, C Härtel $^{1}$, J Spiegler ${ }^{1}$, E Kattner ${ }^{2}$, H Küster ${ }^{3}$, J Möller ${ }^{4}$, D Müller ${ }^{5}$, W Nikischin ${ }^{6}$, H Segerer ${ }^{7}$, C Wieg ${ }^{8}$, A Wense ${ }^{9}$, B Roth $^{10}$, E Herting ${ }^{1}$

${ }^{1}$ Department of Pediatrics, University of Lübeck, Germany, ${ }^{2}$ Kinderkrankenhaus auf der Bult, Hannover, Germany, ${ }^{3}$ Department of Pediatrics, Greifswald University, Germany, ${ }^{4}$ Department of Pediatrics, Saarbrücken Hospital, Germany, ${ }^{5}$ Department of Pediatrics, Hospital of Kassel, Germany, ${ }^{6}$ Department of Pediatrics, Christian Albrecht University, Kiel, Germany, ${ }^{7}$ Kinderklinik St. Hedwig, Regensburg, Germany, ${ }^{8}$ Department of Pediatrics, Aschaffenburg Hospital, Germany, ${ }^{9}$ Altonaer Kinderkrankenhaus, Hamburg, Germany, ${ }^{10}$ Department of Pediatrics, University of Köln, Germany

Background and Aims: Mitochondrial haplotype $\mathrm{H}$ is common in European populations and associated with improved survival and higher core temperatures in adults with gram-negative sepsis (Baudouin et al. Mitochondrial DNA and survival after sepsis: a prospective study. Lancet 2005; 366:2118-2199).

Methods: Genotyping of mitochondrial haplotype $\mathrm{H}$ in a large, prospectively enrolled cohort of VLBW-infants. Infants with different mitochondrial haplotype were compared with regard to body temperature at admission, sepsis prevalence and outcome.

Results: Genotyping was successful in 809 VLBWinfants. Infants carrying the haplotype $\mathrm{H}(\mathrm{n}=351,43 \%)$ were not different to non-carriers with regard to gestational age ( 29.1 vs. 29.1 weeks), birth weight (1110 vs. 1094 grams) or body temperature at admission (36.5 vs. 36.5 degrees centigrade). Non-carriers of mitochondrial haplotype $\mathrm{H}$ had a higher risk of bloodculture-proven sepsis $(24.2 \%$ vs. $17.7 \%, \mathrm{p}=0.025$, 
Fisher's exact test). The rate of sepsis with coagulase negative staphylococci was not different between noncarriers and carriers of mitochondrial haplotype $\mathrm{H}$ $(11.1 \%$ vs. $9.4 \%)$. Sepsis due to other bacteria was significantly more common in non-carriers of mitochondrial haplotype $\mathrm{H}(14.6 \% \mathrm{vs} .8 \%$ in carriers of haplotype $\mathrm{H}, \mathrm{p}=0.004$, Fisher's exact-test). This was also true, if only the prevalence of gram-negative sepsis was analysed $(6.6 \%$ vs. $3.1 \%$ in carriers of mitochondrial haplotype $\mathrm{H}, \mathrm{p}=0.035$, Fisher's exact test). Mortality was not different between non-carriers and carriers of haplotype $\mathrm{H}$ who developed sepsis (13.4\% vs. $7.1 \%)$.

Conclusions: VLBW-infants carrying the mitochondrial haplotype $\mathrm{H}$ have a lower risk of developing bloodculture-positive sepsis during their stay in the hospital.

\section{NEONATAL AND INFANT GROUP B STREPTOCOCCAL INVASIVE DISEASE - THE PORTUGUESE PSU RESULTS}

M T Neto, A Nunes, M Mateus

Portuguese Paediatric Surveillance Unit, Lisbon, Portugal

Background and Aims: Group B Streptococcus (GBS) is the most common cause of invasive disease in the neonate. Our aim was 1) To assess the epidemiology of GBS infection $<90$ days of life; 2) To determine if prophylaxis of early-onset GBS neonatal infection can be based only on risk factors.

Methods: National epidemiologic surveillance. Duration - 4 years - April 1st, 2001 to 31st March, 2005. Case definition: any infant $<90$ days with GBS positive culture in any supposed sterile fluid or place - blood, CSF or joint aspirate.

Results: 241 cases of GBS invasive disease were reported (estimated incidence - 0.5/1000LB). Infection occurred $<7$ days in 199 newborns $(82 \%)$ and $>7$ days in 42 - 28 until 28 days and 14 after. The incidence of early-onset and late-onset infection was respectively $0.44 / 1000 \mathrm{LB}$ and $0.1 / 1000 \mathrm{LB} ; 196$ were term babies (81\%) 166 of whom became hill $<7$ days $(69 \%$ of all). There were 227 positive blood cultures, 46 meningitis and 48 pneumonia. Mothers of 45/166 (27\%) term babies with early-onset infection had intrapartum fever or membranes rupture $>18$ hours. Lethality - 6.6\% $(16 / 241)-6.5 \%<7 \mathrm{~d}$ and $7.1 \%>7 \mathrm{~d} ; 15.5 \%$ in preterm, $4.6 \%$ in term babies, $18 \%$ in VLBW.

Conclusions: GBS infection is predominantly an early infection of the term baby and is rare after the first month of life; its lethality is higher in preterm and VLBW infants. GBS prophylaxis based on risk factors would leave undetected $73 \%$ of term babies with earlyonset disease suggesting that, in Portugal, universal GBS screening should be advised.
BENZYL ALCOHOL, PRESERVATIVE IN

BACTERIOSTATIC 0.9\% NACL, WATER AND

HEPARIN SODIUM SOLUTION, ENHANCES

BIOFILM PRODUCTION IN CLINICAL AND

COMMENSAL STAPHYLOCOCCUS

EPIDERMIDIS STRAINS

V Milisavljevic ${ }^{1}$, L P Tran ${ }^{2}$, J F Miller ${ }^{2}$

${ }^{l}$ Department of Pediatrics, David Geffen School of Medicine at UCLA, Los Angeles, CA, USA, ${ }^{2}$ Department of Microbiology, Immunology and Human Genetics, UCLA, Los Angeles, CA, USA

Background and Aims: S. epidermidis is the most frequent cause of nosocomial infections, particularly in patients with indwelling catheters. Biofilm production has a central role in pathogenesis. PIA, encoded by the icaADBC locus, is the main component of biofilms. We previously demonstrated that ethanol enhances $\mathrm{S}$. epidermidis biofilm production. Bacteriostatic 0.9\% $\mathrm{NaCl}$, water and heparin sodium solution used in hospitals, contain $0.9 \% \quad-1 \%$ benzyl alcohol as preservative. We hypothesized that benzyl alcoholcontaining solutions can enhance biofilm formation in S. epidermidis.

Methods: We studied 10 S. epidermidis strains obtained from blood cultures of infants and hands of nurses in neonatal intensive care unit. All strains had the same PFGE pattern and contained icaADBC locus. Biofilm production was quantified using microtiter plate assay in plain media and media supplemented with bacteriostatic $0.9 \% \mathrm{NaCl}$, water or heparin sodium solution containing benzyl alcohol, as well as with the same solutions without benzyl alcohol (controls).

Results: Despite having an indistinguishable PFGE pattern, tested strains differed in ability to form biofilms. Six strains that were low biofilm producers in plain media displayed a significant enhancement $(p<0.01)$ in biofilm production when media was supplemented with less the $0.7 \%$ benzyl alcoholcontaining solutions. One strain was consistently strong biofilm producer and three strains did not produce biofilms in tested conditions.

Conclusions: 1. Benzyl alcohol, preservative in solutions widely used in the hospitals, enhances production of biofilm by certain S. epidermidis strains. 2. It is a major cause for concern that pathogenic potential of S. epidermidis is influenced by solutions administered through indwelling catheters.

\section{NOSOCOMIAL INFECTIONS IN SMALL FOR GESTATIONAL AGE NEWBORNS WITH BIRTHWEIGHT <1500G: A MULTICENTER ANALYSIS}

D B Bartels ${ }^{1}$, F Schwab ${ }^{2}$, O Dammann ${ }^{1,3}$, C Geffers ${ }^{2}$, S Baerwolff ${ }^{2}$, P Gastmeier, ${ }^{2,4}$

${ }^{I}$ Departments of Obstetrics and Pediatrics, Hannover Medical School, Germany, ${ }^{2}$ Institute of Hygiene, Charité-University Medicine in Berlin, Germany, ${ }^{3}$ Neuroepidemiology Unit, Departments of Neurology, Children's Hospital and Harvard Medical School, Boston, MA, USA, ${ }^{4}$ Institute of Medical Microbiology 
and Hospital Epidemiology, Hannover Medical School, Germany

Background and Aims: Small for gestational age (SGA) infants with very low birthweight (VLBW, $<1500 \mathrm{~g}$ ) are at increased mortality risk in comparison to their appropriate weight for gestational age (AGA) peers, while effects on their morbidity are less clear. The aim of this multicenter analysis was to investigate whether SGA-neonates are at increased risk of nosocomial infections (NI).

Methods: The German national surveillance system for NIs in NICUs includes data from VLBW infants admitted to one of 47 large NICUs. Data from $n=6681$ infants, born 2000-2004, were available. Growth retardation was defined as birthweight $<10$ th percentile, AGA as birthweight between the 10th and 90th percentile. Outcome variables were nosocomial sepsis, pneumonia, and necrotizing enterocolitis (NEC). Univariable data analyses, logistic.

Results: After applying pre-specified inclusion criteria $\mathrm{n}=2918$ SGA- and AGA-neonates (24-regression, and general estimating equation models were performed, the latter to appreciate data clustering within NICUs. 28 weeks) were selected, 13\% ( $\mathrm{n}=392)$ SGA- and $87 \%$ $(\mathrm{n}=2526)$ AGA-infants. Thirty three percent had at least one episode of sepsis, $42 \%$ of the SGA- and $31 \%$ of the AGA infants (adjusted Odds Ratio 1.41, 95\% Confidence Interval 1.05-1.89). Pneumonia was diagnosed in $6 \%, 8.4 \%$ in SGA- and $5.5 \%$ in AGA newborns (adj. OR 1.57, 95\% CI 1.05-2.36). A similar proportion experienced NEC (5\%), 7\% SGA- and 5\% of AGA-infants (adj. OR 1.20, 95\% CI $0.75-1.94$ ). Conclusions: Growth retarded preterm infants appear to be at increased risk of nosocomial infections. These results should prompt more research on possible immunity deficiencies and their sequelae in preterm SGA newborns.

\section{HYPERGLYCAEMIC EPISODES (HE) AS AN EARLY MARKER OF SEPSIS IN PREMATURE INFANTS LESS THAN 30 WEEKS GESTATION AND LESS THAN 1000G BIRTHWEIGHT (ELBW)}

\section{J R Fernandez Alvarez, T Dygas, P Amess, H Rabe}

Trevor Mann Baby Unit, Royal Sussex County Hospital, Brighton, East Sussex, UK

Background and Aims: Early diagnosis of sepsis in preterms is difficult. Platelet count and CRP are specific, but late markers of sepsis. IL-6/IL-8 are sensitive, but expensive early markers. ELBW newborns have 18 times more HE than those $>2000 \mathrm{~g}$. The aim was to investigate the use of $\mathrm{HE}$ in the early diagnosis of sepsis in ELBW newborns.

Methods: Retrospective analysis of data from ELBW newborns with $\geq 1$ HE $(>144 \mathrm{mg} / \mathrm{dl})$ was performed. Onset, duration and frequency of HE, glucose intake, medication and glucose values before, during and after HE were recorded. Frequency of sepsis (pos. blood culture, and/or CRP $>10 \mathrm{mg} / 1$ and/or thrombocytopaenia $<150000$ /microliter) and temporal relation to HE were recorded. Results are given as median.
Results: 8 preterms (26 weeks and $733 \mathrm{~g}$ ) with a total of $12 \mathrm{HE}$ (1.5/patient) were identified. Blood glucose was $180 \mathrm{mg} / \mathrm{dl}$ during HE and $90 \mathrm{mg} / \mathrm{dl} 24 \mathrm{~h}$ before/after HE $(p=0.005$ compared to pre-values and $p=0.002$ compared to post-values). HE occurred on day 4-5 and lasted for 4 days. Glucose-intake was $9 \mathrm{mg} / \mathrm{kg} / \mathrm{min}$. Sepsis was detected 18 times during 53 days of stay (2.3/patient and $1 / 23$ days). Sepsis-workup was performed 3 days after begin of HE $(p=0.025)$. Sepsis was diagnosed 3 days later in $11 / 12 \mathrm{HE}(\mathrm{p}=0.004)$. 11/18 septic episodes presented with HE $(p=0.35)$. Sensitivity and positive predictive value for $\mathrm{HE}$ as early marker of sepsis were $61 \%$ and $92 \%$.

Conclusions: ELBW newborns have HE that may be associated with sepsis. HE seem to indicate sepsis earlier and more specifically than other markers such as CRP and thrombocytopaenia.

\section{NEW DOSAGE SCHEDULE FOR CONTINUOUS- INFUSION VANCOMYCIN THERAPY IN PRETERM NEONATES WITH SUSPECTED GRAM-POSITIVE INFECTIONS}

O Plan ${ }^{1}$, G Cambonie ${ }^{1}, \mathrm{C}_{\text {Milesi }}{ }^{1}$, O Defez ${ }^{2}$, M Pidoux ${ }^{1}$, C Badr ${ }^{1}$, E Sabatier ${ }^{1}$, B Veit-Sauca ${ }^{1}$, J C Picaud ${ }^{1}$

${ }^{I}$ Department of Neonatology, University Hospital, Montpellier, France, ${ }^{2}$ Department of Statistics, University Hospital, Montpellier, France

Background and Aims: A constant infusion rate over $24 \mathrm{~h}$ results in a better bactericidal efficacy of vancomycin since it's action is time-dependent. Proposed dosage schedules (Palowtsky 1998, Borderon 1994) are complex leadind to low serum vancomycin concentrations and unconstant bacteriological efficiency (personal data). We evaluated a new simplified dosage schedule based on body weight and serum creatinine.

Methods: Prospective data collection during routine use of vancomycin in premature neonates. In case of suspected septicemia gram-positive infections blood was collected for culture and creatinine measurement before antibiotic administration. Starting vancomycin dosage was 25 or $15 \mathrm{mg} / \mathrm{kg} / \mathrm{d}$ during the first period and 30 or $20 \mathrm{mg} / \mathrm{kg} / \mathrm{d}$ during the second period whether serum creatinine was below or above $90 \mu \mathrm{mol} / \mathrm{L}$, regardless of gestational or postconceptional age. Then blood sampling was performed $48 \mathrm{~h}$ after the initiation of treatment (blood culture, serum vancomycin). Required therapeutic range was $10-25 \mathrm{mg} / \mathrm{L}$.

Results: Between June 2002 and April 2005, 118 neonates were evaluated (Period 1:76, Period 2:42). At $\mathrm{T} 1,77 \%(91 / 118)$ had serum vancomycin concentrations within the required range, with an improvement between period 1 and period2. Half $(58 / 118,49 \%)$ had confirmed infection. The cure rates of sepsis related to staphylococcus were respectively $75 \%$ and $96 \% 48 \mathrm{~h}$ and 96h after the beginning of antibiotic treatment.

Conclusions: Using that improved new dosage schedule most premature neonates had serum vancomycin concentrations within the required therapeutic range and the bactericial efficacy was satisfactory. 
AMYLIN PEPTIDE LEVELS ARE RAISED IN NEWBORNS WITH FEED INTOLERANCE

V Kairamkonda ${ }^{1}$, A Deorukhkar ${ }^{2}$, R Coombs $^{2}$, R Fraser ${ }^{3}$, T Mayer ${ }^{4}$

${ }^{I}$ Department of Neonatal Intensive Care Unit, University Hospitals of Leicester NHS Trust, Leicester Royal Infirmary, Leicester, Leicestershire, UK ${ }^{2}$ Department of Neonatal Intensive Care Unit, Royal Hallamshire Hospital, Sheffield, Trent, UK

${ }^{3}$ Department of Obstetrics and Gynaecology, Royal Hallamshire Hospital, Sheffield, Trent, UK

${ }^{4}$ Department of Paediatric Intensive Care Unit, Sheffield Children's Hospital, Sheffield, Trent, UK

Background and Aims: Amylin is a novel 37 amino acid peptide that is co-secreted with insulin from the pancreas in response to food intake. As a potent inhibitor of gastric motility it plays an important role in the control of carbohydrate absorption by regulating the efflux from the stomach to the small bowel. Feed intolerance results in prolonged parenteral fluid therapy, increased hospital stay, increased risk of sepsis and consequently increased cost of treatment. We hypothesized that amylin levels may be raised in newborns with feed intolerance. We aimed to determine the relationship between amylin levels and gastric emptying in newborns with feed intolerance.

Methods: Blood from a serial sample of 16 infants greater than 24 weeks gestation was analysed for amylin levels. Feed intolerance was defined as gastric residual volume of greater than $50 \%$ of previous 4 hour feed volume on two consecutive occasions. Eight infants were classified as feed-intolerant (nTOL) and 8 as feedtolerant (TOL).

Results: (Table: mean (SD) and [ranges]). The mean serum amylin concentration was significantly higher in feed intolerant group.

Conclusions: Amylin levels are elevated in newborns with feed intolerance and may play a role in delaying establishment of enteral nutrition in this population.

\begin{tabular}{c|cc|c} 
& nTOL $(\mathrm{n}=8)$ & TOL $(\mathrm{n}=8)$ & p value \\
\hline Amylin (pmol/L) & $\begin{array}{c}\mathbf{2 9 . 4}(23.6) \\
{[10.5-79.8]}\end{array}$ & $\begin{array}{c}\mathbf{2 . 9}(1.1) \\
{[1.4-4.6]}\end{array}$ & $<0.001$ \\
\hline \multirow{2}{*}{$\begin{array}{c}\text { Gestation } \\
\text { (weeks) }\end{array}$} & $\begin{array}{c}31.5(4.3) \\
{[27-38]}\end{array}$ & $\begin{array}{c}32(4.2) \\
{[27-38]}\end{array}$ & 0.8 \\
Weight (grams) & $\begin{array}{c}1825(802) \\
{[1015-3020]}\end{array}$ & $\begin{array}{c}1913(720) \\
{[1435-3200]}\end{array}$ & 0.8 \\
M:F & $1: 1$ & $1: 1$ & 1.0
\end{tabular}

\section{EFFECTS OF IUGR AND POSTNATAL CATCH- UP GROWTH ON ARTERIAL BLOOD PRESSURE, GLUCOSE TOLERANCE AND RENAL FUNCTION IN AGED RATS}

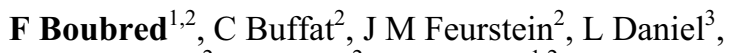
M Tsimaratos ${ }^{2}$, C Oliver $^{2}$, U Simeoni $^{1,2}$

${ }^{I}$ Department of Neonatology, Hospital University La Concepcion, Marseille, France, ${ }^{2}$ Faculty of Medecine, UPRES EA 2193, Marseille, France, ${ }^{3}$ Department of

Histopathology, Faculty of Medicine, Marseille, France
${ }^{4}$ Laboratory of Biology, Les Cordeliers INSERM U356, Paris, France

Background and Aims: Low birth weight is associated with increased risk of arterial hypertension and metabolic diseases in adulthood. Rapid postnatal growth may constitute an additional risk. AIM: to investigate the effects of postnatal overfeeding (OF) after IUGR in aged rats.

Methods: 4 groups of animals were investigated: group I, controls; group II, offspring of dams fed low protein diet (LP, caseine 9\%);group III, postnatal OF (reduction of litter size); group IV, LP rats exposed to postnatal OF. Glomerular number, SBP, intra-peritoneal glucose test, and renal function (GFR) were evaluated at different ages up to 22 months.

Results: (meam+/-SEM). LP rats had $20 \%$ birth weigt and $38 \%$ glomerular number reduction in comparison with control $(\mathrm{p}<0.01)$. $\mathrm{LP}+\mathrm{OF}$ rats caught up the weight of control within the first 15 postnatal days. In comparison with control, SBP was higher in $\mathrm{LP}+\mathrm{OF}$ at 4 wks $(+17 \mathrm{mmHg}, \mathrm{p}<0.05)$ wich remained elevated at 12 months. In comparison with controls, LP+OF had glucose intolerance at 12 months, 2 fold elevated proteinuria at 4 months, progressive decline of GFR with age occuring principally in males, a reduction of kidney weight $(1.84+/-0.1 \mathrm{~g}$ vs $2.06+/-0.1, \mathrm{p}<0.05)$, and increased glomerular volume $(+50 \%)$. Histologic examination showed marked glomerular injury with tubular hypertrophy.

Conclusions: Early catch-up growth in IUGR enhances alterations of SBP, GT and renal function in adult rats. Postnatal overfeeding may amplify single nephron hyperfiltration resulting over a long time to glomerular sclerosis and reduction of renal functions. The gender seems inflence these effects.

\section{CONTINUOUS FEEDING PROMOTES GASTROINTESTINAL TOLERANCE AND GROWTH IN VERY LOW BIRTH WEIGHT INFANTS}

\author{
A L Dsilna ${ }^{1}, \mathrm{~K}$ Christensson ${ }^{1}$, L Alfredsson ${ }^{2}$, \\ H Lagercrantz $^{3}$, M Blennow ${ }^{4}$
}

${ }^{I}$ Division of Reproductive and Perinatal Health Care, Department of Woman and Child Health, Karolinska Institutet, Karolinska University Hospital, Stockholm, Sweden, ${ }^{2}$ Institute of Environmental Medicine, Karolinska Institutet, Karolinska University Hospital, Stockholm, Sweden, ${ }^{3}$ Division of Neonatal Research Unit, Department of Woman and Child Health, Karolinska Institutet, Karolinska University Hospital, Stockholm, Sweden, ${ }^{4}$ Department of Clinical Sciences, Karolinska Institutet, Karolinska University Hospital, Stockholm, Sweden

Background and Aims: Gastrointestinal intolerance is a well known problem among very-low-birth-weight (VLBW) infants. Parenteral nutrition during long periods is therefore needed, which increase the risk for both delayed growth and morbity. Previous studies has shown that feeding given as a slow rate infusion enhances duodenal motor function and decrease energy expenditure when compared to bolus feeding. The aim 
of the study was to compare the effects of continuous versus intermittent feeding on gastrointestinal tolerance and growth in VLBW-infants.

Methods: In a randomized, controlled trial conducted at three neonatal units, seventy premature infants, with a gestational age 24-29 weeks and birth weights $<1200$ grams, were assigned to one of three feeding methods: 1. Continuous nasogastric feeding, 2. Intermittent nasogastric feeding or 3. Intermittent orogastric feeding. Feeding was initiated within 30 hours of birth. Daily enteral and parenteral volumes, caloric and protein intakes, growth, enteral intolerance and clinical complications were collected. Cox regression analysis was used to determine primary outcome, the time to achieve full enteral feeding.

Results: The continuous fed infants achieved full enteral feeding significantly faster than intermittently fed infants, hazard ratio (HR) 1.86 (95\% confidence interval (CI) 1.07-3.22). In stratified analysis according to birth weight, the improvement was even more pronounced in the smallest infants, $<850$ grams, adjusted $\mathrm{HR}=4.13$ (95\% CI: 1.48-11.53). Growth rate was significantly faster among the continuous fed infants ( $\mathrm{p}$ 0.002).

Conclusions: In VLBW-infants continuous feeding seems to be a more beneficial alternative compared to intermittent feeding with regard to gastrointestinal tolerance and growth.

\section{EFFECTS OF AMINO ACID ADMINISTRATION ON ALBUMIN FRACTIONAL SYNTHESIS RATES IN PREMATURE NEONATES ON POSTNATAL DAY TWO}

C H P Van Den Akker ${ }^{1}$, F W J Te Braake ${ }^{1}$, H Schierbeek $^{2}$, T Rietveld ${ }^{3}$, J B Van Goudoever ${ }^{1}$

${ }^{1}$ Department of Paediatrics, Division of Neonatology, , ${ }^{2}$ Department of Paediatrics, Division of Neonatology, Mass Spectrometry Laboratory, ${ }^{3}$ Department of Internal Medicine, Mass Spectrometry Laboratory, Erasmus MC, Rotterdam, The Netherlands

Background and Aims: Recently, we demonstrated that administration of parenteral amino acids (AA) immediately after birth to premature infants is safe and results in positive nitrogen balances via increased protein synthesis. Apart from whole body effects, we wanted to determine organ specific effects of early AA administration. Albumin is an organ specific parameter produced in the liver and often found to be in low concentrations in premature infants. We hypothesised that the fractional synthesis rate (FSR) of albumin increases in premature infants if AA are administered immediately following birth. The FSR represents the fraction that is renewed per unit of time.

Methods: Premature infants $(<1500 \mathrm{~g})$ received the first two postnatal days either solely glucose (control group, $\mathrm{n}=5$ ) or glucose and AA (intervention group, $n=6$ ). On the second day of life, both groups received a primed continuous infusion of [1-13C] leucine and blood was sampled after 5 and 6 hours. Albumin was extracted from the plasma, hydrolyzed, derivatized, and leucine isotopomers were subsequently measured with a gas chromatography - combustion - isotope ratio mass spectrometer (GC-C-IRMS). Plasma alphaketoisocaproate was measured by GC-MS and used as an intracellular leucine precursor.

Results: Birth weight and gestational age were not different between infants in the control $(986 \pm 284 \mathrm{~g}$; $27.9 \pm 2.2 \mathrm{wks})$ and intervention groups $(930 \pm 143 \mathrm{~g}$; $27.8 \pm 1.8 \mathrm{wks})$. The control group had a lower FSR $(14.8 \pm 6.0 \% / \mathrm{d})$ than the infants who also received AA $(23.9 \pm 6.2 \% / d)(p=0.036)$.

Conclusions: AA administration immediately after birth to premature neonates increases the FSR of albumin.

\section{IS CYST(E)INE AN ESSENTIAL AMINO ACID IN TWO-MONTH-OLD VERY LOW BIRTH WEIGHT PRETERM INFANTS?}

M A Riedijk ${ }^{1}, \mathrm{G}$ Voortman ${ }^{2}, \mathrm{R}$ H van Beek ${ }^{3}$, J B van Goudoever ${ }^{1}$

${ }^{1}$ Department of Pediatrics, Division of Neonatology, ${ }^{2}$ Mass Spectrometry Laboratory, Internal Medicine, Erasmus MC-Sophia Children's Hospital, Rotterdam, ${ }^{3}$ Department of Pediatrics, Amphia Hospital, Breda, The Netherlands

Background and Aims: Cyst(e)ine is a non-essential amino acid in adult humans. It can be synthesized from methionine by transsulfuration and this is regulated through the enzyme cystathionase. Cyst(e)ine is considered as a conditionally essential amino acid in preterm infants due to low cystathionase activity. However, the cyst(e)ine requirement in infants is not defined. Aim is to determine the cyst(e)ine requirement in preterm infants with a gestational age of 26 to 29 weeks at the age of 35 to 37 weeks by use of indicator amino acid oxidation (IAAO) technique.

Methods: Twenty-five preterm infants (gestational age $28.1 \mathrm{wk} \pm 1.2 \mathrm{wk}$ SD, BW $1.05 \mathrm{~kg} \pm 0.21 \mathrm{~kg} \mathrm{SD})$ who tolerated full enteral feeding, were randomly assigned to one of the graded cyst(e)ine test diets $(11,22,32,43,65$ $\mathrm{mg}$ cyst(e)ine/100 $\mathrm{ml}$ formula). After 24 hours of adaptation, cyst(e)ine requirement was determined by using [1-13C]phenylalanine hydroxylation. 13CO2 release from [1-13C]phenylalanine was measured by isotopic ratio mass spectrometry and linear regression was used to calculate the cyst(e)ine requirement.

Results: Graded dietary cyst(e)ine levels did not show a significant decrease in fractional [1-13C]phenylalanine oxidation. Linear regression of the data showed that the slope was not different from zero $(p=0.29)$, indicating that cyst(e)ine is not essential.

Conclusions: From these data we can conclude that cyst(e)ine is not an essential amino acid in VLBW premature infants receiving full enteral feeding at postnatal age of two months. These data do not support the hypothesis that cystathionase activity is limited in two-month-old premature infants.

\section{ARE PREBIOTICS EFFECTIVE IN REDUCING THE LEVEL OF NEONATAL HYPERBILIRUBINEMIA?}

M Bisceglia ${ }^{1}$, A Belcastro $^{1}$, V A Poerio ${ }^{1}, \mathrm{U} \mathrm{P}_{\text {Corapi }}{ }^{1}$, F Indrio ${ }^{2}$, R Francavilla ${ }^{2}$, F Raimondi ${ }^{3}$, R Paludetto ${ }^{3}$ 
${ }^{1}$ Neonatal Intensive Care Unit, Crotone Hospital, Crotone, Italy, ${ }^{2}$ Department of Pediatrics, University of Bari, Italy, ${ }^{3}$ Neonatal Intensive Care Unit, University of Naples 'Federico II', Naples, Italy

Background and Aims: To examine whether supplementing formula with oligosaccharide mixture may modulate bilirubin total (BT) levels in formula fed infants during the first weeks of life by modification of intestinal microflora.

Methods: 76 full-term infants were double blind randomly allocated into two groups to receive either supplementation of oligosacharides (GOS/FOS ratio 9:1, $0.8 \mathrm{~g} / 100 \mathrm{ml}(\mathrm{n}=37)$ or maltodexrine $(\mathrm{n}=39)$, from birth. BT (TcB) level was determined by multiwavelength spectral analysis, (BiliCheck) within 2 hours after birth (T0), 24hs (T2), 72 hs (T4) and at completed 28 (T8) days of life. Capillary hematocrit (Ht) was further determined.

Results: Groups were comparable for birth weight, gestational age, maternal age, type of delivery, baseline (T0) serum TcB level, and HT level. Pattern of TcB significantly differed between groups through the study $(\mathrm{p}<0.01$; Anova for repeated measures). Infants in supplemented group exhibited lower plasma TcB levels than placebo group from $72 \mathrm{~h}$ of life onwards : T4 difference was $-2.41 \mathrm{mg} / \mathrm{dl}, \mathrm{p}<0.010$. At $\mathrm{T} 8$, mean $\mathrm{TcB}$ level was $2.4 \mathrm{mg} / \mathrm{dl}$ (gos-fos) vs. $3.0 \mathrm{mg} / \mathrm{dl}$ (placebo). Ht significantly decreased from birth to day 28 in both groups $(\mathrm{P}<0.0001)$ but no difference between groups was found $(\mathrm{P}>0.55)$.

Conclusions: We observed significantly lowers bilirubin level after 72 hours of life in formula fed infants, supplementation of a mixture of gos-fos. It may be hypothesize that stimulating the intestinal growth of bifidobacteria and lactobacilli they enhances bilirubin excretion by reducing it to urobilinoids. Larger randomized trials would be of interest to evaluate prophylactic and therapeutic use of gos-fos in neonatal hyperbilirubinemia.

\section{WHAT FORMULA SHOULD WE PROPOSE FOR VERY LOW BIRTH WEIGHT INFANTS AFTER DISCHARGE FROM HOSPITAL: PRETERM FORMULA OR STANDARD TERM FORMULA?}

J C Picaud ${ }^{1,2}$, O Plan ${ }^{1}$, O Pidoux ${ }^{1}$, B Reygrobellet ${ }^{3}$, F Chapuis $^{4}$, E Lachambre ${ }^{5}$, L D Van Egroo ${ }^{5}$, B L Salle ${ }^{3}$, O Claris $^{3}$

${ }^{I}$ Dept of Neonatology, Arnaud de Villeneuve Hospital, University of Montpellier, France, ${ }^{2}$ Human Nutrition Research Center of Lyon, University of Lyon, France, ${ }^{3}$ Dept of Neonatology, Edouard Herriot Hospital, University of Lyon, France, ${ }^{4}$ Dept of Methodology and Statistics, University of Lyon, France, ${ }^{5}$ Nestle France, Noisiel, France

Background and Aims: As there are still no proposed guidelines about post-discharge nutrition, we evaluated growth and whole-body mineralization in VLBW infants fed preterm (PF) or term (TF) formula.

Methods: Double-blind randomized study in preterm infants randomized at discharge (T0) to be fed PF or TF. Subjects were fed PF or TF until one month after theoretical term (T1). Then, all subjects received $\mathrm{TF}$ until 3 months after theoretical term (T2). Anthropometric data and whole-body Dual-energy Xray Absorptiometry were assessed at $\mathrm{T} 0, \mathrm{~T} 1$ and $\mathrm{T} 2$. Statistical differences between the 2 feeding groups assessed using t-test or Mann-Whitney.

Results: Clinical characteristics were similar between the 2 feeding groups at birth and at T0 $(\mathrm{PF}, \mathrm{n}=23$ : $\mathrm{PNA}=44 \pm 15, \mathrm{BW}=1989 \pm 115 \mathrm{~g}-\mathrm{TF}, \mathrm{n}=24: \mathrm{PNA}=45 \pm 15$ $\mathrm{d}, \mathrm{BW}=2016 \pm 94 \mathrm{~g})$. Body weight at $\mathrm{T} 1(\mathrm{PF}=4533984 \mathrm{~g}$ vs $\mathrm{TF}=4144675 \mathrm{~g}, \mathrm{p}=0.12$ ) and $\mathrm{T} 2$, bone mineral content $(\mathrm{BMC})$ at $\mathrm{T} 1 \quad \mathrm{PF}=7123 \mathrm{~g}$ vs $\mathrm{TF}=60 \pm 16 \mathrm{~g}$, $\mathrm{p}=0.07$ ) tended to be higher in $\mathrm{PF}$ group than in $\mathrm{TF}$ group. At T2, BMC was significantly higher in PF group than in TF group (104 29g vs $8817 \mathrm{~g}, \mathrm{p}=0.03)$. Difference in BMC was related to an increase in bone mineral density and persisted when BMC was expressed as g per kg BW.

Conclusions: Four months after discharge, we observed a better growth and whole-body mineralization in VLBW infants fed PF than in those fed TF during the first two months after discharge. It could be of particular interest at this early stage of development. Study funded by the French National Program for Research and Nestlé France.

\section{RANDOMISED CROSS-OVER STUDY: EFFECT OF TWO DIFFERENT FORTIFIERS ON THE INCIDENCE OF METABOLIC ACIDOSIS IN PRETERM INFANTS}

\author{
N Rochow ${ }^{1}$, A Redlich ${ }^{1}$, H Küster ${ }^{1}$, F Jochum ${ }^{2}$, \\ C Fusch ${ }^{1}$ \\ ${ }^{I}$ Dept of Neonatology, University Children's Hospital, \\ Greifswald, Germany, ${ }^{2}$ Department of Pediatrics, Ev. \\ Waldkrankenhaus Spandau, Berlin, Germany
}

Background and Aims: Breast milk fed preterm neonates need to be fortified to achieve adequate weight gain. The composition of the available fortifiers differs and has a potential influence on acid load. Since the introduction of a new commercially available human milk fortifier in our neonatal unit we observed an increased incidence of metabolic acidosis. Therefore, we compared the effect of two different fortifiers on the development of metabolic acidosis and weight gain.

Methods: Preterm infants on $>100 \mathrm{ml} / \mathrm{kg}$ oral feeds with mothers milk only were randomised and supplemented with fortifiers. Clinical and laboratory parameters were collected using a standardised protocol. In case of acidosis $(\mathrm{BE}<-6 \mathrm{mmol} / \mathrm{L})$ the other fortifier was fed after recovery of the metabolic status.

Results: 34 infants were studied, fortifier $1(\mathrm{n}=15,30.0$ weeks \pm 3.4 , birthweight: $1282 \mathrm{~g} \pm 488$ ), fortifier 2 $(\mathrm{n}=19,29.3$ weeks \pm 3.4 , birthweight: $1247 \mathrm{~g} \pm 464)$. Acidosis (BE: -6.1 to $-9.3 \mathrm{mmol} / \mathrm{L}$ ) developed 3 infants with fortifier 1,6 with fortifier $2(\mathrm{p}=.36)$. Most of these had a birthweight $<1000 \mathrm{~g}$. Acidotic infants $<1000 \mathrm{~g}$ seem to develop a stronger temporary decrease in daily weight gain with longer time of supplementation. Discontinuation of the fortifier resulted in normalization of the acid base status. After cross-over $(n=6)$ all infants developed metabolic acidosis within 4 days. 
Conclusions: The present study shows that breast milk fortifier can have clinical impact on the acid base status and weight gain of preterm neonates.

\section{IS INTRAPARTUM ASPHYXIA PREVENTABLE? AT WHAT COST?}

\section{J C Becher, B J Stenson, A J Lyon \\ Department of Neonatology, Royal Infirmary of Edinburgh, Scotland}

Background and Aims: Recent evidence suggests that asphyxia-related perinatal mortality and morbidity (ARMM) are declining. We wished to examine local delivery unit trends in ARMM and operative deliveries between 1994-2005.

Methods: Records of all full-term inborn admissions were retrospectively reviewed for the diagnosis of 'hypoxic-ischemic encephalopathy' (HIE) and related diagnoses. Accuracy of diagnosis was verified. Threeyear moving averages of HIE, full-term intrapartum stillbirths, delivery room asphyxial neonatal deaths, and full-term operative deliveries were calculated and trends analysed with the Chi-square test.

Results: There were 116 infants with HIE, 7 intrapartum stillbirths and 2 delivery room deaths. ARMM fell progressively from $2.86 / 1000$ births to $0.91 / 1000$ births $(p<0.001)$. The incidence of HIE declined steadily from 2.41 to $0.77 / 1000$ livebirths $(\mathrm{p}<0.001)$. Operative deliveries increased from 28.1$42.7 \%(\mathrm{p}<0.001)$.

Conclusions: ARMM declined significantly over 12 years associated with a large increase in operative deliveries. Expeditious delivery may have prevented a small number of ARMM, but the associated morbidity of an escalating operative delivery rate needs evaluation.

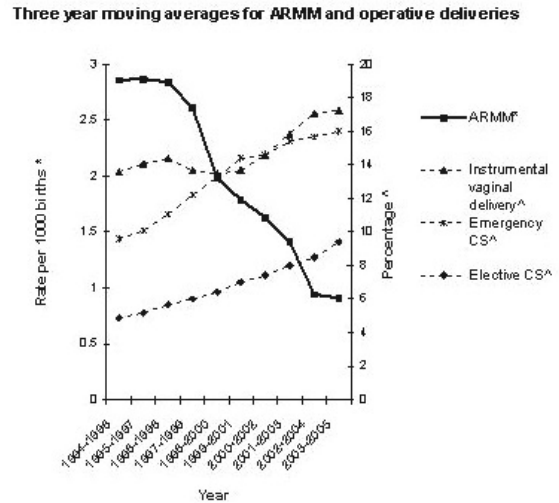

\section{STILLBIRTHS AND SURVIVAL AT 22-31 WEEKS OF GESTATIONAL AGE: A POPULATION- BASED STUDY IN ITALY}

\footnotetext{
M Cuttini ${ }^{1}$, M Da Frè ${ }^{2}$, V Carnielli ${ }^{3}$, F Macagno ${ }^{4}$, D Di Lallo ${ }^{5}$, E Buiatti $^{6}$

${ }^{\text {I} P e d i a t r i c ~ H o s p i t a l ~ B a m b i n o ~ G e s u ̀, ~ R o m e ~(L a z i o), ~ I t a l y ~}$

${ }^{2}$ Regional Health Agency, Firenze (Toscana), Italy

${ }^{3}$ G. Salesi Hospital, Ancona (Marche), Italy, ${ }^{4}$ Civile

Hospital, Udine (Friuli Venezia Giulia), Italy, ${ }^{5}$ Agency

for Public Health, Roma (Lazio), Italy, ${ }^{6}$ Regional Health

Agency, Firenze (Toscana), Italy
}

Background and Aims: Valid estimation of preterm infants' survival to discharge requires studies of geographically defined populations.

Methods: As part of a larger research project on Very Preterm Pregnancies and Births (ACTION), an areabased study was carried out including all births and late terminations of pregnancies occurred in 2003-2004 in four Italian regions: Friuli Venezia Giulia, Toscana, Lazio and Marche. Data on maternal characteristics, pregnancy and, for liveborn, admission to Neonatal Intensive Care Units (NICUs) were collected.

Results: 2410 events were recorded: 1745 livebirths (82\%), 378 stillbirths (18\%) and 287 late terminations (12\%). Children of foreign women contributed to $22 \%$ of total births, and multiples to about $25 \%$. The proportion of livebirths ranged from $18 \%$ at 22 to $94 \%$ at 31 weeks gestation. 1710 infants (98\% of liveborn) were admitted to NICU. Survival to discharge was $82 \%$ among NICUs admissions (from $6 \%$ at 22 to $96 \%$ at 31 weeks), $81 \%$ among livebirths, and only $66 \%$ when all births were considered.

Conclusions: Estimates of survival of preterm infants change according to the selected denominator. These findings have implications for parents' counselling during pregnancy.

\section{ACUTE EFFECTS OF SURGICAL LIGATION OF PATENT DUCTUS ARTERIOSUS (PDA) ON CEREBRAL OXYGENATION AND BLOOD VOLUME IN VERY LOW BIRTH WEIGHT INFANTS}

B M Hüning ${ }^{1}$, B Asfour ${ }^{2}$, S König ${ }^{1}, \mathrm{~N} \mathrm{Hess}^{1}, \mathrm{C} \mathrm{Roll}^{3}$

${ }^{I}$ Department of Pediatrics and Pediatric Neurology, University Children's Hospital, Essen, Germany ${ }^{2}$ Department of Pediatric Thoracic and Cardiovascular Surgery, German Pediatric Heart Institute, Sankt Augustin, Germany, ${ }^{3}$ Department of Neonatology, Children's Hospital Datteln, University of Witten Herdecke, Witten, Germany

Background and Aims: Dopplersonography has shown a sharp increase in diastolic blood flow velocity in cerebral arteries following PDA closure. Using nearinfrared spectroscopy (NIRS) we aimed to assess acute effects of surgical closure on cerebral oxygenation and cerebral blood volume (CBV).

Methods: Ten preterm infants, birth weight $590-1070$ g (median $748 \mathrm{~g}$ ), gestational age 23 - 27 wks (median 24 wks) were studied during surgery (day 12 - 22 of life; median 14). Using NIRS, changes in concentrations of cerebral oxygenated and deoxygenated haemoglobin were measured, and changes in $\mathrm{CBV}$ and cerebral oxygenation were calculated. Heart rate, arterial oxygen saturation (pulse oxymetry), tcPO2, tcPCO2, and blood pressure were registered simultaneously.

Results: During the first two minutes after closure CBV increased significantly $(+0.14 \mathrm{mg} / 100 \mathrm{mg}$ tissue; SE $0.97 ; \mathrm{p}=0.004$ ) and returned to baseline at 2 to 5 minutes. Cerebral oxygenation did not change significantly $(-0.05 \mu \mathrm{mol} / \mathrm{l} ; \mathrm{SE} 0.97 ; \mathrm{p}=0.9)$ but arterial oxygen saturation decreased (2.8\%; SE $0.4 ; \mathrm{p}<0.0001)$. At 5 to 10 minutes after closure tcPCO2 decreased (-3.4 mbar; SE 1.2; $\mathrm{p}=0.02)$ and heart rate increased $(+2.0$ 
bpm; SE 0.7; $\mathrm{p}=0.02$ ). Blood pressure did not change significantly.

Conclusions: NIRS was an appropriate tool to assess changes in CBV and cerebral oxygenation. Surgical closure of PDA induced an acute increase of CBV.

\section{DEEP HYPOTHERMIA IN THE TREATMENT OF NEONATAL ASPHYXIA: PRELIMINARY DATA}

G Cristofori, P Mussini, C Bottura, E Agazzani, R Astolfi, G Compagnoni

Neonatal Intensive Care Unit, 'Carlo Poma' Hospital, Mantua, Italy

Background and Aims: Benefits and safety of mildmoderate hypothermia (34-35,5 $\mathrm{C}$ and 33-34,5 C respectively) to treat perinatal asphyxia are documented. In animal studies, neuroprotective effect seems to be proportional to the depth of hypothermia but with more side effects. The aim of this study is to determine the safety of hypothermia to 30-33 C in term asphyxiated infants. Mortality, major neurologic abnormalities and major RMI cerebral lesions are described, comparing these results with historical controls.

Methods: Term infants admitted for perinatal asphyxia were enrolled within 6 hours after birth and treated with hypothermia (rectal temperatures around 30-33 C) for 72 hours. Neurological abnormalities and RMI cerebral lesions were evaluated.

Results: Fourteen patients were treated with whole body cooling to 30-33 C. The mean gestational age was 39.4 (2.1) weeks, pre-treatment arterial pH $6.9(0.1)$, BE-17.2 (5) and lactate $14.3(5.6) \mathrm{mmol} / \mathrm{L}$. Hypothermia was started at 3.1 (1.6) hours of age and mean rectal temperature was $32.1 \mathrm{C}(0.9)$. Three subjects presented pneumonia on their 2nd-3rd day of life, recovered after antibiotic therapy. Brain RMI documented major cerebral lesions only in one case who also presented a severe psychomotor delay. Comparing these data with clinical outcome and neuroimaging lesions of an untreated group with similar characteristics at birth, we obtained statistically significant better results $(p<0,05)$. We did not observe any difference between the groups treated with different depths (mild-moderate deep) of hypothermia.

Conclusions: By our preliminary data, it appears that hypothermic treatment between $30-33 \mathrm{C}$ is safe and neuroprotective. Further studies are mandatory to confirm these data.

\section{NON-INVASIVE MEASUREMENT OF REDUCED VENTILATION-PERFUSION RATIO AND SHUNT IN INFANTS WITH BRONCHOPULMONARY DYSPLASIA; A PHYSIOLOGICAL DEFINITION OF THE DISEASE}

D Quine $^{1}$, B J Stenson ${ }^{1}$, C M Wong ${ }^{1}$, E M Boyle ${ }^{1}$, J G Jones ${ }^{2}$

${ }^{1}$ Department of Neonatal, Simpson Centre for Reproductive Health, Edinburgh, UK, ${ }^{2}$ Department of Medicine, University of Cambridge, UK
Background and Aims: An objective definition of bronchopulmonary dysplasia (BPD) is required to interpret trial outcomes and provide a baseline for prognostic studies. Reduced ventilation/perfusion ratio (VA/Q) and increased right to left shunt can be determined non-invasively by plotting oxygen saturation (SpO2) against inspired oxygen pressure (PIO2). Reduced VA/Q causes a right shift of the $\mathrm{SpO} 2$ against PIO2 curve. We describe the reduced VA/Q and shunt in infants with BPD.

Methods: 21 preterm infants with BPD were studied at 36 weeks CGA. $\mathrm{PIO} 2$ was varied in steps to cause $\mathrm{SpO} 2$ to vary between $86-94 \%$. Pairs of $\mathrm{SpO} 2$ and $\mathrm{PIO} 2$ values were plotted and analysed to derive reduced $\mathrm{VA} / \mathrm{Q}$ and shunt.

Results: In every infant the $\mathrm{SpO} 2$ vs $\mathrm{PIO} 2$ curve was shifted to the right of normal due to reduced VA/Q. Mean right shift was $16.5 \mathrm{kPa}$ (normal $6 \mathrm{kPa}$ ). Three infants plots enclosed. Shunt was small in all cases mean $8.9 \%$. The degree of right shift was strongly predictive of the PIO2 required to achieve any $\mathrm{SpO} 2$ within the range $86-94 \%$, (R2>0.9), permitting shift and $\mathrm{VA} / \mathrm{Q}$ to be determined from a single pair of $\mathrm{SpO} 2$ and $\mathrm{PIO} 2$ values in this range.

Conclusions: Reduced VA/Q ratio is the dominant gas exchange defect in BPD. This can be described by the right shift of the $\mathrm{SpO} 2$ vs $\mathrm{PIO} 2$ relationship. A single pair of $\mathrm{SpO} 2$ and $\mathrm{PIO} 2$ values predicts $\mathrm{VA} / \mathrm{Q}$ ratio and provides graded physiologically objective measure of BPD disease severity.
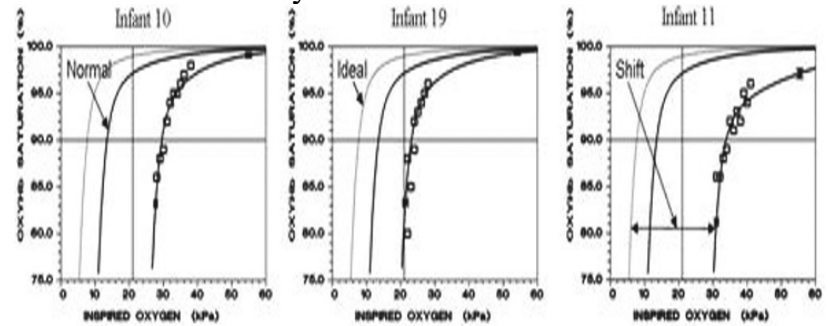

\section{PRETERM BIRTH <25 WEEKS - COSTS AND CONSEQUENCES. AN AUSTRALIAN REGIONAL STUDY}

N P French
H Benninger ${ }^{I}$ Women's and Children's Health Service, King Edward and Princess Margaret Hospitals, Perth, WA, Australia ${ }^{2}$ State Child Development Centre, Perth, WA, Australia

Background and Aims: Controversy continues regarding initiation of life supporting measures in infants born before 25 weeks gestation, with wide variations between units and between different countries. In Western Australia (WA) a long standing permissive approach to the support of these infants and centralisation of perinatal services has allowed us to examine resource use and long term outcome in a regional study of this small group in relation to the wider picture of perinatal care. Aims: 1 . To demonstrate trends in survival $<25$ weeks since 1992. 2. To examine resource use in WA, (hospital days, respiratory support days, estimated dollar costs) 3 . To assess frequency and severity of disability from 1992-2002. 
Methods: Overlapping cohorts were analysed for Survival (1992-2004, $\mathrm{n}=378)$, Resource use (2002-2004, $\mathrm{n}=69$ of all 5,713 neonatal admissions), and Outcome $(1992-2002, \mathrm{n}=151)$. Severe disability was defined as nonambulant cerebral palsy $(\mathrm{CP})$, or $\mathrm{DQ} / \mathrm{IQ}>3$ sd below mean, or blind.

Results: Admissions $<25 \mathrm{w}$ represented $0.1 \%$ of all regional births, $<1.5 \%$ of NICU admissions, $8 \%$ of $<33 \mathrm{w}$ admissions. Survivors at $22 \mathrm{w}(8), 23 \mathrm{w}(48)$ and $24 \mathrm{w}(95)$ represented $5 \%$ of all $<33 \mathrm{w}$ survivors. NICU survival rates increased from $38 \%$ to $71 \%$. $<25 \mathrm{w}$ infants accounted for $8 \%$ of patient stay days, $27 \%$ of respiratory support days, and $11 \%$ of estimated NICU costs. Severe disability occurred in $10 \%$ of survivors. There were no blind or nonambulant $\mathrm{CP}$ cases.

Conclusions: In this regional study, births $<25 \mathrm{w}$ are infrequent, but do account for a disproportionate amount of nursery resources. Ninety percent of the survivors are not severely disabled.

\section{PARENTING STRESS, QUALITY OF MOTHER INFANT INTERACTION AND MATERNAL RESPONSIVENESS IN VERY PREMATURE TWIN AND SINGLETON BIRTHS}

C Glazebrook $^{1}$, C Sheard ${ }^{1}$, S Johnson ${ }^{2}$, A Whitelaw ${ }^{3}$, C Israel ${ }^{3}, \mathrm{~N}_{\text {Marlow }}^{2}$

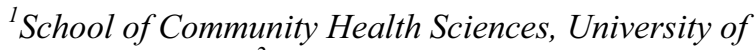
Nottingham, UK, ${ }^{2}$ Divsion of Child Health, University of Nottingham, UK, ${ }^{3}$ Department of Clinical Science at North Bristol, University of Bristol, UK

Background and Aims: The quality of mother-infant interaction is important for very premature (VP) infants who are at risk for cognitive delay. This study aims to investigate the impact of a very premature twin birth on parenting stress, maternal responsiveness and quality of mother-infant interaction.

Methods: Mothers of 17 VP twins $(<32$ weeks gestation), drawn from a parenting intervention study, formed the study sample. The target twin was chosen at random. Each mother was matched for age, educational attainment and parity with a singleton mother. At 3 months corrected age mothers were videotaped whilst attempting to teach their infant a developmentally appropriate task. Videos were rated using The Nursing Child Assessment Teaching Scale (NCATS) to assess the quality of both caregiver and infant interactions. The responsivity subscale of the HOME was also administered and mothers completed the short-form Parenting Stress Index.

Results: During one to one interaction, maternal NCATS scores were similar between groups but twin infants had lower NCATS scores than singletons, indicating less clear and responsive interactions $(p<0.04)$. This effect remained even after controlling for infant factors. Twin mothers had lower HOME scores $(p<0.03)$ and $24 \%$ of twin mothers had clinically high levels of parenting stress compared to none in the singleton group $(\mathrm{p}=0.05)$.

Conclusions: Mothers of twins reported high levels of stress and twins received less responsive day to day care. This may explain the poorer quality of communication shown by twins during one to one interactions and suggests that twin status may be an additional vulnerability factor for very premature infants.

\section{COMBINED THERAPY WITH PHENOBARBITAL AND DELAYED POST- HYPOXIC-ISCHEMIC HYPOTHERMIA: DISSOCIATION OF FUNCTIONAL BENEFIT FROM NEUROPATHOLOGY}

J D Barks ${ }^{1}$, Y Q Liu ${ }^{1}$, F S Silverstein ${ }^{12}$

${ }^{1}$ Department of Pediatrics, University of Michigan, Ann Arbor, MI, USA, ${ }^{2}$ Department of Neurology, University of Michigan, Ann Arbor, MI, USA

Background and Aims: We previously demonstrated that treatment with the Topiramate immediately after unilateral cerebral hypoxia-ischemia (HI) in neonatal rats extended the therapeutic window of post-HI hypothermic neuroprotection, in terms of both function and neuropathology. To begin to address whether this effect of Topiramate is generalizable to other anticonvulsants, we tested phenobarbital (PB) in a similar paradigm.

Methods: P7 rats underwent right carotid ligation followed by $90 \mathrm{~min}$ in $8 \%$ O2. Fifteen min after HI, rats received $\mathrm{PB} 40 \mathrm{mg} / \mathrm{kg}(\mathrm{n}=12)$ or saline vehicle $(\mathrm{n}=11)$. Beginning $3 \mathrm{hr}$ after $\mathrm{HI}$ all rats underwent $3 \mathrm{hr}$ hypothermia (HT) in a $27 \mathrm{C}$ incubator. One wk later lateral vibrissae stimulated forepaw placing was evaluated in 10 trials/side, followed by quantitation of ipsilateral damage severity [\%left-right difference = $100 *(\mathrm{~L}-\mathrm{R}) / \mathrm{L}]$ in cortex, striatum and hippocampus.

Results: On P14, the contralateral vibrissae-stimulated placing deficit typical of HI was attenuated in the $\mathrm{PB}+$ delayed HT $(\mathrm{PB}+\mathrm{dHT})$ group compared to the delayed HT (dHT) group [median (IQR) PB+dHT: 9 (2.5) vs. dHT 5 (5.5), $\mathrm{P}<0.05$ Mann-Whitney]. In contrast damage severity was not attenuated in the $\mathrm{PB}+\mathrm{dHT}$ group, compared to $\mathrm{dHT}$ alone (\%damage mean $\pm \mathrm{SD}$, $\mathrm{PB}+\mathrm{dHT}$ vs. $\mathrm{dHT}$ : cortex, $41.5 \pm 30.5$ vs. $49.5 \pm 30.4$, striatum $44.5 \pm 30.4$ vs. $56.2 \pm 29.7$, hippocampus $52.4 \pm 30$ vs. $62.1 \pm 28.1$, $\mathrm{p}=\mathrm{NS}$ ).

Conclusions: An anticonvulsant dose of PB did not restore the protective effect of delayed hypothermia on post-HI neuropathology, although there was a dissociated beneficial effect on a sensorimotor function measure. Thus the efficacy of Topiramate in extending the therapeutic window of HT does not represent a generalized effect of anticonvulsant therapy in neonatal rodent $\mathrm{HI}$.

\section{NEW TRENDS IN THE MANAGEMENT OF NEONATAL BRAIN INJURY}

\section{Thoresen}

Department of Clinical Sciences, St. Michael's Hospital, Bristol, UK

Perinatal asphyxial encephalopathy remains an important complication in newborn infants occurring in $1-2 / 1000$ births in Europe and is associated with a high risk of death or major neurological and 
neurodevelopmental abnormalities in survivors. Experimental and clinical studies show that following asphyxia, multiple inter-related pathophysiological processes that culminate in necrotic and apoptotic cell death occur over a prolonged period. Experimentally, hypothermia ameliorates these processes and reduces the severity of cerebral injury. Recently completed trials of treatment with moderate induced hypothermia following perinatal asphyxial encephalopathy confirm that clinically significant improvement in outcome is possible. When hypothermia will be used as standard treatment for all infants with severe asphyxia we are faced with several questions. Which early entry criteria do we use? How do we cool? Is head cooling or total body cooling best? Should this intervention only be applied in level III units or is there also a place for treatment in local hospitals? Temperature management after brain injury is important, in particular avoiding rebound hyperthermia which easily occurs when circulation is restored. Number needed to treat was 6 in the 2 large clinical trials published in 20051,2 meaning that 5 out of 6 children treated did not have an effect of hypothermic treatment. Are there more options? Adding additional drugs to hypothermia? There are recent experimental studies suggesting a variety of drugs that might add to the neuroprotection and these will be reviewed. References: 1. Gluckman PD, Wyatt JS, Azzopardi D, Ballard R, Edwards AD, Ferriero DM, Polin RA, Robertson CM, Thoresen M, Whitelaw A, Gunn AJ. Selective head cooling with mild systemic hypothermia after neonatal encephalopathy: multicentre randomised trial. The Lancet 2005;365 (9460):663-670

2. Shankaran S, Laptook A, Ehrenkranz R, Tyson J, MacDonald S, Donovan E, et al. Whole-body hypothermia for neonates with hypoxic-ischemic encephalopathy. NEJM 2005353 1574-84.

\section{B-ESTRADIOL AND PHYTOESTROGENS PROTECT AGAINST OXYGEN-INDUCED CELL DEATH IN DEVELOPING OLIGODENDROCYTES}

B Gerstner ${ }^{1,2}$, N Sucher ${ }^{1}$, U Felderhoff-Mueser ${ }^{2}$, M Obladen ${ }^{2}$, J J Volpe ${ }^{1}$, P A Rosenberg ${ }^{1}$

${ }^{I}$ Department of Neurology, Children's Hospital Boston, Boston, MA, USA, ${ }^{2}$ Department of Neonatology, Children's Hospital Charité, Humboldt University, Berlin, Germany

Background and Aims: In the developing human brain, periventricular leukomalacia (PVL) is the predominant white matter injury underlying the development of cerebral palsy. Our group recently showed that short exposure to high oxygen levels can trigger apoptosis in immature, but not in mature oligodendrocytes (OLs) in vitro. $17 \beta$-estradiol (E2) plays an important role in the development and function of the CNS. The sudden drop in circulating estrogen after birth may aggravate the susceptibility to oxidative stress in premature infants. We examined the effect of E2, and phytoestrogens on hyperoxia-induced cell death in immature rat OLs.

Methods: Primary OL cultures were subjected to $80 \% \mathrm{O} 2$ in the presence or absence of estrogen (10-6-10$10 \mathrm{M})$. LDH assay was used to assess cell viability. OL maturation status and caspase-3 activation were studied by immunochemistry. Immunoblot analysis was used to quantify ER expression, and demonstrated an upregulation of ER- $\alpha$ after E2-pretreatment.

Results: E2, stigmasterol, and $\beta$-sitosterol, but not campesterol or genistein, produced significant dosedependent protection against oxygen-induced apoptotic cell death in OLs. In addition, we studied the impact of E2 on the expression of the pro-apoptotic receptor Fas. Treatment with E2 prevented hyperoxia-induced Fasupregulation. Finally, we found by immunoblot analysis that E2 antagonizes hyperoxia-induced inactivation of ERK1/2 and AKT, key kinases of the MAPK and PI3kinase cell survival promoting pathways, respectively.

Conclusions: These results may be relevant to concerns regarding oxidative injury to the premature infant brain, both at normal and at high oxygen levels, and suggest a possible role for estrogens in the prevention of PVL.

\section{Fas SIGNALLING IN THE NEONATAL RAT BRAIN FOLLOWING EXPOSURE TO OXYGEN}

M Dzietko ${ }^{1}, \mathrm{~V}$ Boos $^{1}$, O Polley ${ }^{1}$, S Endesfelder ${ }^{2}$, M Sifringer ${ }^{2}, \mathrm{~K} \mathrm{Genz}^{1}$, C Bührer ${ }^{3}$, M Obladen ${ }^{1}$, U Felderhoff-Müser ${ }^{1}$

${ }^{I}$ Department of Neonatology, Children's Hospital, Charité Universitätsmedizin Berlin, Germany ${ }^{2}$ Department of Pediatric Neurology, Children's Hospital, Carl Gustav Carus University, Dresden, Germany, ${ }^{3}$ Department of Neonatology, UniversitätsKinderspital Beider Basel, Switzerland

Background and Aims: Advances in neonatal medicine have improved survival of premature and term infants. Unfortunately survivors of premature birth often suffer from neurologic morbidity. Clinical studies have identified hyperoxia as a risk factor for cerebral palsy. Experimental studies show that short exposure to high oxygen levels can trigger neurodegeneration in the developing rat brain. This neuronal death is associated with reduced expression of neurotrophins and decreased concentrations of survival-promoting proteins. To explore further pathogenetic mechanisms, we investigated the impact of supraphysiological oxygen levels on members of the proapoptotic Fas (CD95/APO1) signalling pathway: Fas-Ligand, caspase- 8 , caspase9, caspase-3, FADD and FLIP.

Methods: Six day old rats were exposed with their mothers to $80 \% \mathrm{O} 2$ and were sacrificed at defined time points $(2,6,12,24,48,72 \mathrm{hrs})$. Brains were either examined histologically to visualize degenerating cells or were processed for molecular studies.

Results: Oxygen exposure triggered cell death in the thalamus and cortex peaking at 12 to $24 \mathrm{hrs}$. Increased levels for Fas and Fas-Ligand mRNA were found at 12 and $24 \mathrm{~h}$. Immunoblot assays of thalamus tissue for caspase- 8 were upregulated at $48 \mathrm{hrs}$, for caspase- 9 and FADD at 48 and $72 \mathrm{hrs}$, for Caspase- 3 at 24 and $48 \mathrm{hrs}$ and for FLIP long and short at 24 to $48 \mathrm{hrs}$.

Conclusions: These findings suggest that upregulation of the pro-apoptotic Fas system and his signalling pathway may contribute to oxygen-induced cell death in the developing brain. 
SENSITIZATION BY LIPOPOLYSACCHARIDE TO IN VITRO ISCHEMIA IN A MURINE JUVENILE ORGANOTYPIC HIPPOCAMPAL SLICE CULTURE

T Markus ${ }^{1}$, T Cronberg ${ }^{2}$, C Cilio ${ }^{3}$, D C R Ley ${ }^{1}$,

${ }^{1}$ Department of Pediatrics, Lund University, Sweden, ${ }^{2}$ Department of Obstetrics and Gynecology, Lund University, Sweden, ${ }^{3}$ Department of Experimental Brain Research, Lund University, Sweden, ${ }^{4}$ Department of Neurology, Lund University, Sweden

Background and Aims: Fetal inflammatory response appears an important cause of damage in the immature brain. The hippocampal slice culture enables study of the interaction between induced inflammation and ischemia and of subsequent neuronal damage in the immature brain. Methods: Hippocampal slices from BALB/C mice obtained at P5, placed on Millicell culture inserts and grown in vitro for 8 days. Hippocampal slices co-incubated with/without LPS, 1 microg $/ \mathrm{ml}$ for $24 \mathrm{~h}$ and thereafter subjected to oxygenglucose deprivation for $15 \mathrm{~min}$. Cytokine levels (IL-6, IL-10, TNF-alpha, IFN-gamma, IL-12p40 and MCP-1) and nitric oxide production determined at repeated time points. Cell death was determined with propidium iodide (PI) measured by fluorescent light michroscopy and digital image analysis. Results: Hippocampal slices pre-exposed to LPS before in vitro ischemia exhibited increased PI determined cell death in regions CA1-3 and dentate gyrus from 4 and up to $72 \mathrm{~h}$ after in vitro ischemia $(\mathrm{p}<0.01)$. Immunostaining confirmed increased neuronal depletion and microglial activation in LPS preexposed slices. LPS exposure without subsequent in vitro ischemia was not associated with neuronal cell death. Hippocampal slices secreted increased levels of TNF-alpha, IL-6 and MCP-1 at 12 and $24 \mathrm{~h}$ during LPS exposure $(p<0.01)$ whereas only MCP-1 $(\mathrm{p}<0.01)$ was increased after in vitro ischemia in LPS pre-exposed slices. Conclusions: Exposure to LPS prior to in vitro ischemia increases and extends neuronal death in a juvenile murine hippocampal slice culture. This is associated with increased microglial activation and with increased secretion of MCP-1. Sole exposure to LPS was not associated with neuronal cell death.

\section{ESTROGEN PROTECTS ASTROCYTES AGAINST HYPEROXIA INDUCED APOPTOSIS}

S Huppmann, S Roemer, A Muench, M Berns, M Obladen

Department of Neonatology, Charite Campus Virchow Klinikum, Berlin, Germany

Background and Aims: In premature infants oxygen free radicals are associated with subsequent diseases like retinopathia of prematurity and bronchopulmonal dysplasia. Recent studies in brain tissue samples have shown that nonphysiologic oxygen levels play a key role in induction of apoptosis in the developing brain. As female preterm infants consistently revealed a better neurological outcome then males, estrogen is considered to be neuroprotective in the premature brain. Astrocytes appear to have a critical role in protection and survival of neurons in the brain. As one of the glial cell types they have a great potential for possible involvement in the mediation of estrogen neuroprotective effects. Cell cultures of astrocytes have not yet been investigated concerning their behaviour in hyperoxic environment. The aim of our study was to analyze whether astrocytes in cell cultures are damaged by hyperoxia and whether estrogen can protect them against apoptosis.

Methods: Cell cultures of astrocytes (rat brain glial cells, C6) were cultivated in $80 \%$ compared to $21 \%$ oxygen environment and in different concentrations of 17beta-estradiol. Apoptosis was verified with caspase 3 staining by flow cytometric analysis.

Results: The presence of estrogen receptors alpha and beta in astrocytes was confirmed by RT-PCR. Astrocytes underwent sixfold more apoptosis when cultivated in hyperoxia compared to normoxia. Addition of estrogen reduced apoptosis in hyperoxia by $20 \%$. Conclusions: Hyperoxia induces apoptosis in astrocytes and estrogen seems to be a protecting factor for astrocytes in hyperoxia.

\section{HYPOXIA-ISCHEMIA (HI) MODIFIES POSTNATAL GLIOGENIC ACTIVITIES IN THE RAT CEREBRAL CORTEX}

S V Sizonenko ${ }^{1}$, E Camm $^{1}$, A Dayer ${ }^{2}, \mathrm{~J} \mathrm{Z} \mathrm{Kiss}^{2}$

${ }^{1}$ Department of Pediatrics, University of Geneva, Switzerland, ${ }^{2}$ Department of Fundamental

Neurosciences, University of Geneva, Switzerland

Background and Aims: Neurogenesis is completed after birth, whereas gliogenic activities remain intense during the postnatal period in the rat cortex. Little is known about the effects of $\mathrm{HI}$ on these critical postnatal processes. The aim is to study the glial proliferation after HI injury in the P3 rat parietal cortex (PCx). Methods: P3 rats had right carotid ligation and $30^{\prime}$ exposure to $6 \% \mathrm{O} 2$. Glial markers were used: GFAP, NG2, RIP. Brdu was injected 24h after HI and brains collected $48 \mathrm{~h}, 72 \mathrm{~h}$ and $7 \mathrm{~d}$ after HI. Another group had Brdu injection $7 \mathrm{~d}$ after $\mathrm{HI}$ and brain collected at $8 \mathrm{~d}$. Within the PCx proliferation was measured by counting double-labelled cells. The ipsilateral PCx was compared to the contralateral using the Wilcoxon test. Results: In the ipsilateral PCx: GFAP and RIP were increased at all time-points; NG2 was increased at $48 \mathrm{~h}$ and $72 \mathrm{~h}$. In all groups GFAP/Brdu astrocytes were increased. NG2/Brdu cells in the 24-48h and 24-72h groups and $\mathrm{RIP} / \mathrm{Brdu}$ cells in the $24 \mathrm{~h}-7 \mathrm{~d}$ group were also increased in the HI PCx. In the $7 \mathrm{~d}-8 \mathrm{~d}$ group a significant increase of GFAP/Brdu, NG2/Brdu and RIP/Brdu was present. Conclusions: These data show significant modifications in the glial architecture of the PCx after mild HI injury that could contribute to long-term alterations in cerebral development after neonatal injury. 


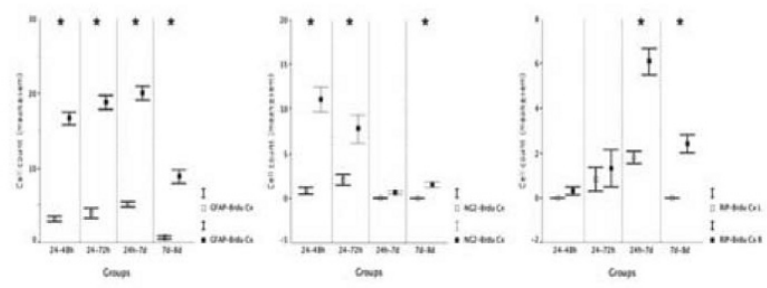

HYPEROXIA CAUSES OXIDATIVE STRESS AND INCREASES NEURAL STEM CELLS PROLIFERATION IN THE MURINE DEVELOPING BRAIN

S Handley, A Sola, X Wang, T Wen, T Genetta, J Moore, M Rogido

Division of Neonatology, Department of Pediatrics, Emory University, Atlanta, GA, USA

Background and Aims: Studies show hyperoxia results in apoptosis in immature murine brains, however, little is known about hyperoxia's effect on neural progenitor/stem cells in developing brains. Our study attempts to determine effects of exposure to $96 \%$ oxygen versus room air on neural progenitor/stem cells in newborn mice.

Methods: Pups expressing green fluorescent protein (GFP) under nestin gene control were randomized into 2 groups ( $\mathrm{n}=5 /$ group). P5 animals were subjected to normoxia (FiO2-0.21), or hyperoxia (FiO2-0.96), for 90 minutes then injected with $\mathrm{BrDu} \times 3$ over 24 hours and euthanized. Apoptotic-like changes were determined by TUNEL staining. Immunohistochemistry was used to assess lipid peroxidation and oxidative DNA damage with 4-Hydroxy-2-nonenal (HNE) and 8-hydroxy-2deoxyguanosine (8OHdG) monoclonal antibodies. Proliferation was assessed using anti-BrdU antibody. Nestin + cells were identified by immunofluorescent microscopy. Cells were counted in 20 fields in cortical and ventricular/subventricular zones (VZ). Values are expressed in mean SD, and normalized with methylgreen staining.

Results: Neural cells showed weak HNE immunoreactivity in cortex and VZ controls. Hyperoxic pups showed increased HNE immunoreactivity and number in cortex $(568 \pm 164$ vs. $55 \pm 24 ; \mathrm{p}=0.0001)$ and VZ ( $311 \pm 103$ vs. $784 \pm 9$; $\mathrm{p}=0.01)$. $8 \mathrm{OHdG}+$ cells were increased in hyperoxia cortex $(96 \pm 47$ vs. $10 \pm 6$, $\mathrm{p}=0.032$ ). Cortical TUNEL+ cells were increased in hyperoxia vs. normoxia $(8901 \pm 1821$ vs. $1930 \pm 717$, $\mathrm{p}=0.0001)$. GFP $+/ \mathrm{BrdU}+$ cortical cells increased in hyperoxia vs. controls (11120 \pm 4536 vs. $1987 \pm 1242$, $\mathrm{p}=0.028$ ).

Conclusions: Data suggests exposure to high oxygen concentrations increase oxidative stress markers and apoptotic-like cell death in developing murine brains. We continue to investigate the increased proliferation of neural progenitor/stem cells in cortex of hyperoxic pups.

\section{HYPOTHERMIA INCREASES ERYTHOPOIETIN RECEPTOR EXPRESSION IN ISOLATED RAT CORTICAL NEURONS}

J E Moore, A Sola, H Peng, M Rogido, S Handley, $\mathrm{T}$ Genetta, T C Wen
Department of Pediatrics, Emory University, Atlanta, GA, USA

Background and Aims: Hypothermia has been shown to improve the outcome of perinatal hypoxic-ischemic encephalopathy (HIE) within the laboratory and clinical setting. Currently the physiologic mechanisms of how hypothermia leads to neuroprotection are not fully understood. Our objective was to evaluate the role of hypothermia on EPO receptor expression and to determine if hypothermia plus EPO has an effect on cellular signaling.

Methods: Isolated cultures of E18 rat cortical neurons were obtained from Genlantis. Cells were fully differentiate for 5 days and then exposed to either hypothermia or allowed to remain at $37 \mathrm{C}$. Anti-rabbit EPO receptor antibody was used in conjunction with fluorescein isothiocyanate (FITC)-conjugated antirabbit $\mathrm{IgG}$ for immunostaining. Calcium signaling was studied with the indicator dye Fluo-4 AM. Results: Using fluorescent microscopy and immunohistochemistry, we identified a more than 3 fold increase in EPO receptor expression in E18 cortical neurons after prior exposure to hypothermia. EPO dependent intracellular calcium signaling was increased by more than 2 fold by prior exposure to hypothermia. Western blot analysis demonstrated a similar increase in EPO receptor expression caused by hypothermia alone as was demonstrated with a 1 hour period of hypoxia. Conclusions: In isolated cortical neurons exposure to hypothermia alone can increase EPO receptor expression, and hypothermia plus EPO combined to significantly increase calcium signaling. Our results suggest that hypothermia-induced EPO receptor upregulation could be a mechanism for the neuroprotective effect of hypothermia previously described. We are currently examining the combined effects of hypoxia followed by hypothermia on EPO receptor expression and cellular signaling.

\section{DOES NICOTINE PROTECT AGAINST BRAIN DAMAGE?}

J H Andresen ${ }^{1}$, R Solberg ${ }^{1}$, B H Munkeby ${ }^{1}$, B Stray Pedersen ${ }^{2}$, O D Saugstad ${ }^{1}$

${ }^{1}$ Dept of Pediatric Research, ${ }^{2}$ Dept of Obstetrics and Gynecology, National Hospital, University of Oslo, Norway

Background and Aims: Inflammation is regarded a main cause of cerebral damage, and is believed to be exacerbated by hypoxia. Nicotine has been shown to have anti-inflammatory and anti-apoptotic properties in experimental studies. We wish to assess the effect of nicotine on newborn piglet's ability to tolerate hypoxia. Methods: Four groups of newborn piglets (12-36 hours) were studied: In two groups animals were randomized to receive either saline or nicotine prior to hypoxia, in two other groups they received nicotine or saline without hypoxia. Global hypoxia was induced by ventilation with $8 \%$ oxygen in N2 until Base Excess $(\mathrm{BE})<-20$ $\mathrm{mmol} / \mathrm{L}$ or mean arterial blood pressure $(\mathrm{MABP})=15$ $\mathrm{mmHg}$. Microdialysis probes were stereotactically inserted into the striatum before the experiment started, 
to measure glycerol, a marker of hypoxic injury. Results: Mean time of hypoxia was $66.5 \mathrm{~min}$. (SD 19.7) in the saline group, and $103.8 \mathrm{~min}$. (SD 28.2) in the nicotine treated group $(\mathrm{p}=0.036)$. $\mathrm{BE}$ and $\mathrm{pH}$ were lower in the nicotine pretreated group $(\mathrm{p}=0.11$ and 0.05$)$ at time of resuscitation, MABP was not significantly different. There were no significant differences at the end of the experiment. Microdialysis showed no differences between the two groups regarding glycerol in striatum.

Conclusions: The difference in time till ended hypoxia could, partly, be explained by the fact that nicotine activates the sympathetic nervous system. There are no indicators of negative effects of nicotine in this study; on the contrary the nicotine treated piglets did not show higher values of glycerol despite longer time till ended hypoxia.

\section{THE A-B-C OF STREPTOCOCCAL INFECTIONS IN THE PICU}

\section{N J G Jansen, A J van Vught}

Pediatric Intensive Care Unit, Dept of Pediatrics, The Wilhelmina Children's Hospital, University Medical Center Utrecht, The Netherlands

The group A streptococcus (GAS), also known as Streptococcus pyogenes, causes a wide range of infections and complications. It is the most frequent cause of bacterial pharyngitis. But it can also cause a variety of cutaneous and invasive infections, such as pyoderma, erysipelas, cellulitis, necrotizing fasciitis, Toxic Shock Syndrome (TSS) and myositis, and other complications such as rheumatic fever and acute glomerulonephritis. Although the global burden of GAS infections it not known it has been described that at least 517.000 people die each year due to severe GAS infections, eg acute rheumatic fever and invasive infections. Therefore, GAS figures among the 10 major human pathogens worldwide, only clearly exceeded by HIV, tuberculosis, malaria and Streptococcus pneumoniae. GAS is a well known cause of severe infections and sepsis/shock in the pediatric intensive care unit (PICU) with a reported mortality rate of $15-25 \%$. The wide range of diseases by GAS with varying severity has been postulated to be due to phenotypic variation in GAS. Differences in virulence factor productions, in ability to invade human epithelial cells and in secretion of high concentrations of exotoxins (A-B-C) are described. However, very recently the difference between a simple GAS pharyngeal disease and a more disseminated, severe disease, eg. Toxic Shock Syndrome or necrotizing fasciitis, is traced to mutations in two regulatory genes. A single mutation in a global two-component signalling locus, the so-called Control of virulence R/S (CovRS) system, alters expression of $10-15 \%$ of the genes. The mutation in CovRS shifts the expression from a pharyngeal transcriptome (PTP) to an invasive transcriptome (ITP), which can result in more invasive disease in human and mice. By ongoing knowledge at the molecular and gene-expression level, it becomes more and more clear how bacteria interact with their hosts to cause a range of diseases that are associated with infection. As translational medicine, from laboratory to bedside (PICU), develops further it will be possible to treat patients in the future more directly at the molecular-level of their disease. Also the development of a vaccine against GAS is a possibility to reduce the colonisation of tonsils and eliminate the dreadful forms of streptococcal infections. In the mean time the treatment of invasive GAS infections includes management of the complications of sepsis, aggressive surgical debridement if a site of infection is identified and antibiotics for the underlying infection. GAS remains susceptible to beta-lactam antibiotics. However, clinical failures of penicillin therapy for streptococcal infections do occur. Therefore, Clindamycin is recommended in invasive GAS infections together with penicillin or a carbapenem. Infections with GAS are still an important cause of morbidity and mortality, especially in less developed countries, and its global importance has been under-estimated. By new developments in molecular and translational medicine in the near future it will be possible to improve outcome in GAS infections substantially.

\section{THE INCIDENCE OF INFECTIONS OF VENTRICULAR RESERVOIRS IN THE TREATMENT OF POST HAEMORRHAGIC VENTRICULAR DILATATION}

A J Brouwer ${ }^{1}$, F Groenendaal ${ }^{1}$, A van den Hoogen ${ }^{1}$, M Verboon-Maciolek ${ }^{1}$, P Hanlo ${ }^{2}$, K Rademaker ${ }^{1}$, L S de Vries ${ }^{1}$

${ }^{1}$ Departments of Neonatology, ${ }^{2}$ Department of Neurosurgery, Wilhelmina Children's Hospital, University Medical Centre Utrecht, The Netherlands

Background and Aims: Infants with a progressive posthaemorrhagic ventricular dilatation (PHVD) have been treated in our neonatal intensive care unit with a ventricular reservoir since 1992. The incidence of infection using this invasive procedure was studied retrospectively.

Methods: Between January 1992 and December 2003, 76 preterm infants were treated with a ventricular reservoir. Infants admitted during two subsequent periods were analysed, group 1:1992-1997 $(n=26)$ and group 2:1998-2003 $(n=50)$. Clinical characteristics and number of reservoir punctures were evaluated. Furthermore the incidence of complications over time was assessed, with a focus on the occurrence of infection of the reservoir.

Results: The number of punctures did not change during both periods. Infection was significantly less common during the second period, 2/50 (4\%), compared to the first period, $5 / 26(19,2 \%), \mathrm{p}=.029$.

Conclusions: The use of a ventricular reservoir is a safe therapy to ensure adequate cerebrospinal fluid (CSF) removal in preterm infants with PHVD. In experienced hands the incidence of infection of the ventricular reservoir or major complications remains within acceptable limits. 
VISUALISATION OF WOUNDS TO IMPROVE THE QUALITY OF WOUND CARE

E J Dekker ${ }^{1}$, B A H Versluis ${ }^{2,3}$

${ }^{I}$ PICU, ${ }^{2}$ Directorate Information, Department of Management and Maintenance Information Systems, ${ }^{3}$ PICU/NICU, Erasmus MC - Sophia Children's Hospital, Rotterdam, The Netherlands

Background and Aims: Physicians and nurses on a Pediatric Intensive Care Unit (PICU) are being confronted with a diversity of patients wounds. Wounds were being observed subjectively by physicians and nurses. A wound care assessment tool was not used. Our PICU is organized with a Patient Data Management System (PDMS). This PDMS system provides the PICU staff the possibility to develop a digital photo registration of the wound that can be downloaded to the PDMS system. The aim of this project is to use the developed digital photo system in the PDMS to guide the process of wound care and eventually improve wound rehabilitation in the critically ill child.

Methods: Photos are made by using a digital camera. After taken the photo of the wound, the photo is downloaded into the Picture Archiving and Communication System (PACS). Photos are linked to the PDMS, to the family name and patient identification number. Photos can be shown on the bedside computer.

Results: With the help of a simple digital registration of a wound in our PDMS, everybody can see and compare the development and the healing process of the wounds of the patient. During the daily medical and nursing rounds the photos are reviewed and if necessary the wound care therapy is changed. This results in an objective wound judgment, assessment and wound care, which prevents mistakes.

Conclusions: Digital photo registration in combination with a PDMS does not only lead to an objective wound care assessment, but also to an unambiguous way of wound care.

\section{THE SPANISH CONNECTION}

M A Gonzalez Caviedes

Madrid, Spain

Abstract not available at the time of printing.

\section{HAVE WE LOST THE BASICS OF OUR NURSING ROLE?}

\section{J Heijstek}

The Netherlands

Abstract not available at the time of printing.

\section{PRESENT AND FUTURE OF SURFACTANT THERAPY. LUCINACTANT'S CONCEPT OF PROPHYLAXIS AND THERAPY}

\section{F Moya}

USA

Abstract not available at the time of printing.
THE 'NEW' APPROACH TO THE

PATHOPHYSIOLOGY OF

BRONCHOPULMONARY DYSPLASIA

S H Abman, J P Kinsella

Department of Pediatrics, University of Colorado School of Medicine, The Children's Hospital, Denver, CO, USA

Background and Aims: Despite surfactant therapy and improvements in perinatal care, premature infants remain at high risk for the development of bronchopulmonary dysplasia (BPD). Strong preclinical data demonstrated that impaired production of nitric oxide (NO) contributes to the pathogenesis of abnormal alveolar and vascular growth in experimental models of $\mathrm{BPD}$, and that prolonged treatment with inhaled NO (iNO) improves lung structure and function in neonatal animals. However, whether iNO therapy could improve long-term outcomes of premature newborns remains uncertain. We hypothesized that prolonged treatment with low doses of iNO would reduce the incidence of BPD without worsening brain injury. We further hypothesized that the effects of iNO therapy may be partly dependent upon the degree of prematurity.

Methods: We conducted an IRB-approved, multicenter randomized trial at 16 centers to determine the effects of iNO in preterm newborns (gestational age $<34$ weeks; BW, $500-1250$ gms) who required intubation and mechanical ventilation during the first 48 hours of life. Newborns were stratified by BW strata (500-749 g, $750-999 \mathrm{~g}$, or $1000-1250 \mathrm{~g}$ ) prior to randomization to iNO (5 ppm) or placebo gas. Assignment remained masked to care providers and families. BPD was defined by an oxygen requirement and abnormal chest $\mathrm{x}$-ray at 36 weeks corrected age. Cranial ultrasounds were obtained prior to enrollment (for baseline incidence of intraventricular hemorrhage), at 7-14 days, and at more than 30 days of age. Power analysis determined that 792 infants were needed to detect a reduction in the incidence of death and BPD from $60 \%$ to $50 \%$, given a statistical power of $80 \%$ and a 2 -sided alpha of 0.05 .

Results: Of the 4562 infants screened, 1921 met enrollment criteria, and 793 infants were enrolled: 398 to receive inhaled NO and 395 for placebo. The most common reason for ineligibility was nCPAP or expected early extubation. Mean BW (786 g), gestational age (25.6 weeks), male gender (53\%), and use of antenatal steroids (79\%) in the iNO group were not different from controls. Age at randomization (30 hours), oxygenation index (5.4), surfactant use (78\%), use of high-frequency ventilation $(29 \%)$ and related baseline characteristics in the iNO patients were also not different from controls. Patients were treated with a mean duration of 12 days. Overall, there was no difference in the incidence of death and BPD; however, for infants between 1000$1250 \mathrm{~g}$, iNO reduced BPD and death or BPD by $50 \%$ $(\mathrm{p}<0.001)$ and $40 \% \quad(\mathrm{p}<0.004)$, respectively. INO therapy also reduced the incidence of IVH, PVL or ventriculomegaly from $24 \%$ to $17 \%(p=0.03)$ for the entire group, but was most marked in the 750-999 $\mathrm{g}$ substratum.

Conclusions: These findings suggest that low dose iNO did not reduce the incidence of BPD for the overall group, but reduced BPD by $50 \%$ in infants over $1000 \mathrm{~g}$ 
at birth. However, iNO markedly reduced signs of brain injury for the overall group. Mechanisms underlying these responses are uncertain, but likely reflect the important influence of early versus later stages of lung development among the premature newborns, and may reflect distal organ effects of iNO therapy.

\section{NOVEL DEVELOPMENTS IN VACCINOLOGY}

\section{R Moxon \\ Oxford Vaccine Group, Department of Paediatrics, University of Oxford, UK}

Immunisation is currently the most effective public health tool for reducing the enormous global morbidity and mortality from infections. In humans, effective vaccines are second only to the availability of clean water in terms of their impact on reducing the burden of infections. In humans, vaccines have eliminated or substantially reduced at least nine major viral and bacterial infections. These successes must be viewed in the context of the huge unmet challenges for which novel developments in vaccines can provide enormous benefits in the future. The challenge of providing improved or novel vaccines comes at a time when the rise in the prevalence of antibiotic resistance poses diabolical problems and the global spread of pathogens is increasingly facilitated by unprecedented population densities in megacities, rapidity of travel, complex food chains and the many natural and human- inflicted disasters that disrupt societal infrastructures. This discussion will focus selectively on some of the potential opportunities provided by the application of 'cutting edge' science for the improvement of our current vaccines and future development of novel vaccines.

\section{VACCINATION AND OTITIS MEDIA}

\section{R Dagan}

Pediatric Infectious Disease Unit, Soroka University Medical Center and the Faculty of Health Sciences, Ben-Gurion University of the Negev, Beer-Sheva, Israel

Background and Aims: Otitis media (OM) is the most common bacterial infection in children aged $<5$ years for which antibiotic treatment is prescribed worldwide. Although most of the time this entity resolves spontaneously it is associated with morbidity, family dysfunction, antibiotic use and burden on the medical system. Efforts to reduce the burden of $\mathrm{OM}$ by vaccination have not been extremely rewarding, but some progress has been made. Methods: Review of the topic. Results: The first obvious step would be to reduce viral infections leading secondarily to OM. In the modern era, the only viral vaccine with proven effect on $\mathrm{AOM}$ is the influenza virus vaccine. Both the inactivated and the live virus showed some effect, but since influenza virus has only a limited season yearly the effect on the overall OM rate is far from being remarkable. Haemophilus influenzae $(\mathrm{Hi}) \mathrm{b}$ vaccine did not reduce $\mathrm{OM}$ since most $\mathrm{Hi}$ causing $\mathrm{OM}$ are nontypable (NTHi) and not Hib. The newly developed pneumococcal conjugate vaccines (PCVs) have all been shown to reduce $>50 \%$ of the $\mathrm{OM}$ caused by the serotypes included in the vaccines, but some replacement with serotypes not included in the vaccines and non pneumococcal organisms was demonstrated to reduce the overall effect of pneumococcal vaccines. The effect of PCV on the reduction of recurrent OM, OM with effusion, the need for ventilation tubes and frequent visits for AOM has been suggested, and the real impact is still being studied. Aiming with PCV at those with established recurrent $\mathrm{OM}$ has proved disappointing. $\mathrm{PCV}$ can reduce $\mathrm{OM}$ caused by antibiotic-resistant S. pneumoniae but the continued overuse of antibiotics is responsible for the increase in antibiotic resistance in non-vaccine serotypes. A newly developed PCV with an outer membrane protein for Hi (PnPD) is suggested to reduce also $\mathrm{OM}$ caused by $\mathrm{Hi}$, but confirmation studies are needed. New protein vaccines with proteins for $\mathrm{Hi}$, $\mathrm{S}$. pneumoniae and $\mathrm{M}$. catarrhalis are under development, but still far from being studied clinically.

Conclusions: Some vaccine can advance OM burden but at least in the next 1-2 decades OM will continue to be an important disease in children.

\section{VACCINATION IN DEVELOPING COUNTRIES}

\section{H Nohynek}

Helsinki, Finland

Abstract not available at the time of printing.

\section{NOVEL VACCINATION STRATEGIES}

\section{H Rümke}

The Netherlands

Abstract not available at the time of printing.

\section{INFLAMMATION, PRETERM DELIVERY AND THE CNS}

\section{H Hagberg}

Perinatal Center, Sahlgrenska Academy, East Hospital, KK, Goteborg, Sweden

Infection causes spontaneous preterm delivery especially at low gestation $(<30$ weeks). These infections can be in the urinary tract, peritoneal cavity (appendicitis), oral cavity (periodontal disease) or be systemic suggesting transplacental passage for some of these infections. More commonly, however, microbes are believed to ascend into the uterine cavity from the lower genital tract. A maternal inflammation is induced with infiltration of polymorphonuclear leukocytes in the chorioamniotic membranes and in the chorion plate of the placenta. Eventually, inflammatory cells accumulate also in the umbilical cord indicating a fetal inflammatory response. Indeed, a multitude of cytokines and chemokines accumulate in the cervicovaginal fluid, amniotic fluid and fetal blood and the levels exhibit an association with preterm delivery and neonatal morbidity. Except for treatment of urinary tract infections, antibiotic treatment of patients with bacterial 
vaginosis or those presenting with preterm contractions have not been successful in prolongation of pregnancy or in reducing neonatal morbidity. This could be due to 1. that preterm birth has multiple causes and, hence, only infectious-related preterm birth could be expected to respond to anti-microbial treatment. 2. Infection induces inflammation which is considered to be critical for induction of preterm birth and neonatal organ damage. Antibiotics may have to be combined with antiinflammatory therapies to be effective, e.g. progesterone and corticosteroids have both proved beneficial and they are both strong anti-inflammatory agents. 3. The infectious/inflammatory process starts early in gestation and treatment at the time of preterm contractions may be too late. Indeed, it has been shown that the presence of ureaplasma Urealyticym or increased cytokines in the amniotic fluid at midgestation predict late abortions/preterm birth. We hypothesize that such chronic "low-intensity" inflammation during pregnancy may be critical not only with respect to preterm birth but also to long-term CNS development, vulnerability and injury. Systemic administration of lipopolysaccharide (LPS) in adult animals induces upregulation of cytokines and activation of microglia in the brain, which was accompanied by a substantial decrease in CNS neurogenesis. High doses of LPS applied between the chorio-amniotic membranes in pregnant mice at embryonic day (ED) 15 (75\% gestation) induces preterm birth. However, much lower doses of LPS at ED 15 does not induce preterm birth but elicits a chronic state of CNS inflammation and the animals develop more extensive brain lesions if later exposed to hypoxiaischemia during postnatal life (Wang et al. 2006). Furthermore, maternal LPS induces inflammation, hypomyelination and cell death in the brain of the postnatal offspring. Intrauterine infection causes preterm birth in a subset of patients. Clinical and experimental studies suggest that the inflammatory process starts early in gestation. The immuno-inflammatory activation during pregnancy may have programming long-term influences on CNS development and vulnerability. References: Romero et al. Semin Neonatol 7:25974,2002; Espinoza et al. AJOG 194:630-7,2006; Hagberg and Mallard, Curr Opin Neurol 18:117-123, 2005; Monje et al. Science 302:1760-1765, 2003; Elovitz et al. Am J Pathol.163:2103-11, 2003; Rousset et al. Pediatr Res. 59:428-33, 2006.

\section{PREVENTION OF INFLAMMATORY DAMAGE - IS A DOUBLE-EDGED SWORD NEEDED TO FIGHT HYDRA IN WONDERLAND?}

O Dammann ${ }^{1,2}$, P Gressens ${ }^{3}$

${ }^{l}$ Perinatal Infectious Disease Epidemiology Unit, Hannover Medical School, Hannover, Germany ${ }^{2}$ Neuroepidemiology Unit, Children's Hospital and Harvard Medical School, Boston, MA, USA ${ }^{3}$ UMR 676 and INSERM-Paris 7 and Service de Neuropediatrie, Hopital Robert Debre, Paris, France

Knowledge continues to accumulate from perinatal and neonatal inflammation research suggesting that each solution to a riddle in this field is accompanied by the appearance of (at least two) new ones. This reminds us of the mythical serpent Hydra, one of whose nine heads was immortal. The beast quickly grew two new heads for each one Hercules chopped off. Recent research suggests that inflammatory processes play a role in most, if not all, neonatal disorders of the brain, eye, lung, and gut. Moreover, systemic inflammation issues require close attention. We should not surrender to complexity, but take it as a stimulation to move ahead. The traditional view that the immune system's primary task is to maintain health by fending off 'non-self' has been challenged by Polly Matzinger (Science, 2002), who suggests to give 'danger' the role of the offender. Her model proposes that alarm signals from stressed tissues rather than specific forms of antigen define the design of the immune response. Although this theory might explain some non-self-tolerance phenomena rather elegantly, we are now obviously left with the task to identify and characterize the alarm signals proposed by the theory. Much of the danger theory revolves around the mechanisms of adaptive immunity (i.e., Tcell activation). Thus, we will need to clarify whether the innate immune response itself constitutes a danger signal, and how much of the danger signal we call 'inflammation' causes damage in and of itself. Finally, we need to figure out how we can prevent the consequences of such danger. For those who consider adult medicine less than straight forward, fetal / neonatal medicine might look like Wonderland - a strange world, where almost nothing is to be taken at face value and apparent paradoxes wait for us at every corner. Here are three rather simplistic examples: antenatal infection reduces postnatal respiratory distress, but increases the risk for longterm bronchopulmonary dysplasia (Watterberg et al, 1996); antenatal glucocorticoids reduce the risk of developmental disability, while neonatal glucocorticoids increase the risk (O'Shea \& Doyle, 2001); maternal, but not fetal, administration of steroids reduce growth (Newnham et al, 1999). In this presentation, we offer speculations about potential future developments in perinatal and neonatal care in order to begin planning preventive intervention. Much of the work that needs to be done involves concepts such as signal detection, characterization of danger, and intervention design. Extreme caution needs to be exerted when working on these issues, because inflammation might (in part) be beneficial. Therefore, antiinflammatory strategies are likely to come from the armamentarium of double-edged swords. According to Greek mythology, Hydra was eventually killed only because Iolaus helped his uncle to chop off the immortal ninth head. Unfortunately, because Hercules had not managed to do it all by himself, the success was not counted towards his ten labors. Still, we believe that it will take exactly this kind of teamwork to eventually reduce the dangers brought to perinatal Wonderland by inflammation.

\section{HYPEROXIC RESUSCITATION INCREASES OXIDATIVE STRESS AND CHANGES IN GENE EXPRESSION}

R Solberg $^{1}$, J Andresen ${ }^{1}$, S Tunheim ${ }^{2}$, G I A Borge ${ }^{3}$, M Bjørås ${ }^{4}$, B H Munkeby ${ }^{1}$, O D Saugstad ${ }^{1}$ 
${ }^{l}$ Department of Pediatric Research, University of Oslo, Rikshospitalet University Hospital, Oslo, Norway ${ }^{2}$ Centre for Occupational and Environmental Medicine, University of Oslo, Rikshospitalet University Hospital, Oslo, Norway, ${ }^{3}$ Norwegian Food Institute, As, Norway ${ }^{4}$ Centre for Molecular Biology and Neuroscience, University of Oslo, Rikshospitalet University Hospital, Oslo, Norway

Background and Aims: Resuscitation at birth with pure oxygen is associated with long term detrimental effects. We tested if there is a dose-response correlation between oxygen level and subsequent changes in antioxidant capacity and gene expression.

Methods: Piglets (12-36h) underwent hypoxia until BE$20 \mathrm{mmol} / 1$ or blood pressure $<15 \mathrm{mmHg}$, and were then resuscitated for $15 \mathrm{~min}$ with $21 \%, 40 \%, 60 \%$ and $100 \%$ oxygen $(n=12, n=10, n=10, n=10$, baseline $n=10)$ and thereafter observed for $1 \mathrm{~h}$. To study gene-expression we used GeneChip ${ }^{\circledR}$ Porcine Genome Array. The Microarrays were performed through isolation of RNA from snap frozen lung tissue. DNA repair activities counteracting the effect of base lesions on DNA, including oxidative damages, were monitored in cellfree protein-extracts.

Results: The $100 \%, 60 \%, 40 \%$ and $21 \%$ group had a mean arterial pO2 value at respectively 41.7, 26.1, 20.0 and $12.4 \mathrm{kPa}$ after $15 \mathrm{~min}$ of resuscitation, $(\mathrm{p}<0.001)$. Total-antioxidant-capacity were significantly lower in lung-tissue from piglets resuscitated with 40,60 or $100 \%$ oxygen compared to $21 \%, \quad(p<0,02)$. The Microarray-study in the room-air and $100 \%$ group, showed significant difference in gene-expression profiles for genes related to cell-replication and inflammatory response. The capacity to repair base lesions on DNA was significantly reduced after resuscitation.

Conclusions: Hypoxia and subsequent resuscitation for 15 min with high percentage oxygen gives a dose dependent hyperoxia with increased oxidative stress and risk of cell damage and long term consequences such as accumulation of mutations in the genome. Hyperoxia leads to changes in expression of genes related to cellreplication and inflammatory response compared to room-air resuscitation.

\section{CHEMOKINE MRNA LEVELS IN PRETERM NEONATES WITH INFLAMMATION}

A Mouchtouri $^{1}$, E Levi ${ }^{1}$, E Petrakou $^{1}$, G Liosis $^{2}$, M Xanthou ${ }^{1}$, S Fotopoulos ${ }^{1}$

${ }^{l}$ Neonatal Immunology Laboratory, B' Neonatal Intensive Care Unit, "Aghia Sophia" Children's Hospital, Athens, Greece, ${ }^{2}$ Neonatal Unit, "Elena Venizelou” Perinatal Centre, Athens, Greece

Background and Aims: Inflammation due to perinatal infection and asphyxia may cause damage to various tissues and very often to the immature brain of the fetus and the newborn. Previously we have shown that the neonatal immune system has the ability to produce increased chemokine protein levels in the serum during the inflammatory response caused by perinatal infection and asphyxia. The aim of our present study was to investigate mRNA levels of the proinflammatory chemokines IL-8 and MCP-1 in infected and asphyxiated neonates.

Methods: Forty-two premature neonates were studied; 11 with perinatal infection (PI), 16 with perinatal asphyxia (PA) and 15, without PA and PI, were used as controls. IL-8 and MCP-1 mRNA levels were investigated in whole blood and in PHA-activated lymphocytes using semi-quantitative PCR and real time PCR, respectively.

Results: IL-8 mRNA levels were significantly increased in whole blood both during PA and PI, while MCP-1 mRNA levels were not. In vitro activated lymphocytes expressed significantly increased IL-8 mRNA levels during PI, whereas no increase was observed during PA. MCP-1 mRNA levels were significantly increased in activated lymphocytes during perinatal asphyxia while no increase was observed during PI.

Conclusions: Our data show that chemokine mRNA levels expressed by activated lymphocytes during inflammation caused by perinatal infections are different to those expressed during perinatal asphyxia. These findings might have important implications during the administration of specific chemokine antagonists in order to prevent or reduce tissue damage caused by inflammation.

\section{EFFECTS OF HYPEROXIA AND NITRIC OXIDE ON ENDOGENOUS NITRIC OXIDE PRODUCTION IN POLYMORPHONUCLEAR LEUKOCYTES}

\author{
T Hoehn ${ }^{1}$, A Gratopp ${ }^{2}$, K Raehse $^{3}$, P Koehne ${ }^{3}$ \\ ${ }^{l}$ Neonatology and Pediatric Intensive Care, Department \\ of General Pediatrics, University Hospital of \\ Duesseldorf, Germany, ${ }^{2}$ Department of General \\ Pediatrics, Charité Virchow Hospital, Humboldt \\ University, Berlin, Germany, ${ }^{3}$ Department of \\ Neonatology, Charité Virchow Hospital, Humboldt \\ University, Berlin, Germany
}

Background and Aims: Exposure towards hyperoxia and nitric oxide (NO) occur frequently during the treatment of neonatal hypoxic pulmonary failure. To analyse the response of neonatal polymorphonuclear neutrophils (PMN) to hyperoxia and NO in vitro.

Methods: Neonatal cord blood was exposed to $100 \%$ oxygen, NO 20ppm plus $100 \%$ oxygen, and NO $20 \mathrm{ppm}$ for 30 minutes. Flow cytometry was used to quantify production of nitric oxide by measurement of the intracellular dye DAF-2 DA.

Results: DAF-2 DA signals following equilibration with room air were used as controls. Exposure to $100 \%$ oxygen increased NO production significantly when compared to NO 20ppm plus $100 \%$ oxygen $(\mathrm{p}=0,031)$ and to NO 20ppm alone $(\mathrm{p}=0,006)$.

Conclusions: Exposure of human PMN cells to hyperoxia induces intracellular upregulation of endogenous NO production, whereas exposure to exogenous NO does not. Hyperoxia - whether in combination with NO or not - can lead to NO-induced apoptosis. This needs to be taken into account in the treatment of clinical conditions requiring high inspiratory concentrations of oxygen and/or inhaled NO. 
IS CHORIOAMNIONITIS ASSOCIATED WITH ADVERSE NEONATAL OUTCOME INDEPENDENT OF ITS EFFECT ON PRETERM BIRTH?

J V Been ${ }^{1}$, R F Kornelisse ${ }^{2}$, I Rours ${ }^{2}$, F Jonkers ${ }^{3}$, A J Schneider ${ }^{4}$, R R de Krijger $^{5}$, L J Zimmermann ${ }^{1}$

${ }^{I}$ Department of Neonatology, Maastricht University Hospital, Maastricht, The Netherlands, ${ }^{2}$ Department of Paediatrics, Erasmus MC, Rotterdam, The Netherlands ${ }^{3}$ Department of Internal Medicine, Maastricht University Hospital, Maastricht, The Netherlands ${ }^{4}$ Department of Obstetrics, Erasmus MC, Rotterdam, The Netherlands, ${ }^{5}$ Department of Pathology, Erasmus $M C$, Rotterdam, The Netherlands

Background and Aims: Several retrospective studies have shown chorioamnionitis to be associated with adverse neonatal outcome. However, findings have been contradictory and despite the fact that chorioamnionitis is an important cause of preterm delivery, few studies have adjusted their data for gestational age. Our aim was to compare short-term outcome parameters between patients with and without chorioamnionitis in a large prospective study.

Methods: Over a two-year period all infants born in the Erasmus MC at $\leq 32$ weeks gestation were prospectively included in this study. Relevant clinical data and placental histopathology were obtained from each infant. Outcome parameters were compared between infants with histological chorioamnionitis (HC) and those without (non-HC).

Results: 303 Patients were included, 119 (39.3\%) of whom had HC. Patient characteristics and outcome parameters are summarised in the table. After correction for gestational age differences, the only relevant outcome parameter significantly associated with $\mathrm{HC}$ was NEC.

Conclusions: In this prospective study, chorioamnionitis was not shown to be associated with adverse neonatal outcome after correction for gestational age. An increased incidence of NEC after $\mathrm{HC}$ was found, but larger studies are needed to confirm this association. Our findings are in contrast with earlier retrospective studies, most of which have not adjusted for gestational age. This study shows that the impact of chorioamnionitis on short-term outcome is mainly mediated through its causal relationship with preterm delivery.

\begin{tabular}{|l|c|c|l}
\hline & non-HC $(n=184)$ & $H C(n=119)$ & $p$-value \\
\hline gestational age (median (IQR)) & $30(28-31)$ & $28(26-30)$ & $<0.001$ \\
\hline birth weight (median (IQR)) & $1080(880-1365)$ & $1110(865-1405)$ & 0.45 \\
\hline$\%$ RDS & 54 & 60 & 0.36 \\
\hline$\%$ BPD at 28 days & 28 & 43 & $<0.05$ \\
\hline$\%$ BPD at 36 weeks & 20 & 24 & 0.59 \\
\hline$\%$ NEC & 1 & 9 & $<0.01$ \\
\hline$\%$ IVH & 11 & 24 & $<0.01$ \\
\hline$\%$ IVH grade 3/4 & 4 & 6 & 0.55 \\
\hline$\%$ CPVL & 3 & 3 & 0.75 \\
\hline$\%$ septicemia & 46 & 50 & 0.50 \\
\hline$\%$ death & 8 & 15 & 0.06 \\
\hline
\end{tabular}

\section{BILE ACIDS IN BRONCHO-ALVEOLAR LAVAGE FLUID OF NEAR-TERM NEONATES WITH SEVERE RDS}

E Zecca ${ }^{1}$, S Baroni ${ }^{2}$, D De Luca ${ }^{1}$, M Marras $^{1}$, G Vento ${ }^{1}$, C Romagnoli ${ }^{1}$
${ }^{1}$ NICU, Division of Neonatology, Catholic University of The Sacred Heart, Rome, Italy, ${ }^{2}$ Institute of Biochemistry, Catholic University of The Sacred Heart, Rome, Italy

Background and Aims: Intrahepatic cholestasis of pregnancy (ICP) is associated with an increased risk of RDS in near term neonates. [1] The diagnosis of bile acid pneumonia has been proposed on the basis of a causative role of bile acids (BA) [2]. Because hyaline membranes have been demonstrated in rabbits after tracheal instillation of BA [3] we hypothesized that abnormally high BA levels in ICP could reverse the reaction of phospholipase-A2 in the alveoli, leading to a lack of surfactant. Finding detectable BA in the lung of affected infants could strongly support this hypothesis.

Methods: We measured BA levels in broncho-alveolar lavage (BALF) of 4 neonates affected by BA pneumonia and in 7 control infants, matched for gestational age, who required mechanical ventilation for reasons different from lung diseases. BA were analyzed, as previously published, [4] in the first day of life. Maternal and neonatal plasma BA levels were also recorded.

Results: BA were detected in BALF of infants affected by $\mathrm{BA}$ pneumonia (median: $0.77 \mu \mathrm{mol} / \mathrm{l}$; i.q. range: $0.43-2.8 ; \mathrm{p}<0.001$ ) and were absent in controls.

Conclusions: Newborn infants from normal pregnancies do not have BA in the lungs. Our study demonstrates that abnormally high BA during ICP pregnancies pass into the alveoli of the newborn and produce a secondary surfactant deficiency reversing the phospholipase-A2 reaction.

\section{LUNG HEALING IN ARDS: ROLE OF ION CHANNELS}

\section{U H Thome}

Department of Pediatrics, University of Ulm, Germany

Background and Aims: The prenatal lung works as an exocrine gland and secretes fluid, which contributes to amniotic fluid and facilitates lung growth. After birth, fluid secretion has to cease as any excess fluid in the alveolar space will hamper gas exchange. Furthermore, excess fluid must be absorbed out of the alveolar space (alveolar fluid clearance). This is one of the most important processes in the transition to extrauterine life, and continuously thereafter. Expression of fluid by the birth canal is of minor importance. Cell physiologic and animal studies have elucidated the transport mechanisms and carrier proteins involved. Sodium ions are taken up by alveolar type II cells, previously known as the producers of surfactant, through epithelial sodium channels located in the apical membrane, and are actively extruded into the interstitial space by $\mathrm{Na}, \mathrm{K}$ ATPases in the basolateral membrane. Water follows passively the resulting osmotic forces. The fluid is removed from the interstitial space by blood capillaries and lymph vessels.

Methods: In respiratory distress syndrome, impaired epithelial transport and increased epithelial leakage result in increased alveolar fluid content, leading to impaired gas exchange and surfactant inactivation. 
Clinical studies have shown that the survival of patients suffering from acute respiratory distress syndrome is dependent on the activity of their alveolar fluid clearance. Likewise, the severity of respiratory distress in premature infants is linked to the activity of epithelial sodium transport mechanisms.

Results: Alveolar fluid clearance is currently much less amenable by therapeutic intervention than surfactant deficiency. Research has been directed towards potential therapies to stimulate alveolar fluid clearance and improve the clinical outcome of patients with severe lung failure. Potential therapies include such diverse options as beta-receptor agonists, steroid hormones, potassium channel activators, diuretics, and transfer of various genes by viral vectors. All these options are currently under investigation in cell culture experiments, animal and human studies. While there is not yet an established therapy available, the first promising randomized trial has been published this year.

Conclusions: The importance of alveolar fluid clearance for surviving severe lung failure is increasingly recognized. The mechanisms have been elucidated, and therapies to improve alveolar fluid clearance are under investigation.

\section{SURFACTANT IN PEDIATRIC ARDS}

\section{Gortner}

Department of Pediatrics, University Hospital, Homburg, Germany

Background and Aims: Secondary surfactant deficiency and increased pulmonary vasculare are the mainstays of pediatric ARDS. This disorder still is characterized by a substantial mortality and se-vere long-term sequelae. It was thus the aim, to assess the efficacy of surfactant treat-ment in pediatric ARDS after having established its efficacy in neonates with RDS.

Methods: Two controlled clinical trials have been conducted, investigating natural surfactant preparations in pediatric $\operatorname{ARDS}(1,2)$, a number of studies described its effects in case-series, however, without enrolling controls.

Results: Main endpoint in study 1 was improvement in oxygenation (pao2/Fio2) at 48 hours, in study 2 ventilator free days and mortality. In study 1 improved oxygenation was observed in surfactant treated children, however, mortality and days on the ventilator were unchanged between both groups. In study 2 , ventilator free days were not significantly different between both groups, however, mor-tality was increased in the placebo group (OR1.32/CI1.15 - 4.85). In both studies, im-proved oxygenation was observed within the first hours of the surfactant treatment. Mean mortality in both studies was $23 \%$ in surfactant treated children (23/97) compared with $40 \%$ (36/90) in the control group. Response to and mortality after surfactant seemed to be influenced most prominently in primary ARDS compared to secondary ARDS, i.e. isolated ARDS without underlying severe illness.

Conclusions: Further trials with clearly defined subgroups of children with primary ARDS should be carried out in order to better describe the effect of surfactant treatment in pediatric ARDS. 1. Möller JC,
Treatment with bovine surfactant in severe acute respiratory distress syndrome in chil-dren: a randomized multicenter study. Intensive Care Med (2003) 29: 437446.2. Wilson DF, Effect of exogenous surfactant (Calfactant) in pediatric acute lung injury. JAMA (2005) Vol. 293, No. 4.

\section{CEREBRAL OXYGENATION (ScO2), FRACTIONAL TISSUE OXYGEN EXTRACTION (FTOE) AND BRAIN ACTIVITY IN PRETERM INFANTS WITH RDS: THE EFFECT OF CPAP VERSUS SIMV AND HFOV}

P M Lemmers ${ }^{1}$, M Toet $^{1}$, L J van Schelven ${ }^{2}$, $\mathrm{F}$ van $\mathrm{Bel}^{1}$

${ }^{1}$ Department of Neonatology, Wilhelmina Children's Hospital, University Medical Centre, Utrecht, The Netherlands, ${ }^{2}$ Department of Biomedical Engineering, Wilhelmina Children's Hospital, University Medical Centre, Utrecht, The Netherlands

Background and Aims: In the etiology of damage to the immature brain, RDS-induced disturbances in cerebral oxygenation, hemodynamics and lack of cerebral autoregulation play an important role. To examine the effect of the type of ventilatory support (CPAP, SIMV, HFOV) on cerebral oxygenation, brain perfusion and activity, arterial $\mathrm{O} 2$ saturation $(\mathrm{SaO} 2)$, NIRS-determined cerebral O2-supply $(\mathrm{ScO} 2)$ and O2extraction (FTOE: $(\mathrm{SaO} 2-\mathrm{ScO} 2 / \mathrm{SaO} 2)$ ), blood pressure (MABP) and amplitude-integrated EEG (aEEG) were monitored during the first 72 hours of life.

Methods: From 36 preterms (GA $<32$ wks) with surfactant-dependent RDS 10, 14 and 12 were treated with CPAP with InSurE procedure (Intubation, Surfactant, Ectubation), synchronized intermittend mandatory ventilation (SIMV) or high frequency oscillatory ventilation (HFOV) respectively. From each patient an average of a $60 \mathrm{~min}$ reliable monitoring period was used every 6 hours (from birth up to day 3) for $\mathrm{SaO} 2, \mathrm{ScO} 2$, FTOE, MABP and aEEG. Correlation coefficients between MABP and $\mathrm{ScO} 2$ and between MABP and FTOE were determined every $15 \mathrm{~min}$ to detect possible lack of autoregulation.

Results: In CPAP/InSurE infants were signifantly higher MABPs and aEEg-activity, $\mathrm{ScO} 2$ and FTOE showed a clearly higher and lower trend respectively and there were less periods of significant correlations between MABP and $\mathrm{ScO} 2 / \mathrm{FTOE}$ as compared to SIMV/HFOV infants.

Conclusions: RDS-treatment using CPAP/InSurE was associated with a better cerebral oxygenation, more stable blood pressures and less periods of lack of cerebral autoregulation.

\section{THE EFFECT OF FUROSEMIDE ON ALVEOLAR EPITHELIAL CELL PROLIFERATION}

B Ackermann ${ }^{1,2}$, A W Flemmer ${ }^{1,2}$

${ }^{1}$ Department of Neonatology, Children's Hospital, Center of Perinatology, LM-University Munich,

Germany, ${ }^{2}$ Center of Pediatrich Research,

LM-University Munich, Germany 
Background and Aims: Furosemide, a potent inhibitor of $\mathrm{Na}-\mathrm{K}-2 \mathrm{Cl}$ cotransporter (NKCC), has been used repetitively for neonates with pulmonary disease. In airway smooth muscle cells it was suggested, that Furosemide inhibits cell proliferation. Yet the effect of Furosemide on alveolar epithelial cell proliferation has not been studied.

Methods: NKCC expression and distribution in alveolar epithelial cells (A549 cells) were assessed by immunoblotting and -staining. To test the effect of Furosemide on cell proliferation, alveolar epithelial cells (A549-cells) were cultured with Furosemide, at 0-1200 $\mu \mathrm{M}$. At 96 hours proliferation was determined by cell count related to seeded cells. Cell DNA-content was studied in permeabilized cells by fluorescence-activated cell sorting (FACS) using propidium iodide. Proliferating cells were defined as high-DNA-cells (double DNA content, G2 / mitotic), whereas low DNAcells were assumed to be in G1-phase.

Results: On both immunoblot and immunostaining NKCC was abundantly expressed in the cells. On confocal microscopy cotransporter was located to the basolateral membrane of the cells. Cell count after 96 hours decreased significantly with increased Furosemide concentration. Cell DNA analysis showed an increase of cells in G1-phase with increased Furosemide. Proportion of proliferating cells (G2 / mitotic) decreased significantly with Furosemide concentration.

Conclusions: Our results suggest that Furosemide, at concentrations comparable to those after Furosemideinstillation, inhibits cell proliferation of alveolar epithelial cells in vitro. However cellular mechanisms of inhibition remain unclear. According to our data the application of Furosemide to the premature lung (e.g. in small infants) might influence lung development, yet further studies are mandatory.

\section{LUCINACTANT (SURFAXIN®) REDUCES REINTUBATION RATES WITH IMPROVED OUTCOMES VS OTHER SURFACTANTS - RESULTS OF TWO RANDOMIZED, CONTROLLED TRIALS}

F R Moya ${ }^{1}$, S Sinha ${ }^{2}$, J Gadzinowski ${ }^{3}$, C Guardia ${ }^{4}$, $\mathrm{J}_{\text {Mazela }}{ }^{4}, \mathrm{G} \mathrm{Liu}^{4}$

${ }^{I}$ Department of Neonatology, Coastal AHEC, Wilmington, NC, USA, ${ }^{2}$ Department of Paediatrics, James Cook University Hospital, Middlesbrough, UK

${ }^{3}$ Department of Neonatology, Poznan University of Medical Sciences, Poznan, Poland, ${ }^{4}$ Discovery

Laboratories, Inc., Warrington, PA, USA

Background and Aims: Many infants who are extubated after initial surfactant administration require reintubation. Repeated or prolonged reintubation may place them at higher risk of adverse outcomes. We examined extubation and reintubation rates and their impact on key clinical outcomes in infants treated with lucinactant vs colfosceril and beractant from the SELECT trial (Moya, et al. Pediatrics. 2005;115:1018) and vs poractant from the STAR trial (Sinha, et al. Pediatrics. 2005;115:1030).

Methods: The rates of initial extubation, subsequent reintubation, and death or BPD were compared using logistic regression adjusted for birth weight stratum and center.

Results: Overall rates of extubation were similar across treatments in both trials (range $80.6 \%-83.9 \%$ for all randomized neonates; $\mathrm{P}=\mathrm{ns}$ ). The reintubation rate following initial extubation was significantly lower $(\mathrm{P}<.05)$ for infants treated with lucinactant compared with beractant or poractant (Table). Proportionally more lucinactant-treated infants who were extubated survived compared with other surfactants, but this difference did not reach significance. Survival without BPD at $36 \mathrm{wk}$ PMA among infants extubated favored lucinactant vs colfosceril ( $72.2 \%$ vs $66.0 \%, \mathrm{P}=.034)$.

Conclusions: Initial extubation rates were similar among infants treated with lucinactant and other surfactants; the rate of subsequent reintubation was significantly lower with lucinactant. Major morbidity and mortality in reintubated patients also were lower with lucinactant, mirroring primary observations previously reported. Supported by Discovery Laboratories.

\begin{tabular}{|c|c|c|c|c|c|}
\hline & \multicolumn{3}{|c|}{ SELECT } & \multicolumn{2}{|c|}{ STAR } \\
\hline & $\begin{array}{l}\text { Lucinactant } \\
\mathrm{N}=436^{\circ}\end{array}$ & $\begin{array}{l}\text { Colfoscent } \\
N=418^{*}\end{array}$ & $\begin{array}{l}\text { Beractant } \\
\mathrm{N}=208^{*}\end{array}$ & $\begin{array}{l}\text { Lucinactant } \\
\mathrm{N}=104^{*}\end{array}$ & $\begin{array}{l}\text { Poractant } \\
\mathrm{N}=106^{*}\end{array}$ \\
\hline Reintubation (\%) & 34.6 & 40.4 & $42.8^{\prime}$ & 32.7 & $47.2^{\prime}$ \\
\hline Mortalify at 28 d/ 36 wk PMA (\%) & $4.1 / 6.2$ & 6.018 .4 & $6.3 / 8.7$ & $1.9 / 3.9$ & $4.7 / 5.7$ \\
\hline
\end{tabular}

\section{HOW SMALL IS SMALL ENOUGH? DETERMINATION OF THE OPTIMAL AEROSOL PARTICLE SIZE FOR INHALATION THERAPY IN PRETERM INFANTS IN VITRO}

S Minocchieri ${ }^{1}$, M A Bachmann ${ }^{1}$, J Burren ${ }^{1}$, R Kaeser ${ }^{3}$, J H Wildhaber $^{2}$, M Nelle ${ }^{1}$

${ }^{I}$ Division of Neonatology, University Children's Hospital, Bern, Switzerland, ${ }^{2}$ Division of Respiratory Medicine, University Children's Hospital, Zurich, Switzerland, ${ }^{3}$ Division of Neonatal and Pediatric Intensive Care, Children's Hospital, Luzern, Switzerland

Background and Aims: As inhaled therapy in preterm infants with IRDS (Infant Respiratory Distress Syndrome) or CLD (Chronic Lung Disease) is difficult, the current therapy consists of Surfactant instilled through a tracheal tube or systemic steroids applied orally or intravenously. Current commercially available inhalation devices are not suitable for this age group. New mesh devices able to produce very small particles may allow age specific aerosol therapy and may be applicable even in preterm infants. This in vitro study assesses the particle size of nebulised aerosols and the distribution in the upper airways and estimates a predicted lung dose for preterm infants.

Methods: Budesonide was nebulised through a standard e-Flow device (Pari GmbH, Starnberg, Germany) with two different perforated vibrating membranes $(3.5 \mu \mathrm{m}$ and $2.5 \mu \mathrm{m}$ pore diameter) attached to an upper airway model of a 32 weeks gestational age preterm infant by a face mask followed by an NG (New Generation) impactor. Particle size distribution as well as lung dose were expressed as a percentage of the total dose and assessed using an HPLC-method.

Results: Median mass aerodynamic diameter (MMAD) of particles reaching the lungs was $1.6 \mu \mathrm{m}$ with a geometric standard deviation (GSD) of 1.5. Comparison 
of the two membranes showed a lung deposition of $17 \%$ \pm 1.055 and $23 \% \pm 1.087$ for the greater $(3.5 \mu \mathrm{m})$ and smaller $(2.5 \mu \mathrm{m})$ pores, respectively.

Conclusions: The difference between the two membranes is statistically significant (Mann-WhitneyU-Test, $\mathrm{p}<0.0001)$. Furthermore, we conclude that aerosols applied to preterm infants of 32 weeks of gestational age should have a MMAD of $<1.6 \mu \mathrm{m}$.

\section{THE EFFECT OF FETAL TRACHEAL OCCLUSION ON LUNG TISSUE MECHANICS IN A RABBIT MODEL FOR DIAPHRAGMATIC HERNIA}

A W Flemmer ${ }^{1}, \mathrm{~J} \mathrm{C} \mathrm{Jani}^{2,4}, \mathrm{~K}^{2,4}$ Hajek $^{1}$, F Bergmann $^{3}$, O J Münsterer ${ }^{3}$, J Deprest ${ }^{2,4}$

${ }^{I}$ Department of Neonatology, Children's Hospital Center of Perinatology Grosshadern, LM-University, Munich, Germany, ${ }^{2}$ Center for Surgical Technologies, Faculty of Medicine, Katholieke Universiteit, Leuven, Belgium, ${ }^{3}$ Department of Pediatric Surgery, LM University, Munich, Germany, ${ }^{4}$ Department of Obstetrics and Gynaecology, Faculty of Medicine, Katholieke Universiteit, Leuven, Belgium

Background and Aims: Diaphragmatic hernia (DH) is associated with high mortality and morbidity. Fetal tracheal occlusion (FETO) has been suggested to accelerate lung growth and development in $\mathrm{DH}$. We evaluated the effect of FETO on lung growth and postnatal lung tissue mechanics.

Methods: Left sided DH was surgically created in fetal rabbits at 23 days of gestation (term 31 days). 5 days later, FETO was performed and compared to non-FETO and sham operated animals. At term, fetuses were delivered by cesarean section. Lung to body weight ratio (LBWR), total lung capacity (TLC), dynamic compliance (Crs) and resistance (Rrs) were measured. In addition we measured lung input impedance by forced oscillation technique (FOT) and lung tissue elastance (HL), tissue resistance (GL), hysteresivity eta (GL/HL) and small airway resistance (Raw) were calculated from impedance data.

Results: LBWR was significantly increased in FETOanimals compared to $\mathrm{DH}(0.024 \pm 0.006$ vs. $0.010 \pm 0.004$ $\mathrm{g} / \mathrm{g}, \mathrm{p}=0.005)$. FETO increased TLC from $16.3 \pm 2.6$ to $19.6 \pm 3.7(\mathrm{p}=0.1)$ and significantly increased $\mathrm{Crs}$ but had no effect on Rrs. FETO had no effect on HL but resulted in increased GL. In contrast, FETO resulted in a significant decrease of $\mathrm{Rn}$ in these animals.

Conclusions: These data suggest that changes of $\mathrm{Crs}$ in $\mathrm{DH}$, due to FETO are rather a result of small airway and lung volume distention. According to our data, FETO potentially increases resistive forces of lung tissue (Gtiss). In our rodent DH-model FETO does not affect lung tissue elastance, deteriorated by DH.

\section{EDUCATION IN THE NEONATAL AND PAEDIATRIC INTENSIVE CARE}

\section{O Frauenfelder}

The Netherlands

Abstract not available at the time of printing.
EVALUATION OF AN INSULIN PROTOCOL TO TREAT HYPERGLYCAEMIA IN THE PICU

M M van de Polder, S B Brand, J J Verhoeven, K F M Joosten

Department of Pediatric Intensive Care, Erasmus MCSophia, Rotterdam, The Netherlands

Background and Aims: In critically ill children hyperglycaemia $(>8 \mathrm{mmol} / \mathrm{L})$ is associated with an increased mortality but it is not known if insulin therapy will improve outcome. Aim of the study was to evaluate a hyperglycaemic treatment protocol trying to lower blood glucose $<8 \mathrm{mmol} / \mathrm{L}$ within 12 hours after start of insulin treatment in critically ill children admitted to the PICU.

Methods: Retrospective evaluation of the incidence and treatment of hyperglycaemia of all children admitted to the PICU in relation to the glucose intake.

Results: In a 4 months period in 80 non-diabetic children blood glucose control was done within 24 hours after admission to the PICU. In $33 / 80$ of the children blood glucose was initially $>8 \mathrm{mmol} / \mathrm{L}$ (mean 10,5 $\mathrm{mmol} / \mathrm{L})$. The glucose intake in only 5 children was according to the protocol (in 6 too high and in 22 too low). Excluded for treatment were 20 children because a second blood glucose was normalised (13x) and various other reasons $(7 \mathrm{x})$. The mean interval between the first and second blood glucose control was $120 \mathrm{~min}$. Eight children were treated according the protocol. Mean duration of treatment was 55 hours (range 8-128 hr). In 7 of the 8 children within 12 hours (mean 10,5 hr) blood glucose was $<8 \mathrm{mmol} / \mathrm{L}$. No hypoglycaemia occurred.

Conclusions: In a high percentage of children admitted to the PICU hyperglycaemia was present but treatment was done in a selected group safely. The glucose intake was only in $15 \%$ according the protocol. The awareness of blood glucose control needs more attention.

\section{A RESEARCH PROGRAM TO EXAMINE EVIDENCE-BASED THERMOREGULATION PRACTICES IN PRETERM NEWBORNS}

M Schindler ${ }^{1}$, F Loersch ${ }^{1}$, S Dahlmann ${ }^{1}, \mathrm{C}_{\text {Herzner }}{ }^{1}$, I Berlet $^{1}$, T Schaible ${ }^{1}$, P Heep ${ }^{2}$, L Lynam ${ }^{2}$

${ }^{1}$ Neonatal Intensive Care, Klinikum Mannheim, Mannheim, Germany, ${ }^{2}$ GE Maternal Infant Care Clinical Research Department, Laurel, MD, USA

Background and Aims: Thermoregulation is a critical component of neonatal care. Yet many of the practices surrounding thermoregulation have not been re-tested despite the marked changes in viability has over the last 4 decades. With this foundation, one should realize that approximately $50 \%$ of global NICUs practice babycontrol philosophies of thermoregulation while the rest practice an air-control philosophy. Is the evidence base all that is should or could be?

Methods: Thirty preterm infants $<1500 \mathrm{~g}$ were randomized to either Giraffe baby control mode or Giraffe air control mode. The Giraffe was set-up and pre-warmed according to manufacturer's guidelines in closed bed/incubator/manual mode. The initial thermal goal was to maintain central skin temperature at 36.5 
degrees $\mathrm{C}$ in both groups and rectal temperature within a range of 36.8 to 37.3 degrees C. Added humidity was controlled at $60-80 \%$. Physiological variables were measured each minute over the period of data collection. Results: The table demonstrates key outcomes in this study population.

Conclusions: In this study, baby control seems to provide better control of preterm newborn infant temperature as compared to air control.

\begin{tabular}{|c|c|c|c|}
\hline Variable & $\begin{array}{c}\text { Baby Mode } \\
\mathrm{N}=7\end{array}$ & $\begin{array}{c}\text { Air Mode } \\
\mathrm{N}=8\end{array}$ & $\mathbf{P}$ \\
\hline $\begin{array}{l}\% \text { time skin temp } 36.5- \\
37.2\end{array}$ & 93 & 84 & .005 \\
\hline Average skin temp & 36.7 & 36.3 & .02 \\
\hline Average rectal temp & 36.75 & 36.5 & .06 \\
\hline Average peripheral temp & 35.1 & 34.12 & .03 \\
\hline $\begin{array}{l}\% \text { time skin-peripheral } \\
\text { skin temp } \leq 2 \text { degrees } \mathrm{C}\end{array}$ & $95 \%$ & $91 \%$ & .04 \\
\hline $\begin{array}{l}\text { Average Baby Mode set } \\
\text { temp }\end{array}$ & 36.6 & $\begin{array}{c}\text { Not } \\
\text { Applicable }\end{array}$ & $\cdots$ \\
\hline $\begin{array}{l}\text { Average Air Mode set } \\
\text { termp }\end{array}$ & Not Applicable & 32.7 & $\cdots$ \\
\hline Average set RH & $65 \%$ & $68 \%$ & .09 \\
\hline
\end{tabular}

\section{THE DEVELOPMENT OF A PROGRAMME TO INCREASE STAFF AWARENESS OF THEIR ROLES AND RESPONSIBILITIES IN RISK MANAGEMENT}

\section{M Thruston, L Singharam Chettair, J Enterkin}

Paediatric Intensive Care Unit, Evelina Childrens' Hospital, Guys' and St Thomas' NHS Foundation Trust, London, UK

Background and Aims: Patients deserve and expect safe care and the identification and prevention of adverse incidents has become an important part of Clinical Governance. Errors are usually complex and caused or exacerbated by many interacting causes. People make mistakes and poor systems increase the likelihood of error. Reporting and monitoring of adverse incidents helps facilitate learning and allow the direction of future preventative strategies.

Methods: A strategy developed and introduced by the PICU Education and Management teams, to reduce adverse incidents, is a programme to increase staffs' awareness of their roles and responsibilities in risk management. The programme includes presentations from the National Patient Safety Agency and the Trust Clinical Risk Team, feedback of local adverse incident follow up and analysis, group work and presentation of their work. Group work may involve a snapshot audit of prescription errors, for example, from which the group will then identify a project they will undertake over several months. Their projects will include findings and proposed action plans and they will present their work to the rest of the PICU team by way of oral and poster presentations.

Results: Staff report; increased awareness of the benefits of reporting and monitoring actual or potential incidents, increased motivation to participate in action planning of preventative strategies and the development of skills and experience of change management. The programme has been well evaluated.
Conclusions: The programme has the effect of staff having a better understanding of the causes of adverse incidents and becoming more aware of the risk management process.

\section{THE NEOSAFE PROJECT: LESSONS FROM A SPECIALTY-BASED INCIDENT REPORTING SYSTEM}

C Snijders $^{1}$, A Molendijk ${ }^{1}$, R A van Lingen ${ }^{1}$, W P F Fetter ${ }^{2}$, T W van der Schaaf ${ }^{3}$

${ }^{1}$ Department of Neonatology, Isala Clinics, Zwolle, The Netherlands, ${ }^{2}$ Department of Neonatology, Free

University Medical Center, Amsterdam, The

Netherlands, ${ }^{3}$ Department of Technology Management, University of Technology, Eindhoven, The Netherlands

Background and Aims: To examine the occurrence and severity of incidents in neonatal intensive care units (NICU's) in The Netherlands; and to identify underlying system factors of incidents related to medication, mechanical ventilation and vascular catheters.

Methods: Study design: Prospective multicenter study. In July 2005, voluntary, non-anonymous, non-punitive incident reporting was introduced in eight level III NICU's and one paediatric surgical intensive care. An incident was defined as any unintended event which (could have) reduced the safety margin for the patient. Multidisciplinary committees analysed incidents pertaining to medication, intravascular catheters and mechanical ventilation for underlying root causes, using the PRISMA methodology ( $\kappa$ statistics of 0.68 for interrater reliability). We describe results of the first six months of reporting.

Results: There were 2728 incident reports describing 2804 incidents, among 1853 admissions. The figure shows incident categories with frequencies. Death was attributed to an incident in two patients. Thirty-one patients experienced major harm with expected increased hospital stay, mainly due to incidents relating to mechanical ventilation $(7 / 31)$ and intravascular catheters (7/31). Out of 2067 minor incidents, 585 (28\%) had the potential to cause major harm. Root causes consisted of $8 \%$ technical, $27 \%$ organizational, $62 \%$ human and $3 \%$ patient-related failures.

Conclusions: A specialty-based, multicenter database can identify useful information about the etiology and severity of incidents in a short period of time, creating the opportunity for system-oriented preventive strategies.

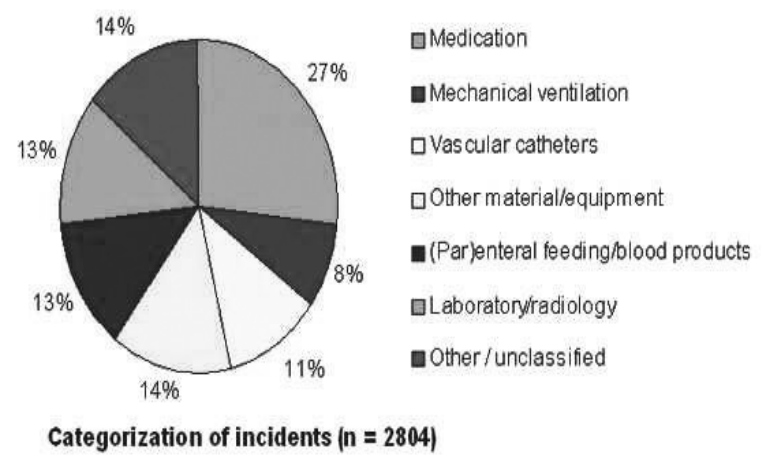




\section{NUTRITIONAL SUPPLEMENTATION DURING PREGNANCY AND LACTATION}

\section{R Bergmann}

Department of Pediatrics, Charité Virchow Klinikum, Berlin, Germany

The nutritional status of pregnant and lactating women has implications not only for their own health, but also for that of their infants. The consequences of insufficient or excessive intake of macro- and micro-nutrients influence the outcome of pregnancy, and may also have long-term effects on the health of the child and the future adult. An optimal supply of all important vitamins and minerals is thus essential for pregnant women. Their requirements with respect to a number of vitamins and minerals are considerably higher than those of women who are not pregnant. As these additional requirements cannot always be satisfied by a normal diet - even in societies with an abundance of food - micronutrient deficiencies occur (hidden hunger), and since detailed individualised nutritional supply levels cannot always be verified, dietary supplements seem advisable for all pregnant women. These supplements may even contain 'functional foods'; foodstuffs whose function goes beyond simply covering nutritional requirements, and which are beneficial to the health of the mother and her infant. The presentation will address the nutrients of special relevance for the pregnant woman and the fetus, and also their importance as supplements during pregnancy.

\section{ABSORBING OR METABOLIZING? THE ROLE OF THE NEONATAL GUT WITH REGARD TO THE DIET}

\section{J B van Goudoever}

Department of Pediatrics, Division of Neonatology, Sophia Children's Hospital, Erasmus Medical Center, Rotterdam, The Netherlands

Background and Aims: The portal-drained viscera (stomach, intestine, pancreas and spleen) have a much higher rate of both energy expenditure and protein synthesis than can be estimated on the basis of their weight. A high utilization rate of dietary nutrients by the portal-drained viscera might result in a low systemic availability, which determines whole-body growth.

Methods: From studies in our multiple catheterisized piglet model we conclude that more than half of the dietary protein intake is utilized within the portaldrained viscera and that amino acids are a major fuel source for the visceral organs.

Results: Specific stable isotope studies reveal that there are large differences in utilization rate amongst the different amino acids. The majority of the results obtained from the piglet studies can be extrapolated to the human (preterm) infant. First-pass, splanchnic uptake of lysine and threonine differ substantially, while non-essential amino acids are oxidized to a great extend in the human gut. Methionine is an important precursor of intestinally derived homocysteine.
Conclusions: Overall, these studies indicate that gut amino acid metabolism has a great impact on systemic availability and hence growth and health in the neonate.

\section{FEEDING THE EXTREMELY LOW BIRTH WEIGHT INFANT}

\section{R J Cooke}

Division of Neonatology, University of Tennessee Center for Health Sciences, Memphis, TN, USA

Nutrition plays a critical role during growth and development; the greater the growth rate the more critical the role, and none more so than that between 24 weeks of gestation and 2-3 months postnatal age, a time when even short periods of malnutrition has long term effects on growth and development. Studies show that under-nutrition and growth retardation are universal in preterm infants; the more immature the infant the greater the degree of under-nutrition and growth retardation. Whether this is entirely preventable or not is unclear. What is clear is that there are 'critical periods' in a preterm infant's life during which the extent of undernutrition and, therefore, growth retardation might be minimized, thus improving outcome in these high-risk infants.

\section{MAKING OF THE BRAIN: GENETIC AND EPIGENETIC REGULATIONS. FROM MOLECULAR BIOLOGY TO CONSCIOUSNESS}

\section{J-P Changeux}

Institut Pasteur, Paris, France

In the course of human evolution, the complexity of brain connectivity increasesd dramatically, while that of the genome did not change significantly. Several genetic mechanisms may plausible account for this paradoxical non-linear relationship such as the spatiotemporal and combinatorial action of developmental genes ie homeotic genes, microcephaly genes and/or several others. Alternatively, the state of activity (evoked/spontaneous) of the developing brain, during foetal life and in the newborn, may contribute to the development of brain organisation via ' epigenetic ' mechanisms such as the selective stabilisation/elimination of developing synapses. Still, such processes are expected to be limited by a ' genetic envelope' which may include genes like those responsible for dyslexia, autism or fragile-X mental retardation. The mechanism of epigenesis by selective stabilisation of synapses was investigated using molecular biology techniques with the nicotinic acetylcholine receptor (nAChR), the best known neurotransmitter-gated ion channel. Studies with mice deleted for defined subunits of the nAChR revealed a contribution of the spontaneous activity of the retina to the development of the adult visual pathways and maps. Moreover, the analysis of the transcriptional regulation of $\mathrm{nAChR}$ genes by electrical activity/neurotrophic factors during motor endplate morphogenesis established causal links between membrane electrical activity and nAChR genes promoters through 
transcription factors and second messenger pathways. In the brain, an efficient in vivo method involving the selective reexpression of defined genes by stereotaxic injections of lentiviral vectors is used to investigate the plasticity of adult brain circuits in cognitive learning. The importance of epigenetic regulation of synapse development (and its pathologies) in early learning processes, in cognitive and/or conscious functions and in cultural evolution, is discussed in the case of the development of the newborn infant and its pathologies such as autism, ADHD, sudden infant death disease. Changeux J.P. The physiology of truth. Harvard University Press: Cambridge, Mass. 2004. Changeux J.P. \& Edelstein S.E. The nicotinic acetylcholine receptors: from molecular biology to cognition. Odile Jacob: Paris 2005. Maskos U., Molles B.E., Pons S., Besson M., Guiard B.P., Guilloux J.P., Evrard A., Cazala P., Cormier A., Mameli-Engvall M., Dufour N., Cloez-Tayarani I., Bemelmans A.P., Mallet J., Gardier A.M., David V., Faure P., Granon S., Changeux J.P. Nicotine reinforcement and cognition restored by targeted expression of nicotinic receptors. Nature, 436:103-107, 2005.

\section{MECHANISM OF BRAIN INJURY AND NEUROPROTECTION}

\section{R C Tasker}

Department of Paediatrics, Cambridge University Clinical School and Department of Paediatric Intensive Care, Addenbrooke's Hospital, Cambridge, UK

Background and Aims: There are a number of mechanisms that appear to contribute to brain injury occurring after focal and global ischaemia. Experimental models have improved our understanding of neuropathology and support a glutamate hypothesis of hypoxic-ischaemic brain damage. Substantial evidence indicates that antagonists of ion channel-linked glutamate receptors can reduce damage. In focal ischaemia, this is true for both N-methyl-D-aspartate (NMDA) antagonists and for non-NMDA (kainate/AMPA) antagonists. In such models, the magnitude of infarct reduction is typically in the order of 25 to $30 \%$. Moving away from the direct effect of the receptor, there are either upstream or downstream sites for drug action. For example, upstream, glibenclamide (acting at the ATP sensitive potassium channel) prolongs the period over which hypoxia can be tolerated. Alternatively, downstream, dantrolene (acting at the endoplasmic reticulum to limit NMDA-initiated, calcium-mediated, calcium release from the ER pool) limits the induction of apoptosis.

Methods: To date, many pharmacological approaches, or combination of approaches, have been applied to animal models, with beneficial effects, which promise efficacy in patients. The NMDA receptor story, although teaching us much about pathophysiology, is an example where basic science has failed to deliver a significant clinical outcome. However, when it comes to extending experimental studies to clinical research trials there are some key questions.

Results: A variety of promising, strongly neuroprotective, agents have been identified by studies in animal models. Despite this advance, translation to clinical practice has not been forthcoming. Unfortunately, the process of converting a promising drug effect in the laboratory to a clinically useful test product may be fraught with difficulty. (1) Do we know that the agent is getting to the site where we think its mechanism of action takes place? (2) Do we understand the mechanism of how a drug or agent is acting in a given experimental model? Although perhaps obvious, this may not always be the case. (3) Does the ability to protect neurons in one animal model extrapolate to similar activity in other animal models? (4) Man may exhibit important differences to the experimental model in relation to disease-, anatomy-, sex- or age-specific factors. (5) Laboratory studies are biased towards inducing poorer outcomes, as this makes quantification easier. (6) Issues concerning the drug itself: What are its effects in the human being?; Can it cross the blood-brain barrier?, How is it metabolised?; What are the interactions?; Is it protein bound? etc.

Conclusions: There are now other areas of acute neurotoxicity that are gaining interest in the wider field of neuroprotection from hypoxia-ischaemia. These include a role for: (1) The cerebral inflammatory response, (2) Ischaemic preconditioning and ischaemic tolerance, and (3) The $\mathrm{pH}$ paradox.

\section{PAIN: NOT ONLY THE BRAIN}

\section{P G Jorens}

Department of (Paediatrics) Critical Care Medicine, Clinical Pharmacology, University Hospital of Antwerp, Edegem, Belgium

Background and Aims: Addressing pain, an inevitable phenomenon from birth until death, in infants and children is an important task for health care workers. Although some progress has been made, many children do not receive adequate attention to their pain.

Methods: Current literature highlights a significant deficiency in our ability to assess pain, to understand the physiopathology and the pharmacokinetics and dynamics of analgesic drugs in this population A review of the topic and the current literature is given.

Results: Children involved in trauma and undergoing major surgery need fast and effective analgesia. Painful procedures are the principal memory in survivors of childhood cancer. However, also neuropathic pain and other forms of chronic pain affect a large number of children. Children with a variety of genetic disorders may develop chronic pain as their life progresses. The reasons for a low availability of data in children are widespread. The diversity and developmental range of the paediatric population have enormous implications for the design and conduct of clinical research and the interpretation of research findings. Inappropriate pain assessment is likely to be an important contributor to the poor pain management in children. The problem of differentiating postoperative pain from fear and anger in toddlers .... Nursing and physician alike remain confused as to the dividing line between conscious sedation and pure pain management. The traditional definitions of pain require self-reporting: a contributing factor is the children's difficulty in expressing their pain 
to those taking care of them in a way that is understood. Behaviour is a useful measure and indicator of pain in neonates and infants and a behavioural pain assessment tool might be necessary. Whatever scale is used, it is important but frequently neglected to ensure that it is appropriate for the cognitive development, personality and condition of the child. Incorporating both pharmacological and non-pharmacological therapies is as necessary in children as in adults. Hardly any paediatric data are available on pharmacokinetics and pharmacodynamics of many drugs and are usually extrapolated from adult data. Recent but limited characterisation of the developmental pharmacokinetics of opioid analgesics, paracetamol and non-steroidal antiinflammatory drugs has however improved dosing in children. Oral solutions can now be used in neonates. Intravenous paracetamol offers increased dosing accuracy. Moreover, the paediatric population has access to unique formulations: the use of topical creams of a local anaesthetic, otic analgesia and rectal administration, although many children dislike the latter route.

Conclusions: As it is recently recognised that pain and its management may have consequences for later painrelated behaviour and perception, all human beings have the right to adequate pain control whether they can selfreport or not. There is an enormous potential for future research and a pressing need for further clinical development in the field of paediatric pain. Current recommendations are frequently not based on the highest possible level of evidence. Although pain measurement and treatment has progressed substantially over the last decade, improvement in all aspects of pain documentation in children is needed to improve pain management and probably outcomes in this precious population.

\section{THE ASSESSMENT OF PAIN IN PRE- OR NON- VERBAL CHILDREN}

\section{S Franck}

Professor and Chair of Children's Nursing Research, Centre for Nursing and Allied Health Professions Research, Institute of Child Health and Great Ormond Street Hospital for Children, London, UK

Despite over 30 years of research regarding measurement of pain in infants and children, there is no consensus regarding the optimal measures for research or to guide clinical management. At last count, there were over 40 published assessment scales for measuring pain in infants and children in the hospital setting. While it has been generally recognized that pain is a complex multidimensional experience, attention in pediatric pain measurement studies has remained largely focused on pain intensity, to the exclusion of measuring the meaning of the pain and its affective and behavioral consequences. There are numerous tools for assessment of acute pain in infants, but few have been shown to be valid for assessment of persistent pain or postoperative pain in critically children. More worrying, there remains minimal integration of pain measurement with pain treatment. Pain intensity scores have been successfully used to demonstrate the efficacy of analgesics in clinical trials. However, none of the published assessment scales provide evidence for implementing one treatment versus another for any given pain score. Possibly as a consequence of that lack of evidence, there is low compliance with pain assessment documentation in many units that have implemented standardized scales. If the measurement of pain does not lead to a treatment decision, and the effectiveness of that decision is not demonstrated by a meaningful change in the scale, what is the utility of the measure? Recent research suggests that specific behavioral and physiological indicators are valid and reliable proxy measures for pain in non-verbal children. The best indicators may vary based on age, severity of illness, degree of neurological impairment, context and type of observer (healthcare professional or parent. Nevertheless, numerous challenges remain in discriminating pain-related signs of distress from other etiologies. Recommendations for pain assessment in preverbal children will be suggested based on current evidence.

\section{NON-PHARMACOLOGICAL PAIN MANAGEMENT IN THE INFANT AND CHILD}

\section{Harth}

\section{PICU, Children's Hospital Johannes Gutenberg University, Mainz, Germany}

Background and Aims: Critically ill children and infants might experience a stay on a PICU with a lot of stressors which may cause pain, discomfort and suffering. Providing comfort to these patients is a core duty of the nursing profession. Optimal pain management is based on a holistic approach which includes the set up of a proper pain diagnosis according to the child's individual needs and its developmental level and it considers both pharmacological and nonpharmacological interventions.

Results: Aim of this lecture is to show up not only the different techniques of non-pharmacological pain management. From a broader perspective some basic but important aspects of non-pharmacological pain management have found their place i.e. in the creation of a family friendly PICU environment or in the definition of primary nursing care goals.

Conclusions: Non-pharmacological pain management methods are well documented in literature and belong to one of the two supporting pillars of pain relief. However, pharmacological and non-pharmacological pain management should never replace one with another but should be applied in synergy.

\section{ADVANCES IN PAEDIATRIC EMERGENCY CARE}

\section{P Biban}

Neonatal and Paediatric Intensive Care Unit, Major City Hospital, Verona, Italy

Abstract not available at the time of printing. 


\section{ASSESSING THE LEVEL OF CONSCIOUSNESS IN CHILDREN: A PLEA FOR THE GLASGOW COMA MOTOR SUBSCORE}

P Van de Voorde ${ }^{1}$, D Lutman ${ }^{2}$, M Peters ${ }^{3}$

${ }^{1}$ Paediatric ICU, University Hospital Gent, Belgium, ${ }^{2}$ CATS, Children's Acute Transport Service, London, UK, ${ }^{3}$ Paediatric ICU, Great Ormond Street Hospital for Sick Children, London, UK

Background and Aims: The Glasgow Coma Scale is not always easy to score and its reliability has been questioned. We hypothesised that the Motor subscore would be at least as effective in predicting outcome from coma in children.

Methods: CATS provides emergency secondary transport of critically ill children (UK). During a 9month period we studied all patients with altered consciousness, not caused by drugs, chronic disease, convulsions or cardio-respiratory failure. For each case we collected the GCS at referral or pre-intubation. Outcome was evaluated upon discharge using a three level ordinal scale (adapted from Fiser DH, J. Ped. 1992; 121:68-74).

Results: 113 patients were identified but in 49 adequate data were not available. There were no significant differences between cases with and without adequate data for inclusion. Of the 64 cases 32 were neurotrauma patients, 32 had other reasons for altered consciousness: CNS infection, diabetic ketoacidosis.... Outcome was good in 44 patients; 8 showed moderate to severe disability; 12 died. Proportional odds models were fitted for various choices of predictive variables: GCS, age, diagnosis... For each we calculated Somers' Dxy as a measure of the predictive accuracy of the model. Overall the difference between all models was small. The highest Dxy value $(0.852)$ was found for the model with the GCS Motor score included linearly.

Conclusions: The Motor subscore has at least the same predictive ability as the total GCS. It is our opinion that, especially in children, the total GCS is unnecessary complicated.

\section{VALUE OF REPEAT CT SCANNING FOR INVESTIGATION OF INTRACTABLE INTRACRANIAL HYPERTENSION IN TRAUMATIC BRAIN INJURY}

\section{E Loh, Q Mok}

Paediatric Intensive Care Unit, Great Ormond Street Hospital, London, UK

Background and Aims: Patients with traumatic brain injury (TBI) frequently require urgent repeat CT scans to determine if there is a surgically treatable cause for their intractable intracranial hypertension. This involves transfer of the ventilated patient from the intensive care unit to the scanning suite, with its antecedent risks. The aim of the study was to determine how frequently these CT scans showed abnormalities where surgery could have improved intracranial hypertension refractory to medical intervention, and if there were clinical parameters that could predict this.

Methods: This was a retrospective study of patients admitted to our tertiary pediatric intensive care unit
(PICU) with TBI requiring intracranial pressure (ICP) monitoring. Patient notes, computerised observation charts and CT scans were reviewed. Scans were reported independently by neuroradiologists not involved in the patients' clinical care.

Results: 88 patients with TBI requiring ICP monitoring were admitted in the 5 years between 2000-2005 to the PICU. The majority had urgent repeat CT scanning because of persistently raised ICP. This led to surgical intervention in only $7(8 \%)$ patients. In all these patients this requirement could have been predicted from haemodynamic variables and other signs suggestive of uncal herniation.

Conclusions: In the majority of patients repeat CT scans did not show any abnormality that resulted in a change in management strategy. In patients that required surgical intervention following CT scanning, there were clear signs suggestive of early uncal herniation. This may be useful in predicting clinically who could benefit from surgical intervention and minimise unnecessary transfers for scans.

\section{THE INFLUENCE OF PRE- CARDIOPULMONARY CEREBRAL RESUSCITATION FACTORS ON SURVIVAL RATE IN CHILDREN}

G van Berlaer, V Van Gorp, S Hachimi-Idrissi

Department of Pediatric Critical Care, Academical Hospital, Vrije Universiteit Brussel, Brussels, Belgium

Background and Aims: The outcome of children after resuscitation for cardiopulmonary arrest is poor. Our aim is to describe a large pediatric population who developed a pre-hospital cardiopulmonary arrest (PCPA) and to outline the influence of the precardiopulmonary cerebral resuscitation (CPCR) factors on survival rate. An evaluation is made of the influence of applied CPCR guidelines on these children's outcome.

Methods: From 1991 till 2005, clinical records of all patients younger than 16 years with PCPA were reviewed. All data were prospectively registered using a uniform standard registry, and adjusted to the Utstein style report. The studied pre-CPCR factors were restricted to age, site and cause of PCPA, bystander CPCR, time to intubation and to administration of drugs, initial cardiac rhythm and respiratory pattern on arrival of the mobile intensive care unit team.

Results: Out of all registered patients with prehospital cardiopulmonary arrest, we review all children. We present the most common sites and causes of PCPA, the number of attempted CPCR and achievement of restoration of spontaneous circulation (ROSC). We compare the influence of initial cardiac rhythms and initial respiratory patterns on the percentage of ROSC and regaining of consciousness. We compare results of CPCR performed by lay bystanders or by EMSpersonnel, skilled in pediatric life support, to study the influence on survival rate and neurological outcome.

Conclusions: Rapid intervention and resuscitation by EMS-bystanders skilled in pediatric life support increases survival rate and neurological outcome in 
children. Therefore CPCR training, appropriate equipment and rapid intervention are mandatory.

\section{TRANSPORT OF NEWBORNS WITH ACUTE LUNG FAILURE ON ECMO}

F Loersch, S T Hien, M Kratz, S Demirakca, C H v. Buch, S T Loff, T H Schaible

NICU, University Childrens Hospital, Mannheim, Germany

Background and Aims: Although ventilation and postpartal management of sick newborns has been optimized, ECMO remains a therapeutic option in the neonatal intensive care of acute lung failure. The ECMO-center Mannheim has performed about 281 ECMO cases since 1987, and currently performs about 25 ECMOs per year. 50 infants per year are evaluated for the option of ECMO therapy. Half of them are born at the center and the others are transported to the ECMO center. Some children are too ill for conventional transport, in which case transport using mobile ECMO is necessary.

Results: 2005 we have installed a special TransportECMO unit on a normal Ferrno Transport system in our ECMO-center, which can be used in a standard ambulance or helicopter. On the unit we use a rollerpump (NovaCirc), a Stephan ventilator, a heating pump (Aquatherm 660) and 6 perfusion pumps. Energy is generated by a UPSU (uninterrupted power supply unit). During the transport the energy comes from the vehicle. The cannulas are placed either by surgeon from the referring hospital or by a surgeon accompanying our transport team. All 6 newborns with was transported on this unit have survived and could later been discharged and left the hospital.

Conclusions: Special equipment and specially trained transport teams are necessary for transporting critically ill children. Transport under ECMO is feasible but remains a high-risk modality that should not replace the prenatal and early postnatal transport to an ECMO center. In some cases this can be a rescue therapy.

\section{INFANTS TRANSFERRED FOR} CONSIDERATION OF EXTRACORPOREAL LIFE SUPPORT (ECLS): PREDICTORS FOR ECLS REQUIREMENT

L Mills ${ }^{1}$, S Rodger ${ }^{1}$, M Liddell ${ }^{2}$, J Simpson ${ }^{2}$, C Davis $^{2}$, C Skeoch $^{1}$, L Jackson $^{1}$

${ }^{1}$ West of Scotland Neonatal Transport Service, Royal Hospital for Sick Children, Glasgow, UK, ${ }^{2}$ Extra Corporeal Life Support Service, Royal Hospital for Sick Children, Glasgow, UK

Background and Aims: ECLS is an established therapy for neonates with a high-risk of death from respiratory failure and is available in 4 UK centres. No standardised criteria for initiating ECLS transfer exist although the oxygenation index (OI) has been used historically. To review factors influencing the decision to commence ECLS for infants transferred to Glasgow as potential candidates for ECLS.
Methods: A retrospective case note review incorporating the West of Scotland Neonatal Transport Database. Factors influencing ECLS requirement were identified using bi-variate correlation. Binary logistic regression was used to identify independent determinants of ECLS requirement. SPSS ${ }^{\circledR}$ was used for all analyses.

Results: Fifty-two infants were referred to the Glasgow ECLS between March 2003 and March 2006, 49 of whom were subsequently deemed suitable for transfer. Thirty infants (61\%) subsequently received ECLS. ECLS requirement correlated strongly with oxygen requirement (FiO2) $(\mathrm{r}=0.47, \mathrm{p}=0.01)$, OI $\quad(\mathrm{r}=0.41$, $\mathrm{p}=0.04)$, and mean airway pressure (MAP) $(\mathrm{r}=0.37$, $\mathrm{p}=0.08$ ) at referral. It did not correlate with $\mathrm{pO} 2$ at referral. Oxygen requirement and OI again correlated with ECLS requirement at arrival at the ECLS centre. Using binary logistic regression analysis, with referral $\mathrm{FiO} 2, \mathrm{MAP}$, and $\mathrm{OI}$ as covariates, $\mathrm{FiO} 2$ was a significant independent predictor of ECLS requirement (OR 1.18; 95\% C.I. 1.04 - 1.33). MAP and OI were not significant predictors in the model.

Conclusions: $\mathrm{FiO} 2$ is a better predictor of the requirement for ECLS than OI at referral. Infants with a high FiO2 who are suitable candidates for ECLS should be discussed early with the ECLS centre to optimise treatment options.

\section{FULMINANT LIVER FAILURE IN INFANTS AND CHILDREN: ABOUT 200 CASES IN A SINGLE CENTER}

P Tissieres, V Haas, L Chevret, S Essouri, D Debray, F Gauthier, P Durand, D Devictor

Department of Pediatrics, Bicêtre Hospital, Le KremlinBicêtre, France

Background and Aims: To report a 20 years experience of managing fulminant liver failure (FLF) in infants and children.

Methods: Retrospective analysis from 1986 to 1996 and prospectively maintained data base since 1996 was used to identify infants and children admitted in a 22 beds multidisciplinary ICU of a University Hospital, national referral centre for pediatric liver disease.

Results: 200 patients were admitted for FLF with a mean age of $4.0 \pm 4.6$ years-old, including 82 infants (41\%) of $<1$ year-old. A third of all FLF in infants occurred within the last three years. In infants, metabolic causes represented $43 \%$ of FLF cases followed by viral FLF (21\%). In children, viral $(28 \%)$ and non-A non-B-non-C FLF (26\%) represented more than half of the cases. Emergency liver transplantation was performed in $44.9 \%$ of all patients ( $52 \%$ of children and $33 \%$ of infants). Overall mortality was $41 \%$, reaching $52 \%$ in infants. $34 \%$ patients undergoing ELT died. Death occurred within a mean time of $11 \pm 19.6$ days after PICU admission. In 43 patients, ELT was contra-indicated, all died. Survival in the 157 patients in whom ELT was performed or was not indicated was $74 \%$.

Conclusions: We report the largest pediatric series of FLF and discuss epidemiology, outcome and management. 


\section{BRAIN INJURY AND DYSFUNCTION DUE TO METABOLIC DISEASES}

\section{F J Eyskens}

Department of Metabolic Diseases, Queen Paola Children's Hospital;Department of Paediatrics, University Hospital of Antwerp, Laboratory for the Study of Metabolic Diseases, Antwerp, Belgium

Background and Aims: Inborn errors of metabolism are genetic disorders due to the deficiency of specific enzymes and proteins involved in catabolic or anabolic pathways, energy metabolism, transport/carriers, ion channels, receptors and signalling. Most of these diseases manifest during (early) childhood and affect the nervous system more often than any other organ system. Early diagnosis is important because treatment, if available, may prevent acute metabolic attacks and mental retardation, epilepsy, severe brain damage, or death; and is also a prerequisite for optimal genetic counselling. The ideal classification of metabolic encephalopathies should be based on cause and pathogenesis. In a recent paper (Ref.: A. Simons, F. Eyskens et al. European Child and Adolescent Psychiatry, 2006) we reported on the intelligence scores and the psychiatric pathology of distinct groups of children with metabolic diseases. Based on this study I would divide the neurometabolic disorders roughly in different subgroups: the "intoxication groupl"(including PKU, galactosemia, organic acidurias and urea cycle disorders), the "substrate and enzymatic disorders of energy metabolism group" (including glycogenoses, neoglucogenesis and fatty acid oxidation disorders that have hypoglycaemia in common), the "mitochondrial respiratory chain defects group", the "storage disorders group" and the"deficiency disorders group". This rough classification based on pathophysiologic processes provides insight in the different mechanisms giving rise to metabolic encephalopathies.

Methods: The study population of the referred paper consisted of 53 children between 0 and 18 years of age. Diagnostic assessment included a semi-structured interview, self-report questionnaires and a standard intelligence test. Case reports of different metabolic diseases not included in the paper will be discussed: Nonketotic Hyperglycinemia, Canavan's Disease, peroxisomal disorders, lysosomal storage disorders, Smith-Lemly-Opitz syndrome, Cerebrotendinous Xanthomatosis, CDG syndrome, L-serine biosynthesis defects, disorders of creatine metabolism, folate receptor antibodies, pyridoxine dependency, and neurotransmitter defects. Besides functional disturbances, developmental structural brain defects, neuronal and glial cell loss, demyelination / leucodystrophy and stroke or stroke-like injury to the brain are common in metabolic diseases. NMR imaging of the brain can reveal specific abnormalities, e.g. Leigh syndrome and Canavan's disease, as well as aspecific abnormalities, e.g. corpus callosum agenesia/ hypoplasia and neuronal migratory disturbances. Special extraneural abnormalities should be looked for: dysmorphic features, cardiac involvement, hepatosplenomegaly, skin disturbances,...

Results: In our study in $40 \%$ of the children older than 5 years, a child psychiatric diagnosis was made. While
CBCL total and internalizing scores did not differ between metabolic disease groups, the CBCL externalizing scores for some groups did (e.g. ADHD), especially in the "intoxication group" with phenylketonuria as the most prevalent disorder. Two fifths of the children showed a below normal intelligence, while a performal-verbal IQ discrepancy was found in half of the children, especially in the "intoxication group". Of the school aged children almost one third attended a special needs school.

Conclusions: Our results suggest substantial psychiatric problems besides neurological disturbances in children with a metabolic disease. Further study on larger groups is warranted, which should enable further comparison of patients affected by specific metabolic diseases improving our insight in the pathogenesis of brain injury and dysfunction.

\section{EFFECT OF FETAL GROWTH RESTRICTION ON BODY COMPOSITION AND HORMONAL STATUS AT BIRTH IN INFANTS OF SMALL AND APPROPRIATE-FOR-GESTATIONAL AGE BIRTH WEIGHT}

R Verkauskiene $^{1}$, J Beltrand ${ }^{1}$, O Claris ${ }^{2}$, D Chevenne ${ }^{3}$, O Sibony $^{4}$, P Gaucherand ${ }^{5}$, C Lévy-Marchal ${ }^{1}$

${ }^{I}$ INSERM U690, Hôpital Robert Debré, Paris, France

${ }^{2}$ Department of Neonatology, Hopital Eduard Heriot, Lyon, France, ${ }^{3}$ Department of Biochemistry, Hôpital Robert Debré, Paris, France, ${ }^{4}$ Department of Obstetrics and Gynaecology, Hôpital Robert Debré, Paris, France ${ }^{5}$ Department of Obstetrics and Gynaecology, Hôpital Eduard Heriot, Lyon, France

Background and Aims: Intrauterine growth retardation (IUGR) has been related to several health risks, generally identified in small-for-gestational age (SGA) individuals. We aimed to evaluate the impact of IUGR on body composition and hormonal status in newborns of small and appropriate-for-gestational age (AGA) birthweight.

Methods: 4 monthly ultrasound examinations were performed in 145 women at risk of IUGR. Intrauterine growth slope (IUGS) was calculated as fetal weight percentiles change and IUGR defined as its reduction by more than 20 percentiles. SGA was defined as birthweight below the 10th percentile. Newborns were stratified into 4 groups: SGA-IUGR $(\mathrm{n}=27)$, 'constitutional' SGA (SGA-C, $\mathrm{n}=21$ ), AGA-IUGR $(\mathrm{n}=42)$ and 'constitutional' AGA (AGA-C, $\mathrm{n}=55$ ). Cord insulin and IGF-I concentrations were determined. DEXA scan was performed at postnatal day 3 .

Results: Fat mass percent were similar between SGA-

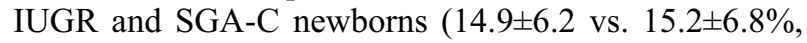
$\mathrm{p}=0.9$ ), but significantly lower in AGA-IUGR vs. AGA$\mathrm{C}$ group (17.0 \pm 6.5 vs. $19.8 \pm 6.6 \%, \mathrm{p}=0.01)$. Insulin concentration was lowest in SGA-IUGR and highest in AGA-C group, but the difference failed to reach statistical significance. IGF-I levels were decreased in SGA-IUGR vs. SGA-C and in AGA-IUGR vs. AGA-C groups $(28.5 \pm 16.5$ vs. $43.9 \pm 26.8 \mathrm{ng} / \mathrm{ml}, \mathrm{p}=0.009$ and $52.8 \pm 29.8$ vs. $65.4 \pm 26.3 \mathrm{ng} / \mathrm{ml}, \mathrm{p}=0.02$ ). In multivariate analysis, birthweight was related to IUGS ( $\mathrm{r}=0.6$, $\mathrm{p}<0.0001)$ and cord IGF-I $(\mathrm{r}=0.22, \mathrm{p}=0.001)$, and 
infant's adiposity to IUGS ( $\mathrm{r}=0.45, \mathrm{p}<0.0001)$, cord insulin $(\mathrm{r}=0.23, \mathrm{p}=0.02)$ and IGF-I concentrations $(\mathrm{r}=0.2, \mathrm{p}=0.04)$.

Conclusions: Fetal growth restriction affects body composition and hormonal parameters even in newborns of normal birthweight, suggesting these individuals could be exposed to similar metabolic risks as SGA.

\section{MATERNAL INSULIN SENSITIVITY IN MID- PREGNANCY AND FETAL GROWTH}

R Verkauskiene $^{1}$, J Beltrand ${ }^{1}$, O Claris ${ }^{2}$, D Chevenne ${ }^{3}$, O Sibony $^{4}$, P Gaucherand $^{5}$, C Lévy-Marchal ${ }^{1}$

${ }^{I}$ INSERM U690, Hôpital Robert Debré, Paris, France ${ }^{2}$ Department of Neonatology, Hôpital Eduard Heriot, Lyon, France, ${ }^{3}$ Department of Biochemistry, Hôpital Robert Debré, Paris, France, ${ }^{4}$ Department of Obstetrics and Gynaecology, Hôpital Robert Debré, Paris, France ${ }^{5}$ Department of Obstetrics and Gynaecology, Hôpital Eduard Heriot, Lyon, France

Background and Aims: The pathway linking intrauterine growth retardation (IUGR) with an increased risk of type 2 diabetes is still not clear. We hypothesised that maternal insulin sensitivity could be related to fetal growth pattern.

Methods: Fetal growth was estimated by four monthly ultrasound examinations from 22 to 36 gestational weeks (GW) in 145 women with normal glucose tolerance. IUGR was defined as a reduction in estimated fetal weight by more than 20 percentiles. Small or appropriate-for-gestational age (SGA/AGA) size was defined as birthweight below/superior the 10th percentile, respectively. At birth, infants were stratified into 4 groups: SGA-IUGR ( $\mathrm{n}=27$ ), 'constitutional' SGA (SGA-C, $\mathrm{n}=21)$, AGA-IUGR ( $\mathrm{n}=42$ ) and 'constitutional' AGA (AGA-C, $n=55$ ). Maternal fasting insulin and glycaemia were determined at 22-24 GW. IGF-I and insulin were measured in cord blood. DEXA scan in newborns was performed at postnatal day 3 .

Results: Maternal insulin resistance index (HOMA) at 22-24 GW was similar in SGA-IUGR and SGA-C groups, but was significantly lower in AGA-IUGR vs. AGA-C group. Maternal HOMA at 22-24 GW was directly related to pre-pregnancy BMI $(\mathrm{r}=0.46$, $\mathrm{p}<0.0001)$, weight gain throughout pregnancy $(\mathrm{r}=0.2$, $\mathrm{p}=0.05)$, infants' birthweight $(\mathrm{r}=0.2, \mathrm{p}=0.05)$ and percent of fat mass $(r=0.3, p=0.016)$, cord insulin $(\mathrm{r}=0.32, \mathrm{p}=0.006)$ and IGF-I concentration $(\mathrm{r}=0.19$, $\mathrm{p}=0.06$ ). In a multivariate analysis, birthweight was best related to cord IGF-I concentration $(r=0.46, p=0.0002)$, gestational age $(\mathrm{r}=0.45, \mathrm{p}<0.0001)$ and maternal HOMA at $22-24 \mathrm{GW}(\mathrm{r}=0.23, \mathrm{p}=0.01)$.

Conclusions: Maternal insulin resistance in midpregnancy is directly related to fetal growth, infants' birthweight and adiposity, probably reflecting a better substrate delivery to fetus.

\section{PLACENTAL GHRELIN RELEASE IN THE DUAL IN VITRO PERFUSION MODEL}

K Linnemann ${ }^{1}$, C Anders ${ }^{1}, \mathrm{~K}_{\text {May }}{ }^{1}$, C Müller ${ }^{1}$, R Trollmann ${ }^{2}$, J Dötsch $^{2}$, W Straube ${ }^{3}$, C Fusch $^{1}$ ',
${ }^{l}$ Department of Neonatology and Pediatric Intensive Care, University of Greifswald, Germany, ${ }^{2}$ Department of Pediatrics, University of Erlangen, Germany,

${ }^{3}$ Department of Obstetrics and Gynecology, University of Greifswald, Germany

Background and Aims: Ghrelin stimulates GH release and appetite, modulates carbohydrate metabolism and insulin secretion and ultimately influences energy balance and fat storage in the human body. The human placenta expresses ghrelin at the mRNA level. It remains unclear if the placenta releases ghrelin, a possibly important growth factor for fetal growth into the fetal or maternal circulation. Aim: Investigation of placental ghrelin release into the fetal or maternal circulation.

Methods: 14 placentas at term; dual in vitro placenta perfusion for $6 \mathrm{~h}$ for determination of ghrelin-, hCG- and leptin- release; and glucose consumption, lactate production, creatinine transfer (controls for normal placental function). Ghrelin mRNA was measured by quantitative PCR.

Results: Ghrelin was released into the maternal and into the fetal circuit. The release rate into the maternal was $130 \pm 100 \mathrm{pg} / \mathrm{g} / \mathrm{h}$ and into the fetal circuit $38 \pm 36$ $\mathrm{pg} / \mathrm{g} / \mathrm{h}$. HCG release rates: maternal $62 \pm 43 \mathrm{IU} / \mathrm{g} / \mathrm{min}$; fetal $0.3 \pm 0.2 \mathrm{IU} / \mathrm{g} / \mathrm{min}$; leptin release rates: maternal: $234 \pm 92 \mathrm{ng} / \mathrm{g} / \mathrm{min}$; fetal $4 \pm 2 \mathrm{ng} / \mathrm{g} / \mathrm{min}$. Normal ranges for glucose consumption, lactate production and creatinine transfer. In addition, ghrelin was clearly expressed in placental homogenates at the mRNA level (ghrelin/ß-actin mRNA: before perfusion $0.87 \pm 0.54$, after perfusion $1.29 \pm 0.33$ ).

Conclusions: For the first time we could demonstrate placental release of ghrelin. It was preferentially released into the maternal circulation $(70 \%)$, but it was also released to a high amount $(30 \%)$ into the fetal circulation. Placental ghrelin is possibly involved in growth and energy metabolism of the growing fetus in concert with other placental hormones especially leptin and resistin.

\section{CARBAMOYL-PHOSPHATE SYNTHETASE POLYMORPHISMS AS A RISK FACTOR FOR NECROTIZING ENTEROCOLITIS IN PREMATURE INFANTS}

R M J Moonen ${ }^{1}$, M E Rubio ${ }^{1}$, N Y P Souren ${ }^{2}$, A D C Paulussen ${ }^{2}$, E Villamor ${ }^{1}$

${ }^{I}$ Department of Paediatrics, University Hospital of Maastricht, The Netherlands, ${ }^{2}$ Department of Population Genetics, University of Maastricht, Maastricht, The Netherlands

Background and Aims: Plasma L-arginine concentrations were found to be lower in preterm infants who developed necrotizing enterocolitis (NEC). Term infants who were homozygous (CC) for a C-to-A nucleotide transversion $(\mathrm{T} 1405 \mathrm{~N})$ in the gene-encoding carbamoyl-phosphate synthetase I (CPSI) had lower Larginine concentrations than infants with the AA genotype (Pearson et al., N Engl J Med 2001; 344:18328 ). The present study tests the hypothesis that this functional polymorphism in CPSI would be related to 
NEC in preterm infants. Methods: Genotypes of the CPSI variants were determined in 16 preterm infants with NEC (Bell stage II or greater, gestational age $\leq 30$ wk), 29 preterm infants without NEC (gestational age $\leq 30 \mathrm{wk})$ and 25 healthy term infants. Results: Sepsis was diagnosed more frequently in NEC infants $(\mathrm{P}=$ 0.031 ), whereas no significant differences in other baseline characteristics and perinatal complications were found between the two preterm populations. Prevalence of the CC genotype was significantly higher in the NEC population (CC:AC:AA = 81\%:12.5\%:6.5\%) than in the term control population (CC:AC:AA $=44 \%: 48 \%: 8 \%$, $\mathrm{P}=0.042)$ but no significant difference $(\mathrm{P}=0.10)$ was found with the preterm control population (CC:AC:AA $=52 \%: 34 \%: 14 \%$ ). The NEC population did not conform to Hardy-Weinberg equilibrium for the CPS1 T1405N polymorphism. Conclusions: Although they are limited by the small sample size, our results suggest that the T1405N variant of CPSI might be associated with the risk of NEC in the preterm infant. Further study is required to confirm these results in a larger cohort.

\section{LEPTIN AND ADIPONECTIN PLASMA LEVELS IN INTRAUTERINE GROWTH RESTRICTED (IUGR) AND APPROPRIATE FOR GESTATIONAL AGE (AGA) FETUSES, NEONATES AND THEIR MOTHERS}

\author{
M Kyriakakou ${ }^{1}$, A Malamitsi-Puchner ${ }^{1}, \mathrm{H} \mathrm{Militsi}^{2}$, \\ T Boutsikou ${ }^{1}$, D Hassiakos ${ }^{1}$, A Margeli ${ }^{2}$, \\ I Papassotiriou ${ }^{2}$, G Mastorakos ${ }^{1}$, C Kanaka-Gantenbein ${ }^{3}$ \\ ${ }^{1}$ Second Dept of Obstetrics and Gynecology, Athens \\ University Medical School, ${ }^{2}$ Dept of Clinical \\ Biochemistry, "Aghia Sophia" Children's Hospital, \\ Athens, Greece, ${ }^{3}$ First Dept of Pediatrics, Athens \\ University Medical School, Greece
}

Background and Aims: Leptin regulates energy balance and exerts an insulin sensitizing effect, while adiponectin increases insulin action in muscle and liver and exerts anti-atherogenic effects. This study investigated the release of these two adipocytokines in the blood of mothers (MP), and their single IUGR or AGA fetuses (UC) and neonates on day 1 (N1) and 4 (N4) postnatally.

Methods: Plasma levels of adipocytokines were measured by radioimmunoassays (LINCO Research, USA) in 20 IUGR and 20 AGA mother-infant sets.

Results: Leptin levels were higher, while adiponectin levels were lower in IUGR compared to AGA mothers $[32.5 \pm 3.7$ vs $23.8 \pm 3.4 \mathrm{microg} / \mathrm{L}$ and $5.4 \pm 0.9$ vs $8.1 \pm 1.6 \mathrm{mg} / \mathrm{L} \quad$ respectively $(\mathrm{p}<0.01)]$. These adipocytokines are positively correlated only in AGA mothers $(\mathrm{r}=0.512, \mathrm{p}<0.02)$. In both groups, offspring's leptin levels decline significantly from $\mathrm{UC}$ to $\mathrm{N} 1$ and stabilized up to N4, and this decline is more pronounced in IUGR, while adiponectin levels demonstrated an inverse profile.

Conclusions: The higher leptin levels in association with lower adiponectin levels in IUGR vs AGA mothers possibly reflect their state of inflammation and chronic stress, which finally results to restricted fetal growth. Moreover, the positive association between leptin and adiponectin levels observed only in AGA mothers could rather indicate that a compensatory mechanism is functional in AGA mothers to counteract the inflammatory state of parturition. This innovative finding may serve to predict outcome in pregnancies at risk. The pronounced leptin decline in IUGR from UC to N1 may result from combination of intrauterine stress, leading to higher fetal levels and decreased adipose tissue, resulting to lower extrauterine levels.

\section{SERUM OSTEOPROTEGERIN AND RANKL CONCENTRATIONS IN THE PERINATAL PERIOD: THE IMPACT OF INTRAUTERINE GROWTH RESTRICTION}

D Briana ${ }^{1}$, A Malamitsi-Puchner ${ }^{1}$, D Gourgiotis ${ }^{2}$, M Boutsikou $^{1}$, L Kontara $^{1}$, A Marmarinos ${ }^{2}, \mathrm{~S} \mathrm{Baka}^{1}$, T Boutsikou ${ }^{1}$, D Hassiakos ${ }^{1}$

${ }^{l}$ Neonatal Division, 2nd Department of Obstetrics and Gynecology, Athens University, Greece, ${ }^{2}$ Research Laboratories, 2nd Department of Pediatrics, Athens University, Greece

Background and Aims: RANKL (receptor activator of $\mathrm{NF}-\mathrm{\kappa B}$ ligand) belongs to the tumor necrosis factor (TNF) ligand family and is crucial for osteoclast formation. Osteoprotegerin (OPG), a member of the TNF receptor superfamily inhibits osteoclastogenesis and bone resorption. This study aimed to investigate RANKL and OPG in full term intrauterine growth restricted (IUGR-below the 10th customized centile) and appropriate for gestational age (AGA) fetuses (UC) and neonates on day 1 (N1) and 4 (N4) postpartum, as well as in their mothers (MS) before delivery.

Methods: RANKL and OPG were measured by enzyme immunoassays in the serum of 20 IUGR and 20 AGA mother-infant sets.

Results: OPG levels were significantly higher in MS than UC, N1 and N4 in both groups (in all cases $\mathrm{p}<0.01$ ) and RANKL levels were significantly higher in N4 than MS, UC and N1 (in all cases $\mathrm{p}<0.02$ ). In the AGA group a negative correlation was documented between $\mathrm{N} 1$ and N4 OPG levels and birthweight $(\mathrm{r}=-0.589, \mathrm{p}=0.006$ and $\mathrm{r}=-0.530, \mathrm{p}=0.016$ respectively) as well as centiles $(\mathrm{r}=-0.640, \quad \mathrm{p}=0.002, \quad$ and $\mathrm{r}=-0.666, \quad \mathrm{p}=0.001$ respectively). Furthermore, a negative correlation between MS RANKL and MS OPG was found $(r=-$ $0.484, \mathrm{p}=0.03$ ) in the AGA group.

Conclusions: In conclusion, maternal OPG levels are increased before delivery, possibly due to placental production. Increased N4 RANKL levels could be attributed to postnatal bone remodeling. The negative association of OPG with birthweight and centile could imply its protective role to diminished bone mineralization. Lastly, the negative correlation of MS RANKL to MS OPG reinforces their role in bone homeostasis. 


\section{ADRENAL STRESS RESPONSE IN PRETERM INFANTS OF MORE THAN 30 WEEKS GESTATIONAL AGE DETERMINED BY URINARY EXCRETION RATES OF GLUCOCORTICOIDS METABOLITES}

M Heckmann ${ }^{1}$, M F Hartmann ${ }^{1}$, B Grofer ${ }^{1}$, R H Boedeker ${ }^{2}$, S A Wudy ${ }^{1}$

${ }^{1}$ Department of General Pediatrics and Neonatology, Center of Child and Adolescent Medicine, Justus Liebig University, Giessen, Germany, ${ }^{2}$ Institute of Medical Statistics, Justus Liebig University, Giessen, Germany

Background and Aims: The aim was to investigate urinary excretion rates of glucocorticoid metabolites $(\mathrm{GM})$ in well and ill preterm infants $(\mathrm{PI})>30 \mathrm{wks}$.

Methods: Illness severity was measured by the score for neonatal acute physiology (SNAP). GM were profiled by gas chromatography-mass spectrometry in 24-h urinary samples. Excretion rates of GM were summed to calculate cortisol production rates (CPR) in 61 well (mean \pm SD: $33.6 \pm 1.7 \mathrm{wks})$ and 20 ill $(33.2 \pm 1.6 \mathrm{wks})$ PI.

Results: During the 1st week of life, SNAP were significantly higher in the group of ill PI $(\mathrm{p}<0.01)$ compared to well PI except day $2(\mathrm{p}=0.06)$. Medians of CPR $(\mu \mathrm{g} / \mathrm{kg} / \mathrm{d}$ per mg creatinine) in ill (well) PI were at 1st day: 31 (28); 2nd day: 24 (28), 3rd day: 27 (26); 5th day: 28 (17); 2nd week: 20 (19); 3rd week: 17 (19); 4th week: 16 (16) and 2nd month: 17 (23). There was a significant correlation between the maximum of SNAP and the maximum of CPR (OR 3.7, 95\%CI 1.2-11.5); $\mathrm{p}=0.02)$ in a regression model which comprised gestational age $(\mathrm{p}=0.004)$, mode of delivery (NS), gender (NS) and the administration of prenatal steroids (NS) as co-variables. The CPR of PI $>30$ wks were 1.5-3 times lower compared to PI $<30$ wks (J Clin Endocrinol Metab 2005;90:5737).

Conclusions: In contrast to PI $<30 \mathrm{wks}$, there was a positive correlation between severity of illness and CPR in PI > $30 \mathrm{wks}$, indicating a maturation of the adrenal stress response. This maturation was concurrently associated with significantly lower CPR compared to PI $<30$ wks.

\section{EVALUATION OF BONE DENSITY AND METABOLISM IN GIRLS WITH PRECOCIOUS AND EARLY PUBERTY DURING TREATMENT WITH GnRH AGONIST}

\author{
A Assa ${ }^{1}$, M Weiss $^{2}$, D Aharoni ${ }^{3}$, A Mor $^{3}$, \\ A Bar-Chaim $^{3}$, L Tsoref ${ }^{4}$, T Schwartz $^{4}$, T Bistrizer ${ }^{1}$ \\ ${ }^{1}$ Pediatric Endocrinology Unit, Assaf Harofeh Medical \\ Center, Zerifin, Israel, ${ }^{2}$ Endocrinology Unit, Assaf \\ Harofeh Medical Center, Zerifin, Israel, ${ }^{3}$ Central \\ Laboratory, Assaf Harofeh Medical Center, Zerifin, \\ Israel, ${ }^{4}$ Sunlight Medical Ltd., Tel Aviv, Israel
}

Background and Aims: Major changes in bone mineral density (BMD) occur during puberty. Few studies have shown no impairment in BMD during GnRH agonist therapy in girls with precocious (PP) and early puberty (EP) using Dual Energy X-ray Absorptiometry (DXA). Quantitative Ultra Sound (QUS) technology, which evaluates bone strength (BS), has not been studied in this group yet. Our objective was to evaluate BMD, BS and bone metabolism in girls with PP/EP during treatment with GnRH agonist.

Methods: We evaluated 25 girls: 10 with $\mathrm{PP}($ age $<8)$ and 15 with EP $(8<$ age $<9)$. BMD was measured by both DXA (Hologic QDR 4500) and QUS (Omnisense 7000P) at baseline, after 6 and 12 months from onset of therapy. Biochemical markers of bone turnover-serum Bone Specific Alkaline Phosphatase (BSALP) and urinary Deoxypyridinoline $\backslash$ Creatinine (DPD $\backslash \mathrm{CR}$ ) were also measured.

Results: Mean lumbar spine (LS) and femoral neck (FN) BMD measured by DXA were significantly low at baseline (LS: $p<0.0001, F N: p<0.002$ ). BS measured by QUS was significantly lower at the radius $(p<0.001)$ but normal at the tibia. There was no significant change in BMD and BS throughout the first year of therapy. Serum BSALP measurements were appropriate for age and pubertal stage at baseline. Urinary $\mathrm{DPD} \backslash \mathrm{CR}$ measurements were significantly high $(\mathrm{p}<0.001)$ at baseline. Both metabolic parameters did not differ significantly during the first year of therapy.

Conclusions: Girls with central PP and EP have low $\mathrm{BMD}$ and $\mathrm{BS}$ for chronological age and increased bone resorption markers. These parameters do not change significantly during the first year of therapy with $\mathrm{GnRH}$ agonist.

\section{INFECTIOUS DISEASES AND THE BRAIN}

\section{N Curtis}

Department of Paediatrics, University of Melbourne, Royal Children's Hospital, Melbourne, Australia

Abstract not available at the time of printing.

\section{TEN-YEAR TENDENCY OF PRIMARY RESISTANCE OF HELICOBACTER PYLORI ISOLATES TO METRONIDAZOLE, CLARITHROMYCIN AND AMOXICILLIN IN CHILDREN}

B V Nguyen ${ }^{1}$, N Kalach ${ }^{2}$, J Raymond ${ }^{3}$

${ }^{1}$ Hanoi Medical University, Hanoi, Vietnam, ${ }^{2}$ Paediatric Saint Antoine Clinic, Saint Paul Hospital, Catholic

University, Lille, France, ${ }^{3}$ Department of Microbiology, Saint Paul-Cochin Hospitals Group, Paris V University, Paris, France

Background and Aims: Good knowledge on susceptibility of Helicobacter pylori (HP) isolates to antibiotics of choix allows improving HP eradication rate in the treatment. The present study was to assess resistance rate to metronidazole (Metro), clarithromycin (Clar) và amoxicillin (Amox) of HP isolates from children, according to sex, age group and period of time. Methods: Retrospective study investigated resistance rate and minimal inhibitory concentration (MIC) of HP isolates from 282 children under 18 years during a period from 1993 to 2002 to Metro using agar disk diffusion method, to Clar and Amox using E-test.

Results: Resistance rate of HP isolates was $37,5 \%$ to Metro, $25,8 \%$ to Clar and $7,3 \%$ to both. No isolate 
resistant to Amox was identified. The rate of resistance to Metro was $52,3 \%$ in isolates from children above 10 years old and $30,5 \%$ in those from younger $(p<0,03)$, and 50,8\% from those isolated between 1993 and 1995, $37,9 \%$ in those from 1996 and 1999 and 21,9\% in those from 2000 and $2002(p<0,004)$. MICs in Metro resistant isolated in 2000-2002 isolates were significantly higher than those in 1996-1999 $(\mathrm{p}<0,005)$ and those in 1993-1995 $(\mathrm{p}<0,0001)$. MICs in Clar resistant isolates in 2000-2002 were significantly lower than those in 1996$1999(\mathrm{p}<0,01)$. MICs in isolates dually resistant to Metro and Clar were significantly higher than those in single resistant ones $(\mathrm{p}<0,01)$ and $\mathrm{p}<0,005$, respectively).

Conclusions: There was a high rate of HP isolates resistant to Metro and Clar in children, according to sex, age group and time while no isolate resistant to Amox was found.

\section{INCREASED RATES OF INVASIVE PNEUMOCOCCAL INFECTIONS IN CHILDREN IN A TERTIARY REFERRAL HOSPITAL}

\author{
J Mayordomo ${ }^{1}$, J Rodríguez ${ }^{1}$, A Fleites ${ }^{2}$, F Pérez $^{2}$, \\ $\mathrm{S}$ Jiménez ${ }^{1}$, A Pérez ${ }^{1}$ \\ ${ }^{l}$ Department of Pediatrics, Hospital Universitario \\ Central de Asturias, Ovideo, Spain, ${ }^{2}$ Department of \\ Microbiology, Hospital Universitario Central de \\ Asturias, Oviedo, Spain
}

Background and Aims: Knowing the epidemiology of invasive pneumococcal disease (IPD) in children is important, mainly when the introduction of antipneumococcal vaccine in inmunization schedules is considered.

Methods: Clinical records of all children under 14 years of age, with confirmed IPD from 1989 to 2005 were reviewed. Main clinical and laboratory data, serogroups, and antimicrobial resistance pattern were analyzed. Serotyping was performed at the National Microbiology Center (Madrid). Rates of IPD were calculated.

Results: There were 83 cases of IPD, and $48 \%$ of the cases occurred in children under 2 years old. Rates of IPD rose from 7.8 to 30.0 cases per 100,000 children in the 1989-1991 and 2003-2005 periods, respectively $(p<0.05)$. Underlying disease was present in $13 \%$ of patients and two patients died. Main sites of infection were: bacteraemia/sepsis $(80.2 \%)$, with associated pneumonia in $34.5 \%$ of them; and meningitis (16.1\%). Serum levels of CRP and procalcitonine were elevated in $83.3 \%$ and $55.6 \%$ of test performed, respectively. Throughout the study, resistance to penicillin declined from $40 \%$ to $14.3 \%(\mathrm{p}<0.036)$, while resistance to erythromycin and cefotaxime has not changed. The most frequent serogroups were 19, 23, 14 and 6, and overall $62.4 \%$ are included in heptavalent vaccine. In the last 4 years, serotypes more frequently isolated were 19A, $18 \mathrm{C}, 6 \mathrm{~A}$ and $19 \mathrm{~F}$.

Conclusions: This study shows that rates of IPD in children have increased in the last 16 years in our hospital. The most prevalent serogroups isolated are included in the heptavalent vaccine, but serotypes 19A and $6 \mathrm{~A}$ also have increased in the last 4 years.

\section{EFFICACY OF A MULTIFACETED EDUCATION PROGRAM ON HAND HYGIENE}

O K Helder ${ }^{1}$, R F Kornelisse ${ }^{1}$, M Op de Weegh ${ }^{2}$, M Wouterson ${ }^{2}$

${ }^{1}$ Division of Neonatology, Erasmus MC - Sophia Children's Hospital, Rotterdam, The Netherlands ${ }^{2}$ Erasmus University Rotterdam, Rotterdam, The Netherlands

Background and Aims: Adequate hand hygiene is widely accepted as the cornerstone in nosocomial infection control. The overall compliance with hand hygiene is considered to be poor in different studies and difficult to enhance. A multifaceted campaign showed to be the best way to improve the behavior towards hand hygiene. We evaluated the effectiveness of an education program on compliance with hand hygiene in a neonatal intensive care unit (NICU).

Methods: The study was performed on the NICU (level IV) of Erasmus MC - Sophia Children's Hospital. The education program (intervention) was based on Centers for Disease Control and Prevention guidelines, the observed practice prior to the intervention and the Theory of Planned Behavior (attitude, subjective norms, perceived behavior control). An interrupted time series study was performed by guided observations of hygienic behavior of health care workers. The mean dry time after disinfection and the amount of used hand alcohol were measured. Two pre-intervention observations took place in the period January 2005 - May 2005 and the first post-intervention observations took place in the period December 2005 - March 2006.

Results: The results of the first $(n=222)$ and second $(n=378)$ pre-intervention observations and one postintervention measurement $(\mathrm{n}=241)$ showed respectively a median (SD) dry time of $5( \pm 7), 7( \pm 9)$ and $12( \pm 8)$ seconds $(\mathrm{P}<.05)$. The amount of used hand alcohol for each unit increased from $51.9-55.3 \mathrm{ml} /$ bed to 65.4 $\mathrm{ml} /$ bed after intervention.

Conclusions: The multifaceted education program seem to improve hand hygiene.

\section{EPIDEMIOLOGY OF INVASIVE FUNGAL INFECTION IN A PICU}

R Chhabra, P Maheshwari, M Nolan, S Nadel, D P Inwald

PICU, St Mary's Hospital, London, UK

Background and Aims: To determine the incidence and risk factors for invasive fungal infection in our Paediatric Intensive Care Unit (PICU).

Methods: Case records of patients admitted from August 2002 to August 2005 were reviewed. Data collected included underlying medical condition, site of infection, associated bacterial and viral infections, antimicrobial and antifungal treatment and mortality.

Results: 1300 patients were admitted to PICU in the study period. 176 of these stayed for $>5$ days. Of these 176, $29(16 \%)$ developed fungal infection, 28 with candida and 1 with aspergillus flavus. $3(10 \%)$ of these had an underlying immune deficiency. Candida species cultured were albicans (23), tropicalis (3), parapsilosis 
(1) and krusei (1). Fungi were cultured from blood (5), urine (10), endotracheal secretions (9) or in catheters in sterile sites (9), either alone or in combination. 26 (90\%) were on antibiotics to cover hospital-acquired infection but only $1(3 \%)$ was on fluconazole prophylaxis. $1(3 \%)$ of patients with fungal infection died. In the remaining 147 patients, $115(66 \%)$ were on antibiotics to cover hospital-acquired infection and $12(8 \%)$ patients were receiving fluconazole prophylaxis. There was no significant difference in the proportion of patients on nosocomial antibiotic cover or on fluconazole prophylaxis between the two groups $(\mathrm{p}=\mathrm{ns}$, Fisher's exact test).

Conclusions: All patients with invasive fungal infection required a prolonged stay of $>5$ days on PICU. Almost all were on treatment with antibiotics to cover hospitalacquired infection at the time of diagnosis. Further work is required to elucidate the role of prophylactic fluconazole in PICU.

\section{PREDICTING SEVERE BACTERIAL INFECTION IN INFANTS AND CHILDREN WITH FEVER WITHOUT A SOURCE}

\section{B Andreola, S Bressan, S Callegaro, M Soligo, T Zangardi, L Da Dalt \\ Department of Pediatrics, University of Padova, Italy}

Background and Aims: Fever without a source (FWS) is a common reason for children to visit the Pediatric Emergency Department (PED) and it remains a challenge for the clinician to early detect severe bacterial infections (SBI). Our aims were to assess the incidence of SBI in an unselected population of children $<36$ months attending our PED and to compare the performance of different biological markers in detecting these infections.

Methods: Over a 12 months period all children aged 7 days - 36 months admitted for FWS were prospectively included. Patients underwent diagnostic tests according to the guidelines in use. Clinical and laboratory parameters - C-Reactive Protein (CRP), White Blood Cell (WBC) count, Absolute Neutrophil Count (ANC) were collected and compared in relationship to microbiological results. Specificity, sensitivity, multilevel likelihood ratios and ROC curve analysis were calculated.

Results: 385 patients were included; 75 (19.5\%) had the final diagnosis of SBI, mostly represented by acute pyelonephritis. No difference was found in the clinical parameters between children with and without a SBI. Of the laboratory parameters CRP obtained the best sensitivity, specificity, positive and negative likelihood ratio values. Area under ROC curve was 0.881 (IC 95\% $0.824-0.925$ ) for CRP, 0.779 (IC $95 \% 0.710-0.838$ ) for WBC count, and 0.782 (IC $95 \% 0.713-0.841$ ) for ANC.

Conclusions: The risk of SBI is still high in this postHIB vaccine era. CRP is superior to clinical parameters and to other more conventional marker in predicting which children have a SBI and need prompt antibiotic treatment.

\section{OROPHARYNGEAL COLONIZATION BY GRAM NEGATIVE PATHOGENS AND ORAL CARE OF CRITICALLY ILL CHILDREN}

M L G Pedreira ${ }^{1}$, L T S Santos ${ }^{1}$, D M Kusahara ${ }^{1}$, M A S Peterlini ${ }^{1}$, A C C Pignatari ${ }^{2}$, A M O Machado ${ }^{3}$

${ }^{1}$ Department of Nursing, Federal University of São Paulo, Brazil, ${ }^{2}$ Infectious and Parasitary Disease, Federal University of São Paulo, Brazil, ${ }^{3}$ Microbiology Laboratory, Federal University of São Paulo, Brazil

Background and Aims: More than $60 \%$ of VAP episodes are related to aerobic gram negative bacteria, predominantly Pseudomonas aeruginosa and Acinetobacter spp. The pathogenesis of VAP can be related to bacteria translocation from oropharynx to tracheobronchial tree. The aim of the study was to describe characteristics of gram negative pathogens colonization of children's oropharyngeal secretion, through different oral care methods.

Methods: Prospective randomized controlled study realized in a University Hospital PICU in the city of S?o Paulo, Brazil. The study was approved by the ethics research committee. The sample was composed of 56 children, $29(51.8 \%)$ in Control Group-CG (nonpharmacological intervention) and $27(48.2 \%)$ in Experimental Group-EG $(0.12 \%$ chlorhexidine $)$. Microbiological cultures of oropharyngeal were obtained 24, 48 and 96 hours after admission and analyzed according to bacterial identification data and antimicrobial resistance.

Results: The majority of the sample was composed of

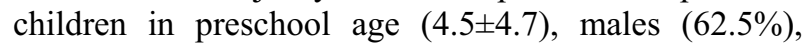
white $(67.8 \%)$, eutrophic $(50.0 \%)$, with surgical diagnosis $(55.3 \%)$ and emergency admissions $(51.8 \%)$. Gram negative were isolated in $39(50 \%)$ of $78 \mathrm{CG}$ cultures and in $43(60.6 \%)$ of the 71 in EG; $28(30.8 \%)$ in CG were pathogens and $26(35.2 \%)$ in EG. In CG $42.8 \%$ and EG $50.0 \%$ of pathogens presented antibiotic resistance to more than 14 groups of antibiotics. The main pathogens (CG;EG) identified were Acinetobacter baumanii $(28.0 \% ; 3.2 \%)$, Pseudomonas aeruginosas (24.0\%;19.4\%), Klebsiella pneumoniae $(20.0 \% ; 32.0 \%)$ and Enterobacter spp $(12.0 \% ; 29.0 \%)$. Conclusions: The use of $0.12 \%$ chlorexidine don't reduce the number of cultures with gram negative pathogens in the EG. Pathogens frequency was different between the studied groups. Acknowledgments: Dr. Pedreira acknowledges support for research by grant 04/133612-FAPESP.

\section{PREVALENCE OF ALLERGIC RHINITIS IN POPULATION OF SCHOOL-AGE CHILDREN}

L D Jorjoliani ${ }^{1}$, N M Adamia ${ }^{2}$, N S Manjavidze ${ }^{2}$, Z A Charkviani ${ }^{2}$, I K Babilashvili ${ }^{2}$

${ }^{l}$ Dept of Allergology and Clinical Immunology, Institute of Pediatrics, ${ }^{2}$ Dept of Pediatrics, Tbilisi State Medical University, Tbilisi, Georgia

Background and Aims: Purpose of the work was to study prevalence of allergic rhinitis and clinical-allergic peculiarities of allergic rhinitis together with bronchial asthma in population of school-age children in Tbilisi. 
Methods: The researches were conducted based on questioning of random and representative groups of school-age children, by the cross-section method of epidemiologic research. At the first stage of research work, there were questioned 3000 children from 6 to 17; at the second stage clinical-allergological researches were conducted with 256 randomly selected children with the signs of rhinitis.

Results: According to the obtained results, prevalence of allergic rhinitis was $13.2 \%$. In $19.1 \%$ of cases, allergic rhinitis was accompanied with bronchial asthma and in $8 \%$ of the patients there was indicated bronchial spasm of physical load (verified as clinically, also through the test with physical load), coughing version of asthma was observed in $4.5 \%$ of children. Rhinitis among Tbilisi children population was $13.2 \%$. There is revealed high frequency $(\mathrm{p}<0.001)$ of late diagnostics and prevalence of light and medium intermitting forms of allergic rhinitis.

Conclusions: Combination of inherited predisposition to allergy with the atopic dermatitis, in the in the anamnesis of children with the increased level of general $\mathrm{IgE}$ in the blood serum may be considered as early diagnostic predictor of atopic phenotype as for the allergic rhinitis, also for its combination with the bronchial asthma.

\section{OCCURRENCE OF WITHDRAWAL SYMPTOMS IN CRITICALLY ILL CHILDREN AFTER LONG- TERM ADMINISTRATION OF SEDATIVES AND/OR ANALGESICS}

\author{
E Ista ${ }^{1}, \mathrm{M}$ van Dijk $^{2}$, D Tibboel ${ }^{2}, \mathrm{M}$ de $\operatorname{Hoog}^{1}$ \\ ${ }^{1}$ Pediatric Intensive Care Unit, ${ }^{2}$ Department of \\ Pediatric Surgery, Erasmus MC-Sophia Children's \\ Hospital, Rotterdam, The Netherlands
}

Background and Aims: Ventilated, critically ill children commonly receive sedative and analgesic drugs to ease their mental burden, anxiety and pain, induced by frightening or painful interventions and environmental factors in the pediatric intensive care unit (PICU). Intravenous opioids (such as morphine and fentanyl) and benzodiazepines (such as midazolam) are the most used drugs for this purpose. Abrupt discontinuation or rapid tapering off after long-term administration may lead to problems of tolerance, physiological or psychological dependence and withdrawal symptoms. The aim of this study was to describe the frequency of withdrawal symptoms and the correlation between total doses of benzodiazepine or opioids and withdrawal symptoms.

Methods: This prospective study was performed in children admitted to the PICU of the Erasmus MC Sophia Children's Hospital, Rotterdam, The Netherlands. Patients were included if midazolam and/or opioids were administrated for more than 5 days. Withdrawal symptoms were assessed using the Sophia Benzodiazepine \& Opioid Withdrawal Scale (SBOWS), which includes all withdrawal symptoms described in the pediatric literature. Withdrawal symptoms were observed by the caregiving nurse.

Results: 2188 observation in 79 children were recorded. Forty-two percent were performed within $24 \mathrm{~h}$ after decreasing or discontinuation of medication (decrease group). Symptoms representing overstimulation of the Central Nervous System such as anxiety, agitation, grimacing, sleep disturbance, increased muscle tension and movement disorder were observed in more than $10 \%$ of the observations. In the category gastrointestinal-dysfunction diarrhea and gastric retention were mostly observed (respectively $14.5 \%$ and $13.0 \%$ ). Tachypnea, fever, sweating and hypertension were observed in more than $13 \%$ of the observations as manifestation of autonomic dysfunction. There are hardly any differences detected in the frequency of the 24 withdrawal symptoms between total dataset and the decrease group. The Spearman Rank Correlation coefficient (rs) for the relation between total doses of midazolam and maximum sum score (of the SBOWS) was $0.51(\mathrm{p}<0.001)$. The correlation between total doses of opioids and the maximum sum score was an rs of $0.39(\mathrm{p}=0.001)$. The correlation between duration of administered medication and maximum sum score was an rs of $0.52(\mathrm{p}<0.001)$.

Conclusions: This study gives a complete overview of the frequency of the 24 withdrawal symptoms in children after decrease and/or discontinuation of benzodiazepines and/or opioids. There is insufficient evidence to state that withdrawal symptoms with a frequency $<10 \%$ can be included in a clinical withdrawal scale. Duration and high doses of benzodiazepines and/or opioids are risk factors for development of withdrawal symptoms in children. The SBOWS should be the basis for further development of a withdrawal assessment tool. Further prospective research of symptoms with low frequency of occurrence and coherence as well as research into the interdependency of symptoms is recommended.

\section{HOW RELIABLE DO WE ASSESS PAIN IN THE LONG RUN?}

J Kats, H de Graaf, M van Dijk

Pediatric Surgical Intensive Care Unit, Erasmus MCSophia's Children Hospital, Rotterdam, The Netherlands

Background and Aims: In 1999 our Pediatric Surgical Intensive Care Unit implemented the COMFORT behavior scale in daily practice combined with a treatment algorithm. All nurses were trained in pain assessment and each had to perform ten assessments with a trained nurse to determine interrater reliability using the linearly weighted Cohen's Kappa. Since than pain assessment became standard of care with. In 2004 and 200514787 combined COMFORT behavior and Visual Analogue Scale pain scores were assessed in 612 patients. Although from the outside this seemed satisfactory we questioned the (interrater) reliability of scoring by the nurses. This study aimed to determine current interrater reliability.

Methods: Nurses assessed COMFORT behavior and a Numeric Rating for pain and distress at bedside. Linearly weighted Cohen's Kappa was calculated and compared with the previous one in eight nurses. In addition the Intraclass correlation coefficient (ICC) for COMFORT total score and NRS pain and distress was calculated. 
Results: Eight nurses assessed 78 paired scores in ten infants. Interrater reliability ranged from 0.21 to 0.64 and was poor to moderate. The ICC for COMFORT total score was 0.60 , for NRS pain 0.55 and for NRS distress 0.46. Analysis on item level showed large discrepancy in scoring for muscle tone (kappa 0.13) and facial tension (kappa 0.23) and acceptable kappa's for crying $(0.73)$ or respiratory response $(0.67)$.

Conclusions: Despite satisfactory frequency of pain assessment, interrater reliability between experienced nurses on our PSICU was poor. In future clear instruction is required to maintain an acceptable level of scoring.

\section{ANALGESIC EFFECT OF TV WATCHING DURING VENIPUNCTURE}

C V Bellieni, G Morgese, G Buonocore, S Perrone

Department of Pediatrics, Obstetrics and Reproduction Medicine, University of Siena, Italy

Background and Aims: We know the importance of parents' collaboration in helping children to cope with pain by their presence and distraction. We recently successfully used this approach in neonatal analgesia. We also know the capturing power of television on children. The difference between these two approaches is that the former is active and involves affectivity, though fear may be transmitted to the child, whereas the latter is passive. Our aim was to assess the analgesic effect of active and passive distraction (parent and television) during venipuncture.

Methods: We studied 69 children aged 7-12 years undergoing venipuncture. The children were randomly divided into three groups: a control group (C) without any distraction procedure, a group TV in which passive distraction was used, using a TV cartoon, and a group $\mathrm{M}$ in which mothers performed active distraction. Both mothers and children scored pain after the procedure.

Results: Main pain levels rated by the children were $23.04(\mathrm{SD}=24.57), 17.39(\mathrm{SD}=21.36)$ and $8.91(\mathrm{SD}=$ 8.65) for $\mathrm{C}, \mathrm{M}$, and TV groups respectively. Main pain levels rated by mothers were $21.30(\mathrm{SD}=19.9), 23.04$ $(\mathrm{SD}=18.39)$ and $12.17(\mathrm{SD}=12.14)$ for $\mathrm{C}, \mathrm{M}$, and $\mathrm{TV}$ groups respectively. Scores assigned by mothers and children indicated that procedures performed during TV watching were less painful $(\mathrm{p}<0.05)$ than control and procedures performed during active distraction.

Conclusions: TV watching was more effective than active distraction. This was due the emotional participation of mothers in the active procedure, or of the distracting power of television.

\section{BIS-GUIDED SEDATION VERSUS STANDARD PRACTICE IN THE PAEDIATRIC INTENSIVE CARE UNIT}

\author{
A S Ramelet ${ }^{1,2}$, A W Duncan ${ }^{3}$, L Flynn ${ }^{3}$

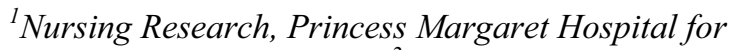 \\ Children, Perth, Australia, ${ }^{2}$ School of Nursing and \\ Midwifery, Curtin University of Technology, Perth, \\ Australia, ${ }^{3}$ Paediatric Intensive Care Unit, Princess \\ Margaret Hospital for Children, Perth, Australia
}

Background and Aims: The Bispectral index (BIS) is a processed EEG parameter that indicates the hypnotic effects of anaesthetic and sedative agents on the brain. BIS may be useful in achieving optimal level of sedation and preventing oversedation in the paediatric intensive care unit (PICU) patient. The objectives of this study were to evaluate the clinical utility of the Bispectral index (BIS) monitoring in titrating sedative agents when compared with Standard Practice (SP) and to determine the correlation of the BIS and the level of sedation, using the Modified Motor Activity Assessment Scale (MMAAS).

Methods: This single blind randomised controlled study was conducted in the paediatric intensive care unit of a tertiary referral hospital in Western Australia. Following parental consent, ventilated children requiring sedation were randomly assigned to the BIS-guided sedation group or the control group (standard practice).

Results: Data from 75 children provided 1168 valid paired observations. The median age was 16.6 months, ranging between 0.9 and 208.7 months. Preliminary results indicated a $10 \%$ increase of midazolam $(0.09$ and $0.1 \mathrm{mg} / \mathrm{Kg} / \mathrm{h}$ ) and opioids (48 and $53 \mathrm{mcg} / \mathrm{Kg} / \mathrm{h}$ ) in the BIS group. The median correlation coefficient between MMAAS and BIS was 0.25 (range -0.78 and 0.87 ) in the control group.

Conclusions: BIS-guided sedation does not seem to help reducing the amount of midazolam in sedated children when compared to the MMAAS. However, these results are preliminary and should be interpreted with caution. Further investigation is required to determine the specific situations in which the BIS could be useful in the management of sedation in the PICU.

\section{SLEEP DISTURBANCES IN CHILDREN HOSPITALIZED IN PEDIATRIC INTENSIVE CARE UNIT (PICU)}

\author{
R Shehadeh $^{1}$, S Anson ${ }^{2}$, G Bar-Joseph ${ }^{1}$, M Halbertal ${ }^{1}$,

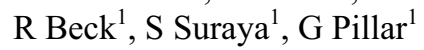 \\ ${ }^{1}$ Rambam Medical Center and Technion Institute of \\ Technology, Haifa, Israel, ${ }^{2}$ Ben-Gurion University of \\ The Negev, Beer Sheva, Israel
}

Background and Aims: Sleep is of great importance to hospitalized patients. Sleep facilitates recovery. PICU's are characterized by intense activity day and night; the work of the nursing staff involves noise and requires light, which may result in sleep disturbances. The purpose of this study was to evaluate sleep disturbances in the PICU. Methods: Sleep patterns of 30 children were monitored by Actigraphy and Manual sound level meter from $7 \mathrm{pm}$ till $7 \mathrm{am}$ the next morning. Factors may interrupt sleep were monitored. Results: Thirty children $(19 \mathrm{M} / 11 \mathrm{~F})$ were observed. All were fully conscious, not treated by any hypnotics or sedatives and no history of sleep disorders. Their mean age was $6 \pm 4$ years. Average total sleep time was $4 \pm 2$ hours, with a sleep efficiency of $40 \pm 21 \%$. A significant negative correlation existed between sleep efficiency and the nursing staff activity level $(\mathrm{r}=-0.53, \mathrm{p}<0.05)$, total general activities $(\mathrm{r}=-0.51, \mathrm{p}<0.05)$, light above the patient $(\mathrm{r}=-0.73$, $\mathrm{p}<0.001)$, the instrumentation level $(\mathrm{r}=-0.56, \mathrm{p}<0.001)$ and the average noise level $(\mathrm{r}=-0.74, \mathrm{p}<0.001)$. 
Similarly, total sleep time correlated negatively with these factors $(\mathrm{r}=-0.49,-0.39,-0.48$ and $-0.62, \mathrm{p}<0.05$ for all. Conclusions: Childrens' sleep pattern in the PICU is severely disturbed. Nursing staff is unaware of the importance of efficient sleep, which facilitates recovery. Future studies should focus on the outcome effects of modifying these preventable adverse factors. Most of the disturbances are preventable during night.

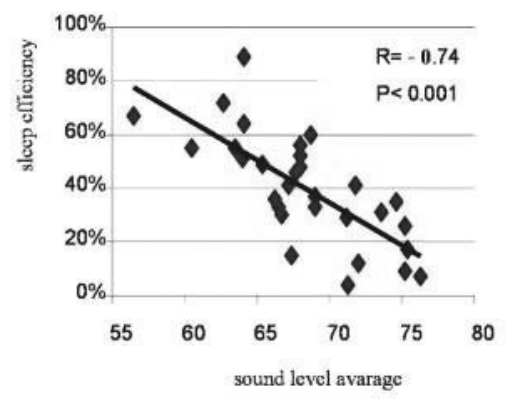

\section{HALLUCINATORY EXPERIENCES REPORTED BY CHILDREN FOLLOWING AN INTENSIVE CARE ADMISSION}

\author{
G A Colville ${ }^{1}, \mathrm{H}_{\text {Tighe }}{ }^{2}, \mathrm{C}$ M Pierce ${ }^{2}$ \\ ${ }^{\text {I} P a e d i a t r i c ~ P s y c h o l o g y ~ S e r v i c e, ~ S t ~ G e o r g e ' s ~ H o s p i t a l ~}$ \\ ${ }^{2}$ PICU, Great Ormond Street Hospital, London, UK
}

Background and Aims: Our aim was to establish, by direct interview, the extent and nature of children's hallucinations following a paediatric intensive care (PIC) admission. We hypothesized that the association between delusional memories and posttraumatic stress reported in adult intensive care survivors would also apply to children.

Methods: The setting was a 20 bed general PIC unit in a tertiary referral Children's Hospital. Children $(n=102)$, aged $7-17 y$, were interviewed 3 months after discharge using the ICU Memory Tool (Jones et al 2000) and the CRIES PTSD scale (Perrin et al 2005).

Results: Thirty-three children (32\%) reported at least one delusional memory. Of these 25 reported having hallucinations and 8 vivid or bizarre dreams. There was a significant association between prevalence of hallucinations and length of time on morphine: $0 / 10$ $(0 \%)$ in the no morphine group, $7 / 45(16 \%)$ in morphine for $<2$ days and $17 / 47(36 \%)$ in the morphine $>2$ days group $\left(\chi^{2}=8.84, \mathrm{p}=0.012\right)$. The association with midazolam was not significant $(\chi 2=2.60, \mathrm{p}=0.279)$. Posttraumatic stress scores at 3 months were significantly associated with the presence of hallucinations $(p=0.002)$ and with emergency admission $(p=0.002)$. Extent of factual memory of admission was not related to subsequent posttraumatic stress.

Conclusions: These results highlight the importance of direct interviews with children in ascertaining the presence of hallucinations after a PIC admission. We propose that improved management of these distressing symptoms in children may lead to a reduction in short term distress and better longer-term psychological adjustment.

\section{THERAPY OF PERSISTENT DUCTUS ARTERIOSUS IN PRETERM INFANTS - WHY, WHEN AND HOW?}

\section{A H G Von Der Wense}

Department of Neonatology and Paediatric Intensive Care, Altonaer Kinderkrankenhaus, Hamburg, Germany

Background and Aims: Patency of the ductus arteriosus (PDA) is one of the most frequent problems complicating the clinical course of very low and especially extreme low birth weight infants (VLBW, ELBW). Thus a large number of preventive, prophylactic or therapeutic strategies have been developed over the last 30 years to minimize the incidence of PDA and its potentially harmful consequences. Recent clinical trials and metaanalyses will be discussed concerning efficacy in closure of PDA as well as side effects of pharmacological treatment and longterm outcome variables.

Methods: Literature Review and Prospective Cohort Study in the Hamburg Ibuprofen Trial.

Results: Ibuprofen (IBU) and Indomethacin (INDO) have been intensively studied for pharmacological closure of PDA. Metaanalyses and several trials have shown comparable efficacy for inducing closure of PDA in VLBW-Infants. Closure rates between 60 and $90 \%$ for VLBW infants and around $40-60 \%$ for ELBW infants have been reported for early treatment of PDA. Acute side effects are favouring IBU, because it has less vasoconstricive potential especially for renal perfusion. The occurence of oliguria is significantly lower for IBU compared to INDO. Furthermore less influence on cerebral perfusion has been shown for IBU, thus avoiding cerebral vasoconstriction. Mesenteric blood flow is significantly lower after treatment with INDO. Two studies have raised concern about a higher rate of chronic lung disease (defined as the need for oxygen therapy at 28 days) for the IBU group. However these findings have not been reported by other groups, and pulmonary outcome at 36 weeks of gestation was not investigated, although this is far more clinically relevant. Optimal timing of treatment still remains controversial. INDO has been shown to reduce the incidence of severe intraventricular hemorrhage (IVH), when used prophylactically during the first days of life. The rate of PDA and surgical intervention was also significantly lower. However long term outcome has been disappointing for the treatment group, showing no reduction of developmental deficit or infantile cerebral palsy. IBU as well has been investigated for prophylactic treatment. Incidence of PDA and surgical closure was also significantly reduced. There was no clear effect on IVH. One trial was stopped after occurrence of severe pulmonary hypertension in three patients. At the moment prophylactic use of INDO is questionable and of IBU is not recommended. Number needed to treat (NNT) for reduction of PDA surgery is 20 and for reduction of severe IVH 25, showing a high proportion of 'overtreated infants' in the prophylactic regimen.

Conclusions: IBU and INDO are both equally effective in pharmacological closure of PDA. IBU has significantly less acute side effects when used in an 'early therapeutic strategy' e.g. from day 3 of life. 
Cerebral perfusion is not altered. Prophylactic use of both drugs is potentially harmful, especially for infants at low risk for development of hemodynamic relevant PDA. Pulmonary hypertension should be excluded prior to treatment with cyclooxigenase inhibitors. Our own treatment strategy derived from the Hamburg Ibuprofen study is presented.

\section{IBUPROFEN TREATMENT OF PATENT DUCTUS ARTERIOSUS IN PRETERM INFANT, EXPERIENCE FROM STOCKHOLM}

\section{S Johansson}

\section{Department of Woman and Child Health, Karolinska Institutet, Stockhom, Sweden}

Background and Aims: Ibuprofen treatment of patent ductus arteriosus (PDA) was introduced at the Karolinska University Hospital, Stockholm, Sweden in 2001. During the years 2001 to 2004 clinical data was prospectively collected on all infant treated with ibuprofen, to assess renal function and clinical outcome. Methods: 83 infants (mean gestational age 27 weeks, standard deviation 2 weeks) with echocardiographic PDA-diagnosis received 98 courses of ibuprofen $(10+5+5 \mathrm{mg} / \mathrm{kg})$. Renal function and water balance were measured by urinary output and s-creatinine, s-urea and s-sodium. Short term closure was assessed by echocardiography 18-24 hours after completed ibuprofen course. Surgical closure rate was assessed at discharge. Bronchopulmonary dysplasia (BPD) was defined as need of supplementary oxygen at 36 postconceptional age.

Results: There was a transient reduction in renal function after the first and second dose of ibuprofen. After the third dose, renal function was not different from pre-treatment values. Short term closure was achieved in most infants, but the rate was lowest (71\%) among 23-24 week infants. Relapses were also common in this group, due to septicemias. Final non-surgical closure rate ranged from $57 \%$ among 23-24 week infants to $94 \%$ among infants born $\geq 29$ weeks. Accordingly, surgical closure rates varied between $43 \%$ to $6 \%$. The BPD rate was $44 \%$ in this cohort.

Conclusions: Ibuprofen PDA-treatment was associated with a transient renal dysfunction. The decrease in renal function was small but surveillance of renal function is indicated during ibuprofen treatment. Short term PDA closure with ibuprofen was achieved in most infants, but final non-surgical closure rate was low among infants born at 23-24 weeks. Whether optimized ibuprofen treatment strategies, such as earlier initation of treatment, could decrease surgical closure rate among the most immature infants remains to be determined. The BPD rate of $44 \%$ was comparable to the rate among indomethacin treated infants in 1997-1999 (45\%).

\section{IBUPROFEN, BILIRUBIN AND ALBUMIN: THE IMPORTANCE OF A BALANCE}

\section{B Van Overmeire}

Neonatology Unit, Department of Pediatrics, Antwerp University Hospital, Belgium
Ibuprofen at a dosing scheme of $10-5-5 \mathrm{mg} / \mathrm{kg}$ at 24 hour intervals is increasingly used as an alternative to indomethacin for the treatment of patent ductus arteriosus in premature infants. Because ibuprofen is highly bound to albumin it may interfere with bilirubin-albumin binding and increase the free fraction of bilirubin, augmenting the risk of bilirubin neurotoxicity.

A large interindividual variation in ibuprofen pharmacokinetics has been demonstrated in newborns. One hour after the first dose, ibuprofen plasma levels are reported to vary from 31 to $280 \mathrm{mg} / \mathrm{L}$. Ibuprofen is mainly metabolized by the cytochrome $\mathrm{P} 450$ complex and more specifically by the CYP2C 8 and CYP2C9 subfamily. Maturation of CYP2C increases fast after birth, reaching $30 \%$ of adult activity by one month of age. Ibuprofen clearance is highly variable among individual neonates and increases with advancing postnatal age. Ibuprofen serum half-lives decreased from 43 hours at age 3 days to 27 hours at age 5 days. Additionally, the pharmacokinetics may also be influenced by the presence of a ductal shunt. The volume of distribution changed more pronounced in infants with a closing ductus.

Ibuprofen is $95 \%$ bound to albumin and may interfere with bilirubin-albumin binding, thereby increasing the free fraction of bilirubin and induce a higher risk of bilirubin encephalopathy. High ibuprofen serum levels have been shown to have comparable displacing effects on free bilirubin as sulfizoxazole, a drug that has caused bilirubin encephalopathy in neonatal infants. Free bilirubin is superior to total bilirubin concentration in predicting the risk for bilirubin toxicity. The risk of inducing kernicterus depends on the concentration of ibuprofen, the binding constants of albumin for ibuprofen and bilirubin, and the bilirubin load at the moment when ibuprofen is administered. Albumin-ibuprofen binding is variable between neonates and probably also changes in time. On the first day after birth the biliubin pool is usually low, but increases steadily in the first week of life. In many neonatal intensive care units patent ductus arteriosus is preferentially treated when first signs of left to right ductal shunt develop, typically at the moment when the bilirubin pool is increased.

Several methods for the measurement of free bilirubin, or indirectly by the assessment of albumin binding, were developed. Recently an improved peroxidase assay has been developed with minimal serum dilution and two enzyme concentrations, yielding more accurate results.

In order to better understand the interactions between ibuprofen, albumin and bilirubin, the concentration of these molecules were analysed in plasma simultaneously with free bilirubin levels, before and after the administration of successive doses of ibuprofen in neonates of varying gestational and postnatal age with patent ductus arteriosus. The infants were followed-up after discharge and were screened for possible hearing deficits. No significant changes of free bilirubin concentrations were observed after the administration of ibuprofen. There was no correlation between these changes and the increase of ibuprofen levels. Free bilirubin increased inversely proportional to birth weight. No clinical signs of bilirubin neurotoxicity could be detected in surviving infants.

First results seem to demonstrate that the risk of bilirubin encephalopathy is not increased after treatment with ibuprofen at a dosing scheme of $10-5-5 \mathrm{mg} / \mathrm{kg}$, with peak 
levels below $60 \mathrm{mg} / \mathrm{L}$ and total bilirubin concentrations below $10 \mathrm{mg} / \mathrm{dL}$. However, when higher doses are applied in sick infants, the risk of kernicterus may markedly increase.

\section{CLINICAL AND IMMUNOLOGICAL EFFECTS OF THE LONG-TERM DIETARY SUPPLEMENTATION WITH A PROBIOTIC FERMENTED MILK IN PRE-SCHOOL CHILDREN ALLERGIC TO INHALANTS}

F Salvini $^{1}$, L Morelli ${ }^{2}$, A Ruscitto ${ }^{1}$, G Radaelli ${ }^{1}$, D Cattivelli ${ }^{2}$, C Agostoni $^{1}, \mathrm{M} \mathrm{Giovannini}^{1}$

${ }^{1}$ Department of Paediatrics, University of Milan, San Paolo Hospital, Milan, Italy, ${ }^{2}$ Microbiology Institute, Università Cattolica del Sacro Cuore, Piacenza, Italy

Background and Aims: Probiotics may have immunomodulatory functions, exerting beneficial effects in allergic disease. We investigated whether prolonged daily consumption of Lactobacillus casei DN114001 fermented milk improve clinical and immunological condition of children aged 3-6 years allergic to inhalants.

Methods: In a randomised-controlled multicentric trial patients were randomly assigned to receive for one year $100 \mathrm{ml}$ per day of L. casei DN-114 001 fermented milk (109 cells $/ \mathrm{ml}$ ), or no probiotics (controls). Clinical evaluations occurred every 3 months. Faecal flora composition was assessed every 6 months (30 treated, 15 controls). Outcome measures were allergy-related signs incidence, infections incidence/severity, IgE-G-A serological levels (baseline vs 12 months).

Results: Participants (187; 92 treated) were similar regarding gestational age, breastfeeding, family smokers, pets, siblings, day-care admission. Supplemented children exibited less rhinitis episodes than controls up to T6 [mean-SD: $0,6(1)$ vs $0,9(1,1)$ at $\mathrm{T} 3, \mathrm{p}=0,031 ; 1(2,6)$ vs $1,6(2)$ at $\mathrm{T} 6, \mathrm{p}=0,027]$. Asthma and infections incidence/severity did not differ between groups. IgE mean \% variation increased in controls at T12 vs baseline $(+25 \% ; \mathrm{p}<0,01)$ but not in the treated group $(+8 \% ; \mathrm{p}=0,59)$. $\operatorname{IgG}$ and $\operatorname{IgA}$ mean $\%$ variation significantly increased in treated group $(+6,3 \%$ and $+11,5 \%$ respectively; $p<0,01)$ but not in controls $(2,4 \%$ and $3,7 \%$ respectively; $p=0,34$ and 0,19 ). Faecal analysis showed Lactobacillus casei DN-114 001 in the gut flora of $\geq 78 \%$ of supplemented children through the study period.

Conclusions: Long-term daily consumption of Lactobacillus Casei DN-114 001 fermented milk may positively influence on the clinical and immunological status of allergic children.

\section{MONTHS OLD INFANTS ARE LEANER IF THEIR MOTHERS RECEIVED LOW DOSE DHA SUPPLEMENTS DURING PREGNANCY AND LACTATION}

R L Bergmann ${ }^{1}$, R Richter ${ }^{1}$, K E Bergmann ${ }^{1}$, J W Dudenhausen ${ }^{1}$, F Haschke ${ }^{2}$

${ }^{1}$ Charité University Hospitals, Berlin, Germany, ${ }^{2}$ Nestlé Nutrition Institute, Vevey, Switzerland
Background and Aims: The supply of LC-PUFAs to the foetus and infant is associated with growth. Our aim was to study the growth of children whose mothers had received a vitamin-mineral-supplement during pregnancy and lactation with additional docosahexaenoic acid (DHA) compared to those without DHA-supplement.

Methods: At 22 weeks of gestation 144 mothers were enrolled to a randomised controlled double blind clinical trial on the effects of a daily intake of a vitamin-mineral supplement up to 37 weeks and voluntarily up to 3 months of lactation. $0.2 \mathrm{~g}$ DHA was added to one supplement (DHA-group). Of the 116 term newborns, measured at ages 1 and 3 months, finally 69 children (no significant selection bias) were followed up to 21 months. For statistical analysis of their growth (length, weight, BMI, head circumference) linear mixed models were used.

Results: At birth, anthropometric values were nearly identical in both groups. But a significant time dependent effect was observed for the DHA-group in weight $(p=0.049)$ and BMI $(p=0.044)$, when taking into account sex, gestational and chronological age of the infant, the mother's education, parity, BMI, gestational weight gain and breast-feeding. After controlling for all influential variables, in 21 months old infants of the DHA-group, weight was significantly lower by $-0.6 \mathrm{~kg}$ $(\mathrm{p}=0.006)$, and BMI by $-0.77 \mathrm{~kg} / \mathrm{m} 2(\mathrm{p}=0.031)$, while head circumference was slightly larger and length was similar in both groups.

Conclusions: The lasting favourable of DHAsupplementation of pregnant and lactating mothers on the BMI of their 21 months old infants could be due to metabolic programming.

\section{GROWTH OF INFANTS BORN FROM HIV POSITIVE MOTHERS FED WITH ACIDIFIED STARTER FORMULA CONTAINING BIFIDOBACTERIUM LACTIS}

\author{
P A Cooper ${ }^{1}$, M Mokhachane ${ }^{1}$, K D Bolton ${ }^{1}$, \\ P Steenhout $^{2}, \mathrm{C} \mathrm{Hager}^{3}$ \\ ${ }^{1}$ Department of Paediatrics, University of the \\ Witwatersrand, Johannesburg, South Africa, ${ }^{2}$ Nestle \\ Nutrition, Nestec Ltd., Vevey, Switzerland, ${ }^{3}$ Nestle \\ Research Center, Nestec Ltd., Lausanne, Switzerland
}

Background and Aims: The choice of formula for infants born to HIV positive mothers in developing countries who choose not to breast feed must be made with the aim of ensuring the least risk of infection and optimal growth. We investigated the effects of acidification and addition of probiotics in two separate but similar studies. Methods: Full term healthy infants born to HIV positive mothers who had decided not to breast feed were randomized within a week of birth to either a standard starter formula, an acidified starter formula or an acidified formula containing probiotics (Bifido-bacterium lactis $2 \times 107 \mathrm{cfu} / \mathrm{g}$ ). The studies were double blind and anthropometric measurements were analyzed up to age 4 months. Results: A total of 333 infants were enrolled in the two studies of whom 271 were followed so that HIV status and growth parameters could be analyzed. There were no 
differences between those fed standard formulas and acidified formulas without probiotics. The 98 infants fed formula containing probiotics gained an average of $2.6 \mathrm{~g} /$ day more than those without probiotics $(95 \% \mathrm{CI}=[0.5 ; 4.7] ; \mathrm{p}=0.015$. A total of 34 infants were HIV positive but there was no effect on weight gain when only HIV positive infants were compared $(-4.3 \mathrm{~g} /$ day $-95 \% \mathrm{CI}=[-11.3 ; 2.7] ; \mathrm{p}=0.22 \mathrm{NS})$. There were no differences in the occurrence of gastrointestinal or respiratory problems. Conclusions: Feeding infants born to HIV positive mothers an acidified starter formula with probiotics was safe and had a positive effect on weight gain. Formulas and funding for these studies was provided by Nestle Nutrition, Switzerland.

\section{EFFECT OF DIETARY SUPPLEMENTATION WITH LC-PUFA, AN OLIGOSACCHARIDE MIX AND L. PARACASEI CNCM I-2116 ON GROWTH OF NEONATAL STRESS RATS}

\section{L Garcia Rodenas ${ }^{1}$, M Moser ${ }^{2}$, G Bergonzelli ${ }^{1}$ \\ ${ }^{1}$ Dept of Nutrition and Health, ${ }^{2}$ Dept of Analytical Bioscience, Nestle Research Center, Lausanne, Switzerland}

Background and Aims: Catch-up growth is essential in preterm infants to ensure adequate development. However, high rates of catch-up growth in early life may increase the risk of obesity later on. We had previously established that a blend of ingredients increased growth recovery after neonatal stress in rats. We aimed to: 1) Identify the ingredient(s) responsible for this effect, and 2) Determine whether the nutritional intervention affected body composition.

Methods: Rat pups were exposed to intermittent maternal deprivation from postnatal days 2 to 14 . After precocious weaning, pups were fed from day 15 till day 35 with either a control diet or a similar diet adapted to contain either LC-PUFA, inulin + galactooligosaccharides, L. paracasei CNCM I-2116 or a blend containing all ingredients. Animals from the nonstressed control group were weaned at day 21 and fed with the control diet until day 35 .

Results: Body weight gain normalized by food intake was lower in stressed animals receiving control diet or individual ingredients than in non-stressed animals. Only the blend diet restored this parameter relative to the control non-stressed animals while no significant differences were found in the fat pad weight between these two groups.

Conclusions: A diet combining LC-PUFA, an oligosaccharide mix and L. paracasei was able to restore the growth rate that had been impaired by neonatal stress and precocious weaning. This was linked to improved food efficiency resulting from the synergy of at least two of the ingredients and was not associated to increased adiposity.

\section{GROWTH OF INFANTS FED A STARTER FORMULA CONTAINING BIFIDOBACTERIUM LONGUM BL999 AND A MIXTURE OF PREBIOTICS}

\author{
G Puccio ${ }^{1}$, C Cajozzo $^{1}$, P Steenhout ${ }^{2}$, F Rochat ${ }^{2}$, \\ M C Fichot ${ }^{2}$, F Meli ${ }^{1}$ \\ ${ }^{l}$ Neonatologia, Istituto di Ostetricia e Ginecologia \\ Dell'Università di Palermo, Italy, ${ }^{2}$ Nestlé Nutrition, \\ Nestec Ltd., Vevey, Switzerland
}

Background and Aims: The aim was to evaluate the effects on growth, tolerance and effects on morbidity of a new starter formula containing a probiotic (B longum BL999) and prebiotics.

Methods: 138 healthy newborns were enrolled and randomized in a prospective double blind study and follow-up to 4 months of age. 69 newborns received the experimental formula containing a probiotic (Bifidobacterium longum ATCC BAA-999, - 2x107 $\mathrm{cfu} / \mathrm{g})$ and $4 \mathrm{~g} / \mathrm{L}$ of a mixture of prebiotics $(10 \%$ short chain FOS, 90\% GOS). The other 69 received a commercially available formula (Nan ${ }^{\circledR}$-Nestlé). Both formulas contained LC-Pufas.

Results: Equivalence in weight gain could be successfully shown. The difference in weight gain from enrolment to 4 months was $+1.1 \mathrm{~g} /$ day in the treatment group, the $90 \%$ confidence interval $(-1.0,3.2)$ being within the equivalence boundaries of $\pm \pm 3.9 \mathrm{~g} /$ day. No statistical difference could be found for length, head circumference and BMI. The experimental group had higher daily intake at the end of the trial $(\Delta=+82.35$ $\mathrm{ml} /$ day, $\mathrm{p}=0.02$ ), presented a higher stool frequency than controls $(\Delta=0.35 \pm 0.14$ /day, $\mathrm{p}=0.018)$, less green $(\mathrm{p}=0.004)$ and more yellow $(\mathrm{p}=0.005)$ stools, less flatulence $(\mathrm{p}=0.051)$ and less constipation $(\mathrm{p}=0.02)$ and showed a trend to have less respiratory tract infections (17 vs. $27, \mathrm{p}=0.088 \mathrm{NS}$ ).

Conclusions: Feeding a starter formula containing a probiotic (B longum BL999) and prebiotics (FOS/GOS) is safe in term of growth development, is well tolerated and has a positive impact on nutritional tolerance, reducing constipation. A tendency to a reduction in the incidence of respiratory tract infections was observed.

\section{NORMOCALORIC ENRICHED FORMULA AFTER TERM DOES NOT AFFECT GROWTH AND BODY COMPOSITION IN PRETERM INFANTS}

\author{
E M Amesz ${ }^{1}$, A Schaafsma ${ }^{2}$, H N Lafeber ${ }^{1}$ \\ ${ }^{1}$ Departments of Pediatrics and Neonatology, $V U$ \\ University Medical Centre, Amsterdam, The \\ Netherlands, ${ }^{2}$ Department of Research and \\ Development, Friesland Foods, Leeuwarden, The \\ Netherlands
}

Background and Aims: Protein and mineral enriched post discharge formula probably improves growth of preterm infants. Differences in growth predominantly seem to develop in the first weeks after discharge. Until what age this formula is beneficial and whether the body composition is influenced is unclear. Growth and body composition of preterm infants fed enriched formula 
until term or 6 months corrected age (CA) are compared.

Methods: At term, preterm infants (bw $\leq 1500$ gram and/or ga $\leq 32$ weeks) were randomized to a post discharge formula $(n=30, P D F)$ or a term formula $(n=26$, TF). A control group of 12 infants was exclusively breast fed until at least 3 months CA (BM). Until term, all infants received preterm formula or fortified breast milk. Anthropometry was performed at 0,3 and 6 months CA. Body composition was measured by DXA at 0 and 6 months $\mathrm{CA}$.

Results: Preliminary results of the first 68 infants participating in this blinded controlled study showed only significant differences in the change in body composition and not in growth (table). The differences were merely found between formula-fed and BM infants and mainly occurred in boys.

Conclusions: Normocaloric enriched formula after term did not affect growth or body composition in formulafed preterm infants. Breast milk fed infants showed less bone acquirement and a smaller increase in fat percentage compared with normocaloric formula-fed infants, however this did not result in a different bone and fat mass at 6 months corrected age.

\begin{tabular}{|c|c|c|c|c|}
\hline & PDF $(\mathrm{n}=30)$ & TF $(n=26)$ & $B M(n=12)$ & p-value \\
\hline $\begin{array}{l}\text { Me an protein intake } \\
\text { (g/kg/day) }\end{array}$ & $2.57 \pm 0.28$ & $2.22 \pm 0.27$ & & 0038 \\
\hline Me an energy intake & $101.1 \pm 11.1$ & $101.3 \pm 12.2$ & & 0.973 \\
\hline Delta BMC (g) & $55.4 \pm 9.2$ & $53.1 \pm 82$ & $43.3 \pm 12.5$ & $0.002^{x}$ \\
\hline Delta BMD $\left(\mathrm{g} / \mathrm{cm}^{2}\right)$ & $0.05 \pm 0.01$ & 0.04 t0.01 & $0.03 \pm 0.02$ & $<0001^{x}$ \\
\hline Delta LM(g) & $15648 \pm 2840$ & $1477.7 \pm 285.9$ & $1449.5 \pm 316.4$ & 0.465 \\
\hline Delta \% FM & $8.5 \pm 2.9$ & $9.2 \pm 3.0$ & $6.3 \pm 3.8$ & $0.034 n$ \\
\hline
\end{tabular}

\section{GENES AND RDS}

\section{Hallman}

\section{Department of Pediatrics, University of Oulu, Finland}

Background and Aims: Respiratory distress syndrome (RDS) is a multifactorial developmental disease caused by lung immaturity and presenting as high-permeability lung edema ("hyaline membrane disease"). It is characterized by a transient deficiency of alveolar surfactant during the first week of life and a secondary lung injury. During the first few days of life, the alveolar surfactant pool size increases in size, reaching the controls within the first week.

Methods: Here we describe the approach how to define the genetic contribution to disease and discuss the polymorphisms some genes that associate with respiratory disease. The candidate genes studied thus far have been chosen on the basis of phenotype respiratory failure in lack-of-function mutations involving genes encoding surfactant proteins (SP) (SP-B, SP-C), on the basis of the regulatory role in the surfactant metabolism and in the host defense (SP-A), and on the basis of the established relationship to another generalized lung disease (G protein-coupled receptor for asthma susceptibility, GPR154 alias GPRA).

Results: The allelic variants of the genes encoding the surfactant proteins (SP) SP-A1, SP-A2, SP-B, and SP-C have been associated with RDS. Specific alleles affect different risk categories, as defined by the degree of prematurity, uterine size, or multiple pregnancy. The main SP-A haplotype, interactively with the SP-B Ile131Thr polymorphism and with constitutional and environmental factors, influence the risk. Case reports on mutations with partially functional SP-B have been published. The genetic susceptibility factors depend on the degree of prematurity at birth, consistent with sequential differentiation of the lung and gestationdependent differences in clinical presentation. According to recent report SP-C 138Asn associated with RDS in extremely preterm infants (Rova et al. Eur J Hum Gen 2004:12;312). In contrast, the near term infants with RDS may share the same susceptibility and protective GPRA haplotypes with asthma populations (Pulkkinen, Haataja, et al. Ann Med 2006 in press). The preferentially type 2 cell expressed genes involved in processing of the surfactant complex (such as ATPbinding cassette transporter $3, \mathrm{ABCA} 3$ ) will likely serve as candidate genes in future studies.

Conclusions: RDS associated with near-term and term births may have a different genetic predisposition and pathobiochemistry compared to RDS after very preterm birth. Other multifactorial lung diseases may share some genetic susceptibility factors with RDS. As we learn more about the molecular consequences of allelic variation, new therapies based on a new generation of surfactant diagnostics and individualized therapies may follow.

\section{GENES AND ARDS}

\section{J-D Chiche}

France

Abstract not available at the time of printing.

\section{PLACENTAL ADENOVIRAL GENOME PCR DETECTION AND PLACENTAL HISTOLOGY IN PRETERM AND TERM DELIVERIES}

E A Tsekoura ${ }^{1}$, A Konstantinidou ${ }^{2}$, D Kafetzis ${ }^{1}$, N Spanakis ${ }^{3}$, S Papadopoulou ${ }^{3}$, D Yfanti ${ }^{3}$, N Nikolaidis ${ }^{4}, \mathrm{~A} \mathrm{Tsakris}^{3}$, A Antsaklis ${ }^{5}$

${ }^{1}$ Department of Paediatrics, Medical School, University of Athens, Greece, ${ }^{2}$ Department of Pathology, Medical School, University of Athens, Greece, ${ }^{3}$ Department of Microbiology, Medical School, University of Athens, Athens, Greece, ${ }^{4}$ NICU Department, Papageorgiou Hospital, Thessaloniki, Greece, ${ }^{5} 1$ st Department of Obstetrics and Gynecology, 'Alexandra' Maternity Hospital, Medical School University of Athens, Greece

Background and Aims: Intrauterine infection is an important cause of spontaneous preterm birth. However, evidence based etiology of viral infection is still lacking. This study aims to test the hypothesis that detection of adenovirus in placenta tissue is associated with preterm birth and to determine the placental histopathological findings.

Methods: Placentas were collected from 193 deliveries, 71 premature deliveries and 122 at term. Detection of adenovirus genome was tested by PCR assay. Placental histology and immunohistochemistry with monoclonal antibody CD45 were evaluated for signs of inflammatory reaction in the positive for adenovirus samples. 
Results: Adenoviral genome was isolated in 54/193 of placentas $(28 \%)$. The frequency of placental adenovirus detection in preterm versus term was significantly increased $\{(29 / 71=41 \%$ versus $25 / 122=20 \% ; \mathrm{P}=0.002$, $\mathrm{OR}=2.6(95 \%$ CI $1.4-5.1)\}$. Stratification by gestational age (GA) revealed a stronger association as GA decreased (GA $\leq 29$ weeks: $\mathrm{OR}=2.8 ; 95 \% \mathrm{CI}$ 1.1-7.0 and GA 30-33: OR $=2.7 ; 95 \%$ CI 1.1-6.5). In the subgroup 34-36 weeks GA the association was no longer significant $(\mathrm{OR}=2.6 ; 95 \%$ CI $0.9-7.0)$. Adenoviral genome detection followed the seasonal variation of adenovirus respiratory infections (beginning of March to end of June). Chronic villitis was present in 39.5\% of adenovirus-positive placentas without any difference between term and preterm births.

Conclusions: Our results indicate that there is an association between placental adenoviral genome detection and spontaneous early premature birth. Histological placental infection does not appear to be the only mechanism inducing preterm labor. In addition, the presence of adenoviral DNA does not necessarily result in placental inflammation.

\section{IMPAIRED CELLULAR IMMUNITY MAY EXPLAIN HIGHER INCIDENCE AND SEVERITY OF RESPIRATORY TRACT INFECTIONS IN CHILDREN WITH DOWN SYNDROME}

C J M Broers, R J B J Gemke, S G M Lamers, M E Weijerman, A M van Furth

\section{Department of Pediatrics and Infectious Diseases, VU University Medical Center, Amsterdam, The Netherlands}

Background and Aims: Children with Down syndrome have an increased susceptibility to infections, probably due to an altered cellular and humoral immune response. In this study the frequency of infections and hospitalization of children with Down syndrome was compared to healthy sibs and related to differences in their cellular immunity.

Methods: Parents of 62 children with Down syndrome and 57 of their healthy sibs were interviewed about the frequency of infections and hospitalizations due to infection. Also T-cell characteristics were analysed in both groups.

Results: The frequency of respiratory tract infections and associated hospitalization were higher in children with Down syndrome $(p<0.05$; Table 1$)$. The absolute counts of total lymphocytes, T-lymphocytes, CD4 positive T-lymphocytes and CD8 positive Tlymphocytes were lower in Down children, especially in the age group 2-5 years ( $<<0.05$; data not shown).

Conclusions: The frequency of respiratory tract infections and associated hospitalization is increased in children with Down syndrome, compared to their healthy sibs. Lower T-cell counts in children with Down syndrome (partially) explain this finding.

\begin{tabular}{|c|c|c|c|c|c|}
\hline & $\begin{array}{c}\text { Down } \\
\text { syndrome } \\
(n=62)\end{array}$ & $\begin{array}{l}\text { Sibling } \\
(n=57)\end{array}$ & p-value & $\begin{array}{l}\text { odds } \\
\text { ratio }\end{array}$ & $95 \% \mathrm{Cl}$ \\
\hline \multicolumn{6}{|c|}{ Patient characteristics } \\
\hline Sex (\% male) & $39 / 62(63 \%)^{*}$ & $24 / 57(42 \%)^{*}$ & 0.03 & & \\
\hline Age (in years) & $7.8 \pm 5.1^{\star *}$ & $9.3 \pm 5.5^{\star *}$ & 0.12 & & \\
\hline \multicolumn{6}{|c|}{ Recent infections (past 4 weeks, $\% \geq 1$ period) } \\
\hline $\begin{array}{l}\text { Respiratory tract } \\
\text { infection }\end{array}$ & $39 / 62(63 \%)$ & $20 / 57(35 \%)$ & 0.0033 & 3.14 & $1.48-6.64$ \\
\hline Other infections & $12 / 62(19 \%)$ & $6 / 57(11 \%)$ & 0.21 & 2.04 & $0.71-5.86$ \\
\hline \multicolumn{6}{|c|}{ History of infections ( $\% \geq 1$ period) } \\
\hline $\begin{array}{l}\text { Higher respiratory } \\
\text { tract infection }\end{array}$ & $61 / 62(98 \%)$ & $49 / 57(86 \%)$ & 0.014 & 9.96 & $1.20-82.4$ \\
\hline $\begin{array}{l}\text { Lower respiratory } \\
\text { tract infection }\end{array}$ & $30 / 62(48 \%)$ & $1 / 57(2 \%)$ & $<0.0001$ & 52 & $6.83-403.6$ \\
\hline $\begin{array}{l}\text { Urinary tract } \\
\text { infection }\end{array}$ & $8 / 62(13 \%)$ & $3 / 57(5 \%)$ & 0.21 & 2.67 & $0.67-10.6$ \\
\hline Gastroenteritis & $31 / 62(50 \%)$ & $24 / 57(42 \%)$ & 0.46 & 1.38 & $0.66-2.83$ \\
\hline Meningitis & $0 / 62(0 \%)$ & $1 / 57(2 \%)$ & 0.48 & 0.30 & $0.012-7.55$ \\
\hline Hospitalization & $32 / 62(52 \%)$ & $4 / 57(7 \%)$ & $<0.001$ & 14.1 & $4.56-43.8$ \\
\hline
\end{tabular}

\section{NEONATAL RESPIRATORY DISTRESS: CHANGES OVER 30 YEARS IN A GEOGRAPHICALLY DEFINED POPULATION}

M Roth-Kleiner ${ }^{1}, \mathrm{~J} \mathrm{Ersch}^{2}$, P Baeckert ${ }^{3}$, H U Bucher ${ }^{2}$

${ }^{1}$ Division of Neonatology, Department of Pediatrics, Centre Hospitalier Universitaire Vaudois, Lausanne, Switzerland, ${ }^{2}$ Clinic of Neonatology, University Hospital of Zurich, Switzerland, ${ }^{3}$ Spital Bethanien, Zurich, Switzerland

Background and Aims: To evaluate trends of neonatal respiratory distress $(\mathrm{RD})$ regarding incidence, treatment and mortality in a geographically defined population over 30 years.

Methods: A questionnaire was sent to all primary, secondary and tertiary neonatal units in Switzerland every ten years between 1974 and 2004 asking for the number of hospitalized patients with $\mathrm{RD}$ and hyaline membrane disease (HMD), their treatment and mortality. RD was defined as the presence of two of the following symptoms for more than 15 minutes: tachypnoe $(>60 / \mathrm{min})$, nasal flaring, retractions, central cyanosis in room air and expiratory grunting.

Results: Number (mean: 5'300/year) and rate of hospitalized newborns (7\%) was stable over the 30 years. The proportion of patients with RD increased from $1.9 \%$ to $3.8 \%$ of all lifeborns (mean: $78^{\prime} 800 /$ year) and from $29.7 \%$ to $52.8 \%$ of all hospitalized newborns. The rate of HMD remained stable at around $8.5 \%$ of all hospitalized patients. Treatment of $\mathrm{RD}$ changed significantly over the three decades: mechanical ventilation decreased from $31.4 \%$ to $16.3 \%$, use of CPAP increased from $8.8 \%$ in 1994 to $25.9 \%$ in 2004 and application of surfactant in HMD (introduced after 1984) raised to $48 \%$ in 1994 and $53 \%$ in 2004 . Mortality of patients with RD decreased from $15.5 \%$ (1974) to $3.5 \%$ (2004).

Conclusions: Whereas the introduction of preventive and therapeutic means helped to stabilize the rate of HMD and to decrease mortality over the last three decades, the incidence of neonatal $\mathrm{RD}$ has almost doubled in the same period, in particular in the population with birth weight of $>2500 \mathrm{~g}$. 


\section{A TWO CENTRE GENETIC ASSOCIATION STUDY IN PICU CHILDREN WITH AND WITHOUT SIRS}

K M Illing ${ }^{1}$, V J Pappachan ${ }^{1}$, M L Allen ${ }^{3}$, M J Rose-Zerill ${ }^{2}$, R Agbeko ${ }^{3}$, H Rutkowska ${ }^{1}$, K Cathie $^{1}$, T G Coulson ${ }^{1}$, P Wilson ${ }^{1}$, J W Holloway ${ }^{2}$, M J Peters ${ }^{3}$

${ }^{I}$ Peadiatric Intensive Care Unit, Southampton General Hospital, Southampton, UK, ${ }^{2}$ Infection, Inflammation and Repair Division, University of Southampton, Southampton, UK, ${ }^{3}$ Paediatric Intensive Care Unit, Great Ormond Street Hospital for Children NHS Trust, London, UK

Background and Aims: The incidence of SIRS may have a genetic predisposition. Functionally significant polymorphisms (SNPs) in genes encoding Macrophage Inhibitory Factor (MIF: -173) the RAGE receptor (AGER 374A) the TLR4 receptor (+896A) were studied in children admitted to 2 PICUs after informed consent and LREC approval.. All of these SNPs down regulate the host's ability to mount an inflammatory response and should be more prevalent in children without SIRS.

Methods: A total of 225 children from 2 centres were recruited. Phenotype/genotype relationships were analysed (225 children) using Pearson $\chi^{2}$ statistics. Phenotype was defined by the presence or absence of SIRS as well as SOFA, PELOD and PIM scores. Genotyping was performed using Taqman ${ }^{\mathrm{TM}}$ PCR.

Results: These are presented in Table 1.

Conclusions: Despite previously described associations between MBL polymorphisms locus and susceptibility to SIRS we were unable to demonstrate a similar association with the 2 SNPs studied.

\begin{tabular}{|c|c|c|c|c|c|c|c|c|c|}
\hline & & & \multicolumn{3}{|c|}{ RAGG qumatype soded } & \multicolumn{3}{|c|}{ MIIF qenatype saded } & Tatal \\
\hline & & & $\pi$ & TA & AA & GG & $G c$ & $c c$ & \\
\hline \multirow[t]{2}{*}{ SIRS Phondetyos } & SIRS & Number[\%] & $103[6 ; .2]$ & $42[27.8]$ & $6[4,0]$ & $197[70.9]$ & $39[25.8]$ & $5[3,3]$ & 151 \\
\hline & $\begin{array}{l}\text { NON- } \\
\text { SIRS }\end{array}$ & Number [\%] & $47[63.5]$ & $22[29.7]$ & $5[6.8]$ & $53[71.6]$ & $19[25.7]$ & $2[2.7]$ & 74 \\
\hline \multicolumn{3}{|c|}{ 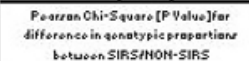 } & \multicolumn{3}{|c|}{0.602} & \multicolumn{3}{|c|}{0.906} & \\
\hline
\end{tabular}

\section{NEW EVIDENCES OF GENOTYPE-PHENOTYPE CORRELATIONS IN METACHROMATIC LEUKODYSTROPHY}
A Biffi ${ }^{1,2}, \mathrm{M} \mathrm{Cesani}^{1}$, U Del Carro ${ }^{3}$, F Fumagalli ${ }^{2,3}$, C Baldoli ${ }^{4}$, S Gerevini ${ }^{4}$, M Falautano ${ }^{5}$, S Canale ${ }^{5}$, G Comi $^{2,3}$, M G Roncarolo ${ }^{1,2}$, M Sessa ${ }^{1,3}$
${ }^{1}$ San Raffaele Telethon Institute for Gene Therapy, Pediatric Clinical Research Unit (HSR-TIGET PCRU), San Raffaele Scientific Institute, Milan, Italy, ${ }^{2}$ Vita Salute University, San Raffaele Scientific Institute, Milan, Italy, ${ }^{3}$ Neurology Unit, San Raffaele Scientific Institute, Milan, Italy, ${ }^{4}$ Neuroradiology Unit, San Raffaele Scientific Institute, Milan, Italy,
${ }^{5}$ Neuropsychology Unit, San Raffaele Scientific Institute, Milan, Italy

\section{Background and Aims: Metachromatic} Leukodystrophy (MLD) is a rare and fatal demyelinating lysosomal storage disorder, due to the inherited deficiency of the lysosomal enzyme arylsulfatase A (ARSA). Currently no valid therapeutic options are available for affected patients. Previously described correlations between clinical presentation and either residual enzymatic activity or common mutations resulted respectively poorly informative or helpful in predicting patient's prognosis only in a minority of MLD patients harboring common mutations.

Methods: We analyzed the complete ARSA gene in 23 MLD patients that we followed with periodical clinical and instrumental evaluations.

Results: By examining the data obtained from neurophysiologic and neuroradiologic evaluations of our patients' cohort and by critical reevaluation of the existing literature, we demonstrated the occurrence of a precise correlation between ARSA gene mutations and age of onset and disease progression. This correlation was evident not only for common mutations, but, most importantly, for rare ones. Moreover, in the absence/delay of molecular diagnosis, or in case of newly identified mutations, the involvement of peripheral nervous system since disease onset resulted a sensitive prognostic marker for prediction of a severe progression.

Conclusions: These data provide information on disease prognosis and management, which can be useful for prenatal testing, and patients' selection for current and future therapeutic options, such as hematopoietic stem cell transplantation and gene therapy.

\section{PRIVACY AND CONFIDENTIALITY IN THE CARE OF CHILDREN}

\section{Weindling}

Liverpool Womens Hospital, Liverpool, UK

Abstract not available at the time of printing.

\section{THE DECLARATION OF HELSINKI}

\section{F P Crawley}

Good Clinical Practice Alliance - Europe and Member, Ethics Working Group, Confederation of European Specialists in Paediatrics, Kessel-Lo, Belgium

The Declaration of Helsinki (1964-2004) from the World Medical Association provides the cornerstone for ethics in biomedical research. This document has become the focal point for debate regarding the ethical acceptability of the conditions for medical research. Helsinki has played a determining role in the development of international and national guidelines and regulations in research. In the area of paediatric research, Helsinki has posed new questions and challenges, and it has both reflected and pushed the development of US, European, and international considerations regarding the development of health interventions for children. Within Europe, and CESP in particular, the Declaration of Helsinki has played a pivotal role in reflections on, and the promotion of, ethical research in children. The presentation will show the impact of the Declaration of Helsinki and the international discussion on ethics in biomedical research on paediatric research in Europe. 


\section{CROSS AGENCY WORKING AND INFORMATION SHARING}

\section{T Berney}

\section{Prudhoe Hospital, Prudhoe, Northumberland, UK}

Medical confidentiality, a contract that is at the heart of the doctor-patient relationship, is complicated by the inclusion of other parties: A. On the child's part, this is an inevitable element for, as a minor, his/her interests are represented by the parent. As the child moves through adolescence, their right to privacy requires the exclusion of the parent from the confidential relationship, a process that is complicated by anything that impairs the child's autonomy (notably intellectual disability). Events such as abuse by the parents or parental psychiatric disturbance, mean that their can be so great a divergence between parental interests and the child's. If the child does not have the competence to be a full partner in the confidential relationship, the doctor may find that this is then with a proxy outside the family. B. On the doctor's part, good medical care increasingly requires a team approach by an ill-defined and variable group that is multidisciplinary and multiagency. The boundaries of these teams often are so diffuse and extensive as to be incomprehensible to a child and family. They extend to include not just the people the patient actually sees treating them but also laboratory and pharmacy staff and, linked I a more indirect fashion,. people such as the student and the administrator. The team may include people employed by other agencies - notably teacher and social worker the discipline not necessarily being a guide to the employer. The difficulty is not only that different agencies have different concepts and thresholds of confidentiality but also that they have different reasons for their involvement; they have their own agendas. A concrete indicator of confidentiality is the circulation list for clinical letters for it indicates the boundary of confidentiality for that particular matter. Especially where it includes the patient (a practice recently introduced in England) for the practice defines the extent to which the patient has the authority to control the distribution of personal information. The letter also defines their status in relation to their parents - are they addressed to the patient or to the parents - as well as that of other professionals involved in their care. Certain issues regularly present the paediatrician with their own difficulties such as the pregnant minor who does not want their family to know, and the family in conflict (short of clear-cut child abuse) who do not want other agencies to be brought in. Child abuse brings its own particular problems where the paediatrician can find disclosure that is in his patient's best interests (to prevent further injury to the child) shifting to disclosure that is in the public interest (to prevent injury to other children). Although the determinant of the degree of disclosure is governed by the 'need to know,' partial disclosure is not simple and, once the process has started, it can be very unclear as to who has what responsibility for continued confidentiality.

\section{PRIVACY AND CONFIDENTIALITY IN CHILDREN'S HEALTHCARE}

\section{P Thornton}

Pear Tree Surgery, Kingsbury, Warwickshire, UK

It would be difficult to name a democracy in the Western World that pays less respect to confidential medical information than the United Kingdom." It would be easy to dismiss such criticism if it hadn't come from such an authoritive source. Dr John Havard was speaking in 1989. He was then the secretary of the British Medical Association (BMA), a barrister as well as being qualified in medicine. In 2006, day in day out large numbers of patients divulge highly sensitive information to doctors and the information does not seem to leak out into the public domain. From the perspective of the doctor in clinical practice it is easy to assume that is no great problem with medical confidentiality. So was Havard wrong? Do patients with hold important information from clinicians? What has happened to standards, custom and practice in the interim? Does current practice meet the requirements of the Data Protection and Human Rights Acts? How will the migration to electronic records improve or diminish privacy for patients? At its Annual Representative Meeting in June, the BMA changed its policy. They now demand that patients should give explicit consent before information is transferred on to the national database an "opt-in". Connecting for Health are recommending that information be transferred to the national spine having simply informed patients by leaflet of the database proposals. They regard it as sufficient to give patients the facility to "opt-out". But the "opt out" they are offering is simply the use of software safeguards to block the sharing of information via the database. It remains their intention that "People should not be offered the choice to opt out of recording their healthcare data on National Care Records service."And this is against a the background of connecting for health documents which confirm that the software safeguards on offer - metaphorically dubbed "sealed envelopes" are not yet written and tested, will not be available in time for the initial proposed uploads of summary information from GP systems, will never work in respect of scanned images of correspondence and can be over ridden, contrary to the wishes of the patient. Conflated with all of this we have the proposed national Identity register and the two children's registers by which information about children and their parents will be required to be shared. 
PNEUMOCOCCAL CONJUGATE VACCINE: IMPACT OF PREVENAR IN NORTH AMERICA

\section{J D Kellner}

Alberta, Canada

The 7-valent pneumococcal conjugate vaccine (PCV7, Prevenar) was licensed and recommended for routine use in all infants and high-risk children in the USA in 2000 and in Canada in 2001. Prevenar has been very effective in both countries. Data from post-licensure studies will be presented that show Prevenar has been highly effective to prevent invasive pneumococcal disease in vaccine recipients. Data will also be presented to show the large indirect effect (herd immunity) in unimmunized children and adults. Overall, more than 2 cases of invasive pneumococcal disease are prevented indirectly for every case prevented directly. Another benefit of Prevenar has been a reduction in the prevalence of antibiotic resistant pneumococcal infections. The impact of replacement disease with nonvaccine serotypes has been small.

\section{ROTAVIRUS INFECTIONS-THE VIRUS AND ITS DISEASE, WHAT ARE WE TALKING ABOUT?}

\section{K Johansen}

\section{Department of Virology, Immunology and Vaccinology} Swedish Institute for Infectious Disease Control, Sweden

Rotavirus infections are the leading cause of severe dehydrating gastroenteritis, responsible for $\sim 600,000$ deaths per year worldwide in children less than 5 years of age. Almost all children in developed and developing countries acquire their first episode of rotavirus-induced infection before their fifth birthday. Although $>80 \%$ of deaths due to rotaviruses occur in children in developing countries significant morbidity and occasional deaths occur in developed countries. Examination of rotavirusinduced annual hospitalisation incidence from several industrialized countries demonstrated a median rate of 445 per 100000 children. The spectrum of symptoms that rotaviruses induce ranges from mild, watery diarrhoea of limited duration to fever, profuse diarrhoea and frequent vomiting that may result in severe dehydration leading to electrolyte imbalance and possibly death. It is difficult to predict which child will develop severe disease and complications to rotavirus-induced infections in industrialized countries are poorly quantified. Rotavirus is a well-known cause of community-acquired diarrhoea but it is less well recognized as a major cause of nosocomial infection in children although it is found consistently to be one of the most common causes of hospital-acquired diarrhoea. Two proteins of the outer capsid (VP4 and VP7) induce neutralizing antibodies and are used for genotyping, G-typing (the glycoprotein VP7) and Ptyping (the protease cleaving protein VP4). Due to the segmented nature of the rotavirus genome (consisting of 11 segments of dsRNA) rotaviruses can reassort during infection with multiple virus strains. Rotaviruses have been detected in many species of wild and domestic animals. Theoretically $\sim 110$ reassortments are possible but it has been shown repeatedly in studies from different parts of the world that G1, G2, G3, G4 and G9 contribute to $>95 \%$ of all rotavirus-induced diarrhoea. It will be difficult to eradicate rotaviruses this is why a reasonable goal for rotavirus vaccination is to eliminate severe disease and hospitalization and decrease the total number of rotavirus-induced infections.

\section{PAEDIATRIC ROTAVIRUS GASTROENTERITIS: TIME FOR VACCINATION!}

\section{F Gimenez-Sanchez}

Advisory Committee of Vaccines, Spanish Association of Pediatrics, Paediatric Infectious Diseases, Torrecardenas Hospital, Almeria, Spain

The availability of rotavirus vaccination to reduce Paediatric Rotavirus Gastroenteritis (PRG) burden in Europe has stimulated efforts to characterize the real burden of rotavirus. It is estimated that PRG results in relatively few deaths per year in Europe, however, nearly every child in Europe is infected with rotavirus by age 5 years and the majority will have gastroenteritis, resulting in about 2.8 million cases cared at home, 700,000 outpatient visits and 87,000 hospitalisations. A prospective, observational study (REVEAL) conducted in seven study areas (Belgium, France, Germany, Italy, Spain, Sweden, UK) between October 2004 to September 2005 confirmed the high burden of rotavirus infections in Europe: $95 \%$ of PRG cases were in children above 6 months of age with more than $50 \%$ of hospitalisations and emergency department visits due to PRGE. The mean duration of hospitalisation varied between 2.5 to 5 days with at least one parent who had to miss work in $66 \%$ of families with a hospitalised child. In addition to causing morbidity and mortality in children, Paediatric Rotavirus Gastroenteritis creates a major economic burden on health care systems and families in Europe. Recent study (REVEAL) found that five globally common rotaviruses serotypes (G1,G2,G3,G4 \& G9) represent more than $98.5 \%$ of the circulating rotavirus strains in Europe. Almost two decades ago, studies demonstrated that rotaviruses serotypes $(\mathrm{G} 1, \mathrm{G} 2, \mathrm{G} 3, \mathrm{G} 4)$ were predominant with an emerging novel rotavirus type: G9. To effectively reduce Paediatric Rotavirus Burden, a rotavirus vaccine should have demonstrated its efficacy against those five predominant rotavirus serotypes. Despite the significant morbidity and costs associated with Rotavirus in Europe, there is always a need to raise awareness of the rotavirus disease among European physicians and policy-makers in order to help the decision-making process about recommendation and use of rotavirus vaccination in each country. In industrialised countries, if widely used, rotavirus vaccination could substantially reduce the number and associated costs of child hospitalisations and clinical visits for acute diarrhoea as well as office visits and burden on the family.

\section{ROTATEQ®: ENSURE SUCCESSFUL VACCINATION}

\section{P Heaton}

Merck Research Laboratories, USA

The pentavalent human-bovine (WC3) reassortant rotavirus vaccine (RotaTeq ${ }^{\circledR}$ - Merck \& Co., Inc.) was approved by the US Food and Drug Administration and 
the European Medicines Agency earlier this year, with registration and/or filing in multiple countries. The vaccine, which is provided in a ready-to-use liquid formulation, was demonstrated to be generally well tolerated and efficacious in Phase III studies conducted in 11 countries worldwide, preventing $98 \%$ of severe rotavirus gastroenteritis and reducing rotavirus-related hospitalizations and emergency visits by $95 \%$. Reductions in rotavirus-related health care contacts were comparable across geographic regions and across different serotypes including G1, G2, G3, G4, and P1A[8]-containing G9 strains. The vaccine did not show evidence of an increase in intussusception and was well tolerated with respect to other adverse experiences of special clinical interest for rotavirus vaccines, including fever, vomiting, and diarrhea. This presentation will provide an update of the clinical safety and efficacy data from the Phase III studies.

\section{HOME OXYGEN DELIVERY AND TREATMENT}

H F Ayyash ${ }^{1}$, H I Alfaneck ${ }^{2}$, A Herrick ${ }^{3}$

${ }^{I}$ Department of Paediatrics, Doncaster Royal Infirmary, Doncaster, South Yorkshire, United Kingdom, ${ }^{2}$ PCT

Health Care, Chesterfield, North Derbyshire, United

Kingdom, ${ }^{3}$ CNDRH, North Derbyshire, United Kingdom

Background and Aims: As the structure and financing of health and pharmaceutical care changes, families provide increasingly complex home oxygen treatment for their chronically ill children who are oxygen dependent.

Methods: We describe the clinical care of a child who was born prematurely and developed severe respiratory distress syndrome requiring ventilation in special care baby unit.

Results: The Child stayed in special care baby unit for several weeks and developed chronic lung disease. Once the decision was made that the patient is fit to go home on oxygen treatment, a planning meeting took place for discharge. An individualized care plan was drawn up. Prior to discharge, the parents received advice on the use of equipments, administration of medication and oxygen treatment. The factors contributing to create a serious stress within a family, the impact of chronic lung disease and delivery of home oxygen treatment on the family and the child will be discussed.

Conclusions: Families require information and assistance in using home oxygen. Adequate support for care giving families extends well beyond teaching them medical,pharmaceutical and technical care.It is the role of doctors,pharmacists and nurses to advocate for restructuring of health services on the use of home oxygen treatment for the chronically ill patients. 
- 120 - 
EUROPEAN ACADEMY OF PAEDIATRICS

Barcelona, Spain, 0ctober 7-10, 2006

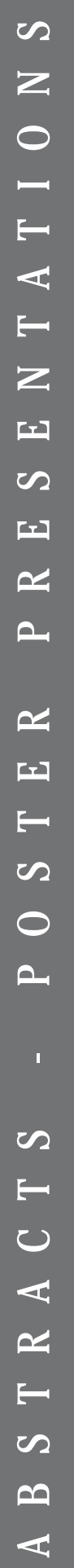

A B S T R A C T S POSTER PRESENTATIONS 
-122 - 


\section{NEUROTOXICITY OF HYPEROXIA IN CORTICAL NEURONAL CULTURE}

M Toennessen, M Berns, B Gerstner, M Obladen

Department of Neonatology, Charité Virchow Hospital, Berlin, Germany

Background and Aims: A growing body of evidence indicates that hyperoxia affects apoptotic processes especially in unmature neuronal cells of premature infants. In the developing rat brain and in cell culture hyperoxia has been identified to cause neurodegeneration. We therefore examined the effects of hyperoxia in cultured rat neuronal cells at different maturation stages.

Methods: Primary cortical neuronal cultures were prepared from Wistar rat embryos at 18 days gestation and exposed to hyperoxia $(80 \%)$ for $48 \mathrm{~h}$ at different maturation stages (division 7 for immature neuronal culture, and division 14 for mature neuronal culture). Control cells were kept in normoxia. Cell viability was assessed by the methyltetrazolium method (MTT) and lactate dehydrogenase release (LDH). Cells showed morphologic changes consistent with apoptosis. Involvement of caspases was shown by assessment of caspase-3-like-activity, and accumulation of reactive oxygen species (ROS) was shown by CM-H2DCF-DAassay.

Results: Hyperoxia reduced cell viability by $29.1 \%$ vs controls $(\mathrm{p}<0.05)$ at division 7 and by $18,3 \%$ vs controls $(p<0.05)$ at division 14 whereas LDH release significantly increased. Exposure to hyperoxia leads to oxidative stress and time dependent accumulation of ROS [127.5\% after $6 \mathrm{~h}$ exposure, and $202.8 \%$ after $24 \mathrm{~h}$ exposure to hyperoxia vs controls $(p<0.05)$ ]. Hyperoxia caused a significant increase of caspase-3-like-activity after a $24 \mathrm{~h}$ period $(150.9 \%)$ vs control $(\mathrm{p}<0.05)$.

Conclusions: We conclude that a hyperoxia leads to apoptosis of especially immature neurons. We speculate that in premature infants oxygen exposure may exacerbate neuronal damage and contribute to neurological impairment.

\section{THE CANNABINOID AGONIST WIN 55,212-2 REDUCES BRAIN DAMAGE AFTER EXPERIMENTAL HYPOXIA-ISCHEMIA IN NEWBORN RATS}

J Martínez-Orgado ${ }^{1}$, D Fernández-López ${ }^{3}$, R Pazos ${ }^{2}$, R Tolón ${ }^{2}$, M A Moro ${ }^{3}$, J Romero ${ }^{2}$, I Lizasoain ${ }^{3}$

${ }^{\text {I}}$ Area Pediatria-Neonatología, Fundación Hospital Alcorcón, Madrid, Spain, ${ }^{2}$ Laboratorio de Apoyo a la Investigación, Fundación Hospital Alcorcón, Madrid, Spain, ${ }^{3}$ Departamento de Farmacología, Facultad de Medicina, Universidad Complutense de Madrid, Spain

Background and Aims: To assess the neuroprotective effect of the cannabinoid WIN55212 after experimental hypoxia-ischemia (HI) in newborn rats.

Methods: Left common carotid artery was ligated and sectioned in anesthetized 7-day-old Wistar rats, which later inhaled $8 \% \mathrm{O} 2$ for 120 minutes. Then, single doses of vehicle (0.5\% DMSO; $\mathrm{n}=10)$, WIN55212 $(0.1 \mathrm{mg} / \mathrm{kg}$; $\mathrm{n}=13), \mathrm{WIN}+$ the CB1 antagonist SR141716A (3mg/kg; $\mathrm{n}=6)$ or WIN + the CB2 antagonist SR144528 $(2 \mathrm{mg} / \mathrm{kg}$; $\mathrm{n}=6$ ) were administered. Control group had sham surgery without hypoxia or treatment. Brain magnetic resonance imaging (T2WI, DWI, ADC maps) was performed at $24 \mathrm{~h}, 72 \mathrm{~h}$ and 7 days after HI (Biospec 47/40, 7 Tesla). Then, rats were perfused with $4 \%$ paraformaldehyde and brains removed for Nissl staining and GFAP inmmunofluorescence.

Results: $24 \mathrm{~h}$ after HI, vehicle rats showed in MRI an ipsilateral area of mixed edema (T2WI augmented and ADC reduced; $17.2 \pm 2.6 \%$ of brain volume), which evolved to necrosis at $7 \mathrm{~d}$ (T2WI and ADC augmented; $16.4 \pm 1.6 \%$ ). In WIN, MRI at $24 \mathrm{~h}$ was similar to vehicle, but damaged area evolved to T2WI hyperintense area reduction $(6.6 \pm 1.2 \%$, ANOVA $\mathrm{p}<0.05)$ and $\mathrm{ADC}$ normalization. In agreement, number of viable neurones increased and reactive gliosis was reduced in cortex in WIN as compared with vehicle. The antagonism of $\mathrm{CB} 1$ or $\mathrm{CB} 2$ receptors reversed the effects of WIN.

Conclusions: Administration of the cannabinoid WIN55212 after HI reduced brain damage seemingly by promoting the salvage of viable tissue. Supported by grants SEN2000, FIS-PI021540, FIS-PI030304, SAF 2004-00237, and SAF2005-05960.

\section{NEUROTOXICITY OF BILIRUBIN AND IBUPROFEN IN CORTICAL NEURONAL CULTURE}

M Berns, M Toennessen, P Koehne, M Obladen

Department of Neonatology, Charité, Campus Virchow Klinikum, Berlin, Germany

Background and Aims: Preterm infants are on risk for bilirubin associated brain damage. In cell cultures bilirubin causes neuronal apoptosis and necrosis. Ibuprofen is used for closing the ductus arteriosus which usually occurs at an age when hyperbilirubinemia is on its maximum. Ibuprofen interferes with bilirubinalbumin-binding. We therefore examined the effects of bilirubin in combination with ibuprofen in rat neuronal cultures.

Methods: Primary cortical neuronal cultures were prepared from Wistar rat embryos at 18 days gestation and exposed to ibuprofen $(7.5-1000 \mu \mathrm{g} / \mathrm{ml})$ alone or in combination with bilirubin $(1-10 \mu \mathrm{M})$ for $48 \mathrm{~h}$. Control cells were kept in normoxia. Cell viability was assessed by the methyltetrazolium method (MTT) and lactate dehydrogenase release (LDH). DNA-fragments as marker of apoptosis was measured by elisa.

Results: Incubation with increasing concentrations of ibuprofen reduced cell viability by $68 \%$ vs controls $(\mathrm{p}<0.05)$ above a concentration of $125 \mu \mathrm{g} / \mathrm{ml}$ whereas LDH release increased from $29 \%$ to $36 \%$ above 62.5 $\mu \mathrm{g} / \mathrm{ml}(\mathrm{p}<0.01)$. Placebo did not affect cell viability. Coincubation with bilirubin $(10 \mu \mathrm{M})$ and $250 \mu \mathrm{g} / \mathrm{ml}$ ibuprofen caused significant $(\mathrm{p}<0.01)$ additional loss of MTT cleavage and increased LDH release. DNAfragmentation increased likewise.

Conclusions: We conclude that coincubation of ibuprofen in suprtherapeutic and bilirubin in low concentration results in stronger detrimental effects on neurons than ibuprofen or bilirubin alone. Our results 
suggest that exposure of preterm infants to ibuprofen may increase the risk for bilirubin encephalopathy.

\section{RESVERATROL, A RED WINE CONSTITUENT, OFFERS NEUROPROTECTION FOLLOWING NEONATAL HYPOXIA-ISCHEMIA IN RATS}

\author{
F Karalis ${ }^{1}$, E Spandou ${ }^{1}$, C Simeonidou ${ }^{1}$, \\ I Papadopoulou $^{1}$, V Soubasi ${ }^{2}$, O Guiba-Tziampiri ${ }^{1}$ \\ ${ }^{1}$ Dept of Physiology, ${ }^{2}$ Dept of Neonatology, Faculty of \\ Medicine, Aristotle University of Thessaloniki, Greece
}

Background and Aims: Previous studies have shown that resveratrol, a polyphenolic compound found in grapes and wine, has anti-oxidant and anti-inflammatory properties in experimental models of ischemiareperfusion injuries. Aim of this study was to investigate whether resveratrol: a) has neuroprotective effect on hypoxia-ischemia (HI) induced brain damage in neonatal rats and b) exerts its action through inhibition of inducible nitric oxide synthase (iNOS).

Methods: At postnatal day 7 rats were separated into 3 groups: A (control) sham operated, B (HI) ligated and exposed to hypoxia for $1 \mathrm{~h}$ and $\mathrm{C}$ (R-HI) post-treated with resveratrol $(90 \mathrm{mg} / \mathrm{kg}$ in 2 equal doses). At P10, based on the gross morphologic appearance of the infarction, brains were grouped in 4 categories (normal, mild, moderate and severe). Histological analysis was performed at the level of dorsal hippocampus according to a semiquantitative 5-point scale. iNOS expression was estimated using immunohistochemistry.

Results: Neonatal HI resulted in extensive neuronal damage that was limited after resveratrol administration $(p<0.05)$. The majority of the R-HI group exhibit normal to mild damage and histologic evaluation showed a significant protection of cells in the cerebral cortex and hippocampus. Immunohistochemical study showed that $1 \mathrm{~h}$ of hypoxia led to the increased iNOS immunoreactivity in the hippocampus and the boundaries of the damaged cerebral cortex whereas resveratrol diminished iNOS expression.

Conclusions: Resveratrol attenuated the effects of $\mathrm{HI}$ on the neonatal brain by reducing the progression of neuronal injury. iNOS inhibition seems to be implicated in its action. The results of the present study are encouraging for the contribution of this natural phytoalexin to neuroprotection.

\section{PRESERVATION OF THE BLOOD-BRAIN- BARRIER BY MAGNESIUM SULFATE TREATMENT AFTER PERINATAL ASPHYXIA IN FETAL LAMBS}

F Goñi de Cerio ${ }^{1}$, A Alvarez ${ }^{1}$, F J Alvarez ${ }^{2}$, N Martínez ${ }^{1}$, A Caballero ${ }^{3}$, M C Rey-Santano ${ }^{2}$, V E Mielgo ${ }^{2}$, A Valls I Soler ${ }^{2,4}$, E Hilario ${ }^{1}$

${ }^{\prime}$ Dept of Cell Biology and Histology, School of Medicine, University of Basque Country, ${ }^{2}$ Research Unit on Pediatrics, Cruces Hospital, Basque Country ${ }^{3}$ Dept of Neuroscience, ${ }^{4}$ Dept of Pediatrics, School of Medicine, University of Basque Country, Spain
Background and Aims: The astroglial protein S100 is a marker of blood-brain-barrier (BBB) leakage and an early indicator of brain damage. To evaluate the neuroprotective efficacy of $\mathrm{MgSO} 4$ treatment in a hypoxic-ischemic event (HI) using protein $\mathrm{S} 100$ as brain damaged tissue-marker in preterm lambs.

Methods: 20 fetal lambs (133 days) were randomly assigned to: One control group (sacrificed 3 hour after delivery) and three hypoxic-ischemic groups (one immediately sacrificed and two others receiving or not $400 \mathrm{mg} / \mathrm{kg}$ of MgSO 4 during 20 minutes and sacrificed 3 hours after HI event). Injury was induced by partial occlusion of umbilical cord during 60 minutes. Brains were fixed, divided in different regions, disgregated and immunolabelled for protein S100. Samples were analyzed by flow citometry and positive cells were evaluated. One-factor ANOVA was performed $(\mathrm{p}<0.05)$. Results: HI group sacrificed immediately after the injury (0h) did not show differences of S100 levels in comparison to control one in all brain regions (cortex: $56.8 \pm 13$ vs. $59 \pm 16 \%$; inner zones: $57.4 \pm 9.3$ vs. 56.6 $\pm 12.1 \%$, respectively). However, S100 levels were significantly decreased in the group sacrificed 3 hours after $\mathrm{HI}$ injury in comparison to control and $\mathrm{h} \mathrm{h} \mathrm{HI}$ group (cortex: $8.6 \pm 5.2 \%$; inner zones: $8.2 \pm 5.5 \%$ ). The administration of $\mathrm{MgSO} 4$ give rise to values similar to control group (cortex: $63.6 \pm 9.7 \%$; inner zones: $53.4 \pm$ 9.5\%).

Conclusions: Our results show that $\mathrm{MgSO} 4$ treatment after the HI event maintains protein S100 levels similar to control group, suggesting that $\mathrm{MgSO} 4$ treatment avoids the BBB dysfunction. Grants: 9/UPV00077.32715330-2003.

\section{CEREBRAL INJURY IN A FULL-TERM NEWBORN WITH HYPOXIC ISCHEMIC ENCEPHALOPATHY (HIE): WHAT'S THE BEST INSTRUMENTAL TOOL?}

\author{
S Soffritti ${ }^{1}$, G Ancora ${ }^{1}$, F Sandri ${ }^{1}$, L Marvulli ${ }^{1}$, \\ L Nardi ${ }^{2}$, N Bisacchi ${ }^{2}$, B Mostacci ${ }^{3}$, R Lodi $^{3}$, \\ B Barbiroli $^{3}$, G Faldella ${ }^{1}$

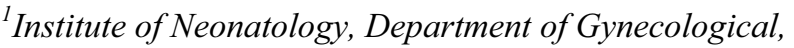 \\ Obstetrical and Pediatric Sciences, Bologna, Italy, \\ ${ }^{2}$ Psicology, Department of Gynecological, Obstetrical \\ and Pediatric Sciences, Bologna, Italy, ${ }^{3}$ Department of \\ Clinical Medicine and Applied Biotechnology, Bologna, \\ Italy
}

Background and Aims: Defining outcome in HIE is important, especially in Sarnat grade II, for counselling parents and early therapeutic intervention.

Methods: Case report: Female, outborn, GA 41 weeks, spontaneous delivery, AS 2-3-5, umbilical artery $\mathrm{pH}$ 7.1, BE -16 mmol/L, meconium stained amniotic fluid, intubated and ventilated at birth. Referred to our NICU at 2 hrs of life for asphyxia and meconium inhalation. Sarnat 2 HIE was diagnosed on admission. At $12 \mathrm{hrs}$ of life, clinical seizures appeared, amplitude-EEG showed a discontinuous normal voltage pattern that recovered to normal (CNV) at $20 \mathrm{hrs}$ with epileptic activity until 25 hrs. At 4 days, after crises of apnoea and bradycardia, aEEG showed a CNV pattern with recurrent seizures. Traditional EEG showed left temporal regions spikes. 
At 6 days $1 \mathrm{H}-\mathrm{MRS}$ detected a reduction of NAA/Cr and an increase of lactate and lipids in the left frontalparietal white matter; a reduction of ADC was observed in the same areas. First day cranial ultrasound showed parasagittal hyperechogenicity no longer visible after 2 wks. 1H-MRS and DWI at 6 months were normal.

Results: Outcome showed no sensorineural deficits; general movements, neurological examination and psychomotor development were normal at 6 months (GQ 125).

Conclusions: Although ultrasound, DWI and 1H-MRS were all abnormal in the $1 \mathrm{st}$ wk of life, the patient showed a normal outcome at 6 months of age. The only normal examination in the first $24 \mathrm{hrs}$ of life was the aEEG, suggesting, as reported in literature, the high accuracy of this tool in predicting outcome.

\section{EFFECTS OF ERYTHROPOIETIN ON CEREBRAL BLOOD CIRCULATION MEASURED BY NEAR-INFRARED SPECTROSCOPY IN PRETERM NEONATES}

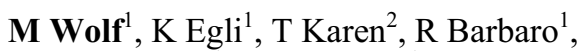 \\ A S Bauschatz ${ }^{1}$, H U Bucher ${ }^{1}$ \\ ${ }^{l}$ Clinic of Neonatology, University Hospital Zurich, \\ Switzerland, ${ }^{2}$ Department of Neonatology University \\ Childrens Hospital Tuebingen, Germany
}

Background and Aims: In a randomized controlled trial erythropoietin (epo) was given to preterm infants to study potential neuroprotective effects. Within this framework the aim was to assess, whether the relatively high dose of epo affects the cerebral blood circulation.

Methods: Sixteen newborn infants with $28.8 \pm 2.2$ weeks gestational age and $1167 \pm 382 \mathrm{~g}$ birthweight were enrolled and received either $3000 \mathrm{U} / \mathrm{kg}$ epo (epo-group, $\mathrm{N}=10$ ) or placebo (placebo-group, $\mathrm{N}=6$ ) i.v. A near infrared spectroscopy sensor (Hamamatsu ${ }^{\mathrm{TM}}$ NIRO-300) was placed on the head of each infant. The differences between the baseline before the injection and the first (period A) and second $10 \mathrm{~min}($ period $\mathrm{B})$ and the following $30 \mathrm{~min}$ (period $\mathrm{C}$ ) after injection were calculated for tissue oxygenation index (TOI) and hemoglobin index (THI) and compared between the two groups.

Results: The demographic data was similar between both groups, only the Apgar score at $1 \mathrm{~min}$ was significantly lower for the placebo-group. The following differences \pm SE were observed for the periods $A, B, C$, respectively: TOI (in \%): $-1.7 \pm 1.0,-4.3 \pm 2.1,-5.8 \pm 2.7$, THI (in $\mu \mathrm{M}$ ): $0.1 \pm 1.9,-2.2 \pm 4.2,-3.9 \pm 4.5$. None of the differences was statistically significant $(\mathrm{p}>0.05)$, although for all differences in TOI there was a trend $(p<0.1)$. The initial TOI before the injection was considerably but not significantly higher by $10.6 \pm 6.7 \%$ in the epo-group, which may be a factor contributing to the decrease in TOI in this group. Despite the decrease the mean TOI in the epo-group remained higher than in the placebo-group throughout the observation period.

Conclusions: Our preliminary results suggest that erythropoietin may affect cerebral blood circulation.

\section{NEAR INFRARED SPECTROSCOPY (NIRS), CEREBRAL BLOOD FLOW AND HEMODYNAMIC CHANGES IN HYPOXIC PIGLETS}

\author{
B Loureiro ${ }^{1}$, M C Rey-Santano $^{2}$, V E Mielgo ${ }^{2}$, \\ E Gastiasoro $^{2}$, A Valls-I-Soler ${ }^{1}$, F J Alvarez ${ }^{2}$ \\ ${ }^{1}$ Department of Pediatrics, Cruces Hospital, Barakaldo, \\ Bizkaia, Spain, ${ }^{2}$ Research Unit, Cruces Hospital, \\ Barakaldo, Bizkaia, Spain
}

Background and Aims: NIRS is a successful technique to measure cerebral oxygenation, but its use is limited to cortical zones. So, the combination with experimental procedures, like transient time flow or microspheres injection, is a better approach to determine regional or total cerebral perfusion. To compare cortical oxygenation measured by NIRS and transient time flow of hypoxic piglets induced by $8 \% \mathrm{FiO} 2$.

Methods: 6 piglets with 5-9 postnatal days were used (86/609 EC). Animals were tracheostomised and IPPV was established. Lung mechanics (VT, Raw, CL, din) were determined. Femoral artery catheter was placed to obtain blood samples, to measure cardiac output (CO), heart rate (HR) and mean arterial pressure (MAP). A transient time flow probe was placed around carotid artery to measure cerebral blood flow (CF). Cerebral oxygenation was determined by NIRS. Hypoxia was induced by reduction of $\mathrm{FiO} 2$ at $8 \%$ during $20 \mathrm{~min}$. Data represented by mean $\pm \mathrm{SD}$. One-factor ANOVA was performed $(\mathrm{p}<0.05)$.

Results: After hypoxia, gas exchange was $\mathrm{PaO} 2: 38 \pm 11$ mmHg; PaCO2: 41 \pm 10 mmHg; $p H: 7.1 \pm 0.1$. No changes were observed on lung mechanics. Hypoxia induced a decreased on: $\mathrm{CO} 1.0 \pm 0.2$ vs $0.5 \pm 0.3$ $\mathrm{L} / \mathrm{min} / \mathrm{kg}$; CF $77 \pm 35$ vs $38 \pm 23 \mathrm{~mL} / \mathrm{min}$; HR $259 \pm 22$ vs $177 \pm 56 \mathrm{bpm}$; MAP $88 \pm 11$ vs $31 \pm 5 \mathrm{mmHg}$. Satured (O2Hb: $-0.3 \pm 1.9$ vs $-20.4 \pm 9.9$ ), desatured (cHb: $0.5 \pm 2.3$ vs $9.6 \pm 2.2$ ) and total (HHb: $0.4 \pm 1.4$ vs $29.9 \pm 9.8$ ) hemoglobin demonstrated a significantly change.

Conclusions: Transient changes on cerebral oxygenation are demonstrated by NIRS. Hypoxia produced a severe hemodynamic compromise, correlated to a decrease on cerebral blood flow and oxygenation. Grants: C03/11; FIS PI030987 \& $\mathrm{BIO}$ 05/LF/13.

\section{PERFORMANCE OF A SEIZURE DETECTION ALGORITHM FOR NEONATES ON TWO- CHANNEL EEG DATASET}

M A Navakatikyan ${ }^{1}, \mathrm{C}$ G Spooner ${ }^{2}$, C Menache Starobinski ${ }^{3}$, J M Zempel $^{4}$, T E Inder ${ }^{5}$

${ }^{1}$ Department of Research, BrainZ Instruments Ltd, Auckland, New Zealand, ${ }^{2}$ Department of Neurology, Starship Children's Hospital, Auckland, New Zealand ${ }^{3}$ Department of Pediatric Neurology, Children's Hospital, Geneva, Switzerland, ${ }^{4}$ Pediatric Epilepsy Center, St Louis Children's Hospital, St. Louis, MO, USA, 'Department of Pediatrics, St Louis Children's Hospital, St. Louis, MO, USA

Background and Aims: An on-line neonatal seizure detection algorithm (Navakatikyan et al) based on wave- 
sequence analysis previously compared favourably against the Gotman et al (1997) algorithm (Sensa software 5.4) on a dataset of conventional multi-channel EEG. However, devices with a limited number of channels are important for long-term monitoring and ICU. Aim of the study was to evaluate the Navakatikyan algorithm on a two-channel EEG dataset.

Methods: The Navakatikyan algorithm was assessed on 56 one-hour two-channel EEG files (BRM2 Brain Monitor, BrainZ Instruments Ltd, New Zealand). The records were independently marked for seizures by four pediatric neurologists. Performance of the Navakatikyan algorithm was compared to the performance of the Gotman algorithm against each of the four specialists. Performance of the specialists was also compared against each other. Conservative and liberal assessments of algorithm performance were used, based on detected seizure duration.

Results: Conservative assessment: Sensitivities of the Navakatikyan algorithm, the Gotman algorithm and the specialists were 59,16 and $76 \%$, respectively. Positive predictive values (PPV) were 63, 56 and $76 \%$. Correlations were $0.56,0.25$ and 0.71 . Liberal assessment: Sensitivities were 82,70 and $84 \%$. PPVs were 70,59 and $84 \%$. Rates of false positives per hour were $0.7,2.8$ and 1.2, respectively. Thus, in all categories the Navakatikyan algorithm performed better than the Gotman algorithm, with similar performance to that of the neurologists.

Conclusions: The Navakatikyan algorithm performed well and provides a basis for major improvements in neonatal seizure detection. References: Navakatikyan at al. Clin. Neurophysiol.(accepted for publication). Gotman et al. Clin. Neurophysiol. 1997;103(3):356-62.

\section{CEREBRAL FUNCTION MONITORING WITH AMPLITUDE INTEGRATED EEG IN NEONATAL ECMO}

D Blanco Bravo, P Chimenti Camacho,

S Zeballos Sarrato, B Bernardo Atienza, S Villar, I Marsinyach, B Arias Novas, S Caballero, M Sanchez Luna, V Perez-Sheriff

Department of Pediatrics, Hospital General Universitario Gregorio Marañon, Madrid, Spain

Background and Aims: During the last few years it is possible to use continuous EEG to monitor brain function. ECMO represent a high risk procedure where monitoring cerebral function could be critical to help clinical decisions. Compare Cerebral Function Monitoring (CFM) with classical two channel EEG and biespectral index (BIS) in newborns during ECMO support.

Methods: Prospective observational study with CMF using the Olympic CMF 6000 and continuous EEG with CSA and BIS. The quality and characteristic of the traces and diagnostic support were analyzed.

Results: From July 2003 until January 2006, 22 newborns on ECMO were studied. $44,5 \%$ of them were Cardiac ECMO in congenital cardiac malformations during post surgery. $13,6 \%$ of all patients required cardiopulmonary resuscitation or had severe hypoxemia before ECMO. Seizure activity was recorded in 31\% of all. Mean duration of CFM was 120 hours and $26 \mathrm{~min}$, the dominant patter was the discontinuous normal voltage pattern. The low voltage in the left brain hemisphere by edema did not modified mono-channel aEEG. Only when high doses of analgesic agents were used the amplitutude of the traces were reduced: burst suppression and continuous low voltage with BIS of $<30$. Only seizures accompanied by worsening of base activity anticipate severe neurological complications confirmed by neuro-image.

Conclusions: CFM is a helpful monitoring system in patients on ECMO. Gives information about seizures activity and have a prognostic value depending on the background patterns.

\section{THE EFFECT OF INHIBITION OF NEURONAL SYNTHETASE OF NITRIC OXIDE ON NEURONAL APOPTOSIS, IN AN EXPERIMENTAL MODEL OF SEIZURES, DURING DEVELOPMENT}

I Papadopoulou ${ }^{1}$, E Spandou ${ }^{1}, \mathrm{C} \mathrm{Simeonidou}^{1}$, F Karalis ${ }^{1}$, I Liangouris ${ }^{1}$, N Grigoriadis ${ }^{3}$, G Karkavelas ${ }^{2}$, O Guiba-Tziambiri ${ }^{1}$

${ }^{1}$ Department of Physiology, Medical School, Aristotle University of Thessaloniki, Greece, ${ }^{2}$ Department of Pathology, Medical School, Aristotle University of Thessaloniki, Greece, ${ }^{3}$ Laboratory of Experimental Neurology and Neuroimmunology, Department of Neurology B, AHEPA Hospital, Thessaloniki, Greece

Background and Aims: Nitric oxide is involved in the pathophysiology of seizures in a multifunctional way. The aim of this study is to evaluate the effect of nNOS inhibition on seizures-induced neurodegeneration, by examining the expression of BAX, an apoptosis marker. Methods: Wistar rats (10-11-d-old) were divided into 4 groups: A, B, C and D (control). Groups A, B and C were subjected to hypoxic conditions. At P21-P22, rats of groups $\mathrm{B}$ and $\mathrm{C}$ were administered with kainic acid (KA $10 \mathrm{mg} / \mathrm{kg}$ i.p) and group $\mathrm{C}$ was pre-treated with $7 \mathrm{NI}$ $(50 \mathrm{mg} / \mathrm{kg}$ i.p). The BAX and nNOS expression was examined by immunohistochemistry in the rat hippocampus at 4,24,48 and 72 hours after KA administration and prolonged seizures.

Results: In the H/KA group, nNOS expression is increased at $4 \mathrm{~h}$, compared to control, with especially strong immunostaining in normal neurons at $24 \mathrm{~h}$. In the $7 \mathrm{NI}$ group, expression is obviously reduced at $4 \mathrm{~h}$, increased at 24h, although at lower levels than group B. At $48 \mathrm{~h}$, both group B and C present the baseline level. In H/KA group the induction of BAX immunoreactivity is apparent at $4 \mathrm{~h}$, with stronger immunostaining in degenerated neurons, becomes more intense at $24 \mathrm{~h}$, both in normal and dying neurons and is reduced after $48 \mathrm{~h}$ in intact neurons. In comparison with the 7NI group, there is a moderate difference starting at 24 hours, in favor of the 7NI group.

Conclusions: The inhibition of nNOS following the administration of 7NI had a moderate effect on the BAX expression, indicating a slight attenuation of seizuresinduced apoptotic cell death. 
IL1BETA PROMOTER POLYMORPHISMS ASSOCIATION WITH CEREBRAL PALSY

H Kapitanovic Vidak ${ }^{1}$, S Kapitanovic ${ }^{2}$

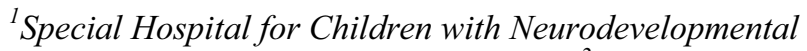
and Motor Difficulties, Zagreb, Croatia, ${ }^{2}$ Division of Molecular Medicine, Rudjer Boskovic Institute, Zagreb, Croatia

Background and Aims: Cerebral palsy (CP) is a nonprogressive motor disorder caused by white matter damage in the developing brain. It is often accompanied with neurocognitive and sensory disabilities. The cause and pathogenesis of $\mathrm{CP}$ is multifactorial and continues to be poorly understood. Chorioamnionitis, clinically silent or manifest, has been reported to be a risk factor for $\mathrm{CP}$ both in term and preterm infants. IL1beta gene was mapped to chromosome $2 \mathrm{q} 14$ and a few polymorphisms associated with altered IL1beta production have been described. Increased IL1beta levels have been found in peripheral blood in septic neonates and can be associated with the development of cerebral palsy. The aim of our study was to estimate allelic frequency for two promoter SNPs in IL1beta gene, -31 and -511 in the children with $\mathrm{CP}$.

Methods: DNAs obtained from peripheral blood of 27 $\mathrm{CP}$ patients and 162 unrelated healthy volunteers were genotyped for the IL1beta -31 and -511 SNP using realtime PCR TaqMan ${ }^{\circledR}$ SNP genotyping assays.

Results: IL1beta allele variants $-31 \mathrm{~T}$ and $-511 \mathrm{C}$ were more common in the population with cerebral palsy in the comparison to healthy volunteers.

Conclusions: The significance of the association between IL1beta gene polymorphisms and cerebral palsy has to be investigated in the future studies. This work was supported by grant \#0098108 from the Ministry of Sciences and Technology Republic of Croatia.

\section{CRANIAL ULTRASONOGRAPHY (US) AND PREDICTION OF OUTCOME IN TERM NEWBORNS WITH HYPOXIC ISCHEMIC ENCEPHALOPATHY (HIE)}

\section{G Ancora ${ }^{1}$, S Soffritti ${ }^{1}$, F Sandri $^{1}$, E Mazzoni ${ }^{1}$, N Bisacchi ${ }^{2}$, L Nardi $^{2}$, B Benassi ${ }^{1}$, G Faldella ${ }^{1}$ ', \\ ${ }^{l}$ Institute of Neonatology, Department of Gynecological, Obstetrical and Pediatric Sciences, Bologna, Italy, ${ }^{2}$ Psicology, Department of Gynecological, Obstetrical and Pediatric Sciences, Bologna, Italy}

Background and Aims: Studies about prognostic value of cerebral US have consisted of small numbers of patients and have showed contrasting results. US is a safe and greatly used bedside examination and it is useful to continue to report data about its prognostic value.

Methods: 26 newborns affected by HIE (Sarnat grading) were studied by US in the first $72 \mathrm{hrs}$ of life. Pathological US were defined having diffuse hyperecogenicity and slit ventricles, isolated parasagittal hyperecogenicity, talamic and basal ganglia hyperecogenicity. At 12-18 months of life a motor evaluation and a psychomotor assessment (Griffith test) were performed by an expert psychiatrist and psychologist. Visual and hearing function were evaluated during the first year of life. Motor outcome was classified as normal, transient hypertonia and cerebral palsy.

Results: 17 patients had a normal motor outcome, 6 developed transient hypertonia and 3 cerebral palsy. PPV of US for CP and transient hypertonia were $50 \%$ (CI 24-50\%) and $75 \% \quad$ (CI $47-91 \%) \quad(\mathrm{p}<0.01)$, respectively; NPV were $100 \%$ (CI 91-100\%) and $83 \%$ (CI $71-91 \%) \quad(\mathrm{p}<0.01)$. Among brain lesions, parasagittal hyperecogenicity resulted in normal outcome, basal ganglia hyperecogenicity in transient hypertonia and diffuse brain damage in CP. Mean (SD) general quotient in normal and abnormal US were 103 (12) and 72 (30) $(\mathrm{p}=0.006)$, respectively. Visual impairment was detected in 8 children and was not predictable by US ( $\mathrm{p}>0.05$ ).

Conclusions: Early US imaging is quite accurate in predicting $\mathrm{CP}$ in patients affected by HIE, especially when diffuse brain edema is detected. Isolated parasagittal or basal ganglia hyperecogenicity do not correlate with poor outcome.

\section{IN VIVO QUANTIFICATION OF SOD ACTIVATION IN NEWBORN MICE}

\author{
H H Odland ${ }^{1}$, G Dohlen ${ }^{1}$, E Thaulow ${ }^{2}$, O D Saugstad ${ }^{1}$ \\ ${ }^{l}$ Department of Pediatric Research, National Hospital, \\ Oslo, Norway, ${ }^{2}$ Department of Pediatric Cardiology, \\ National Hospital, Oslo, Norway
}

Background and Aims: Superoxide dismutase-1 (SOD-1) plays an important role in oxygen radical brain homeostasis. Neonatal transgenic mice overexpressing SOD-1 are more susceptible to hypoxic injury than adult transgenic mice. The activity of SOD-1 is higher in full term infants than in preterm infants. SOD-1 activity in newborn transgenic mice was assessed for 10 days.

Methods: 6 heterozygote litters with a total of 33 light producing transgenic reporter mice (CD-1-Tg(Sod1luc)Xen), containing a $15.5 \mathrm{~kb}$ mouse superoxide dismutase-1 (SOD-1) gene promoter, were imaged using the Xenogen imaging system. This includes a CCD camera to detect luminescence in luciferin injected mice. SOD-1 Activity (luminescence expressed as photons) was observed primarily in the brain, liver and skin.

Results: Newborn mice show a near 2-fold activity of SOD-1 in the brain during the first day of life compared to the activity at 10 days, with a significant rise from day 1 compared to day $5(\mathrm{p}<0.0001)$. The activity peaked at day 5 with a 2.5 -fold increase compared to the activity at day $10(\mathrm{p}<0.0001)$.

Conclusions: There is a statistically significant dynamic change in SOD-1 during the first days of life. This may play an important role in oxygen tolerance in the newborn. 


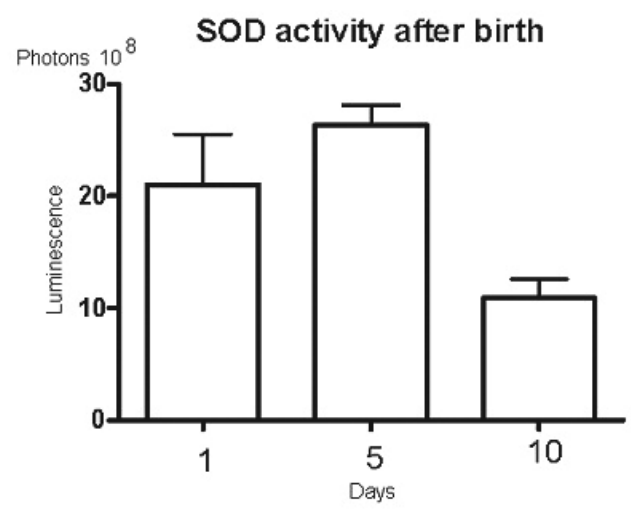

\section{EFFECT OF CHANGES IN END EXPIRATORY PRESSURE ON BRAIN AND UPPER BODY FLOW IN VENTILATED NEWBORNS}

\author{
K A de Waal ${ }^{1}$, N Evans ${ }^{1}$, D A Osborn ${ }^{1}$, M Kluckow ${ }^{2}$ \\ ${ }^{I}$ Department of Neonatal Medicine, Royal Prince Alfred \\ Hospital, ${ }^{2}$ Department of Neonatology, Royal North \\ Shore Hospital, Sydney, NSW, Australia
}

Background and Aims: Applying positive pressure ventilation to premature infants may diminish cerebral blood flow and cardiac output. Low brain and upper body flow (superior vena cava flow, SVC) is a risk factor for cerebral ischaemia and intraventricular haemorrhage in preterm infants, with previous research showing mean airway pressure as a significant determinant of SVC flow. It is not known whether this is a direct effect of positive pressure ventilation or a reflection of severity of lung disease. We investigated the effect of increasing PEEP on systemic blood flow at different gestations and with increasing postnatal age.

Methods: Ventilated newborns were measured before and 10 minutes after increasing PEEP from 5 to 8 cmH2O. Simultaneous echocardiographic and respiratory function measurements were collected between 3 and 9 hours of age, then daily if still ventilated.

Results: Sixty-nine studies in 50 infants were performed. Overall, increased PEEP was associated with a significant reduction in right ventricular output (mean difference $-12 \mathrm{ml} / \mathrm{kg} / \mathrm{min}$ ), but not in SVC flow, or left pulmonary or middle cerebral artery mean velocities. Lung compliance was increased and lung resistance decreased after 10 minutes of higher PEEP (mean difference $+0.08 \mathrm{ml} / \mathrm{cmH} 2 \mathrm{O} / \mathrm{kg}$ and $-16 \mathrm{cmH} 2 \mathrm{O} / \mathrm{L} / \mathrm{s}$ ). There was a weak positive correlation between change in SVC flow and change in lung compliance.

Conclusions: A short-term increase in PEEP does not lead to a significant change in systemic blood flow in most infants. The intervention can improve dynamic lung function, especially airway resistance. Improvements in compliance tend to be associated with improvements in blood flow.

\section{APO B GENE POLYMORPHISMS IN CZECH PATIENTS WITH ATHEROSCLEROSIS AND CHILDHOOD OBESITY}

I J Mazura ${ }^{1,2}$, H Svobodova $^{2}$, P Sedlak ${ }^{1,2}$, P Blaha $^{2}$, $\mathrm{J}_{\text {Zvarova }}{ }^{1}$
${ }^{1}$ Institute of Computer Science AS CR, Prague, Czech Republic, ${ }^{2}$ Department of Anthropology and Human Genetics, The Faculty of Science, Charles University, Prague, Czech Republic

Background and Aims: Atherosclerosis and childhood obesity are the most frequent disorders in Czech population. Influence of the genetic factors on atherosclerosis and obesity is known. We analyzed relationship between anthropometric, biochemical values and genetic polymorphisms in apolipoprotein B gene.

Methods: We collected and tested from January 1999 to December 2004, 51 patients with artherosclerosis and 99 patients with childhood obesity. In both groups we analyzed by basic molecular genetic techniques (blood sample DNA isolation, polymerase chain reaction, restriction fragment length polymorphisms method and electrophoresis) two promotor Apo B gene polymorphisms.

Results: Anthropometric and biochemical data were compared with tested genetic polymorphisms (Mendelian randomization). Linkage between low density lipoprotein level ( $\mathrm{T}$ allele presented) and cholesterol, LDL and apolipoprotein B levels (CC genotype presented) were observed different.

Conclusions: We suppose that atherosclerosis and childhood obesity are pathological units influenced by wide range of genes. Possible linkage between Apo B gene promotor polymorphisms occurrence and biochemical levels of relevant protein products is described. Genotype - phenotype relationship and anthropometric consequences of tested gene polymorphisms are discussed. This study was supported by Ministry of Education, Youth and Sport, Czech Republic, grant no. 1M06014.

\section{OXYGEN SATURATION IN NEONATES. COMPARISON OF THREE DIFFERENT PULSE OXIMETERS WITH ARTERIAL MEASUREMENTS}

M M Indrebø ${ }^{1}, \mathrm{~K}_{\text {Liestol}}{ }^{2}, \mathrm{~L}$ I Brundvand ${ }^{1}$, R Lindemann ${ }^{1}$

${ }^{I}$ Department of Paediatrics, Ulleval University Hospital, Oslo, Norway, ${ }^{2}$ Institute of Informatics, University of Oslo, Norway

Background and Aims: There are several brands of pulse oximeters, but accuracy and reliability differ and there is lack of validation in neonates. We wanted to compare the accuracy of three pulse oximeters compared with arterial oxygen saturation $(\mathrm{SaO} 2)$, and time consumption to a stable value.

Methods: The measurements were performed on neonates who needed an arterial line. $\mathrm{SaO} 2$ was measured from blood sample taken from an umbilical artery catheter and analysed with a RadiometerABL735. The pulse oximeters used were NellcorOxiMaxN-550. MasimoSETRadical $^{\mathrm{TM}}$. MiniCorrOximeterBCI3402. 42 measurements were performed on 21 patients, GA 23.441.3 weeks, age 1-25 days. On each infant, two different random monitors were used, on two different feet. The 
time until a stable $\mathrm{SpO} 2$ was registered, and a $\mathrm{SaO} 2$ was measured.

Results: The monitors had a tendency to show lower values when $\mathrm{SaO} 2$ was high and opposite when $\mathrm{SaO} 2$ was low. The mean absolute deviation was 2.79, 1.96 and 3.19 respectively. Gender, gestational age, age and blood pressure did not influence the results. There were no significant differences in time to stable values.

Conclusions: Presumed that arterial oxygen saturation is the true value, there were significant difference between the monitors in accuracy and reliability. This is of importance when used as a screening tool for congenital heart diseases.
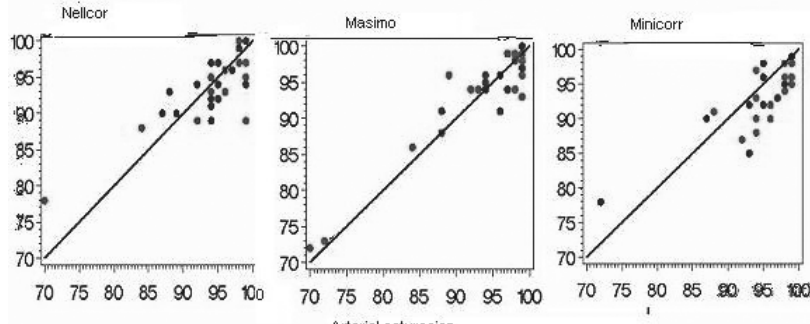

\section{CARDIAC TUMORS IN CHILDHOOD}

\section{B Varan ${ }^{1}, \mathrm{~K}_{\text {Tokel }}{ }^{1}$, I I Cetin ${ }^{1}$, U A Orun ${ }^{1}$, S Mercan $^{2}$, S Aslamaci ${ }^{2}$}

${ }^{I}$ Department of Pediatric Cardiology, Baskent University, Faculty of Medicine, Ankara, Turkey

${ }^{2}$ Department of Cardiovascular Surgery, Baskent

University, Faculty of Medicine, Ankara, Turkey

Background and Aims: Cardiac tumors are rare in infants and children but are a serious cause of morbidity and mortality. We aimed to evaluate the clinical characteristics of our patients with cardiac tumor.

Methods: 13 patients with cardiac tumor diagnosed between 1994 and 2005 were evaluated retrospectively. There was a clear male predominance, $\mathrm{M}: \mathrm{F}=12: 1$. Three patients also had congenital heart disease (1 ASD, 1 VSD, and 1 VSD and pulmonary stenosis).

Results: Age range was 5 days to 3 years. Clinical presentation was with a cardiac murmur in 7 patients, syncope in two, and convulsion in one. In two patients echocardiography was made for other reasons. One patient was diagnosed with antenatal ultrasonography. Four patients underwent surgery for resection of the tumor and only biopsy was made in two. Histopathologic diagnosis was rhabdomyoma in three, fibroma in one, myxoma in one, and malignant mesenchymal tumor in one patient. Seven patients had been diagnosed by echocardiography. Four patients had tuberosclerosis and three of them had rhabdomyoma. The patient with tuberosclerosis and multiple cardiac masses with no histopathological diagnosis died due to intractable supraventricular tachycardia. The patient with malignant mesenchymal tumor died after the operation. One patient with a mass in the left ventricle who could not have operation was learned to die later on.

Conclusions: Cardiac tumors are rare in children and should be suspected in case of cardiac murmur, syncope, and cerebrovascular events. Prognosis is usually poor in unresectable cases.

\section{FALSE TENDONS AS A ANATOMIC SUBSTRATE FOR IDIOPATHIC VENTRICULAR TACHYCARDIA}

M I Kelmendi, R H Bejiqi

Department of Cardiology, University Children's Hospital, Prishtina, Serbia and Montenegro

Background and Aims: Left ventricular false tendons (FT) are fibrous or fibromuscular bands that stretch across the left ventricle from the septum to the free wall. They are the most frequent cause of innocent murmur in childhood and can be seen in up to $50 \%$ of children examined with $2 \mathrm{D}$ echo. Numerous reports indicate the incidence of the coexistence of left ventricular false tendons and premature ventricular contractions. The aim of this study is to determine the incidence of false tendons in children with heart murmur and to stress the link between false tendons and idiopathic ventricular tachicardia in some of those cases.

Methods: 2015 children with heart murmur are examined at University Children's Hospital Echo lab, using Doppler echo with it's modalities.

Results: Most of examined children showed a various congenital and acquired heart disease, while in 274 pts $(13.6 \%)$ were detected only left ventricular false tendons. There were different types of FT: thin, thick, longitudinal, transversal, multiple .... A small number of pts with false tendons have other congenital or acquired heart disease, and 220 of these patients (80.3\%) were judged to have no underlying heart disease. Of 220 subjects with FT, $12(5.5 \%)$ manifested premature ventricular contractions; two children of this group underwent catheter ablation.

Conclusions: Like other authors data, our results indicate false tendons may play an etiologic role in the genesis of premature ventricular complexes in apparently normal heart.

\section{QUALITY OF LIFE IN CHILDREN WITH HEART ARRHYTHMIAS}

G V Dubova, N V Nagorna, Y V Bordyugova

Department of Post-Diploma Education, Donetsk State Medical University, Donetsk, Ukraine

Background and Aims: The heart arrhythmias (HA) make influence not only a physical condition of the child, but also on psychology of his behaviour, emotional reactions, his place in a social life that is why the effective help to this patients is impossible without the evaluation of quality of life (Qol). To study the Qol in children with HA.

Methods: 97 children (53 girls and 44 boys) at the age 6-18 years old with different HA were examined. All children completed the own special questionnaire 'Assessment of the quality of life in children with heart arrhythmias' (UA, Pat. 12262, A61B10/02, №200509883).

Results: At receipt actively showed complaints only half of patients with HA, at other examined they have been revealed at testing. Decrease in Qol have noted in $78,9 \pm 5,4 \%$ of children with HA, thus in $35,1 \pm 6,3 \%$ of children it has been considerably lowered. Main causes 
of decrease in Qol were disturbances of psychoemotional status $(85,6 \pm 3,6 \%)$ : the increased anxiety, irritability, various disturbances of a dream, fears, fast fatigue and the general weakness, melancholy, grief. Disturbances of health's state have noted in 78,9 $\pm 5,4 \%$ of patients with HA due to headache, cardiac pain, dizziness, frequent pulse.

Conclusions: Disturbances of psycho-emotional and the vegetative status in children with HA are such significant for the patients, as the symptoms of arrhythmia. Received data grounded the inclusion to the therapy of the children with HA the psychotherapeutic measures, vegetative system stabilizing and psychopharmacological medicines with the purpose to optimize children's treatment, as an alternative to antiarrhythmic drugs, dangerous in this age.

\section{CHOLESTEROL SCREENING IN AN AT RISK PAEDIATRIC POPULATION}

\author{
M J O'Grady ${ }^{1}$, A M Brown ${ }^{2}$, M B O'Neill ${ }^{1}$ \\ ${ }^{1}$ Department of Paediatrics, Mayo General Hospital, \\ Castlebar, Ireland, ${ }^{2}$ Department of Cardiac \\ Rehabilitation, Mayo General Hospital, Castlebar, \\ Ireland
}

Background and Aims: Elevation of low-density lipoprotein (LDL) cholesterol is a recognised risk factor for premature atherosclerosis and such elevations have their antecedents in the paediatric population. This study evaluated the frequency of elevated LDL cholesterol in children and adolescents in whom one parent had a history of premature ischaemic heart disease.

Methods: The Cardiac Rehabilitation database at Mayo General Hospital was searched between $2001-2005$ to determine individuals with a history of premature $(<55$ years) ischaemic heart disease (IHD). Parents were subsequently surveyed to determine the number of children. Inclusion criteria were children and adolescents $8-18$ years having one parent with premature IHD. Patients had a fasting lipid profile and results were classified as acceptable, borderline/high or high in accordance with American Academy of Pediatrics (AAP) guidelines. Those with high levels were screened for secondary causes of hyperlipidaemia. Normative data inclusive of height and weight were obtained and parental risk factor profiles were ascertained.

Results: Fifty-two parents had a total of 97 children and adolescents who were eligible for screening. Ninetythree initially consented and fasting lipid profiles were obtained in 82 children $(84.5 \%)$. Reasons for nonparticipation included potential school loss and needle phobia. Prevalences of high and borderline/high LDL cholesterol were (7/82) $8.5 \%$ and (17/82) $20.7 \%$ respectively.

Conclusions: Given the high incidence of abnormal results, screening of high risk children and adolescents is prudent, however follow-up care must be offered to this population.

\section{THE INTRACELLULAR PATHOGENS INFECTIONS IN CHILDREN WITH HEART ARRHYTHMIAS}

O V Pshenychna ${ }^{1}$, N V Nagorna ${ }^{1}$, Y I Yulysh ${ }^{2}$

${ }^{1}$ Chair of Pediatrics, Department of Post-Diploma Education, Donetsk State Medical University, Donetsk, Ukraine, ${ }^{2}$ Chair of Propedevtic Pediatrics, Donetsk State Medical University, Donetsk, Ukraine

Background and Aims: Problem of heart arrhythmias during the last decennial event is one of the most actual in children's cardiology. In last years appears all more given in favour of participations of intracellular infectious agents in genesis and rates of progressing number of cardiovascular diseases. Purpose: To study the presence of row the intracellular pathogens infections beside the children with heart arrhythmias.

Methods: 52 children ( 20 boys and 32 girls) at the age from 6 month to 16 years with different heart arrhythmias were examined. All children the serological diagnostics of specific antibody of class IgG to row of infectious agents (herpes simplex virus, cytomegalovirus, Epstein-Barr virus, chlamydia pneumoniae) was conducted; the DNA of this infectious agents defined a method of polymerase chain reactions.

Results: The diagnostically significant subtitles of antibody of class IgG to different intracellular pathogens infections are discovered beside all examined children with heart arrhythmias $(100,0 \%)$. The DNA of the intracellular pathogens infections took place in 40 $(76,9 \%)$ children with heart arrhythmias, herewith herpes simplex virus I type beside $23(45,0 \%)$ patients, Epstein-Barr virus - beside $16(30,0 \%)$ children, the association of Epstein-Barr virus and herpes simplex virus I type - beside $13(25,0 \%)$ examined patients.

Conclusions: The results of conducting studies are indicative of intracellular pathogens infections in 100\% children with heart arrhythmias. The received data allowed authors to suppose a probability of a heart arrhythmias occurrence as a result of pathological influence of intracellular infections. It is reason for expansions of diagnostic complex with the purpose to optimize of their treatment.

\section{VENTRICULAR ARRHYTHMIAS - DIAGNOSIS AND TREATMENT}

K Kuzevska-Maneva, R Kacarska, B Gjurkova, S Krstevska, V Micevska

Department of Cardiology, University Children's Hospital, Skopje, Macedonia

Background and Aims: Ventricular arrhythmias (VA) in children are less common than supraventricular and they are occurring more frequently after repairing of congenital heart disease, especially of complex CHD. The aim of the study is assessment of clinical features and treatment of VA.

Methods: The used methods were: Clinical examination, 12 channels electrocardiography, 2D Doppler Collor echocardiography and Holter ECG.

Results: For the period of 10 year (1996-2005) we found out VA in $48 \mathrm{pts}$, mean aged $8,7 \mathrm{y}+/-2,4$. Three 
of pts had ventricular tachycardia (VT), the other 45 premature ventricular contraction $(\mathrm{PVC})>=$ Lown class III. All children with VT had symptoms, but in group of children with PVC only 10 had a chest pain and discomfort. Structurally normal heart had 17 pts, secondary cardiomyopathia 2, Sy WPW 3, CHD after repair 26 (T. Fallot 8, Atrial septal defect 7, ventricular septal defect 5, transposition of the great arteries 4, canalis atrioventricularis 1 and double outlet right ventricle 1). Children with PVC were treated with Amiodarone 25/45, Propafenon 12/45, and Propranolol $1 / 45$; in a group with VT one child was treated with Lidocaine and the other 2 with electrocardioversion. PVC were successfully treated in $42 / 48 \mathrm{pts}, 3 / 48$ had resistant PVC. VT was stopped and converted in sinus rhythm in all three children. Later one child of them died from chronic heart failure.

Conclusions: VA is very dangerous heart rhythm disturbance especially in children with heart diseases. For these reason they must be discovered at time and treated to avoid sudden death.

\section{EFFICACY OF SILDENAFIL FOR TREATMENT OF PULMONARY HYPERTENSION IN PEDIATRIC CARDIAC SURGERY}

M Chkhaidze, I Metreveli, A Tsintsadze, M Tevzadze

Cardiac Intensive Care Department, Jo Ann Medical Center, Tbilisi, Georgia

Background and Aims: During last years increased the number of publications describing the efficacy of Sildenafil in treatment of infants and children with pulmonary hypertension $(\mathrm{PH})$ of different etiologies. In this regard we present two cases in which sildenafil was used to treat acute $\mathrm{PH}$ in infants after $\mathrm{CPB}$ cardiac surgery, as a last resort, instead of switching patient on ECMO, for lack of iNO (only one iNO delivery system is available in our facility and in both cases it was occupied by another patient).

Methods: Two infants with VSD, 9 and 2 months old (second one with Down syndrome) were presented after cardiac surgery in which PH developed postoperatively within 48 hours. Despite of hyperventilation, high FiO2, deep sedation and I.V. nitroglycerin, $\mathrm{PH}$ persisted, after approval of ethic committee and written consent of parents, sildenafil $0,5 \mathrm{mg} / \mathrm{kg}$ every 6 hours via NG tube was started. Systemic, pulmonary arterial pressures, arterial pulsoximetry, $\mathrm{ABG}$ were measured during study period.

Results: In one patient MPAP decreased by $51,3 \%$ after $40 \mathrm{~min}$. and in second - by $42,7 \%$ after $120 \mathrm{~min}$. of sildenafil administration, without any changes of systemic hemodynamics. Nitroglycerin was discontinued after 24 hours of stability. Patients were extubated on postoperative days VII and V, discharged from hospital on days XII and XIV, respectively.

Conclusions: We discuss potential use of sildenafil for treatment of PH after open heart surgery in infants, particularly under circumstances where, due to limited resources, iNO is not available. However, more research is needed to establish efficacy of sildenafil.

\section{THROMBOCYTOPENIA-AGENESIS RADII}

R Bejiqi ${ }^{1}$, R J Ratkoceri ${ }^{1}$, M Kelmendi ${ }^{2}$, N Zeka ${ }^{3}$, H Bejiqi ${ }^{4}$

${ }^{l}$ Department of Intensive Care, Paediatric Clinic, UCC Prishtina, Prishtina, Kosovo, ${ }^{2}$ Department of Cardiology, Paediatric Clinic, UCC Prishtina, Prishtina, Kosovo, ${ }^{3}$ Department of Neurology, Paediatric Clinic, UCC Prishtina, Prishtina, Kosovo

${ }^{4}$ Health House, Prishtina, Kosovo

Background and Aims: TAR- Syndrome is autosomrecessive inherited disease, more often in girls. It is manifested since birth with a group of symptoms dominated by hypopplasio radii billateralis $(100 \%)$, more rare of ulnas in (20\%). Very often this syndrome is accompanied with CHD (ASD or TOF). Thrombocytopenia dominated from the beginning and presented anaemia is in correlation with haemorrhagic diathesis. There are presented two girls with TARsyndromes from the same mother, but from two marriages.

Methods: History, x-ray of extremitas, haematological findings, echocardiography.

Results: History: In first marriage mother has one healthy boy and one with TAR-syndrome verificated. This girl has died when she was sixth months old of intracranial haemorrhagic. First husband also died in accident. In her second marriage mother has another girl with morphology of TAR-syndrome. X-Ray of extremitas: In both girls radius billateralis are absent and second girl has also aplasio humeri billateralis. Lab: Both girls has had haemorrhagic purpura since the second month of life and extreme thrombocytopenia has been registrated $\left(30^{*} 10^{\wedge} 9 / \mathrm{L}\right)$, haemostatic factors are in order, anaemia is presented $(\mathrm{Hgb}-60 \mathrm{~g} / \mathrm{L}, \mathrm{HCT}-\mathrm{o} .18$, WBC-38*10^9/L). Echocardiography: First girl has restrictive ASD, Cardiogram of both girls was regular.

Conclusions: Girls are from two different marriages in different times (difference is five years) and for this fen omen it has no explanation.

\section{CONGENITAL CARDIAC DEFECTS WITH 22Q11 DELETION}

A Butnariu ${ }^{1}, \mathrm{M} \mathrm{Marc}{ }^{2}, \mathrm{~L}_{\text {Bachner }}{ }^{1}, \mathrm{~L}$ Savu
O Motica

Background and Aims: Estimates suggests that the 22q11 deletion occurs in approximately 1 in 4000 live births. The majority of patients have conotruncal anomalies within DiGeorge syndrome, velocardiofacial syndrome and conotruncal anomaly face syndrome. Microdeletion of chromosomal region 22q11 is recognised by fluorescence in situ hybridization technique. The aim of the study was to determine the frequency of the 22q11 deletion in children with conotruncal cardiac defects.

Methods: There were evaluated fifty children diagnosed with conotruncal anomalies who were admitted to the 
Department of Cardiology in the Third Pediatric Clinic, Cluj-Napoca. Nine of them presented additional extracardiac clinical features. Fluorescence in situ hybridization (FISH) analysis was performed to detect 22q11 deletion.

Results: The forms of congenital cardiac defects included in the study were: tetralogy of Fallot in 28 patients (56\%), transposition of great arteries in 10 cases (20\%), double outlet right ventricle in 8 children (16\%) and total anomalous pulmonary venous connection in 4 patients (8\%). Additional extracardiac defects were represented by narrow palpebral fissures, congenital coloboma, velopharyngeal dysfunction, simian crease, auricular anomalies, defects of the nasal pyramid, mental delay, motor retardation and situs inversus. The FISH test evidenced the 22q11 microdeletion in 9 patients (18\%), 7 of them with additional extracardiac anomalies.

Conclusions: The frequency of 22q11 deletion in patients with conotruncal cardiac defects was of $18 \%$, the majority of these had additional extracardiac anomalies, mainly facial defects.

\section{PULMONARY HYPERTENSION IN A PATIENT WITH 11q DELETION DISORDER}

L J A Corel ${ }^{1}$, Y van Bever ${ }^{2}$, M Witsenburg ${ }^{3}$, D Tibboel ${ }^{1}$

${ }^{I}$ Department of Pediatric Surgery, Erasmus MC Sophia Children's Hospital, Rotterdam, The Netherlands ${ }^{2}$ Department of Clinical Genetics, Erasmus MC, Rotterdam, The Netherlands, ${ }^{3}$ Department of Pediatric Cardiology, Erasmus MC - Sophia Children's Hospital, Rotterdam, The Netherlands

Background and Aims: The 11q deletion disorder (Jacobsen syndrome) is a rare genetic disorder with variable phenotype (Grossfeld, P.D. et al. Am J Med Genet A. 2004 Aug 15;129(1):51-61). Cardiac anomalies has been described: mainly ventricular septal defect (VSD) or left sided obstructive lesions. So far pulmonary hypertension (PHT) has never been reported in patients with Jacobsen syndrome.

Methods: Observational study (case report).

Results: Case report: a female patient with antenatal suspected cardiac anomalies. Postnatally, ultrasound revealed a ventricular and atrial septal defect while the ductus arteriosus was still open. Other findings were mild dysmorphic features, one-sided renal dysplasia and thrombocytopenia. Chromosomal analysis showed deletion of chromosome 11q. Cardiac catheterization at age 3 weeks confirmed severe PHT $(120 \mathrm{mmHg}$; aortic pressure $70 \mathrm{mmHg}$ ), restrictive VSD and ductus and no aortic arch obstruction at that time. PHT was rapidly progressive and did not respond to oxygen and nitric oxide. Extra corporeal membrane oxygenation (ECMO) was instituted and additionally treatment with Sildenafil, Bosentan and Prostacyclin was started. After 2 weeks ECMO treatment was stopped. Due to anuria and weak leg pulses an aortic arch gradient was suspected. Repeat cardiac catheterization now revealed a hypoplastic transverse arch with an important gradient. Emergency balloon dilatation was attempted. Unfortunately, the patient died of a profuse bleeding few hours after balloon dilatation. Autopsy was not performed.

Conclusions: The finding of severe pulmonary hypertension adds to the possibly lethal features in Jacobsen syndrome, while aortic arch obstruction may occur at an unusual level (transverse aorta).

\section{THE MORPHOLOGICAL STRUCTURE OF HEART IN CHILDREN WITH CONGENITAL HEART-DISEASES IN COMBINATION WITH A INTRACELLULAR INFECTION}

N V Nagorna, K V Vinogradov

Department of Post-Diploma Education, Donetsk State Medical University, Donetsk, Ukraine

Background and Aims: To study the morphological structure of heart of children with congenital heartdiseases (CHD), associated with a intracellular infection.

Methods: 15 children ( 8 girls, 7 boys) from 5 month to 14 years with the different types of CHD (atrial septal defect, ventricular septal defect, coarctation of the aorta, patent ductus arteriosus, diseases of Fallot) were inspected. Specific IgG to herpes simplex virus, cytomegalovirus, Epstein-Barr virus, chlamydia pneumoniae, toxoplasma gondii were determined by the method of enzyme immunoassay. A polymerase chain reaction (PCR) to those exciters in the serum and intraoperative bioptates of the tissues of heart was conducted. The morphological study of intraoperative bioptates of the tissues of heart was conducted by a light microscopy.

Results: For all inspected children with CHD specific $\mathrm{IgG}$ is discovered in diagnostically meaningful titles. Thus in $7(58,3 \%)$ cases found out IgG to the different associations of the explored intracellular exciters. Accordant to the data of PCR of serum, the DNA of the explored exciters found out in $4(26,7 \%)$ examined patients, in intraoperative bioptate of endomyocardium in $3(20,0 \%)$ children with CHD. At morphological research of intraoperative bioptates of the tissues of heart the signs of inflammatory changes were exposed in $93,3 \%$ children. In $10(66,7 \%)$ children displays of acute inflammatory process took place. The markers of the earlier inflammatory process - at $4(26,7 \%)$ examined patients.

Conclusions: Results testify that presence in the organism of children with CHD persistence infections can be accompanied with inflammatory changes in tissues of heart.

\section{A REVIEW OF THE USE OF PROPHYLACTIC INDOMETHACIN AND A COMPARISON WITH OTHER STRATEGIES}

S Harigopal ${ }^{1}$, D Anand ${ }^{2}$, M Kubwalo ${ }^{1}, \mathrm{M} \mathrm{Tan}^{2}$, J Turley $^{1}, \mathrm{~K}$ Poole ${ }^{1}$, N Subhedar ${ }^{1}$

${ }^{l}$ Neonatal Unit, Liverpool Women's Hospital, UK,

${ }^{2}$ University of Liverpool, $U K$

Background and Aims: Prophylactic indomethacin (pIND) is effective in reducing symptomatic PDA 
(sPDA) requiring medical or surgical closure. Our aim was (1) to determine whether the short term effects of pIND were replicable in our population and (2) to compare the likely effectiveness of 3 different strategies for preventing or treating SPDA.

Methods: We reviewed case notes of babies with a birth weight of $<1250 \mathrm{~g}$ treated with pIND. Information was collected on the incidence of sPDA requiring a further course of indomethacin and/or ductal ligation and these rates were compared with previously reported rates of sPDA. Three strategies of preventing or treating SPDA in babies $<1250 \mathrm{~g}$ were modelled using these data: (1) prophylaxis; (2) symptomatic treatment and (3) 'selective' prophylaxis of extremely preterm babies.

Results: During a 12 month period, 124 babies were treated with pIND. The rate of sPDA (ie. failure rate of pIND) was $16 \%$ and the incidence of ductal ligation was $4 \%$. These figures are consistent with previously reported data. For a hypothetical population of 100 babies $<1250 \mathrm{~g}$, the table summarises potential benefits against indomethacin exposure:

Conclusions: The short-term efficacy of prophylactic indomethacin in our unselected population was similar to that in previously reported trials. Targetting prophylaxis at a higher risk population of extremely preterm infants may allow reduced exposure to indomethacin but risks increasing the number of ductal ligations.

\begin{tabular}{|l|c|c|c|c|}
\hline Strategy & $\begin{array}{l}\text { Total courses of } \\
\text { indomethacin }\end{array}$ & $\begin{array}{l}\text { Total sPDA } \\
\text { requiring } \\
\text { medicalsurgical } \\
\text { treatment }\end{array}$ & $\begin{array}{l}\text { sPDA } \\
\text { requiring } \\
\text { ductal } \\
\text { ligation }\end{array}$ & $\begin{array}{l}\text { Benefit } \\
\text { (courses/ligation) }\end{array}$ \\
\hline $\begin{array}{l}\text { Prophylaxis } \\
<1250 \mathrm{~g}\end{array}$ & 116 & 16 & 3 & 39 \\
\hline $\begin{array}{l}\text { Symptomatic } \\
\text { treatment } \\
<1250 \mathrm{~g}\end{array}$ & 40 & 40 & 8 & 5 \\
\hline $\begin{array}{l}\text { 'Selective' } \\
\text { prophylaxis } \\
\text { for } \leq 27 \mathrm{w}\end{array}$ & 72 & 22 & 5 & 14 \\
\hline
\end{tabular}

\section{LEFT VENTRICULAR MYXOMA: AN UNUSUAL LOCALIZATION CAUSED CARDIAC ARREST}

S V Yildirim ${ }^{1}$, R Turkoz ${ }^{2}$, B E Guc ${ }^{3}$, O Gulcan ${ }^{2}$, K Tokel ${ }^{1}$

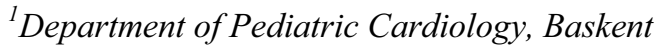
University, Adana Teaching and Medical Research Center, Adana, Turkey, ${ }^{2}$ Department of Cardiovascular Surgery, Baskent University, Adana Teaching and Medical Research Center, Adana, Turkey, ${ }^{3}$ Department of Pediatrics, Baskent University, Adana Teaching and Medical Research Center, Adana, Turkey

Background and Aims: Primary cardiac tumors are rare, and myxomas constitute about $50 \%$ of primary cardiac tumors in patients of all ages. Myxomas are mostly localized in the left atrium (75\%), and generally friable, pedinculated, gelatinous benign tumors often presents with cardiac obstruction and emboli. Here, we report a case of myxoma localized in left ventricular free wall caused syncope and cardiac arrest in a child.

Methods: An 11 years-old girl admitted to our hospital cause of syncope, cardiac arrest, and following consciousnesses. We learned that she was previously healthy; after the beden eğitimi lesson she had chest pain and in the hospital she suddenly felt down with cardiac arrest. After the cardiopulmonary resuscitation she transported to our hospital while she was intubated. We evaluated the patient, and echocardiography showed an intracardiac pedinculated tumor localized in the left ventricle free wall below the papillary muscle. The tumor was totally resected by transmitral approach; it was pathologically a simple myxoma. The child was discharged after 5 days surgery without complication.

Conclusions: A very rare localization of myxoma, a primary cardiac tumor, was presented because of unusual clinical picture that ventricular fibrillation and cardiac arrest. Cardiac tumors must be thought in such clinical conditions.

\section{THE ROLE OF ECHOCARDIOGRAPHY ON EVALUATION OF THE SEVERITY OF MITRAL REGURGITATION}

M I Kelmendi, R H Bejiqi

Department of Cardiology, University Children's Hospital, Prishtina, Kosova

Background and Aims: Echocardiography with Doppler has recently emerged as the method of choice for the non-invasive detection and evaluation of the severity and etiology of valvular regurgitation. There are many, qualitative and quantitative echocardiographic parameters that can provide assessment of valvular regurgitation.

Methods: A retrospective study of children with rheumatic mitral regurgitation, examined at University Children's Hospital, Prishtina. Following recommendations of the American Society of Echocardiography, six qualitative and quantitative parameters are used for assessing mitral valve regurgitation: regurgitant jet area, vena contracta, PISA, pulmonary artery pressure, pulmonary venous flow pattern and left atrium size.

Results: There have been diagnosed 74 children with rheumatic mitral regurgitation, $50(67.5 \%)$ of them as a pure mitral regurgitation and 24 (32.5\%) combined with aortic or tricuspid regurgitation. Using mentioned criteria, the mitral regurgitation quantification has been calculated as: mild in 36 pts (48.6\%), moderate in 31 $(41.9 \%)$ and severe regurgitation in $7(9.4 \%)$.

Conclusions: The aproach to the evaluation of mitral regurgitation severity ideally integrates multiple parameters rather than depending on a single measurement. this helps minimize the effects of technical or measurement errors that are inherent to each method previously discussed.

\section{PATENT DUCTUS ARTERIOSUS IN VERY LOW BIRTHWEIGHT INFANTS}

M Vera ${ }^{1}$, A Riverola ${ }^{1}, \mathrm{~J}_{\text {Moreno }}{ }^{1}, \mathrm{~F}_{\text {Prada }}{ }^{2}, \mathrm{~J}_{\text {Bartrons }}{ }^{2}$, $\mathrm{X} \mathrm{Mayol}^{3}$, X Krauel ${ }^{1}$

${ }^{l}$ Neonatal Unit, Agrupació Sanitaria Hospital Sant Joan de Deu, Hospital Clinic, Barcelona, Spain, ${ }^{2}$ Department of Paediatric Cardiology, Agrupació Sanitaria Hospital Sant Joan de Deu, Hospital Clinic, Barcelona, Spain ${ }^{3}$ Department of Paediatric Cardiac Surgery, Agrupació Sanitaria Hospital Sant Joan de Deu, Hospital Clinic, Barcelona, Spain 
Background and Aims: Patent Ductus Arteriosus (PDA) is a frequent complication in the clinical course of Very Low Birthweight Infants (VLBW). Our aim was to evaluate PDA in preterm infants in our Neonatal Intensive Care Unit.

Methods: Retrospective review from data base of inborn preterm infants ( $<33$ weeks) admitted from $01 / 01 / 95$ to $31 / 12 / 05$. Diagnosis of PDA: 1) Birth weight $(\mathrm{BW})>1000$ gr., with clinical signs, confirmed by echocardiography, 2) BW $<1000$ gr., all had echocardiography for diagnosis in the first 48-72 hours of life. We analyzed epidemiologic data, clinical features, echocardiographic findings, treatment and outcome.

Results: Of 730 babies of $<33$ weeks admitted into the NICU, 148 had PDA (incidence 20.2\%). 75 were male. Mean BW: 933 +/- 256 g, gestational age: $27.2+/-2$ weeks. Clinical features: 60/148 (40.5\%) infants were asymptomatic, 88 symptomatic: murmur 60/148 $(40.5 \%)$, signs of congestive heart failure signs 21 (14\%), other 7. Echocardiography: mean diameter PDA $2.14+/-0.7 \mathrm{~mm}$, pulmonary hypertension $100(67.5 \%)$. Treatment: 128 (86\%) indomethacin, 8 ibuprofen, ductal closure achieved in $88(68 \%)$ and $3(37 \%)$ cases respectively. 30 infants needed surgical ligation. 40 $(31.2 \%)$ patients presented side effects after indometacin (renal and digestive). Global mortality: $21.6 \%$.

Conclusions: Incidence of PDA is high in preterm infants. Clinical presentation is in most of the patients either absent or mild. Early echocardiography diagnoses half the cases, before clinical signs appear. Indomethacin is the main treatment with an acceptable rate of ductus closure, but frequent side effects.

\section{EVALUATION OF NUTRITIONAL STATUS AND CALORIE INTAKE IN PAEDIATRIC INTENSIVE CARE}

\author{
B Bockenkamp ${ }^{1}$, P Jouvet $^{1}$, A Proietti ${ }^{1}$, V-A Pelletier ${ }^{2}$ \\ ${ }^{I}$ Department of Paediatric Intensive Care, Montreal \\ University, Sainte-Justine Hospital, Montreal, QC, \\ Canada, ${ }^{2}$ Department of Paediatrics, Montreal \\ University, Sainte-Justine Hospital, Montreal, QC, \\ Canada
}

Background and Aims: The benefit of nutritional support in critically ill children gains growing awareness, however assessment of the nutritional status is not yet part of the routine admission. More recently studies have emphasised the search for barriers to adequate nutrition. The objectives are to assess the nutritional status upon admission to the paediatric intensive care unit (PICU), to evaluate the prescribed and received calories during PICU stay and to identify factors confounding the actual intake.

Methods: During the 4-month-study-period patients with an expected PICU stay of $\geq 72$ hours were prospectively included. On admission anthropometric measurements were performed. Daily calories prescribed and actually delivered were recorded and compared to estimated energy requirements (EER). The reasons for decreasing the nutritional support were registered in parallel.
Results: Fifty-two out of 298 patients (17.4\%) were included. Sixty percent (31/52) were acutely or chronically malnourished according to the definition of Waterlow. The median ratio of actual calories delivered to EER was below 0.8 on day 3, 7, 14 and 21 in $68 \%$, $17 \%, 13 \%$ and $7 \%$ of the cases, respectively. A difference $\geq 5 \mathrm{cal} / \mathrm{kg} /$ day between prescribed and delivered calories was observed in 231/589 of recorded days $(39 \%)$ for enteral nutrition (EN) and $0.1 \%$ for parenteral nutrition. The decrease of EN was mainly due to extubation, imaging studies and procedures.

Conclusions: Our study shows that despite a high prevalence of malnutrition among the PICU population, EER are not covered in a proportion of patients because of ongoing interventions. We believe that specific strategies may prevent this "procedure-induced" calorie deficit.

\section{DAILY ORAL SUPPLEMENTATION WITH L. PARACASEI CNCM I-2116 LEADS TO A DOWN - MODULATION OF MILK PROTEIN HYPERSENSITIVITY IN MICE}

S Pecquet, S Chibani-Chennoufi, F Rochat, A Mercenier

Department of Nutrition and Health, Nestlé Research Center, Lausanne, Switzerland

Background and Aims: Cow's milk allergy is one of the leading causes of food allergy in children. Sensitisation to milk proteins occurs early in life, leading to atopic symptoms. Some clinical studies showed that probiotic treatment of "at risk" infants might prevent them from allergic symptoms, in particular from atopic dermatitis. Here, we use a milk hypersensitivity mouse model to evaluate beneficial effect of the probiotic L. paracasei CNCM I-2116 on the onset of atopic symptoms.

Methods: $\mathrm{C} 3 \mathrm{H} / \mathrm{HeJ}$ mice were sensitised 4 times with whey proteins associated with cholera toxin, once a week. All trial long, mice were receiving either L. paracasei CNCM I-2116 $(5.108 \mathrm{cfu} / \mathrm{mL})$ or maltodextrine (matrix control) in drinking water. One week after last sensitisation, mice were orally challenged with b-lactoglobulin and symptoms (scratching, puffiness and activity loss) were followed for $45 \mathrm{~min}$. At sacrifice, blood and faeces were collected for seric IgE, IgG1, MMCP1, histamine and faecal IgA. Faecal probiotic were recurrently counted during trial.

Results: Mice treated with maltodextrine showed a high symptom score (4.1) compared to L. paracasei CNCM I2116 treated mice (1.3). This significant difference could be correlated to a similar trend observed with MMCP1, histamine, total IgE and specific IgG1 levels.

Conclusions: This preliminary data first indicates that this experimental model is suitable to evaluate preventive effect of probiotics in allergy. Secondly, this study provides the evidence that probiotics can modulate food hypersensitisation in mice. Indeed, we demonstrate that daily administration of L. paracasei CNCM I-2116 protect mice from cow's milk protein hypersensitivity. 


\section{PRETERM INFANTS: BODY COMPOSITION ASSESSMENT DURING THE FIRST THREE MONTHS OF CORRECTED AGE}

P Roggero, P Piemontese, M L Giannì, A Orsi, O Amato, V Puricelli, S Montella, F Mosca

NICU, Clinica Mangiagalli (IRCCS), University of Milan, Italy

Background and Aims: Body composition (BC) assessment in premature infants is important for nutritional support as protein intake influences body fat retention. To examine postnatal changes in $\mathrm{BC}$ in preterm compared to full term infants.

Methods: BC (\%fat) was assessed using a new air displacement system (PEA POD, LMI Concord, CA) in 19 Caucasian PT (mean GA 31 wks $\pm 2,4$; mean BW $1332 \mathrm{~g} \pm 370$ ) and in 52 Caucasian FT exclusively breast fed. In PT fat mass was evaluated at term adjusted age, 1,2 and 3 months of corrected age and in FT within 72 hours of life, at 1,2 and 3 months. The protein/100Kcal intake in PT up to term adjusted age: 3.1-2.7 g. Thereafter PT were fed standard formula.

Results: The mean fat of PT at term adjusted age was higher than that of FT at birth $(13.5 \% \pm 3.6$ vs $6.8 \% \pm 3.94, \mathrm{p}<.001)$. No significant difference was found between mean fat of PT and FT at $1(19.3 \% \pm 4.3$ vs $17.2 \% \pm 4.27), 2(23.1 \% \pm 2.3$ vs $23.7 \% \pm 3.9)$ and 3 months $(24.6 \pm 4.1$ vs $27.3 \% \pm 4.03)$. Mean weight was lower in PT at term adjusted age compared to FT at birth $(2577 \pm 281$ vs $3236 \pm 443,7 \mathrm{p}<.001)$ and in PT compared to FT assessed at 1 month. (3755 \pm 501 vs $4359 \pm 622.5$ $\mathrm{p}<.05$ ).

Conclusions: Our data show that PT have fat mass at term-adjusted age higher than FT at birth. Body weight assessment does not provide information about BC. High protein and energy intake could play an important role in body fat retention in the first week of postnatal life.

\section{MARKERS OF INSULIN RESISTANCE SYNDROME IN OBESE CHILDREN AND ADOLESCENTS}

E Papadopoulou, E Giahnakis, M Avanidi, N M Fitrolaki, H Maniadaki, M Kalmanti

Department of Pediatrics, University of Crete, Heraklion, Crete, Greece

Background and Aims: The prevalence of metabolic syndrome (MS) or insulin resistance syndrome (IRS) is high among obese children and adolescents, and increases with worsening obesity. All or some of the following characterizes the IRS: obesity, impaired glucose metabolism, dyslipidemia, and hypertension. The use of hematological and non-hematological markers to screen for IRS in this population remains a necessity.

Methods: We reviewed data from 76 obese children, including 33 females (43.4\%) and 43 males (56.6\%), with a mean age of 10.3 years (range 3-16), who were followed in our Childhood Obesity Clinic. BMI $\left(\mathrm{kg} / \mathrm{m}^{2}\right)$, waist circumference (WC, in $\mathrm{cm}$ ), fasting glucose ( $\mathrm{fG}$, in $\mathrm{mg} / \mathrm{dL}$ ), fasting insulin (fI, in $\mu \mathrm{U} / \mathrm{mL}$ ), HOMA-IR index [Homeostasis Model Assessment-Insulin Resistance $=\mathrm{fI} \times \mathrm{fG} / 22.5]$, systolic and diastolic blood pressure (BP), fasting triglycerides (fT, in $\mathrm{mg} / \mathrm{dL}$ ), and atheromatic index (AI=total cholesterol/HDL) were measured in every patient. Descriptive statistics and difference of means of variables (independent samples T test) were used. Correlations were considered as significant at the 0.01 or 0.05 levels (2-tailed).

Results: A number of correlations between the markers examined for IRS were observed: 1) fI is strongly correlated with WC and BMI; 2) HOMA-IR is strongly correlated with WC, fG, BMI, and fI; 3) Systolic and diastolic BP are significantly correlated with WC, BMI, fI and HOMA-IR. There was no a positive association of fT and AI with the other markers of IRS.

Conclusions: Waist circumference may be a noninvasive and low-cost marker of insulin resistance syndrome, whereas fasting triglycerides and atheromatic index probably cannot be used as early marker of this syndrome.

\section{THE EFFECT OF CHRONIC FETAL UNDERNUTRITION AND HYPOXEMIA ON THE FETAL RESPONSE TO ACUTE HYPOXIA}

T Mulder, E Villamor, Z Gura, C E Blanco,

Department of Pediatrics, University Hospital

Maastricht, The Netherlands

Background and Aims: In most species fetal glucocorticoid activity increases late in gestation and is closely associated with maturational processes, essential for postnatal survival. However, fetal exposure to excess glucocorticoids is one mechanism suggested to contribute to adult cardiovascular disease. Objective: To describe the effect of chronic under nutrition and hypoxia on fetal glucocorticoid levels in an mother independent animal model.

Methods: Three groups of fertilised eggs of White Leghorn chicken were incubated: 1) Chronic hypoxia group: incubation in a hypoxic $(15 \% \mathrm{O} 2)$ environment 2) Malnutrition group: incubation after removal of $10 \%$ of the egg's albumin in a normoxemic environment 3) Control group: incubation in a normoxemic environment without albumin removal. Plasma corticosterone was measured in blood samples from the chorio-allantoic artery. In each group 10 chick embryos were measured at day $10,12,14,16$ and 18 of incubation (complete incubation time 21 days).

Results: Both chronic under nutrition and hypoxia caused a significant reduction of embryo weight (E18: $24.6 \mathrm{~g}$ vs $20.5 \mathrm{~g}$ and $21.9 \mathrm{~g}$ respectively) with significant sparing of the heart and brain weights. Control chicken embryos showed an increase in corticosterone levels late in incubation which was attenuated by chronic hypoxia ( $25.9 \mathrm{nmol} / 1$ vs $15.3 \mathrm{nmol} / \mathrm{l})$ but not by under nutrition.

Conclusions: Chronic hypoxia resulted in a reduced surge for glucocorticoids. Chronic under nutrition had no effect. This gives support to the hypothesis that maternal glucocorticoids are responsible for the fetal exposure to excess glucocorticoids in fetal growth retardation. 


\section{OXIDATIVE STRESS MARKERS IN REFLEX TEARS OF CHILDREN WITH INSULIN DEPENDENT DIABETES MELLITUS}

M I Eliopoulou ${ }^{1}$, N M Nikolakopoulou ${ }^{1}$, B E Spiliotis ${ }^{1}$, A M Exarchou ${ }^{2}$, C D Georgakopoulos ${ }^{2}$, F Lamari ${ }^{3}$, V S Gartaganis $^{4}$, N K Karamanos ${ }^{4}$

${ }^{I}$ Pediatric Endocrine Unit, Department of Pediatrics, University of Patras, Greece, ${ }^{2}$ Department of Ophthalmology, University of Patras, Greece ${ }^{3}$ Department of Pharmacy, University of Patras, Greece, ${ }^{4}$ Laboratory of Biochemistry Department of Chemistry, University of Patras, Greece

Background and Aims: To develop a novel method for the simultaneous determination of uric acid (a lowmolecular weight antioxidant) and of malondialdehyde (MDA, indicator of lipid peroxidation) in reflex tears of healthy and children with insulin dependent diabetes mellitus (IDDM) by high performance capillary electrophoresis.

Methods: Tear samples were collected with preweighted strip filter papers, extracted with a low $\mathrm{pH}$ phosphate buffer containing EDTA and were stored at $70^{\circ} \mathrm{C}$ in the presence of 2,6-di-tetrabutyl-4methylphenol (BHT). Tears' proteins were removed after centrifugation through centricon-3 membranes. Recovery values for all analytes were over $90 \%$. Analyte separation is achieved in a $25 \mathrm{mM}$ borate buffer, $\mathrm{pH} 10.0$, containing $100 \mathrm{mM}$ sodium dodecyl sulfate (SDS) at the temperature of $25 \mathrm{oC}$ and $20 \mathrm{kV}$ (normal polarity). Reproducibility tests measuring both migration times and peak areas gave low relative standard deviation values. Calibration graphs were linear and the detection limits, defined as a signal to noise ratio of $3: 1$, were are 0.50 , and 1.25 micromol for uric acid and MDA, respectively.

Results: Preliminary results showed significantly lower levels of uric acid in tears of diabetic children (median 5.27, range 1.28-27.02 micromol/L) than those of healthy subjects (median 28.3, range 9.6-34.6 micromol/L). Levels of MDA in tears of diabetic children (median 0.86, range 0-3.79 micromol/L) were significantly higher compared to normal controls (median 0.01 range 0-0.69 micromol/L).

Conclusions: The results suggest an increased oxidative stress in tear film of children with IDDM which may render them more susceptible to ocular surface disorders.

\section{PULMONARY STRETCH RECEPTOR ACTIVITY RELATED TO THE ENERGY STORAGE OF NORMAL AND SURFACTANT-DEPLETED LUNGS DURING SPONTANEOUS BREATHING}

\section{R Sindelar, G Sedin, A Jonzon \\ Department of Women's and Children's Health,} University Children's Hospital, Uppsala, Sweden

Background and Aims: Vagal input from slowly adapting pulmonary stretch receptors (PSRs) is important for establishing regular breathing, normal surfactant function and normal pulmonary gas exchange at birth with the least work of breathing and without overextending the lung. Our aim was to study the response of PSRs to changes in energy storage of the lungs under conditions encountered in newborn infants.

Methods: Single units of PSRs were recorded in seven anaesthetised, spontaneously breathing young cats, before and after lung lavage and then after surfactant instillation. PSRs were classified as high threshold (HT) and low threshold (LT) receptors. Instantaneous impulse frequency (fimp) of PSRs was related to transpulmonary pressure (Ptp), tidal volume (VT), and inspiratory time. The PSR fimp was normalised ( $\mathrm{fN}$ ) to the energy storage of the lung during inspiration (sigmadelta $\mathrm{P} * \mathrm{~V}$ ).

Results: Before lung lavage, all PSRs had the same fN $(0.41 \pm 0.03$ impulses*s-1/L*kPa*10-4; \pm S.E.M. $)$, which decreased after lung lavage $(0.17 \pm 0.07$ impulses*s$\left.1 / \mathrm{L} * \mathrm{kPa}^{*} 10-4\right)$ and increased after surfactant instillation to the pre-lavage level $(0.45 \pm 0.12$ impulses*s$1 / \mathrm{L} * \mathrm{kPa}^{*} 10-4 ; \mathrm{p}<0.01$, respectively). A high inverse relationship between $\mathrm{fN}$ and inspiratory time was observed for HT PSRs only, and was lost after lung lavage and not re-established after surfactant instillation. Conclusions: All PSRs responded similarly to the energy storage of the lung in all lung conditions studied, where more peripherally situated receptors (HT) were sensitive to the rate of accumulated energy in normal lungs only. Thus the lung protective and regulatory function of PSRs might be related to the energy storage of the lung.

\section{PREVENTION OF ATOPIC DERMATITIS IN MICE BY ORAL SUPPLEMENTATION WITH} LACTOBACILLUS RHAMNOSUS CGMCC1.3724

A Tanaka $^{1}, \mathrm{~J}_{\text {Benyacoub }}{ }^{2}$, S Pecquet $^{2}$, S Blum $^{2}$, A Mercenier $^{2}, \mathrm{H}_{\text {Matsuda }}{ }^{1}$

${ }^{1}$ Laboratory of Veterinary Molecular Pathology and Therapeutics, Division of Animal Life Science, Institute of Symbiotic Science and Technology, Tokyo, Japan ${ }^{2}$ Department of Nutrition and Health, Nestle Research Center, Nestec Ltd., Lausanne, Switzerland

Background and Aims: Epidemiological studies have pointed to a role of the intestinal microbiota in atopic diseases in infants. Increasing evidences suggest that intake of some probiotic bacteria reduces risk of atopic dermatitis (AD) in children. The NC/Nga mouse model of $\mathrm{AD}$ has an environmental etiology, which probably includes microbial stimuli. Thus representing an appropriate model for testing potential efficacy of dietary interventions. The aim of this study was to evaluate the effect of a probiotic strain Lactobacillus rhamnosus CGMCC1.3724 on the development of $\mathrm{AD}$ in $\mathrm{NC} / \mathrm{Nga}$ mice and document the potential underlying mechanism.

Methods: Pregnant NC/Nga mice received either sterile drinking water or water containing probiotics one week before delivery and this treatment was maintained until weaning. Weaning pups $(\mathrm{n}=10$ per group) received water containing or not probiotics. Pups were transferred and kept into non-air controlled environment until 12 weeks of age. Clinical skin severity scores, scratching behavior, plasma concentrations of total $\mathrm{IgE}$, and IFN-g and IL-10 mRNA expression levels in dorsal skin biopsies were examined in the two groups of pups. 
Results: Oral administration of $L$. rhamnosus CGMCC1.3724 was able to prevent the development of high AD clinical scores. The scratching frequency was also reduced. Plasma IgE levels were significantly lower in the probiotic-supplemented group compared to controls. Skin's expression levels of IFN-g in probioticsupplemented mice were much higher than those of control mice. IL-10 levels remained unchanged between the two groups.

Conclusions: Continuous supplementation with $L$. rhamnosus CGMCC1.3724 prevented the development of $\mathrm{AD}$ in $\mathrm{NC} / \mathrm{Nga}$ mice when given to pregnant mothers and pups.

\section{EXCLUSIVE BREAST FEEDING RISES AFTER DISCHARGE FROM MATERNITY IN GREECE}

C Bakoula ${ }^{1}$, A Veltsista ${ }^{1}$, G Kavadias ${ }^{1}$, A Fretzayas ${ }^{2}$, A Prezerakou $^{3}$, P Nicolaidou ${ }^{2}$

${ }^{1}$ First Department of Paediatrics, Aghia Sofia Children's Hospital, ${ }^{2}$ Third Department of Paediatrics, Attikon University General Hospital, ${ }^{3}$ Second Department of Paediatrics, P. \& A. Kyriakou Children's Hospital, University of Athens, Greece

Background and Aims: To monitor the rates of exclusive breast feeding in Greek maternities and four weeks after discharge, and to determine whether or not supplementation in the hospital may alter to exclusive breastfeeding once discharged.

Methods: We questioned 4310 Greek mothers from seven representative hospitals on the fourth day in maternity and postpartum. Odds ratios were calculated to estimate the effects of health system, sociodemographic, psychosocial and environmental factors on the initiation of exclusive breast feeding and on the possible change from partial and formula feeding in the hospital to exclusive breast feeding at home, one month after discharge.

Results: In maternities, the breast-feeding initiation rate was $85 \%$, whereas the rate of exclusive breast feeding was only $23 \%$. The initiation of exclusive breast feeding was significantly positively associated with delivery at a hospital with Baby-Friendly characteristics, early infantto-breast contact, rooming-in, vaginal delivery, young maternal age, living in a less crowded home, high education of paternal grandmother, previous experience of breast feeding and delivery in autumn-winter. One month postpartum the rate of breast feeding declined slightly to $79 \%$, whereas exclusive breast feeding rose to $61 \%$. The strongest determinants for the above rise of exclusive breastfeeding were lack of rooming-in and previous breastfeeding experience. Women with no experience were less likely to change to exclusive breast feeding compared to primiparae.

Conclusions: The re-establishment of exclusive breast feeding after hospital discharge suggests that hospital practices have a reversible impact as women are capable of overcoming a bad start in hospital.
EARLY NUTRITION IS ASSOCIATED WITH GROWTH AND PULMONARY FUNCTION AT 1 YEAR IN PREMATURE INFANTS WITH BRONCHOPULMONARY DYSPLASIA

K Husseini ${ }^{1}$, S Isambert ${ }^{1}, \mathrm{C}$ Huon $^{1}$, M Mokhtari ${ }^{1}$, M Chaussain $^{2}$, C Dupont ${ }^{1,3}$, A Lapillonne ${ }^{1,3}$

${ }^{1}$ Department of Neonatology, Saint Vincent de Paul Hospital, Paris, France, ${ }^{2}$ Department of Respiratory Physiology, Saint Vincent de Paul Hospital, Paris, France, ${ }^{3}$ Rene Descartes Paris 5 University, Paris, France

Background and Aims: The aim of the study is to determine if nutrition during the first month of life is significantly associated with growth and/or pulmonary function at 1 year of infants with bronchopulmonary dysplasia (BPD).

Methods: Growth and pulmonary function at 1 year were prospectively assessed for 27 preterm infants (mean GA $\pm \mathrm{SD}=27.6 \pm 1.9 \mathrm{wks}$; birth weight $=$ $851 \pm 174 \mathrm{~g}$ ) with BPD (i.e., O2 requirement for more than 28 days). Growth z-scores were calculated by using the EuroGrowth Project growth curves. Functional residual capacity (FRC) was assessed $(n=27)$ with the nitrogen washout method and the partial forced expiratory flow (Vmax FRC) $(n=23)$ with the jacket method. Total energy and protein intakes during the first 5 weeks of life were determined from the medical chart. Results: Height and weight $\mathrm{z}$-score at 1 year were significantly correlated with the first 2 weeks cumulative protein intake $(\mathrm{r}=0.485, \mathrm{p}=0.016)$ and 5 weeks energy intake $(\mathrm{r}=0.436, \mathrm{p}=0.033)$, respectively. FRC expressed as percent of predicted value, but not Vmax FRC, was significantly correlated with the first 2 $(\mathrm{r}=0.447, \mathrm{p}=0.029)$ and 5 weeks protein intake $(\mathrm{r}=0.445$, $\mathrm{p}=0.029$ ). By using a stepwise regression model, which included gender, birth weight z-score, postnatal age, and in-utero growth, the first 2 and 5 weeks protein intake, respectively, were the strongest predictors for height zscore and FRC at 1 year.

Conclusions: In preterm infants with BPD, early protein intake is significantly associated with catch-up growth in length and pulmonary function at 1 year of age.

\section{THE INFLUENCE OF DIFFERENT VENTILATORY MODES ON CALORIMETRY USING A NEW COMPACT MODULAR METABOLIC MONITOR IN CRITICALLY ILL CHILDREN}

G Briassoulis, E Mihailoudi, E Vasilaki, A M Spanaki

Paediatric Intensive Care Unit, University Hospital of Heraklion, Crete, Greece

Background and Aims: To assess the influence of different ventilatory modes on $\mathrm{VCO} 2, \mathrm{VO} 2$, and respiratory quotient (RQ) using a new compact modular metabolic monitor (M-COVX) and its impact on measured resting energy expenditure (MEE).

Methods: The REE was measured at three different modes of ventilation (PRVC, SIMV, BIVENT) and compared to equations commonly used to predict resting 
energy expenditure (REE) in mechanically ventilated children.

Results: 103 pulmonary gas exchange measurements were recorded using the M-COVX monitor at the three ventilatory modes, sequentially. The patients were sedated and/or paralyzed and in a stable condition. Each measurement consisted of five consecutive 1-min readings of $\mathrm{VO} 2$ and $\mathrm{VCO} 2$. MEE did not differ significantly between PRVC $(879 \pm 21 \mathrm{kcal} / \mathrm{d})$, SIMV $(897 \pm 21 \mathrm{kcal} / \mathrm{d}), \quad$ BIVENT $(945 \pm 26 \mathrm{kcal} / \mathrm{d})$. RQ $(.82 \pm .01)$. and VCO2 $(123 \pm 4 \mathrm{ml} / \mathrm{min})$ were higher in the BIVENT mode when compared to PRVC $(.78 \pm .01$, $\mathrm{p}=.002$ and $102 \pm 2 \mathrm{ml} / \mathrm{min}, \mathrm{p}<.0001$, respectively). All three modes displayed good agreement, and only energy intake and temperature were independently associated with MEE $(p<.001)$. When paired differences between equations and calorimetry were tested, CaldwellKennedy equation showed the lowest mean MEE-REE difference $(-65 \pm 13 \mathrm{kcal} / \mathrm{d}, \mathrm{p}<.0001)$ and Seashore and Schofield equations exhibited the highest $95 \%$ Confidence Interval of the Difference (-473.7 to -421 $\mathrm{kcal} / \mathrm{d}$ and -307 to $-226 \mathrm{kcal} / \mathrm{d}$ for upper and lower CI, respectively, $\mathrm{p}<.0001)$.

Conclusions: The M-COVX is not influenced by the different modes of ventilation in clinically stable, adequately sedated critically ill children. None of the commonly used equations is suitable to predict REE in various modes of mechanical ventilation.

\section{MATERNAL AND PERINATAL FACTORS AFFECTING BREAST FEEDING INITIATION IN CRETE, GREECE}

\author{
F Ladomenou $^{1}$, A G Kafatos ${ }^{2}$, E Galanakis ${ }^{1}$ \\ ${ }^{I}$ Department of Paediatrics, University of Crete, \\ Greece, ${ }^{2}$ Department of Social Medicine, University of \\ Crete, Greece
}

Background and Aims: Understanding the factors that promote or prevent from breast feeding initiation in a given society may substantially contribute to better breastfeeding rates. The aim of this study was to assess breast feeding rates in Crete, Greece and to identify maternal and perinatal factors influencing the maternal decision to breastfeed or not breastfeed.

Methods: A prospective cohort study using both a questionnaire and follow-up telephone interviews. Setting: Public and private maternity wards of Heraklion, Crete, Greece. A sample of 1,049 mothers in early parturition was interviewed during their stay in the maternity ward. Investigated variables included demographics, family size, past breast feeding experience, social support, tobacco use and presence of complications during pregnancy and labor.

Results: Of the 1049 participants, 942 (89.7\%) intended to breastfeed their infants. Of these 942 mothers, 619 $(65.7 \%)$ were still breastfeeding at month 1 post-partum but only $161(17.1 \%)$ were still breastfeeding at month 6 post-partum. Maternal factors negatively affecting breast feeding initiation were indigenous vs. immigrant mothers $(p<0.05)$, advanced age $(p<0.05)$, caesarean section $(p<0.05)$, lack of breastfeeding experience $(p<0.0001)$ and tobacco use $(p<0.001)$. Infant-related factors negatively affecting breast feeding initiation were prematurity $(\mathrm{p}<0.005)$, admission to neonatal ward $(\mathrm{p}<0.0001)$ and male gender $(\mathrm{p}<=0.05)$.

Conclusions: Our results suggest that several of the factors negatively affecting breastfeeding initiation rates are liable to early intervention and prevention. Health professionals involved in perinatal medicine share a considerable part of responsibility for the low breastfeeding rates.

\section{FEEDING AUGMENTATION IMPACT ON LEPTIN SECRETION IN PRETERM INFANTS}

M D Dasopoulou ${ }^{1}, \mathrm{C}$ G Costalos ${ }^{1}$, E M Constantellou ${ }^{2}$, F T Siannis ${ }^{3}$

${ }^{I}$ Neonatal Department, 'Alexandra' Hospital, Athens, Greece, ${ }^{2}$ Endocrinology Department, 'Agios

Panteleimon', Nikeas Hospital, Athens, Greece,

${ }^{3}$ Biostatistics Unit, Cambridge, UK

Background and Aims: Leptin is a hormone excreting from adipose tissue and bound to hypothalamus. Leptin is implicated in appetite control, temperature upregulation and energy expenditure. Our study's aim was to determine the impact of feeding on leptin secretion in a population of preterm neonates.

Methods: Leptin study was a prospective one, enrolling 87 preterms born at the hospital. Ethics Committee Approval and parental informed consent were obtained. Eligible neonates were all preterms $(<37$ weeks of gestation, according to Dubowitz criteria). All neonates were fed within 4-6 hours after birth the same volume according to weight and day. For example on day 1 they received $30 \mathrm{ml} / \mathrm{kg}$ with an increment of $30 \mathrm{ml} / \mathrm{kg} / \mathrm{per}$ day till maximum $250 \mathrm{ml} / \mathrm{kg} /$ per day. Neonates were fed the same formula and breast fed infants were excluded as well as those with respiratory distress, birth injury, vomiting, diarrhea, necrotising enterocolitis. Blood sampling during the first 6 hours of life and repeated sampling on day 26 of feeding for serial leptin measurements.

Results: 87 neonates mean gestational age 34.13 (SD: 1.04), mean birth weight 2047.73 gr (SD: 217.15) were enrolled in the study. 87 were sampled on day 1 of life and 76 on day 16. Mean leptin value on day 1 was 2.44 pmol (SD: 5.845), and on day 16 mean leptin value was 1.477 pmol (SD: 1.240). T-test for paired samples gave statistic difference in $95 \%$ confidence interval with (2tail significance: 0.029 ) on leptin value between day 1 and 16.

Conclusions: Leptin is greatly influenced by food augmentation in preterm infants.

\section{ANTIOXIDANT AND PEROXIDE CONTENT OF MILK FROM MOTHERS WHO DELIVERED AT TERM AND PRETERM NEWBORN INFANTS}

D Turoli, M Condo', R Zanini, R Bellu'

NICU, Ospedale 'Manzoni', Lecco, Italy

Background and Aims: Evidence indicates that source of oxidants can be a challenge to the immature antioxidant defenses of the newborn infant and that human milk contains many antioxidant considered to 
prevent diseases mediated by oxygen free radicals in very low birth weight (VLBW) infants. In previous studies we investigated human and formula milk antioxidant and peroxide load. In the present study we investigated antioxidant and peroxide load of preterm vs term human milk.

Methods: We measured with spectrophotometric methods the lipid peroxides (LPOx), conjugated diene (CD) and T-BARS (pro-oxidants) and the total antioxidant activity (TAA) content of 26 milk samples, 10 from mothers who delivered at term newborns and 16 from mothers who delivered preterm (24-32 weeks gestational age) newborns. Mean values and standard deviations were calculated and analyzed by Student's t-test.

Results: Results are shown in table. No significant differences were noted between term and preterm human milk.

Conclusions: Milk from mothers who delivered term or preterm did not show any significant differences as regard to total antioxidant content and peroxide load, thus indicating similar resistance to oxidative stress. These in vivo data agree with in vitro studies (Friel JK, Pediatr Res 2002).

\begin{tabular}{|c|c|c|c|c|c|c|}
\hline \multirow{4}{*}{$\begin{array}{l}\text { LPOX ( } \mu \mathrm{mol} / \mathrm{L}) \\
\mathrm{CD}(\mu \mathrm{mol} / \mathrm{L})\end{array}$} & \multicolumn{2}{|c|}{ Term milk } & \multicolumn{2}{|c|}{$\begin{array}{c}\text { Preterm } \\
\text { milk }\end{array}$} & \multirow[b]{2}{*}{$t$} & \multirow[b]{2}{*}{$\mathbf{P}$} \\
\hline & Mean & SD & Mean & SD & & \\
\hline & 6.15 & 1.75 & 5.40 & 1.90 & 1.00 & 0.32 \\
\hline & 24.33 & 9.62 & 17.09 & 11.77 & 1.73 & 0.11 \\
\hline TBARS & 3.17 & 0.77 & 3.55 & 0.58 & 143 & ח \\
\hline TAA ( $\mu \mathrm{mol} / \mathrm{L})$ & 3.37 & 0.58 & 3.66 & 1.15 & 0.73 & 0.46 \\
\hline
\end{tabular}

\section{COMPARISON OF AXILLARY, ABDOMINAL AND SKIN SHIN TEMPERATURE AS WELL AS SPECTROSCOPIC CAPILLARY REACTIVITY (CR) MEASUREMENTS FOR EARLY DETECTION OF SEPSIS IN EXTREMELY LOW BIRTH WEIGHT INFANTS (ELBW)}

\author{
J R Fernandez Alvarez ${ }^{1}$, F E Lawson ${ }^{1}$, P Amess ${ }^{1}$, \\ H Jungmann ${ }^{2}$, H Rabe ${ }^{1}$ \\ ${ }^{1}$ Trevor Mann Baby Unit, Royal Sussex County \\ Hospital, Brighton, East Sussex, UK, ${ }^{2}$ Institute for \\ Cancer Research, University of Bochum, Nordrhein- \\ Westfalen, Germany
}

Background and Aims: Microcirculation is impaired in sepsis. The non-invasive spectroscopic device Mediscan-2000 detects changes in CR earlier than clinical capillary refill time in sepsis. It measures total haemoglobin in capillaries using a probe with a short pathlength. In term newborns measurement of central/peripheral temperature gradient helps recognising sepsis. The axilla is useful for core temperature monitoring in afebrile neonates. Aim was to assess the value of axillary and central/peripheral temperature measurements in ELBW newborns in comparison to $\mathrm{CR}$ measured by Mediscan-2000 in sepsis.

Methods: Axillary, abdominal skin and shin temperatures were recorded when bloods taken for sepsis. Within $2 \mathrm{~h}$ spectroscopic measurement of $\mathrm{CR}$ was performed at the forearm using a defined pressure stimulation. Time between end of pressure stimulation and resulting peak of $\mathrm{CR}$ was calculated. Infants served as their own controls without sepsis. Results are given as median.

Results: 10 septic ELBW newborns (27 weeks, 990g) were recruited. CR increased from $4.5 \mathrm{~s}$ without sepsis to 6s during sepsis $(p=0.017)$. No significant change in axillary, skin or shin temperatures and skin-shin gradient. Axillary-shin or skin-shin gradients were equally significantly different $(\mathrm{p}=0.005)$ with and without sepsis. CR correlated significantly with each other $(\mathrm{r}=0.67, \mathrm{p}=0.035)$ during both periods. No significant correlations of the temperatures and/or gradients except for axillary and abdominal skin temperature during sepsis $(\mathrm{r}=0.85, \mathrm{p}=0.002)$.

Conclusions: ELBW newborns have central/peripheral temperature differences that do not vary with sepsis despite changes in microcirculation suggesting an incomplete temperature control mechanism. Axillary and abdominal skin temperature and skin-shin gradient are not helpful detecting sepsis.

\section{DO PRENATAL GROWTH AND ILLNESS INFLUENCE POSTNATAL UNDERNUTRITION?}

I Dorronsoro Martin ${ }^{1}$, M Saenz de Pipaon Marcos ${ }^{1}$, J Perez Rodriguez ${ }^{1}, \mathrm{R}$ Madero Jarabo ${ }^{2}$, M Biarge Martinez ${ }^{1}$, J Quero Jimenez ${ }^{1}$

${ }^{1}$ Department of Neonatology, La Paz Hospital, Madrid, Spain, ${ }^{2}$ Department of Research, La Paz Hospital, Madrid, Spain

Background and Aims: Describe postnatal growth of preterm very low birth weight infants (VLBWI). Evaluate the impact of prenatal growth, maturity, acute and chronic illness in postnatal growth.

Methods: Retrospective analysis of VLBWI with gestational age (GA) under 33 weeks born at a single institution between 2002 and 2005. Growth was evaluated at birth, 28 days of postnatal life and 36 weeks of postconceptional age. Acute illness was defined as the need of mechanical ventilation for more than three days (MV), high frequency ventilation (HFV) or late sepsis. Chronic illness was defined as bronchopulmonary dysplasia (BPD). Prenatal growth was evaluated by $\mathrm{Z}$ score at birth. We defined small for gestational age (SGA) as birth weight (BW) under 10 percentil (Alexander curves). Statistical analysis was performed using SPSS.

Results: 472 newborns were included. BW was $1072,6 \pm 252$ grams and GA $28,7 \pm 2,2$ weeks. $18,8 \%$ were SGA. $61,5 \%$ needed MV; $21 \%$ HFV; $27,2 \%$ had late sepsis and $17,2 \%$ BPD. oAll infants had lower $Z$ scores at 36 weeks than at birth. Difference of $Z$ score between birth and 36 weeks was higher in babies under 29 GA. Acute, chronic illness and SGA favours postnatal undernutrition (PU). In multivariant logistic analysis, birth weight $Z$ score protected against $\mathrm{PU}$ (OR 0, 16); lower GA $(\mathrm{OR}=4,3)$, need of HFV $(\mathrm{OR}=3,1)$ and $\mathrm{BPD}$ $(\mathrm{OR}=4,3)$ increased the risk of $\mathrm{PU}$.

Conclusions: oPU is universal in VLBWI. oIn utero growth protect against PU. Inmaturity, acute and chronic illness are risk factors for PU. oAll infants should be monitored specially those with higher risk. 
THE EFFECT OF BODY MASS INDEX ON THYROID FUNCTION IN ADOLESCENT GIRLS

M H Eftekhari ${ }^{1}$, F Shidfar $^{2}$

${ }^{l}$ Dept of Nutrition, Shiraz University of Medical Sciences, School of Health, ${ }^{2}$ Dept of Nutrition, Iran University of Medical Sciences, School of Health, Tehran, Iran

Background and Aims: Little is known about thyroid function in obese adolescents. A possible relationship between thyroid function and adipose tissue metabolism in human has been suggested. To examine the relationship between thyroid hormones and body mass index. Methods: A cross-sectional study was carried out in Lar province and its' vicinity in south of Iran. By stepwise random sampling from all public girls' high schools, 227 subjects (aged 12 - 21) were selected. Serum samples were collected and assayed for Triiodothyronine (T3), thyroxine (T4), thyroid stimulating hormone (TSH), reverse triiodothyronine (rT3), free thyroxine (FT4), free triiodothyronine (FT3). Weight and height were measured and body mass index (BMI) were calculated (weight / height ${ }^{2}$ ). Subgroup analysis was done according to body mass index. Results: TSH and rT3 were correlated with BMI $(\mathrm{r}=0.66, \mathrm{P}=0.001$ and $\mathrm{r}=0.65, \mathrm{P}=0.001$ respectively). Adolescent girls with BMI $>25 \mathrm{~kg} / \mathrm{m}^{2}$ showed higher serum TSH and rT3 than subjects with BMI $<25 \mathrm{~kg} / \mathrm{m}^{2}(\mathrm{P}=0.001)$. Conclusions: Our data showed that, although thyroid function was normal in the studied subjects, TSH and rT3 were positively correlated to BMI. TSH and rT3 could present a marker of altered energy balance in overweight and obese adolescent girls.

\section{CIRCULATING PEPTIDE YY (PYY) 3-36 CONCENTRATIONS IN PRETERM AND FULLTERM INFANTS}

T Siahanidou ${ }^{1}$, H Mandyla ${ }^{1}$, H Militsi ${ }^{2}$, I Papassotiriou ${ }^{2}, \mathrm{G}_{\text {Chrousos }}{ }^{1}$

${ }^{1}$ First Dept of Pediatrics, Athens University Medical School, ${ }^{2}$ Dept of Clinical Biochemistry, "Aghia Sophia" Children's Hospital, Athens, Greece

Background and Aims: The gut hormone peptide YY (PYY) 3-36, one of the two main isoforms of PYY, has been suggested to posses an antiorexigenic action in animals and human adults. However, its circulating levels and function have not been studied in neonates. The aim of this study was to determine the circulating concentrations of PYY3-36 in preterm and full term infants and to examine their correlations with the infants' anthropometric characteristics, food intake, growth rates and the circulating levels of hormones involved in the regulation of metabolism, total PYY, ghrelin, leptin, insulin and adiponectin.

Methods: Serum levels of the above mentioned hormones were measured by RIA in 62 preterm infants (gestational age $32.0 \pm 2.1$ weeks, postnatal age $40.9 \pm 14.8$ days) and 15 full term infants of a comparable postnatal age. All the infants were formulafed on demand.
Results: PYY3-36 concentrations were significantly higher in preterm $(543.7 \pm 157.6 \mathrm{ng} / \mathrm{L})$ than full term infants $(350.9 \pm 114.1 \mathrm{ng} / \mathrm{L}, \mathrm{p}<0.001)$. PYY3-36 accounted for $48 \%$ and $42 \%$ of total PYY basal plasma immunoreactivity in preterm and full term infants, respectively, and correlated negatively with the infants' BMI and positively with serum ghrelin concentrations in preterm and full term infants. There was no significant correlation between PYY3-36 and caloric intake, weight gain or any of the other hormones studied.

Conclusions: PYY3-36 accounts for a high percentage of total PYY immunoreactivity in neonates, similarly to adults. Along with the orexigenic peptide ghrelin it seems to play a central role in the regulation of metabolism; however, its specific functions and physiologic significance in neonates remain to be elucidated.

\section{A BRIEF DELAY IN CLAMPING THE UMBILICAL CORD AS FIRST STEP IN RESUSCITATING PRETERM INFANTS - A META-ANALYSIS}

\author{
H Rabe ${ }^{1}$, G Reynolds ${ }^{2}$, J Diaz-Rossello ${ }^{3}$ \\ ${ }^{1}$ Department of Neonatology, Brighton and Sussex \\ University Hospitals, Brighton, UK, ${ }^{2}$ Department of \\ Women's and Child Health, The Canberra Hospital, \\ Canberra, Australia, ${ }^{3}$ Latin American Centre for \\ Perinatology, Hospital de Clinicas, Montevideo, \\ Uruguay
}

Background and Aims: The optimal timing of clamping the umbilical cord in preterm infants at birth is the subject of continuing debate. New studies were published since the last systematic review on the effects of a brief delay in cord clamping time on the outcome of babies born prematurely was published and a new review is warranted.

Methods: Retrospective meta-analysis of randomised trials in preterm infants. Data collected from published studies systematically searched and analysed according to the rules and regulations of the Cochrane collaboration. Inclusion: All infants born below 37 weeks gestation and enrolled into randomised trials of delayed cord clamping versus immediate cord clamping after birth.

Results: Ten studies describing a total of 454 preterm infants were identified which met the inclusion and assessment criteria. Major benefits of the intervention were higher circulating blood volume during the first 24 hours of life, less need for blood transfusions $(p=$ $0.004)$ and less incidence of intraventricular haemorrhage $(\mathrm{p}=0.002)$.

Conclusions: The procedure of a delayed cord clamping time of at least 30 seconds is safe to use and does not compromise the preterm infant in the initial post partum adaptation phase. It should be introduced as a routine procedure. 
KINETICS OF HEMATOPOIETIC LINEAGE

RECONSTITUTION IN HIGH-LEVEL CHIMERAS OBTAINED BY PRENATAL BONE MARROW TRANSPLANTATION

J C Chen ${ }^{1}$, P Y Chang ${ }^{1}$, M O Muench ${ }^{2}$

${ }^{I}$ Department of Surgery, Chang Gung Children's

Hospital, Taoyuan, Taiwan, ${ }^{2}$ Department of Laboratory Medicine, University of California, San Francisco, San

Francisco, CA, USA

Background and Aims: This study aimed at analyzing host and donor-derived hematopoiesis of mixed chimeras, created by prenatal bone marrow transplantation (PBMT), without the influence of cytoablation and immunosuppression.

Methods: Gestational day $14 \mathrm{FVB} / \mathrm{N}$ (H-2Kq) fetuses were transplanted with $\mathrm{C} 57 \mathrm{BL} / 6(\mathrm{H}-2 \mathrm{~Kb})$ marrow cells at a dose of 5-10x106. Recipients with chimerism levels $>10 \%$, at 1 month old, were examined monthly for donor-derived CD3, CD4, CD8, CD45R, CD11b and Gr1 cells.

Results: Ten high-level chimeras were enrolled (Fig a). The levels of different lineage antigens didn't fluctuate over 6 months except for significantly lower levels of $\mathrm{CD} 8+(\mathrm{P}=0.004)$ and $\mathrm{CD} 45 \mathrm{R}+$ cells $(\mathrm{P}=0.002)$ at month 1 (Fig b). Apart from CD45R ( $\mathrm{P}=0.003)$, all lineage antigens displayed steady fractions of donor-derived cells within 6 months (Fig c). In donor-cell population, $\mathrm{CD} 11 \mathrm{~b}+(\mathrm{P}=0.001)$ and $\mathrm{Gr} 1+(\mathrm{P}<0.001)$ cells showed significantly higher expression, reciprocally with lower expression of $\mathrm{CD} 3+(\mathrm{P}=0.002)$ and $\mathrm{CD} 8+(\mathrm{P}<0.001)$ cells, at month 1 than at later time-points (Fig d).

Conclusions: Successful engraftment by PBMT started as higher expression of donor myeloid cells and disproportionate domination of donor B-cells. Overall, lymphoid reconstitution was superior to myeloid reconstitution, mirroring the clinical success of PBMT only in diseases with lymphoid but not myeloid defects.

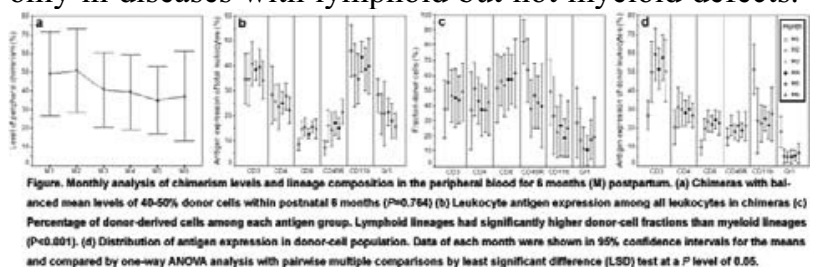

\section{PRE-NATAL RETROPERITONEAL MASS: HIGHLY DIFERENTIATED TERATOMA OR FETUS IN FETU?}

H Santos $^{1}$, H B Tavares ${ }^{1}$, I Carvalho ${ }^{1}$, A Furtado ${ }^{2}$, A João ${ }^{3}$, L Ferraz $^{3}$, G Ferreira ${ }^{1}$, F Valente ${ }^{4}$, P Sousa $^{5}$

${ }^{I}$ Department of Pediatrics, Centro Hospitalar de Vila Nova de Gaia, Portugal, ${ }^{2}$ Department of Pathologic Anatomy, Centro Hospitalar de Vila Nova de Gaia, Portugal, ${ }^{3}$ Neonatology Unit, Department of Pediatrics, Centro Hospitalar de Vila Nova de Gaia, Portugal, ${ }^{4}$ Department of Gynaecology and Obstetrics, Centro Hospitalar de Vila Nova de Gaia, Portugal

${ }^{5}$ Department of Paediatric Surgery, Centro Hospitalar de Vila Nova de Gaia, Portugal

Background and Aims: Several cases reports in the literature brought to discussion the distinction criteria between fetus in fetu and teratoma. It has been suggested that considerable overlap exist between the two conditions, making sometimes their distinction a complex task.

Methods: We present a clinical case of a boy, born at 34 weeks, evaluated for a capsulated abdominal mass with left uretero-hydronephrosis, diagnosed at the 31 st week.

Results: He was only son of young, healthy, nonconsanguineous parents. Pregnancy complicated with gestational diabetes. Birth by caesarean section, APGAR 5/8/8, adequate anthropometry for gestational age. A post-natal CT scan showed an expansive retroperitoneal lesion, from kidneys to pelvis, sacro and coccyx, with solid, cystic and fatty areas; aspirative biopsy was inconclusive; he underwent surgery on day 9 with partial resection of the lesion; it revealed a $33 \mathrm{~g}$, $6 \times 4 \times 4 \mathrm{~cm}$ fragment with a broken capsule, cystic and solid components, with mature neuro-epithelial (encephalic, choroidal plexus, ependyma), adipose and foetal epithelial tissues; no immature tissue found. A new procedure to remove the remaining, 8 months later, resulted in a 133g, 9x8 $55 \mathrm{~cm}$ mass, partially capsulated, yellowish, with small cavities fulfilled with brown material; mature tissues, with organized components of nervous system, respiratory and digestive apparatus, liver pancreas, thymus, nervous ganglia, skin and adnexa, musculoskeletal and brown adipose tissues; cartilage and several focus of calcification.

Conclusions: This case presents features of both teratoma and fetus in fetu. The assumption of one of the diagnosis has prognostic implications, with resulting differences in clinical follow up and expectations about the child's future health.

\section{ANTITHROMBIN LOSS IN CHILDREN WITH CHYLOTHORAX}

V Bernet ${ }^{1}, \mathrm{~K}_{\text {Waldvogel }}{ }^{1}$, V Cannizzaro ${ }^{1}, \mathrm{M} \mathrm{Albisetti}^{2}$

${ }^{I}$ Department of Pediatric and Neonatal Intensive Care, Childrens University Hospital Zurich, Switzerland,

${ }^{2}$ Department of Hematology, Childrens University Hospital Zurich, Switzerland

Background and Aims: Chylothorax is an accumulation of lymphatic fluid in the pleural space and commonly develops after cardio-thoracic surgery or secondary to thrombosis and/or high pressure in the superior vena cava. Antithrombin (AT) is the most important natural inhibitor of the coagulation cascade. While inherited deficiency of AT is rare in the general population, acquired deficiency is frequently observed in patients with liver disease, nephrotic syndrome, and after major surgery, particularly cardiac surgery. The study determines whether increased AT loss is present in newborn and children with chylothorax after cardiac surgery.

Methods: The plasma and pleural effusion samples of children with chylothorax and with non-chylous pleural effusion were assayed for AT activity. Chylous samples were collected on day 2 to 4 postoperatively when chylothorax diagnosis was confirmed.

Results: Ten children with chylothorax and five children with non-chylous pleural effusion were 
investigated. Mean antithrombin activity was statistically significant increased in chylous samples $(32.2 \pm 11.4 \%)$ compared to non-chylous samples (14.4 \pm 13.9$)$, and significantly decreased in plasma of children with chylothorax $(44.6 \pm 15.4 \%)$ compared to children with non-chylous pleural effusion $(69.9 \pm 22.4 \%)$. Seven of ten children with chylous and none of the children without chylous developed thrombosis $(\mathrm{p}<0.007)$.

Conclusions: Increased loss of AT is present in children with chylothorax, potentially predisposing these children to an increased risk of thrombosis. Thus, repeated AT substitution on a regular base should be considered in critically ill children with chylothorax.

\section{WAY OF DELIVERY AS WELL AS MODE OF FEEDING SEEM TO INFLUENCE THE FECAL LEVEL OF CALPROTECTIN IN NEONATES}

S J Moltu ${ }^{1}$, M Eggesboe ${ }^{2}$, B Kvenshagen ${ }^{3}$, M K Fagerhol $^{1}$, J Rugtveit ${ }^{1}$

${ }^{1}$ Ullevål University Hospital, Oslo, Norway, ${ }^{2}$ Institute of Public Health, Oslo, Norway, ${ }^{3}$ Central Hospital Østfold, Fredrikstad, Norway

Background and Aims: To study the fecal level of calprotectin in relation to mode of delivery and feeding, both factors reported to influence type of bacterial gut colonization and thus potentially also the granulocyte reponse.

Methods: We measured fecal calprotectin values in spot samples of faeces from 244 infants at age 4, 10, 30 and 120 days. Wilcoxon signed rank test and Mann-Whitney $\mathrm{U}$ Test were used for comparisons.

Results: Calprotectin levels increased from day 4 to day 30 in all infants $(p=0.007)$, followed by a significant decrease by day $120(p<0.001)$. The formula fed infants had lower calprotectin levels at day 30 than those breastfed $(\mathrm{p}=0.01)$, and the initial rise in calprotectin level from day 4-10 was lower in the infants delivered by $\mathrm{C}$-section compared to those delivered vaginally $(p=0.009)$, also when stratified according to mode of feeding $(\mathrm{p}=0.022)$. A stratified analysis among the infants delivered by $\mathrm{C}$-section demonstrated a lower fecal calprotectin average between day 10 and 30 in the formula versus breast fed group $(p=0.013)$. An effect of formula feeding in the infants delivered vaginally (7/160) could not be calculated because of too small numbers included so far.

Conclusions: The initial increase in calprotectin level has previously been reported and may reflect increased migration of neutrophile granulocytes into the gut lumen as an early immunologic adaption to the first bacterial colonization. The levels though seem influenced both by mode of delivery and feeding, possibly due to associated differences in the bacterial colonization of the gut and the resulting innate immune response.

\section{SUSTAINED, FUNCTIONAL AND MATURE CD8+ T CELL POPULATIONS AFTER VIRAL INFECTION IN INFANCY}

H Komatsu ${ }^{1}$, A Inui ${ }^{2}$, T Sogo ${ }^{2}$, T Fujisawa ${ }^{3}$
${ }^{I}$ Department of Paediatrics, Sakura Hospital, Toho University, Sakura, Chiba, Japan, ${ }^{2}$ Department of Paediatrics, Yokohama Sakae Kyosai Hospital, Yokohama, Kanagawa, Japan, ${ }^{3}$ Department of Paediatrics, Atami Hospital, International University of Health and Welfare, Atami, Shizuoka, Japan

Background and Aims: Cellular immunity plays a crucial role in the cytomegalovirus (CMV) infection, and substantial populations of CMV-specific $\mathrm{T}$ cells accumulate throughout life. However, although CMV infection occurs during childhood, little is know about the quality of $\mathrm{T}$ cell responses in pediatric populations.

Methods: We screened a large Japanese population (1036 individuals) and analysed the CD8+ $\mathrm{T}$ cell responses to $\mathrm{CMV}$, comparing these to responses in children, young and elderly adults. The frequencies, phenotype and function CD8+ $\mathrm{T}$ cells for two immunodominant epitopes from pp65 were measured cross-sectionally.

Results: We observed consistently high frequency and phenotypically 'mature' (CD27 low, CD28 low, CD45RA+) CMV-specific CD8+ $\mathrm{T}$ cell responses in children, including those studied in the first year of life. These CD8+ T cells retained functionality across all age groups, and showed evidence of memory 'inflation' at a population level through adult life.

Conclusions: CMV consistently elicits a very strong $\mathrm{CD} 8+\mathrm{T}$ cell response in infants and large pools of CMV specific $\mathrm{CD} 8+\mathrm{T}$ cells are maintained throughout childhood. The presence of CMV may considerably mould the CD8+ T cell compartment over time, but the relative frequencies of CMV-specific cells do not show evidence of a population-level increase during childhood and young adulthood. This contrasts with the marked expansion ('inflation') of such CD8+ T cells in older adults. The strong and sustained responses to CMV observed in children suggest that vaccine strategies aiming to elicit protective CD8 $+\mathrm{T}$ cell responses to major pathogens (such as $\mathrm{HIV}, \mathrm{HCV}$, malaria or CMV itself) might be successful in younger children.

\section{ANTI-ANNEXIN V ANTIBODIES IN WOMEN WITH SPONTANEOUS ABORTIONS AND PREMATURE BIRTHS}

K Malickova $^{1,2}$, R Svancarova ${ }^{1}, \mathrm{~K}$ Masova ${ }^{1}$, I Janatkova ${ }^{1}$

${ }^{1}$ Laboratory of Clinical Immunology and Allergology, Institute of Clinical Biochemistry and Laboratory Diagnostics, General Faculty Hospital and 1st Faculty of Medicine, Charles University, Prague, Czech Republic, ${ }^{2}$ Institute of Immunology and Microbiology, General Faculty Hospital and 1st Faculty of Medicine, Charles University, Prague, Czech Republic

Background and Aims: Annexin V is a phospholipidbinding glycoprotein with anticoagulant activity. It is expressed by trophoblast cells and its preferential binding substrate is phosphatidylserine. As the gamete is fertilized, the trophoblast expresses phosphatidylserine for binding to the endometrial surface. In the normal embryo, annexin $\mathrm{V}$ binds to phosphatidylserine as a 
protective anticoagulant mechanism. In women with recurrent fetal losses, placental annexin $\mathrm{V}$ is decreased. We have conducted a study on the prevalence of serum anti-annexin $\mathrm{V}$ antibodies in relation to pregnancy failures in women with and without the history of fetal losses and/or premature births.

Methods: We have screened serum samples of 13 women $(29.7+/-2.3$ years of age $)$ with no antecedent of thromboembolic or autoimmune diseases but manifest recurrent pregnancy losses and/or premature births; 14 serum samples of women (33.7 +/- 4.2 years) with sterility but without history of fetal losses; and serum samples of 14 women $(38.4+/-7.3$ years) with SLE and no coincidental history of reproductive failure. $\operatorname{IgG}$ and IgM anti-annexin $\mathrm{V}$, anti-cardiolipin, antiphosphatidylserine and anti-ethanolamine antibodies were determined in all serum samples, with no awareness of the outcome of pregnancies.

Results: Patients with pregnancy losses and/or premature births showed significantly increased levels of IgG anti-annexin $\mathrm{V}$ antibodies as compared to the patients without miscarriages/premature deliveries $(\mathrm{p}=0.005)$. Neither IgG/IgM anti-cardiolipin, beta-2glycoprotein-1, phosphatidylserine, phosphatidylethanolamine levels nor IgM anti-annexin levels were significantly different between the groups.

Conclusions: Anti-Annexin V antibodies were the only ones significantly associated with child losses. Acknowledgements: This work was supported by the Czech Ministry of Education Project MSM0021620807.

\section{TREATMENT OF NON-HODGKIN LYMPHOMA WITH BFM PROTOCOLS IN HUNGARY - EXPERIENCE OF FIFTEEN YEARS}

J Müller, Z Jakab, M Csóka, H Erlaky, T Constantin, A Ponyi, G T Kovács

\section{2nd Department of Pediatrics, Semmelweis University, Budapest, Hungary}

Background and Aims: In the last 15 years Hungarian children with non-Hodgkin lymphoma (NHL) were treated according to the Berlin-Frankfurt-Münster (BFM) German protocols. Aim of the present study was to summarize our experience with these protocols, to assess the survival rates and to compare the Hungarian data with the international results.

Methods: Between 1990 and 2004230 children, diagnosed with NHL were treated according to BFM protocols in Hungary. The male-to-female ratio was $2.59: 1$, the mean age at the time of diagnosis was 10 years and 1 month.

Results: Among the 230 children, $28(12 \%)$ had stage I disease, $65(28 \%)$ stage II, $92(40 \%)$ stage III and 45 (20\%) stage IV. Ninety-one children had non-B, 108 BNHL and 31 anaplastic large cell lymphoma (ALCL). Twenty-eight patients had relapse after a mean time of 13 months from the time of the initial diagnosis. In the over mentioned period, 16 children underwent autologous stem-cell transplantation. Nine patients with B-NHL got anti-CD-20 immunotherapy. Five year overall survival (OS) of our patients is $78 \% \pm 3 \%$, eventfree survival (EFS) is $75 \% \pm 3 \%$, the mean follow-up time of 6 years. The 5-year OS and EFS rates were not statistical different in the three histology groups (OS: $72 \% \pm 5 \%, 83 \% \pm 4 \%$ and $80 \% \pm 7 \%$ in non-B-, B-NHL and ALCL; EFS: $69 \% \pm 5 \%, 81 \% \pm 4 \%$ and $74 \% \pm 8 \%$ in non-B-, B-NHL and ALCL).

Conclusions: NHL has quite good prognosis among the malignant pediatric diseases. The Hungarian results are comparable with other international data. In the last five years the OS is about 10 percent better than it was before.

\section{RE-IMMUNIZATION STRATEGY IN CHILDREN RECOVERED FROM ACUTE LYMPHOBLASTIC LEUKAEMIA (ALL)}

\author{
I Stryjewska $^{1}$, M Golebiowska ${ }^{1}, \mathrm{~K}$ Zeman $^{2}$ \\ ${ }^{l}$ Copernicus Memorial Hospital, Children Vaccination \\ Centre, Lodz, Poland, ${ }^{2}$ Medical University, Lodz, \\ Poland
}

Background and Aims: It is still unclear what the best re-immunization strategy for children recovered from ALL is. Recently, the loss of humoral immunity to viral antigens has been described, suggesting the need for revaccinating patients. This study aimed to evaluate serological and immunological status in children recovered fom ALL.

Methods: The study involved 30 children aged 5 to 17 , after chemotherapy which ended from 12 months to 5 years before. Before the leukaemia was diagnosed all of them had been immunized according to Polish National Immunization Program. We analyzed levels of serum antibodies against 3 polio viruses, measles, mumps and rubella by enzyme immunoassays. IgG, IgA, IgM serum concentrations and some parameters of cell mediated immunity were determined. Non-immune children were given boosters and revaccination responses were studied.

Results: (1) Approximately $70 \%$ of investigated children did not have a protective level of antibodies against 3 polio viruses, $80 \%$ did not show protective level against measles despite immunization before ALL diagnosis. (2) Analysis of post re-immunization response showed high percentage of children with protective level of antibodies: for polio 1 and $2-100 \%$, polio $3-90 \%$, measles and mumps $87 \%$, rubella $100 \%$.

Conclusions: (1) No significant impairment of humoral and cell mediated immunity as a possible contraindication for the use of attenuated vaccine was found. (2) Our finding of loss in antibodies in children treated with chemotherapy suggests that reimmunization is necessary after the treatment is completed. Children recovered from ALL should be vaccinated according to individual immunization schedule based on the results of serological and immunological testing.

\section{ROUTINE SCREENING COAGULATION TESTS AT ADMISSION AND DEVELOPMENT OF HAEMORRHAGE IN HIGH-RISK NEWBORNS}

C De Felice ${ }^{1}$, A Del Vecchio ${ }^{2}$, M C Carbone ${ }^{1}$, E Cervo ${ }^{1}$, F Bagnoli ${ }^{1}$, G Latini ${ }^{2,3}$

${ }^{1}$ Neonatal Intensive Care Unit, Azienda Ospedaliera

Universitaria Senese, Siena, Italy, ${ }^{2}$ Division of 
Neonatology, Perrino Hospital, Brindisi, Italy, ${ }^{3}$ Clinical Physiology Institute, National Research Council of Italy (IFC-CNR), Lecce Section, Italy

Background and Aims: Consumption coagulopathy (CC) is characterized by the abnormally regulated activation of procoagulant pathways resulting in decreased levels of hemostatic components. Here, we tested the hypothesis of a subclinical CC status in highrisk infants admitted to the neonatal intensive care unit (NICU) who subsequently developed hemorrhage (H).

Methods: A total of 88 , consecutively admitted, newborns (gestation: $32.9 \pm 3.93$ wk; birthweight: $1927 \pm 835 \mathrm{~g}$ ), were screened for coagulation. The coagulation screening panel, performed $<1$ st hour after birth, included: prothrombin time (PT), International Normalized Ratio (INR), activated partial thromboplastin time (aPTT), fibrinogen, proteins $\mathrm{C}$ and $\mathrm{S}$, antithrombin III (AT3), D-dimers (D-D), and platelet (PLT) counts. Absolute values were converted into multiple of medians (MoMs) for gestational age. Hematocrit (Hct) was also recorded.

Results: $\mathrm{H}$ occurred in $14(15.9 \%)$ cases at a mean time of $1.5 \pm 0.77$ days from admission, with severe lifethreatening bleeding in 4 cases $(4.5 \%$ of the total; $28 \%$ of the bleeding group). Development of $\mathrm{H}$ was associated with a significantly prolonged aPTT $(3.0$ MoMs, $95 \%$ CI: $1.63-3.0$ vs. 0.49 MoMs, $95 \%$ CI: $0.43-$ $0.56, \quad \mathrm{p}<0.0001)$ and high frequency of thrombocytopenia (PLT $<150,000 / \mathrm{mmc}$ ) at the time of admission $(28.6 \%$ vs. $1.3 \%, \mathrm{p}=0.0007)$. Gestational age, birth weight, gender ratio, PT, INR, AT3, D-D, proteins $\mathrm{C}$ and $\mathrm{S}$, and initial Hct were comparable between groups ( $\mathrm{p}>0.22$ ).

Conclusions: These findings suggest that (i) development of $\mathrm{H}$ is significantly associated with early laboratory signs of asymptomatic compensated $\mathrm{CC}$; and (ii) the combination of aPTT and platelet counts could provide a first level screening to identify the condition.

\section{IDIOPATHIC PULMONARY HEMOSIDEROSIS - RARE CAUSE OF IRON DEFICIENCY ANEMIA IN CHILDHOOD}

\section{K G Martinova, O N Muratovska, S R Glamocanin, Z S Trajkova-Antevska, B A Coneska-Jovanova, S T Koceva}

Department of Hematology-Oncology, University Children's Hospital, Skopje, Macedonia

Background and Aims: Idiopathic pulmonary hemosiderosis (IPH) is uncommon in children. A triad of hemoptysis, iron deficiency anemia (IDA) and diffuse pulmonary infiltrates characterized pulmonary hemosiderosis.

Methods: The subject of this study were 43 patients with IPH diagnosed from 1969 to 2005 at the Hematology-Oncology department, Pediatric clinic in Skopje. Statistical analysis was performed.

Results: Mean age at diagnosis was $6 \pm 3,64$ years, the gender distribution was almost equal. At diagnosis all patients had anemia and pulmonary infiltrates, 26 $(60,4 \%)$ had dyspnea, $10(23,2 \%)$ had tachycardia, 5
$(11,6 \%)$ had hemoptysis, and $16(37,2 \%)$ had icterus. The level of hemoglobin was usually between $40-70$ $\mathrm{gr} / 1$. The diagnosis was based on history, presence of anemia and characteristic chest $\mathrm{x}$-ray, and was confirmed by showing macrophages laden with hemosiderin in gastric washings or bronchoalveolar lavage. The initial treatment consisted of prednisone in 40 patients $(93 \%)$. Ten patients $(23,2 \%)$ required longterm corticosteroids because of recurrent attacks and 17 patients $(39,5 \%)$ required other immunosuppressants (imuran or leuceran) in addition to prednisone to control their hemoptysis. Eight patients $(18,6 \%)$ died of acute massive pulmonary hemorrhage, 3 patients have been cured (with long-term follow up of 22,25 and 30 years and being without treatment of 18,11 and 19 years respectively). Five-year survival for IPH patients in our study was $80 \%$ (by Kaplan Meier method).

Conclusions: Sudden decrease in hemoglobin and hematocrit associated with the onset of active respiratory disease is strongly suggestive of IPH. Hemosiderin laden macrophages are diagnostic for IPH. Early and long-term immunosuppressive therapy may improve the prognosis.

\section{RETICULOCYTE HAEMOGLOBIN CONTENT IN THE DIAGNOSIS OF IRON DEFICIENCY IN CHILDREN}

M E Mateos ${ }^{1}$, J De La Cruz ${ }^{2}$, E Lopez-Laso ${ }^{1}$, M D Valdes ${ }^{3}$, A Nogales $^{4}$

${ }^{I}$ Department of Pediatrics, Reina Sofia Universitary Hospital, Cordoba, Spain, ${ }^{2}$ Department of

Epidemiology, Doce de Octubre, Universitary Hospital, Madrid, Spain, ${ }^{3}$ Department of Hematology, Doce de Octubre, Universitary Hospital, Madrid, Spain,

${ }^{4}$ Department of Pediatrics, Doce de Octubre

Universitary Hospital, Madrid, Spain

Background and Aims: Identification of iron deficiency (ID) is essential to prompt early treatment to prevent long-term systemic complications, including cognitive impairment in children. Reticulocyte haemoglobin content $(\mathrm{CHr})$ provides a real time assessment of erythropoietic activity. The diagnostic efficiency of $\mathrm{CHr}$ was investigated for the detection of ID in a pediatric population using five different cutoff levels of biochemical markers.

Methods: A descriptive transversal study of an urban population aged 6 months to 14 years-old with risk factors for ID or ID anemia was carried out. Levels of red blood cell indices, $\mathrm{CHr}$, serum ferritin, trasferrin saturation, transferrin, serum iron and total iron binding capacity were measured.

Results: Samples were obtained for 237 children (mean age 63.7 months, male:female ratio 1.08:1). ID and ID anemia mean prevalence was $17 \%$ and $5 \%$, respectively. A multiple stepwise logistic regression analysis identified $\mathrm{CHr}$ as the best multivariate predictor of iron deficiency. Each unit increase in $\mathrm{CHr}$ lowered the risk of iron deficiency by $63 \%(1-\mathrm{OR})$. A CHr cutoff value of $25 \mathrm{pg}$ had a sensitivity of $86 \%, 88.8 \%$ and $97.4 \%$ and a specificity of $81.4 \%, 81.4 \%$ and $77.6 \%$ for the diagnosis of ID, ID with or without anemia and ID anemia, respectively. 
Conclusions: A CHr level of less than 25 pg was the strongest predictor of ID and ID anemia. Our study supports that a panel based exclusively on hematologic indices including $\mathrm{CHr}$ is a valid alternative to the traditional biochemical panel for the diagnosis of ID with or without anemia in children.

\section{IRON DEFICIENCY ANEMIA IN PEDIATRIC HIGH RISK POPULATION: INTERVENTIONAL STUDY}

L Jaber ${ }^{1,2}$, M Balaom ${ }^{3}$, F Tebe ${ }^{3}$, A Tayeh ${ }^{3}$, Y Meirovitz $^{1,3}$, A Bader ${ }^{3}$, S Rigler ${ }^{3}$

${ }^{I}$ Neurology Clinic, Schneider Children's Medical Center of Israel, Petah Tikva, ${ }^{2}$ The Bridge to Peace Community Pediatrics Center, Taibe, ${ }^{3}$ Clalit Health Services, Israel

Background and Aims: Iron deficiency anemia is a prevalent health problem world wide. We aimed to assess if health education programme could be used with exiting health resources to reduce the incidence of IDA in a population in high risk.

Methods: We performed a prospective cohort study. A total of 310 recorded on the child health computer registers were participated. Children were recruited at one month age and randomized into control and intervention groups. Families in the intervention group received specific health education information at two six and eight month age by face to face contact, giving information on iron and iron deficiency anemia and their prevention and dietary health education. The control group received standard health education as delivered by nurses in the well baby clinic. Hemoglobin, ferriten, $\mathrm{MCV}$ and iron estimation and the estimation of the diet at one year age were also performed. The data analyzed by EP info using X2 test for analyzing different groups.

Results: A total of 287 children completed the study. Fifty four $(38 \%)$ of the control group and forty two (29\%) of the intervention group were anemic as defined by hemoglobin less than $11 \mathrm{gr} / \mathrm{dl}$ (P value: N.S). NO significant difference was found in the values of ferritin, MCV and hemoglobin in the two groups. There was no difference in the iron content of the diet offered to the two groups of children.

Conclusions: Effective prevention strategies are needed and further research is warranted.

\section{CORD BLOOD IGE (CB-IGE) LEVELS AND THE DEVELOPMENT OF ALLERGY DURING THE FIRST YEAR OF LIFE}

\section{A J Sybilski, M Odalska \\ Department of Pediatrics and Neonatology, Central Clinical Hospital MSWiA, Warsaw, Poland}

Background and Aims: It is crucial to have a method for selecting high risk infants and preventing atopic disorders. Probably essential significance has a CB-IgE. The goals of this study were: Appreciation whether CB $\operatorname{tg} E$ and $\operatorname{sgE}$ predicts the development of atopic diseases in early infancy and analyze the factors affecting on CB-IgE.
Methods: 207 randomized term neonates were being included in a prospective cohort study, 173 responses were obtained. Total and specific (for children's food, wheat/grass and HDM) CB-IgE levels were determined using the immunoassay test. The cohort was observed prospectively for 12 months and we performed questionnaires (after birth, in 6 and 12 month).

Results: CB-IgE level was between 0,0-23,08 IU/ml (mean 0,549 IU/ml). We detected $\mathrm{sIgE}$ in 34 newborns (40 positive tests). $42 \%$ of the infants had atopic symptoms. The major disease was atopic dermatitis (36,4\%). The hazard for developing atopy symptoms was higher in boys, children with family history of atopy and passive tobacco smoke exposure. The pregnant complications, caesarean section and early eggs intake increased risk for onset atopy diseases. Exclusive breast-feeding was protective factor. The male gender, the pregnant complication and number of sibling increased total CB-IgE levels. No correlation was seen between family history of atopy, parental smoking and CB-IgE.

Conclusions: There is no correlation between total and specific CB-IgE levels and presence of atopic symptoms. Total and specific CB-IgE level with atopy family history cannot be used as the indicators for selecting high risk of infants. The best method for selecting high risk infants is precise medical history.

\section{TREATMENT RESULTS OF 614 PATIENTS WITH HODGKIN'S DISEASE}

M Buyukpamukcu ${ }^{1}, \mathbf{A} \operatorname{Varan}^{1}$, C Akyuz ${ }^{1}$, L Atahan ${ }^{2}$, G Kale $^{3}$, T Kutluk ${ }^{1}$, Y Koksal ${ }^{1}$, B Yalcin ${ }^{1}$

${ }^{1}$ Department of Pediatric Oncology, Institute of Oncology, ${ }^{2}$ Department of Radiation Oncology, Faculty of Medicine, ${ }^{3}$ Department of Pediatric Pathology, Faculty of Medicine, Hacettepe University Ankara, Turkey

Background and Aims: To evaluate the clinical characteristics, treatment regimens, and outcome of the patients with Hodgkin's disease.

Methods: 614 patients diagnosed with Hodgkin's disease between 1972 and 2006 were retrospectively evaluated. All patients were treated with chemotherapy with/o radiotherapy. Chemotherapeutic regimens included COPP derivatives (cyclophosphamide or mustargen + oncovin + prednisolone + procarbazine), ABVD (Adriamycin + Bleomycin + Dacarbazine + Vinblastine), or alternated ABVD/COPP. Staging was done according to Ann Arbor classification system and histopathologic examination was done according to Rye system. Mean and median values were used for the demographic characteristics. Kaplan-Meier survival curves were used for survival analysis. The patient groups were compared in terms of survival duration using a log-rank test.

Results: There were 452 males and 162 females with a median age of 8 years ( 2 to 21 ). 183 patients had B symptoms. Stage distributions were $165,185,145$, and 119 patients in stage I, II, III, and IV, respectively. Histopathologic subtypes were mixed cellularity, nodular sclerosis, lymphocytic predominance, lymphocytic depletion, and unclassified in $344,90,62$, 
46, 71 patients, respectively. Overall (OS), and eventfree survival (EFS) rates in whole group were 83 and $60 \%$. OS rates were $79 \%, 95.6 \%, 91.8 \%$ in regimens of COPP $(\mathrm{n}=402), \quad$ ABVD $(\mathrm{n}=94)$, and alternated ABVD/COPP $(n=65) \quad(p=0.02)$. The other significant prognostic factors on survival were stage $(\mathrm{p}=0.0001), B$ symptoms $(\mathrm{p}=0.0001)$, and age $(\mathrm{p}=0.01)$.

Conclusions: This is one of the largest series in a single center. ABVD regimen has the best survival rates and should be used for treatment of patients with Hodgkin's disease.

\section{INTERLEUKIN-18 AS A MEDIATOR OF SYSTEMIC JUVENILE IDIOPATHIC ARTHRITIS (JIA)}

M Jelusic ${ }^{1}$, L Tambic Bukovac ${ }^{1}$, I K Lukic ${ }^{2}$, K Dubravcic ${ }^{3}$, I Malcic ${ }^{1}$, D Batinic ${ }^{3}$

${ }^{l}$ Department of Paediatrics, University Hospital Centre, Zagreb, Croatia, ${ }^{2}$ Department of Anatomy and Croatian Institute for Brain Research, Zagreb University School of Medicine, Zagreb, Croatia, ${ }^{3}$ Deprtment of Laboratory of Immunology, University Hospital Centre, Zagreb, Croatia

Background and Aims: The immunoregulatory abnormalities, including abnormal cytokine production, are thought to be important in the pathogenesis of JIA. However, the key mediators have not yet been elucidated. To determine the serum and synovial fluid levels of IL-18 in patients with JIA, and to explore the association of those levels with the status and activity of disease.

Methods: Blood samples were obtained from 81 children with JIA (31 with oligoarticular, 33 with polyarticular and 17 with systemic JIA). Synovial fluid samples were collected from 16 children with pauciarticular JIA. Concentrations of IL-18 were determined using commercial kits

Results: Patients with systemic JIA had higher serum levels of IL-18 than patients with other forms of JIA or control children, both during the active (median, range: $6240,1600-78750 \mathrm{pg} / \mathrm{mL})$ and inactive $(1615,513-$ $3270 \mathrm{pg} / \mathrm{mL}$ ) phase of disease (ANOVA, $\mathrm{P}<0.05$ ). Levels of IL-18 in sera of children with pauciarticular JIA $(255,89-4342 \mathrm{pg} / \mathrm{mL})$ were similar to the corresponding synovial fluid levels $(217,89-1245$ $\mathrm{pg} / \mathrm{mL}$ ). Serum levels of IL-18 were proportional to the ESR and CRP, but inversely proportional to the haemoglobin levels.

Conclusions: As IL-18 appears to be an important mediator of systemic JIA, its inhibition might be a base for a successful therapeutic approach.

\section{MICROCYTIC ANEMIA IN CHILDREN IN POLAND AS A SYMPTOM OF THE THALASSEMIA}

T Jackowska ${ }^{1}$, E Wagiel $^{1}$, M Ciebiera $^{1}$, E Zdebska ${ }^{2}$, B Burzynska ${ }^{3}$, M Maciag ${ }^{3}$

${ }^{I}$ Department of Pediatric Hematology and Oncology, Medical University, Warsaw, Poland, ${ }^{2}$ Department of Biochemistry, Institute of Hematology and
Transfusiology, Warsaw, Poland, ${ }^{3}$ Institute Biochemistry and Biophysics, PAS, Warsaw, Poland

Background and Aims: The thalassemias, a group of inherited disorders of hemoglobin synthesis. This disease was regarded as very rare in Poland. The aim of the present investigation was to investigate thalassemia in children who had been referred to a Hematological Clinic in Warsaw for hypochromic anemia.

Methods: 25 children (14 male and 11 female), aged 1- 17 years (average 7.7 years) with microcytic anemia, who had unsuccessfully been treated by family doctors with ferrous were examined for thalassemia. Levels of iron, ferritin, TIBC, transferrin saturation, bilirubin hemoglobin A2 (HbA2) and hemoglobin F (HbF) were evaluated. Family blood count was also evaluated.

Results: Average hemoglobin level was $11.15 \mathrm{~g} / \mathrm{dl}$ $(9.2-11.8 \mathrm{~g} / \mathrm{dl})$ and erythrocyte count was $5.52 \mathrm{mln} / \mathrm{mm} 3$ (5.65-6.3 $\mathrm{mln} / \mathrm{mm} 3)$. Average mean cell volume (MCV - 63.5 fl /55-75 fl/) and mean cell hemoglobin (MCH - $20.25 \mathrm{fl} / 55-75 \mathrm{fl} /$ ) were below normal. Average MCHC, RDW, iron, ferritin and bilirubin levels were normal. $\mathrm{HbA} 2$ had increased in 8 children and $\mathrm{HbF}$ in 3 children. There was an increase in both $\mathrm{HbA} 2$ and $\mathrm{HbF}$ in 10 children. In three children genotypes confirmed the presence of mutations. Thalassemia $\alpha$ was detected in one of the children (M.G.) and thalassemia $\beta$ in another. No clinical symptoms of thalassemia were found in any of the children. In 11 adult relations similar deviations were observed in blood count (low $\mathrm{Hb}, \mathrm{MCV}$ and hight RBC level). Thalassemia had not been diagnosed in these people earlier.

Conclusions: Thalassemia should be considered in differential diagnosis of children with microcytic anemia in Poland.

\section{THE EFFECTS OF BRONCHIOLITIS ON PLATELETS}

V Vlacha $^{1}$, G Feketea ${ }^{2}$

${ }^{1}$ Department of Pediatrics, University Hospital of Patras, Greece, ${ }^{2}$ Department of Pediatrics, Hospital of Amaliada, Greece

Background and Aims: It is well known that the number of platelets is related to many factors including infections. The aim of this study was to evaluate the effects of bronchiolitis on platelets.

Methods: The admitted patients diagnosed with bronchiolitis and had at least one complete blood count (CBC) performed from the same Coulter analyzer were included in this study. The $\mathrm{CBC}$, the sedimentation rate (SR) and the clinical characteristics such as age and days of hospitalization, were recorded.

Results: From 116 patients studied, $63.8 \%$ had thrombocytosis (platelet number $>500 \mathrm{x} 103 \mathrm{cells} / \mathrm{mm} 3$ ). The mean platelet number (PIt) was $452 \pm 154$ $\mathrm{x} 103$ cells $/ \mathrm{mm} 3$. The patients had mean SR $24 \pm 17$ $\mathrm{mm} / \mathrm{h}$, mean white blood count (WBC) $10.9 \pm 4$ $\mathrm{x} 103$ cells $/ \mathrm{mm} 3$ and mean hemoglobin $(\mathrm{Hb}) 11.3 \pm 1.6$ $\mathrm{gr} / \mathrm{dl}$. The Plt had positive linear relationship with the patients' age $(\mathrm{R} 2=0.210, \mathrm{p}<0.0001)$ and the $\mathrm{SR}$ $((\mathrm{R} 2=0.167, \mathrm{p}<0.0001)$. However, negative linear relationship was shown between the Plt and the $\mathrm{Hb}$ 
$(\mathrm{R} 2=0.183, \mathrm{p}<0.0001)$. Statistical significant differences were reported comparing the Plt of the patients with high SR $(>50 \mathrm{~mm} / \mathrm{h})$ and low SR $(<50 \mathrm{~mm} / \mathrm{h})(\mathrm{p}=0.009)$ and the patients with high WBC $(>15 \times 103$ cells $/ \mathrm{mm} 3)$ and low WBC $(<15$ x103cells $/ \mathrm{mm} 3), \quad(\mathrm{p}=0.001)$. Interestingly, significant difference was shown comparing the SR value of the patients with high Plt $(>500 \times 103 \mathrm{cells} / \mathrm{mm} 3)$ and low Plt $(<500 \times 103$ cells $/ \mathrm{mm} 3),(\mathrm{p}=0.0001)$.

Conclusions: Thrombocytosis is a very common finding in patients with bronchiolitis. The age of the patient and the degree of inflammation are affecting the platelet number.

\section{A SIMPLE RISK SCORE TO PREDICT INTRAVENOUS IMMUNOGLOBULIN UNRESPONSIVENESS IN PATIENTS WITH KAWASAKI DISEASE}

T Kobayashi ${ }^{1}$, Y Inoue ${ }^{1}$, K Takeuchi $^{2}$, Y Okada ${ }^{1}$, $\mathrm{K}_{\text {Tamura }}{ }^{1}, \mathrm{~T}_{\text {Tomomasa }}{ }^{1}$, T Kobayashi ${ }^{3}$, A Morikawa $^{1}$

${ }^{I}$ Department of Pediatrics and Developmental Medicine, Gunma University Graduate School of Medicine, Maebashi, ${ }^{2}$ Department of Health and Welfare, Takasaki University of Health and Welfare, Takasaki, ${ }^{3}$ Department of Cardiology, Gunma Children's Medical Center, Shibukawa, Gunma, Japan

Background and Aims: Predictions of intravenous immunoglobulin (IVIG) unresponsiveness in Kawasaki disease (KD) are difficult. The aim of this study was to establish predictive models of being an IVIG nonresponder. Methods: We reviewed clinical records of 546 consecutive KD patients (development data set). A predictive model was estimated from multiple logistic regression analysis and additional validation was performed on an independent dataset of 204 consecutive KD patients (validation data set). To increase the usefulness of this risk stratification in clinical practice, we approximated it with a simple scoring model where integer score points were assigned to each variable.

Results: A seven variable logistic model was constructed, including day of illness at initial treatment, age in months, percentage of white blood cells representing neutrophils, platelet count, and serum aspartate aminotransferase, sodium, and C-reactive protein, that generated an area under the receiveroperating-characteristics curve of 0.84 and 0.90 for the development and validation data sets, respectively. Using both data sets, the seven variables were used to generate a simple scoring model that gave an area under the receiver-operating-characteristics curve of 0.85 . For a cutoff of 0.15 or more in the logistic regression model and 4 points or more in the simple scoring model, sensitivity and specificity were $86 \%$ and $67 \%$ in the logistic model; and $86 \%$ and $68 \%$ in the simple scoring model. The kappa statistic is 0.67 , indicating good agreement between logistic and simple scoring models.

Conclusions: Our predictive models showed high sensitivity and specificity in identifying IVIG unresponsiveness among KD patients.

\section{WHOLE BLOOD GENE EXPRESSION IN RESPIRATORY SYNCYTIAL VIRUS BRONCHIOLITIS}

H O Fjaerli ${ }^{1}$, G Bukholm ${ }^{2}$, A Krog ${ }^{2}$, C Skjaeret ${ }^{2}$, M Holden $^{3}$, B Nakstad ${ }^{1}$

${ }^{1}$ University of Oslo, Faculty Division Akershus University Hospital, Department of Paediatrics, Akershus University Hospital, Nordbyhagen, Norway

${ }^{2}$ Institute of Clinical Epidemiology and Molecular Biology, Akershus University Hospital, Nordbyhagen, Norway, ${ }^{3}$ Norwegian Computing Center, Oslo, Norway

Background and Aims: Respiratory syncytial virus is a major cause of viral bronchiolitis during infancy. Host response is important for disease susceptibility and severity but not yet fully elucidated. Methods: To study this response we used microarray to measure mRNA gene expression in whole blood of five male infants hospitalised with RSV, subtype B, bronchiolitis versus five one year old male controls exposed to RSV during infancy without bronchiolitis. Gene expression levels were further evaluated in a quantitative real-time PCR study with TaqMan Low Density Array cards in an extended population of 18 infants hospitalised with this disease. Results: Among the 30 genes most differentially expressed by microarray nearly $50 \%$ were involved in immunological processes. Among these were the upregulated interferon stimulated genes IFI27, IFI44, EIF2AK2, IFI44L, OAS3 and G1P2 and the downregulated genes CLC, HLA-DQA1 and HLADRB4 (tab). When performing PCR on some of these genes IFI27 was upregulated in all but one infant, and CLC was downregulated in all 18 infants. Conclusions: IFI27 is upregulated and CLC is downregulated in whole blood of infants hospitalised with RSV, subtype $\mathrm{B}$, bronchiolitis when compared with one year old controls exposed to RSV without bronchiolitis during infancy.

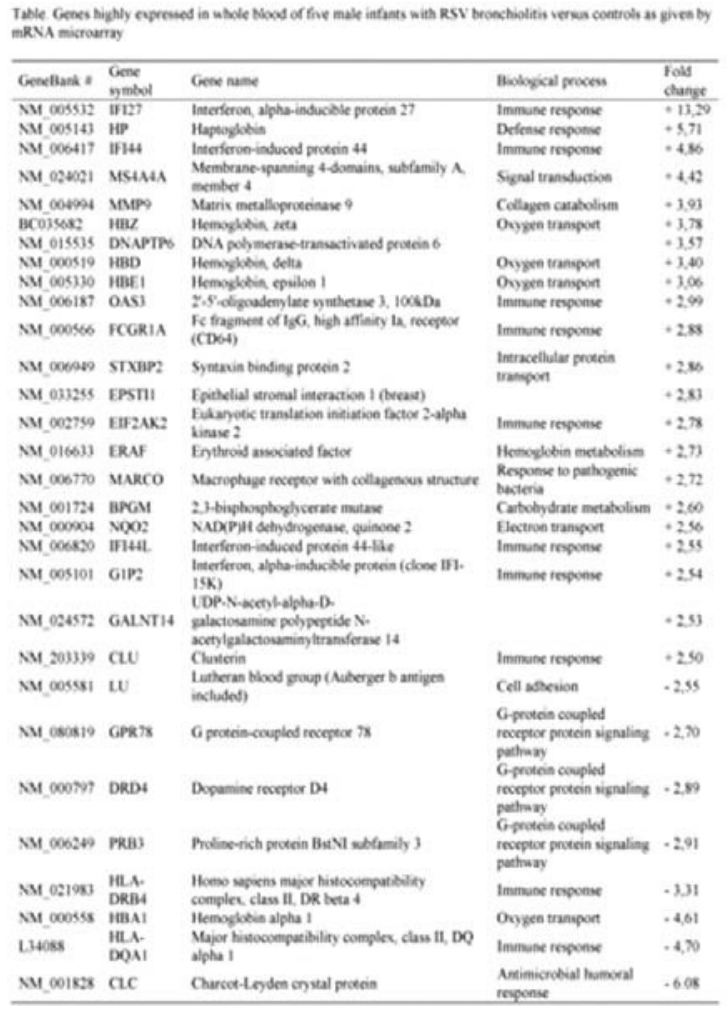


IMMUNOGENICITY AND TOLERABILITY OF 1 AND 2 DOSES OF PROQUAD ${ }^{\circledR}$ (MEASLES, MUMPS, RUBELLA, VARICELLA VACCINE)

M G Goveia, B J Kuter, J Hartzel, W R Williams, M L H Brown, K A Eves, S O Kloper, F Schodel

Merck Research Laboratories, West Point, PA, USA

Background and Aims: Measles, mumps, rubella, and varicella are serious viral diseases worldwide. A combination vaccine, ProQuad $\AA$, was developed for their prevention.

Methods: 5446 children, 12-23 months, received a single dose of ProQuad ${ }^{\circledR}$ in 4 clinical studies. A subset of 1035 children received a second dose of ProQuad $\mathbb{}$ $\sim 3$ months later. M-M-R ${ }^{\circledR} I I$ and VARIVAX ${ }^{\circledR}$ were provided as control. Serum was obtained prior to and 6 weeks after each vaccination and antigens were evaluated by ELISA or gpELISA. Safety was evaluated for 6 weeks postvaccination.

Results: The rate of seroconversion $(\geq 1.25$ gpELISA units $/ \mathrm{mL}$ ) to varicella was $97.9 \%$ and $99.5 \%$ after one and two doses, respectively. Titers $\geq 5$ gpELISA units $/ \mathrm{mL}$ generally correlate with protection against varicella disease; the rate was $91.2 \%$ and $99.4 \%$ after one and two doses, respectively. Responses to measles, mumps, and rubella were $\geq 97.4 \%$ after one and two doses. The response rates to all 4 antigens after a single dose of ProQuad ${ }^{\circledR}$ were similar to those after M-M$\mathrm{R} \circledast \mathrm{II}$ and VARIVAX ${ }^{\circledR}$. The proportion of subjects with at least one adverse experience (AE) was comparable between ProQuad $\AA$ recipients and recipients of $M$ $\mathrm{R}{ }^{\circledR} I I$ and VARIVAX ${ }^{\circledR}$. The most commonly reported vaccine-related systemic AEs after ProQuad ${ }^{\circledR}$ were fever, irritability, measles-like rash and varicella-like rash. Fever was transient without long-term sequelae. The AE incidence following a second dose was generally lower than following the first dose.

Conclusions: ProQuad $\AA$ is generally well tolerated and highly immunogenic for measles, mumps, rubella, and varicella following one and two doses, at levels which have been shown to correlate with protection.

\section{ACTIVATION ANTIGENS, T CELL PROLIFERATION AND GAMMA INTERFERON PRODUCTION IN EARLY DIAGNOSIS OF CONGENITAL TOXOPLASMOSIS}

L Ciardelli ${ }^{1}$, V Meroni ${ }^{2}$, L Bollani ${ }^{3}$, M A Avanzini $^{1}$, C Tinelli ${ }^{4}$, F Garofoli ${ }^{1}$, A Gasparoni ${ }^{5}$, A Serra ${ }^{3}$, M Stronati ${ }^{3}$

${ }^{1}$ Research Laboratories, Neonatal Immunology and Paediatric Oncohematology, IRCCS Policlinico San Matteo, Pavia, Italy, ${ }^{2}$ Department of Infectious Diseases, University of Pavia, IRCCS Policlinico San Matteo, Pavia, Italy, ${ }^{3}$ Neonatal Intensive Care Unit, IRCCS Policlinico San Matteo, Pavia, Italy, ${ }^{4}$ Biometric Unit, IRCCS Policlinico San Matteo, Pavia, Italy ${ }^{5}$ Neonatal Intensive Care Unit, Spedali Civili, Brescia, Italy

Background and Aims: Congenital infection with Toxoplasma gondii is mainly subclinical, it initially goes unnoticed, but if it is untreated, babies can later develop sequelae. Until now, postnatal diagnosis was made by the detection of specific antibodies, nevertheless transplacentally acquired maternal IgG can interfere at this level and neonatal IgM or IgA production can be largely variable. Furthermore, current diagnostic methods are not sufficiently sensitive and frequently fail to establish the diagnosis before one year of age: tests for IgM and $\operatorname{IgA}$ antibodies identify up to $75 \%$ infected babies. The aim of our study was to evaluate the specific cellular immunity to $T$. gondii antigens in newborns with different immunological assays, in order to provide an accurate diagnosis of congenital infection as early as possible.

Methods: We tested IFN-g production, $\mathrm{T}$ lymphocyte proliferation and the expression of activation antigens CD25 and HLA-DR on T lymphocyte membrane on infected and non infected neonates at different ages.

Results: All the employed assays showed good sensitivity and specificity with the cut-off values defined in the study. Furthermore, immunological tests let us find 2/9 infected neonates which were negative with serological tests before three months of age. Moreover, we observed that specific $\mathrm{T}$ lymphocyte response increased with age in treated infected neonates, suggesting that therapy does not affect lymphocyte activity.

Conclusions: Our results confirm the usefulness of cellular immunity evaluation in association with serological investigations for an early diagnosis of congenital toxoplasmosis.

\section{USEFULNESS OF TUBERCULIN SKIN TEST FOR DIAGNOSIS OF LATENT TUBERCULOSIS INFECTION AMONG SCHOOL AGED INTERNATIONALLY ADOPTED CHILDREN}

O Gkika, G Z Zantopoulos, N Manakou, S Liosi, P Anastasopoulou, A Giannisi, Z Patrona

Department of Paediatrics, General Hospital of Korinthos, Korinthos, Greece

Background and Aims: Tuberculosis remains a leading cause of death from any single infectious disease accounting for over a quarter of all avoidable deaths. Children with latent tuberculosis infection (LTBI) represent the future reservoir from which adult cases will arise. This study aims to describe the incidence and to investigate the usefulness of tuberculin skin test (TST) for diagnosis of LTBI among internationally adopted children of our area.

Methods: TST reaction size was recorded for 100 school aged internationally adopted children ranging in age from 6 to 14 years old. Two groups of children were identified: First group (A) consisted of 54 children that were born in Greece and weren't BCG immunized and the second group (B) included 46 children who were born in a TB endemic country, had at least one foreign parent and were BCG immunized at birth.

Results: The mean age of children was 7.1 years. In 44 (81.4\%) children of group A and $30(65.2 \%)$ of group B was found induration $\leq 5 \mathrm{~mm}$. In $22 \%$ of children of both groups was confirmed LTBI. Chest radiography was done for all children with induration of $15 \mathrm{~mm}$ or more and for BCG- immunized children with induration 
$\geq 10 \mathrm{~mm}$. None of our patients was found to have active TB disease.

Conclusions: The result indicates that the use of TST in the diagnosis of LTBI may not be affected by BCG vaccination at birth. The prevalence of latent tuberculosis infection among internationally adopted children is high and warrants an expansion of contact investigation. TB prevention strategy targeting children of foreign origin is imperative.

\section{LEVELS OF ANTICAPSULAR ANTIBODIES BEFORE AND AFTER THE ADMINISTRATION OF HEPTAVALENT PNEUMOCOCCAL CONJUGATE VACCINE IN GREEK CHILDREN}

\author{
A A Gatsouli ${ }^{1}$, E V Liatsis ${ }^{2}$, F A Haliotis ${ }^{1}$, \\ M N Tzanoudaki ${ }^{2}$, S A Avlonitis ${ }^{1}$, M G Kanariou ${ }^{2}$ \\ ${ }^{1}$ Second Department of Pediatric Clinics, Aghia Sophia \\ Children's Hospital, Athens, Greece, ${ }^{2}$ Departments of \\ Immunology and Histocompatibility, Aghia Sophia \\ Children's Hospital, Athens, Greece
}

Background and Aims: The recent wide application of the 7-valent pneumococcal conjugate vaccine (7-PCV) has contributed in the prevention of invasive pneumococcal disease. And in certain countries is recommended for all children under 2 years of age. The schedule for 7-PCV is 4 doses if started under 7 months of age but older children need less doses. This study is designed to evaluate the quantitative response of the antibody against Pneumococcal capsular polysaccharide, before and after the administration of 7-PCV in children 1-4 years old and the correlation of the results with age and dose regimen.

Methods: Blood was drawn from 22 Greek children before and 3-4 weeks after the administration of 7-PCV. The children were classified in two age groups: A:6 children 12-23 months and B:16 children 2-4 years old. Group A received two 7-PCV doses, while Group B received one dose of the vaccine. Pneumococcal anticapsular antibodies (anti-PCP) were measured in serum by an enzyme immunoassay (ELISA). Antibody response to 7-PCV was estimated using an antibody increase factor (IF) which was calculated as follows: $\mathrm{IF}=$ anti-PCPafter7-PCV/anti-PCPbefore 7-PCV.

Results: Group A children had significantly higher IF score compared to the older children $(\mathrm{P}=0.008)$. A 3 -fold increase in antibody levels was observed in $4 / 6$ $(66,7 \%)$ and 3/16 (18,75\%) Group A and B respectively. Conclusions: The administration of two 7-PCV doses results in satisfactory anti-PCP levels in children of 12-23 months of age. In older children, the antibody response to the administration of one 7-PCV dose was not considered to be satisfactory. Further studies will support the need or not for a booster dose in older children.

\section{CHANGES OF IMMUNITY PARAMETERS CAUSED BY CONGENITAL OR EARLY POSTNATAL CYTOMEGALOVIRUS INFECTION}

J Dort $^{1}$, V Gutova ${ }^{2}$, E Dortova $^{1}$, M Svecova $^{3}$
${ }^{1}$ Department of Neonatology, Charles University Hospital, Plzen, Czech Republic, ${ }^{2}$ Institution of Immunology and Allergy, Charles University Hospital, Plzen, Czech Republic, Institution of Microbiology, Charles University Hospital, Plzen, Czech Republic

Background and Aims: CMV is the most frequent cause of congenital infections. Prematurity itself brings immunodeficiency due to immaturity of immune system that can facilitate infection process. Moreover, CMV infection can further compromise the immune system of premature infants. To assess changes of humoral and cellular immunity parameters in infants with congenital and early postnatal cytomegalovirus (CMV) infection. To use the results as a tool for better timing of vaccination in premature infants.

Methods: Assessment of humoral and cellular immunity parameters - IgG, IgA, IgM levels, T and B lymphocytes, NK cells, T lymphocytes subpopulations, $\mathrm{T}$ lymphocytes transformation after mitogen stimulation - in a group of infants with congenital or early acquired postnatal CMV infection at 1 year of corrected age. Results are compared with age specific normal values. The intensity of urine CMV shedding served as marker of the CMV infection activity. The examination was made from treatment reasons in the sake of patients, not for study reasons.

Results: 10 children with active CMV infection were examined. Hypoimmunoglobulinemia IgA was found in all cases, combined $\operatorname{IgG}$ and $\operatorname{IgA}$ in 2 and $\operatorname{IgG}, \operatorname{IgA}$ and IgM in 4 cases. The results of cellular immunity examination show dysregulation rather than deficiency. The intensity of virus urine shedding was very high in patients with organ involvement.

Conclusions: Infants with congenital or early postnatal CMV infection can have compromised immunity. The immunity assessment and the intensity of virus shedding can be a useful tool for the proper timing of vaccination with vivid vaccines especially in premature infants.

\section{PROTECTION OF IMMUNODEFICIENT CHILDREN FROM HAEMOPHILUS INFLUENZAE INFECTIONS}

K S Mironska, S Peova, K Stavric, L Kareva

Department of Immunology, Clinic for Children's Diseases, Skopje, Macedonia

Background and Aims: Haemophilus Influenzae is one of the most important bacterial agents of upper respiratory tract infections, and one of the main causes of severe invasive bacterial disease in children under 6 years of age. With introduction of conjugated polisaharide Hib vaccine, protection from this serious agent is achieved. The aim was to protect our immunodeficient patients or patients with suspected immunodeficiency suffering of frequent upper respiratory tract infections caused with Haemophilus Influenzae type $\mathrm{b}$ with $\mathrm{Hib}$ vaccine, because regular immunization against this agent in our country is not introduced yet.

Methods: On the Department of Immunology at the Clinic for Children's Disease in Skopje, 67 patients, aged from 1 to 5 years, were immunized with 
conjugated Hib vaccines. They were followed up during the next 24 hours, and then, every 3 months in the next two years.

Results: There were not adverse post vaccine reactions during the first 24 hours or later. During the next two years, Haemophilus Influenzae type b was not isolated, neither in nose nor oropharynx and no one case of Hib disease had appear, except in one case, when after one year of immunization, Haemophilus Influenzae type $b$ was found again in upper respiratory tract, but without Hib disease manifested.

Conclusions: Immunization with conjugated Hib vaccine of our patients came out as safe and effective, resulting decreased morbidity connected with Hib disease. Immunization with conjugated Hib vaccine in all our immunodeficient patients is necessary, as well as introduction of this Hib vaccine in regular program for immunization in our country.

\section{TOXIC SHOCK SYNDROME VERSUS KAWASAKI DISEASE}

C Goni-Orayen ${ }^{1}$, M Herranz-Aguirre ${ }^{1}$, A Perez-Martinez ${ }^{2}$, J Rodriguez-Ozcoidi ${ }^{1}$, G DuranUrdaniz $^{3}$, A J Cabria-Fernandez ${ }^{3}$

${ }^{1}$ Pediatric Service, ${ }^{2}$ Pediatric Surgery Service, Virgen del Camino Hospital, Pamplona, ${ }^{3}$ Pediatric Service, Garcia Orcoyen Hospital, Estella, Navarra, Spain

Background and Aims: Diagnosis of toxic shock syndrome (TSS) is based on clinical criteria. It has similarities with diseases that are mediated by toxins, both from the clinical and the immunological points of view. Proinflammatory cytokines play a role in the pathogenics, similar to what occurs in Kawasaki's disease (KD), which is also diagnosed clinically.

Methods: A four-year-old girl with high fever, vomiting, abdominalgia, and headache for 48 hours, was referred to our hospital. She presented conjunctival hyperemia without secretion, very hyperemic oral mucosa, dry cracking lips and palmoplantar erythema. On admission temperature was $39 \mathrm{C}$, heart rate 145 bpm, and blood pressure $95 / 60 \mathrm{mmHg}$. She had not urinated since the night before admission.

Results: Laboratory tests showed: leucocytosis, thrombocytopenia, disseminated intravascular coagulation, high plasma C-reactive protein level and elevated ESR. Renal and hepatic study was normal. Creatine kinase levels and antistreptolysin-O titres were normal. Blood, urinary and cerebrospinal fluid cultures were negative. Echocardiograph was normal. The rheumatic disease study was negative. Needed intensive care and was administered dopamine, cefotaxima and teicoplanina, intravenous immunoglobulins and aspirin. Remained feverish and with periungueal desquamation. The polymorphous skin rash eased in one week. Favorable evolution, presenting generalized skin desquamation at 2 weeks.

Conclusions: Our patient was diagnosed with TSS and was treated aggressively. Given the diagnostic doubt about atypical KD endovenous gammaglobulins were administered, due to the higher cardiological risk and because children with TSS can benefit from its administration. Similarities with syndromes triggered by bacterial antigens make differential diagnosis very difficult on occasions.

\section{INFECTIONS 'FACTORS OF PROVOCATION FOR SMALL BLOOD VESSELS ENDOTHELIAL INJURY' M. KAWASAKI}

Z Rajcanovska, B Filipova, K Bakieva- Bojcevska, Z Trpkovski, V Trpkovska

Zdravstven Dom Negotino, Faculty of Medicine, Skopje, Negotino, Macedonia

Background and Aims: The endothelium of blood vessels has important role in forming of immunological response, its modulation and regulation. The immunological response has manifestation of vasculitis, purpura, etc. Our aim is to present 'the path' of our patient to the final diagnosis and all differential diagnostic problems.

Methods: Our patient was 11 months male baby, extremely upset, with high temperature and enlargedswollen neck noduli lymphatici. $\mathrm{He}$ has been ambulatory treated with antibiotics for seven days without any results. The second day after hospitalization exanthema rash showed up all over the body. The treatment with anti allergic therapy showed no results, too. Punction of the neck noduli lymphatici was realized without any suppuration - it eliminated neck's phlegmona. The consultation with ORL specialist and infectiologist from Clinical center- Skopje didn't give final diagnosis. The baby was transported to the Clinic of Pediatrics in Skopje in difficult condition. Several diagnoses have lead us to diagnosis of M. Kawasaki.

Results: The treatment with immunoglobulin and aspirin has shown the first positive results.

Conclusions: M. Kawasaki was noticed for the first time in Japan 1967 with unknown etiology. The patient was male baby less than 5 years. The provocation factor is staphylococcus endotoxin B. Complete clinical investigation is necessary for determining the final diagnosis of the immunological response of the endothelium and realization of the necessary therapy.

\section{COMPARISON OF MAGNETIC RESONANCE IMAGING AND SCINTIGRAPHY IN THE DIAGNOSIS OF OSTEOMYELITIS IN CAT- SCRATCH DISEASE}

V Rozmanic ${ }^{1}$, D Miletic ${ }^{2}$, K Manestar ${ }^{1}$, S Kamber ${ }^{1}$, S Banac ${ }^{1}, \mathrm{~N}$ Cace $^{1}$

${ }^{l}$ Paediatric Clinic, University of Rijeka, Rijeka, Croatia, ${ }^{2}$ Clinic for Radiology, University of Rijeka, Rijeka, Croatia

Background and Aims: Typical cat-scratch disease (CSD) is a self limiting infectious disease present with lymphadenopathy in a patient with a history of cat contact. Cases of bone involvement in patients with catscratch disease are rare.

Methods: We describe a case of a 11 year old boy with prolonged fever, inguinal lyphadenitis and osteomyelitis after being scratched by a kitten. 
Results: The physical examination revealed enlarged lymph node in right inguinal. Serum titer for Bartonella henselae was positive (IgM 80, IgG 1024). Scintigraphy revealed a pathological focus of 8 th rib and right parietal bone. MRI of the skeletal bone revealed pathological changes of 8th vertebra with associated rib and changes on the right iliac bone. After observing MRI signs of bone lesion a combination of rifampicin and azithromycin in period of 6 weeks was given.

Conclusions: Magnetic resonance imaging (MRI) is the best methods for osteomyelitis providing detailed anatomical information especially in vertebral and pelvis osteomyelitis. Our case confirms reports that MRI has an excellent diagnostic accuracy and is more reliable for the detection and evaluation of the bone lesions of CSD osteomyelitis than scintigraphy and computerised tomography.

\section{INFECTIVE MONONUCLEOSIS AMONG CHILDREN FROM 0 TO 15 YEARS OF AGE}

\author{
L Kuculovska $^{1}$, T Stosic ${ }^{2}$, P Kuculovski ${ }^{3}$, M Petrovic ${ }^{1}$ \\ ${ }^{1}$ Emergency Centre, Skopje, Macedonia, ${ }^{2}$ Student \\ Hospital, Skopje, Macedonia, ${ }^{3}$ GRANIT Construction \\ Co, Skopje, Macedonia
}

Background and Aims: Infective mononucleosis is a viral disease caused by EBV (Epstein-Barr virus). It is characterised with changes of lymphocyte in fact monocyte segment and clinical expression of higher body temperature, tonsylopharingitis and an enlargement of lymphatic glands, splenogalis, and appear of atypic lymphocyte in the peripheral blood. EBV is lymphocytropic toward B-lymphocytes which are in same time receptors $\mathrm{C} 3 \mathrm{~d}$ components of complements. The injection of EBV in the epitel cells and B-lymphocytes is by tonsils and Waldeyers ring in oropharings. When the virus is in B-lymphocytes, it can cause cytolysis in those cells (sometimes this infection is missing - when the DNA virus is build in the infected genome of infected B-lymphocytes.

Methods: Laboratory tests, atypic lymphocyte and monocyte, specific reactions, evident disease, materials from clinic for children disease - Hematooncology, Childrens Hospital - Bukurest/Skopje (Macedonia).

Results: With aim to get a complete knowledge of expansion of this type of illness from October until December, which have been already written: of 137 sick people, from the age of 0-4 years are 39, and from 11-15 years old are 98 infected.

Conclusions: Bad economical environment is a cause for appearance of subclinical form of infective mononucleosis in the early age of life, and also among the puberty population.

\section{C-REACTIVE PROTEIN IN KAWASAKI DISEASE AS A CARDIOVASCULAR PROGNOSTIC FACTOR}

\author{
A F El-Hassanien ${ }^{1}$, H A Al-Ghiaty ${ }^{2}$ \\ ${ }^{l}$ Department of Pediatrics, Faculty of Medcicine, \\ El-Mansoura University, El-Mansoura, Egypt
}

${ }^{2}$ Department of Pediatrics, Faculty of Medcicine, Benha University, Tukh, Kaliobia, Egypt

Background and Aims: Coronary sequelae that persist after Kawasaki disease (KD) have been associated with obstructive changes of coronary blood vessels in adolescents and young adults. However, little is known about the association between sequelae late after $\mathrm{KD}$ and inflammatory markers, which are potential mediators and markers for atherogenesis. The aim of our study to evaluate the prognostic role of CRP as an inflammatory marker in patients with $\mathrm{KD}$ as regards late cardiac complications.

Methods: Retrospective study was performed to test the hypothesis that coronary sequelae are associated with elevated levels of inflammatory markers especially CRP in patients after $\mathrm{KD}$ (3 and 6 months). Levels of highsensitivity $\mathrm{C}$-reactive protein (CRP) was reviewed 53 patients diagnosed as classical $\mathrm{KD}$ and divided into 3 groups according to the coronary artery lesions (CALs): KD patients without CALs $(\mathrm{n}=40)$; KD with regressed CALs $(\mathrm{n}=8)$; and KD with persistent CALs, such as persistent aneurysms, stenosis, and occlusion $(\mathrm{n}=5)$.

Results: CRP levels were significantly elevated in a KD group with persistent CALs compared with other KD groups. Regression analyses supported the association between the coronary artery lesions and elevated level of CRP.

Conclusions: The persistence of coronary lesions after $\mathrm{KD}$ was independently associated with elevated level of CRP suggesting that inflammation may be a novel functional aspect of coronary artery diseases late after $\mathrm{KD}$.

\section{VACCINATION STATUS AMONG GIPSY CHILDREN OF A RURAL AREA IN GREECE}

N Manakou, S Liosi, O Gkika, P Anastasopoulou, G Z Zantopoulos, E Garou, A Giannisi, Z Patrona

Department of Paediatrics, General Hospital of Korinthos, Korinthos, Greece

Background and Aims: Immunization is an effective strategy to reduce morbidity and mortality among children. The objective of the study is to determine the vaccination status among gipsy children of our region.

Methods: The study was carried out to assess the vaccination coverage by comprehensive analysis of routinely collected child immunization cards of gipsy outpatients ranging in age from 2 months to 14 years old, who were admitted to the emergency paediatric department of our hospital for the period 2003-2005. The sample consisted of 425 children (203 males and 222 females).

Results: Complete age-appropriate vaccination coverage for all vaccines recommended by the National Immunisation Program was $21 \%$. Immunization coverage (three doses) was $55 \%$ for diphtheriapertussis- tetanus, $58.7 \%$ for poliomyelitis, $35 \%$ for Haemophilus influenza and $61.3 \%$ for hepatitis B and $42.8 \%$ for first dose measles- rubella- mumps. Immunization coverage was $88 \%$ for measles (one dose) and $46.8 \%$ for tuberculosis.

Conclusions: A high proportion of gipsy children of all ages did not have an age appropriate vaccination status. 
The immunization coverage is too low to prevent epidemics in our region. Targeted parental education and timely vaccination strategies would be of essential value for individual and community protection against common preventable diseases.

\section{AUDIT OF CENTRAL LINE INFECTIONS ON PICU}

D J G Sidaras ${ }^{1}$, O Bagshaw ${ }^{2}$

${ }^{\text {I}}$ PICU, Alder Hey Hospital, Liverpool, ${ }^{2}$ PICU, Birmingham Children's Hospital, Birmingham, UK

Background and Aims: A working party had been formed in a tertiary children's hospital to look at the management of central venous lines. Information on positive cultures was available from the microbiology department. However there was a lack of denominator information i.e. number of lines inserted, type of lines, average duration of placement, reasons for removal etc. An audit was undertaken on PICU to address some of these issues.

Methods: Information on the patients, line and infection data was collected over a 2 months period (11/04-01/05) on all PICU patients who had a central venous line during their stay. Data continued to be collected until the line was removed.

Results: More than half of the 124 line episodes were in cardiac patients, $16 \%$ of all patients were immunocompromised. $1 / 3 \mathrm{rd}$ of the lines inserted were heparinised. Nearly $60 \%$ of the lines were placed into the internal jugular veins, $35 \%$ into the femoral veins. $2 / 3$ rds of the lines were in situ for less than 7 days, 15 lines were removed because of suspected infection. Of these only 5 were the source of the infection. However on routine cultures of line tips $1 / 3 \mathrm{rd}$ were colonised significantly without causing clinical concern.

Conclusions: Our audit did not show significant microbiological differences in the type of central line used (heparinised versus non-heparinised), site of insertion nor how long the line was in situ. The high rate of significant colonisation without clinical correlate led to the recommendation to suspend the routine microbiological work-up of the line tips.

\section{HIGH FREQUENCY OF VIRAL INFECTIONS IN CHILDREN UNDER THREE YEARS WITH COMMUNITY-ACQUIRED PNEUMONIA}

\author{
E Oñate ${ }^{1}$, G. Cilla ${ }^{2}$, E G Pérez-Yarza ${ }^{1}$, D Vicente ${ }^{2}$, \\ I Olaciregui ${ }^{1}, \mathrm{M} \mathrm{Montes}^{2}$ \\ ${ }^{I}$ Pneumology Unit, Department of Pediatrics \\ ${ }^{2}$ Department of Microbiology, Hospital Donostia, San \\ Sebastián, Spain
}

Background and Aims: Community-acquired pneumonia (CAP) is a frequent cause of children's hospitalization. Few studies have systematically analyzed the presence of viruses in children with CAP, main objective of this work.

Methods: Prospective study in children between 1-36 months of age diagnosed with CAP (clinical and radiology) that required emergency attention between october-1-2004/september-30-2005. Inhaled nasopharynx virus influenza A/B, RSV, Parainfluenza, Adenovirus, Metapneumovirus (hMPV), Coronavirus and Rhinovirus presence were investigated by means of cell culture and/or polymerase chain reaction.

Results: 241 children presented CAP. 77 cases were not included because of partial data and/or paternal non consent. The 164 included cases (91 male children and 72 female children), had an average age of 19.7 months (2-36 months) and 50 cases $(30 \%)$ were under 12 months. Viruses were identified in 104 cases (63\%), 37 in children under 12 months (74\%) and 67 in children above 12 months $(59 \%)(\mathrm{p}=0.06)$. The most frequent virus was the rhinovirus with 27 cases $(25,9 \%)$ followed by RSV (24\%) and other virus (33.6\%). Coinfections were found in 17 patients (16.3\%). 85/164 children were admitted (58 infected with virus), 8 of them to the PICU ward. A virus was found in 26 out of 34 who needed oxygen therapy $(76 \%)$ (the most frequent RSV, $n=8)$. There were no deaths.

Conclusions: Viruses are frequently detected in children under 3 years with CAP, being Rhinovirus and RSV the most prevalent. The results suggest that the viruses are cause or cofactors in most of the cases of CAP among children.

\section{PSYCHOLOGICAL FUNCTIONING AND HEALTH RELATED QUALITY OF LIFE IN ADULTHOOD FOLLOWING PRETERM BIRTH}

\begin{abstract}
S R Dalziel ${ }^{1,2,3}, \mathrm{~V} \mathrm{~K} \mathrm{Lim}^{4}$, A Lambert ${ }^{4}$, D McCarthy ${ }^{4}$, V Parag ${ }^{3}$, A Rodgers ${ }^{3}$, J E Harding ${ }^{2}$

${ }^{I}$ Great Ormond Street Hospital, London, UK, ${ }^{2}$ Liggins Institute, ${ }^{3}$ Clinical Trials Research Unit, ${ }^{4}$ Department of Psychology, The University of Auckland, New Zealand
\end{abstract}

Background and Aims: To determine if preterm birth alters socioeconomic status, psychological functioning and health related quality of life (HRQoL) in adulthood.

Methods: Prospective follow-up of 192 adult offspring whose mothers took part in a randomised controlled trial of antenatal betamethasone for the prevention of neonatal respiratory distress syndrome (66 born at term, 126 born preterm). Cognitive functioning was assessed using the Wechsler Abbreviated Scale of Intelligence. Working memory and attention was assessed using the Benton Visual Retention Test, the Paced Auditory Serial Addition Test and the Brown Attention Deficit Disorder Scale. Psychiatric morbidity was assessed using the Beck Depression Inventory II, the State-Trait Anxiety Inventory and the Schizotypy Traits Questionnaire. Handedness was assessed using the Edinburgh Handedness Inventory. HRQoL was assessed using the Short Form 36 Health Survey.

Results: Moderate prematurity (median gestation 34 weeks, mean birth weight $1,946 \mathrm{~g}$ ) did not alter later marital status, educational attainment, socioeconomic status, cognitive functioning, working memory, attention, or symptoms of anxiety or schizotypy at thirty-one years of age. Prematurity was associated with fewer symptoms of depression and higher levels of satisfaction in three of the eight HRQoL domains measured (bodily pain, general health perception and social functioning). 
Conclusions: Adults who were born moderately preterm have socioeconomic status, psychological functioning and HRQoL consistent with those who were born at term. This good long-term outcome cannot be extrapolated to those with early childhood disability or very low birth weights.

\section{A NEUROIMAGING INVESTIGATION OF LANGUAGE FUNCTIONS IN PRETERM CHILDREN WITH PERINATALLY ACQUIRED BRAIN LESIONS}

G B Northam ${ }^{1}$, F Liegeois ${ }^{1}$, A Morgan ${ }^{1}$, J S Wyatt ${ }^{2}$, B Vollmer ${ }^{3}$, T Baldeweg ${ }^{1}$

${ }^{I}$ Developmental Cognitive Neuroscience Unit, Institute of Child Health, ${ }^{2}$ Department of Paediatrics, University College London Medical School, The Rayne Institute,

${ }^{3}$ Neurosciences Unit, Institute of Child Health, University College London, UK

Background and Aims: The cognitive outcome of preterm children with perinatal brain injury is still poorly understood. Injury often involves periventricular white matter tracts, which are essential for speech and language functions. The study aimed to carry out a comprehensive assessment of speech, language and literacy abilities in preterm children aged 10-16 years with perinatal brain injury, and investigate associations with structural and functional imaging findings.

Methods: A cross sectional study of a cohort of children born $<33$ weeks gestation treated at a single tertiary neonatal unit. Children with unilateral or bilateral lesions on neonatal cranial ultrasound were eligible for inclusion in the study. Outcome was assessed using standardised tests of auditory processing (SCAN-A), speech (VMPAC, GFTA) and language functions including tests of grammar (TROG-2), expressive and receptive language (CELF-3 UK, EOWPVT, ROWPVT), and phonological (CTOPP) and literacy (WORD) abilities. Neuroimaging included conventional structural MRI (T1, T2 weighted sequences) for visual assessment of lesions, diffusion tensor imaging (DTI) for assessment of white matter integrity, and functional MRI (fMRI) to determine language lateralisation and reorganisation.

Results: Preliminary data in ten cases demonstrated a wide range of language skills from low to high levels of functioning, and normal speech outcome in the absence of a relationship to standard neuroradiological findings.

Conclusions: There is variability in the profile of language abilities in pre-term children with perinatal brain injury, which does not appear to be associated with findings on conventional structural MRI. However, it is expected that quantitative DTI measures will be more sensitive to white matter injury.

\section{DIFFUSION TENSOR IMAGING IN EXTREMELY LOW GESTATIONAL AGE INFANTS. A POPULATION BASED STUDY FROM STOCKHOLM}

B Skiöld ${ }^{1}$, J Bengtsson ${ }^{2}$, S Horsch ${ }^{1}$, B Hallberg ${ }^{3}$, Z Nagy ${ }^{2}$, B Nordell ${ }^{2}$, A Nordell ${ }^{2}$, M Mosskin ${ }^{4}$, $\mathrm{H}_{\text {Lagercrantz }}{ }^{1}, \mathrm{M}$ Blennow ${ }^{3}$, U Ådén ${ }^{1}$
${ }^{1}$ Institution Women and Child Health, Karolinska Institute, Stockholm, Sweden, ${ }^{2}$ Department of Medical Physics, Karolinska University Hospital, Stockholm, Sweden, ${ }^{3}$ Institution CLINTEC, Karolinska Institute, Stockholm, Sweden, ${ }^{4}$ Department of Neuroradiology, Karolinska University Hospital, Stockholm, Sweden

Background and Aims: The aim of this study was to investigate white matter (WM) abnormalities in a cohort of extremely low gestational age infants (ELGA $<27+0$ w) born in Stockholm 2004-2006.

Methods: 61 infants underwent magnetic resonance imaging (MRI, Philips Intera 1.5T) at term-equivalent age and diffusion tensor imaging (DTI, 6 diffusion directions) data of high quality was acquired in 32 infants with mean gestational age (GA) $25+3 \mathrm{w}(23+5-$ $26+6)$. Four regions of interest were chosen: The genu and the splenium of corpus callosum, the posterior limb of the internal capsule (PLIC) and the total WM at the level of centrum semiovale. Apparent Diffusion Coefficient (ADC) and Fractional Anisotropy (FA) values were measured.

Results: FA and ADC values were not influenced by GA at birth. Mean FA of the total white matter was lower in infants with diffuse excessive high signal intensities (DESHI) than in infants without DESHI $(\mathrm{p}<0.05)$. Moreover, low FA values in PLIC correlated to low Apgar scores $(\mathrm{p}<0.05)$.

Conclusions: We have obtained DTI in a cohort of ELGA infants. FA and ADC values did not change with GA, possibly due to the narrow range of GA in this cohort. In agreement with a previous study (Counsell et al, Pediatrics 2006), we found changes in diffusion in infants with DESHIs, indicating altered WM organisation. Interestingly, FA values in the PLIC at term-equivalent age also varied with Apgar score, indicating early impact on WM microstructure in a region where MRI findings correlates to later outcome.

\section{LONG-TERM EFFECT OF ZINC SUPPLEMENTATION ON GROWTH AND MENTAL DEVELOPMENT OF PRETERM INFANTS AT FOUR YEARS OF AGE}
N M Díaz-Gómez ${ }^{2}$, E Doménech ${ }^{1}$, F Barroso ${ }^{1}$, C Cortabarría ${ }^{1}$, I Ayala $^{1}$
${ }^{I}$ Department of Pediatrics, Faculty of Medicine, University of La Laguna, Tenerife, Spain, ${ }^{2}$ Nursing School, University of La Laguna, Tenerife, Spain

Background and Aims: Previous studies have shown that zinc supplementation has a positive short-term influence on growth; however, the long-term effect of zinc on growth and mental development of preterm infants has not been well studied, and these were our aims.

Methods: We studied 40 preterm infants fed with a standard term-infant formula supplemented with zinc (SG, $\mathrm{n}=20, \mathrm{BW}: 1645+440 \mathrm{~g} ; \mathrm{GA}: 32.2+2.3 \mathrm{w}$ ) or without supplementation (CG, $\mathrm{n}=20$, BW: $1700+326 \mathrm{~g}$.; GA: $32.1+2.0 \mathrm{w}$ ) from 36 weeks postconceptional age until 6 months corrected postnatal age (CA). At 9 and 12 months $\mathrm{CA}$ and at 4 years of age anthropometric variables and total body water (TBW) estimated by 
bioelectrical impedance (BI) were measured. Mental development (McCarthy scale) was evaluated at four years of age.

Results: On admission the SG and CG (W: $2.27+0.28$ vs $2.28+0.14 \mathrm{~kg}$; length: $45.2+2.0$ vs $45.9+1.6 \mathrm{~cm}$ ) did not differ in the variables studied. Analysis of variance test for repeated measurements showed significant differences between groups in length $(\mathrm{p}=0.005)$ and TBW ( $\mathrm{p}=0.02$ ), with higher values in the $\mathrm{SG}$ at 9 (length: $72.6+1.9$ vs $70.9+2.0 \mathrm{~cm}$; TBW: $4.5+0.5$ vs $4.1 \pm+0.4 \mathrm{~kg}$ ) and 12 months (length: $77.0+2.5$ vs $74.8+2.1 \mathrm{~cm}$; TBW: $6.0+0.7$ vs $5.6+0.6 \mathrm{~kg}$ ) and at 4 years of age (length: $105.1+3.3$ vs $102.5+3.0 \mathrm{~cm}$; TBW: $10.5+1.0$ vs $9.7+1.0 \mathrm{~kg}$ ). There were no significant differences between groups in the mental age $(4.1+0.6$ vs $4.3+0.3$ years).

Conclusions: Premature infants receiving zinc supplementation showed increased long-term linear growth at four years of age, but no difference in mental development was observed.

\section{EVIDENCE OF A SENSITIVE PERIOD FOR OLFACTORY LEARNING BY HUMAN NEWBORNS}

\author{
O Romantshik ${ }^{1}$, R H Porter ${ }^{2}$, H Varendi $^{1}$ \\ ${ }^{1}$ Department of Paediatrics, University of Tartu, Tartu, \\ Estonia, ${ }^{2}$ CNRS-INRA, UMR 6175, Nouzilly, France
}

Background and Aims: The olfactory system is functional at birth and learned responses to odours have been demonstrated during the first postnatal days. Labour contractions and vaginal delivery activate the locus coeruleus and result in a massive release of norepinephrine that rapidly declines during the first 1-2 hours after birth. Norepinephrine and locus coeruleus activity have been implicated in olfactory learning in rodent pups. We therefore hypothesized that a brief window of time immediately after delivery may be a particularly sensitive period for the development of olfactory learning by human neonates.

Methods: Vaginally delivered infants were exposed to an odorant for $30 \mathrm{~min}$. Beginning 4 -54 min. after birth (Early exposure) or 12-h post-partum (Late exposure). Several days later they were tested for their responses to the exposure odour versus an unfamiliar odour or an odourless control stimulus.

Results: Infants that had been exposed to an odorant immediately after birth spent significantly more time oriented towards that scent rather than a novel odour, or an odourless stimulus, during subsequent test trials. In contrast, infants in the Late exposure condition did not respond preferentially to the exposure odour.

Conclusions: Infants in the Early exposure group, but not those in the Late exposure group, became familiar with the exposure odour and retained a memory trace of it during the test trials. Newborn infants are physiologically prepared for efficient olfactory learning within a limited interval of time shortly after birth.
IS HISTOLOGICAL CHORIOAMNIONITIS A NEUROPROTECTIVE FACTOR FOR ELBW?

F Decobert, D Ciocca, V Champion, G Dassieu, C Danan

Centre Hospitalier Intercommunal de Creteil, Creteil, France

Background and Aims: The rapid evolution in the care of extremely premature babies requires evaluation of neurological outcome of infants treated. This study aims to evaluate known and possible risk factors of poor neurological outcome in a population of inborn ELBW $<28 w k$ GA. Using an ongoing systematic study of placentas, we tried to evaluate the role of histological chorioamnionitis (CAM) as a risk factor for poor neurological outcome.

Methods: Retrospective monocentric study. Neurological outcome was assessed at minimum age one year or later. A logistic regression model was used to analyse perinatal data, medical interventions and postnatal morbidity. Histological analysis of the placenta for CAM was available for $90 \%$ of the population.

Results: 133 inborn ELBW <28wk GA were admitted between 2000 and 2004. Among 105 survivors, 92 $(87.6 \%)$ underwent neuro-developmental evaluation at 25 months median age (11-65 months). 25 (27.1\%) had an abnormal neurological examination; strabismus or need for visual correction 7 , isolated motor dysfunction: 7, global developmental delay:11. Factors associated $(p<0.1)$ with poor neurological outcome were: venous umbilical catheterisation, postnatal systemic steroids, fluid load before day 3, and length of mechanical ventilation, central venous catheterisation or Doxapram therapy. Surprisingly, the absence of histological CAM $(\mathrm{p}=0,03)$ was also associated with abnormal neurological outcome and remains the only independent risk factor for poor neurological outcome after multivariate analysis. The analysis of clinical versus histological CAM as risk factors was not done.

Conclusions: Risk factors for abnormal neurological outcomes in this population include common factors and more unusual factors such as the absence of histological CAM.

\section{QUALITY OF GENERAL MOVEMENTS AT POSTNATAL DAY 1 IN PRETERM INFANTS}

J L M Bruggink, H J ter Horst, T Tameling, C V Hulzebos, A F Bos

Department of Pediatrics, Division of Neonatology, University Medical Center Groningen, Groningen, The Netherlands

Background and Aims: A reliable method to assess the presence of brain damage in infants is Prechtl's method of the qualitative judgement of general movements (GMs). The quality of GMs in preterm infants at postnatal day 1 has not been investigated so far. Our aim was to determine the quality of GMs in preterm infants at postnatal day 1 , and its relationship with perinatal variables. 
Methods: In 27 preterm infants, video recordings of GMs were performed within $24 \mathrm{~h}$ after birth and at weekly intervals. The quality of GMs was assessed as normal, abnormal or hypokinesia (H). Normal GMs are movements that involve the whole body in a complex sequence with variable intensity, force and speed. Abnormal GMs lack complexity and variability. $\mathrm{H}$ is defined as absence of GMs during at least 45 minutes recording.

Results: All infants showed GMs of abnormal quality at postnatal day 1 . Eleven infants (41\%) were hypokinetic. $\mathrm{H}$ occurred more often in infants born before 30 weeks of GA than in infants born after 30 weeks $(10 / 17$ vs $1 / 10, p<0.05$ ). Perinatal metabolic acidosis (base excess $\leq-5$ ) occurred more often in hypokinetic infants than in infants with abnormal GMs (10/11 vs $7 / 16, \mathrm{p}<0.05)$. $\mathrm{H}$ disappeared in all infants, and 13 infants had normal GMs after 2 weeks.

Conclusions: $H$ is common at postnatal day 1 in preterm infants, especially at gestational ages $<30$ weeks. Perinatal hypoxia, reflected in metabolic acidosis, may affect the quality of GMs at postnatal day

\begin{tabular}{l|ccc|l} 
& H & A & N & Total \\
\hline Day 1 & 11 & 16 & 0 & 27 \\
Days 8 & 2 & 11 & 11 & 24 \\
Day 15 & 0 & 9 & 13 & 22 \\
Abbreviations: & H=hypokinesia, A=abnormal & GMs. N=normal $\mathrm{GMs}$ &
\end{tabular}

\section{BEHAVIOURAL PROBLEMS IN EXTREMELY PRETERM INFANTS}

P Kutz ${ }^{1}, \mathrm{~S} \mathrm{Horsch}^{2}$, C Roll ${ }^{1}$

${ }^{I}$ Department of Neonatology, Children's Hospital Datteln, University Witten/Herdecke, Witten, Germany

${ }^{2}$ University Children's Hospital, Essen, Germany

Background and Aims: Behavioural problems in extremely preterm infants had not been paid much attention to, although parents often focus on this issue. We aimed to examine the prevalence of behavioural problems in a cohort of extremely preterm infants.

Methods: Data from all liveborn infants $(\mathrm{GA}<27 \mathrm{wk})$ born 2000 to 2002 at University Hospital Essen were recorded. Survivors had been followed-up by clinical examination and Bayley Scales II. Additionally, at 17 to 59 months corrected age, child behaviour was evaluated by Child Behaviour Check List (CBCL) filled out by the mothers.

Results: 79 infants were admitted. Forty-six (88\%) of 52 survivors were followed-up. Birth weight: $360 \mathrm{~g}$ $1300 \mathrm{~g}$ (median 680g), gestational age: 22-26 wk (median 25 wk). Behaviour Rating Scales of Bayley II were questionable in 7 and non-optimal in 3 children. 30 (83\%) mothers of the 36 children who showed a normal behaviour in the Bayley Scales filled out the CBCL: 5 (17\%) children showed an abnormal total problem score (1 'clinical range', 4 'borderline'). 12 (40\%) children were rated abnormal in one or several CBCL-Scales (7 in $>2$ scales), mainly for the 'withdrawn' and the 'emotionally reactive' scales. In the DMS-Oriented Scales 8 children showed abnormal behaviour (4 'clinical range').

Conclusions: CBCL was a helpful tool to detect behavioural problems missed by Bayley Scales II. 33\% of our extremely preterm infants with a normal score in
Bayley Behaviour Rating Scales showed behavioural problems in CBCL-Scales.

\section{OUTCOME OF INFANTS $<27$ WEEKS GESTATIONAL AGE}

\author{
L Kühn ${ }^{1}$, P Kutz ${ }^{2}$, C Roll ${ }^{1}$ \\ ${ }^{1}$ University Children's Hospital, Essen, Germany \\ ${ }^{2}$ Department of Neonatology, Children's Hospital \\ Datteln, University of Witten/Herdecke, Witten, \\ Germany
}

Background and Aims: Follow-up data show a constantly high rate of disability among extremely preterm infants. Therefore, it is important to assess short- and long-term outcome regularly to detect changes.

Methods: Data from liveborn infants $(\mathrm{GA}<27 \mathrm{wk})$ born $01 / 2003$ to $06 / 2004$ were recorded. Children were assessed at 18 months (17-22) corrected age by clinical examination, Bayley Scales II, Child Behaviour Check List (CBCL), assessment of height, body weight, head circumference.

Results: 41 infants were born. Birth weight: $355 \mathrm{~g}$ $1100 \mathrm{~g}$ (median $711 \mathrm{~g}$ ). 14 infants $(34 \%)$ died, 8 in the delivery room (22wk: $5 / 5 ; 23 w k: 3 / 8), 6$ in the NICU (24wk: 4/10; 25wk: 1/9; 26wk: 1/9). There were ten cases of IVH (I 5, II 4, III 1) and 15 of BPD among the 27 survivors. Follow-up is completed in 21 infants (23 wk: 4/5; 24 wks: 6/6; 25wks: 5/8; 26 wks: 6/8). Bayley Scales: Mental development-Scores (MDI) were $>84$ in 17, 84-70 in 2, and $<70$ in 2 infants. Motor development-Scores (PDI) were $>84$ in 17, 84-70 in 3, and $<70$ in 1 infant. Behaviour Rating Scales were questionable in 1 and non-optimal in 2 children. CBCL of 11 children showed normal behaviour in 7 children, abnormal (clinical) in 1 , and behaviour at risk (borderline) in 3 children. Height, body weight, and head circumference were below 2 SD in $(3 / 21),(5 / 21)$, (7/21). One child died three months after assessment.

Conclusions: 18-months outcome data of this recent cohort of extremely preterm infants were better than expected. However, neurodevelopment has to be reevaluated at school age.

\section{ASSOCIATION BETWEEN IL-6 -174 POLYMORPHISMS AND CYSTIC PERIVENTRICULAR LEUKOMALACIA IN PRETERM INFANTS}

A Radinger ${ }^{1}$, B Resch $^{1}$, C Mannhalter ${ }^{2}$, B Horvath ${ }^{2}$, A Binder ${ }^{1}$, W Zenz ${ }^{1}$, W Walcher ${ }^{3}$, B Pertl ${ }^{3}$, W D Mueller ${ }^{1}$

${ }^{1}$ Department of Paediatrics, Medical University Graz, Graz, Austria, ${ }^{2}$ Department of Laboratory Medicine, Medical University Vienna, Vienna, Austria ${ }^{3}$ Department of Gynaecology and Obstetrics, Medical University Graz, Graz, Austria

Background and Aims: The fetal inflammatory response syndrome plays a central role in the pathogenesis of white matter damage, i.e. cystic periventricular leukomalacia (PVL) in the preterm 
infant. High levels of proinflammatory cytokines including interleukin-6 (IL-6) have been observed in autopsies of brains of these infants. Thus, an association between IL-6 -174 (G/G, G/C or C/C) polymorphism, affecting IL-6 transcription rate and plasma cytokine levels, and development of PVL might be hypothesized.

Methods: The study group (cases) compromised infants with diagnosis of cystic PVL out of a single centre cohort from 1988 to 2005 and their parents. The control group (controls) included healthy newborns born between April and June 2002. Analysis of IL-6 -174 polymorphisms was performed in cases and their parents from venous blood samples after parental informed consent and in controls from umbilical cord blood samples. Samples were stored at -200 Celsius. IL-6 genotyping was performed using a polymerase chain reaction-restriction fragment length polymorphism (PCR-RFLP) technique. The study was approved by the local ethic committee. Results: Fifty-two cases (and 44 mothers) were enrolled and compared to 395 controls. Differences regarding $\mathrm{G} / \mathrm{G}, \mathrm{C} / \mathrm{C}$, and $\mathrm{G} / \mathrm{C}$ polymorphisms were not significant between groups. An increased rate of the IL-6-174 (C/C) polymorphism was found in mothers of cases with history of chorioamnionitis $(7 / 23,30.4 \%$; $\mathrm{p}=0.0192)$.

Conclusions: $\mathrm{We}$ found a significant association between IL-6-174 (C/C) polymorphism in mothers with chorioamnionitis and preterm birth with subsequent development of cystic PVL. In case of preterm PROM knowledge of this polymorphism might influence timing of delivery.

\section{PROGNOSTIC VALUE OF CEREBRAL ULTRASOUND ISOLATED VENTRICULOMEGALY AT DISCHARGE IN VLBW INFANTS}

\author{
M Lopez ${ }^{1}$, J de la Cruz ${ }^{2}$, C R Pallas ${ }^{1}$, E Gomez ${ }^{1}$, \\ C Gallego ${ }^{3}$, D Lora ${ }^{2}$
}

${ }^{1}$ Department of Neonatology, ${ }^{2}$ Clinic Epidemiology

Unit, ${ }^{3}$ Deparment of Radiology, Hospital 12 de Octubre, Madrid, Spain

Background and Aims: Parenchymal lesions and intraventricular haemorrhages in neonatal cerebral ultrasound (US) are known as the most informative neonatal prognostic findings. The prognostic value of US ventriculomegaly is less documented. To estimate developmental delay (DD) and cerebral palsy (CP) occurrence at two years in children with isolated ventriculomegaly at discharge vs. no abnormal US findings.

Methods: Cohort study of VLBW or $<32$ weeks infants born in a single center (1991-2002). Data were collected prospectively on US protocolised exams at 2, 7, 28 days and at discharge: for each type of lesion, the more severe was included. DD and motor development were assessed at two years as part of a standardised follow-up programme. Results: 1015 VLBW babies were born during the 12 year study period; from this analysis were excluded 190 who died, 29 with major malformations, 15 with incompleted US protocol, 90 lost-to-follow-up and 259 with abnormal neonatal US findings other than isolated ventriculomegaly. Assessment at 2 years was performed on 346 children with normal neonatal US and on 87 children with isolated ventriculomegaly. Children with ventriculomegaly had shown lower birthweight, lower GA and higher morbidity. DD was present in $9.2 \%(32 / 346)$ of the children with normal US and in $20.6 \%(18 / 87)$ of the children with isolated ventriculomegaly (odds ratio $2.6 ; 1.3-5.0$ ). No differences were found in $\mathrm{CP}$ rates between groups. Conclusions: Children with isolated ventriculomegaly showed a two-fold increased risk of developmental delay compared with children with no US abnormalities at discharge.

\section{TRIPLETS' DEVELOPMENTAL DISADVANTAGE - IS IT ALL ABOUT PERINATAL MORBIDITY?}

\author{
A M Graca ${ }^{1}$, C B Lima ${ }^{2}$, P Gomes ${ }^{3}$, F Torgal Garcia ${ }^{2}$, \\ $\mathrm{R}$ Gouveia $^{2}$, L J Da Silva ${ }^{1}$ \\ ${ }^{I}$ Neonatal Unit, Department of Pediatrics, ${ }^{2}$ Child \\ Developmental Clinic, Department of Pediatrics, \\ Hospital de Santa Maria, ${ }^{3}$ Department of Preventive \\ Medicine, Faculty of Medicine, University of Lisbon, \\ Lisbon, Portugal
}

Background and Aims: The risks of triple pregnancies are related to perinatal morbidity, but several other factors can influence development. The aims of this study are: 1) To identify significant perinatal and postnatal variables on triplets' development; 2) To compare development of triplets followed at the Developmental Clinic with those not followed and with age-matched controls.

Methods: We assessed retrospectively 15 sets of triplets born between 1998 and 2004. The triplets were divided in Group A (9 sets) followed at Developmental Clinic and Group B (6 sets), called for evaluation later. Medical records were reviewed for perinatal variables. Griffiths' scale was used for developmental assessment. Both groups were compared with each other and with age-matched control groups. Statistical analysis was performed with SPSS 12, considering significant $\mathrm{p}<0.05$.

Results: Statistical analysis comparing neonatal variables and Griffiths' total developmental quotient (DQ) is shown in Table 1. When compared to agematched controls DQs from group A were statistically similar, while DQs from group B were significantly lower.

Conclusions: Despite a more severe perinatal course, followed triplets had higher DQs than not followed ones. Better outcomes of babies that were followed could relate to anticipation of developmental issues and increased support to the family. Social-familial factors should be taken into account and subject to further research. Triplets, whatever their perinatal course, should be followed after discharge. 


\begin{tabular}{|lccc|}
\hline Gestational age (we eks) & Group A & Group B & $\mathrm{p}$ \\
Birth weight (grams) & 30,9 & 32,7 & 0,03 \\
5 minute Apgar score & 1378 & 1810 & 40,01 \\
Male sex & 9,2 & 9,7 & NS \\
Mechanical ventilation & $51,9 \%$ & $50 \%$ & NS \\
Nasal CPAP use & $40,7 \%$ & $16,7 \%$ & NS \\
Surfactant use & $55,6 \%$ & $22,2 \%$ & 0,03 \\
Sepsis & $51,9 \%$ & $33,3 \%$ & NS \\
Anemia & $29,6 \%$ & $0 \%$ & 0,01 \\
PDA & $51,9 \%$ & $11,1 \%$ & $\square, 01$ \\
ROP & $7,4 \%$ & $5,6 \%$ & NS \\
Length of stay (days) & $18,5 \%$ & $0 \%$ & 0,05 \\
DQ & 38,5 & 24,5 & 0,02 \\
\hline Table 1 - Statistical analys is & 104,1 & 90,2 & 0,01 \\
\hline
\end{tabular}

\section{EFFICACY OF SOLKOSERYL IN TREATMENT OF NEWBORNS WITH HYPOXIC-ISHEMIC ENCEPHALOPATHY}

F S Shamsiev, B A Burov

\section{Tashkent Medical Institute of Postgraduate Education, Tashkent, Uzbekistan}

Background and Aims: Hemorheological and metabolic disorders in hypoxic-ischemic encephalopathy require the beginning of early correction of these disorders as soon as possible and explains the search of new methods of treatment for newborns with this pathology.

Methods: To evaluate efficacy of Solkoseryl in the complex therapy of hypoxic-ischemic encephalopathy in newborns. Thirty newborns with hypoxic encephalopathy were studied. Solkoseryl was prescribed in dose $0.3-0.5 \mathrm{ml}$ ( $4 \%$ solution) per $\mathrm{kg} /$ body mass in a day. The indications for prescription were marked hypoxic-ischemic disorders identified with use of neurosonography, computed tomography and dopplerography. Solkoseryl was prescribed during the period of ear rehabilitation of hypoxic-ischemic disorders. The course of treatment was 10-20 injections. If it was necessary the course of therapy repeated every 4-6 weeks. Therapy was performed in 30 preterm infants.

Results: There is noted positive dynamics of neurological disorders regress: restoration of consciousness with appearance of attempts of visual contact with a child. Improvement of emotional sphere, restoration of the functions such as swallowing and suckling. The methods of neurovisualization were used for diagnosis of stopping of postischemic necrosis, as well as increase in blood flow velocity in the vertebrobasilar bed.

Conclusions: The active strategy in relation to infants with heavy hypoxic-ischemic disorders in the neonatal period including metabolic therapy with preparation Solkosery resulted in improvement of prognosis that is confirmed both by neurosonographic monitoring and dopplerography findings.

\section{INFLUENCE OF THERAPEUTIC TOUCH IN THE DEVELOPMENT OF PREMATURE NEWBORNS}

F León Larios, R Domínguez Rosales,

M J Garzón Real, M T Ruíz Pérez,

A A Rosado Poveda, M I Peñaloza Gómez,

E Gonzales Caro

Neonatal Intensive Care Unit, Virgin Macarena

University Hospital, Seville, Spain
Background and Aims: To analyse the effectiveness of therapeutic touch on weight, number of postnatal complications, hospital stay and parent satisfaction of premature newborns.

Methods: A random, controlled, experimental study in the Neonatal Intensive Care Unit in Virgin Macarena University Hospital of premature newborns who are randomly assigned to the control group and who fulfil the established protocol of the inclusion criteria for the following two years of study. To determine the sample size, weight gain is used as the main variable. We are considering approximately 80 subjects in the study (40 per group), to try to find a minimum relevant difference of 50gr, considering an alpha 0.025 as a significant level, and a unilateral character of the test hypothesis potency at $80 \%$. Data collection is done in a specifically designed registry, where the measures of variable results are reflected: Weight, postnatal complications, hospital stay, parent satisfaction and control variables. Statistical descriptions for the experimental and control group will be calculated. In order to compare quantitative information between the groups, a Student $\mathrm{T}$ test for independent samples or non parametric test U of MannWhitney.

Results: To date there are 11 children included in the study with a predicted 40 total by October, 2006, which will provide more conclusive results.

Conclusions: The conclusions will be presentable upon the inclusion of up to 40 participants as outlined in the results.

\section{MAXIMUM LENGTH SEQUENCE BAER IN PRETERM INFANTS AFTER PERINATAL HYPOXIA-ISCHAEMIA}

\section{Z D Jiang ${ }^{1,2}$, D B Brosi ${ }^{1}$, X M Shao ${ }^{2}$, A R Wilkinson ${ }^{1}$ \\ ${ }^{I}$ Department of Paediatrics, University of Oxford, John Radcliffe Hospital, Oxford, UK, ${ }^{2}$ Children's Hospital, Fudan University, Shanghai, China}

Background and Aims: Our understanding of the influence of perinatal hypoxia-ischaemia (HI) on the brainstem in preterm infants remains very limited. To further the understanding we examined functional status of the auditory brainstem following high-rate click stimulation in preterm infants.

Methods: The subjects were 35 preterm infants who had clinical signs of perinatal $\mathrm{HI}$ and a depression of Apgar score ( $\leq 6$ at $5 \mathrm{~min}$ ), with gestation 28-36 wks. The controls were 35 age-matched preterm infants with no perinatal problems. Brainstem auditory evoked response (BAER) was studied using maximum length sequence at 37-42 wks postconceptional age.

Results: The preterm infants with perinatal HI showed a significantly prolonged wave $\mathrm{V}$ latency at the highest click rate 910/s (ANOVA $\mathrm{p}<0.05$ ) and prolonged I-V interval at very high rates $455 / \mathrm{s}$ and $910 / \mathrm{s}(\mathrm{p}<0.01$ and 0.001 ). I-III interval was similar to the controls. III-V interval was significantly prolonged at all click rates $(91-910 / \mathrm{s})$, particularly at the very high rates $(\mathrm{p}<0.05$ 0.001). III-V/I-III interval ratio also increased significantly at all rates. Wave III amplitude was smaller at all rates $(\mathrm{p}<0.05-0.01)$. Wave $\mathrm{V}$ amplitude was significant smaller at 455/s and 910/s $(\mathrm{p}<0.01,0.001)$. 
These abnormalities became more significant with the increase in click rate.

Conclusions: BAER variables, mainly reflecting central auditory function, in preterm infants after $\mathrm{HI}$ were generally near normal at lower rates of clicks but abnormal at very higher rates (455/s and 910/s). Central impairment due to perinatal $\mathrm{HI}$ in preterm infants is unlikely to recover completely within a relatively short period.

\section{NEONATAL HYPOTONIA: PREVALENCE, SURVIVAL AND DIAGNOSTIC PROCEDURE}

\author{
G H J M Leyten ${ }^{1}$, LA Bok ${ }^{2}$, M J de Kleine ${ }^{2}$ \\ ${ }^{I}$ Deventer Hospital, Deventer, The Netherlands \\ ${ }^{2}$ Maxima Medical Center, Veldhoven, The Netherlands
}

Background and Aims: The differential diagnosis of neonatal hypotonia is extensive. This complicates the diagnostic process, also the absence of a practical quickscan diagnostic procedure delays the diagnostic process. Survival is uncertain. Our aim was to evaluate retrospectively the relative prevalence, causes and survival of neonatal hypotonia in newborns admitted to a level three NICU.

Methods: A retrospective cohort study of neonates presenting with neonatal hypotonia from January 1990 to January 1994 and from December 1996 to December 1999. Patients were subdivided into practical categories of neonatal hypotonia and the mortality rate was determined. The diagnostic procedure was evaluated.

Results: Discharge records of 134 patients with neonatal hypotonia were found. The study cohort was subdivided into the following 7 practical categories: asphyxia and/or intracranial bleeding $(\mathrm{n}=97)$, infection $(\mathrm{n}=5)$, medication $(\mathrm{n}=3)$, neuro-metabolic disorders $(\mathrm{n}=8)$, chromosomal disorders $(\mathrm{n}=7)$, syndromal disorders $(\mathrm{n}=8)$ and idiopathic neonatal hypotonia with total recovery $(n=6)$. Total mortality rate was $45 \%$ $(\mathrm{n}=60)$. The mortality rate in each category: asphyxia and/or intracranial bleeding $40 \%(\mathrm{n}=39)$, infection $60 \%$ $(\mathrm{n}=3)$, medication $33 \%(\mathrm{n}=1)$, neuro-metabolic disorders $100 \%(\mathrm{n}=8)$, chromosomal disorders $57 \%(\mathrm{n}=4)$, syndromal disorders $63 \%(\mathrm{n}=5)$.

Conclusions: The majority of neonatal hypotonia was acquired perinatal, especially by asphyxia and/or intracranial bleeding. 17,5\% was caused by an innate disorder. Mortality rate by innate disorders was very high, 17 out of 23 (74\%). All patients with a neurometabolic disorder died. In all patients with neonatal hypotonia the diagnostic procedure should include cerebral MRI and EEG; secondly metabolic and genetic assessment is indicated in hypotonic patients without asphyxia/intracranial bleeding or perinatal infection.

\section{DEVELOPMENTAL AGE INFLUENCES PATHOPHYSIOLOGICAL RESPONSE TO EXPERIMENTAL DIFFUSE TRAUMATIC BRAIN INJURY}

R Vink ${ }^{1,2}$, T Chang ${ }^{2,3}$, A I Faden ${ }^{2}$, F A Ahmed ${ }^{2}$, M I Cruz $^{2}$, B Stoica ${ }^{2}$, I Cernak ${ }^{2}$
${ }^{1}$ Discipline of Pathology, University of Adelaide, Adelaide SA, Australia, ${ }^{2}$ Department of Neuroscience, Georgetown University, Washington, DC, USA

${ }^{3}$ Departments of Pediatrics and Neurology, Children's National Medical Center, George Washington University Medical School, Washington, DC, USA

Background and Aims: Traumatic brain injury (TBI) is the most frequent cause of mortality and disability in children between 0-14 years of age. Although diffuse TBI represents the most common type of pediatric head trauma, there are few available experimental models to address the developmentally dependent response to such injury. We utilized a new rat model of diffuse TBI to study the graded response to head injury as a function of developmental age.

Methods: TBI was induced in 7-day old rats using the Cernak diffuse injury model. Edema formation was assessed by diffusion-weighted magnetic resonance imaging, bioenergetic state by magnetic resonance spectroscopy, blood brain barrier permeability by extravasation of Evans blue, functional outcome by motor and cognitive tests, and morphological changes by histology and immunohistochemistry.

Results: Injury induced vasogenic brain edema beginning $20 \mathrm{~min}$ post-injury and becoming primarily cytotoxic by $24 \mathrm{~h}$. There were no significant changes in brain energy metabolism after moderate trauma, suggesting a greater capacity of the immature brain to maintain normal cerebral bioenergetics after injury compared to adult animals. Diffuse morphological changes were found in the cortex, hippocampus, thalamus, and cerebellum of injured pups, including activation of microglia, caspase-3 and apoptosis inducible factor (AIF). These histological and histochemical changes were associated with long- term motor and cognitive deficits.

Conclusions: Our findings underscore the differences in the pathophysiology of TBI between young animals and adults and suggest that this new experimental model may be useful for evaluating differences in injury mechanisms and therapy as a function of developmental age.

\section{CONGENITAL MALFORMATIONS - INCIDENCE AND DISTRIBUTION AMONG ORGAN SYSTEMS}

M Kalajdzieva-Zip, J Guleva, B Mihajlovic, V Delovska, T Pensovska, S Jusufi, M Jegeni

Department of Neonatology, Special Hospital for Gynaecology and Obstetrics CAIR, Skopje, Macedonia

Background and Aims: Congenital malformations (CM) are defined as abnormal structure of the organism resulting from disrupted embryogenesis. Regarding different criteria and authors, the incidence of $\mathrm{CM}$ at newborns is between $2-7 \%$. The aim of this paper was to determine the incidence of $\mathrm{CM}$ at liveborns delivered at SHGO Cair, Skopje, during the five years period (2000-2004). Also, the distribution among organ systems had been analysed.

Methods: A retrospective analysis of 19097 liveborns delivered at SHGO Cair, during 2000-2004, has been 
performed. Database (Access 2000) from Neonatal Unit has been used. The incidence and percentage of $\mathrm{CM}$ among different systems have been determined.

Results: Among a total of 19097 leveborns, 736 or $3,85 \%$ have had CM. The incidence between different years was: $4,28 \%$ in $2000,3,92 \%-2001,3,79 \%-2002$, $3,20 \%-2003$ and 3,98\% in 2004. Regarding different organ systems, the distribution was: $58,1 \%$ of all $\mathrm{CM}$ were the anomalies of musculoskeletal system, $14,6 \%$ cardiopathies, $8,1 \%$ - anomalies of uro-genial system, $7,2 \%$ - CM of gastro-intestinal system, etc.

Conclusions: During the five years period, the incidence of $\mathrm{CM}$ is $3,85 \%$, which is in accordance with data from literature. Among years, the incidence varies from 3,2 to $4,3 \%$. The anomalies of musculoskeletal system are the most frequent, followed by those from cardiovascular, uro-genital, gastro-intestinal and central nervous system. Congenital malformations still remain an important medical and social problem requesting more serious nationwide engagement.

\section{NEONATAL AND MATERNAL CIRCULATING VISFATIN LEVELS IN NORMAL AND INTRAUTERINE GROWTH RESTRICTED PREGNANCIES}

A Malamitsi-Puchner ${ }^{1}$, D Gourgiotis ${ }^{2}$, M Boutsikou ${ }^{1}$, S Baka ${ }^{1}$, D Briana $^{1}$, L Kontara $^{1}$, M Kyriakakou $^{1}$, A Marmarinos $^{2}$, D Hassiakos ${ }^{1}$

\section{${ }^{I}$ Neonatal Division, 2nd Department Obstetrics and Gynecology, Athens University, Athens, Greece \\ ${ }^{2}$ Research Laboratories, 2nd Department of Pediatrics, Athens, Greece}

Background and Aims: Visfatin is a recently discovered cytokine that is preferentially produced in visceral adipose tissue. Its role appears to be controversial; on one hand, it facilitates fat deposition and increases in states of insulin resistance; on the other hand, it has been documented to exert insulin-mimetic effects in various tissues. This study aimed to investigate visfatin levels in full-term intrauterine growth restricted (IUGR- below the 10th customized centile) and appropriate for gestational age (AGA) fetuses (UC) and neonates on day 1 (N1) and 4 (N4) postpartum, as well as in their mothers (MS) before delivery.

Methods: Blood serum visfatin levels were measured in 20 IUGR and 20 AGA mother-infant sets by enzyme immunoassay.

Results: N1 and N4 visfatin levels were significantly higher in IUGR compared with AGA neonates $(\mathrm{p}=0.011$ and $\mathrm{p}=0.026$, respectively). In the AGA group, a positive correlation was demonstrated between N1 visfatin levels and infant centiles $(\mathrm{r}=0.452, \mathrm{p}=0.045)$; additionally, a similar correlation was found between N4 visfatin levels and infant centiles, as well as birth weight $(\mathrm{r}=0.455, \mathrm{p}=0.044$, and $\mathrm{r}=0.558, \mathrm{p}=0.011$, respectively). Finally, in the IUGR group, a negative correlation was documented between MS visfatin levels and infant centiles $(\mathrm{r}=-0.643, \mathrm{p}=-0.002)$.

Conclusions: Insulin resistance in IUGR neonates could possibly explain high N1 and N4 visfatin levels. The strong association between visfatin levels and centiles in
AGA neonates could be attributed to its production in adipose tissue. Pathology in pregnancy, leading to IUGR, could also be responsible for maternal visfatin levels.

\section{AUTOMATIC IDENTIFICATION OF CLINICALLY SIGNIFICANT CHANGES IN THE VITAL SIGNS OF NEONATES}

J Quinn ${ }^{1}$, C K I Williams ${ }^{1}$, B Wefers ${ }^{2}$, N McIntosh ${ }^{2}$

${ }^{1}$ School of Informatics, University of Edinburgh, Edinburgh, UK, ${ }^{2}$ School of Clinical Sciences and Reproductive Health, University of Edinburgh, Edinburgh, UK

Background and Aims: Alarms in a neonatal intensive care unit are triggered by vital signs measurements crossing preset thresholds. This is a crude way of drawing attention to changes in the physiological state of an infant, having low specificity and being affected by artifactual measurements. We aimed to construct a statistical model which could be used to infer clinically significant physiological changepoints at the cotside.

Methods: A factorial switching Kalman filter (FSKF) was used to model monitoring data. In previous work such a model has been used to identify monitoring artifacts. An extra factor (referred to as the 'X-factor') was added, accounting for observations not corresponding to either a baby's baseline dynamics or to any known artifactual patterns. Given test data, the FSKF can then infer the probability that the X-factor was active at any point. Eight test periods of monitoring data, 1-3 hours in duration and containing different types of physiological change, were taken from 4 infants. Measurements consisted of heart rate, blood pressures, and $\mathrm{SpO} 2$ sampled at $1 \mathrm{~Hz}$. An experienced clinician annotated the times that significant physiological changes occurred, which was used as a gold standard.

Results: FSKF inferences were compared against the gold standard using Receiver Operating Characteristics (ROC) curves. Evaluations were performed for different categories of physiological change, with the results shown in the table.

Conclusions: The FSKF model can be effective in identifying some types of clinically significant physiological changes in neonates.

\begin{tabular}{|l|l|l|}
\hline & Area under ROC curve & Equal error rate \\
\hline $\begin{array}{l}\text { Reaction to changing } \\
\text { ventilation settings }\end{array}$ & 0.96 & 0.08 \\
\hline Handling effects & 0.98 & 0.07 \\
\hline Onset of IVH & 0.95 & 0.10 \\
\hline Bradycardia & 0.97 & 0.08 \\
\hline
\end{tabular}

\section{PULSE OXIMETER USE IN NEONATAL RESUSCITATIONS: A VIDEO ANALYSIS}

G Bernstein, M R Rasmussen, D M Kaegi, P M Tillson, E E Stout, C Joseph, P R Wozniak

NICU, Sharp Mary Birch Hospital for Women, San Diego, CA, USA

Background and Aims: P.Ox display of $\mathrm{HR}$ and $\mathrm{SpO} 2$ is a useful guide during intubation attempts (Intub) and 
surfactant administration (Surf). Video-recording of resuscitations enables critical review and quality improvement.

Methods: We evaluated $\mathrm{HR}$ and $\mathrm{SpO} 2$ values during resuscitations of 33 infants $<1250 \mathrm{gm} ; 28$ were intubated for surfactant. We video-recorded P.Ox readings combined with the baby using a specially designed mobile video system.

Results: Infants arrived on the bed at $22.5+/-8 \mathrm{~s}$. Initial P.Ox readings were at $1 \mathrm{~min} 49 \mathrm{~s}+/-30 \mathrm{~s}$ after birth (all by $3 \mathrm{~min}$ ); $24+/-25 \mathrm{~s}$ (Median $14 \mathrm{~s}$ ) after sensor connected. Initial HR: $<100 \mathrm{bpm}$ in $9 / 33,>100 \mathrm{bpm}$ in $24 / 33$; and $\mathrm{SpO} 2:<60 \%$ in $12 / 33,60-80 \%$ in $7 / 33$, $>80 \%$ in $14 / 33$. HR remained $>100 \mathrm{bpm}$ during $39 / 42$ Intub, and $\mathrm{SpO} 2$ remained within $15 \%$ of baseline in $39 / 42$ Intub. After successful intubations, 3/28 had delayed improvement of $\mathrm{SpO} 2$ until ETT pulled back; all other 25/28 had increasing $\mathrm{SpO} 2$ within 5-10 s of completion. Surf was tolerated well in $24 / 28$. However, 2 infants (both $750-999 \mathrm{gm}$ ) decreased $\mathrm{SpO} 2$ to $77 \%$ and $68 \%$, and 2 infants (both 500-749 gm) decreased $\mathrm{SpO} 2$ to $36 \%$ and $27 \%$ - the latter with bradycardia related to insufficient ventilation and weaned $\mathrm{FiO} 2$. All recovered with increased ventilation and $\mathrm{FiO} 2$.

Conclusions: $\mathrm{HR}$ and $\mathrm{SpO} 2$ readings were readily obtained and helped guide adjustments to ventilation and $\mathrm{FiO} 2$, timing and duration of Intub attempts, as well as aid assessment of ETT position and tolerance to Surf administration. Supported by Nellcor (Tyco).

\section{NEONATAL OUTCOMES IN ASSISTED TWIN PREGNANCIES: INTRACYTOPLASMIC SPERM INJECTION (ICSI) VERSUS IN VITRO FERTILIZATION (IVF)}

\section{A A Zuppa, V D'Andrea, A Scorrano, F Cota, C Romagnoli \\ Department of Pediatrics, Division of Neonatology, Catholic University of Sacred Heart, Rome, Italy}

Background and Aims: The Assisted Reproductive Techniques (ART) have become a routine infertility treatment. International data suggest the high rates of multiple pregnancies, prematurity, low birth-weight, and neonatal morbility in ART conceived births. The aim of this study is to compare perinatal and neonatal outcomes in IVF twin pregnancies to those conceived by ICSI delivered at Gemelli Hospital.

Methods: We studied 29 assisted twin pregnancies: 12 ICSI and 17 IVF. All twins were evaluated for prenatal outcomes (maternal age, pregnancy complications and placentation) and neonatal outcomes (gestational age, birth-weight, small-for-gestational-age (SGA), birthweight discordance $>20 \%$, depression at birth, major neonatal diseases, NICU admissions and neonatal mortality).

Results: Data concerning prenatal outcomes, gestational age, birth-weight, rate of SGA, birth-weight discordance, depression at birth and neonatal mortality, were similar in the two groups. A significantly higher rate of ICSI twins affected by major neonatal diseases $(50 \%$ vs $20.6 \%, \mathrm{p}<0.05)$ and of NICU admissions $(50 \%$ vs $11.8 \%$, p 0.002) was observed comparing the two groups. No significant differences were observed in prenatal outcomes, gestational age and birth-weight matching the twins of the two groups according to the presence of major neonatal diseases and NICU admission. In the ICSI group we observed: one case of anencephaly, two cases of pharmacologically treated PDA and one case of surgically treated NEC.

Conclusions: Our study suggests a significantly higher rate of neonatal morbidity in ICSI twins than IVF twins. The adverse neonatal outcomes in ICSI group could be due to micromanipulation of gametes. Further studies are necessary to confirm these findings.

\section{THE INFLUENCE OF INCUBATOR HUMIDITY ON WEIGHT GAIN OF PREMATURE INFANTS}

\author{
O K Helder ${ }^{1}$, J B Van Goudoever ${ }^{1}$, P G M Mulder ${ }^{2}$ \\ ${ }^{I}$ Department of Pediatrics, Division of Neonatology, \\ University Medical Center Rotterdam, Rotterdam, The \\ Netherlands, ${ }^{2}$ Department of Epidemiology and \\ Biostatistics, University Medical Center Rotterdam, \\ Rotterdam, The Netherlands
}

Background and Aims: Very low birth weight infants need close control of their thermal environment in order to maintain their optimal temperature. Transepidermal water loss plays an important role in energy loss in case the humidity setting is inadequate. An optimal humidity protocol could lead to a reduction of time to regain birth weight. Aim of this study is to compare the effect of a mandatory and free chosen incubator humidity strategy to increase weight in preterm infants within the first two weeks of life.

Methods: Two groups of premature infants of 24-30 weeks gestational age and with a birth weight less than 1500 gram were studied in a prospective randomized controlled way. The Kaplan-Meier Survival Analysis was used to compare days to regain birth weight.

Results: 136 infants were enrolled, 71 infants in the control group and 65 infants in the experimental group. The demographic characteristics were not statistically different between both groups birth weight $(0.981+$ 0.245 vs $0.991+0.213) \mathrm{kg}$, mean gestational age (27.7 weeks +1.7 vs $27.7+1.6$ ). Analysis shows an equal number of days to regain birth weight in the control and experimental group (mean 9 vs. 9 days $95 \%$ CI 8-10 vs 7-11). In addition the mean infants central temperature during the first 14 days was not significant different.

Conclusions: Both humidification strategies are associated with similar outcomes.

\section{SHORTER HOSPITAL STAY FOR MODERATELY PRETERM INFANTS}

\author{
M Altman ${ }^{1}$, M Vanpée $^{2}$, A Bendito ${ }^{2}$, M Norman $^{1}$ \\ ${ }^{1}$ Clintec, Karolinska Institute, Stockholm, Sweden \\ ${ }^{2}$ Department for Women and Child Health, Karolinska \\ Institute, Stockholm, Sweden
}

Background and Aims: To determine length of hospital stay (LOS) for moderately preterm infants during the last twenty years and to identify factors affecting the number of bed-days. 
Methods: Review of LOS for all infants delivered between 30 to 34 gestational weeks during 1983, 1988, 1993, 1998 and 2002. Exclusion criteria: life threatening abnormalities, chromosomal anomalies and death during hospitalization.

Results: 564 included infants accounted for $20 \%$ of admissions and $48 \%$ of bed-days in the neonatal unit. Between 1983 and 2002, maternal age and use of nasal continuous positive airway pressure increased, use of antibiotics and mechanical ventilation decreased, whereas distributions for gestational age, birth weight, gender, smallness for gestational age, low Apgar score or RDS incidence did not change. For healthy inborn singletons discharged at home, LOS decreased from 1983 (28 $\pm 11 \mathrm{~d}$, mean \pm SD values) to $2002(14 \pm 7 \mathrm{~d}$, $\mathrm{p}<0.05$ ). Infants born more immature had longer LOS, but postconceptional age at discharge did not differ between age groups.

Conclusions: LOS for moderately preterm infants has decreased as a result of individualized neonatal care and organization of homecare support. Shorter LOS is of benefit for the family, prevents overcrowding in the NICU and has important economic implications.

\section{INTERIM REPORT OF OUTCOMES FROM A MULTI-NATIONAL INTERNET-BASED POSTGRADUATE EDUCATION PROGRAMME IN NEONATOLOGY}

M A Hall ${ }^{1}$, M Cuttini $^{2}$, G Greisen ${ }^{3}$, M Marlow ${ }^{4}$, A Schulze ${ }^{5}$, S L Smith ${ }^{1}$, P Truffert ${ }^{6}$, A Valls I Soler ${ }^{7}$

${ }^{I}$ Department of Neonatal Medicine, The Princess Anne Hospital, Southampton, UK, ${ }^{2}$ Unit of Epidemiology, Paediatric Hospital Bambino Gesù, Rome, Italy

${ }^{3}$ Department of Neonatology, Rigshospitalet, Copenhagen, Denmark, ${ }^{4}$ School of Human Development, University of Nottingham, Department of Child Health, Queen's Medical Centre, Nottingham, UK ${ }^{5}$ Department of Obstetrics and Gynaecology, Division of Neonatology, Klinikum Grosshadern, University of Munich, Munich, Germany, ${ }^{6}$ Department of Neonatal Medicine, Hospital Jeanne de Flandre, University Hospital, Lille, France, ${ }^{7}$ Neonatal Intensive Care Unit, Department of Paediatrics, Hospital de Cruces, Basque Country University Medical School, Bilbao, Spain

Background and Aims: The provision of a comprehensive programme of postgraduate education for trainees in neonatology can be a challenge for individual centres. Reasons for this include the small numbers of trainees in any one hospital and time constraints. The Internet offers the potential for the development of educational programmes which are standardised, flexible and widely accessible, irrespective of time and place. We report initial data concerning a multi-national European Commission funded project designed to provide a collaborative educational programme covering topics defined in the European training syllabus.

Methods: Six modules, incorporating a range of multimedia and case-based scenarios, have been developed for delivery within a "virtual learning environment". Topics include: neurology, ethics, epidemiology, nutrition, respiration and early care of the very low birthweight baby. Cross-cultural collaborative learning is promoted by internet-based discussions which are facilitated by a team of online tutors. Recruitment of trainees was via e-mail contact with members from the European Society of Paediatric Research (ESPR).

Results: 107 doctors from 15 countries have been enrolled. At the programme mid-point $77 \%$ were still participating of whom $67 \%$ provided evaluation data: $90 \%$ reported that the programme provides "added value" to their training, characterised as an "increase in the level of knowledge, the "sharing of knowledge and experience" and the "quality of the learning experience". The main limiting factor has been time.

Conclusions: Neonatal trainees require flexible, structured educational opportunities involving the sharing of clinical practice and experience. Online learning may help meet this demand and provide a focus for future ESPR educational programmes.

\section{IDENTIFICATION OF C-KIT-POSITIVE CELLS IN THE HUMAN UMBILICAL VEIN}

\author{
N Linke ${ }^{1}$, Z Novák $^{2}$, M Krecsmarik ${ }^{1}$, É Fekete ${ }^{1}$, \\ $\mathrm{H} \mathrm{Orvos}^{3}$, A Pál ${ }^{3}$, M Bagyánszki ${ }^{1}$ \\ ${ }^{I}$ Department of Zoology and Cell Biology, University of \\ Szeged, Szeged, Hungary, ${ }^{2}$ Department of Pediatrics, \\ University of Szeged, Szeged, Hungary, ${ }^{3}$ Department of \\ Obstetrics and Gynecology, University of Szeged, \\ Szeged, Hungary
}

Background and Aims: Steady blood flow between the foetus and the placenta is crucial for normal prenatal development. Since with the exception of the foetal end the umbilical cord lacks innervation, we hypothesised the presence of specialized pacemaker cells in the wall of the umbilical vessels that might contribute to the coordinated function of smooth muscle cells. C-kit protein is considered to be a specific marker for pacemaker cells in the gastrointestinal tract, known as interstitial cells of Cajal (ICCs) and c-kitimmunoreactive pacemaker cells have recently been discovered in the rabbit portal vein.

Methods: C-kit antibody was used on paraffin and on ultrathin sections of umbilical veins obtained from normal-term vaginal deliveries. C-kit-immunoreactivity was visualized by diaminobenzidine (DAB) and $5 \mathrm{~nm}$ gold-conjugated antibody.

Results: In paraffin sections a dense network of c-kitimmunoreactive cells was visible under the endothelial cell layer. The c-kit-immunoreactive cells had an irregular shape and numerous long processes extending to neighbouring cells. At the ultrastructural level, c-kitimmunoreactivity was most often seen in the cell processes. DAB precipitate and gold particles were localized around the endoplasmic reticulum, transport vesicles and caveolae.

Conclusions: Based on their c-kit-immunoreactivity and on their morphological characteristics we assume that the c-kit-labelled cells in the wall of the umbilical vein are ICC-like cells. ICC-like cells in human blood vessels have not been identified previously. In the human umbilical vein, the ICC-like cells may play an important role in the regulation of peristaltic-like waves of 
umbilical smooth muscle cells thereby ensuring a steady foeto-placental blood flow.

\section{THE CONCEPT OF CHRONIC PAIN IN THE NEONATE, A DELPHI SURVEY}

\section{J L M Van Ganzewinkel}

NICU, Máxima Medical Centre, Veldhoven, The

Netherlands

Background and Aims: A literature review revealed that the concept of chronic pain in the newborn has hardly received attention in pain research. Conceptualisation is required to develop evidence based pain assessment methods and to validate medical and nursing interventions.

Methods: A Delphi survey consisting of 3 rounds was conducted in an international and multidisciplinary panel of experts. The first round consisted of three openended questions and aimed at identifying the opinion of the experts on the definition, aetiology and diagnostic determinants of chronic pain. The two subsequent rounds aimed at reaching consensus on the opinions.

Results: Four of the originally 11 invited experts entered the study. 3 experts were included following recommendation by originally invited experts. In defining chronic pain the factor time was found to be essential. Necrotising enterocolitis is thought to be an aetiological determinant. None of the diagnostic determinants seemed to have sufficient sensitivity and specificity to be useful in the assessment of chronic pain. Several experts stated that chronic pain and stress were interrelated.

Conclusions: This study showed some methodological imperfections in both design and process. It is hardly possible to reach valid conclusions. We propose to speak in terms of 'prolonged pain', not chronic pain. This study suggests that prolonged pain in the newborn is pain lasting longer than 7 days. Prolonged pain is suggested to result from or can even exist in absence of noxious stimuli. Necrotising enterocolitis is suggested to be an important aetiological determinant. In this study, no diagnostic determinant with sufficient sensitivity and specificity was identified.

\section{HOW GOOD IS NEONATAL HIP EXAMINATION?}

M Vandeleur ${ }^{1}$, J F Murphy ${ }^{1}$, V B Donoghue ${ }^{2}$

${ }^{I}$ Department of Neonatology, National Maternity Hospital, Dublin, Ireland, ${ }^{2}$ Department of Radiology, The Childrens University Hospital, Dublin, Ireland

Background and Aims: Neonatal clinical examination for developmental dysplasia of the hip (DDH) remains controversial in relation to its sensitivity and specificity. This study evaluates the accuracy of neonatal hip examination in detection of truly dislocated hips.

Methods: Infants born National Maternity Hospital January 2004- December 2005 included. Following clinical suspicion of dislocated/dislocatable hip by consultant paediatrician, hip ultrasound day 3 performed. Infants with dislocated hips with dysplasia on ultrasound, treated with Pavlik harness; infants with dysplasia or mild instability followed with 8 week ultrasound or referred to orthopaedics. 8 week hip ultrasound performed for all infants with stable hips on exam but who met following criteria: (1) 1st degree relative with DDH; (2) breech; (3) persistant 'click'. All ultrasounds performed by same consultant paediatric radiologist (VBD).

Results: 15988 infants born during study period. 58 with unstable hips day 2 examination had day 3 ultrasound. Scan findings: 43 infants had dislocated hip(s), 5 had normal acetabuli with mildly subluxable hip(s), 2 had dysplasia, 8 normals. Thus neonatal exam for truly dislocated hips had positive pickup rate: $74.14 \%(43 / 58)$ and false positive rate: $25.86 \%(15 / 58)$. $45(77.59 \%)$ infants treated with Pavlik harness, 13 (22.41\%) followed with serial ultrasounds, no active intervention required. Based on above criteria, 1604 hip ultrasounds performed at 8 weeks. Scan findings: 4 $(0.25 \%)$ infants had dislocated hips, $50(3.12 \%)$ had dysplasia. In high risk infants, hip exam day 2 had false negative rate: $0.25 \%$.

Conclusions: Use of early hip ultrasound to inform the management of infants with clinically unstable hips reduces risk of unnecessary treatment by abduction splinting.

\section{SERUM ALBUMIN AS A PREDICTOR OF MORTALITY IN PRETERM INFANTS}

\author{
I Morris, E J Molloy \\ Department of Neonatology, National Maternity \\ Hospital, Dublin, Ireland
}

Background and Aims: Albumin has long been recognised as a predictor of patient outcome in adult medicine. However, its use as a prognostic tool remains less clear in paediatrics, particularly in neonates. We aimed to evaluate the use of serum albumin in neonates as a predictor of morbidity and mortality.

Methods: All neonates $<1500 \mathrm{~g}$ delivered in a tertiary referral centre were included over a 1 year period. The relationship between earliest and lowest recorded serum albumin levels and mortality and morbidity (incidence of IVH, NEC, and length of hospital stay) was evaluated.

Results: 115 infants were included with a mean (standard deviation) gestational age of 28.3 (2.7) weeks and birth weight of 1070 (275)g. There was a statistically significant association between lowest albumin levels and mortality $(\mathrm{p}=<0.001)$, which remained when adjusting for birth weight and gestational age $(\mathrm{p}=0.002)$. There was also an association with incidence of NEC ( $\mathrm{p}=0.027)$ although this did not remain with adjustment $(\mathrm{p}=0.067)$. There was no significant association between lowest albumin and either IVH $(\mathrm{p}=0.298)$, or length of hospital stay ( $\mathrm{p}=0.379$ ).

Conclusions: Although serum albumin did not predict morbidity, there was a statistically significant association with neonatal mortality in preterm infants. 


\section{EARLY BLOOD LACTATE CONCENTRATION AND CRIB SCORE AS PREDICTORS OF MORTALITY IN VERY LOW BIRTH WEIGHT BABIES}

\section{Phillips, C Dewhurst, A P Ganesan, H Isaac, R W Cooke, C W Yoxall \\ Department of Neonatology, Liverpool Women's Hospital, Liverpool, UK}

Background and Aims: Early identification of very low birth weight (VLBW) babies with a high risk of mortality may be beneficial. Blood lactate concentrations ([L]) have been shown to be associated with adverse outcomes in other critically ill patient groups. The aim of this study was to investigate the predictive value of [L] in VLBW babies and to compare this with CRIB score.

Methods: All babies admitted to the neonatal unit with a birth weight of less than 1501 grams during 2001-2004 were included. CRIB score, [L] in the first 12 hours and clinical outcome were obtained.

Results: Out of 409 babies, CRIB score was available in 327 babies and [L] in 374 babies. First [L] was significantly higher in babies who died compared to those who survived (median (range) 9.17 (1.26 to 21.1) $\mathrm{mmol} / \mathrm{L}$ vs 3.64 (0.67 to 17.9$) \mathrm{mmol} / \mathrm{L}, \mathrm{p}<0.0001)$. The highest [L] in the first 12 hours also was significantly higher in those who died compared to those who survived (10.20 (3.37 to 26$) \mathrm{mmol} / \mathrm{L}$ vs 3.84 (1.05 to 20.7) $\mathrm{mmol} / \mathrm{L}, \mathrm{p}<0.0001)$. The area under the ROC curves for predicting death for first [L] and highest [L] respectively were 0.772 (95\% CI $0.694-0.850)$ and 0.854 (95\% CI $0.799-0.908$ ). For CRIB score, the area under the ROC curve was 0.932 (95\% CI 0.893-0.968). Conclusions: Early hyperlactataemia is a predictor of death in VLBW babies. However, it does not appear to be better than CRIB score.

\section{FETAL ACID-BASE STATUS AT BIRTH AND EARLY NEONATAL OUTCOME AFTER GENERAL AND NEURAXIAL ANAESTHESIA IN WOMEN UNDERGOING ELECTIVE CAESAREAN SECTION AT TERM}

\author{
G Tonni ${ }^{1}$, C De Felice ${ }^{2}$, A Ventura ${ }^{1}$ \\ ${ }^{I}$ Division of Obstetrics and Gynecology, Guastalla Civil \\ Hospital, AUSL Reggio Emilia, Reggio Emilia, Italy \\ ${ }^{2}$ Department of Neonatology, Policlionic Hospitla 'Le \\ Scotte', University of Siena, Siena, Italy
}

Background and Aims: A prospective study involving 900 uneventful, term, singleton pregnancies undergoing elective Caesarean section. The aim of the study was to evaluate if acid-base status at birth and early neonatal outcome were statistically different in women undergoing general vs spinal and epidural anesthesia.

Methods: Acid-base assessment at birth was derived from blood samples obtained either from umbilical cord artery and vein. Neonatal outcome measurements considered were 1- and 5-min Apgar score $\leq 3$, transfer to NICU, need for ventilation, neonatal seizures, hypotonia and coma, neonatal hypoglicemia and survival.
Results: Fetal oxygenation as expressed by an umbilical cord artery PO2 showed higher values in women under general when compared to both spinal and epidural anaesthesia. Newborns in the epidural group demonstrated an higher $\mathrm{pH}$ higher BB. Early neonatal outcome showed statistical significant differences with regards to neonatal depression (1-min Apgar score $\leq 3$ ) between general anaesthesia and spinal and need for assisted ventilation, between general and spinal and epidural group.

Conclusions: General anaesthesia showed the highest umbilical artery $\mathrm{PO} 2$ values although epidural anaesthesia can be considered the method of choice in terms of highest metabolic parameters and lowest neonatal complications. General anaesthesia was associated with the highest incidence of neonatal depression although transient in duration and not severe in degree.

\section{END TIDAL CARBON MONOXIDE (ETCO) EXCRETION IN INFANTS EXPOSED TO PHOTOTHERAPY}

\author{
M Barak ${ }^{1,2}$, Y Littner $^{1,2}$, S Dollberg ${ }^{1,2}$, F Mimouni ${ }^{1,2}$ \\ ${ }^{l}$ Department of Neonatology, Tel Aviv Sourasky Medical \\ Center, Lis Maternity Hospital, ${ }^{2}$ Sackler Faculty of \\ Medicine, Tel Aviv, Israel
}

Background and Aims: It has been suggested that phototherapy might increase hemolysis, due to increase in heme oxygenase activity and/or oxidative injury to the red blood cells membrane. The objective of the study was to test the hypothesis that ETCO concentrations, an index of hemoglobin degradation, increase during phototherapy and decrease after its interruption in jaundiced neonates.

Methods: ETCOc concentration was measured using a $\mathrm{CO}$ breath analyzer, in 38 infants who developed hyperbilirubinemia requiring phototherapy. ETCOc measurements were performed before and at 12,24 and 48 hours of phototherapy and again before phototherapy interruption and 12 hours afterward. Analyses were by repeated measures ANOVA.

Results: Mean ETCOc before phototherapy was $2.4 \pm 0.6$ $\mathrm{ppm}$. No significant change in ETCOc occurred at 12, 24 and 48 hours of phototherapy $(2.3 \pm 0.6 \mathrm{ppm}$, $2.5 \pm 0.8 \mathrm{ppm}$ and $2.8 \pm 1 \mathrm{ppm}$ respectively), neither 12 after phototherapy interruption. Nine out of 38 infants (23\%) had ABO incompatibility (positive direct Coombs test). Mean ETCOc before phototherapy was higher in these infants than in the others $(2.8 \pm 0.4$ compared with $2.2 \pm 0.5 \mathrm{ppm})(\mathrm{p}=0.006)$, but remained also stable during the study period $(2.3 \pm 1.1 \mathrm{ppm}$, $3.2 \pm 0.8 \mathrm{ppm}, 3.3 \pm 1 \mathrm{ppm}$ and $3.0 \pm 1.1 \mathrm{ppm}$ at 12,2448 hours of phototherapy and 12 hours after its interruption, respectively).

Conclusions: Phototherapy does not appear to increase the rate of hemolysis, as demonstrated by the lack of change in ETCO concentration during phototherapy and after its interruption. Even in the presence of an existing hemolytic process, phototherapy does not affect the rate of hemoglobin degradation. 


\section{APGAR SCORES AND EARLY NEONATAL BIOCHEMISTRY}

C De Felice ${ }^{1}$, G Tonni ${ }^{2}$, A Del Vecchio ${ }^{3}$, G Latini ${ }^{3,4}$

${ }^{I}$ Neonatal Intensive Care Unit, Azienda Ospedaliera Universitaria Senese, Siena, ${ }^{2}$ Div. of Obstetrics and Gynecology Guastalla Civil Hospital, AUSL Reggio Emilia, ${ }^{3}$ Div. of Neonatology, Perrino Hospital, Brindisi, ${ }^{4}$ Clinical Physiology Institute, Nat'l Research Council of Italy (IFC-CNR), Lecce Section, Italy

Background and Aims: The 10-point Apgar score has successfully been used to assess the conditions and prognosis of newborn infants since $>50$ years. However, the relationship between Apgar scores and early neonatal biochemistry remains unclear. Methods: A total of 750 newborns (gestational age: $38.77 \pm 2.25 \mathrm{wk}$; birth weight $3195 \pm 610 \mathrm{~g}$ ) were evaluated for Apgar scores at 1-min (APG-1) and 5-min (APG-5) of life. Biochemical data were assessed by micro-methods on capillary blood sampling $1 \mathrm{~h}$ after birth. Associations between APG-1 or APG-5 and biochemical variables were tested using Spearman's coefficient of rank correlation (rho). Results: APG-1 was positively correlated with blood $\mathrm{pH}(\mathrm{rho}=0.258, \mathrm{p}=0.0097), \mathrm{pO} 2$ $(0.269, \mathrm{p}=0.0345), \mathrm{O} 2$-saturation $(0.271, \mathrm{p}=0.0068)$, $\mathrm{O} 2-\mathrm{Hb}(0.278, \mathrm{p}=0.0055)$, Co-Hb (0.214, $\mathrm{p}=0.0328)$, $\mathrm{Na}+(0.241, \mathrm{p}=0.0161), \operatorname{ABE}(0.211, \mathrm{p}=0.0345)$, and HCO3- $(0.226, p=0.0242)$, while was inversely related to $\mathrm{Hb}-\mathrm{H} \quad(-0.248, \mathrm{p}=0.0136)$ and met- $\mathrm{Hb} \quad(-0.257$, $\mathrm{p}=0.0101)$. APG-5 was positively correlated with blood $\mathrm{pH}(0.336, \mathrm{p}=0.0008), \mathrm{pO} 2(0.328, \mathrm{p}=0.0010), \mathrm{O} 2-$ saturation $(0.337, \mathrm{p}=0.0008), \mathrm{O} 2-\mathrm{Hb}(0.341, \mathrm{p}=0.0007)$, $\mathrm{Co}-\mathrm{Hb}(0.212, \mathrm{p}=0.0349), \mathrm{Na}+(0.230, \mathrm{p}=0.0217), \mathrm{ABE}$ $(0.278, \mathrm{p}=0.0055)$, and HCO3- $(0.285, \mathrm{p}=0.0045)$, while was inversely correlated with $\mathrm{Hb}-\mathrm{H}(-0.328, \mathrm{p}=0.0011)$ and met-Hb (-0.295, $\mathrm{p}=0.0032)$. APG-5, but not APG-1, was positively related to Hct $(0.226, p=0.0238)$. No significant relationships with $\mathrm{pCO} 2$, total $\mathrm{Hb}, \mathrm{Hb}-\mathrm{F}$, glucose, $\mathrm{Na}+, \mathrm{K}+, \mathrm{Ca}++, \mathrm{Cl}-$, lactate, total bilirubin, or SBE were evidenced. Conclusions: APG-1 and APG-5 are significantly linked to newborn's oxygenation, although the strength of the observed associations is weak.

\section{THE SKIN COLOR COMPONENT OF THE APGAR SCORE: A QUANTITATIVE SPECTROPHOTOMETRIC APPROACH}

C De Felice ${ }^{1}$, A Barducci ${ }^{2,3}$, P Corrocher ${ }^{4}$, G Tonni ${ }^{5}$, G Latini $^{6,7}, \mathrm{R} \mathrm{Casini}^{8}$

${ }^{I}$ Neonatal Intensive Care Unit, Azienda Ospedaliera Universitaria Senese, Siena, ${ }^{2}$ Institute of Applied Physics "Nello Carrara", Nat'l Research Council of Italy, Florence, ${ }^{3}$ Dept of Information Engineering, University of Siena, ${ }^{4}$ IntexSystem, Prato, ${ }^{5}$ Div. of Obstetrics and Gynecology, Guastalla Hospital, Guastalla-Reggio Emilia, ${ }^{6}$ Div. of Neonatology, Perrino Hospital, Brindisi, ${ }^{7}$ Clinical Physiology Inst., Nat'l Research Council of Italy (IFC-CNR), Lecce Section, ${ }^{8} \mathrm{Te} \&$ Co, Prato, Italy

Background and Aims: The value of the subjective color component of Apgar score remains controversial.
Our aim was to objectively evaluate skin color in the Apgar color classes: 0 ("blue" or pale) -1 ("pink" body with "blue" extremities)-2 (entire body "pink").

Methods: Skin color at 1, 5, and 10 min was rated in Caucasian term newborns $(\mathrm{n}=50)$ and simultaneously measured at 3 body sites (abdomen, hand, and foot) with a portable spectrophotometer unit (400-700 nm wavelengths range; standard D65 illuminant). "Pink" control newborns ( $\mathrm{n}=50$; age: $4 \mathrm{~h}$ ) were also measured. Spectral reflectance curves were analyzed and distances $(\Delta \mathrm{E})$ between colors for different body sites and color classes were calculated (Colormedica software package, IntexSystem).

Results: The $\Delta \mathrm{E}$ between Apgar color classes 0-1 and $1-2$ were $4.16 \pm 1.05$ and $4.62 \pm 0.18$ for the abdomen site, i.e., $>4$-folds the psychophysical limit of $\sim 1 \Delta \mathrm{E}$. By contrast, a significant overlap among color classes for the extremities was observed ( $\mathrm{p} \geq 0.25)$. $\Delta \mathrm{E}$ between the Apgar color class 2 (i.e., "pink") and controls) was $8.81 \pm 5.64$ (body site: abdomen) that is almost 9-folds the psychophysical limit.

Conclusions: (1) Color variations among the Apgar's color classes are extremely wide, with significant overlapping due to subjective classification; (2) color class 2 is significantly different from the "pink" color, as rated outside the early postnatal period, and the pink color as intended in standard colorimetry. These data indicate that the color component of the Apgar score should be replaced by objective measures.

\section{CANDIDA VERSUS BACTERIAL LATE-ONSET SEPSIS IN VERY LOW BIRTH WEIGHT INFANTS IN ISRAEL: A NATIONAL SURVEY}

I R Makhoul ${ }^{1}$, Y Bental ${ }^{2}$, M Weisbrod ${ }^{2}$, P Sujov ${ }^{1}$, A Lusky $^{3}$, B Reichman ${ }^{3}$

${ }^{I}$ Department of Neonatology, Rambam Medical Center, Haifa, Israel, ${ }^{2}$ Department of Neonatology, Laniado Hospital, Netanya, Israel, ${ }^{3}$ Women and Children's Health Research Unit, Gertner Institute, Tel-Hashomer, Israel

Background and Aims: Sepsis due to fungi accounts for $9-12 \%$ of late-onset sepsis (LOS) among very low birth weight (VLBW) infants, and is associated with significant morbidity and mortality. The aim of this population-based study was to evaluate the perinatal and neonatal risk factors for fungal LOS, as compared to bacterial LOS, in VLBW infants.

Methods: This is a population-based observational study including all VLBW infants born in all 28 NICUs in Israel. From 1995 through 2002, the Israel National VLBW infant database gathered information on 11830 infants. The study population comprised 3054 infants with one or more events of LOS. Univariate analysis and logistic regression models were used to compare infants with fungal sepsis only $(\mathrm{n}=179)$ and those with bacterial sepsis only $(\mathrm{n}=2630)$, regarding various perinatal and neonatal risk factors.

Results: The mean birth weight and gestational age of infants with Candida LOS were significantly lower (940 $\pm 271 \mathrm{~g} ; 27.1 \pm 2.5$ weeks) than those of the bacterial LOS group $(1027 \pm 255 \mathrm{~g} ; 28.3 \pm 2.5$ weeks $)(\mathrm{p}<0.001)$. Logistic regression analysis shows that Candida sepsis, 
as compared to bacterial sepsis, was independently associated with decreasing gestational age and bronchopulmonary dysplasia (BPD). In addition, BPD only (OR 1.84; 95\% C.I.:1.03-3.23) and BPD with postnatal steroid therapy (OR 2.66; 95\% C.I.: 1.59-4.46) were independently associated with an increased risk for Candida sepsis.

Conclusions: Compared to bacterial LOS, Candida LOS in VLBW infants was independently associated with decreasing gestational age and bronchopulmonary dysplasia (BPD), especially when BPD was accompanied with postnatal steroid therapy.

\section{MATERNAL CYTOMEGALOVIRUS (CMV) NEUTRALIZING ANTIBODIES AND CONGENITAL INFECTION}

A Y Yamamoto ${ }^{1}, \mathrm{~N}^{\mathrm{R}}$ Barbieri ${ }^{1}$, V M Wagatsuma ${ }^{1}$, $\mathrm{G}_{\text {Duarte }^{2}, \text { M M Mussi-Pinhata }}{ }^{1}$

${ }^{1}$ Department of Pediatrics, ${ }^{2}$ Department of Gynecology and Obstetrics, Faculty of Medicine of Ribeirão Preto, University of São Paulo, Hospital das Clínicas, Ribeirão Preto, São Paulo, Brazil

Background and Aims: CMV specific humoral immunity in pregnant women may play a role against viral reactivation and congenital infection. We compared CMV neutralizing (NTR) antibody titers between transmitter ( $\mathrm{T}$ ) and non-transmitter (NT) mothers.

Methods: Serum samples were obtained in the first weeks of pregnancy (median= 13 weeks) and at delivery from 26 mothers of infants identified as congenitally infected by CMV (T) and 26 mothers of non-infected infants (NT). CMV- neutralizing activity was quantified by a microneutralization assay in the presence of guinea pig complement with the AD169 CMV strain. In a subset of (T) mothers the neutralization assay was also performed with the viral isolate from the respective infant.

Results: Maternal age was similar in both groups (median=19 years). All mothers were found to be CMV seropositive at first weeks of gestation. By then, lower geometric mean titer of neutralizing activity was detected in $\mathrm{T}$ mothers $(87$; range: $8-256)$ than in NT mothers $(131 ; 32-256)(\mathrm{p}<0.01)$. Significant NTR titer increments were detected in both groups from gestation to delivery. However, reflecting higher increments among $\mathrm{T}$ mothers, geometric mean titers were similar in $\mathrm{T}$ (262; range: 64-512) and NT (236; 128-512) mothers $(p=0.17)$ at delivery. The use of infant $\mathrm{CMV}$ isolates for testing NTR activity in T mothers revealed comparable results to those found with CMV AD169 strains.

Conclusions: Low titers of neutralizing antibodies to CMV in the first weeks of gestation are a correlate of transmission of intrauterine CMV infection suggesting that high titers are protective of its occurrence. Funded by FAPESP.

\section{CAN WE REDUCE ANTIBIOTIC USAGE IN THE} TERTIARY NEONATAL UNIT

E M Stuwe ${ }^{1}$, M Horan ${ }^{1}$, R Mittal ${ }^{1}$, H Ibrahim ${ }^{1}$, $\mathrm{S}$ Paulus ${ }^{1}$, T J Neal ${ }^{2}$, C W Yoxall ${ }^{1}$

${ }^{I}$ Neonatal Unit, Liverpool Womens Hospital, Liverpool, Merseyside, UK, ${ }^{2}$ Department of Medical Microbiology, Royal Liverpool University Hospital, Liverpool,

Merseyside, UK

Background and Aims: Reducing the use of antibiotics in non-infected individuals has been identified as a potential strategy to reduce the emergence of multiresistant pathogens in the hospital environment. In response to local research and audit findings we have altered our practice: Antibiotics are stopped after 36 hours if cultures are negative and the baby is well. A direct computer link between the blood culture analyser and the unit allows 24 hour access to blood culture status. An audit was performed to measure the impact of these changes on the number of antibiotic doses administered on our unit.

Methods: The case notes of a random 5\% sample of babies with blood cultures taken before and after the new policies were examined. Demographics, clinical outcomes and number of antibiotic doses received by each baby were recorded.

Results: Gestational age, birth weight, gender and age were similar in both groups. There was no significant difference in the duration of hospitalisation or mortality rate between the 2 groups. The median (range) number of antibiotic doses per baby in 1998 was 25 (0 to 231) versus $14(0$ to 233$)$ in $2004(\mathrm{p}=0.021)$. The estimated reduction in total doses is 11000 per year (30 per day). The pattern of antibiotic use had also changed.

Conclusions: Significant reduction of the number of antibiotic doses administered can be safely achieved, decreasing nursing workload and babies' exposure to the hazards of intravenous drug therapy, and possibly helping to reduce the chances of the emergence of antibiotic resistant bacteria.

\section{CLINICAL AVAILABILITY OF NEONATAL BLOOD CULTURE RESULTS IN LESS THAN 48 HOURS}

E A M Roell-Schorer, E J d'Haens, A J de Beaufort, A J Sprij

Department Neonatology, Leiden University Medical Centre, Juliana's Children's Hospital, The Hague, The Netherlands

Background and Aims: Neonates at risk for sepsis receive empirical antimicrobial treatment for 72 hours, pending negative culture results. Benefits to minimise treatment duration are evident. The Bactec 9240 detects $100 \%$ positive blood cultures within 30 hours. Thus, can length of antibiotic therapy be shortened in these neonates? The aim of this study is to determine the time at which neonatal blood cultures can be considered negative.

Methods: In a one-year (2005) prospective study we collected 249 blood cultures from neonates admitted to our neonatal unit. Time to positivity (TTP) (the time 
between culture sampling and the moment the neonatologist received the results) was calculated. Incubation time was automatically registered.

Results: 48 out of 249 blood cultures were positive (20\%). 98\% (47/48) of the positive cultures were detected within 48 hours (figure). One sample had a TTP of 50 hours. Maximum incubation time was 29,6 hours.

Conclusions: In clinical practice neonatal blood cultures can be considered negative at 48 hours. We suggest that antibiotic treatment for 48-hours in asymptomatic neonates is sufficient. Logistic optimisation can further reduce treatment duration.

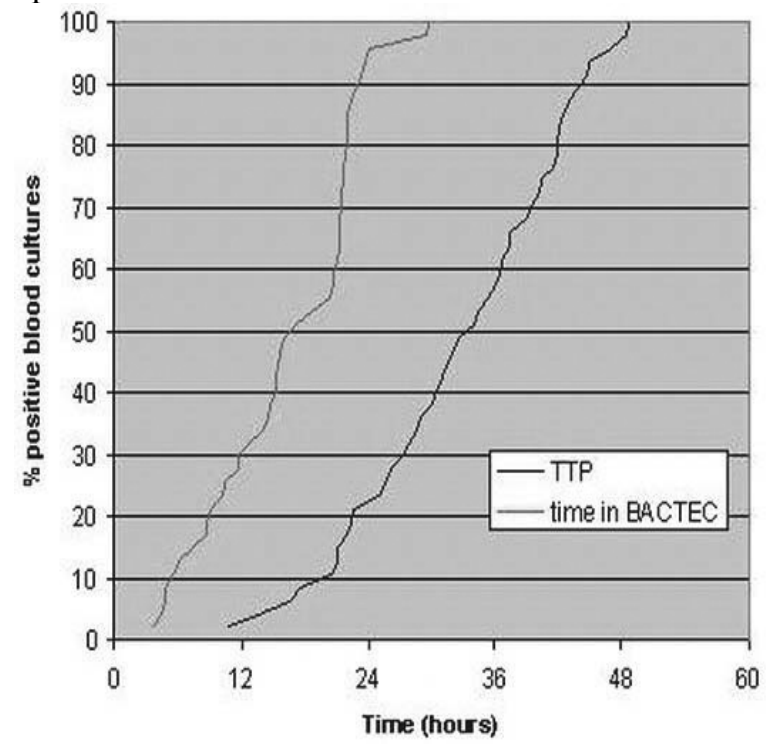

\section{IMMIGRATION FROM EASTERN EUROPE: REAPPEARANCE OF CONGENITAL SYPHILIS IN THE ITALIAN POPULATION}

E Tridapalli, M G Capretti, M Stella, G Brighi, F Vitali, G Faldella

Department of Preventive Paediatrics and Neonatology, St. Orsola-Malpighi General Hospital, University of Bologna, Bologna, Italy

Background and Aims: To evaluate the prevalence of syphilis in pregnancy and congenital syphilis (CS) in an Italian city, in relation to the increased immigration flow.

Methods: A prospective surveillance study was carried out in Bologna, Italy, from November 2000 to July 2005. All pregnant women were screened for syphilis at delivery by enzymatic immunoassay. Positive samples were then analysed by $\mathrm{T}$. pallidum haemoagglutination assay (TPHA), Western Blot (WB) and rapid plasma reagin (RPR) tests. Infants born to seropositive mothers were enrolled in a prospective follow-up.

Results: During the study period 16932 women gave birth to 17238 infants. 79 infants were born to 70 mothers seropositive at delivery. The syphilis seroprevalence in pregnant women was 0.41 , but the immigrant women were more likely to be seropositive than Italian women $(p=.0001) .8$ women $(11.4 \%), 7$ from Eastern Europe, were found positive only at delivery as they did not receive any prenatal care. Eight infants $(11.6 \%)$ born to mothers found to have syphilis at delivery were all asymptomatic. 5 had both reactive
IgM WB and RPR tests and were considered as infants with highly probable disease. All 5 infants were born to mothers coming from Eastern Europe. Three out of five were preterm.

Conclusions: The prevalence of CS in our area is 0.33 per 1000 live births, and it rises to 5.9 per 1000 live births in infants born to mothers from Eastern Europe. Therefore a closer prenatal screening for syphilis is advisable for immigrant women to prevent CS.

\section{TREATMENT OF NEONATES WITH CENTRAL VENOUS CATHETER RELATED COAGUALSE- NEGATIVE STAPHYLOCOCCAL SEPSIS USING CONTINUOUS-INFUSION VANCOMYCIN THERAPY}

\section{A B Kapetanakis, S Bradley, K Farrer \\ Regional Neonatal Unit, St George's Hospital, London, $U K$}

Background and Aims: Coagulase negative staphylococci (CONS) are the most common cause of catheter related blood stream infection (CR-BSI) in neonates. Removal of the central venous catheters (CVC's) is often necessary. Attempts to treat using conventional vancomycin dosing without removal of the CVC's have limited success. Vancomycin exhibits time dependent activity. Continuous infusion (CI) of the drug improves serum concentrations and bactericidal activity. Methods: Prospective data collection from infants treated for CR-BSITreatment was commenced using CI Vancomycin administered via the CVC. All received a loading dose $(15 \mathrm{mg} / \mathrm{kg})$ followed by CI. The starting doses were $15 \mathrm{mg} / \mathrm{kg} /$ day- $30 \mathrm{mg} / \mathrm{kg} /$ day depending on initial serum creatinine level. Blood cultures where obtained before and during the antibiotic treatment, CRP and serum creatinine were monitored. Vancomycin levels were obtained at 24-48 hour intervals and the dose adjusted accordingly. Target Vancomycin concentrations were $15-25 \mathrm{mcg} / \mathrm{ml}$.

Results: Mean ( \pm Standard Deviation) 47 infants were treated.7 had Broviac (BC), 40 Percutaneous-inserted Catheters (PICC's) 59 treatment episodes were evaluated (14 in the BC group, 45 in the PICC group). The gestational age was $25.1( \pm 3.2)$ weeks, the birth weight was $0.74( \pm 0.8) \mathrm{KgPICC}$ 's where in place for 22 $( \pm 0.9)$ days and the BC's for $70.6( \pm 19)$ days. The treatment duration was $7.8( \pm 0.7)$ days in the infants with PICC,13.2 ( \pm 6.6$)$ days in the infants with BC. All patients had clinical improvement before the antibiotic course was completed.

Conclusions: CONS CR-BSI was successfully treated using CI vancomycin allowing for CVC's retention. CI vancomycin may offer advantages in treatment of CONS sepsis when the CVC cannot be removed.

\section{ROLE OF ROUTINE LUMBAR PUNCTURES IN NEONATAL SEPSIS - ARE THEY JUSTIFIED?}

R F Kornelisse ${ }^{1}$, C E P M Duijsters ${ }^{2}$

${ }^{l}$ Division of Neonatology, Department of Pediatrics, Erasmus MC - Sophia Children's Hospital, University Medical Center, Rotterdam, The Netherlands 
${ }^{2}$ Division of Neonatology, Department of Pediatrics, Máxima Medisch Centrum, Veldhoven, The Netherlands

Background and Aims: Neonatal meningitis has a high mortality and frequent neurological sequelae. The importance of a lumbar puncture (LP) as part of the diagnostic evaluation of neonates with suspected sepsis has been subject of debate. Neonatologists often defer the LP when evaluating the very low birth weight (VLBW) infant $(<1500$ gram). We evaluated the utility of LP's done routinely as part of complete workup in neonatal sepsis.

Methods: A retrospective study was conducted to evaluate LP's performed in VLBW infants admitted between January 1, 1997, and January 1, 2004 who survived for at least 3 days at the neonatal intensive care unit (NICU) of Erasmus MC - Sophia Children's Hospital. Excluded were patients with posthemorrhagic hydrocephalus requiring treatment. Late-onset meningitis was defined by a CSF culture positive for bacteria or fungi.

Results: Of 1443 VLBW infants, 798 LP's were performed in 544 patients. Thirteen infants had lateonset meningitis acquired at the NICU. Thirty-one percent of the infants had seizures. The cultured microorganisms from CSF causing meningitis were $\beta$ hemolytic streptococcus group B $(n=1)$, Escherichia coli $(n=3)$, Klebsiella pneumoniae $(n=1)$, Staphylococcus aureus $(n=3)$, Coagulase-negative Staphylococci $(n=3)$, Candida albicans $(n=2)$. Only 1 of $13(8 \%)$ infants with meningitis had negative blood cultures.

Conclusions: Since only $1,6 \%$ of all LP's showed meningitis, it is questionable whether LP's should be routine investigation in VLWB infants suspected of an infection. We suggest performing a LP in the presence of seizures and/or in patients with blood cultures positive for non-CoNS microorganisms.

\section{TRANSMISSION OF CYTOMEGALOVIRUS TO PRETERM INFANTS THROUGH BREAST MILK}

Z Horvath, E Polonkai, T Kovacs, N Katona, B Kovacs-Paszthy, J Kovacs, G Balla

Department of Obstetrics, Division of Neonatology, University of Debrecen, Debrecen, Hungary

Background and Aims: Breastfeeding and breast milk has many known advantages, both to newborns and to mothers. However, breast milk can also be the source of infections transmitted from mothers to newborns, and CMV transmission is probably the most common one. Premature infants who acquire postnatal CMV infection may develop a sepsis-like syndrome characterized by respiratory distress, jaundice, hepato-splenomegaly, neutropenia, thrombocytopenia, and lymphocytosis. The aim of our study was to estimate the significance of the postnatal CMV infection in preterm infants.

Methods: In order to estimate the rate of severe CMV infection the records of 413 preterm infants with birth weight under $1500 \mathrm{~g}$ were reviewed retrospectively. Own breast milk was universally administered from the first day of life.

Results: The number of infants who had symptoms and laboratory abnormalities that might have caused by
CMV was 30. Two of them had sepsis-like syndrome. There is no screening for CMV among infants fed by breast milk in our protocol, antibody measurement is done when CMV is the possible causative agent of the clinical and laboratory abnormalities. Only 5 infants of the 30 presented positive result. There was no infant death linked to CMV among the 413 preterm neonates.

Conclusions: In the light of the low number of serious cases probably attributable to $\mathrm{CMV}$, the fresh breast milk may have potential risk for CMV infection in premature infants, but the well known advantages of breast milk outweigh this effect. Methods for milk treatment and prevention of CMV infection are still being under evaluation.

\section{IMPACT OF MATERNAL CHORIOAMNIONITIS ON OUTCOMES IN 28-32 WEEKS GESTATION INFANTS}

\section{S Bhargava, M Khashu}

\section{$B C$ Children's Hospital and University of British Columbia, Vancouver, BC, Canada}

Background and Aims: Histological chorioamnionitis has been associated with preterm birth, major perinatal morbidity and neonatal death. We investigated whether clinical diagnosis of chorioamnionitis in a preterm cohort would impact on need for resuscitation, neonatal mortality and infant mortality.

Methods: This is a retrospective analysis of data from the BC Perinatal Database Registry and Perinatal Reporting Tool of the BC Reproductive Care Program. We compared need for resuscitation at delivery, neonatal and infant mortality among 28-32 weeks gestation infants born between 1st April 1999 and 31st March 2002, with and without a clinical diagnosis of maternal chorioamnionitis. Statistical analysis was performed with chi-square test. Resuscitation was defined as use of IPPV by mask or ETT, chest compression, or drugs for resuscitation.

Results: A total of 1141 live born infants were delivered at 28-32 weeks gestation during the study period. Outcome measures are depicted in the table.

Conclusions: A clinical diagnosis of maternal chorioamnionitis increased the risk of need for resuscitation suggesting that these infants were born in a worse clinical condition. However, neonatal and infant mortality was not affected by a clinical diagnosis of maternal chorioamnionitis in this preterm cohort.

\begin{tabular}{|c|c|cc}
\hline NEONAT AL OUT COME & $\begin{array}{c}\text { CLINICAL DIA GN OSIS OF } \\
\text { CHORIOAMIIONIT IS }\end{array}$ & $\begin{array}{c}\text { CHORIOAMNIONIT IS } \\
\text { NOT SUSPE CTED }\end{array}$ & P value \\
\hline Need for resurcitation & $29 \%$ & $39 \%$ & 0.04 \\
Neonatal mortality & $1.7 \%$ & $2.9 \%$ & 0.4 \\
\hline Infant mortality & $1.7 \%$ & $3.4 \%$ & 0.3 \\
\hline
\end{tabular}

\section{EFFECT OF MATERNAL STEROID ON ANTIMICROBIAL PEPTIDES ACTIVITIES IN AMNIOTIC FLUID}

H Yoshio $^{1}$, M Yamada ${ }^{2}$, M Yoshida ${ }^{2}$, A Takeuchi ${ }^{1}$,

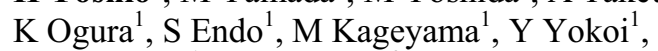
Y Yamauchi $^{1}$, B Agerberth ${ }^{3}$ 
${ }^{I}$ Department of Neonatalogy, Okayama Medical Center, Okayama, Japan, ${ }^{2}$ Department of Virology, Okayama Medical School, Okayama, Japan, ${ }^{3}$ Department of Medical Biochemistry and Biophysics, Karolinska Institute, Stockholm, Sweden

Background and Aims: Antimicrobial peptides are present in amniotic fluid and their activities increase with perinatal infection. Our aim is to examine whether the maternal steroid treatment affects the antimicrobial peptides activities in amniotic fluid.

Methods: Amniotic fluids from eleven infants were examined. Five of all were with maternal steroid and six without steroid. The gestational ages and birth weights of the former and later groups were $30 \pm 1.8 \mathrm{w}$ and 1063 $\pm 391 \mathrm{~g}, 33 \pm 2.5 \mathrm{w}$ and $1658 \pm 625 \mathrm{~g}$, respectively. Maternal betamethazone $(12 \mathrm{mg})$ treatments were intramuscularly carried out twice before birth. The collected amniotic fluid was adjusted with $0.1 \%$ trifluoroacetic acid (TFA). For enrichment of proteins/peptides these solutions were passed through OASIS columns at $4 \mathrm{C}$ and eluted peptides/proteins were lyophilized. These samples were analyzed for antimicrobial activity against Bacillus megaterium (strain Bm11), using an inhibition zone assay. Lysozyme activity also was examined with Micrococcus luteus cell walls. LL-37 was measured with ELISA.

Results: The diameters of inhibition zone assay against Bm11 with and without maternal steroid were $4.8 \pm 0.4$ and $5.2 \pm 0.6 \mathrm{~mm}$, respectively, and they were no significant. There were also no significant in lysozyme activity between the two groups. On the other hand, the LL-37 concentrations with maternal steroid were significantly lower than without one $(5.0 \pm 2.5$; $11.8 \pm 6.0 \mathrm{ng} / \mathrm{ml}, \mathrm{p}<0.05)$.

Conclusions: In this study, the maternal steroid treatment does not strongly seem to affect antimicrobial peptides activities in amniotic fluid, but partially seem to affect the peptides that are inductive with inflammation such as LL-37.

\section{MOTHER TO CHILD TRANSMISSION OF THE HUMAN IMMUNODEFICIENCY VIRUS (HIV) IN PARMA: A RETROSPECTIVE STUDY}

C Ruspaggiari ${ }^{1}$, P L Bacchini ${ }^{1}$, E Romanini ${ }^{1}$, I Dodi ${ }^{2}$, G C Izzi $^{2}$, G Bevilacqua ${ }^{1}$

${ }^{1}$ Department of Gynaecology, Obstetrics and Neonatology, University Hospital, Parma, Italy

${ }^{2}$ Infective Disease Section of Pediatrics and Oncoematology, University Hospital, Parma, Italy

Background and Aims: We carried out a retrospective study on the decreasing rate of vertically transmitted (mother to child) HIV infection, after following a specific protocol.

Methods: Since the year 1997 the protocol consists in: administering Zidovudin (following the ACTG076 protocol) to both the pregnant woman and the newborn, programmed cesarean delivery at the $38^{\wedge}$ week of pregnancy and prohibiting breast feeding. A series of controls were carried out in all newborns from the moment of birth till the age of 12 months.
Results: From January 1997 to December 2005, we examined 58 newborn babies; after the first year followup just a child $(1,7 \%)$ resulted positive for HIV and this was the child delivered naturally through the vaginal canal due to a precipitated birth. We analysed and compared these results with those during the period from 1994 to 1996 when this protocol was not yet adopted and it resulted that out of the 36 children delivered during this period, the HIV virus was transmitted in $5(13,9 \%)$ cases. A cause to an increase in premature births in the presence of both HIV and other sexually or blood transmitted diseases (hepatitis, condylomatosis, genital herpes, syphilis) as was being found in 32 pregnant women, i.e. from $13 \%$ in women with only HIV infection to $25 \%$ in those with HIV associated to other diseases.

Conclusions: In conclusion we believe that this protocol is efficient in modifying the number of vertically transmitted infections and we therefore sustain that it is absolutely important carrying out an HIV test in every pregnant woman.

\section{CASPOFUNGIN THERAPY OF VERY LOW BIRTH WEIGHT INFANTS WITH PERSISTENT INVASIVE CANDIDIASIS}

G Dimitriou ${ }^{1}$, S Tzifas ${ }^{1}$, A Mermiga ${ }^{1}$, I Giannakopoulos $^{1}$, V Pavlou ${ }^{1}$, A Darzentas ${ }^{1}$, $\mathrm{M} \mathrm{Marangos}^{2}$, M Christofidou ${ }^{3}, \mathrm{~S}_{\text {Mantagos }}{ }^{1}$

${ }^{l}$ Neonatal Intensive Care Unit, Department of Paediatrics, School of Medicine, University of Patras, Patras, Greece, ${ }^{2}$ Department of Infectious Diseases, School of Medicine, University of Patras, Patras, Greece, ${ }^{3}$ Department of Microbiology, School of Medicine, University of Patras, Patras, Greece

Background and Aims: Invasive candidiasis is an important cause of morbidity and mortality in very low birth weight (VLBW) infants in neonatal intensive care units. Occasionally, despite administration of amphotericin B invasive candidiasis is complicated by persistent fungemia. Caspofungin is a novel antifungal agent. The experience of caspofungin use is very limited in neonates. The aim of this study was to evaluate the effectiveness of caspofungin in VLBW $(<1500$ gr) infants with persistent candidemia.

Methods: Retrospective analysis of the blood cultures in the Neonatal Intensive Care Unit of University of Patras was performed.

Results: During the last year six VLBW infants presented with invasive infection from candida species, five from candida albicans and one from candida parapsilosis. Initially, liposomal amphotericin was started in a dose of $4-5 \mathrm{mg} / \mathrm{kg} /$ day. Despite the removal of existing central lines the candidemia persisted in four infants and caspofungin was added in a dose of 1 $\mathrm{mg} / \mathrm{kg} /$ day for the first two days and $2 \mathrm{mg} / \mathrm{kg} /$ day for the next days. In two of them with fungal endocarditis caspofungin was ineffective and replaced with fluconazole, after 24 and 15 days of treatment, respectively. In the rest two of the infants the blood cultures became negative and they received caspofungin for a total of 4 weeks. 
Conclusions: The introduction of new antifungal drugs could be useful in cases of preterm infants with persistent fungemia.

\section{ALTERNATIVE APPROACH TO THE INFECTION CONTROL IN MATERNITY NURSERIES: APPLICATION OF SPECIFIC BACTERIOPHAGES FOR PREVENTION AND THERAPY OF BACTERIAL INFECTIONS IN NEONATES}

M Mshvildadze ${ }^{1,2}$, S Tsertsvadze ${ }^{1}$, M Tediashvili $^{1,3}$, I Davitaia $^{1,2}$

${ }^{l}$ Association of Neonatologists of Georgia, Tbilisi, Georgia, ${ }^{2}$ Chachava Research Institute for Perinatal Medicine, Obstetrics and Gynecology, Tbilisi, Georgia

${ }^{3}$ George Eliava Institute of Bacteriophage, Microbiology and Virology, Tbilisi, Georgia

Background and Aims: Neonatal bacterial infections result in considerable morbidity and mortality. Antibiotics still remain major treatments. Occurrence of resistant bacterial strains increases every year. Bacteriophages and antibacterial substances of plant origin may be considered as alternative medicine. To examine the epidemiology of neonatal microflora and study sensitivity of separated strains on antibiotics, bacteriophages and Tea antibacterials.

Methods: Specimens were collected at Neonatology Departments of Maternity Hospitals in Tbilisi during 10 months. Smears were collected from Women's vagina before labor, from the neonates' oral cavity and nose immediately after birth and within 12, 24, 48 and 72 hours. Processing of specimens was performed by standard bacteriological methods. In some cases identification was made by API-tests. Sensitivity of the isolated strains to antibiotics was studied by disc diffusion method and phage sensitivity - by spot test followed by titration using double layer method.

Results: 75 bacterial cultures were isolated from 78 mothers and newborns, 57 from them were identified. The gram-negative bacterial flora (mostly Pseudomonas aeruginosa) was prevalent in pregnant women, in the hospital environment and in the neonates in first 48 hours. After 48 hours from birth gram-positive bacteria (prevalence of Staphylococcus aureus) were revealed. Resistance to antibiotics was revealed in $72 \%$ of strains. The susceptibility to various bacteriophages (Pyophage, Intestyphage, Enterophage, SPS-phage) showed high phage $(>50 \%)$ and tea antibacterials-sensitivity.

Conclusions: The results demonstrated high potential of using bacteriophage and Antibacterials of plant origin preparations for prevention and complex (together with antibiotics) treatment of bacterial infections.

\section{RETINOPATHY OF PREMATURITY}

C C Loureiro, L Winckler

Pediatric Service, Hospital de Santo André, Leiria, Portugal

Background and Aims: Retinopathy of prematurity (ROP) is a vasoproliferative retinopathy that might affect premature children and, in its most severe form, result in the detachment of the retina and blindness. Our aim was to characterize the children admitted in our intermediate care unit (UCEP) with criteria to be submitted to ROP screen, and the ophthalmologic evaluation and orientation they were submitted to.

Methods: Descriptive retrospective study involving children born (in-born/out-born) with gestational age (GA) $\leq 30$ week's and/or with $\mathrm{BW} \leq 1500 \mathrm{~g}$ between July/1999-June/2005, admitted at UCEP. The results were obtained by means of consultation of the individual child hospital process.

Results: Of 141 children studied, the GA has varied between 24 and 37 week's and the BW balanced between $470 \mathrm{~g}$ and $1710 \mathrm{~g}$. Eighty six percent of children were screened and in $14 \%$ of cases no screening report was found. Nineteen percent of screened children had ROP. Twenty four percent of children with ROP had BW inferior to $1250 \mathrm{~g}$ and inferior to $1000 \mathrm{~g}$ in $36 \%$ of cases. Seventy one percent of ROP cases were stage 1 disease and $21 \%$ were stage 2 or greater. Children with stage 1 ROP disease had a mean GA of 28 week's with a mean BW of $1017 \mathrm{~g}$ while stage 2 ROP mean GA was 26 week's and the mean BW was $742 \mathrm{~g}$. ROP spontaneous regression has occurred in $70 \%$ of cases and $22 \%$ of children developed cicatricial lesion.

Conclusions: ROP prevalence is greater in children with smaller GA and BW. In most cases of low severity disease spontaneous resolution has occurred.

\section{PREDICTORS AND EMPIRIC ANTI-MICROBIAL THERAPY OF LATE-ONSET SEPSIS IN THE NEONATAL INTENSIVE CARE UNIT}

T Smolkin ${ }^{1}$, I R Makhoul ${ }^{1}$, R Hanna-Elias ${ }^{1}$, I Kassis ${ }^{2}$, A Tamir ${ }^{3}$, P Sujov $^{1}$

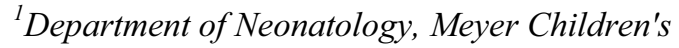
Hospital, Rambam Medical Center, Haifa, Israel ${ }^{2}$ Pediatric Infectious Diseases Unit, Meyer Children's Hospital, Rambam Medical Center, Haifa, Israel ${ }^{3}$ Department of Epidemiology, Rappaport Faculty of Medicine, Technion, Haifa, Israel

Background and Aims: Whenever late-onset sepsis (LOS) is suspected, empiric anti-microbial therapy (AMT) is initiated after sepsis work up. To evaluate the clinical, interventional and laboratory predictors of LOS, and the appropriateness of empiric AMT in neonatal LOS.

Methods: Medical records of all neonates admitted to a tertiary NICU during a 1-year period were reviewed. Of 352 neonates, 84 developed 96 suspected septic events beyond 3 days of age. LOS definition: clinical signs of sepsis + positive blood and/or urine and/or CSF culture. Clinical and laboratory abnormalities at onset of sepsis, AMT instituted, and microbiological data were recorded.

Results: Of the 96 events of suspected LOS, 26 (27.0\%) had positive blood cultures, with coagulase-negative Staphylococcus (CoNS), Klebsiella and Candida accounting for $22 / 26(84.7 \%)$ of these events. In four out of 75 events $(5.3 \%)$, urine culture was positive. Logistic regression multivariate analysis showed that birth weight $(\mathrm{OR}=0.9,95 \% \mathrm{CI}$ : 0.82-0.99; $\mathrm{p}=0.031)$, 
apnea/bradycardia $\quad(\mathrm{OR}=3.16, \quad 95 \% \mathrm{CI}: \quad 1.08-9.25$; $\mathrm{p}=0.036)$, and platelet count $<100,000 / \mathrm{mm} 3(\mathrm{OR}=7.04$, $95 \%$ CI:1.21-40.9; $\mathrm{p}=0.03)$ were significantly associated with LOS. In 26/29 (89.7\%) of proven septic events, empirical AMT was started after sepsis work up, mostly vancomycin 18/29 (62.0\%), imipenem 14/29 (48.3\%), and amphotericin B 9/29 (31.0\%). The causative pathogen was sensitive to the prescribed AMT in 24/26 (92.3\%) of proven septic events.

Conclusions: Significant predictors of LOS include lower birth weight, apnea/bradycardia and thrombocytopenia. Empirical AMT was initiated in 90\% of proven septic events and was appropriate in $92.3 \%$. Empiric coverage of CoNS, Klebsiella and fungi should be considered, based on infant's condition and local microbiological data.

\section{SURVEY OF ANALGESIA AND ANAESTHESIA FOR RETINOPATHY OF PREMATURITY}

\author{
D Anand ${ }^{1}$, B Etuwewe $^{1}$, D Clark $^{2}, \mathrm{C}$ W Yoxall ${ }^{1}$

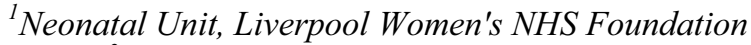 \\ Trust, ${ }^{2}$ Aintree Hospital NHS Trust, Liverpool, UK
}

Background and Aims: In UK, there are nationally agreed guidelines for screening for retinopathy of prematurity (ROP), treatment thresholds and the maximum interval between the identification of threshold disease and treatment. There are no guidelines for analgaesia or anaesthesia for the baby during treatment and we are aware of differences in clinical practise across UK. The present survey was conducted across UK regional neonatal units (NNU) to evaluate the management of babies during ROP treatment and to assess associated problems.

Methods: A questionnaire on anaesthesia for ROP treatment was sent to 21 regional NNU in UK.

Results: 15 of $21(71.4 \%)$ NNU responded. 5 units $(33.3 \%)$ use general anaesthesia (GA) in theatre. 2 units (13.4\%) use sedation and/or local anaesthesia (SLA) in the NNU. 8 units $(53.3 \%)$ use sedation, paralysis and ventilation (SPV) on the NNU. 4 of the 5 units that use GA in theatre have reported delay in treatment beyond the recommended time because of lack of theatre availability. Both units that use SLA on the NNU reported the need for emergency intubation in some infants. In the 8 units that used SPV on the NNU, there were no delays in treatment and the only reported adverse event was the need for emergency reintubation in one infant at one centre.

Conclusions: The findings from this survey suggest that SPV on the NNU provides safe care for babies having ROP treatment and avoids delays caused by availability of anaesthetic or theatre resources.

\section{ROLE OF INSULIN GROWTH FACTOR AND CENTRAL RETINAL ARTERY DOPPLER IN THE RETINOPATHY OF PREMATURITY}

\footnotetext{
A Perez-Muñuzuri ${ }^{1}$, J R Fernandez-Lorenzo ${ }^{1}$, M I Martinez-Soto ${ }^{1}$, M J Blanco-Teijeiro ${ }^{2}$, J M Fraga-Bermudez ${ }^{1}$
}

${ }^{1}$ Servicio de Neonatologia, ${ }^{2}$ Servicio de Oftalmologia, Hospital Clinico Universitario, Santiago, Spain

Background and Aims: The retinopathy of prematurity (ROP) is the first cause of blindness in the infant population. Many factors have been legated to the pathogenesis of this illness. Methods: A prospective study with premature babies of less than 32 weeks and / or less than 1500 grs was designed. We have determined every week since the delivery till 39 weeks of corrected gestational age the seric levels of insulin growth factor type 1 (IGF1) and the echo-doppler of the central retinal artery. An ophthalmologic exam was made to check if ROP was present. Results: 35 patients included, 12 with retinopathy and 23 with not. Mean weight at delivery was 1130 grs and 1460 grs respectively $(p<0.05)$. There was not difference between both groups in function of the sex, mean gestational age, childbirth type, oxygenation time, bronchopulmonary dysplasia, intracranial haemorrhage, patent ductus arteriosus, erythropoietin treatment and central retinal artery echodoppler. Children with retinopathy had more sepsis $(p<0.05)$ and lower levels of IGF1. Conclusions: Seric levels of IGF1 at the 3rd week after delivery are predictive of ROP $(\mathrm{p}<0.05)$. Low weight at delivery and sepsis during the first weeks of life are clearly related with retinopathy. No difference was found in doppler of central retinal artery, probably due to the complexity of the technique and its low reproducibility.

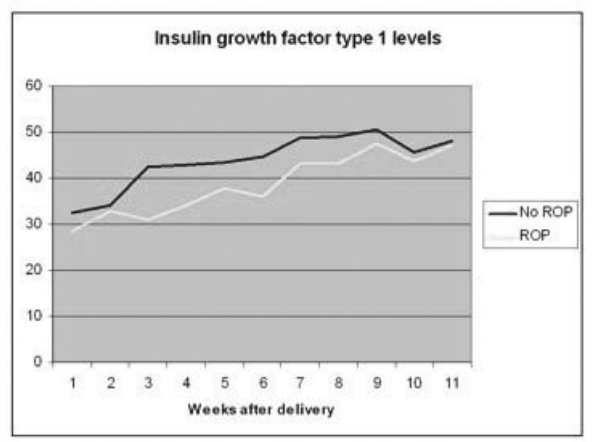

\section{ROP SCREENING CRITERIA NEED TO BE ADAPTED TO THE LOCAL POPULATION - ROMANIAN EXPERIENCE}

T Ciomartan ${ }^{1}$, C Nascutzy ${ }^{2}$, I Vătavu ${ }^{2}$, D Stoenescu ${ }^{2}$, I Anca $^{1}, \mathrm{~S}$ Stoicescu ${ }^{3}, \mathrm{M} \mathrm{Ceauşu}^{4}, \mathrm{~A} \mathrm{Culcer}^{5}$, G Sardescu ${ }^{6}$

${ }^{1}$ PICU, ${ }^{2}$ Dept of Ophthalmology, ${ }^{3}$ Neonatology Unit,
Institute for Mother and Child Care 'Polizu',
${ }^{4}$ Neonatology Unit, Clinical Hospital "I. Cantacuzino",
${ }^{5}$ Neonatology Unit, University Emergency Hospital,
${ }^{6}$ Neonatology Unit, Emergency Hospital "St.
Pantelimon", Bucharest, Romania

Background and Aims: 1 . To assess the incidence of ROP, including severe forms of the disease that required treatment in preemies included in a regional screening programme. 2. To assess whether the screening criteria used in developed countries are appropriate for our population.

Methods: All babies born Sept 2002 - Dec 2005 with gestational age $(\mathrm{GA})<=32$ weeks and birth weight $(\mathrm{BW})<=1500 \mathrm{~g}$, plus infants with GA $>32$ weeks and 
BW $>1500 \mathrm{~g}$ with perinatal complicated progress were included in study.

Results: 884 preemies were examined - 447 (50.5\%) had ROP, 122/884 (13.8\%) with severe ROP requiring laser photocoagulation. The latter had mean \pm SEM GA was $29.2 \pm 0,336$ wks (range $25-33$ ) and mean \pm SEM BW $1245 \pm 52$ g (range $750-1800)$. In addition, 15 infants presented at the first examination with advanced stages of ROP ( $>=4)$. Among those with ROP, 48/447 $(10.7 \%)$ had GA $>32$ weeks and 113/447 (25.3\%) had BW $>1500$ g. Similarly, of those $122 / 447$ (27.3\%) with severe ROP, 7/122 (5.7\%) had GA > 32 weeks and $30 / 122(24.6 \%)$ had BW $>1500 \mathrm{~g}$, in recent months with an increased incidence of ROP in babies with BW $>1800 \mathrm{~g}$. These would have been missed if we would have applied exclusively selection criteria used in developed countries.

Conclusions: Screening criteria published and used in the developed countries are too narrow for our population. We propose the extension of these criteria, for Romania, to GA $<=34$ weeks and $\mathrm{BW}<=2000 \mathrm{~g}$.

\section{IN VITRO DNA DAMAGE AND REPAIR CAPACITY AFTER OXIDATIVE STRESS}

R Ciardelli $^{1}$, K De Bont ${ }^{2}$, I Decordier ${ }^{2}$, G Delanghe ${ }^{1}$, I Van Herreweghe ${ }^{1}$, M Kirsch-Volders ${ }^{2}$, D Haumont ${ }^{1}$

${ }^{I}$ Neonatal Unit, St-Pierre University Hospital, Brussels, Belgium, ${ }^{2}$ Laboratory of Cellular Genetics, Free

University of Brussels, Brussels, Belgium

Background and Aims: Oxidative stress (OS) might be an important contributor to neonatal complications. Effects of OS on DNA damage are not well documented in this age group. Aim of the study: To investigate in vitro DNA damage and repair after OS in healthy term newborns compared to adults and to analyse the importance of genotypes in the DNA repair systems.

Methods: Lymphocytes from cord blood and adult peripheral blood from ten mother-daughter couples were exposed in vitro to $\mathrm{H} 2 \mathrm{O} 2$. Genotoxic effects were tested using alkaline comet assay and micronucleus assay. Secondly genotypes of some important repair genes were defined for all individuals.

Results: Initial DNA damage was similar for neonates compared to their mothers but because of a better DNA repair capacity final DNA damage was lower for newborns. When correlating DNA damage to the different genotypes, only MTHFR222 variant genotype had a significant negative effect. The amount of fixed DNA damage was not significantly different. Multivariate analysis showed a significant protective effect of maternal antioxidant supplementation during pregnancy.

Conclusions: Term newborns tend to have a better DNA repair capacity after OS compared to adults. A better knowledge of the influence of the genotypes of DNA repair genes should allow a better understanding of individual susceptibility to OS. This is a preliminary study to assess healthy newborn's sensitivity to DNA damage after OS. Further studies concerning the premature infants are planned.
THE EFFECTS OF ANTENATAL STEROID THERAPY AND MODES OF DELIVERY TO ERYTHROCYTE ANTIOXYENZYMES ACTIVITY IN PRETERM INFANTS WITH GESTATIONAL AGE 25 TO 30 WEEKS

R H Fu ${ }^{1,2}$, P H Yang ${ }^{2}$, R Lien², M C Chiang ${ }^{2}$, C C Chiang ${ }^{2}$, Y H Cho

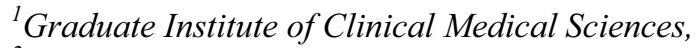
${ }^{2}$ Division of Neonatology, Department of Pediatrics, ${ }^{3}$ Research Institute, Chang Gung Children's Hospital, Taoyuan, Taiwan-R.O.C.

Background and Aims: Antenatal maternal steroid therapy has been proved to improve the pulmonary maturation in preterm infants. However, few data on antioxidant ability have been noted. Our aims are to evaluate the effects of therapy and delivery modes to erythrocyte antioxyenzymes activity of preterm infants.

Methods: Prospective study was performed from June 2001 to May 2002. Preterm infants with gestational age 25 to 30 weeks were included in this study. Blood was taken from umbilical arterial line or radial artery within the first 24 hours. Erythrocytes were collected by centrifugation and washed with cold saline. Erythrocyte antioxyenzymes (SOD, catalase, cellular glutathione peroxidase) activity were measured by Bioxytech SOD525, Catalase-520, and GPx-340 and corrected by the hemoglobin level. Antenatal steroid therapy, mode of delivery, cumulative oxygen administration, and patient's data were collected from chart review. Student $t$ test and ANOVA were used to evaluate groups of data. Results: 34 preterm infants were included in our study. Among them, 16 did and 12 did not receive antenatal steroid therapy. 6 cases were uncertain. There were no differences in gestational age, enzyme activity, and cumulative oxygen administration among the 3 groups. Birth weight of treatment group was significantly lower $(p=0.015)$. Of all cases, 9 were vaginal delivery and 25 were caesarean section. The erythrocyte SOD activity was lower in caesarean section group $(\mathrm{p}=0.021)$.

Conclusions: Antenatal maternal steroid therapy didn't seem to affect the activity of erythrocyte antioxyenzymes of preterm infants. Infants delivered through caesarean section have lower SOD activity, which may indicate lower oxidative stress.

\section{IMPACT OF DELIVERY TYPE IN THE HYDROPEROXIDE MEMBRANE LEVELS AND ERYTHROCYTARY CATALASE ACTIVITY IN PREMATURES DURING THE EARLY NEONATAL PERIOD}

F Contreras-Chova ${ }^{1}$, A Bonillo-Perales ${ }^{3}$, A Molina-Carballo ${ }^{1}, \mathrm{~J}_{\text {Ochoa-Herrera }}^{2}$, R Pérez-Iáñez ${ }^{1}$, E Narbona-López ${ }^{1}$, M González-Ripoll ${ }^{3}$, J Uberos-Fernández ${ }^{1}$, E De Araujo-Nepomuceno², A Muñoz-Hoyos ${ }^{1}$

${ }^{1}$ Departamento Pediatría, Hospital Clínico San Cecilio, ${ }^{2}$ Instituto de Nutrición y Tecnología de los Alimentos, Granada, ${ }^{3}$ Departamento de Pediatría, Hospital Torrecárdenas, Almería, Spain 
Background and Aims: To analyze the repercussion that delivery type (spontaneous vaginal vs Cesarean section) could have on oxidative stress levels, quantified by hydroperoxide concentration in erythrocyte membranes, as well as in the antioxidant catalase activity in preterm newborns (PTN), in the first week of life.

Methods: 84 PTN with gestational ages between 26-36 weeks, and birth weight between 630-2500 g. Samples were obtained from umbilical cord and at $3 \mathrm{~h}, 72 \mathrm{~h}$ and 7 days of life, measuring hydroperoxide membrane levels $(\mathrm{H} 2 \mathrm{O} 2, \mathrm{ng} / \mathrm{ml})$, as well as the catalase erythrocyte activity. The preterms were subdivided in two groups: Group A, 27 neonates born by spontaneous vaginal delivery and Group B, 57 newborn delivered by Cesarean section. Statistical analysis: t Student test.

Results: H2O2: We report a greater level of hydroperoxides in umbilical cord erythrocytes of preterms delivered by Cesarean section, $(p<0,005)$. From $3 \mathrm{rd}$ h of life, the evolution and levels of $\mathrm{H} 2 \mathrm{O} 2$ are comparable between both groups. Catalase: Major level of enzymatic catalase activity in preterms borns by spontaneous vaginal delivery, especially in umbilical cord and at $3 \mathrm{rd} \mathrm{h}$ of life, with significant differences $(p<0,005)$ in umbilical cord.

Conclusions: The birth by Cesarean delivery section induces a disbalance between oxidative stress levels and its compensating enzymatic mechanisms in preterms; that is rapidly compensated in the first hours of life, presenting later a similar evolution in both delivery types.

\section{EVOLUTION OF MEMBRANE ERYTHROCYTE HYDROPEROXIDES LEVELS IN NEONATAL PERIOD: COMPARATIVE ANALYSIS BETWEEN TERM AND PRETERM NEWBORNS}

F Contreras-Chova ${ }^{1}$, J Ochoa-Herrera ${ }^{2}$, R Pérez-Iáñez ${ }^{1}$, A Molina-Carballo ${ }^{1}$, A Muñoz-Hoyos ${ }^{1}$, E Narbona-López ${ }^{1}$, J Uberos-Fernández ${ }^{1}$, E De Araujo-Nepomuceno ${ }^{2}$, M González-Ripoll ${ }^{3}$, A Bonillo-Perales ${ }^{3}$

${ }^{I}$ Departamento de Pediatría, Hospital Universitario San Cecilio, ${ }^{2}$ Instituto de Nutrición y Tecnología de Alimentos, Granada, ${ }^{3}$ Departamento de Pediatría, Hospital Torrecárdenas, Almería, Spain

Background and Aims: The knowledge about the oxidative stress levels in newborn babies of very low weight $(<1500 \mathrm{~g}$ at birth $)$ and his evolution in the first days is still very scarce, for what we realize a comparative study of the levels of membrane erythrocyte hydroperoxides in newborns at term and preterm, analyzing his evolution in the first week of life. Methods: 147 newborns subdivided in three groups: Control (CG): 63 newborn at term of weight adequate to gestational age; group A (GA), 57 very-low-birthweight preterms $(<1500 \mathrm{~g}$; VLBW); group B (BG): 27 preterm of $1500-2500 \mathrm{~g}$. Blood samples of umbilical cord and at $3 \mathrm{~h}$ of life (in all groups), and at $72 \mathrm{~h}$ and 7 days in preterm groups, to quantify membrane erythrocyte hydroperoxides (H2O2) levels. Statistic: Two way (groups, hours of life) ANOVA.
Results: $\mathrm{H} 2 \mathrm{O} 2$ values minors in the $\mathrm{CG}$ that in both groups of prematures, in umbilical cord and at $3 \mathrm{~h}$ of life, with $\mathrm{p}<0,005$ in both determinations. The evolution of $\mathrm{H} 2 \mathrm{O} 2$ levels in the first 7 days was similar in both groups of preterms, with mean concentration greater in the BG and significant differences at $72 \mathrm{~h}$ and 7 days.

Conclusions: There are greater levels of oxidative stress in preterms than at term. Surprisingly, the concentration is higher in $>1500 \mathrm{~g}$ group than in VLBW group. We need new studies that extend the knowledge on this fact.

\section{CLINICAL SIGNIFICANCE OF PRENATAL DETECTION OF A SINGLE UMBILICAL ARTERY}

I Sánchez Fernández ${ }^{1}$, A Hernández Saavedra ${ }^{3}$, M Vázquez Ortiz ${ }^{1}$, A Martín Ancel ${ }^{2}$, Krauel Vidal ${ }^{2}$, J Pou Fernández ${ }^{1}$, R Jiménez González ${ }^{1}$

${ }^{1}$ Department of Pediatrics, ${ }^{2}$ Department of Neonatology, ${ }^{3}$ Department of Gynecology and Obstetrics, Hospital Sant Joan de Déu, Barcelona, Spain

Background and Aims: Single umbilical artery (SUA) is one of the most frequent congenital malformations in humans. Associated malformations are usually diagnosed prenatally or neonatally. Postnatal development of children with SUA is studied.

Methods: Case-control study. Cases: SUA fetuses (diagnosed by prenatal ultrasonography) during the period 2002-2004 in a third level reference Pediatric hospital. Two matched controls per case (same day, same ecographist, alleatory match). Data: revision of clinical records and interview with parents.

Results: 40 Cases, 80 Controls. 15\% Cases, 1'49\% Controls died during the prenatal and perinatal period (all of them diagnosed prenatally as having severe anomalies). Small for gestational age and intrauterine growth retardation: $25 \%$ Cases, 6'25\% Controls. Even when no associated anomalies were diagnosed, SUA newborns weighted less. Prematurity: 15'63\% Cases, $12 ' 12 \%$ Controls. Weight percentile after 1 year of life: p 49'2 Cases, p 56'35 Controls. Age at walking: 13'61m Cases, $12^{\prime} 38 \mathrm{~m}$ Controls; $7^{\prime} 5 \%$ of cases could not walk at 14 months or more (all controls did). Urinary tract infection (UTI): 10\% Cases, 1'47\% Controls. A renal pelvic dilatation detected prenatally, hipospadias and preauricular appendices diagnosed at birth and a colonic malformation diagnosed at 2 years of age are found in the case group. No other malformations are observed postnatally. Hospital admissions: $20^{\prime} 69 \%$ Cases, $8^{\prime} 82 \%$ Controls.

Conclusions: Our study suggests that SUA detected prenatally is associated with malformations and perinatal death, low birthweight and prematurity. Children with an isolated SUA (no associated anomalies detected) weight less, start walking later and suffer more UTI and hospital admissions.

\section{ROLE OF OXIDATIVE STRESS AND RED BLOOD CELL DEFORMABILITY IN INTRAUTERINE GROWTH RETARDATION}

Z Hracsko ${ }^{1}$, H Orvos ${ }^{2}$, Z Novak ${ }^{3}$, I S Varga ${ }^{1}$ 
${ }^{l}$ Department of Genetics and Molecular Biology, University of Szeged, Szeged, Hungary, ${ }^{2}$ Department of Obstetrics and Gynecology, University of Szeged, Szeged, Hungary, ${ }^{3}$ Department of Pediatrics, University of Szeged, Szeged, Hungary

Background and Aims: Many neonatal diseases are associated with oxidative stress. It is likely that intrauterine growth retardation (IUGR) is also connected with the attack of free radicals. We presumed that IUGR is a consequence of a foetomaternal blood flow disorder. Red blood cells (RBCs) have specific properties, which enable them to reach all tissues. The membrane structure is developed to result in a special shape and elasticity. If the integrity of the membrane is damaged, RBCs lose this shape and elasticity and may undergo haemolysis. Consequently, the tissues obtain less oxygen and nutriment. Our study deals with lipid peroxidation (LP) and rheological properties of the RBC membranes.

Methods: 156 full-term mature neonates of either sex, born between weeks 37 and 40 were included, 127 of them with normal weight $(3425 \pm 450 \mathrm{~g})$ and 29 neonates with IUGR $(2125 \pm 305 \mathrm{~g})$. We determined the erythrocyte ghost damage, the LP and filtration speed of the RBCs.

Results: LP in the control group was $0.055 \mathrm{nM}$ $\mathrm{MDA} / \mathrm{mg}$ protein, while that in the IUGR group was $0.13 \mathrm{nM} \mathrm{MDA} / \mathrm{mg}$ protein. LP of erythrocyte ghost was $5.9 \mathrm{nM} \mathrm{MDA} / \mathrm{ml}$ in the control and $9.01 \mathrm{nM} \mathrm{MDA} / \mathrm{ml}$ in IUGR group. The rheological parameters of control neonates had more favourable values than that of IUGR newborn (relative filtration speed: 0.67 vs. 0.59 ; cell transit time: 9.56 vs. 11.69 ; clogging rate: 5.04 vs. 3.14 $\times 10-3$ ). All differences are significant.

Conclusions: We found a relationship between the LP in the foetomaternal blood stream and IUGR.

\section{COMPARISON OF A NEW TRANSCUTANEOUS BILIRUBINOMETER (BILIMED) WITH SERUM BILIRUBIN MEASUREMENTS IN PRETERM AND FULL-TERM INFANTS}

\author{
T Karen ${ }^{1,2}$, H U Bucher ${ }^{1}$, J C Fauchère ${ }^{1}$ \\ ${ }^{l}$ Clinic of Neonatology, University Hospital Zurich, \\ Zurich, Switzerland, ${ }^{2}$ Department of Neonatology, \\ University Children's Hospital Tuebingen, Tuebingen, \\ Germany
}

Background and Aims: The gold standard to asses hyperbilirubinaemia in neonates remains the serum bilirubin measurement. Unfortunately, this is invasive, painful, and costly. Bilimed ${ }^{\circledR}$, a new transcutaneous bilirubinometer, suggests more accuracy compare to the existing non-invasive bilirubinometers because of its new technology, it includes different ethnic skin colors, does not need contact with the skin during measurement, and generates no additional costs per measurement. Aim was to assess the agreement between this new transcutaneous bilirubinometer and serum bilirubin.

Methods: The Bilimed ${ }^{\circledR}$ was applied on the sternum of preterm and term infants, including different ethnic colors of skin. Serum bilirubin concentration was determined simultaneously. The agreement between both methods was assessed by the method of BlandAltman.

Results: A total of 117 measurements in 99 term newborn infants (group1), 47 measurements in 37 preterm infants between 34 - 36 6/7 gestational weeks (group 2) and 21 measurements in 13 preterm infants between 28 - 33 6/7 gestational weeks (group 3) were performed. The mean deviation and variability (+/-2SD) of the transcutaneous values and serum bilirubin in group 1 were: $-14(+/-144)$ (figure 1), in group 2: +16 $(+/-91)$ and in group 3: $-8(+/-76) \mathrm{mcmol} / \mathrm{l}$. Although most points lie within the limits of agreement, these limits are too wide to be acceptable in a clinical setting. Moreover, there seems to be a trend towards less good agreement with increasing bilirubin values.

Conclusions: Despite its new technology the new transcutaneous bilirubinometer has no considerable advantages, and more specifically no greater agreement compared to the existing non invasive bilirubinometer.

\section{NEONATAL READMISSION FOR PRIMARY HYPERBILIRUBINEMIA}

M Kaplan ${ }^{1,2}$, R Bromiker ${ }^{1,3}$, M S Schimmel ${ }^{1,3}$, C Hammerman ${ }^{1,3}$

${ }^{I}$ Department of Neonatology, Shaare Zedek Medical Center, Jerusalem, ${ }^{2}$ Hebrew University, Jerusalem, ${ }^{3}$ Ben Gurion University of The Negev, Be'er Sheva, Israel

Background and Aims: In the absence of a measure for assessing the incidence or severity of post-discharge bilirubin morbidity, surveillance of the rate of hospital readmission has been suggested to assess this potential.

Methods: The rate of readmission for neonatal hyperbilirubinemia was determined in term and nearterm neonates cared for in the well baby nurseries of the Shaare Zedek Medical Center. Severity of hyperbilirubinemia and etiological categories were compared between those who required phototherapy either during birth hospitalization or readmission.

Results: During 2001-2002 there were 19,437 live births, of whom $342(1.8 \%)$ were treated with phototherapy. 74 of these $(0.38 \%$ of total $)$ were readmitted for primary phototherapy. Mean \pm SD plasma total bilirubin at onset of phototherapy was 14.7 $\pm 3.0 \mathrm{mg} / \mathrm{dL}$ (upper range $21.0 \mathrm{mg} / \mathrm{dL}$ ) in the birth hospitalized neonates and $18.5 \pm 1.7 \mathrm{mg} / \mathrm{dL}$ (upper range $26.0 \mathrm{mg} / \mathrm{dL})$ in those readmitted $(\mathrm{p}<0.0001)$. Four required exchange transfusion (3 during birth hospitalization and 1 readmitted). Mean weight change from birth to readmission was $-3.5 \pm 1.7 \%$. Among those treated during the birth hospitalization the majority had a hemolytic etiology for their jaundice whereas in $52 \%$ of the readmitted neonates breast feeding was the only identifiable risk factor. No infant in either group had signs of bilirubin encephalopathy.

Conclusions: Potentially severe post-discharge hyperbilirubinemia requiring readmission may occur in the absence of identifiable predictive risk factors other than breastfeeding. All neonates should be vigilantly observed for hyperbilirubinemia in the immediate postdischarge period. 
UNRECOGNIZED MILD ALTERATIONS IN THE METABOLISM OF BILIRUBIN MAY UNDERLIE SIGNIFICANT HYPERBILIRUBINEMIA

S Fernández Ureña ${ }^{1}$, A Barrio Merino ${ }^{2}, \mathrm{~F} \mathrm{Cava}^{3}$, A Martín-Ancel ${ }^{4}$

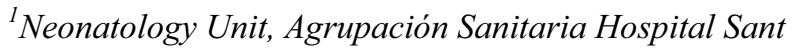
Joan de Deu, Hospital Clinic, Barcelona, Spain

${ }^{2}$ Pediatrics Unit, Fundación Hospital Alcorcón, Madrid, Spain, ${ }^{3}$ Laboratory Unit, Fundación Hospital Alcorcón, Madrid, Spain, ${ }^{4}$ Neonatology Unit, Agrupación Sanitaria Hospital Sant Joan de Deu, Hospital Clinic, Barcelona, Spain

Background and Aims: A salient feature of recent guidelines for the management of neonatal hyperbilirubinemia is the assessment of risk for severe hyperbilirubinemia in part by means of evaluation of clinical risk factors. However, clinical risk factors are not apparent in many cases with severe hyperbilirubinemia. Objective: To analyze the incidence, etiology, treatment, and outcome of term and near-term newborns $(\mathrm{GA} \geq 35)$ with bilirubin levels $\geq 20$ $\mathrm{mg} / \mathrm{dl}$.

Methods: Newborns were identified using computer databases from a cohort of 14,129 infants (1998-2005). Data on clinical manifestations, etiology, treatment, and follow-up were obtained.

Results: 78 newborns had bilirubin levels $\geq 20 \mathrm{mg} / \mathrm{dl}$ (5.5 in 1000 newborn infants); none $\geq 29 \mathrm{mg} / \mathrm{dl}$. No acute neurological symptoms were observed in any infant. One required exchange transfusion. A cause for hyperbilirubinemia was identified in 37 infants $(47 \%)$ : 14 isoimmunization (9 O/A, 4 O/B, $1 \mathrm{Rh}), 9$ dehydration, 2 infection, 3 neonatal hepatic disease, 9 other. Most infants did not require in-hospital follow-up; among those followed, 5 showed unexpected elevation of transaminases, 2 of them had alfal-antitrypsin deficiency (Pi:MS, Pi:MZ). No differences were found between infants with and without identified cause for gestational age, maximum bilirubin, and days of phototherapy; birth weight was lower in infants with identified cause ( $2952 \pm 364$ vs $3189 \pm 482$, $\mathrm{p}<0.05)$.

Conclusions: 1) Unrecognized mild alterations in the metabolism of bilirubin might underlie significant hyperbilirubinemia without apparent cause. Follow-up including hepatic function tests might help to identify families at risk for severe hyperbilirubinemia of genetic origin. 2) Adequate management of known risk factors, such as $\mathrm{Rh}$ isoimmunization, precludes severe hyperbilirubinemia in these infants.

\section{VALIDATING A COMPUTER TOOL WHICH HELPS TO USE THE AAP GUIDELINE ON HYPERBILIRUBINEMIA}

A Balaguer, M Camprubí, M Milá, R Collell, R Jimenez-Feijoo, F Pagone, J Escribano

Unitat de Neonatología Hospital Sant Joan de Reus, Reus, Spain

Background and Aims: The AAP guidelines for evaluating the risk of severe hyperbilirubinemia in the newborn changed in 2004. The clinical decision to initiate treatment with phototherapy or exchange transfusion is subject to many errors. Objectives Aware of this problem, we designed a spreadsheet (NeoBili), which helps to interpret bilirubin levels according to the infant's age in hours and helps to take into account the clinical risk factors.

Methods: A randomised controlled trial was conducted with simulated clinical cases. In this preliminary study, one hundred and eighty simulated cases were performed by two groups, one of paediatricians and another of paediatric residents. The main variables measured were the number of errors ("minor" and "major") and the time taken to make the decision.

Results: Without computer support, 32 "minor" errors out of 90 cases were made. The number of errors decreased to 13 when NeoBili was used $(\mathrm{p}=0.002)$. Major errors also decreased when the computer was used (from 6 to 2 out of 90 simulations), although this difference was not significant $(\mathrm{p}=0.27)$. As far as the time taken to make the decision, the group of paediatric was the one that benefited most from using the computer: the time taken to solve 10 cases reduced from $16.25 \mathrm{~min}$. to $10.5 \mathrm{~min}$.

Conclusions: The test we carried out with simulated clinical cases shows that many errors are made in the management of hyperbilirubinemia. Neobili helped clinicians to make fewer errors and also saved them time.

\section{CORRELATION OF GENDER AND MODE OF DELIVERY WITH NEONATAL JAUNDICE}

G Z Zantopoulos, O Gkika, S Liosi, P Anastasopoloulou, A Giannisi, N Manakou, C Rossas, I Anastasopoulos, Z Patrona

Department of Paediatric, General Hospital of Korinthos, Korinthos, Greece

Background and Aims: Neonatal jaundice (NJ) is a common condition and usually a benign transitional event that resolves without treatment. The aim of this study was to determine the correlation of gender and mode of delivery with the values of bilirubin in healthy full- term neonates.

Methods: Serum total bilirubin (TSB) measurements were initially made at the 24th hour of life and repeated daily for the next 4 days) in 198 full-term healthy breast feeding newborns born in a regional hospital and correlated with gender and mode of delivery. $(n=198$, males $=104$ - females $=94$, median Birth Weight $=3230$ g).

Results: Clinically detectable jaundice was present in $177(84.8 \%)$ newborns. When comparing males with females, differences were detected in max TSB $(6.74+/-$ 2.8 vs. $7.56+/-2.3 \mathrm{mg} \%$ ). TSB level of $\geq 6 \mathrm{mg} / \mathrm{dl}$ at 24 $+/-6 \mathrm{hr}$ was presented in $50(25.2 \%)$ infants. Of these $29(58 \%)$ were females and $21(42 \%)$ males, and 30 $(60 \%)$ were born after spontaneous delivery and 20 $(40 \%)$ after caesarean section and $9(18 \%)$ were defined to have significant hyperbilirubinemia, and phototherapy treatment was started. Compared with newborns not noted to be jaundiced on the first day, newborns noted to be jaundiced within 24 hours were more likely to receive phototherapy (18\% vs. $1.2 \%)$. 
Conclusions: Maximum value of TSB in our sample was higher in females when compared with males. Although no differences were detected in mode of gestation of total sample, spontaneous delivery correlated with influencing of TSB on the first day.

\section{THE IMPACT OF BACTERIEMIA ON JAUNDICE WHICH REQUIRED EXCHANGE TRANSFUSION IN NEONATES}

\author{
N Tekin, A Aksit \\ Department of Pediatrics, Division of Neonatology, \\ Eskisehir Osmangazi University Medical Faculty, \\ Eskisehir, Turkey
}

Background and Aims: In recent years the need for exchange transfusion in neonatal jaundice was decreased with improvement in perinatal care in Turkey. $\mathrm{Rh}$ incompatibility cases reduced while the cases with unknown etiology increased. This study was designed to evaluate the incidence of bacteriemia in the infants who received exchange transfusion in the last two years.

Methods: This study was performed in Osmangazi University Medical Faculty Hospital which is a tertiary level perinatal center. We reviewed the charts of the infants with a gestational age $>/=36$ weeks who received exchange transfusion for indirect hyperbilirubinemia. Sixty one infants were found suitable for evaluation from January 2004 to November 2005. Mean gestational age was $39.1 \pm 1.31$ weeks, and birth weight was $3187.5 \pm 573.63$ grams. As our routine procedure, blood cultures of all the infants were taken before and after the exchange transfusion and antibiotic treatment were started.

Results: Mean value of bilirubin level was $26.69 \pm 6.48$ on admission who received exchange transfusion. Sixteen infants had a positive coombs test with ABO incompatibility in 9 and $\mathrm{Rh}$ incompatibility in 6 of them. In remaining 45 infants, pathogen microorganisms were detected in 22 of the blood cultures taken just before the exchange transfusion, $\mathrm{S}$ aureus was the most common one. None of the infants had clinical evidence of infection and the mean duration of hospital stay was $5.47 \pm 3.2$.

Conclusions: We suggested that the infection could be the triggering factor for hyperbilirubinemia and treatment with exchange transfusion in the early, asymptomatic stage of infection prevented the progression of disease.

\section{A CASE OF RHIZOMELIC CHONDRODYSPLASIA PUNCTATA, TYPE I}

\section{N Tekin, A Aksit, S Hekim \\ Department of Pediatrics, Division of Neonatology, Eskisehir Osmangazi University Medical Faculty, Eskisehir, Turkey}

Background and Aims: To report a case of rhizomelic chondrodysplasia punctata and discuss differential diagnosis of this rare condition from other chondrodysplasias.
Methods: A 12-day-old male infant whose anthropometric measurements all below the 10th percentile presented with respiratory distress. He had rhizomelic micromelia, joint contractures, pes equinovarus deformity and bilateral cataracts, low grade systolic heart murmur on physical examination and roentgenogram showed short humerus and femur, punctate calcifications and abnormal ossification in metaphyses and epiphysis. Echocardiography revealed secundum ASD and PDA.

Results: Presence of short humerus and femur, punctate calcification of the cartilage, congenital cataracts and respiratory problems are satisfactory for clinical diagnosis and to distinguish from other types of punctate calcifications such as $\mathrm{X}$ linked chondrodysplasia punctata, the multiple forms of Zellweger syndrome, maternal ingestion of certain anticoagulants in early pregnancy.

Conclusions: Rhizomelic form of chondrodysplasia punctata is a rare multisystem peroxisomal disorder. Usually diagnosis is based on clinical and radiological criteria, but demonstrating biochemical abnormalities; impairment of plasmalogen biosythesis and elevated phytanic acid will be helpful to assess the underlying mechanism.

\section{THE WALKER-WARBURG PHENOTYPE AND GENOTYPE AMONGST IRISH TRAVELLERS}

\author{
A M Murphy ${ }^{1}$, M Sheridan $^{2}$, M B O'Neill ${ }^{3}$, \\ A G Green ${ }^{4}$ \\ ${ }^{I}$ National Centre for Medical Genetics, Our Lady's \\ Hospital for Sick Children, Dublin, Ireland \\ ${ }^{2}$ Department of Neonatology, Our Lady's Hospital for \\ Sick Children, Dublin, Ireland, ${ }^{3}$ Department of \\ Paediatrics, Mayo General Hospital, Castlebar, Co. \\ Mayo, Ireland, ${ }^{4}$ The Conway Institute School of \\ Medicine and Medical Sciences, University College, \\ Dublin, Ireland
}

Background and Aims: Walker-Warburg syndrome is characterised by congenital muscular dystrophy in conjunction with severe brain malformations and ocular abnormalities. Also known as HARD +/- E syndrome hydrocephalous (H). Agyria (A), retinal dysplasia (RD) with or without encephaloceole (+/-E) the condition is usually lethal within the first few months of life. This extremely rare autosomal recessive congenital muscular dystrophy is one of more than 35 genetic conditions now known to occur in the highly consanguineous Irish Traveller Population.

Methods: We report a family (including a detailed family pedigree) from the Irish Traveller Community who have 2 infants diagnosed with the dystroglycanopathy; 4 of mother's siblings were also affected.

Results: The genotype (POMT1 mutation) and phenotype are described.

Conclusions: Identification of the unique mutation in this ethnic group allows timely prenatal diagnosis, management and genetic counselling. 


\section{HEPATIC NEONATAL HEMANGIOENDOTHELIOMA}

M García Peris ${ }^{1}$, M Aguar Carrascosa ${ }^{1}$, I Izquierdo Macián ${ }^{1}$, E Pérez Santolaya ${ }^{,}$, $\mathrm{J} \mathrm{J} \mathrm{Vila} \mathrm{Carbó}^{2}$, F Morcillo Sopena ${ }^{1}$

${ }^{1}$ Neonatal Intensive Care Unit, Pediatric Hospital La Fe, Valencia, Italy, ${ }^{2}$ Department of Pediatric Surgery, Pediatric Hospital La Fe, Valencia, Italy

Background and Aims: Hepatic tumors in children are uncommon (2-3\% of pediatric tumors). However, infantile hemangioendothelioma is the most frequent benign vascular liver tumor in infancy. Although they are usually benign tumors, they may be life-threatening if haemodynamic or hematologic complications are associated.

Methods: Case report.

Results: A preterm newborn (35 g.e.) presented with a neonatal depression and palpable abdominal mass. The patient had signs of heart failure, anemia (hematocrito $33 \%)$ and thrombocytopenia (59.000 platelets $/ \mathrm{mm} 3)$; coagulation tests proved Quick index of $43 \%$. Serum $\alpha$ fetoprotein level was $9451 \mathrm{ng} / \mathrm{mL}$. Angio-magnectic resonance demonstrated a left intraperitoneal tumor in close relationship with the left hepatic lobe. Vascular study showed involvement of celliaco trunk and upper mesenteryc artery and drainage by dilated suprahepatic left vein. The tumor was completely removed surgically. It was necessary to resect the left hepatic lobe and transverse colon. Macroscopy view showed a gray-red exophytic tumor and an arterio-venous malformation. Histologic analysis demonstrated hemangiondothelioma with necrotic areas and not infiltrated limits. Fifteen days after surgery the patient presented acholic depositions, jaundice and hypertransaminasemia, study slope.

Conclusions: Most of these tumors spontaneously regress. Patients with small lesions don't need treatment, but those with big size tumors or symptomatic require aggressive management with surgical or medical treatment (steroids, alpha-interferon or vincristine). In this case, surgical resection was indicated because of haemodynamic inestability and tumor size. Despite the benign histological findings of these tumors, some hemangioendotheliomes can develop malignant behaviour, so a long term follow-up is important for the evolution of treatment response.

\section{AUDIT OF TRISOMY 13 AND 18 CASES OVER A TEN YEAR PERIOD IN IRELAND}

\section{N Ni Shuibhne, R Howell, A J Green, S A Lynch \\ The National Centre for Medical Genetics, Our Lady's Hospital for Sick Children, Dublin, Ireland}

Background and Aims: In Western Europe the majority of cases of trisomy 13 and 18 are detected antenatally through routine anomaly ultrasound screening. Routine screening is not uniform in Ireland and antenatal detection is much lower.

Methods: We performed an audit of cases of trisomy 13 and 18 made by our Cytogenetics department from 1995-2005.
Results: There were 90 cases of Trisomy 13 diagnosed from 1995-2005. Of these only 28 were diagnosed by amniocentesis and three by chorionic villus sampling (CVS). There were twelve cases of trisomy 13 during that time period seen by clinical geneticists. Of these cases, the mean maternal age was 36 years. Five had abnormal antenatal ultrasounds, mean birth weight was $2.4 \mathrm{~kg}$, mean gestation 30/40, two had caesarean sections and survival ranged from three days to two months. There were 177 cases of trisomy 18seen in the same period of ten years. Of these 77 were diagnosed through amniocentesis and four cases through CVS. There were 16 cases of Trisomy 18 seen by clinical geneticists. The mean maternal age was 33 years. Only two babies had evidence of intrauterine growth retardation on antenatal ultrasound. Mean birth weight was $1.7 \mathrm{~kg}$, mean gestation 37/40, four babies had caesarean sections and survival ranged from one hour to two weeks.

Conclusions: Antenatal detection of trisomy 13 and 18 is still low in Ireland. Antenatal detection can facilitate conservative management. We recommend offering routine antenatal anomaly ultrasound scan at midgestation.

\section{IMMATURE SUCKING PATTERNS IN INFANTS OF DIABETIC MOTHERS}

\author{
R Bromiker ${ }^{1}$, A Rachamim ${ }^{2}$, C Hammerman ${ }^{1}$, \\ M Schimmel $^{1}$, M Kaplan ${ }^{1}$, B Medoff-Cooper ${ }^{3}$ \\ ${ }^{I}$ Department of Neonatology, Shaareh Zedek Medical \\ Center, Jerusalem, Israel, ${ }^{2}$ School of Medicine, \\ Hadassah Hebrew University, Jerusalem, Israel \\ ${ }^{3}$ School of Nursing, University of Pennsylvania, \\ Pennsylvania, PA, USA
}

Background and Aims: Infants of diabetic mothers (IDMs) frequently feed less well than normal counterparts. Our goal was to evaluate sucking patterns in IDMs to objectively demonstrate any difference in these patterns.

Methods: Term healthy IDMs whose mothers were managed with either insulin or diet alone (insulin and diet groups respectively) were compared to matched normal infants (control group). Sucking parameters were recorded over 5 minutes' bottle feedings with the Kron's Apparatus on the third day of life. Student's t-test was used to compare continuous variables and $\mathrm{X} 2$ test for categorical variables.

Results: The study included 47 IDMs (16 insulin group and 31 diet group) and 55 normal infants (control group). There were significant differences in the total number of sucks and total number of bursts between the insulin and the control groups (table 1). The insulin group averaged 5.2 fewer bursts and 42 fewer sucks $(\mathrm{P}=0.013$ and $\mathrm{P}=0.04$ respectively). However, there were no differences between the diet and the control groups.

Conclusions: A poorer feeding performance was found among infants of the insulin group but not of the diet group. This poorer performance during the early neonatal period may have been due to immaturity of the sucking mechanism. To the best of our knowledge, this 
is the first report of nutritive sucking behavior in IDMs.

Table 1 Sucking parameters of IDMs (insulin or diet groups) and healthy controls, measured over 5 minutes feeding

\begin{tabular}{|l|l|l|l|}
\hline Sucking Parameter & Insulin & Diet & Control \\
\hline N sucks & $115 \pm 65^{*}$ & $152 \pm 71$ & $157 \pm 73$ \\
\hline N bursts & $14.5 \pm 6.5^{* *}$ & $18.3 \pm 6.6$ & $19.7 \pm 7.9$ \\
\hline S/B & $9.6 \pm 11.4$ & $9.5 \pm 8.1$ & $9.9 \pm 12$ \\
\hline SW (sec) & $.38 \pm .08$ & $.37 \pm .08$ & $.38 \pm .08$ \\
\hline IBW (sec) & $11.5 \pm 7.5$ & $8.7 \pm 4.6$ & $8.6 \pm 4.2$ \\
\hline Pmax (mm/Hg) & $70 \pm 39$ & $61 \pm 27$ & $69 \pm 40$ \\
\hline
\end{tabular}

*P $=0.04,{ }^{* *} \mathrm{P}=0.013$

N Sucks: number of sucks, N Bursts: number of bursts S/B: Sucks per burst, SW: suck width

IBW: interburst width Pmax: mean maximum nressure

\section{NUTRIENT DEFICITS IN VLBW INFANTS: CURRENT NUTRITION PRACTICE IS NOT GOOD ENOUGH}

S K Somisetty, M Khashu, M Paterson, L Hawkes, A Urs, M Vaithianathan

Neonatal Unit, Leicester Royal Infirmary, University Hospitals of Leicester NHS Trust, Leicester, UK

Background and Aims: Under nutrition and extra uterine growth restriction is a major problem in very low birth weight (VLBW) infants. We evaluated daily nutrient intake in VLBW infants during first four weeks of life and compared with the recommended dietary intake (RDI) to quantify nutrient deficits accrued in the neonatal period.

Methods: Daily nutrient intakes were analysed in VLBW infants (birth weight $\leq 1250 \mathrm{~g}$ ) admitted to our NICU between $01 / 01 / 05$ and $31 / 12 / 05$. Neonates with major congenital malformations and abdominal surgical problems were excluded. Actual intake of calories and protein (both enteral and parenteral) was subtracted from RDI $(120 \mathrm{Kcal} / \mathrm{kg} / \mathrm{day}, 3.8 \mathrm{~g}$ of protein $/ \mathrm{kg} / \mathrm{day})$ and nutritional deficits calculated. Data are expressed as mean \pm standard deviation.

Results: During the study period, 47 infants (birth weight $926 \pm 49.5 \mathrm{~g}$; gestational age $28 \pm 3.5$ weeks) were eligible for inclusion. The weekly and cumulative energy and protein intakes and deficits during the neonatal period are depicted in the table.

Conclusions: Our results corroborate previous evidence that current nutrition practice leads to significant nutrient deficits in VLBW infants. Although there was progressive improvement, deficits were still significant, especially for protein. Since sub-optimal nutrition may be rate limiting on clinical outcome, there is urgent need to evaluate and practice more aggressive nutritional management in this fragile population.

\begin{tabular}{|l|l|l|l|l|}
\hline & $\begin{array}{l}\text { Energy } \\
\text { intake } \\
(\mathrm{Kcal} / \mathrm{kg} / \text { day })\end{array}$ & $\begin{array}{l}\text { Weekly } \\
\text { energy } \\
\text { deficit } \\
(\mathrm{Kcal} / \mathrm{kg} / \mathrm{wk})\end{array}$ & $\begin{array}{l}\text { Protein } \\
\text { intake } \\
(\mathrm{gm} / \mathrm{kg} / \text { day })\end{array}$ & $\begin{array}{l}\text { Weekly } \\
\text { protein } \\
\text { deficit } \\
(\mathrm{gm} / \mathrm{kg} / \mathrm{wk})\end{array}$ \\
\hline Wekk 1 & $59.4 \pm 10.3$ & -424.2 & $1.25 \pm 0.04$ & 17.85 \\
\hline Week 2 & $104.4 \pm 9.4$ & -109.2 & $2.92 \pm 0.33$ & 6.16 \\
\hline Week 3 & $121 \pm 2.0$ & +7 & $3.39 \pm 0.08$ & 2.87 \\
\hline Week 4 & $128.7 \pm 5.0$ & +60.9 & $3.57 \pm 0.35$ & 1.61 \\
\hline $\begin{array}{l}\text { Cumulative } \\
\text { deficit for } \\
1^{\text {st } 4 \text { weeks }}\end{array}$ & & $465.5 \mathrm{Kcal} / \mathrm{kg}$ & & $28.49 \mathrm{gm} / \mathrm{kg}$ \\
\hline
\end{tabular}

PLASMA CONCENTRATIONS OF ARGININE AND ASYMMETRIC DIMETHYLARGININE IN PREMATURE INFANTS: A POSSIBLE EXPLANATION OF NECROTIZING ENTEROCOLITIS

R M van Elburg ${ }^{1}$, M Richir ${ }^{2}$, M P C Siroen ${ }^{2}$, W P F Fetter ${ }^{1}$, F Quik ${ }^{1}$, R J Nijveldt ${ }^{2}$, B J Smit ${ }^{4}$, T Teerlink ${ }^{3}$, P A M van Leeuwen ${ }^{2}$

${ }^{l}$ Department of Pediatrics Subdivision of Neonatology, VU University Medical Center, Amsterdam, The Netherlands, ${ }^{2}$ Department of Surgery, VU University Medical Center, Amsterdam, The Netherlands ${ }^{3}$ Department of Clinical Chemistry, VU University Medical Center, Amsterdam, The Netherlands ${ }^{4}$ Department of Neonatology, Emma Children's Hospital Academic Medical Center, Amsterdam, The Netherlands

Background and Aims: Several studies have described reduced plasma concentrations of arginine, the substrate for nitric oxide synthase (NOS) in infants with necrotizing enterocolitis (NEC). It is currently not known what the level of the plasma concentrations of the endogenous NOS inhibitor asymmetric dimethylarginine (ADMA) is in patients with NEC. We investigated whether plasma concentrations of arginine, ADMA and their ratio differ between premature infants with and without NEC, and within the NEC group between survivors and non-survivors.

Methods: In a prospective case-control study, arginine, and ADMA concentrations were measured in 10 premature infants with NEC (mean (SD) gestational age 197 (15) days, birthweight 1089 (367) g), and 10 matched control infants (gestational age 201 (14) days, birthweight 1063 (209) g), admitted to the Neonatal Intensive Care Unit.

Results: In the premature infants with NEC, arginine and ADMA concentrations, and the arginine/ADMA ratio were lower, compared to infants without NEC: median 21.40 vs $48.15, \mathrm{p}=0.001 ; 0.587$ vs 0.821 , $\mathrm{p}=0.015$; and 36.55 vs $68.82, \mathrm{p}=0.023$ respectively. In the NEC group, arginine and the arginine/ADMA ratio were lower in non-surviving than in surviving infants: median 14.65 vs $33.75, \mathrm{p}=0.01$; and 32.02 vs 47.47 , $\mathrm{p}=0.038$ respectively.

Conclusions: In premature infants with NEC not only the NOS substrate arginine, but also the endogenous NOS inhibitor ADMA and the arginine/ADMA ratio are lower than in infants without NEC. In addition, a low arginine and arginine/ADMA ratio are associated with mortality in infants with NEC. A diminished nitric oxide production may be involved in the pathology of NEC.

\section{POPULATION BASED EPIDEMIOLOGY OF NECROTIZING ENTEROCOLITIS ON VERY LOW BIRTH WEIGHT INFANTS IN PORTUGAL, 1996-2004}

D Virella ${ }^{1}$, G Rocha $^{2}$, G Silva $^{2}$, H Guimarães $^{2}$, R N Muito Baixo Peso ${ }^{3}$

${ }^{1}$ UCIN, Hospital de Dona Estefânia, Lisboa, Portugal

${ }^{2}$ Serviço se Neonatologia, Hospital de São João, Porto,

Portugal, ${ }^{3}$ Secção de Neonatologia da Sociedade

Portuguesa de Pediatria, Lisboa, Portugal 
Background and Aims: Necrotizing Enterocolitis (NEC) is a worrisome acquired gastrointestinal disease in VLBWI. We aim to monitor the population epidemiology and clinical outcomes of NEC in VLBWI. Methods: The National Registry of VLBWI Database (1996-2004) was used for NEC-related morbidity and mortality risk analysis. Bell's stage II-III cases were considered.

Results: In 1996-2004, 8567 VLBWI (2952 ELBWI) were registered in Portugal. The incidence of NEC was 765 VLBWI (8,3\%), 324 with NEC II-III (3,8\%), 194 among ELBWI (6,6\%). While global incidence of NEC and NEC II-III showed a wide annual variation, incidence of NEC IIIb significantly reduced $(r=-0,73)$. There was significant association of NEC II-III to gestational age $<30$ weeks, birth weight $<1000 \mathrm{~g}$, umbilical arterial catheter and indometacin administration. $111(34,4 \%)$ of NEC II-III cases were submitted to surgery. NEC caused death of $0,95 \%$ of the VLBWI and $2 \%$ of ELBWI. Lethality in stages II-III was $25 \%$ and $43 \%$ in stage IIIb. The lethality observed in the newborns with stage IIIb was reduced to $36,5 \%$ when submitted to surgery (RR 0,$63 ; 95 \% \mathrm{CI} 0,43-0,92$ ). The risk of death was $49 \%$ for NEC IIIb ELBWI, but it lowered to $39 \%$ when submitted to surgical intervention (RR 0,56; 95\%CI 0,38-0,82).

Conclusions: Data from a complete national population-based dataset certainly explain differences from previous studies based exclusively on VLBWI treated in level III NICU. A reduction of the incidence of NEC IIIb is apparent. The reduction of the death risk among grade IIIb ELBWI when submitted to surgery denotes the importance of early diagnosis and intervention.

\section{EFFECTS OF ORAL STIMULATION AND SUPPORT ON SUCKING PERFORMANCE OF PRETERM INFANT}

\author{
A Chemin ${ }^{1}$, L Da Nobrega ${ }^{2}$, A Henrot $^{1}$, M Boiron $^{2}$, \\ E Saliba $^{1,3}$ \\ ${ }^{1}$ NICU, Clocheville Hospital, Tours, France \\ ${ }^{2}$ Laboratory of Physiology and Digestive Motility, \\ Faculty of Medicine, Tours, France, ${ }^{3}$ INSERM U 619, \\ François Rabelais University, Tours, France
}

Background and Aims: The preterm infant frequently experiences oral feeding difficulties, because of brain stem immaturity and oral disorders due to inadequate oral stimulations in neonatal period (poor and painful stimuli). Oral stimulations before feeding or oral support measures seem to enhance suck and swallow coordination of the preterm infant. The aim of our study was to assess the beneficial effects of oral stimulation and support on preterm sucking performance.

Methods: 51 preterm infants born before 34 weeks gestationnel age were randomised into 3 experimental groups according to oral stimulation program and oral support measures, realised independently or combined, and control group. The oral stimulation program was administered once per day for 14 consecutive days, before tube enteral feeding then the bottle feeding was introduced. Oral support was administered once per day, during transition from tube to independent oral feeding.
Suction's strength (exprimed in mmHg) and frequency (measured in percentage of all sucking burst length during the entire recording) were regularly evaluated by a pressure transducer.

Results: The sucking strength increased significantly (cf. graph) and sucking rate had a tendency to increase $(p=0.1)$. These effects were earlier and longer in the time when oral stimulation program and oral support measures were associated.

Conclusions: A prefeeding oral stimulation program and oral support measures, provided combined or indepedently, enhance sucking performance of preterm infant in regard to sucking strength and frequency.

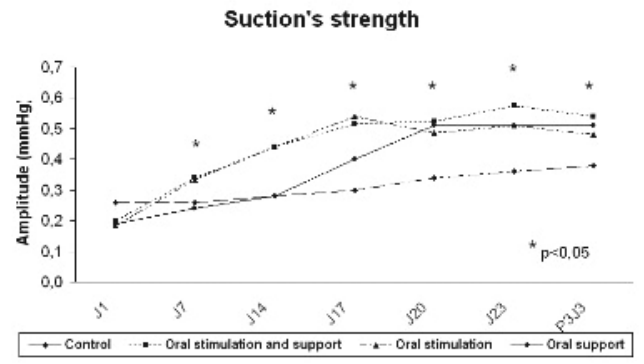

\section{DIAPHRAGMATIC ENDURANCE FOLLOWING ABDOMINAL DEFECT REPAIR IN GASTROSCHISIS INFANTS}

G Dimitriou, C Agakidis, G F Rafferty, A Greenough

Division of Asthma, Allergy and Lung Biology, Guy's, King's and St Thomas' Medical School, London, UK

Background and Aims: It has been shown that diaphragmatic strength postoperatively is impaired in infants with gastroschisis. Diaphragmatic tension-time index (TTdi), which describes the pressure-generating activity of the diaphragm, is a measure of diaphragmatic endurance and hence a high TTdi can predict diaphragmatic fatigue. The aim of this study was to test the hypothesis that infants who had surgical repair of gastroschisis would have impaired diaphragmatic endurance.

Methods: Twenty gastroschisis and twenty control infants, median gestational age 37 weeks were studied. TTdi was assessed in gastroschisis infants and controls matched to gestational age. TTdi was calculated as the product of the mean to the maximum transdiaphragmatic pressure (Pdimean/Pdimax) and the inspiratory duty cycle (Ti/Ttot). Transdiaphragmatic pressure (Pdi) was calculated by subtraction of oesophageal from gastric pressure measured using a Gaeltec dual pressure catheter during quiet breathing. The Pdimean during a breath was electronically determined over the inspiratory time interval where the Pdimax was the largest transdiaphragmatic pressure generated during airway occlusions performed at the end of a crying effort. The inspiratory duty cycle was calculated using the oesophageal and transdiaphragmatic pressure signals.

Results: The gastroschisis infants had higher TTdi than the controls (median 0.053 range 0.026 to 0.081 versus median 0.040 , median 0.022 to 0.084 , respectively, $\mathrm{p}=$ 0.0186 ). 
Conclusions: These results suggest that shortly after the abdominal wall repair, gastroschisis infants have impaired diaphragmatic endurance.

\section{EFFECTS OF ORAL GLUTAMINE SUPPLEMENTATION ON THE INTESTINAL PERMEABILITY AND GROWTH OF PRETERM INFANTS}

\author{
S Sevastiadou, A Puchner-Malamitsi, E Stratiki, \\ L Kastanidou, M Skouroliakou, A Antsaklis, \\ C Kostalos
}

Department of Neonatology, 'Alexandra' University Hospital, Athens, Greece

Background and Aims: The aim of the study was to evaluate the effect of Glutamine supplementation on the intestinal permeability of preterm infants and contribution to growth. Methods: In a prospective randomized study, 74 healthy premature neonates of less than 2000gr. birth weight, born at gestational age of 26 - 34 weeks, were included. Fourty one neonates (Glutamine group) received a preterm formula, supplemented with Glutamine 0,3gr. per kg, whereas the other thirty four neonates were fed with non supplemented formula (control group). Lactulose/mannitol excretion ratio in urine samples was used to measure the intestinal permeability and was performed three times: a) at birth b) at the age of 7days c) at the age of 1-month. Results: There was no signifact difference in anthropometric measurements between the two groups, at the time of birth. At the age of 1-month, the head circumference and the weight were statistically higher in Glutamine group: ( $\mathrm{HC} \mathrm{p}<0,001$ ) (WG $p=0,001$ ) as compared to the control group. The $\mathrm{L} / \mathrm{M}$ ratio, although lower in the Glutamine group, did not reach however statistical significancy $(p=0,392)$.

Conclusions: The administration of Glutamine leads to significant increase of the anthropometric parameters and to lower intestinal permeability. Further studies will be needed using higher doses of glutamine in order to confirm preliminary results.

\section{EMERGENCY DRAINAGE OF ABDOMINAL COMPARTMENT SYNDROME IN SEVERE NECROTISING ENTEROCOLITIS IMPROVES CARDIAC OUTPUT AND RESPIRATORY FUNCTION}

\author{
J Brierley $^{1}$, N Hall ${ }^{2}$, A Pierro ${ }^{2}$, M Peters ${ }^{1}$ \\ ${ }^{l}$ Paediatric and Neonatal Intensive Care, ${ }^{2}$ Department \\ of Paediatric Surgery, Great Ormond Street Hospital, \\ London, UK
}

Background and Aims: Abdominal distension in severe necrotising enterocolitis (NEC) may cause an abdominal compartment syndrome (ACS). In other scenarios (e.g. abdominal trauma) multi-organ failure (MOF) is accelerated by a reduction in venous return and increased systemic vascular resistance secondary to the ACS. While semi-elective abdominal drainage is a recognised approach to perforated NEC, the acute haemodynamic impact of emergency abdominal drainage (EAD) has not been reported. We hypothesised that ACS can be a contributor to the MOF of NEC and that EAD improves cardiorespiratory function immediately in these most severely ill premature infants. Methods: Six infants (corrected gestation 25-33 weeks, weight $0.77-2.2 \mathrm{Kg}$ ) with stage III NEC, and profound cardiorespiratory failure (despite volume resuscitation $>$ $60 \mathrm{ml} / \mathrm{kg}$, epinephrine $>0.5 \mathrm{mcg} / \mathrm{kg} / \mathrm{min}$ ) on admission had clinical ACS and underwent EAD. Cardiac Index (cardiac output/body surface area) was assessed with the non-invasive USCOM device immediately prior to and within $6 \mathrm{~min}$ of drainage. Oxygenation index (OI $=$ FiO2xMean Airway Pressure $/ \mathrm{PaO} 2)$ was noted before and $<60$ min after drainage. Results: Emergency abdominal drainage was associated with a rapid improvement in cardio-respiratory status in each case without other interventions. Initial measures of cardiac index were low in every case but increased by $71-283 \%$, $(p=0.03$ Wilcoxon signed ranks, Figure). High initial oxygenation index values (median 56 ), fell by between $27-56 \%, \quad(p=0.03$. Wilcoxon signed ranks). Conclusions: Abdominal compartment syndrome contributes to the severity of multiple organ failure in severe NEC. Emergency abdominal drainage is associated with an immediate improvement in cardiorespiratory status.

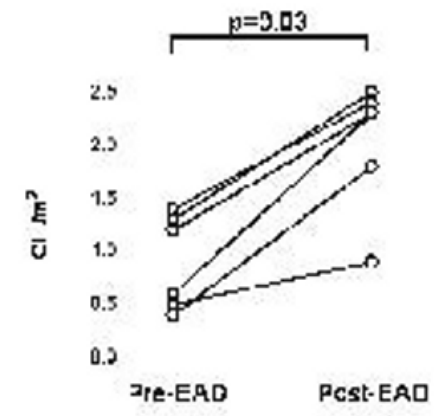

\section{EFFECTS OF ORAL BIFIDOBACTERIUM LACTIS ON THE INTESTINAL PERMEABILITY AND THE GROWTH OF PRETERM INFANTS}

\author{
E Stratiki ${ }^{1}$, S Sevastiadou ${ }^{1}, \mathrm{~K}$ Stamouli ${ }^{1}$, \\ ${\mathrm{O} K a s t a n i d o u^{2}, \text { M Skouroliakou }^{2}, \text { C Kostalos }}^{1}$ \\ ${ }^{1}$ Department of Neonatology, General Hospital \\ 'Alexandra', 'Department of Science of Nutrition and \\ Dietetics, Harakopio University, Athens, Greece
}

Background and Aims: The aim of the study was to evaluate the effect of Bifidobacterium lactis supplementation on the intestinal permeability of preterm infants and its contribution to growth.

Methods: In a prospective randomized study, 75 healthy premature neonates of less than 2000 gr. birth weight, born at gestational age of 26-36 weeks, were included. Forty-one neonates (probiotic group) received a preterm formula to which B.lactis was added, whereas the other thirty-four neonates were fed with an unsupplemented pre-term formula (control group). The intestinal permeability was examined three times using a sugar permeability absorption test, by introducing orally mannitol and lactulose and assessing their concentration in urine samples: a) at birth b) seven days after the initiation of feeding and, c) at the age of 1 month or 2125 days after the onset of enteral feeding. 
Results: Head circumference was statistically higher in infants receiving probiotic $(\mathrm{P}=0,001)$ at the age of one month. Between groups, comparison showed that $\mathrm{L} / \mathrm{M}$ ratio in the probiotic group was significantly lower in comparison with the control group $(\mathrm{p}=0,003)$ due to decrease in lactulose absorption from the gut.

Conclusions: The administration of the probiotic B.lactis to preterm leads to significant decrease of intestinal permeability (or increase of the gut barrier). This could be one of the mechanisms through which probiotics reduce bacterial translocation and allergic disease.

\section{NEONATAL DEHYDRATION - WHY INCREASING INCIDENCE?}

\author{
M Reilev, O Pryds, K Børch \\ Department of Childcare, Hvidovre Hospital, Hvidovre, \\ Denmark
}

Background and Aims: Neonatal dehydration with hypernatraemia is a serious condition with risk of cerebral damage and death. The aim of this study was to evaluate the incidence of dehydration among readmitted neonates and to determine related complications.

Methods: A retrospective study was conducted at Hvidovre Hospital over a 5-year period to identify term or near-term infants ( $>35$ weeks of gestation) who were admitted with a weight loss $>10 \%$. Infants admitted between the third and the 14th day of life having a discharge diagnosis code indicating dehydration were included.

Results: During the period a total of 89 infants were admitted with dehydration -24 had hypernatraemia. They were born primarily to primiparous women $(72.3 \%)$ and 9 were heavy for gestational age (HGA). All were breastfed and only three received supplementation prior to readmission. The weight loss ranged between 270 and 1100 grams (corresponding to $10.1-29.7 \%$ of birth weight) with a serum sodium concentration being proportionally related to the relative weight loss (range: 135-175 mmol/l). Lethargy and jaundice were frequent symptoms. Cerebral complications occurred for eight infants and two developed permanent brain damage. There were no deaths. The incidence of dehydration increased from 2.1 to 4.9 during the period.

Conclusions: Breastfeeding should be encouraged but supplementation may be necessary for some neonates. Infants born to primiparous women and HGA infants need special attention. Infants at risk could be identified by regular weighing from day $4-5$ at latest. Supplementation and follow-up should be instituted if necessary.

\section{INTESTINAL PROTECTION BY ACTIVATION OF PPAR GAMMA}

C Kluthe, K Klinghammer, R Wauer, F Guthmann

Department of Neonatology, Charité Campus Mitte, Berlin, Germany
Background and Aims: Prematurity remains the main but less understood risk factor for necrotising enterocolitis (NEC) - the most devastating intestinal emergency in premature infants. The molecular mechanisms of immaturity are still unclear, nutrition with breast milk is known to act prophylactic. We aimed to specify prophylactic peptides, which are developmentally expressed, and induced by activation of transcriptional factor PPARg.

Methods: Intestinal expression of haeme oxygenase-1 (HO-1), and treefoil factor 3 (TFF3) was measured by quantitative PCR and western blot analysis in rats before (d18, d20 post conceptionem), and after birth (d2 p.n.) comparing the effects of PPARg activation (oral application of Pioglitazone to the mother). Early enterocolitis was induced in dam-fed newborn rats by hypercapnia/reoxygenation $(\mathrm{H} / \mathrm{R})$ in Pioglitazone treated animals. Capase 3 activation was detected calorimetically.

Results: Unlike to d18 rats we found a significant increase in intestinal TFF3 expression after PPARg activation in $\mathrm{d} 20$ rats. $48 \mathrm{~h}$ after $\mathrm{H} / \mathrm{R}$ we saw an anctivtion of Caspase 3 in dam fed animals. This effect could be abrogated by maternal application of Pioglitazone, and was associated with an increase in HO-1 expression in newborn rats.

Conclusions: Oral application of Pioglitazone to rats during pregnancy provides an effective strategy to induce intestinal TFF3, which is known to act protective in the intestine. After birth PPARg activation induces a rise in HO-1 expression and ameliorates harming effects of $\mathrm{H} / \mathrm{R}$. By this Pioglitazone acts protective via two peptides and could reveal a new strategy in the prevention of NEC.

\section{SERUM AND BREAST MILK LEPTIN AND GHRELIN LEVELS IN BREAST AND FORMULA FED NEWBORNS}

\author{
E Onal $^{1}, \mathrm{~N}_{\text {Okumus }}{ }^{1}, \mathrm{C}$ Turkyilmaz ${ }^{1}$, Y Atalay ${ }^{1}$, \\ S Elbeg ${ }^{2}, \mathrm{H}^{2}$ Pasaoglu ${ }^{2}$ \\ ${ }^{1}$ Department of Pediatrics, Gazi University Medical \\ School, Ankara, Turkey, ${ }^{2}$ Department of Clinical \\ Biochemistry, Gazi Uinversity Medical School, Ankara, \\ Turkey
}

Background and Aims: The aim of this study was to investigate serum and breast milk leptin, ghrelin, insulin, GH ve IGF concentrations before and after feeding in exclusively breastfed and formula fed neonates.

Methods: The serum and breast milk leptin, ghrelin, insulin, GH ve IGF concentrations were measured before (fasting) and 30 minutes after feeding (postprandial) in 22 breastfed and 23 formula fed term infants at Gazi University Hospital. The study was approved by local ethical committee.

Results: The mean gestational age, birth weight and postnatal age of infants were similar in groups. No difference was found between breast milk leptin, insulin, GH ve IGF concentrations before and after feeding. However, the mean fasting breast milk ghrelin concentration was higher than postprandial levels (mean $\pm \mathrm{SD} ; 1215,2 \pm 692,2 \mathrm{pg} / \mathrm{mL}$ vs $785,2 \pm 666,7$ 
$\mathrm{pg} / \mathrm{mL})(\mathrm{p}<0.05)$. Postprandial serum GH, IGF and insulin levels were higher than fasting levels in both groups $(\mathrm{p}<0.05)$. Fasting serum ghrelin concentrations $(2515,9 \pm 592,2 \mathrm{pg} / \mathrm{mL})$ were higher than postprandial serum concentrations $(951,2 \pm 384,2 \mathrm{pg} / \mathrm{mL})(\mathrm{p}=0.000)$ in breastfed infants. No difference was found between the serum concentrations of ghrelin before and after feeding $(1639,1 \pm 435,7 \mathrm{pg} / \mathrm{mL}$ vs $1427,2 \pm 365,4$ $\mathrm{pg} / \mathrm{mL}$, respectively) in formula fed infants ( $\mathrm{p}>0.05$ ). In both groups, fasting and postprandial serum leptin levels were similar ( $\mathrm{p}>0.05)$.

Conclusions: It is concluded that ghrelin but not leptin seems to be an important factor for stimulating feeding. It is also an interesting finding that formula feeding did not affect serum ghrelin levels in contrast to breast feeding.

\section{A STUDY OF EPIDERMAL GROWTH FACTOR IN SALIVA OF NORMAL PRETERM INFANTS - IMPLICATIONS FOR MANAGEMENT OF NEC?}

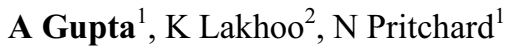 \\ ${ }^{I}$ Neonatal Unit, John Radcliffe Hospital, Oxford, UK \\ ${ }^{2}$ Department of Paediatric Surgery, John Radcliffe \\ Hospital, Oxford, UK
}

Background and Aims: Epidermal growth factor (EGF) is an amino acid polypeptide that has been shown to promote mucosal healing and intestinal growth in experimental models and has a potential role in the aetiology and treatment of NEC. We have previously shown that EGF measurement in saliva was relatively consistent and reproducible as compared to other bodily fluids. The aim of this study was to determine the normal levels of salivary EGF in a cohort of pre-term infants.

Methods: With appropriate ethical approval and informed consent, saliva was collected using a suction catheter from all normal pre-term infants admitted to our unit over a 9 month period. The samples were immediately frozen at $-20 \mathrm{C}$ and analysed using an ELISA assay. All results are expressed as mean values with standard deviation.

Results: A total of 60 samples were collected and analysed from 25 babies. The mean gestation and weight of the patients was $32.2(+/-5.50)$ weeks and 1954.44 $(+/-1045.7)$ grams respectively. None of the patients had clinical or serum markers of sepsis or NEC. All infants were given maternal breast milk started at the mean age of $5(+/-3.47)$ days. The mean EGF values were 676.95 $(+/-168.47) \mathrm{pg} / \mathrm{ml}$. There was no correlation between the EGF values and differences in gestation, birth weight or timing of initiating enteral feeds.

Conclusions: This study provides a useful baseline of salivary EGF values in normal pre-term infants. This data may have important implications for understanding the aetiology of NEC and in the design of novel therapies for this condition.

\section{IMPROVING THE NUTRITIONAL INTAKE OF THE VERY LOW BIRTH WEIGHT (VLBW) INFANT}

M C Chua ${ }^{1}$, W Y Teo ${ }^{2}, \mathrm{~J} \mathrm{M} \mathrm{Gomez}^{1}$, J Y Soh ${ }^{3}$

${ }^{1}$ Department of Neonatology, KK Women's and Children's Hospital, Singapore, ${ }^{2}$ Department of Paediatric Medicine, KK Women's and Children's Hospital, Singapore, ${ }^{3}$ Department of Paediatric Medicine, National University Hospital, Singapore

Background and Aims: To reduce mean duration of time taken for VLBW infants (birth weight $<1500$ grams) to attain a weight of $2 \mathrm{~kg}$, by $15 \%$ over 6 months.

Methods: A multidisciplinary team involving neonatologists, nurse managers and dietitian was formed. Nutritional interventions were introduced based on evidence in the literature. New parenteral nutrition policies (early initiation of total parenteral nutrition, rapid advancement of amino-acids, lipids, glucose) and new enteral nutrition policies (standardization of definition of minimal enteral nutrition, revision of rate of feed increment, encouragement of use of breast milk, introduction of ad-lib feeding, use of preterm milk formula instead of term milk formula when breast milk was not available) were implemented using a clinical pathway in December 2004. An audit on the clinical pathway was carried out from December 2004 to May 2005.

Results: 59 VLBWs were enrolled in study. Mean weights on days 14 and 21 showed an improving trend indicating a possible effect of revised parenteral and enteral nutrition policies. There was a $21 \%$ reduction in the time taken to reach a discharge weight of $2 \mathrm{~kg}$ ( 60 days versus 47.6 days). The incidence of necrotising entercolitis remained low despite more aggressive enteral feeding guidelines.

Conclusions: We achieved a $21 \%$ reduction in the time taken to achieve a weight of $2 \mathrm{~kg}$. Early discharge means a shortened length of stay, reducing cost of hospitalization. Good nutrition ensures a good start in life for this group of vulnerable infants. The use of evidence-based medicine and a multidisciplinary approach helped to improve our clinical practice.

\section{GASTROTONOMETRIC EXAMINATIONS WITH A NEW, EASILY APPLICABLE PROBE IN INFANTS REQUIRING INTENSIVE CARE}

\author{
G Tálosi ${ }^{1}, \mathrm{~J} \mathrm{Kaszaki}^{2}, \mathrm{D}$ Boda ${ }^{1}$
}

${ }^{1}$ Department of Paediatrics, University of Szeged, Szeged, Hungary, ${ }^{2}$ Department of Experimental

Surgery, University of Szeged, Szeged, Hungary

Background and Aims: Gastrotonometry, a method developed for the measurement of the partial pressure of carbon dioxide $(\mathrm{PgCO} 2)$ in the gastrointestinal tract. The main field of application is to monitor the condition of patients in critical states. Although it is widely applied in adult intensive care, its use is limited in paediatrics. Our main aim was to develop a method with 
which gastrotonometric examinations can be performed on neonates and infants, without difficulties.

Methods: A newly developed balloon-free probe was used. 29 neonates and infants requiring intensive care were monitored (age: 2 - 456 days, weight: 1200 $6700 \mathrm{~g}$ ). Paediatric Index of Mortality scores, $\mathrm{PCO} 2$ gap values, acid base parameters and intramucosal $\mathrm{pH}$ values were measured/calculated. Results of surviving and non-surviving patients were compared with unpaired, two-tailed Student's test.

Results: Examinations could be easily performed. Out of the 29 patients 7 died. PIM scores $(35.1 \pm 19.6 \%$ versus $14.6 \pm 14.8 \%$ ), as well as PCO2 gap values $(13.48 \pm 9.30 \mathrm{mmHg}$ versus $8.43 \pm 6.54 \mathrm{mHg})$ and systemic-intramucosal $\mathrm{pH}$ differences $(0.124 \pm 0.074$ versus $0.079 \pm 0.054$ ) were significantly higher in the non-surviving group of patients.

Conclusions: The new gastrotonometric probe is easily applicable even in infants. Residents and nurses can perform the examinations, too. Worsening gastrotonometric parameters may predict adverse outcome.

\section{VERTICAL HIV EXPOSURE - A NEW RISK FACTOR FOR NECROTIZING ENTEROCOLITIS?}

T Schmitz ${ }^{1}, \mathrm{~K}$ Weizsaecker ${ }^{2}$, C Feiterna-Sperling ${ }^{3}$, E Eilers $^{1}$, M Obladen $^{1}$

${ }^{I}$ Department of Neonatology, Campus Virchow Klinikum, Universitaetsmedizin Berlin Charité, Berlin, Germany, ${ }^{2}$ Department of Obstetrics, Campus Virchow Klinikum, Universitaetsmedizin Berlin Charité, Berlin, Germany, ${ }^{3}$ Department of Pediatric Pulmonology and Immunology, Campus Virchow Klinikum,

Universitaetsmedizin Berlin Charité, Berlin, Germany

Background and Aims: Classical risk factors for necrotizing enterocolitis (NEC) are prematurity, hypoxia/ischemia, cardiac malformation, anaemia, and formula feeding. In a recent study, an association between maternal HIV infection and NEC has been observed in preterm neonates (Desfrere et al. 2005). We report about a term infant that developed NEC without identifiable risk factors other than exposure to maternal HIV infection and to antiretroviral medication.

Methods: Case report.

Results: The 21 year old G1 P1 from Kamerun started using nevirapine, lamivudine and zidovudine at 31 gestational weeks, viral load decreased from 55.800 to $64 \mathrm{cop} / \mathrm{ml}$ prenatally, CD4-cells were normal. Postnatal adaptation of the newborn was uneventful, birthweight was $3015 \mathrm{~g}$, APGAR scores at 1,5 and 10 minutes were 6,10 and 10 , respectively. The male infant was delivered by elective Cesarean section at $37+4$ weeks of gestation to an HIV positive mother. Postnatal transmission prophylaxis was performed with intravenous zidovudine $1.3 \mathrm{mg} / \mathrm{kg}$ body weight four times daily. On the fifth day of life, the infant developed clinical signs of sepsis, interleukin-6 of 50.000ng/nl and granulocytopenia of $1.3 / \mathrm{nl}$ confirmed the diagnosis. An abdominal x-ray revealed pronounced intestinal pneumatosis. Besides antibiotic treatment and feeding abstinence, symptoms deteriorated, and the infant underwent surgical resection of perforated and necrotic bowel. Re-anastomosis was performed at four weeks of age. Vertical HIV-1 infection could be excluded after three consecutive HIV-DNA polymerase chain reaction tests at three months.

Conclusions: Exposure to HIV and antiretroviral drugs is associated with NEC in preterm and term infants.

\section{AN UNEXPECTED CAUSE OF GASTRIC PERFORATION IN A TERM NEONATE}

P Korhonen $^{1}$, M Helminen ${ }^{1}$, T Iber ${ }^{1}$, A Abram², O Tammela ${ }^{1}$

${ }^{l}$ Department of Paediatrics, Tampere University

Hospital, Tampere, Finland, ${ }^{2}$ Department of

Paediatrics, Kanta-Häme Central Hospital, Hämeenlinna, Finland

Background and Aims: Neonatal gastric perforation is a rare occurrence of often unknown etiology. Our aim is to explore a case of a term-born neonate with gastric perforation.

Methods: Fungal and bacterial cultures of blood and peritoneal fluid. Periodic acid-Schiff (PAS) staining of tissue specimens.

Results: A 37-week boy of $3050 \mathrm{~g}$ birth weight was born vaginally after an uneventful pregnancy. In the 2 nd day of life he became irritable and febrile, and antibiotic treatment was initiated. Abdominal distention developed within 4 hours; X-rays showed pneumoperitoneum. Laparotomy revealed a gastric perforation with peritonitis. Recurrent perforations were sutured at 9 and 12 days. Candida albicans grew from peritoneal fluid at 2 and from blood at 9 days. PAS staining revealed fungal spores and filaments in the muscular gastric wall. Fluconazole was started at 9 days, and liposomal amphotericin B at 12 days. Granulocyte colony stimulating factor was administered. At 3 weeks, the wound and fascia ruptured. Coagulase-negative Staphylococcus species grew from the peritoneal fluid. Due to infection the fascia was not reconstructed until at 4 weeks. Wide-spread skin necrosis, severe respiratory problems and bleeding tendency developed. Fungal blood cultures remained positive, and fluconazole was substituted with caspofungin. Granulocyte transfusions were administered. The infection subsided gradually. The first negative fungal blood culture was obtained at 23 days, and antifungal treatment discontinued at 53 days. The wound healed well. No increased susceptibility to infections was noted by one year of age. Conclusions: Fungal infection and early antifungal therapy should be considered in term newborns with gastric perforation.

\section{ANTHROPOMETRICAL PARAMETERS OF CHILDREN AGED 5-6 YEARS AND FACTORS INFLUENCING CHILD GROWTH}

M Kherkheulidze, N Manjavidze

Department of Pediatrics, State Medical University, Tbilisi, Georgia 
Background and Aims: Aim of the study was to assess present nutritional status of children aged 5-6 years and to identify possible factors influencing growth pattern.

Methods: The cross-sectional study covered randomly selected 754 children of 5-6 years old. The percentiles and $\mathrm{Z}$ scores of weight for age and for height, height for age, BMI and the percentile and Z score of BMI were calculated using EPI INFO program. To identify factors influencing child growth were used special questionnaire.

Results: The results revealed prevalence of children with overweight. The growth depend on the child sex, the high weight for age is more frequent in girls while height for age in boys. Approximately 13 per cent of children's BMI is under 5 percentile, 18 percent of children have risk of overweight and 16 percent have overweight. Genetic factors such as low anthropometrical indices of parents, poor feeding practices of the children are suggested reasons for the low BMI. Length and weight of child at birth showed significant correlations with height at 5 years. Socioeconomic factors appeared to be of little importance as determinants of growth. At the same time income and per capita food expenditure was positively correlated with nutritional status.

Conclusions: We can conclude that factors influencing child growth can be considered as risk factors for growth and could be used to identify households at risk of malnutrition, as well as for overweight.

\section{EPIDEMIOLOGIC CHARACTERISTICS OF CHILDREN'S POISONING CASES ADMITTED TO A REGIONAL HOSPITAL IN GREECE}

P Anastasopoulou, N Manakou, A Giannisi, G Z Zantopoulos, S Liosi, O Gkika, Z Patrona

Department of Paediatric, General Hospital of Korinthos, Korinthos, Greece

Background and Aims: Poisoning represents one of the most common medical emergencies encountered in childhood. This study was performed to contribute epidemiological data regarding poisonings in childhood.

Methods: Medical records of 707 children admitted in our paediatric department for acute poisoning, were studied. The mean age of the population was $3.2 \pm 1.2$ years. Results: During the period 1996- 2005, 707 patients, with acute poisoning were admitted in Paediatric Ward $(4.71 \%$ of total admissions). The number of cases increased over the 10 -year period. The seasonal distributions in poisoning patients suggested a peak on summer $(30.3 \%)$ and lower numbers on spring $(25.6 \%)$, winter $(22.3 \%)$ and autumn (21.8\%). Male exposures were more prevalent than females (57.1\%vs.42.9\%) The etiology most often involved was: accidental $(95.9 \%)$, followed by intentional $(2.9 \%)$ and undetermined $(1.2 \%)$. The most commonly implicated substance categories recorded were: drugs $49.1 \%$ (347/707), especially paracetamol, nonsteroidal antiinflammatory drugs, antihistamines and antibiotics, cigarette $14.1 \%$ (100/707), agricultural drugs $13.2 \%$ (94/707), detergents $12.4 \%$ (88/707), alcohol 4\% (28/707), other hazard factors $7.2 \%(50 / 707)$ such as carbohydrates, carbon monoxide inhalation and plants.
In the majority of cases, complications were mild or absent and no deaths were recorded.

Conclusions: Accidental poisoning exposures from drugs, cigarettes and household and agricultural products remain a significant problem. Severe symptoms have rarely been observed in drug poisonings in childhood. The frequency of accidental poisoning in childhood merits more widespread public education aimed at preventing exposure to toxic substances.

\section{DO SOCIOECONOMIC FACTORS EXPLAIN WHY MATERNAL SMOKING DURING PREGNANCY IS HIGHER IN A MORE DEVELOPED CITY OF BRAZIL?}

\author{
V S Ribeiro ${ }^{1}$, A A M Silva ${ }^{2}$, M A Barbieri ${ }^{3}$, B Bettiol $^{3}$, \\ V M F Aragão ${ }^{1}$ \\ ${ }^{l}$ Department of Pediatrics, University of Maranhão, São \\ Luís, Maranhão, ${ }^{2}$ Department of Public Health, \\ ${ }^{3}$ Department of Pediatrics, University of São Paulo, \\ Ribeirão Preto, São Paulo, Brazil
}

Background and Aims: The prevalence of smoking during pregnancy in Ribeirão Preto, one of the richest cities in the country, was significantly higher $(21.4 \%)$ than in São Luís (5.9\%), a less developed city. To assess which variables, especially socioeconomic factors, explain the higher prevalence of smoking during pregnancy in the more developed city.

Methods: Two cohort studies were conducted, one in Ribeirão Preto, in 1994, on a sample of 2846 puerperae, and the other in São Luís, in 1997/98, on a sample of 2445 puerperae. Logistic regression was used to analyze the interactions between the factors studied and the city. Results: In multivariable analysis, the variables associated with smoking habit in São Luís were a household with five or more persons (OR 1.72), having five or more children (OR 2.10), smoking habit of the companion (OR 2.20) and age of 35 years or more (OR 1.98). Age of less than 20 years was a protective factor (OR 0.55). In Ribeirão Preto there was association with low educational level (OR 2.18) and with smoking habit of the companion (OR 3.25). Receiving prenatal care was a strong protective factor (OR 0.24). There was a significant interaction between city and mother working outside the home, maternal age and prenatal care visits. However the variables explained little of the difference in maternal smoking between the two cities.

Conclusions: The variables of the model, especially income or educational level, provided little explanation of the higher prevalence of smoking during pregnancy in the more developed city. Funded by FAPESP and CNPq.

\section{DOES THE PHYSICIAN FACTOR EXPLAIN WHY CESAREAN SECTION RATES ARE HIGHER IN THE SOUTHEAST THAN IN THE NORTHEAST REGION OF BRAZIL?}

\author{
V S Ribeiro ${ }^{1}$, A A M Silva ${ }^{2}$, M A Barbieri ${ }^{3}, \mathrm{H} \mathrm{Bettiol}^{3}$ \\ ${ }^{l}$ Department of Pediatrics, University of Maranhão, São \\ Luis, Maranhão, Brazil, ${ }^{2}$ Department of Public Health, \\ University of Maranhão, São Luis, Maranhão, Brazil
}


${ }^{3}$ Department of Pediatrics, University of São Paulo, Ribeirão Preto, São Paulo, Brazil

Background and Aims: Brazil has the highest cesarean section rate in the world which is higher in a more developed region, the Southeast, as compared to a less developed one, the Northeast. To assess which variables explain the higher cesarean section rate in the more developed region.

Methods: 2,846 puerperae were evaluated in Ribeirão Preto (RP), in the Southeast, in 1994, and 2,445 in São Luís (SL), in the Northeast region. A Poisson regression model was used to calculate non-adjusted and adjusted risk estimates. Variables were also adjusted one at a time in a combined model, including the variable 'city' in order to identify which variables could explain the difference in the rate between the two cities.

Results: The cesarean section rate was $50.8 \%$ in RP and $33.7 \%$ in SL. Practically the same variables were associated with cesarean delivery in the two towns: age of 35 years or more (RR 1.45 for RP and 1.54 for SL), adequate prenatal care (RR 1.24 and 1.29) and delivery performed by the same doctor who had provided prenatal care (RR 2.17 and 1.66). Association with marital status was observed only in São Luís (RR 1.19). Adjusted analysis in a combined model revealed that the variables 'category of admission' and 'the same doctor providing prenatal care and performing delivery' explained together $90.2 \%$ of the difference in cesarean section rate between the two cities.

Conclusions: Difference in cesarean section rate between the two cities was explained predominantly by the physician factor. Funded by FAPESP and CNPq.

\section{CHILDREN RESPIRATORY SYSTEM AND ATMOSPHERIC ENVIRONMENT}

\author{
T Ferreira $^{1}, \mathrm{~N} \mathrm{Batista}^{1}, \mathrm{C}_{\text {Louro }}{ }^{2}, \mathrm{R}_{\text {Cerdeira }}{ }^{2}$, \\ L Coelho $^{2}$, G Garcia $^{2}$, C Gouveia ${ }^{2}$ \\ ${ }^{l}$ Paediatric Service, Hospital Nossa Senhora do \\ Rosário, Barreiro, Portuga, ${ }^{2}$ Mechanical Department, \\ Escola Superior de Tecnologia, Setúbal, Portugal
}

Background and Aims: Air pollution affect children in a particular way, since their respiratory system is still in development and airways are more vulnerable. The aim of this work is to investigate atmospheric environment effects, such as air pollution and meteorological conditions, on a children population, between 0 and 15 years old, who attend Barreiro city hospital urgencies with respiratory problems.

Methods: Pollutants and meteorological variables were measured by stations distributed in the city. To study the possible relation between environmental factors and children health, children number admitted in paediatric urgency service, their symptomatology, meteorological variables and atmospheric pollutants, descriptive statistics was made and Pearson correlation coefficients were calculated.

Results: In the studied period, 958 children were admitted in city Hospital urgency with respiratory problems, being $28 \%$ for cough, $40 \%$ for respiratory syndrome and $32 \%$ for asthma. A negative correlation between children number and temperature and a positive correlation with relative humidity was observed. Concerning the relation between children number and pollutants concentration, weak but positive correlations were found for $\mathrm{CO}$ and NOx. Remain pollutants were negatively correlated.

Conclusions: The complexity of intervenient factors between health and atmospheric environment relation, are the reason for such weak correlations. Time factors such as weekdays, annual seasonality, epidemics among others are called confusion factors and will be introduced in further studies to achieve better correlations.

This work referenced POCTI/MGS/47247/2002 was performed in the framework of POCTI program financed by FCT of Portugal and FEDER program. The authors also wish to acknowledge CCDR-LVT, IM, IGEO and EDP for provided information.

\section{RISK FACTORS FOR CARDIOVASCULAR DISEASE IN PEDIATRIC RENAL TRANSPLANT RECIPIENTS}

T Ferreira, M Chaves, C Simão

Pediatric Nephorology Unit, Pediatric University

Clinic, Santa Maria Hospital, Lisbon, Portugal

Background and Aims: Cardiovascular disease is an important cause of morbidity and mortality and can compromise the graft survival. Aims: Evaluate the prevalence of the risk factors for cardiovascular disease, including hypertension, obesity, dyslipidemia and hyperhomocysteinemia and search possible correlation between themselves.

Methods: Prospective transversal study in patients between 0 and 19 years old. Hypertension was considerate when systolic or diastolic blood pressure was greater then the 95th centile for age, sex and height and when was introduced antihypertensive therapy. Obesity with BMI $>25$; Hypercholesterolemia serum total cholesterol > $200 \mathrm{mg} / \mathrm{dl}$, Hypertriglyceridemia serum total triglycerides $>200 \mathrm{mg} / \mathrm{dl}$, hyperhomocysteinemia serum total homocysteine $>11,3 \mu \mathrm{mol} / 1$. Descriptive statistics was made with SPSS-12.0 and Pearson correlation coefficients were calculated.

Results: In population of 22 patients were identified at less one risk factor in $19(86 \%)$ and in 17 had 2 or more risk factors. Hypertension was the alteration more prevalent 19/22 (86\%), after hyperhomocysteinemia in $12(54,5 \%)$ and Hypercholesterolemia in 11/22 (50\%). Obesity and Hypertriglyceridemia were found in 6 $(27,2 \%)$ and $4(18,1 \%)$ respectively. Positive and significantly correlation were found for Hyperhomocysteinemia and Hypercholesterolemia $(\mathrm{r}=0,44, \mathrm{p}=0,035)$.

Conclusions: The prevalence of risk factors for cardiovascular disease is high and is frequent association between risk factors. In these study positive correlation were found for Hyperhomocysteinemia and Hypercholesterolemia. It is necessary improve this alterations for to assure greater graft survival with best life quality for patients. 


\section{BIRTH WEIGHT AS A RISK FACTORS FOR OBESITY IN CHILDHOOD}

\author{
I Bralic ${ }^{1}$, M Kojundzic Tiljak ${ }^{2}$, U Rodin ${ }^{3}$ \\ ${ }^{1}$ Pediatric Outpatient Clinic, Trogir, Croatia \\ ${ }^{2}$ Andrija Štampar School of Public Health, School of \\ Medicine, University of Zagreb, Zagreb, Croatia \\ ${ }^{3}$ Croatian National Institute of Public Health, Zagreb, \\ Croatia
}

Background and Aims: The obesity in Croatia, likewise in all over the world, is getting wide proportions. The early recognition of children with higher risk of obesity should contribute to the prevention of different chronic diseases. The relation between the birth weight (BW) and the total overweight (TO) (BMI $\geq 85$ centils) and obesity (BMI $\geq 95$ centils) at 7,11 and 15 years of age were analysed.

Methods: Prospective study of child growth has been made on children $(n=1.721)$ growing up in the same social-economical situation in Trogir, Croatia. The BW prediction on TO or obesity during childhood was estimated by logistical regression.

Results: The incidence of TO and obesity didn't differ according the sex $(p=0.999)$ and age $(p=0.999)$. The examines with $\mathrm{BW} \geq 4000 \mathrm{~g}$ showed higher incidence of TO $(14.8 \%$ vs $9.62 \%$ vs $5 \%)$ and obesity $(11,72 \%$ vs $3.33 \%$ vs $3.86 \%$ ) compared to the children of the same age whose BW was 2500-3999g and $<2500 \mathrm{~g}$ ( $<<0.001$ all). For boys aged 11, the increased $\mathrm{BW}$ by $100 \mathrm{~g}$ increased the obesity risk by $10.6 \%(\mathrm{p}=0.022)$ and girls aged 15 by $18.2 \%(\mathrm{p}=0.015)$. The increased $\mathrm{BW}$ by 100 $\mathrm{g}$ increased the TO risk by $8.9 \%$ for girls aged 11 $(\mathrm{p}=0.006)$, for boys aged 15 by $10 \%(\mathrm{p}=0.007)$ and for girls aged 15 by $11.1 \%(\mathrm{p}=0.011)$.

Conclusions: TO and obesity manifestations during childhood is significantly connected with BW values. While analysing the obesity factor during childhood, we recommend that $\mathrm{BW}$ should also be taken into consideration, especially for children with $\mathrm{BW} \geq 4000 \mathrm{~g}$.

\section{EVALUATION OF LDL, HDL AND APOA LIPID FRACTIONS IN CHILDREN AS RISK FACTORS FOR ATHEROGENESIS}

\author{
D P Nikolic ${ }^{1}$, Z Milincic ${ }^{2}$, S Simeunovic ${ }^{2}$, \\ S Nedeljkovic ${ }^{2}$, M Vukotic ${ }^{2}$ \\ ${ }^{I}$ School of Medicine, University of Belgrade, Belgrade, \\ Serbia, ${ }^{2}$ University Childrens Hospital, University of \\ Belgrade, Belgrade, Serbia
}

Background and Aims: YUSAD study is prospective five-year study that begun in 1998. Aim of our study was to monitor levels of LDL, HDL and ApoA lipid fractions between boys and girls during 5 year period within YUSAD study group.

Methods: We analyzed separately LDL, HDL, ApoA1 and ApoA2 lipid fractions in two populations of children (10 years population and 15 years population). Results were compared with nomograms for age and gender.

Results: In 3525 children age of 10 years (49.82\% girls and $50.18 \%$ boys) and in 3439 children age of 15 years (49.98\% girls and $50.02 \%$ boys), LDL, HDL, ApoA1 and ApoA2 lipid fractions were (median value \pm standard deviation): Girls: LDL 2,73 $\pm 0,81$ and $2,48 \pm 0,90 ;$ HDL $1,42 \pm 0,37$ and 1,53 $\pm 0,48 ;$ ApoA1 $1,54 \pm 0,28$ and $1,45 \pm 0,25 ; \quad A p o A 2 \quad 0,38 \pm 0,06$ and $0,34 \pm 0,35$. Boys: LDL 2,63 $\pm 0,74$ and $2,28 \pm 0,86$; HDL $1,45 \pm 0,37$ and $1,44 \pm 0,44 ;$ ApoA1 $1,60 \pm 0,30$ and $1,37 \pm 0,28 ; \mathrm{ApoA} 20,39 \pm 0,06$ and $0,37 \pm 0,71$.

Conclusions: Higher values of LDL and HDL lipid fractions but not significant were detected in girls in both age populations except for HDL levels in 10 year age group. Higher values of ApoA1 and ApoA2 lipid fractions but not significant were detected in boys in both age populations except for ApoA1 levels in 15 year age group.

\section{SLEEP-WAKE DISTURBANCES AMONG ROMANIAN CHILDREN 3 TO 16 YEARS OLD}

O Falup-Pecurariu, I Dumitru, I Popescu, O Andreescu, C Falup-Pecurariu

Children's Hospital, Transilvania University, Brasov, Romania

Background and Aims: There are no data regarding prevalence of sleep-wake disturbances in Romanian population. The aim of this study was to estimate the prevalence and risk factors of sleep-wake disturbances among children 3 to 16 years old.

Methods: This cross-sectional study was carried out in Brasov County inwhich there are 400.000 inhabitants. All the 6 districts were involved, schools from eachdistrict were randomly selected and we performed systematic sampling for determination of 17 schools. We used modified Pediatric Sleep Questionnaire (PSQ) - validated and reability scale. Romanian translation was done and stability of the questionnaire was tested on a study group of 43 patients before the study. We evaluated 725 children's with a mean age of $11,85 \pm 5,03$ years.

Results: There were 357 females (49,25\%). Habitual snorers were in 69 cases from which 28 females $(40,57 \%)$. Excessive daytime sleepiness evaluated by Epworth Sleepiness Scale was 8,2 +/- 3,4 overall, 11,23 $+/-5,8$ in habitual snorer group and 6,2 +/- 2,4 in nonsnorer group $(\mathrm{p}<0,01)$. Fragmented sleep at least 2 nights per week in $21,09 \%$ of cases, sleep talking at least 2 nights per week in $20,13 \%$, nightmares in $8,82 \%$. Difficulty falling asleep was founded as a rare problem in $52,27 \%, 1-2$ times per week in 3,31\%, 3-4 times in $6.06 \%$ and $5-7$ times in 4,82\%. Co-sleeping was a rare problem in $21,51 \%, 1-2$ times per week in $19,71 \%, 3-4$ times in 29,10\%, 5-7 times per week in 15,44\%.

Conclusions: Sleep-wake disturbances are frequent and unrecognized pathology.

\section{FAMILIAL LEAD POISONING FROM TRADITIONAL TEAPOT}

N Ranguelov ${ }^{1}$, D Petit ${ }^{2}$, C Delaet ${ }^{1}$, C Sykes ${ }^{3}$, F Claeys ${ }^{3}$, M Toppet ${ }^{1}$

${ }^{l}$ Hôpital Universitaire des Enfants Reine Fabiola, Bruxelles, Belgium, ${ }^{2}$ Laboratoire Intercommunal de Chimie et de Bactériologie de Bruxelles, Bruxelles, 
Belgium, ${ }^{3}$ Scientific Institute of Public Health, Bruxelles, Belgium

Background and Aims: Lead poisoning is still very common in our countries and children are the first affected because of the ingestion of old paint chips and dust containing a high percentage of lead. The ingestion of contaminated tap water or the use of kohl are among other well-known potential sources. We identified a relatively unknown source of lead intoxication involving a young moroccan child and his family: The metal teapot.

Methods: A thorough discussion with the family lead us to think to the traditional metallic teapot used by the whole family as source of the intoxication. Lead concentration in spout, lid and brewed tea from 8 similar teapots was tested.

Results: A high blood lead level (BLL) confirmed the lead intoxication of a Moroccan child. The BLL of 18 other members of his family aged from 1 day to 43 years were also abnormal. Testing for classical sources of lead intoxication was negative. The analysis on similar teapots showed high lead contents with a large variation of lead concentrations in relation with models.

Conclusions: These kind of metallic teapots as source of lead intoxication represents a real public health problem because these kinds of teapots are frequently used by migrant population and in the catering sector. Therefore, we want to draw attention to this relatively unknown potential source of lead, namely the teapots, particularly in regards to young children and to the aspects of familial poisoning.

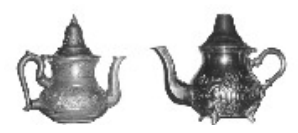

\section{KNOWLEDGE OF TOXICITY OF ELEMENTAL MERCURY AMONG INNER-CITY POPULATION}

P O Ozuah, J E Romm

\section{Children's Hospital at Montefiore, Albert Einstein College of Medicine, Bronx, NY, USA}

Background and Aims: Published reports suggest that inner city populations may be exposed to elemental mercury via ritualistic usage. It is not known if this population is aware of the risks of mercury exposure. Objective: To assess awareness of risks associated with use of elemental mercury among an inner city population.

Methods: Cross-sectional survey of subjects recruited from a community in which reports suggest ritualistic use of elemental mercury may be prevalent. A 23 -item questionnaire was interviewer-administered to all subjects. We inquired about awareness of potential toxicity associated with different ritualistic uses of elemental mercury, knowledge of the routes of exposure, and methods to limit exposure.

Results: 1000 subjects participated, of whom $43 \%$ were African American and 42\% were Hispanic or Latino. $47 \%$ of participants did not know that boiling mercury may result in poisoning, $44 \%$ did not know that burning mercury was harmful, and $40 \%$ were unaware that sprinkling mercury in the house was harmful. Similarly, $81 \%$ did not know that children breathe in more vapors than adults living in the same household, $71 \%$ did not know that mercury should not be flushed down the toilet, and $47 \%$ did not know that mercury vapors were harmful. $88 \%$ of subjects incorrectly thought that most cases of mercury poisoning were caused by handling mercury with bare hands.

Conclusions: A substantial number of subjects did not know that practices associated with ritualistic uses of mercury might lead to toxicity.

\section{FACTORS AFFECTING SEXUAL BEHAVIOR OF GREEK ADOLESCENTS}

\author{
A Zervoudakis ${ }^{2}$, F Michas $^{4}$, A Papadopoulou ${ }^{2}$, \\ G Michas $^{3}$, C Tsoumakas ${ }^{1}$, P Georgiopoulou ${ }^{2}$ \\ ${ }^{l}$ Faculty of Nursing, University of Athens, Athens, \\ Greece, ${ }^{2}$ Department of Pediatrics KAT General \\ Hospital, Athens, Greece, ${ }^{3}$ Medical School, University \\ of Athens, Athens, Greece, ${ }^{4}$ Medical School, University \\ of Patra, Patra, Greece
}

Background and Aims: It is known that adolescents' behavior is different than that of other pediatric age groups and they tend to take risks regardless of consequences. Aim of the study was to determine factors affecting risky behavior among sexually active Greek adolescents.

Methods: Of 413 adolescents (159 boys and 216 girls), which came for various reasons to the outpatient adolescent clinic over a period of 18 months and answered a specific questionnaire, 142 reported to be sexually active ( 75 boys and 67 girls), mean age 17,5 years. Of them only $63 \%$ of the boys and $74 \%$ of the girls reported using any form of protection. Data analysis was made with SPSS 8.0.

Results: More boys than girls are sexually active under the age of $18(\mathrm{P}=0.028)$ and start sexual activity at a younger age $(\mathrm{P}=0,005)$. Boys starting sex at an older age tend more frequently to use contraception $(\mathrm{P}=0,015)$. Adolescents drinking alcohol were more likely to be smoking cigarettes $(\mathrm{P}=0,0001)$. Adolescents drinking and/or smoking were more likely to have sexual activity ( $\mathrm{P}=0,0001$ for both). Relations with parents did not show any correlation with initiation of sexual activity though there was a correlation $(\mathrm{P}=0,06)$ between poor relationship with parents and amount of consumed alcohol.

Conclusions: Drinking alcohol and smoking cigarettes were significantly correlated with sexual activity under the age of 18. It also appears that other factors, outside the home and the relations with parents are affecting initiation of sexual activity in Greek adolescents.

\section{FEBRILE SEIZURES: A RETROSPECTIVE STUDY, EPIDEMIOLOGY AND CLINICAL FEATURES}

A N Filias, M I Eliopoulou, N M Nikolakopoulou, D A Papanastasiou, S Mantagos

Department of Pediatrics, University of Patras, Patras, Greece 
Background and Aims: Febrile seizures are common cause of admission and hospitalisation in a paediatric clinic in Greece.

Methods: We performed a retrospective study to examine the epidemiology and clinical features of hospitalised children with febrile seizures admitted in a 5-year period. A total of 248 children, median age 1.5 (range 0.5-11.5 yrs), were included in our study.

Results: In $50 \%$ of the cases, the convulsions occurred in children under $2 \mathrm{yrs}$ and in $82 \%$ it was the first episode. The ratio of boys to girls was 1.8:1. In $4.6 \%$ of cases, there was a family history of febrile seizures. The average duration of seizures was 2 min (range 0-9 min) with a predominance of generalised tonic-clonic crisis. The episode occurred $8 \mathrm{~h}$ (range $0-96 \mathrm{~h}$ ) from the onset of fever $(39.3 \pm 0.69 \mathrm{oC})$ and in $96 \%$ was not recurrent. The duration of hospitalisation was $3 \mathrm{~d}$ (range 1-14d). There was a seasonal variation with a predominance of winter (33\%) and spring (28\%) months. Lumbar puncture was performed in $14 \%$ of cases. Upper respiratory tract viral infection (44\%), tonsilitis (18\%), otitis media (15\%), gastroenteritis $(9 \%)$ were the most common causes of fever. Anticonvulsant therapy was needed in $2.7 \%$ of patients.

Conclusions: Febrile seizures usually occur in otherwise normal children and are brief, generalised and relatively benign. First febrile seizures should be evaluated by a physician to rule out serious underlying disease. A careful history and thorough physical examination are essential. Treatment of most febrile seizures consists of fever control and parental counseling.

\section{MYOTENOFASCIOTOMY TREATMENT FOR CHILDREN WITH CEREBRAL PALSY}

\section{G Nazarov}

\section{Medical University of Moscow, Moscow, Russia}

Background and Aims: The contractures of muscles are an important consequence of spasticity that children with CP suffer. These contractures are retracted fibres of soft tissues that have no more vitality, like, muscle fibres, fasciaes, aponeurotic fibres, muscle tendon fibres and ligaments produced by the degenerative process of dystrophy and this has a negative influence on all areas of the locomotor apparatus functions. The aim of this valuation is to demonstrate the possible optimisation of $\mathrm{CP}$ children with spastic forms of rehabilitation.

Methods: The Miotenofasciotomy eliminates muscle contractures of upper and lower extremities and those in the postural muscles of the body. Clinical methods for medical examination and recordings were made to 107 Spanish patients with CP who were operated using this surgery treatment. The period of time they were operated is from March 2005 until April 2006. These patients had different forms of CP like, spastic forms and mixed (spastic-hiperkinnetic and spastic-distonic forms).

Results: In all cases, children obtained positive results due to the elimination of muscle contractures, for example, liberation of movements, elimination of several forms of deformities in upper and lower extremities, improvement of balance and postures, and in some of the cases, they have been able to start walking with walkers, crutches or by themselves.

Conclusions: The Miotenofasciotomy, together with other therapies, optimise the rehabilitation of children with spastic forms of CP.

\section{PULMONARY COMPLICATIONS IN SPINAL MUSCULAR ATROPHIES}

\author{
G Sur $^{1,2}$, V Salajan ${ }^{1}$, D T Gireada ${ }^{1}$, L Sur $^{2}$

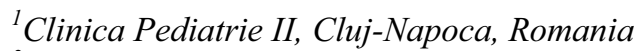 \\ ${ }^{2}$ University of Medicine, Cluj-Napooca, Romania
}

Background and Aims: The authors intend to present the pulmonary complications of various forms of muscular atrophies. These complications have been severe in type I and II of the disease and only transitory in type III. These complications have consisted of bacterial pulmonary infections that finally ended in death in the two cases with type I and II disease.

Methods: There are presented 3 cases of spinal muscular atrophy with 3 types of disease. A 1 month old infant with generalized hypotonia having the appearance of a doll. The final diagnosis is Werdnig-Hoffmann disease type 1 . The second case, a 7 month old infant is brought for a severe generalized hypotonia beginning at the age of 6-7 months. Established the diagnosis: Spinal muscular atrophy type 2 . The third case is seen for a severe scoliosis and in the end the diagnosis was type 3 spinal muscular atrophy.

Results: In the first case the diagnosis was established by molecular genetics which showed mutations of the gene coding SMN on chromosome 5. The evolution was lethal in the second case the diagnosis was established by muscular biopsy, which showed denervated skeletal muscles with atrophy had congenital hypothyroidism and rickets as associated pathology. The evolution was finally lethal. In the third case the infections have been controlled and the child maintained the motor acquisitions.

Conclusions: The evolution of cases with spinal muscular atrophy is usually severe, with an elevated mortality rate.

\section{IDIOPATHIC INTRACRANIAL HYPERTENSION IN CHILDREN}

K Chandanos, P Neonaki, M Mavrokosta, S Stefanaki, T Tsekoura, A Sxoinaki, A Tsilimigaki-Christaki

Pediatric Clinic, Venizeleio General Hospital, Iraklio, Crete, Greece

Background and Aims: Idiopathic intracranial hypertension (I.I.H.) is uncommon in children. It is critical to recognize the warning signs and symptoms to prevent devastating ophthalmologic complications. Obesity has been reported as an important risk factor for I.I.H.

Methods: We report two cases of obese adolescent patients who were diagnosed with I.I.H. Case 1: A 13year-old obese male, with a Body Mass Index (BMI) of 32,8 presented with diplopia and neck pain during the last 15 days. Cerebrospinal fluid (CSF) pressure was 
$870 \mathrm{mmH} 20$, with normal CSF content. Case 2: A 13year-old obese female, with a BMI of 28 presented with headache, nausea and occasional vomiting during the last 10 days and diplopia the last 2 days. She referred use of minocyclin as treatment for acnes. CSF pressure was $420 \mathrm{~mm}$ H20, with normal CSF content and blood pressure. In both cases neurologic examination revealed VI cranial nerve palsy, ophthalmologic examination revealed papilledema, blood chemistry was normal and brain MRI was unremarkable. Both patients started treatment with acetazolamide and were put on a low calorie diet. During the following 3 months papilledema gradually resolved and treatment was tapered while they remained symptom free.

Results: In both cases patients were obese (>95th percentile), although the use of minocyclin of the second patient may have contribute in the development of IIH.

Conclusions: Obesity may constitute a more relevant risk factor for $\mathrm{IIH}$ in the pediatric and adolescent population in the next years, as its prevalence increases among that population.

\section{EVALUATION OF THE SPECIFIC DYSMORPHIC FEATURES IN MULTIMALFORMATIVE SYNDROMES}

E Sukarova-Angelovska, M Kocova, M Krstevska-Konstantinova

Department of Endocrinology and Genetics, Pediatric Clinic, Skopje, Macedonia

Background and Aims: The combination of minor and major dysmorphic malformations is unique in various multimalformative syndromes. Some of them are wellknown and easy to recognize, but in the majority of the cases evaluation of spectrum of present anomalies is time-consuming and difficult. Some of the minor dysmorphic stigmata (hypertelorism, epicantus, clinodactylia) are more frequent and present in many syndromes, thus are not informative. Appearance of specific pattern of dysmorphic features (lobulated tongue, creases of the ear lobules, iris spots) can help in syndrome delineation.

Methods: Evaluation of 250 children with various congenital malformations was done. The children were divided in 4 groups and subgroups according to the etiology. Malformations and combination of malformations (both minor and major) of head, neck, extremities and organs were analyzed.

Results: The mean number of minor anomalies in the group of children with chromosomal disorders was 38 and 24 per child in the group of nonchromosomal syndromes. The most frequent minor anomalies were eye slanting and space $(21 \%)$, ear position and modeling $(16 \%)$ and hair pattern (14\%), finger length and abnormal dermatoglyphes (28\%). The significant association in several combinations between minor and major anomalies was noticed, such as hypertelorism and heart defect; posteriorly rotated ears with kidney anomalies, etc. In some syndromes specific minor malformations were found and discussed.

Conclusions: In the era of powerful, but expensive genetic testing, it is important to set the investigation in proper direction. Recognizing the pattern of minor and major malformations in children with multimalformative syndromes is essential step in delineating the specific syndrome.

\section{MEASUREMENT OF PAIN CAUSED BY VENIPUNCTURE IN CHILDREN UNDER THREE YEARS OLD}

I Ledesma, R Álvarez, L Regueras, C Iglesias, M Neira, M Roson, L M Rodriguez

Servicio de Pediatria, Hospital de León, León, Spain

Background and Aims: Venipuncture is the painful procedure more used in pediatrics and in that way it's seen for allied health personnel and for parents. This study measures the pain caused by venipuncture in children under three years old, judging the differences which can be attributed to sex.

Methods: Prospective, cross-sectional and observational study in 30 infants, with a mean age of 16,3 months, which venipuncture was carried out to performing a renal scan. Bell pain rating scale was applied in all of them, which takes into account behavioral parameters facial expression, motor activity, body language, cry and solace and biological ones - heart rate (HR), blood pressure (BP) and $\mathrm{O} 2$ saturation (SAT); and pain is classified in: Absence of pain, moderate pain and intense pain. Results were compared using the MannWhitney U, Wilcoxon and $\chi^{2}$ test, as appropriate.

Results: Venipuncture didn't cause measurable pain in $26,6 \%$ of children; it caused moderate pain in $56,6 \%$ and intense pain in $16,6 \%$. There was no difference between sexes in the intensity of pain detected by total score on the scale $(p=0,54)$ or by distribution in pain ranges $(p=0,89)$. Venipuncture caused a significant increase in HR $(p=0,025)$ and in BP $(p<0,0001)$. There weren't significant changes in SAT and behavior sings in any of the children.

Conclusions: Venipuncture cause moderate or intense pain around of $75 \%$ of our patients. In our group of children (under 3 years old) we haven't detected measurable differences in pain caused by venipuncture which depends on sex.

\section{ONE PATIENT, TWO RARE DISEASES!}

S M Nunes ${ }^{1}, \mathrm{H} \mathrm{Silva}^{1}, \mathrm{M} \mathrm{Marques}^{1}, \mathrm{~A}$ Silva ${ }^{1}, \mathrm{I}_{\text {Cunha }}{ }^{1}$,

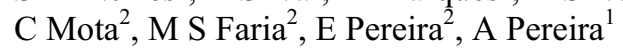

${ }^{1}$ Department of Neonatology, Hospital de São Marcos, Braga, Portugal, ${ }^{2}$ Department of Nephrology, Hospital de Crianças Maria Pia, Porto, Portugal

Background and Aims: The presence of two genetic disorders in a single patient is uncommon. We describe a case of a child with Finnish type congenital nephrotic syndrome diagnosed in the neonatal period and a urea cycle disorder diagnosed later.

Results: Newborn admitted at Neonatal Unity at D2 because of respiratory distress. Euthocic delivery at 35th week of gestational age. Large placenta. Diagnostic of nephrotic syndrome at 8 th of life. Infectious causes of congenital nephrotic syndrome were excluded. He started therapy with albumin, furosemid, aspirin, 
captopril, levotiroxine, calcium carbonate, D vitamin, imunoglobulin infusions and erythropoietin. At 2 month the renal biopsy confirmed Finnish type nephrotic syndrome and began indometacin. Because of arterial hypertension, he was medicated with losartan and nifedipine. At 3 month, he presented with respiratory distress, seizures, multiorgan failure and coma. Of the laboratorial analysis we emphasize severe metabolic acidosis and ammonia $>1000 \mathrm{ug} / \mathrm{dl}$. The child started peritoneal dialysis and was medicated with arginine, carnitine, phenylbutyrate and benzoate. Metabolic defect workup: increased urine and plasma argininosuccinic acid levels, increased plasma citrulline levels and diminished plasma arginine levels. Diagnose confirmed with Argininosuccinic acid lyase assay. Cerebral magnetic resonance showed '...global atrophy...'. He had several catheter sepsis. At 12 month he died of a Steven Johnson Syndrome.

Conclusions: Finnish type nephrotic syndrome and Argininosuccinate lyase deficiency are rare autosomal recessive diseases. In the first, an intensive medical treatment followed by renal transplantation, leads to a remarkable reduction of mortality. In the second, early diagnosis of hyperammonaemia is necessary to improve survival.

\section{URINARY TRACT INFECTIONS (UTI) IN CHILDREN. HOW NECESSARY IS IT TO OBTAIN A URINE CULTURE UNDER TREATMENT?}

D Karakaidos, G Triantafyllidis, A Salapata, A Papadimitropoulou, P Zosi, F Papadelis, C Karis

Department of Pediatrics, General Hospital of Nikea, Piraeus, Greece

Background and Aims: Obtaining a urine culture sample on the 3rd day of treatment in a child with a urinary tract infection (UTI) in order to assess response to therapy has become standard practice in pediatric departments. The aim of our study was to examine whether that practice is necessary to perform in a child who otherwise has signs of clinical improvement and is receiving the appropriate antibiotic therapy (according to the sensitivity of the isolated pathogen).

Methods: We examined the records of children with UTIs admitted to our department during a five year period (2000-2005). Urinary cultures were taken in 310 children (138 boys-172 girls, 15 days-14 yrs old) with established UTIs on the 3rd day of a treatment compatible with sensitivity of the offending pathogen to the appropriate antibiotic. $16.1 \%$ of cultures were taken as freshly voided urine, $0.6 \%$ with a suprapubic puncture, $12.9 \%$ with the use of a catheter and $70.3 \%$ using a bag.

Results: $307 / 310$ (99\%) came up with either sterile or negative cultures on the 3 rd day. Only $3(1 \%)$ continued to have positive cultures. Further evaluation revealed the presence of a renal abscess in 1, bilateral double pelvis and ureters with ureterocystic junction stenosis in the other and the last had developed resistance to the initial amoxicillin regimen.

Conclusions: Urinary cultures taken under treatment are totally unnecessary in children who otherwise show improved clinical status and should only be reserved for cases with none or partial improvement as well as those with major congenital renal abnormalities.

\section{URINE MICROALBUMIN, ALPHA1- MICROGLOBULIN AND BETA2- MICROGLOBULIN IN CHILDREN WITH HEMATURIA WITHOUT OBVIOUS PROTEINURIA}

D Karakaidos $^{1}$, G Karagrigoriou ${ }^{1}$, G Triantafyllidis ${ }^{1}$, A Drakatos $^{2}, Z_{\text {Tseflika }}{ }^{1}$, E Tasiopoulou $^{1}, \mathrm{C}_{\text {Karis }}{ }^{1}$

${ }^{1}$ Department of Pediatrics, General Hospital of Nikea, Piraeus, Greece, ${ }^{2}$ Department of Biochemistry, Children's Hospital "P\&A. Kyriakou”, Athens, Greece

Background and Aims: Isolated significant hematuria in children can be a sign of numerous causes, benign or not. At those children an evaluation of renal function can be made with the use of urine beta2-microglobulin (b2-MG) and alpha1-microglobulin (a1-MG) which are indices of renal tubular damage and urine microalbumin (L) which is an index of renal glomerular damage. Our aim was to measure b2-MG, a1-MG and L in children with hematuria in a routine urinalysis without obvious proteinuria.

Methods: First morning void urine samples were collected from 678 healthy children (403 boys and 275 girls) 7-12 years old and from 72 children (37 boys and 35 girls, mean age 9,9 $\pm 1,8 \mathrm{yrs}$ ) with hematuria without proteinuria in urinalysis. Beta2-microglobulin, alpha1microglobulin and microalbumin levels were measured with the nephelometry method and expressed as $\mathrm{mg} / \mathrm{L}$.

Results: There was a statistically significant elevation of microalbumin and alpha1-microglobulin in children with hematuria (median values of 51,8 and 7,74 respectively) in relation with healthy children (median values 13,2 and 5,58 respectively). No significant correlation was found for beta2-microglobulin between the two groups.

Conclusions: Children with hematuria in urinalysis have elevated values of microalbumin and alpha1microglobulin, indicating mild renal damage.

\section{NEPHROLITHIASIS IN ECTOPIC KIDNEY}

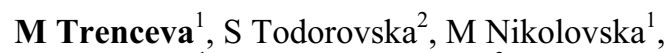 \\ S Smugreska ${ }^{1}$, K Cekic-Dimovska $^{3}$, R Hadzi-Manceva $^{4}$, \\ V Tasic ${ }^{5}$ \\ ${ }^{1}$ Pediatric Service, Health Center Skopje, Skopje, \\ Macedonia, ${ }^{2}$ Pediatric Office, PZU Pantelejmon, \\ Skopje, Macedonia, ${ }^{3}$ Pediatric Office, PZU Pinokio, \\ Skopje, Macedonia, ${ }^{4}$ Pediatric Service, Remedica \\ Hospital, Skopje, Macedonia, ${ }^{5}$ Department of Pediatric \\ Nephrology, Children's Hospital Skopje, Skopje, \\ Macedonia
}

Background and Aims: Ectopic kidney is poorly visualized with ultrasound. Other imaging methods are mandatory when the patients present with microhematuria and non-specific low abdominal pains. The aim of this work was to present a boy who developed hydronephrosis in ectopic kidney. 
Methods: Standard clinical laboratory and radiographic methods including ultrasonography, radionuclide studies, IVP and CT scanning.

Results: A ten year old boy presented with nonglomerular hematuria. Ultrasound scan, radionclide studies and IVP revealed a well functioning ectopic left kidney (pelvic ectopia). Four years after diagnosis of ectopia the boy complained to lower abdominal pains and urinalyses showed non-glomerula microscopic hematuria. The US poorly visualized the left kidney, on plain X-ray there was a calculus, which was located in the pyelon of the left kidney causing obstruction and hydronephrosis (IVP, CT scan, diuretic scintigraphy). The boy underwent surgery for extraction of the calculus. Conclusions: Children with ectopic kidneys and non-glomerular hematuria should undergo additional imaging studies. Ultrasound scanning does not allow good visualization of calculi and hydronephrosis in ectopic kidneys.

\section{CAKUT IN A FATHER AND HIS CHILD BORN AFTER IN VITRO FERTILIZATION}

\author{
M Gligorova $^{1}$, A Sofijanova ${ }^{2}$, Z Gucev $^{2}$, A Jancevska ${ }^{2}$, \\ N Ristoska-Bojkovska ${ }^{2}$, V Tasic ${ }^{2}$ \\ ${ }^{I}$ Department of Pediatrics, General Hospital, Strumica, \\ Macedonia, ${ }^{2}$ Children's Hospital, Clinical Center, \\ Skopje, Macedonia
}

Background and Aims: There is evidence that congenital anomalies of the kidneys and urinary tract (CAKUT) have genetic basis. The aim of this study is to present a family in which the father and his child, who was born after in vitro fertilization (IVF), were found to have CAKUT. Methods: Ultrasound, radionuclide and $\mathrm{X}$ ray studies were performed to define the type of CAKUT. Results: The index case was born after IVF. He was the very one of three babies who survived. He was treated in the ICU for the immaturity and respiratory distress. As a consequence of neonatal brain damage, he developed cerebral paralysis. On imaging, he was found to have unilateral hydronephrosis on the right side. An ureterocella was demonstrated with ultrasound, IVP and VCUG and the boy underwent surgery. The postoperative course was uneventful and his renal function was normal. The both parents were screened for CAKUT. The father was found to have small hypoplastic right kidney. Conclusions: IVF is incriminated for higher incidence of congenital anomalies in babies born after this procedure. In our case we can speculate about genetic basis of the CAKUT in the parent and his child; it is known that CAKUT may be associated with anomalies of funiculus spermaticus, which is responsible for father's infertility.

\section{CYSTINE UROLITHIASIS IN A CHILD WITH VESICOURETERAL REFLUX}

S Smugreska ${ }^{1}, \mathrm{~S}$ Todorovska ${ }^{2}, \mathrm{M}$ Trenceva ${ }^{1}$, K Cekic-Dimovska ${ }^{3}$, R Hadzi-Manceva ${ }^{4}$, V Tasik

${ }^{I}$ Pediatric Service - Dracevo, Health Center Skopje, ${ }^{2}$ Pediatric Office, PZU Pantelejmon, ${ }^{3}$ Pediatric Office, PZU Pinokio, ${ }^{4}$ Pediatric Service, Remedica Hospital,
${ }^{5}$ Department of Pediatric Nephrology, Children's Hospital Skopje, Macedonia

Background and Aims: Vesicoureteral reflux (VUR) is a common urinary tract malformation. Occasionally urolithiasis may be found in children with VUR. The aim of this work was to present an association (by chance) of two urologic conditions in a child.

Methods: Standard clinical, biochemical, and imaging studies for evaluation of the kidneys and urinary tract.

Results: One year old male infant presented with symptomatic urinary tract infection. Cystographic study revealed left sided vesicoureteral refllux grade III. The child was given regular hemoprophylaxis. After three years of conservative treatment, the reflux resolved. Laboratory studies revealed persistent microscopic hematuria of non-glomerular origine. The ultrasound scanning did not find any abnormality. Six months later moderate left sided hydronephrosis was noted. This prompted X-ray study which demonstrated big calculus in the lower portion of the left ureter. Metabolic studies established cystinuria. The calculus was surgically removed. On surgery prevesical stesnosis of the left ureter was seen. The child was given alkali therapy and new calculi were not seen during the follow up.

Conclusions: Persistent non-glomerular hematuria should prompt investigation of the urinary tract. Although ultrasound is a non invasive method, it is insufficient to demonstrate ureteral stones, for this purpose X-ray studies are still needed.

\section{NUTCRACKER PHENOMENON IN A CHILD WITH NON-GLOMERULAR HEMATURIA}

O Nikolovska ${ }^{1}$, S Cekovska $^{2}$, P Korneti ${ }^{2}$, S Dzikova $^{3}$, V Tasic ${ }^{4}$

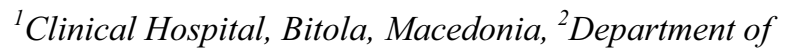
Biochemistry, Medical School, Skopje, Macedonia

${ }^{3}$ Department of Nephrology, University Clinical Center, Skopje, Macedonia, ${ }^{4}$ University Children's Hospital, Skopje, Macedonia

Background and Aims: Hematuria represents a serious differential diagnostic problem to the pediatrician. The aim of this study was to describe a pediatric patient with recurrent attacks of gross hematuria, who was found to suffer from nutcracker-phenomenon.

Methods: The source of hematuria was evaluated by the use of SDS-PAG electrophoresis of urinary proteins using frozen urine sample. Doppler ultrasound was performed to diagnose nutcracker phenomenon.

Results: A 10 year old boy had recurrent short lasting episodes of painless gross hematuria. In one attack urine sample was obtained and frozen until analysis. SDSPAG electrophoresis showed non-glomerular type of hematuria on the basis of presence of clear fractions of apolipoprotein A-1, IgG and alfa-2-macroglobulin. This indicated searching for non-glomerular causes of the bleeding. Ultrasound and Doppler study demonstrated nutcracker phenomenon (left renal vein entrapment between aorta and superior mesenteric artery).

Conclusions: Pediatricians should think about nutcracker phenomenon as a cause of non-glomerular bleeding. SDS-PAG electrophoresis is a useful tool for 
differentiation glomerular from nonglomerular hematurias, particularly when fresh-voided urine samples are not available.

\section{INFANTILE ONSET POMPE DISEASE: CASE REPORT}

N Slaveska, N Pop-Jordanova, S Fustik, T Jakovska, A Demerdzieva

Department of Metabolism, University HospitalPediatric Clinic, Skopje, Macedonia

Background and Aims: Pompe disease also known as Glycogen storage disease type II (GSD-II), is a rare autosomal recessive lisosomal disorder caused by inherited deficiency of acid alpha - glucosidase. The most severe is the classic infantile-onset form with prominent cardiomegaly, hipotonia, and death priop to first year of life. We report an infant with the classical course of the infantile-onset Pompe disease, who died at 4,5 months of age due to pneumonia and hypertrophic cardiomyopathy.

Methods: The diagnosis was based on physical examination, cardiology investigation, serum CPK assay, and autopsy study.

Results: The patient was admitted to the hospital at 3,5 months of age due to generalized muscle weakness, feeding and respiratory difficulties. Rapidly increased hypotonia, respiratory distress, progressive macroglossia, and cardiomegaly characterized progression of the disease. Left ventricular thickening increased over time. EKG showed a shortened PR interval and large QRS complexes. Despite the profound hypotonia muscles had firm and hypertrophic appearance.

Conclusions: Death occurred within one month of hospitalization due to cardiorespiratory failure. On autopsy glycogen had accumulated in the liver, myocardium, and skeletal muscle.

\section{TRIPLICATED COLLECTING SYSTEM, A RARE UROLOGICAL MALFORMATION}

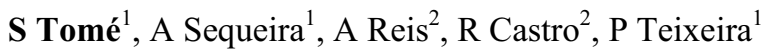 \\ ${ }^{l}$ Paediatric Service, Hospital São João de Deus, Vila \\ Nova de Famalicão, Portugal, ${ }^{2}$ Paediatric Surgery, \\ Hospital Maria Pia, Porto, Portugal
}

Background and Aims: Urinary tract infections occur in $3-5 \%$ of girls and $1 \%$ of boys and have been considered an important risk factor for the development of renal insufficiency. Some anatomical abnormalities of the urinary system predispose to infection, and their correction is sometimes necessary to solve the problem. The authors report a clinical case of a rare urological malformation in a girl with multiple urinary infections.

Methods: Revision of the clinical case by medical and image registrations.

Results: Girl, 10 years old, was admitted at the age of 8 for investigation of frequent urinary infections and urological malformation presented on image evaluation. Their parents were addicted to drugs, and the pregnancy was not under surveillance. Delivery took place in the hospital at term. Good height and weight evolution and no pathological important events until the age of 6 years old, when she had the first urinary infection. Subsequent evaluation revealed a triplicated collecting system with upper pole ureterohydronephrosis, and a left upper pole nephrectomy was realized. The patient did not have any other urogenital anomaly.

Conclusions: Despite ureteral duplication is a frequent urological anomaly, the presence of three ureters occurring in the same side, in a complete or incomplete form, is a true rarity. Since the first description of ureteral triplication by Wrany in 1870 , about 100 cases have been reported and paediatric cases are fewer. The authors report this case in spite of it rarity and the difficulty of the diagnosis. A thorough evaluation of such an anomaly is essential for effective management.

\section{A COMPREHENSIVE CONSERVATIVE MANAGEMENT FOR END-STAGE RENAL FAILURE}

\section{A J Al Mosawi}

Department of Pediatrics, University Hospital in Al Kadhimiyia, Baghdad, Iraq

Background and Aims: The appropriate dietary and pharmacologic management (conservative measures) of patients with chronic renal failure (CRF) includes: protein and phosphorus restriction, calorie and water soluble vitamins supplementation, phosphate binders, etc. With appropriate dietary and pharmacologic management, patients with non-terminal chronic renal failure (CRF) can be maintained surprisingly well, and the transition from non-terminal CRF to end-stage-renal failure ESRF represents a small decrement of renal function resulting in a large physiologic hurdle for the patient. Conservative measures can only be successful in non- terminal CRF, and patients with ESRF can't sustain life in the absence of renal replacement therapy. The addition to these effective traditional measures, an agent enhance fecal nitrogen excretion can possibly bridge this gap resulting from this small decrement of renal function obviating the need for RRT for some period of time. A novel regimen combining the traditional conservative management of CRF with addition of Acacia gum (AG) $1 \mathrm{~g} / \mathrm{kg} /$ day has been reported to provide patients with ESRF dialysis freedom. Aim: To describe the longest period of dialysis freedom (3-year) achieved in history with this novel form of RRT.

Methods: An 11-year-old girl with ESRF who initially required 4 sessions of intermittent peritoneal dialysis to control uremic symptoms despite conservative measures. Thereafter, managed with a novel regimen combining the traditional conservative management of CRF including low protein diet with addition of Acacia gum (AG) $1 \mathrm{~g} / \mathrm{kg} /$ day. One year dialysis and improve well- being was achieved [Published in October issue of Pediatric Nephrology 2004]. This patient continued the previously described regimen for additional two years.

Results: During these 2 years she continue in experiencing improved well- being and good participation in outdoor activities, had never been acidotic or experienced any uremic symptoms. A maximum urea level of $127 \mathrm{mg} / \mathrm{dl}$ and a serum 
creatinine of $5 \mathrm{mg} / \mathrm{dl}$ were noted during a period of decreases adherence to dietary restriction and less compliance to AG therapy. Periods of decreased compliance with other pharmacologic therapies such as iron and erythropoietin, calcium carbonate, and onealphacalcidol. Was associated with hypochromic microcytic anemia and renal osteoystrophy. These problems of anemia and renal ostedystrophy were solved by returning to the strict adherence to all of the items of the regimen.

Conclusions: This is the first paper describing three years dialysis freedom in a child with ESRF.

\section{EARLY ONSET END-STAGE RENAL FAILURE IN OCULO-CEREBRO-RENAL PHENOTYPE: NOVEL OCCURRENCE}

\section{A J Al Mosawi \\ Head of the Department of Pediatrics, University Hospital, Al Kadhimiyia, Baghdad, Iraq}

Background and Aims: The Oculo-cerebro-renal syndrome (OCRS) is a rare disorder characterized by congenital ocular and cerebral defects, and renal tubular dysfunction with later renal onset of renal failure during the fourth. The paper reports the novel occurrence of early end-stage renal failure (ESRF) in OCRS phenotype.

Methods: From 1994 to 2005 four patients (two boys and two girls) with OCRS phenotype were observed. All of them have congenital cataract(s), hypotonia and hyporeflexia and renal dysfunction.

Results: The ocular abnormalities in three patients were similar to those reported in the classical OCRS of Lowe consisting of cataract(s) and glaucoma. One patient (boy) has immature cataracts, and bilateral congenital chorioretinal hypoplasia. The 2 boys have nystagmus during infancy, in one of them nystagmus was not present at the time of referral. All the patients have hypotonia and hyporeflexia characteristic of Lowe OCRS. In one patient (girl) hypotonia and hyporeflexia occurred late and was not present during the first 3 years of life. Three patients have global developmental delay. One patient has normal IQ and her language was well developed. All the patients reached ESRF during the first 6 years of life and required dialysis. All of them have microscopic hematuria, albuminuria, and casturia suggesting glomerular disorder. None of them have evidence of renal tubular dysfunction. Three patients died from uremia during the first decade of life. It was not possible to follow the fourth patient.

Conclusions: This is the first paper reporting early onset of ESRF during the first decade of life.

\section{UNILATERAL RENAL AGENESIS ASSOCIATED WITH 76TH CASE OF COFFIN-SIRIS SYNDROME IN THE WORLD}

\author{
A J Al Mosawi \\ Head of Department of Pediatrics, University Hospital \\ Al Kadhimiyia, Baghdad, Iraq
}

Background and Aims: Coffin Siris syndrome (CSS) is a rare clinical syndrome of unknown etiology, first described by Coffin GS and Siris E in 3 unrelated girls with mental retardation and absent nails and terminal phalanx of the fifth finger in 1970 . Until now only 75 cases have been reported. Renal agenesis has been reported infrequently in CSS.

Methods: Patient: A 4 years old dysmorphic and mentally retarded girl. She was born at 30 weeks of gestation by cesarean delivery because of lack of progress of labour. Her weight was $1.75 \mathrm{~kg}$. During infancy feeding difficulties was prominent. Her development was retarded with no language development, gross motor milestones was slightly better than other fields of development. She didn't have any urinary complaint. Her growth was retarded with all of her parameters below the third centiles. She has coarse facial features with thick eyebrows, mild left ptosis big mouth, thick prominent lips facial hypertrichosis and low nuchal hair line. Her hands showed single palmer creases, fingers and nails were small; the terminal phalanges of her fingers were small with hypoplastic changes more severe on the little finger of the right hand.

Results: Radiological examination showed hypoplastic distal phalanges with rudimentary terminal phalanx of the right fifth digit. Abdominal sonography revealed absence the right kidney with no other abnormality. Brain CT scan and chromosomal analysis showed normal findings has been reported from USA, Europe, Turkey, Japan, and India.

Conclusions: 76th patient with CSS, and the first Arab with CSS, associated unilateral renal agenesis, an infrequently reported association.

\section{ASSESSMENT OF FEVER, WBC, ESR AND CRP IN THE FIRST URINARY TRACT INFECTION IN CHILDREN}

\section{A Nickavar \\ Department of Pediatrics, Hazrat Rassoul Hospital, Tehran, Iran}

Background and Aims: Urinary tract infection (UTI) is one of the most common infections in children, which in the form of acute pyelonephritis with delayed and improper treatment leads to kidney scarring, hypertention and renal failure. The present study was done for the evaluation of important differentiating parameters in pyelonephritis and cystitis.

Methods: This cross sectional study was performed in 96 patients with the first attack of UTI during 3 years. Acute pyelonephritis diagnosed by DMSA scan in 71 cases. Parameters such as fever, ESR, CRP, and WBC were analysed in these two disease.

Results: Most of the patients were female. $75 \%$ of cases had less than 5 years and the most prevalent symptoms were fever, dysuria, urinary frequency, incontinence, and flank pain. The most common isolated germ was $\mathrm{E}$ coli. Bilateral renal involvement was reported in 48 patients in DMSA scan. CRP (qualitative) was positive in 40 cases of pyelonephritis $(14 \% 1+, 7 \% 2+, 35 \% 3+$, $35 \%$ negative and undetermined in $9 \%$ ) and 6 with cystitis $(8 \% 2+, 16 \% 3+, 64 \%$ negative and $12 \%$ 
undetected). Fever, ESR and WBC were not important statistical parameters in differentiating pyelonephritis and cystitis.

Conclusions: CRP is the most valuable laboratory parameter which could be used reliably for the assessment of pyelonephritis.

\section{URINARY TRACT INFECTION AND SIMPLE FEBRILE CONVULSION}

N Pashapour ${ }^{1}$, A Macooie ${ }^{1}$, S Gol Mohammadlou ${ }^{2}$

${ }^{l}$ Department of Pediatrics, Imam Hospital, Oromieh, Iran, ${ }^{2}$ Kosar Hospital, Oromieh, Iran

Background and Aims: Investigation of urinary tract infection (UTI) in first simple febrile convulsion is controversy. The aim of this study was to evaluate the incidence of urinary tract infection in children with simple febrile convulsion

Methods: All children with diagnosis of first simple febrile convulsion that admitted to hospital of Imam, Oromieh, Iran between January 2003 and December 2004 were included in the study. A first simple febrile convulsion and UTI work-up including lumbar puncture, complete blood count, CRP, ESR, blood glucose, serum calcium, serum electrolytes, renal function tests, urinalysis, urine culture, blood culture were performed in all patients. Chest X-ray, EEG and CT scan were requested in suspected cases. Patients with UTI, defined as more than 100,000 colony-forming units per milliliter of a single pathogen obtained by midstream urine collection, were evaluated for renal imaging.

Results: Two hundred fifty one consecutive cases aged 6-60 months were evaluated. The simple febrile convulsion work-up of patients other than UTI did not demonstrate any significant underlying disorder. Renal function tests were within normal levels in all patients. Four urinary tract infections with grade 4 reflux were diagnosed

Conclusions: It is of importance that UTI was presented initially with simple febrile convulsion. It is well known that UTI is a common cause of high fever. Urine culture should be considered in work-up of the children with first simple febrile convulsion.

\section{URETHRAL AND HYDRONEPHROSIS IN CIRCUMCISION WITH RING PROCEDURE}

\author{
R Gaffari ${ }^{1}$, H Gaffari ${ }^{2}$, M Makani ${ }^{1}$ \\ ${ }^{I}$ Gamar Bany Hashem Hospital, Khoy, Iran, ${ }^{2}$ Health \\ Ministry, Tehran, Iran
}

Background and Aims: The prevention and notice of using ring procedure in circumcision especially in early infancy.

Methods: Case study: The patients that circumcision is done with ring they have had fullness in kidney before of ring dropping.

Results: 1 . We have to use ring with caution because it may be give hydronephrosis or urination problem especially before dropping of ring. 2. The infant with ring shouldn't be closed with diaper. 3 . The ring method can be used only with serious observation.
Conclusions: The neonate and infants have narrow duct for urination and they closed with diaper and when they undergo to ring procedure they urination and kidney problem is increased. So it is better we don't do the ring procedure in early infant or it have to do with caution.

\section{INCREASED INCIDENCE OF URETHRAL OBSTRUCTION WITH RING CIRCUMSICION METHOD}

\author{
R Gaffari ${ }^{1}$, H Gaffari ${ }^{2}$, M Makani ${ }^{1}$ \\ ${ }^{I}$ Gamar Bany Hashem Hospital, Khoy, Iran, ${ }^{2}$ Health \\ Ministry, Tehran, Iran
}

Background and Aims: The aggressive and severe supervision have to do in patients with ring circumcision because it may induce obstruction in urinogenitary duct. Applying of ring method in circumcision should be done with precaution.

Methods: We studied this article with case study. In my patients that undergone ring circumcision, when we used from catheter they have not any problem but after removing catheter they have had fullness in U.G system. Results: 1 . The ring method is not very good procedure in circumcision especially in early infancy because it may be induced or increased urethral obstruction.

Conclusions: In my study, all patients when we use catheter in urinary system, the patient have no problem but when we removed it the patient have fullness in U.G system in scan and sonography. We noticed when we use catheter the patients had no problem in sono and scan but after removing catheter they found problem in scan and sonography.

\section{NORMAL VALUES OF URINE MICROALBUMIN, ALPHA1-MICROGLOBULIN AND BETA2-MICROGLOBULIN IN HEALTHY CHILDREN}

D Karakaidos $^{1}$, G Triantafyllidis ${ }^{1}$, G Karagrigoriou ${ }^{1}$, A Drakatos $^{2}$, G Kafalidis ${ }^{1}$, E Paraskaki $^{2}$, C Karis ${ }^{1}$

${ }^{1}$ Department of Pediatrics, General Hospital of Nikea, Piraeus, Greece, ${ }^{2}$ Department of Biochemistry, Children's Hospital "P\&A. Kyriakou”, Athens, Greece

Background and Aims: It is well established that, for both children and adults, elevated values of urine beta2microglobulin (b2-MG) and alpha1-microglobulin (a1MG) are indices of renal tubular damage and elevated urine microalbumin (L) is an index of renal glomerular damage. Measurement of these indices at first morning void urine samples is corresponding quite accurately with the 24 hour urine sample and is much more convenient to obtain in children. Our aim was to evaluate the normal range of $\mathrm{L}, \mathrm{b} 2-\mathrm{MG}$ and $\mathrm{a} 1-\mathrm{MG}$ in healthy children using first morning void urine samples.

Methods: First morning void urine samples were collected from 678 healthy children (384 boys and 294 girls) 7-12 years old. Children with a history of urinary tract infections or other renal disease, with febrile disease the last 15 days, abnormal findings in urinalysis or drug treatment of any kind were excluded from our study. Beta2-microglobulin, alpha1-microglobulin and 
microalbumin were measured with the nephelometry method.

Results: See table.

Conclusions: Knowledge of normal value range of urine beta2-microglobulin, alpha1-microglobulin and microalbumin in healthy children is essential in order to evaluate renal tubular and glomerular function.

\begin{tabular}{|c|c|c|c|c|c|c|c|}
\hline Age & $\mathrm{n}$ & \multirow{2}{*}{$\begin{array}{c}\mathrm{L}(\mathrm{mg} / \\
\mathrm{L}) \\
50^{\text {th }}\end{array}$} & \multirow{2}{*}{$\begin{array}{l}\mathrm{L}(\mathrm{mg} / \\
\mathrm{L}) \\
95^{\text {th }}\end{array}$} & \multirow{2}{*}{$\begin{array}{c}\begin{array}{c}\text { Al-MG } \\
(\mathrm{mg} / \mathrm{L})\end{array} \\
50^{\mathrm{th}}\end{array}$} & \multirow{2}{*}{$\begin{array}{c}\begin{array}{c}\text { Al-MG } \\
(\mathrm{mg} / \mathrm{L})\end{array} \\
9^{\text {th }}\end{array}$} & \multirow{2}{*}{$\begin{array}{c}\begin{array}{c}\text { B2-MG } \\
(\mathrm{mg} / \mathrm{L})\end{array} \\
50^{\text {th }}\end{array}$} & \multirow{2}{*}{$\begin{array}{r}\begin{array}{c}\text { B2-MG } \\
(\mathrm{mg} / \mathrm{L})\end{array} \\
9^{\text {th }}\end{array}$} \\
\hline \multicolumn{2}{|c|}{ Percentiles } & & & & & & \\
\hline Boys 7-8 & 144 & 7,1 & 18,1 & $<5,2$ & 6 & $<0,2$ & $<0,2$ \\
\hline Boys 9-10 & 122 & 6,3 & 26,8 & $<5,2$ & 8,7 & $<0,2$ & $<0,2$ \\
\hline Boys 11-12 & 118 & 7,1 & 29,3 & $<5,2$ & 9,3 & $<0,2$ & 0,25 \\
\hline Girls 7-8 & 150 & 7,4 & 27,2 & $<5,2$ & 5,5 & $<0,2$ & $<0,2$ \\
\hline Girls 9-10 & 72 & 10 & 36 & $<5,2$ & 5,6 & $<0,2$ & $<0,2$ \\
\hline Girls 11-12 & 72 & 8,3 & 24,8 & $<5,2$ & 6,5 & $<0,2$ & $<0,2$ \\
\hline
\end{tabular}

\section{CONGENITAL HYDRONEPHROSIS PRESENTING AS ACUTE PYONEPHROS}

N Ristoska Bojkovska, D Kuzmanovska, V Tasic, E Sahpazova

Department of Nephrology, Children's Hospital, Skopje, Macedonia

Background and Aims: Congenital urinary tract anomalies are often in children. We present a case of late detected urinary tract malformation. We investigated a 12 years boy with fever and urinary tract infection. The ultrasound examination showed ureterohydronephrosis on the left side and one $4 \times 3 \mathrm{~cm}$ cystic formation. Acute pyelonephritis was severe with abdominal and lumbal pain, high temperature and laboratory findings for developing urosepsis.

Methods: CT abdomen showed hydronephrotic left kidney with reduction of the parenchyma and pyonephros. Radionuclide renal scanning (DMSA) was pathological with a functional left kidney. Conventional radio-opaque voiding cystourethrography (VCUG) confirmed the diagnosis of stenosis prevesicalis with ureterohydronephrosis of the left side.

Results: The patient was undergoing on surgical treatment with percutaneus nephroureterostomia. The performed pyelography from the nephroureterostomia showed the results of dilated ureter $(24 \mathrm{~mm})$ with stenosis prevesicalis on the left side. The surgeon had drained 10 liters liquid out of the kidney.

Conclusions: We had stabilised the clinical symptoms. After one month drainage of the kidney we did not have better function of the kidney and we had done the unilateral nephrectomia. The patient has been stabilized without any further urinary tract infection. Urinary tract malformations should be recognised early after birth not as a late complication.

\section{PULMONARY EXPRESSION OF VASCULAR ENDOTHELIAL GROWTH FACTOR (VEGF) IN NEWBORN RATS EXPOSED TO HYPOXIA AND RECOVERED IN HYPEROXIA OR AIR}
A Remesal ${ }^{1}, \mathrm{C} \mathrm{Pedraz}^{1}$, D Ludeña ${ }^{2}$, L San Feliciano ${ }^{1}$, M J Hernández ${ }^{1}$
${ }^{1}$ Neonatal Unit, Department of Pediatrics, ${ }^{2}$ Department of Cellular Biology and Pathology, Salamanca
University, Salamanca, Spain

Background and Aims: VEGF is a potent endothelial cell growth factor expressed in normal lung tissue. This study was performed to investigate the expression of VEGF in development rat lung under basal conditions and after hypoxia and oxidative stress.

Methods: We evaluated pulmonary VEGF expression in rats at 0 and 14 days of life. At 4-8 hours of life newborn rats were exposed in sequential way during 2 hours in hypoxia, and recovered in $>95 \%$ oxygen or $21 \%$ oxygen; the control group were exposed in $21 \%$ oxygen. For VEGF inmunostaining, the lungs were fixed by intratracheal instillation $4 \%$ paraformaldehyde and paraffin embedded for tissue sections. Inmunohistochemistry was performed on lung tissue sections by using 1:100 dilution of rabbit anti-human VEGF. We used a semiquantitative assessment of VEGF inmunostained, were assigned a relative value from 0 (minimal) to 4 (most intense).

Results: The 14 days groups had decreased VEGF inmunostaining compared with 0 days group in the same situation. The group exposed to hypoxia had increased VEGF inmunostaining. The group recovered in $>95 \%$ oxygen had decreased VEGF inmunostaining compared with the group recovered in $21 \%$ oxygen in the alveolar epithelium, cells of the interstice and cells of the bronchi; this find was strong in 14 days samples.

Conclusions: The VEGF is expressed during postnatal rat lung development. The hypoxia may increase VEGF expression. These date suggest that acute oxidant injury in newborn rat lung resulted in decreased epithelial VEGF expression.

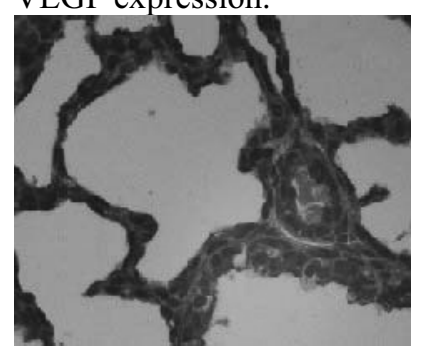

\section{AORTIC NARROWING AFTER PRETERM BIRTH - AN MRI STUDY}

\author{
A K Edstedt Bonamy ${ }^{1}$, J Bengtsson ${ }^{2}$, H De Keyzer ${ }^{3}$, \\ $Z_{\text {Nagy }}{ }^{1}$, H Lagercrantz ${ }^{1}$, M Norman ${ }^{1,4}$ \\ ${ }^{1}$ Department of Woman and Child Health, Karolinska \\ Institutet, Stockholm, Sweden, ${ }^{2}$ Department of Medical \\ Physics, Karolinska University Hospital, Stockholm, \\ Sweden, ${ }^{3}$ Huddinge Children's Hospital, Karolinska \\ University Hospital, Stockholm, Sweden, ${ }^{4}$ Institution for \\ Clinical Science, Intervention and Technology, \\ Karolinska Institutet, Stockholm, Sweden
}

Background and Aims: Preterm birth might permanently affect vascular structure and growth. We have previously found reduced diameter in the distal part of the aorta of adolescents born preterm. In the present study, we hypothesized that subjects born preterm have smaller aortas throughout the vessel course and investigated this hypothesis using MRI.

Methods: 88 healthy adolescents (mean age $15 \mathrm{y}$ ) were included in the study; 46 born preterm weighing less than $1500 \mathrm{~g}$ at birth and 42 controls born at term. Each participant underwent a single scanning session on a 1.5 
T MR scanner. The protocol included a pulse-gated Fiesta sequence with 25 images within the heart cycle. Six $5 \mathrm{~mm}$ thick slices were positioned $4.5 \mathrm{~cm}$ apart, along the descending aorta. The first slice was positioned just below the iliac bifurcation. The aortas were segmented using a subpixel-precision Active Contour Model implemented in MATLAB (Mathworks, Natick, Massachusetts, USA), permitting automatic calculations of end-diastolic aortic cross sectional areas. Blood pressure and heart rate were also recorded.

Results: Subjects born preterm had 15.4-19.8\% narrower aortic lumen on all levels, as compared to controls $(\mathrm{p}<0.001)$. This difference remained highly significant when correcting for body surface. Adolescents born preterm also had higher systolic and diastolic blood pressure. In multivariate analysis, BP correlated to height and preterm birth, but not to aortic luminal area or gender.

Conclusions: Preterm birth is associated with general aortic narrowing and higher BP in adolescence. The long-term cardiovascular consequences of these findings remain to be studied.

\section{LOW FOLATE IN PREGNANCY - A PREVENTABLE PERINATAL RISK FACTOR FOR CORONARY HEART DISEASE?}

H Martin, B Lindblad, M Norman

Karolinska Institutet, Stockholm, Sweden

Background and Aims: Low folate levels during pregnancy correlate with low birth weight (LBW), a perinatal risk factor for later coronary disease. Our aim was to study the relations between levels of folate, vitamin $\mathrm{B} 12$ and homocysteine (Hcy) and vascular endothelial dysfunction - a key factor in early pathophysiology of coronary heart disease - in newborn infants with low or normal birth weight, and their mothers.

Methods: We used a laser-Doppler technique to measure skin perfusion before and after transdermal iontophoresis of acetylcholine ( $\mathrm{ACh}$; an endotheliumdependent vasodilator). Red blood cell (RBC) folate, vitamin B12 and Hcy levels were measured in all subjects.

Results: The perfusion response to ACh was lower $(\mathrm{p}<0.001)$ in LBW infants $(\mathrm{n}=30$, mean birth weight $2394 \mathrm{~g})$ than in normal birth weight controls $(\mathrm{n}=52$, mean birth weight $3571 \mathrm{~g}$ ). The neonatal ACh response correlated with the RBC folate levels, both in infants $(\mathrm{r}=0.43, \mathrm{p}=0.003)$ and their mothers $(\mathrm{r}=0.36, \mathrm{p}=0.004)$. We found no significant difference between the folate levels of LBW and control infants $(p=0.11)$ but the mothers of LBW infants had lower RBC folate levels than mothers of control infants $(p=0.02)$. The levels of vitamin B12 and Hcy were similar in the two groups and no correlations were found between the levels of vitamin B12 or Hcy and endothelial function.

Conclusions: The data presented here provide the first evidence for a relation between low folate levels in pregnancy and vascular endothelial dysfunction in the developing human fetus. Low folate levels late in pregnancy may be a preventable perinatal risk factor for coronary heart disease.
EFFECTS OF ORAL AND VAGINAL INDOMETHACIN ON PULMONARY NITRIC OXIDE AND ENDOTHELIN-1 IN FETAL RABBITS

K D Beharry ${ }^{1}$, W Fortson ${ }^{2}$, H D Modanlou ${ }^{1}$

${ }^{1}$ Department of Pediatrics, University of California Irvine, Irvine, CA, USA, ${ }^{2}$ Department of Obstetrics and Gynecology, Miller Children's Hospital, Long Beach, CA, USA

Background and Aims: Antenatal indomethacin is associated with PPHN. Nitric oxide (NO) is a pulmonary vasodilator and its inhibition results in elevated pulmonary vascular resistance. Endothelin-1 (ET-1) is a vasoconstrictor that mediates pulmonary hypertension. Using a rabbit model for preterm birth, we compared the effects of oral and vaginal indomethacin on NO, cGMP, ET-1, and big ET-1 in fetal rabbit lungs.

Methods: Pregnant rabbits ( $\mathrm{n}=6 /$ group) were induced with RU486, prior to administration of oral indomethacin (OI, $20 \mathrm{mg}$ ); oral vehicle $(\mathrm{OV})$; vaginal indomethacin (VI, $20 \mathrm{mg}$ in cocoa butter); or vaginal cocoa butter (placebo, VP) once daily for 2 days. At delivery, fetal lung NO metabolites (NOx), cGMP, ET1, and big ET-1 were measured.

Results: OI increased fetal lung NOx levels $(60.6 \pm 1.5$ $\mu \mathrm{mol} / \mathrm{mg}$ protein, $\mathrm{p}<0.01)$ compared to OV $(41.2 \pm 4.1)$, VI (42.7 \pm 1.4$)$, and VP $(31.7 \pm 1.8)$. OI caused a similar increase in ET-1 levels $(9.6 \pm 1.3 \mathrm{pg} / \mathrm{mg}$ protein, $\mathrm{p}<0.01)$ compared to OV $(3.83 \pm 1.22)$, VI $(1.5 \pm 0.5)$, and VP (3.58 \pm 1.1$)$. cGMP levels ( $\mathrm{pmol} / \mathrm{mg}$ protein) were higher with VI $(23.9 \pm 1.9, \quad \mathrm{p}<0.05)$ than OI $(16.6 \pm 1.3)$ treatment. VI treatment also resulted in higher big ET-1 levels $(0.24 \pm 0.05 \mathrm{pg} / \mathrm{mg}$ protein, $\mathrm{p}<0.01)$ compared to OI (0.04 \pm 0.04$)$, OV $(0.04 \pm 0.02)$, and VP $(0.0 \pm 0.0)$. Data are mean \pm SEM.

Conclusions: Maternal administration of oral indomethacin increases fetal pulmonary ET-1 levels, an effect that may be responsible in part, for pulmonary hypertension. Increased NO production may be a compensatory response to the ET-1-induced vascular tone. Vaginal indomethacin does not appear to have similar effects on NO and ET-1 despite elevated big ET1 and cGMP.

\section{INTERCELLULAR ADHESION MOLECULE 1 (ICAM-1, CD54) AND PLATELET- ENDOTHELIAL CELL ADHESION MOLECULE- 1 (PECAM-1, CD31) EXPRESSION ON HUMAN UMBILICAL VEIN ENDOTHELIAL CELLS (HUVEC) IN OXIDATIVE STRESS INDUCED BY TUMOR NECROSIS FACTOR-ALFA}

M Szczepanski ${ }^{1}$, M Kamianowska ${ }^{1}, \mathrm{~K}$ Wnuczko ${ }^{1}$, M Pietruczuk ${ }^{2}$, E Skrzydlewska $^{3}, \mathrm{~S}$ Wolczynski ${ }^{4}$

${ }^{1}$ Department of Neonatology, ${ }^{2}$ Department of Haematological Diagnostics, ${ }^{3}$ Department of Nonorganic and Analytical Chemistry, ${ }^{4}$ Department of Gynecological Endocrinology, Medical University of Bialystok, Poland

Background and Aims: Tumor necrosis factor- $\alpha$ $(\mathrm{TNF}-\alpha)$ is one of the main cytokines of inflammatory 
and immunological response. It displays cytotoxic activity by generation of reactive oxygen species (ROS) in cell, which disturbs prooxidation - antioxidation balance, thus causing oxidative stress which initiates lipid peroxidation processes. The latest research proved that ROS are involved in the regulating of ICAM-1 and PECAM-1 expression on the endothelial cells. In the present study, we examined an effect of oxidative stress (determining by malonyldialdehyde content) induced by TNF- $\alpha$ on ICAM-1 and PECAM-1 expression on human umbilical vein endothelial cells (HUVEC).

Methods: Human umbilical vein endothelial cells were cultured in a standard medium (M 199). The culture cells were incubated for 6 hours with a TNF- $\alpha(10,20$, $40 \mathrm{ng} / \mathrm{ml})$. In the HUVEC lysate, malonyldialdehyde (MDA), marker of fatty acids peroxidation, was measured by HPLC method. Expression levels of ICAM-1 and PECAM-1 were quantified by flow cytometry.

Results: It was proved that MDA content in the control culture cells was $12,92 \pm 1,02 \mathrm{nmol} / \mathrm{mg}$ protein. In the cultures stimulated by TNF- $\alpha$ in concentrations 10, 20, $40 \mathrm{ng} / \mathrm{ml}$, MDA content increased to $14,34 \pm 1,05$; $16,48 \pm 1,21$ and $17,39 \pm 3,01 \mathrm{nmol} / \mathrm{mg}$ protein respectively. In the control culture cells expression of ICAM- 1 and PECAM- 1 amounted to $28,4 \pm 0,55 \%$ and $20,9 \pm 0,35 \%$ respectively. After HUVEC stimulation with $10,20,40 \mathrm{ng} / \mathrm{ml} \mathrm{TNF}-\alpha$, expression of ICAM-1 and PECAM-1 increased statistically significantly to $65,1 \pm 3,76$ and $80,4 \pm 3,32 ; 70,0 \pm 3,94$ and $83,4 \pm 2,27$; $73,1 \pm 5,30$ and $86,5 \pm 0,69$ respectively for ICAM- 1 and PECAM-1.

Conclusions: Tumor necrosis factor $-\alpha$ induced ICAM- 1 and PECAM-1 expression on HUVEC by generating of oxidative stress.

\section{THE INFLUENCE OF ERYTHROPOIETIN (EPO) ON INTERCELLULAR ADHESION MOLECULE 1 (ICAM-1, CD54) AND PLATELET- ENDOTHELIAL CELL ADHESION MOLECULE- 1 (PECAM-1, CD31) EXPRESSION ON HUMAN UMBILICAL VEIN ENDOTHELIAL CELLS (HUVEC) IN OXIDATIVE STRESS INDUCED BY HIGH G}

\section{Szczepanski $^{1}$, M Kamianowska $^{1}, \mathrm{~K}$ Wnuczko ${ }^{1}$, M Pietruczuk $^{2}$, E Skrzydlewska ${ }^{3}$, S Wolczynski ${ }^{4}$ \\ ${ }^{1}$ Department of Neonatology, Medical University of Bialystok, Poland, ${ }^{2}$ Department of Haematological Diagnostics, Medical University of Bialystok, Poland, ${ }^{3}$ Department of Nonorganic and Analytical Chemistry, Medical University of Bialystok, Poland, ${ }^{4}$ Department of Gynecological Endocrinology, Medical University of Bialystok, Poland}

Background and Aims: High glucose concentrations disturb oxidation-reduction reaction leading to the increase of reactive oxygen species production. ROS are involved in ICAM-1 and PECAM-1 expression on endothelial cells. Erythropoietin displays cytoprotective effects. We examined an influence of oxidative stress induced by high glucose concentrations on ICAM-1 and PECAM-1 expression and an effect of Epo.
Methods: Some HUVEC were pretreated (24 hours) with Epo $(20 \mathrm{IU} / \mathrm{ml})$. The control culture cells and the cells from Epo were incubated for 48 hours with glucose in concentrations 5,$5 ; 11,1$ and $22,2 \mathrm{mmol} / \mathrm{l}$. In the HUVEC lysate malonyldialdehyde, was measured by HPLC. Expression of ICAM-1 and PECAM-1 were quantified by flow cytometry.

Results: In the cultures stimulated by glucose (concentrations: 11,1 and $22,2 \mathrm{mmol} / \mathrm{l}$ ), MDA content increased form $12,92 \pm 1,02$ (control culture) to $18,15 \pm 1,26$ and $21,50 \pm 2,49 \mathrm{nmol} / \mathrm{mg}$ protein respectively. It was significantly statistically higher than MDA content in the cultures pretreated with Epo $15,06 \pm 1,12$ and $18,98 \pm 1,56 \mathrm{nmol} / \mathrm{mg}$ protein respectively. $60+60+45$ After HUVEC stimulation with glucose (concentrations: 11,1 and 22,2 $\mathrm{mmol} / \mathrm{l}$ ) expression of ICAM-1 and PECAM-1 increased from $28,4 \pm 0,55$ to $29,6 \pm 3,99$ and $47,2 \pm 4,80$ for ICAM- 1 and from $20,9 \pm 0,35$ to $26,2 \pm 3,33$ and $44,88 \pm 5,16 \%$ for PECAM-1. It was significantly statistically less than ICAM-1 and PECAM-1 expression in the cultures

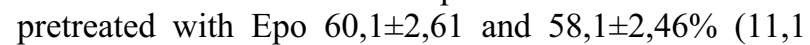
$\mathrm{mmol} / 1$ of glucose) and $60,4 \pm 1,51$ and $57,9 \pm 1,24 \%$ ( $22,2 \mathrm{mmol} / \mathrm{l}$ of glucose) respectively.

Conclusions: Erythropoietin inhibits oxidative stress induced by high glucose concentrations. High ICAM-1 and PECAM-1 expression on HUVEC pretreated with Epo is a consequence of higher stimulation activity of Epo in comparison to high glucose concentrations.

\section{EFFECT OF MATERNAL MAGNESIUM SULFATE ON RED CELLS MEMBRANE LIPID PEROXIDATION - IS THERE A CORRELATION WITH CLINICAL OUTCOME IN VLBWN?}

E M Gulczynska ${ }^{1}$, J Gadzinowski ${ }^{1}$, M Kesiak ${ }^{1}$, B Sobolewska $^{1}$, A Grodzicka ${ }^{1}$, L Zylinska ${ }^{2}$

${ }^{I}$ Department of Neonatology, Research Institute of Polish Mother's Memorial Hospital, Lodz, Poland ${ }^{2}$ Department of Molecular Neurochemistry, Medical University of Lodz, Poland

Background and Aims: Magnesium sulfate is nowadays suggested as a neuroprotective agent in VLBW. We evaluated whether they might have an effect on lipid peroxidation of erythrocytes membranes. Methods: The study groups consist of neonates $(\leq 1500 \mathrm{~g})$ born from mother receiving prenatally magnesium sulphate $(n=24)$ and control group $(n=20)$ without MgSO4. Lipid peroxidation in erythrocyte ghosts was determined as thiobarbituric acid reacting substances (TBARS). We have compared the TBARS level assessed in umbilical blood cord and after $24 \mathrm{~h}$ of life. Simultaneously, the differences in neonatal outcome between antenatally exposed to $\mathrm{MgSO} 4$ neonates $(\mathrm{n}=78)$ and control group $(\mathrm{n}=293)$ VLBW neonates were analyzed.

Results: After the birth, the mean $\mathrm{Mg} 2+$ serum concentration in control group was $0.73 \mathrm{mM}$, and $1.27 \mathrm{mM}$ after $24 \mathrm{~h}$ of life. In MgSO4-treated group, these values were 1.13 and 1.38 , respectively. In erythrocyte membranes of control group the amount of TBARS was twice as high (mean 1,84; and 1.99 after 24 h) as in $\mathrm{Mg}$-treated newborns during examined period 
(mean 1.21 and $1.34 / 10 \mu \mathrm{g}$ of protein, respectively). A statistically significant correlation was found between antenatal Mg treatment and BPD $(\mathrm{r}=-0,23, \mathrm{p}<0,001)$ as well as severe IVH $(\mathrm{r}=-0,18, \mathrm{p}<0,05$; only in group 1000-1500g). These observations were confirmed by multiple logistic regression analysis which also revealed that both BPD and severe IVH were negatively associated with Mg-administration.

Conclusions: Our results demonstrate that prenatal administration of magnesium sulfate significantly altered lipid peroxidation measured as a TBARS. It is possible that observed effect could explain the reduced rate of BPD and severe IVH in magnesium treated group. Supported in part by the Grants no. 5P05E 09224 from the State Committee for Scientific Research, and no. 503-686-2 from the Medical University of Lodz.

\section{FLEXIBLE FIBEROPTIC BRONCHOSCOPY IN THE NEONATAL INTENSIVE CARE UNIT}

\author{
E Arbel $^{1}$, D Kohelet ${ }^{1,2}$ \\ ${ }^{I}$ Department of Neonatology, Edith Wolfson Medical \\ Center, Holon, Israel, ${ }^{2}$ Sackler School of Medicine, \\ Tel-Aviv University, Tel-Aviv, Israel
}

Background and Aims: Bronchoscopy is probably under-used procedure, usually because its diagnostic potential in the Neonatal Intensive Care Unit (NICU) is underrated. The study was carried out to evaluate the contribution of flexible fiberoptic bronchoscopy (FFB) and BAL to the clinical management of patients in a NICU. Setting and design: A retrospective study based on medical records in a nine-bed NICU of a tertiary care hospital.

Methods: Twenty seven procedures were performed, for the following causes: search for airways anatomic pathologies (7); treatment of atelectasis and installation of surfactant (6); BAL (7), noisy breathing (2), aid to difficult intubation (1), aid to difficult extubation (3) and tracheotomy evaluation (2).

Results: Eleven procedures had more than one cause. Airway pathology was observed in 11 procedures. BAL results changed antimicrobial treatment in 4 of 7 cases, with clinical improvement in 3 cases. Treatment of atelectasis was successful in 6 of 6 cases. No procedurerelated mortality, life-threatening complications, or significant changes in patient status occurred.

Conclusions: Anesthesia and mechanical ventilation make FFB so safe that the NICU patient must be gravelly ill, or have gross reduction in his respiratory reserve, before bronchoscopy becomes dangerous. FFB should be considered to be a NICU staff expertise.

\section{POSTNATAL CHANGES OF PULMONARY PRESSURES AND DUCTUS ARTERIOSUS DIAMETERS CAN PREDICT LATTER NECESSITY FOR PDA TREATMENT}

T Kovács, N Katona, M Bérces, Z Horváth, E Polonkai, B Kovács-Pászthy, G Balla

Division of Neonatology, Department of Obstetrics, University of Debrecen, Hungary
Background and Aims: Haemodynamic impact of ductal shunting occurs earlier than clinical symptoms develop. Timing of PDA treatment remains controversial.

Methods: We conducted a prospective analyses in infants who were $\leq 29$ weeks of gestation. In 24 infants (mean: $760 \mathrm{~g}, 25.5$ weeks) serial echocardiography were performed on 1,2,3,7 postnatal days, and when PDA was clinically suspected. The diameter of ductus arteriosus (DA) was assessed, and pulmonary vascular resistance (PVR) was estimated (velocity of tricuspid and pulmonary insufficiency, pulmonary acceleration time, direction and velocity of ductal shunting). PDA treatment was indicated when clinical or echocardiographic signs of volume overload were present. We collected data on presence of IVH grade IIV and BPD. Patients were divided into two subgroups at the end of study period: infants who later developed PDA necessiating treatment (group $\mathrm{I}, \mathrm{n}=8$ ) and who didn't (group II, $\mathrm{n}=16$ ).

Results: No infant found with elevated PVR on the 3rd postnatal day. That time occurance of ductal diameter above $1.5 \mathrm{~mm}$ were $75 \%$ in group I. On contrary we found only one infant with PDA $(1.2 \mathrm{~mm})$ in group II. BPD and IVH grade I-IV were more common in group I than in group II $(62.5 \%$ versus $31.25 \%$ and $50 \%$ versus $25 \%)$.

Conclusions: In ELBW infants PVR falls in much the same time frame as in full-term infants, on the day 3 PVR is low. DA diameter measured on day 3 can predict persistent ductal patency. Symptomatic, or even some forms of presymptomatic PDA treatment given later than day 3 may be ineffective in prevention of PDA associated adverse outcomes.

\section{EFFECT OF SERUM FROM PREECLAMPSIA MOTHER AND HER NEONATAL CORD BLOOD ON THE ACTIVITY OF SOLUBLE ADHESION MOLECULES AND APOPTOSIS IN CULTURED HUMAN UMBILICAL VENOUS ENDOTHELIAL CELLS}

S Y Kim ${ }^{1}$, H S Kim ${ }^{1}$, C S Chun ${ }^{2}$

${ }^{1}$ Department of Pediatrics, Catholic University of Korea, Holy Family Hospital, Pucheon, South Korea ${ }^{2}$ Department of Pediatrics, Catholic University of Korea, Kang-Nam St. Mary's Hospital, Seoul, South Korea

Background and Aims: It is proposed that endothelial cell dysfunction, as a central pathogenic feature of preeclampsia is caused by circulating unknown factors produced by placenta in the blood of women with preeclampsia. In this study we investigated the effect of such factors in the serum from preeclamptic mother or her neonatal umbilical vein on the activity of sICAM-1 and apoptosis in cultured human umbilical venous endothelial cells.

Methods: Quantitive determinations of sICAM-1 and apoptosis were detected from isolated and cultured HUVEC supernatants which were incubated with serum samples for 48 hours. The serum samples were collected from preeclamptic mother and her neonatal cord blood 
in pairs according to gestational age and were compaired to nonpreeclamptic control groups.

Results: 1) sICAM-1 levels was significantly higher levels in the maternal groups compared to corresponding cord groups $(\mathrm{P}<0.001)$. The preterm maternal groups showed higher levels than term maternal groups $(\mathrm{P}<0.001)$. The preeclamptic preterm mother groups showed elevated level compared to other mother groups and the existence of preterm delivery affects sICAM-1 level more importantly than the existence of preeclampsia. 2) In TUNEL stain: Preeclamptic preterm mother group showed increased number of apoptotic nuclei compaired to other groups. The neonatal cord groups of preterm preeclampsia showed no apoptotic body on cultured HUVEC.

Conclusions: Unknown circulating factors in preeclamptic preterm mother activate expression of sICAM-1 and apoptosis in cultured human umbilical venous endothelial cells. But the fetal circulation may not be affected by the factor(s) that lead to disturbed endothelial cell function in women with preterm preeclampsia.

\section{IBUPROFEN TREATMENT OF PATENT DUCTUS ARTERIOSUS IS ASSOCIATED WITH TRANSITORY RENAL FUNCTION IMPAIRMENT}

S Johansson, A Rakow, M Bartocci, M Norman, M Vanpee, B Jonsson

\section{Department of Woman and Child Health, Karolinska Institutet, Stockholm, Sweden}

Background and Aims: PDA-treatment with ibuprofen has been associated with little renal function impairment. We assessed urinary function during ibuprofen treatment, in a clinical setting.

Methods: 83 infants (mean GA 27 wks, SD=2 wks) with echocardiographic PDA-diagnosis received ibuprofen $(10+5+5 \mathrm{mg} / \mathrm{kg})$. Pharmacological closure rate was $80 \%$.

Results: There was a transient reduction in renal function after the first and second dose (Table 1). After the third dose, renal function was not different from pretreatment values.

Conclusions: Ibuprofen PDA-treatment is associated with transient renal dysfunction. This decrease in renal function seems small but surveillance of renal function is indicated during ibuprofen PDA-treatment.

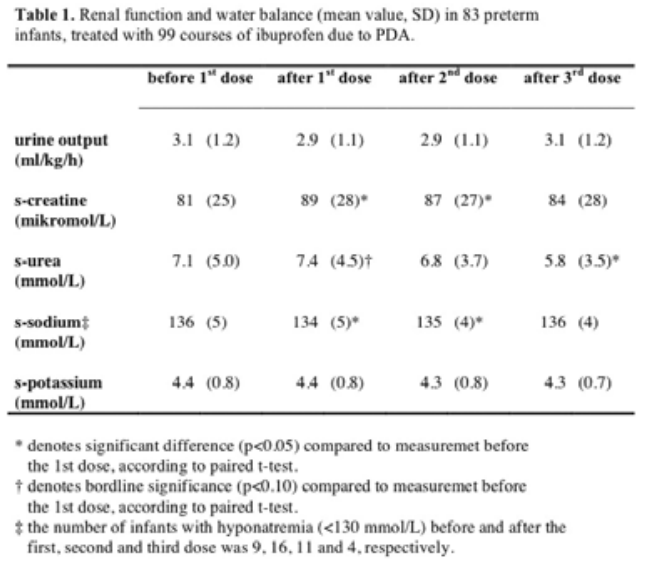

SURGICAL PDA LIGATION IS COMMON AMONG 23-24 WEEK PRETERM INFANTS INITIALLY TREATED WITH IBUPROFEN

S Johansson, A Rakow, M Bartocci, M Norman, M Vanpee, B Jonsson

Department of Woman and Child Health, Karolinska Institutet, Stockholm, Sweden

Background and Aims: Most published data on ibuprofen treatment of patent ductus arteriosus (PDA) have included few infants born at 23-24 gestational weeks. We determined PDA closure rates, stratified by gestational age, in a cohort of preterm infants with PDA, admitted to Karolinska University Hospital.

Methods: During 2001-2004, 83 consecutive infants (mean gestational age 27 weeks, $\mathrm{SD}=2$ weeks) received ibuprofen treatment due to echocardiographically confirmed PDA. Short term closure was assessed by echocardiography 18-24 hours after completed ibuprofen course. Surgical closure rate was assessed at discharge.

Results: Short term closure was achieved in most infants, but the rate was lowest (71\%) among 23-24 week infants. Relapses were also common in this group. Final non-surgical closure rate ranged from 57\% among 23-24 week infants to $94 \%$ among infants born $>28$ weeks. Accordingly, surgical closure rates varied between $43 \%$ to $6 \%$.

Conclusions: Short term PDA closure with ibuprofen was achieved in most infants, but final non-surgical closure rate was low among infants born at 23-24 weeks. Whether optimized ibuprofen treatment strategies, such as earlier initation of treatment, could decrease surgical closure rate among the most immature infants remains to be determined.

\section{EARLY ECHOCARDIOGRAPHIC FINDINGS AS PREDICTORS OF PATENCY OF DUCTUS ARTERIOUSUS}

P Kwinta ${ }^{1}$, A Rudzinski ${ }^{2}$, P Kruczek ${ }^{1}$, Z Kordon $^{2}$, J J Pietrzyk $^{1}$

Department of Pediatrics, Jagiellonian University, Cracow, Poland, ${ }^{2}$ Department of Pediatric Cardiology, Jagiellonian University, Cracow, Poland

Background and Aims: Prophylactic treatment with prostaglandin synthetase inhibitors (PSI) can unnecessarily exposes infants to a drug. Alternative to routine prophylactic use of PSI is early treatment of patent ductus arteriosus (PDA). The aim of the study was evaluation of prognostic value of early echocardiographic study with respect to PDA in later life.

Methods: 60 preemies with mean birthweight $1087 \mathrm{~g}$ were included into prospective study. The cardiac scans were performed at the entry into the study (at the age 12-48 hour of life) and further in case of suspicion of PDA or obligatory at the 7th and 28th day of life. There was no prophylactic or treatment use of PSI during the study. Newborns were divided into 2 cohorts: With significant left to right shunt requiring ligation of PDA $(n=16)$ or without significant PDA $(n=44)$. 
Results: At the entry into the study the mean internal diameter of DA ( 2.6 vs $0.9 \mathrm{~mm} / \mathrm{kg} ; \mathrm{p}<0.01)$, mean CI Ao (2.96 vs $2.371 / \mathrm{min} . / \mathrm{m} 2 ; \mathrm{p}<0.01)$ and $\mathrm{fE}$ (43 vs $34 \mathrm{~m} / \mathrm{s} ; \mathrm{p}=0.02$ ) were significantly higher in the treated group. There was no difference in mean values of other echocardiographic parameters studied. Sensitivity, specifity, predictive value of selected variables were presented in the table.

Conclusions: Early echocardiographic study possesses negative predictive value for persistence of DA and by this means can help to avoid unnecessary use of prostaglandin synthetase inhibitors in VLBW infants.

\begin{tabular}{|l|l|l|l|l|l|}
\hline & Cut off point & Sensivity & Specifity & PPV & NPV \\
\hline DA diameter & $>1,5 \mathrm{~mm} / \mathrm{kg}$ & $94 \%$ & $73 \%$ & $57 \%$ & $97 \%$ \\
\hline $\mathrm{ClAo}$ & $>2,5 \mathrm{I} / \mathrm{min} . / \mathrm{m} 2$ & $82 \%$ & $64 \%$ & $46 \%$ & $91 \%$ \\
\hline $\mathrm{fE}$ & $>36 \mathrm{~m} / \mathrm{s}$ & $70 \%$ & $62 \%$ & $38 \%$ & $86 \%$ \\
\hline
\end{tabular}

\section{PERIPHERAL FRACTIONAL OXYGEN EXTRACTION AND TISSUE OXYGENATION INDEX IN TERM NEONATES WITH IN UTERO EXPOSURE TO SMOKING}

G Pichler ${ }^{1}$, B Urlesberger ${ }^{1}$, J Heizinger ${ }^{1}$, P Klaritsch ${ }^{2}$, J Kutschera $^{1}$, H Zotter ${ }^{1}$, W Müller ${ }^{1}$

${ }^{1}$ Division of Neonatology, Department of Pediatrics, Medical University of Graz, Austria, ${ }^{2}$ Division of Obstetrics, Department of Obstetrics and Gynecology, Medical University of Graz, Austria

Background and Aims: Aim of the present study was to analyse the influence of in utero exposure to smoking on peripheral oxygenation in healthy term neonates.

Methods: 13 term healthy neonates with in utero exposure to smoking were compared to 13 term healthy neonates without in utero exposure to smoking. Measurements were performed during first week of life. Neonates were matched for gestational age, actual weight and postnatal age at time of measurement. Peripheral oxygenation was measured by near infrared spectroscopy (NIRS) in combination with the venous occlusion method on the left forearm. Fractional oxygen extraction (FOE) and tissue oxygenation index (TOI) were analysed. Central and peripheral temperatures were measured continuously. By means of pulsoximetry heart rate and peripheral arterial oxygen saturation were measured continuously. Arterial blood pressure was measured before and after venous occlusions.

Results: FOE tended to be higher and TOI tended to be lower in the group with in utero exposure to smoking $(0.38 \pm 0.07 ; 66 \pm 6 \%)$ compared to the group without in utero exposure $(0.34 \pm 0.06 ; 63 \pm 9 \%)$. FOE and TOI did not change with age in the group with in utero exposure to smoking and, whereas FOE increased and TOI decreased significantly with increasing age in the group without in utero exposure. There was no significant difference between the two groups in birth weight, arm circumferences, arterial blood pressure, arterial oxygen saturation and heart rate.

Conclusions: In these preliminary results healthy term neonates demonstrated influence of in utero exposure to smoking on FOE and TOI in the first week of life.

\section{BIRTHWEIGHT AND GESTATIONAL AGE ARE PREDICTORS OF DUCTAL CLOSURE FOLLOWING IBUPROFEN TREATMENT}

G J Richards, A Johnson, G F Fox, M E Campbell

Neonatal Intensive Care Unit, St Thomas' Hospital, London, UK

Background and Aims: Lower gestational age is a recognised risk factor for the development of a clinically significant patent ductus arteriosus (PDA). Rates of successful PDA closure with indomethacin treatment are reduced in extremely premature infants. There are no data describing the rate of PDA closure following intravenous (IV) ibuprofen treatment in the most immature infants.

Methods: Data were collected on all babies $<1000 \mathrm{~g}$ birthweight admitted to a tertiary NICU who received IV ibuprofen for treatment of a clinically symptomatic PDA. A successful outcome was defined as PDA closure following a course of ibuprofen treatment, data were analysed for factors influencing treatment outcome.

Results: We identified 132 babies who had a PDA treated with IV ibuprofen, mean birthweight $( \pm \mathrm{SD})$ was $752 \pm 135 \mathrm{~g}$ and gestational age $25.6 \pm 1.4$ weeks. The overall PDA closure rate was $48.5 \%$ (64/132). Infants who failed to close their PDA were significantly lighter $(p=0.012)$ and less mature $(p=0.011)$. Risk factors significantly associated with reduced PDA closure rates were gestational age $<26$ weeks (OR $0.23,95 \%$ CI 0.11-0.49; $<0.001$ ); and birthweight $<750 \mathrm{~g}$ (OR 0.30, CI $0.15-0.61 ; \mathrm{p}=0.002)$. Neither age at treatment nor sex significantly affected treatment outcome.

Conclusions: The rates of PDA treatment failure following a course of IV ibuprofen were $67.2 \%$ in infants $<750 \mathrm{~g}$ birthweight and $68.1 \%$ in infants $<26$ weeks gestation. These data demonstrate that the efficacy of IV ibuprofen therapy for inducing PDA closure in this population of high risk babies is both birthweight and gestational age dependant.

\section{THE COST-UTILITY OF PALIVIZUMAB IN CHILDREN WITH CONGENITAL HEART DISEASE IN GERMANY}

\author{
M Nuijten $^{1}$, M Lebmeier $^{2,3}$, W Wittenberg ${ }^{3}$ \\ ${ }^{1}$ Ars Accessus Medica, Erasmus University Rotterdam, \\ Rotterdam, The Netherlands, ${ }^{2}$ University of Sheffield, \\ Sheffield, UK, ${ }^{3}$ Global Health Economics and Outcomes \\ Research, Abbott GmbH \& Co. KG, Ludwigshafen, \\ Germany
}

Background and Aims: To assess the cost-utility of Palivizumab, a prevention against respiratory syncytial virus (RSV) in children with congenital heart disease (CHD) in a German health care setting.

Methods: A decision tree model was used to estimate the cost-effectiveness of Palivizumab. The data sources included published literature, the Palivizumab pivotal trials, official price/tariff lists and national population statistics. The study was conducted from the perspective of the health care purchaser (HCP) (primary analysis) and society (secondary analysis). 
Results: From the perspective of the HCP, the incremental cost-effectiveness ratio (ICER) was $€ 5,013$ /quality adjusted life year (QALY) without discounting; and was $€ 18,266 / \mathrm{QALY}$ discounted. The results from the societal perspective were substantially more cost-effective. The ICER was $€ 2,615 / \mathrm{QALY}$ without discounting, and $€ 9,529 / \mathrm{QALY}$ with discounting. Probabilistic sensitivity analyses confirmed the robustness of the model.

Conclusions: This study showed that Palivizumab provides a cost-effective prevention against RSV infections in children with CHD in Germany.

\section{SURGICAL PDA LIGATION ACUTELY REDUCES VENTILATORY REQUIREMENTS IN BABIES $<1500 G$ BIRTHWEIGHT}

\section{S A Hoodbhoy, H Cutting, M E Campbell}

Neonatal Intensive Care Unit, St Thomas' Hospital, London, UK

Background and Aims: A patent ductus arteriosus (PDA) is associated with increased pulmonary morbidity in premature babies. There are few published data describing the acute effects of PDA ligation on ventilatory status in this population.

Methods: Mechanically ventilated infants $<1500 \mathrm{~g}$ birthweight undergoing surgical PDA ligation were studied. Acid-base variables, ventilatory and haemodynamic data were collected pre-operatively, then at 3 and 24 hours post ligation.

Results: 32 infants were studied, mean $( \pm \mathrm{SD})$ birthweight $761.6 \pm 170 \mathrm{~g}$, gestation $25.6 \pm 1.4$ weeks and age at surgery $33.7 \pm 12.9$ days. There were no significant changes in $\mathrm{pH}, \mathrm{PaCO} 2$, lactate or mean blood pressure over the study time period. Ventilatory parameters significantly decreased following surgery (see Table).

Conclusions: These data demonstrate a significant decrease in mean airway pressure within 24 hours of PDA ligation. These changes likely reflect improved pulmonary compliance consequent upon reduced ductal shunting.

\begin{tabular}{|l|c|c|}
\hline & $\begin{array}{c}\text { Mean Airway Pressure } \\
\left(\mathbf{c m} \mathbf{H}_{z} \mathbf{0}\right)\end{array}$ & $\mathbf{F i O}_{\mathbf{2}}$ \\
\hline Pre op & $9.90 \pm 1.56$ & $0.36 \pm 0.09$ \\
\hline 3 hours post & $9.46 \pm 1.52$ & $0.33 \pm 0.12$ \\
\hline 24 hours post & $9.06 \pm 1.44^{*}$ & $0.35 \pm 0.11$ \\
\hline
\end{tabular}

\section{ACUTE MYOCARDIAL INFARCTION - A RARE BUT TREATABLE COMPLICATION IN THE NEONATE}

\author{
M Ehlen $^{1}$, B Wiebe ${ }^{1}$, M Kästner ${ }^{2}$, P Zartner ${ }^{2}$, \\ W Wiebe ${ }^{2}$, M Schneider ${ }^{2}$ \\ ${ }^{I}$ Department of Neonatology and Paediatric Intensive \\ Care, Asklepios Klinik St. Augustin, St. Augustin, \\ Germany, ${ }^{2}$ Department of Paediatric Cardiology and \\ Congenital Heart Disease, DKHZ, Asklepios Klinik St. \\ Augustin, St. Augustin, Germany
}

Background and Aims: Acute myocardial infarction in preterm and term neonates usually is a rare event associated to a high mortality rate. Apart from neonatal myocarditis and anatomical malformations of the coronary arteries it may occur after thromboembolic occlusion of the coronary arteries.

Methods: We report on two neonates with acute left heart failure after myocardial infarction due to thromboembolic occlusion of the left coronary artery (LCA).

Results: In both cases the diagnosis could be established via immediate echocardiography at the bedside. Rapid onset of systemic thrombolysis with $\mathrm{r}$ TPA after cardiopulmonary resuscitation succeeded in restoration of the myocardial function. In the first case recurrent thrombi at the apex of the left ventricle and adherent to the atrioventricular septum occurred. In the other case the thrombus could be dissoluted entirely. Both infants developed arrhythmias, i.e. complete AVblock as well as ventricular and supraventricular extrasystoles. The first patient died in terminal heart failure after futile intensive care management including inotropes and antiarrhythmic drugs while the arrhythmias of the second infant could be treated successfully. In both cases no underlying thrombogenic disorder could be detected.

Conclusions: Due to the foudroyant course of cardial decompensation the verification of an acute myocardial infarction in the neonate is often only detectable in postmortem examination. Immediate echocardiography at the bedside may reveal thromboembolic occlusion of the LCA as a possible cause. Systemic thrombolysis with r-TPA is estimated to be the only curative therapy. Furthermore, a rapid initiation of advanced intensive care management is mandatory.

\section{THE EFFECT OF DEEP MASSAGE ON WEIGHT GAIN OF LOW BIRTH WEIGHT INFANTS}

\author{
M Golchin, P Taheri, P Rafati
}

\section{Faculty of Nursing and Midwifery, Isfahan Medical Sciences University, Isfahan, Iran}

Background and Aims: The aim of study was determination of the effect of deep massage method on weight gain of LOW infants.

Methods: This is a quasi experimental study which was conducted on 54 LOW infants in hospitals was conducted by convenient sampling method. They were assigned to two groups of 27 infants. One of the groups received deep massage respectively. This group was compared with a control group receiving no massage. Criteria for inclusion in the study were: birth weight $<2500 \mathrm{~g}$, age $<18$ days, breast-feeding, the Iranian race, absence of diseases. Data was collected through observations, interviews and measuring weight by special scales of infants weighing (seco). Each infant in the treatment group received deep massage for three 15minute periods, 30-45 minutes after feeding in the morning, afternoon and evening for 10 consecutive days. Weight of infants was measured in the beginning of the study and 10 days after it. To data was analysis by $\mathrm{t}$-test and paired t-test.

Results: The groups did not differ significantly on matched variables. Means of weight gain within groups, before and after of the study were statistical different. Also $t$ test showed a significant difference between two 
groups. The treatment group was significantly gaining weight better than control group.

Conclusions: These findings show that deep massage enhances weight gain in low birth weight infants. Thus, it can decrease infant's mortality and morbidity, the two most important indexes of health in the world.

\section{RELATION BETWEEN FAMILIES FUNCTIONING AND SELF CONCEPT, A COMPARATIVE STUDY IN HIGH SCHOOL BOYS AND GIRLS OF ISFAHAN (IRAN)}

\section{Golchin}

\section{Faculty of Nursing and Midwifery, Isfahan Medical Sciences University, Isfahan, Iran}

Background and Aims: Determining relation between families functioning and self concept in high school children.

Methods: The study was done according to cross sectional backward manner. 892 male and female students were chosen randomly from different high schools of the Isfahan city. They filled out Questionnaire for Self Concept based on Rajers character test and also BLOOM Questionnaire for family functioning. Family functioning were classified in three levels: good, intermediate, and poor. They also were classified according to their normal distribution of normal condition of society including one SD Data were analyzed by SPSS using Spearman Coefficient correlation and $\mathrm{t}$ test.

Results: Most of boys described level of family function to be good (41\%), and most of girls (37.4\%) reported the same item to be intermediate. Results of $t$ test showed that family functioning for girls and boys were significantly different $(\mathrm{t}=-7.4, \mathrm{P}=0.016)$, also results of Spearman Coefficient correlation showed significant relation between family functioning and selfconcept of youth $(\mathrm{r}=-0.4, \mathrm{P}<0.0001)$.

Conclusions: This study confirms the Significant difference between family functioning for high school girls and boys, may be due to self-sense of limitation for girls or their sense about family attention to boys, so they are falsely unsatisfied. This study confirms reverse relationship between family functioning and selfconcept; we found that the more desired family functioning, the more desired would be self-concept and the above findings are compatible with other findings in setting like this.

\section{'IT'S LIKE ONE OF THOSE THINGS THAT SPIRAL DOWN. IT'S LIKE IN YOUR BODY. IT'S LIKE SPIDERMAN': CHILDREN'S UNDERSTANDING OF GENETICS}

\author{
F M Ulph ${ }^{1}$, C Glazebrook ${ }^{2}$, E J Townsend ${ }^{3}$ \\ ${ }^{1}$ IGBiS, University of Notthingham, Nottingham, UK \\ ${ }^{2}$ School of Community and Health Sciences, University \\ of Nottingham, Nottingham, UK, ${ }^{3}$ School of Psychology, \\ University of Nottingham, Nottingham, UK
}

Background and Aims: Children are increasingly being exposed to genetic information, but how they make sense of genetics, or how best to communicate with children about genetics, remains unclear. Thus, this study examines children's perceptions of genetic illnesses and understanding of probabilistic information. Methods: Children's perceptions of genetics were explored through a write-and-draw task. A probabilistic choice task including label, percentage, ratio and pie chart formats examined understanding of probability. One condition included a mixture of formats.

Results: 106 children aged 7.01 to 11.06 years old completed the probabilistic choice task. Age significantly affected understanding of all formats, except from percentage. 10-11 year olds understood pie charts better than other formats whilst mixed formats significantly decreased understanding $(\mathrm{p}<.001) .7-9$ year old children understood labels, percentages and pie charts significantly better than ratios $(\mathrm{p}=.001)$ or mixed formats $(\mathrm{p}=<.001)$. Thirty-seven children completed the write-and-draw task. Analysis showed children actively construct their understanding of genetics, gathering information from various informal sources. Genetic tests were predominantly understood as paternity tests which appeared to be linked to coverage in daytime chat-shows that children watched. Children evidenced learning the language of genetics, but their understanding often lagged behind their vocabulary.

Conclusions: Format significantly affects comprehension of probabilistic information. All children understood pie charts the best. The write-and-draw task provided vital insights into children's understanding and possible anxieties regarding genetics, highlighting the influence of media in the development of such knowledge. This study represents an important first step in understanding how to communicate with children about genetics.

\section{THE MAGIC OF THE WORDS: THE IMPACT OF STORYTELLING ON SICK CHILDREN}

\section{Andresen, H S Korsgaard, C Blix}

The Children's Program 4074, National Hospital, Copenhagen, Denmark

Background and Aims: Children and their parents need all possible support to cope with the challenges they face through long term treatment. Storytelling have a comeback, which has inspired to let two professional storytellers, 'The Blue Witch' of 'Bowler Granny', tell stories for sick children. The aim of the study was to get experience with the impact of storytelling on severe sick children. An intermediate aim was to stimulate the children's desire for reading, and to give both children and their parents a coping strategy through their long term stay at the hospital.

Methods: Data was collected through observations and videoshuts of the episodes with storytelling, and from questionnaires to the parents, and the logbooks of the storytellers. The target group were children between 3 and 9 years old, who spoke Danish, and who were treated for a severe, acute or chronic, disease. Data was collected through 3 weeks. 5 children were followed in between 3 and 8 story episodes (13 hours of videotaping). 
Results: Children, parents and staff found that storytelling had an important impact on the child's wellbeing and coping with the hospital stay. Storytelling increased the quality of the contact between children and their parents. Some children formulated their difficult situation, by engaging themselves in forming the story. Parents read more books for their child, than they did before. The staff found that the method was useful in their spare contact with the child.

Conclusions: The pilot study point at storytelling as meaningful coping strategy for children suffering from serious diseases and further investigation is relevant.

\section{ATTITUDES OF NURSE PROFESSIONALS AND NURSING STUDENTS TOWARD CHILDREN WITH DISABILITIES}

\section{Matziou ${ }^{1}$, H Brokalaki, P Galanis, E Gymnopoulou, C Tsoumakas}

Faculty of Nursing, University of Athens, Greece

Background and Aims: Today, some people still address children with disabilities with prejudice that leaves them with unhappy feelings. This problem becomes more intense when health professionals and especially nurses have not the proper sensitivity and attitude toward them. We aimed to study perceptions and attitudes of nurse professionals and nursing students towards children with disabilities.

Methods: The study population included 228 (52\%) first year nursing students, $90(20 \%)$ postgraduate nursing students $\kappa a 1123$ (28\%) nurse professionals who work in pediatric hospitals in Athens. Participants have answered the questionnaire of Yuker, Block and Younng. Statistical analysis was performed with statistical package SPSS 13.0 and has included t-test and Analysis of Variance (ANOVA).

Results: The mean age of first year nursing students was 17,8 years, of postgraduate nursing students 27 years and that of nurse professionals 35,3 years. Of the participants $81 \% \quad(n=359)$ were women. Women presented with more positive feelings toward the children with disabilities compared to men $(\mathrm{p}=0,04)$. Postgraduate nursing students were more positive toward disabled children compared the other two groups $(\mathrm{p}=0,00)$, while the attitude of first year nursing students was more positive compared to that of nurse professionals $(p=0,047)$. Age did not have statistically significant relation.

Conclusions: Nurse professionals, despite the fact that they come more often in contact with handicapped children, maintain more negative attitude toward them compared with the other two groups. There is a need for constant systematic informative intervention in order to achieve better social acceptance of children with disabilities.

\section{CONSIDERING FAMILY CENTRED CARE}

H Haslund $^{1}$, I Doj ${ }^{2}$, M Andersen $^{3}$, A Hansen $^{4}$, R Maastrup ${ }^{3}$, E Anthon $^{5}$

${ }^{\text {I}}$ NICU, Aalborg University Hospital, Aalborg, Denmark ${ }^{2}$ NICU, Odense University Hospital, Odense, Denmark
${ }^{3}$ NICU, Rigshospitalet, University Hospital, Copenhagen, Denmark, ${ }^{4}$ NICU, Glostrup University Hospital, Glostrup, Denmark, ${ }^{5}$ NICU, Hillerod University Hospital, Hillerod, Denmark

Background and Aims: In Denmark sick newborns and premature infants are separated from their mothers (and fathers) when admitted at NICU/PICU. This has been practise in the past and still is. Parents do not in all NICUs have unlimited access to their infants. When transported in ambulances they are not allowed either. The aim for most Danish NICUs care is that parents should have an unlimited access, to their children. We suggest that this will enable the parents to obtain self confidence in abilities to take care of their child, and thus be able to act and decide advocating their child's best interests. As a result the families regain the autonomy and their parental competence, which was disturbed by hospitalisation in NICU.

Methods: A national SIG-group organized under The Danish Paediatric Nurse Association, has during the last years had focus on facilitating the implementation of Family Centred Care. As a group we have taught and informed about this subject, during national conferences and teaching sessions using expert nurses, psychologist, master NIDCAP-Trainers, family- therapists and hospital priests.

Results: We have noticed an increased attention towards the subject and there has been an overwhelming participation at our courses both in number and quality of debate.

Conclusions: Considering the ideal of 'family centred care' we recognize there is still a long way to go in changing 'ward cultures' as well as physical realities (including room for a mothers bed with her infant or child). However we consider NICU care in Denmark moving forward in the right direction.

\section{NURSING ATTITUDE TOWARDS END OF LIFE DECISIONS - HOW WE DEAL WITH IT!}

M Cicak Novak, M Novak

Division for Neonatology and Intensive Care, Department of Pediatrics, University Hospital Center Zagreb, Croatia

Background and Aims: Most deaths in pediatric intensive care unit are not unexpected; they follow limitation or withdrawal of life-sustaining treatment (LST). The nursing stuff attitude towards such decisions between nurses in neonatal, pediatric, adult neurosurgical intensive care unit, and general pediatric ward was investigated.

Methods: A prospective, descriptive study based on a survey was conducted. A questionnaire was sent to nurses from all the mentioned divisions. The attitude about active/passive euthanasia; nursing care at the end of life; pain management; symptom management; ethical/legal issues; cultural and especially religious consideration in end-of life care; communication with parents/family and family presence; grief, loss, and bereavement; achieving quality at the end of life. The opinion about nursing role in LST withdrawal decisions was investigated. 
Results: Summary statistics is presented.

Conclusions: Uniformly, most of the participants considered active euthanasia as an at least unacceptable practice, but opinions about LST withdrawal differed significantly between nurses in pediatric/neonatal units, general pediatric wards and adult units. Interestingly, the opinions about nursing role in LST withdrawal decision making differed between the groups, but between the members of the groups, too.

\section{PARENTING IN NICU}

\section{W Wellejus, B Pallesgaard}

\section{Hvidovre Hospital, Copenhagen, Denmark}

Background and Aims: In Denmark the parents are alowed in the NICU 24 hours a day. The staff offer a lot of time, teaching the parents how to nurse their infant meening bathing the infant, changing the diper, taking care of the tubefeeding and many other things. The parents learn all these things very early in the pathway. This means that the infant very ealy are nursed mainly from their parents hands.

Methods: Even very sick infants will be nursed and held by their parents as much as possible. It is very important for us, that the infants as erly as possible are taken out of the incubator to lay on their mothers or fathers chest, so they can share the feeling of oneanothers skin ('kangaru-method'). We do that even with infants in the respirator. In very rare cases, where the infants need to stay in the incubator (eg. with thoraxdrains), we teach the parents how to sit by the incubator with their hands quiet around the infant.

Results: By having the parents in the unit as much as possible, continuity is gained regarding who is touching and handling the infant and the parents are ealy in the infants life, which in many ways is a caotic beginning of life for the sick ore premature born infant. Another good thing is that the milk-production increases, so the mother are able to give her own milk to her own baby.

Conclusions: Many advantages for both infants and parents physically, psychologically and socially.

\section{END OF LIFE CARE IN A PICU: PARENTAL AND STAFF PERSPECTIVES}
A Tagarro, P Dorao, R Tapia, S Moraleda, P López, T Moreno, C Schüffelmann, F Ruza
PICU, La Paz Children's Hospital, Madrid, Spain

Background and Aims: To evaluate the end-of-life care in a PICU from parental and staff point of view, to compare perceptions, and to get information to design proper guidelines.

Methods: Parents of patients died from 2001 to 2003 and staff completed one questionnaire designed to test the information, the environment, the accuracy of care in the moment of death, and bereavement care.

Results: A total of 43 PICU workers (69\%) filled the questionnaire. Finally, 29 questionnaires were obtained from interviews or mail (30\% of possible). Parents judged information better than staff, especially $(p<0,05)$ relating therapy choices, understanding, and prognosis.
Around $80 \%$ of respondents demanded one only spokesman. Near $60 \%$ of respondents thought that visiting time was not sufficient, parents are informed of imminent death for a not familiar doctor, and parents guess that children are going to die before being informed of this possibility. Seventy-six per cent of parents judged as positive to be by their children's side when died versus $35 \%$ of staff $(p<0,05)$. Almost $75 \%$ of staff states that withdrawal of life support is raised not often enough. Seventy-two per cent of staff thinks that psychological support is necessary for parents, vs. one third of parents.

Conclusions: We have got a complete perspective of the end-of-life care we provide. We have identified skills and pitfalls about of information, family role, emotional support for families and staff, and withdrawal of life support. We have tried to use this information to improve by several actions, including the design of a clinical pathway for end-of-life care.

\section{CHILDREN WITH CHRONIC DISEASES: A CHALLENGE TO FAMILY THERAPIES}

\author{
C Pogonidis ${ }^{1}$, E Xenodoxidou ${ }^{2}$, I Christodoulou ${ }^{3}$, \\ C Vaiopoulos ${ }^{1}, \mathrm{~K}_{\text {Godosidis }}{ }^{4}, \mathrm{M}$ Livaditis ${ }^{4}$, \\ T K Konstantinidis ${ }^{5}$ \\ ${ }^{1}$ Xanthi Hospital, Thrace, Greece, ${ }^{2}$ Sismanoglion \\ Hospital, Komotini, Thrace, Greece, ${ }^{3}$ Papanikolaou \\ General Hospital, Thessaloniki, Greece, ${ }^{4}$ Department of \\ Psychiatry, Medical School, Democritus University of \\ Thrace, Greece, ${ }^{5}$ Laboratory of Hygiene and \\ Environment Protection, Democritus University of \\ Thrace, Greece
}

Background and Aims: Aim of this study is to focus in problems arising in families with children suffering from chronic diseases

Methods: In total 17 families were studied in Thrace, Greece. A questionnaire was completed assessing children and parents' everyday problems. A special concern was given to brothers and sisters of children with a chronic disease. We did not ask any questions to the young patients themselves.

Results: In total, 21 healthy children of the study families were asked, with a median age of $10,4 \pm 2,7$ years old. The $90 \%$ accepted that the sickness of their brother/sister had affected dramatically their lives. "Living like - ill" was the referred problem by $77 \%$ of children. The $34 \%$ had been bothered by the special lifestyle determined by numerous Hospital admissions of a family member, while $60 \%$ had noticed a big share of parents' care was always missing. A 5\% stated that they were afraid of getting sick and die. Parents' answers were matching to their children impressions, as they accepted that the psychological impact on their families was crucial for their lives. Behavioral problems $(\sim 50 \%)$, development and learning difficulties (25\%) of the healthy children were reported. All parents accepted that they were incapable to provide equal attention and care to their children.

Conclusions: Family care should be given in families with children suffering from chronic diseases. General Doctors, social workers and Hospital services might be the ideal functional parts of the unsolved puzzle. 


\section{CAN PALLIATIVE CARE OUTREACH OFFER} AN ALTERNATIVE TO PICU?

\section{A P Anderson, F Craig \\ Great Ormond Street Hospital, London, UK}

Background and Aims: Clinicians have a duty not to ask families to consent to a treatment that does not have a realistic chance of recovery. We investigate the scope for a palliative care outreach service and determine if this may help children and families avoid inappropriate PICU admission.

Methods: A retrospective case note review of children, known to the palliative care service, who were referred to PICU, by their local hospital during an acute illness.

Results: Over a two year period, six children, known to the palliative service, between 2-18 months prior to the acute presentation at their local hospital, were identified. Treatment of acute deterioration had been previously discussed with all families. Two had agreed to a 'do not resuscitate order' (DNR). The remainder said they would only make a decision when the time arose. Palliative care service was contacted directly by five local hospitals and in one case by the family. Palliative care provided outreach to all 6 families on the day of request and each case was discussed with PICU. In two cases, admission was felt to be appropriate. The two admitted to PICU were both discharged home within $48 \mathrm{hrs}$. Of the remaining four children: 3 died in their local hospital and one survived their acute episode. At follow no families regretted their decision.

Conclusions: PICU should be offered when there is a realistic chance of a reversible component of the acutely presenting symptom or illness. Involvement of palliative care, as an outreach service, during an acute episode, can prevent inappropriate PICU admissions.

\section{THE OROPHARYNGEAL COLONIZATION OF CHILDREN AT THE ADMISSION IN A PEDIATRIC INTENSIVE CARE UNIT}

M L G Pedreira ${ }^{1}$, M I Gomes ${ }^{1}$, D M Kusahara ${ }^{1}$, M A S Peterlini ${ }^{1}$, M Abramczyk ${ }^{2}$

${ }^{I}$ Department of Nursing, Federal University of São Paulo, São Paulo, Brazil, ${ }^{2}$ Department of Pediatric, Federal University of São Paulo, São Paulo, Brazil

Background and Aims: The oropharyngeal colonization of critical care patients has been described as capable of increasing nosocomial infections. The oropharynx is usually considered the main reservoir of bacterial colonies in the upper airways. The aim of this study was to verify the oropharyngeal colonization of children at the first 24 hours of admission in a PICU.

Methods: Prospective study realized in a University Hospital PICU in the city of São Paulo, Brazil. The study was approved by the ethics research committee. The sample was composed of 32 children. Microbiological cultures were obtained during the first 24 hours after children's admission, and collected swabbing by tonsils and pharyngeal areas. The collected oropharyngeal secretion were inoculated on culture media and incubated according to atmosphere, temperature, time and humidity standards.
Results: The children presented an average of 3.6 years old, $62.5 \%$ were male, and $78.1 \%$ received mechanical ventilation. Pathogens were identified in $12(37.5 \%)$ of the 32 oropharyngeal cultures analyzed. The pathogens identified in this cultures were: Klebsiella pneumoniae (21.4\%), Staphylococcus aureus (21.4\%), Pseudomonas aeruginosa (14.4\%), Enterobacter spp (14.4\%), Escherichia coli (7.1\%), Proteus mirabillis (7.1\%), Acinetobacter baumanii (7.1\%) and Morganella morganii $(7.1 \%)$. Clinical characteristics were analyzed according to the identification of Normal (NF) or Pathogenic Flora (PF): Length of stay in hospital (NF18.0 days; PF26.7 days) and PICU (NF5.7 days; PF8.2 days); elective (NF76.5\%; PF23.5\%) or emergency (NF46.7\% ; PF53.3\%) admission; clinical (NF55.5\%; PF45.5\%) or surgical (NF75.0\%; PF25.0\%) diagnosis.

Conclusions: The main pathogens identified were gram negative and presence of pathogenic flora increased hospital and PICU length of stay. Acknowledgments: Dr. Pedreira acknowledges support for research by grant 04/13361-2-FAPESP.

\section{CLINICAL UTILITY OF THE MULTIDIMENSIONAL ASSESSMENT PAIN SCALE}

\section{A S Ramelet}

School of Nursing and Midwifery, Curtin University of Technology and Nursing Research, Princess Margaret Hospital for Children, Perth, WA, Australia

Background and Aims: Even if an assessment tool has sound psychometric properties, if it is not feasible for use in a clinical setting or does not provide useful clinical information, then it is of little value to clinicians. This study aimed to evaluate the clinical utility of the Multidimensional Assessment Pain Scale (MAPS) for critically ill young preverbal children.

Methods: Usefulness of the MAPS was tested in a convenience sample of 19 children aged between 0 and 31 months who had major surgery at a tertiary referral hospital in Western Australia. The other components of clinical utility, such as ease of use, format and scoring, were evaluated by 17 staff nurses who completed an anonymous 5-point Lykert type scale questionnaire.

Results: MAPS scores decreased significantly by 4 points ( $40 \%$ of total score) after the administration of a potent dose of morphine $(\mathrm{p}<0.001)$. Internal consistency of the MAPS was poor (Cronbach's alpha 0.26), but would increase to 0.80 if the physiologic item was deleted. However, the actual decreases of heart rate, systolic, mean, and diastolic arterial pressure after a potent bolus of morphine $(p<0.001)$ suggested that this item should be modified rather than deleted. The MAPS, as evaluated by 17 nurses ( $57 \%$ response rate), proved to be clinically useful, with a median of 2 (range 4 ) in all components.

Conclusions: This study showed that the MAPS is sensitive to analgesics and clinically useful, yet the item "vital sign" required some amendments. For clinical application, it is recommended to use the revised version of the MAPS. 
COMPLICATION AND OUTCOME IN CHILDREN REQUIRING PEDIATRIC INTENSIVE CARE UNIT FOR STAPHYLOCOCCUS AUREUS PNEUMONIA

G N Feketea, A J Patsoura, A G Eliades, D D Gionis, J H Papadatos

PICU, “P.\&A. Kyriakou” Children's Hospital, Athens, Greece

Background and Aims: Despite advances in antibiotic therapy strategies and in pediatric intensive care, Staphylococcus aureus pneumonia remains a serious infection.

Methods: Treatment, complication and outcome of children with severe Staphylococcus aureus pneumonia were reviewed.

Results: Eight children (age 1-29 months) were identified with $\mathrm{S}$. aureus pneumonia over the 3-year study period ( $1,3 \%$ of all admission). The mean age was 7,25 months (range 1-29), 5/8 children-under 3 months. Two children $(25 \%)$ were presented with CA-MRSA. Reasons for ICU admission were respiratory failure hypoxemia (8/8), convulsions (2/8), and septic shock (3/8). Three children required inotropic support but none patient had endocarditis. Renal failure was not occurred. A coagulopathy was seen in 5 children on presentation. $5 / 8$ children required mechanical ventilation for mean 10,8 days (range 4-26). Despite aggressive treatment with Teicoplanin or Vancomycin (4/8) two children died while $3 / 8$ children had complications requiring prolonged antimicrobial therapy. Teicoplanin or Vancomycin was the initial treatment in $4 / 8$ cases and few hours later in the other $4 / 8$. The decisions were taken when susceptibility results were known or due to poor clinical course. Six children presented pneumothorax and/or pleural empyema witch needed drainage, 5 by thoracocentesis and 1 by thoracotomy.

Conclusions: Initial empirical antibiotic treatment for infant with suspected pneumonia and/or pleural empyema for which S.aureus is a possible pathogen, should include an agent that is active against the CAMRSA. Infants under 3 months old with S.aureus pneumonia need usually PICU. Aggressive drainage of purulent collection or surgical incision and drainage of large empyema is preferable.

\section{DOES CLIMATE CHANGE INFLUENCE BLOOD TSH CONCENTRATIONS IN HEALTHY NEWBORNS?}

C De Felice ${ }^{1}$, L Peruzzi ${ }^{2}$, M Musaro' ${ }^{2}$, L Cecchi ${ }^{3}$, M Morabito $^{3}$, G Latini ${ }^{4,5}$, G Tonni ${ }^{6}$, G Bartolini ${ }^{3}$, G Maracchi ${ }^{7}$, S Orlandini ${ }^{3}$ ${ }^{I}$ Neonatal Intensive Care Unit, Azienda Ospedaliera
Universitaria Senese, Siena, Italy, ${ }^{2}$ Laboratory of
Pediatric Endocrinology, Department of Pediatrics,
Obstetrics, and Reproductive Medicine, University of
Siena, Siena, Italy, ${ }^{3}$ Interdepartmental Centre of
Bioclimatology, University of Florence, Florence, Italy
${ }^{4}$ Clinical Physiology Institute, National Research
Council of Italy (IFC-CNR), Lecce, Italy, ${ }^{5}$ Division of
Neonatology, Perrino Hospital, Brindisi, Italy, ${ }^{6}$ Division
of Obstetrics and Gynaecology, Guastalla Civil Hospital
- AUSL Reggio Emilia, Reggio Emilia, Italy, ${ }^{7}$ Institute of Biometeorology, National Research Council of Italy (IBIMET-CNR), Florence, Italy

Background and Aims: Emerging concern exists about possible health effects of climate change. Summer 2003 is known as the hottest in the last 400 years in Northern Europe. Although a possible effect of high air temperature on TSH levels has been previously reported in experimental models, to date no information exists on this phenomenon in human newborns. We tested the hypothesis that neonatal blood TSH concentrations on day 3 are inversely related with the air temperature, as assessed on a congenital hypothyroidism $(\mathrm{CH})$ mass screening program.

Methods: Whole blood T4 and TSH concentrations on day 3 were evaluated in 32,091 newborns in SouthEastern Tuscany, Italy, sampled from January 1999 to December 2003. Monthly air temperatures for 9 weather stations covering the geographical area of birth were obtained from the regional database. Cases with proven $\mathrm{CH}$ or other known thyroid diseases were excluded from the study. T4 and TSH concentrations on filter paperadsorbed whole blood specimens (DELFIA) were evaluated.

Results: Year 2003 was associated with decreased median TSH concentrations as compared to the preceding years $(\mathrm{p}=0.003)$, and significant relationships between monthly TSH and temperature (minimum: $\mathrm{r}=-$ $0.5438, \quad \mathrm{p}<0.0001$; median: $\mathrm{r}=-0.5066, \mathrm{p}=0.0001$; maximum $\mathrm{r}=-0.5260, \mathrm{p}<0.0001)$ were observed. By contrast, no significant relationship between blood T4 and environmental temperature was evidenced. TSH was found to be thermostable at $47 \mathrm{C}$ for 7 days.

Conclusions: Our findings indicate relationship between high environmental temperature and early low neonatal TSH concentrations. The observation reveals a previously unrecognized, possible direct effect of climate change on human health, whose possible longterm consequences need to be investigated.

\section{FRESH-FROZEN PLASMA AS A SOURCE OF EXOGENOUS IGF-1 TO THE VERY PRETERM INFANT}

I Hansen-Pupp ${ }^{1}$, E Engström², A Niklasson ${ }^{2}$, V Fellman ${ }^{1}$, A Hellström ${ }^{3}$, C Löfqvist ${ }^{2}$, D Ley ${ }^{1}$

${ }^{I}$ Department of Pediatrics, Lund University Hospital, Lund, Sweden, ${ }^{2}$ Department of Pediatrics, Institute of Clinical Sciences, Göteborg, Sweden, ${ }^{3}$ Department of Ophtalmology, Institute of Neuroscience and Physiology, Göteborg, Sweden

Background and Aims: Preterm birth is followed by an immediate decrease in circulatory levels of IGF-1. The aim of the study was to evaluate effect of intravenous administration of fresh frozen plasma (FFP) on levels of IGF-1 in preterm infants.

Methods: FFP from adult donors was administered to 17 infants (mean GA $25.5 \mathrm{wks}$ ) at a median (range) age of 3 (1-29) days. Serum IGF-1 was analyzed in administered FFP, in neonatal blood before and after FFP transfusion, and 6, 12, 24 and $48 \mathrm{~h}$ later. 
Results: FFP was administered with a mean (SD) volume of $10.9(2.0) \mathrm{ml} / \mathrm{kg}$ during 90-240 min. Mean (range) IGF-1 concentration in administered FFP was 127 (77-171) ng/ml. Immediately after FFP administration, mean levels of IGF-1 had increased from 12 to $26 \mathrm{ng} / \mathrm{ml}(\mathrm{p}<0.001)$. Mean levels of IGF-1 remained higher at $6 \mathrm{~h}(16 \mathrm{ng} / \mathrm{ml})(\mathrm{p}=0.003)$ and $12 \mathrm{~h}$ $(16 \mathrm{ng} / \mathrm{ml})(\mathrm{p}=0.012)$ after transfusion, as compared with levels before transfusion. Mean (SEM) levels see fig.

Conclusions: Infusion of plasma in neonates elevates IGF-1 levels to a variable degree during 12 but not 24 hours. The results may serve as a basis for further studies on the role of IGF-1 as a neuro- and vasoprotective agent in preterm infants.

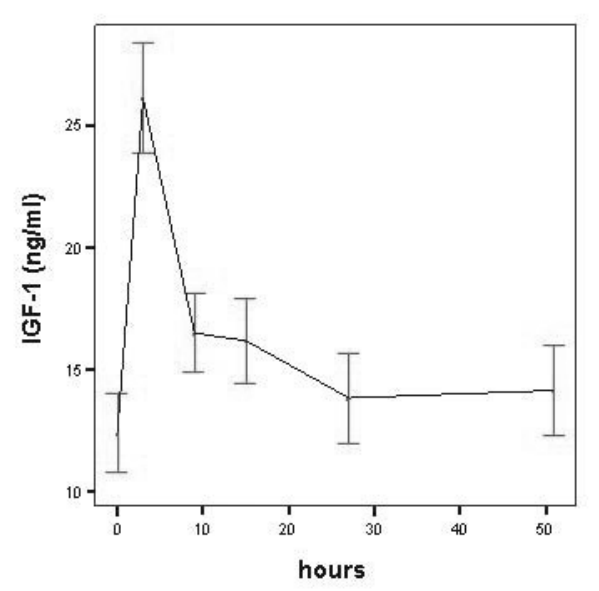

\section{CONTINUOUS GLUCOSE MONITORING IN VERY LOW BIRTHWEIGHT INFANTS}

\author{
I Iglesias Platas ${ }^{1}$, M Thió Lluch ${ }^{1}$, \\ N Pociello Almiñana ${ }^{2}$, A Morillo Palomo ${ }^{1}$, \\ M Iriondo Sanz ${ }^{1}, X_{\text {Krauel Vidal }}{ }^{1}$

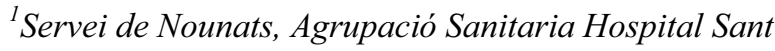 \\ Joan de Déu, Hospital Clínic, Barcelona, Spain \\ ${ }^{2}$ Servei de Pediatría, Hospital Sant Joan de Déu, \\ Barcelona, Spain
}

Background and Aims: Definition of optimal levels of blood glucose in newborns remains controversial. Very Low Birth Weight (VLBW) infants are at a higher risk for disturbances in glucose homeostasis. Hypoglycaemia may worsen neurodevelopmental outcomes, while hyperglycaemia contributes to risk of intraventricular haemorrhage and sepsis. Our aims were to study actual levels of blood glucose in VLBW infants and to validate a method of continuous glucose monitoring in these patients.

Methods: Extremely premature VLBW infants receiving intensive care were recruited within the first 24 hours from delivery. A subcutaneous sensor connected to a Continuous Glucose Monitoring System ${ }^{\circledR}$ (Medtronics) was maintained during 7 days or until dysfunction. Management of patients followed usual standard protocols.

Results: 38 patients (20 male and 18 female) were included over 17 months. Mean gestational age was $27,58 \pm 2,06$ weeks. Mean weight, length and cranial circumference were $962,22 \pm 199,47 \mathrm{~g}, 35,85 \pm 2,90 \mathrm{~cm}$ and $25,24 \pm 1,96 \mathrm{~cm}$. Perinatal history and complications were the usual in extremely premature babies. Continuous monitoring lasted an average of 7,84 $\pm 1,99$ days per patient. Hyperglycemia was detected in 22 $(57,90 \%)$ patients and lasted a mean of $20,33 \pm 30,13$ hours, while 14 (36,8\%) presented with hypoglycaemia, for a mean of $2,45 \pm 2,3$ hours. Some of these values were not detected by intermitent sampling.

Conclusions: Continuous glucose monitoring reveals frequent alterations of glucose levels in VLBW infants, more than expected by intermittent capillary sampling. Further studies should clarify relevance of hyper and hypoglycaemia in short and long term outcomes of premature babies. Real-time continuous glucose monitoring may be useful in management of VLBW infants under intensive care.

\section{INCIDENCE AND ETIOLOGICAL STUDY OF NON-NOSOCOMIAL NEONATAL PNEUMONIA}

\author{
R Lien ${ }^{1}$, H R Huang ${ }^{1}$, P H Yang ${ }^{1}$, T Y Lin ${ }^{2}$, \\ Y C Huang ${ }^{2}$ \\ ${ }^{1}$ Divisions of Neonatology, Department of Pediatrics, \\ Chang Gung Children's Hospital, Chang Gung \\ University, Taoyuan, Taiwan-R.O.C., ${ }^{2}$ Division of \\ Infectious Disease, Department of Pediatrics, Chang \\ Gung Children's Hospital, Chang Gung University, \\ Taoyuan, Taiwan-R.O.C.
}

Background and Aims: There's a lack of clinical information as regard to the incidence and etiology of neonatal pneumonia due to uncertainty of case definitions, rate differences in patient populations, and ascertainment bias. To establish the incidence and investigate the common infectious etiologies of nonnosocomial neonatal pneumonia in northern Taiwan. Methods: This is a retrospective study by reviewing medical records from 2000 to 2005. Those who had the diagnosis of pneumonia with disease onset prior to or upon admission, or no later than the first 96 hours of hospital stay, who also had simultaneous laboratory studies for infectious microorganism and changes on the chest $\mathrm{x}$-ray, were included in the study. They were divided into early and late onset pneumonia, indicating pneumonia in infants younger and older than 48 hours of age.

Results: The total admission to NICU was 11000 for the study period. There were 223 non-nosocomial pneumonia (2.0\% of NICU admissions), 35 of them early and 188 late. In the early pneumonia, they were all of bacterial origin, including Gr. B streptococcus (GBS) (16), Staph. Aureus (5), E. coli (3), Listeria (3). In the late pneumonia, there were 123 by RSV, 16 by Chlamydia, and 49 (26\%) by bacteria: Non-typable H. influenzae (12), E.coli (12), Staph. Aureus (10), and other pathogens (15).

Conclusions: The results of this study serve as valuable guides for selecting antimicrobial agents for nonnosocomial neonatal pneumonia. All the early and $26 \%$ of the late onset neonatal pneumonia are caused by bacteria, in which GBS and nontypable H. Influenzae are the leading causes. 
PERMANENT HISTOPATHOLOGICAL CHANGES ON THE HEART AFTER NEONATAL DEXAMETHASONE TREATMENT. STUDIES IN 4, 8 AND 50-WEEK OLD WISTAR RATS

W B de Vries ${ }^{1}, \mathrm{M} \mathrm{P} \mathrm{Bal}^{1,2,3}$, P Homoet-van der Kraak ${ }^{4}$, P Steendijk ${ }^{2}$, F R Van Der Leij ${ }^{3}$, J Baan ${ }^{2}$, M F M Van Oosterhout ${ }^{4}, \mathrm{~F}$ van Bel ${ }^{1}$

${ }^{I}$ Department of Neonatology, University Medical Center Utrecht, Utrecht, The Netherlands, ${ }^{2}$ Department of Cardiology, Leiden University Medical Center, Leiden, The Netherlands, ${ }^{3}$ Department of Pediatrics, University of Groningen, Groningen, The Netherlands

${ }^{4}$ Department of Pathology, University Medical Center Utrecht, Utrecht, The Netherlands

Background and Aims: Despite beneficial effects of neonatal Dexamethasone (Dex) in preterm infants with chronic lung disease on the short term, there are multiple side-effects such as transient myocardial hypertrophy and hypertension. We reported a temporary suppression of cardiomyocyte proliferation in neonatal rats after neonatal Dex, possibly inducing persistent alterations in cardiac morphology (Basic Res Cardiol 2006). Our aim was to perform histopathological investigations after neonatal Dex in the juvenile and adult rat heart.

Methods: Rat pups received Dex on day 1, 2 and 3 of life $(0.5,0.3$ and $0.1 \mathrm{ug} / \mathrm{g}$ ip). Control pups received saline (Sal). At 4, 8 and 50 week of life the rats were sacrificed ( $\mathrm{n}=8 /$ group). Anatomic data were collected and heart tissue sections were stained with HE, Cadherin-PAS and Sirius Red for cardiomyocyte morphometry and collagen determination.

Results: Cardiomyocyte length of the Dex-treated rats was significantly increased compared to control in all three age groups. In the 8- and 50-week-old Dex-treated rats this was associated with an increased cross sectional area and volume, indicating cellular hypertrophy. Collagen content increased with age and was higher in the Dex groups (only significant at 50 weeks).

Conclusions: Neonatal Dex affects normal growth of the heart resulting in hypertrophy of cardiomyocytes and increased collagen deposition in the adult rat heart. Previous studies in rats have shown premature death and suggested cardiac failure. Therefore cardiovascular follow-up for preterm infants treated with glucocorticosteroids is mandatory.

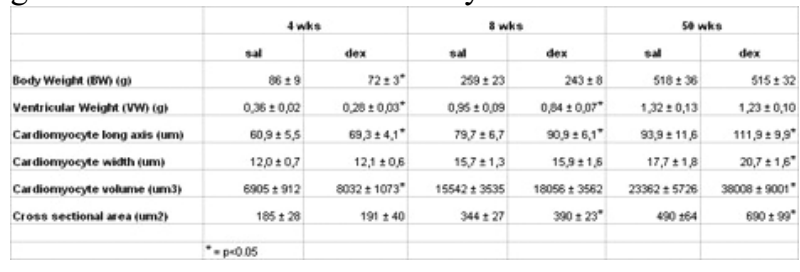

\section{MELATONIN AND RETARDATION OF CNS DEVELOPMENT IN IUGR NEWBORN INFANTS}

\section{Evsyukova}

Department of Physiology and Pathology of Newborn of D.O. Ott Research Institute of Obstetrics and

Gynecology RAMS, St. Petersburg, Russia
Background and Aims: The aim of this study is to assess the role of melatonin (MT) in pathogenesis of retardation CNS development and perinatal pathology in IUGR newborn infants.

Methods: Clinical and laboratory investigations with electropoligraphic characteristics of the cycle organization of sleep were performed in 112 IUGR and 63 normal full term neonates (control group). Expression of MT in platelets and their aggregation activity were investigated. Excretion of the main MT metabolite 6-sulfatoxymelatonin (6SMT) in diurnal $(09.00$ to $21.00 \mathrm{~h})$ and nocturnal urine $(21.00$ to $09.00 \mathrm{~h})$ in $1,4,10$ days of life were determined.

Results: The results showed that the excretion of 6SMT was significantly lower in IUGR neonates in diurnal and nocturnal urine $(4,5 \pm 1,0 \mathrm{ng} / \mathrm{ml}$ and $3,6 \pm 1,1 \mathrm{ng} / \mathrm{ml})$ than in the control group infants $(14,1 \pm 2,2 \mathrm{ng} / \mathrm{ml}$ and $7,7 \pm 1,7$ $\mathrm{ng} / \mathrm{ml}$ respectively) during first 10 days of life. Platelet's expression of MT was lowed and their aggregation activity was increased in IUGR neonates which had a 24 week delay in the formation of passive, active tonuses and an inborn reflexes. It combined with the nondifferentiated state in the sleep structure and correlated with functional disturbances of CNS, hemodynamic disorders and microcirculation in lungs. Retardation of psychomotor development took place by the end of the first year of life.

Conclusions: MT as the neurotransmitter and endogenous antioxidant plays an important role in the adaptation to hypoxia and development CNS function in early ontogenesis. It may potentially be useful for restore and development CNS function in IUGR neonates.

\section{CONTINUOUS COMPUTER ANALYSIS OF SPO2 AND ARTERIAL BLOOD GASES IN VERY LOW BIRTH WEIGHT INFANTS (VLBWI) WITH PDA}

P Kruczek ${ }^{1}$, P Stoch ${ }^{3}$, A Rudzinski ${ }^{2}$, P Kwinta ${ }^{1}$, Z Kordon $^{2}$, W Wajs ${ }^{3}$, J J Pietrzyk ${ }^{1}$

${ }^{I}$ Department of Pediatrics, Collegium Medicum, Jagiellonian University, Krakow, Poland, ${ }^{2}$ Department of Pediatric Cardiology, Collegium Medicum, Jagiellonian University, Krakow, Poland, ${ }^{3}$ Department of Automatics, Institute of Metallurgy and Mining,

Krakow, Poland

Background and Aims: Respiratory distress syndrome (RDS) - severe consequence of prematurity leads to further complications including persistent ductus arteriosus (PDA). The goal of the study is to test hypothesis that severity of RDS increases risk of PDA in VLBWI.

Methods: 51 VLBWI admitted to Neonatal Intensive Care Unit till the second day of life between January 2004 and October 2005 were included into study group. Blood gases were checked on admission and in day 3, pulsoximetry saturations ( $\mathrm{SpO} 2)$ were continuously recorded during first week of hospitalization. Chest Xray (CXR) was taken on admission, the severity of RDS was graded (scale 0-4). Echocardiographic evaluation of hemodynamic significance of PDA was performed in day 1 and 7, the study group was divided into PDA (-) 
and $\mathrm{PDA}(+) . \mathrm{SpO} 2, \mathrm{FiO} 2 / \mathrm{pO} 2, \mathrm{pH}$ and severity of RDS in CXR were compared in both groups.

Results: Results are shown in the table.

Conclusions: PDA $(+)$ patients revealed higher grade of RDS in CXR, lower: pO2/FiO2 in day 1 and $\mathrm{pH}$ in the day 3. They also had longer and deeper episodes of desaturations. The severity of RDS increases risk of PDA in VLBWI.

\begin{tabular}{|c|c|c|c|c|c|}
\hline \multirow[t]{2}{*}{ Parameter } & \multicolumn{2}{|c|}{ PDA (-) } & \multicolumn{2}{|c|}{ PDA (+) } & \multirow{2}{*}{$\begin{array}{c}p \\
\text { value }\end{array}$} \\
\hline & Mean & $95 \mathrm{Cl}$ & Mean & $95 \mathrm{Cl}$ & \\
\hline $\mathrm{pH}$ day 3 & 7.32 & $7.30-7.35$ & 7.25 & $7.20-7.30$ & 0.002 \\
\hline $\begin{array}{l}\mathrm{pO}_{2} / \mathrm{FiO}_{2} \text { on admission } \\
{[\mathrm{mm} / \mathrm{Hg}]}\end{array}$ & 216 & $163-268$ & 142 & 83-201 & 0.05 \\
\hline RDS grade on chest $X$-ray & $\begin{array}{c}1,3 \text { Median } \\
1\end{array}$ & $0.8-1.9$ & $\begin{array}{c}2.6 \text { Median } \\
3\end{array}$ & $2.0-3.2$ & 0.003 \\
\hline Area $\mathrm{SpO}_{2}<90 \%$ & 54230 & $\begin{array}{l}34450- \\
74010 \\
\end{array}$ & 84881 & $\begin{array}{l}56658- \\
113104 \\
\end{array}$ & 0.05 \\
\hline $\begin{array}{l}\text { \% of recording time with } \\
\mathrm{SpO}_{2}<85 \%\end{array}$ & 1.0 & $0.66-1.40$ & 1.56 & $1.03-2.09$ & 0.05 \\
\hline
\end{tabular}

\section{VOLUME OF ACTIVITY AND NURSES' STAFFING LEVEL IN NEONATAL INTENSIVE CARE UNITS. RESULTS OF A REPEATED MEASURE CROSS SECTIONAL STUDY IN ITALY}

\section{Corchia, S M Orlando}

Department of Medical and Surgical Neonatology, Neonatal Intensive Care Unit, Bambino Gesù Children's Hospital, Rome, Italy

Background and Aims: Volume of activity and staff workload in Neonatal Intensive Care Units (NICUs) could be relevant to the quality of care and outcome of high risk infants. The aims of our study were to describe the distribution of some variables reflecting the volume of activity of Italian NICUs, and to investigate the relationship between these variables and the staffing level.

Methods: One hundred and four NICUs participated in a nationwide investigation from May to December 2005. The following data were collected monthly at specified points in time, representing both weekdays and weekends, and including all possible nursing shifts: Number of ventilated or NCPAP-cared infants (intensive patients, IPs), Number of nurses in the intensive care section (ICN) of each NICU, and Infant to Staff ratio (ISr), calculated dividing IPs by ICN. Medians and Interquartile ranges (IQR) of each variable were calculated, and their distribution and possible variations over time were assessed.

Results: The distribution of the relevant variables did not vary significantly over time; therefore pooled data were used for further evaluation. Medians (IQR) were: 2 (1.12-3.31) for IPs; 2 (1.50-2.69) for ICN; and 1 (0.651.50) for ISr. The mean values of ISr were $0.44,0.92$, 1.26 , and 1.74, respectively, from the lowest to the highest quartile of IPs' distribution $(\mathrm{p}<0.05$, Bonferroni test).

Conclusions: In Italian NICUs the Infant to Staff ratio is less favourable when the volume of activity is high, suggesting an inefficient distribution of human resources.

\section{BLOOD PRESSURE (BP) WAVES ARE DUE TO NORADRENALINE SURGES IN NEWBORN INFANTS}

B Wefers, S Cunningham, J Quinn, N McIntosh

Department of Neonatology, University of Edinburgh, Edinburgh, UK

Background and Aims: Maintenance of BP within a 'normal range' is important for perfusion of vital organs and avoidance of perfusion related insults to the neonatal brain. Hypertensive neonatal BP waves have been described by our research team and have been linked to more severe neonatal illness. An increased risk of cerebral pathology in these infants has been postulated. However the mechanism behind these waves remained unclear (Cunningham et.al., 1993). We investigated plasma catecholamine levels when hypertensive BP waves were observed.

Methods: Trained clinical staff notified the investigator when hypertensive BP waves were seen on the computerised trend monitoring system (CPTM). Blood samples were taken at the peak and trough of BP waves from the indwelling arterial line used to monitor the infant's BP. Catecholamine levels were analysed using High Performance Liquid Chromatography with dual electrode coulochem detection. Mean BP recorded by CPTM at the time of sampling was correlated to plasma adrenaline, noradrenaline and dopamine levels using the Wilcoxon signed ranked test.

Results: In 5 infants ( 7 paired samples) the mean BP and noradrenaline levels were significantly correlated $(\mathrm{p}=0.021)$, while mean BP and dopamine levels were not $(\mathrm{p}=0.487)$. Adrenaline was mainly undetectable. The association between plasma noradrenaline levels and mean BP was independent of absolute plasma noradrenaline levels and concurrent therapeutic dopamine infusion (2 infants).

Conclusions: Hypertensive BP waves in neonates are induced by a surge in plasma noradrenaline; the reason for this surge has not been elicited.

\section{LONG TERM PHYSICAL EFFECTS IN SURVIVORS OF PEDIATRIC INTENSIVE CARE}

\author{
H Knoester ${ }^{1}$, M B Bronner ${ }^{2}$, A P Bos ${ }^{1}$ \\ ${ }^{1}$ Pediatric Intensive Care Unit, Emma Children's \\ Hospital, Academic Medical Center, Amsterdam, The \\ Netherlands, ${ }^{2}$ Psychosocial Department, Emma \\ Children's Hospital, Academic Medical Center, \\ Amsterdam, The Netherlands
}

Background and Aims: The development of pediatric intensive care (IC) has contributed to improved survival in critically ill children. Little is known about long term physical sequelae in pediatric IC survivors, but they might resemble the findings in adult IC survivors. Awareness of sequelae due to the original illness and its treatment may result in changes in management during the acute phase. The purpose of this study was to evaluate physical sequelae of survivors of Pediatric Intensive Care Units (PICU).

Methods: From December 2002 through October 2005 all acute admissions to our PICU, were examined in the 
out-patient follow-up clinic, three months after discharge. A pediatric intensivist evaluated physical sequelae by standardized history taking and physical examination.

Results: 116 of 250 eligible patients were evaluated. Patient characteristics, PIM II score, length of stay and length of mechanical ventilation did not differ statistically significant between participants and nonparticipants. 55 patients suffered a chronic illness after discharge. Ten patients complained of concentration disturbances, 17 of behavioral problems, and 10 of sleeping problems. Nine of 40 school-age children had problems at school. Neurological abnormalities were seen in 24 children, post thrombotic syndrome after a central venous catheter in 3 and hoarseness after extubation in 3 children.

Conclusions: PICU survivors have various, but substantial physical sequelae three months after discharge. Almost $50 \%$ of patients have a chronic illness and almost $20 \%$ of patients have neurological problems. Further research is warranted to determine risk factors and methods to prevent sequelae due to intensive care treatment.

\section{SHOULD WE CONTINUE CARE AFTER 1 MONTH STAY IN THE ICU?}

S Naghib ${ }^{1,2}$, C Van Der Starre ${ }^{1}$, E Van Der Voort ${ }^{2}$, S Gischler ${ }^{1}$, K F M Joosten ${ }^{2}$, D Tibboel ${ }^{1}$

${ }^{I}$ Department of Pediatric Surgery, Erasmus MC-Sophia Children's Hospital, Rotterdam, The Netherlands

${ }^{2}$ Department of Pediatrics, Erasmus MC-Sophia

Children's Hospital, Rotterdam, The Netherlands

Background and Aims: Overall mortality in PICU is 4$7 \%$, but the mortality in the group children with a length of stay of more than 1 month ( $>4$ times median duration of stay) might be much higher.

Methods: Children up to the age of 18 who fulfilled the criteria of prolonged stay ( $>28$ days) were included in our University children's hospital and multidisciplinary intensive care unit. The design was a retrospective analysis over a 3-year interval (2003-2005).

Results: A total of 120 children (equal gender) had a prolonged stay. Mean length of stay was 82 days (range 29-500). The mean age was 2 years (range $0-18$ years). Main underlying diagnoses were congenital: heart diseases and gastrointestinal diseases and acquired and congenital malformations of the airway with a total of $48 \%$ of all admission days. Thirty two children died (26\%), mean hospital stay until death 75 days (range 30220). Among the nonsurvivers, 5 of 32 children were withhold from life support. In 15 of 32 cases the terminal event preceding death was withdrawal of lifesustaining treatment.

Conclusions: Children with prolonged stay at the ICU have a higher unexpected incidence of mortality. A multidisciplinary management approach of the care and cure for these children is needed to reach maximum opportunities. We suggest the institution of multidisciplinary staff meetings after a prolonged stay of 28 days, which are automatically announced by our patient data management system in order to reach better medical insight and to determine guidelines and boundaries of future treatment modalities.

\section{ORGAN DONATION FROM A PEDIATRIC INTENSIVE CARE UNIT IN GREECE}

\author{
F C Nikolaou, K G Papazoglou, J H Papadatos
}

Intensive Care Unit, Children's Hospital 'P\&A. Kyriakou', Athens, Greece

Background and Aims: Current demand for organs far surpasses availability all over the world, waiting lists are long and many people die before the organ they desperately need becomes available. The purpose of this paper is to estimate the number of potential donors and the organ donation/transplantation rate in the pediatric population.

Methods: All deaths in the PICU at the Children Hospital "P \& A Kyriakou" during the last ten years were checked for brain death (BD). These patients were valued as possible organ and tissue donors. The number of donors and the causes for not donation were estimated.

Results: During the study period 191 children died, of whom $101(52.8 \%)$ were confirmed as brain stem death. The main cause of $\mathrm{BD}$ was the traumatic brain injury (TBI) $(39.6 \%)$. Potential organ donors were 47 (46.5\%) and $23(22.8 \%)$ were potential tissue donors. Medical contraindications to organ donation had 31 (30.7\%). Donors according to update criteria became 11 children $(15.7 \%)$. The main causes of no-donation were: (a) death during the first day of admission in PICU (11.4\%) (b) family's refusal (14.5\%) (unfamiliar idea, religious causes) (c) judicial reasons (5.7\%) (child abuse) (d) inability in approach the donor family (53\%) (14 organ donors and all tissue donors).

Conclusions: Children with TBI are the most frequently BD potential donors. The organ donation rate could increase with the reduction of losses due to donor management (further work in medical stuff), reduction the family's refusal (education in school and community, publicity) and increase the family's approach.

\section{LONG TERM PSYCHOLOGICAL EFFECTS IN SURVIVORS OF PEDIATRIC INTENSIVE CARE}

M B Bronner ${ }^{1}$, H Knoester ${ }^{2}$, A P Bos ${ }^{2}$, M A Grootenhuis ${ }^{1}$, B F Last ${ }^{1}$

${ }^{I}$ Department of Psychosocial, Emma Children's Hospital AMC, Amsterdam, The Netherlands, ${ }^{2}$ Department of Pediatric Intensive Care Unit, Emma Children's Hospital AMC, Amsterdam, The Netherlands

Background and Aims: Admission to a Pediatric Intensive Care Unit (PICU) is a stressful event associated with both psychological and physical long term consequences. Posttraumatic Stress Disorder (PTSD) has been frequently mentioned as a psychological consequence. The purpose of this study was to examine the prevalence of PTSD in families after an acute PICU admission and to determine the 
contribution of different medical risk factors to the development of PTSD.

Methods: From December 2002 through October 2005 we did follow-up research in the Emma's children's hospital, Amsterdam. Families filled out PTSD questionnaires three months after discharge. A total of 143 families participated in this study with 27 children older than 8 years, 140 mothers and 107 fathers.

Results: Data-analysis showed that in total 9 (33.3\%) of the 27 children had scores in the clinical range (scores $\geq 38$ ) meaning further treatment is indicated. From these 9 children $3(11.1 \%)$ met the criteria for severe PTSD (scores $\geq 47$ ). For parents, the complete triad of PTSD clusters could be diagnosed in $15.0 \%(n=21)$ of the mothers and $9.3 \%(\mathrm{n}=10)$ of the fathers. In 6 families PTSD was diagnosed in both parents. Furthermore the analysis showed no associations between the PTSD scores in families and the medical risk factors.

Conclusions: An unexpected admission to a PICU is a stressful event associated with a heightened risk for developing PTSD in families. These results underline the importance of systematic psychological follow-up research in the PICU. Further research is necessary to identify risk factors for PTSD in families.

\section{PREDICTORS OF SURVIVAL IN INFANTS TRANSFERRED FOR CONSIDERATION OF EXTRACORPOREAL LIFE SUPPORT (ECLS)}

L Mills ${ }^{1}$, S Rodger ${ }^{1}$, M Liddell ${ }^{2}$, J Simpson ${ }^{2}, \mathrm{C}_{\text {Davis }}{ }^{2}$, C Skeoch $^{1}$, L Jackson ${ }^{1}$

${ }^{1}$ West of Scotland Neonatal Transport Service, Royal Hospital for Sick Children, Glasgow, UK, ${ }^{2}$ Extra Corporeal Life Support Service, Royal Hospital for Sick Children, Glasgow, UK

Background and Aims: ECLS is an established therapy for neonates with a high risk of death from respiratory failure and is available in $4 \mathrm{UK}$ centres. No standardised criteria for initiating ECLS transfer exist although the oxygenation index (OI) has been used historically. Aim: To review factors influencing the mortality of infants transferred to Glasgow as potential candidates for ECLS.

Methods: A retrospective case note review incorporating the West of Scotland Neonatal Transport Database. Factors influencing survival were identified using bi-variate correlation. Binary logistic regression was used to identify independent determinants of survival. SPSS $®$ was used for all analyses.

Results: Fifty-two infants were referred to the Glasgow ECLS between March 2003 and March 2006, 49 of whom were subsequently deemed suitable for transfer. No deaths occurred during transfer. Referral diagnoses included Meconium Aspiration Syndrome $(n=16)$, Persistent Pulmonary Hypertension of the Newborn $(\mathrm{n}=17)$, Septicaemia $(\mathrm{n}=4)$, and Congenital Diaphragmatic Hernia $(n=7)$. Thirty infants $(61 \%)$ subsequently received ECLS. Of the 49 infants transferred $86 \% \quad(n=42)$ survived to discharge. No clinical or laboratory factors were identified that significantly predicted survival. In particular, indices of oxygenation at the time of referral $(\mathrm{OI} \mathrm{r}=0.04, \mathrm{p}=0.76$; FiO2 $\mathrm{r}=0.10, \mathrm{p}=0.94)$, base hospital blood gas parameters and arrival blood gas parameters did not predict survival.

Conclusions: Numerical predictors of illness severity, such as the OI, are poor determinants of survival following ECLS. The condition of the infant, based upon clinical experience, must remain the best indicator of need for transfer.

\section{END-OF-LIFE DECISIONS AND CONCOMITTANT DRUG USE IN THE NEONATAL INTENSIVE CARE UNIT OF THE ERASMUS MC-SOPHIA CHILDREN'S HOSPITAL}

M Williams, R F Kornelisse, B J Smit, J B Van Goudoever ${ }^{1}$

Department of Pediatrics, Erasmus MC - Sophia Childrens Hospital, Rotterdam, The Netherlands

Background and Aims: Many neonatologists in European countries set limits to intensive care treatment (Cuttini, 2000). In Belgium and The Netherlands approximately $60 \%$ of all deaths are preceded by an end-of-life decision (Provoost 2005 and Vrakking 2005). The end-of-life decision is sometimes followed by the use of drugs to alleviate pain and discomfort. A retrospective and prospective study to get more insight into reasons for withdrawal and medication prescribed was performed.

Methods: Many neonatologists in European countries set limits to intensive care treatment (Cuttini, 2000). In Belgium and The Netherlands approximately $60 \%$ of all deaths are preceded by an end-of-life decision (Provoost 2005 and Vrakking 2005). The end-of-life decision is sometimes followed by the use of drugs to alleviate pain and discomfort. A retrospective and prospective study to get more insight into reasons for withdrawal and medication prescribed was performed.

Results: The proportion of neonatal deaths (2002-2004) that were preceded by end-of-life decisions was 61/119 $(51 \%)$. Drugs to alleviate pain and discomfort were used but most patient files did not give exact information about medication dosage or its clinical effect. In the prospective study of 2005 (Jan-Sept) 42 infants died, in $26(61 \%)$ an end-of-life decision was made. 22/26 $(85 \%)$ patients received drugs to in the period after withdrawal of treatment.

Conclusions: The practice of end-of-life decisions in neonates is stable. Drugs seem to be used more frequently in 2005, which might be caused by improved registration or an increase in use because of an altered attitude towards suffering around death.

\section{EFFICACY OF EUTECTIC MIXTURE OF LOCAL ANESTHETICS IN REDUCTION OF VENIPUNCTURE PAIN IN CHILDREN}

\author{
E Stefanaki, G N Konstantinou, C Petraki, \\ A Tsilimigaki-Christaki, T Tsekoura
}

Department of Second Pediatric, Venizelio General Hospital, Heraklio, Crete, Greece 
Background and Aims: Acute pain caused by common minor procedures, such as venipuncture, can be a major source of distress for hospitalized children. Our objective is to explore the efficacy of a mixture of lidocaine and prilocaine cream [Eutectic mixture of local anesthetics (EMLA)] and of other social parameters such as educational status in reducing pain during venipuncture.

Methods: 97 children aged from 3 to 14 years old, who were hospitalized and required venipuncture, were enrolled in a randomized, single blind placebo controlled trial. They were randomized in two-treatment groups 1) EMLA cream, 2) placebo cream. Either EMLA or placebo cream were applied 60 minutes before the procedure. Children rated the pain using the revised Wong- Baker Faces pain scale with scores ranging from 0 to 10 .

Results: Age was found to be high correlated with pain score (p-value $<0,001$ ), which was also found to be statistically significant higher in children in the placebo group $(p<0,001)$. Children accompanied by mothers with university education experienced less pain than those with mothers of elementary education $(\mathrm{p}=0,022)$. Finally it is interesting that $10,8 \%$ of the children of the EMLA group reported pain scores $>8$ while $32,6 \%$ of the children of the placebo group reported pain scores $<2$.

Conclusions: EMLA is effective in minimizing procedural pain during venipuncture with better results in older children who have mothers with university education. However the final outcome is affected by various individual factors and it is difficult to be predicted in advance.

\section{A CASE OF ACCIDENTAL STAGGERED PARACETAMOL OVERDOSE}

K K Z Aabideen, L S Moulton, M Loscertales, J Sills

Department of Paediatrics, Whiston Hospital,

Liverpool, UK

Background and Aims: Liquid paracetemol formulations are available in $120 \mathrm{mg} / 5 \mathrm{ml}$ dosages over the counter in UK. With their common usage in children, parents assume that doses abroad are the same. This can lead to accidental overdose with therapeutic intent.

Methods: Case Report.

Results: A 14 months old boy was admitted with threeday history of vomiting and diarrhoea. On closer questioning the parents reported that during their holiday in Spain one week previously, Spanish hospital prescribed paracetamol (fig1) containing $100 \mathrm{mg} / \mathrm{ml}$ to control the pyrexia. As the written instruction and the explanation of the dosage were in Spanish, the parents did not understand clearly. They had therefore been giving $5 \mathrm{ml}$ of Spanish concentrated paracetamol assuming that it contained only $120 \mathrm{mg}$. This child weighed $10.4 \mathrm{Kg}$; therefore he was receiving nearly $300 \mathrm{mg} / \mathrm{Kg} /$ day of Paracetamol. He was treated with Nacetylcysteine, as advised by the National Poisons Information Service (NPIS).

Conclusions: Accidental overdoses have been reported with 'therapeutic intent'1, 2. This case highlights the importance of taking a full drug history in every patient including not only how much has been prescribed, but how much has actually been taken. In a society where holidays abroad are very common, this also highlights the need for good communication between the doctor and patient especially if it is in foreign language.

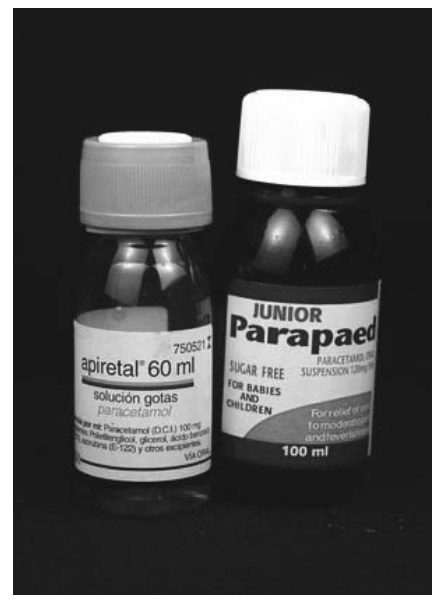

\section{AUDITORY EVENT-RELATED POTENTIALS IN PRETERM CHILDREN AT FIVE YEARS OF AGE}

K Mikkola $^{1}$, E Kushnerenko ${ }^{2,3}$, E Partanen ${ }^{2}$, S Serenius-Sirve $^{1}$, J Leipälä ${ }^{1,4}$, M Huotilainen $^{5,2}$, V Fellman ${ }^{1,6}$

${ }^{1}$ Hospital for Children and Adolescents, University of Helsinki, Helsinki, Finland, ${ }^{2}$ Cognitive Brain Research Unit, Department of Psychology, University of Helsinki, Helsinki, Finland, ${ }^{3}$ Centre for Brain and Cognitive Development, School of Psychology, Birkberg College, London, UK, ${ }^{4}$ Department of Pediatrics, University of Kuopio, Kuopio, Finland, ${ }^{5}$ Helsinki Collegium for Advanced Studies, University of Helsinki, Helsinki, Finland, ${ }^{6}$ Department of Pediatrics, University of Lund, Lund, Sweden

Background and Aims: Atypical auditory event-related potentials (AERPs) of preterm infants correlated with 2year development (Fellman et al. Pediatr Res.2004;56:291-7). We compared the 5-year AERPs and neurocognition to infant AERPs.

Methods: Preterm small (SGA, $\mathrm{n}=13$; birthweight $844 \pm 287 \mathrm{~g}, \quad 28.1 \pm 2.3$ gestational weeks, gw) and appropriate for gestational age (AGA, $\mathrm{n}=14 ; 1002 \pm 236$ $\mathrm{g}, 27.0 \pm 1.9 \mathrm{gw})$, and control $(\mathrm{n}=13 ; 3856 \pm 466 \mathrm{~g}$, $40.3 \pm 1.6 \mathrm{gw}$ ) children were assessed at $0,6,12$ months, and 5 years with a frequency odd-ball using a standard 100 -ms harmonic tone of $500-\mathrm{Hz}$, and a deviant tone of $750-\mathrm{Hz}$ of fundamental frequency. At age five novel sounds representing environmental noises were added. The full-scale intelligence quotient (FS-IQ) of the preterm children was assessed (WPPSI-R).

Results: The FS-IQs (mean \pm SD) were $97 \pm 24$ (SGA) and $98 \pm 16$ (AGA). Positive peak amplitude at $100 \mathrm{~ms}$ both to frequency and novel deviants were smaller in SGA than in controls $(\mathrm{P}<0.001$ and $\mathrm{P}<0.04)$, and tended to be smaller than in AGA $(\mathrm{P}<0.07$ and $\mathrm{P}<0.06)$. Negative peak at $250 \mathrm{~ms}$ to novel sound tended to be more negative in SGA than in AGA $(\mathrm{P}<0.08)$. Negative peak at $250 \mathrm{~ms}$ correlated inversely $(\mathrm{P}<0.05)$ with FS-IQ 
to frequency deviant in electrodes $\mathrm{Cz}(\mathrm{r}=-0,51)$ and $\mathrm{Pz}$ $(\mathrm{r}=-0.45)$ and to novel sound in $\mathrm{C} 3, \mathrm{C} 4$, and $\mathrm{Pz}(\mathrm{r}=-0.43$ to -0.44). Preterm had smaller amplitude responses than control children to standard stimuli and frequency deviants at latency windows 150-250 and 250-350 ms at different study ages.

Conclusions: AERP abnormalities may suggest different pattern of brain maturation in preterm children.

\section{RELATIONSHIPS OF DEVELOPMENT FUNCTIONS AND TEMPERAMENTS IN CHILDREN WITH CEREBRAL PALSY}

C L Chen ${ }^{1}$, C H Chen ${ }^{1}, \mathrm{C}$ Y Wu ${ }^{2}$, W Y Liu ${ }^{1}$, A M K Wong ${ }^{1}$, S W Chou ${ }^{1}$, S F T Tang ${ }^{1}$, C Y Chung ${ }^{1}$

${ }^{l}$ Department of Physical Medicine and Rehabilitation, Chang Gung Memorial and Children Hospital, Chang Gung University, Tao-Yuan, Taiwan, ${ }^{2}$ Department of Occupational Therapy, Chang Gung University, TaoYuan, Taiwan

Background and Aims: Children with cerebral palsy (CP) had varieties in the temperaments and developmental functions. This study investigated the relationships of developmental functions and temperaments in children with $\mathrm{CP}$.

Methods: We collected 126 children with spastic CP and 66 children with normal-development children, aged 3-6 years. Children with $\mathrm{CP}$ were divided into 3 groups: Hemiplegia $(n=21)$, diplegia $(n=60)$, and quadriplegia $(\mathrm{n}=45)$. All children completed Chinese Children Temperamental Inventory (CCTI) $(9$ temperament dimensions), and Chinese Children Developmental Inventory (CCDI) (8 functional domains) assessments. Children with $\mathrm{CP}$ also received assessments of $\mathrm{CP}$ classification and Gross Motor Functional Classification System (GMFCS). The one way ANOVA and correlation analysis were used for statistical analysis. It was considered as statistically significant if $\mathrm{P}<0.05$.

Results: Children with CP showed lesser approach and persistence, lower adaptability, and higher responsive threshold than control group $(p<0.05)$. And children with spastic quadriplegia showed lower adaptability and lesser persistence than $\mathrm{CP}$ with diplegia or hemiplegia $(\mathrm{p}<0.05)$. Correlation analysis showed development quotients (DQs) of all developmental functions were highly correlated with GMFCS scales and CP classifications $(p<0.01)$. The DQs of most developmental functions were positively correlated with adaptability and persistence $(\mathrm{p}<0.05)$, and were negatively correlated with responsive threshold $(\mathrm{p}<0.05)$.

Conclusions: Some temperaments varied in children with $\mathrm{CP}$ having different classifications. The CP severity and classification played dominant roles in determining all developmental functions in children with CP. The temperaments, particularly adaptability, persistence, and responsive threshold, were also related to most developmental functions.

\section{LONG-TERM NEUROFUNCTIONAL DISTURBANCES AFTER SEIZURES: A POSSIBLE IMPLICATION OF NITRIC OXIDE}

I Papadopoulou ${ }^{1}, \mathrm{E} \mathrm{Spandou}^{1}, \mathrm{C} \mathrm{Simeonidou}^{1}$, T Lourbopoulos ${ }^{3}$, A Giannakopoulou ${ }^{3}$, N Grigoriadis ${ }^{3}$, I Liangouris $^{1}$, G Karkavelas ${ }^{2}$, O Guiba-Tziampiri ${ }^{1}$

${ }^{I}$ Department of Physiology, Medical School, Aristotle University of Thessaloniki, Thessaloniki, Greece

${ }^{2}$ Department of Pathology, Medical School, Aristotle University of Thessaloniki, Thessaloniki, Greece

${ }^{3}$ Laboratory of Experimental Neurology and Neuroimmunology, Department of Neurology B', AHEPA Hospital, Thessaloniki, Greece

Background and Aims: Prolonged seizures during development may cause permanent dysfunction of neurons and mossy fiber sprouting, resulting to longterm cognitive dysfunction. Inhibition of NO production is suggested to result in both neuroprotection and neurodestructive outcome. Our aim is to examine the implication of NO in long-term neurological disturbances, caused by hypoxia/kainic acid induced prolonged seizures.

Methods: Wistar rats (10-11-d-old) were divided into 4 groups: A, B, C and D (control) A, B, C groups were subjected to hypoxia, induced by decreasing $\mathrm{O} 2$ concentration from $7 \%$ to $4 \%$ within 15 minutes. At P21-P22, rats of groups B and C were administered with $\mathrm{KA}(10 \mathrm{mg} / \mathrm{kg}$ i.p.) Animals of group C were pre-treated with 7-Nitroindazole $(50 \mathrm{mg} / \mathrm{kg}$ i.p). Visualization of the mossy fibers sprouting was examined with Timm staining at PND75. Cognitive function was assessed with the passive avoidance and the water-maze tests for 5 consecutive days, at PND 65 and 70, respectively.

Results: In the passive avoidance test, the 7NI group presented better performance compared with the H/KA group, particularly the last day of trials. In the watermaze test, all the examined groups significantly improved their performance during the trial period and especially 7NI group reached the level of control groups. According to Timms staining scores, 7NI attenuated the mossy fiber sprouting during adulthood.

Conclusions: Our findings indicate that hypoxic seizures during development followed by prolonged seizures at later stages cause axonal growth and neurological dysfunction and suggest that NO may modulate seizure-induced hippocampal mossy fiber sprouting and play a role in long-term disturbances, observed in cognitive function.

\section{PROGNOSIS AT SCHOOL AGE OF FETUSES WITH ISOLATED MILD VENTRICULOMEGALY (IMVM)}

G Ancora ${ }^{1}$, A Lanzoni ${ }^{1}$, F Sandri ${ }^{1}$, F D'Alberton ${ }^{2}$, G L Pilu ${ }^{3}$, S Soffritti ${ }^{1}$, E Ferretti $^{4}$, G Faldella $^{1}$

${ }^{1}$ Department of Gynecological, Obstetrical and Pediatric Sciences, Institute of Neonatology, Bologna, Italy, ${ }^{2}$ Psychology, Department of Gynecological, Obstetrical and Pediatric Sciences, Bologna, Italy ${ }^{3}$ Institute of Obstetrics and Gynecology, Department of Gynecological, Obstetrical and Pediatric Sciences, 
Bologna, Italy, ${ }^{4}$ Neonatal Intensive Care Unit, University of Sherbrooke, Sherbrooke, QC, Canada

Background and Aims: Prognosis at school age of fetuses with IMVM, defined as cerebral ventricles with a 10-15 $\mathrm{mm}$ atrial diameter, remains unknown, despite conjecture that there is functional deterioration with age. To study the outcome for school age children that had been diagnosed prenatally with IMVM.

Methods: We studied 25 cases of IMVM obstetric diagnoses from the ultrasound lab archives. After birth, isolated VM was confirmed in 23 cases; in 2 cases VM was associated with periventricular leukomalacia and Simpson-Golabi-Behmel syndrome, respectively. We were able to contact 21 out of 25 patients for follow-up. Outcome was assessed at age 6-8 years with the Wechsler Intelligence Scales for Children and the test of Visuo-Motor Integration (VMI) in 14 children and in 7 others by structured telephone interviews.

Results: Mean (SD) IQ and VMI score were 110.8 (12.1) and 110.6 (14.9), respectively in 12/14 children; $2 / 14$ children, in which VM resulted not isolated after birth, were not evaluable due to severe psychomotor deficit. All the 7 children analyzed by telephone had a normal psychomotor development, good school performance and no need for a supporting teacher.

Conclusions: Of the 21 children followed at school age, $19(90.5 \%)$ showed normal outcome while $2(9.5 \%)$, where VM was not isolated after birth, showed severe psychomotor delay. Our results suggest that there is no functional deterioration with age associated with confirmed postnatal diagnosis of IMVM. These data are very useful in helping counseling parents with a fetus affected by IMVM.

\section{THE VALUE OF ASSOCIATED RISK FACTORS IN THE FORMATION OF MINIMAL BRAIN DYSFUNCTION IN CHILDREN OF YOUNG SCHOOL AGE}

\section{S Shamansurov, M Gulyamova, N Mirsaidova}

\section{Tashkent Medical Institute of Postgraduate Education, Tashkent, Uzbekistan}

Background and Aims: The significance of the problem of early identification and medico-social rehabilitation of children with minimal brain dysfunction (MBD) is explained by growing worsening of the state of nervous-psychic health of children, including increase in frequency of intellectual and speech deviations. Purpose: To identify and study risk factors of MBD in children of young school age.

Methods: Investigation was carried out on 57 patients with MBD and 20 healthy children aged from 3 to 5 years. Studying of pre- and perinatal medical history of patients and healthy children the pathological factors were the following: anemia in mother during pregnancy (64\% and 59\%), toxicosis (52\% and $47 \%$ ), pathological labors $(29 \%$ and $8 \%)$, risk of pregnancy interruption $(20,5 \%$ and $12 \%)$, frequent respiratory diseases $(8,8 \%$ and $3,6 \%)$, group of $\mathrm{Rh}$-factor $(2,3 \%$ and $0 \%)$, birth injury $(33,7 \%$ and $17 \%)$, gestosis $(8,8 \%$ and $3,3 \%)$.

Results: Studying of medical history of healthy children showed the presence of above-mentioned risk factors but of less severity. Anemia and toxicoses of mothers during pregnancy were almost similar both in group of study and healthy children. However, in group of sick children in the medical history there was noted additionally risk factors with birth trauma of different severity degree which, probably, in combination had reliable unfavorable effect on pregnancy and labor outcome.

Conclusions: Analysis of data obtained allowed to conclude that for development of MBD the combination of some factors but not single are of importance that requires from neurologists, neonatologists and pediatricians to concentrate their attention for management of this category of patients.

\section{SALIVARY CORTISOL AND SERUM INTERLEUKIN (IL)-6 CONCENTRATIONS AFTER MOTOR VEHICLE ACCIDENTS PREDICT POSTRAUMATIC STRESS DISORDER IN CHILDREN AND ADOLESCENTS}

\section{P Pervanidou ${ }^{1}$, G Kolaitis ${ }^{2}$, S Charitaki ${ }^{2}$, A Margeli $^{3}$, I Papassotiriou $^{3}$, J Tsiantis ${ }^{2}$, C H Bakoula ${ }^{1}$, G P Chrousos ${ }^{1}$ \\ ${ }^{1}$ First Department of Pediatrics, , ${ }^{2}$ Department of Child Psychiatry, Athens University Medical School, Athens, ${ }^{3}$ Department of Clinical Biochemistry, "Aghia Sophia" Children's Hospital, Athens, Greece}

Background and Aims: Posttraumatic stress disorder (PTSD) describes a syndrome of distress that develops after exposure to events or circumstances that involved actual death, injury or a threat to the physical integrity of oneself or others. This study examined prospectively posttraumatic stress symptomatology in relation to neuroendocrine and inflammatory factors after the event in children and adolescents hospitalized for road accidents

Methods: 60 children and adolescents involved in motor vehicle accidents (MVAs) were studied immediately after hospitalization, and at 1 month and 6 months later. Both parents and self-reported instruments were used: Children's PTSD-Reaction Index and Kiddie-SADS semi-structured interview. Serum cortisol, interleukin-6 and plasma catecholamine concentrations were measured with appropriate methodology. Furthermore, salivary cortisol was measured in samples collected serially five times a day.

Results: a) $38 \%$ of victims presented with PTSD at the 1 month assessment and $20 \%$ at the 6 month follow-up. b) Salivary cortisol concentrations at $12.00,18.00$ and $21.00 \mathrm{hrs}$ were significantly elevated $(\mathrm{p}=0.034, \mathrm{p}=0.006$ and $\mathrm{p}=0.007$ ) immediately after the accident, in those who subsequently developed PTSD at the 1 month assessment. Salivary cortisol levels at baseline were even higher in those presented with PTSD 6 months later: $(12.00: p=0.003,15.00$ hrs: $p=0.014,18.00 \mathrm{hrs}$ : $\mathrm{p}=0.004$ and $21.00 \mathrm{hrs} p=0.000)$. IL- 6 concentrations after the accident were elevated in those who developed PTSD 1- and 6-months later $(\mathrm{p}=0.003$ and $\mathrm{p}=0.0001$ respectively).

Conclusions: Increased evening and night salivary cortisol and/or increased serum IL-6 concentrations 
immediately after the accident, could be predictive markers of subsequent PTSD development in children.

\section{FACTORS INFLUENCING LATER GROWTH OUTCOME IN PRETERM INFANTS WITH CYSTIC PERIVENTRICULAR LEUKOMALACIA}

F Gallini, L Maggio, F Cota, P Papacci, F Serrao, C Romagnoli

\section{NICU, Catholic University, Rome, Italy}

Background and Aims: Causes of growth failure in children with severe neurological impairment remain often unclear. Our aim was to study, in preterm infants with cystic periventricular leukomalacia (CPVL), the prevalence and risk factors of poor growth at 2 years of corrected age.

Methods: The population consisted of all preterm admitted to our NICU from 1997 to 2003 with cranial ultrasound evidence of periventricular cystic white matter lesions. Growth failure at 2 years was defined, in infants with weight $\mathrm{z}$ score at birth $\geq-2$, as $\mathrm{z}$ score $<-2$; in those with weight $\mathrm{z}$ score at birth already $<-2$, as $\mathrm{z}$ score fall of another standard deviation.

Results: Out of the 26 children who were studied (GA $29.9 \pm 2.9$ wks, BW1340 \pm 530 g) 14 showed growth failure (W zs $-3.2 \pm 1.0$ ) and $12 \operatorname{did} \operatorname{not}(\mathrm{W}$ zs $-0.6 \pm 0.8$ ). During the hospital stay, growth retarded infants had similar baseline characteristics, early growth patterns, prevalence of intraventricular hemorrhage and grade of CPVL. During the follow up period, the frequency of rehospitalization $(2.5 \pm 0.6$ vs $0.75 \pm 0.7 ; \mathrm{p}: 0.03)$, oral feeding impairment $(36 \%$ vs $0 \%, \mathrm{p}<0.05)$, and the proportion of infants not able to walk independently $(50 \%$ vs $0 \%, p<0.05)$ resulted significantly higher in the growth retarded group.

Conclusions: At 2 years of corrected age $53.8 \%$ of premature infants with CPVL were growth retarded. In this population risk factors associated with growth failure were oral feeding impairment, rehospitalization and severe motor disability.

\section{QUANTITATIVE ANALYSIS OF PERFORMANCE IN THE FARNSWORTH- MUNSELL 100 HUE TEST IN CHILDREN WITH NORMAL COLOR VISION}

C De Felice ${ }^{1}$, F Scali $^{2}$, A Barducci ${ }^{3,4}$, P Corrocher $^{5}$, G Latini $^{6,7}, \mathrm{R}$ Casini $^{8}$

${ }^{I}$ Neonatal Intensive Care Unit, Azienda Ospedaliera Universitaria Senese, Siena, Italy, ${ }^{2}$ Semeiologia dei Parametri Funzionali Della Visione, U.O.C. Clinica Oculistica, Azienda Ospedaliera Universitaria Senese, Siena, Italy, ${ }^{3}$ Institute of Applied Physics "Nello Carrara", National Research Council of Italy, Florence, Italy, ${ }^{4}$ Department of Information Engineering, University of Siena, Siena, Italy, ${ }^{5}$ IntexSystem, Prato, Italy, ${ }^{6}$ Division of Neonatology, Perrino Hospital, Brindisi, Italy, ${ }^{7}$ Clinical Physiology Institute, National Research Council of Italy (IFC-CNR), Lecce Section, Italy, ${ }^{8} \mathrm{Te} \& \mathrm{Co}$, Prato, Italy
Background and Aims: Resolution between colors is based on $\Delta \mathrm{E}$, an euclidean distance in the non-euclidean perceptual color space. The Farnsworth-Munsell 100 Hue (FM100-H) test is a widely used qualitative tool for screening and classification of color vision anomalies. However, the relationship between test performance and quantitative color variables is unclear. Here, we tested color vision in healthy children as a function of the quantitative color variables of different hues in the FM100-H test.

Methods: Color vision was tested in a total of 41 healthy children aged 9 to 11 years, using the FM100-H test (Munsell Color Co.), consisting in rearranging in sequence 85 different color caps according to hue. All the caps were measured using a portable portable spectrophotometer unit (400-700 nm wavelengths range; standard D65 illuminant) and color was expressed in terms of CIE- $\mathrm{L}^{*} \mathrm{a} \mathrm{b}^{*}$ and $\mathrm{L} * \mathrm{C}^{*} \mathrm{H}^{*}$ color coordinates. $\Delta \mathrm{E}$ values between consecutive colors were computed using a color software package (Colormedica, IntexSystem). Test results were expressed as squared total error sum $(\sqrt{T E S})$ and percentage of errors in the sequence.

Results: All children had test results within normal limits for age ( $\sqrt{T E S}=9.54 \pm 2.29)$. Error percentage for single sequences $(21,95 \%$, inter-quartile range: 9.732.93) was unrelated to $\Delta \mathrm{E}(\mathrm{rho}=-0.001, \mathrm{p}=0.9923), \Delta \mathrm{L}$ $(\mathrm{rho}=0.040, \mathrm{p}=0.7186) \Delta \mathrm{a}(\mathrm{rho}=0.207, \mathrm{p}=0.0594), \Delta \mathrm{b}$ $(\mathrm{rho}=-0.107, \mathrm{p}=0.3309)$ or $\Delta \mathrm{H}(\mathrm{rho}=-0.084, \mathrm{p}=0.4453)$, while was inversely related to saturation differences $(\Delta \mathrm{C}: \mathrm{rho}=-0.297, \mathrm{p}=0.0067)$ between consecutive hues and color axis (blue-yellow errors $>$ red-green errors, $\mathrm{p}=0.0024$ ).

Conclusions: Performance in the FM100-H test is based on saturation differences between consecutive hues and axis.

\section{SCREENING SYMPTOMS OF ATTENTION DEFICIT DISORDER WITH OR WITHOUT HYPERACTIVITY IN 4639 SCHOOL CHILDREN BY MEANS OF RATING SCALE IV AND ABBREVIATED CONNOR'S FORM}

\author{
L Jaber $^{1,2,3}$, S Samara ${ }^{1,2,3}$, A Shuper ${ }^{1,3}$ \\ ${ }^{I}$ Neurology Clinic, Schneider Children's Medical Center \\ of Israel, Petah Tikva, Israel, ${ }^{2}$ The Bridge to Peace \\ Community Pediatrics Center, Taibe, Israel, ${ }^{3}$ Clalit \\ Health Services, Israel
}

Background and Aims: The prevalence of ADHD world wide is controversial. Although most studies place it in 3-5\%. Publications in English language regarding this issue in Arab children are lacking. We aimed by this study to evaluate the prevalence rate of ADHD in Israeli Arab school children.

Methods: The instruments we used for screening for symptoms of ADHD were the ADHD rating scale IV and the abbreviated Connor's scale. The sample of the study that was taken included 4639 school children of both sexes between 6-12 years age from 11 schools in three locations in the center of Israel. Location I is a village of less than 10,000 residents, location II is a small town of 22000 residents and location III is a big 
town of 35000 residents. The forms were filled by the teachers or by psychological consultants and teachers.

Results: The analysis of the data obtained showed high prevalence of ADHD in these children. It was $22.7 \%, 16.3 \%$ and $16.9 \%$ in locations I, II, III when the DSM IV scale was used and $18.3 \%, 13.2 \%$ and $11.5 \%$ when the abbreviated Connor's scale was used, respectively. Boys scored consistently higher than girls in both instruments used.

Conclusions: The prevalence of ADHD seems high in this population and further studies and controlled, should be conducted to determine the adequate prevalence of this disorder.

\section{CLINICAL CHARACTERISTICS AND OUTCOME OF CONVULSIVE STATUS EPILEPTICUS (CSE) COMPARED TO SHORTER SEIZURES IN 234 CHILDREN}

\author{
P Haapala $^{3}$, A-L Kuusela ${ }^{1}$, K Eriksson ${ }^{2,3}$ \\ ${ }^{1}$ Department of Pediatrics, Tampere University \\ Hospital, Tampere, Finland, ${ }^{2}$ Pediatric Research \\ Centre, Medical School, University of Tampere, \\ Tampere, Finland, ${ }^{3}$ Pediatric Neurology Unit, Tampere \\ University Hospital, Tampere, Finland
}

Background and Aims: To explore differences in background factors and outcome between convulsive status epilepticus (CSE, seizures lasting over 30 minutes) and shorter convulsions in children.

Methods: Retrospectively reviewed medical records and treatment charts of all children (up to 16 years) who had been admitted to the Pediatric Emergency Room or Pediatric Intensive Care Unit of Tampere University Hospital, between 1993 and 2002, due to convulsive seizures lasting over 5 minutes.

Results: There were 187 episodes of CSE and 152 seizure episodes lasting shorter time in 234 children with mean follow-up time of 2.0 years after the index seizure episode. CSE associated significantly with acute and remote symptomatic etiologies (vs. idiopathic or febrile etiology, $\mathrm{p}<0.001)$. Eleven (5.9\%) CSE and 39 $(25.6 \%)$ seizures lasting 5-29 minutes ended spontaneously $(\mathrm{p}<0.001)$. The treatment delay (time from the beginning of seizure to the anticonvulsive treatment) was 20 minutes in CSE group and 7 minutes in the comparison group $(\mathrm{p}=0.08)$. Mortality in these two groups was $1.7 \%$ and $0.4 \%$, and new epilepsy occurred in $12.8 \%$ and $20.0 \%$, respectively. Other neurological morbidity was associated with etiology $(p=0.004)$ but not with seizure duration.

Conclusions: The etiology of the seizure episode was the major determinant for neurological outcome and it was significantly different in CSE group and in 5-29minute seizure group. Rapidly induced treatment may prevent seizure prolongation and the overall outcome of prolonged seizures in children seems to be good.

\section{NEONATAL HYPOXIA AND LONG-TERM NEUROPSYCHOLOGICAL OUTCOME: A FOLLOW-UP STUDY INTO LATE CHILDHOOD AND ADOLESCENCE}

D Gosar ${ }^{1}$, D Neubauer $^{1}, \mathrm{~V}$ Tretnjak $^{1}$, T Bregant $^{1}$,
M Derganc

${ }^{1}$ Department of Child, Adolescent and Developmental Neurology, Division of Paediatrics, University Medical Center, Ljubljana, Slovenia, ${ }^{2}$ Department of Paediatric Surgery and Intensive Care, Division of Surgery, University Medical Center, Ljubljana, Slovenia

Background and Aims: Neonatal encephalopathy due to perinatal hypoxia-ischaemia is often associated with alterations in the development of specific cognitive abilities. This cognitive impairment may only be recognized when examining specific areas of cognition. The aim of our study was to examine later neuropsychological functioning in children who, as newborns, had been included in the study of different biochemical markers of hypoxia and ischaemia in the serum and CSF.

Methods: Twenty-one children who suffered perinatal hypoxia took part in the study and completed a 9neuropsychological-test-battery (WISC-III, WMS-R, VMI, Rey complex figure, Trail making test, Corsi block-tapping task, COWAT, Test of verbal fluency and Stroop colour test). The children were between 11 and 16 years old (average 13). Their neonatal indicators of perinatal hypoxia-ischaemia included Apgar scores, ultrasound findings and different biochemical markers (lactate, lactate dehydrogenase and creatin kinase) in the serum and CSF.

Results: With regard to reference values, the group of children included in the study, displayed the most cognitive deficits in visuo-motor skills and executive function. Neuropsychological test results correlated with creatin kinase in the CSF $(r=.33)$ and serum $(r=.45)$, memory scores being more closely associated with LDH in the CFS $(r=.41, p=.06)$ and $\operatorname{serum}(r=.54, \mathrm{p}=.01)$. Apgar scores and ultrasound findings had less predictive power.

Conclusion: The results indicate that biochemical markers of neonatal hypoxia-ischaemia creatin kinase in CSF and serum and lactate dehydrogenase in the serum are better long-term predictors of neuropsychological impairment than Apgar scores and ultrasound findings.

\section{AGE OF INDEPENDENT GAIT IN CHILDREN WITH DOWN SYNDROME}

T Campos, A Saianda, R Botelho, R Gouveia,

\section{Baptista}

Department of Paediatrics, Universitary Hospital of Santa Maria, Santa Maria Hospital, Lisbon, Portugal

Background and Aims: To evaluate age of independent gait in children with Down Syndrome followed at the Child Development Department of the University Hospital of Lisbon.

Methods: Clinical reports of 125 children with Down Syndrome were retrospectively reviewed for demographic data, age of mother at birth of child, 
chromosome defects, age of beginning of intervention programme, associated disorders, age of independent gait and developmental assessment at 12 and 36 months of age using the Ruth Griffiths Mental Developmental Scale.

Results: Mean of age of mother at birth of the child was 31.1 years with pre-natal diagnosis in five cases. Mean age of children at the beginning of the intervention programme was 9.15 months (in the 78 cases in which this item was recorded). Mean age of independent gait was 24.12 months in the entire sample and 25.3 in children with heart disease (12 of which had heart surgery). As for development assessment, mean GQ score at 12 months was $76.14 \%$ and $66.4 \%$ at 36 months.

Conclusions: No data existed in Portugal concerning mean age of independent gait in children with Down Syndrome, but our results are similar to those described in literature. Presence of heart disease, which could play delay developmental skills, did not significantly influence age of independent gait. Probably, on the first years of life, more time of the intervention programme should be spent with verbal and non verbal cognition rather than with motor skills, which come naturally.

\section{LIFE QUALITY IN WEST SYNDROME PATIENTS}

\author{
E Soler Fuentes ${ }^{1}$, G Naranjo Pelayo ${ }^{2}$ \\ ${ }^{1}$ Hospital Pediátrico de Palma Soriano, de Palma \\ Soriano, Cuba, ${ }^{2}$ Hospital Pediátrico Docente Infantil \\ sur de Santiago de Cuba, Santiago de Cuba, Cuba
}

Background and Aims: From ancient times people have considered epilepsy as a very dark disease, also the people who suffered it. This kind of people have leaved in a very bad situation because of the people reject, they have a poor quality of life even if they were childs with any known about life.

Methods: We applied an Spanish scale to know how do this patients live, how do they react with the environment, school, family and doctors. We made a 10year-old-study to get as much information as we could and to know how to work with all those patients.

Results: We find out that CAVE, the scale we used have to be modified to be able to apply in our country, because most of the times the results didn't show what was really happening with these kind of patients or their relatives.

Conclusions: We suggest that the scale be modified and apply in all the epileptic patients we see in consults, not only our group of West Syndrome if it is real that they were the most affected group, also their relatives.

\section{COMPARING THE EFFECTIVENESS OF DIFFERENT DOSES OF BOTULINUM TOXIN ON IMPROVEMENT OF WALKING AND REDUCING THE SPASTICITY IN SPASTIC DIPLEGIC CEREBRAL PALSY}

M Javadzadeh ${ }^{1}$, A Akbari ${ }^{2}$, A Zadeh-Vakili ${ }^{3}$, A Moghtaderi ${ }^{4}$, F Mahvelati ${ }^{5}$
${ }^{1}$ Department of Pediatric, Zahedan University of Medical Sciences, Imam Ali Hospital, Zahedan, Iran ${ }^{2}$ Department of Physiotherapy, Zahedan University of Medical Sciences, Imam Ali Hospital, Zahedan, Iran ${ }^{3}$ Department of Genetics, Zahedan University of Medical Sciences, Imam Ali Hospital, Zahedan, Iran ${ }^{4}$ Department of Neurology, Zahedan University of Medical Sciences, Khatam Hospital, Zahedan, Iran ${ }^{5}$ Department of Pediatric Neurology, Shahid Beheshti University of Medical Sciences, Mofid Hospital, Tehran, Iran

Background and Aims: Spasticity of lower limbs is a challenging problem for children with motor impairment due to cerebral palsy. For many years, treatment has centered on the use of physiotherapy and orthoses to overcome these problems. Intramuscular botulinum toxin (BT-A) offers a targeted form of therapy to reduce spasticity in specific muscle groups, though the high cost is a limiting factor for it's ready application in the developing countries. We tried to compare the effectiveness of it's different doses to choose the least expensive regimen which produces the desired effect.

Methods: Forty-five patients with spastic diplegia were enrolled, and were randomly divided into 3 groups each with 15 patients. Patients in the first group received 5 units $/ \mathrm{kg} / \mathrm{leg}$ of BT-A, and the patients in the 2nd and 3rd groups received 10 and 15 units $/ \mathrm{kg} /$ leg of BT-A respectively. The outcome was assessed six weeks later by video gait analysis, gross motor function measure (GMFM), and passive ankle dorsiflexion.

Results: Video gait analysis and GMFM showed the best results in the 2nd group with the 10units $/ \mathrm{kg} / \mathrm{leg}$ dose. The difference in passive dorsiflexion was not statistically significant in the 3 groups.

Conclusions: The study gives further support to the use of intramuscular botulinum toxin as an adjunct to conventional physiotherapy and orthoses to reduce spasticity in children with spastic diplegia. Since this therapy is considered as a relatively expensive treatment in developing countries, and considering that the best outcome was seen with $10 \mathrm{units} / \mathrm{kg} / \mathrm{leg}$ of BT-A, we discourage using higher doses of the drug for these patients.

\section{ORTHOPAEDIC SURGERY ON EQUINE FEET FOR CHILDREN WITH SPASTIC CEREBRAL PALSY}

\author{
I G Nazarov ${ }^{1}$, I G Nazarov ${ }^{2}$ \\ ${ }^{l}$ Medical University of Moscow, Moscow, Russia \\ ${ }^{2}$ University of Pediatric Surgery, Moscow, Russia
}

Background and Aims: The deformities of the equine feet of children with $\mathrm{CP}$ are the consequences of contractures of soft tissues. The elimination of these contractures that would permit increasing the capacity of movement would favour the development of the motor system.

Methods: the surgical intervention that I practice to eliminate the contractures of the soft tissues for 10 years, consists of performing small surgical incisions in the contracted fibers. In order to carry this out, one uses a sharp scalpel that allows one to surgically intervene 
just subcutaneously. The patient will find the postoperation to experience very little trauma. In order to conduct this research, 188 equine feet were operated on 120 children. Traditional methods were used for the medical research before and after these operations and at the end of 1-4 years after the operations were performed.

Results: The results demonstrate that in 181 operated feet, 90 degrees and less of the angle of the tibia-foot was decreased. In addition, the surface of the sole of the foot was increased. Also, the elimination of the pain syndrome, verticalization of the patients was proven to improve the balance, the capacity of movement and displacements. In the remaining 7 feet, beneficial results were found as well, but to a lesser degree.

Conclusions: This research show that it is necessary that orthopaedic surgery has an important place in the treatment of equine feet in order to optimize the rehabilitation of the children with Spastic Cerebral Palsy.

\section{PSYCHIATRIC SYMPTOMS IN ADOLESCENTS BORN BY MOTHERS WHO SMOKED IN PREGNANCY}

M S Indredavik ${ }^{1}$, P Romundstad ${ }^{2}$, A M Brubakk ${ }^{3}$, T Vik ${ }^{2}$

${ }^{I}$ Department of Neuroscience, Norwegian University of Science and Technology, Trondheim, Norway, ${ }^{2}$ Department of Public Health and General Practice, Norwegian University of Science and Technology, Trondheim, Norway, ${ }^{3}$ Department of Laboratory Medicine, Children's and Women's Health, Norwegian University of Science and Technology, Trondheim, Norway

Background and Aims: To study the association between smoking in pregnancy and psychiatric symptoms in the adolescent offspring.

Methods: 84 adolescents born by women who had been randomly selected before week 20 of pregnancy as participants in a multicenter study were examined at age 14 years. At enrolment of the pregnant mothers, 32 of 84 indicated that they were smokers. At follow up the adolescents' scores on the Achenbach System of Empirically Based Assessment (ASEBA), Autism Spectrum Screening Questionnaire (ASSQ), ADHDRating Scale IV and Children's Global Assessment Scale (CGAS) were recorded. In addition IQ was estimated through four subscales of WISC-III. Socioeconomic status (SES) was assessed through Hollingshead's Two factor Index, and maternal distress using the Symptom Checklist-90-R.

Results: Adolescents who were born by smokers had significantly higher total scores on all instruments when scored by mother (Table), father or teacher than adolescents of non-smokers. All caregivers consistently scored adolescents of smokers higher on the ASEBA subscores delinquent and aggressive behaviour, and social problems.

Conclusions: Smoking in pregnancy is associated with more psychiatric symptoms in adolescence, especially rule-breaking and aggressive behaviour, and other social problems.

\begin{tabular}{|l|c|c|c|l|}
\hline Table & N & ASEBA $^{*}$ & ASSQ $^{*}$ & ADHD $^{*}$ \\
\hline Non-smoker & 52 & $5.7(3.8-8.8)$ & $1.9(1.3-2.8)$ & $3.2(2.0-5.1)$ \\
\hline Smoker & 32 & $10.6(7.1-15.8)$ & $3.3(2.2-4.8)$ & $4.9(3.1-7.7)$ \\
\hline p-values & & $<0.01$ & $<0.01$ & $<0.05$
\end{tabular}

*Numbers are geometric mean and $95 \% \mathrm{CI}$, adjusted for sex, birth weight, SE single parent, mothers' use of alcohol, mothers' age and mothers' mental health.

\section{NEUROLOGIC PATIENTS ADMITTED FOR RESPIRATORY COMPLICATIONS - A RETROSPECTIVE ANALYSIS IN A TERTIARY REFERRAL UNIT}

A S Cochino ${ }^{1}$, $\mathrm{P}_{\text {Avram }}^{2}$, A V Cochino ${ }^{2}, \mathrm{M} \mathrm{Craiu}^{2}$

${ }^{1}$ Emergency Children Hospital 'Gr. Alexandrescu', Bucharest, Romania, ${ }^{2}$ Institute for Mother and Child Care (IMCC) 'A. Rusescu', Bucharest, Romania

Background and Aims: Children with cerebral palsy have functional prognosis dependent mainly on their neurologic impairment, as long as life expectancy is related to respiratory complications. We analyzed respiratory complications of the children with cerebral palsy admitted in IMCC.

Methods: Retrospective study of a case series of 24 children with cerebral palsy admitted for respiratory episodes, compared with a control group, age matched, neurologically unafected. We studied risk factors, airway protection reflexes, reccurence frequency, longof-stay, clinical forms, complications, treatment, outcome.

Results: The mean age was 62.53 months $(0-216)$, with sex ratio of 1.4:1 (M:F). See table. These children presented more severe respiratory infections episodes, sometimes protracted. Two patients had documented tuberculosis. Respiratory failure and athelectasis were the most frequent complications.

Conclusions: (1) Distrophy and abandon are frequently associated risk factors. (2) Airway protection reflexes impairment was present in $91.66 \%$; in some cases we tried to improve them using captopril. (3) Almost 100\% had lower airway infection. (4) High reccurence rate may lead to antibiotic resistance; in $54.16 \%$ expensive antibiotics were needed (e.g. cephalosporines, vancomycine). (5) Long-of-stay and parenteral antibiotic treatment were longer in the study group. (6) More than two thirds presented at least one serious complication. (7) Mortality was higher in the study group.

\begin{tabular}{|lll|}
\hline & Study group & Control goup \\
\hline Distrophy/ severe distrophy & $83.33 \% / 50 \%$ & $32 \% / 8 \%$ \\
\hline Abandon & $70.83 \%$ & $8 \%$ \\
\hline Apnea & $29.16 \%$ & $12 \%$ \\
\hline G ag reflex impairment & $68 \%$ & 0 \\
\hline L ong-of-stay (mean) & 31.12 days & 7.44 days \\
\hline L ower airw ay infection & $95.83 \%$ & $76 \%$ \\
\hline Length of parenter al antibiotic treatm ent (m ean) & 19.16 days & 5.68 days \\
\hline Mechanical ventilation (patients) & 3 & 0 \\
\hline Deaths & 3 & 0 \\
\hline
\end{tabular}

PROTEIN 14-3-3 IN THE CSF OF NEWBORN RATS FOLLOWING HYPOXIA-ISCHAEMIA

Z Kecskes ${ }^{1,4}$, M Mulchandani ${ }^{2}$, A Kent ${ }^{1,4}$, J Dahlstrom $^{3,4}$, I Hendry ${ }^{2}$ 
${ }^{1}$ Department of Neonatology, The Canberra Hospital, Canberra, ACT, Australia, ${ }^{2}$ Division of Neuroscience, John Curtin School of Medical Research, Canberra, ACT, Australia, ${ }^{3}$ Department of Anatomical Pathology, The Canberra Hospital, Canberra, ACT, Australia

${ }^{4}$ Australian National University Medical School, Canberra, ACT, Australia

Background and Aims: A hypoxic-ischaemic insult is one of the major causes of morbidity and mortality in term infants. To initiate neuroprotective interventions, early indicators of brain damage are required. The protein 14-3-3 family are a group of highly conserved proteins ubiquitously expressed in a wide range of organisms. In spite of reports in adult animals and humans, protein 14-3-3 has never been measured following a hypoxic-ischaemic insult in a newborn animal experiment. We measured protein 14-3-3 in the CSF of newborn rats following a $\mathrm{HI}$ insult .

Methods: 10-day-old rats were exposed to hypoxiaischaemia using the Levine model. CSF was collected regularly after the insult. A control group was subjected to sham surgery but not to the HI insult. Protein 14-3-3g was quantified by Western blotting. The rats were sacrificed and the brains taken for histological examination.

Results: In the control group, protein 14-3-3 did not increase significantly over time. In the study group, there was a significant increase in protein14-3-3 as early as 2 hours after the end of the insult, peaking at 4 to 6 hours and returning to basal levels by 72 hours.

Conclusions: Protein 14-3-3 increases in the CSF of newborn rats following a $\mathrm{HI}$ insult. The presence of protein 14-3-3 in CSF is a good indicator for neuronal loss or damage. Before protein 14-3-3 may be used to guide clinicians to accurately predict outcome and time of an insult to potentially initiate neural rescue therapies, these results have to be verified in humans and protein 14-3-3 measured in blood.

\section{THE PREVALENCE OF CAMPYLOBACTER JEJUNI IN PATIENTS WITH GASTROENTERITIS IN SHIRAZ, IRAN}

\author{
M Motamedifar ${ }^{1}$, P Hassanzadeh ${ }^{2}$ \\ ${ }^{1}$ Department of Bacteriology and Virology, Shiraz \\ Medical School, Shiraz, Iran, ${ }^{2}$ Department of Biology, \\ Shiraz University, Shiraz, Iran
}

Background and Aims: Campylobacter jejuni (C. jejuni) is known as a common causative agent of acute bacterial gastroenteritis in the world. It is not distinguishable by a routine coproculture. The prevalence of C. jejuni among other bacterial agents of acute diarrhea is investigated in this cross- sectional study.

Methods: In a period of three months, 114 fecal specimens were collected from patients aged 2-58 years with acute diarrhea, referring to Nemazi hospital, Shiraz, Iran. All samples were tested for identifying bacterial pathogens causing acute diarrhea.

Results: Of 40 positive samples for bacterial enteric pathogens, the overall prevalence of C. jejuni was $9.6 \%$ compared to Salmonella spp and Shigella spp with
$15.8 \%$ and $9.6 \%$ respectively. Maximum prevalence of C. Jejuni was observed in patients aged 11-15. All of these agents of diarrhea were most frequent in females $(\mathrm{P}<0.05)$.

Conclusions: This study revealed the significance $(\mathrm{P}<0.05)$ of $\mathrm{C}$. jejuni among other bacterial agents of acute gastroenteritis in Shiraz, Iran.

\section{INFANTIL COLIC: AETIOLOGY, PROGNOSIS AND CRITERIA FOR DIAGNOSIS}

\author{
A Sofijanova ${ }^{1}, \mathrm{~K}_{\text {Piperkova }}{ }^{2}$, A Sajkovski $^{1}$, \\ D Fidanovski ${ }^{1}$, A Hristovski ${ }^{1}$, L Koic $^{1}$ \\ ${ }^{I}$ Department of Intensive Care Unit, Pediatric Clinic, \\ Skopje, Macedonia, ${ }^{2}$ Department of Neonatology, \\ Pediatric Clinic, Skopje, Macedonia
}

Background and Aims: Infantil colic is one of the most commonly encountered problems in the early neonatal period. Inspite of everything the aetiology of the disorder is still an enigma. Natural course of the infantile colic, with a spontaneous disapperance of symtoms at 3-4 months of age, makes research on this disorder more difficult than usually. Still, it is a loss that intestinal function has not been monitored over a longer period in order to study whether the abnormality persists later in infancy or childhood.

Methods: Questionnaires.

Results: The occurrence of infantil colic has been studied at 100 neonates who came for a normal check up after suffering a neonatal risk period at our department. Our conclusion about the main reason for the origin of infantil colic presented was: 1 . inadequate interaction between mother and infant $25 \%$; 2 . hypertonicity and immaturity of gastrointestinal tract $35 \% ; 3$. Reaction to cow's milk or other food constituens in 50\%. According to the prognosis on the behaviour and temperament scales the only difference was that the colic infants were more emotional than the other infants. There were some basic criteria for association with the infantil colic like: sleep disorders, fussiness, aggresiveness and mostly crying. Also they refused certain meals.

Conclusions: We need to make a follow up study in order to find the clue to the enigma of infantil colic that probably lies in the physiology of the problem.

\section{IRON DEFICIENCY IS THREE TIMES MORE PREVALENT IN YOUNG CHILDREN IN NEW ZEALAND THAN IN EUROPE}

\author{
C R Wall ${ }^{1}$, C C Grant ${ }^{2}$, D Brunt ${ }^{2}$, R Scragg ${ }^{3}$, \\ S Crengle $^{4}$ \\ ${ }^{1}$ Institute of Food Nutrition and Human Health, Massey \\ University, Auckland, New Zealand, ${ }^{2}$ Department of \\ Paediatrics, University of Auckland, Auckland, New \\ Zealand, ${ }^{3}$ Department of Community Health, University \\ of Auckland, Auckland, New Zealand, ${ }^{4}$ Department of \\ Maori Health, University of Auckland, Auckland, New \\ Zealand
}

Background and Aims: Recent studies from Europe and the USA indicate that iron deficiency (ID) is present in approximately $7 \%$ of children less than two years old. 
Previous prevalence estimates of ID in New Zealand (NZ) have used hospitalised samples or biased community samples. The aims were to estimate the prevalence of ID in NZ children aged 6 to 23 months and the factors associated with ID.

Methods: An ethnically stratified random sample of well children aged 6 to 23 months from Auckland, NZ. Iron status was determined in 380 children enrolled from 1999 to 2002. ID was defined as abnormal values for 2 or more of serum ferritin $(<10 \mathrm{mg} / \mathrm{L})$, iron saturation $(<10 \%)$ and red cell distribution width $(>14 \%)$. Logistic regression analysis was used to calculate odds ratios and from these, relative risks (RR) \& 95\% confidence intervals (CI) were estimated.

Results: ID was present in $24 \%$ (95\% CI 19-29\%). In a multivariate analysis there was an increased risk (RR, 95\% CI) of ID associated with an elevated C-reactive protein $(2.20,1.02-3.42)$, low serum vitamin A (2.38, 1.23-3.53), enrolment during summer (2.48, 1.14-3.98), increased body mass $(2.86,1.32-4.55)$, breastfeeding after age six months $(2.21,1.13-3.67)$, and not receiving iron-fortified formula $(2.08,1.04-3.30)$.

Conclusions: ID is prevalent in the first two years of life in NZ. Seasonal and dietary rather than demographic factors identify children at increased risk. To reduce the prevalence of ID in NZ iron supplementation of breast fed infants and the use of iron-fortified formula past age 12 months should be considered.

\section{VITAMIN D DEFICIENCY: PREVALENT IN THE SUNNY SOUTH PACIFIC}

\author{
C C Grant ${ }^{1}, \mathrm{C}$ R Wall ${ }^{2}$, R Scragg ${ }^{3}$, S Crengle ${ }^{4}$ \\ ${ }^{1}$ Department of Paediatrics, University of Auckland, \\ Auckland, New Zealand, ${ }^{2}$ Institute of Food Nutrition and \\ Human Health, Massey University, Auckland, New \\ Zealand, ${ }^{3}$ Department of Community Health, University \\ of Auckland, Auckland, New Zealand, ${ }^{4}$ Department of \\ Maori Health, University of Auckland, Auckland, New \\ Zealand
}

Background and Aims: In recent years Vitamin D deficiency has re-emerged as a child health issue in Europe, the USA and also the Middle-East. New Zealand is a sunny country that has never considered the need for routine vitamin D supplementation. To estimate the prevalence of low vitamin D in young children and the factors associated with low vitamin D.

Methods: An ethnically stratified random sample of well children aged 6 to 23 months resident in Auckland, New Zealand. Serum 25-hydroxy-vitaminD3 was measured in 353 children enrolled from 1999 to 2002. Low vitamin $\mathrm{D}$ was defined as less than $27.5 \mathrm{nmol} / \mathrm{L}$. Logistic regression analysis was used to calculate odds ratios and from these, relative risks (RR) \& 95\% confidence intervals (CI) were estimated.

Results: Low vitamin D was present in $46(10 \%, 95 \%$ CI 7-13\%) of 353 children. After adjustment for sunlight exposure, gestation, number of siblings, household crowding and income there was an increased risk (RR, 95\% CI) of low vitamin D status associated with enrolment during winter (16.53, 1.02-46.06), Pacific Island $(9.79,2.74-21.94)$ or Maori ethnicity $(5.80,1.51$ -
16.13) and not having any infant or follow-on formula (6.07, 2.87-9.37).

Conclusions: Low vitamin D is prevalent in the first two years of life in New Zealand. The prevalence varies with season. Children of non-European ethnicity and children who don't receive iron fortified formula are at increased risk. Infant vitamin D supplementation should be considered as part of New Zealand's child health policy.

\section{PLASMA HOMOCYSTEINE LEVELS IN CHILDREN WITH GROWTH HORMONE DEFICIENCY}

G Triantafyllidis ${ }^{1}$, D Karakaidos ${ }^{1}$, M Solidaki ${ }^{2}$, P Zosi $^{1}$, N Kouskounelou ${ }^{1}$, E Velissaridou ${ }^{2}$, C Karis ${ }^{1}$

${ }^{I}$ Department of Pediatrics, General Hospital of Nikea, Piraeus, Greece, ${ }^{2}$ Department of Biochemistry, General Hospital of Nikea, Piraeus, Greece

Background and Aims: Elevated homocysteine levels have been identified as an independent risk factor for atherosclerosis. The purpose of our study was to evaluate the possible effect of growth hormone deficiency as well as recombinant human growth hormone (rhGH) replacement therapy on serum homocysteine (Hcy) levels in children with growth hormone deficiency.

Methods: 11 children ( 8 boys and 3 girls) with a mean age of $10.6 \pm 1.98 \mathrm{yrs}$ and established growth hormone deficiency $(<10 \mathrm{ng} / \mathrm{ml})$ on at least two provocative tests (Insulin, clonidine or glucagon) comprised the study group. Serum homocysteine levels were measured before and at 2, 3, 6 and 12 months of rhGH therapy which was administered at a dose of $0.5 \mathrm{IU} / \mathrm{kg} /$ week S.C. All children were instructed to receive the same appropriate diet for their age for at least 7 days before blood sampling. The Wilcoxon-signed rank test was used for comparison purposes.

Results: Our evidence show significantly reduced homocysteine levels by 12 months of therapy. Results are summarized on the table. ${ }^{*}$ Data represent median values.

Conclusions: rhGH therapy significantly reduces total homocysteine levels by 12 months of therapy. However, given the small number of patients, further studies will be required to clarify whether these results may significantly influence prevention of cardiovascular disease in adulthood.

\begin{tabular}{|l|l|l|l|l|l|l|}
\hline $\begin{array}{l}\text { Patients } \\
\mathrm{n}=11\end{array}$ & $\begin{array}{l}\text { Pretreatment } \\
(\mu \mathrm{mol} / \mathrm{L})\end{array}$ & 2 mon. & 3 mon. & 6 mon. & 12 mo. & $\begin{array}{l}\text { Sig.(2- } \\
\text { tailed) }\end{array}$ \\
\hline $\begin{array}{l}\text { homocysteine } \\
\text { levels }\end{array}$ & $5.80^{*}$ & 5.10 & 4.87 & 5.17 & 4.96 & $\mathrm{p}<0.05(\mathrm{~S})$ \\
\hline
\end{tabular}

\section{THE EFFECT OF RECOMBINANT HUMAN GROWTH HORMONE THERAPY ON CHOLESTEROL, LIPOPROTEIN A (LP(A)) AND APOLIPROTEIN A AND B LEVELS OF GROWTH HORMONE DEFICIENT CHILDREN}

G Triantafyllidis ${ }^{1}$, D Karakaidos ${ }^{1}$, N Kouskounelou ${ }^{1}$, G Hajigeorgiou ${ }^{1}$, G Liakos $^{2}$, E Velissaridou ${ }^{2}$, C Karis $^{1}$ 
${ }^{l}$ Department of Pediatrics, General Hospital of Nikea, Piraeus, Greece, ${ }^{2}$ Department of Biochemistry, General Hospital of Nikea, Piraeus, Greece

Background and Aims: Growth hormone participates in the regulation of serum lipid and lipoprotein levels. The study's aim evaluates the possible effects of rhGH treatment on cholesterol, lipoprotein $\mathrm{A}$ and apolipoprotein A and B levels of children with established growth hormone deficiency.

Methods: 11 children ( 8 boys, 3 girls) with a mean age of $10.6 \pm 1.98 \mathrm{yrs}$ and established growth hormone deficiency $(<10 \mathrm{ng} / \mathrm{ml})$ on at least two provocative tests (Insulin, clonidine or glucagon) comprised the study group. Serum total cholesterol (HDL+LDL), $\mathrm{Lp}(\mathrm{A})$ as well as apoA and apoB levels were measured before and at 2, 3, 6 and 12 months of rhGH therapy administered at a dose of $0.5 \mathrm{IU} / \mathrm{kg} /$ week S.C. All children were instructed to receive the same appropriate diet for their age for at least 7 days before blood sampling.

Results: Our evidence show significantly reduced total cholesterol levels along with reduced LDL levels and significantly elevated HDL levels by 12 months of therapy. Additionally, Lp(A) levels significantly increase, whereas apoA and apoB remained unaltered. (See table for results).*median.

Conclusions: rhGH therapy significantly reduces total cholesterol levels and induces a significant increase of protective LpA levels. A larger confirmatory cohort study is under way for verification of the above.

\begin{tabular}{|l|l|l|l|l|l|l|}
\hline $\begin{array}{l}\text { Patients } \\
\mathrm{n}=11\end{array}$ & $\begin{array}{l}\text { Total } \\
\text { cholesterol } \\
\mathrm{mg} / \mathrm{dl}\end{array}$ & $\begin{array}{l}\mathrm{LDL} \\
\mathrm{chol} . \\
\mathrm{mg} / \mathrm{dl}\end{array}$ & $\begin{array}{l}\mathrm{HDL} \\
\mathrm{chol} . \\
\mathrm{mg} / \mathrm{dl}\end{array}$ & $\begin{array}{l}\mathrm{Lp}(\mathrm{A}) \\
\mathrm{mg} / \mathrm{dl}\end{array}$ & $\begin{array}{l}\text { apoA } \\
\mathrm{mg} / \mathrm{dl}\end{array}$ & $\begin{array}{l}\mathrm{apoB} \\
\mathrm{mg} / \mathrm{dl}\end{array}$ \\
\hline before & $174^{*}$ & 104 & 50 & 5.98 & 155 & 83 \\
\hline 2 months & 175 & 105 & 53 & 6.14 & 158 & 85 \\
\hline 3 months & 170 & 100 & 58 & 8.10 & 154 & 82 \\
\hline 6 months & 167 & 95 & 65 & 8.00 & 146 & 79 \\
\hline 12 months & 165 & 97.5 & 66 & 8.60 & 159 & 83 \\
\hline Sig.(2tailed) & $<0.05(\mathrm{~S})$ & $<0.05(\mathrm{~S})$ & $<0.05(\mathrm{~S})$ & $<0.05(\mathrm{~S})$ & $<0.05(\mathrm{~S})$ & $>0.05(\mathrm{NS})$ \\
\hline
\end{tabular}

\section{THYROID DYSFUNCTION AND CHILDHOOD OBESITY}

E Papadopoulou, E Dilanas, E Giahnakis, M Avanidi, E Paraskakis, H Maniadaki, M Kalmanti

Department of Pediatrics, University of Crete, Heraklion, Crete, Greece

Background and Aims: Childhood obesity may be associated with disorders of thyroid gland. We investigated the frequency of thyroid dysfunction (TD) in overweight and obese children.

Methods: We reviewed data from 135 obese and overweight children, including 65 females $(48.5 \%)$ and 70 males $(51.4 \%)$, with a mean age $10.7 \pm 3.12$ years, followed in our Childhood Obesity Clinic. The mean weight was $61.6 \pm 22.7 \mathrm{~kg}$ and the mean BMI was $29 \pm 4.9$ $\mathrm{kg} / \mathrm{m}^{2}$. TSH $(\mu \mathrm{IU} / \mathrm{mL})$, FT3 $(\mathrm{pg} / \mathrm{mL})$, FT4 $(\mathrm{ng} / \mathrm{dL})$, thyroid antithyroglobulin, and thyroid antiperoxidase antibodies, were measured in every patient. The existence of positive TD family history was recorded. Subjects were stratified according to age-specific groups (A: 2.5-10yrs, B: 10-17 yrs), and BMI-subgroups $(1: \mathrm{BMI}<25,2: 25 \leq \mathrm{BMI}<30,3$ : $\mathrm{BMI} \geq 30$ ).

Results: TD was detected in 31 children $(22.9 \%)$, with a positive TD-family-history in 7 of these $(22.5 \%)$. There were 2 patients with decreased TSH, 18 with eythyroidism and elevated TSH, 3 with elevated TSH and positive thyroid autoantibodies or abnormal thyroid hormones, 4 with elevated FT3, and 4 patients with positive thyroid autoantibodies. In group A (52/135, $38.5 \%), 13 / 52$ children (25\%) had TD (subgroups A1: $23.8 \%, \mathrm{~A} 2: 25 \%, \mathrm{~A} 3: 28,5 \%)$. In group B $(83 / 135$, $61.4 \%), 18 / 83$ children $(21.4 \%)$ had TD (subgroups B1: $16.6 \%$, B2: $16.2 \%$, B3: $27.5 \%$ ). There was no statistical significant correlation of TD with the age-specific groups or the BMI-subgroups of the obese children.

Conclusions: One out of four obese or overweight children presents thyroid dysfunction, particularly elevated TSH levels and/or positive thyroid autoantibodies. A thyroid screening has to be included in the diagnostic approach of the obese children.

\section{CONTRAST SENSITIVITY CHANGES IN TYPE 1 DIABETIC CHILDREN WITHOUT RETINOPATHY}

M I Eliopoulou ${ }^{1}$, C D Georgakopoulos ${ }^{2}, \mathrm{P} \mathrm{Nanos}^{2}$, A M Exarchou $^{2}$, A N Filias ${ }^{1}$, B E Spiliotis ${ }^{1}$

${ }^{1}$ Pediatric Endocrine Unit, Department of Paediatrics, University of Patras, Patras, Greece, ${ }^{2}$ Department of Ophthalmology, University of Patras, Patras, Greece

Background and Aims: To evaluate the visual abnormalities preceding the retinopathy in children with insulin-dependent diabetes mellitus (IDDM) with contrast sensitivity testing (CS).

Methods: Sixty patients with IDDM (age range 8-18 years, mean $13.73 \pm 3.15$ years) with a mean duration of disease $5.9 \pm 3.8$ years and a mean $\mathrm{HbA} 1 \mathrm{c}$ of $8.26 \pm 1.93 \%$ were studied. All patients had normal visual acuity $(>$ or $=1.0)$ and no visible diabetic retinopathy. Control group consisted of 35 healthy subjects (age range 8-18 years; mean $12.1 \pm 3.11$ years) with normal visual acuity. Ophthalmoscopy and retinal photography were used to determine the presence or absence of diabetic retinopathy. CS was assessed with a CSV-1000 contrast testing instrument, testing for four spatial frequencies, 3 , 6, 12 and 18 cycles per degree (cpd). Patients' CS values were correlated to the variables age, duration of disease and glycemic control (mean value of the last four determinations of $\mathrm{HbAlc}$ ).

Results: Contrast sensitivity function was significantly impaired in patients with IDDM. The reduction was statistically significant in all spatial frequencies compared with the control group $(p<0.05)$. Contrast thresholds were inversely related to HbA1c levels in 3 and $6 \mathrm{cpd}$ (linear regression $\mathrm{r}=-0.38, \mathrm{p}<0.05$ and $\mathrm{r}=-0.33, \mathrm{p}<0.05$, respectively) but age and duration of disease did not show any significant correlation.

Conclusions: Patients with IDDM demonstrate abnormal contrast sensitivity. The results suggest that an early dysfunction in visual pathway may occur without visible retina involvement. CS testing can detect early changes in the visual function of diabetic children. 
OVERWEIGHT AND OBESITY IN SPANISH PREPUBERTAL CHILDREN ARE RELATED TO EXCESS OF FAT IN THE DIET, LOW PHYSICAL ACTIVITY, HIGH BLOOD LIPIDS LEVELS AND ATHEROGENIC RISK

E Martin-Bautista ${ }^{1}$, M Martin-Lagos ${ }^{1}$, M Martin-Matillas ${ }^{1}, \mathrm{~V}$ Dolz ${ }^{1}$, T Pedrosa ${ }^{1}$, A Ocon ${ }^{1}$, J Quer $^{2}$, M Rivero ${ }^{2}$, C Campoy ${ }^{1}$

${ }^{I}$ Departament of Paediatric, School of Medicine, University of Granada, Granada, Spain, ${ }^{2}$ Grup D'Estudis Nutricionals I Alimentació (GENA), Barcelona, Spain

Background and Aims: This study evaluated the relationship between diet, anthropometry, blood lipids and atherogenic risk in 70 healthy Spanish children between 6-12 years.

Methods: Evaluation of the diet, anthropometry (body mass index-BMI, skinfolds and perimeters) and PA proofs were made. Spectrophotometry: total cholesterol (TC), cholesterol joined to high (HDLc) and very low (VLDLc) density lipoproteins, triglycerides (TG) in $\mathrm{mg} / \mathrm{dL}$. Friedwald's formula: (LDLc). Atherogenic ratios $(\mathrm{ATI} 1)=\mathrm{LDLc} / \mathrm{HDLc}$ and $(\mathrm{ATI} 2)=(\mathrm{VLDLc}+\mathrm{LDLc})$ HDLc. Statistical analysis: Student/Welch ' $t$ ' test, Pearson and Spearman. ${ }^{*}: \mathrm{p}<0.05 ;{ }^{* *}: \mathrm{p}<0.01$.

Results: $100 \%$ of the children had excess intake of total fat $(>80 \mathrm{~g} /$ day $)$ and saturated fatty acids $(>8 \mathrm{~g})$ and the $80 \%$ showed excess of cholesterol $(>300 \mathrm{mg} /$ day $)$ in their diet. The $57 \%$ had excess intake of kilocalories $(>2450 \mathrm{kcal} /$ day) but deficiencies of calcium $(90 \%)$, iodine (76\%) and phosporum (22\%). Boys: Overweight: $26.2 \%$, obesity: $7.1 \%$. Girls: Overweight: $25 \%$, obesity: $10.7 \%$. Correlations established: BMI and TG, VLDLc, ATI1 y ATI2 $\left(\mathrm{r}=0.389^{* *} ; \mathrm{r}=0.290^{* *} ; \mathrm{r}=0.434 * *\right.$, $\left.\mathrm{r}=0.553^{* *}\right)$, respectively; BMI and HDLc $\left(\mathrm{r}=-0.366^{* *}\right)$. The relation of the BMI to measured skinfolds (biceps, triceps, subescapular and abdominal) were: $\mathrm{r}=0.860^{* *}$, $\mathrm{r}=0.859^{* *}, \mathrm{r}=0.826^{* *}$ and $\mathrm{r}=0.788^{* *} .11 .1 \%$ of children had TC> P95 $(200 \mathrm{mg} / \mathrm{dl}), 14.8 \%$ of them had TG $>$ P95 $(110 \mathrm{mg} / \mathrm{dl})$ and $3.7 \% \mathrm{had}$ LDLc $>$ P95 $(150 \mathrm{mg} / \mathrm{dl}) .30 \%$ of the children didn't practise PA and $27 \%$ PA frequently.

Conclusions: A high \% of the children studied showed overweight and obesity, high BMI, plasma TG, VLDLc, elevated AT and low HDLc. These factors could be influenced by the diet and the PA and could predispose to future cardiovascular diseases in the adulthood. Supported by UNIBISCUITS International.

\section{ARE THERE ETHNIC DIFFERENCES IN NEONATAL OUTCOME FOR VERY PRETERM BABIES? RESULTS FROM THE ITALIAN ACTION STUDY}

D Di Lallo ${ }^{1}, \mathrm{M}$ Da Frè ${ }^{2}, \mathrm{E}_{\text {Buiatti }}{ }^{2}, \mathrm{~V}$ Carnielli ${ }^{3}$, C Corchia ${ }^{4}$, L Gagliardi ${ }^{5}$, F Macagno $^{6}$, S Miniaci $^{7}$, $\mathrm{M} \mathrm{Cuttini}^{8}$

\footnotetext{
${ }^{I}$ Agency for Public Health, Rome (Lazio), Italy

${ }^{2}$ Regional Health Agency, Firenze (Toscana), Italy

${ }^{3}$ G. Salesi Hospital, Ancona (Marche), Italy, ${ }^{4}$ Pediatric

Hospital Bambino Gesù, Rome (Lazio), Italy, ${ }^{5}$ Versilia

Hospital, Lucca (Toscana), Italy, ${ }^{6}$ Civile Hospital,
}

Udine (Friuli Venezia Giulia), Italy, ${ }^{7}$ Pugliese-Ciaccio Hospital, Catanzaro (Calabria), Italy

Background and Aims: In Italy about $8 \%$ of newborns are born to foreign mothers. Despite growing concerns about ethnic inequalities in pregnancy outcome, little is known about the health of these infants when born severely preterm. This study compares neonatal outcomes of Foreign Mothers' (FMs) and Italian Mothers' (IMs) very preterm babies in a large areabased national cohort.

Methods: The study population consisted of 2897 infants, gestational age 22-31 wks, born in six Italian regions during 2003-2004 and admitted to NICUs. Socio-demographic maternal information, including citizenship, and perinatal data were collected as part of the Italian project on Very Preterm Pregnancies and Births (ACTION). The main outcomes were in-hospital mortality and a combination of mortality and severe morbidity (intraventricular hemorrhage intraventricular of grade III-IV, periventricular leukomalacia and bronchopulmonary dysplasia) at discharge. The Cox proportional hazards and logistic regression analysis were used to model the two outcomes.

Results: 628 infants ( $22 \%$ of total) were born to FMs. Compared to IMs' infants, they were more often severely preterm $(38.1 \%$ vs $28.7 \%$ in $22-27$ wks category); less often from multiple pregnancy $(22.8 \%$ vs $29.1 \%$ ); experienced a lower use of prenatal steroids $(71.4 \%$ vs $80.5 \%)$, and higher in-hospital mortality $(20.2 \%$ vs $16.6 \%)$ and combined mortality/morbidity ( $34.2 \%$ vs $31 \%$ ). However, when potential confounders were adjusted for, FMs' babies had no greater risk of mortality (HR $0.92 ; 95 \%$ CI $0.74-1.15$ ) or mortality/morbidity (OR 0.88; 95\% CI 0.68-1.13).

Conclusions: When relevant risk factors are taken into account, neonatal outcomes of FMs' very preterm infants were comparable to those of IMs.

\section{EVALUATION OF ANDROGEN AND PROGESTERONE LEVELS OF WOMEN WITH PREECLAMPSIA IN THIRD TRIMESTER}

S Golmohammadlou ${ }^{1}$, S Salari ${ }^{2}$, N Pashapour ${ }^{3}$

${ }^{1}$ Kosar Hospital, Oromieh, Iran, ${ }^{2}$ Medical Faculty, Oromieh, Iran, ${ }^{3}$ Department of Pediatrics, Medical Faculty, Oromieh, Iran

Background and Aims: Pre eclampsia along with its complications continues to be one of the major causes of maternal and fetal morbidity and mortality. In spite of so many researches, the pathology of pre eclampsia has not yet been fully elucidated. The purpose of the study was to determine whether maternal serum levels of androgen and progesterone, are higher in patient with pre eclampsia than in matched control subjects.

Methods: Serum progesterone, total testosterone, free testosterone and dehydroepiandrosterone levels were measured in 19 subjects in third trimester of pregnancy with documented pre eclampsia and 17 healthy normotensive women with similar maternal and gestational age. All subjects were primigravida women with singleton pregnancy who were visited in Kosar Medical center in Uromiyeh. 
Results: There were no significant difference between two groups in maternal age, gestational age and body mass index. Progesterone and free testosterone levels were significantly lower $(\mathrm{P}=0.01)$ in patients with pre eclampsia $(75.1 . \pm 8.6 \mathrm{ng} / \mathrm{dl}$ and $2.27 \pm 1.74 \mathrm{pg} / \mathrm{dl}$, respectively) than in control group $(111.6 \pm 9.71 \mathrm{ng} / \mathrm{dl}$ and $3.73 \pm 1.31 \mathrm{pg} / \mathrm{dl}$, respectively). There were no significant difference in total testosterone and dehydroepiandrosterone levels between cases $(1.02 \pm 0.10 \mathrm{ng} / \mathrm{dl}$ and $0.99 \pm 0.13 \mu \mathrm{g} / \mathrm{dl}$ respectively) and controls $\quad(1.37 \pm .019 \quad \mathrm{ng} / \mathrm{dl}$ and $0.98 \pm 5.15 \mu \mathrm{g} / \mathrm{dl}$ respectively.

Conclusions: Accentuated sex hormone binding globulin increase in pre eclampsia is the cause of significant decreased free testosterone of pre eclamptic cases. Levels of progesterone were pathologically and significantly lower in pre eclamptic cases than control women with similar age, gestational age and body mass index. Keyword: Pre eclampsia, androgen, progesterone, pregnancy, mortality.

\section{GROWTH HORMONE DEFICIENCY IN SHORT STATURED CHILDREN}

\section{S Abtahi, M R Khazaee}

Department of Pediatrics, Mashhad Azad University, 22 Bahman Hospital, Mashhad, Khorasan, Iran

Background and Aims: One of the causes of growth failure is Growth Hormone Deficiency, that usually shows a growth pattern of less than 2 inches a year. Sometimes there is a deficiency of other pituitary hormones resulting in Hypopituitarism.

Methods: A cross-sectional survey. A total of 293 children were referred for growth hormone evaluation. All the patients underwent thorough clinical evaluation including recording of height, weight, presence of chronic diseases and malnutrition. Twenty children were excluded for various reasons. Screening for $\mathrm{GH}$ deficiency was carried out by exercise stimulation test and/or L-dopa stimulation test. The children with GH level $<10 \mathrm{mIU} / \mathrm{L}$ on screening tests were provisionally considered to have GH deficiency. These children underwent insulin tolerance test (ITT).

Results: Out of 273 children, 189 were subjected to both exercise and L-dopa stimulation test, while 60 patients underwent only exercise stimulation test. The remaining 24 patients were subjected to L-dopa administration alone because of their physical weakness or inability to perform exercise. Sixty-six patients exhibited GH levels $<10 \mathrm{mIU} / \mathrm{L}$ on screening tests. The frequency of GH deficiency was found to be $24.17 \%$ on screening. Out of these 66 patients, 54 underwent an ITT, while 12 patients did not report back for the test. Thirty-eight children showed lack of response on ITT, therefore, after ITT, the frequency of GH deficiency was revealed to be $13.9 \%$.

Conclusions: GH deficiency is responsible for about $14 \%$ cases of short stature in a hospital setting. Screening tests showed a positive predictive value of $70.4 \%$ and a false positivity of $6.8 \%$.

\section{A SURVEY OF NEONATAL PARENTERAL NUTRITION PRESCRIPTION IN ENGLAND, SCOTLAND AND WALES}

A Grover ${ }^{1}, \mathrm{M}_{\mathrm{K}} \mathrm{Khashu}^{2}$, V Kairamkonda ${ }^{2}$, A Muhkerjee ${ }^{2}$

${ }^{1}$ Department of Paediatrics, Northampton General Hospital, Northampton, UK, ${ }^{2}$ Neonatal Unit, Leicester Royal Infirmary, Leicester, UK

Background and Aims: Extrauterine growth retardation is a major clinical problem in very low birth infants (VLBW). Parenteral Nutrition (PN) serves to achieve rapid maximal nutrition in early postnatal life. Since there is no consensus with regard to optimal neonatal PN practice, we aimed to ascertain current practice regarding prescribed PN glucose, protein and lipid intake in early postnatal period in the VLBW in the United Kingdom.

Methods: Survey questionnaire was emailed to hospital neonatal pharmacists serving level 3 and major level 2 units in the UK. Survey data items were static numerical information regarding $\mathrm{PN}$ prescription until establishment of full PN. Data are expressed as median and range.

Results: A response was obtained from 46 (71.8\%) units (70\% of level 3 and $75 \%$ of level 2). 25 out of 46 centres (54\%) initiated PN on day 1. Full PN was achieved by day 5 (range 4-10). Comparison of prescribed PN with RDA demonstrated a deficit of $477.9 \mathrm{kcal} / \mathrm{kg}$ and $1.65 \mathrm{gm} / \mathrm{kg}$ nitrogen protein in the first 10 days of life (see table).

Conclusions: There is diverse practice with regard to neonatal PN prescription in the UK. In view of difficulties in establishment of enteral feeding in VLBW, current PN practice would entail a significant calorie and protein deficit during early postnatal life and warrants a review.

\begin{tabular}{|l|l|l|l|l|}
\hline Day & $\begin{array}{l}\text { Glucose infusion } \\
\text { rate }(\mathrm{mg} / \mathrm{kg} / \mathrm{min})\end{array}$ & $\begin{array}{l}\text { Nitrogen Protein } \\
\text { (gm/kg/day) }\end{array}$ & $\begin{array}{l}\text { Lipid } \\
\text { (gm/kg/day) }\end{array}$ & $\begin{array}{l}\text { Non Protein } \\
\text { Calories } \\
\text { (Kcal/kg/d) }\end{array}$ \\
\hline 1 & $4.16(3.47-8.30)$ & $0.07(0-0.29)$ & $0.0(0.0-2.0)$ & 23.9 \\
\hline 2 & $6.25(4.68-9.70)$ & $0.15(0.0-0.42)$ & $1.0(0.0-2.0)$ & 46.0 \\
\hline 3 & $7.99(6.25-11.10)$ & $0.28(0.07-0.47)$ & $1.5(0.5-3.0)$ & 61.0 \\
\hline 4 & $9.55(6.77-12.5)$ & $0.32(0.11-0.47)$ & $2.0(1.0-3.6)$ & 75.0 \\
\hline 5 & $9.72(7.2-12.5)$ & $0.35(0.14-0.50)$ & $3.0(1.5-4.0)$ & 85.9 \\
\hline 6 & $9.72(7.56-14.5)$ & $0.40(0.2-0.60)$ & $3.0(2.0-4.0)$ & 85.9 \\
\hline 7 & $9.74(7.39-14.3)$ & $0.42(0.2-0.60)$ & $3.0(2.0-4.0)$ & 86.1 \\
\hline 8 & $9.74(7.39-14.0)$ & $0.42(0.25-0.60)$ & $3.0(2.0-4.0)$ & 86.1 \\
\hline 9 & $9.74(7.39-13.8)$ & $0.42(0.25-0.60)$ & $3.0(2.0-4.0)$ & 86.1 \\
\hline 10 & $9.88(7.39-13.7)$ & $0.42(0.28-0.60)$ & $3.0(2.0-4.0)$ & 86.1 \\
\hline
\end{tabular}

\section{THE INFLUENCE OF AGE, DISEASE SEVERITY AND MEDICAL INTERVENTIONS ON THE GROWTH HORMONE-INSULIN-LIKE GROWTH FACTOR ONE (GH-IGF-I) AXIS BEFORE AND AFTER PAEDIATRIC CARDIAC SURGERY}

M den Brinker ${ }^{1}$, A C Hokken-Koelega ${ }^{3}$, R J Van Thiel ${ }^{5}$, A J Bogers ${ }^{5}$, W Helbing ${ }^{4}$, M J Wijers ${ }^{5}$, W C Hop ${ }^{6}$, D C Van Der Kaay ${ }^{3}$, K F Joosten ${ }^{2}$

${ }^{l}$ Department of Paediatrics, Erasmus MC - Sophia Children's Hospital, Rotterdam, The Netherlands ${ }^{2}$ Department of Paediatrics, Division of Paediatric Intensive Care, Erasmus MC - Sophia Children's Hospital, Rotterdam, The Netherlands, ${ }^{3}$ Department of Paediatrics, Division of Endocrinology, Erasmus MCSophia Children's Hospital, Rotterdam, The 
Netherlands, ${ }^{4}$ Department of Paediatrics, Division of

Paediatric Cardiology, Erasmus MC - Sophia

Children's Hospital, Rotterdam, The Netherlands

${ }^{5}$ Department of Cardiothoracic Surgery, Erasmus MC,

Rotterdam, The Netherlands, Rotterdam, The

Netherlands, ${ }^{6}$ Department of Epidemiology and

Biostatistics, Erasmus MC, Rotterdam, The Netherlands, Rotterdam, The Netherlands

Background and Aims: To evaluate GH-IGF-I axis before and after paediatric cardiac surgery and to determine influencing factors.

Methods: Observational study with 49 children undergoing cardiac surgery divided according to age into: infants $(n=19)$, young children $(n=20)$ and teenagers $(n=10)$. Measurements were: $\mathrm{GH}$ profiles after surgery and IGF-I, IGFBP-3 and IGFBP-1 levels before, at end of surgery, 12 and 24 hours thereafter.

Results: At start of surgery, IGF-I and IGFBP-3 SDscores were lower than reference values and related to low weight SD-scores and cyanosis. At end of surgery, mean-GH levels were elevated and related inversely to high-dose glucocorticoid and dopamine administration, but not to IL-6 levels. Glucose levels were higher in patients who received glucocorticoids than in those who did not. At end of surgery, IGF-I and IGFBP-3 SDscores related to the percentage plasma administered during surgery, and not to IL-6 or GH levels. At start of surgery, IGFBP-1 related to insulin levels, but at end of surgery to IL-6 levels. Twenty-four hours after cardiac surgery, IGF-I, IGFBP-3 SD-scores and IGFBP-1 levels returned to the initial values at start of surgery.

Conclusions: At start of surgery low IGF-I related to underweight and low IGFBP-3 to cyanosis. Glucocorticoids and dopamine had significant influence on GH and percentage plasma on IGF-I and IGFBP-3 levels, whereas IL-6 levels had no significant influence. Glucocorticoid administration during surgery was associated with higher glucose levels. Already the day after surgery, IGF-I, IGFBP-3 and IGFBP-1 levels had returned to the initial values at start of surgery.

\section{URINARY TRACT INFECTIONS IN MALNOURISHED CHILDREN}

\section{Rahimkhani, F Vaezzadeh \\ School of Medical Sciences, Tehran University of Medical Sciences, Tehran, Iran}

Background and Aims: Malnutrition is common in developing and under developing countries and Urinary tract infection (UTI) is a well-recognized complication in malnourished children.

Methods: We examined the incidence of UTI, bacteriuria and pyuria in malnourished children. Of 61 patients (19 boys), 1 had severe, 15 had moderate and 45 had mild malnutrition. A normally nourished control matched for age, sex and presence of fever was included. Clean catch midstream urine specimens from patients and control group were examined microscopically and cultured.

Results: The incidence of UTI, bacteriuria and pyuria in malnourished subjects was $44 \%, 44 \%$ and $13 \%$ respectively. The incidence of UTI, bacteriuria and pyuria in control children was $19 \%, 19 \%$ and $11 \%$ respectively. E. coli is predominantly an organism isolated in patient and control group.

Conclusions: Our observations show that malnourished children are at risk for UTI. Urinalysis and urine culture is useful for screening for UTI in these subjects.

\section{INCREASING INCIDENCE OF TYPE I DIABETES MELLITUS IN GREEK CYPRIOT CHILDREN AND ADOLESCENTS IN 2000-2004}

N Skordis ${ }^{1}$, I Bacopoulou ${ }^{1}$, M Toumba $^{1}$, T Apsiotou ${ }^{1}$, $\mathrm{S} S$ Stavrou$^{1}$, T Georgiou ${ }^{1}$, S C Savva ${ }^{2}$

${ }^{1}$ Pediatric Endocrine Unit, Makarios Hospital, Nicosia, Cyprus, ${ }^{2}$ Research and Education Institute of Child

Health, Nicosia, Cyprus

Background and Aims: The incidence of type I diabetes mellitus (T1DM) has recently increased in some countries. The purpose of this study is to ascertain the incidence of T1DM in our population during 1990-2004, and detect any increasing trend.

Methods: All cases of newly diagnosed T1DM children under the age of 15 years were registered and relevant information was obtained. The population demographic data were provided by the Ministry of Finance and statistical analysis was processed with SPSS.

Results: The mean overall annual incidence of T1DM during this 15 -year period was: $11.95 / 100000$ with a statistically significant increase in the last five years. The incidence during the 5-year periods was as follow: (90-94): 10.50/100000, (95-99): 10.80/100000 (p: 0.55) and (00-04): 14.80/100000 (p: 0.0014). The overall female: male ratio was 1.06:1. There is a gender influence on the age of onset as more males manifest T1DM before age $6 \mathrm{yr}$ and after $13 \mathrm{yr}$. There was a significant variation in the number of newly diagnosed patients during spring compared to autumn $(\mathrm{p}=0.023)$ and winter $(\mathrm{p}=0.002)$. There is also a statistically significant difference in cases diagnosed in summer compared to winter $(p=0.012)$. Seasonal variation was marginal for patients diagnosed in the preschool age (0-4.99 years) and interestingly abolished in cases diagnosed during the period of high incidence 2000-2004.

Conclusions: The overall incidence of T1DM in Cyprus has increased during the last 5 years. The gender differences in the age of onset could be explained by the combination of genetic loading and pubertal hormonal changes.

\section{CURRENT DIETARY HABITS, PHYSICAL ACTIVITY AND OBESITY AMONG PRESCHOOL CHILDREN IN CRETE}

P Neonaki, M Mavrokosta, K Skoypas, E Stefanaki, G Vlachaki, E Kokori, A Tsilimigaki-Christaki

Pediatric Clinic, Venizeleio General Hospital, Iraklio, Crete, Greece

Background and Aims: Mediterranean diet (a greater intake of olive oil, cereals, legumes, fruits and vegetables) and physical activity seem to have been 
modified. We examined trends in diet, activity and the prevalence of obesity among a population of preschool children in our city.

Methods: Questionnaires about dietary habits, outdoor physical activity and time spent on TV, were completed by the parents of 380 children aged 3-6 years. 100 of the children were weighted and measured for height and their BMI was calculated.

Results: $43(11 \%)$ out of 380 children never take breakfast. $41(11 \%)$ consume junk food every day and 19 (5\%) never consume junk food. Only $145(38 \%)$ children consume fruit and vegetables daily and 7 (2\%) never eat fruit and vegetables. $90 \%$ of the children share at least one meal with their whole family, but the rest $10 \%$ share none. $258(68 \%)$ watch TV every day and $34 \%$ spend 2-3 hours daily on TV. 140 (37\%) rarely play outside and only $20(5 \%)$ play more than 3 hours per day in parks or playgrounds. BMI was estimated for 100 children, 52 were boys. $33 \%$ of those were overweight (BMI 75th - 95th percentile) and $12 \%$ were obese (BMI $>95$ th percentile).

Conclusions: The diet of our children, and their physical activity have been influenced by the western lifestyle. Those changes result in high prevalence of obesity in preschool children. This should lead prevention strategies to focus on promoting Mediterranean lifestyle which has been proven healthier.

\section{DAILY CALCIUM (CA) INTAKE OF GREEK SCHOOL-AGED CHILDREN}

D Karakaidos, G Kafalidis, G Triantafyllidis, F Papadelis, M Salapata, H Koufopoulou, C Karis

Department of Pediatrics, General Hospital of Nikea, Piraeus, Greece

Background and Aims: Adequate $\mathrm{Ca}$ uptake in childhood is essential in preventing osteoporotic changes later in adulthood. The aim of our study was the determination of the amount of daily calcium intake of school aged children of our country.

Methods: We diligently investigated the possible $\mathrm{Ca}$ sources contained in the diet of 712 children (368 boys344 girls) 6-12 years old living in an urban district by filling in a special questionnaire after interviewing their parents.

Results: The daily $\mathrm{Ca}(\mathrm{mg})$ intake per age and sex appears on the table. $95.8 \%$ of children 6 yrs old have adequate calcium intake (above the recommended for age, $>450 \mathrm{mg} / 24 \mathrm{~h}$ ), along with $81.8 \%$ of children $7-10$ yrs old $(>550 \mathrm{mg} / 24 \mathrm{~h})$. On the contrary, only $31,6 \%$ of children 11-12 yrs old have adequate daily calcium intake (>800mg/24h).

Conclusions: Daily calcium intake is satisfactory up to the age of 10. However, it decreases significantly thereafter, making the threat of osteoporosis more likely in future.

\begin{tabular}{|l|l|l|l|l|l|l|}
\hline & & $\begin{array}{l}\text { Boys } \\
\mathrm{mg} / 24 \mathrm{~h}\end{array}$ & & $\begin{array}{l}\text { Girls } \\
\mathrm{mg} / 24 \mathrm{~h}\end{array}$ & $\begin{array}{l}\text { Total } \\
\mathrm{mg} / 24 \mathrm{~h}\end{array}$ & $\begin{array}{l}\text { Recommended } \\
\text { intake(mg/24h) }\end{array}$ \\
\hline $\begin{array}{l}6 \\
\varepsilon \tau \dot{\mathrm{v} v}\end{array}$ & $\mathrm{n}=52$ & $867 \pm 233$ & $\mathrm{n}=44$ & $773+268$ & $(96) 824+249$ & $(>450 \mathrm{mg}) 95,8 \%$ \\
\hline $\begin{array}{l}7-10 \\
\varepsilon \tau \dot{\mathrm{v}}\end{array}$ & $\mathrm{n}=296$ & $710 \pm 264$ & $\mathrm{n}=244$ & $740+237$ & $(540) 724+252$ & $(>550 \mathrm{mg}) 81,8 \%$ \\
\hline $\begin{array}{l}11- \\
12 \\
\varepsilon \tau \dot{\omega} v\end{array}$ & $\mathrm{n}=20$ & $500+131$ & $\mathrm{n}=56$ & $765+278$ & $(76) 695+272$ & $(>800 \mathrm{mg}) 31,6 \%$ \\
\hline
\end{tabular}

\section{HEPATOSPLENIC AND MULTIFOCAL BONE MARROW INVOLVEMENT IN CAT-SCRATCH DISEASE}

D Vukelic, N Schoenwald, B Benic

University Hospital for Infectious Diseases, Zagreb, Croatia

Background and Aims: We describe a child with prolonged fever of unknown origin and prominent skeletal pain who had hepatic and splenic abscesses and multifocal bone disease caused by Bartonella infection.

Methods: Radiologic studies, including plain films, radionuclide scintigraphy and computed tomography, yielded positive results.

Results: Microbiologic diagnoses were established serologically.

Conclusions: Several atypical presentations of catscratch disease (CSD) have been recognized, including encephalopathy, Parinaud's oculoglandular disease, hepatic and splenic abscesses, osteomyelitis with lytic bone lesions, neuroretinitis and prolonged fever without source.

\section{HEMORRHAGIC SHOCK AND ENCEPHALOPATHY SYNDROME}

M F Nunes, L Oliveira, S Sarafana, V Silva, L Ventura, J P Vieira, D Barata

Department of Pediatric Intensive Care, Hospital de Dona Estefânia, Lisbon, Portugal

Background and Aims: Hemorrhagic shock and encephalopathy syndrome is a severe disease of unknown aetiology with significant mortality and neurological morbidity. It affects previously healthy infants of less than 1 year of age.

Methods: Clinical case.

Results: We describe a previously healthy five-monthold infant found unresponsive and with gasping respirations by his mother. He was taken to the emergency department of the regional hospital where he developed seizure activity. Physical examination revealed auricular temperature of $41^{\circ} \mathrm{C}$, gasping breathing pattern, hypotension and poor perfusion. Later then, he started to bleed from puncture sites. He initiated antiepileptic drugs, cardiovascular and respiratory support measures and was transferred to our facility. The seizure activity was refractory to benzodiazepines, phenytoin and responded to fenobarbital. Laboratory data revealed severe metabolic acidosis, abnormal renal functions, hypoglicemia, consumption coagulopathy and increased transaminase and CPK levels. Computed tomography of the head revealed no mass, hemorrhage or midline shift. Cerebrospinal fluid analyses were normal. Blood, urine and cerebrospinal fluid cultures were negative. Ammonia levels were normal. Serologic test for hantaviruses and arboviruses were negative. He required continuous midazolan infusions, phenytoin and fenobarbital for seizure control, vasopressor and blood transfusions for cardiovascular support and mechanical ventilation. Despite aggressive management, his neurological condition deteriorated with signs of raised 
intracranial pressure and died on the 7 th day of admission.

Conclusions: Although rare, hemorrhagic shock and encephalopathy syndrome should be considered in the differential diagnosis of shock, convulsions and coma, coagulopathy, fever, metabolic acidosis and hepatorenal dysfunction.

\section{MULTI ORGAN FAILURE IN CHILDREN DUE TO ACUTE WOOD PRESERVATIVES POISONING}

\author{
E Pietraszek-Jezierska ${ }^{1}, \mathrm{M} \mathrm{Migdal}^{1}, \mathrm{~S}$ Prokurat $^{2}$, \\ A Siwinska-Ziolkowska ${ }^{3}$ \\ ${ }^{1}$ Department of Anaesthesiology and Intensive Care, \\ Childrens Memorial Health Institute, Warsaw, Poland \\ ${ }^{2}$ Department of Nephrology and Kidney \\ Transplantation, Childrens Memorial Health Institute, \\ Warsaw, Poland, ${ }^{3}$ Department of Forensic Medicine, \\ Medical Academy, Warsaw, Poland
}

Background and Aims: In Poland acute poisoning in children is estimated of approximately 80000 cases a year. $1,5 \%$ of them require PICU admittance, 120 of them die. One of the most serious clinical courses leading to multi organ failure is observed in acute chromium-copper intoxication. The aim of the study was to analyse the outcome of such cases.

Methods: Three children admitted to our PICU during the last 5 years with signs of multi organ failure following accidental digestion of wood preservative products. Two girls (age 4 and 11 years) and one boy (age 5) referred to the PICU in 47, 80 and 94 hours after intoxication.

Results: On admission cardiovascular, renal and liver failure as well as ARDS and brain oedema had been diagnosed. The oldest girl required CPR immediately and died 1 hour later. Two other patients were electively intubated and ventilated using PRVC mode. Both of them were qualified for urgent combined liver-kidney transplantation. As a bridge before transplantation the extracorporeal MARS procedure was started. In each case two MARS sessions were performed. Despite of intensive treatment both children died in 137 and 51 hours after admission (184 and 145 hours after intoxication). At the meantime no required organ for the transplantation was available. Post mortem analysis confirmed lethal chromium and copper concentration in blood and tissues.

Conclusions: Despite of significant progress in multi organ failure the outcome of chromium-copper acute intoxication still remains fatal.

\section{PATENT DUCTUS ARTERIOSUS - INCIDENCE, RISK FACTORS AND TREATMENT OPTIONS}

\author{
A I Toma ${ }^{1}$, D F Albu ${ }^{2}$, M Mitran ${ }^{2}$, M Scheiner ${ }^{1}$, \\ A Zamfirescu ${ }^{4}, \mathrm{M} \mathrm{Iordachescu}^{3}$ \\ ${ }^{1}$ Department of Neonatology, Panait Sarbu Obstetrics \\ and Gynecology Hospital, Bucharest, Romania, \\ ${ }^{2}$ Department of Obstetrics, Panait Sarbu Obstetrics and \\ Gynecology Hospital, Bucharest, Romania, ${ }^{3}$ Department \\ of Pediatrics, Mother and Child Institute, Bucharest,
}

Romania, ${ }^{4}$ Department of Pediatrics, 'Victor Gomoiu' Hospital, Bucharest, Romania

Background and Aims: To find the incidence of the Patent Ductus Arteriosus (PDA) in our unit, the time of occurrence and the risk factors in order to establish a strategy for prevention.

Methods: The population studied was represented by the Very Low Birth Weight Infants (VLBW) born between January 2004-January 2006. The diagnosis of PDA was established both clinically and by ultrasound. The treatment options were represented by fluid restriction only or fluid restriction + indomethacin i.v.

Results: In the study period, there were born 112 VLBW infants. The incidence of PDA was 29.5\% (31 cases). There were 2 period at risk for the occurrence of PDA - days of life 2-3 (12 cases) and 14 (7 cases). The risk factors identified were represented by the excess of fluids administered to the PDA group in the first 2 days of life $(\mathrm{p}<0.004)$ and fluids over $140 \mathrm{ml} / \mathrm{kg}$ in days $13-14(\mathrm{p}<0.0056)$. From the 31 cases, the date rate was $5 / 16$ in the early group (days 2-3) and 2/15 in the rest of the babies. The fluid restriction was efficient in closing the PDA in 20/31 cases and Indomethacin (1 course of 3 doses) closed the PDA in another 4 cases.

Conclusions: PDA appears in the case of excess fluid administration in a VLBW neonate. The periods of maximum risk are days 2-3 of life and day 14 of life. Fluid restriction resulted in closure of $2 / 3$ of the PDA.

\section{AUDIT OF PICU FOLLOW-UP CLINICS}

F Rajah ${ }^{1}$, B Pai $^{2}$, M O'Meara ${ }^{1}$, J L Luntley ${ }^{3}$, J Alderson $^{1}$, S M Whiteley ${ }^{1}$

${ }^{1}$ Paediatric Intensive Care Unit, St James's University Hospital, Leeds, UK, ${ }^{2}$ St Peter's Hsopital, Chertsey, Surrey, United Kigndom, 'Alberta Children's Hospital, Calgary, AB, Canada

Background and Aims: Mortality is an easily measurable outcome following PICU. We were interested in identifying other less easily measurable adverse outcomes including physical and psychological morbidity in patients and their carers. In January 2002 we established a PICU follow-up clinic to identify these outcomes and offer appropriate support.

Methods: Patients admitted to a tertiary PICU (oncology, hepatology, renal and general paediatric) ventilated for $>24$ hours, and alive at three months post discharge were offered clinic appointments. We carried out a retrospective review of clinic records of 54 consecutive patients invited to clinic during 2002-3.

Results: Of 54 patients, 31 (56.4\%) attended, 22 $(40.7 \%)$ failed to attend and 1 patient died before attendance. Patients were more likely to attend if they lived locally ( $54.8 \%$ attenders v $46.5 \%$ nonattenders), or were admitted from the general paediatric population with respiratory or infectious diseases (65.38\%). Reasons for non attendance included continued hospitalisation and ongoing outpatient treatment under a tertiary specialist. In $18(58 \%)$ of attenders there were no problems. Morbidity was identified in 10 (32\%) of patients. Soft tissue injury including pressure sores (4), nail bed injury (1) extravasation injury (1) were 
commonest. 1 patient suffered an arterial injury requiring revascularisation. 4 patients complained of severe emergence phenomenon. 1 patient, 1 sibling and 4 parents were identified as suffering Post-traumatic stress disorder.

Conclusions: PICU follow-up clinics can provide useful information regarding physical and psychological morbidity in patients and carers. Patients are more likely to attend if they live locally and are not requiring long term specialist follow up.

\section{EFFECT OF DIRECT ADMINISTRATION OF GROWTH HORMONE ON RAT CHONDRO- COSTAL CARTILAGE - AN ANIMAL MODEL FOR THE STUDY OF PECTUS EXCAVATUM}

\author{
A Radulescu ${ }^{1,2}$, E S Boia ${ }^{1}$, M C Popoiu ${ }^{1}$, O Adam ${ }^{1}$, \\ R Iacob ${ }^{1}$ \\ ${ }^{1}$ Department of Pediatric Surgery, Children's Hospital \\ 'Louis Turcanu', Timisoara, Romania, ${ }^{2}$ Department of \\ Pediatric Surgery Research, Children's Hospital \\ Columbus, Columbus, OH, USA
}

Background and Aims: Pectus excavatum a chest wall deformity seen in children is determined by the excess growth of the chondrocostal cartilage. A new nonsurgical alternative approach for treatment of this malformation could consist in control of the cartilage growth.

Methods: The local and systemic effects of human growth hormone, injected at the surface of intact chondrocostal cartilage in Sprague Dawley rats were studied. Two weeks old rats $(\mathrm{n}=16)$ were divided into an experimental $\mathrm{E}$ and a control $\mathrm{C}$ group. The chondrocostal cartilages of the right hemi thorax were injected every two days for 21 days. Body weight and blood concentration of the growth hormone, was measured one hour after the injection. Chondro-costal cartilage was harvested for histological analysis.

Results: Blood analysis one hour after local injection, showed a significant increase in growth hormone levels in the experimental group compared with the control (C) group ; E group; $61.14 \pm 35.87 \mu$ U.I. / ml vs. C group; $0.15 \pm 2.56 \mu \mathrm{U} . \mathrm{I}$. $/ \mathrm{ml}, \mathrm{p} 0.003$ The growth hormone injected, did not influence the body weight of the rats in the experimental group when compared with the control group E ; $26.12 \pm 25.52$ gr. vs. C $22.87 \pm 34.98$ gr., p 0.7953 Histological analysis confirmed the presence of ossification and calus formation at the site of injection and modified shape of the cartilage was noted at gross inspection.

Conclusions: These results encourage us to further investigate the possibility of a locally applied therapy in case of pectus excavatum.

\section{OUTCOMES OF PATIENTS REQUIRING PAEDIATRIC RESPIRATORY ECMO SUPPORT}

N Pathan, E Smith, A Goldman, K Brown

Department of Cardiac Critical Care, Great Ormond Street Hospital, London, UK
Background and Aims: Paediatric respiratory is one of the most diverse groups of patients supported with ECMO, with a total of 3,064 paediatric respiratory cases on the ELSO registry, and an overall survival to discharge of $56 \%$. There is little published descriptive data on this group. Our aims were to analyse the background and clinical course of children requiring respiratory ECMO support, in particular to assess the effects of pre-existing co-morbidity on ECMO need and outcome, and the changes in respiratory ECMO support over the last 12 years in our institution.

Methods: Retrospective review of paediatric respiratory ECMO cases at Great Ormond Street Hospital between July 1992 - December 2005, with no cardiac lesions or primary cardiac reason for ECMO support.

Results: 123 cases were supported over the study period. The overall survival to hospital discharge was $61.8 \%$. Median duration of ECMO support was 9 days. A high proportion $(50.4 \%)$ of children had pre-existing co-morbidities. Of patients who survived discharge from hospital, there was a late survival of $59.3 \%$, with post discharge deaths ranging from 14 days to 4.4 years after ECMO support. Amongst patients with comorbidities, there was significantly lower survival in those with chromosomal defects, sickle cell disease, tracheal patients, and patients with haematological or oncological diagnoses.

Conclusions: A high proportion had a pre-existing medical condition that put them at increased risk of critical illness. Co-morbidity was also associated with a high proportion of late mortality. Survival, as expected, depends on underlying condition and reason for ECMO, ranging from $31-100 \%$.

\section{BODY MASS INDEX AND CHANGING IN BLOOD PRESSURE AS RISK FACTORS FOR CORONARY DISEASE}

D P Nikolic ${ }^{1}, Z_{\text {Milincic }}^{2}$, S Simeunovic ${ }^{2}$, D Simeunovic $^{3}$, M Bajeetic ${ }^{4}$

${ }^{1}$ School of Medicine, University of Belgrade, Belgrade, Serbia and Montenegro, ${ }^{2}$ University Childrens Hospital, University of Belgrade, Belgrade, Serbia and Montenegro, ${ }^{3}$ Clinical Center of Serbia, Belgrade, Serbia and Montenegro, ${ }^{4}$ Institute of Pharmacology, University of Belgrade, Belgrade, Serbia and Montenegro

Background and Aims: In our study we monitored children from YUSAD study group that begun 8 years ago. Aim of our study was to determine the movement of both systolic and diastolic blood pressure in children and Body Mass Index (BMI) during 5 year period.

Methods: Clinical examination was performed and blood pressure (BP) was measured. Results were compared with nomograms for age and gender.

Results: Systolic BP was analyzed in 4243 children. Diastolic BP was analyzed in 3048 children. During 5 years of follow-up period in $3.55 \%$ of children systolic BP worsened and in $2.92 \%$ of children systolic BP improved. In $1.93 \%$ of children diastolic BP worsened 
and in $0.78 \%$ of children diastolic BP improved. In 3525 children age of 10 years $(49.82 \%$ girls and $50.18 \%$ boys) and in 3439 children age of 15 years ( $49.98 \%$ girls and $50.02 \%$ boys), BMI was (median value \pm standard deviation): Girls: BMI 16,96 $\pm 2,91$ and $20,54 \pm 3,41$. Boys: BMI 17,16 $\pm 2,82$ and $17,18 \pm 2,98$.

Conclusions: During 5 year period increase and decrease in values of systolic BP were frequent than same changements of diastolic BP. During same period elevation of BMI in girls compared to boys was detected.

\section{THE RISK OF CARDIO-VASCULAR DISEASES AT CHILDREN}

\section{Tomic, S Smugreska, S Asani, L Nikoloska \\ Childrens Department, City Hospital, Tetovo, Macedonia}

Background and Aims: Cardio-vascular diseases have become a leading in the world. Arterioscleriscleroses starts during childhood, but clinical occurrence is later in life. Aim of this work is to show risk factors for occurrence of arteriosclerosis and cardio-vascular diseases at children.

Methods: Interview screening of 250 children (age of 13 and 14) of their weight, frequency of sport or fitness activity, time spent watching television, nutritious habits and smocking in the family.

Results: From total number of screened, 130 were girls, 120 boys. Obesity was found with $7 \%$ of girls and $13 \%$ of boys.

Conclusions: An average child at the age of 13 ate junk food, watch TV for 3-5 hours a day, doesn't do sports (or unsatisfactory), and is passive smoker. So, still in the school age all factors of risk for arterioscleroses are present. There is need of huge action for promoting healthy lifestyle and eliminating bad habits. This will not only prevent cardio-vascular diseases, but it will also bring more quality and balance in life.

\begin{tabular}{|c|c|c|c|c|}
\hline \multirow{2}{*}{$\begin{array}{l}\text { Froposy of } \\
\text { pandicing spats }\end{array}$} & m pots & 1-2ting/ wouk & $30 \times$ mont $t=1$ & \\
\hline & 497 & $33 x$ & $18 x$ & \\
\hline \multirow{2}{*}{$T=\mathrm{TV}$} & 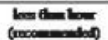 & 1-3 lows itury & 3.5 lowes/ding & -5 \\
\hline & $17 \pi$ & 397 & $30 x$ & $11 \%$ \\
\hline \multirow{2}{*}{ 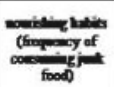 } & 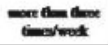 & $1.2 \mathrm{in}$ an wot & Dexterex & \\
\hline & $35 \%$ & $30 \%$ & $2 \pi x$ & \\
\hline \multirow{2}{*}{ (finpoly of } & $n=\alpha \cos \alpha$ & $1-2$ in ate not & 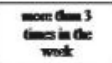 & \\
\hline & $5 \pi x$ & $11 \%$ & $2 \%$ & \\
\hline \multirow{2}{*}{ 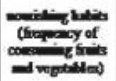 } & mex arver & motinfory & Adyly best & \\
\hline & $10 \%$ & $15 x$ & $75 x$ & \\
\hline \multirow{2}{*}{ mingenty } & 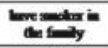 & bodichem & $\operatorname{molum}_{\operatorname{mox}}$ & moturie in \\
\hline & $34 \mathbf{x}$ & 23X & $17 x$ & $26 \%$ \\
\hline
\end{tabular}

\section{MANAGEMENT OF POST SURGICAL CHYLOTHORAX IN A TERTIARY CARDIOLOGY CENTER}

C Yaliwal $^{1}$, A Sohal ${ }^{2}$, V Agarwal ${ }^{2}$

${ }^{1}$ Torbay General Hospital, Torquay, UK, ${ }^{2}$ Alder Hey Hospital, Liverpool, UK
Background and Aims: The aim of this study was to audit the management of post cardiac surgical chylothorax in children presenting in Tertiary Cardiology Center and compare the practice against the local guidelines.

Methods: Records of children presenting to Alder Hey Children's hospital Cardiology ward for Open Heart Cardiac surgeries during Jan 2000 - Dec 2004 were randomly analysed using a structured chylothorax pathway proforma covering assessment and management. Retrospective, Single Institute, Random case note analysis.

Results: 72 children developed chylothorax as post operative complication and records of 35 were randomly selected and analysed.11/35 (31.4\%) were neonates and $12 / 35(34.2 \%)$ were between 1-12 months age group. Fallot's repair (14\%) and Cavo- pulmonary shunt $(11.4 \%)$ were a common cause for chylothorax. 21 $(60 \%)$ were tested for chyle due to the macroscopic appearance of pleural fluid and $7(20 \%)$ for effusions persisting for more than 48hours. 26 (74\%) were managed on Monogen feeds alone, 4 (11.4\%) with Octreotide, 1 with TPN and $3(8.5 \%)$ did not require any treatment. The median number of days chest drains were in-situ in all the cases was 12 (Range 4-27 days) and the length of stay in hospital with uncomplicated chylothorax was 21 days (Range 6-49). The morbidity associated with chylothorax resulting in prolonged length of stay in hospital was 21 days (Range 6-49) and prolonged chest drain in-situ was 12 days (Range 4 27). In Monogen resistant cases, Octreotide remains an effective alternative for treatment as observed in 4 (11.4\%) cases.

Conclusion: Monogen feeds remain the first line management of post cardiac surgical chylothorax.

\section{PULMONARY HYPERTENSION, HEART FAILURE AND NEUTROPENIA DUE TO DIAZOXIDE THERAPY}

D Yildizdas ${ }^{1}, \mathrm{~S} \mathrm{Erdem}^{2}, \mathrm{M} \mathrm{Yilmaz}^{3}$, O Kücükosmanoglu ${ }^{2}$, B Yuksel ${ }^{4}$

${ }^{1}$ Department of PICU, Çukurova University Faculty of Medicine, Adana, Turkey, ${ }^{2}$ Department of Cardiology, Çukurova University Faculty of Medicine, Adana, Turkey, ${ }^{3}$ Department of Pediatrics, Çukurova University Faculty of Medicine, Adana, Turkey, ${ }^{4}$ Department of Endocrinology, Çukurova University Faculty of Medicine, Adana, Turkey

Background and Aims: Primary persistent hyperinsulinaemic hypoglycemia of infancy is a rare disorder which is currently difficult to treat. Diazoxide treatment remains the mainstay of medical therapy. Tolerance of diazoxide is usually excellent, but several side effects of this drug have been described.

Methods: We present 4 month old girl who developed pulmonary hypertension, heart failure and neutropenia during diazoxide therapy. The patient was 3 months old infant, affected by persistent and severe hypoglycemia. Hyperinsulinism diagnosed and she was treated with octreotide $(10 \mu / \mathrm{kg} /$ day $)$. Then diazoxide $(15 \mathrm{mg} / \mathrm{kg} /$ day $)$ was added, because of inadequate control of blood glucose. After ten days later, she became dyspneic. 
Echocardiography revealed moderate pulmonary hypertension, RA/LA enlargement and LV enlargement. Although she administered iloprost, her clinical condition worsened and referred to our pediatric intensive care unit.

Results: On admission to pediatric intensive care unit, she had tachycardia, dyspnea, cyanosis, low oxygen saturation and CVP $15 \mathrm{cmH} 2 \mathrm{O}$. She was intubated and required mechanical ventilation and her white blood cell count: $3900 \mathrm{~mm} 3$. Because of side-effects, diazoxide was stopped on the 13th day. During the next three days his respiratory and hemodynamic status dramatically improved and she was weaned from mechanical ventilation. Control white blood cell count was 8800 $\mathrm{mm} 3$ and control echocardiography showed reduction of PAP to $20 \mathrm{mmHg}$ and resolution of atrial and ventricular enlargement.

Conclusions: Pediatric physicians should be in mind of pulmonary hypertension, heart failure and neutropenia developing during diazoxide therapy.

\section{PREVALENCE OF CONGENITAL HYPOTHYROIDISM IN THE GREEK CYPRIOT POPULATION: RESULTS OF THE NEWBORN SCREENING PROGRAM 1990 - 2003}

N Skordis ${ }^{1}$, M Toumba ${ }^{1}$, S C Savva ${ }^{2}$, A K Nicolaou ${ }^{3}$, $\mathrm{A} \mathrm{Nicolaou}^{3}, \mathrm{M} \mathrm{Vogazianos}^{3}, \mathrm{M} \mathrm{Iacovidou}^{3}$, A Argyriou ${ }^{3}$

${ }^{1}$ Pediatric Endocrine Unit, Makarios Hospital, Nicosia, Cyprus, ${ }^{2}$ Research and Education Foundation of Child

Health, Nicosia, Cyprus, ${ }^{3}$ Center of Preventive

Pediatrics, Limassol, Cyprus

Background and Aims: The purpose of this report is to evaluate the results of the screening program for Congenital Hypothyroidism $(\mathrm{CH})$ in the Greek Cypriot population, which was introduced in 1990.

Methods: During 1990 - 2003, 127530 neonates were screened with TSH determination. Permanent $\mathrm{CH}$ was proven after discontinuation of treatment at age 3 years. SPSS was used for statistical analysis

Results: Permanent $\mathrm{CH}$ was diagnosed on 70 infants incidence $1 / 1820$, with a female/male ratio $2.05 / 1$. The most common clinical findings were: Omphalocele (61\%) and large anterior fontanelle (49\%). The more delayed the bone maturation, the lower were initial T4 levels $(\mathrm{p}=0.005)$. Bone maturation was more delayed in thyroid agenesis $(\mathrm{p}=0.049)$. Scintigraphy of the thyroid with TC 99 revealed: Ectopia in 38\%, thyroid agenesis in $36 \%$, thyroid hypoplasia $24 \%$ and dyshormonogenesis in $1.7 \%$. Children with transient $\mathrm{CH}$ had initially lower T4 and higher TSH values, although initial TSH levels failed to predict the nature of $\mathrm{CH}$. Children with transient $\mathrm{CH}$ required less thyroxine dosage to maintain normal thyroid hormone levels. The TSH level was normalized before the age of 2 months with a starting L-thyroxine dose of $10 \mathrm{mcg} / \mathrm{kg} /$ daily. The developmental outcome of the children is within the normal range.

Conclusions: The incidence of $\mathrm{CH}$ in Greek Cypriots is $1 / 1820$ live births. The most common etiology is thyroid dysgenesis. Initial T4 levels correlated with the degree of skeletal maturation, and the etiology. Initial TSH level, although lower in children with transient $\mathrm{CH}$, could not predict the nature of $\mathrm{CH}$.

\section{THE EXPRESSION OF VEGF AND ITS RECEPTORS IN THE DEVELOPING HUMAN DUCTUS ARTERIOSUS}

S C Weber ${ }^{1}$, C Rheinlaender ${ }^{1}$, E Strauss ${ }^{1}$, C Peiser ${ }^{1}$, N Sarioglu ${ }^{2}$, M Obladen ${ }^{1}$, P S Koehne ${ }^{1}$

${ }^{1}$ Department of Neonatology, Charité, VirchowKlinikum, Universitätsmedizin Berlin, Berlin, Germany ${ }^{2}$ Department of Paidopathology, Charité, VirchowKlinikum, Universitätsmedizin Berlin, Berlin, Germany

Background and Aims: Programmed proliferative degeneration of the human fetal ductus arteriosus (DA) in preparation for its definite postnatal closure has a large developmental variability and is controlled by several signalling pathways. VEGF plays an important role in the formation of the neointima and vasa vasorum ingrowth during permanent closure of the DA.

Methods: We examined ductal protein expression of VEGF, VEGF-R1 and VEGF-R2 in 51 human fetal autopsy DA specimens between 11-38 weeks of gestation which were collected over 12 months at the Charité Department of Paidopathology. According to their histological maturity specimens were classified using a new maturity score. Immunohistochemistry was performed on paraformaldehyde-fixed specimens following a standard protocol. Immunohistochemical staining was evaluated semiquantitatively by three independent observers using a discrete scale with 0.5 intervals varying from ' 0 ' for no staining to ' 3 ' for strong staining.

Results: VEGF, VEGF-R1 and VEGF-R2- staining was detected in all three maturity stages. VEGF-staining was localized perinuclearly in all three vascular regions and did not alter during development. VEGF-R1 expression was profound in the endothelium in early maturity stages and decreased during development. In contrast VEGF-R2 predominated in later developmental stages in the muscular layer.

Conclusions: Our results emphasize the importance of VEGF as a mediator during proliferative degeneration of the fetal ductus and support the hypothesis that VEGF$\mathrm{R} 1$ is required for normal blood vessel development during embryogenesis while VEGF-R2 is the predominant receptor in later angiogenic signalling.

\section{SERUM LEPTIN LEVELS OF CHILDREN WITH CONGENITAL HEART DEFECTS WITH LEFT TO RIGHT SHUNT AND RELATION WITH HEMODYNAMIC AND GROWTH PARAMETERS}

B Varan ${ }^{1}$, A Olali ${ }^{1}$, I Cetin ${ }^{1}$, U A Orun ${ }^{1}$, M Yucel $^{2}$, S Kinik ${ }^{3}, \mathrm{~K}$ Tokel $^{1}$

${ }^{l}$ Department of Pediatric Cardiology, Baskent University, Faculty of Medicine, Ankara, Turkey ${ }^{2}$ Department of Biochemistry, Baskent University, Faculty of Medicine, Ankara, Turkey, ${ }^{3}$ Department of Pediatric Endocrinology, Baskent University, Faculty of Medicine, Ankara, Turkey 
Background and Aims: To investigate the relation of serum leptin levels with nutritional status and with hemodynamic and echocardiographic parameters in patients with left-to-right shunt.

Methods: From January till December 2004, 40 patients with left-to-right shunt aged 3 months to 2 years old were included in the study. Cardiac diagnoses included 20 ventricular septal defect (VSD), 5 atrial septal defect (ASD), 6 patent ductus arteriosus (PDA) and 9 patients with a combination of VSD, ASD or PDA. The patients above $80 \%$ of their ideal weight for height were group 1 and those below $80 \%$ were group 2. Echocardiographic parameters included left ventricular mass index $(\mathrm{LVM} / \mathrm{h} 2.7)$ and relative wall thickness. Pulmonary artery pressure and ratio of pulmonary to systemic flow (Qp/Qs) were determined during cardiac catheterization. The serum leptin, glucose and insulin levels were measured after 8 hours of fasting.

Results: Serum leptin levels were lower in patients with body weight less than $80 \%$ of ideal weight for height than those above $80 \%(2.27 \pm 1.20$ vs $2.58 \pm 0.92 \mathrm{ng} / \mathrm{mL}$, $\mathrm{p}=0.041)$ and in patients with pulmonary hypertension $(\mathrm{PH})$ than those without $(2.15 \pm 1.28$ vs $2.67 \pm 0.80$, $\mathrm{p}=0.011)$. The LVM index was greater in patients with $\mathrm{PH}$. Serum leptin levels showed a negative correlation with pulmonary artery pressure, Qp/Qs, and LVM index.

Conclusions: Patients with left-to right shunt lesions having $\mathrm{PH}$ and high pulmonary to systemic flow ratio have lower weight and height. Their serum leptin levels are also lower which may be related to their cardiac hypertrophy.

\section{SUPPORTING FAMILIES OF CHILDREN WITH SEVERE CONGENITAL HEART DISEASE AT HOME USING VIDEOCONFERENCING}

\author{
B A McCrossan, G J Morgan, B Grant, A Sands, \\ B Craig, F Casey \\ Department of Paediatric Cardiology, Royal Belfast \\ Hospital for Sick Children, Belfast, UK
}

Background and Aims: For parents of children with complex congenital heart disease, the immediate period following discharge from hospital is an anxious time. Many families feel isolated and need access to professional help at times other than their regular review. This study aims to evaluate the feasibility of videoconferencing to support families at home and its effect on parental anxiety and use of NHS services.

Methods: Randomised controlled clinical trial comparing a videoconferencing protocol with regular telephone contacts or "usual" out patient follow up currently provided. Inclusion criteria: 1. Severe congenital heart defect and 2. Significant support anticipated after discharge. Qualitative and quantitative data (including O2 saturations \& STAI questionnaires) collected to assess impact of videoconferencing service on families' quality of life and use of NHS services.

Results: 50 families: Videoconference support $n=21$, Telephone support $n=16$, "Usual follow up" $n=13$. Age range: 1 month -3 years. No. videoconferences: Range 6-15, Mean 8. 100\% parents felt the videoconferences beneficial and reassuring. $72 \%$ of videoconference advice would have been different if telephone only available, 58\% telephone advice may have been different if videoconferencing available. Preliminary results suggest videoconferencing provides significant benefits over telephone follow-up in providing reassurance and support. No situations of clinical risk or near misses. Initially, start up and running costs were high but these are now significantly reduced.

Conclusions: Initial results suggest that videoconferencing provides significant benefits over telephone follow-up in providing reassurance and support to families of children with severe congenital heart disease. There are obvious applications for this study in other medical specialities.

\section{LATE EFFECTS ON CARDIAC FUNCTION IN SEPTIC SHOCK SURVIVORS}

J J Sol ${ }^{1}$, H Knoester ${ }^{1}$, I M Kuipers ${ }^{2}$, S A Clur ${ }^{2}$, A P Bos ${ }^{1}$

${ }^{1}$ Pediatric Intensive Care Unit, EKZ AMC, Amsterdam, The Netherlands, ${ }^{2}$ Department of Pediatric Cardiology, EKZ AMC, Amsterdam, The Netherlands

Background and Aims: Septic shock (SS) is a lifethreatening disease, characterized by impaired myocardial contractility and loss of vascular tone, often requiring treatment with vasocontrictive agents (VA). Data on the long term effects of SS and VA on the developing myocardium in SS-survivors are lacking. We hypothesize that the combination of the underlying illness and the side-effects of its treatment will result in permanent damage of the developing heart in children.

Methods: SS survivors admitted to our tertiary PICU between 1995 and 2004 who had received $\geq 24$ hours administration of VA's were included. Cardiac function was evaluated by ECG in rest and during exercise (when $>7$ years), 24-hours-ECG-registration and echocardiography. Age at admission and follow-up, severity of illness (PIM II score), length of PICU stay were evaluated.

Results: Ninety of 124 eligible patients were evaluated. In 14 patients abnormalities were detected; such as episodes of ventricular extrasystole during and after exercise $(n=3)$, rhythm disturbances on 24-hours-ECG registration $(\mathrm{n}=2)$, mild left ventricular hypertrophy $(\mathrm{n}=1)$ and mild systolic dysfunction of the right ventricle $(n=8)$ on echocardiography. Patient characteristics were not statistically different between patients with and without abnormalities.

Conclusions: A $15 \%$ prevalence of ventricular dysrhythmia and/or mild dysfunction of the right ventricle is seen in 90 SS survivors, 1-10 yrs after admission. Since ventricular rhythm disturbances could lead to sudden cardiac death and the meaning of this dysfunction of the right ventricle in developing children is not known, long term follow-up of survivors of SS in childhood is essential. 


\section{CHRONIC GRANULOMATOUS DISEASE IN PAEDIATRICS: 25 YEARS EXPERIENCE IN A SINGLE CENTRE}

C Margareto ${ }^{1}$, P Soler $^{1}, \mathrm{O}$ Asensio ${ }^{2}, \mathrm{M} \mathrm{Hernández}^{1}$, I Caragol $^{1}, \mathrm{~T}^{\mathrm{T}}$ Español ${ }^{1}$

${ }^{1}$ Pediatric Immunology Unit, Vall D'Hebron Hospital, Barcelona, Spain, ${ }^{2}$ Paediatric Pneumology Unit, Sabadell Hospital, Barcelona, Spain

Background and Aims: Chronic Granulomatous Disease (CGD), an inherited disorder of phagocytic cells, results from inability of phagocytes to undergo the respiratory burst to kill certain types of bacteria and fungi leading to life-threatening infections. Our aim was reviewing clinical, analytical and epidemiological features in patients diagnosed of CGD in our hospital.

Methods: clinical charts of 12 patients diagnosed during childhood in our centre between 1980 to 2005 were retrospectively reviewed.

Results: All males. Median age at diagnose: 36.5 months. Onset symptoms: lymphadenitis and cervical abscesses 3/12 (Staphylococcus aureus, Serratia liquefaciens y Klebsiella sp.), pneumonia 3/12, (Rhodococcus equi, Salmonella typhimurium and Pneumocistis jiroveci), osteomyelitis 1/12 (Aspergilus sp.), septicaemia 1/12 (Staphylococcus aureus), urinary tract infections 1/12 (Klebsiella sp.), aphtous ulcers of the mouth $1 / 12$ and Crohn like colitis 1/12. Initial diagnostic by Nitroblue Tetrazolium Test (NBT) and confirmed by oxidation flux citometry test $9 / 12$ and genetics (gp91phox) 8/12. Patients presented 83 infections: abscesses (26), pneumonia (10), lymphadenitis (9), enteric infections (7), septicaemia (4), osteomyelitis (3) and others (24). Responsible microorganisms (38): Aspergillus sp. (10), Staphylococcus sp. (5), Salmonella sp (4), Pseudomonas aeruginosa (4), and others (15). Patients were treated with interferon-gamma $(5 / 12)$ and received prophylactic itraconazole (9/12) and/or cotrimoxazole (11/12). Four patients died: 2 septicaemia (invasive aspergillosis, unidentified negative gram bacillary infection), 1 disseminated leishmaniasis, 1 kidney transplant complications.

Conclusions: clinical suspicion and flow citometry are basic in diagnosing CGD patients and related familiars. Specific prophylaxis and medical care should be promptly established to prevent severe infections. Interferon-gamma has been intermittently used although its use remains controversial.

\section{SHORT TERM OUTCOME OF PEDIATRIC HEMATO-ONCOLOGY PATIENTS ADMITTED TO THE PICU AND VALIDATION OF A NOVEL RISK SCORE TO ASSESS ACUTE MORTALITY}

B Houthoofd $^{1}$, J Verlooy ${ }^{2}$, V Bordon ${ }^{2}$, A De Jaeger ${ }^{3}$, C Dhooge ${ }^{2}$

${ }^{1}$ Department of Pediatrics, Ghent University Hospital, Ghent, Belgium, ${ }^{2}$ Department of Hemato-Oncology, Ghent University Hospital, Ghent, Belgium

${ }^{3}$ Department of Pediatric Intensive Care, Ghent

University Hospital, Ghent, Belgium
Background and Aims: The decision to treat children with cancer on a Pediatric Intensive Care Unit (PICU) is difficult because they suffer from an acute life threatening illness superimposed on a chronic life threatening disease. Recent data show improvement in outcome for critically ill cancer patients requiring PICU support. However, commonly used risk scores fail to predict acute mortality in these patients. To assess acute outcome of pediatric hemato-oncology patients admitted to the PICU for reasons other than routine postoperative care and to validate a new risk score.

Methods: Retrospective analysis.

Results: 90 PICU admissions (2000-2005) of 67 children with hemato-oncological disease. 18 children died on the PICU (mortality rate 20\%). 28 admissions (31\%) occurred during or after Hematopoietic Stem Cell Transplantation (HSCT) with higher mortality rate (36\%). In the HSCT group, 4 patients died from disease progression and 6 from transplant related complications. In the non HSCT group 6 children died from disease progression. For 56 admissions sufficient data were available to calculate Pediatric Risk of Mortality (PRISM) score and a novel risk score described by Meyer et al. Median PRISM score was higher in non survivors $(9$, range $0-18)$ as compared to survivors $(4$, range $0-20$ ) with significant overlap. Novel risk scores varied from 0 to 4 in non survivors (median 1) and from 0 to 5 in survivors (median 1).

Conclusions: Good short term survival $(80 \%)$. Worse outcome when admission after HSCT (64\%). Disease progression and HSCT related complications mean causes of death. Novel risk score failed to distinguish survivors from non survivors.

\section{CLINICAL VALUE OF CYSTATIN-C DETERMINATION IN CHILDREN WITH LYMPHOMA}

Y Koksal ${ }^{1}$, A Varan ${ }^{1}$, G Hascelik², M Buyukpamukcu ${ }^{1}$

${ }^{I}$ Department of Pediatric Oncology, Institute of Oncology, Hacettepe University, Ankara, Turkey

${ }^{2}$ Department of Microbiology and Clinical

Microbiology, Faculty of Medicine, Hacettepe

University, Ankara, Turkey

Background and Aims: Cystatin, a proteinase inhibitor, is involved in the intracellular catabolism of proteins. We aimed to investigate the changes and clinical value of the serum Cystatin C (CysC) concentration in children with lymphomas.

Methods: 23 newly diagnosed patients with lymphoma were included in this study. Male/female ratio was $15 / 8$ with a median age of 8 years (range: 3-17 years). Eleven patients were Hodgkin's lymphoma (HL) and 12 were non-Hodgkin's lymphoma (NHL). Cystatin C was studied at the time of diagnosis and in remission.

Results: In whole group, $\mathrm{CysC}$ concentrations at diagnosis and remission were $0.92 \pm 0.28 \mathrm{mg} / \mathrm{L}$ and $0.89 \pm 0.2 \mathrm{mg} / \mathrm{L}$, respectively ( $\mathrm{p}=0.7$ ). In NHL group, $\mathrm{Cys} \mathrm{C}$ concentrations at diagnosis and remission were $0.87 \pm 0.25 \mathrm{mg} / \mathrm{L}$ and $0.86 \pm 0.18 \mathrm{mg} / \mathrm{L}$, respectively $(\mathrm{p}=0.9)$. The $\mathrm{CysC}$ concentration of the HL group at diagnosis and remission were $0.92 \pm 0.14 \mathrm{mg} / \mathrm{L}$ and $0.98 \pm 0.36 \mathrm{mg} / \mathrm{L}$, respectively $(\mathrm{p}=0.67)$. No significant 
difference was observed between HL $(0.95 \pm 0.35 \mathrm{mg} / \mathrm{L})$ and NHL $(0.94 \pm 0.33 \mathrm{mg} / \mathrm{L})$ groups statistically. Cystatin $\mathrm{C}$ concentrations in localized and advanced disease were $1.07 \pm 0.38 \mathrm{mg} / \mathrm{L}$ and $0.89 \pm 0.3 \mathrm{mg} / \mathrm{L}$, respectively $(\mathrm{p}=0.31)$. Cystatin $\mathrm{C}$ concentrations of the patients with localized and advanced HL were $1.11 \pm 0.4 \mathrm{mg} / \mathrm{L}$ and $0.77 \pm 0.14 \mathrm{mg} / \mathrm{L}$, respectively, $(\mathrm{p}=0.1)$. In one patient with localized NHL, CysC concentration was 0.85 $\mathrm{mg} / \mathrm{L}$, whereas in patients with advanced NHL, CysC concentration was $0.95 \pm 0.35 \mathrm{mg} / \mathrm{L}$.

Conclusions: Even though patient population is a small group in this study, CysC level was higher in localized than advanced disease. This study has been supported by a grant from Terry Fox Research Foundation

\section{RBC TRANSFUSIONS IN THE PEDIATRIC INTENSIVE CARE DEPARTMENT: THE EFFECT OF EDUCATION}

\section{Bogaert, K F M Joosten}

Department of Pediatric Intensive Care, Erasmus MCSophia Children's Hospital, Rotterdam, The Netherlands

Background and Aims: A paucity of clinical evidence with respect to red blood cell (RBC) transfusion in critically ill children has resulted in a large variation in transfusion practice and guidelines among paediatric intensivists. The lack of consensus might result in the use of liberal hemoglobin $(\mathrm{Hb})$ thresholds for RBC transfusions. However, in critically ill adults a restrictive transfusion strategy $(\mathrm{Hb}<4.3 \mathrm{mmol} / \mathrm{L})$ has been shown to be at least as effective as and possibly superior to a liberal regime. The aim of the study was to evaluate the effect of education on the transfusion practice in our PICU.

Methods: All RBC transfusions prescribed between April 2005 and March 2006 with respect to age, $\mathrm{Hb}$ threshold and transfusion volume were analysed and compared with a similar survey performed between April 2004 and April 2005. All first RBC transfusions per child per admittance were included in the analysis.

Results: 30 of the 540 admitted children received a RBC transfusion (5.6\%) compared to 56 of 591 children admitted $(9.5 \%)$ in the year previously. The average $\mathrm{RBC}$ transfusion threshold was $4.6 \mathrm{mmol} / \mathrm{L}$ (range 2.8 6.3) compared to $5.2 \mathrm{mmol} / \mathrm{L}$ (range 2.6-6.6). A transfusion threshold of $\mathrm{Hb}<5.6$ and $<5.2 \mathrm{mmol} / \mathrm{L}$ was recorded in $94 \%$ and $83 \%$ of these children, respectively, versus $78 \%$ and $52 \%$ in the year previously.

Conclusions: After education a trend towards lower $\mathrm{Hb}$ transfusion thresholds was noticed even in the absence of $\mathrm{RBC}$ transfusion guidelines. We hypothesize that the introduction of universal RBC transfusion guidelines will maximize this policy.

\section{SCREENING HEALTHY INFANTS IN THE POLISH COUNTRYSIDE FOR IRON DEFICIENCY}

T Jackowska ${ }^{1}$, J Dziadek ${ }^{2}$
${ }^{1}$ Department of Pediatric Hematology and Oncology, Medical University, Warsaw, Poland, ${ }^{2}$ Family Practice, Gruta, Poland

Background and Aims: Iron deficiency anemia (IDA) is the most common nutritional problem in the world. The aim of the study was to assess the frequency of iron deficiency (ID) and the occurrence of IDA in children treated by family doctor in the Polish countryside.

Methods: A prospective observational cohort study of 146 healthy 7-to 48-month-old-infants from a village, primary care clinic were screened for ID between September 2005 and November 2005, and followed up for a median of 24 months. ID (serum iron $<50 \mu \mathrm{g} / \mathrm{dl}$ ) and IDA (hemoglobin $<11 \mathrm{~g} / \mathrm{dl}$ or/and serum iron $<50 \mu \mathrm{g} / \mathrm{dl}$ ) were diagnosed. No symptoms of anemia were noted. Health Found only these tests are available for doctors in such clinics.

Results: Of 146 infants enrolled, 13 (11\%) had ID and 23 (15.7\%) had IDA. Average hemoglobin (Hb-12.3) levels, erythrocyte count (RBC-4.8), mean cell volume (MCV-78.1) and serum iron (Fe-81) were normal. $\mathrm{Hb}$ level $<11 \mathrm{~g} / \mathrm{dl}$ was observed in 16 children. Iron level $<$ $50 \mathrm{ug} / \mathrm{dl}$ (normal range 50-170) was detected in 13 children. Ferrous treatment was started in 25 children.

Conclusions: We suggest that all children aged 12-48 months should be screening for IDA. Determination of hemoglobin is not the optimal way to identify children at risk from effects of iron deficiency as it identify patients who are iron-deficient but are not anemic.

\section{COEXISTENCE OF HEREDITARY SPHEROCYTOSIS AND ALPHA- THALASSAEMIA TRAIT: CASE REPORT}

T Jackowska ${ }^{1}$, M Maciag ${ }^{2}$, E Zdebska ${ }^{3}$, E Wagiel $^{1}$, E Mendek-Czajkowska ${ }^{3}$, J Spychalska $^{3}$, A Sajkowska ${ }^{3}$, B Burzyńska

${ }^{1}$ Department of Paediatric Haematology and Oncology, Medical University, Warsaw, Poland, ${ }^{2}$ Institute Biochemistry and Biophysics, PAS, Warsaw, Poland ${ }^{3}$ Department of Biochemistry, Institute of Haematology and Transfusiology, Warsaw, Poland

Background and Aims: Hereditary spherocytosis (HS) is the most common haemolytic anaemia in Poland. Until recently, it has been thought, that thalassaemia is not present among Polish population.

Methods: A 10-month-old girl, whose mother, grandmother and aunt suffer from HS, was admitted for diagnosis of anaemia. To check for HS we performed AGLT and EMA test, which binds specifically to the band 3 at lysine- 430 and specific proteins' defects by quantification of erythrocyte membrane proteins (calculated as $\mu \mathrm{g} / 106$ cells) by SDS-PAGE method. To evaluate the genetic basis of thalassaemia, we isolated the DNA from leukocytes and we sequenced the promoter region, 5'UTR,3'UTR,3exons and its flanking regions of the $\beta$-globin gene. Subsequently, we used PCR reaction to detect $\alpha$-thalassaemia deletions.

Results: Successive blood chemistry tests (performed at follow-ups) showed microcytic anaemia (Hb:10.6$2.3 \mathrm{~g} / \mathrm{dl} ; \quad \mathrm{RBC}: 4.59-5.17 \mathrm{M} / \mu \mathrm{L} ; \quad \mathrm{MCV}: 55.2-63.1 \mathrm{fl}$; MCH:23.1-22.4pg) with marginally elevated 
reticulocytes (17-22\%). Bilirubin level was normal or slightly elevated $\quad(0.5 \mathrm{mg} \%-2.1 \mathrm{mg} \%)$. Lactic dehydrogenase was elevated (LDH:733U/L). Ferrum level was first decreased $(46 \mu \mathrm{g} / \mathrm{dl})$, but returned to normal after treatment $(83 \mu \mathrm{g} / \mathrm{dl})$. The initially normal ferritin level $(43,4 \mu \mathrm{g} / \mathrm{dl})$, increased subsequently to $167 \mu \mathrm{g} / \mathrm{dl}$. AGLT was abnormal (170sec.)-less than 5 minutes. EMA was abnormal (61.04\%), with protein band 3 deficiency. We diagnosed hereditary spherocytosis. The MCV and MCH were markedly below the normal levels, diagnostic investigations for thalassaemia were performed. The levels of $\mathrm{HbA2}-3 \%$ and $\mathrm{HbF}-0,6 \%$ were normal. Direct sequencing of $\beta$ globin gene did not show any nucleotide changes in DNA sequence. The results of the PCR assays showed the $3.7 \mathrm{~kb}$ deletion one of $\alpha$-globin gene, indicating $\alpha+$ thalassaemia.

Conclusions: We diagnosed $\alpha$-thalassaemia $(\alpha \alpha /-\alpha 3.7)$ in a girl with hereditary spherocytosis.

\section{THE EFFECTS OF INFLAMMATION ON PLATELET INDICES}

\author{
V Vlacha $^{1}$, G Feketea ${ }^{2}$ \\ ${ }^{I}$ Department of Pediatrics, University Hospital of \\ Patras, Patras, Greece, ${ }^{2}$ Department of Pediatrics, \\ Hospital of Amaliada, Amaliada, Greece
}

Background and Aims: It is well known that the inflammation affects the platelet number. The aim of this study was to evaluate the effects of inflammation on platelet indices.

Methods: The admitted patients in a 12 month period, having at least one complete blood count (CBC) from the same coulter analyzer, were included in this study. The CBC, including the platelet indices: mean platelet volume (MPV), platelet distribution width (PDW) and platelet crit (PltCr) and the ESR were recorded.

Results: 1548 patients were included in the study. They had mean platelet number (Plt) $413 \pm 174 \quad \mathrm{x}$ 103 cells $/ \mathrm{mm} 3$ and mean ESR $32 \pm 27 \mathrm{~mm} / \mathrm{h}$. The Plt and platelet indices showed statistically significant differences between the patients with high ESR $(>50 \mathrm{~mm} / \mathrm{h})$ and the ones with low ESR $(<50 \mathrm{~mm} / \mathrm{h})$ : (Plt $\mathrm{p}<0.0001$, MPV $\mathrm{p}=0.04$, PDW $\mathrm{p}=0.02$, PltCr $\mathrm{p}<0.01)$. Grouping the patients according to their WBC; Group I: WBC $<4$ x 103cells $/ \mathrm{mm} 3$, Group II: WBC $4-15 \mathrm{x}$ 103cells/mm3, Group III: WBC $>15$ x 103cells $/ \mathrm{mm} 3$ ) statistically significant differences of the above parameters were recorded among the three groups: Plt $(p<0.0001)$, MPV $(p<0.0001)$, PDW $(p<0,0001)$, PltCr $(p<0.0001)$. Interestingly, the differences remained significant even when the platelet parameters were compared between the patients with high WBC $>15 \mathrm{x}$ 103 cells $/ \mathrm{mm} 3$ and low $\mathrm{WBC}<15$ x $103 \mathrm{cells} / \mathrm{mm} 3$. However, no differences were documented between the neutropenic and non-neutropenic patients.

Conclusions: The degree of inflammation affects the platelet number and the platelet indices.

\section{OUTCOME OF CHILDREN WITH HAEMATOLOGICAL / ONCOLOGICAL DISORDERS ADMITTED TO A TERTIARY PAEDIATRIC INTENSIVE CARE UNIT (PICU)}

R Jarugula, S Shah, D Pauldhas, M O'Meara, S M Whiteley

Paediatric Intensive Care Unit, St James University Hospital, Leeds, UK

Background and Aims: To assess outcome of children admitted to a tertiary PICU with Haematological / Oncological disorders between January 2000 - 2005.

Methods: Retrospective review of case notes. Patients admitted solely for stem cell harvest were excluded.

Results: 183 patients, mean age 7.4 years (range 3 weeks - 19 years) with Haematological / oncological disorders were admitted during this period. Main diagnoses were Leukaemia (36.6\%), Lymphoma $(16.9 \%)$, soft tissue tumours (28.4\%), other haematological disorders (8.2\%), CNS tumours (5.5\%) and bone tumours $(5.5 \%)$. Indications for admission included respiratory failure $(38.4 \%)$ haemodynamic support $(22.4 \%)$ post operative care $(20.8 \%)$, others (18.4\%). Febrile neutropenia $(42.6 \%)$ and culture proven sepsis (33.3\%) were common problems. Bacterial sepsis was identified in (63.9\%) fungal (18\%), viral $(6.5 \%)$ and mixed infections in $(11.4 \%)$. Mean duration of stay was 4.6 days. (Range 4 hours - 57 days) Overall mortality rate was $17.5 \%$. (Range $8.7-25 \%$ per year). Mortality was highest in the bone tumour group $(25 \%)$ and lowest in the CNS tumour group (0\%). 13 patients $(7.1 \%)$ required renal replacement therapy. The mortality in this group was $53.8 \%$. Febrile neutropenia and culture proven sepsis were associated with increased mortality $(21.8 \%$ and $26.2 \%)$. Mortality was highest in patients with mixed (71.5\%) and fungal (36.4\%) infection. Patients with negative cultures were more likely to be discharged $<48$ hours $(62.2 \%$ v $34.1 \%)$.

Conclusions: Overall mortality in haematology / oncology patients admitted to PICU is $17.5 \%$. Increased duration of stay, culture positive sepsis and renal replacement therapy are associated with worse outcome.

\section{FOOD ALLERGY: CAN PRICK TEST OR CAP VALUES PREDICT THE RESULT OF AN ORAL PROVOCATION CHALLENGE?}

S J Quevedo Teruel, M Centeno Jiménez, M F Sánchez Mateos, C Blanco Rodríguez, L Echeverría Zudaire

Unidad de Neumología y Alergia Infantil, Servicio de Pediatría, Hospital Severo Ochoa, Leganés (Madrid), Spain

Background and Aims: Cow's milk protein, egg and fish are the most common causes of food allergy. Oral provocation challenge is election test for diagnosis. The objective of this study is to know if we can establish a cut off point in prick test and specific IgE values to avoid oral provocation challenge and its possible adverse effects.

Methods: We studied three groups of patients that had allergy to cow's milk protein, egg and fish with a CAP 
value higher than 0,35 or prick test higher than $3 \mathrm{~mm}$. We collected their prick test and CAP values and we analysed with ROC curves which cuttoff point we can establish over of which it is very likely a positive result of open oral provocation challenge. It is used SPSS 13.0 version program.

Results: We collected 255 patients with cow's milk protein allergy, 125 with egg allergy and 57 with fish allergy. It was impossible to establish a cut off for prick and CAP values in patients with cow's milk protein, egg and fish allergy because of the cut off obtained is the highest value in all cases. (Cow's milk protein: Prick: 16mm, CAP: 100; Egg: Prick: 11mm, CAP: 3,86; Fish: Prick: $17 \mathrm{~mm}, \mathrm{CAP}: 12,5$ ).

Conclusions: There is not a CAP or prick test value over than which we can predict a positive result of oral provocation challenge. At present oral provocation challenge is the election test to diagnosis food allergy.

\section{CLINICAL AND GENETIC HETEROGENEITY IN FANCONI ANEMIA}

\section{Bali, A Godo, Q Kora, E Anastasi, M Xhafa, A Babameto \\ Department of Pediatrics, UHC 'Mother Theresa', Tirana, Albania}

Background and Aims: FA, an autosomal recessive chromosomal instability disorder, characterized by congenital anomalies, is the commonest type of inherited bone marrow failure syndrome. Objective: To describe clinical and genetic features in three patients with FA.

Methods: Clinical and genetic examinations was performed.

Results: Girl 10 year old, twin from a homozygote pregnancy. In the skin some cafe-au-lait spots $(>11)$ paraxial right lateral polydactyly. Pancytopenia; bone marrow: aplastic hemoglobin electrophoresis: $\mathrm{Hb}$ F$16.5 \%$ hypometropy. Karyotype: about $8 \%$ of analyzed cells with chromatics and isochromatics, chromosomal breakings and gaps. By the induction with DEB the number of cells with instability increased in $10 \%$ with the presence of radial figures of asymmetric type between the non homologue chromosomes. Girl, the other twin. Café-au-lait spots $(>14)$, lingual frenulum. $\mathrm{Hb}$ F-15.2\% Karyotype: $20 \%$ of cells the same abnormality increased in $22 \%$ by the induction with DEB. Boy 4 years old: vescico-urethral reflux, at voiding cystography, horseshoe kidney, pelvic position. Café-au-lait spots, $(>11)$. The thumbs of the right hand rudimental; thumb of left hand-distal phalange duplex. Hb F- $11.4 \%$; kariotype: $2 \%$ of analyzed cells - the same alterations, increased in $12 \%$, by the induction with DEB, presence of bicentric chromosome.

Conclusions: New discoveries in molecular biology and genetics have unveiled some of the mysteries of bone marrow failure syndromes. FA is a rare disorder with variable organ abnormalities, some relatively common, but some other rarely associated with the disease recognition of unusual pathologies is important in order to improve the diagnostic.

\section{FACTOR SUPPLETION AND CONTROL IN THREE PREMATURE NEONATES WITH HAEMOPHILIA}

K E Kraft ${ }^{1}$, R Verlaak ${ }^{2}$, P P T Brons ${ }^{3}$

${ }^{I}$ Department of Pediatrics, Rijnstate Hospital, Arnhem, The Netherlands, ${ }^{2}$ Department of Pediatrics, Canisius Wilhelmina Hospital, Nijmegen, The Netherlands

${ }^{3}$ Department of Pediatric Hemato-Oncology, University Children's Hospital, Nijmegen, The Netherlands

Background and Aims: We report the first case of factor suppletion therapy in a premature infant with haemophilia B and the first case of haemophilia A in a premature born twin. Factor suppletion, control regime and the question whether to use a central venous catheter are discussed.

Methods: The administration of recombinant factor concentrate was started immediately after birth in all cases, to prevent the occurrence of intracranial haemorrhage (ICH) and haemorrhage due to iatrogenic injuries. Control of factor levels took place via heel stabs or venipuncture in two cases. These infants were given factor suppletion with minimal monitoring of the factor level. The third infant received a central venous catheter (CVC), which provided continuous access and intensive monitoring of the factor level.

Results: No bleeding, thrombosis or inhibitor formation associated with factor suppletion therapy was noted in any of the three cases. A CVC-related sepsis was seen which needed withdrawal of the CVC. ICH was seen in two infants in spite of factor suppletion therapy. However, in one of these cases, ICH was related to diffuse intravasal clotting accompanying a sepsis in absence of a CVC. This lead to the death of the infant, in spite of optimal treatment.

Conclusions: Recombinant factor concentrate seems to be safe and effective for treatment of haemophilia in premature infants. It may reduce the occurrence of ICH. The first choice of venous access for factor suppletion and control should be a peripheral vein. Moreover, when it concerns a prophylactic therapy, insertion of a CVC is not indicated.

\section{RISK FACTORS FOR INTRAOCLUAR ADVANCED RETINOBLASTOMA}

C Leal-Leal, R Rivera-Luna, M Flores-Rojo, J Amador-Zarco, M Gonzalez

Clinica de Retinoblastoma - INP, Mexico DF, Mexico

Background and Aims: Retinoblastoma (RB) is the most frequent malignant eye tumor in childhood. There is no international agreement about risk factors for metastatic disease. The aim of this paper is to present the results from a retrospective study of patients diagnosed and treated with advanced eye disease from Mexico.

Methods: An analysis was carried out reviewing the clinical characteristics of patients with unilateral advanced ocular stages of RB, treated between 1994 and 2004. All patients were treated with ocular enucleation as first treatment, The data included for the analysis was: age at diagnosis, sex, ocular staging, anatomic site 
of tumor involvement within the eye (choroids, lamina cribosa), chemotherapy treatment scheme and status at the last contact.

Results: From 1990 to 2004, 195 patients were diagnosed and treated with advanced ocular RB; age range was from 1 to 169 months (mean=29.27). The pathologist report showed choroidal involvement in 166 (83.4\%) cases, and lamina cribrosa involvement in 66 (33.2\%) cases. Received chemotherapy 113 (56.8\%) patients and $86(43.2 \%)$ did not received any schema. Overall survival was of $96.98 \%$ at 187 months of follow up; there were no differences in overall survival within or between groups when we compared choroidal or lamina cribrosa affection ( $\log$ rank $=1.70, \quad \mathrm{DF}=3$, sig=.6368).

Conclusions: In our series as others, choroidal and post lamina cribrosa involment are not risk factors to developing metastatic disease could be treated only with enucleation.

\section{ALLERGIC RHINITIS AND COCKROACH SENSITIZATION}

\author{
M Nabavi $^{1}$, M H Bemanian ${ }^{2}$ \\ ${ }^{1}$ Semnan Medical University, Semnan, Iran \\ ${ }^{2}$ Yazd Medical University, Yazd, Iran
}

Background and Aims: Allergic rhinitis is an increasing problem worldwide. Aeroallergens are the most important allergens as the cause of respiratory allergies. Air pollution and outdoor and indoor allergen exposures may be the cause, and indoor allergens are more important especially in children and adults with perennial allergic rhinitis. Cockroach allergens are among the most important and increasingly observed cause of sensitization in allergic patients.

Methods: In our study 258 patients with allergic rhinitis were studied and skin prick test performed to determine the allergen sensitizations.

Results: In 137 patients (53\%) cockroach allergy were detected and in 22 patients $(9 \%)$ it was the only sensitization. In 102 patients (38\%), other allergens, mostly aeroallergens including botanical allergens, mites and moulds were causative. 19 patients (9\%) showed no sensitizations. In the second stage of the study skin prick test was performed on cockroachsensitized patients for 2 types of cockroaches, Bellattella germanica \& Bellattela Americana. 68 patients (49\%) showed sensitization to both types, 49 patients $(36 \%)$ were sensitized to B. germanica and 20 patients $(15 \%)$ to B. Americana.

Conclusions: B.germanica with $45 \%$ prevalence in all patients with allergic rhinitis were shown to be one of the most prevalent aeroallergens leading to signs and symptoms of allergic rhinitis.

\section{HUMORAL IMMUNITY IN CHILDREN FROM TRANSYLVANIA REGION, ROMANIA}

\author{
M Marc, N Miu, A Butnariu, O Maftei \\ Pediatric Clinic II, Cluj-Napoca, Romania
}

Background and Aims: The characteristics of humoral immunity in healthy children are well known from birth through adolescence and so are its modifications in different pathological states. We aimed to study the humoral immunity in healthy children in the geographical region of Transylvania.

Methods: We realised a 5 years prospective study on 193 healthy children of different ages. The criteria of inclusion in the group were: the absence of any infectious pathology during the last 3 months and the absence of any chronical disease that would modify the immune status. The values obtained have been compared to those of a similar group in other regions. The statistical processing has been made in the EPIINFO-5 programme, the comparison between values has been made with the aid of the multifactorial variable test.

Results: We have set the limits of the values, the average and the Standard Deviation according to age groups, concerning the seric level of the main immunoglobuline classes. Analyzing similar groups from two different regions of Romania, we didn't find any significant differencies. The comparision with values of similar studies from literature shows significant differences for the same age groups.

Conclusions: In area influenced by the same environmental factors, with a relatively homogeneous population, genetically and socio-economically, the immune status is set between limits that can be considered normal only for that area. In order to estimate the immune status of a certain subject, reference has to be made to another witness of the same age, in the same geographical area.

\section{DEEP VEIN THROMBOSIS IN CHILDREN. A CASE REPORT}

K K Z Aabideen ${ }^{2}$, M Ogendele ${ }^{2}$, I Ahmad ${ }^{2}$, R Keenan ${ }^{1}$

${ }^{I}$ Department of Haematology, Aldery Hospital, Liverpool, UK, ${ }^{2}$ Department of Paediatrics, Whiston Hospital, Liverpool, UK

Background and Aims: Spontaneous Deep Vein Thrombosis (DVT) is extremely rare $(1 / 51,000)$ in children. We report a case of Spontaneous DVT of left popliteal vein in a 5-year-old boy.

Methods: He presented to us with one-day history of left calf pain, refusal of walking, hot, tender, and swollen left calf. In view of the high D-Dimer and clinical presentation, Venography was performed and this confirmed the diagnosis of DVT of left popliteal vein. There was no clinical evidence of pulmonary embolism (PE). No cause was found on extensive investigations.

Results: He was managed with anticoagulants and discharged home on oral Warfarin.

Conclusions: This case highlights that though the DVT is very rare in paediatric patients, it does occur. Paediatricians must consider DVT in their differential diagnosis when children present with leg pain. DVT is rare, but can be serious, and treatment guidelines validated in children are not available. There is a need for an evidence based approach using information from International Registries. 
CLINICAL CORRELATES OF EARLY READMISSION FOR SICKLE CELL DISEASE VASO-OCLUSSIVE CRISIS

C C Skae, D Brijlall, M McGuire, P O Ozuah

Children's Hospital at Montefiore, Albert Einstein College of Medicine, Bronx, NY, USA

Background and Aims: Some patients admitted for sickle cell vasoocclusive crisis (VOC) are readmitted within 14 days. We wondered whether there were differences between those patients with frequent admissions/year versus those without. Objective: To identify the clinical correlates of patients with early readmission for VOC.

Methods: Case-control cohort study between 1/1/2000 and $12 / 1 / 2004$. Cases were readmitted within 14 days, but did not have frequent yearly admissions for VOC. Controls were readmitted within 14 days and had 5 or more admissions for VOC in a given calendar year. Cases and controls were matched for age, gender, and ethnicity. Bivariate analyses and conditional logistic regression assessed relative contribution of independent variables.

Results: Of 132 early readmissions, 44 were cases and 88 were controls. Cases/Controls were successfully matched (mean age 13 yrs vs. 14.8 yrs; for both $50 \%$ were male, 92\% Black). Cases were significantly more likely to have higher pain scores in the 24 hours preceding discharge, including maximum pain score $/ 24$ hours (3.6 for cases vs. 2.4 for controls; $p=.048$ ), and average pain score/24 hours (2.9 vs. $1.7 ; \mathrm{p}=.018)$. No significant differences were found between the groups in mean length of stay for index admission (6.1 vs. 5.4), admission hemoglobin ( 8.5 vs. 9.5), days to readmission (5.1 vs. 6.4), mean LOS for readmission (6.5 vs. 6.0), and discharge hemoglobin (8.7 vs. 9.3).

Conclusions: Early readmission for VOC in patients without frequent admissions for VOC was associated with higher pain scores in the 24 hours preceding discharge.

\section{CORRELATION BETWEEN INITIAL} SYMPTOMS OF FOOD ALLERGIES AND CLINICAL MANIFESTATION IN ORAL PROVOCATION CHALLENGES. ARE THEY SAFE?

\author{
M F Sánchez Mateos, C Blanco Rodríguez, \\ S J Quevedo Teruel, M Centeno Jiménez, \\ B García Cuartero, A González Vergaz, \\ L Echeverría Zudaire
}

Servicio de Pediatría, Hospital Severo Ochoa, Leganés, Madrid, Spain

Background and Aims: Cow's milk protein, eggs, dried fruits and fish are the most common causes of food allergy in pediatric age. Opened oral provocation challenge is esencial for diagnosis, but when it is positive, could we predict clinical syntoms? The objetive is to describe if initial symptomalogy of disease can predict what kind of symptoms we will found in case of positive oral provocation.
Methods: We made a prospective study of oral provocation challenges that we did in our Pediatrics Service in year 2005. Variables collected were food involved, initial symptoms, oral provocation test symptoms and severity of them compared with previous. Results: 366 oral provocation challenges achieved. We found $116(31,7 \%)$ with a positive result; skin manifestations $(53,4 \%)$, gastrointestinal $(18,1 \%)$, multiorganic $(22,4 \%)$ y anaphylaxia $(6 \%)$. These symptoms were similar than initial in $50 \%$ of cases, more severe in $25,9 \%$ and less important in $10,3 \%$. In function of food more frecuently implicated: Egg: changes $50,8 \%$; severe $34,9 \%$; similar $61,9 \%$; light $3,2 \%$. Cow's milk protein: changes $73,1 \%$; severe $26,9 \%$; similar $53,8 \%$; light $19,2 \%$. Fish: changes $30,8 \%$; severe $7,7 \%$; similar 76,9\%; light $15,4 \%$.

Conclusions: 1 . Egg, cow's milk and fish are the more common food allergy in our population. 2. Cow's Milk Protein were the least predectible oral provocation challenges of all in symptoms during the test. 3. Egg was the food with more severe symptoms in a bigger number of cases.

\section{ROLE OF TLR4 AND CD14 IN LPS-INDUCED IL- 8, TNF-ALPHA AND IL-10 RELEASE IN NEONATES}

E Levi, A Mouchtouri, K Xini, S Fotopoulos, M Xanthou

Neonatal Immunology Laboratory, B NICU, 'Agia Sophia' Childrens Hospital, Athens, Greece

Background and Aims: Toll-like receptor (TLR)-4 and CD14, expressed on leukocytes, bind to LPS on pathogens and trigger cytokines release. Previously we demonstrated increased TLR4 expression and IL-8 release following LPS stimulation in neonates compared to adults. However, the role of TLR4 and CD14 in LPSinduced cytokine release is not clear. Aim: To investigate CD14 expression and the role of TLR4 and CD14 in LPS-induced IL-8, TNF-alpha and IL-10 release in neonates.

Methods: Peripheral blood from 15 preterm, 15 full term neonates and 15 adults was cultured with LPS. CD14 expression on leukocytes was measured by flow cytometry. TLR4 and CD14 function was inhibited using blocking antibodies. Cytokine release was assayed by ELISA.

Results: Neonates exhibited increased CD14 expression following LPS stimulation, as compared to adults. Blocking of TLR4 inhibited LPS-induced IL-8 release significantly more in preterms $(38 \%)$ than in adults (14\%). CD14 blockade resulted in an even greater inhibition $(80 \%)$ on IL-8 release, similar in all groups. Blocking of both TLR4 and CD14 did not result in a further increase in the inhibition of IL-8 release. TLR4 blockade inhibited (60\%) LPS-induced TNF-alpha and IL-10 release, while CD14 blockade completely inhibited (100\%) TNF-alpha and IL-10 release in all groups.

Conclusions: Preterms appear to dependent more on TLR4 for IL-8 release than adults. CD14 is critical for LPS-induced IL-8, TNF-a and IL-10 release, whereas the role of TLR4 is less prominent, suggesting that other 
TLRs are also involved. LPS-induced IL-8 release depends not only on CD14 and TLR4 signalling but also on other immune receptors.

\section{CYTOKINE INDUCTION BY STREPTOCOCCUS PNEUMONIAE: POLYSACCHARIDE CAPSULE IMPEDES TLR-DEPENDENT CYTOKINE PRODUCTION}

\author{
C Neeleman ${ }^{1}$, T Sprong ${ }^{2}$, R Petru ${ }^{1}, \mathrm{M}$ van Deuren ${ }^{2}$ \\ ${ }^{I}$ Department of Pediatric Intensive Care, Radboud \\ University Medical Centre Nijmegen, Nijmegen, The \\ Netherlands, ${ }^{2}$ Department of Internal Medicine, \\ Radboud University Medical Centre Nijmegen, \\ Nijmegen, The Netherlands
}

Background and Aims: Despite advances in critical care, morbidity and fatality rates of invasive pneumococcal disease remain high. Cytokines play a crucial role in the pathogenesis of pneumococcal infection. We investigated the role of the polysaccharide capsule and pneumococcal surface protein A (PspA), both established virulence factors, in cytokine production using serotype 3 strain WU2 (caps $+/ \mathrm{PspA}+$ ), its PspA-negative encapsulated mutant JY1123 (caps+/PspA-) and its unencapsulated PspA-negative mutant DW3.8. (caps-/PspA-).

Methods: Cytokine production by human PBMCs and peritoneal macrophages (PM) of TLR2 and -4 knockout mice and NFkB dependent CD25-expression on TLR2 and -4 transfected $\mathrm{CHO}-$ cells.

Results: WU2 (caps+/PspA+) and its mutant JY1123 (caps+/PspA-) induced similar amounts of cytokines in human PBMC, whereas the unencapsulated mutant DW3.8 (PspA-) was significantly more potent. A TLR4 inhibitory antibody significantly inhibited $\mathrm{TNFa}$ production by DW3.8 but not by the encapsulated maternal strain WU2. In PM of TLR2-deficient mice, cytokines by the WU2 strain were reduced to baseline levels, whereas significant production was seen by the capsule-negative DW3.8. PM of TLR4 deficient mice showed an attenuated response to DW3.8. Similar CD25 was induced in TLR2 transfected CHO-cells by WU2 and DW3.8.

Conclusions: The capsular polysaccharide impedes TLR-mediated cytokine production by S. pneumoniae. In the presence of capsule, signaling is mainly via TLR2, whereas in the absence of capsule other - more potent - pathways are engaged, and TLR4 is of importance in this signaling.

\section{ARE WE GETTING BETTER? DRAMATIC CHANGES IN MORTALITY IN CHILDREN WITH SEPSIS AND PURPURA IN ROTTERDAM}

S C A T Verbruggen, C M P Buysse, M de Hoog, J A Hazelzet, K F M Joosten

Pediatric Intensive Care, Erasmus MC-Sophia Children's Hospital, Rotterdam, The Netherlands

Background and Aims: Meningococcal sepsis and septic shock is a fulminant disease in young, previously healthy children. In our PICU, overall mortality due to sepsis and purpura was $16.1 \%$ over the past 18 years. Since 2001 a marked drop in mortality was observed.

Methods: Between 1988 and 2006 all patients (1 month -18 yrs) consecutively admitted with sepsis and purpura were analysed. Retrospective comparison of two time periods (1989-2001 (A) and 2001-2006 (B)) of inotropic and vasopressor use in relation with severity of disease and outcome.

Results: 285 patients were admitted with sepsis and purpura, 45 non-survivors (16.1\%). 218 patients were admitted in period $\mathrm{A}$ and 67 patients were admitted in period B. Between period A and B there was no significant difference in age (mean 5 vs. 5,2 yrs, range 0,3 - 17 vs. $0,3-15,8$ ), sex and PRISM score (18 vs. 20) but a significant difference in mortality ( $20 \%$ vs. $1 \%)$. Dopamine was significantly more used in time period A $(p<0.05)$. In time period B the level of norepinephrine used was significantly higher $(0.2$ vs. 0.6 $\operatorname{microgram} / \mathrm{kg} / \mathrm{min}(\mathrm{p}<0.05))$. There was no difference in dobutamine use, the combination of dobutamine and norepinephrine and overall vasopressor score in the 2 time periods.

Conclusions: We observed a dramatic decrease in mortality in children with meningococcal sepsis since 2001 with a change in the use of dopamine and the level of norepinephrine. It is questionable of the changes in inotropic use might have influenced mortality and others factors have to be determined.

\section{THROMBOLYTIC THERAPY IN MENINGOCOCCAL SEPSIS WITH SERIOUS LIMB ISCHEMIA}

T L M Walk ${ }^{1}$, J Lemson ${ }^{1}$, T Sprong ${ }^{2}$, $\mathrm{J} G$ van der Hoeven ${ }^{1}$

${ }^{I}$ Department of Intensive Care Medicine, Radboud University Nijmegen Medical Centre, Nijmegen, The Netherlands, ${ }^{2}$ Department of General Internal Medicine, Radboud University Nijmegen Medical Centre, Nijmegen, The Netherlands

Background and Aims: Fulminant meningococcal sepsis (FMS) is a life threatening infection that can lead to shock and disseminated intravascular coagulation (DIC). Although mortality has decreased during the last decade morbidity is still high and $10-20 \%$ of the survivors suffer from disabling limb ischemia because of arterial thrombotic occlusion. Therefore thrombolytic therapy could have therapeutic possibilities. We describe our experiences with this treatment.

Methods: As an adjunct to our normal intensive care management we treated four children with FMS and severe limb ischemia with thrombolytic therapy using recombinant tissue plasminogen activator (rt-PA).

Results: The table depicts various patient characteristics. Thrombolytic therapy consisted of rt-PA in a dose of 0.25 to $0.5 \mathrm{mg} / \mathrm{kg} / \mathrm{hr}$ for several hours. All children were also treated with fresh frozen plasma. Three patients had a marked clinical response with return of peripheral pulsations and improvement of skin perfusion shortly after initiation of rt-PA therapy. No patient had signs of cerebral bleeding. One patient died with extensive necrosis and all children suffered from adrenal insufficiency. 
Conclusions: Thrombolytic therapy with rt-PA can potentially prevent from serious disabling limb amputations. However the benefits must always be weighted against possible bleeding complications.

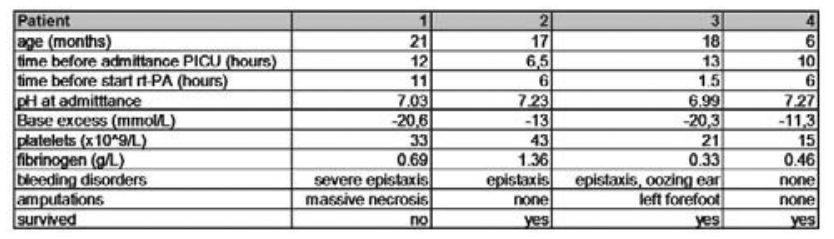

\section{CLINICAL FEATURES OF FULMINANT LATE- ONSET SEPSIS IN A NEONATAL INTENSIVE CARE UNIT AND ANTIMICROBIAL RESISTANCE}

\author{
C S Kim ${ }^{1}$, S L Lee ${ }^{1}$, W D Kim² \\ ${ }^{I}$ Department of Pediatrics, Keimyung University School \\ of Medicine, Daegu, Korea, ${ }^{2}$ Department of Pediatrics, \\ Fatima Hospital, Daegu, Korea
}

Background and Aims: This study was undertaken to investigate clinical features of fulminant (lethal within 72 hours of onset of illness) late-onset sepsis (LOS) in the neonatal intensive care units (NICU) and antimicrobial resistance.

Methods: Retrospective analysis of the medical records was conducted of LOS (occurring after 3 days of age) in infants in a NICU over a 5-year period (2001-2005).

Results: The mainly causative organisms of LOS were S. aures $(27 \%)$, S. epidemidisis (22\%), Candida sp. (19\%), Enterococcus sp. (7\%) and Klebsiella sp. (7\%), and fulminant infection was developed in $11(4 \%)$ of them. Frequency of fulminant LOS was highest for Klebsiella sp., 5 of 24 (21\%), and followed by E. coli, 1 of $10(10 \%)$. Although Gram-positive organisms caused 211 (59\%) episodes of LOS, fulminant sepsis was considerably low: Enterococcus sp. with $4 \%$ and S. aures with $2 \%$. Also Candida sp. had a 3\% rate there. In rate of antimicrobial resistance, S. aures was $74 \%$ to oxacillin and Enterococcus sp. was $23 \%$ to vancomycin. Resistant rate to cefotaxime of Klebsiella sp. and E. coli was $46 \%$ and $33 \%$ respectively, and all $6(18 \%)$ organisms causing fulminant sepsis of them were resistant to cefotaxime.

Conclusions: Fulminant LOS in the neonates is strongly associated with Klebsiella infection $(\mathrm{P}<0.005)$, and a proper drug choice is necessary based on the major pathogens and antimicrobial resistance patterns in each one of the NICUs to prevent acute death.

\section{A RANDOMIZED STUDY OF ORAL CARE INFLUENCE ON OROPHARYNGEAL COLONIZATION OF CRITICALLY ILL CHILDREN}

D M Kusahara, M A S Peterlini, W B Carvalho, M L G Pedreira

Federal University of São Paulo, São Paulo, Brazil

Background and Aims: The oropharyngeal colonization by endogenous flora or pathogens obtained exogenously from the intensive care environment has been described as one of the major routes for acquiring VAP. Oral care intervention has been described as capable to prevent VAP in adult patients. The aim of this study was to analyze the influence of oral care interventions on oropharyngeal colonization of critically ill children.

Methods: Prospective randomized controlled study realized in a PICU in the city of São Paulo, Brazil. The research was approved by the ethics in research committee. The sample was composed of 56 children, $29(51.8 \%)$ in the Control Group-CG (mechanical intervention) and $27(48.2 \%)$ in the Experimental Group-EG $(0.12 \%$ chlorhexidine). Microbiological cultures of oropharyngeal secretions was obtained 24 (CI), 48 (CII) and 96 (CIII) hours after admission.

Results: No significant difference in the groups was verified regarding children's characteristics: age $(\mathrm{p}=0.854)$, gender $(\mathrm{p}=0.945)$, skin color $(\mathrm{p}=0.983)$, nutritional status $(\mathrm{p}=0.531)$, clinical conditions of mouth $(p=0.414)$ and teeth $(p=0.343)$ and, infections $(p=0.222)$ at admission. The main microorganisms identified were Streptococcus viridans, Staphylococcus epidermidis, Moraxella spp, Staphylococcus aureus, Pseudomonas aeruginosus, Acinetobacter baumanni, Enterobacter ssp, and Klebsiella pneumoniae. No global significant influence of oral care on microorganisms colonization was observed: CI to CII $(\mathrm{p}=0.085)$ and CII to CIII $(\mathrm{p}=0.927)$. An increase of pathogens was observed in CG at CII (33.3\%) and CIII (30.8\%); in EG increase was only observed at CIII (25.0\%).

Conclusions: There was no significant differences on the global analyzes of pathogens colonization in the studied groups, nonetheless a lower increase of pathogens was observed in the EG. Acknowledgments: Dr. Pedreira acknowledges support for research by grant 04/13361-2- FAPESP.

\section{EARLY DETECTON OF BACTERIAL INFECTIONS IN HOSPITAL AND AMBULATORY SETTINGS}

\author{
S Radulovic $^{1}$, N Pejovic Mandic ${ }^{2}$ \\ ${ }^{1}$ Institute for Disease of Children Clinical Center of \\ Montenegro, Podgorica, Serbia and Montenegro \\ ${ }^{2}$ Private Pediatric Practise Doktorica Mica, Podgorica, \\ Serbia and Montenegro
}

Background and Aims: White blood cells (WBC, Creactive protein (CRP) and sedimentation rate (SE) are important predictors of bacterial infections. Evaluation of WBC, CRP, SE, and fever (F) in early diagnosis of bacterial infection.

Methods: This study involved 198 hospitalised and 96 treated in primary pediatric care settings. Hospitalised patients are divided in two groups. 1a with serious bacterial infection (SBI), $1 \mathrm{~b}$ viral infections. Outpatients are also divided in two groups. 2a bacterial infections and $2 \mathrm{~b}$ viral infections. Diagnostic criteria where blood culture, urine culture, cerebro spinal fluid culture, pharynheal swab, chest x-ray. Values of WBC, CRP, SE and $\mathrm{F}$ where estimated at the beginning of the disease and are compared with definitive diagnosis. Beginning 
of disease is defined to be 3,69th day for hospitalised patients and 2, 69th day for outpatients.

Results: Values of WBC, CRP, SE and F are compared between each others, such as between the groups (Mann-Whitney $\mathrm{U}$ test, ANOVA). There is no significant difference between hospitalised and outpatients concerning $\mathrm{WBC}$ and SE. 1a group has significantly higher $\mathrm{WBC}$ and $\mathrm{SE}$ than $1 \mathrm{~b}$ group $(p<0.001) 1$ a group has significantly higher SE than $1 b$ group $(\mathrm{p}<0.001)$, and $2 \mathrm{a}$ group has significantly higher $\mathrm{SE}$ than $1 \mathrm{~b}$. Fever and CRP where significantly higher in hospitalised than outpatient group $(\mathrm{p}<0.001) .1 \mathrm{~b}$ group has significantly lower fever than $2 \mathrm{a}$ group and $2 \mathrm{~b}$ group $(p<0.001)$. 1a group and $2 \mathrm{a}$ group have significantly higher CRP than $1 \mathrm{~b}$ group and $2 \mathrm{~b}$ group.

Conclusions: CRP and fever are better early predictors of bacterial infections than SE and WBC.

\section{NOSOCOMIAL INFECTIONS AMONG VENTILATED PRETERM INFANTS}

\section{E Petkovska, S Jancevska}

\section{Department of Neonatology, University Clinic of Gynecology and Obstetrics, Skopje, Macedonia}

Background and Aims: Neonatal nosocomial infection (NI) is a very important cause of mortality and morbidity among ventilated infants in neonatal intensive care units (NICU). The aim of this study was to calculate the incidence and to identify the risk factors for NI among ventilated preterm infants in NICU.

Methods: We performed a prospective study during a period of 2 years $(2003 / 2004)$ among ventilated preterm infants in NICU at University Clinic of Gynecology and Obstetrics in Skopje-tertiary care center for 'transportin-utero'. Infections were defined as nosocomial when occurring $>48$ hours after birth.

Results: 123 discharged patients who were ventilated are included. 87/123 (70.7\%) had conventional mechanical ventilation (CMV) and 36/123 (29.3\%) had nasal continuous positive airway pressure (NCPAP) treatment. 31/123 (25.2\%) patients experienced $40 \mathrm{NI}$ during this period. 16/36 (44.4\%) NCPAP ventilated patients had NI vs. 15/87 (17.2\%) patients treated with CMV ( $<<0.05$ ). Sepsis represents the most common NI, followed by ventilator-associated pneumonia (VAP). 9/15 $(60 \%)$ of patients with NI treated with CMV had VAP vs. $2 / 16(12.5 \%)$ of NCPAP ventilated ones $(p<0.05)$. The rate of sepsis was not significantly different between two ventilated groups of patients. Pseudomonas aeruginosa and Klebsiella pneumonie were the most frequently isolated microorganisms from endotratracheal positive aspirates, while Streptococcus epidermidis and Staphylococcs aureus were the microorganisms most frequently isolated in positive blood cultures.

Conclusions: This study demonstrated a high incidence of NI among ventilated preterm infants, related to type of mechanical ventilation, interventions and process of care in our NICU. The results will be used for further surveillance and improving of prevention strategies in our NICU.
MENINGOCOCCAL CLUSTERS - NOT GONE YET!

N Ni Shuibhne, P Finnegan, A Nicholson

Department of Paediatrics, Our Lady of Lourdes Hospital, Drogheda, Co. Louth, Ireland

Background and Aims: Meningococcal $\mathrm{C}$ vaccine introduced in Ireland in October 2000 has had a major impact in reducing mortality in Meningococcal sepsis. Clusters of group B disease have not been reported in Ireland.

Methods: We describe a cluster of Meningococcal group B serogroup diseases occurring in the Northeastern Health board region.

Results: A six-week-old male infant presented to the emergency department with a disseminated pupuric rash. Despite aggressive fluid resuscitation, inotropic agents, and ventilation the infant died four hours after presentation. Meningococcal PCR revealed group B serogroup. Four hours later on the same day, a fifteenmonth-old female infant presented with a one-day history of vomiting, pyrexia, and petechial rash. Meningococcal PCR grew group b serogroup with the same subtype as the first case. The third case was a fourteen- month old infant who had been unwell the previous week and had a 12-hour history of petechial rash, pyrexia and lethargy. Meningococcal PCR again revealed serogroup $b$ with same subtype. The fourth case, a ten-month-old female infant was a first cousin of the third case and had been in contact with him 48 hours prior to his admission. Meningococcal PCR again revealed group B serogroup however with a different subtype.

Conclusions: Our cluster of four cases of serogroup $b$ invasive Meningococcal disease in one week constitutes a cluster hitherto unreported in Ireland. This cluster highlights the ongoing burden of group B disease and the challenge of finding an effective vaccine.

\section{SELECTIVE FLUCONAZOLE PROPHYLAXIS IN HIGH RISK BABIES TO REDUCE INVASIVE FUNGAL INFECTION}

B A McCrossan ${ }^{1}$, E McHenry ${ }^{2}$, F O'Neill ${ }^{3}$, D G Sweet ${ }^{1}$, $\mathrm{G} \mathrm{Ong}^{2}$

${ }^{1}$ Regional Neonatal Intensive Care Unit, Royal Maternity Hospital, Belfast, Northern Ireland

${ }^{2}$ Department of Microbiology, Royal Victoria Hospital, Belfast, Northern Ireland, ${ }^{3}$ Department of Pharmacy, Royal Victoria Hospital, Belfast, Northern Ireland

Background and Aims: Invasive fungal infection is an increasingly common cause of mortality and morbidity in very low birth weight (VLBW) babies. We aimed to evaluate the impact of selective fluconazole prophylaxis on incidence of invasive fungal infection, examine cost implications and emergence of fluconazole resistance in Neonatal Intensive Care.

Methods: Retrospective study of babies $<1500 \mathrm{~g}$ birth weight admitted to NICU in a period 1 year before and after implementation of an antifungal prophylaxis guideline. Eligibility: Birth weight $<1500 \mathrm{~g}$ with an additional risk factor: (1) Colonization of candida 
species from surface sites with a central venous catheter, (2) 3rd generation Cephalosporin treatment or (3) total duration of antibiotic therapy $>10$ days. Prophylaxis consists of: Fluconazole $6 \mathrm{mg} / \mathrm{kg}$ for 3 weeks. Dose interval every 72 hours during the first 2 weeks of life. Thereafter, dose interval reduced to 48 hourly until 3 weeks old when daily fluconazole is given.

Results: One hundred and twenty-one and 107 VLBW infants admitted in year before and after guideline. Data available in 110 and 100 charts respectively. Six (6/121) babies developed invasive fungal sepsis before the guideline compared with only one (1/107) after. The cost of prophylaxis was less than that of treating infants with fungal sepsis prior to the guidelines. There was no evidence of emerging resistance to standard anti-fungal treatments.

Conclusions: Selective anti-fungal prophylaxis has reduced invasive fungal sepsis in our unit. Prophylactic therapy with fluconazole is relatively inexpensive. There was no evidence of fluconazole resistance emerging.

\section{UNCOMMON LIFE-THREATENING COMPLICATIONS OF CHILDHOOD STREPTOCOCCAL SEPSIS}

\author{
M Piastra ${ }^{1}, \mathrm{~S}$ Pulitanò ${ }^{1}$, G Zorzi ${ }^{1}$, A Tempera ${ }^{1}$, \\ C Pedace ${ }^{1}$, G Polidori $^{1}$, G De Rosa ${ }^{2}$, D Pietrini ${ }^{1}$ \\ ${ }^{\text {I}}$ Pediatric Intensive Care Unit, Catholic University 'A. \\ Gemelli' Hospital, Rome, Italy, ${ }^{2}$ Department of \\ Pediatric Cardiology, Catholic University 'A. Gemelli' \\ Hospital, Rome, Italy
}

Background and Aims: Even in the antibiotic era, streptococci infected healthy infants/children may require PICU admission because of serious and lifethreatening conditions. Differently from multi-resistent Gram negative bacteria or fungi, which are increasingly recovered in PICU setting, antibiotic susceptibility did not represent the main problem we encountered in our 3 patients with streptococcal sepsis.

Methods: Illustrative cases. Setting: Pediatric Intensive Care Unit at a University Hospital.

Results: Clinical experiences including 3 sepsis episodes from different streptococcal strains are reported. All children were previously healthy and no predisposing factors were identifyied. Main PICU complications and their management are illustrated in Table 1.

Conclusions: Streptococcal infections remain diffusely present among pediatric population, while severe and life-threatening cases have become consistently rarer. Even in previously healthy infants/children - in the absence of antibiotic-resistant streptococcal species (due to increasing penicillin resistance or intermediate susceptibility) - a rapidly evolving picture of sepsis and septic shock may occur. Emergency physicians and intensivist should be aware of these uncommon complications, needing a complex physiopathological support besides antimicrobials. In our experience, only a highly individualised approach permitted to achieve a significant improvement of the patient clinical status.

\begin{tabular}{|c|c|c|c|}
\hline Clinical characteristics & case 1 & case 2 & case 3 \\
\hline Age (months)/sex & $42 / \mathrm{F}$ & $20 / \mathrm{F}$ & $<1 / M$ \\
\hline clinical history & scarlet fever & - & - \\
\hline $\begin{array}{l}\text { Previous antimicrobial } \\
\text { therapy }\end{array}$ & yes (azythromycin) & no & no \\
\hline Clinical onset & acute & acute & acute \\
\hline Causative agent & S. pyogenes & S. viridans & S. agalactiae B \\
\hline Penicillin susceptibility & yes & yes & yes \\
\hline clinical presentation & $\begin{array}{l}\text { Acute respiratory fallure, } \\
\text { pleural empyema }\end{array}$ & $\begin{array}{l}\text { Cardiogentic snock, } \\
\text { consumption } \\
\text { coagulopathy }\end{array}$ & $\begin{array}{l}\text { Purulent meningitis, coma, } \\
\text { status epilepticus }\end{array}$ \\
\hline Respiratory failure & severe & severe & moderate \\
\hline Intubation timing & ED (emergency dept) & ED & PICU \\
\hline $\begin{array}{l}\text { Mechanical respiratory } \\
\text { support }\end{array}$ & yes & yes & yes \\
\hline ventilation mode & Pressure control & PRVC & PRVC \\
\hline $\begin{array}{l}\text { Maximal settings } \\
\text { (PIP/PEEP) }\end{array}$ & $36 / 14$ & $25 / 8$ & $18 / 5$ \\
\hline $\mathrm{PaO2} /$ FiO2 [first $24 \mathrm{hrs}$ ] & $<80$ & 150 & 350 \\
\hline Hemodynamic impairment & moderate & severe & moderate \\
\hline Inotropic support & + (dopamine) & $\begin{array}{l}+++ \text { (dobutamine- } \\
\text { enoximone) }\end{array}$ & + (dopamine) \\
\hline Main PICU complication & ARDS, bronchopleural tistula & Cardiogenic shock, DIC & $\begin{array}{l}\text { Cerebral sinovenous } \\
\text { thrombosis }\end{array}$ \\
\hline Individualised approach & Different lung ventilation & $\begin{array}{c}\text { Enoximone-activ. protein } \\
\mathrm{c}\end{array}$ & Unfractionated heparin \\
\hline PICU stay (days) & 96 & 7 & 20 \\
\hline
\end{tabular}

\section{PROCALCITONIN - A VALUABLE DIAGNOSTIC MARKER IN MENINGOCOCCAL DISEASE}

G D Mills ${ }^{1}$, H M Lala ${ }^{1}$, M R Oehley ${ }^{1}$, A B Craig ${ }^{2}$, $\mathrm{K}$ Barratt $^{3}$, D Hood ${ }^{4}, \mathrm{C}$ N Thornley ${ }^{6}$, A Nesdale ${ }^{7}$, N E Manikkam ${ }^{2}$, P Reeve ${ }^{5}$

${ }^{1}$ Department of Infectious Diseases, Waikato District Health Board, Hamilton, New Zealand, ${ }^{2}$ Department of Paediatrics, Waikato District Health Board, Hamilton, New Zealand, ${ }^{3}$ Department of Molecular Biology, Waikato District Health Board, Hamilton, New Zealand ${ }^{4}$ Waikato Public Health Service, Waikato District Health Board, Hamilton, New Zealand, ${ }^{5}$ Department of General Medicine, Waikato District Health Board, Hamilton, New Zealand, ${ }^{6}$ Auckland Regional Public Health Service, Auckland District Health Board, Auckland, New Zealand, ${ }^{7}$ Wellington Regional Public Health, Hutt Valley District Health Board, Lower Hutt, New Zealand

Background and Aims: Meningococcal disease (MCD) is responsible for a large number of deaths among children worldwide. Diagnosing meningococcal septicaemia is simple when it presents with classical signs, especially the purpuric rash. However, meningococcaemia may present as a febrile illness mimicking common childhood illnesses. In these circumstances, misdiagnosis results in significant morbidity or death. This study assesses the diagnostic accuracy of procalcitonin in the setting of meningococcal disease.

Methods: Clinical and laboratory data in two emergency department cohorts (A and B) were analysed between 2002 and 2005, within New Zealand's serogroup B epidemic.

Results: Cohort A consisted of 84 children $(<14$ years old), with confirmed MCD. Cohort B consisted of 1003 consecutively recruited emergency department febrile children, with 18 cases of MCD diagnosed. Within the MCD cohort (cohort A), the procalcitonin geometric mean was $21.6 \mathrm{ng} / \mathrm{mL}$. The overall sensitivity of procalcitonin, using a $2.0 \mathrm{ng} / \mathrm{mL}$ cut-off (as defined by the ROC analysis in cohort B), was 0.96 (95\% CI 0.90 0.99). Procalcitonin was correlated with whole blood meningococcal load $(\mathrm{r}=0.47)$ and Glasgow Meningococcal Sepsis Prognostic Score ( $\mathrm{r}=0.42)$. Mean whole blood meningococcal load was $3.7 \log 10$ 
$\mathrm{cfu} / \mathrm{mL}$. Within the febrile presentation cohort (cohort B) the specificity of procalcitonin in MCD was 0.84 (95\% CI $0.82-0.86$ ), positive and negative likelihood ratios were 6.0 and 0.05 respectively, and sensitivity was 0.94 (95\% CI $0.73-0.99)$. The area under the ROC curve was 0.93 (SE 0.04).

Conclusions: Procalcitonin is an important clinical tool in children with non-specific febrile illnesses when the possibility of meningococcal disease is present. The diagnostic accuracy surpasses other laboratory markers.

\section{EVOLUTION OF HOSPITALIZATIONS OF PATIENTS WITH ONGOING VARICELLA IN A FRENCH CHILDREN HOSPITAL}

\section{A Mandelcwajg, B Quinet, B Castello, N Parez, E Grimprel \\ Consultation, Urgences, Pathologie Infectieuse et Tropicale, Paris, France}

Background and Aims: In the absence of universal varicella vaccination in France, we describe the clinical features of paediatric varicella patients admitted through the emergency department (ED) of the ArmandTrousseau Children hospital in Paris and the evolution in reasons of admission during a longitudinal period of eleven years.

Methods: Retrospective cohort study from March 1990 to February 2001.

Results: 108 children were hospitalized for varicella during these 11 years, of whom, $64(60 \%)$ were under 2 years of age. All but one were immunocompetent. The main cause of admission was the presence of at least one complication $(83,3 \%)$ classified as: cutaneous (34\%, cutaneous superinfections), digestive (19,8,\%, mainly food intolerance), respiratory $(17,6 \%$, mainly bronchiolitis and pneumonia), neurological (15,3\%, mainly febrile seizures) and Ear-Nose-Throat complications $(8,8 \%$, mainly acute otitis media). During the studied period, the annual number of hospitalizations increased. The rate of complications as a cause for hospitalization remained stable but cutaneous superinfections emerged as the first complication.

Conclusions: 108 children were hospitalized for varicella during these 11 years, of whom, $64(60 \%)$ were under 2 years of age. All but one were immunocompetent. The main cause of admission was the presence of at least one complication $(83,3 \%)$ classified as: Cutaneous $(34 \%$, cutaneous superinfections), digestive $(19,8, \%$, mainly food intolerance), respiratory $(17,6 \%$, mainly bronchiolitis and pneumonia), neurological (15,3\%, mainly febrile seizures) and Ear-Nose-Throat complications (8,8\%, mainly acute otitis media). During the studied period, the annual number of hospitalizations increased. The rate of complications as a cause for hospitalization remained stable but cutaneous superinfections emerged as the first complication.

\section{HANDWASHING PRACTICES AND BACTERIAL HAND FLORA IN THE NURSING STUDENTS DOING APPLICATIONS IN PEDIATRICS UNITS}

$\mathrm{T} \mathrm{Nemut}^{1}$, N Dede Çinar ${ }^{1}, \mathrm{C}$ Dede $^{2}$

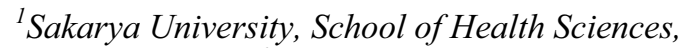
Sakarya, Turkey, ${ }^{2}$ Sakarya University, Vocational School of Healt, Sakarya, Turkey

Background and Aims: Pediatrics units are risky units regarding the nosocomial infections. The aim of this study is to evaluate the hand washing practices and bacterial hand floras of the student nurses doing applications in pediatrics units.

Methods: The study was carried out in March 2006, with the participation of 41 students. The data regarding the hand washing practices of the students was collected by means of the questionnaire. In the study the hand flora samples were taken. After putting $0.9 \% \mathrm{NaCl}$ in glass tubes and sterilizing in autoclave, the samples were taken from the hand palms and fingers by rubbing with a sterile cotton-rod. The sowing was carried out in a way that one colony fell to $5 \%$ sheep blood agar. The samples were evaluated after a 48-hour incubation period in a $5-10 \% \mathrm{CO} 2$ environment in $37 \mathrm{C}$. The percentage method and chi-square test were used in the statistical evaluation.

Results: Of the samples taken pre hand washing, coagulase-negative staphylococci (CNS) reproduced in $58.6 \%$, while alpha hemolytic streptococcus reproduced in $2.4 \%$. Of the samples taken post hand washing, CNS reproduced in 39\%, alpha hemolytic streptococcus reproduced in $2.4 \%$. There found to be a statistically meaningful difference between the floras before and after the hand washing $(\mathrm{p}<0.05)$.

Conclusions: In order to prevent the contamination of the probable nosocomial pathogens, the subjects of proper hand washing techniques and the protection against the hospital infections should be given importance in the clinic education of the students.

\section{DETERMINATION OF ANTIBIOTIC RESISTANCE OF COMMON BACTERIAL NEONATAL PATHOGENS IN A NICU WITHIN A TWO YEAR PERIOD (2004-2005)}

G Triantafyllidis, G Kafalidis, M Theodoraki, H Koufopoulou, N Kouskounelou, I Labadaridis

Neonatal Intensive Care Unit, General Hospital of Nikea, Piraeus, Greece

Background and Aims: Neonatal infections constitute the leading cause of neonatal morbidity and mortality. The study's aim was to detect possible changes in antibiotic resistance of the most common bacterial pathogens involved in early (EO) $(<72 \mathrm{~h})$, late $(72 \mathrm{~h}-28 \mathrm{~d})$ (LO) and very late onset $(>28 \mathrm{~d})$ (VLO) neonatal infections in a NICU within a two year period (20042005).

Methods: 44 positive blood culture confirmed septicemias were recorded in 37 neonates (full term+premature) out of 656 admissions (6.7\%) in 2 years. Of those $9 \%, 61.3 \%$ and $29.5 \%$ were EO, LO and VLO infections respectively. Staph.epidermidis was the 
predominant isolated pathogen in each of these periods followed by group B Streptococcus in EO infections, Enterobacter cloacae, Citrobacter div. Klebsiella sp, Candida and Enterococcus in LO infections and Enterobacter cloacae, Enterococcus sp. Klebsiella sp, and Candida in VLO infections.

Results: Staph.epidermidis proved resistant to $\beta$ lactams, whereas emerging resistance developed to trimethoprim/sulfamethoxazole, gentamycin, fusidic acid and clindamycin. However, it remained highly sensitive to teichoplanin and vancomycin. Although Enterobacter cloacae has developed partial and/or complete resistance to $\beta$-lactams, cephalosporins, aminoglycosides, aztreonam and piperacillin / tazobactam, it is sensitive to carbapenems. Citrobacter behaves in a similar manner displaying complete resistance to $\beta$-lactams, cephalosporins, aminoglycosides and aztreonam, partial resistance to piperacillin/tazobactam and increased sensitivity to the use of carbapenems and cefoxitin. Finally, Enteroccocus is sensitive to most antibiotic categories ( $\beta$-lactams, aminoglycosides, carbapenems) whereas Klebsiella and Candida respond to the use of piperacillin/tazobactam, carbapenems and flucytosine, respectively.

Conclusions: Knowledge of emerging resistance to antibiotics is imperative in planning treatment and therapeutic modalities for infection control.

\section{DETECTION OF NEISSERIA MENINGITIDIS DNA FROM SKIN BIOPSY USING REAL-TIME PCR: USEFULNESS IN THE ETIOLOGICAL DIAGNOSIS AND EPIDEMIOLOGY OF PURPURA FULMINANS}

P Staquet $^{1}$, L Lemee ${ }^{2}$, E Verdier ${ }^{3}$, A Chadie ${ }^{1}$,

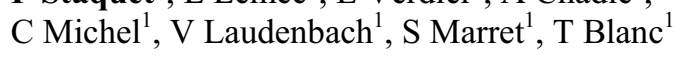

${ }^{I}$ Pediatric Intensive Care Unit, University Hospital of Rouen, France, ${ }^{2}$ Department of Bacteriology, University Hospital of Rouen, France, ${ }^{3}$ Department of

Dermatology, University Hospital of Rouen, Rouen, France

Background and Aims: The aim of the present work was to evaluate the usefulness of a real-time PCR assay for the detection of Neisseria meningitidis $(\mathrm{Nm})$ and genogrouping on skin lesion biopsies in patients with purpura fulminans (PF).

Methods: Monocentric retrospective study. All patients admitted between January 2000 and January 2006 to ICU with a final diagnosis of PF and for which skin biopsy and blood cultures were performed were included. These samples were used for culture and realtime PCR amplification. Quantitative variables were compared using McNemar's test. $p$ value of $<0.05$ was considered significant.

Results: Forty patients (31 children and 9 adults) were included. When Nm PCR was performed on skin biopsy (34 cases), results were positive in $100 \%$ of the cases compared to only $14,7 \%$ for skin culture $(\mathrm{p}=0.0001)$. PCR genogrouping on skin biopsy was positive in $58,8 \%$ of the cases compared to $14,7 \%$ for skin culture $(p=0.007)$. When Nm PCR was performed both on skin biopsy and blood (17 cases), results for skin biopsy were positive in $100 \%$ of the cases compared to $58.82 \%$ for blood ( $\mathrm{p}=0.0233$ ). PCR genogrouping on skin biopsy was positive in $58,8 \%$ of the cases compared to $35,3 \%$ for blood ( $\mathrm{p}=0.045)$.

Conclusions: In all the samples (skin biopsy, blood, and cerebrospinal fluid) PCR was better than methods of culture. In suspected PF cases, skin biopsy provides a better chance to identify $\mathrm{Nm}$ and its genogroup than blood, especially when PCR methods are used. This could help the implementation of public health interventions, especially concerning a vaccination policy.

\section{BLOOD CULTURE ISOLATES DURING 10 YEARS IN A TERTIARY PEDIATRIC INTENSIVE CARE UNIT}

\section{F Duque, J Campos, A Dinis, L Carvalho, J F Neves \\ Paediatric Intensive Care Unit, Coimbra's Paediatric Hospital, Coimbra, Portugal}

Background and Aims: Sepsis is a common cause of morbidity and death in critically ill patients, and blood culture (BC) samples are drawn in an effort to identify a responsible pathogen and guide therapeutic. The authors analysed positive BC from the Paediatric Intensive Care Unit (PICU) and the annual evolution of antimicrobial susceptibility.

Methods: All medical records and database of children with BC 48h after admission, from 1996 to 2005 were retrospectively reviewed.

Results: The total number of children admitted during this period was 3469. A BC was obtained from 1512 children and yielded a pathogen in 186 cases $(12,3 \%$ positive $\mathrm{BC} ; 5,4 / 100$ admissions). The mean distribution of positive BC per year was 18,6 (range from 36 in 1997 to 8 in 2004). Gram-positive bacteria dominated with coagulase-negative staphylococci (CoNS) as the most frequent isolate in 67 cases (32\%). Following CoNS, the 4 most frequent isolates were: E. coli in $15(8 \%)$, Pseudomonas aeruginosa in $10(5,4 \%)$, Enterococcus in $10(5,4 \%)$ and Staphylococcus aureus in $11(5,9 \%)$. There were $4 / 11$ cases (36\%) of Methicillin-resistant S. aureus (MRSA). Penicillin-resistant Streptococcus Pneumoniae hasn't been found. An intravascular catheter was present more often when CoNS were isolated as compared to other organisms.

Conclusions: Our microbiological pattern is dominated by gram-positive flora, which is in agreement with previous studies. MRSA increased from $21 \%$ (in a prior 10 -year survey of our PICU) to $36 \%$, being all susceptible to vancomycin. Prevention and control strategies should focus on MRSA, among others.

\section{NEUROLOGICAL COMPLICATIONS OF VARICELLA INFECTION IN IMMUNOCOMPETENT CHILDREN: A 19 YEAR REVIEW}

A Méndez Echevarría ${ }^{1}, \mathrm{~F}$ Baquero-Artigao ${ }^{1}$, N Hernández ${ }^{1}, \mathrm{R}_{\text {Velázquez }}{ }^{2}, \mathrm{~F}$ Del Castillo ${ }^{1}$, M J García-Miguel $^{1}$ M J Garcia-Miguel 
${ }^{1}$ Pediatric Infectious Diseases Unit, Hospital La Paz, Madrid, Spain, ${ }^{2}$ Department of Pediatric Neurology, Hospital La Paz, Madrid, Spain

Background and Aims: To describe the neurological complications of varicella infection in immunocompetent children.

Methods: Clinical records of patients older than 1 month of age admitted with varicella from 1987 to 2005 were reviewed. Immunodepressed patients were excluded.

Results: During the study, 374 patients were admitted with varicella, 61 with neurological complications $(16 \%)$. Cerebellitis was the most frequent complication observed $(\mathrm{n}=19)$ and the one which appears the latest (6.2 \pm 2.7 days from the beginning of exanthema). Febrile seizures appear early $(1.7 \pm 0.9$ days $)$ and occur in younger children than encephalitis and meningitis do. The CSF analyse was normal in cerebellitis. $80 \%$ of meningitis presented more than $100 \mathrm{cels} / \mathrm{mL}$ leukocyte count and an elevated protein concentration $(>90$ $\mathrm{mg} / \mathrm{dL}$ ) in CSF sample. $66 \%$ of encephalitis cases presented a moderated increase of protein concentration (45-90 mg/dL) and of leukocyte count. (10-100 cels $/ \mathrm{mL})$. Varicella-zoster virus serology was made in CSF in 5 cases ( 3 meningitis and 2 encephalitis), being positive in 4. PCR was made in CSF in 3 patients with encephalitis, being negative in all of them. Admission time was shorter in seizures ( $5 \pm 2$ days) and longer in encephalitis (10.8 \pm 4 days). All cases cured with out sequels. In the first 15 years, 3.6 cases/year were diagnosed and in the last 4 years, 1.7 cases/year.

Conclusions: Age of patient, time pass from the beginning of exanthema, symptoms and CSF analyse allow us to distinguish the three more frequent complications. These seem to be decreasing, probably due to the increase rate of vaccination.

\section{TRANSFERS OF NEWBORNS WITH BIRTH ASPHYXIA EXPERIENCE OF PAEDIATRIC INTENSIVE CARE TRANSPORT TEAMS IN THE PARIS AREA}

N Lodé ${ }^{1}$, P Jones ${ }^{1}$, J L Chabernaud ${ }^{2}$, J Lavaud ${ }^{3}$, V Henry Larzul ${ }^{4}$, A Ayachi $^{5}$

${ }^{1}$ SMUR, Robert Debré APHP, Paris, France, ${ }^{2}$ SMUR, Antoine Beclère APHP, Clamart, France, ${ }^{3}$ SMUR, Necker Enfants Malades APHP, Paris, France, ${ }^{4}$ SMUR, CH de Pontoise, France, ${ }^{5}$ SMUR, CH Montreuil, France

Background and Aims: We aimed to determinate the incidence of term newborns with birth asphyxia transported by the transport teams of Paris area, to analyse the difficulties of management and identify which of them require new therapeutic strategies.

Methods: A prospective study was performed among term babies from six months in 2002 in paediatric transport team units. Inclusion criteria were the association of one criterion of foetal distress during delivery and at least one criterion of neonatal asphyxia or one criterion of anoxic-ischemic encephalopathy. Data were analysed with Epi-info.

Results: 1576 newborns were transported. 101 were included (mean gestational age: 39,3 +/ -1,7 weeks; mean birth weight: $3155+/-627 \mathrm{~g}$ ). Half of them were born in level one maternities. 87 received resuscitation care and 20 had a10 min Apgar $<5$. For 50\% of them, the request for transfer was $55 \mathrm{~min}$ after birth and transportation of the neonates was completed after 3 hours. 86 were referring with ventilatory support. Four were included in hypothermia protocol at arrival in intensive care unit, 31 had a severe encephalopathy, 22 had multiple organ failure, 26 died. Babies with $10 \mathrm{~min}$ Apgar score $<5$, or who arrived in intensive care unit after 3 hours of life had more severe morbidity.

Conclusions: Paediatric transport teams transfer babies with critical initial state. Neurological and systemic complications are severe. Request for transfer should be made earlier in order to enable a better impact of new neuroprotective strategies.

\section{ASPIRATION RISK OF SEDATING CHILDREN AFTER INGESTION OF ORAL CONTRAST FOR CT IMAGING STUDIES}

K P Mason ${ }^{1,2}$, S E Zgleszewski ${ }^{1}$, M D'Angelo ${ }^{2}$, P J Fontaine $^{2}$, D Zurakowski ${ }^{3}$

${ }^{1}$ Department of Anesthesia, Children's Hospital Boston, Boston, MA, USA, ${ }^{2}$ Department of Radiology, Children's Hospital Boston, Boston, MA, USA, ${ }^{3}$ Department of Biostatistics, Children's Hospital Boston, Boston, MA, USA

Background and Aims: Pediatric CT imaging frequently requires the administration of oral contrast. Dilute gastrograffin can be consumed (either orally or via nasogastric tube) up to $500 \mathrm{ml}$ volume, with a typical "NPO" time interval of 30-90 minutes between ingestion and acquisition of CT images. Many pediatric patients require sedation to ensure immobility. The aspiration risk of pediatric patient's with oral contrast who undergo subsequent sedation for CT imaging, has never been specifically studied.

Methods: At Boston Children's Hospital, there is a defined sedation protocol for patient's undergoing CT sedation after ingestion of oral gastrograffin. There are defined clinical criteria which must be met for a patient to receive sedation. Absolute contraindications would include uncontrolled/unpredictable vomiting or gastroesophageal reflux, or a positive swallow study. Sedation consists of intravenous pentobarbital with fentanyl, if needed. A computerized sedation database tracks patient demographics and outcome. This database was retrospectively queried for aspiration events on all patients who received oral contrast with subsequent sedation since 1997.

Results: Since 1997, there have been a total of 4.126 sedations for CT imaging. 492 patients received oral contrast $1.5 \%$ dilute gastrograffin. There was one incident of vomiting which occurred after the study, with no subsequent sequela. Patient suffered no hypoxia, oxygen desaturation, need for airway management (positive pressure ventilation or endotracheal intubation) or clinically recognized aspiration.

Conclusions: The ingestion of dilute oral gastrograffin prior to pediatric sedation for CT imaging studies appears to be safe for patients who do not have uncontrolled gastroesophageal reflux or vomiting. 


\section{PATTERN OF PRESCRIBING MEDICATIONS BY PAEDIATRICIANS AND CHILD PSYCHIATRISTS}

H F Ayyash ${ }^{1}$, M Omran $^{1}$, R Verity ${ }^{2}$, M Thomas ${ }^{2}$

${ }^{I}$ Department of Paediatrics, Doncaster Royal Infirmary, Doncaster, South Yorkshire, UK, ${ }^{2} C A M H S$ and Adult Mental Health Team, Rotherham, Doncaster \& South Humber NHS Trust, UK

Background and Aims: To find out if there are differences in the regime of prescribing of stimulant and non-stimulant medications in Attention Deficit Hyperactivity Disorder (ADHD) patients who are treated by Paediatricians and Child Psychiatrists.

Methods: We reviewed the case notes of ADHD patients aged 15 to 16 years old who were treated in a Paediatric Service in Doncaster and a neighbouring CAMHS Service in Rotherham. A total of 71 patients were identified. Forty four $(62 \%)$ children were treated by paediatricians and $27(38 \%)$ were treated by Psychiatrists.

Results: Short acting Methylphenidate was prescribed more by Paediatricians than by Psychiatrists (61\% versus $11 \%$ ) respectively. Long acting Methylphenidate was prescribed more by Psychiatrists than by Paediatricians $(74 \%$ versus $18 \%)$ respectively. Dexamphetamine was given to $20 \%$ of patients attending Paediatric Services compared to none $(0 \%)$ patients attending CAMHS Services. Atomixitine was given to $7.4 \%$ of patients attending CAMHS compared to none $(0 \%)$ patients attending the Paediatric Services.

Conclusions: Psychiatrists tend to prescribe more the newer generation long acting stimulant (Concerta) and non-stimulant (Atomixitine) medications, which are known to be more expensive but also more preferable by patients and teachers as they are given once daily in the morning. The new NICE guidelines 2006 on the use of medications for ADHD should be used step by step by Paediatricians and Psychiatrists in order to avoid huge differences in prescribing, reduce cost of spending on drugs if necessary, and increase patients' confidence in our treatment regime.

\section{CHILDREN'S CHOICE OF MEDICINE - A TWO COUNTY EXPERIENCE}

S J Perera ${ }^{1}$, S Banerjee ${ }^{2}$

${ }^{I}$ Department of Paediatrics, Southend University Hospital, Westcliff on sea, Essex, UK, ${ }^{2}$ School and Child Health, Ramsgate, Kent, UK

Background and Aims: ADHD is a lifespan disorder and there is often a need for long term medication. Involving children and their carers in the choice of formulations and delivery methods promotes concordance. Data on children's choice of ADHD medication is scanty. The objective of this survey was to investigate the children's views and choice of formulations, delivery methods, duration of action and the preferred mode and source of receiving information about medication and side effects.

Methods: Views of randomly selected children attending ADHD clinics in South Essex and East Kent was obtained using an anonymised questionnaire. Each responses was rated from 0-10 (Likert scales).

Results: 122 questionnaires were analysed using SPSS version 10 . Around $20 \%$ children preferred syrup. $80 \%$ wanted a flavoured medication. (40\% strawberry flavour). $94 \%$ wanted a round tablet. ( $70 \%$ pea size). $42 \%$ wanted the effect of the drug to last 24 hours. Only $1 \%$ wanted information from the Doctors. $86 \%$ preferred the cartoons followed by the internet as a mode of receiving information.

Conclusions: Children's choice of medicine can be diverse in nature. Majority of children in an ADHD clinic sample preferred a pea size, round tablet of strawberry flavour that worked for 24 hours. Larger studies of this nature are warranted to improve children's drug compliance.

\section{THE IMPACTS OF MATERNAL POSTPARTUM DEPRESSION ON INFANTS DURING WELL- CHILD FOLLOW-UP VISITS}

\author{
F Simsek Orhon ${ }^{1}$, B Ulukol ${ }^{1}$, A Soykan ${ }^{2}$ \\ ${ }^{I}$ Department of Social Pediatrics, Ankara University, \\ School of Medicine, Ankara, Turkey, ${ }^{2}$ Department of \\ Psychiatrics, Ankara University, School of Medicine, \\ Ankara, Turkey
}

Background and Aims: Postpartum depression have important implications for both mother and child health. The aim of this study was to evaluate the associations of postpartum depression both with infants' feeding, sleep, growth, and developmental patterns, and with infants' behavioral states, as perceived by such mothers at each visit during follow-up.

Methods: The study was conducted at the 1st, 2nd, 4th, 6th, 9th, and 12th well-child visits. One hundred and three mothers and infants were participated to the study during the first month. At the end of the study sixtyeight mother-infant pairs regularly attended the whole visits. Self-report questionnaires have been applied to mothers with the purpose of eliciting infant feeding and sleep patterns, maternal sleep patterns, and the maternal perception of infant behavior at each visit. Denver II Developmental Screening test has been employed to infants, and the Edinburgh Postpartum Depression Scale (EPDS) has been employed to mothers at each visit.

Results: Mothers with higher depression scores were more inclined to report infant feeding and sleep problems, and infant colic. They perceived their infants more hardly controlled, unhappy, disturbed, frequently crying, and hardly-cared. The infants' daily weight gain was found sufficient. EPDS mean scores at the first month visit and the second month visit were relatively higher.

Conclusions: Mothers with depressive symptoms especially at early stages after delivery may define problems associated to their infants' feeding, sleep, colic, and behavior. Well-child visits provide an appropriate setting for diagnosing maternal postpartum depression, and preventing probable negative impacts of such depression on infants. 


\section{HOME VISITS FOR EVALUATION OF DIFFERENT EDUCATION METHODS TO PREVENT CHILDHOOD INJURY}

B Ulukol, F Simsek Orhon, S Usubutun, S Baskan Gulnar

Department of Social Pediatrics, Ankara University, School of Medicine, Ankara, Turkey

Background and Aims: We know that exposure to and absorption of safety education help inspire effective personal action to reduce the injury risks. The aim of this study was examine the effectiveness of two different child accident prevention education program to engagement in home safety practices prevent injuries to pre-school children's family.

Methods: This study was performed in Ankara, Turkey. Seventy-four parents were coming for well-child visit to Ankara University, Social Pediatrics Department for their children, were participated to the study. Fortyseven parents (almost mothers) were taken an interactive education program for home safety practices prevent injuries in the hospital. An education brochure was just given to other twenty-seven parents. One month later, home safety practices of these two groups' parents were evaluated with a home visit and researchers measured knowledge level of the mothers about injury risks.

Results: The knowledge levels of the mothers in interactive education group were significantly higher than the other mothers'. There were many hazards identified in the homes studied, but no difference between both groups. However, safety improvement was significantly higher in the interactive education group.

Conclusions: Parental attitudes are an important consideration in designing successful injury interventions. Our results suggest that the focused to home safety education in hospital was an effective intervention in knowledge of parents and home safety practices for prevent injuries of their children. Keywords: childhood injury, home safety, parent education

\section{AMERICAN ADVOCACY FOR PEDIATRIC RESEARCH AND EDUCATION}

\author{
M Genel $^{1}, \mathrm{~K}$ Hendricks ${ }^{2}$ \\ ${ }^{I}$ Yale Child Health Research Center, Department of \\ Pediatrics, Yale University School of Medicine, New \\ Haven, CT, USA, ${ }^{2}$ American Academy of Pediatrics, \\ North Washington, DC, USA
}

Background and Aims: Securing public support for medical research and education depends heavily upon developing effective communication with policymakers, elected and appointed, the media and the public at large. Effective advocacy also requires the research community to coordinate efforts and to present a coherent, unified message. Methods: In North America, three major societies representing pediatric investigators, scholars and academic administrators are the American Pediatric Society, Assn of American Pediatric Chairs (AMSPDC) and Society for Pediatric Research. For 22 years advocacy/public affairs of these three major societies have been conducted by a joint Public Policy Council (PPC) with two members from each who also serve as representatives to the AAMC Council of Academic Societies and to the American Academy of Pediatrics (AAP) Committee on Federal Government Affairs. Logistical and advocacy support is provided by the AAP capital office and activities are coordinated via a monthly conference call. Results: As measured by impact at the annual spring meeting of the Pediatric Academic Societies (PAS), the PPC has had significant success. A comprehensive report of legislative and government affairs relevant to research and education appears in the annual PAS meeting program. The PPC has organized a major plenary symposium for 13 years on topics such as training of subspecialty pediatricians, clinical trial registries, ethics of research in children and resident work hours. A legislative breakfast has been conducted for 20 years. Most significantly, the PPC has been a vehicle to unite the American pediatric community to work collectively for common goals. Conclusions: Coordination of public policy/advocacy activities by three major American pediatric socities through a common Public Policy Council has enhanced visibility within the academic community and more effectively serves academic pediatrics.

\section{COMPUTERIZED TRIAGE SYSTEM IN PRIMARY CARE}

N Yahagi ${ }^{1}$, H Fujita ${ }^{2}$, I Kato ${ }^{3}$, J Ishihara ${ }^{1}$

${ }^{I}$ Yokohama Municipal Citizen's Hospital, Yokohama, Japan, ${ }^{2}$ Kawasaki Municipal Hospital, Kanagawa, Japan, ${ }^{3}$ Grand Medical Associates Corporation, Tokyo, Japan

Background and Aims: Clinical decision making has three integrated phases: diagnosis, assessment of severity, and management. Clinical decision making is difficult due to the overlapings of the various types of conditions. We developed a computerized triage system (CTS) based on diagnosis algorithm and have validated it's accuracy.

Methods: All children visited the Pediatric Department of the Yokohama Municipal Citizens' Hospital for the year 2004. The patient's guardian input the patient's general conditions and symptoms into the CTS. The system then measured the severity level: mild/moderate/severe (includes life threatening level). After the examination of the patient, the Physician input the severity level. We verified and analyzed the difference between the CTS' data and the Physician's data.

Results: The data used were the 1367 randomly sampled from the 20851 patients' visitings. The severity level measured by the CTS was: mild 804, moderate 316 and severe 247. The severity level given by the Physician was: mild 1107, moderate 238 and severe 22 . We put them into the 'required-for-treatment' group or 'no-treatment-needed' group for analysis. Sensitivity was $100 \%$, specificity was $72.6 \%$, positive predictive value (PPV) was $46.1 \%$ and negative predictive value was $100 \%$. 
Conclusions: Approximately $50 \%$ of the patients was over-triaged by the system, but there was no underestimate. In the case of the severity level judged mild, by the CTS, was accurate. This system will not only support the patient, but also the Resident's decision making for severity. In the near future, we are sure that new CTS will provide both specificity and PPV over $80 \%$.

\section{PERFORMANCE OF THE PEDIATRIC RISK OF MORTALITY - PRISM III 24H IN A GREEK PICU}

E A Volakli ${ }^{1}, \mathrm{M} \mathrm{Sdouga}^{1}, \mathrm{M}_{\text {Tamiolaki }}{ }^{1}, \mathrm{M} \mathrm{Kotsiou}^{1}$, A Violaki $^{1}$, M Reizoglou $^{2}$, A Antoniades ${ }^{3}, \mathrm{M} \mathrm{M} \mathrm{Giala}^{4}$

${ }^{1}$ Pediatric Intensive Care Unit, Hippokration General Hospital, Thessaloniki, Greece, ${ }^{2}$ Department of Anesthesiology, Aristotle's University of Thessaloniki, Gennimatas General Hospital, Thessaloniki, Greece ${ }^{3}$ Organ Tsansplantation Unit, Aristotle's University of Thessaloniki, Hippokration General Hospital, Thessaloniki, Greece, ${ }^{4}$ Department of Anesthesiology, Aristotle's University of Thessaloniki, Ahepa University Hospital, Thessaloniki, Greece

Background and Aims: To evaluate the performance of the PRISM III $24 \mathrm{~h}$ predictive model in our PICU setting.

Methods: Prospective observational cohort study of 300 consecutive pts. Prism III $24 \mathrm{~h}$ predicted mortality rate was estimated with the free for 60 days PICUEs software version 3.2 trial. Values are mean \pm SEM, $p$ value was set at .05 .

Results: $92.3 \%$ of our pts were medical pediatric emergencies. Mean age was $54.26 \pm 2.8$ mo, mean PRISM III $24 \mathrm{~h}$ was $8.97 \pm 0.45$ given a predicted mortality rate of $11.16 \pm 0.19$. Overall mortality rate was $9.7 \%$. The performance of PRISM III scores were assessed through discrimination and calibration measures. Utilisation of receiver operating characteristics (ROC) curve showed good discrimination capability of the PRISM III scoring system for the whole population $(.892, \mathrm{p}=.00$; C.I.: .821 to .963$)$. The stratified performance for all age groups of the PRISM III score in predicting mortality has been evaluated by the Hosmer and Lemeshow's Goodness -of- Fit Test and showed a good calibration capability in the whole population $\left(\chi^{2}(3)=.234, \mathrm{p}=.972\right)$. The Standardised Mortality Ratio (SMR) was .87. Efficiency at admission (mortality risk $>1 \%$ ) was $76.7 \%$.

Conclusions: PRISM III $24 \mathrm{~h}$ performed well in predicting the overall mortality on the whole population of our PICU for all age groups. Effectiveness and efficiency of our PICU population was met by an international standard.

\section{THE TINIEST BABIES REGISTRY}

\section{E F Bell ${ }^{1}$, D K Zumbach ${ }^{2}$}

${ }^{I}$ Department of Pediatrics, University of Iowa, Iowa City, IA, USA, ${ }^{2}$ Health Information Systems, University of Iowa, Iowa City, IA, USA
Background and Aims: Infants weighing less than 400 $\mathrm{g}$ at birth rarely survive, and little information is available on the characteristics and outcomes of the few survivors. In 2000, we developed the Tiniest Babies Registry, a web-based registry of survivors with birth weight below $400 \mathrm{~g}$. The purpose of the Registry was to collect and make available information on these extraordinary infants.

Methods: Reports of surviving infants with birth weight below $400 \mathrm{~g}$ were collected from medical and lay publications, both print and web reports, and additional cases were submitted by parents or medical personnel to the Tiniest Babies Registry web site. In recent years, most cases have been submitted by the parents and validated by a treating neonatologist.

Results: The Registry is located at http://www.medicine.uiowa.edu/tiniestbabies. On 1 April 2006, the Registry contained 75 infants with birth weights ranging from 260 to $397 \mathrm{~g}$ and gestational ages from 23 to 34 weeks. All infants in the Registry were small for gestational age; $58(77 \%)$ are girls. The continents of birth are North America 57, Europe 14, Asia 4. The decades of birth are 1930s 3, 1980s 7, 1990s 26, 2000s (6 years) 39. Most children have developmental sequelae and lingering health issues. The majority who have reached school age are in regular classes, although some require special educational services.

Conclusions: The Tiniest Babies Registry provides a repository of information about the world's smallest surviving infants, those born weighing less than $400 \mathrm{~g}$. The predicament of these extremely vulnerable children is daunting but not hopeless.

\section{AIDS ORPHANS - OPTIMISING CARE FOR HIV INFECTED CHILDREN AND THEIR FAMILIES}

\section{Neves, M J Brito}

\section{Department of Paediatrics, Hospital Fernando Fonseca, Amadora, Portugal}

Background and Aims: The large number of HIVinfected women of childbearing age has resulted in a secondary epidemics of AIDS ORPHANS defined as a child who has lost one or both parents. A family care providing specialist and counseling services for families living with HIV. The purpose of this study was to characterize the problems concerning orphan hood.

Methods: Retrospective analysis, of orphans children born to HIV mothers, between 1999 and 2004 (6 years). Sociodemographic data, nuclear family history, legal issues and social situation were analysed.

Results: A total of 405 HIV families were observed, with 429 children. Less than a quarter (24\%) of children were cared for by both parents, $53 \%$ by mother alone and $11 \%$ by guardians or foster parents. There are 43 $(10 \%)$ of orphans and $12(27,9 \%)$ of them are HIV1 infection. The majority of the parents $38(88,3 \%)$ came from a Portuguese Speaking African Country and 17 $(44,7 \%)$ were illegal in our country. All of them have poor social-economical status and $18,6 \%$ have poor housing and stay at slum. Fourteen $(32,5 \%)$ of orphans are adopted inside family and $5(11,6 \%)$ siblings are severely separated and adopted from different families. 
The most of all $(95,3 \%)$ have support from the social service and two children return to its native country.

Conclusions: A strategy of care should be shared with primary care, pediatricians should become aware of local laws and community resources, and initiate discussion early in the course of parenteral illness to facilitate planning for the future care and custody of children.

\section{PATIENT SAFETY LEADERSHIP WALKROUNDS - A WAY TO MAKE SAFETY FOR CHILDREN IN A HOSPITAL SETTING}

\author{
B N Simonsen ${ }^{1}$, A M Carlsen ${ }^{2}$ \\ ${ }^{1}$ Juliane Marie Centre for Children, Women and \\ Reproduction, Rigshospitalet, Copenhagen, Denmark \\ ${ }^{2}$ Semi-Intensive Paediatric Unit, Juliane Marie Centre, \\ Rigshospitalet, Copenhagen, Denmark
}

Background and Aims: Patient Safety Leadership Walkrounds is an effective method to develop and maintain a safety culture. Walkrounds contribute to identify latent conditions within the system. Unlike active failures, latent conditions can be identified and remedied before an adverse event occurs. This leads to proactive rather than reactive risk management. We chose walkrounds, because the clinical setting, as a semi-intensive paediatric unit, is a high-risk area for sentinel events. The aim of this project is to proceed a self-sustaining culture of safety whereby each individual, is preoccupied with safety, and armed with the skills to evaluate his or hers environment for potential harm, and make appropriate changes.

Methods: It's a quality improvement design. Before and after description is done by a Hospital Survey Safety Culture Questionnaire. The intervention is three walkrounds during the period from September 2005 to September 2006.

Results: The first safety culture survey indicates that overall perception of patient safety is positive. The communication is open, and the answers indicate that the culture is a nonpunitive response to error. Yet there's room to improvement. Presently we have finished two walkrounds and identified up to nine safety issues. The issues have potential to harm patients. The first theme we analyzed was the patient record. The second walkround initiate qualitative interviews to investigate parents and children perspective of safety.

Conclusions: It's clear that the concept proceed a culture of safety. It visualized immediately for leaders in a constructive way, what nurses, doctors, children and their parents in an every day occurrence are concerned about.

\section{SIGNALLING INHERITED AND CONGENITAL CONDITIONS AT AGE 0-4 YEARS}

\author{
E J Siderius ${ }^{1}$, D J A Bolscher ${ }^{2}$, J J Roord ${ }^{3}$ \\ ${ }^{I}$ Department of Pediatric, Ziekenhuis Hilversum, \\ Hilversum, The Netherlands, ${ }^{2}$ Youth Health Care, \\ Thuiszorg Oost Veluwe, Apeldoorn, The Netherlands \\ ${ }^{3}$ Department of Pediatric, VU University Medical \\ Center Amsterdam, Amsterdam, The Netherlands
}

Background and Aims: In the Netherlands $95 \%$ of all children age 0-4 year are participating in a preventive public health care system. A. What are the possibilities of this preventive program in screening on inherited diseases with consequences for the family members and/or the child? B. What is the frequency of complex congenital disorders, demanding specific care and how is this provided?

Methods: During a period of 5 months a genetic trained paediatrician replaced the preventive child health care physicians in 92 different health care sessions. Standard procedures were followed including standard family history, physical examination and motor and speech development.

Results: 1085 children were examined: A) *22 (2\%) children showed on physical examination a symptom, possibly part of a syndrome*16 (1.4\%) family histories were positive for disorders with a risk for the child and or other family members. 7 parents were aware of the risk. B) *7 $(0.7 \%)$ children had a disorder that needed multidisciplinary care. They visited 3-6 different healthcare providers from the academic working sub specialist to the local dietician on the basis of each symptom separately. One child received integrated care. Conclusions: With a family-history-questionnaire in a child health preventive screening program genetic health risks for child and family members can be detected. Once a risk is established care is divided between different (sub) specialists and other local healthcare providers. Possibilities of prevention on the basis of genetics need adjustment of health care systems and training of healthcare providers.

\section{PRIMARY CARE IN PEDIATRIC TRAUMA - THE EXPERIENCE OF GENERAL DOCTORS IN THRACE, GREECE}

E Xenodoxidou ${ }^{1}$, C Pogonidis ${ }^{1}, \mathrm{~L}_{\text {Borgi }}{ }^{1}$, I Christodoulou $^{1}$, A Koukouliata ${ }^{1}$, O Koutsogiannis ${ }^{1}$, T K Konstantinidis ${ }^{2}$

${ }^{1}$ Health Center of Iasmos, Xanthi, Greece, ${ }^{2}$ Laboratory of Hygiene and Environment Protection, Democritus University of Thrace, Greece

Background and Aims: Pediatric trauma is the first cause of death in children. General Medicine has been strengthened as a specialty throughout Greece during past decade. Aim of this study is to report the experience of General Practitioners in pediatric trauma in Thrace, Greece.

Methods: In total 1250 cases of pediatric trauma were treated in Health Center of Iasmos during past decade. The children were 767 male, and 483 female, with a median age of $8 \pm 2,57$ years old. General Practition applied was reported and evaluated. A categorization in three groups was made, according to age: A $(<2$ months), B (1-5 years old) and C (5-14 years old).

Results: Falls from adults' bed were the first cause of pediatric trauma in group A, burns were the first cause in group B, and falls outside home were first in group C. There were no deaths and major trauma cases were transported to Hospitals of Thrace. The percentage of need for immediate transportation was $22,16 \%$, while recommendations for visiting a Hospital in case of 
arising symptoms were given in $32,24 \%$. The rest of cases were treated by general doctors. Information to schools and parents was given in annual meetings and events organized by the Health Center of Iasmos in cooperation with the Scientific Committee of Hospitals of Thrace.

Conclusions: Pediatric trauma is a very common problem for peripheral Health Centers. General Doctors have managed to offer primary care to injured children, decide and organize transportation to Hospital when needed and contribute in prevention via educational events.

\section{SPECTRUM OF SERVICES IN A PEDIATRIC, NON-UNIVERSITY HOSPITAL: THE DRG (DIAGNOSIS RELATED GROUPS) PERSPECTIVE}

\section{L Losa}

Department of Pediatrics, Ostschweizer Kinderspital, St. Gallen, Switzerland

Background and Aims: The reimbursement of hospitals by means of patient classifications schemes is actively discussed in Switzerland. In view of the experiences from Germany, considerable problems with financing are to be feared equally in Switzerland particularly for pediatric hospitals and departments.

Methods: The patient data for 2004 and 2005 were processed by a DRG-Grouper (APDRG-Grouper version 1.5, swiss-cw version 5.1). The resulting DRG-data were analysed using MS-Excel tables.

Results: 6258 cases were grouped. Out of 641 possible DRGs, 264 got at least one case. $28 \%$ of the cases were grouped in Major Diagnostic Categories (MDC) 6 and 4 (diseases and disorders of the respiratory or gastrointestinal system). The DRGs 777 (disorders of the digestive tract without complications, age $<18$ years) and 775 (bronchitis and asthma, without complications, age $<18$ years) represented $22 \%$ of the cases. With a cost weight (cw version 5.1) of 0.403 and 0.304 respectively these DRGs are among those with the lowest valuation. The Case Mix Index (CMI) of the hospital was 0.794 in 2004 and 0.832 in 2005.

Conclusions: The DRG analysis shows the following features: 1. High in homogeneity (many DRG groups /few cases). 2. Few low-valued DRGs with many patients. 3. The gross CMI of a tertiary children's hospital is comparable to that of a medium-sized adult's hospital without tertiary services. It is obvious to pediatric health professionals that this constellation will be the likely origin of financial problems but this has still to be proven by detailed data on services and costs.

\section{IMPROVING THE COMPLETENESS OF DATA IN A CLINICAL INFORMATION SYSTEM}

B A H Versluis ${ }^{1,2}, \mathrm{M}$ W Herrewijn ${ }^{2}, \mathrm{~W}$ G Ista ${ }^{2}$, $\mathrm{J} \mathrm{A} \mathrm{Hazelzet}^{2}, \mathrm{M}$ De $\mathrm{Mul}^{3}$

${ }^{1}$ Directorate Information, Department of Management and Maintenance Information Systems, Erasmus MC, Rotterdam, The Netherlands, ${ }^{2}$ PICU/NICU, Erasmus $M C$ - Sophia Children's Hospital, Rotterdam, The
Netherlands, ${ }^{3}$ Institute of Health Policy and Management, Erasmus MC, Rotterdam, The Netherlands

Background and Aims: Implementing and maintaining a Patient Data management System (PDMS) is an ongoing process. Eight years after the implementation of the PDMS, we experienced that complete and accurate recording of data was not self-evident. As missing or unvalidated data may negatively influence care of the patients we used a multilevel approach to improve completeness. The aim is to improve the documentation of the clinical data by the PICU physicians and nurses.

Methods: On the technical level, we have tailored the PDMS to meet our recording needs, by adding icons that launched a number of automatic data entries to the system. After a procedure, e.g. an arterial line insertion, the application will, after clicking an icon, launch an orderset and document an event automatically. By a 'breakthrough'-approach physicians and nurses were stimulated to improve documentation and to do this on an unambiguous manner. During the process we collected different real time variables related to procedures and compared these with the imported events by the PICU staff. Every month, the results were presented graphically to the team.

Results: Since the implementation, September 2005, correctly documentation of intubation procedures in patients increased of 20 to $73 \%$. Arterial procedures increased of 29 to $63 \%$. However, we also learned that completeness needs continuous attention, as we noticed a decrease in completeness in months when the research team did not present the graph to the staff.

Conclusions: Both technical improvements and personal strategies are needed to improve documentation completeness in a PDMS. Motivation of the staff needs continuous attention.

\section{CHILDREN UNDER THREE YEARS OF AGE PRESENTING TO THE EMERGENCY DEPARTMENT WITH SKULL AND LONG BONE FRACTURES, ARE WE RECOGNISING THE SIGNS OF NON-ACCIDENTAL INJURY?}

T W Bourke, V K Kumar, N P Corrigan

Department of Paediatrics, Altnagelvin Area Hospital, Londonderry, Northern Ireland, UK

Background and Aims: A child injury surveillance proforma exists in our emergency department to help distinguish accidental injuries from those requiring further expert assessment. We wished to establish if this proforma was being used effectively in patients with identifiable risk factors for non-accidental injury (NAI).

Methods: All patients under three years of age with skull and long bone fractures attending our emergency department in a one-year period were identified. Notes were retrospectively audited for documentation of proven risk factors for NAI.

Results: 389 children under 3 attended with injuries in the study period of which $56(14 \%)$ met the inclusion criteria. Notes were available for $56(100 \%)$ patients. Incomplete documentation of risk factors was noted in $18(32 \%)$. One or more risk factors were noted in 23 
(46\%). The child injury surveillance proforma was partially or fully completed in only $6(23 \%)$ of these. A social services referral was made for $1(4 \%)$.

Conclusions: The child injury surveillance proforma is not being completed in many children at potential risk of NAI. This may contribute to under-recognition of cases requiring referral for further expert assessment. Appropriate use of a child injury surveillance proforma combined with a comprehensive system of child protection training may help prevent this.

\section{NEAR INFRARED SPECTROSCOPY IDENTIFIES A SIGNIFICANT PATENT DUCTUS ARTERIOSUS IN PRETERM INFANTS}

\author{
M P Sherman ${ }^{1,2}$, M A Underwood ${ }^{1}$, J M Milstein ${ }^{1}$ \\ ${ }^{l}$ Department of Pediatrics, University of California, \\ Davis, CA, USA, ${ }^{2}$ Department of Pediatrics, Southern \\ Illinois University, Springfield, IL, USA
}

Background and Aims: Patent ductus arteriosus [PDA] is a frequent and potentially pathologic condition in preterm infants. A simple bedside screening tool to assess ductal patency would assist in the care of extremely low birthweight [ELBW] infants. Our aim was to study the utility of near-infrared spectroscopy [NIRS] to identify a hemodynamically significant PDA in ELBW infants.

Methods: Tissue oxygen saturation was measured by NIRS in the lungs, brain, skeletal muscle and kidney of 20 ELBW infants. Comparisons were made between the tissue saturation in these organs and the presence or absence of a significant PDA by echocardiography. All studies were performed within the first 2 weeks of life.

Results: A tissue saturation less than 50 in the kidney identified a significant PDA on the initial evaluation (positive predictive value $[\mathrm{PPV}]=.86$, negative predictive value $[\mathrm{NPV}]=.92, \mathrm{P}<.01)$ and in paired comparisons before and after closure of the PDA with indomethacin $(\mathrm{P}=.05)$. A ratio of the tissue saturation in the lung to the tissue saturation in the kidney of $>1.0$ predicted a significant PDA on the initial evaluations $(\mathrm{PPV}=.85, \mathrm{NPV}=.83, \mathrm{P}<.01)$ and on all evaluations $(\mathrm{PPV}=.93, \mathrm{NPV}=.78, \mathrm{P}<.01)$. This ratio also declined significantly after closure of the PDA with indomethacin $(\mathrm{P}=.01)$.

Conclusions: A portable NIRS device shows encouraging efficacy in identifying a hemodynamically significant PDA in ELBW infants and in assessing pharmacologic closure of the PDA.

\section{THE PROGNOSTIC VALUE OF AMPLITUDE INTEGRATED EEG IN NEONATAL SEPSIS/MENINGITIS}

\section{H J ter Horst, H de Vries, A F Bos}

Department of Pediatrics, University Medical Center Groningen, Groningen, The Netherlands

Background and Aims: Amplitude integrated EEG (aEEG) is a powerful tool to predict neurological outcome following asphyxia. Neonatal sepsis/meningitis is also known to cause mortality and neurological morbidity. We aimed to investigate the longitudinal course and prognostic value of aEEG traces in newborns suffering sepsis/meningitis.

Methods: Medical records of 13 infants that had suffered from sepsis/meningitis and had aEEG recordings were retrospectively investigated. Mean gestational age was 39,3 weeks; birth weight 3490 grams. 12 infants suffered from meninigitis (11 bacterial [2 E. Coli/9 GBS], 1 viral [enterovirus]); 1 infant had an E. Coli-sepsis. AEEG recordings were assessed by pattern recognition: background pattern, presence of sleep wake cycling (SWC) and epileptic activity (EA) were appraised. Recordings during the first 72 hours were used for analysis. 2 infants died; 3 had an abnormal neurologic development and 8 were normal at follow up.

Results: 6 aEEGs had low voltage background patterns (flat trace [FT], continuous low voltage [CLV] or burst suppression [BS]). A normal background pattern (discontinuous [DNV] or continuous normal voltage $[\mathrm{CNV}]$ ) was predictive for a normal outcome (MannWhitney U; $\mathrm{p}<0.05$ ). 7 infants had epileptic activity (EA) on their aEEGs. EA on aEEG was significantly more frequent in infants with an adverse outcome (Mann-Whitney U; p < 0.05). Sleep wake cycling (SWC) appeared in 10 infants. Appearance of SWC was more frequent in infants with normal outcome. (MannWhitney U; $p<0.01$ ).

Conclusions: Severely abnormal background patterns (FT, CLV and BS), EA and absence of SWC on aEEG are predictive for adverse outcome in infants suffering from neonatal sepsis/meningitis.

\section{AMPLITUDE INTEGRATED EEG IN PRETERM INFANTS WITH HYPOTENSION AND PERIVENTRICULAR LEUCOMALACIA}

H J ter Horst ${ }^{1}$, T Tameling ${ }^{1}$, L A van Eykern ${ }^{2}$, A F Bos ${ }^{1}$

${ }^{I}$ Department of Pediatrics, University Medical Center Groningen, Groningen, The Netherlands, ${ }^{2}$ Department of Developmental Neurology, University Medical Center Groningen, Groningen, The Netherlands

Background and Aims: Amplitude integrated EEG (aEEG) changes with gestational age (GA). Brain activity is dependent on cerebral perfusion and oxygenation. Brain ischemia is a major factor contributing to development of periventricular leucomalacia (PVL). We aimed to investigate if PVL and hypotension influence aEEG in preterms during the first weeks of life.

Methods: AEEG was recorded in 13 infants with GA of 26-32,9 weeks (median $31 \mathrm{wk}$ ), birth weight was 6351805 gram (median $1200 \mathrm{~g}$ ). Longitudinal aEEG was digitally recorded at weekly intervals until discharge. AEEG was assessed by pattern recognition. In addition the highest, lowest and mean values of the 5th, 50th and 95th centile of aEEG amplitudes were calculated. PVL was diagnosed by serial cranial ultrasound and hypotension by the necessity for vaso-active agents.

Results: 56 recordings (median of 3 (range 2-8) per infant) were assessed. AEEGs were mainly discontinuous with sleep wake cycling during first 
recording. With increasing postmenstrual age continuity increased, also reflected by an increase of the mean values of 5th and 50th centiles $(\mathrm{r}=0.46, \mathrm{p}<0.001$ and $\mathrm{r}=0.61, \mathrm{p}<0.01$ respectively). Infants with PVL grade I $(\mathrm{n}=5)$ had lower values for lowest 5 th and highest 5 th and 50th centiles $(p<0.05)$. Infants suffering hypotension $(n=3)$ had lower values for all centiles but one (lowest 95th centile) $(\mathrm{p}<0.05)$.

Conclusions: Digital recording makes it possible to assess aEEG in more detail. In case of hypotension and PVL grade I aEEG amplitudes are lower. This may be the result of lower cerebral perfusion and might have implications for managing hypotension in preterms.

\section{REGIONAL BLOOD FLOW ADAPTATION IN THE WEEK FOLLOWING SURGICAL PDA LIGATION}

\section{S Hoodbhoy, H Cutting, M E Campbell}

Department of Neonatal, Guy's and St Thomas' NHS Foundation Trust, London, UK

Background and Aims: Abnormalities in regional blood flow have been reported in babies with a patent ductus arteriosus (PDA). Our aim was to describe regional vascular adaptation to surgical PDA ligation in infants $<1500 \mathrm{~g}$.

Methods: Doppler measurements of average velocity (AV) from middle cerebral (MCA), celiac (CA), and superior mesenteric (SMA) arteries were performed preoperatively, at 3-6 hours, 24 hours and 1 week post ligation.

Results: We studied 13 infants, birthweight $802+/-222 \mathrm{~g}$, GA $25.9+/-1.3$ weeks and age at surgery $28+/-10.4$ days. Data are presented as mean (+/-SD) or median (IQR). (* $\mathrm{p}<0.05$ vs pre-op)

Conclusions: AV increased significantly in all vessels; however the pattern of change differed between vessels suggesting region specific adaptations. These data suggest lush blood flow in the splanchnic bed immediately after surgery and may indicate increased vulnerability to gut injury in the early post-operative period.

\begin{tabular}{|l|c|c|c|}
\hline & \multicolumn{3}{|c|}{ AV (cm/sec) } \\
& MCA & CA & SMA \\
& & & \\
\hline Pre op & $20.2 \pm 6.1$ & $33.8(27.7,41.1)$ & $17.5 \pm 3.5$ \\
\hline 3 hrs & $23.7 \pm 8.8$ & $46.3(42.0,54.2)^{*}$ & $32.8 \pm 12.9 *$ \\
\hline $\mathbf{2 4}$ hrs & $25.9 \pm 5.0$ & $49.7(39.3,58.4)$ & $35.2 \pm 8.9 *$ \\
\hline 1 week & $27.2 \pm 9.9 *$ & $45.8(33.8,54.1)$ & $31.9 \pm 9.2^{*}$ \\
\hline
\end{tabular}

\section{CEREBRAL BLOOD FLOW VELOCITY BY PULSED DOPPLER ULTRASOUND IN FULL TERM INFANTS WITH ASPHYXIA}

\author{
M Julkunen ${ }^{1,2}$, M Janas ${ }^{2}$, R L Seppänen ${ }^{3}$, \\ O Tammela ${ }^{1,2}$
}

${ }^{I}$ The Pediatric Research Centre, University of Tampere, Tampere, Finland, ${ }^{2}$ Department of Paediatrics, Tampere University Hospital, Tampere, Finland, ${ }^{3}$ Unit of Physical Medicine and Rehabilitation, Tampere University Hospital, Tampere, Finland
Background and Aims: To evaluate the correlation between cerebral blood velocities by pulsed Doppler ultrasound, severity of hypoxic ischaemic encephalopathy (HIE) and outcome at 1 year's age in asphyxiated newborns.

Methods: Thirty term infants (GA 37-42 weeks) with birth asphyxia (5 min Apgar 5 or less) and 30 healthy age-and sex-matched controls underwent pulsed Doppler ultrasound examinations three times during the first 24 hours of life. Systolic cerebral blood flow velocities (SCBFV) of anterior cerebral (ACA), basilar (BA) and internal carotid (ICA) arteries were measured. HIE was graded using modified Sarnat criteria (stage 03). Motor development by Alberta Infant Motor Scale (AIMS) was assessed at 1 year's age.

Results: The SCBFVs during the first 24 hours of life were similar in infants with or without HIE. Asphyxiated infants with abnormal outcome at 1 year's age $(n=6)$ had significantly higher SCBFV in all three arteries at 24 hours of age than asphyxiated infants with normal outcome $(\mathrm{n}=21)$; in the ACA $(50.8 \mathrm{~cm} / \mathrm{s}(17.5)$ vs. $32.5 \mathrm{~cm} / \mathrm{s}(6.6), \mathrm{p}=0.034)$; in the BA $(59.3 \mathrm{~cm} / \mathrm{s}$ (12.9) vs. $38.5 \mathrm{~cm} / \mathrm{s}(9.2), \mathrm{p}=0.001$; in the ICA $(56.2$ $\mathrm{cm} / \mathrm{s}(6.8)$ vs. $41.0 \mathrm{~cm} / \mathrm{s}(9.9), \mathrm{p}=0.04)$. AIMS points (maximum 58) at 1 year's age were significantly lower in asphyxiated infants with abnormal outcome than other asphyxiated infants (40.3 (7.6) vs. 53.7 (4.0), $\mathrm{p}=0.003$ ).

Conclusions: High SCBFV during the first 24 hours of life seems to predict adverse outcome better than HIE symptoms in infants with birth asphyxia.

\section{ELECTROENCEPHALOGRAPHIC RESPONSE TO PROCEDURAL PAIN IN HEALTHY NEWBORN INFANTS}

E Norman ${ }^{1}$, I Rosén ${ }^{2}$, V Fellman ${ }^{1,3}, \mathrm{~S}$ Vanhatalo ${ }^{4}$, K Stjernqvist $^{5}$, O Ökland ${ }^{6}$, L Hellström-Westas ${ }^{1}$

${ }^{1}$ Department of Paediatrics, Institution of Clinical Science, Lund University Hospital, Lund, Sweden ${ }^{2}$ Department of Clinical Neurophysiology, Institution of Clinical Science, Lund University Hospital, Lund, Sweden, ${ }^{3}$ Department of Paediatrics, University Hospital of Helsinki, Helsinki, Finland, ${ }^{4}$ Department of Clinical Neurophysiology, University Hospital of Helsinki, Helsinki, Finland, ${ }^{5}$ Department of Psychology, Lund University, Lund, Sweden, ${ }^{6}$ Department of Paediatrics, Alesund Hospital, Alesund, Norway

Background and Aims: A recent study (Fernandez et al, 2003) reported that painful or unpleasant stimuli in newborns result in measurable changes in frontal theta (3-6 Hz) activity, attenuated by oral glucose. We aimed at investigating the type and magnitude of frontal EEG responses to nociceptive stimuli in a larger group, undergoing different types of noxious procedures.

Methods: Healthy term infants were investigated $(\mathrm{n}=72), 28$ (group 1) underwent a heel prick (non skinbreaking procedure from the Neonatal Behavior Assessment Scale). Prior to the heel-prick, 16 of the 28 infants received oral glucose and 12 water (randomised, double-blind). Group $2(\mathrm{n}=21)$ underwent a venous blood sample from the dorsum of the hand, and group 3 $(n=23)$ capillary heel blood sampling for metabolic 
screening. EEG was recorded from five derivations (F3, $\mathrm{F} 4, \mathrm{Cz}, \mathrm{P} 3$ and P4), the infants were videotaped and assessed with the Premature Infant Pain Profile (PIPP). EEG power $(\mu \mathrm{V} 2)$ within the theta band $(3$ to $6 \mathrm{~Hz})$ was calculated, an asymmetry index between right and left frontal activity was derived using natural logarithms. Non-parametric tests were used.

Results: In group 1, infants given glucose had lower PIPP than those given water $(p<0.05)$. There were no consistent correlations between PIPP, PNA or gestational age, and EEG power measures. There was no change in EEG asymmetry; i.e. absolute and relative theta power for right frontal activity in comparison to left frontal activity.

Conclusions: The present results demonstrate that EEG theta activity is not informative in assessing infant's response to noxious stimulus.

\section{ORAL HYPERTONIC GLUCOSE FOR ANALGESIA IN PRETERM NEWBORNS}

\section{Z Badiee}

Neonatologist, Assistant Professor of Pediatrics, Isfahan University of Medical Sciences, Alzahra Hospital, Isfahan, Iran

Background and Aims: A number of studies have shown that orally administered sweet tasting solutions reduce signs of pain during painful procedures in the term newborns. The aim of the present study was to compared the pain reducing effect of orally administered glucose with that of placebo during venipuncture in preterm infants.

Methods: A Randomized, double- blind, placebo controlled trial was carried out at the neonatal intensive care unit at the Alzahra Hospital, Isfahan, Iran from June to September 2005. Thirty six preterm newborns (gestational age 28-34 weeks; postnatal age (1-14 days) undergoing venipuncture for clinical purposes, were enrolled in the study. Each infant was assessed two times receiving $1 \mathrm{ml} 30 \%$ glucose or $1 \mathrm{ml}$ sterile water by mouth, 2 minutes before venipuncture. The behavioral pain reactions were scored with the Premature Infant Pain Profile and duration of crying and heart rate were measured.

Results: The PIPP scores were significantly lower in the glucose group compared with the placebo group (8.94 \pm 3.013 VS $11.44 \pm 3.42$ respectively, $\mathrm{P}<0.001$ ). The duration of crying in the first 3 minutes was significantly lower in $30 \%$ glucose compared with placebo group $(24.1 \pm 18.48$ VS $39.6 \pm 26.8$ respectively, $\mathrm{P}=0.002$ ).

Conclusions: We found that $30 \%$ glucose may be a useful and safe analgesic for minor procedures in preterm newborns.
CHANGES IN CEREBRAL OXYGENATION AS MEASURED BY NEAR-INFRARED

SPECTROSCOPY DURING EARLY POSTNATAL ADAPTATION IN NEWBORNS DELIVERED BY VACUUM EXTRACTION

T Karen ${ }^{1,2}$, M Wolf $^{1}, \mathrm{R} \mathrm{Nef}^{1}$, D Haensse ${ }^{1}, \mathrm{H}$ U Bucher ${ }^{1}$, G Schulze $^{1}$, J C Fauchère ${ }^{1}$

${ }^{1}$ Clinic of Neonatology, University Hospital Zurich, Zurich, Switaerland, ${ }^{2}$ Department of Neonatology, University Children's Hospital Tuebingen, Tuebingen, Germany

Background and Aims: Newborns delivered by vacuum extraction quite often represent a group of infants with a haemodynamic instability. The conventional means to measure the haemodynamic status are not sensitive enough to assess the severity of general, and more specifically of cerebral circulatory imbalance. Near-infrared spectroscopy was used to study cerebral tissue oxygenation during postnatal adaptation in these infants.

Methods: 15 newborn infants delivered by vacuum extraction (group 1) due to fetal distress were enrolled in this study. Immediately after birth and again after 12-24 hours of life, a near-infrared sensor (NIRO-300TM Hamamatsu Japan) was placed over the right forehead of the infant. The tissue haemoglobin index (THI), oxygenation index (TOI), arterial oxygen saturation (pre-ductal tcSaO2) and heart rate (HR) were recorded. Mean values were calculated for the first 0-5, 5-10 and 10-15 minutes and for the measurement at 12-24 hours (median 21 hours) of life and compared by Wilcoxon test to 15 healthy newborns infants delivered by elective caesarean section (group 2).

Results: No statistically significant differences of THI, TOI, $\mathrm{tcSaO} 2$ and HR were observed between the two groups. There was a trend for higher mean values of HR and THI in infants born by vacuum extraction (group 1). By 21 hours, the THI remained higher by 55,3\% $(=19.7$ $\mu \mathrm{M})$ in group 1 compared to group 2. This difference was not significant $(\mathrm{p}=0.108)$, possibly due to the large variability within the groups.

Conclusions: The postnatal adaptation of cerebral oxygenation in newborn infants delivered by vacuum extraction following fetal distress is similar to healthy infants.

\section{ANTENATAL FAST MRI IN THE PRENATAL DIAGNOSIS OF FETAL MALFORMATIONS}

G Tonni ${ }^{1}$, P P Pattacini ${ }^{2}$, C De Felice ${ }^{3}$, A Ventura ${ }^{1}$

${ }^{I}$ Division of Obstetrics and Gynecology, Guastalla Civil Hospital, AUSL Reggio Emilia, Reggio Emilia, Italy

${ }^{2}$ Division of Radiology, Guastalla Civil Hospital, AUSL Reggio Emilia, Reggio Emilia, Italy, ${ }^{3}$ Department of Neonatology, Policlinic Hospital 'Le Scotte', University of Siena, Siena, Italy

Background and Aims: To evaluate the role of integrating ultrasound with antenatal ultrafast MRI (Magnetic Resonance Imaging) in the prenatal diagnosis of fetal malformations during 2 nd and 3 rd trimester of pregnancy. 
Methods: 2D-3D ultrasound (US) and targeted ultrafast MRI have been used to detect a panel of fetal malformations.

Results: Two cases of bilateral severe hydrocephalus of whom one associated with Dandy-Walker syndrome, one case of cleft lip and palate of whom the latter undiagnosed by 2D-3D US, one case of right lung sequestration, one case of placental pathology, one case of renal dysplasia with associated ureterocele and one case of meconium peritonitis due to ileal atresia were diagnosed.

Conclusions: Targeted antenatal ultrafast MRI enhance the prenatal diagnosis of fetal malformation especially those cases regarding the definition of brain structures, soft tissues and abdominal mass. The integration of ultrasound with antenatal MRI may lead to better definition of the anatomical landmarks of the fetal malformations thus improving the prenatal counselling of the patient and the parental decision-making strategy.

\section{SERIAL CEREBRAL BLOOD FLOW PROFILES OF PRETERM AND TERM NEONATES}

S Abbasi ${ }^{1}$, K Finnegan, J S Gerdes

Department of Pediatrics, Pennsylvania Hospital, Children's Hospital, University of Pennsylvania, Philadelphia, PA, USA

Background and Aims: Cerebral blood flow (CBF) disregulation as measured by Pourcelot's resistive index (RI) is GA dependent and may play a significant role in the development of poor neurologic outcome of infants with intraventricular hemorrhage (IVH) and hypoxicischemic encephalopathy (HIE). Objective: To generate normal reference data for neonates of different $\mathrm{BW}$ and GA, and compare it to those with cerebral pathology.

Methods: Spectral Doppler blood flow profiles of the anterior cerebral (ACA) and middle cerebral (MCA) arteries were obtained using an Acuson XP color and Duplex Doppler units with a 5.0/7.5 MHertz specter probe. RI was calculated as (peak systolic velocity-end diastolic velocity)/peak systolic velocity.

Results: Data from 400 normal infants (Group1, BW $1923 \pm 686 \mathrm{~g}$, GA $32.7 \pm 3.3 \mathrm{wks})$ are compared to 46 with Grade I-II IVH (Group 2, BW 1567 $\pm 765 \mathrm{~g}$, GA $30.1 \pm 4.0 \mathrm{wks}$ ), 14 with Grade III-IV IVH (Group 3, BW $985 \pm 345 \mathrm{~g}$, GA $26.7 \pm 2.5 \mathrm{wks}$ ), and 12 with HIE (Group 4, BW $2481 \pm 1048 \mathrm{~g}$, GA 34.9 $\pm 5.1 \mathrm{wks})$. ACA RI's for groups 1 to 4 were $0.74 \pm 0.08,0.75 \pm 0.10,0.71 \pm 0.11$, $0.67 \pm 0.12 \mathrm{SD}$ respectively. MCA RI's for groups 1 to 4 were $0.73 \pm 0.07, \quad 0.77 \pm 0.07, \quad 0.72 \pm 0.06, \quad 0.67 \pm 0.12 \mathrm{SD}$ respectively. In normals, mean RI decreased significantly with increasing GA and BW. RI decreased significantly with increased severity of cerebral pathology. Neurodevelopmental screening at 2 years was abnormal for $52 \%$ of HIE infants, $18 \%$ of grade $3-4$ IVH/PVL infants as compared to $4 \%$ of matched controls. Lowest RI $(<0.5)$ correlated with mortality and worst long-term outcome.

Conclusions: Doppler ultrasonography of cerebral arteries may provide a means of identifying infants at risk for cerebral vascular insult and poor outcomes.
ENDOTRACHEAL SUCTIONING AND CEREBRAL HEMODYNAMICS IN NEONATES

C Michel ${ }^{1}$, P Staquet ${ }^{1}$, M Arsac ${ }^{1}$, S Marret $^{1}$, D Eurin ${ }^{2}$, T Blanc ${ }^{1}$, V Laudenbach ${ }^{1}$

${ }^{I}$ Department of Neonatal Medicine, Charles Nicolle University Hospital, Rouen, France, ${ }^{2}$ Department of Pediatric Radiology, Charles Nicolle University

Hospital, Rouen, France

Background and Aims: In adults under mechanical ventilation, endotracheal suctioning (ES) increases cerebral blood flow (CBF). In neonates, the effects of ES on CBF are poorly described. To evaluate the effects of $\mathrm{ES}$ on $\mathrm{CBF}$ velocity (CBFV) in ventilated neonates, analyzing the respective influence of i) gestational age and ii) perinatal asphyxia (PA).

Methods: 26 ventilated neonates, born at different term, aged 1 to 48 hours, without sedation, with PA or not ('PA' and 'no PA' groups) were prospectively enrolled after parental informed consent. CBFV was measured in the pericalleous artery during 2 successive ES. The primary end point was the variation of the mean velocities $(\mathrm{Vm})$. Results were analyzed with Student T-test or Wilcoxon-Mann-Whitney test.

Results: Basal Vm was higher in the 'PA' group when compared to the 'no PA' group $(18,2 \pm 5,9 \mathrm{~cm} / \mathrm{s}$ versus $12,8 \pm 4,6 \mathrm{~cm} / \mathrm{s}$, mean $\pm \mathrm{SD}, \mathrm{p}=0,019)$. In the 'no $\mathrm{PA}^{\prime}$ group, $\mathrm{Vm}$ increased by $32,5 \pm 20,3 \%$ after the 1 st ES $(\mathrm{p}<0,001) . \mathrm{Vm} 2 \mathrm{mn}$ after the 1st ES remained increased when compared to basal Vm. The 2nd ES tended to further increase $\mathrm{Vm}$. In the 'PA' group, Vm did not vary significantly after ES. Gestational age did not influence these results.

Conclusions: We observed an increase in CBFV, probably due to increased $\mathrm{CBF}$, after ES in ventilated newborns without PA. PA was associated with a higher CBFV apart from any ES but the ES then did not induce any further variation. Analyzing the effects of sedatives on this PA-related elevated CBF, the pathophysiological significance of which remains unknown, is an interesting matter of research.

\section{EFFECTS OF DIFFERENT PHASES OF SURGICAL REPAIR OF CONGENITAL DIAPHRAGMATIC HERNIA (CDH) ON CEREBRAL HAEMODYNAMICS EVALUATED BY NEAR INFRARED SPECTROSCOPY (NIRS)}

A Dotta, A Braguglia, F Campi, J Rechichi, I Capolupo, S Lozzi, C Corchia, P Bagolan, M Orzalesi

Department of Medical and Surgical Neonatology, Bambino Gesù Children's Hospital, Rome, Italy

Background and Aims: Surgery for $\mathrm{CDH}$ induces a reduction in cerebral blood volume and oxygenation. Our aim was to identify in which phase of the surgical procedure these changes occur preferentially.

Methods: Fourteen CDH infants were operated at a median age of 3.8 days. Surgery was divided into 3 phases: Removal of viscera from the chest (P1), Closure of diaphragm (P2), Repositioning of viscera into the abdomen (P3). SaO2, Heart Rate and Mean Arterial 
Blood Pressure (MABP) were continuously monitored and, if necessary, $\mathrm{FiO} 2$ was adjusted to maintain $\mathrm{SaO} 2$ $>80 \%$. Changes in Tissue Oxygenation Index (TOI, \%) and total cerebral haemoglobin $(\mathrm{tHb}, \mathrm{mM} / \mathrm{l})$, with respect to initial baseline, were assessed by NIRS. Values obtained at the beginning and at the end of each phase were expressed as means $\pm \mathrm{SD}$ and compared by the Student's t-test for paired values.

Results: $\mathrm{FiO} 2, \mathrm{SaO} 2$ and $\mathrm{MABP}$ did not change significantly during surgery. The lowest value of TOI was reached at $\mathrm{P} 1$ in 1/14 patients, at $\mathrm{P} 2$ in 9/14 and at P3 in 4/14 babies. During P1, TOI and $\mathrm{tHb}$ did not change significantly; during P2 TOI $(70.7 \pm 10.8$ vs $64.4 \pm 11.2 \%, \mathrm{p}<0.01)$ and $\mathrm{tHb}(+1.7 \pm 0.01$ vs $-2.6 \pm 0.01$, $\mathrm{p}<0.05)$ decreased significantly; during P3 TOI decreased further initially (63.4 \pm 9.3$)$ and recovered partially at the end $(66.4 \pm 10.3)$.

Conclusions: Our results show that, for cerebral haemodynamics, the most critical phase of surgical repair of $\mathrm{CDH}$ is the closure of the diaphragmatic defect, when the compression of the inferior vena cava is more marked and could impair venous return.

\section{EXTERNAL VENTRICULAR DRAINAGE WITH TEFLON SCREW FOR TREATMENT OF RAPIDLY PROGRESSIVE POSTHEMORRAGIC HYDROCEPHALUS IN PREMATURE NEWBORN}

A Franceschi, M Tumolo, G Ottonello, L Nahum, A Moscatelli, S Casanova, G Silvestri

Dipartimento di Anestesia e Rianimazione Pediatrica, Istituto Giannina Gaslini, Genova, Italy

Background and Aims: Thirteen premature newborn, birthweight $1250 \mathrm{~g}$ (745-1965) with rapidly progressive posthemorragic hydrocephalus were treated by external ventricular drainage with teflon screw. Seven patients suffered from intra ventricular hemorrage grade 3 and six grade 4 .

Methods: The drainage was placed at day $8,4+/-2$ after intra ventricular hemorrage occurred and was kept in place 33,4+/- 6 days, the longest period being 46 days. Monitoring of intracranial pressure was performed while the drainage was in place.

Results: One of thirteen patients died for causes unrelated to external ventricular hemorrage. Twelve patiens survived. In 3 of 12 surviving patients progressive posthemorragic hydrocephalus recurred after removal of the drainage and required definitive shunt implantation. For the remaining 9 patients no further therapy was necessary. Bacterial cultures from cerebro spinal fluid were negative in 11 patients and positive in 2 patients. No chemical or biochemical evidence of ventriculitis was noted. In 3 patients neurological development was classified as normal, 8 patients experienced mild or moderate paresis and development delay; one patient was severely retarded. Visual deficits were absent in 7 patients, in one patient total blindness was present and in 4 patients strabismus.

Conclusions: This study reports a $25 \%$ incidence of hydrocephalus recurrence and definitive shunt implantation. Precoucious drainage, monitoring of intracranial pressure, daily cerebral echograpy and cerebro spinal fluid protein less than $100 \mathrm{mg} / \mathrm{dl}$ before external ventricular drainage removal are probably useful means to achieve a low rate of definitive shunt implantation.

\section{EFFECTIVENESS OF A BRIEF COURSE IN INTERPRETING AMPLITUDE INTEGRATED ELECTROENCEPHALOGRAMS IN TERM NEWBORN}

\begin{abstract}
A Soliz, B Chandler, P Valencia, I Alfonso,
\end{abstract} E Vasconcellos

Miami Children's Hospital, Miami, FL, USA

Background and Aims: Routine monitoring and interpretation of newborn brain activity using conventional electroencephalogram (EEG) in Neonatal Intensive Care Units (NICUs) is difficult. Amplitudeintegrated electroencephalogram (aEEG) technology may help NICU personnel evaluate neonatal brain activity in a rapid, non-invasive, and cost-effective manner. Whether NICU personnel could learn to recognize patterns of aEEG/EEG tracings in term newborns after a brief introductory training.

Methods: Eleven physicians and twenty seven nurses without training in brain monitoring interpretation participated in a pre-test, a brief interactive training course to interpreter neonatal aEEG/EEG patterns, and a follow-up post-course test to interpret these tracings. Twenty five tracings of normal function, brain injury, seizures, and artifacts obtained from a 2 channel aEEG/EEG 'Brain $Z$ monitor' were shown to participants. Twelve questions were given pre and post training. Answers were blindly scored. Chi-square was used for statistical analysis.

Results: Four hundred fifty six answers were analyzed. $\mathrm{We}$ found significant improvement in correct interpretations of aEEG/EEG monitor tracings between the pre and post test respectively: Normal tracing 10/38 vs. $38 / 38$ ( $<00.001)$; bilateral brain injury $1 / 38$ vs. $37 / 38$ $(\mathrm{p}<0.001)$; unilateral brain injury $7 / 38$ vs. $34 / 38$ $(\mathrm{p}<0.001)$; seizures $1 / 38$ vs. $31 / 38 \quad(\mathrm{p}<0.001)$; status epilecticus $1 / 38$ vs. $34 / 38(\mathrm{p}<0.001)$; artifact $1 / 38$ vs. $36 / 38(\mathrm{p}<0.001)$.

Conclusions: This study shows that a brief instructional course on interpretation of aEEG/EEG tracings can be effective in improving NICU personnel's recognition of normal and abnormal term neonatal brain patterns.

\section{NEUROIMAGING IN NEONATAL HYPOGLYCAEMIA}

C Ruiz Serrano ${ }^{1}$, M Blanco Rodríguez ${ }^{1}$, R Benítez Fuentes ${ }^{1}$, M Velázquez De Cuéllar Paracci ${ }^{1}$, A Leal Orozco ${ }^{1}$, N Domínguez Garrido ${ }^{1}$, D Quiñones Tapia ${ }^{2}$, L Soriano Guillén ${ }^{1}$, M Bernácer Borja ${ }^{1}$

${ }^{l}$ Department of Pediatrics, Fundación Jiménez Díaz, Madrid, Spain, ${ }^{2}$ Department of Neuroradiology, Fundación Jiménez Díaz, Madrid, Spain

Background and Aims: Neuroradiological pictures and clinical patterns of two cases of severe neonatal hypoglycaemia: Case 1. A term male three days old with 
poor feeding in the last 12 hours. He showed signs of dehydration, neurological deterioration and generalized seizures associated with severe hypoglycaemia and metabolic acidosis. Case 2: A term male born by cesarean delivery after an uneventful pregnancy that presented micropenis, respiratory distress and severe hypoglycaemia whenever glucose infusion was reduced or interrupted. Endocrine study revealed multiple pituitary hormone deficiencies.

Methods: Brain imaging at 1.5 Tesla consists of saggital T1, axial TSE T2, coronal FLAIR and diffusion. Case 1: the follow-up MRI included 3D T1 and venous MRA. Case 2: the sella was evaluated with high resolution $\mathrm{T} 13 \mathrm{D}$.

Results: Case 1: Simetric diffuse effacement of posterior parieto-occipital sulci was associated with $\mathrm{T} 2$ hyperintensity, increased diffusion, and decreased ADC indicating citotoxic edema. An abnormal deep parafacine left vein is noted with absent straight sinus and vein of Galen. Mielination and cortical structures were normal. The abnormal vein was confirmed on MRA, draining to a small irregular posterior superior sagital sinus. The torcula and transverse sinuses were normal. Case 2: The cerebrum, sulci and mielination were normal. The sella was small, with ectopic bright spot in diencephalon and absent pituitary stalk.

Conclusions: There are few reports relating neuroimaging findings in neonatal hypoglycaemia. We report two cases of severe neonatal hypoglycaemia where neuroimaging demostrated significant findings on MRI.

\section{THE EFFICACY OF BRAIN ULTRASOUND EXAMINATION FOR DIAGNOSTICS OF INTRAUTERINE INFECTIONS}

\section{N Sapanadze, M Kherkheulidze, N Kavlashvili \\ State Medical University, Tbilisi, Georgia}

Background and Aims: Intrauterine infections are one of the main causes of neonatal and infant morbidity and mortality. Early diagnostics and appropriate treatment of these diseases is very important. The aim of the study was to evaluate the efficacy of brain ultrasound examination (BUE) for diagnostics of intrauterine infections.

Methods: We studied 0-3 month's age 150 children with diagnosis of sepsis, perinatal encephalopathy, intracranial hypertension, and prolonged neonatal jaundice. BUE was conducted for every patient and in case of special changes that characterized infections (calzificats, edema, periventricular ishemia, microcysts, and ventriculomegalia) were studied special Ig M and $\mathrm{IgG}$ by immunofermental assay. The elevation of specific antibodies more then 4 times in second analyzes comparing to first one was considered as diagnostic criteria of intrauterine infections.

Results: The results of the study showed that 32 patients $(36 \%)$ had the changes, that characterized infections and in all these patients the ethnologic factors of intrauterine infections (cytomegalovirus, herpes, toxoplazma, chlamidia) by immuno-fermental assay were discovered. In the Control group the patients with no changes of the
BUE intrauterine infections were diagnosed only in 3 cases (chlamidia, herpes).

Conclusions: We conclude that BUE is cost-effective method for early diagnostic of the intrauterine infections.

\section{THE CONTRIBUTION OF NEUROIMAGING IN NEONATAL PATHOLOGY}

\section{G C Zaharie}

Department of Neonatology, University of Medicine and Pharmacy 'Iuliu Hatieganu', Cluj-Napoca, Romania

Background and Aims: The investigation want to reevaluate neuroimaging in newborn infants with neonatal hypoxic pathology.

Methods: Complete neurologic examination, EEG, transfontanellar ultrasonography, CT or MRI were carried out in 8 newborns with severe neonatal asphixia 10 days after birth and at the age of 3 months.

Results: MRI was performed at the age of 10 days in 5 newborns with neurologic syndrome since the first day of life, revealing various aspects of cerebral ischemia. At the age of 3 months, in three of these, the neurologic examination revealed spastic paraplegia, MRI detecting marked brain atrophy. In one case with normal neurologic examination, EEG and MRI were within normal range at the age of 10 days, at 3 months changes in the neurologic picture were noted, with the occurrence of spastic paraplegia, changes in the EEG, the MRI aspect remaining normal. In one case with neonatal asphixia and DIC, the neurologic examination and EEG were within normal range, with multiple superficial and deep hemorrhagic foci at MRI, and at the age of 3 months the neurologic examination and EEG being normal and MRI much improved. Cerebral hemorrhage degree IV with secondary hydrocephalia was diagnosed in 2 premature infants aged 29 and 30 weeks respectively, with improvements in the CT after performing ventriculo-peritoneal shunt. Cerebral malformations were diagnosed based on the MRI and CT performed.

Conclusions: MRI correlated with the neurologic picture in $87.5 \%$ of the cases. Neuroimaging is a marker of the course of cerebral distress.

\section{SENSORIAL SATURATION FOR NEONATAL ANALGESIA}

C V Bellieni, D M Cordelli, S Perrone, G Buonocore

Departments of Pediatrics, Obstetrics and Reproduction Medicine, University of Siena, Siena, Italy

Background and Aims: Sensorial Saturation is a procedure in which touch, massage, taste, voice, smell and sight compete with pain, producing almost complete analgesia during heelprick in neonates. SS is an apparently complex manoeuvre, but when correctly explained it is easily learnt. In the present paper we studied its feasibility, assessing whether a long training is really needed to achieve good results.

Methods: We enrolled 66 consecutive babies and divided them randomly into three groups which received 
the following forms of analgesia: glucose plus sucking (A), sensorial saturation performed by nurses (B); sensorial saturation performed by mothers (C). We did not use perfume on the caregivers' hands, so that babies could smell the natural scent of the hands. We assessed pain level by the $\mathrm{ABC}$ scale.

Results: Median scores of groups A, B and C were: 1 $(0-6), 0(0-4)$ and $0(0-6)$ respectively. Mean scores were: $0.6,0.6$ and 1.7 and standard errors were 0.38 , 0.22 and 0.32 respectively. Scores of groups B and C were significantly lower than that of $A(p=0.03$ and 0.006 respectively). No significant difference was found between values of scores of groups $\mathrm{B}$ and $\mathrm{C}$.

Conclusions: Even without the use of perfume on the hands, SS was effective as an analgesic manoeuvre. It made no difference whether SS was performed by mothers who applied it for the first time or experienced nurses: SS is rapid to learn and any caregiver (mother, pediatrician or nurse) can effectively use it.

\section{HIGH PREVALENCE OF VITAMIN D DEFICIENCY IN NEWBORN INFANTS OF DARK SKINNED OR VEILED MOTHERS IN THE NETHERLANDS}

S H Dijkstra ${ }^{1}$, A Van Beek ${ }^{2}$, J W Janssen ${ }^{3}$, L H M de Vleeschouwer ${ }^{2}$, E L T van den Akker ${ }^{4}$, W A Huysman ${ }^{1}$

${ }^{1}$ Department of Paediatrics, ${ }^{2}$ Department of Gynaecology, ${ }^{3}$ Department of Clinical Chemistry, Sint Franciscus Gasthuis, ${ }^{4}$ Department of Paediatrics, Erasmus MC-Sophia Children's Hospital, Rotterdam, The Netherlands

Background and Aims: Pregnant women with hyper pigmented skin or wearing veils are at risk of vitamin D deficiency. The prevalence of vitamin D deficiency in pregnant women and their newborn infants is unknown. Our aim is to determine the prevalence of vitamin D deficiency in newborns of mothers at risk in the Netherlands.

Methods: All pregnant women visiting our outpatient clinic of the obstetric department between April 2004 and February 2006 were encountered to participate in our study. The risk group consisted of women with tinted or dark skin and women wearing veils. The control group consisted of Caucasian women. Information was obtained through a questionnaire about pigmentation of skin, race, sun exposure, dressing habits, use of vitamins and patient history, including clinical symptoms of vitamin D deficiency. Blood samples were collected from the mothers at the end of third trimester. The umbilical cord blood was tested on 25-hydroxyvitamin D3, 1,25- dihydroxyvitamin D3, alkaline phosphatase, parathyroid hormone, calcium and phosphorus, in 87 newborns. Vitamin D deficiency in the newborn was defined as 25-hydroxyvitamin D3 $<25$ $\mathrm{nmol} / \mathrm{l}$.

Results: Of 87 newborn infants 37 were vitamin D deficient $(16 \%$ of the newborns of Caucasian mothers, $39 \%$ of the newborns of mothers with tinted skin, $44 \%$ of the newborns of mothers with dark skin and $91 \%$ of the newborns of veiled mothers).
Conclusions: Vitamin D deficiency is frequently seen in newborns born to mothers at risk. Clinical implications of vitamin $\mathrm{D}$ deficiency for the newborn infants are unknown and need further investigation.

\section{POSTNATAL BLOOD PRESSURE IN PRETERM INFANTS WITH AND WITHOUT HISTOLOGICAL CHORIOAMNIONITIS: A PROSPECTIVE STUDY}

J V Been ${ }^{1}$, N Akinturk ${ }^{1}$, M Karaduman ${ }^{1}$, I Rours ${ }^{2}$, R F Kornelisse ${ }^{2}$, A J Schneider ${ }^{3}$, R R de Krijger ${ }^{4}$, L J Zimmermann ${ }^{1}$

${ }^{I}$ Department of Neonatology, Maastricht University Hospital, ${ }^{2}$ Department of Pediatrics, ${ }^{3}$ Department of Obstetrics, ${ }^{4}$ Department of Pathology, Erasmus MC, Rotterdam, The Netherlands

Background and Aims: Chorioamnionitis is an important cause of preterm birth and is associated with major neurological morbidity. We hypothesised that chorioamnionitis in preterm infants is associated with postnatal hypotension, a known etiological factor in the development of neurological morbidity.

Methods: Over a two-year period all infants born in the Erasmus MC at $\leq 32$ weeks gestation were included. Relevant clinical data and placental histopathology were obtained from each infant. Blood pressure values were recorded during the first three postnatal days, clustered in four-hour periods, and compared between patients with histological chorioamnionitis (HC) and those without (non-HC).

Results: 303 Patients were included, 119 (39.3\%) of whom had HC. HC patients had significantly lower diastolic and mean blood pressures at several time points during the first three days after birth. The largest differences were present in the diastolic blood pressure between 36 and 72 hours. However, patients with $\mathrm{HC}$ had a significantly lower gestational age $(28.2 \pm 2.0$ versus $29.6 \pm 1.7, \mathrm{p}<0.001$ ). When blood pressures were compared after stratification for gestational age no significant differences were present. There were no differences in treatment with vasopressors, inotropes or volume expansion between the two groups. Also, the incidence of septicemia during the study period was similar.

Conclusions: After correction for gestational age differences, there was no association between histological chorioamnionitis and blood pressure during the first three postnatal days in preterm infants born at $\leq 32$ weeks gestation. Thus, low early postnatal blood pressure values do not seem to explain the increased incidence of neurological morbidity after chorioamnionitis in preterm infants.

\section{SMALL FOR GESTATIONAL AGE BIRTH AND RISK OF HYPERTENSION IN ADULT LIFE: TWO UNRECOGNIZED CHILDREN POPULATIONS?}

C De Felice ${ }^{1}$, M Strambi ${ }^{4}$, B De Capua ${ }^{2}$, R Tassi ${ }^{3}$, D Costantini $^{2}$, A Del Vecchio ${ }^{5}$, G Chitano ${ }^{6,7}$, G Latini $^{5,8}$ 
${ }^{1}$ Neonatal Intensive Care Unit, Azienda Ospedaliera Universitaria Senese, Siena, Italy, ${ }^{2}$ Clinical Audiology Unit, Azienda Ospedaliera Universitaria Senese, Siena, Italy, ${ }^{3}$ Laboratory of Ultrasonology, Azienda Ospedaliera Universitaria Senese, Siena, Italy ${ }^{4}$ Departments of Pediatrics, Obstetrics and Reproductive Medicine, Azienda Ospedaliera Universitaria Senese, Siena, Italy, ${ }^{5}$ Division of Neonatology, Perrino Hospital, Brindisi, Italy ${ }^{6}$ Department of Cardio-Thoracic, University of Pisa, Pisa, Italy, ${ }^{7}$ Euro Mediterranean Scientific Biomedical Institute (ISBEM), Brindisi, Italy, ${ }^{8}$ Clinical Physiology Institute, National Research Council of Italy (IFC-CNR), Lecce Section, Brindisi, Italy

Background and Aims: Emerging clinical and experimental evidence indicates a link between small size at birth and cardiovascular risk in adult life. The relationship with hypertension remains controversial. The aim of this study was to evaluate the physiological day/night variations of blood pressure in two subgroups of children as a function of the posterior communicating arteries (PCoAs).

Methods: A total of 26, randomly selected SGA children participated to the study (age, $\mathrm{M} \pm \mathrm{SD}: 9.45 \pm$ $2.08 \mathrm{yr}$ ). A 24-h ambulatory blood pressure monitoring (SpaceLabs 90207) was carried out and the population was subdivided into 2 groups, based on transcranial doppler evidence of bilateral non-functioning PcoAs (PcoA-positive vs. bil-PCoA-negative). Day-to-night variations in systolic (SBP), diastolic (DBP) and mean (MBP) blood pressure were the main outcome measures. Results: $65.4 \%$ children were found to be PcoApositive vs. $34.6 \%$ bil-PCoA-negative. The groups were comparable for blood pressure percentiles for age, gender, gestational age at birth, birth weight SDS, and auxometric growth $(\mathrm{p} \geq 0.35)$. PcoA-positive children showed significantly reduced day-night variations in SBP $(7.16 \pm 4.93 \%$ vs. $16.6 \pm 5.55 \%, \mathrm{p}=0.0002)$, DBP $(14.28 \pm 8.92 \%$ vs. $24.96 \pm 7.62 \%, \mathrm{p}=0.0056)$ and MBP $(9.84 \pm 8.49 \%$ vs. $21.2 \pm 7.31 \%, p=0.0024)$, had a significantly higher frequency of history of pregnancyinduced hypertension $(\mathrm{p}=0.0144)$, and their mothers showed a significantly increased right-side carotid intima-media thickness $(\mathrm{p}=0.0277)$, as compared to the bil-PcoA-negative population.

Conclusions: Our data suggest the existence of two phenotypically distinct SGA children populations, at possibly different risk of developing hypertension in adult life.

\section{HIGH PLASMA CORTISOL AND ACTH CONCENTRATIONS IN VERY-LOW-BIRTH- WEIGHT INFANTS WITH SMALL THYMUS AT BIRTH}
C De Felice ${ }^{1}$, P Toti $^{4}$, M Musarò $^{2}$, L Peruzzi ${ }^{2}$, P Paffetti ${ }^{2}$, L Pasqui ${ }^{3}$, R Magaldi ${ }^{5}$, M Rinaldi, G Rinaldi ${ }^{5}$, G Grilli $^{6}$, F Bagnoli ${ }^{1}$, G Latini ${ }^{7,8}$
${ }^{1}$ Neonatal Intensive Care Unit, Section of Neonatology,
Azienda Ospedaliera Universitaria Senese, Siena, Italy
${ }^{2}$ Pediatric Laboratory, Azienda Ospedaliera
Universitaria Senese, Siena, Italy, ${ }^{3}$ Endocrinology

Laboratory Azienda Ospedaliera Universitaria Senese, Siena, Italy, ${ }^{4}$ Department of Human Pathology and Oncology, University of Siena, Siena, Italy, ${ }^{5}$ Neonatal Intensive Care Unit, Azienda Ospedaliera Universitaria, Foggia, Italy, ${ }^{6}$ Radiology Unit, Azienda Ospedaliera Universitaria, Foggia, Italy, ${ }^{7}$ Division of Neonatology, Perrino Hospital, Brindisi, Italy, ${ }^{8}$ Clinical Physiology Institute, National Research Council of Italy (IFC-CNR), Lecce Section, Italy

Background and Aims: An acute thymic involution in human fetuses and newborns has been described in very-low-birth-weight (VLBW) infants with histological chorioamnionitis. However, its underlying mechanisms remain to be clarified. We tested the hypothesis that an activation of the hypothalamo-pituitary-adrenals (HPA) axis in VLBW infants with acute thymic involution at birth.

Methods: 107 VLBW newborns $(28.7 \pm 3.1$ wk gestation; $1078 \pm 292 \mathrm{~g}$ ) were examined. Thymic size was measured on standard chest radiographs at birth, and expressed as the ratio between the transverse diameter of the cardiothymic image at the level of the carina (CT) and that of the thorax (T). CT/T $<0.28$ was considered to indicate small thymus. Plasma cortisol (Ortho-Clinical Diagnostics) and ACTH (DPC Immunolyte) concentrations were determined on days 1 (d-1) and 7 (d-7), and at 1 month (mo-1).

Results: $32.7 \%(35 / 107)$ of the newborns showed a $\mathrm{CT} / \mathrm{T}<0.28$. As compared with those with normal thymic size, infants with small thymus had significantly increased cortisol on d-1 ( $\sim 4.5$-folds) [median: 19.0 (95\% C.I:13.04-37.81) vs. 4.19 (3.21-5.34) $\mu \mathrm{g} / \mathrm{dL}$, $\mathrm{p}<0.0001)]$ and d-7 ( 1.7-folds) [7.96 (5.73-9.93) vs. $4.48(2.33-8.17) \mu \mathrm{g} / \mathrm{dL}, \mathrm{p}=0.0293)]$, together with higher ACTH concentrations on d-1 ( 1.8-folds) [25.6 $(15.32-60.58) \quad$ vs. $14.0 \quad(10.70-18.16) \quad \mathrm{pg} / \mathrm{mL}, \quad \mathrm{p}=$ 0.0180)]. No significant differences for cortisol at mo-1 $(\mathrm{p}=0.9485)$, or ACTH concentrations on $\mathrm{d}-7(\mathrm{p}=0.6713)$ and mo-1 $(\mathrm{p}=0.5006)$ were evidenced.

Conclusions: Our data indicated the presence of a previously unrecognized activation of the HPA axis life in VLBW newborns with a small thymus at birth.

\section{ALLERGIC DISEASES AND ATOPY UP TO THE AGE OF 5-7 YEARS IN THE VERY LOW BIRTHWEIGHT INFANTS}

\author{
P Kwinta, G Lis, M Klimek, E Cichocka-Jarosz, \\ J J Pietrzyk \\ Department of Pediatrics, Jagiellonian University, \\ Cracow, Poland
}

Background and Aims: Factors present in early life can increase the risk of asthma, allergic rhinitis (AR), and atopic dermatitis (AD). The aim of the study was analysis of the prevalence of wheezing and diagnosis of: asthma, $\mathrm{AR}$, and $\mathrm{AD}$ in 5-7 years old very low birthweight infants.

Methods: 108 children with mean birthweight $1090 \mathrm{~g}$ were evaluated by standardized ISAAC (International Study of Asthma and Allergies in Childhood) questionnaire at the age of 5-7 years. Total IgE level, specific IgE, lymphocyte Th1/Th2 ratio and skin prick 
tests were done. Prevalence of allergic disorders among VLBW infants was compared to ISAAC regional data.

Results: The prevalence of wheezing ever (47.2 vs $28.6 \%)$ and wheezing last year (25 vs $13.6 \%$ ) were higher in the VLBW infants. Number of children with asthma diagnosis was higher in the VLBW group (23.1 vs $5.8 \%$ ). The prevalence of AR ever symptoms ( $34.3 \mathrm{vs}$ $33.6 \%$ ) and established AR diagnosis (19.4 vs $21.1 \%$ ) were similar in both cohorts. The prevalence of $\mathrm{AD}$ ever and $\mathrm{AD}$ in the last year was lower in the VLBW group (16 vs $25,2 \%$, and 5.5 vs $15.3 \%$ ). Elevated IgE levels were detected in $31 \%$, positive prick test occurred in $13.6 \%$, and lymphocyte Th1/Th2 ratio $<1$ in $21.5 \%$ of VLBW infants.

Conclusions: Higher prevalence of wheezing ever, wheezing last year and diagnosed asthma, along with similar prevalence of $\mathrm{AR}$ and $\mathrm{AD}$ in both cohorts suggest that symptoms of asthma in 5-7 years old VLBW infants are associated with persistent bronchial hyperreactivity rather than atopic asthma. (Study partially supported by Nutricia Research Foundation).

\section{HEALTH UTILISATION OF VERY PRETERM INFANTS TO AGE 4}

N C Austin ${ }^{1}$, J Laney ${ }^{2}$, C Spencer ${ }^{1}$, D Gray ${ }^{1}$, T E Inder ${ }^{3}$, L Woodward $^{4}$

${ }^{\text {I}}$ Christchurch Women's Hospital, Christchurch, New Zealand, ${ }^{2}$ Christchurch School of Medicine, University of Otago, Christchurch, New Zealand, ${ }^{3}$ Washington University, St. Louis, MO, USA, ${ }^{4}$ Department of Psychology, University of Canterbury, Christchurch, New Zealand

Background and Aims: Very preterm infants are known to have ongoing health problems after discharge with respiratory illnesses and neurodevelopmental delay being common. This study examines the health service contacts of a representative regional cohort of very preterm infants to 4 years.

Methods: Between July 1998 and November 2000, 100 preterm babies were recruited and 104 term born control infants were selected when aged 2. General Practitioner (GP), hospital outpatient and admissions from discharge to 4 years were collated and compared.

Results: Complete data was available for 92 very preterm and 71 controls children. Up to age 2 both groups had similar rates of GP contacts despite preterm children having more frequent hospital outpatient visits (most frequently for neurodevelopmental follow-up) and hospital admissions, After age 2 the GP visits became significantly higher in the preterm children $(p<0.001)$. Respiratory diagnoses were significantly higher in the very preterm children for admissions and GP visits $>2$ years. Ophthalmology, otolaryngology and orthopaedics were the predominant surgical specialties accessed as outpatients and inpatients particularly after age 2 . From age 2 years hospital admission continued to be significantly higher in the preterm $(\mathrm{p}<0.001)$.

Conclusions: Babies born very preterm have more frequent GP and outpatient visits and hospital admissions up to age 4 years. Compared to age $<2$, respiratory illnesses have reduced as reason for hospital admissions but increased as reason for GP visits in the preterm group. Surgical specialties were used more frequently at age $2-3$ years. These findings confirm the high health utilisation of very preterm infants to age 4 .

\section{PRENATAL BETAMETHASONE HAS NO EFFECT ON RENAL DRUG CLEARANCE IN THE FIRST MONTH OF LIFE}

K Allegaert $^{1}$, J N van den Anker $^{2}$, A Debeer ${ }^{1}$, N H Holford ${ }^{3}$, G Naulaers ${ }^{1}$, B Anderson ${ }^{4}$

${ }^{1}$ Neonatal Intensive Care Unit, University Hospital Gasthuisberg, Leuven, Belgium, ${ }^{2}$ Departments of Paediatrics, Pharmacology and Physiology, George Washington University School of Medicine and Health Sciences, Washington, DC, USA, ${ }^{3}$ Department of Clinical Pharmacology, University of Auckland, Auckland, New Zealand, ${ }^{4}$ Department of Anaesthesiology, University of Auckland, New Zealand

Background and Aims: There is conflicting evidence on the effect of prenatal betamethasone administration on renal drug clearance $[1,2]$. We therefore wanted to document and quantify the impact of betamethasone administration on vancomycin in the first month of life. Methods: Population pharmacokinetics of vancomycin in neonates were estimated in a cohort of 214 premature neonates (24-34 weeks) with a postnatal age of 11.9 (1-27) days. Covariate analysis included weight, postmenstrual age (PMA), serum creatinine, use of inotropes, positive blood culture, use of respiratory support, administration of ibuprofen as well as maternal betamethasone administration.

Results: A one-compartment linear disposition model with zero order input and first order elimination was used to describe data on 604 observations. Clearance increased from $0.897 \mathrm{~L} / \mathrm{h} / 70 \mathrm{~kg}$ at 24 weeks PMA to $2.02 \mathrm{~L} / \mathrm{h} / 70 \mathrm{~kg}$ by 34 weeks. Between subject variability was $18.6 \%$, between occasion variability $12.2 \%$. The use of ibuprofen reduced clearance (18\%). Overall 82\% of the variability was predictable. Size explained $49 \%$, PMA $18 \%$ and renal function $34 \%$. There was no effect of betamethasone on vancomycin clearance.

Conclusions: Size, renal function and postmenstrual age are the major contributors to clearance variability in premature neonates. Prenatal betamethasone administration had no independent effect on vancomycin clearance in the first month of postnatal life. [1] Van den Anker JN et al. Ped Research 1994;36:578-81[2] Allegaert $\mathrm{K}$ et al. $\mathrm{Br} \mathrm{J}$ Clin Pharmacol 2006;61:39-48.

\section{PRETERM INTRAUTERINE GROWTH RESTRICTION AND EARLY POSTNATAL PROTEIN METABOLISM}

R M Reynolds ${ }^{1}$, M S Reece ${ }^{1}$, R E Ridout ${ }^{2}$, P J Thureen ${ }^{1}$

${ }^{1}$ Department of Pediatrics, University of Colorado Health Sciences Center, The Children's Hospital, Denver, CO, USA, ${ }^{2}$ Tripler Army Medical Center, Tripler AMC, HI, USA 
Background and Aims: There is evidence that IUGR fetuses alter in utero nutrient metabolism in order to survive their environment, and this fetal adaptation is associated with long term health disorders. The ability to identify a population of IUGR neonates who demonstrate abnormal protein metabolism would enable an investigation of infant feeding strategies that could ameliorate long-term adverse health outcomes.

Methods: The goal of this study was to determine if preterm infants with definitive IUGR demonstrate abnormal postnatal nitrogen metabolism in the immediate postnatal period when compared to AGA infants of comparable gestational age. All infants received exclusive parenteral nutrition. IUGR infants were pair matched with AGA controls for the following parameters by order of importance to the study outcome: protein intake, gestational age, hours of age at study, energy intake. The primary outcome variable was protein balance as determined by the nitrogen balance method.

Results: Data from 16 IUGR/AGA pairs are shown in the table. Analysis by paired t-test, though mean + SEM data is presented; $p<0.05$ considered significant.

Conclusions: Although this study was not able to detect differences in estimated protein accretion rates in IUGR vs AGA preterm neonates, these differences may exist, and studies using different experimental strategies may

\begin{tabular}{|c|c|c|c|}
\multicolumn{1}{c}{ be able to define } & \multicolumn{1}{c}{ these } & \multicolumn{2}{c|}{ differences. } \\
\hline & IUGR & AGA & p-value \\
\hline Gestational age (weeks) & $28.5 \pm 0.4$ & $28.3 \pm 0.5$ & NS \\
\hline Age at study (hours) & $37.2 \pm 1.4$ & $40 \pm 2.1$ & NS \\
\hline Biith weight (grams) & $741 \pm 0.03$ & $1.21 \pm 0.07$ & $p<0.000006$ \\
\hline Protein intake (g/kg/day) & $1.82 \pm 0.21$ & $2.14 \pm 0.18$ & NS \\
\hline Protein balance (g/kg/day) & $0.63 \pm 0.21$ & $0.62 \pm 0.16$ & NS \\
\hline
\end{tabular}

\section{PERINATAL FACTORS INFLUENCING LATER GROWTH OUTCOME IN VERY PRETERM INFANTS}

L Maggio, F Gallini, F Cota, F Matronola, S Costa, C Romagnoli

\section{NICU, Catholic University, Rome, Italy}

Background and Aims: Poor growth during the early years is well documented in children born prematurely but the relation between perinatal morbidity and subsequent growth retardation is poorly understood. Our aim was to determine, in children born AGA at GA $\leq 30$ weeks, the main perinatal factors predictive of growth patterns at corrected age of 36 months.

Methods: The growth outcome of 133 infants (GA $27.8 \pm 1.5 \mathrm{wks}$, BW $1017 \pm 208 \mathrm{~g}$ ) discharged from our NICU between 1996 and 2002 was assessed at 36 months of corrected age. Poor growth was defined as a Zs. $<-1.28$.

Results: At 36 months of age the children were slightly smaller on average in the three growth measures: $\mathrm{Z}$ scores were $-1.02 \pm 1.29$ for Weight, $-0.90 \pm 1.09$ for Height and $-0.72 \pm 1.07$ for Head Circumference; $45.9 \%$ of children had poor growth in $\mathrm{W}, 34.6 \%$ in $\mathrm{H}$ and $30.3 \%$ in HC. During hospitalization weight growth retarded children showed higher CRIB score (3.7 vs 2.6; $\mathrm{p}<0.05$ ), more days of oxygen therapy (40.4 vs 25.1 ; $p$
$<0.05)$ and a greater Zs fall between birth and discharge $(-1.06$ vs $-0.81 ; \mathrm{p}<0.05)$. Multiple logistic analysis identified ZsW change between birth and discharge (OR 0.44; Ic95\% 0.24,0.81), male gender (OR 0.11; Ic95\% $0.05,0.53$ ) and ZsW at birth (OR 0.08; Ic95\% 0.01,0.65) as the best predictors for poor later growth. A similar model was predictive for $\mathrm{H}$ and $\mathrm{HC}$ poor growth.

Conclusions: In very premature infants growth during early postnatal period could predict the growth retardation at 3 years of corrected age.

\section{SOMATIC PARAMETERS OF THE NEWBORNS IN RELATIONSHIP TO CORD BLOOD LEPTIN CONCENTRATION}

J Wojculewicz ${ }^{1}$, H Jasiel-Wojculewicz ${ }^{2}$, P Krygier ${ }^{1}$, J Szczapa ${ }^{1}$

${ }^{l}$ Department of Neonatal, Medical University, Gdansk, Poland, ${ }^{2}$ Department of Hypertension and Diabetology, Medical University, Gdansk, Poland

Background and Aims: The aim of the study was estimation of the leptin level in the umbilical cord blood in newborns in connection to their gestational age, gender and somatic parameters.

Methods: A cohort of 301 newborns born between 01.01.04 - 30.06.05 in Neonatal Dept. Medical University of Gdańsk was examined. Antropometric measurements were taken. Samples of the umbilical cord blood were collected after birth and leptin concentration was analysed by standard RIA method.

Results: We stated significantly lower leptin level in prematures $(6,88+6,49 \mathrm{ng} / \mathrm{ml})$ comparing to mature children $(13,8+9,81 \mathrm{ng} / \mathrm{ml})(\mathrm{p}<0,01)$. Taking birth weight into account as a nutritional state criterium we affirm that the lowest leptin concentration was observed in $<3000 \mathrm{~g}$ group $(8,41+6,07 \mathrm{ng} / \mathrm{ml})$. The significantly highest level $(\mathrm{p}<0,01)$ was observed in $>4000 \mathrm{~g}$ group $(20,52+12,83 \mathrm{ng} / \mathrm{ml})$. Significantly higher $(\mathrm{p}<0,05)$ leptin level was observed in female children $(14,9+9,4 \mathrm{ng} / \mathrm{ml}$ vs $12,6+10,13 \mathrm{ng} / \mathrm{ml})$. There were no differences in P.I. between premature and mature newborns. P.I. was significantly higher in male newborns. In the age of 6 and 12 months $86 \%$ of newborns with birth weight $>90$ percentile were still characterized by the same $>90$ body mass percentile.

Conclusions: Both the small and the large birth weight are connected with similarly low and high leptinaemia. According to ' fetal programming' theory large birth weight causes higher probability of patophysiological (especially obesity) disturbances. The risk of diabetes in future is also more often in these children.

\section{ELEVATED IRON STORES IN PREGNANT WOMEN: IS THERE A PERINATAL RISK IN OFFSPRING? (PRELIMINARY STUDY)}

V Soubasi-Griva ${ }^{1}$, S Petridou ${ }^{1}, \mathrm{C}$ Tsantali ${ }^{2}$, $\mathrm{K}_{\text {Mitsakis }}{ }^{1}, \mathrm{~K}$ Gourzioti $^{2}$, V Drossu-Agakidou ${ }^{1}$

${ }^{l} 1$ st Department of Neonatology, Hippokration Hospital, Aristotle University of Thessaloniki, Thessaloniki, Greece, ${ }^{2}$ Laboratory of 1 st Department of Pediatrics, 
Hippokration Hospital, Aristotle University of Thessaloniki, Thessaloniki, Greece

Background and Aims: The risks and benefits of prophylactic iron supplementation in pregnant women who are not iron deficient remains a source of controversy. Elevated iron stores during pregnancy have recently been linked to maternal complications and perinatal risks to their offspring (Scholl 2005, Viteri 2005). The aim of this study was to determine if there is an association between maternal iron status, under prophylactic iron supplementation, and perinatalneonatal outcomes.

Methods: Full blood count and serum ferritin concentrations were measured at delivery in 42 high risk neonates (IUGR, IDM, prematures) (group A), and 29 healthy term neonates (group B) and their mothers. Serum soluble transferin receptor (TfR) was measured at birth in all neonates. Each group was divided in 3 subgroups according to mothers' ferritin levels.

Results: Iron repleted mothers were found only in group A. The incidence of gestational diabetes (GD) and/or IUGR was higher $(\mathrm{p}<0.04)$ in mothers with high ferritine levels $(\mu \mathrm{g} / \mathrm{l})$ (table). Ferritin concentration was significantly lower in mothers compared to their neonates $(\mathrm{p}<0.0001)$. High risk neonates (group A) had lower ferritin levels compared to healthy term neonates $(p<0.0005)$. Neonatal TfR levels $(\mathrm{mg} / \mathrm{L})$ were higher in group A compared to group B ( $\mathrm{p}<0.05)$.

Conclusions: Elevated iron stores during pregnancy might predispose to GD and IUGR. Maternal iron deficiency does not affect iron status in the neonate. In high risk neonates iron stores are lower than in term neonates.

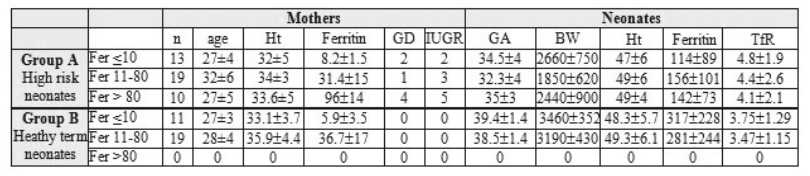

\section{SALIVARY CORTISOL IN VLBW- ADOLESCENTS BEFORE AND AFTER MENTAL STRESS}

E Elsmén ${ }^{1}$, A K Edstedt Bonamy ${ }^{2}$, M Norman ${ }^{2}$, L Hellström-Westas ${ }^{1}$

${ }^{I}$ Department of Pediatrics, Institution of Clinical Sciences, Lund University, Lund, Sweden, ${ }^{2}$ Department of Woman and Child Health, Karolinska Institute, Stockholm, Sweden

Background and Aims: Low neonatal plasma cortisol is associated with morbidity in very preterm infants. Later, other studies show increased salivary cortisol responses in such infants, and children born preterm are at risk for higher blood pressure. The aim was to investigate salivary cortisol responses and blood pressure in adolescents born preterm before and after MRI, a potentially stressful situation.

Methods: Forty-nine adolescents, age 12-17 years, born preterm (birth weight $<1500 \mathrm{~g}$ ) and 43 controls born at term, age 12-16 years. Salivary cortisol was measured before and after MRI. Subjects also answered a validated questionnaire, including 12 items, about perceived stress. Systolic and diastolic blood pressures were measured before MRI.

Results: Before MRI median (range) salivary cortisol was $4.6 \mathrm{nmol} / \mathrm{L}(1.2-39)$ in the preterm group and 3.7 $\mathrm{nmol} / \mathrm{L}(0.8-192)$ in controls $(\mathrm{p}=0.87)$, and after MRI $2.9 \mathrm{nmol} / \mathrm{L} \quad(0.8-30)$ and $3.5 \mathrm{nmol} / \mathrm{L} \quad(1.0-186$, respectively $(\mathrm{p}=0.68)$. Most children expressed little stress in relation to the MRI, the preterm group scored lower for feeling of pressure before $(\mathrm{p}=0.04)$, but also expressed feelings of less energy after the MRI $(p=0.028)$. There were no differences between the groups in blood pressure in this study population. There were no gender differences in salivary cortisol or blood pressure, but in boys born preterm pre-MRI salivary cortisol and systolic blood pressure correlated $(\mathrm{rs}=0.6$, $\mathrm{p}<0.004)$.

Conclusions: This study did not find significant differences in stress responses between adolescents born preterm $(\mathrm{BW}<1500 \mathrm{~g})$ and controls born at term.

\section{TOTAL CHOLESTEROL TO PONDERAL INDEX RATIO AS A MARKER OF IMPAIRED LIPID METABOLISM IN IUGR VERSUS AGA FETUSES AND NEWBORNS}

T Boutsikou ${ }^{1}$, A Malamitsi-Puchner ${ }^{1}$, M Kyriakakou ${ }^{1}$, $\mathrm{H} \mathrm{Militsi}^{2}$, A Margeli ${ }^{2}$, D Hassiakos ${ }^{1}$, I Papassotiriou ${ }^{2}$, G Mastorakos $^{1}$, C Kanaka-Gantenbein ${ }^{3}$

${ }^{1}$ Neonatal Division, 2nd Department Obstetrics and Gynecology Athens University, Medical School, Athens, Greece, ${ }^{2}$ Department of Clinical Biochemistry, 'Aghia Sophia' Children's Hospital, Athens, Greece, ${ }^{3} 1$ st Department of Pediatrics, Athens University, Medical School, Athens, Greece

Background and Aims: According to the 'fetal origin of adult disease' hypothesis, intrauterine growth restriction (IUGR) is associated with later hypercholesterolemia and occurrence of the metabolic syndrome. This study aimed to investigate lipid status during intrauterine and early postnatal life in 20 IUGR (birth weight below the 10th customized centile) and 20 appropriate for gestational age (AGA) fetuses (UC), and newborns on days 1 (N1) and 4 (N4), and to assess the ratio of total cholesterol levels to the ponderal index $\left[\mathrm{PI}=\right.$ weight $(\mathrm{kg}) /$ height $\left.(\mathrm{m})^{3}\right]$, which is an anthropometric method, used to diagnose impaired fetal growth.

Methods: Total cholesterol, HDL and triglyceride levels were measured by an automatic clinical chemistry analyzer (ADVIA 1650, Bayer Diagnostics, Tarrytown, NY, USA). LDL was determined by using the Friedewald equation.

Results: Absolute total cholesterol, LDL, HDL and triglyceride levels were similar in both groups at all time points studied (UC, N1 and N4). The ratio of total cholesterol to PI was significantly increased in IUGR versus AGA fetuses $(2.94+/-0.87$ versus $2.46+/-0.49$., $\mathrm{p}=0.042)$ and neonates on day $1(3.06+/-0.83$ versus 2.58+/-0.54, $\mathrm{p}=0.039$ ).

Conclusions: Although lipid levels do not differ between both groups, total cholesterol to ponderal index ratio is increased in IUGR versus AGA fetuses and newborns. This finding possibly indicates existence of hypercholesterolemia and impaired fetal liver function, 
evident from the early postnatal life and due to the adverse intrauterine environment. Thus, the 'fetal origin of adult disease' hypothesis is reinforced.

\section{THE CLINICAL STUDY ON INFANTS OF DIABETIC MOTHERS (IDMS) FOR 20 YEARS IN TOKYO WOMEN'S MEDICAL UNIVERSITY}

\author{
I Sakuma $^{1}$, N Takahashi ${ }^{1}$, M Shiraishi ${ }^{1}, \mathrm{~S} \mathrm{Kusuda}^{1}$, \\ H Nishida $^{1}$, M Sanaka ${ }^{2}$, Y Omori ${ }^{2}$ \\ ${ }^{I}$ Neonatal Division, Maternal and Perinatal Center, \\ Tokyo Women's Medical University, Tokyo, Japan \\ ${ }^{2}$ Diabetology and Metabolism Division, Diabetes \\ Center, Tokyo Women's Medical University, Tokyo, \\ Japan
}

Background and Aims: The clinical spectra of infants of diabetic mothers (IDMs) encountered in the past 20 years were retrospectively analyzed to clarify the prognosis.

Methods: IDMs were divided into three groups according to years born. Group 1 consists of 166 infants born from 1984 to 1989, Group 2 of 192 infants from 1994 to 1997, and Group 3 of 173 infants from 2000 to 2003. The frequencies of complications such as heavy for dates, prematurity, asphyxia, hypoglycemia, polycythemia, hypocalcemia, hyperbilirubinemia, congenital malformations, respiratory dysfunctions, and myocardial hypertrophy were determined. Mortality rate were also evaluated.

Results: During the study period $754(12.5 \%)$ IDMs were born out of 6042 births. Major congenital malformations were observed in 3.8\% infants including $1.5 \%$ cardiac anomalies. Each one case of VACTER association and caudal regression syndrome were born from untreated mothers during early pregnancy. Two infants died due to VACTER association and extreme prematurity. The rates of all complications in Group 2 were lower than Group 1, but they reascended in Group 3 , which included highest frequency of the mothers complicated with retinopathy and/or nephropathy (12.7\% (22/173)). Among 22 pregnancies, 15 were terminated prematurely for the treatment of mothers, and eventually 6 of them were born $<1500 \mathrm{~g}$.

Conclusions: Even under the tight control at our special Diabetes Center, the incidences of complications in IDMs from mothers untreated at the beginning of pregnancy were not low enough. We emphasize further improvements in health education and treatment for diabetic women before and during pregnancy.

\section{WEIGHT GAIN DURING HOSPITALIZATION IN PREMATURE NEWBORNS. A FIFTEEN YEAR RETROSPECTIVE STUDY}

\author{
G Bustos ${ }^{1}$, E Gómez ${ }^{1}$, M López ${ }^{1}$, de la Cruz ${ }^{2}$, \\ J Rodriguez ${ }^{1}$, C R Pallás ${ }^{1}$ \\ ${ }^{\text {I}}$ Servicio de Neonatología, Hospital Universitario 12 de \\ Octubre, Madrid, Spain ${ }^{2}$ Unidad de Epidemiología \\ Clínica, Hospital Universitario 12 de Octubre, Madrid, \\ Spain
}

Background and Aims: To assess the differences in weight gain during hospitalization in premature newborn.

Methods: Design: retrospective comparison from 1990 to 2004, in three year periods. Population: newborn 32 week of gestation or less, born within the hospital. Exclusion criteria: death during hospitalization, major congenital malformation, transfer to other centres and hospitalization of less than 14 days. Study variables: date of birth, birth and discharge weight and respective $\mathrm{z}$ score ( $Z 1$ and $Z 2)$, gestation, number of foetuses, sex, CRIB score, bronchopulmonary dysplasia, necrotizing enterocolitis, postnatal corticoids treatment, diuretic treatment, hospital stay and mortality.

Results: A total of 1192 premature were included and 552 excluded. Comparing the five three-years periods, there were not differences in Z1, sex distribution, CRIB and hospital stay. Mortality decreased and bronchopulmonary dysplasia increased from 1990 to 1995, with no significant changes from 1996 to 2004. Daily weight gain and $\mathrm{z}$ difference $(\mathrm{Z} 2 \mathrm{-Z1}$ ) were worse during 1996-98 period (14.2 g/day and -1.74 respectively) and better during 2002-04 (16.74 g/day and $-1,33$ respectively), $(\mathrm{p}<0.001)$. These differences were also significant in all birth weight and gestational groups. Postnatal treatment with corticoids was $14.6 \%$ during 1996-98 period and $0.3 \%$ during 2001-04 $(\mathrm{p}<0.001)$, and diuretics treatment $23.4 \%$ and $12.6 \%$ respectively $(\mathrm{p}<0.001)$.

Conclusions: Medical practices influence weight gain during hospitalization. An 24\% improvement on z score difference (Z2 - Z1), comparing 1996-98 and 2002-04 periods, is coincidental with a dramatic decrease in corticoids and diuretics postnatal treatments, and probably, with less liquids restriction.

\section{EFFECT OF PLACENTAL INSUFFICIENCY ON POSTNATAL MORBIDITY IN MODERATELY PRETERM INFANTS}

\section{R Mikalsen, M Olsen, T Farstad \\ Department of Neonatology, Akershus University Hospital, Nordbyhagen, Norway}

Background and Aims: The aim of this study was to determine if the degree of the placental insufficiency is associated with intestinal dysfunction and if this affects infants growth and length of hospital stay.

Methods: All infants between 30- 34 weeks Gestation Age (wGA), were divided into two groups based on the results of Doppler studies. Patients with documented normal +/- 2 SD Pulsatility Index (PI) in arteries umbilicalis were allocated to Group I while patients with PI> +2 SD were in Group II.

Results: A total of 56 infants were identified. Four had lethal malformation and were excluded. Eleven patients had inconclusive Pulsatily Index (PI). 33 patients had $\mathrm{PI} \pm 2$ SD (Group I) and eight Group II (PI $>+2 \mathrm{SD}$ ). All infants received mothers own milk or bank milk from day one.

Conclusions: We found correlation between placental insufficiency and postnatal intestinal dysfunction in premature infants. Neonates with Pulsatility Index $>+2$ 
Hippokration Hospital, Aristotle University of Thessaloniki, Thessaloniki, Greece

Background and Aims: The risks and benefits of prophylactic iron supplementation in pregnant women who are not iron deficient remains a source of controversy. Elevated iron stores during pregnancy have recently been linked to maternal complications and perinatal risks to their offspring (Scholl 2005, Viteri 2005). The aim of this study was to determine if there is an association between maternal iron status, under prophylactic iron supplementation, and perinatalneonatal outcomes.

Methods: Full blood count and serum ferritin concentrations were measured at delivery in 42 high risk neonates (IUGR, IDM, prematures) (group A), and 29 healthy term neonates (group B) and their mothers. Serum soluble transferin receptor (TfR) was measured at birth in all neonates. Each group was divided in 3 subgroups according to mothers' ferritin levels.

Results: Iron repleted mothers were found only in group A. The incidence of gestational diabetes (GD) and/or IUGR was higher $(\mathrm{p}<0.04)$ in mothers with high ferritine levels $(\mu \mathrm{g} / \mathrm{l})$ (table). Ferritin concentration was significantly lower in mothers compared to their neonates $(\mathrm{p}<0.0001)$. High risk neonates (group A) had lower ferritin levels compared to healthy term neonates $(p<0.0005)$. Neonatal TfR levels $(\mathrm{mg} / \mathrm{L})$ were higher in group A compared to group B ( $\mathrm{p}<0.05)$.

Conclusions: Elevated iron stores during pregnancy might predispose to GD and IUGR. Maternal iron deficiency does not affect iron status in the neonate. In high risk neonates iron stores are lower than in term neonates.

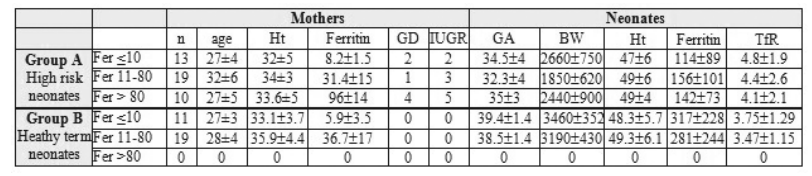

\section{SALIVARY CORTISOL IN VLBW- ADOLESCENTS BEFORE AND AFTER MENTAL STRESS}

E Elsmén ${ }^{1}$, A K Edstedt Bonamy ${ }^{2}$, M Norman ${ }^{2}$, L Hellström-Westas ${ }^{1}$

${ }^{I}$ Department of Pediatrics, Institution of Clinical Sciences, Lund University, Lund, Sweden, ${ }^{2}$ Department of Woman and Child Health, Karolinska Institute, Stockholm, Sweden

Background and Aims: Low neonatal plasma cortisol is associated with morbidity in very preterm infants. Later, other studies show increased salivary cortisol responses in such infants, and children born preterm are at risk for higher blood pressure. The aim was to investigate salivary cortisol responses and blood pressure in adolescents born preterm before and after MRI, a potentially stressful situation.

Methods: Forty-nine adolescents, age 12-17 years, born preterm (birth weight $<1500 \mathrm{~g}$ ) and 43 controls born at term, age 12-16 years. Salivary cortisol was measured before and after MRI. Subjects also answered a validated questionnaire, including 12 items, about perceived stress. Systolic and diastolic blood pressures were measured before MRI.

Results: Before MRI median (range) salivary cortisol was $4.6 \mathrm{nmol} / \mathrm{L}(1.2-39)$ in the preterm group and 3.7 $\mathrm{nmol} / \mathrm{L}(0.8-192)$ in controls $(\mathrm{p}=0.87)$, and after MRI $2.9 \mathrm{nmol} / \mathrm{L} \quad(0.8-30)$ and $3.5 \mathrm{nmol} / \mathrm{L} \quad(1.0-186$, respectively $(\mathrm{p}=0.68)$. Most children expressed little stress in relation to the MRI, the preterm group scored lower for feeling of pressure before $(\mathrm{p}=0.04)$, but also expressed feelings of less energy after the MRI $(p=0.028)$. There were no differences between the groups in blood pressure in this study population. There were no gender differences in salivary cortisol or blood pressure, but in boys born preterm pre-MRI salivary cortisol and systolic blood pressure correlated $(\mathrm{rs}=0.6$, $\mathrm{p}<0.004)$.

Conclusions: This study did not find significant differences in stress responses between adolescents born preterm $(\mathrm{BW}<1500 \mathrm{~g})$ and controls born at term.

\section{TOTAL CHOLESTEROL TO PONDERAL INDEX RATIO AS A MARKER OF IMPAIRED LIPID METABOLISM IN IUGR VERSUS AGA FETUSES AND NEWBORNS}

T Boutsikou ${ }^{1}$, A Malamitsi-Puchner ${ }^{1}$, M Kyriakakou ${ }^{1}$, $\mathrm{H} \mathrm{Militsi}^{2}$, A Margeli ${ }^{2}$, D Hassiakos ${ }^{1}$, I Papassotiriou ${ }^{2}$, G Mastorakos $^{1}$, C Kanaka-Gantenbein ${ }^{3}$

${ }^{1}$ Neonatal Division, 2nd Department Obstetrics and Gynecology Athens University, Medical School, Athens, Greece, ${ }^{2}$ Department of Clinical Biochemistry, 'Aghia Sophia' Children's Hospital, Athens, Greece, ${ }^{3} 1$ st Department of Pediatrics, Athens University, Medical School, Athens, Greece

Background and Aims: According to the 'fetal origin of adult disease' hypothesis, intrauterine growth restriction (IUGR) is associated with later hypercholesterolemia and occurrence of the metabolic syndrome. This study aimed to investigate lipid status during intrauterine and early postnatal life in 20 IUGR (birth weight below the 10th customized centile) and 20 appropriate for gestational age (AGA) fetuses (UC), and newborns on days 1 (N1) and 4 (N4), and to assess the ratio of total cholesterol levels to the ponderal index $\left[\mathrm{PI}=\right.$ weight $(\mathrm{kg}) /$ height $\left.(\mathrm{m})^{3}\right]$, which is an anthropometric method, used to diagnose impaired fetal growth.

Methods: Total cholesterol, HDL and triglyceride levels were measured by an automatic clinical chemistry analyzer (ADVIA 1650, Bayer Diagnostics, Tarrytown, NY, USA). LDL was determined by using the Friedewald equation.

Results: Absolute total cholesterol, LDL, HDL and triglyceride levels were similar in both groups at all time points studied (UC, N1 and N4). The ratio of total cholesterol to PI was significantly increased in IUGR versus AGA fetuses $(2.94+/-0.87$ versus $2.46+/-0.49$., $\mathrm{p}=0.042)$ and neonates on day $1(3.06+/-0.83$ versus 2.58+/-0.54, $\mathrm{p}=0.039$ ).

Conclusions: Although lipid levels do not differ between both groups, total cholesterol to ponderal index ratio is increased in IUGR versus AGA fetuses and newborns. This finding possibly indicates existence of hypercholesterolemia and impaired fetal liver function, 
age, height, weight, parity, smoking habits and infant sex. Estimated marginal means are presented in the table below. Each of the 7 sub-domains showed similar associations except for 'barriers to housing and services'.

Conclusions: Gestational age and birth weight are associated with IMD and some, but not all of its subdomains. Studies evaluating perinatal outcome could use IMD to take account of specific patterns of deprivation.

\begin{tabular}{|l|c|c|c|}
\hline Outcome & IMD Lower quartile (95\% CI) & IMD Higher quartile (95\% CD) & P Value \\
\hline $\begin{array}{l}\text { Gestational } \\
\text { Age (Weeks) }\end{array}$ & $(38.5-38.8)$ & 38.9 & 0.000 \\
\hline $\begin{array}{l}\text { Birth weight } \\
\text { (grams) }\end{array}$ & 3215 & $(38.7-39.0)$ & \\
\hline
\end{tabular}

\section{VLBWI FROM MULTIPLE PREGNANCIES IN PORTUGAL, 1996-2004}

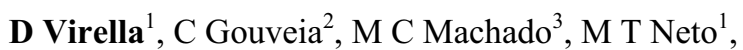 \\ R N Muito Baixo Peso ${ }^{4}$ \\ ${ }^{1}$ UCIN, Hospital de Dona Estefânia, Lisboa, Portugal \\ ${ }^{2}$ Departamento de Pediatria, Hospital de Dona \\ Estefânia, Lisboa, Portugal, ${ }^{3}$ Serviço de Pediatria, \\ Hospital Fernando Fonseca, Amadora, Portugal \\ ${ }^{4}$ Secção de Neonatologia da Sociedade Portuguesa de \\ Pediatria, Lisboa, Portugal
}

Background and Aims: Multiple pregnancies, both spontaneous and iatrogenic, are an important component of VLBWI incidence. Our aim was to monitor epidemiology and clinical outcomes of VLBWI from multiple pregnancies.

Methods: Data from the National Registry of VLBWI (1996-2004) was used for risk analysis of morbidity and mortality of VLBWI from multiple pregnancies. VLBWI singletons were considered as having basal risk. VLBWI from highest order multiple pregnancies $(>4)$ were not considered. Analysis of iatrogenic multiple pregnancies was not a purpose.

Results: Amongst the 8567 registered VLBWI, 2249 (26\%) were multiples (1852 twins, 346 triplets, 40 quadruplets and 11 of higher order). Since 2001, the rate of multiples has been decreasing. Some major outcome risk indicators, as mortality and HMD, were better for triplets and quadruples than for singletons and twins. No difference was proved for less frequent events, such as surfactant administration, PDA, IVH 3-4, NEC II-III or NEC-related surgery. Perinatal risk factors were consistently less severe among triplets and quadruples than singletons and twins: absence of prenatal care, absence of prenatal steroids, iatrogenic prematurity, CRIB, NTISS, gestational age, and birth weight. Level of neonatal care was similar whichever the number of foetuses was.

Conclusions: During this period, the improved prenatal and perinatal care of VLBWI from higher order multiple pregnancies was associated to lower than expected mortality and morbidity risks (even lower than single pregnancies). The role of assisted reproduction techniques can not be analysed from these database. Optimal prenatal and perinatal care should be achieved on every pregnancy, despite the number of foetuses.
THE EFFECT OF NIDCAP® INTRODUCTION IN A DUTCH LEVEL III NEONATAL INTENSIVE CARE UNIT ON SHORT-TERM CLINICAL OUTCOME

J M Wielenga ${ }^{1}$, B J Smit ${ }^{2}$, M P Merkus ${ }^{3}$, J H Kok $^{1}$

${ }^{1}$ Department of Neonatology, Emma Children's Hospital, Academic Medical Center, Amsterdam, The Netherlands, ${ }^{2}$ Department of Neonatology, Erasmus MC-Sophia Children's Hospital, University Medical Center, Rotterdam, The Netherlands, ${ }^{3}$ Department of Pediatric Clinical Epidemiology, Emma Children's Hospital, Academic Medical Center, Amsterdam, The Netherlands

Background and Aims: The aim of the present study was to evaluate the short-term clinical outcomes of NIDCAP ${ }^{\circledR}$ as compared to conventional care $(C C)$.

Methods: A prospective phase lag cohort study performed in a tertiary level NICU in the Netherlands. Infants born before 30 weeks of gestational age were included, 26 infants in the CC group and 25 infants in the NIDCAP ${ }^{\circledR}$ group. Primary outcome of the study was length of NICU stay.

Results: In the CC group mean days of NICU stay was 38 days (SD 25, range 9-99), in the NIDCAP group this was 49 days (SD 28, range 13-123) $(\mathrm{p}=0.131)$. Birth weight, gestational age, and pneumonia were statistically significant independent determinants of length of NICU stay; they explained $55 \%$ of variation. No difference in days of mechanical ventilation was seen. The NIDCAP group had significantly more days of respiratory support: median (IQR) days: 53 (32.5$90.0)$ versus $22(10.5-62.8)(\mathrm{p}=0.021)$. After adjustment in a multivariate regression model for the significant differences between both groups no difference in days of respiratory support were seen anymore. Cranial ultrasound findings differed significantly: in the NIDCAP group only one infant with severely abnormal findings, in the CC group 7 infants (plus 2 deceased, treatment withdrawn due to severe neurological condition) had severely abnormal findings $(\mathrm{p}=0.054)$.

Conclusions: The results of the present study provide no evidence for a beneficial effect of NIDCAP ${ }^{\circledR}$ care on length of NICU stay, respiratory support, feeding progress and growth. A possible beneficial effect was seen with respect to cranial ultra sound findings.

\section{IMPACT OF DELIVERY ROOM CARDIOPULMONARY RESUSCITATION (DR- CPR) ON AGE AT THE TIME OF DEATH (LOS) IN INFANTS $<1,200 G$ (ELBW)}

A Castillo, R Deulofeut, A Sola

Emory University, Atlanta, GA, USA

Background and Aims: Delayed mortality may be associated with prolonged suffering and also with emotional, economic and resource costs. The influence on this variability of DR-CPR has not been investigated. The objective was to analyze if the LOS in ELBW is different in those who received DR-CPR compared to those who died but did not require DR-CPR.

Methods: Analysis of all ELBW who died and had been born at two Emory perinatal centers in the last 5 years. 
Exclusion criteria were major congenital malformations and provision of comfort care. DR-CPR was defined as chest compression and/or epinephrine use in the delivery room (DR).

Results: 87 infants met enrollment criteria; 26 of them (30\%) received DR-CPR. 25\% died in first day (d); $18 \%$ at $1-3 \mathrm{~d} ; 5 \%$ between $4-7 \mathrm{~d} ; 29 \%$ between $8-28 \mathrm{~d}$ and $23 \%>28 \mathrm{~d}$ of age. The DR-CPR group was similar to the no DR-CPR group in birth weight [726 \pm 160 vs. $731 \pm 176]$ and gestational age $[24.8 \pm 1.9 \mathrm{vs} .25 .3 \pm 2.2]$. The average LOS was widely variable, $[21.8 \pm 47$ vs. $30.7 \pm 56.9 ; \mathrm{p}=0.669]$. Death in the DR $[27 \%$ vs. $0 \% ; \mathrm{p}=0.000]$, in the first 12 hours of life [38\% vs. $7 \% ; \mathrm{p}=0.001]$ and in the first $\mathrm{d}(42 \%$ vs. $20 \% ; \mathrm{p}=0.047)$ was more frequent in DR-CPR group. Death after 28d was $11.5 \%$ in DR-CPRvs. $26.6 \%$ in no DR-CPR infants $(\mathrm{p}=0.2)$; and the median LOS was $156.00 \mathrm{dvs} 41.00 \mathrm{~d}$, respectively.

Conclusions: A large proportion of the infants $<1,200 \mathrm{~g}$ who die do so after a prolonged LOS. Exposure to DRCPR is associated with a higher proportion of early mortality. Infants exposed to DR-CPR who survive $>28 \mathrm{~d}$ die after a prolonged LOS. It remains to be determined if criteria can be identified to avoid delaying the inevitable.

\section{NASOGASTRIC TUBE POSITION IN NICU CHILDREN}

\section{J de Boer, B J Smit}

Department of Pediatrics, Division of Neonatology, Erasmus MC - Sophia, Rotterdam, The Netherlands

Background and Aims: In the NICU, nasogastric tube placement is an essential procedure that is considered relatively safe. In adult patients however, tube placement errors are common, with tubes being positioned in the esophagus, the intestines, and the respiratory tract. Incidentally even perforations into the pleural space are mentioned. Errors may occur both during insertion and due to migration over time. In neonates only few studies on tube placement were performed. Improper positions were mentioned in 38\% to $55 \%$ of cases (Weibley et al., 1987). With regard to patient safety, these are alarming numbers. To establish the validity of these figures for our own NICU, we conducted a retrospective study of tube position on $\mathrm{X}$-rays in a one-year patient cohort.

Methods: The first available X-ray from all NICU patients with visible nasogastric tube position admitted through 2005 was reviewed. Trained medical students categorized them according to previously set decision rules as 'correct', 'incorrect' or 'sub-optimal' (in the stomach but excessively long).

Results: Until now, 99 X-rays were classified $( \pm 30 \%$ of the patient cohort). Kappa was .68, indicating substantial inter-rater agreement. Correct placement was concluded in $32 \%$, incorrect placement in $19 \%$, and suboptimal placement in $32 \%$ of cases.

Conclusions: As misplacement appears to be a relevant problem in our NICU, this study will be continued. Proper standardized methods for placement and checking the position of nasogastric tubes should be developed and refined to improve patient safety.

\section{SHOULD VERY PRETERM BABIES BE GIVEN} HYDROCORTISONE AT BIRTH?

\section{Watkinson, C Rose}

Neonatal Unit, Birmingham Heartlands Hospital, Birmingham, UK

Background and Aims: To measure the incidence of early (first 24 hours of life) hypotension in very preterm $(<27$ weeks) infants. To review hydrocortisone in its treatment.

Methods: An analysis of hypotension in 52 babies, median (range) gestation 25 (23-26) weeks. Hypotension was defined as a mean blood pressure (mmHg) less than the gestation (weeks).

Results: 32 (62\%) were hypotensive. 22 received fluid aliquots of $10 \mathrm{ml} / \mathrm{kg}$. All of these also had dopamine, 12 dobutamine and 5 norepinephrine. Those receiving norepinephrine were already on $>20 \mu \mathrm{g} / \mathrm{kg} / \mathrm{min}$ of dobutamine, and dobutamine was started only after the dopamine reached $>20 \mu \mathrm{g} / \mathrm{kg} / \mathrm{min} .13$ of the 21 , including all who had dobutamine and norepinephrine, received hydrocortisone. Of the 10 hypotensive babies who did not receive a fluid bolus all received dopamine, two had hydrocortisone. Overall 15 (29\%) of the babies received hydrocortisone at $2.5 \mathrm{mg} / \mathrm{kg} 6$ hourly.19 (59\%) of the hypotensive babies died, compared to $4(20 \%)$ of 20 non-hypotensive babies $-($ difference $=39 \% ; 95 \% \mathrm{CI}$ $16,50 \%)$.

Conclusions: Early hypotension was associated with a significantly higher mortality. Hydrocortisone is an effective treatment for neonatal hypotension but it takes some hours to work, and we gave it late in the therapy sequence. Nearly a third of the babies received it. Would its administration during resuscitation reduce the incidence of early hypotension in the very preterm infant? The potential risks of hydrocortisone given in this way are undefined. There is therefore equipoise that would justify a RCT of hydrocortisone at birth of very preterm babies.

\section{SURVIVAL AT THE LIMITS OF VIABILITY - PREDICTIVE VALUE THE APGAR SCORE AT MINUTE 1}

\section{O Genzel-Boroviczeny, J Hempelman}

\section{Department of Neonatology, University Childrens Hospital LMU, Munich, Germany}

Background and Aims: Is an APGAR 1 at minute 1 predictive for survival of extremely premature infants, who have been actively resuscitated and admitted to an neonatal intensive care unit?

Methods: Chart review of all infants with gestational ages $(\mathrm{GA})<27$ completed weeks over a 5 year period. Values as median $[95 \% \mathrm{CI}]$.

Results: Of the 237 infants, 29 had APGAR scores 1at minute (G1) and $208>1$ (G2). There was no sig. difference in delivery modus, but infants in G1 were intubated more frequently ( $93 \%$ vs $77 \%, \mathrm{p}=0,04)$ and earlier (2 minutes $[2,3 ; 6,7]$ vs 5 min $[7,5 ; 10])$. Despite earlier intervention mortality was sig. higher in G1 (62\% vs $17 \% ; p<0,0001)$, but age at death did not differ (G1: $3,5 \mathrm{~d}[1 ; 30]$ vs. G2: $6 \mathrm{~d}[6 ; 44]$. There was a small but sig. difference in GA (24 wks $[23,9 ; 24,7]$ vs 25 wks 
$[24,7 ; 25,0] \mathrm{p}=0,008)$ and birthweight $(617 \mathrm{~g}[579 ; 678]$ vs $700 \mathrm{~g}[694 ; 752] \mathrm{p}=0,02)$ Subgroup analysis of survivors $(\mathrm{n}=11)$ and non-survivors $(\mathrm{n}=18)$ of the G1 infants revealed birth weight and sex as the best predictors of survival. With an APGAR 1 at the first minute a male infant with GA 23 weeks and BW $500 \mathrm{~g}$ has a mortality rate of $92 \%$.

Conclusions: Despite successful resuscitation, infants at the limits of viability have a very poor prognosis for survival when presenting with bradycardia, cyanosis and no respiratory efforts $(A P G A R=1)$ at birth. This information may be helpful for prenatal counseling and for the decision making in the delivery room.

\section{TWIN TRANSFUSION SYNDROME CAUSING CUTANEOUS ERYTHROPOIESIS}

\section{F Duque, J Campos, R Henriques}

Neonatology Unit, Maternidade Daniel de Matos, Coimbra's University Hospital, Coimbra, Portugal

Background and Aims: In the newborn infant, the most common cause of blueberry muffin skin lesions is cutaneous erythropoiesis, due to intrauterine viral infection. Also have been described in congenital leukaemia and neuroblastoma, and rarely reported in twin transfusion syndrome.

Methods: We report a case of female twins born to a 38-year-old blood type O, Rh-positive mother, by caesarean section, at 33 weeks of a monochorial gestation. Maternal rubella and cytomegalovirus titers indicated immunity at two weeks of gestation. Pregnancy was not complicated by known viral exposures, maternal illness or rashes.

Results: Twin A, who weighed $1.965 \mathrm{~g}$ was noted to be plethoric. Twin B, a $1.250 \mathrm{~g}$ newborn, was extremely pale, had hepatomegaly and bluish-red papular skin lesions, with 3-6 $\mathrm{mm}$ in diameter, distributed over the face, trunk and limbs. She was intubated and ventilated. Initial laboratory evaluation showed a hematocrit of $13.7 \%$ and haemoglobin $4.0 \mathrm{~g} / \mathrm{dl}$ (twin A hematocrit was $73.1 \%$ and haemoglobin $24.6 \mathrm{~g} / \mathrm{dl}$ ), WBC count was $16.900 / \mu \mathrm{l}$ and the platelet count was $85.000 / \mu 1$. The patient received a transfusion of packed RBCs. She had congestive heart failure treated with inotropics and metabolic acidosis. Cytomegalovirus and syphilis serologic testing were negative. She had a favourable clinical evolution, remained well after discharge and spontaneous resolution of the cutaneous eruption occurred.

Conclusions: Cutaneous erythropoiesis in this case of twin transfusion syndrome is considered to be due to persistence or reactivation of fetal dermal erythropoies, secondary to prolonged severe intrauterine anemia.

\section{SIMULTANEOUS MEASUREMENT OF SIX CYTOKINE LEVELS IN THE SERUM OF NEONATES WITH OR WITHOUT EARLY ONSET NEONATAL INFECTION}

\footnotetext{
M Labenne ${ }^{1}$, G Lizard ${ }^{2}$, B Gouyon ${ }^{3}$, C Ferdynus ${ }^{3}$, J B Gouyon ${ }^{1}$
}

${ }^{1}$ Pédiatrie 2, CHU de Dijon, France, ${ }^{2}$ INSERM U498, CHU de Dijon, France, ${ }^{3}$ DIM, CHU de Dijon, Dijon, France

Background and Aims: Antibiotic therapy is frequently initiated at birth, because the is no rapid and reliable diagnostic test for identifying the infected neonates. To determine prospectively the negative predictive value of simultaneous measurement of 6 cytokines for diagnosing early onset neonatal infection (EONI).

Methods: Neonates were included if they required biological and bacteriological evaluation for a presumptive diagnosis of EONI within 6 hours of life. After parental consent, a simultaneous measurement of 6 cytokines (IL-1 $\beta$, IL-6, IL-8, IL-10, IL-12 and TNF $\alpha$ ) was performed using the Cytometry Bead Array (CBA) technique. Exhaustive data were collected from the medical record of each neonate to allow classification in 3 diagnostic categories (definite, probable, excluded).

Results: Neonates were included if they required biological and bacteriological evaluation for a presumptive diagnosis of EONI within 6 hours of life. After parental consent, a simultaneaus measurement of 6 cytokines (IL-1 $\beta$, IL-6, IL-8, IL-10, IL-12 and TNF $\alpha$ ) was performed using the Cytometry Bead Array (CBA) technique. Exhaustive data were collected from the medical record of each neonate to allow classification in 3 diagnostic categories (definite, probable, excluded.

Conclusions: The measurement of IL-6 and IL- 8 levels, using the CBA technique, should be effective in reducing unnecessary antibiotic therapy at birth.

\section{EVALUATION AND INCIDENCE OF EARLY ONSET SEPSIS IN OUR NEONATAL UNIT DURING THE LAST TWO YEARS}

V Delovska-Stojkova, B Mihajlovic-Dimovska, J Guleva, M Kalajdzieva-Zip, T Pensovska

Department of Neonatology, Special Hospital of Gynaecology and Obstetrics, Skopje, Macedonia

Background and Aims: Early neonatal sepsis can be caused by an extraordinary variety of microorganisams. Early recognition, early diagnosis and treatment is essential, because the risk of morbidity and mortality can develop. The aim of our paper is to inform for incidence of early onset sepsis in our neonatal unit during last two years.

Methods: The incidence of early onset sepsis was evaluated by retrospective analysis, using our database results, enrolling all high risk for neonatal sepsis newborns and positive blood cultures before 48 hours of live.

Results: During last two years with approximate 6425 deliveries, we studied 193 neonates clinically and microbiologically. Bacterial sepsis was confirmed in 6 cases. Between them 4 were premature and 2 were mature newborns.

Conclusions: We conclude that incidence of the early onset neonatal bacterial sepsis is higher in population of preterm, than in population of the term newborns $0,96 \%$. Results that we found are similar in literature. 


\section{HERPES SIMPLEX VIRUS INFECTION IN THE NEONATE: DIAGNOSIS, TREATMENT AND PREVENTION}

\author{
M Akbarzadeh $^{1}$, R Akbarzadeh $^{2}$, R Akbarzadeh ${ }^{1}$ \\ ${ }^{I}$ Department of Psychology, Russian University of \\ Economics and Culture, Moscow, Russia, ${ }^{2}$ Mashhad \\ University of Medical Sciences, Mashhad, Iran
}

Background and Aims: Neonatal herpes simplex virus (HSV) infection is one of the life-threatening infections of newborns. Infections are categorized by extent of disease into skin/eye/ mouth, central nervous system and disseminated disease categories. Each disease category is responsible for roughly one third of neonatal infections. We aim in this article guideline on the diagnosis, prevention and effective management of neonatal (HSV) infection.

Methods: This paper focuses on the advances that have been made in our understanding of the pathogenesis, diagnosis, treatment, and prevention of (HSV) infections.

Results: Many of the infections result from asymptomatic cervical shedding of virus after a primary episode of genital HSV in the third trimester. Infection may occur in uterus, by transplacental or ascending infection, by exposure to genital lesions during delivery, or postnatal from relatives or attendants. Most cases of neonatal HSV disease are caused by HSV type 2 . Diagnosis of neonatal infection requires a very high level of clinical awareness. Direct immunofluorescence and cultures are reliable diagnostic tests. Polymerase chain reaction (PCR) is a powerful diagnostic tool that has supplanted brain biopsy as the modality of choice for diagnosing HSV CNS disease.

Conclusions: Careful physical examination and appropriate investigations of the infant should accurately identify the infection in the majority of cases. Monitoring of hydration and renal function as well as meticulous supportive care of a very sick infant is also required. Treatment is recommended where diagnosis is confirmed or there is a high level of suspicion.

\section{BLOOD CULTURE RESULTS IN NEWBORNS}

\section{G Mijovic $^{1}$, S Crnogorac ${ }^{2}$, N Jokmanovic ${ }^{2}$ \\ ${ }^{1}$ Institute of Public Health, Podgorica, Serbia and Montenegro, ${ }^{2}$ Clinical of Gynecology and Obstetrics, Clinical Center of Montenegro, Podgorica, Serbia and Montenegro}

Background and Aims: Newborns are in risk of acute bacterial infection for numerous reasons. One of the risks is preterm delivery. Morbidity and mortality are also higher in preterm newborns. To estimate how often the bacteria is found in umbilical cord blood in preterm and term newborns.

Methods: We prospectively reviewed umbilical cord blood samples of all preterm newborns and randomly chosen term newborns during 3 months period in Clinical of Gynecology and Obstetrics, Podgorica.

Results: 123 samples are examined, out of which 24 are from preterm and 99 from term newborns. 4 out of 99 were positive and among preterms, none had positive result.
Conclusions: This investigation has demonstrated that bacterias are not found more often in preterm than in term newborns. Therefore, we can conclude that other factors have role in appearance of infection in preterm newborns.

\section{RESPIRATORY SYNCICIAL VIRUS IN THE NEONATAL INTENSIVE CARE UNIT - CLINICAL AND ECONOMIC IMPACT}

T A Aguiar, M A Ferreira, I M Leal, M R Barroso, M H Carreiro, M C Machado

Departamento de Pediatria, Hospital Fernando Fonseca, Amadora, Portugal

Background and Aims: Respiratory Syncicial Virus (RSV) infections are rare in Neonatal Intensive Care Units (NICU). However, nosocomial outbreaks occur, leading to major morbidity among premature babies, especially those with chronic lung disease (CLD). Characterization of RSV infection in the NICU, clinical and economic evaluation.

Methods: Characterization of RSV infection in the NICU, clinical and economic evaluation.

Results: Twenty four $(2,8 \%)$ out of 865 neonates had RSV infection: 21 cases were studied. Incidence was higher in $2003(71,4 \%)$, during January $(5 / 21)$ and December (3/21). The mean age of infected infants was 40 days with mean gestational age (GA) and birth weight of 29 weeks and $1273 \mathrm{~g}$ respectively. Mean weight at onset of symptoms was $1629 \mathrm{~g}$. Six infants (28\%) had CLD. Three infants (14,3\%) required intubation and one $(4,8 \%)$ non invasive mechanical ventilation. There were no deaths morbidity had additional charges: Expenses with diagnosis tests and a prolonged stay of 49 days, with costs of 16959 Euros.

Conclusions: Reduction in RSV infection in 2004 was probably due to restriction of visits and reinforcement of prevention measures. Severity of infection was related to lower GA $(p=0,002)$ and $\mathrm{BW}(\mathrm{p}=0,011)$, lower weight at onset of the symptoms $(\mathrm{p}=0,001)$. Morbidity lead to a more prolonged stay of length with economic, clinical and social repercussions.

\section{A NEW PHENOTYPICAL VARIANT OF INTRAUTERINE GROWTH RESTRICTION?}

C De Felice ${ }^{1}$, R Tassi $^{2}$, B De Capua ${ }^{3}$, M Gentile $^{8}$, L Quartulli ${ }^{6}$, G Tonni ${ }^{9}$, D Costantini ${ }^{5}$, M Strambi ${ }^{4}$, G Latini ${ }^{6,7}$

${ }^{1}$ Neonatal Intensive Care Unit, Azienda Ospedaliera Universitaria Senese, Siena, Italy, ${ }^{2}$ Laboratory of Ultrasonology, Department of Neurosciences, Azienda Ospedaliera Universitaria Senese, Siena, Italy, ${ }^{3}$ Clinical Audiology Unit, Dipartimento Cefalico-Scheletrico, Azienda Ospedaliera Universitaria Senese, Siena, Italy ${ }^{4}$ Departments of Pediatrics, Obstetrics, and Reproductive Medicine, University of Siena, Siena, Italy ${ }^{5}$ Neonatal Audiologic Screening Service, USL7, Siena, Italy, ${ }^{6}$ Division of Neonatology, Perrino Hospital, Brindisi, Italy, ${ }^{7}$ Clinical Physiology Institute, National Research Council of Italy (IFC-CNR), Lecce, Italy ${ }^{8}$ Medical Genetics Unit, Ospedale Di Venere, Bari, 
Italy, ${ }^{9}$ Division of Obstetrics and Gynecology, Guastalla Civil Hospital, AUSL Reggio Emilia, Reggio Emilia, Italy

Background and Aims: Emerging evidence indicates a link between intrauterine growth restriction (IUGR) and major adult-onset diseases. Here, we describe previously unrecognized, phenotypical features in children with idiopathic IUGR/SGA.

Methods: A total of 77 Italian children (age: $9.45 \pm$ 2.08 years) with antenatally diagnosed IUGR and SGA birth were examined, and subdivided into two groups ("variant" vs. controls) according to auricle morphology deviation from normal. The following variables were determined: 1) external ear auricle geometry [maximum width of longest axis (LA); shortest axis (SA); distance of SA from the lowest auricle point (SA-D); SA/LA and LA/SA-D ratios]; 2) posterior communicating arteries (PCoAs) function (increased flow velocity in the posterior cerebral artery $>20 \%$ during ipsilateral compression of the common carotid artery) (transcranial doppler ultrasonography; Philips Sonos 5500); 3) articular mobility (Beighton's 9-point scale); 4) skin softness; and 5) distortion product evoked otoacoustic emissions (DPOAEs) (ILO 292 DP Echoport).

Results: A total of 27/77 (35.06\%) children showed a variant auricle shape. Lower SA-D/LA ratio and higher SA/LA and SA-D asymmetry indices $(p<0.0001)$, excess of bilaterally non-functioning PCoAs $(p<0.0001)$, higher Beighton's scores $(p<0.0001)$, excessive skin softness $(\mathrm{p}<0.0001)$, and evidence of bilateral DPOAEs notches $(\mathrm{p}<0.0001)$, as compared to the control IUGR/SGA subjects.

Conclusions: The phenotype of the described IUGR/SGA variant is likely the consequence of a large developmental field disturbance occurring early in embryogenesis. We propose that the observed phenotypical constellation may represent an unrecognized variant of IUGR. Further research is needed in order to assess its frequency, geographic distribution and possible relationships with long-term prognosis.

\section{EFFECTIVENESS OF THE NRP PROGRAM IN GEORGIA}

M Kherkheulidze ${ }^{1}$, K Nemsadze ${ }^{1}$, N Kavlashvili ${ }^{1}$, E Kandelaki $^{2}$, N Sapandze ${ }^{1}$, T Manjavidze ${ }^{1}$, M Macharashvili², D Toklikishvilii ${ }^{2}$

${ }^{1}$ State Medical University, Tbilisi, Georgia

${ }^{2}$ M. Guramishvili Pediatric Clinic, Tbilisi, Georgia

Background and Aims: The study aims to assess the impact of neonatal resuscitation program (APA/AHA) on the management and outcome of the birth asphyxia and to determine differences between the approaches to resuscitation in trained and untrained maternity houses.

Methods: The multi-center study was conducted in 12 randomly selected maternity houses; I group include (8 trained institutions) and the control II group (4 untrained institutions). Information was collected using special record form.

Results: The results emphasized significant difference in conducting of resuscitation procedures between groups. The nessessity of resuscitation in I group was
$8,2 \%$, while in II group $14,3 \%$. In the second group initial steps of resuscitation were conducted only in $4,6 \%$, in I group in all cases of resuscitation. The usage of medications was higher in II group $(8,8 \%)$ then in I $(0,79 \%)$. For infants of I group requiring resuscitation statistical significance is achieved between the 1 and 5 minute Apgar score, $(p<0.05)$, comparing to II group. The incidence of RDS, Asphyxia and MAS was higher in the II group, as well as referrals to high level hospitals. In II group thermal control was neglected body temperature was measured only in $26,6 \%$ compared to I group $(72,7 \%)$.

Conclusions: The introduction of NRP was associated with significant improvement in evaluation and management of the neonates in delivery room.

\section{DO WE GET MORE HIGH RISK NEWBORNS?}

\section{E M Zisovska, J T Zivkovik, L B Spasova}

Department of Neonatology, Obstetric and Gynecology Clinic, Skopje, Macedonia

Background and Aims: Annual perinatal data show changes in some perinatal indicators, especially for high risk newborns who takes the greatest part in morbidity and mortality rates. The aim is to present prematurity rate, the incidence of Small for Gestational Age babies (SGA) and Large for GA babies (LGA) in non selected neonatal population throughout Macedonia in last 5 years (2000-2004) and to find the trends.

Methods: Annual perinatal reports, retrospective population study using epidemiological method.

Results: the most striking is the decreasing number of live births in last five years, starting from 25255 (2000) to 22836 (2004) (decrease of $10,6 \%$ for 5 years). Unfortunately, the prematurity rate has an increasing tendency, from $5,1 \%$ (2000) to $6,3 \%$ (2004), an increase of $23 \%$, and this group has the highest risk for survival and morbidity. The percent of SGA babies has increased from $9,45 \%$ to $10,6 \%$, (increase $12 \%$ ), and LGA from $6,8 \%$ to $7,4 \%$ (increase $8,8 \%$ ). On the other side, there is a great improvement of the obstetric care, and the percent of pregnant women who have 5 and more antenatal controls (as standard) is higher, in 2000$48,8 \%$ and in $2004-60,8 \%$. This fact suggests that despite the better obstetric and perinatal care, the number of high risk newborns is getting higher.

Conclusions: : There are a lot of reasons why we get these results (social, economic, etc). Although at the first sight it is not too great increase, it means a lot, because the trend towards getting more risky newborns is obvious and happened within short period of investigation.

\section{NEONATAL HEMOCHROMATOSIS - SUCCESSFUL SHORT TIME OUTCOME}

M L Mägi ${ }^{1}$, A Ormisson ${ }^{2}$

${ }^{1}$ Children's Clinic, Tartu University Hospital, Tartu, Estonia, ${ }^{2}$ Department of Pediatrics, University of Tartu, Tartu, Estonia

Background and Aims: Neonatal hemochromatosis $(\mathrm{NH})$ is a rare disease of gestation associated with fetal 
liver injury and extrahepatic siderosis. The etiology of $\mathrm{NH}$ is not fully understood, but the gene mutation that causes NH has not been identified. The alloimmune etiology has been suggested.

Methods: We describe a case of NH in a term baby with successful short time outcome.

Results: The baby was born on 40th week of gestation with birthweight $3754 \mathrm{~g}$ and Apgar score 8/7/7 with manifested haemorrhagic syndrome. Early neonatal period complicated with severe anemia, thrombocytopenia $(18 \times 10 \psi / 1)$, hepatosplenomegalia, respiratory disorders and hepatic insufficiency, which culminated in remarkable cholestasis (S-Bil-conj $249 \mathrm{mmol} / \mathrm{l}$; S-AST $630 \mathrm{U} / 1, \mathrm{~S}-\mathrm{ALT} 516 \mathrm{U} / 1$ ) and severe iron overload (SFerritin $7417 \mathrm{mg} / \mathrm{l}, \mathrm{S}-\mathrm{TRF}$ sat $99,1 \%$ ) at the age of 2 weeks. Cholestasis and iron deposition was confirmed by liver biopsy. Hemotransfusion, thrombomass and intravenous immunglobulin transfusions were applied. DNA-based test for the gene mutation showed the child to be a heterosygote for the mutation C282Y. Primary possible etiological factors as hepatotropic infections, metabolic and autoimmune diseases were excluded. With iron reduction therapy (Desferal, antioxidants, erythropoetin), laboratory findings (total bilirubin, aminotransferases, ferritin and transferrin saturation) gradually returned to normal at the age of 4-5 months. The baby had normal neurodevelopment and normal hepatic function nonetheless moderate hepatosplenomegaly at the end of first half-year of life.

Conclusions: Severe NH was diagnosed after birth by clinical, biochemical and liver biopsy findings. On the bases of successful recovery from severe $\mathrm{NH}$ the alloimmune neonatal hemochromatosis can be suggested.

\section{GROWTH-RESTRICTION AND FETAL DISTRESS - AN INDEPENDENT RISK FACTOR FOR MATERNAL AND NEONATAL MORBIDITY}

\section{E Petkovska, S Jancevska}

\section{Department of Neonatology, University Clinic of Gynecology and Obstetrics, Skopje, Macedonia}

Background and Aims: Growth-restricted infants do not tolerate the stress of delivery a well as normally grown infants. They are more prone to intrapartum distress, operative way of delivery and perinatal asphyxia. The aim of this study was to evaluate the contribution of the fetal distress and growth-restriction as combined pathology to maternal and neonatal morbidity.

Methods: We have done a prospective study of early maternal and neonatal complications on 160 growthrestricted infants and compared with 200 normal pregnancies. We used SPSS 1.1 and Statgraf for WIN 2.1 statistical programs. Results were compared by Pearson Chi-Square $(p<0.05)$ and Logistic regression analyses.

Results: Operative way of delivery had $52(66.3 \%) / 54$ growth-restricted pregnancies with fetal distress vs. 47 (44.34\%)/106 growth-restricted pregnancies without fetal distress $(\mathrm{p}<0.05)$. Perinatal asphyxia had 37 $((68.52 \%) / 54$ growth-restricted infants with fetal distress vs. $20(18.87 \%) / 106$ growth-restricted infants without fetal distress $(\mathrm{p}<0.05)$. The combination of growth-restriction and fetal distress was found to be an independent risk factor for cesarean section (OR 32.64;95\%CI:7.55-141.0) and perinatal asphyxia (OR 9.36;95\% CI:4.41-19.86).

Conclusions: Growth-restricted fetuses should be monitored carefully and continuously during delivery. In the face of deteriorating fetal status, a cesarean section should be performed. Because growth-restricted infants are more prone to hypoxemia during delivery, neonatal team capable of managing perinatal asphyxia should be available at the time of delivery. Growth-restriction and fetal distress as combined pathology is multiplicity the risk for early maternal and neonatal morbidity. Close collaboration between obstetricians and neonatologist is essential for adequate care of the growth-restricted fetuses.

\section{THE USE OF $20 \%$ ALBUMIN IN THE NEONATAL INTENSIVE CARE UNIT}

I Morris, E J Molloy

Department of Neonatology, National Maternity Hospital, Dublin, Ireland

Background and Aims: There is a paucity of evidence for the use of $20 \%$ albumin in neonates. We aimed to evaluate $20 \%$ human albumin use in neonates and assess its efficacy in the management of hypoalbuminemia.

Methods: All infants treated with 20\% albumin infusion in a tertiary referral maternity hospital were included. Records were reviewed to identify patient demographics, indications for albumin treatment, means of administration, duration of hospital stay, mortality, morbidity and any evidence or suggestion of adverse reactions to albumin administration.

Results: 30 infants (9 Female, 21 Male) were included with a mean gestational age of $32.2+/-5.3$ and birth weight of $1893+/-1136 \mathrm{~g}$. The mean number of hours ventilated was 263 hours and 17 had positive blood cultures. There was a wide variation in albumin use in a single institution. Concurrent frusemide increased in urine output was observed $(\mathrm{p}=0.02)$ and albumin levels increased following albumin infusion (mean rise $6.67 \mathrm{~g} / \mathrm{l}$, $\mathrm{p}=<0.05$ ) for 48hours. No adverse events were associated with albumin administration.

Conclusions: Neonatal serum albumin can be increased by $20 \%$ albumin infusion albeit briefly and urine output enhanced by concurrent frusemide administration. However prospective randomised controlled trials are needed to further assess efficacy of $20 \%$ human albumin administration in neonates and devise standardized clinical guidelines.

\section{NON SPECIFIC PRESENTATIONS OF NEONATAL URINARY TRACT INFECTION}

P A Valencia, B Chandler, A Soliz

Division of Neonatology, Miami Children's Hospital, Miami, FL, USA

Background and Aims: Urinary tract infections (UTI) in neonates can result in significant acute and long term complications. Febrile neonates are routinely screened 
for UTI; but neonates with UTI could be afebrile and present with nonspecific symptoms or be asymptomatic and the diagnosis could be missed. The purpose of this study was to describe the different presentations of UTI in neonates admitted from home to our Neonatal Intensive Care Unit (NICU) at Miami Children's Hospital (MCH).

Methods: We reviewed the medical records of newborn infants from 37 to 44 weeks post conceptional age at the time of admission from home to our NICU and diagnosed with UTI for the time period from 2000 to 2006. UTI was defined as the presence of greater than 10,000 colony forming units of bacteria in a urine sample obtained by urethral catheterization.

Results: Seventy patients were included in the study. The most frequent presentations of illness were: fever $(49 \%)$, irritability $(27 \%)$, emesis $(18 \%)$, and jaundice $(10 \%)$, Of 36 afebrile patients, 11 presented with emesis $(30 \%), 5$ with jaundice (13\%), and 3 with irritability $(8.3 \%)$. Other presentations included decreased oral intake, hypoactivity, diarrhea, apnea and hypotonia. Only 1 patient was asymptomatic. The presentation of emesis without fever as sign of UTI was more frequent in the past 2 years when screening of more babies with emesis began.

Conclusions: The diagnosis of UTI in neonates who present with symptoms other than fever needs to be considered. Afebrile infants with emesis and other nonspecific symptoms should be screened for UTI.

\section{SERUM CYSTATIN C LEVELS IN INTRAUTERINE GROWTH RESTRICTED AND APPROPRIATE FOR GESTATIONAL AGE FETUSES AND NEONATES}

L Kontara ${ }^{1}$, A Malamitsi-Puchner ${ }^{1}$, D Gourgiotis ${ }^{2}$, M Boutsikou $^{1}, \mathrm{M} \mathrm{Kyriakakou}^{1}, \mathrm{~K}$ E Nikolaou ${ }^{1}$, A Marmarinos $^{2}, \mathrm{~S} \mathrm{Baka}^{1}$, D Hassiakos ${ }^{1}$

${ }^{I}$ Neonatal Division, 2nd Department Obstetrics and Gynecology, Athens University, Medical School, Athens, Greece, ${ }^{2}$ Research Laboratories, 2nd Department of Pediatrics, Athens University, Medical School, Athens, Greece

Background and Aims: Cystatin C, an endogenous marker of renal function, correlating better with glomerular filtration rate (GFR) than creatinine, presents higher serum levels immediately after birth, followed by a considerable decrease, due to postnatal renal maturation. Animal studies have shown that intrauterine growth restriction (IUGR) is associated with reduction in nephron number and increased glomeruli size, leading to hyperfiltration and hypertension later in life. This study aimed to investigate serum cystatin levels in IUGR $(<10$ th customized centile, due to gestational pathology) and appropriate for gestational age (AGA) full term fetuses and neonates on postnatal day 1 and 4 , as well as their mothers.

Methods: Serum cystatin C levels were measured by enzyme immunoassay in 20 IUGR and 20 AGA motherinfant sets.

Results: Fetal Cystatin C levels were significantly higher in the AGA than in the IUGR group $(p=0.001)$. No correlation was found in the IUGR and AGA group between maternal levels, and fetal, neonatal day 1 or neonatal day 4 levels.

Conclusions: In conclusion, fetal cystatin $\mathrm{C}$ serum levels are lower in the IUGR group, possibly due to increased glomeruli size and subsequent hyperfiltration. In addition, triggered renal maturation, consequent to intrauterine stress could be also responsible. The lack of correlation between fetal and maternal cystatin $\mathrm{C}$ levels may imply its exclusively neonatal origin, as it has been previously reported that this substance does not cross the placental barrier.

\section{CONGENITAL MESOBLASTIC NEPHROMA (CMN) IN A PREMATURE CHILD ASSOCIATED WITH MATERNAL HYPERTENSION DURING PREGNANCY. A CASE REPORT AND REVIEW OF LITERATURE}

\author{
K Euchler ${ }^{1}$, S M Pfister ${ }^{1}$, W Back ${ }^{2}$, T H Schaible ${ }^{1}$, \\ M Dürken ${ }^{1}$ \\ ${ }^{1}$ Department of Pediatrics, University Hospital \\ Mannheim, Mannheim, Germany, ${ }^{2}$ Department of \\ Pathology, University Hospital Mannheim, Mannheim, \\ Germany
}

Background and Aims: To describe a case of congenital mesoblastic nephroma $(\mathrm{CMN})$ in a premature child in conjunction with maternal hypertension during pregnancy including prenatal diagnosis, complications, and treatment.

Methods: Presenting signs and symptoms of mother and child, diagnosis, treatment and outcome of the patient.

Results: Hypertension was found in a 25 year old pregnant women without any other symptoms or signs of preeclampsia. Fetal ultrasound examination showed polyhydramnion and an abdominal tumor arising from the right kidney. By 30 weeks of gestation the boy was born spontaneously, birth weight was $1450 \mathrm{~g}$. Physical examination revealed a right upper quadrant mass. Due to respiratory distress syndrome diagnosed shortly after birth the patient needed mechanical ventilation for 3 days. Hypertension with a maximum systolic blood pressure of $129 \mathrm{mmHg}$ and elevated serum renin concentration of $10858 \mu \mathrm{U} / \mathrm{ml}$ (normal 11-147 $\mu \mathrm{U} / \mathrm{ml}$ ) was found at day 3 . Abdominal ultrasound and magnetic resonance imaging confirmed a tumor of 4,2 x 3,9 x 4,2 $\mathrm{cm}$ arising from the right kidney. On day 19 after delivery a right-sided tumor nephrectomy was performed. The blood pressure immediately returned normal without any treatment. Histologically, tumor specimens were classified as classic, focally cellular CMN.

Conclusions: Unexplained maternal hypertension may be the presenting symptom of congenital mesoblastic nephroma.

\section{RANDOMISED CONTROLLED TRIAL OF SEVOFLURANE FOR INTUBATION IN NEONATES}

S Hassid ${ }^{1}, \mathrm{C} \mathrm{Nicaise}^{1}, \mathrm{~F} \mathrm{Michel}^{1}$, R Vialet ${ }^{1}$, L Thomachot $^{1}$, P Lagier ${ }^{1}, \mathrm{C}$ Martin $^{2}$ 
${ }^{1}$ Réanimation Infantile, Hôpital Nord, Marseille, France ${ }^{2}$ Département D'Anesthésie-Réanimation, Hôpital Nord, Marseille, France

Background and Aims: To determine if sevoflurane, an inhaled anesthetic agent, can be used with safety and efficacity for premedication of intubation in term and preterm neonates.

Methods: A prospective randomised controlled nonblinded study in a tertiary neonatal intensive care unit. Minute by minute heart rate (HR), mean arterial blood pressure, transcutaneous oxygen saturation $(\mathrm{SpO} 2)$, and number of bradycardia (HR $<100 \mathrm{bpm})$ and desaturation ( $\mathrm{SpO} 2<85 \%$ for more than 30 seconds) were noted from 5 minutes before to 10 minutes after intubation. Operator experience, facility and number of attempts were collected.

Results: 33 neonates (19 in the study group and 14 in the control group) were randomly allocated to receive sevoflurane (concentrations varying from 2 to $5 \%$ ) or no premedication (preoxygenation with $100 \%$ oxygen alone) before their intubation. No major adverse events were noted in the study group (hypotension $(37,5 \% \mathrm{vs}$ $37,5 \%$, NS), number of desaturation $(37,5 \%$ vs $44,5 \%$, NS)). Hypertension $(56,3 \%$ vs $25 \%, \mathrm{p}=0,04)$ and incidence of bradycardias $(44,4 \%$ vs $8,3 \%, \mathrm{p}<0,01)$ were greater in the control group. Intubation was easier in the study group : no movements: $95,5 \%$ vs $28 \%$ $(p<0,005)$, good glottis visualisation: $73 \%$ vs $33 \%$ $(\mathrm{p}=0,013)$. The failure rate was lower in the study group ( $25 \%$ vs $39 \%$ ), but this difference was not statistically significant.

Conclusions: Premedication for intubation with sevoflurane in neonates is well tolerated, even in the less mature. It facilitates the condition of intubation and leads to less adverse events. Other studies are necessary to confirm these preliminary results.

\section{CLINICAL RELIABILITY OF TWO PAIN SCALES FOR NEWBORNS}

C V Bellieni, S Perrone, F Bagnoli, G Buonocore

Department of Pediatrics, University of Siena, Siena, Italy

Background and Aims: We studied the reliability of two of them.

Methods: We studied two groups of babies during a heelprick. The first group of 20 premature babies was studied using the PIPP scale, and the second group of 20 term babies was studied with the NIPS scale. We compared the pain scores given by nurse A who took the blood sample with those given by nurse $\mathrm{B}$ who was present during heelprick and those of nurse $\mathrm{C}$ who watched later the video clip of the blood sampling.

Results: NIPS: $8 / 20$ scores were different between nurse A and nurse C; but only in 1 case this difference was $>2$. In the case of nurse $\mathrm{B}$, the differences from nurse $C$ were $12 / 20$ and also in this case only 1 baby was scored with a score difference $>2$. The scores of nurse B did not differ significantly from those of nurse A. PIPP: 16/20 scores were different between nurse A and nurse $C$; in 9 case this difference was $>2$. In the case of nurse $\mathrm{B}$, the differences from nurse $\mathrm{C}$ were 17/20 and in this case 6 babies obtained a score difference $>2$. The number of scores of nurse A diverging from reference was not statistically significant.

Conclusions: Though both scales are good for research studies, further efforts to find reliable tools to assess neonatal pain in a clinical set are needed.

\section{NEONATAL DISEASES RESEARCH THROUGH SURVEILLANCE: THE CANADIAN EXPERIENCE}

D Grenier ${ }^{1}$, J-A Doherty ${ }^{2}, \mathrm{~S}$ Srikanthan ${ }^{1}$

${ }^{1}$ Canadian Paediatric Society, Ottawa, ON, Canada

${ }^{2}$ Public Health Agency of Canada, Ottawa, ON, Canada

Background and Aims: Encephalopathy incidence due to severe neonatal hyperbilirubinemia $(\mathrm{NH})$ has increased, attributed to conservative jaundice management and earlier newborn discharge. Neonatal herpes simplex virus (HSV) infection has changed in prevalence, presentation and infection types. Mortality remains high despite improved diagnostic tests and antiviral therapy. NH and HSV study results were compared on incidence, maternal and newborn profiles, and burden of illness to better define clinical and public health impacts. Timely diagnosis of congenital cytomegalovirus (CMV) infection remains important.

Methods: Over 2400 paediatricians monthly report rare diseases according to preset protocols. Confidentiality is mandatory. All studies receive ethical approval.

Results: The CPSP confirmed $259 \mathrm{NH}$ cases in term neonates. A high percentage of discharged newborns $(72 \%)$ were readmitted at an early age of five days with high-unconjugated bilirubin (median: $471 \mu$ mole/L). Majorities were only breastfed (81\%) with over representation of non-white ethnic groups. Exchange transfusions were performed in $22 \%$. Evidence of clinical bilirubin encephalopathy was present at discharge in 5\%. Canada established a national incidence rate of 5.9/100,000 live births for HSV with fatality rates of $16 \%$. HSV-1 disease accounted for $63 \%$ of cases. Localized disease to skin, eye and mucosa represented $60 \%$ of cases. High percentage of mothers were unaware of previous HSV infection prior to delivery and mothers under 20 years of age were disproportionately represented. A survey pre-CMV study documented diagnostic approaches of respondents.

Conclusions: Systematic analyses and interpretation of this surveillance identified important medical and public health actions that will benefit children and their families.

\section{PRETERM NEWBORNS MORTALITY PREDICTION}

P A Tavares Junior ${ }^{1}$, M D Salgado Quintans ${ }^{2}$

${ }^{1}$ Universidade Federal Fluminense, Niteroi City, RJ, Brazil, ${ }^{2}$ Hospital Estadual Pereira Nunes, Niteroi City, RJ, Brazil

Background and Aims: Based on the analysis of newborns illness severity were introduced mortality prediction scores. The objective was to compare the prediction efficiency for mortality and disabilities 
(chronic lung disease, hypoxic-ischemic brain injury and prematurely retinopathy), among two methods applied on low birth weight newborns. They are the Clinical Risk Index for Babies (CRIB) and the Score for Neonatal Acute Physiology Perinatal Extension (SNAPPE-II).

Methods: Were included 60 newborns with birth weight less than $1500 \mathrm{~g}$ and/or gestational age under 32 weeks. We also compared mortality with birth weight, gestational age, APGAR and others risk factors.

Results: The average birth weight was $1083 \mathrm{~g}$ (S.D.323g) and gestational age of 29w (S.D. of 2.7). To APGAR, 31.7\% had a score lower than 7 in the 5th life minute. For the CRIB, the found average value was 6.48 (S.D 6.1) and, for the SNAPPE-II was 29.1 (S.D. 27.0). These variables had statistics significance for mortality $(p<0.001)$ with qui-square test. The gestational age lower than 30 weeks, showed a relative risk of 2.46 $(\mathrm{CI}=1.16-5.23)$, the birth weight a R.R. of $4.7(\mathrm{CI}=1.28$ 17.24), the 5th minute APGAR $<7$ a $R R$ of 2.0 (CI=1.11-3.58), the CRIB with R.R. of 2.48 (CI=1.434.27) and the SNAPPE-II with R.R. of 3.58 ( $\mathrm{CI}=1.22$ 10.45). After logistic regression, only the birth weight showed significance $(\mathrm{p}=0.01)$. Neither methods predict disabilities.

Conclusions: We can conclude that birth weight was the most efficient variable to predict mortality in these studied premature newborns. However, we indicate the CRIB for initial illness severity evaluation due to its easier use.

\section{HEALTH PROFESSIONALS' ATTITUDES TO CARING FOR EXTREMELY PREMATURE INFANTS}

\author{
J Swift $^{1}$, C Glazebrook ${ }^{1}$, N Marlow ${ }^{2}$ \\ ${ }^{I}$ School of Community Health Sciences, University of \\ Nottingham, Nottingham, UK, ${ }^{2}$ Division of Child \\ Health, University of Nottingham, Nottingham, UK
}

Background and Aims: Although the limits of survival for premature infants are continually being pushed back, infants born extremely prematurely are characterized by high rates of mortality and neurodevelopmental disability. Consequently the treatment of extremely premature infants is contentious and health professionals' views may vary, with possible implications for both clinical practice and infant outcomes. This study aims to use qualitative methods to investigate health professionals' attitudes to the care of extremely premature infants.

Methods: Semi-structured interviews were conducted with UK health professionals involved in the care of extremely premature infants. Interviews were transcribed verbatim and subjected to theme analysis.

Results: Major themes emerging from the data included: Parental Involvement in the Decision Making Process - a number of participants held strong and opposing views regarding the appropriateness and feasibility of allowing parents to be involved in care decisions; Infant Quality of Life - participants' care decisions were strongly influenced by infants' quality of life rather than endorsing life at all costs; Treatment Protocols - participants' views regarding the appropriateness of strict rules and protocols in the care of extremely premature infants varied; Depersonalization - some participants described disengaging from emotionally difficult situations; Cost of Care - several participants questioned whether the resources involved in caring for extremely premature infants could be justified.

Conclusions: Health professionals' attitudes to the care of extremely premature infants are multidimensional and often polarized. Quantitative research is needed to examine factors influencing views and to determine the impact of practitioners' attitudes on clinical decision making and infant outcomes.

\section{THE CARE OF PREMATURE INFANTS AT THE END OF THE XIX CENTURY}

\author{
A Martín ${ }^{1}, \mathrm{E} \mathrm{Ponsell}^{1}$, P Ferrer De Sant Jordi ${ }^{1}$, \\ V Ferrer $^{2}$ \\ ${ }^{I}$ Dpt. de Enfermería y Fisioterapia, UIB, Palma de \\ Mallorca, Spain, ${ }^{2}$ Dpt. de Psicología, UIB, Palma de \\ Mallorca, Spain
}

Background and Aims: The care of premature infants is a matter of a great importance and of considerable interest during the last two centuries. In 1898 premature births ranged from 15 to 30 per cent of all births in Europe and in USA. The aim of this paper is to describe the recommendations published in some scientific articles about the care of premature infants at the end of the XIX century in Europe.

Methods: The recommendations written in these three articles: Incubators and milk laboratory feeding (1897); the care and feeding of premature infants (1898) and The care of premature and feeble infants (1898) have been analyzed.

Results: As Flacker said 'the principles to be observed in the care of premature babies can be deduced from de conditions of intrauterine life'. The technological advances as the incubators or the artificial feeding were the mean purposes that the authors written in their papers. The temperature of a child born must be conserved with an incubator as the employed by Denuce (1857), Credé (1884) or Tarnier (1880). Breastfeeding was recommended as the best way to fed premature infants but when this option was impossible, laboratory milk, was chosen despite to be very expensive for some families.

Conclusions: As the papers analyzed show, the physicians were worried about the high rate of premature babies in their countries, so they tried to answer to the babies needs with the technology available at that time, such as incubators and laboratory milk.

\section{LONGITUDINAL, NORMATIVE END TIDAL CARBON MONOXIDE MEASUREMENTS IN PRETERM NEONATES}

F A Camfferman ${ }^{1}$, C A Blok ${ }^{1}$, T G Krediet ${ }^{1}$, H J Vreman ${ }^{2}$, R Marges ${ }^{1}$, D K Stevenson ${ }^{2}$, F van Bel $^{1}$

${ }^{1}$ Department of Neonatology, University Medical Center, Utrecht, The Netherlands, ${ }^{2}$ Department of Pediatrics, Division of Neonatal and Developmental 
Medicine, Stanford University School of Medicine, Stanford, CA, USA

Background and Aims: End-tidal carbon monoxide production, corrected for ambient $\mathrm{CO}$ (ETCOc), is associated with severity of respiratory distress syndrome (RDS) in premature infants and may be used in the prediction of chronic lung disease. In term infants ETCOc measurements are validated as parameter for hemolytic disease. However, normal values for ETCOc among preterm infants are not available. To obtain these, we measured ETCOc in term and preterm infants during the first week of life and compared these with ETCOc during hemolysis.

Methods: Using the $\mathrm{COCO} 2$ Puff End-Tidal Breath Analyzer (Everest Biomed Instr, Chesterfield, MO) ETCOc ( $\mathrm{ppm}$ ) was measured in healthy preterm and term infants from non-smoking mothers, at days 1, 3, 5 and 7 , together with serum bilirubin and hematocrit (Ht).

Results: 61 infants were included: $24 \mathrm{GA} \leq 32$ wks; 20 GA 32-37 wks; 13 healthy term infants and 4 with AOhemolytic disease. Mean \pm SD ETCOc values were not different between groups (Figure). ETCOc was increased during hemolysis (days 1 and 3), showing higher values than in healthy preterm and term infants $(\mathrm{p}<0.001)$.

Conclusions: ETCOc measurements are similar in healthy preterm infants and term infants during the first week of life. ETCOc is increased during hemolysis.

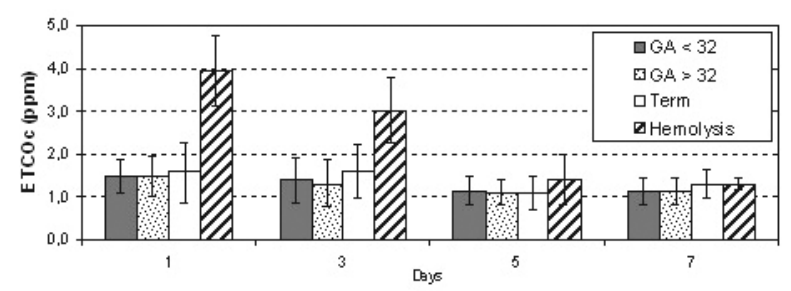

\section{MEDICATION ERRORS IN A NEONATAL INTENSIVE CARE UNIT (UCIN): THE EFFECT OF ITS REGISTRATION ON THE DRUG ERROR RATE}

\section{A Campino Villegas, I Lopez de Heredia I Goya, M C Lopez Herrera, M Garcia Franco, A Valls I Soler \\ Neonatal Intensive Care Unit, Department of Paediatrics, Hospital de Cruces, Barakaldo, Bizkaia, Spain}

Background and Aims: Medication errors occur in relation to the many steps involved in the process of drug use in neonates. To err is human, so errors should not be considered as punishable acts, but rather there causes should be analysed to prevent new incidents. We aim to report the effect on the error rate of the simple review and registration of medication use, before a specific preventive strategy was implemented.

Methods: Deviations from drug use related to legibility, dose, units, route of administration, and dosing intervals were prospectively registered in a level III regional NICU. The study was planed in three steps: a pilot phase $(\mathrm{n}=122$ drug prescriptions and their transcriptions by nurses) to know the incidence of errors; a full phase $(n=4,182$ observations) before any intervention was implemented, and a final one to measure the effect of the preventives on error rate. We report the results of the first two phases.

Results: Prescription errors were detected in $35.2 \%$ and $20.6 \%$, of the pilot and full phase, respectively. The incidence of all types of drug prescription errors was smaller in the second phase, except for route of administration. The rate of transcription errors was similar in both phases ( 21.5 vs $23.7 \%)$.

Conclusions: We observed that the presence of a person reviewing and registering the drug administration records had itself a positive impact on the overall drug administration process. This should be taken in consideration when evaluating the efficacy of specific interventions to prevent medication errors.

\section{METHODS OF MEASUREMENT OF GLUCOSURIA IN NEWBORNS}

J Bekhof, C Deiman, B Kollen, H L M van Straaten

Amalia Department of Pediatrics/Neonatology, Isala Clinics, Zwolle, The Netherlands

Background and Aims: In newborns measurement of glucosuria by means of a visually readabile teststrip (glucostick) is a frequently used test. The newborn's urine can be collected by putting a piece of cloth in the diaper. After compression of the wetted cloth urine can be evacuated with a syringe and subsequently applicated to the teststrip (dripstick method). Alternatively teststrips are frequently interpreted after pressing the strip in a wet diaper for a few seconds (diaperstick method). The aim of this study was to compare both methods in measuring glucosuria.

Methods: In 47 premature newborns 201 urinesamples ere tested both with the diaperstickmethod and the dripstickmethod. Two different manufacturers were used: Multistick (Bayer) and Combur3Test (Roche). Results of both type of strips are categorized as $0,+,++$, +++ or ++++ , in ascending degree of glucosuria. All samples were interpreted by the same investigator.

Results: The proportion of agreement between the diaperstickmethod and the dripstickmethod was 0,35 in the 100 samples tested with the Multistick and 0,64 in the 101 samples tested with the Combur3Test. The weighted Kappa using the Multistick was 0,54 (95\%-CI $0,42-0,66)$ and using the Combur3Test $0,45(95 \%$-CI $0,34-0,55)$.

Conclusions: In both the Multistick and the Combur3Test the agreement between the dripstickmethod and the diaperstick method is moderate. We speculate that the dripstickmethod is more sensitive for the measurement of glucosuria in newborns than the diapermethod.

\section{METABOLIC BONE DISEASE IN PRETERM INFANTS: COMPARISON BETWEEN QUANTITATIVE ULTRASOUND IN BONES AND DUAL X-RAY ABSORPTIOMETRY (DEXA)}

J C Fauchere ${ }^{1}$, T Karen ${ }^{1,4}$, K Huerzeler ${ }^{1}$, S Das-Kundu ${ }^{1}$, H U Bucher ${ }^{1}$, G Goerres $^{3}$, D Uebelhart ${ }^{2}$ 
${ }^{I}$ Clinic of Neonatology, University Hospital Zurich, Zurich, Switzerland, ${ }^{2}$ Clinic of Rheumatology, Osteoporosis Centre, University Hospital Zurich, Zurich, Switzerland, ${ }^{3}$ Division of Nuclear Medicine, Department of Radiology, University Hospital Zurich, Zurich, Switzerland, ${ }^{4}$ Department of Neonatology, University Children's Hospital Tuebingen, Tuebingen, Germany

Background and Aims: Fetal bone accumulation in calcium and phosphorus occurs in the last trimester of pregnancy. Thus, preterm infants, and specially those undergoing intensive care and thereby periods of non optimal nutrition are at risk of osteopenia. Measuring the speed of ultrasound (SOS) in bones is a promising, bed-side and radiation-free means of assessing bone mineralization with the potential to replace the current bone-mineral density assessment techniques which produce ionizing radiation.

Methods: SOS was measured using the Sunlight Omnisense Premier 7000® (mean of 3 cycles) in the right tibial cortex of premature infants born before 32 wks gestation at 4 weeks of age. Bone mineral content (BMC; $\mathrm{g}$ ) and bone mineral density (BMD; $\mathrm{g} / \mathrm{cm} 2$ ) were determined the same day by the DEXA ODR-1500

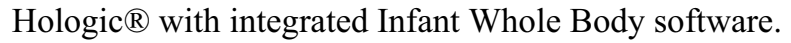

Results: A total of 60 measurements of SOS and 20 assessments of osteodensitometry (DEXA) were performed in 20 preterm infants (median GA 30 3/7 wks, BW $1175 \mathrm{~g}$ ). The intra-rater reliability of SOS measurements was 0.904 . The linear relationship between SOS and tibial BMC was $y=-0.0017 x+5.764$; $\mathrm{r} 2=0.071$, and between SOS and tibial BMD $\mathrm{y}=-0.011 \mathrm{x}$ $+0.082 ; \mathrm{r} 2=0.043$.

Conclusions: There was no close relationship between SOS and BMC or BMD as measured over the tibia. This may be due to the fact that SOS and DEXA measure different aspects of bone mineralization and structure, and therefore that they cannot be used interchangeably.

\section{MITOCHONDRIAL ENERGY GENERATING SYSTEM IN CRITICALLY ILL NEONATES}

J Zeman, T Honzik, H Hansikova, L Wenchich, M Pejcnochova, M Magner, M Zapadlo, R Plavka

Department of Paediatrics and Neonatology, Faculty of Medicine, Charles University, Prague, Czech Republic

Background and Aims: Inadequate capacity of energy production due to delayed biosynthesis and proper assembly of respiratory chain complexes in the inner mitochondrial membrane may play an important role in the neonatal morbidity, especially in very premature neonates.

Methods: The activities and amount of respiratory chain (RC) complexes and pyruvate dehydrogenase (PDH) were analyzed in isolated muscle mitochondria obtained at autopsy in 19 premature neonates using spectrophotometric and radioenzymatic methods, Western blotting and Blue-native electrophoresis. Two groups of children recommended to muscle biopsy at the age of 0.5-2 and 3-18 years served as controls.

Results: In premature neonates, the protein amount of PDH and RC complexes I, III, IV and V were lower in all analyzed samples in comparison with older children.
The activities of PDH and RC complexes I+III, II+III, III and IV were lower in premature neonates in comparison with older children. No differences were found for complex II, only the activity of complex I was higher in premature neonates than in older children. PDH activity was lower in premature neonates with hyperlactacidemia $>5.9 \mathrm{mmol} / 1$ in comparison with neonates with normal or mild increase of lactate.

Conclusions: Our study suggests, that in critically ill premature neonates the activities and amount of respiratory chain complexes and $\mathrm{PDH}$ in muscle mitochondria are lower in comparison with older children. The lower functional capacity of energy production in premature neonates is influenced by the delay in maturation of mitochondrial complexes combined with increased protein degradation during infection. This work was supported by IGA-MZ NR/8065/3.

\section{ASYMMETRIC DIMETHYLARGININE AND NEONATAL ADAPTATION}

G Vida ${ }^{1}$, E Sulyok $^{2}$, T Ertl ${ }^{1}$, J Martens-Lobenhoffer $^{3}$, S Bode-Böger ${ }^{3}$

${ }^{I}$ Department of Obstetrics and Gynecology, Faculty of Medicine, University of Pécs, Pécs, Hungary, ${ }^{2}$ Institute of Health Promotion and Family Health, Faculty of Health Sciences, University of Pécs, Pécs, Hungary ${ }^{3}$ Department of Clinical Pharmacology, University of Magdeburg, Magdeburg, Germany

Background and Aims: Recent evidences suggested that elevated plasma asymmetric dimethylarginine (ADMA) levels can be regarded as a marker of endothelial dysfunction that mediates cardiovascular morbidity. It may have a role in the cardiopulmonary adaptation of the neonate. The present study was undertaken to investigate the perinatal NO metabolism by measuring 1-arginine, ADMA, and symmetrical dimethylarginine (SDMA) in cord blood, and in peripheral plasma of the neonate.

Methods: Ten newborn infants of healthy mothers were selected for the study. The mean birth weight and gestational age of the newborn infants were $3330 \mathrm{~g}$ and 39.6 weeks (ranges $2560-4070 \mathrm{~g}$ and $39-40$ weeks), respectively. Blood samples were taken from the umbilical vein and artery, and by venepuncture on the second day of life into tubes in ice water containing EDTA. Plasma concentrations of 1-arginine, ADMA and SDMA were measured by liquid chromatography-mass spectrometry method.

Results: L-arginine concentration in venous cord blood $(73.89 \pm 4.37 \mu \mathrm{mol} / \mathrm{l}$,$) was significantly higher than that$ of umbilical artery $(63.03 \pm 4.15 \mu \mathrm{mol} / 1 ; \mathrm{p}<0.01)$. By the second postnatal day l-arginine concentration markedly fell to a value of $34.78 \pm 4.32 \mu \mathrm{mol} / 1 \quad(\mathrm{p}<0.001)$. The pattern of ADMA concentrations was similar to that of 1-arginine, its highest level was found in the umbilical vein $(1.06 \pm 0.08)$ followed by a slight decline in umbilical artery $(0.90 \pm 0.09 ; \mathrm{p}<0.03)$ and by a further fall on day 2 postnatally $(0.66 \pm 0.05 ; \mathrm{p}<0.001)$. A statistically significant veno-arterious difference was seen in cord blood SDMA levels $(\mathrm{p}<0.05)$, but their postnatal fall did not occur. 
Conclusions: Our data suggest that fetoplacental ADMA has an important role in the circulatory adaptation of the neonate.

\section{ASSESSMENT OF PRETERM AT TERM- ADJUSTED AGE AND FULL-TERM INFANTS BONE STATUS WITH QUANTITATIVE ULTRASOUND}

\section{P Roggero, P Piemontese, M L Giannì, A Orsi, O Amato, V Puricelli, S Montella, F Mosca \\ NICU, Clinica Mangiagalli (IRCCS), University of Milan, Milan, Italy}

Background and Aims: Preterm infants (PT) have an increased risk of low bone mass. Ultrasound velocity [speed of sound (SOS)] has been proposed as a non invasive, non ionizing and portable method of evaluation of bone status. Several observations in PT at birth show a significantly lower SOS than in term infants (FT). To compare SOS of PT assessed at term adjusted age to SOS of FT assessed within 72 hours of life.

Methods: 29 PT (13 males; mean gestational age (GA) 31.2 wk; mean birth weight (BW) $1361 \mathrm{~g} \pm 329$; and 82 FT infants (46 males; mean BW $3218 \mathrm{~g} \pm 418$ ) at birth. Body length (BL) was measured to the nearest $0,1 \mathrm{~cm}$ on a Harpenden neonatometer (Holtain Ltd, UK) and knee-heel length (TL) was measured using a heel-knee caliper (Chasmors Ltd, UK). Bone status was evaluated in the midpoint of the right tibia by quantitative ultrasound (CS probe), Sunlight Omnisense (Sunlight Medical Ltd, Israel) and expressed as the mean of three SOS values assessed by the same operator.

Results: Mean SOS \pm SD in PT was significantly lower than SOS in FT $(2894 \pm 214$ vs $3042 \pm 138$; $\mathrm{p}<0.001)$. GA $(r=0.50: p<0.05)$ was significantly correlated to $\mathrm{SOS}$ in PT. No difference was found related to BW, BL, TL, gender, and being SGA.

Conclusions: Bone mineralization in PT is significantly lower than in FT. Our preliminary data suggest that ultrasound velocity could be a non invasive procedure, suitable for longitudinal studies in order to monitor bone status in patients at high risk of bone breakability.

\section{CONTINUOUS INSULIN INFUSION IN EXTREMELY-LOW-BIRTH-WEIGHT NEWBORNS UNDER 26 WEEKS OF GESTATIONAL AGE}

M Berces, E Polonkai, Z Horvath, N Katona, T Kovacs, B Kovacs-Paszthy, J Kovacs, G Balla

Department of Obstetrics, Division of Neonatology, University of Debrecen, Debrecen, Hungary

Background and Aims: To provide normal glucose homeostasis for extremely immature newborns at the early postnatal age represents a significant therapeutic goal for the neonatal intensive care team. Physiologic blood glucose levels and metabolism are critical factors for disease-free survival. We were seeking early clinical and laboratory signs which could detect or predict disturbances of glucose metabolism and indication for insulin treatment.
Methods: In a retrospective analysis we studied 169 extremely immature newborns with post menstrual gestational age less than 26 weeks. Continuous insulin infusion was initiated for those who developed hyperglycemia, hyperkalemia. Each day of the first two weeks of life insulin-, glucose intake were recorded besides the parameters of ventilation therapy, blood gas, ion values, glucose-, lactate concentrations, temperature support, and other vital signs, treatments.

Results: Insulin treatment was initiated for 64 newborns of the 169 extremely immature patients. The survival rate for those patients who do not required insulin was $68 \%$, and for those who needed continuous insulin infusion was $61 \%$. The insulin treatment group was divided into two arms, the first who survived and the second who does not survived the neonatal age. Our results showed that besides glucose and potassium levels, the metabolic acidosis, high blood lactate, low carbon dioxide (independent from ventilatory therapy), low body temperature, especially in the not surviving group are significant risk factors for insulin treatment.

Conclusions: Early insulin intervention is critical for controlling high glucose and potassium levels, and in the decision making process the above introduced risk factors might have benefits.

\section{NEONATAL SCREENING FOR CONGENITAL HYPOTHYREOIDISM (NSCH), PHENYLKETONURIA (NSPKU) AND CONGENITAL ADRENAL HYPERPLASIA (NSCAH): FOUR YEAR DATA}
Z Gucev $^{1}$, A Jancevska ${ }^{1}$, G Jovanov ${ }^{2}$, A Sisko ${ }^{2}$, A Trenevska ${ }^{2}$, L Tasevska-Rmus $^{1}$, B Taseva $^{1}$, V Vankovska ${ }^{1}$
${ }^{I}$ Department of Endocrinology, Pediatric Clinic, Medical Faculty Skopje, Skopje, Macedonia
${ }^{2}$ Health Center Skopje, Skopje, Macedonia

Background and Aims: Importance of performing neonatal screening programs for congenital hypothyreoidism, phenylketonuria and congenital adrenal hyperplasia for Macedonian children.

Methods: NSCH was introduced in the Republic of Macedonia in April 2002. 51200 newborns from the Republic (exceptions Skopje and Bitola with children screened in the neighboring pediatric laboratory) were screened. For 10 months NSPKU and NSCAH also functioned and 28600 children were screened for PKU, 9258 for CAH. A new design of the filter paper and instructions were applied. Education was organized in the delivery wards throughout Macedonia. A central laboratory was set out and links with a reference laboratory were established. Blood was collected (days 2-5 after birth) on filter paper and enzymatically assayed for TSH, phenylalanine and 17 hydroxy progesterone.

Results: Seven children were screened positive with high values of TSH. Venous samples confirmed the diagnosis (low T4 and high TSH values). The thyroid was hypoplasic/ectopic on ultrasound. In five cases treatment begun in the first two weeks of life. Two unfortunate exceptions were due to a temporary lack of reagents or change in a home address. 7 detected hypothyroid children out of 51200 screened suggest an 
incidence of 1:7314 newborns. No case of PKU or CAH was detected. So far no false-positive or false-negative results were noticed. No recalls were realized for PKU or CAH. However, 56 children $(9.14 \%)$ with border line screening results for TSH were recalled for additional tests which proved to be within normal range.

Conclusions: The data suggest a need for institualisation of the screening programs.

\section{EXCESS OF IODINE DURING THE NEONATAL PERIOD: BE CAREFUL, EVEN VERY LITTLE MAY BE TOO MUCH}

\section{S Ares ${ }^{1}$, M Saenz De Pipaon ${ }^{1}$, G Morreale De Escobar ${ }^{2}$, J Quero ${ }^{1}$

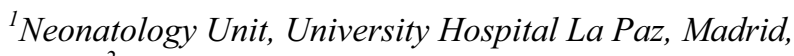 Spain, ${ }^{2}$ Department of Molecular Endocrinology, \\ Instituto de Investigaciones Biomedicas Alberto Sols, Madrid, Spain}

Background and Aims: There are several causes for alterations of thyroid function. Quite prominent are iodine deficiency during the perinatal period, and exposure to an iodine excess, caused by iodinecontaining antiseptics $(10,000 \mu \mathrm{g}$ of iodine $/ \mathrm{mL})$ and radiologic contrast media (250- $370 \mathrm{mg}$ of iodine $/ \mathrm{mL}$ ). The aim is to bring to the attention, that even a minor iodine-overload may impair thyroid function during a period of development when thyroid hormones are very important for the brain.

Methods: Thyroid function and iodine excretion were analysed at different postnatal ages.

Results: No dysfunction was found in the Screening Program for congenital hypothyroidism before excess iodine exposure, and with urinary iodine excretions below $50 \mu \mathrm{g} / \mathrm{L}$. After exposure, iodine in serum was $>100 \mu \mathrm{g} / \mathrm{dL}$ and in urine $200-6000 \mu \mathrm{g} / \mathrm{L}$. Transient hypothyroidism (Total serum thyroxine $<4 \mathrm{ng} / \mathrm{dL}$ and thyroid stimulating hormone levels $>10 \mathrm{mU} / \mathrm{L}$ ) was present in 4 patients after skin disinfection during surgical procedures, 3 patients after gastrografin, 2 patient after angiography; another 2 patients presented transient hyperthyrotropinaemia. Treatment with thyroxine was started in all patients.

Conclusions: The precocious diagnosis and treatment of the alterations of thyroid function associated with iodine excess, could have beneficial effects in the prevention of developmental abnormalities. We suggest a protocol to evaluate thyroid function when iodine compounds are used, either topically, as enema, or intravenously, because of the blocking effect of iodine excess in the thyroid gland. The risk of transient hypothyroidism may be avoided by the use of iodine-free compounds. Chlorhexidine should be used for skin disinfection and surgery.

\section{MANAGEMENT OF NEONATAL HYPERGLYCAEMIA: GLUCOSE RESTRICTION IS STILL IN VOGUE!}

A L Mukherjee ${ }^{1}$, V Kairamkonda ${ }^{1}$, M Khashu $^{1}$, A Grover ${ }^{2}$
${ }^{1}$ Department of Neonatology, Leicester Royal Infirmary, Leicester, UK, ${ }^{2}$ Department of Paediatrics, Northampton General Hospital, Northampton, UK

Background and Aims: Although hyperglycaemia is a common problem in VLBW infants, there is a lack of consensus with regard to its management. We investigated current practice regarding management of neonatal hyperglycaemia across the UK. Our aim was to ascertain the working definition and management strategy of neonatal hyperglycaemia in major neonatal units across the UK.

Methods: Survey questionnaire was emailed to consultants working in units with $>6$ NICU cots. Results: $50 \%$ of 50 units have responded to date. $84 \%$ respondents considered that incidence of hyperglycemia is maximum in infants $<1 \mathrm{~kg}$. $32 \%$ respondents use blood glucose alone [median $>10$ (range $7-15 \mathrm{mmol} / \mathrm{L})$ ], $44 \%$ respondents use glucose $>10 \mathrm{mmol} / \mathrm{L}$ and glycosuria, and $24 \%$ use glucose $>10 \mathrm{mmol} / \mathrm{L}$, glycosuria and osmotic diuresis as their working definition of hyperglycemia. Except one unit, all others use a combination of decreased glucose delivery and insulin for management of hyperglycemia.

Conclusions: There is a wide variation in practice regarding management of neonatal hyperglycaemia. The median glucose delivery rate conforms to optimal delivery of $6-9 \mathrm{mg} / \mathrm{kg} / \mathrm{min}$ and suggests that a high glucose delivery is not the predominant factor leading to neonatal hyperglycaemia. The high proportion of units practising glucose restriction warrants a review of optimal nutritional strategy in this already malnourished fragile population.

\begin{tabular}{|c|c|c|c|c|c|c|c|c|c|c|}
\hline Day of TPN & 1 & 2 & 3 & 4 & 5 & 6 & 7 & 8 & 9 & 10 \\
\hline $\begin{array}{l}\text { Median } \\
\text { glucose }\end{array}$ & 4.1 & 6.2 & 7.9 & 9.5 & 9.7 & 9.7 & 9.7 & 9.7 & 9.7 & 9.8 \\
\hline $\begin{array}{l}\text { infusion } \\
\text { rate }\end{array}$ & $\begin{array}{l}3.4 \\
\text { to }\end{array}$ & $\begin{array}{l}4.6 \\
\text { to }\end{array}$ & $\begin{array}{l}6.2 \\
\text { to }\end{array}$ & $\begin{array}{l}6.7 \\
\text { to }\end{array}$ & $\begin{array}{l}7.2 \\
\text { to }\end{array}$ & $\begin{array}{l}7.5 \\
\text { to }\end{array}$ & $\begin{array}{l}7.5 \\
\text { to }\end{array}$ & $\begin{array}{l}7.5 \\
\text { to }\end{array}$ & $\begin{array}{l}7.5 \\
\text { to }\end{array}$ & $\begin{array}{l}7.5 \\
\text { to }\end{array}$ \\
\hline $\begin{array}{l}(\mathrm{mg} / \mathrm{kg} / \mathrm{min}) \\
\text { (range) }\end{array}$ & 8.3 & 9.7 & 11 & 12.5 & 12.5 & 14 & 14 & 14 & 14 & 14 \\
\hline
\end{tabular}

\section{EXTRAORDINARILY RAPID DESCENT OF NON PALPABLE TESTES AT BIRTH - A PROSPECTIVE STUDY ON 5250 LIVE MALE NEWBORNS}

M Barak $^{1,2}$, F Mimouni $^{1,2}$, I Berger $^{1,2}$, S Dollberg $^{1,2}$,
D Mandel $^{1,2}$

${ }^{1}$ Department of Neonatology, Sourasky Tel Aviv Medical Center, Lis Maternity Hospital, Tel Aviv, Israel

${ }^{2}$ Sackler Faculty of Medicine, Tel Aviv, Israel

Background and Aims: Cryptorchidism occurs in 2$5 \%$ of full term boys at birth. Because an undescended testis may represent an atrophic or vanishing testis, it has been suggested to use ultrasonography or even MRI to verify its presence. The objective of this study was to evaluate the rate of testicular descent in full term infants with at least 1 non palpable testes at birth.

Methods: 5250 male infants were checked during a period of 14 months. Infants with non palpable unilateral or bilateral testis at the first examination, were reexamined daily until their discharge (day 2-3 of life) and weekly thereafter until the age of 2 weeks. Premature infants delivered before 37 weeks of 
gestation or those with additional genital anomalies were excluded form the study.

Results: Forty one male infants were diagnosed as having at least one non palpable testicle. In 8 infants both testes were non palpable. 5 out of 41 diagnosed infants ( 5 out of 49 testes) were lost to follow up. Of the 44 remaining testes, 26 (59\%) became palpable within 3 days. Thirteen additional testes were palpable 4-14 days later, 39 out of $44(88 \%)$ were palpated within 2 weeks. By 2 weeks of age all non-palpable testes that became palpable were located in the inguinal canal.

Conclusions: A majority ( $88 \%$ ) of non-palpable testes at birth descend into the inguinal canal within 2 weeks. We speculate that reexamination of these infants at the age of 2 weeks will preclude unnecessary imaging studies in most of them.

\section{DRIED WHOLE BLOOD SPOTS SPECTROPHOTOMETRY IN NEWBORNS WITH CONGENITAL HYPOTHYROIDISM}

C De Felice ${ }^{1}$, M Musarò ${ }^{2}$, L Peruzzi ${ }^{2}$, A Barducci $^{3,4}$, P Corrocher $^{5}$, G Latini ${ }^{6,7}, \mathrm{R}$ Casini $^{8}$

${ }^{1}$ Neonatal Intensive Care Unit, Azienda Ospedaliera Universitaria Senese, Siena, Italy, ${ }^{2}$ Laboratory of Pediatric Endocrinology, Department of Pediatrics, Obstetrics and Reproductive Medicine, University of Siena, Siena, Italy, ${ }^{3}$ Institute of Applied Physics "Nello Carrara", National Research Council of Italy, Florence, Italy, ${ }^{4}$ Department of Information Engineering, University of Siena, Siena, Italy, ${ }^{5}$ Intex System, Prato, Italy, ${ }^{6}$ Division of Neonatology, Perrino Hospital, Brindisi, Italy, ${ }^{7}$ Clinical Physiology Institute, National Research Council of Italy (IFC-CNR), Lecce Section, Italy, ${ }^{8}$ Te\&Co, Prato, Italy

Background and Aims: Early diagnosis and treatment of congenital hypothyroidism $(\mathrm{CH})(\sim$ 1/3000-1/4000 live births) prevent severe mental retardation in affected patients. Only $\sim 5 \%$ of the $\mathrm{CH}$ infants are detected by clinical criteria before the results of the T4/TSH population screening. As anemia is a frequent finding in $\mathrm{CH}$ and depends on the degree of hypothyroidism, we tested the hypothesis of a decreased reflectance in the red section of the visible spectrum on blood spots from $\mathrm{CH}$ newborns.

Methods: Spectrophotometry on filter paper-adsorbed dried blood spots was performed for $16 \mathrm{CH}$ newborns and 322 matched control newborns. Coordinates in the standard CIE-L*a*b* and $\mathrm{L}^{*} \mathrm{C} * \mathrm{H}^{*}$ space colors and spectral reflectance curves were obtained (portable spectrophotometer; 400-700 $\mathrm{nm}$ wavelengths range; standard D65 illuminant) and computed by a color software package (Colormedica, IntexSystem). Accuracy of spectrophotometry in identifying $\mathrm{CH}$ samples was evaluated by Receiver Operating Characteristic (ROC) curve analysis.

Results: $\mathrm{CH}$ blood showed lower $\mathrm{a}^{*}, \mathrm{~b}^{*}$, and $\mathrm{C}^{*}$ values, and higher $\mathrm{H}^{*}(\mathrm{p}<0.0001)$, with a significantly lower reflectance in the $600-700 \mathrm{~nm}$ wavelengths range, as compared to control samples $(\mathrm{p}<0.0001)$, whereas no significant differences were observed for the adsorbing paper samples. Values of $\mathrm{H}^{*}>45.1$ identified $\mathrm{CH}$ with 100\% sensitivity, $87.3 \%$ specificity (AUC: 0.957 , 95\%
C.I.: $0.930-0.976, \quad \mathrm{p}<0.0001)$, while $\mathrm{a}^{*} \leq 12.87$ discriminated $\mathrm{CH}$ specimens from controls with $100 \%$ sensitivity and $87.3 \%$ specificity (AUC:0.939, 95\% C.I.: 0.908-0.962, $\mathrm{p}<0.0001)$.

Conclusions: These findings could help $\mathrm{CH}$ screening centres in selecting samples with highest priority, hence possibly further anticipating the time at diagnosis and hormonal replacement treatment in affected newborns.

\section{INFLUENCE OF EARLY HYPERGLYCEMIA TO CLINICAL COURSE AND OUTCOME IN PRETERM INFANTS}

\author{
C A Lee, Y S Wee, E K Yoo, K H Lee
}

Department of Pediatrics, Pochon CHA University, Bundang CHA General Hospital, Sungnam, Kyunggi, South Korea

Background and Aims: We can find the hyperglycemia at the condition of acute illness easily. There is a report that hyperglycemia cause the increase of morbidity and mortality of many diseases. The purpose of this study is to know how can the hyperglycemia influence to the preterm infants 48 hours after be born.

Methods: A retrospective chart review for 77 premature infants who were admitted to Pochun CHA University neonatal intensive care unit between January 2002 and March 2005 was done. They are all less than gestational age 30 weeks. Infants were divided into two groups depending upon their glucose level. One is the hyperglycemia infants group whose glucose level is at least one time more than $150 \mathrm{mg} / \mathrm{dL}(\mathrm{n}=38)$, the other is the non-hyperglycemia infants group whose glucose level is less than $150 \mathrm{mg} / \mathrm{dL}(\mathrm{n}=39)$.

Results: The average of the glucose level in the hyperglycemia infants group is $222 \pm 65 \mathrm{mg} / \mathrm{dL}$, and the non-hyperglycemia infants group is $112 \pm 22 \mathrm{mg} / \mathrm{dL}$. A death rate of the hyperglycemia infants group is $42.1 \%$ but the non-hyperglycemia infants group is $12.8 \%$. The death rate of the hyperglycemia infants group is higher than non-hyperglycemia infants group definitely $\mathrm{P}<0.01$ ). There are no differences between two groups of the rest which are initial clinical characteristics, clinical features, clinical courses and complications. Conclusions: We can bring to a conclusion that the early hyperglycemia influence to the death rate of the less than 30 weeks infants, and more positive treatment of the early hyperglycemia will be help to the improvement of the clinical progress.

\section{ORGANOCHLORINE COMPOUNDS AND DOCOSAHEXAENOIC ACID DURING PRENATAL EXPOSURE}

C Campoy ${ }^{1}$, I Machado ${ }^{1}$, M Jimenez ${ }^{2}$, A Abril ${ }^{1}$, M F Olea-Serrano ${ }^{3}$, M Parrilla ${ }^{1}, \mathrm{~N} \mathrm{Olea}^{2}$

${ }^{1}$ Department of Paediatrics, School of Medicine, University of Granada, Granada, Spain, ${ }^{2}$ Department of Radiology and Nuclear Medicine, School of Medicine, University of Granada, Granada, Spain, ${ }^{3}$ Department of Nutrition and Bromatology, University of Granada, Granada, Spain 
Background and Aims: The present study examines the relationship between the prenatal exposure to Organochlorine Compounds (OCs) and their bioaccumulate in fatty tissues with the LC-PUFA n3 plasma concentrations.

Methods: 72 Spanish mother-baby pairs were analysed. Plasma phospholipids $n-3$ and n-6 PUFAs (in $\mathrm{mg} / \mathrm{dl}$ ) and OCs (in $\mathrm{ng} / \mathrm{ml}$ ) were obtained in mother's and neonates' plasma using Gas Chromatography-Mass Spectrometry. The study has the corresponding ethical approval. ANOVA, General Lineal Model and correlations were done using SPSS Version 12.0. $*: \mathrm{p}<0.05$.

Results: OCs were found in different concentrations in all the samples studied \{Lindane: $88 \%$, Vinclozolina: 96\%, DDE: 99\%, o,pDDT: 96.5\%, Endosulfan sulphate: 97\%..\}. Plasma OCs concentrations were closely correlated between mothers and their neonates. Positive correlations between linoleic acid, acid araquidonic (AA), as well as eicosapentaenoic acid (EPA) with some of the OCs analysed were found. Some of the OCs analysed resulted significantly lower in the mothers who had higher levels of plasma phospholipids DHA at delivery (underaverage of DHA vs upperaverage of DHA: Endosulfan-II: $\quad 0.43 \pm 0.25$ vs $0.20 \pm 0.03 *$; Endosulfan-eter: $3.63 \pm 2.91$ vs $0.22 \pm 0.05 *$ ). However, most of the OCs resulted significantly higher depending on plasma phospholipids AA concentrations at delivery \{underaverage of AA vs upperaverage of AA: Endosulfan-I: $0.08 \pm 0.015$ vs $0.18 \pm 0.04^{*}$; Dieldrin: $0.22 \pm 0.05$ vs $0.66 \pm 0.13 * ; 0.54 \pm 0.05$ vs $\left.0.91 \pm 0.16^{*}\right\}$.

Conclusions: Mothers with high DHA levels and low AA levels in plasma had less concentrations of OCs and consecuently less amount of these contaminants achieved the foetus during pregnancy. This study is partially financed by INMA Spanish Network of Excellence. Ref. $n^{\circ}$ G03/176. 2003-2006.

\section{THE EFFECT OF RH-DNASE ON MECONIUM VISCOSITY}

\author{
W J M van Heel ${ }^{1}$, M J Jansen ${ }^{2}$, A J Sprij ${ }^{2}$, \\ A J de Beaufort ${ }^{2}$ \\ ${ }^{1}$ Department of Pediatrics, Erasmus MC-Sophia \\ Children's Hospital, Rotterdam, The Netherlands \\ ${ }^{2}$ Neonatal Unit, Juliana Childrens Hospital, The Hague, \\ The Netherlands
}

Background and Aims: Around 5\% of infants born through meconium stained amniotic fluid develop meconium aspiration syndrome (MAS). Meconium, a highly viscous substance, causes small airway obstruction leading to airtrapping and atelectasis, in addition to effects of local inflammation. Meconium contains gastrointestinal secretions, cellular debris and proinflammatory factors. DNA could contribute to the viscosity of meconium, analogous to the situation in sputum in cystic fibrosis patients. RhDNase treatment of these patients induces mucolysis and improves airway patency. Meconiumlysis based on DNA degradation may create new therapeutic approaches in newborns with MAS. We hypothesized that rhDNase decreases meconium viscosity.
Methods: Sterile meconium samples were incubated with rhDNase or PBS as control. Final concentrations of substrate varied from 250 to 1000 microgram per gram meconium. Viscosity was measured with the CAP 2000 viscometer. For each sample, the arithmetic mean of three measurements was calculated.

Results: Meconium viscosity (in mPa.s) decreased significantly (figure 1) with rhDNase and control ( $p<0.000 ; 95 \%$ CL: $14.8 \%-98.5 \%$ ). There was no significant decrease in viscosity between meconium treated with $250 \mu \mathrm{g}$ and $1000 \mu \mathrm{g}$ rhDNAse or PBS $(\mathrm{p}=0.106$; 95\%CL: $-4.4 \%-12.1 \%)$.

Conclusions: In vitro, both rhDNase and PBS decrease meconium viscosity significantly, an effect attributable to dilution as no difference was found between rhDNAse and PBS. Possibly, the rhDNAse is inactivated by meconium or the DNA content in meconium is too low. Moreover, other factors than DNA could be primarily responsible for the visco-elastic properties of meconium.

Figure 1

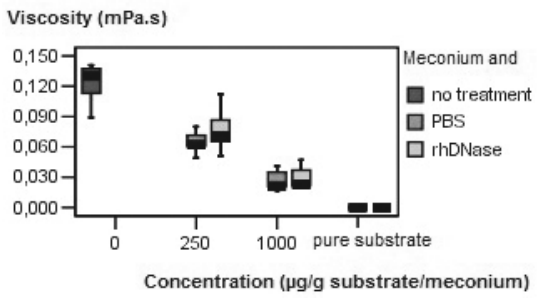

\section{GLUTATHIONE FRACTIONAL SYNTHESIS RATES IN PRETERM INFANTS}

F W J te Braake ${ }^{1}, \mathrm{H}_{\text {Schierbeek }}{ }^{2}, \mathrm{~K}$ de Groof $^{1}$, A Vermes ${ }^{3}$, J B van Goudoever ${ }^{1}$

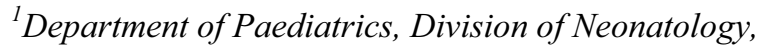
Erasmus MC - Sophia Children's Hospital, Rotterdam, The Netherlands, ${ }^{2}$ Department of Paediatrics, Mass Spectrometry Laboratory, Erasmus MC - Sophia Children's Hospital, Rotterdam, The Netherlands ${ }^{3}$ Hospital Pharmacy, Erasmus MC, Rotterdam, The Netherlands

Background and Aims: Oxidative stress is associated with severe complications of prematurity, such as bronchopulmonary dysplasia, periventricular leukomalacia, and necrotising enterocolitis. Glutathione is the major intracellular antioxidant with a mean fractional synthesis rate (FSR) of $65 \%$ in healthy adults [Lyons, 2000]. The FSR reflects the fraction that is renewed per unit of time. Our aim was to determine the FSR of glutathione in preterm infants, receiving only glucose, on the second postnatal day.

Methods: Preterm infants with a birth weight $<1000$ gram were given a primed continuous infusion of [1-13C]glycine. Blood was sampled after 4, 5, and 6 hours. $[1-13 \mathrm{C}]$ glycine incorporated in glutathione was measured with liquid chromatography - istope ratio mass spectrometry (IRMS). As a precursor for glutathione synthesis, intracellular free [1-13C]glycine enrichment was measured with gas chromatography IRMS.

Results: Glutathione FSR of eight preterm infants (birth weight $807 \pm 130 \mathrm{~g}$, gestational age $271 / 7 \pm 15 / 7$ weeks), receiving only glucose $(6.0 \mathrm{mg} /(\mathrm{kg} \times \mathrm{min}))$, were 
measured on postnatal day 2. Glycine enrichment reached a plateau within 4 hours after start of administration (4.55 \pm 0.99 mole percent excess). Glutathione FSR was found to be $44 \pm 5 \%$ /day.

Conclusions: Preterm infants, receiving glucose only, have a low FSR of glutathione as compared to healthy adults.

\section{RISK FACTORS FOR GROWTH FAILURE IN VERY LOW BIRTH WEIGHT INFANTS}

\author{
R C Silveira, M G Oliveira, R S Procianoy
}

Department of Pediatrics, Newborn Section, Universidade Federal do Rio Grande do Sul, Hospital de Clínicas de Porto Alegre, Porto Alegre, RS, Brazil

Background and Aims: Some VLBW infants present growth retardation in infancy. Aim: Evaluate risk factors for growth failure at 1 year corrected age of newborns with birth weight $\leq 1500$ grams.

Methods: A cohort of newborns with birth weight $\leq 1500$ grams admitted between $11 / 2003$ to $12 / 2004$ was followed up to 12 months corrected age. Weight, length and head circumference were plotted on NCHS curves. The group was divided in relation to $\mathrm{Z}$ score: $<2 \mathrm{SD}$ and $\geq 2$ SD. Birth weight, gestational age, adequacy to gestational age, length of hospital stay, maternal education, hospital readmission were compared between both groups. Birth weight $\leq 750$ grams, presence of PIVH and/or PVL, clinical sepsis and maternal education $\leq 9$ years were also analyzed.

Results: 116 patients were admitted, 32 died, 14 never returned to follow up clinic, 5 died during the follow and 11 were lost. 54 newborns were included in the study. Head circumference, length and weight were $<2$ $\mathrm{SD}$ in 6, 7 and 9 newborns respectively. No studied risk factor was associated to low head circumference or length. Low gestational age was associated to weight $<2 \mathrm{SD}(\mathrm{p}=0.024)$. There was a significant statistical association between one year low weight and birth weight $\leq 750$ grams, presence of PIVH and/or PVL, clinical sepsis and maternal education $\leq 9$ years $(p=0.037)$.

Conclusions: Birth weight $\leq 750$ grams, presence of PIVH and/or PVL, clinical sepsis and maternal education $\leq 9$ years are important risk factors for growth failure in VLBW infants.

\section{EFFECTS OF EARLY ENTERAL NUTRITION ON VERY LOW BIRTHWEIGHT (VLBW) AND EXTREMELY LOW BIRTHWEIGHT (ELBW) INFANTS}

F M Tandoi, C Negri, G Calciolari, L Leva, P Battaini, P Rancan, M Agosti

\section{U.O. Nido, Neonatologia e Terapia Intensiva Neonatale/Ospedale 'Filippo del Ponte', Varese, Italy}

Background and Aims: Advantages of early enteral nutrition on preterm infants are widely described in literature. In the year 2000, in our unit, we started to introduced nutritional guidelines, to promote enteral nutrition in VLBW and ELBW. Aim of the study is to verify the effects of nutritional changes on several clinical indicators, confronting 1998-99 and 2003-04 periods.

Methods: All ELBW (group A) and VLBW (group B) infants consecutively admitted to our NICU in the twoyears period of 1998-99 - newborn under older nutritional guidelines - and 2003-04 - implemented guidelines - were included in the study. Significant changes of policies regarded introduction of human milk bank, schedule for increasing enteral nutrition and management of feeding intolerance. A comparison between the two year-periods was performed inside both groups A and B. Between two-year periods, as clinical indicators, we analyzed: Days under parenteral nutrition, (total and partial), regaining of birthweight, type of feeding at discharge, growth patterns during hospitalization (average growth) and at discharge (weight, length and head circumference), days of hospitalization, incidence of surgical necrotizing enterocolitis (NEC). For statistical purposes we used the c-square test, Student's t-test and Mann-Whitney test.

Results: Most relevant differences regarded: Days of hospitalization, gestational age at discharge, days of parenteral nutrition, average growth during hospitalization.

Conclusions: Our results confirm literature about advantages of early enteral nutrition on VLBW and ELBW. In daily practice positive effects was evident even for nursing management, money and resource saving, "care" practices.

\section{SECONDARY COPPER DEFICIENCY AS A DIFFERENTIAL DIAGNOSIS OF SEVERE OSTEOPENIA IN A VLBW INFANT WITH MULTIPLE PATHOLOGICAL FRACTURES}

\author{
B Wiebe ${ }^{1}$, C H Fremerey ${ }^{1}$, U Pohlmann ${ }^{1}$, M Ehlen ${ }^{1}$, \\ P Bartmann $^{2}$ \\ ${ }^{1}$ Department of Neonatology and Paediatric Intensive \\ Care, Asklepios Klinik St. Augustin, St. Augustin, \\ Germany, ${ }^{2}$ Department of Neonatology, University of \\ Bonn, Bonn, Germany
}

Background and Aims: Copper, through its role as a cofactor for lysyl oxidase is essential for cross-links in collagen and elastin. Studies in copper deficient animals demonstrated that impaired mechanical strength was related to defects in the collagen component of the bone thus facilitating pathological fracturing of the bone.

Methods: We report on a female preterm infant of 25th week of gestation with multiple spontaneous fractures most probably due to alimentary copper deficiency.

Results: In the tenth week of life a spontaneous fracture of the femur developed, shortly afterwards multiple fractures of both upper and lower limbs were observed. After exclusion of vitamin D deficiency as well as close observation of calcium/phosphate metabolism low copper levels $(5.6 \mu \mathrm{mol} / 1$ normal range $5.0-11.6 \mu \mathrm{mol} / \mathrm{l})$ and ceruloplasmin levels $(0.084 \mathrm{~g} / 1$, normal range 0.15 $0.56 \mathrm{~g} / \mathrm{l})$ were detectable. Radiological findings showed no evidence of osteogenesis imperfecta but signs compatible to copper deficiency. At this time the infant was fed with a copper depleted formula because of severe cholestasis. After augmented substitution of 
copper, no more fractures occurred and bone changes regressed.

Conclusions: Apart from a central position in the collagen metabolism copper is required for the function of at least 30 more proteins including superoxide dismutase, cytochrome $\mathrm{C}$ oxidase, tyrosinase and dopamine-beta-hydroxylase. As sufficient copper stores are established only in the last period of gestation very premature infants are at risk for copper deficiency leading to impaired mechanical strength of the developing bones. In severe osteopenia of prematurity copper deficiency should be considered in the differential diagnosis.

\section{PERINATAL MORBIDITY OF FULLTERM NEWBORNS WITH INTRAUTERINE GROWTH RESTRICTION}

B Mihajlovic-Dimovska, M Kalajdzieva-Zip, V Delovska-Stojkova, J Guleva, T Pensovska

Department of Neonatology, Special Hospital of Gynaecology and Obstetrics, Skopje, Macedonia

Background and Aims: Newborns with intrauterine growth restriction, because of placental insufitiention and chronic hypoxia, have high risk for asphyxia and other pathological conditions. Aim of the study is to establish perinatal morbidity of full term IUGR babies, by analysis of frequency of perinatal asphyxia, meconium aspiration syndroma (MAS) and hypooxicishemic encephalopathy (HIE).

Methods: 3190 liveborn have been analysed in the study, born in 2004, in SHGO-Cair, Skopje. Full term IUGR babies are $282(8,8 \%)$, and full term babies with normal fetal growth are $2764(77,2 \%)$. Computer data and neonatal histories data have been analysed.

Results: Perinatal morbidity has been analysed up to appearance of asphyxia, MAS and HIE. Full term IUGR babies have higher frequency of these conditions in contrasting to normal growth babies: 1 . Asphyxia at 7 $(2,48 \%)$ babies vs. $36(1,3 \%) ; 2$. MAS at $2(0,7 \%)$ babies vs. $10(0,36 \%)$; 3 . HIE at $2(0,70 \%)$ vs. 8 $(0,28 \%)$.

Conclusions: Full term IUGR babies have higher frequency of pathological conditions like asphyxia, MAS and HIE in contrasting to full term babies with normal fetal growth. To prevent these conditions, there is need for introducing an adequate antenatal and intrapartal management, by: antenatal screening for fetal growth restriction, early detection of fetal distress, pregnancy interruption in the cases where is necessary and adequate neonatal resuscitation.

\section{VACCINATION AGAINST HEPATITIS A DURING AN OUTBREAK}

R Martins ${ }^{1}$, P Gregorio ${ }^{2}$, A Tavares ${ }^{2}$, H Cargaleiro ${ }^{2}$, A Verde $^{2}$, C Portilheiro ${ }^{2}$, I Simões ${ }^{2}, \mathrm{C} \mathrm{Amaral}^{2}$, P Roque $^{2}$, P Correia ${ }^{1}$, M J Brito ${ }^{1}$

${ }^{1}$ Department of Paediatrics, Hospital Fernando Fonseca, Amadora, Portugal, ${ }^{2}$ Public Health, Amadora, Portugal
Background and Aims: The hepatitis A vaccine is available in Portugal, but not worldwide. The prevalence of this infection is decreasing in developed countries but outbreaks can occur, usually among socio-economic deprived groups like gipsy communities. Objectives: To evaluate the development of a hepatitis A outbreak and the preventive measures taken to its control.

Methods: The prevention procedures carried out between the identification of the index case and the end of the outbreak were analysed.

Results: Hepatitis A virus infection (HAV) was identified in 7 gipsy children aged between 3 and 11 years-old, between October and December 2005. Some of these children were members of the same family and others attended the same school in a suburban neighbourhood of Lisbon. After the alert launched by the hospital, the local health authorities started a community intervention with the involvement of Town Hall, local schools and a non-governmental agency. In January 2006 a vaccination programme was initiated in the gipsy community and in the local school. The programme occurred in 3 stages because 1 new case was found after the first stage and other case after the second. In the local school, HAV vaccines were given to $71(100 \%)$ children and $14(78 \%)$ employees. In the community 197 (71\%) children were vaccinated. The global coverage was $79 \%$. The outbreak was successfully controlled.

Conclusions: The notification of some infectious diseases like HAV infection is obligatory in Portugal. This is very important in public health because it can allow fast and effective measures to outbreak control.

\section{ROTAVIRUS ACUTE GASTROENTERITIS: CLINICAL BURDEN AND PREDICTORS OF SEVERITY IN A PEDIATRIC EMERGENCY DEPARTMENT}

S Callegaro, G Rossi, B Andreola, M Perin, N Mainini, L Da Dalt

Department of Pediatrics, University of Padova, Padova, Italy

Background and Aims: Rotavirus gastroenteritis still causes clinical and social burden in all developed countries, but Italian epidemiological data are old and inaccurate. Our aims therefore were to evaluate the impact of acute gastroenteritis in children $<5$ years, in terms of access to a Pediatric Emergency Department (PED) and hospitalization; to compare epidemiologic and clinical characteristics of rotavirus related gastroenteritis to those caused by other pathogens.

Methods: This is a one-year prospective observational study, including children $<5$ years, admitted with community acquired gastroenteritis to our PED. Demographic, clinical, socio-economical data were collected; rotavirus was detected by an immunocromatographic rapid stool test. We performed descriptive two-variable analyses to compare rotavirus and non-rotavirus gastroenteritis and a multiple logistic regression for predictors of hospitalization.

Results: Annual access rate to our PED due to acute gastroenteritis resulted 4.1/100 inhabitants $<5 y ; 46,9 \%$ of them were infected by rotavirus. Annual rate of 
hospitalization was 5,2/1000, with rotaviral positivity of $56 \%$. In comparison to other pathogens, rotaviral infection was associated with higher clinical severity number of daily diarrheic episodes, number of vomiting, level of fever, severity of dehydration, metabolic acidosis, ketonuria). At the multi-variable analysis, predictors of hospitalization resulted age $<3$ months $(\mathrm{OR}=70.89)$ and presence of moderate-severe dehydration $(\mathrm{OR}=3.87)$.

Conclusions: In our area rotavirus gastroenteritis represents an important cause of admission to PED and of hospitalization, with higher severity in respect to other pathogens. These results can be useful in planning strategies of prevention and of active immunization.

\section{OUTBREAK OF MEASLES IN WEST ATHENS}

A Vontzalidis, P Korovessi, K Avgeri, M Psalti, T Poukoulidou, I Papadea, E Papadakis

Department of Pediatrics, Thriasion General Hospital, Athens, Greece

Background and Aims: Measles continues to be a menace to millions of children worldwide. Currently, the World Health Organization (WHO) has set up a number of programs to reduce measles mortality worldwide by $50 \%$ by the end of 2005 . The last 25 years 4 major outbreaks of measles occurred in Greece, last being on 1996. In late November 2005 a cluster of confirmed measles cases was reported among preschool children in North of Greece.

Methods: This abstract describes an outbreak, 3 months later in West Athens (South Greece) and examines the characteristics of the children affected, with particular reference to immunisation history and socioeconomic status.

Results: From February 2006 to April 2006, 30 cases were diagnosed in our hospital. $40 \%$ of all cases were aged under 12 months, $90 \%$ were 5 years or under. All cases had no record of receiving any measles vaccine. All children were from a racial/ethnic minority (gypsies), having parents with low educational status, and belonging to a very poor household. Serological analysis (measles specific IgM) was carried out on 10 patients blood samples. Urinary and saliva samples of these patients were also tested for measles virus by reverse transcription-polymerase chain reaction. (RTPCR). $30 \%$ of cases needed hospitalisation. $1 \%$ were transferred to a Paediatric Infectious Disease Department.

Conclusions: Unvaccinated children are clustered geographically and socioeconomically. Greece needs to reach the WHO objective of measles elimination by 2010 and the interventions need to be specifically designed and targeted toward racial/ethnic minorities who choose for their children not to receive any vaccinations.

\section{INTRAFAMILIAL TRANSMISSION OF HELICOBACTER PYLORI INFECTION IN CHILDREN OF HOUSEHOLDS WITH MULTIPLE GENERATIONS IN VIETNAM}

B V Nguyen ${ }^{1}, \mathrm{~K}$ G Nguyen ${ }^{1}$, C D Phung ${ }^{2}$, N Kalach ${ }^{3}$, O Kremp ${ }^{3}$, J Raymond ${ }^{4}$, K Okrainec ${ }^{5}$, G Vidal-Trecan ${ }^{6}$

${ }^{l}$ Department of Paediatric, Hanoi Medical University, Hanoi, Vietnam, ${ }^{2}$ Digestive Disease Division, National Institute of Hygiene and Epidemiology, Hanoi, Vietnam

${ }^{3}$ Paediatric Saint Antoine Clinic, Saint Paul Hospital, Catholic University, Lille, France, ${ }^{4}$ Department of Microbiology, Saint Paul-Cochin Hospital Group, Paris, France, ${ }^{5}$ Department of Public Health, McGill University, Montreal, QC, Canada, ${ }^{6}$ Department of Public Health, Saint Paul-Cochin Hospital Group, Paris, France

Background and Aims: Intra familial pathway has been considered as an important way for person-toperson transmission of $\mathrm{H}$. pylori infection. However, very few studies involved all household members into study population. The present study aimed at investigating the relationship between $\mathrm{H}$. pylori infection in children and the other household members.

Methods: This community-based cross-sectional study was carried out on 533 participants from 135 households with multiple generations living in the same household.

Results: H. pylori infection in children was found significantly associated with the infection in mothers [OR (95\% CI): 2.50 (1.19 - 5.26)], even after being adjusted for sex, age group and sibling number [adjusted OR $(95 \% \quad \mathrm{CI}): 2.47 \quad(1.12 \quad$ - 5.47)]. It was also significantly associated with the infection in both parents [adjusted OR (95\% CI): 4.14 (1.29 - 13.23)]. No significant association between $\mathrm{H}$. pylori infection in the father, grandparent(s), uncle or aunt with that in their children was found.

Conclusions: Results from the present study showed intra familial transmission in a multi-generation population and supported the hypothesis of person-toperson transmission of $\mathrm{H}$. pylori infection.

\section{MENINGOCOCCAL DISEASE - EIGHT YEAR REVIEW OF CARE DELIVERED BY A PAEDIATRIC INTENSIVE CARE UNIT}

K C Pike ${ }^{1}$, V S Garikapati ${ }^{2}$, R L Mehta ${ }^{3}$, M J Marsh ${ }^{2}$

${ }^{I}$ Department of Paediatrics, Poole Hospital, Poole, Dorset, UK, ${ }^{2}$ Department of Paediatric Intensive Care, Southampton General Hospital, Southampton, Hampshire, UK, ${ }^{3}$ School of Medicine, University of Southampton, Southampton, Hampshire, UK

Background and Aims: This presentation describes the epidemiology, clinical course and outcome of children admitted to a tertiary paediatric intensive care unit (PICU) with Meningococcal disease (MD). Temporal trends are explored and three predictors of survival are compared.

Methods: Retrospective case note analysis of all PICU admissions diagnosed clinically with MD. Data collected: demographics, mortality prediction scores, treatment, and outcome. 
Results: Between 1997 and 2005 the PICU admitted 106 children with MD. The median age was 2.5 years. Fifty-four percent of admissions were male. There were 6 deaths, (mortality 5.7\%). Detailed information was available for 102 patients. Of these, 17 suffered predominantly meningitis and 79 predominantly septicaemia, 6 had mixed disease. Ninety-two children required mechanical ventilation, 74 required inotropes and 14 required haemofiltration. Eighty-four percent of survivors had no lasting morbidity. Compared to the group that died or survived with disability, intact survivors had significantly lower mean (SD) PRISM score 11.9 (6.5) vs. 19.9 (8.2), $\mathrm{p}<0.001$, Glasgow Meningococcal Septicaemia Prognostic (GMSP) score 5.4 (2.7) vs. 8.2 (3.6), $\mathrm{p}<0.001$ and Platelet-Neutrophil product $2,156(2,038)$ vs. $884 \quad(1,133)$, p $<0.01$. Referrals from within the hospital had lower mean PRISM 9.4 (6.7) compared to outside referrals PRISM 15.1 (7.4), $\mathrm{p}<0.001$. Logistic regression proved PRISM best predicted survival in the group as a whole, although GMSP best predicted survival of meningitis.

Conclusions: Increasingly, due to centralisation of MD care, patients referred to PICU have poorer prognostic scores and require more intensive treatment. Prognostic scores are useful but should reflect the disease process to which they are applied.

\section{ROTAVIRUS GASTROENTERITIS: EPIDEMIOLOGICAL DATA FROM A REGIONAL HOSPITAL}

\author{
M Raes ${ }^{1}$, P Aerssens ${ }^{1}$, P Alliet $^{1}$, P Gillis ${ }^{1}$, M P Verjans ${ }^{1}$, \\ R Cartuyvels ${ }^{2}$, K Magerman ${ }^{2}$, A Mewis $^{2}$ \\ ${ }^{l}$ Department, of Pediatrics, Virga Jesseziekenhuis, \\ Hasselt, Belgium, ${ }^{2}$ Clinical Microbiology Laboratory, \\ Virga Jesseziekenhuis, Hasselt, Belgium
}

Background and Aims: Two new vaccines against rotavirus (RV) infection will be available in Belgium later this year. Both are very effective in reducing the rate of serious rotavirus gastroenteritis and associated hospitalisations. In Belgium no official data are available about the burden from rotavirus disease. The data from the last three rotavirus gastroenteritis seasons in our department were retrospectively analysed.

Methods: All stool samples, sent to our clinical microbiology laboratory between June 2002 and June 2005 for the detection of RV were taken into account: Result (positive/negative), date of sample, age of the patient, hospitalisation characteristics.

Results: see fig 1

Conclusions: National data are necessary to calculate the health profit and financial repercussion of the introduction of a vaccine against rotavirus. The results will depend on the vaccination schedule and the vaccine coverage. If the vaccine protects almost totally against hospitalisation, a substantial reduction in hospitalisation days can be expected for most pediatric wards.

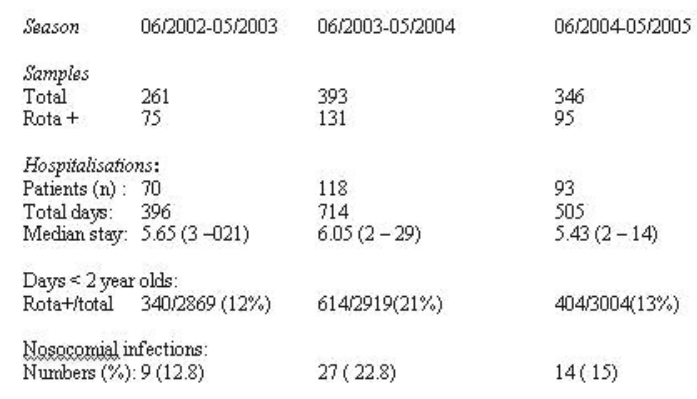

\section{EPIDEMIOLOGICAL ASPECTS OF CHILDHOOD ASEPTIC MENINGITIS IN WESTERN GREECE DURING 2001 TO 2005}

I Giannakopoulos, K Makrakis, D Papanastasiou, A Giannakopoulos, A Ellina, S Mantagos

Department of Pediatrics, School of Medicine, University of Patras, Patras, Greece

Background and Aims: To study the demographic and epidemiologic data of aseptic meningitis in the childhood population of Achaia, a prefecture of Western Greece during the last five years.

Methods: Children (age $<14$ years) with aseptic meningitis admitted to the Pediatric Clinic and/or reported to the regional Department of Health from $1 / 1 / 01-31 / 12 / 2005$ and coming from Achaia were included in the study. The demographic and epidemiologic data were analysed. Diagnosis was based on clinical picture, elevated CSF white cells with lymphocyte predominate and negative CSF and blood culture. No patient received antibiotic treatment.

Results: During 2001 to 2005 there were 115 (48.7\%) patients with aseptic meningitis among the 236 meningitis patients. Seventy-five $(65.2 \%)$ of them were males and $40(34.8 \%)$ females. The mean age of the patients was $7.55+3.95$ (range 0.08 to 14 ) years. Fifty patients $(43.5 \%)$ were recorded during the summer and $44(38.3 \%)$ during the spring. Exacerbations of the disease were observed during 2001; there were recorded $80(69.6 \%)$ cases. The annual incidence of the disease per 100000 children was 39.5 , while during the exacerbation-period of 2001 reached 137.8. Complete recovery without complications occurred in all patients.

Conclusions: Aseptic meningitis is common during summer and spring. The annual incidence during the last five years was 39.5 with exacerbation occurring during 2001. The disease affects young children, more often males than females. The course was favourable in all cases.

\section{BACTERIAL MENINGITIS CONTINUES TO REMAIN RARE IN RURAL AREAS OF W. GREECE DURING 2001 T0 2005}

I Giannakopoulos ${ }^{1}, \mathrm{~K}_{\text {Makrakis }}{ }^{1}$, A Ellina ${ }^{1}$, D Papanastasiou ${ }^{1}$, A Giannakopoulos ${ }^{2}$, M Leotsinidis ${ }^{3}$, S Mantagos ${ }^{1}$

${ }^{1}$ Department of Pediatrics, School of Medicine, University of Patras, Patras, Greece, ${ }^{2}$ Department of Health of Western Greece, Patras, Greece, ${ }^{3}$ Laboratory of Public Health of Western Greece, Patras, Greece 
Background and Aims: In an earlier study it was established that during the decade 1991 to 2000 bacterial meningitis was rare in rural areas of $\mathrm{W}$. Greece. This study was conducted in order to investigate if the incidence of bacterial meningitis in children during 2001 to 2005 continues to remain low in rural regions and high in urban and suburban settings in the same areas of W. Greece.

Methods: Included were all children with proven or suspected bacterial meningitis in W. Greece from 2001 to 2005 . There were 42 children with proven and 79 children with suspected bacterial meningitis. All cases of proven or suspected bacterial meningitis had pleiocytosis in the cerebrospinal fluid with predominance of polymorphonuclear leucocytes and compatible clinical picture. Proven bacterial meningitis fulfilled in addition one or more of the following criteria: (a) positive blood/CSF culture, (b) positive Gram stain or latex agglutination.

Results: The annual incidence of proven bacterial meningitis was 1.95 (38 cases) per 10,000 children in urban areas, 1.92 ( 2 cases) in suburban and $0.23(2$ cases) in rural areas. In cases of suspected bacterial meningitis the incidence per 10,000 children was 3.39 (66 cases), 7.69 ( 8 cases) and 0.58 (5 cases), respectively. Only two of the 38 cases of Haemophilus influenzae type b, Neisseria meningitidis or Streptococcus pneumoniae meningitis came from rural areas.

Conclusions: Children living in rural areas continue to be spared from bacterial meningitis compared to those living in urban and suburban regions, since bacterial meningitis appeared extremely rare in the rural settings.

\section{PREVALENT BACTERIA ISOLATED FROM NEWBORN INFANTS IN INTENSIVE CARE NURSERY OF NEMAZEE HOSPITAL, SHIRAZ, IRAN, 2002-2003}

\author{
M Motamedifar ${ }^{1}$, Z Hashemizadeh $^{2}$ \\ ${ }^{l}$ Department of Bacteriology and Virology, Shiraz \\ Medical School, Shiraz, Iran, ${ }^{2}$ Nemazee Hospital, Shiraz \\ University of Medical Sciences, Shiraz, Iran
}

Background and Aims: Bacterial infection is common among very low birth weigh (VLBW) infants as a consequence of weak immune system. In this study an analysis of infections in the intensive care nursery of the Shiraz Nemazi hospital was made over a two year period.

Methods: In this cross-sectional study the data were extracted from files of 416 infants admitted in nursery of the Shiraz Nemazi hospital during a two year period (2002-2003). The frequencies of different infections, prevalent isolated bacteria along with their demographic data were analyzed.

Results: Among 416 VLBW infants 262 (63\%) were male and $154(37 \%)$ were female. The most prevalent infections were pulmonary and gastrointestinal infections. The mean hospitalization time was 7 days with the range of 1-64 days and the mean age of infection was 5 days with the range of 1-30 days. $19.2 \%$ of infants diagnosed to be suffered from infection and $79.8 \%$ from other non-infectious diseases. The most frequent microbes isolated were: Escherichia coli 9.1\%, Acenitobacter spp 7.7\%, Kelebsiella spp 4.8\%, Pseudomonas Spp 4.3\%, Staphylococcus aureus 3.4\%, Candida albicans $1.9 \%$, Staphylococcus epidermidis $1.6 \%$, Streptococcus faecalis $1.4 \%$ and Enetrobacter spp $1.1 \%$.

Conclusions: This study confirms the necessity of expanding management programs for prevention of nosocomial infections, improving sampling and microbial isolation procedures and precise clinical diagnosis.

\section{AIDS IN CHILDREN FOR VERTICAL TRANSMISSION IN CUBA}

I Gonzalez Nuñez, M Diaz Jidy, J Perez Avila

Atención Medica, Instituto Pedro Kourí, Hospital Ciudad de la Habana, Ciudad de la Habana, Cuba

Background and Aims: Since 1986 a controlled program is established in primary health care in Cuba to reduce vertical transmission.

Methods: Each HIV+ pregnant woman who decides to keep her pregnant receive ZDV; $600 \mathrm{mg}$ daily from week 14 to 38 when Caesarean operation is carried out. During his first 6 weeks, the child receives AZT $(2 \mathrm{mg} / \mathrm{Kg} / \mathrm{dose})$ every 6 hours. Evolution of these children is made in IPK external office, where they are studied to determine HIV infection Infected children are treated with antiretrovirals, according to opportunistic diseases, CD4 and viral load. Quantification of CD4 is determined by flow cytometry, using a FACScan cytometer from Becton Dickinson. Quantification of viral RNA levels was made through the Nuclisens system from Biomerieux.

Results: A total of 1386 seropositive women have been reported since January 1, 1986 to February 28, 2006 (18, $6 \%$ ) of all the seropositive cases of the country (1386/ $7109), 216(15,5 \%)$ have given birth a total of 233 children (15 women have delivered twice and 2 have twins); 26 of 233 are $\mathrm{HIV}+(11,1 \%), 22$ classified as AIDS $(22 / 26=84,6 \%), 13$ are under treatment with HAART; $9(9 / 26=34,6 \%)$ died and 4 are asymptomatic. No infection was demonstrated in 126 children by PCR and Western Blot $(126 / 233=54,0 \%)$ and $78(78 / 233=33$, $4 \%)$ are still under study.

Conclusions: The program and prevention and control of vertical transmission is effective as the amount of infected children is low similar to that developed countries.

\section{IMMUNIZATION STATUS REGARDING HEPATITIS B OF CHILDREN 2 TO 6 Y. O. IN THE ISLAND OF THERA, GREECE}

S S Katinakis, M Triantafyllou

Department of Pediatrics, Thera Health Center, Thera, Greece

Background and Aims: Immunizations are vital for the prevention of infections in pediatrics. Aim of this study was to determine the immunization status of children 2 to 6 years of age regarding Hepatitis B, in the Greek island of Thera (Santorini). 
Methods: Data were collected from the immunization records of 591 children (304 boys and 287 girls) (332 Greek) aged 2 to 6 years, who were examined for various reasons at the public Health Center of Thera, during a period of 6 months (1-11-2005 to 30-4-2006). Fully immunized were considered the children who had received three doses of the vaccine with at least 6 months interval between the first and the third dose.

Results: $96.6 \%$ of the children were fully immunized, $2.8 \%$ were incompletely immunized and only $0.7 \%$ had not received any dose of Hepatitis B vaccine. Among the Greek children, $97.3 \%$ were fully immunized, while the foreign children were fully immunized at a $95.3 \%$ rate. Most children lacking doses of vaccination were among the younger ones, which shows that they were just neglecting to follow the vaccination schedule strictly, while 2 out of the 4 unimmunized children had parents who refused to vaccinate their children as a principle.

Conclusions: In the island of Thera, Hepatitis B immunization is carried out at a satisfactory rate and we are contented with the compliance of the people, both Greek and foreign.

\section{BREASTFEEDING AND NASAL CARRIAGE OF STAPHYLOCOCCUS AUREUS}

F Ladomenou ${ }^{1}$, S Maraki ${ }^{2}$, A Georgiladakis ${ }^{2}$, Y Tselentis $^{2}$, E Galanakis ${ }^{1}$

${ }^{I}$ Department of Paediatrics, University of Crete, Crete, Greece, ${ }^{2}$ Department of Clinical Bacteriology, Parasitology, Zoonoses and Geographical Medicine, University of Crete, Crete, Greece

Background and Aims: Breastfeeding is well known to affect neonatal and infantile colonization by microorganisms. Nasal carriage of Staphylococcus aureus is a risk factor for invasive disease. The aim of the study was to assess the protective role of breastfeeding on the nasal carriage of S. aureus.

Methods: We conducted a prospective communitybased study of breastfeeding in Crete, Greece. A randomised sample of 1049 pairs (mother-infant) was formed. The epidemiology of staphylococcal carriage was examined for 62 healthy infants (31 fully, 17 partially breast-fed and 14 bottle fed) at six months after birth.

Results: Among the 31 exclusively breast-fed infants, 9 were colonized by S. aureus strains, of whom 3 were methicillin-resistant. Among the 31 partially breast-fed or bottle-fed infants, 7 were colonized by S. aureus strains, of whom 6 were methicillin-resistant. Exclusive breastfeeding was associated with somehow higher rates of S. aureus carriage (odds ratio 1.40; 95\% confidence intervals 0.45 to 4.41 ), however MRSA carriage was higher among partially breast-fed and bottle-fed infants than in their exclusively breast-fed peers (odds ratio 2.24; $95 \%$ confidence intervals 0.51 to 9.91 ).

Conclusions: The findings of this small cohort suggest that exclusively breast-fed infants, although equally or even more often colonized by S. aureus, present with decreased frequency of methicillin-resistant strains. Larger cohorts are required to demonstrate this protective effect of breastfeeding, if any.
MORBIDITY OF DISEASE WHO ARE LIABLE TO REGULAR VACCINATION IN A PERIOD OF TEN YEARS

\section{R A Brezovska}

\section{Pediatrics Medical Center, Gostivar, Macedonia}

Background and Aims: Review of the representation of contagious diseases that are liable to regular vaccination in Gostivar. Review of morbidity of Varicelae that is not liable to obligatory vaccine and observe the effects of vaccination and the need to bring regular vaccination for Varicelae.

Methods: In R. Macedonia is conducting obligatory vaccination against: TBC Morbili, Rubeola, Parotitis Epidemica, Diptheria, Tetanus, Pertusis, Poliomielitis, and not for Varicelae. Against Hepatitis B the vaccination is obligatory from November 2005. To elaborate the material the facts were taken from monthly reports and reporting lists of sick people in period from 1996 until 2005. The city of Gostivar has around 110.000 people from mixed national and social structure and the morbidity we get from formula.

Results: The contagious diseases which are obligate for vaccination have very low morbidity and that is for high surround of $95 \%$ vaccined people. In 2005 there is appearance of Parotitis in epidemic form on people born in 1990-93. That is for low vaccination from 35\% because of lack of vaccine against Parotitis from independence of the state. There has not been ill people from Poliomielitis and Diptheria, but 2 people died from Tetanus in 2003 and 2005. There is high morbidity from Varicelae 250 cases in year of children until 7 years old but also there is cases of adults.

Conclusions: With vaccination successfully are prevented contagious diseases. Considering the fact that high morbidity from Varicelae especially in early child age is necessary to bring in continuer vaccination against Varicelae.

\section{MOLECULAR EPIDEMIOLOGY AND INCIDENCE OF PNEUMOCOCCAL PNEUMONIA IN CHILDREN ATTENDED IN A REFERENCE PEDIATRIC HOSPITAL IN BARCELONA (SPAIN)}

C Munoz-Almagro ${ }^{1}, \mathrm{C} \mathrm{Polo}^{1}, \mathrm{I}_{\text {Jordan }}{ }^{2}, \mathrm{C}_{\text {Esteva }}{ }^{1}$, S Hernandez-Bou ${ }^{2}$, C Latorre ${ }^{1}$, J J Garcia ${ }^{2}$, W Hausdorff ${ }^{3}$, A B Brueggemann ${ }^{4}$

${ }^{l}$ Department of Microbiology, University Hospital Sant Joan de Deu, Barcelona, Spain, ${ }^{2}$ Department of Paediatrics, University Hospital Sant Joan de Deu, Barcelona, Spain, ${ }^{3}$ GlaxoSmithKline Biologicals, Rixensart, Belgium, ${ }^{4}$ Department of Zoology, University of Oxford, Oxford, UK

Background and Aims: The aim of this study was to determine the molecular epidemiology and incidence of pneumonia caused by Streptococcus pneumoniae during a 5 years period.

Methods: Retrospective study including patients aged $<19$ years old with clinical diagnosis of pneumonia or empyema with S.pneumoniae isolated by culture from 2000 to 2005. Annual incidence rates were calculated per 100,000 children $<19$ years old from Health Area of 
our center. Serotype and Multi Locus Sequence Typing (MLST) was made to a subset of strains. Pre-PCV7 period was defined as 2000-2002 and the post-PCV7 was defined as 2003-2005.

Results: A total of 48 episodes of pneumococcal pneumonia were identified in 47 patients, mean age $3,8 y$. Strains were recovered from blood (17), pleural efussions (25), both (2) and bronchoalveolar lavage (4). Annual incidence of pneumococcal pneumonia increased from 2,4 per 100.000 children during prePCV7 period to 5,9 episodes per 100.000 during postvacunal period. Most frequent serotypes were serotype 1 (12 cases), 14 (10 cases), 5 (5 cases) and 19A (5 cases). Strains serotype distribution varied between first period and second: 7 -valent vaccine serotypes (7VS) $(45,5 \%$ vs. $25,7 \%)$, vaccine-related (7VRS) $(18,2 \%$ vs. $20 \%)$ and non-vaccine serotypes (7NVS) $(36,4 \%$ vs $54,3 \%)$. MLST performed showed that the majority of serotype 1s were of known serotype 1 sequence types (ST 306 and ST 304).

Conclusions: The increase of pneumonia and empyema in our area may be associated with infection due to nonvaccine serotypes. Serotype 1 clones may be related to this fact.

\section{PHYSICIANS' AND PARENTS' ATTITUDES TOWARDS VARICELLA VACCINATION IN EUROPE: THE OVALE SURVEY}

\author{
F A Allaert ${ }^{1}$, A Blanc ${ }^{2}$, Y Megard ${ }^{2}$ \\ ${ }^{I}$ Cenbiotech CHRU Dijon, France and McGill \\ University Montreal, QC, Canada, ${ }^{2}$ Sanofi Pasteur \\ MSD, Lyon, France
}

Background and Aims: Describe the physicians' and parents' opinions about the chicken pox vaccination.

Methods: Cross sectional pharmaco-epidemiological study conducted in three European countries (France, Italy, Germany) with the same protocol including a physician opinion questionnaire, a clinical form describing the vaccine and a parents' self administered questionnaire.

Results: 186 French, 509 German and 170 Italian paediatricians have participated to the study and respectively 878 French, 2380 German and 182 Italian patients. Among the paediatricians involved, respectively $52.2 \%, 67.7 \%$ and $39.0 \%$ systematically recommended chickenpox vaccination for children and estimate that $56.2 \%, 70.3 \%, 63.6 \%$ of parents accept it. Among those who accepted, the most relevant arguments in convincing them are: The availability of an effective and well-tolerated vaccine $(78.7 \%, 78.7 \%$, $77,7 \%$ ) and information on the seriousness of the disease $(67,8 \%, 77.4 \%, 73,5 \%)$. Paediatricians indicate that the main reasons why parents refuse are the perception that chickenpox is a harmless disease $(82.6 \%, 88.1 \%, 51.0 \%)$. A sub-analysis of parents who were initially reluctant showed that refusal was essentially due to the fear of vaccine complications $(57.6 \%, 58.8 \%, 92.9 \%)$ as well as the increasing number of vaccinations $(37.5 \%, 45.4 \%, 42.9 \%) .22 .5 \%, 25.0 \%$ and $36.1 \%$ of the practitioners administered chickenpox vaccine at the same time than MMR vaccine. For some parents, acceptance could potentially be enhanced by the co-administration of varicella vaccine with MMR.

Conclusions: In the three European countries parents readily accept chickenpox vaccination when the practitioner recommends it and/or when they are appropriately informed both about the potential severity of varicella disease and the safety of the vaccine.

\section{INFANTS BORN TO HIV-SEROPOSITIVE MOTHERS: EXPERIENCE OF 20 YEARS} E Tridapalli, M G Capretti, M Stella, G Brighi,
G Faldella

Department of Preventive Paediatrics and Neonatology, St. Orsola-Malpighi General Hospital, University of Bologna, Bologna, Italy

Background and Aims: Paediatric HIV infection is mainly the consequence of perinatal transmission. Since 1994 has been adopted antiviral-prophylaxis in the mothers and in the newborns (P076). In developed countries, these measures associated with cesarean delivery have reduced the transmission rate from $36 \%$ to $2 \%$.

Methods: We conduced a retrospective study from 1985 to 2005 in St. Orsola Hospital to evaluate the different incidence of HIV perinatal transmission.

Results: During the study period 173 HIV-seropositive women delivered 194 newborns. 88\% came from Italy and 50\% acquired infection from drug abuse. After 2002 the source of infection changed; most women acquired HIV sexually. $37 \%$ of mothers were co-infected with HCV. After 1996, $82 \%$ of the women completed antiviral-prophylaxis during the prenatal and intrapartum period, as did $89 \%$ of newborns. 2 babies had low birth weight $(<1500 \mathrm{gr})$, and 2 babies were less than 30 weeks of GA. HIV infection was diagnosed in 17 newborns $(8.7 \%)$. Since 1997 there have been 5 cases of infected children; in four cases the mother was not treated during pregnancy and labour while all the babies underwent a correct prophylaxis.

Conclusions: 1) the number of mothers treated with antiviral prophylaxis during pregnancy and labour progressively and significantly increased (8\% after 1996 vs $21 \%$ before 1996). 2) Also the number of newborns with completed antiviral prophylaxis significantly increased ( $89 \%$ after 1996 vs $29 \%$ before 1996) 3) Consequent cumulative incidence decreased with the implementation of guidelines: after 1995 only 5 babies infected/ 85 babies at risk (5.9\%), before 199512 infected/ 109 babies at risk (11\%).

\section{SYMBIOSIS OF GIARDIA AND HEPATITIS B VIRUS}

\author{
R Gaffari ${ }^{1}$, H Gaffari ${ }^{2}$, M Makani $^{1}$ \\ ${ }^{1}$ Gamar Bany Hashem Hospital, Khoy, Iran, ${ }^{2}$ Health \\ Ministry, Tehran, Iran
}

Background and Aims: We studied this subject from 5 years ago and understand these aims. 1. The Jiardia infection susceptible the patient for hepatitis B virus. 2 . With complete and aggressive treatment of giardia the risk of hepatitis B virus is reduced. 
Methods: This problem is completed inquired and is continued from text book especially pharmacology, immunology, pathology.

Results: We will have to be serious with Jiardia infection and taking medicine in this infection should be totally completed. When Jiardia infection is eradicated, the risk of infection with hepatitis B is decreased.

Conclusions: Giardia have symbiosis with hepatitis B virus. The risk for other is decreased, when one of them is cured. Jiardia vs symbiosis with hepatitis B virus. This problem is cleared ours and we can prove it.

\section{VENTILATORY SUPPORT EVOLUTION IN BRONCHIOLITIS INFANTS ADMITTED TO PICU}

J Lozano, E Esteban, I Jordan, M Pons, Z Martinez de Compañon, J Caritg, A Palomeque

Pediatric Critical Care Unit, Hospital Sant Joan de Deu, Barcelona, Spain

Background and Aims: About $15-20 \%$ of infants with bronchiolitis are admitted at PICU, and $30-40 \%$ of these cases become mechanical ventilated. To analyze bronchiolitis ventilatory support characteristics, the last years.

Methods: Prospective and observational study from infants with bronchiolitis admitted at PICU during 20042005. We compared data obtained with 2002-03 period.

Results: Data from 72 patients were collected. 45 were male $(62.5 \%)$ with a mean age of 1.93 months $(\mathrm{SD} \pm 2.69)$. In the $34.7 \%$ of cases, risk factors for bronchiolitis were found. Almost one quarter were premature at birth, whom 6 received specific anti Respiratory Syncitial Virus (RSV) antibodies. RSV was positive in $52.8 \%$ of cases, with significant differences from 2002-03 period (79.2\%), $\mathrm{p}<0.03$. Exclusive noninvasive ventilation with nasal CPAP or BiPAP was required in $22 \%$ of all cases, and $12.5 \%$ previous to intubation. Duration of non-invasive ventilation was 1.9 days range 1-8 days. Mechanical ventilation was required in 38 cases $(52.8 \%)$ and high frequency ventilation was used in two infants. Indication for mechanical ventilation was respiratory distress in $60 \%$ and apnea in $21.1 \%$. No differences were found regard ventilation requirements and risk factors, or SRV diagnosis, nor previous VNI. Mean stay in PICU was 5.3 days $(\mathrm{SD} \pm 4.94)$. Including gravity score, all results were comparable with 2002-2003. Last period active humidification was systematically used, the time on mechanical ventilation was lower but without statistically different (5.13 versus 6.8 ).

Conclusions: Non-invasive ventilotherapy is useful in bronchiolitis. Decreases in duration of mechanical ventilation may be related to the systematic use of humidification.

\section{VOLUME CONTROL VENTILATION IN EXTREMELY LOW BIRTH WEIGHT INFANTS - A RANDOMIZED CONTROLLED TRIAL}

J Singh $^{1}$, S Gupta $^{1}$, P Clarke ${ }^{2}$, S K Sinha ${ }^{1}$
${ }^{1}$ Directorate of Neonatal Medicine, The James Cook University Hospital, Middlesbrough, UK, ${ }^{2}$ Hope Hospital, Salford, UK

Background and Aims: Preterm infants with variable lung compliance are at risk of volume induced lung injury. Volume controlled ventilation (VCV) differs from pressure limited ventilation (PLV) in terms of gas delivery target, which is tidal volume in VCV rather than pressure in PLV. This study was planned to compare the efficacy and safety of VCV to PLV in very premature infants with respiratory distress syndrome (RDS) requiring mechanical ventilation.

Methods: 109 newborns weighing 600-1500 $\mathrm{g}$ and 24-32 weeks gestation with RDS were randomized to either VCV or PLV, and treated with a standardized protocol. The two modalities were compared by determining the time required to achieve pre-determined 'success' criteria, based on either the Alveolar-arterial oxygen gradient $[<13 \mathrm{kPa}]$ or the mean airway pressure $[<8 \mathrm{~cm} \mathrm{H2O}$. Analysis was done on an intention to treat basis.

Results: Out of 109 babies enrolled, there were 57 in the VCV group and 52 in the PLV group. Mean time to reach success criteria was $23 \mathrm{hrs}$ in VCV group compared to $33 \mathrm{hrs}$ in PLV ( $\mathrm{p}=0.15$ ). However this difference between VCV and PLV was more striking in babies weighing less than $1000 \mathrm{~g}$ ( $21 \mathrm{vs.} 58 \mathrm{hrs}$; $\mathrm{p}=0.03$ ) and amongst those who had moderate to severe respiratory failure ( $27 \mathrm{vs} 59 \mathrm{hrs} ; \mathrm{p}=0.03$ ) respectively. There were 5 deaths in the VCV group and 10 in PLV $(p=0.10)$. The incidence of other complications was similar in two groups.

Conclusions: VCV is safe and efficacious in extremely low birth weight infants and may have advantages over traditional methods of ventilation.

\section{PRESSURE SUPPORT VENTILATION IN PRETERM BABIES - A RANDOMIZED CROSSOVER TRIAL}

\author{
S Gupta $^{1}$, S K Sinha ${ }^{1}$, S M Donn ${ }^{2}$ \\ ${ }^{1}$ Directorate of Neonatal Medicine, The James Cook \\ University Hospital, Middlesbrough, UK, ${ }^{2}$ Division of \\ Neonatal-Perinatal Medicine, University of Michigan \\ Health System, Ann Arbor, MI, USA
}

Background and Aims: Pressure Support Ventilation (PSV) is a patient-triggered, pressure-limited, flowcycled mode of ventilation which provides an inspiratory "pressure boost" during spontaneous breathing. This study was planned to evaluate the effect of varying levels of pressure support with synchronized intermittent mandatory ventilation (SIMV). Methods: In randomized crossover trial, breath-to-breath pulmonary mechanics data were collected from 10 premature infants during three modes of ventilation SIMV alone, SIMV with partial PSV, and SIMV with full PSV. Full PSV provided 5-8 ml/kg and Partial PSV 2.5-4 ml/kg of expired Tidal volume (Vte). Results: A total of 19,112 breaths were analysed. The Minute Ventilation (Ve) and Respiratory Rate (RR) improved with the addition of pressure support (Table 1). Conclusions: Pressure Support Ventilation improves the efficiency of breathing during mechanical 
ventilation and the improvement is proportional to the level of support provided.

Table 1: PSV and Pulmonary mechanics

\begin{tabular}{|c|c|c|c|}
\hline Study parameter & SIMV & $\begin{array}{l}\text { SIMV + } \\
\text { Partial PSV }\end{array}$ & $\begin{array}{l}\text { SIMV + } \\
\text { Full PSV }\end{array}$ \\
\hline No. of analyzed breaths & 7203 & 6320 & 5589 \\
\hline Total Ve/kg (ml) & $270.1 \pm 0.84$ & $332.4 \pm 0.90^{*}$ & $391.7 \pm 1.1 * \#$ \\
\hline Spontaneous Ve/kg (ml) & $143.2 \pm 0.64$ & $201 \pm 0.67^{*}$ & $256.8 \pm 1.1 * \#$ \\
\hline Spontaneous RR (per min) & $51.9 \pm 0.13$ & $45.0 \pm 0.14^{*}$ & $38.8 \pm 0.16 * \#$ \\
\hline
\end{tabular}

\section{NASAL CONTINUOUS POSITIVE AIRWAY PRESSURE (N-CPAP) DOES NOT CHANGE CARDIAC OUTPUT IN PRETERM BABIES}

\author{
B Moritz ${ }^{1}$, M Fritz ${ }^{1}$, C Mann ${ }^{2}$, B Simma \\ ${ }^{1}$ Pädiatrie, LKH Feldkirch, Feldkirch, Austria \\ ${ }^{2}$ Neonatologie, KH Chur, Chur, Switzerland
}

Background and Aims: Invasive mechanical ventilation impairs cardiac output in children and adults. $\mathrm{N}-\mathrm{CPAP}$ is widely used in neonatal intensive care, as yet, hemodynamic consequences have not been investigated.

Methods: A prospective study to assess the hemodynamic effects has been conducted in 21 preterm babies ( 8 males, 13 females $)<1500 \mathrm{~g}$ by twodimensional M-mode and pulsed Doppler echocardiography during and one hour after discontinuation of n-CPAP.

Results: Gestational age was $28.0 \pm 1.9$ weeks (mean \pm SD), birth weight $1000 \pm 238 \mathrm{~g}$, age at study entry $200 \pm 155$ hours, total maintenance fluid $154 \pm 42$ $\mathrm{mL} / \mathrm{kg} /$ day, level of $\mathrm{n}$-CPAP was $4.4 \pm 0.9 \mathrm{cmH} 2 \mathrm{O}$. None of the babies received inotropic support. N-CPAP did not cause any significant difference in any parameters measured (Wilcoxon Test): stroke volume $3.1 \pm 1.0 \mathrm{~mL}$ (without n-CPAP) vs. $3.1 \pm 1.0 \mathrm{~mL}$ (with $\mathrm{n}$-CPAP), cardiac output $500 \pm 176 \mathrm{~mL} / \mathrm{min}$ vs. $487 \pm$ $156 \mathrm{~mL} / \mathrm{min}$, left ventricular diastolic diameter $1.24 \pm$ $0.14 \mathrm{~cm}$ vs. $1.22 \pm 0.15 \mathrm{~cm}$, fractional shortening $0.29 \pm$ $0.04 \%$ vs. $0.30 \pm 0.05 \%$, aortic velocity-time integral $8.70 \pm 1.65 \mathrm{~cm}$ vs. $8.64 \pm 1.80 \mathrm{~cm}$. Level of $n-C P A P$ did not influence cardiac output (Kruskal-Wallis).

Conclusions: N-CPAP (up to $7 \mathrm{cmH} 2 \mathrm{O}$ ) has no hemodynamic effect in preterm babies. Our data imply that there is no need to withhold n-CPAP support in order to avoid circulatory compromise in small preterm babies.

\section{ACUTE RESPIRATORY DISTRESS SYNDROME: THE EXPERIENCE OF A PAEDIATRIC INTENSIVE CARE UNIT}

T Caldeira ${ }^{1}$, S Figueiredo ${ }^{2}, \mathrm{~F}_{\text {Cunha }}{ }^{3}, \mathrm{~J} \mathrm{Carvalho}^{3}$, L Almeida Santos ${ }^{3}$, A Ribeiro ${ }^{3}$

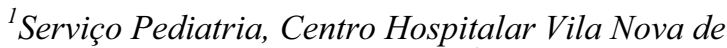
Gaia, Vila Nova Gaia, Portugal, ${ }^{2}$ Serviço Pediatria, Centro Hospitalar Alto Minho, Viana do Castelo, Portugal, ${ }^{3}$ Departamento Pediatria, Unidade Cuidados Intensivos Pediatricos, Hospital S. João, Porto, Portugal
Background and Aims: The acute respiratory distress syndrome (ARDS) is an inflammatory process which develops following a multitude of direct or indirect lung insults, manifested by refractory hypoxemia and respiratory failure, still associated with high morbidity and mortality rates. The aim of this study was to investigate the incidence, predisposing factors, clinical course, management and outcomes in children with ARDS, and to identify variables related with higher mortality.

Methods: We retrospectively reviewed medical records of all children discharged with the diagnosis of ARDS in our tertiary paediatric intensive care unit (PICU), from 2001 to 2005. Those who met the American-European Consensus definition criteria of ARDS were included.

Results: Twenty-nine children were identified as having ARDS, representing $2.7 \%$ of all PICU admission. Median age (interquartile range) was 29 months (14-60.5). The most common predisposing factors were sepsis/septic shock $(34,5 \%)$ and pneumonia (34.5\%). The mean values $( \pm \mathrm{SD})$ of $\mathrm{PaO} 2 / \mathrm{FiO} 2$, oxygenation index and $\mathrm{A}-\mathrm{aDO} 2$ on the 1 st $24 \mathrm{~h}$ were $85.3 \pm 29.1$, $26.9 \pm 11.0$ and $490.7 \pm 109.3$, respectively. The means values $( \pm \mathrm{SD})$ of maximal peak inspiratory pressure (PIP), airway median pressure (MAP) and positive endexpiratory pressure (PEEP) were $38.9 \pm 5.8,20.6 \pm 4.5$ and $7.98 \pm 2.5 \mathrm{cmH} 2 \mathrm{O}$, respectively. Mortality rate was $34.5 \%(n=10)$. Duration of PICU's stay and ventilator treatment was shorter for nonsurvivors $(p<0.05)$.

Conclusions: There were no significant differences in respiratory parameters between survivors and nonsurvivors. We speculate that early mortality is more related to the severity of the underlying disease than with the respiratory failure itself.

\section{HELIOX IN PEDIATRIC EMERGENCY AND CRITICAL CARE SETTING}

\section{A Deep, M Choudhary, P Habibi \\ PICU, St. Mary's Hospital, London, UK}

Background and Aims: Heliox-21 has a lower density than air which allows movement of gas with less driving pressure and better ventilation down to the alveolar level Methods: Cases managed with heliox in emergency department and PICU of a tertiary care hospital.

Results: Heliox in Post-Extubation Stridor: A 10 month old infant was admitted with respiratory failure. Following extubation she developed stridor and was treated with heliox-21. Patient rapidly settled following heliox-21 therapy, stridor score decreased to 4/11 from 9/11 and after 5 hours heliox-21 was stopped. Patient required only 1 dose of nebulised adrenaline and heliox21 was used as the driving gas at a flow rate of $12 \mathrm{l} / \mathrm{min}$. Heliox in Asthma: A 2 year old was admitted with an acute asthma Wood's asthma score was $7 / 10$. He was on high doses of salbutamol and aminophylline. He was started on heliox-21 and after 20 minutes there was an improvement in clinical condition. and reduction in asthma severity score to $4 / 10$. Heliox-21 therapy was continued for 6 hours, requirements for bronchodilators decreased and he did not require ventilation. Heliox in Croup: 2 year old girl presented with marked respiratory distress. Stridor score on admission was $7 / 11$ and 
heliox-21 was commenced immediately in A\&E. There was increase in saturation to $94 \%$ within 10 minutes of starting heliox-21. Stridor score fell to zero after 7 hours of starting heliox and the patient did not require further adrenaline nebulisers.

Conclusions: It has a potential for use as 1 st line for many respiratory conditions in children averting need for PICU.

\section{SURFACTANT INSTILLATION VIA BRONCHOSCOPY IN PRETERM INFANTS WITH RESPIRATORY DISTRESS SYNDROME}

P Biban, M Benedetti, M Soffiati, C Ghizzi, F Zaglia, M Bolognani, P Bonetti, P Santuz

\section{Neonatal and Paediatric Intensive Care Unit, Major City Hospital, Verona, Italy}

Background and Aims: Surfactant therapy is widely used in newborn infants with respiratory distress syndrome (RDS). As a rescue treatment, it generally requires tracheal intubation and mechanical ventilation. We investigated the safety and efficacy of surfactant instillation through a fiberoptic bronchoscope, thus avoiding intubation.

Methods: Preterm newborns $(<36$ weeks GA) with $\mathrm{RDS}$, requiring nasal $\mathrm{CPAP}$ and $\mathrm{FiO} 2>30 \%$ to maintain $\mathrm{SatO} 2>92 \%$, were eligible for the study. After topical anaesthesia (1\% lidocaine) and mild sedation (midazolam $0.1 \quad$ mg.kg-1), a fiberoptic endoscope with operative channel (Wolf $2.5 \mathrm{~mm}$, Pentax $3.5 \mathrm{~mm}$ ) was inserted into the trachea. $200 \mathrm{mg}$. kg-1 of natural surfactant (Curosurf®, Chiesi) were directly instilled into the airway. Immediately after instillation, a short course of manual bagging was provided, in order to favour a better distribution of surfactant. Parental informed consent and approval by the local review committee were obtained.

Results: 13 newborns (6 male) with RDS were enrolled in a 24-month period. Median weight and gestational age (range) were $1470 \mathrm{~g}$ (770-2360) and $31 \mathrm{GA}$ (24-34). The procedure was well tolerated and no complications were observed. In two patients the procedure was briefly interrupted due to mild desaturation episodes. All infants showed a prompt improvement of oxygenation, respiratory distress and radiological findings. 10/13 patients were subsequently managed without the need of tracheal intubation.

Conclusions: We suggest direct instillation of surfactant via bronchoscopy is a feasible, safe and effective technique in newborns with moderate RDS. Larger controlled studies are needed to compare this method with the standard administration by tracheal intubation.

\section{SYNCHRONIZED NONINVASIVE NASAL VENTILATION IN PRETERM NEWBORNS. PILOT STUDY}

C Ramos Navarro, M Sanchez Luna, C Menendez, M L Franco, B Bernardo, E Zamora, S Marin, C López Menchero, V Perez Sheriff

Department of Pediatrics, Hospital General Universitario Gregorio Marañón, Madrid, Spain
Background and Aims: Synchronized nasal ventilation (SNIPPV) could be a useful method to prevent in some situations the need of intubation and minimize the need for prolonged mechanical ventilation in order to reduce ventilator-induced trauma and oxygen toxicity. To analyzed the efficacy and safety of SNIPPV to prevent intubation in RDS of prematurity. The study was designed as a preliminary pilot study prior to a randomized controlled trial.

Methods: Prospective observational study of non invasive respiratory support in preterm newborns with RDS, using a flow sensor synchronizing nasal ventilator, 'Giulia'. Initial settings were: PIP limited at 16-17 cmH2O; PEEP $5 \mathrm{cmH} 2 \mathrm{O}$; Ti $0,35 \mathrm{~s}$; respiratory frequency of back-up of $35 \mathrm{rpm}$; FiO2 adjusted to keep saturations at $88-92 \%$. We analyzed the need of invasive mechanical ventilation as failure of SNIPPV, the incidence of air leak and gastrointestinal side effects.

Results: SNIPPV was used in 8 patients: 6 after surfactant administration during the first 2 hours of live and two as a rescue therapy after extubation failure in nasal-CPAP. The gestational age range from 25 to 34 weeks. Two patients required mechanical ventilation secondary to increased oxygen requirement. 6 patients were successfully ventilated during 48 hours. There were no adverse effects.

Conclusions: SNIPPV using a flow sensor device for triggering in an assist /control can be used in some preterms with RDS. The technique is safe and easy to use. Comments: We are conducting a randomized controlled trial to compare SNIPPV to n-CPAP to prevent invasive mechanical ventilation.

\section{EARLY CONTINUOUS DISTENDING PRESSURE FOR RESPIRATORY DISTRESS SYNDROME IN PRETERM INFANTS. PROSPECTIVE STUDY}

P Chimenti Camacho, E Sanz Lopez, M Sanchez Luna, R Chacón, C Ramos, M Franco, G Zeballos Sarrato, A De L Huerga, M L Serrano, V Perez-Sheriff

Department of Pediatrics, Hospital General Universitario Gregorio Marañon, Madrid, Spain

Background and Aims: The application of a continuous distending pressure has been show to have some benefits in the treatment of preterm infants with respiratory distress syndrome. To determine if apply early continuous distending pressure (CPAP) at delivery prevents intubation and surfactant therapy in preterm infants and to identify adverse effects associated with failure of early CPAP therapy.

Methods: Prospective observational study comprising liveborn infants with birthweight $<1500$ g. Exclusion criteria were respiratory effort absence at birth, congenital malformations and outdoor delivery. CPAP was applied with Neopuff® device immediately after delivery to establish a functional residual capacity, maintained during transport to the NICU and then switch to Infant Flow Driver ${ }^{\circledR}$ Nasal CPAP system. Therapy success was defined as no requirement endotracheal intubation in the first 72 hours of life.

Results: 32 infants received CPAP at delivery room (gestational age 28+6 [25 - 33] weeks; birth weight 
1190 [894 - 1500] g; 66\% male and 34\% female). 9 infants (28\%) required intubation and surfactant therapy. The differences between failure and success groups were: GA, BW, chorioamnionitis prevalence and antenatal steroids therapy; only GA was statistically significant $(p 0,049)$. In early CPAP successful group, none of the infants showed adverse effects as pulmonary air leak, intraventricular haemorrhage, or retinopathy of prematurity.

Conclusions: Respiratory support in premature infants based on early CPAP is a safe method even in extremely premature infants.

\section{RESPIRATORY SUPPORT FROM DELIVERY OF PRETERM NEONATES AND CHRONIC LUNG DISEASE OF INFANCY}

E Sanz Lopez, C Ramos Navarro, M Sanchez Luna, S Fernandez De Miguel, C Lopez Menchero, I Marsinyach Ross, A De La Huerga, V Perez-Sheriff

Department of Pediatrics, Hospital General Universitario Gregorio Marañon, Madrid, Spain

Background and Aims: Mechanical ventilation is a major contributing factor of Chronic Lung Disease of Infancy (CLD). To evaluate the outcome of preterm babies with respiratory distress syndrome (RDS) and to determine weather the need for conventional ventilation could be reduced with the use of nasal CPAP from delivery or the combination of nasal CPAP following Intubation for surfactant administration and extubation (INSURE).

Methods: Retrospective study in the NICU of the HGU 'Gregorio Marañón' (January 2004-December 2005) of all inborn premature infants of less than 32 weeks GA and RDS. We describe 4 respiratory support groups: 1: Oxygen therapy (nasals prongs), 2: Nasal CPAP from delivery, 3: INSURE, 4: Early surfactant followed by mechanical ventilation ( $>2$ hours). Surfactant was given when $\mathrm{FiO} 2>30 \%$ was reached.

Results: 202 infants were analysed and treated as follows: Group 1: 49 (24,3\%), group 2: $30(14,9 \%)$, group 3: $47(23,3 \%)$, and group 4: $76(37,6 \%)$. The rate of air leak was $2 \%, 13,3 \%, 0 \%, 22,4 \%$ for groups $1,2,3$ and 4. Mechanical ventilation was initiated in $23,3 \%$ of group 2 and $17 \%$ of 3 . The rate of CLD: $0 \%, 6,7 \%$, $8,5 \%, 34,2 \%$ for groups $1,2,3$ and 4 . Significant differences in the rate of CLD were found when the diagnostic chorioamnionitis were present $(p=0,000$ Fisher exact test) and between groups 3 and 4 $(p=0,001$.$) . Logistic regression showed that this$ significance could not be explained by GA.

Conclusions: Compared with the group of mechanical ventilation, INSURE was significantly associated to less BPD, independent of the gestational age.

\section{HIGH FREQUENCY OSCILLATORY VENTILATION: FOUR YEARS EXPERIENCE IN A PEDIATRIC INTENSIVE CARE UNIT}

V Anacleto, C Abadesso, P Nunes, E Almeida, H Loureiro, H Almeida

PICU, Department of Paediatrics, Hospital Fernando Fonseca, Amadora, Portugal
Background and Aims: High frequency oscillatory ventilation (HFOV) is an important advance in the treatment of children with respiratory distress. Advantages include less ventilator-induced lung injury and less incidence of air-leak. Our purpose was to describe the experience with HFOV (Sensormedics 3100A) in our unit.

Methods: Retrospective study of all children ventilated in HFOV (January 2002-December 2005). Demographic data, blood gases, ventilatory parameters and complications during conventional ventilation (CV) and HFOV analysed.

Results: Thirty four children ventilated with HFOV, mean age of 5,2 $\pm 7,47$ months (min 0,$3 ; \max 36$ ). Pneumonia diagnosed in 26, bronchiolitis in 5, ARDS in 15 and MODS in 5. Etiologic agent isolated in 22: VSR in $9(26,5 \%)$ and adenovirus in $5(14,7 \%)$. Twenty nine ventilated in $\mathrm{CV}$ before HFOV (mean maximum

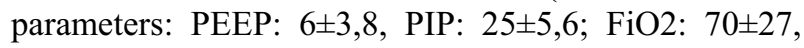
RR: $57,4 \pm 5,6$. HFOV initiated due to persistent hypercapnia with respiratory acidosis in $22(64,7 \%)$ and severe hypoxemia/hypercapnia in 7. Mean maximum parameters in HFOV were: amplitude: $52 \pm 12,6$; MAP $19 \pm 4,5$; frequency 9,7 $\pm 0,9 ; \mathrm{FiO} 2: 74,5 \pm 26,2$ and a mean duration of $4,3 \pm 4,1$ days. Significant improvement in capilar $\mathrm{pH}$ and $\mathrm{pCO} 2$ occurred at 1,6,12 and $24 \mathrm{~h}$ after HFOV onset. $(p<0,05)$. Nine air-leak syndromes occurred (7 pneumothorax: 3 occurring in $\mathrm{CV}$ and 3 during ventilation with inflating bag). Mortality rate was $32,4 \%$.

Conclusions: HFOV represents an additional option for acute respiratory failure, facilitating $\mathrm{CO} 2$ elimination and avoiding aggressive conventional ventilation. It should be used as a protective lung strategy, and not only as a rescue option. No complications directly related with it occurring in our study.

\section{NON-INVASIVE VENTILATION IN A PAEDIATRIC INTENSIVE CARE UNIT}

C Abadesso, E Almeida, P Nunes, C Silvestre, H Loureiro, H Almeida

PICU, Department of Paediatrics, Hospital Fernando Fonseca, Amadora, Portugal

Background and Aims: Non-invasive ventilation (NIV) is being increasingly used in children with acute respiratory failure, preventing complications associated with mechanical ventilation. We have recently introduced NIV for children with acute respiratory failure. This study describes our experience.

Methods: Prospective study of all patients who underwent NIV (November 2005 to March 2006). Demographic data analysed. Heart and respiratory rate, $\mathrm{SaO} 2$, blood gases evaluated before and at 2, 612 , and 24 hours after NIV.

Results: Thirty five patients included (36 NIV trials). Mean age 6,3 $\pm 20,6$ months (median: 1 ; min 0,3; max 120 months). CPAP used in 32 and BiPAP in 4 patients. Diagnosis: bronchiolitis in $26(72,3 \%)$, pneumonia in 7 $(19,4 \%)$ patients. Indications for NIV: acute hypercapnic and hypoxemic respiratory failure (33), apnea (4), acute exacerbation of pulmonary chronic disease (3), upper airway obstruction (2). There was a 
significant improvement in respiratory and heart rates, $\mathrm{pH}, \mathrm{pCO} 2$ at 6,12 and 24 hours after NIV onset $(p<0,05)$ (table). Mean duration of NIV was $51,8 \pm 40,6$ hours. Sedation with chloral hydrate used in $17(47,2 \%)$. Six patients required conventional mechanical ventilation. There were no major complications related with NIV.

Conclusions: NIV can be effective for children and infants with acute respiratory failure, preventing some patients from deteriorating and/or from being ventilated.

\begin{tabular}{|l|c|c|c|c|c|}
\hline & Pre-NTV & $2 \mathrm{~h}$ & $6 \mathrm{~h}$ & $12 \mathrm{~h}$ & $24 \mathrm{~h}$ \\
\hline $\begin{array}{l}\text { Respiratory } \\
\text { ratte (bpm) }\end{array}$ & $59,6 \pm 17,6$ & $50,3 \pm 15,4$ & $47,8 \pm 15,5$ & $46,7 \pm 10$ & $43,7 \pm 10,2$ \\
\hline $\begin{array}{l}\text { Heart rate } \\
\text { (bpm) }\end{array}$ & $160 \pm 24,4$ & $154 \pm 18,0$ & $148,4 \pm 18,3$ & $144 \pm 12,4$ & $141 \pm 16,4$ \\
\hline $\mathrm{pH}$ & $7,28 \pm 0,06$ & $7,32 \pm 0,06$ & $7,33 \pm 0,6$ & $7,35 \pm 0,4$ & $7.37 \pm 0.5$ \\
\hline $\mathrm{pCO} 2$ & $63.1 \pm 11,3$ & $61,9 \pm 12.6$ & $58 \pm 9,7$ & $53,9 \pm 4,7$ & $53 \pm 6,7$ \\
\hline
\end{tabular}

\section{THE ROLE OF HFOV IN PEDIATRIC INTENSIVE CARE - AN ANALYSYS OF THE SITUATION IN THE CZECH REPUBLIC}

D Blazek, P Pavlicek, D Hechtova, K Dlask

Department of Anaesthesiology and Intensive Care Medicine, University Hospital Motol, Prague, Czech Republic

Background and Aims: HFOV was introduced to clinical use in the early eighties and after 1984 first clinical trials on HFOV ventilation were performed. In 1995 HFOV was approved by FDA as a medical tool for treating ARDS in children. Since then several ICU in the Czech Republic uses this ventilatory strategy in treatment of severe respiratory failure in children.

Methods: We used a general questionaire to investigate the use of HFOV in treatment of severe respiratory failure in children in Prague (4 workplaces), Brno, Olomouc and Ostrava. The age spectrum of patients, indications for use of HFOV, how early the method was applied and the efficiency of HFOV were followed. The data from each workplace were processed and results compared.

Results: The experience in use of HFOV in treatment of respiratory failure in the Czech Republic differ. HFOV is a standart part of nearly all treatment protocols in neonatal RDS therapy. The use of the method in higher age groups of patients is slightly different in different ICU's.

Conclusions: The use of HFOV in the Czech Republic is recognised as one of the standards in treatment of severe respiratory failure. More common is its use in neonatal RDS therapy; the criteria, timing and indication of this method in treatment of ALI/ARDS in higher age groups are not in general agreement/united.

\section{HOME-BASED PULMONARY VENTILATION - THE DEVELOPMENT OF HOME CARE VENTILATION PROGRAM IN THE CZECH REPUBLIC}

D Blazek, K Dlask, P Pavlicek, D Hechtova

Department of Anaesthesiology and Intensive Care Medicine, University Hospital Motol, Prague, Czech Republic
Background and Aims: There has been a dramatic progress in the artificial lung ventilation since its origins in the fifties. The application of the newest technical discoveries enabled to develop ventilators providing not only the basic lung ventilation but also allowing for a significant comfort to the patient during prolonged periods of the ventilation. The development of his type of respirators represented the beginning of the era of home-based ventilation.

Methods: The transition from hospital-based to homebased ventilation required to address economic, medical, technical and psychosocial aspects. The centers for education and training of the patient's family members have been formed in the large state and university hospitals.

Results: It was shown that: a) $\mathrm{HCV}$ is more economic than long term hospitalization. b) HCV represents a significant improvement in the quality of life of a chronically ventilated patient. The program of home care ventilation is being developed in the Czech Republic for last seven years. Our PICU program involves currently five permanet patients and many others are beeing enrolled all over the Czech Republic. The most common diagnosis are neuromuscular disorders, cystic fibrosis, sleep apnea syndrom and deformities of the skeleton, posttraumatic spinal chord lesion etc.

Conclusions: The program of the $\mathrm{HCV}$ is developing succesfully. We have overcame the initial legislative and funding problems. The HCV became an accepted trend in the Czech Republic and its importance is due to all advantages on a rise.

\section{PREDICTING EXTUBATION OUTCOME IN PREMATURE INFANTS USING A WEB-BASED ARTIFICIAL NEURAL NETWORK}

\author{
M Mueller ${ }^{1}$, C L Wagner ${ }^{2}$ \\ ${ }^{1}$ Department of Biostatistics, Bioinformatics and \\ Epidemiology, Medical University of South Carolina, \\ Charleston, SC, USA, ${ }^{2}$ Department of Pediatrics, \\ Medical University of South Carolina, Charleston, SC, \\ USA
}

Background and Aims: Decision-making whether or not to extubate a premature infant is extremely complex and based largely on clinical experience. A decisionsupport tool can be used to aid inexperienced clinicians in their decision-making and assist in decreasing the number of false-positive (i.e. infants extubated too early) and false-negative cases. Aim: To test a previously developed web-based decision-support tool employing an artificial neural network (ANN).

Methods: An ANN developed for the prediction of extubation outcome in mechanically ventilated premature infants was tested using data from 102 infants with birth weights (BW) ranging from 590-3200 grams. Proportions of failed and successful extubations were compared for clinicians and the ANN for the entire sample and by BW categories $(<900 \mathrm{~g}, \geq 900$ and $\leq 1500 \mathrm{~g},>1500 \mathrm{~g}$ ). 
Results: 80 infants were determined as ready for extubation. Of those, 13 infants had to be reintubated within 48 hours of extubation. Overall the ANN predicted $84 \%$ correctly, $79 \%$ successful extubations and $5 \%$ failures. $11 \%$ were incorrectly classified as successful and $5 \%$ as failures. Overall agreement between experienced clinicians and the ANN was $63 \%$ $(\mathrm{p}<0.0001)$; for infants with BW $<900 \mathrm{~g}$ the agreement was $58 \%$ compared to $44 \%$ for birth weight $>1500 \mathrm{~g}$ and $84 \%$ for infants with BW between $900 \mathrm{~g}$ and $1500 \mathrm{~g}$.

Conclusions: The ANN as a predictive tool can provide decision-support to inexperienced clinicians in determining when to extubate a premature infant in any NICU. Further enhancement of the ANN predictions through training with newly collected data for BW below $900 \mathrm{~g}$ and above $1500 \mathrm{~g}$ will create potential for integration with broader clinical management systems.

\section{EFFECT OF PARTIAL LIQUID VENTILATION ON PULMONARY RECRUITMENT IN ASPHYCTIC PRETERM LAMBS}

\author{
N Martínez ${ }^{1}$, F J Alvarez ${ }^{2}$, A Alvarez ${ }^{1}$, \\ F Goñi de Cerio $^{1}$, J Bilbao ${ }^{3}$, M C Rey-Santano ${ }^{2}$, \\ V E Mielgo ${ }^{2}$, M A Gomez-Solaetxe ${ }^{4}$, A Valls I Soler ${ }^{2,5}$, \\ E Hilario $^{1}$ \\ ${ }^{I}$ Department of Cell Biology and Histology, School of \\ Medicine, University of Basque Country, Basque \\ Country, Spain, ${ }^{2}$ Research Unit on Pediatrics, Cruces \\ Hospital, Basque Country, Spain, ${ }^{3}$ Department of \\ Biostatistics, School of Medicine, University of Basque \\ Country, Basque Country, Spain, ${ }^{4}$ Department of \\ Biomedical Engineering, University of Basque Country, \\ Basque Country, Spain, ${ }^{5}$ Department of Pediatrics, \\ School of Medicine, University of Basque Country, \\ Basque Country, Spain
}

Background and Aims: Partial liquid ventilation is an successful experimental therapy used in the respiratory distress syndrome in immature newborns. To investigate the influence of partial liquid ventilation on pulmonary recruitment and on brain damage in an experimental model of asphyctic preterm lambs.

Methods: 12 fetal lambs of 125-133 days of developmental age (term: 145 days) were subjected to hypoxic-ischemic event (HI) by partial occlusion of umbilical cord during 60 minutes. Animals were randomly allocated to: Control group, sacrificed immediately after $\mathrm{HI}$ event (Control, $\mathrm{n}=3$ ), controlled mechanical ventilation group (CMV, $n=6)$ and partial liquid ventilation group (PLV, $\mathrm{n}=3$ ) treated with 30 $\mathrm{ml} / \mathrm{kg}$ of perfluorodecalin and both sacrificed after 3 hours. Lungs and brains were fixed by perfusion and embedded in paraffin wax. Lungs sections were stained with hematoxylin-eosin for morphometric studies (percentage of air space, alveolar surface, alveolar perimeter and wall thickness). Brain sections were immunostained by TUNEL method for apoptotic cell count. One-factor ANOVA was performed $(\mathrm{p}<0.05)$.

Results: In the lung, both ventilatory therapies showed significant differences in all the studied parameters in comparison to the control group. Moreover, PLV group improved the alveolar expansion in relation to the CMV group (alveolar surface: $3925 \pm 1322$ vs. $2146 \pm 1010$ $\mu \mathrm{m} 2$; alveolar perimeter: $283 \pm 61$ vs. $249 \pm 65 \mu \mathrm{m})$. In relation to brain lesion, CMV group showed a higher apoptotic cell count $(83 \pm 11)$ in comparison to both control (18 \pm 8$)$ and PLV groups $(34 \pm 12)$.

Conclusions: Our results suggest that PLV improves the alveolar recruitment without adverse effect on the brain injury in preterm lambs. Grants: 9/UPV00077.32715330-2003.

\section{HIGH FREQUENCY JET VENTILATION IMPROVES GAS EXCHANGE IN EXTREMELY PREMATURE INFANTS WITH EVOLVING BRONCHOPULMONARY DYSPLASIA}

R Plavka ${ }^{1}$, M Dokoupilova ${ }^{1}$, L Pazderova ${ }^{1}$, P Kopecky $^{1}$, V Sebron ${ }^{1}$, M Zapadlo ${ }^{1}$, M Keszler ${ }^{2}$

${ }^{l}$ Department of Neonatology, General Faculty Hospital, Prague, Czech Republic, ${ }^{2}$ Division of Neonatology, Georgetown University, Washington, DC, USA

Background and Aims: Extremely preterm infants often develop chronic lung disease (CLD) characterized by heterogeneous aeration, poorly supported, floppy airways and air trapping. High-frequency jet ventilation (HFJV) with high end-expiratory pressure (optimal lung volume strategy, OLVS) may improve airway patency, lead to better gas distribution, improve gas exchange and facilitate extubation. To explore, in a pilot trial, the effect of HFJV on oxygenation, ventilation, and ease of extubation in preterm infants with evolving CLD and refractory respiratory failure (RRF).

Methods: From 9/02 to 10/04 twelve episodes of RRF developed in 10 ventilated extremely immature infants with evolving CLD (10 on conventional and 2 on highfrequency oscillation). Chorioamnionitis was confirmed in all infants, patent ductus arteriosus was ligated in 5 patients and Ureaplasma urealyticum was cultured from trachea in 4 patients. HFJV (Life Pulse, Bunnell Inc) with OLVS was initiated when oxygenation index (OI) $>10$ or tidal volume $(\mathrm{Vt}) \ngtr 7 \mathrm{ml} / \mathrm{kg}$ were required to maintain $\mathrm{PaCO} 2<60 \mathrm{mmHg}$. Inspiratory time 0.02 and frequency $310-420 / \mathrm{min}$ were set initially with adjustment of pressure amplitude to keep $\mathrm{PaCO} 2$ 45-55 $\mathrm{mmHg}$. Ventilatory stabilization and weaning from mechanical ventilation with extubation to nasal CPAP were the goals of this approach. Gas exchange data were analyzed by ANOVA for repeated measures.

Results: Ten patients on 11 occasions of RRF were extubated to nasal CPAP successfully in a median of 15.5 days. Nine of ten patients survived (1 died of Cantrell's Pentalogy), all required supplemental $\mathrm{O} 2$ at 36 weeks. $\mathrm{PaCO} 2$ fell within 1 hour after the initiation of HFJV and OI decreased by 24 hours. Both remained significantly lower until successful extubation $(\mathrm{p}<0.02)$.

Conclusions: Compared to conventional ventilation or HFOV, HFJV used with OLVS improves gas exchange and may facilitate weaning from MV in extremely immature infants with evolving CLD. These encouraging pilot data support a larger clinical trial. 
RESPIRATORY DISTRESS SYNDROME IN NEONATAL INTENSIVE CARE UNIT

\section{T Neskovska, R D Stojkovska}

Department of Neonatology, City Hospital, Kumanovo, Macedonia

Background and Aims: Respiratory distress syndrome (RDS) is one of the most common pathologies in neonatal ICU and a significant cause of neonatal mortality. Elaboration of RDS in newborns according to the etiology, treatment and outcome.

Methods: Newborns delivered at the Gynaecology and Obstetrics Ward in Kumanovo during the period of 2003 and 2004. Clinical, laboratory, microbiological and radiology methods.

Results: 3365 newborns were treated at the Nursery Ward. $225(6.68 \%)$ were found to suffer from RD. According to aetiology they have been categorized in the following way: 1 . Pulmonary origin *RDS through immaturity $-158(70.22 \%) *$ RDS through co natal Bronchopneumonia - $10(4.44 \%) *$ Transitory tachypnea - $4(1.77 \%) *$ Aspiration Bronchopneumonia - 3 (1.33\%) *Labour asphyxia - 32 (14.22\%) 2. Extra-pulmonary origin *Phrenic hernia - $2(0.88 \%) *$ Cardiopathio congenita - $2(0.88 \%) *$ Sepsis - 14 (6.22\%) Apart from applying appropriate therapy, all newborns had undergone oxygen-therapy in order to meet the newborn needs and the severity of the disease. The main goal in treating newborns suffering from RD was maintaining acid-base status, saturation $\mathrm{SaO} 2$, from $88-92 \%$ at the initial period up to $92-98 \%$. In order to achieve satisfactory level, heated and moistened oxygen was administrated by using special suit and mask. Out of the total number of newborns suffering from RDS, 178 (79.11\%) were sent to tertian level for further treatment. Death rate at RD is high and counts $83.9 \%$.

Conclusions: RDS in the examined population was common cause for admission to the ICU and a cause for death as well.

\section{SUCROSE 24\% DURING HEELSTICK LESS EFFECTIVE FOR INFANTS BEYOND NEONATAL AGE?}

H te Beest, I van't Wout, M van Dijk

Pediatric Surgical Intensive Care Unit, Erasmus MCSophia Children's Hospital, Rotterdam, The Netherlands

Background and Aims: Oral sucrose has a known analgesic effect in (premature) neonates during heelstick. Contradictory results have been reported concerning the efficacy of sucrose during painful procedures in older infants. We conducted a study aimed to determine the usefulness and efficacy of sucrose in neonates and infants younger than 4 months on our Pediatric Surgical Intensive Care Unit, a group which comprizes $46 \%$ of total patients.

Methods: Eligible children received $1 \mathrm{ml}$ of sucrose $24 \%$ through a syringe in combination with a pacifier 2 minutes before heelstick. During the procedure, a nurse assessed pain using the COMFORT Behavior Scale and noted heart rate and oxygen saturation (registered every 30 seconds) for comparison with baseline values (mean of previous 6 hours). COMFORT behavior scores exceeding 16 indicate pain.

Results: During 10 weeks 38 assessments were performed in 17 infants (4 girls and 13 boys) with mean postnatal age 24 days (range 2 to 108 days). The median pain score was 14 (range 9 to 24). Pain scores exceeded 16 , and thus indicated pain, in 11 observations $(29 \%)$, in three of which the infant underwent suctioning just before or during the procedure. Median heart rate increase was 16; oxygen saturations did not change substantially. The correlation between postnatal age and mean COMFORT behavior score during heelstick was $0.59(95 \%$ CI 0.15 to $0.83, \mathrm{n}=17)$.

Conclusions: Administering sucrose before heelstick to infants seems less effective than in neonates. However, our sample was small and we will need more data to confirm our observations.

\section{TREATMENT OF HYPERGLYCAEMIA IN CRITICALLY ILL CHILDREN}

S B Brand, M M van de Polder, J J Verhoeven, K F M Joosten

Department of Pediatric Intensive Care, Erasmus MCSophia, Rotterdam, The Netherlands

Background and Aims: To study current strategies of national PICU's in treatment of hyperglycaemia in critically ill children. To design a protocol for treatment of hyperglycaemia with insulin for critically ill children. Methods: Survey by a questionnaire sent to the 8 PICU's in the Netherlands. Literature search of protocols used in adult studies. Retrospective analysis of children treated with insulin in the past years on our PICU.

Results: In 2 of the 7 PICU's a protocol was present. Treatment starts in 4 PICU's after two blood glucosemeasurements $>10 \mathrm{mmol} / \mathrm{l}$, in one $>8,3 \mathrm{mmol} / 1$ and in one $>11 \mathrm{mmol} / \mathrm{l}$. In 6 of the 7 PICU's a standard amount of glucose was given on admission. The start doses of insulin was in 5 PICU's $0.02 \mathrm{IU} / \mathrm{kg} / \mathrm{hr}$, in one PICU between 0,05 and $0,1 \mathrm{IU} / \mathrm{kg} / \mathrm{hr}$ and in one PICU the start doses varied. In 6 of the 7 PICU's control of blood glucose after start treatment was done after 1 hour All centres are willing to participate in a national (nurse driven) protocol. The final design of our protocol consisted an age dependent standard glucose intake (2-6 $\mathrm{mg} / \mathrm{kg} / \mathrm{min}$ ), an insulin starting dose depending from the initial glucose level $(0,02-0,05 \mathrm{IU} / \mathrm{kg} / \mathrm{hr})$. The dose of insulin was adjusted depending from the decrease or increase of the glucose level.

Conclusions: A protocol was designed based on standard glucose intake and varying the amount of insulin depending from the level of glucose and the decrease and increase of glucose levels. Most PICU's are willing to participate in a national nurse driven protocol.

\section{ESTABLISHING A KNOWLEDGE CENTRE FOR BREASTFEEDING INFANTS WITH SPECIAL NEEDS}

\section{R Maastrup}


Knowledge Centre for Breastfeeding Infants With Special Needs, Rigshospitalet University Hospital, Copenhagen, Denmark

Background and Aims: Premature infants, sick newborns, infants and children with a variety of diagnoses have problems in establishing breastfeeding and maintaining breastfeeding. The National Hospital in Denmark, Rigshospitalet, is establishing a Knowledge Centre for Breastfeeding Infants with Special Needs. Successful fundraising has made a 3 years project possible.

Methods: The Knowledge Centre gather, systematize and develop knowledge and experiences both at the Rigshospitalet as well as national and international. Together with literature searches this will be the basis for guidelines in breastfeeding infants with special needs. In cooperation with the neonatal and paediatric departments the Knowledge Centre will develop practice in Rigshospitalet that care, treatment and counselling in relation to breastfeeding infants with special needs, will be uniform, evidence based and off high quality. The Knowledge Centre will identify areas off research and bring up to date the latest knowledge in the area. One topic of interest is breastfeeding premature infants still in need off CPAP-treatment. The homepage off the Knowledge Centre will ensure knowledge sharing.

Results: Establishing the Knowledge Centre began September 2005.

\section{VALIDATION OF THE IRMA TRUPOINT BLOOD ANALYSIS SYSTEM FOR USE IN NEONATAL/PEDIATRIC ECMO APPLICATIONS}

R Groenewegen, J Wierema, D Tibboel

PICU, Erasmus Medical Center, Sophia Children's Hospital, Rotterdam, The Netherlands

Background and Aims: Extracorporeal Membrane Oxygenation (ECMO), a high technology intervention for newborns and children suffering from severe cardiopulmonary disorders is associated with significant morbidity. Close surveillance of blood gases and electrolytes is required to ensure physiologic function. An evaluation was conducted to assess the bedside IRMA TRUpoint ${ }^{\circledR}$. Blood Analysis System (NJ, USA), when used by nursing and medical staff to monitor ECMO patients.

Methods: Blood collected from neonatal and pediatric ECMO patients was analyzed for blood gases and electrolytes using the TRUpoint and the laboratory reference analyzer, ABL Radiometer (Copenhagen, Denmark). Blood gas data were evaluated by linear regression, electrolytes for statistical difference $(\mathrm{p}<0.05)$ by Student's t-test.

Results: See Table.

Conclusions: Blood gases and electrolytes all show strong agreement with the laboratory analyzer. Results from critical tests can be used to assess ECMO patient status and modulate therapeutic strategies in a similar manner whether the result is from the TRUpoint or the laboratory. One major advantage of using a bedside device is to obtain data within minutes from blood sampling. This eliminates delays inherent to sending samples for laboratory analysis, allowing faster response time for critical interventions.

\begin{tabular}{|l|c|c|c|c|c|c|}
\hline \multicolumn{1}{|c|}{ Analyte } & n & Mean & Range & Bias & r & p \\
\hline $\mathrm{pH}$ & 43 & 7.41 & $7.27-7.54$ & -0.02 & 0.960 & \\
\hline $\mathrm{PCO}_{2}(\mathrm{kPa})$ & 41 & 5.13 & $3.39-8.28$ & 0.36 & 0.969 & \\
\hline $\mathrm{PO}_{2}(\mathrm{kPa})$ & 40 & 16.38 & $8.59-46.65$ & 1.73 & 0.995 & \\
\hline $\mathrm{Na}^{+}(\mathrm{mmol} / \mathrm{L})$ & 21 & 136 & $132-143$ & 5.3 & & 0.946 \\
\hline $\mathrm{K}^{+}(\mathrm{mmol} / \mathrm{L})$ & 19 & 3.72 & $3.10-4.20$ & 0.15 & & 0.733 \\
\hline $\mathrm{Ca}^{++}(\mathrm{mmol} / \mathrm{L})$ & 19 & 1.17 & $0.82-1.29$ & 0.18 & & 1.000 \\
\hline
\end{tabular}

\section{NASAL CONTINUOS POSITIVE AIRWAY PRESSURE (N-CPAP)}

\section{B Pallesgaard, L T Larsen}

\section{NICU, Hvidovre Hospital, Copenhagen, Denmark}

Background and Aims: In Denmark infants with lung injuries and breathing problems have previously been treated with N-CPAP for many years. Even extreme premature infants and infants with severe lung problems can be treated with N-CPAP. Respirator is often avoided when using N-CPAP or only used for a short time if the infant needs Surfactant. Mechanical Ventilation is practically never used more than one week.

Methods: Our N-CPAP system is almost like infantflow just simpler and not as heavy and big. The N-CPAP treatment is often introduced with $6-8 \mathrm{~cm}$ of water or flow $12-13 \mathrm{~L} / \mathrm{min}$ and reduced to $4 \mathrm{~cm}$ or $8 \mathrm{~L} / \mathrm{min}$ to decline the treatment.

Results: The treatment with N-CPAP is more gentle for the infants lungs. In Denmark infants seldom have Broncho-pulmonal-dysplasia - not even extreme premature infants.

Conclusions: Socially it is easier for the parents to handle their infant - regarding bathing, changing diaper or to sit with their infant. Psychologically N-CPAP is more gentle for the infant because it isn't medicated in any way. It can move freely and has very little or no pain and discomfort.

\section{PROBLEM SOLVING SKILLS RELATED WITH BABY CARE OF MOTHERS WHO HAVE NORMAL AND PREMATURE NEWBORNS}
S Kuguoglu ${ }^{1}$, N Dede Çinar $^{2}$, A Ergun ${ }^{3}$
${ }^{I}$ Department of Pediatric Nursing, Marmara University College of Nursing, Uskudar, Istanbul, Turkey
${ }^{2}$ Department of Pediatric Nursing, Sakarya University School of Health Sciences, Sakarya, Turkey
${ }^{3}$ Department of Community Health Nursing, Marmara
University College of Nursing, Uskudar, Istanbul,
Turkey

Background and Aims: In the literature it is important for mothers to problem solving related with taking care of their newborns. Because of that, nurses have to identify if and when mothers need support after the assessment of the care ability of mothers performed by nurses. The purpose of this study was to assess and compare the problem solving skills related with baby care of mothers who have normal or premature newborns. 
Methods: This study is a descriptive and comparative study. The research to place at the government hospital in Sakarya. The samples in the research were volunteer participant mothers who have normal neonates and premature newborns. All of the test subjects were healthy newborns, normal or premature. There were 43 of normal newborns and 43 of premature newborns chosen for the study. Data was obtained using an 'Introductory Information Form' and 'Problem-Solving Skills Assessment Questionnaire: How I Deal with Problems Regarding Care of my Baby'.

Results: In a test taken by 43 woman with normal newborn babies. The average points scored reached $198.16 \pm 19.57$. In the same test taken by 43 women with premature born babies, the average points scored was $157.18 \pm 35.23$. Significant difference was found between two groups $(\mathrm{t}=6.66 ; \mathrm{p}=0.03)$.

Conclusions: Mothers who have premature babies need more support to develop their problem solving skills regarding care of their baby.

\section{THE EFFECT OF CHRONIC FETAL UNDERNUTRITION AND HYPOXIA ON FETAL PLASMA GLUCOCORTICOIDS}

T Mulder ${ }^{1}$, E Villamor $^{1}$, R Hermans $^{2}$, Z Gura $^{1}$, C E Blanco ${ }^{1}$

${ }^{1}$ Department of Pediatrics, ${ }^{2}$ Department of Pharmacology and Toxicology, Maastricht University, Maastricht, The Netherlands

Background and Aims: Fetal growth restriction is associated with chronic fetal under nutrition and hypoxemia and is known to result in life long alterations in HPA and other hormone axis (2004, Fowden \& Forhead). Aim: To study the effect of chronic under nutrition and hypoxia on the fetal response to acute stress late in gestation.

Methods: Three groups of fertilised eggs of White Leghorn chicken were incubated: 1) Chronic hypoxia group: incubation in a hypoxic $(15 \% \mathrm{O} 2)$ environment. 2) Malnutrition group: incubation after removal of $10 \%$ of the egg's albumin in a normoxemic environment. 3) Control group. At day 18, the eggs were exposed to a 5 -minute challenge of acute hypoxia (5\% of oxygen). Plasma corticosterone and catecholamines were measured at baseline and after the acute hypoxic challenge.

Results: Chronic hypoxia resulted in elevated catecholamine levels in baseline conditions (epinephrine 2.7 vs $0.7 \mathrm{nmol} / \mathrm{l}$ ) whereas corticosterone level was decreased ( 15.3 vs $25.9 \mathrm{nmol} / \mathrm{l})$. After the acute hypoxic insult catecholamine levels increased markedly without any difference between the groups. Corticosterone increased also, however to a significant lower level in the chronic hypoxia group (18,9 vs $34.6 \mathrm{nmol} / \mathrm{l})$. Chronic under nutrition had no influence on the stress response.

Conclusions: Chronic fetal hypoxemia resulted in elevated baseline catecholamine levels and decreased corticosterone levels. In response to acute hypoxia catecholamine release was unaffected but corticosteron release was blunted. Chronic under nutrition did not affect baseline levels nor the response to acute hypoxia.
THE EFFICACY OF INTRA-TRACHEAL RHDNASE IN THERAPY IN THE TREATMENT OF VENTILATED CHILDREN WITH STATUS ASTHMATICUSAND BRONCHIOLITIS WITH PERSISTENT RESPIRATORY ACIDOSIS

M McDougall, S Colthurst, A Durward, S M Tibby, I A Murdoch

\section{Evelina Childrens Hospital, London, UK}

Background and Aims: Mucous plugging of the airways may play a central role in ventilated patients with status asthmaticus and bronchiolitis where respiratory acidosis is progressive despite optimising conventional therapy. In both conditions airway secretions may be rich in extracellular DNA.1,2. The aim of this study was to demonstrate if the mucolytic action of intra-tracheal DNAse could improve gas exchange in a cohort of ventilated patients with refractory hypercarbia $(\mathrm{pCO} 2>8 \mathrm{kPa}, \mathrm{pH}<7.2)$.

Methods: Retrospective review of patients meeting criteria.

Results: See chart.

Conclusions: Intratracheal DNAse therapy was associated with a significant reduction in arterial $\mathrm{pCO} 2$ in a cohort of patients with severe airway obstruction and respiratory acidosis in both asthma and bronchiolitis. This is most likely due to enhanced mucous clearance and hence reduction in airflow obstruction. Further studies are warranted.
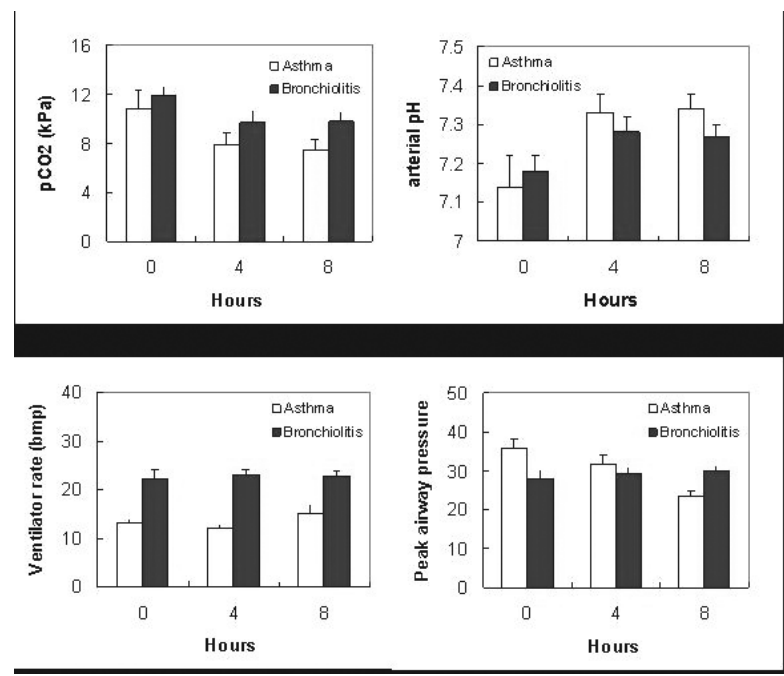

\section{INTRAVENOUS SALBUTAMOL THERAPY AND KETONURIA: A DOSE DEPENDENT METABOLIC ADVERSE EFFECT}

P Maheshwari, R Chhabra, P Ramnarayan

PICU, St Mary's Hospital, London, UK

Background and Aims: To study the metabolic adverse effects of intravenous salbutamol in acute severe asthma.

Methods: Consecutive referrals of acute severe asthma to a regional transport service, who were on intravenous salbutamol were studied. Patients were divided in 2 groups based on salbutamol infusion rate (group 1: $\leq 3$ $\mathrm{mcg} / \mathrm{kg} / \mathrm{min}$, group 2: $3-5 \mathrm{mcg} / \mathrm{kg} / \mathrm{min}$ ). Heart rate, respiratory rate, blood glucose levels, serum bicarbonate 
levels, base deficit, serum lactate and urine ketones on dipstix at 4 and 12 hours after starting salbutamol infusion were recorded.

Results: Both the groups were comparable with regard to age and weight. All patients in both the groups had received hydrocortisone; 2 patients in group 1 and 7 in group 2 were on aminophylline infusion; 2 patients in group 1 and 8 patients in group 2 had received magnesium sulphate bolus intravenously. There was significant ketonuria in group 2 compared to group 1 (Fischer exact test, $\mathrm{p}$ value $<0.01$ ). There was no significant difference between the groups with respect to aminophylline infusion or magnesium sulphate ( $\mathrm{p}$ value $>0.05$ ). Blood glucose and base deficit were increased although there was no significant difference in both the groups. There was no significant difference in heart rate ( $p$ value 0.319$)$ and respiratory rate ( $p$ value 0.269 ) in both the groups. There was no significant difference in serum bicarbonate ( $\mathrm{p}$ value $>0.05$ ) and serum lactate ( $p$ value $>0.05$ ) at 4 and 12 hours after starting salbutamol infusion.

Conclusions: These findings suggest that salbutamol infusion in doses $>3 \mathrm{mcg} / \mathrm{kg} / \mathrm{min}$ causes significant ketonuria associated with acidosis.

\section{ENDOTRACHEAL ANESTHESIA IN A CHILD WITH BRONCHIAL ASTHMA}

\section{R D Stojkovska, L P Neskovska, S M Stankovic}

Department of Paediatrics, Public Hospital, Kumanovo, Macedonia

Background and Aims: Bronchial asthma, a frequent disease in the childhood is a serious problem in anesthesiology. The basic problem is endotracheal intubations as a possible provocative factor of bronchospasam in the tracheobronchial three.

Methods: The aim of this study is to present a case a of six year old boy, bronchial asthma patient of 4 years, who had to be urgently operated on appendix. The patient at the moment has been in a symptom-free period, the last asthmatic episode and the last glucocorticoid administration had been 3 months ago.

Results: The patient was premeditated by Amp. Atropin 0,3 mg, Amp. Phenergan $20 \mathrm{mg}$, Amp. Dalantin $40 \mathrm{mg}$; i.m. single dose of Solu-Cortef $100 \mathrm{mg}$ was applicated i.v. to prevent bronchospasm during the intubation. The intubations was done in deep anesthesia with Thiopental $100 \mathrm{mg}$ and Pavulin $1 \mathrm{mg}$ (initial barbiturate narcosis) 30 minutes after the premedication. $3 \%$ halothane was given as an initial dosage, $1-2 \%$ was given as a maintaining dosage. The appendectomy was performed with in $30 \mathrm{~min}$. While the duration of the narcosis was 50 minutes. The extubation was done in deep anesthesia by Halothane - at the end of inspirium to prevent laryngo-spasm. Due to preventive steps there was not any attack of bronchospasam during the operation and the postoperative course.

Conclusions: The patients with bronchial asthma are serious problem for the Anaesthesiologists. Preventive steps (adequate premedication and deepness of the narcosis, the moment of the etubation) are necessary to avoid respiratory complications.

\section{EXERCISE HYPOTHESIS IN ASTHMA IN} YOUNG ADOLESCENTS

E Vlaski ${ }^{1}$, K Stavric $^{2}$, R Isjanovska ${ }^{3}$, M Kimovska $^{4}$, L Seckova $^{1}$

${ }^{1}$ Department of Pulmonology, University Children's Hospital, Skopje, Macedonia, ${ }^{2}$ Department of Immunology, University Children's Hospital, Skopje, Macedonia, ${ }^{3}$ Institute of Epidemiology and Biostatistics with Medical Informatics, Skopje, Macedonia

${ }^{4}$ Department of Intensive Care, University Children's Hospital, Skopje, Macedonia

Background and Aims: Regarding the suggestion that regular exercise and physical fitness are protective against asthma, the study was aimed to explore the impact of sedentary regimen, through duration of television (TV) watching, on asthma in young adolescents.

Methods: Self-reported data obtained through ISAAC phase 3 questionnaire on asthma and environmental questionnaire from 3,026 children aged 13/14 years from randomly selected schools in Skopje, the capital of Macedonia, were analysed. The relationship between wheeze or asthma 'ever' (W or A), current wheeze (W12), current speech-limiting wheeze (WS12), current exercise-induced wheeze (EW12), current dry nightcough (NC12) and daily TV watching, adjusted for body mass index, indoor air pollution, cat and dog ownership, was investigated. Odds ratios in logistic regression for statistic analysis of data were performed.

Results: A positive association between TV watching more than 3 hours daily and W12 (OR 1.38, 95\% CI 1.04-1.82 $\mathrm{p}=0.026$ ), and EW12 (OR 1.37, 95\% CI 1.10$1.71 \mathrm{p}=0.006$ ) was established. A significant association between TV watching and asthma severity or everdiagnosed asthma was not found.

Conclusions: It seems that being sedentary and lack of physical fitness increase the risk of current wheeze and exercise-induced wheeze in young adolescents.

\section{ATOPIC DERMATITIS AND FAMILY HISTORY OF ALLERGIC DISEASES IN ASTHMATIC AND NON ASTHMATIC CHILDREN}

I B Sokolovski, J B Minov

Department of Pediatrics, Public Health Center, Skopje, Macedonia

Background and Aims: Bronchial asthma is the most frequent chronic diseases in childhood. It is well known fact that infants suffered from atopic dermatitis (AD) and/or positive family history of allergic diseases have higher risk to develop asthma. The aim of our study is to compare the prevalence of $\mathrm{AD}$ and positive family history of allergic diseases in children with asthma and nonasthmatic children.

Methods: Personal history of AD and family history of allergic diseases were compared at 60 children with asthma (36 boys and 24 girls) aged 10-14, mean age $12,5(3,8)$ and equal number of nonasthmatic children matched by sex and age. The data obtained were statistically analysed by descriptive and inferential methods. 
Results: AD precedes asthma in $45 \%$ of children with asthma ( $46 \%$ of the boys and $42 \%$ of the girls). The prevalence of $\mathrm{AD}$ was significantly higher in asthmatic compared to nonasthmatic children $(45 \%$ vs $11 \%$ $\mathrm{p}<0,01)$. Positive family history of allergic diseases had $37,5 \%$ of children with asthma (38,5 of the boys and 35,7 of the girls) and its prevalence was significantly higher compared to nonasthmatic children $(37,5 \%$ vs $2,5 \%, \mathrm{p}<0,01)$.

Conclusions: Our results confirm the role of $\mathrm{AD}$ and positive family history of allergic diseases as risk factors for childhood asthma.

\section{ALLERGIC RHINITIS IN CORRELATION WITH ASTHMA BRONCHIALE IN CHILDHOOD}

\section{S V Stankovic}

Department of Paediatrics, General Hospital, Kumanovo, Macedonia

Background and Aims: The aim of the study is to present the incidence of the allergic rhinitis in correlation with the asthma bronchiale in childhood.

Methods: The date for our investigation were received from the medical documentation at The Paediatric Pulmonology Ambulance in Kumanovo. The diagnosis was determined on the basis of anamnesis, clinical and laboratory examinations according standard diagnostic criteria from diagnosing allergic rhinitis and asthma bronchiale.

Results: We monitored 165 patients (from 1 to 14 years) with diagnosed asthma bronchiale. The majority of them 89 or $53,9 \%$ had additional symptoms of allergic rhinitis. In 26 or $29,2 \%$ of the cases had both disorders appeared at the same time. 47 or $52,8 \%$ of the patients get allergic rhinitis first and 16 or $18 \%$ asthma. The most common allergic rhinitis was found in age group of 6-14 years.

Conclusions: Asthma bronchiale is frequently accompanying by allergic rhinitis. Our results correspond with the literature, it is proved that allergic rhinitis is precipitating factor for asthma bronchiale. Early diagnosis of allergic rhinitis is of a great importance due to tamely undertaking of preventive measures and treatment of allergic rhinitis as real risk for development of asthma bronchiale.

\section{CONTROLLING PAEDIATRIC ASTHMA - OUR EXPERIENCE}

S V Stankovic, A S Sulejmani, M S Mihajlovska, R D Stojkovska

Department of Paediatrics, General Hospital, Kumanovo, Macedonia

Background and Aims: Good compliance and education the children and their parents are very important for controlling child asthma. We want to present our results of 8 year's work in paediatric asthma following the GINA guidelines.

Methods: We selected our patients in two groups to base oneself on monitoring their asthma control. Results: In first group had 137 children with nearly controlled asthma. There were good compliance with themselves asthma management plan, by regular monthly visits, ongoing education and working together with children and their parents. In second group had 93 children with uncontrolled asthma. 37 of them had not any daily anti-inflammatory therapy, 29 had inappropriate inhalation technique and 25 didn't use spacer or face mask. In 41 patients therapy didn't correlate with asthma severity. In 19 cases was found parents fear of side effects, in 33 had financial difficulties in families that limited therapy. Also in 17 case persisted in keeping their pets and 39 had parents' smokers. In 43 patients were identified more then one factor for uncontrolling asthma. In I group we noted regular visits in all patients (at 1-6 month) but in II group $79 \%$ patients went to doctor only worsening of them disease. PEF variability were less then $20 \%$ (I group) versus greater then $20-30 \%$ (II group). In I group we found diminished emergency visits (by $71 \%$ ) and hospitalizations (by 49\%) in relation II group during the same period. That showed significantly reduction cost of care in I versus II group.

Conclusions: This study demonstrated that good doctorpatient partnership is the key to controlled child asthma.

\section{NASAL AND BLOOD EOSINOPHILIA IN CHILDREN WITH BRONCHIAL ASTHMA, SEASONAL AND PERENNIAL ALLERGIC RHINITIS}

R M Markova, R T Kabakchieva, P I Perenovska

Pediatric Clinic, University Hospital 'Alexandrovska', Sofia, Bulgaria

Background and Aims: Allergic diseases are a major reason for blood eosinophilia among children and adult population. Cytological assessment of nasal smear and determination of eosinophil count in differential analysis of blood are important laboratory methods in diagnostic approach of allergic diseases.

Methods: Children were divided into three groups. First group included 37 patients with bronchial asthma with mean age 5.3 years (mean eosinophil percentage in nasal smear- $4 \%$ and $2 \%$ eosinophils in blood differential analysis). Second group was formed of 11 children with both asthma and seasonal allergic rhinitis with mean age 9.3 years (mean percentage of eosinophils in nasal smear was the highest in this group $-7 \%$, in differential count $-3 \%$ ). And third group include 5 patients with asthma and perennial allergic rhinitis with mean age 12.8 years (mean percentage of eosinophils in nasal smear $-5 \%$, blood eosinophils $-3 \%$ ).

Results: Analyzing results from the three groups regarding patient's age is obvious that mean age in second and third group is higher than in the first group. In the same groups is higher the percentage of mean nasal and blood eosinophilia. The highest nasal eosinophilia is found in children with asthma and seasonal allergic rhinitis.

Conclusions: Determination of nasal and peripheral (blood) eosinophilia are important diagnostic methods in children with bronchial asthma and co-existing seasonal or perennial allergic rhinitis. 


\section{BRONCHIAL HYPERREACTIVITY IN CHILDREN WITH ASTHMA UNDERGOING FOOD CHALLENGES}

A Krogulska, K Wasowska-Królikowska, J Dynowski, B Trzezwinska

Department of Allergology, Gastroenterology and Nutrition for Children, Medical University Lodz, Poland

Background and Aims: It has been shown that food allergy does not always manifest within the respiratory tract in such as obvious way as: Dyspnea, cough or wheezing. The aim of the study was to evaluate the influence of food allergens on bronchial reactivity in children with asthma challenged with food.

Methods: A total of 95 patients (age range 6 to 18 years, mean 10 years) with atopic asthma were evaluated for food allergy by means of questionnaire, skin prick testing, specific IgE tests (Pharmacia CAP System FEIA) and DBPCFC. Bronchial hyperreactivity was measured by methacholine inhalation challenges performed before and after DBPCFC in 34 asthmatic patients suspected of food allergy.

Results: Food allergy was confirmed by DBPCFC in 11 asthmatic children. The mean FEV1 before and after food challenges in the positive group was $2.25 \pm 0.871$ and $2.15 \pm 1.021$ respectively which was not significantly different $(\mathrm{p}>0.05)$. The mean $\mathrm{PC} 20$ in positive group was $1.41 \pm 1.39 \mathrm{mg} / \mathrm{ml}$ and $0.96 \pm 0.93 \mathrm{mg} / \mathrm{ml}$ before and after food challenge respectively which was not significantly different $(p>0.05)$. In the negative group the mean PC20 was $2.07 \pm 3.34$ and $2.06 \pm 3.35$ before and after challenge respectively $(\mathrm{p}>0.05)$.

Conclusions: Food allergy was present in $11.6 \%$ children with asthma. Food challenges in these children increased bronchial reactivity, without exacerbation. Food allergies in asthmatic children can present not only with most commonly reported gastrointestinal symptoms and skin rash, but also with increased bronchial reactivity. Evaluation of food allergy in patients with asthma is indicated.

\section{BRONCHIAL HYPERREACTIVITY IN CHILDREN WITH ASTHMA}

\section{A Krogulska, K Wasowska-Królikowska}

Department of Allergology, Gastroenterology and Nutrition for Children, Medical University Lodz, Poland

Background and Aims: Bronchial hyperreactivity (BHR) is a characteristic feature of asthma, but is neither specific nor constant. Bronchial hyperreactivity can be evaluated by methacholin inhalation challenge (MIC) which assesses a non-specific airway hyperresponsiveness. The aim of the study was to evaluate bronchial hyperreactivity in children with asthma and its correlation with clinically relevant parameters such as age, sex, weight, height, parameters of ventilation, atopy, inhaled corticosteroids (ICS) and clinical course.

Methods: A total of 74 patients (age range 6 to 18 years) with atopic asthma were evaluated for bronchial hyperreactivity, using methacholin challenge testing (two-minute tidal breathing dosing protocol).
Results: On the basis of the performed study, BHR was recognized in $68(91.9 \%)$ children. Mild hyperreactivity was found most often. Borderline values of PC20 were determined in $10.8 \%$ of children, normal values of PC20 in $8.1 \%$ of children. PC20 positively correlated with ventilation parameters of small bronchi, but did not correlate with other spirometric parameters. PC20 had a positive correlation with positive skin prick tests. The correlation between BHR and therapy with ICS was detected. There was no correlation between PC20 and age, sex, weight, height.

Conclusions: Increased BHR was related to episodes of infections and dyspnea. BHR is more common in children with positive skin prick test. Therapy with ICS reduces BHR.

\section{EXERCISE TEST (FEEDING, SWALLOWING, STEPPING, CRYING) IN RAPID AND ACCURATE DIAGNOSIS}

R Gaffari, H Gaffari

Gamar Bany Hashem Hospital, Khoy, Iran

Background and Aims: We did exercise tests with crying, swallowing, stepping and feeding them that we found many patients whom they haven't had symptoms in normal activity but after introduction of exercise the symptoms appeared.

Methods: Case Study.

Results: The Exercise test is a very useful tool in rapid diagnosis in respiratory problems.

Conclusions: We did investigations.

\section{ALLERGY MARCH AND THE POSSIBILITIES OF INFLUENCING THE PROCESS}

G Sur $^{1,2}$, V Salajan ${ }^{1}$, D T Gireada ${ }^{1}$, C R Popescu ${ }^{1}$, L Sur ${ }^{2}$

${ }^{1}$ 2nd Paediatric Clinic, Cluj-Napoca, Romania

${ }^{2}$ University of Medicine 'Iuliu-Hatieganu', Cluj-Napoca, Romania

Background and Aims: It is known that atopic individuals may present one or more allergic entities, beginning with the cow milk proteins allergy, continuing with atopic dermatitis, the allergic rhinitis, than bronchial asthma. The authors' objective is to find the ways to stop the allergy march.

Methods: In this study were included all the patients hospitalized in Paediatric Clinic II Cluj-Napoca between 2003-2005, who presented at least one of the allergic features. There were applied measures to prevent the expression of other ways of going on of the allergic march. Therefore, at the patients which presented allergic symptoms at cow milk proteins, these proteins were replaced with hydrolyzed hypoallergenic proteins; at the patients with atopic dermatitis there were initiated preventive measures to remove the offending allergens from the patient's environment, and specific treatment with antihistaminic drugs and topic treatment with cortisone and elidel.

Results: In Pediatric Clinic II there were registered during the period of two years 114 patients with bronchial asthma, 46 patients with atopic dermatitis, 38 
with cow milk proteins allergy, 57 patients with bronchial asthma and rhinitis and 21 cases of allergic rhinitis. From the 38 cases diagnosed with cow milk allergy, only five of them evolved subsequent with atopic dermatitis because of disregarding of the diet and medication indicated. From 46 patients with atopic dermatitis, only two developed allergic rhinitis and none of them asthma.

Conclusions: The use of appropriate preventive and therapeutic strategies stops or limits the development of more allergic entities on the same individual.

\section{ALLERGIC RHINITIS AND EXERCISE HYPOTHESIS IN YOUNG ADOLESCENTS}

\author{
L Seckova $^{1}$, E Vlaski $^{1}, \mathrm{~K}_{\text {Stavric }}{ }^{2}$, M Kimovska $^{1}$, \\ R Isjanovska ${ }^{3}$

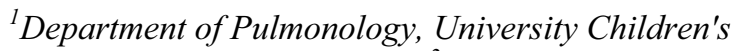 \\ Hospital, Skopje, Macedonia, ${ }^{2}$ Department of \\ Immunology, University Children's Hospital, Skopje, \\ Macedonia, ${ }^{3}$ Department of Epidemiology, Institute of \\ Epidemiology and Biostatistics with Medical \\ Informatics, Skopje, Macedonia
}

Background and Aims: As a protective effect of regular exercise and physical fitness against allergic diseases is suggested, the study was aimed to examine the influence of sedentary regimen, through duration of television (TV) watching, on allergic rhinitis in young adolescents.

Methods: The self-reported data obtained through ISAAC phase 3 questionnaires from 3,026 young adolescents aged 13/14 years from randomly selected schools in Skopje were analysed. The relationship between allergic rhinitis symptoms 'ever', current allergic rhinitis symptoms, current allergic rhinoconjunctivitis symptoms, interference with daily activities because of current rhinitis symptoms, hay fever 'ever' and daily TV watching, adjusted for body mass index, indoor air pollution, cat and dog ownership, was investigated. Odds ratios in binary logistic regression for statistic analysis of data were performed.

Results: A positive association between TV watching more than 3 hours daily and allergic rhinitis symptoms 'ever' (OR 1.21, 95\% CI 1.02-1.43 p=0.029) was established. A significant association between TV watching and current allergic rhinitis symptoms, their severity or ever-diagnosed hay fever was not found.

Conclusions: It seems that being sedentary and lack of physical fitness do not influence allergic rhinitis in young adolescents.

\section{PLASTIC BRONCHITIS AS A RESULT OF ASTHMA AND BRONCHIECTASIS AT THE SAME TIME: WHAT IS THE FIRST?}

\section{Maneva, Z Sarevska, L Stojanovska \\ Institute for Respiratory Diseases in Children-Kozle, Skopje, Macedonia}

Background and Aims: Plastic bronchitis is defined as a presence of acute or recurrent development of bronchial casts, which causes obstruction of airways, associated between the other with asthma or bronchiectasis.

Methods: A 14 years old girl, otherwise healthy, who had cough during last 4 months, was admitted. She was treated four times to her age of $16 \mathrm{yr}$. The lung findings were from pneumonia to repeated pleuropneumonies left clinically, on X-ray and echo chest.

Results: Lab investigations (blood count, sweet and TB tests, echinococcosis, and blood culture) were normal. Sputum: M.catarrhalis or Str.pneumoniae. Functional tests- from normal to obstruction and restriction. Skin prick test was positive to Dermatophagoides. Fiberoptic and rigid bronchoscopy: bronchial casts in the left bronchial tree. Pathological findings: Fragments consisting fibrin and eosinophilic infiltrates. Patient was initially treated with standard therapy for pneumonia and bronchial obstruction, later with topic steroid. She was well one year, than she needs admissions several times because of progression of disease when were found out this typically signs of plastic bronchitis. At the end of the course of disease left bronchiectasis was developed. Finally, results of her healthy is unknown, she never had come to us, again.

Conclusions: Our case shows pneumonia as first condition, asthma as second, and finally, repeated left pleuro-pneumonies with plastic bronchitis were the base for development of bronchiectasis. We think that in that case, circulus viciosus was made. Bronchoscopic exploration is the unique method in diagnosis and therapy of bronchitis plastica.

\section{PNEUMOMEDIASTINUM DUE TO SEVERE ASTHMA ATTACKS IN CHILDREN}

\section{Petrusevska-Kolekevska, B Manceva, B Dimceva}

Institute for Respiratory Diseases in Children-Kozle, Skopje, Macedonia

Background and Aims: Although pneumotorax may occur in patients with asthma it rarely occurs spontaneously during an acute exacerbation in children To show four children with pneumomediastinum because of uncontrolled and under diagnosed asthma disease.

Methods: In a period of ten years four children in our Institute appeared with pneumomediastinum due to asthma attacks. All necessary investigations were done.

Results: All of the patients had sudden onset of symptoms, positive family history for atopy, positive skin prick tests and increased IgE levels in serum. Two of them have had pneumo-mediastinum in the last year (asthma attacks were ignored from parents and physicians). None of them was asthma prevented. In three patients additional diagnosis was pneumonia.

Conclusions: Lack of recognition of life-threatening asthma in children can have devastating consequences. In efficient implementation of guidelines for treating asthma still exist.

\section{SAFETY OF CORTICOSTEROIDS STEP DOWN IN ASTHMATIC CHILDREN}

E Dilanas, E Paraskakis, A Moissiadi, D Mamoulakis, K Kalmanti 
Department of Pediatrics, General University Hospital, Heraklio, Crete, Greece

Background and Aims: Asthma treatment with corticosteroids should be guided by symptoms and lung function. Once asthma is controlled, the dose of corticosteroids should be reduced to the lowest possible and effective dose according to the standard guidelines. Symptoms often deteriorate in well-controlled asthmatics after a step down in therapy. We aimed to assess the safety and the lung function changes in asthmatic children after steroid step down.

Methods: 54 children (35 male) with moderate persistent asthma aged (mean \pm SD) $10.13 \pm 3.29$ yrs that their inhaled corticosteroids (ICS) were reduced in a 36 month interval were studied. Asthma control, quality of life and medication were recorded in all children.

Results: In $18.5 \%(10 / 54)$ of the patients receiving median (range) 450 (200-1000) mcg budesonide the ICS were stopped. There was no difference in FEV1\% predicted (mean $\pm \mathrm{SD})$ before and after the steroid discontinuation ( $108.7 \pm 6$ vs $115.7 \pm 9, p=0.62)$. In $44 / 54$ $(81.5 \%)$ of the asthmatics the median (range) dose of ICS was reduced significantly [500(200-1000) vs 320 $(160-750) \mathrm{mcg}, \mathrm{p}<0.0001)$. The FEV1\% predicted (mean $\pm \mathrm{SD}$ ) of those patients after the steroid step down was slightly increased (109.8 \pm 18.9 vs $120 \pm 18.36$, $\mathrm{p}=0.012$ ). In none of the 54 asthmatic children asthma control was lost or any change at their quality of life was exhibited, after the decrease or discontinuation of their ICS treatment.

Conclusions: The reduction of inhaled corticosteroids seems to be safe for the asthmatic children and the young patient should be revaluated every 3-6 months to avoid treatment with doses of ICS much higher than the effective dose for the asthma severity.

\section{QUALITY OF LIFE IN CHILDREN WITH ATOPIC DISEASES}

V Rozmanic, K Manestar, Z Korotaj, S Banac, I Zubovic, V Ahel

\section{Pediatric Clinic, University of Rijeka, Rijeka, Croatia}

Background and Aims: The aim of this study was to evaluate quality of life $(\mathrm{QoL})$ in pediatric patients with atopic diseases.

Methods: We evaluated QoL in 132 asthma patients by using Pediatric Asthma Quality of Life Questionnaire (PAQLQ), 122 patients with allergic rhinitis by using Pediatric Rhiniconjuctivitis Quality of Life Questionnaire (PRQLQ) and 40 patients with atopic dermatitis by using Childhood Atopic Dermatitis Impact Scale (CADIS). Severity of atopic diseases was made with GINA (for asthma), ARIA (for allergic rhinitis) and SCORAD (for atopic dermatitis) classification.

Results: The evaluation and analysis of questionnaires showed that there is a statistically significant correlation of overall QoL scores and severity of atopic diseases.

Conclusions: This study shows that children with atopic diseases have significant impairment on HRQoL. With medical categorization of severity of atopic diseases, which is priory based severity of symptoms, we can assess a degree of HRQoL impairment.

\section{WHEEZING EPISODES AND VASCULAR ANOMALIES IN CHILDHOOD}

O Zafirovski, J Buzarov, S Galbova, M Caparoska, V Stevic

Institute for Respiratory Diseases in Children - Kozle, Skopje, Macedonia

Background and Aims: Congenital cardiovascular anomalies are rare but treatable cause of stridor in the paediatric population. It has been estimated that up to $3 \%$ of the population have congenital anomalies of the aortic arch complex and we feel that many patients currently being diagnosed as having recurrent croup or atypical asthma with recidivate wheezing episodes must be excluded from this group.

Methods: We had 10 cases of congenital vascular anomalies causing tracheobronchial compression and wheezing episodes with following diagnosis: Arcus aortae duplex (2); right sided aortal arch with left ligamentum arteriosum and ductus arteriosus persistence (1); Anomalous arteria innominata (6); Anomalous initial point of the left pulmonary arteria (1). Presenting symptoms included stridor $(100 \%)$, persistent cough $(70 \%)$, chronic dyspnoea $(70 \%)$, recurring respiratory tract infections (80\%) and dysphagia (20\%).

Results: Direct laryngoscopy and bronchoscopy were diagnostics in all cases. They were our investigation of choice. Two (20\%) underwent aortopexy, $3(30 \%)$ had division of the non-dominant aortic arch and $5(50 \%)$ were managed conservatively. They were followed for up to six years post-operatively and $80 \%$ had complete resolution of their symptoms.

Conclusions: In recidivate wheezing episodes we have to think about vascular anomalies, especially in small children. We feel that only a high clinical index of suspicion will enable these potentially fatal anomalies to be diagnosed.

\section{UROLOGICAL MALFORMATIONS DURING THE PERIOD 1997-2005}

A Huerga, C López Menchero, I Marsinyach, P Chimenti, D Blanco, S Caballero, B Arias, M L Serrano, M L Franco, B Bernardos, M Sánchez Luna, V Pérez Sheriff

\section{Neonatology Unit, Department of Pediatrics, Hospital General Universitario Gregorio Marañon, Madrid, Spain}

Background and Aims: Study the incidence of urinary malformations (UM) during the period 1997-2005.

Methods: A total number of 42361 newborns born alive were delivered during this period in our hospital. Babies that had been diagnosed during pregnancy were observed and confirmed the malformation. In addition all newborns that had to be hospitalized underwent abdominal ultrasound.

Results: The total number of malformations was 630 $(14,87 \%), 255$ (40,51\%) had to be hospitalized and 375 $(59,48 \%)$ did not need hospitalization. UM has had an increment in incidence during the years studied in this period. Year $19977 / 4183(0,64 \%)$ versus year 2006 $166 / 9173(1,80 \%)$. All the incidence index studied were elevated. Less than 2500 gr. 159/859 (18,50\%); less 
than 37 weeks 166/859 (19,32\%) and incidence of twins was $37 / 859(4,30 \%)$; low birth weight $37 / 859$ (6,98\%). The relation male/female was $3: 1$.

Conclusions: The increment in incidence during the years studied seems to be due to a more accurate diagnose in pregnancy. The data obtained leads us to the conclusion that the real incidence of UM must be much higher than that obtained from the papers in this matter. The more accurate prenatal diagnosis corresponded to Q60 (CIE 10) and Q61. The concern is that only 9/25 urinary infections had prenatal diagnosis of UM.

\section{HUMAN PARECHOVIRUS TYPE 3 RESPONSIBLE FOR TWO FATAL CASES OF NEONATAL ENCEPHALITIS}

\author{
C D Aarts-Tesselaar, P Govaert, A Vossen, \\ M de Hoog, K F M Joosten
}

Department of Paediatric Intensive Care, Erasmus MC, Sophia Children's Hospital, Rotterdam, The Netherlands

Background and Aims: Picornaviruses form a diverse group of small, non-enveloped, single-stranded RNA viruses. Parechoviruses belong to the picornavirus family and are well known human pathogens responsible for several clinical manifestations including encephalitis in neonates and young children. We report two cases of fatal neonatal encephalitis caused by human parechovirus type 3 (HPEV3).

Methods: Two neonates ( 3 and 5 weeks old) were referred to the paediatric intensive care unit because of respiratory insufficiency and refractory convulsions irresponsive to therapy. Despite intensive care and extensive anticonvulsant treatment, both children developed general brain damage. No curative treatment was available and both children died. Investigation by EEG, ultrasound and MRI was conducted to explore the extensiveness of brain damage. Throat, nasopharyngeal and rectal swabs, cerebrospinal fluid and serum was collected and analysed by PCR and immunofluorescence assay.

Results: Numerous EEG's revealed progressive multifocal epileptic activity in both children. Ultrasound documented discrete ventriculomegaly and hyperechoic white matter. MRI confirmed extensive loss of white matter, diffuse edema, slit ventricles and white basal ganglia, verifying (viral) encephalitis. HPEV3 was isolated from the throat and nasopharyngeal swabs and its genome was detectable in the cerebrospinal fluid. In addition, HPEV3 was found in post mortal samples of brain and lung tissue. The samples were negative for other tested viruses.

Conclusions: Human parechoviruses can be responsible for severe central nervous system infections leading to death in neonates. Extensive viral cultures and visualisation of the brain were clue to final diagnosis.

\section{HOSPITALIZATIONS FOR VARICELLA IN LISBON, PORTUGAL, 2005-2006. A PROSPECTIVE STUDY}

J F Neves ${ }^{1}$, C Gouveia $^{1}$, R Martins ${ }^{2}$, M J Brito ${ }^{2}$, A Leça ${ }^{1}$
${ }^{1}$ Unit of Infecciology, Hospital de Dona Estefânia, Lisbon, Portugal, ${ }^{2}$ Department of Paediatrics, Hospital Fernando da Fonseca, Amadora, Portugal

Background and Aims: Varicella is normally a benign disease of childhood, but has potentially serious complications. Varicella epidemiology is changing with increasing use of varicella vaccine and data concerning hospitalizations is still incomplete. Our aim is to evaluate the complications of varicella in hospitalized children and its relation with age, type of exposure, underlying disease and the previous therapy.

Methods: Prospective study from January 2005 to March 2006, of children and adolescents hospitalized with varicella in Hospital Dona Estefânia and Hospital Fernando Fonseca. Demographic, clinical features, laboratory, therapy and evolution were analysed, using SPSS for Windows.

Results: During the study period 58 children were hospitalized. The median age was 2,5 years (range 10 days to 13 years). Nine $(15,5 \%)$ children had underlying disease. The reason for admission was complicated varicella in $45(77,6 \%)$; varicella during hospitalization in $5(8,6 \%)$; nosocomial varicella in $4(6,9 \%)$ and risk of severe varicella in 4 cases $(6,8 \%)$. The complications were cutaneous in 27 patients $(46,6 \%)$, neurological in 7 $(12,1 \%)$; respiratory in $6(10,3 \%)$, digestive in $3(5,2 \%)$ and osteoarticular in $2(3,4 \%)$. The mean length of hospital stay was 6 days (range: 2 to 122 days) and the mean associated cost per patient was 1996 Euros.

Conclusions: This study suggests a high morbidity associated with varicella and its complications, as well as high social and economic costs of this disease. The national survey that has been set up since January 2006 in Portugal is important on the definition of subsequent strategies for the country.

\section{CSF CHARACTERISTICS IN PCR PROVEN VIRAL MENINGITIS}

J Rajah $^{1}$, T El-Hassan ${ }^{1}$, G Ritchie ${ }^{2}$

${ }^{1}$ Department of Pediatrics, Sheikh Khalifa Medical City (SKMC), Abu Dhabi, United Arab Emirates

${ }^{2}$ Department of Molecular Biology, Sheikh Khalifa Medical City (SKMC), Abu Dhabi, United Arab

Emirates

Background and Aims: The aim of our study was to model the typical CSF findings of enteroviral meningitis ( $80 \%$ of all aseptic meningitis) in children with the gold standard of diagnosis, the PCR.

Methods: 34 children between 3 months and 12 years admitted to SKMC from January 2005 to January 2006 were included. The diagnostic criteria were the presence of RT-PCR enterovirus RNA (Roche Lightcycler) and CSF pleocytosis of greater than $5 \mathrm{cell} / \mathrm{mm} 3$.

Results: The median length of stay (LOS) was 48 hours. The mean (SD) serum WBC was 12.2 (3.6) and serum PMN 77\% (12.3). By multiple regression with LOS as the dependent variable and using CSF PMN, CSF glucose, serum $\mathrm{WBC}$ and serum PMN as predictors, only CSF PMN was included in the model, with higher CSF PMN being associated with shorter LOS $(\mathrm{t}=$ 3.389, $\mathrm{P}=0.002$ ). 
Conclusions: It is not unusual for neutrophils to dominate in enteroviral meningitis. This description may help physicians better evaluate their decision to start or withhold antibiotics and avoid unnecessary comorbidity.

\begin{tabular}{|c|c|}
\hline CSF Characteristics & Mean (SD) \\
\hline WBC (cells/mat') & $92.5^{*}$ \\
\hline $\mathrm{RBC}\left(\mathrm{cella} / \mathrm{mm} \mathrm{m}^{2}\right)$ & $5^{*}$ \\
\hline Neutrophils (\%) & $58.7(305)$ \\
\hline Lymphocytes (\%) & $412(30.4)$ \\
\hline Gloccese (munol/) & $3.69(0.61)$ \\
\hline CSF/Serum glucose ratio & $0.61(0.09)$ \\
\hline Protein (el) & $0.43(0.21)$ \\
\hline
\end{tabular}

"median for non nomal distribution

\section{INVASIVE PNEUMOCOCAL DISEASE AMONG CHILDREN IN MEXICO CITY DURING 1997- 2004: SEROTYPE DISTRIBUTION, ANTIBIOTIC SUSCEPTIBILITY, EPIDEMIOLOGY AND RISK FACTORS TO MORTALITY}

\author{
L E Espinosa ${ }^{1}$, C Lopez $^{2}$, L V Jimenez ${ }^{3}$, D Gomez ${ }^{1}$ \\ ${ }^{\text {I} B a c t e r i o l o g i a, ~ H o s p i t a l ~ D r . ~ M a n u e l ~ G e a ~ G o n z a l e z, ~}$ \\ Mexico D.F., Mexico, ${ }^{2}$ Pediatria, Hospital de la \\ Beneficiencia Española, Mexico D.F., Mexico \\ ${ }^{3}$ Bacteriologia, Hospital Infantil de Mexico, \\ Mexico D.F., Mexico
}

Background and Aims: In Mexico, there are limited data that describe the burden of invasive pneumococcal disease (IPD), serotype distribution, antibiotic susceptibility and epidemiology. Knowledge resistance is important for determining appropriate therapy for the patient presenting to the hospital with an illness for which Streptococcus pneumoniae (Sp) is a possible cause.

Methods: Is an analytic study of 168 hospitalized patients without pneumoccal vaccine and with IPD who had Sp isolated in a sterile fluid between January 1997 and august 2004.

Results: In 168 patients with IPD, 107 male; we isolated $168 \mathrm{Sp}$ : 73 blood, 41 cerebrospinal fluid, 25 pleural fluid, 11 peritoneal fluid, 6 joint fluid, 8 urine and 4 mastoid fluid. Were $60.1 \%$ of the patient with underlying disease. The case fatality rate was $28.5 \%$, associated with pneumococcal serotype and without association with antibiotic susceptibility. Overall rates of resistance, were as follows: penicillin, 62.5\%; and cefotaxima $23.3 \%$. Underlying disease was significantly associated with mortality (OR 5.4 (2.5-18.3); IC 95\%: $\mathrm{p}<0.05)$. The serotypes significantly associated with mortality were: 14 . 6B, 23F, 6A, 19F and 19A.

Conclusions: Trend analysis indicated that mortality in IPD have association with underlying disease. Five Sp serotypes whit significant association with mortality are in a 7-valent pneumoccocal conjugate vaccine (PCV7). This fact is important because the PCV7 application should be key for mortality prevention in IPD. The high rates of resistant to $\beta$-lactamics make necessary the creation of a National System for knowledge of serotypes and resistant of $\mathrm{Sp}$ in our country.

\section{SECONDARY PROPHYLAXIS WITH CLINDAMYCIN IN PEDIATRIC PATIENTS WITH RECURRENT SKIN ABCESSES. A PROSPECTIVE STUDY (2002-2006)}

S Melendo, M Nicolás, M Mumany, M Miserachs, P Soler, C Figueras

Pediatric Infectious Disease Unit, Vall D'Hebron Hospital, Barcelona, Spain

Background and Aims: Recurrent skin abscesses are relatively common in paediatrics. Use of clindamycin therapy as secondary prophylaxis in adults has been described for more than 20 years. However, pediatric literature is scarce. Our aim was to describe efficacy, tolerability and adverse effects of oral clindamycin in immunocompetent children with recurrent skin abscesses.

Methods: Medical charts of 16 patients attending our centre from March 2002 to March 2006 were prospectively reviewed. Group A received oral clindamycin $(150 \mathrm{mg} / 1.73 \mathrm{~m} 2 /$ day qd) during 3 months. Group B did not receive clindamycin because inability to swallow capsules, intolerance or family decision. Topic mupirocine was used in nasal carriers of S. aureus (SA).

Results: Ten patients received clindamycin, 6 did not. No patient presented immunity abnormalities (normal neutrophils oxidative burst, IgE and CD18). Sex: A: $60 \%$ females; B: $67 \%$ females. Median age at diagnose: A: 10 yo; B: 6 yo. Underlying skin abnormalities: A: 5/10; B: 1/6. Median pre-treatment skin infections/patient: A: 6; B: 3. Surgical drainage: A: 7/10; B: 2/6. Nasal carriers of SA: A: 1/9; B: 2/6. Skin infections in relatives: A: 7/10; B: 4/6. Nasal carriers of SA relatives: A: 2/9; B: 2/6. Post-clindamycin follow-up in Group A: 5 months (1-14m);1 mild cellulitis. Followup in Group B: 5 months $(1-15 \mathrm{~m})$ : no further skin infections.

Conclusions: Oral clindamycin could be effective as secondary prophylaxis in pediatric patients with recurrent skin abscesses. Lower incidence and severity of previous infections in group B could explain their good evolution. Future studies are needed to determine the role of prophylactic clindamycin in these patients.

\section{PASSIVE SMOKING IN CHILDHOOD AND THE PERCEPTIONS OF PARENTS}

I M Velissariou, N G Papadopoulos, D A Kafetzis, P Saxoni-Papageorgiou

${ }^{1}$ 2nd Department of Paediatrics, University of Athens, 'P and A Kyriakou' Children's Hospital, Athens, Greece

Background and Aims: Children are frequent passive smokers due to the active smoking of their guardians. Our aim was to quantify the exposure of children to smoke, to assess this exposure with regards to family demographics and to present the perceptions of parents regarding passive smoking of their children.

Methods: One hundred children (from 2.5 to 14 years old, median age 4.5+/- 1.2) were enrolled that presented either with chronic cough or for a routine follow-up.

Results: Data were collected from 12 fathers and 88 mothers, with a median age of $35+/-6.2$. Ten percent 
have finished primary education, $30 \%$ secondary education and $60 \%$ had an academic degree. Eighty percent lived in a city, while $20 \%$ in a rural area, $70 \%$ in a block of flats and $30 \%$ in a detached house. Sixty two fathers were active smokers (median age that they started smoking 17.49+/- 1.92, median number of cigarettes per day $22.58+/-14.11$ ) and 47 mothers (median age that they started smoking $18.95+/-2.44$, median number of cigarettes per day 18.05+/- 10.55). Fifty eight percent of smoking parents smoke in the house and of them $68 \%$ smoke in the presence of their child. Of the 100 families, $67 \%$ allow guests to smoke indoors, $95 \%$ believe that passive smoking is harmful for their child, while $80 \%$ state that parental smoking is a bad imitating influence for their child.

Conclusions: Passive smoking is quite frequent even in children with respiratory symptoms and reflects their parents' perceptions regarding this habit.

\section{RECURRENT CHEST INFECTIONS AND PRIMARY PULMONARY TUMORS}

\section{M Velissariou, E Latoufi}

Paediatric Intensive Care Unit, Athens Medical Centre, Athens, Greece

Background and Aims: Primary tumors of the lung are extremely rare in children and can occasionally present as recurrent lower respiratory tract infections.

Methods: We present 2 cases of rare lung neoplasms that were enrolled over a 3 year period, a 9 year old boy and a 10 year old girl. Both children had documented previous hospitalizations in the last few months due to recurrent chest infections. Both cases were subjected to a bronchoscopy that revealed a space occupying lesion which was blocking the right main bronchus and the left main bronchus, respectively. Biopsies were collected. Computed tomography of the lung confirmed the presence of a tumor.

Results: Both patients had a surgical exploration, during which both tumors were removed following bronchotomy of the right and left main bronchus, respectively. The histological findings were suggestive of adenocarcinomas of low differentiation. A complete surgical excision was accomplished in both cases, hence neither received any other treatment. Both had an unremarkable postoperative period. Until the present time, neither has shown any evidence of relapse.

Conclusions: Bronchoscopy and computed tomography may be of use in children with recurrent episodes of lower respiratory tract infections, as they may reveal the presence of a rare primary pulmonary tumor that would otherwise remain undiagnosed.

\section{THE MOST FREQUENT CAUSES FOR PNEUMONIA IN THE EARLY INFANTILE AGE}

\author{
S Asani, V Tomik \\ Detsko Oddelenie-Opsta Bolnica, Tetovo, Macedonia
}

Background and Aims: Respiratory diseases are the frequent pathology in the infantile age. Aim: To present the representation of the most frequent causes for pneumonia.
Methods: A group of 215 patients was analysed, all hospitalized in the Children's Department in the period from 2002-2004 in the age of 0-3 years.

Results: Anamnestically $95,0 \%$ of the children had information for cough, 93\% were treated ambulant with antibiotic. Auscultatory at $52 \%$ of the cases were with crepitations, and Rtg-finding with present parenchyma changes. On all the children a bacteriological examination was realised on a drainage secretion At $49 \%$ (105) of the children the finding was positive. Among them the most frequent bacteriological causes were: -Streptococcus pneurnoniae among 39, 9 children -Hemoplilus influensae 36 children -Staphylococcus aureus 27 children -Pseudornonas auriginosa 2, 0 children According to the examination, children were put on an antibiotic therapy.

Conclusions: Pneumonia in the early children's age still has a significant role in the low-respiratory pathology.

\section{MYCOPLASMA PNEUMONIAE INFECTION - A SIX YEAR RETROSPECTIVE REVIEW}

\author{
A C Peres, P Rodrigues, A S Simões, A Casimiro, \\ F Cunha
}

Department of Paediatric, Reynaldo dos Santos Hospital, Vila Franca de Xira, Portugal

Background and Aims: Atypical pneumonia is the main clinical presentation of infection to Mycoplasma pneumoniae, but this bacteria is also responsible for other manifestations with or without respiratory involvement. The purpose of this study is to evaluate the epidemiology, presentation, clinical curse, therapeutic and prognosis of this infection.

Methods: A retrospective review of 20 cases of infection to Mycoplasma pneumoniae, confirmed by ELISA, hospitalized in a six year period (February of 2000 to February of 2006) in a paediatric unit.

Results: During the study period we identified 20 cases in children aged 9 months to 13 years (mean age 6 years), $50 \%$ female. Ten days was the average interval between the initial symptoms and the admittance. Before hospitalization $50 \%$ of the children were on antibiotic therapies. In $75 \%$ fever was present and in $55 \%$ pneumonia was the clinical presentation (3 with atelectasis and 1 with pleural effusion). In four children there were other respiratory involvements. Five children had skin involvement and four had osteoarticular manifestations. Empiric antibiotic was prescribed in $70 \%$ of the cases. The clinical course was good in all patients but two of them had a slow evolution towards complete resolution.

Conclusions: With this study we pretend to demonstrate the heterogeneity of the clinical presentation of the infection by Mycoplasma pneumoniae and the frequent good prognosis with spontaneous resolution. 
COMMUNITY ACQUIRED PNEUMONIA - THE MOST FREQUENT CAUSE FOR HOSPITALIZATION IN CHILDHOOD AND MOST OFTEN ETIOLOGICAL DIAGNOSTIC PROBLEM

L Stojanovska, M Maneva, L Nikolova, O Stefanovska

Institute for Respiratory Diseases in Children-Kozle, Skopje, Macedonia

Background and Aims: Community acquired pneumonia (CAP) is still actual problem and the most often cause for hospitalization of children in our country. The aim of this study is to analyses dynamics, frequency and diagnosis of this disease.

Methods: From the total number of 1750 patients at the Department for acute respiratory diseases in preschool and school children, 1458 (83.3\%) histories of patients with confirmed pneumonia were elaborated, retrospectively. In the 5 years period till 2006 yr., number of hospitalizations was as follows: 220/320/310/263/345.

Results: Patients were at the age of 3 to 16 yr. (mean 8.5 yr.). Dominant symptoms were high temperature in $70 \%$, rough cough $-91 \%$, difficult breathing in $12.4 \%$, hemopthysis $-1.3 \%$. Auscultatory finding with crepitations was presented in $49 \%$ of the patients. Lung radiograms contributed to pneumonia with right lung domination. As diagnostic we used echo-sonography and bronchological exploration on the breathing tree (therapeutically, too). Laboratory findings were in contribution with bacterial pneumonia. Etiologic diagnosis was made only in $460(31.5 \%)$ patients with pneumonia. The most frequent causes were Str.pneumonia-210 (45.6\%), H.influenzae-190 (41.3\%), M.catarrhalis- 45 (9.7\%), M.pneumoniae- 11 (2.3\%). Massive pneumonia was presented in 137 (9.36\%) patients (with atelectasis-19 (16.7\%) and pleura involving -34 (24.8\%). Treatment duration was approximately 7 to 10 days with favorable outcome, except in 5 children.

Conclusions: CAP actuality without tends of decreasing was shown in this study. CAP etiologic diagnosis is a problem, too, especially important in order to fast, adequate diagnosis, therapy and sequels prevention (particularly in severe forms of pneumonia).

\section{CYST ECHINOCOCCOSIS - PEDIATRIC PROBLEM IN CONTINUUM}

O Stefanovska, L Nikolova, L Stojanovska, Z Sarovska

Institute for Respiratory Diseases in Children-Kozle, Skopje, Macedonia

Background and Aims: To evaluate the frequency of cyst echinococcosis (CE) in children.

Methods: Medical documentation from the department for acute respiratory diseases was retrospectively analyzed for a period January 2000 to December 2005 yr.

Results: The total number of treated children was 2178 . From them, $36(1.65 \%)$ were with CE. The frequency of the disease, regarding each year properly was almost constant; age of patients: $3-14$ yr. (middle age $=9 \mathrm{yr}$.). Urban area place of living - $22(61.5 \%)$ children. Lower socioeconomic background and bed hygiene standards 20 (55.5\%) Cysts localization: in lungs-36 (100\%), in liver-15 (41.6\%), in brain- 1 (2.7\%). Surgical treatment with good outcome - $36(100 \%)$ children.

Conclusions: CE is our permanent medical and social problem, also. Imperative is to emphasis preventive activities included in a national and regional net of control programs.

\section{DEVELOPMENT OF A NOVEL IMMUNOASSAY FOR THE DETECTION OF HUMAN METAPNEUMOVIRUS IN CLINICAL SPECIMENS}

\author{
K Barry-Murphy ${ }^{1}$, T Sloots ${ }^{2}$, S Reid ${ }^{1}$, G Elliott ${ }^{1}$ \\ ${ }^{I}$ Biotrin International Ltd., Dublin, Ireland \\ ${ }^{2}$ Queensland Paediatric Infectious Diseases Laboratory, \\ Royal Children's Hospital, Brisbane, QLD, Australia
}

Background and Aims: Human Metapneumovirus (hMPV) has been described as a causative agent for acute respiratory tract infections and is considered one of the main causes of hospitalisation for such infections in young children worldwide. Currently, there is a requirement for an antigen detection assay for respiratory screening. Here we describe an antigen detection system utilising a combination of monoclonal antibodies directed to major hMPV proteins.

Methods: The performance characteristics of the immunoassay system were evaluated using panels of confirmed hMPV positive or negative respiratory samples.

Results: The prototype assay results exhibited close correlation to PCR data. A high level of specificity was demonstrated with a panel of potentially cross-reactive, non-hMPV, specimens. The newly developed immunoassay has been found to be reactive with hMPV subgroups 1 and 2 of genotypes A and B from viral culture.

Conclusions: This efficient, specific assay may have useful implications in hMPV detection in respiratory specimens and/or in culture confirmation.

\section{SMOKING BEHAVIORS AND OPINIONS TOWARDS CHILDREN'S PASSIVE SMOKING AMONG GREEK PARENTS}

C Tsoumakas ${ }^{1}$, A Papadopoulou ${ }^{2}$, P Georgiopoulou ${ }^{2}$, A Zervoudakis ${ }^{2}, \mathrm{~V}$ Matziou ${ }^{1}$

${ }^{I}$ Faculty of Nursing, University of Athens, Athens, Greece, ${ }^{2}$ Department of Pediatric, KAT General Hospital, Athens, Greece

Background and Aims: Tobacco smoke is one of the most important preventable environmental risk factors to which children are exposed. Though most parents are aware of the importance of protecting their children from tobacco smoke exposure and of strategies for prevention, children's exposure mainly transpires at home. Objective of the study was to reveal attitudes among Greek parents of school aged children towards children's tobacco smoke exposure. 
Methods: : Data derived from a sample of smoking and nonsmoking parents $(\mathrm{n}=938)$ of 6 to 13 years old children living in Attica. They answered a questionnaire on their opinions and attitudes regarding children's passive smoking. Data analysis was made with SPSS 10.0 .

Results: $90.9 \%$ of parents said that they were informed about tobacco smoke's effects on health. In $63 \%$ of families there was one parent who smoked and in $25 \%$ both parents were smokers. Of them, 59\% smoke indoors. 3 out of 5 parents stated that they allow their guests to smoke indoors. Fathers use to smoke in the car in the presence of their children more than mothers do and $64.8 \%$ of them stated that their children complained about that at least once in the past.

Conclusions: Results indicate that Greek parents need more specific scientific guidance from experts, even though the majority of them stated that they are well informed and they take some kind of precaution. There is great need for more intense efforts (e.g. policies, mass media campaigns) to convince smokers to quit or at least avoid indoors smoking.

\section{RSV BRONCHIOLITIS IN PEDIATRIC INTENSIVE CARE UNIT}

G N Feketea, G G Sideri, A G Vasilopoulos, J H Papadatos

PICU, P.\&A. Kyriakou Children's Hospital, Athens, Greece

Background and Aims: To determine the demographic characteristics, seasonal distribution, and complication associated with RSV bronchiolitis in PICU.

Methods: Twenty-five children with RSV infection were admitted on PICU over a 3-year period study. Their medical charts were reviewed.

Results: Males were affected more that females with a ratio 2,12:1. The epidemy appeared in December and disappeared in May, with a peak of incidence during March ( $40 \%$ of cases). More than half of hospitalized children $(56 \%)$ were between 35-60 days of age. The indications for ICU admission were respiratory distress in $76 \%$ of cases, apnea $(20 \%)$ and sepsis (4\%). One child presented with pneumotorax. Mean PICU length of stay was 6,44 days (SD 3,92). Most of children had no underlying disease (76\%). A small percentage (16\%) of children presented fever. Six children from the 25, needed mechanical ventilation (mean 7,3 days) because of apnea (33\%) or severe respiratory distress $(66 \%)$. One child with positive urine culture was infected by RSV during hospitalization for urine infection. No children had positive microbiological cultures upon PICU admission. Three children had seizures during their hospitalization; one of them had febrile convulsions and the other two manifested seizures after their extubation.

Conclusions: Now days, most of the neonates treated in a NICU are immunized against RSV with palivizumab. That is the reason why the number of neonates having RSV infection and underlying disease has decreased. A risk factor for sever RSV infection remain small age. Very few infants had a bacterial infection as a secondary infection after RSV bronchiolitis.
MYCOPLASMA PNEUMONIAE RESPIRATORY INFECTONS IN CHILDREN

\author{
L Serbo ${ }^{1}$, A Kondi ${ }^{1}$, L Myftari ${ }^{1}$, A Cobo ${ }^{2}$ \\ ${ }^{1}$ Department of Pediatrics, CHU Tirana, Tirana, \\ Albania, ${ }^{2}$ Pediatric Hospital, Fier, Albania
}

Background and Aims: Presentation of some epidemiologic, clinical, roentgen graphic aspects in Mycoplasma pneumoniae respiratory infections and their diagnostic.

Methods: In all 33 children diagnosed and treated for M. pneumoniae respiratory infections, in pneumoallergology pediatric service, CHU Tirana, during period October 2004 - May 2005. Diagnosis of M. pneumoniae infections is based on positive specific IgM\&IgG antibodies for M. pneumoniae.

Results: In 82 serologic tests done, results that 33 of them, $40.2 \%$ are infected with $\mathrm{M}$. pneumoniae. The age of sick children varies between 2 months- 13 years. The age most affected is between $4-9$ years $(42.4 \%)$, age 1-4 year $(21.2 \%)$, age 1 month-1 year $(18.2 \%)$, age 9-14 years $(18.2 \%)$ of cases. Clinical aspect of $\mathrm{M}$. pneumoniae infections has been varied: Bronchopneumonia in $48.5 \%$, pneumonia in $12.1 \%$, bronchiolitis $18.2 \%$, tracheo-bronchitis $9.1 \%$ and spasmodic cough $12.1 \%$ of cases. The essential clinical sign has been cough in $100 \%$ of cases, fever in $30.3 \%$ of cases. Roentgenographic examination has described interstitial bronchopneumonia in $48.5 \%$ of cases, pneumonic infiltrates in $18.2 \%$, hilar lymphadenopatia in $18.2 \%$, pleural fluid in $6 \%$, normal aspect in $9.1 \%$ of cases. Positive serology for IgM was in $66.7 \%$ and positive for IgM\&IgG in $33.3 \%$ of cases, which demonstrates that a great number of patients have recidivate infections or have been diagnosed after 1-2 months. In $39.4 \%$ of cases M.Pneumoniae infections have been associated with pathology like TBC, viral hepatitis, asthma, bronchectasie, leucosis.

Conclusions: It's evident an elevation of $M$. pneumoniae respiratory infections. A high percentage of this infection is presented in infants of first months of life.

\section{CLINICAL AND IMMUNOLOGICAL PECULIARITIES OF ASTHMA IN CHILDREN}

T A Kryuchko, O Y Tkachenko, I V Ivanina

Department of Hospital Pediatrics, Ukrainian Medical Dental Academy, Poltava, Ukraine

Background and Aims: Modern scientific investigations in pediatric immunology allowed to obtain new data concerning pathogenic aspects of asthma formation. But some questions of clinical and immunological accordance remain still undiscussed. The aim of the work was to investigate clinical and immunological peculiarities of asthma in children.

Methods: Clinical, allergological, instrumental, immunological (CD3, CD4, CD8, CD23, IgA, M, G, E, IL-4, $\gamma$-INF) were used.

Results: Constant increase of CD23 cells $(p<0.001)$ quantity in blood serum in children with asthma was revealed both in attack and remission period. CD23 cells content correlates with increase of $\operatorname{IgE}$ concentration 
$(\tau=0.32 ; \mathrm{p}<0.001)$ and decrease of serum IgA level $(\tau=0.29 ; \mathrm{p}<0.01)$. The dysbalance of cytokines was found even if the quantity dispersion of T-cells kept normal ratio: increase of IL-4 level $(p<0.001)$ and decrease of . $\gamma$-INF $(p<0.01)$.

Conclusions: Firm immunological changes such as hypofunction of I type T-helpers and stimulation of II type T-helpers in children with asthma were found.

\section{AFEBRILE SEIZURE AS PRESENTATION OF A METABOLIC DISORDER}

H Santos $^{1}{ }^{\text {, I Valente }}{ }^{1}$, S Freitas $^{2}$, L Vilarinho
T Oliveira

${ }^{I}$ Department of Pediatric, Hospital de São Sebastião, Santa Maria da Feira, Portugal, ${ }^{2}$ Neonatology Unit, Department of Pediatric, Hospital de São Sebastião, Santa Maria da Feira, Portugal, ${ }^{3}$ Instituto de Genética Médica Jacinto Magalhães, Porto, Portugal

Background and Aims: Biotin is a coenzyme for four carboxylases, intervening in gluconeogenisis, fatty acid synthesis and amino acid catabolism. The defect in biotin metabolism has variable manifestations, including hypotonia, seizures, cutaneous changes, deafness and alopecia.

Methods: We presented a clinical case of a six month old boy, referred for single seizure without fever.

Results: He was only son of young, healthy, nonconsanguineous parents. Born by caesarean section at 40 weeks of gestation, Apgar 9/10; CMV seroconversion during 2nd trimester; CMV antigen in urine was negative. Ophthalmologic and auditory tests were normal. Transfontanelar ultrasonography showed small subependimary haemorrhage, with complete resolution at 6 months. In the 3rd month, discrete global hypotonia was described, without other findings. By 6 months he suffered a single afebrile seizure, with spontaneous resolution. The clinical observation, EEG and cerebral MRI where normal; the metabolic study revealed elevation of ammonia and traces of 3-hidroxi-isovaleric acid; serum biotiniadse activity was diminished (3,24 $\mathrm{nmol} / \mathrm{min} / \mathrm{ml}$ ) - biotinidase deficiency. During this period it was observed a discrete delay in language area, without other alteration in growth and development. He initiated supplementation with biotin $5 \mathrm{mg}$ at 14 months and never had other episode of seizures; now with 25 months he as a completely normal growth and development for his age.

Conclusions: The biotinidase deficit is a disorder with gradual manifestations, and may have episodes of remission, associated with varying degrees of biotin in diet. This diagnosis must be considered precociously in children with neurological disorders because late diagnosed patients often have psychomotor retardation and permanent neurological damage.

\section{INFLUENCE OF THE APOLIPOPROTEIN E GENOTYPE ON THE COGNITIVE DEVELOPMENT AFTER TRAUMATIC BRAIN INJURY IN CHILDHOOD}

M Baz Bartels ${ }^{1}, \mathrm{~S}_{\text {Vlaho }}{ }^{1}, \mathrm{~V}_{\text {Boda }}{ }^{1}, \mathrm{~S} \mathrm{Parbel}^{1}$,

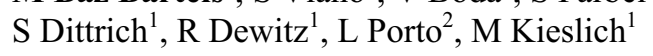

${ }^{1}$ Abteilung für Neuropädiatrie, Klinik für Kinder-und Jugendheilkunde des Universitätsklinikums Frankfurt am Main, Frankfurt am Main, Germany, ${ }^{2}$ Klinik für Neuroradiologie des Universitätsklinikums Frankfurt am Main, Frankfurt am Main, Germany

Background and Aims: As there are differences in cognitive recovery after similar traumatic brain injuries in childhood, the pathophysiology can not be longer explained only by the known prognostic factors as: injury mechanism, -type, -severity and location. Our study investigated the influence of apolipoprotein (Apo) E- polymorphism as modulating genetic factor in posttraumatic regeneration, emphasizing the significance on cognitive development.

Methods: After an Apo E typing, 38 adults, who had suffered a severe traumatic brain injury in childhood, received neurological, cognitive and magnetic resonance imaging (MRI) investigations by a standardized protocol. The mean age of the patients was 23 years with a mean of 17 years follow- up time after the initial brain injury.

Results: 8 (21\%) had an Apo E4 allele. While they showed retrospectively a longer and more severe initial post- traumatic course with longer vigilance disturbances, no significant differences in neurological disability and MRI examinations could be found. Whereas the Apo E4- group showed statistically significant worse cognitive results in the "Diagnosticum für Cerebralschädigung (DCS)" and the HamburgWechsler- intelligence- test (HAWIE), emphasizing concentration, figural memory skills and staying power.

Conclusions: The ApoE- polymorphism influences the post- traumatic outcome, increasing the risk of a poorer cognitive development, specially for concentration, memory and staying power skills through the ApoE 4allele. It therefore implicates a genetically determined, inborn vulnerability to traumatic brain lesions.

\section{OUR EXPERIENCE WITH THE MANAGEMENT OF THE CADAVERIC ORGAN DONORS IN THE PEDIATRIC INTENSIVE CARE UNIT AND ANAESTHESIA DURING ORGAN HARVEST}

D Blazek, P Pavlicek, D Hechtova, K Dlask

Department of Anaesthesiology and Intensive Care Medicine, University Hospital Motol, Prague, Czech Republic

Background and Aims: In the last years there has been seen the ever increasing number of the patients awaiting an organ transplant as the only curative treatment of their disease shifts the off-age limits of suitable cadaveric organ donors to both higher age limits (above age of 60) as well as lower ones in the case of pediatric donors.

Methods: This report presents authors' experience based on the care of 36 cadaveric donors since 1993.

Results: The authors suggest some principles of the care of the potential donor in the pediatric intensive care unit, specifically in comparison to the care of the adult donor. A special attention is given to the issues of assessment of the 'brain death', the tests prior to the PGA and the actual arrangement of the cerebral PGA. The importance of the continuity of the intensive care before and also 
after establishing the diagnosis of brain death is emphasized. The stress is put on the ventilation, cardiovascular system, arrhythmias, hemostasis, body temperature, infection prevention and intensive continuous monitoring of the vital functions of both the recipient and donor. Specific details of anaesthesia during the procedure are discussed.

Conclusions: In the last section the authors address variety of sensitive issues regarding legislation, previous informed consent, the consent of the pediatric patient's family and the influence of their involvement on the number of successful donations. They also discuss the maintenance of the medical record and overall approach to the management of the pediatric cadaveric donor in the settings of intensive care unit.

\section{ALBUMIN DIALYSIS FOR PREVENTION AND TREATMENT OF HEPATIC ENCEPHALOPATHY IN CHILDREN}

G Trittenwein, J Golej, G Burda, M Hermon, H Boigner, G Mostafa, A Pollak

\section{PICU, University Children's Hospital, Vienna, Austria}

Background and Aims: Hepatic encephalopathy remains a harassing challenge in pediatric critical care. High mortality and the risk of permanent neurological damage explain the need for a rapid available effective detoxification procedure particularly if liver transplantation cannot be carried out in short time. Albumin dialysis was used successfully in adults for this indication already.

Methods: In 3 children albumin dialysis was performed (using the MARS system in two patients and single pass albumin dialysis in one) to prevent (in one child with proven mushroom intoxication) and to treat (in two children suffering from biliary atresia and from autoimmune hepatitis) hepatic encephalopathy documented clinically and by EEG.

Results: Values of age and weight of patients were 20, 7 months and 16 years; 15, 8 and 39 kilograms. Values and admission were: ALAT (133, 3840, $52 \mathrm{U} / 1)$, bilirubin $(0,4 ; 18,89 ; 1,72 \mathrm{mg} / \mathrm{dL})$, INR $(0,97 ; 5,83 ; 1,9)$ and ammonia $(21,75,84 \mathrm{mcmol} / \mathrm{l})$. All children survived without neurological deficit.

Conclusions: Albumin dialysis can be performed successfully also in children in order to prevent or to treat hepatic encephalopathy.

\section{QUALITY OF LIFE IN CHILDREN WITH ATTENTION DEFICIT/HYPERACTIVITY DISORDER BEFORE AND AFTER TREATMENT WITH METYLPHENIDATE}

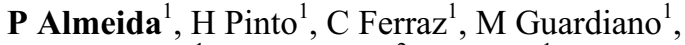 \\ M C Teixeira ${ }^{1}$, A M Coelho $^{2}$, V Viana ${ }^{1}$, \\ M J Guimarães ${ }^{1}$ \\ ${ }^{l}$ Departamento de Pediatria, Hospital de S. João, Porto, \\ Portugal, ${ }^{2}$ Serviço de Psiquiatria, Hospital de S. João, \\ Porto, Portugal
}

Background and Aims: Attention Deficit/Hyperactivity disorder, which affects between $3 \%$ and $5 \%$ of children, is characterized by different levels of hyperactivity, impulsivity and inattention that give rise to important academic, social and emotional problems. The aim of treatment is to improve symptoms and well being for the child and close contacts. The authors propose to study symptoms severity and quality of life of children with attention deficit/hyperactivity disorder before and after starting treatment with metylphenidate.

Methods: The sample included 40 children with the diagnosis of attention deficit/hyperactivity disorder, aged between 6 and 10 years old, who were referred to the pediatric development and psychology consultation. The Conners Questionnaire was answered by parents and teachers before and one month after the child started treatment with metylphenidate, to confirm diagnosis and to evaluate symptoms severity. The Pediatric Quality of life Inventory was used to measure child's functioning on four major domains (physical, emotional, social and academic) and was answered only by parents before and after starting treatment.

Results: The study evidenced a strong correlation between symptoms severity and quality of life. In overall, after starting treatment there was a reduction in symptoms and improvement in the quality of life, specially on emotional, social and academic levels.

Conclusions: Attention deficit/Hyperactivity disorder in children has an important impact on quality of life, which could substantially interfere with their future. Both symptoms measurement and knowledge about those quality of life domains which are mostly affected are important to define treatment needs.

\section{AUTISM AND RELATED DISORDERS: ASSESSMENT AND INTERVENTION IN A PEDIATRIC SERVICE}

A M Coelho ${ }^{1}, \mathrm{C} \mathrm{Ferraz}^{2}, \mathrm{H}$ Pinto $^{2}, \mathrm{M} \mathrm{Guardiano}^{2}$, M C Teixeira ${ }^{2}$, P Almeida ${ }^{2}$, V Viana ${ }^{2}$, M J Guimarães $^{2}$

${ }^{l}$ Serviço de Psiquiatria, Hospital de S. João, Porto, Portugal, ${ }^{2}$ Departamento de Pediatria, Hospital de $S$. João, Porto, Portugal

Background and Aims: Autism and related pervasive developmental disorders are characterized by patterns of delay and deviance in the development of social, communicative and cognitive skills. These conditions present particular challenges for clinicians and require care and coordination of multiple professionals. The authors propose to study clinical features and outcome of children with diagnosis of autism and related disorders, two years after starting specific intervention.

Methods: The sample included 80 children with diagnosis of autism and related disorders. Their clinical reports were reviewed and data was collected regarding clinical features, laboratory and imagiological evaluation, differential diagnosis, treatment plan and multidisciplinary intervention. The Childhood Autism Rating Scale, by the time of diagnosis and after two years of follow-up, has been evaluated. A standardized parent interview has been performed concerning their point of view about improvement in social and communicative behavior and autonomy.

Results: In overall, after starting specific multidisciplinary intervention, there was an 
improvement in problematic behavior, social skills and relationships, and educational functioning.

Conclusions: Long term evaluation and monitoring of autistic children requires a team of qualified professionals: pediatricians, child psychiatrists, psychologists, therapists and special educators, establishing goals for educational/social intervention, symptoms improvement and family counselling and support.

\section{VANISHING TWIN: A POSSIBLE CAUSE OF CEREBRAL IMPAIRMENT}

D Anand ${ }^{1}$, M J Platt ${ }^{2}$, P O Pharoah ${ }^{2}$

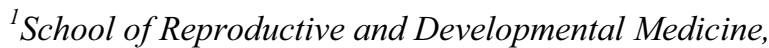
University of Liverpool, Liverpool Women's Hospital, Liverpool, UK, ${ }^{2}$ Department of Public Health, University of Liverpool, Liverpool, UK

Background and Aims: Fetal death in a twin conception during second and third trimester is associated with increased risk of cerebral injury in the surviving twin (ST). The aim of this study is to test the hypothesis that even early fetal losses, the vanishing twin (VT), may be associated with an increased risk of cerebral impairment in the ST.

Methods: 362 pregnant women attending Liverpool Women's Hospital between May 1999 and May 2001 were recruited. Women were classified according to the first ultrasound scan into three groups - 1) VT group 2) Twin group 3) Singleton group. VT group is further classified into 'Definite' and 'Possible'. Children from these pregnancies were assessed at 1 year of age for their development and neurological function using the Griffiths Mental and Developmental Scales and Optimality score.

Results: Children from 229 pregnancies (63.2\%) attended the assessment. Information on children from further $21(5.8 \%)$ pregnancies is obtained through a review of hospital records. Cerebral impairment is noted in 2 children from VT group, 2 from the Twin group and none from the singleton group. When cases with 'Definite' VT are considered there is a significant difference between the VT and singleton group (RR 6.1 [CI 1.5 to $8.3 ; p=0.03]$ ).

Conclusions: Although, when cases with 'Definite' VT were examined, there is a statistically significant association with cerebral impairment in the ST, it is possible that this may be a statistical type I error. Further studies with larger sample size are required to establish a definitive conclusion.

\section{WHEN SHOULD PHOTOTHERAPY BE STOPPED. A PILOT STUDY COMPARING TWO TARGETS OF SERUM BILIRUBIN CONCENTRATION}

M Barak ${ }^{1,2}$, F Mimouni $^{1,2}$, I Berger ${ }^{1,2}$, D Mandel ${ }^{1,2}$, S Dollberg ${ }^{1,2}$

${ }^{I}$ Department of Neonatology, Sourasky Tel Aviv Medical Center, Lis Maternity Hospital, Tel Aviv, Israel

${ }^{2}$ Sackler Faculty of Medicine, Tel Aviv, Israel

Background and Aims: AAP made recommendations about when phototherapy (PT) should be initiated, but not about when it should be discontinued. We compared two groups of jaundiced newborns randomized to one of two targets of bilirubin (TSB) concentration for photodiscontinuation.

Methods: Term infants under phototherapy were randomly assigned to two TSB groups for photointerruption: a high threshold group (photo-interruption when bilirubin decreased to $1 \mathrm{mg} / \mathrm{dL}$ below the limit for starting phototherapy and a low threshold group (when bilirubin decreased to $3 \mathrm{mg} / \mathrm{dL}$ below that limit).

Results: Mean bilirubin at phototherapy start was similar in both groups. Bilirubin rebound at 10 and 28 hours after photo-interruption was similar in both groups. Phototherapy duration and length of stay were shorter in the high than in the low threshold group ( $p=0.03$ and $p=0.05$, respectively). Re-phototherapy was needed similarly in both groups. In the presence of hemolysis or G6PD deficiency, $28 \%$ of infants required re-phototherapy compared with $8.3 \%$ when such factors were not present $(\mathrm{p}=0.066)$.

Conclusions: Phototherapy may be shortened by using higher bilirubin limits for photo-interruption. In hemolytic or G6PD deficient infants, they should be followed for longer periods since a substantial number may need re-phototherapy.

\begin{tabular}{|c|c|c|c|}
\hline \multicolumn{4}{|c|}{ Main study results } \\
\hline & $\begin{array}{l}\text { High threshold } \\
\text { group }\end{array}$ & $\begin{array}{l}\text { Low threshold } \\
\text { group }\end{array}$ & $\begin{array}{l}\mathrm{P} \text { - } \\
\text { value }\end{array}$ \\
\hline TSB at PT initiation (m g/d1) & $14.1 \div 3.2$ & $15.3 \pm 3.3$ & 0.167 \\
\hline $\begin{array}{l}\text { TSB below AAP recom mended level for } \\
\text { initiation of PT(m g'd } 1 \text { ) }\end{array}$ & $1.68 \pm 0.72$ & $4.07 \pm 1.02$ & $<0.001$ \\
\hline TSB rebound at $10 \pm 3.7$ hours $(\mathrm{mg} / \mathrm{dl})$ & $0.11 \pm 1.50$ & $0.28 \pm 1.30$ & 0.96 \\
\hline TSB rebound at $28 \pm 10$ hours ( $\mathrm{m}$ g/di) & $1.12 \pm 1.70$ & $0.68 \pm 2.13$ & 0.24 \\
\hline Duration of phototherapy (hours) & $22 \pm 13$ & $27 \pm 12$ & 0.03 \\
\hline Length of hospital stay (hours) & $84 \pm 29$ & $94 \pm 24$ & 0.05 \\
\hline $\begin{array}{l}\text { Inf ants requiring additional course of } \\
\text { photother apy, } \mathrm{N} \circ(\%)\end{array}$ & $5(20)$ & $5(18)$ & 0.58 \\
\hline
\end{tabular}

\section{SEVERE COMPLICATIONS ASSOCIATED WITH THE USE OF SYNACTHEN IN THE MANAGEMENT OF INFANTILE SPASMS}

\author{
N Ni Shuibhne ${ }^{1}$, J Mc Menamin ${ }^{1}$, D Coleman ${ }^{2}$, \\ P Oslizlok $^{2}$, D Webb ${ }^{1}$ \\ ${ }^{1}$ Department of Neurosciences, Our Lady's Hospital for \\ Sick Children, Dublin, Ireland, ${ }^{2}$ Department of \\ Cardiology, Our Lady's Hospital for Sick Children, \\ Dublin, Ireland
}

Background and Aims: The onset of infantile spasms is a neurological emergency requiring urgent treatment. Recent research indicates a superior efficacy of steroids over vigabatrin as first line treatment of infantile spasms, except in infants with Tuberous Sclerosis.

Methods: We describe five infants with serious adverse effects whilst on synacthen therapy, two of which were life threatening.

Results: Three infants were diagnosed with idiopathic infantile spasms and two had pre-existing brain injury. Age at onset was between 6 weeks and nine months. Steroids were commenced after vigabatrin in 2 cases and as third line in 3 cases because of resistant seizures. We used Synacthen depot $0.5 \mathrm{mg}$ alternate days (equivalent to 40iu ACTH) following the recommendations of this UK study. All our infants became seizure free on Synacthen therapy and hypsarrythmia resolved in all infants. Adverse effects began between 3 and 8 weeks of treatment and included excessive weight gain and hypertension in five by day 
18 , and hypertrophic cardiomyopathy in four infants by day 21. Two of these infants had associated respiratory distress and cardiac failure. Two infants had evidence of steroid myopathy. All infants required anti hypertensive therapy. Hypertension and hypertrophic cardiomyopathy resolved on cessation of Synacthen and institution of beta-blocker and diuretic treatment. Steroid myopathy and weight gain were more resistant to treatment.

Conclusions: Synacthen is effective in the treatment of infantile spasms but we would caution against its use beyond 2-3 weeks. Echocardiography should be considered in infants who develop hypertension on steroids.

\section{LABORATORY EVALUATION OF CHILDREN WITH FEBRILE CONVULSIONS}

S Liosi, N Manakou, O Gkika, G Z Zantopoulos, A Giannisi, P Anastasopoulou, Z Patrona

Department of Paediatric, General Hospital of Korinthos, Korinthos, Greece

Background and Aims: Febrile Seizures (FS) are the commonest cause of convulsions in children under five years of age. The purpose of this study was to perform an analysis of a group of children with FS in order to determine the correlation between FS and the results of laboratory examination.

Methods: We reviewed clinical records of 50 children aged 11 months to 5 years old, who were admitted for episode of febrile convulsion in our paediatric department, between January 2004 and December 2005. We have studied serum levels of sodium, potassium, calcium, phosphorus, and magnesium and blood levels of glucose in our sample.

Results: Of the 50 children, 35 had simple and 15 had complex initial febrile seizures. The median age was 27 months. Total calcium levels were lower in those children with simple convulsions in comparison with those having complicated convulsions $(9.3+/-1.22 \mathrm{mg} / \mathrm{dl}$, $\mathrm{n}=35$, and $9.5+/-1.63 \mathrm{mg} / \mathrm{dl}, \mathrm{n}=15$, respectively), but the serum potassium, phosphorus and magnesium levels showed no statistically significant differences between the patient groups. The sodium concentrations were lowest in children with simple convulsions in comparison with those having complicated convulsions $(137.22+/-2.76 \mathrm{mmol} / \mathrm{lt}$ and $138.42+/-2.58 \mathrm{mmol} / \mathrm{lt}$, respectively). In $72.43 \%$ of children blood levels of glucose were $>110 \mathrm{mg} / \mathrm{dl}$.

Conclusions: Serum levels of sodium and calcium were related to type of febrile convulsions. Blood glucose levels were significantly elevated in children with febrile seizures. These findings suggest that electrolytes serum levels should be measured in all children with febrile seizures, especially when the history is not clear or unknown.

\section{NON-TRAUMATIC INTRACRANIAL HAEMORRHAGE IN PICU: A 12-YEAR SURVEY}

M Piastra ${ }^{1}$, G Zorzi $^{1}$, S Pulitanò ${ }^{1}, \mathrm{E}_{\text {Caresta }}{ }^{1}$, A Tempera ${ }^{1}$, G Polidori $^{1}, \mathrm{C}$ Di Rocco ${ }^{2}$, L Massimi $^{2}$, R Barbaro ${ }^{1}$, D Pietrini ${ }^{1}$
${ }^{1}$ Pediatric Intensive Care Unit, Catholic University Gemelli Hospital, Rome, Italy, ${ }^{2}$ Department of Pediatric Neurosurgery, Catholic University Gemelli Hospital, Rome, Italy

Background and Aims: Haemorrhagic stroke in infants and children represents both an important cause of morbidity/mortality and an emerging area for clinical research. Differently from adult cerebrovascular disease, intracranial haemorrhage (ICH) in pediatrics has different causes, locations and mechanisms. Our aim is to analyse the clinical features, risk factors and outcomes of a large cohort of infants/children with ICH admitted to a tertiary hospital PICU over a 12-year period.

Methods: We perform a retrospective review of all nontraumatic ICH admitted to the PICU of Catholic University Gemelli Hospital, Rome, between January 1993 and January 2005. Prematurity ICH was ruled out. During the study period 98 patients with non-traumatic ICH were admitted. All cases were investigated with neuroimaging (cerebral CT, MRI or angiography) and other diagnostic procedures as required.

Results: Vascular malformations accounted for $46 \%$ of ICH admissions (followed by $16 \%$ each for malignancy/coagulopathies, about $11 \%$ each for infections/undetermined). Overall PICU mortality was $24.5 \%$ (24/98), largely depending on diagnosis $(15.5 \%$ vascular malformations, $50 \%$ malignancy, $31.2 \%$ coagulopathies/congenital syndromes, $40 \%$ infections, $27.2 \%$ undetermined origin). Neurological impairment/need for respiratory support at presentation were predictive of poor outcome in PICU.

Conclusions: Epidemiological studies revealed that $\mathrm{ICH}$ resulted as common as ischemic strokes in children, even when neonatal ICH and traumatic lesions were ruled out. Recently, an increasing interest regarding ICH has been raised by innovative therapy introduction (i.e. recombinant factor VII) for limitation of early hemorrhage growth, reducing the high risk from continued bleeding. Further studies are required in pediatric emergency and intensive care to evaluate its possible application.

\section{PSYCHOMETRIC EVALUATION OF ROMANIAN VERSION OF QOLIE-AD-48}

O Falup-Pecurariu ${ }^{1}$, D Nagy ${ }^{2}$, I Dumitru ${ }^{2}$, A Patriche ${ }^{2}$, C Falup-Pecurariu ${ }^{2}$

${ }^{1}$ Department of Pediatrics, Transilvania University, Brasov, Romania, ${ }^{2}$ Department of Neurology, Transilvania University, Brasov, Romania

Background and Aims: Measurements of healthrelated quality of life is an important issue in epilepsy. There are actually several psychometric validated scales in order to evaluate this aspects. Quality of Life in Epilepsy Inventory for Adolescents (QOLIE-AD-48) it's a self-reported scale used by adolescents for evaluation quality of life. The aim of this study was to evaluate psychometric properties of Romanian version of QOLIE-AD-48.

Methods: We translated and back translated original version of QOLIE-AD-48 and adapt culturally for Romanian people. The 8 subscales - epilepsy impact, 
memory/concentration, attitudes toward epilepsy, physical functioning, stigma, social support, school behaviour, health perceptions, and a total summary score were evaluated at 204 Romanian adolescents with epilepsy.

Results: Internal consistency reliability estimated by Cronbach's alpha coefficient was 0.81 for the summary score and between range of 0.75 to 0.90 for subscales. We performed test-retest reliability at 3 weeks period and we found a 0.79 for the summary score. Overall patients with more severe and more frequent seizures and lower socioeconomic status reported a poorer quality of life. We identified risk factors for each of 8 subscales.

Conclusions: Quality of life of epileptic patients could be measured using the Romanian version of QOLIEAD-48 that is a valid instruments. Future studies are needed to compare this scale with others scales evaluating quality of life in epilepsy.

\section{ASSESSMENT OF PREVENTIVE THERAPY IN CHILDREN WITHDRAWN FROM ANALGESIC REBOUND HEADACHE}

\author{
A Zadeh-Vakili ${ }^{1}$, M Javadzadeh ${ }^{2}$ \\ ${ }^{l}$ Department of Genetics, Zahedan University of \\ Medical Sciences, Ali-Asghar Hospital, ${ }^{2}$ Department of \\ Pediatric Neurology, Zahedan University of Medical \\ Sciences, Imam Ali Hospital, Zahedan, Iran
}

Background and Aims: Chronic daily headaches can be a difficult problem in children as well as adults. About half of the cases of chronic daily headaches in adults are thought to be due to medication-overuse headache, and treatment consists of discontinuation of these analgesics. Since many patients are also treated with preventive agents at the time of analgesic withdrawal, it is difficult to determine whether discontinuation alone is the most effective treatment.

Methods: A prospective study was performed to evaluate the outcomes of 22 children (ages 5-15 years) with medication-overuse headache 1 month after withdrawal of analgesics: 10 children received daily doses of a preventive medication, and 12 received no preventive medication. Headache reduction was assessed 1 month later.

Results: There was no significant difference in the percentage, with $90 \%$ or greater headache reduction at 1 month between children who were solely treated by withdrawal of analgesic drugs and those receiving preventive medications ( $60 \%$ vs $54 \%$, respectively). We did not find any influence of age or gender on outcomes. A previous duration of headaches over 1 years was negatively correlated with overall outcome.

Conclusions: We advise discontinuation of analgesics in all children with medication-overuse headache, without the necessity for starting daily preventive agents concurrently. Only if headaches persist after 1 month, the preventive therapy may be added.

\section{PERIPHERAL PARESIS OF FACIAL NERVE IN CRETE, GREECE}

M Avanidi, E Galanakis, P Vorgia, M Kalmanti
Department of Paediatrics, University Hospital, Heraklion, Crete, Greece

Background and Aims: Peripheral paresis of facial nerve is usually a complication, of acute or chronic inflammation of middle ear, but also can be idiopathic. In rare cases it can be associated with ear herpes Zoster virus. The aim of the present study is the assessment of influence of corticosteroids on the physical course of the facial nerve peripheral paresis in children, admitted to the referral University Hospital of Heraklion, during the 5-year period 2001-2005 were included. Irrelevantly of the reason caused the disease, the administration of corticosteroids seems not to affect hospitalization time and the decline of symptoms.

Methods: 11 children were studied ( 9 girls and 2 boys) with age ranging from 20 months to 11 years (mean 9.02). Blood and virological tests were held in all children. Ophthalmologic assessment was held in 7 children, otorhinolaryngologic assessment in 9 children, 2 children underwent MRI investigation and $\mathrm{CT}$ was done in 1 child.

Results: Evidence of viral origin was found in 1 (CMV) and of bacterial origin in 5 (Rickettsia conorii, Mycoplasma pneumoniae in 3,2 cases respectively). At 5 children, blood and virological results were negative. Ophthalmologic and otorhinolaryngologic assessment, CT and MRI did not demonstrate any pathological signs. Conclusions: More girls were affected by the disease. Hospitalization term lasted 2 days. Corticosteroids neither affected the hospitalization time nor seem to promote the remission of symptoms. None of the children showed reversion or complications. The disease isn't characterized by considerable geographic variations in etiology.

\section{STATUS EPILEPTICUS: A FREQUENT CAUSE OF ADMISSION IN PICU}

\author{
A Violaki, M Kotsiou, M Sdouga, H Volakli, \\ G Ladopoulos, M Tamiolaki
}

\section{PICU Department, Ippokration General Hospital of} Thessaloniki, Thessaloniki, Greece

Background and Aims: Status Epilepticus (SE) is a frequent cause of admission in PICU. In order to determine the causes of refractory SE as well as to evaluate the effectiveness of treatment protocols, we studied retrorpectively the files of patients admitted to our PICU with the diagnosis of SE in a 3 years period.

Methods: During the last 3 years, 44 patients were admitted to our PICU with SE, a number that reflects $10 \%$ of total admissions. Thirty four (78\%) of these patients were transported sedated and incubated from other hospitals. Midazolam was the main anesthetic agent used. Effectiveness of treatment was confirmed by clinical criteria and continuous EEG monitoring.

Results: Acute symptomatic SE mainly due to CNS infection was documented in 20 cases $(45,4 \%)$, idiopathic SE in 2 cases (4.5\%) and febrile SE in 8 cases $(18,1 \%)$. SE as manifestation of chronic neurological disorder was observed in 14 cases $(31,8 \%)$. Eight patients were intubated because of respiratory depression and the rest because of refractory SE. There were 4 deaths, 4 patients recovered without other 
anticonvulsant treatment and 36 patients recovered with one or more anticonvulsant medications.

Conclusions: CNS infection and chronic neurological disorders are the main causes of SE in children. First line treatment is often not fully applied because of respiratory depression or fear of IV administration of a benzodiazepine in the upsence of proper monitoring. As a result a conciderably high number of patients are intubated, sedated and transported to PICU.

\section{POSTERIOR CRANIAL FOSSA TUMOR}

M C Espinheira, S Soares, M Guardiano, A Maia, C Rego, M J Gil da Costa, M M Campos, J E Guimarães

Departament of Pediatrics, Hospital São João, Porto, Portugal

Background and Aims: The eradication of congenital syphilis has remained an elusive goal of public health policy. Wordwide, syphilis remains as a significant cause of neonatal morbidity and mortality. Neurosyphilis is believed to occur in 60 percent of these infants. Neurosyphilis is a pleomorphic disease, causing clinical pictures that occasionally mimic other better known neurological diseases.

Methods: The authors present a clinical case of a six year-old girl, brought to the emergency department complaining of headaches, balance disturbances, dysarthria and vomiting, that evolved for the course of three weeks. Initial cerebral computerized tomography scan showed a posterior cranial fossa lesion, with mass effect.

Results: Complementary study was performed in order to rule out neoplastic and inflammatory/infectious disease. CSF examination by polymerase chain reaction was positive for Treponema pallidum and hystopathologic findings of the brain biopsy suggested features of syphilitic gumma. The treatment consisted of Penicilin $G$ and dexametasone, with favorable clinical and imagiologic outcome.

Conclusions: Cerebral gumma is considered a rare complication of neurosyphilis in children. The authors' intention is to emphasize the importance of considering the diagnosis of syphilitic gumma in children with neurologic symptoms and a previous history of congenital syphilis. Polymerase chain reaction has proven to be a useful tool in establishing this often difficult diagnosis.

\section{MANAGEMENT OF REFRACTORY CONVULSIVE STATUS EPILEPTICUS IN THE PEDIATRIC INTENSIVE CARE UNIT}

E Vasilaki, A M Spanaki, E Mihailoudi, D Fitroulaki, $G$ Briassoulis

\section{Paediatric Intensive Care Unit, University Hospital of Heraklion, Heraklion, Crete, Greece}

Background and Aims: The optimal treatment of refractory convulsive status epilepticus (RCSE) has not been defined. The aim of our study was to determine the characteristics and treatment modalities in the management of RCSE in a PICU.
Methods: Nineteen children with RCSE are described. The incidence was $2.7 \%$ of total admissions in a five year period and the age at the onset ranged between 40 days and 14 years (12 girls, 7 boys). Clinical characteristics and treatment algorithms were correlated with the need for mechanical ventilation (MV), length of stay (LOS) and length of MV (LOMV).

Results: The most frequent primary diseases were neurodegenerative diseases $(38.9 \%)$ and encephalitis/encephalopathy (33.3\%), mostly presented as generalized refractory status $(72.2 \%)$. Five $(27.8 \%)$ children were controlled with phenytoin, phenobarbital, or valproate. The third line anticonvulsive treatment in PICU included continuous midazolam infusion $44.4 \%$, high dose phenobarbital 5.6\%, and propofol or pentothal (burst suppression coma) $16.7 \%$. Half of the patients required MV (50\%), 16.7\% had $>4$ days LOMV and $21.1 \%>10$ days LOS. None of the patients died. Regarding the need for MV, only primary disease $(p<.05)$ and degree of anticonvulsive treatment $(p<.04)$ differ significantly. In a logistic regression model, only primary disease was independently correlated with the need for mechanical ventilation support $(\mathrm{p}<.0001)$.

Conclusions: Third-line treatment is used in $3 / 4$ of children with RCSE. The need for mechanical ventilation is influenced by primary disease and the degree of treatment. An ideal new drug for RCSE having the ability to stop seizures more effectively and safely than current drugs is urgently needed.

\section{APPLICATION OF EEG MONITORING IN PICU}

M Kotsiou, A Violaki, H Volakli, M Sdouga, E Kontopoulos, M Tamiolaki

\section{PICU, Hippokration Hospital, Thessaloniki, Greece}

Background and Aims: The use of continuous EEG monitoring has been proposed for evaluation of increased ICP, ischemic events, level of sedation, status epilepticus, non convulsive seizures, metabolic disorders, intracerebral tumors and all etiologies of coma. We present the use of continuous EEG monitoring with the Infinity Express EEG pod, Siemens SC 9000 XL monitor in the assessment of treatment results in patients with status epilepticus treated in our PICU.

Methods: Continuous EEG monitoring was applied in 8 patients aged from 2 months to 9 years admitted in our ICU during the last two years. Status epilepticus was the main symptom in all patients and the underlying disease was encephalitis in 2 patients, metabolic disorder in 1, hypoxic encephalopathy in 1 , sepsis in 1 and in 3 patients no profound etiology was found. All patients needed sedation and mechanical ventilation.

Results: Continuous EEG monitoring proved to be useful in determining the duration and level of pentobarbital coma needed in 3 of our patients with intractable status epilepticus. It also helped in the recognition of subclinical seizures in 5 patients. Continuous EEG monitoring proved to be valuable for the evaluation of anticonvulsant treatment with combination of 3 drugs in 3 patients, 2 drugs in 1 patient and 1 drug in 4 patients. It has also proved the 
effectiveness of pyridoxine administration as anticonvulsive therapy in 3 patients $<1$ year old.

Conclusions: Continuous EEG monitoring provided valuable information about subclinical seizures, effectiveness of treatment and level of anesthesia in our patients.

\section{ADEQUACY OF FINAPRES TECHNOLOGY FOR DETECTING BLOOD PRESSURE CHANGES IN CHILDREN}

J Lemson ${ }^{1}$, C M Hofhuizen ${ }^{1}$, A E A Hemelaar ${ }^{1}$, S K Singh ${ }^{2}$, O Schraa ${ }^{3}$, G J Scheffer ${ }^{4}$

${ }^{I}$ Department of Intensive Care Medicine, Radboud University Nijmegen Medical Centre, Nijmegen, The Netherlands, ${ }^{2}$ Department of Thoracic Surgery,

Radboud University Nijmegen Medical Centre, Nijmegen, The Netherlands, ${ }^{3}$ BMEYE, Amsterdam, The Netherlands, ${ }^{4}$ Department of Anesthesiology, Radboud University Nijmegen Medical Centre, Nijmegen, The Netherlands

Background and Aims: Finapres technology allows for continuous non-invasive measurement of finger blood pressure and has proven to be accurate in adults. A prototype pediatric device (PFINAP) has been developed and accuracy in children has been demonstrated in an earlier study. Aim of this study was to assess the adequacy of PFINAP in detecting blood pressure changes in children.

Methods: Fifteen anesthetized children with a median age of 11 months ( 2 months -7 years) and a median body weight of $10 \mathrm{~kg}(4-31)$ undergoing congenital cardiac surgery were included in the study. PFINAP and intra arterial blood pressure (IAP) were recorded simultaneously during the procedure. The four largest IAP variations within a 5 minute interval were identified from each procedure. Afterwards, PFINAP was compared with IAP for these blood pressure changes.

Results: In 17 of 27 attempts (63\%) a non-invasive signal was obtainable. The average bias (PFINAP IAP) for mean arterial pressure was $-8,8 \mathrm{mmHg}$ (SD $7,2)$. The figure shows the changes in PFINAP compared to the changes in IAP for mean arterial pressure. The correlation is 0.98 (spearman) with $\mathrm{p}<$ 0.0001 .

Conclusions: This prototype pediatric Finapres device closely follows arterial blood pressure changes in children. However, further technical development is necessary before clinical application is feasible during surgery.

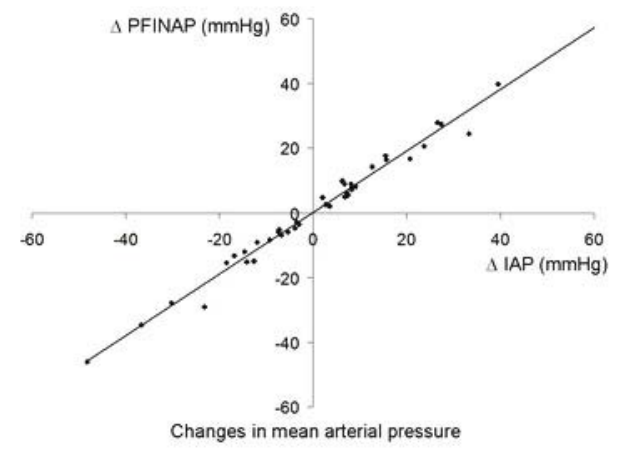

FEASIBILITY OF PULSE CONTOUR CARDIAC OUTPUT IN CRITICALLY ILL CHILDREN

J Lemson, A E A Hemelaar,

H N M van de Sant-van Alst, J G van der Hoeven

Department of Intensive Care Medicine, Radboud University Nijmegen Medical Centre, Nijmegen, The Netherlands

Background and Aims: Cardiac output (CO) measurement in critically ill children is cumbersome, but may improve hemodynamic management. Following a primary calibration, arterial pulse contour cardiac output (PCCO) provides a fast and accurate beat-to-beat measurement of $\mathrm{CO}$ in adults with special relevance to changes in CO. Aim of this study was to determine the accuracy of PCCO in critically ill small children.

Methods: We prospectively studied 13 mechanically ventilated and sedated children with a median age of 2.3 years ( 2 months -8 years) in our paediatric intensive care unit. Transpulmonary thermodilution (TPTD) with ice cold saline was considered clinical gold standard. Measurements were performed using the PiCCOplus system with software version 6 (Pulsion Medical Systems) incorporating both PCCO and TPTD techniques. PCCO and TPTD were recorded simultaneously and relative changes were compared at various intervals.

Results: A total of 144 paired measurements were analysed. Mean CO was $2,2 \mathrm{l} / \mathrm{min}(0.5-5.3 \mathrm{l} / \mathrm{min})$. With a mean interval of 7 hours (10 min $-47 \mathrm{hrs}$ ) changes in PCCO were compared to changes in TPTD. The figure shows these relative changes. The correlation coefficient (Pearson) was $0.5(\mathrm{p}<0.01)$.

Conclusions: In critically ill children the arterial pulse contour method is not an accurate method to track changes in cardiac output.

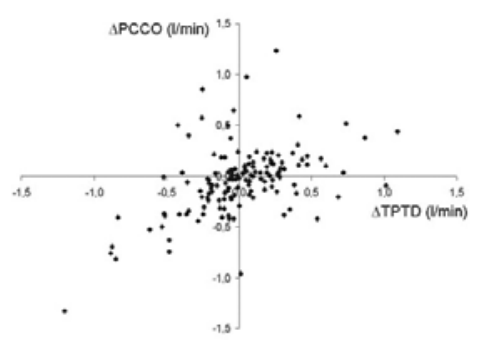

\section{RELATIONSHIP BETWEEN BNP VALUES AND CLINICAL SEVERITY INDICATORS AFTER PEDIATRIC CARDIAC SURGERY}

J C López-Menchero ${ }^{1}$, A Alcaraz ${ }^{1}$, C Romero ${ }^{2}$,

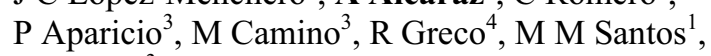
E Maroto $^{3}$

${ }^{I}$ Pediatric Intensive Care Unit, Hospital General Universitario Gregorio Marañón, Madrid, Spain ${ }^{2}$ Department of Clinical Chemistry, Hospital General Universitario Gregorio Marañón, Madrid, Spain ${ }^{3}$ Department of Pediatric Cardiology, Hospital General Universitario Gregorio Marañón, Madrid, Spain ${ }^{4}$ Department of Pediatric Cardiac Surgery, Hospital General Universitario Gregorio Marañón, Madrid, Spain 
Background and Aims: The few studies conducted in children suggest that BNP could be a useful biomarker in the diagnosis and follow-up of cardiac function after surgery. The aim of the present study was to measure BNP in children undergoing cardiac surgery and to evaluate its relationship with their clinical outcome.

Methods: Prospective observational study in 55 consecutive patients admitted to the pediatric intensive care unit (PICU) after cardiac surgery. BNP levels were determined by an inmunochemiluminiscent assay (ADVIA Centaur, Bayer) at four different times: before surgery (T0) and on first, second and third day after surgery (T1, T2, T3). BNP concentrations were examined in relation to: mean duration of PICU stay (MS), days on mechanical ventilation (MV), inotropic score (IS) $(\mu \mathrm{gr} / \mathrm{Kg} / \mathrm{min}$ dopamine $\mathrm{x} 1+$ dobutamine $\mathrm{x} 1+$ epinephrine $\mathrm{x} 100$ ) and worst PELOD score (PS) at 24, 48 and 72 hours following surgery. Data are shown as median (P25-P75), differences were assessed by the Mann-Whitney $U$ test and correlation between variables by Spearman rank correlation test.

Results: We found that postoperative maximum BNP values were significantly correlated with clinical parameters of severity. Furthermore, those patients who stayed at the PICU $>4$ days, were on MV $>24 \mathrm{~h}$, with IS $>5$, lactate $>2 \mathrm{mmol} / \mathrm{L}$ or PELOD score $>11$ showed higher BNP concentrations in the early postoperative period.

Conclusions: BNP levels on the first day after surgery were related with the severity of heart failure. Therefore, postoperative BNP could be a useful marker in the assessment and management of cardiac function in pediatric patients.

\section{LONGITUDINAL STUDY OF BLOOD PRESSURE AND BAROREFLEX SENSITIVITY IN PEDIATRIC ONCOLOGIC PATIENTS}

\author{
H Hrstková ${ }^{1}$, N Honzíková ${ }^{2}$, B Fiser ${ }^{2}$, Z Nováková ${ }^{2}$, \\ E Závodná ${ }^{2}$, St'astná ${ }^{1}$ \\ ${ }^{1} 1$ st Department of Paediatrics, Faculty of Medicine, \\ Masaryk University, Brno, Czech Republic \\ ${ }^{2}$ Department of Physiology, Faculty of Medicine, \\ Masaryk University, Brno, Czech Republic
}

Background and Aims: The aim of the present study was the analysis of blood pressure regulation in children and adolescents after treatment with cardiotoxic antracyclines for oncological diseases.

Methods: Thirty one subjects treated with antracycline (A) and 11 subjects treated with different drugs (nonA) were investigated twice (interval between investigations from 1 to 9 years) in the range between 1 and 18 years after the treatment. Baroreflex sensitivity (BRS) was determined from 5 minutes beat-to-beat recording of systolic (SBP), diastolic blood pressure (DBP) and interbeat intervals (IBI). The values of all these parameters were compared with the values obtained by the identical method in 339 healthy children and adolescents (N). Because the investigated subjects were of different age the measured values were normalized for age 16 years by linear regression and only normalized values were further analyzed.
Results: There was no difference between A, nonA and $\mathrm{N}$ in BRS and IBI. SBP and DBP were significantly lower in A (A: $102 . \pm 10.7 / 59.7 \pm 8.9$ versus $\mathrm{N}$ : $114.1 \pm 12.4 / 69.0 \pm 9.5 \mathrm{mmHg} ; \mathrm{p}<0.001 / \mathrm{p}<0.001$; mean from two investigations). SBP but not DBP was also lower in nonA $(102.7 \pm 15.2 / 64.5 \pm 11.9 \mathrm{mmHg}$; $\mathrm{p}<0.01 /$ no significant) than in $\mathrm{N}$. The correlation coefficient between time after treatment and SBP in A was -0.11 in SBP (insignificant), and -0.06 in DBP (insignificant). It indicates that there is not a trend to the normalization.

Conclusions: The anthracyline oncological therapy in children decreases SBP and DBP and within 20 years after treatment there is not a trend toward normal values. BRS was not influenced by antracycline treatment. Supported by grant IGA:NR8006.

\section{SUPERIOR VENA CAVA FLOW IN HEALTHY TERM INFANTS}

\author{
A Lee $^{1}$, E Nestaas $^{1,2}$, D Fugelseth ${ }^{1}$, L Brunvand $^{1}$ \\ ${ }^{1}$ Barneintensiv Avdeling, Barnesenteret, Ullevål \\ Universitetssykehus, Oslo, Norway, ${ }^{2}$ Barneavdelingen, \\ Sykehuset Vestfold HF-Tønsberg, Tønsberg, Norway
}

Background and Aims: The risk of impaired neurodevelopmental outcome remains a challenge in the treatment of very premature infants. Disturbances of cerebral blood flow play an important role in the pathogenesis of intraventricular haemorrhage and periventricular leukomalacia. A patent ductus arteriosus (PDA) may cause hypoperfusion of the immature brain. Kluckow et al. have shown that systemic blood flow can be estimated by measuring flow in the superior vena cava (SVC). The aim of this study was to gather experience with this method, and to investigate the relation between ductal flow and SVC-flow in healthy term infants.

Methods: Healthy term infants were enrolled consecutively during spring 2005 and underwent echocardiographic investigation on the first, second and third day of life. SVC-flow was measured according to the method of Kluckow et al.

Results: 49 infants (25 male) were enrolled. Median gestational age was 41 weeks and mean birthweight was 3670 g. 27 complete data sets were evaluated. Reasons for dropout were pneumothorax (1), VSD (1), phototherapy (3), early discharge (1), and various technical problems (16). Mean SVC-flow was 59, 52 and $45 \mathrm{ml} / \mathrm{kg} / \mathrm{min}$ on the first, second and third day. On day one, there was a trend towards increasing SVC-flow with decreasing ductal flow.

Conclusions: With some practice, SVC-flow measurement can be performed as part of a standard neonatal echocardiographic investigation. In healthy term infants with normal closure of the duct, SVC-flow seems to be rather constant during the first three days of life. Further studies are needed to investigate the influence of a PDA on SVC-flow. 
HIGH FREQUENCY OSCILLATORY

VENTILATION DOES NOT IMPAIR CARDIAC

OUTPUT IN INFANTS WITH ISOLATED

RESPIRATORY FAILURE

J Brierley, T Thiruchelvam, M Peters

Paediatric Intensive Care Unit, Great Ormond Street Hospital, London, UK

Background and Aims: The impact of the higher mean airway pressure (MAP) during high frequency oscillatory ventilation (HFOV) compared to conventional ventilation on haemodynamics remains uncertain. HFOV may decrease right ventricular venous return due to higher intrathoracic pressures or lost respiratory muscle pump. Newer non-invasive techniques allow accurate haemodynamics in smaller children than previously possible. We hypothesised HFOV would have no significant impact on Cardiac Output $(\mathrm{CO})$ expressed-Cardiac Index $=\mathrm{CO} /$ body surface area (CI) or Blood Pressure (BP).

Methods: Ten isolated respiratory failure infants had baseline haemodynamic assessment before HFOV and after 1 and 5 mins of HFOV using continuous wave non-invasive Doppler ultrasound. (USCOM). Diagnoses: 6 RSV, 1 metapneumovirus, 1 adenovirus and 2 pneumococcal pneumonia. No volume administered or inotropes (all $<5 \mathrm{mcg} / \mathrm{kg} / \mathrm{min}$ dopamine) altered during study.

Results: MAP pre $=19.7+2.8$ post $=21.6+2.4$. Mean change of cardiac index from baseline $-0.031 / \mathrm{min} / \mathrm{m} 2$ $(95 \% \mathrm{CI}=-0.15$ to $+0.08, \mathrm{p}=0.63$, paired t-test $)$ at $1 \mathrm{~min}$, and $-0.07(-0.21$ to $+0.07, p=0.4)$ at 5 mins

Conclusions: No statistically or clinically significant impact of HFOV on CO is shown as measured HFOV effects fall within expected errors of the technique. The increased MAP and effects of HFOV mechanics do not decrease $\mathrm{CO}$ in infants with single-organ respiratory failure. Concerns over deleterious effects of HFOV on $\mathrm{CO}$ appear unfounded in cardiovascularly stable infants, despite high MAPS. Although some fall in BP occurred this is not the important parameter.

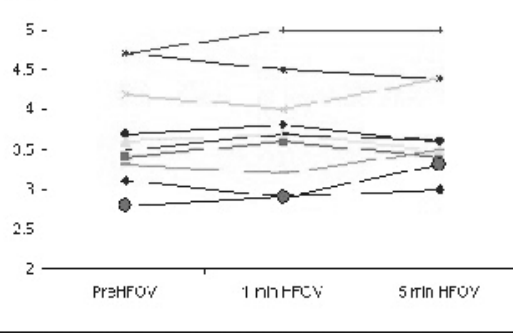

\section{THE ROLE OF PRO B-TYPE NATRIURETIC PEPTIDE IN EARLY DIAGNOSIS AND PREDICTING OUTCOME OF PATENT DUCTUS ARTERIOSUS IN PREMATURE NEONATES}

\author{
A F El-Khuffash ${ }^{1}, \mathrm{M}^{\text {Ambruso }}{ }^{2}, \mathrm{M}^{2}$ Culliton ${ }^{2}$, \\ $\mathrm{J} \mathrm{Loyta}^{3}, \mathrm{~K}$ Walsh ${ }^{4}$, EJ Molloy ${ }^{1}$ \\ ${ }^{1}$ Department of Paediatrics, National Maternity \\ Hospital, Dublin, Ireland, ${ }^{2}$ Department of Biochemistry, \\ National Maternity Hospital, Dublin, Ireland \\ ${ }^{3}$ Department of Biostatistics, University College Dublin, \\ Dublin, Ireland, ${ }^{4}$ Department of Paediatric Cardiology, \\ Our Lady's Hospital for Sick Children, Dublin, Ireland
}

Background and Aims: B-type natriuretic peptide (BNP) and pro-atrial natriuretic (pro-BNP) are both secreted by the atrium in response to volume loading. BNP is useful in predicting the magnitude of shunting through a patent ductus arteriosus (PDA) in premature neonates. We hypothesized that pro-BNP may be useful in the early diagnosis of a PDA and in monitoring treatment response.

Methods: Preterm neonates with weights $<1500 \mathrm{~g}$ and $</=30$ weeks underwent an echocardiogram on day 1 and day 3 of life coupled with a pro-BNP assay. Neonates who did not have a PDA underwent a repeat echo and BNP measurement on day 5. In patients who received treatment for a PDA, an echo and BNP were repeated 48 hours after completion of treatment.

Results: A total of 14 neonates [mean (SD) weight 1083g (259), mean gestation 27 weeks (1.8)] had a total of 39 paired echocardiographs and Pro-BNP levels assays. Infants that required PDA treatment had a significantly higher pro-BNP level (pmol/L) on day 3 of life which decreased post treatment to levels similar to the non PDA group.

Conclusions: Elevated Pro-BNP is a good early predictor of the presence of a haemodynamically significant duct. Day one levels may have a role in predicting which neonates will develop a PDA. Successful closure of PDA is reflected by a decrease in the pro-BNP levels to values comparable to those of neonates with no duct and may have a role in replacing echo to confirm duct closure.

\begin{tabular}{|c|c|c|c|}
\hline Pro-BHP & Day 1 & Day 3 & Day 5 \\
\hline $\begin{array}{c}\text { No PD A } \\
\mathbf{n = 9}\end{array}$ & 2981 & 1821 & 3156 \\
\hline $\begin{array}{c}\text { PDA } \\
\mathbf{n}=\mathbf{5}\end{array}$ & 8880 & 16413 & 2793 \\
\hline D value 1 & 0.17 & 0.01 & 0.92 \\
\hline
\end{tabular}

\section{THE ROLE OF LEFT VENTRICULAR OUTPUT IN MAINTAINING ADEQUATE SYSTEMIC PERFUSION IN THE PRESENCE AND ABSENCE OF A PATENT DUCTUS ARTERIOSUS IN PREMATURE INFANTS}

\author{
A F EL-Khuffash ${ }^{1}, \mathrm{~J} \mathrm{Loyta}^{2}, \mathrm{~K}$ Walsh ${ }^{3}, \mathrm{EJ} \mathrm{Molloy}^{1}$ \\ ${ }^{1}$ Department of Paediatrics, National Maternity \\ Hospital, Dublin, Ireland, ${ }^{2}$ Department of Biostatistics, \\ University College Dublin, Dublin, Ireland, \\ ${ }^{3}$ Department of Paediatric Cardiology, Our Lady's \\ Hospital for Sick Children, Dublin, Ireland
}

Background and Aims: Patent Ductus Arteriosus (PDA) is a significant cause of morbidity in premature neonates. We aim to assess the significance of LVO in maintaining good perfusion in the descending aorta (DAo) and the superior mesenteric artery (SMA) in premature neonates and demonstrate the negative impact of a PDA on systemic blood flow in the setting of poor LVO.

Methods: Premature neonates less than $1500 \mathrm{~g}$ were included in this prospective observational study. Echocardiographic evaluation was carried out on days 1, 3 and 5 of life. In neonates requiring treatment of PDA, a further echo was carried out to confirm closure. LVO, systolic and mean velocities of the SMA and DAo were measured. 
Results: 14 neonates underwent 42 echos with 126 measurements of LVO coupled with DAo and SMA flow. 6 neonates improved their LVO in the first three days of life compared to 8 neonates that that did not [mean (SD) birth weight $1.127 \mathrm{~g}$ (209), and $1.016 \mathrm{~g}$ (204) respectively]. Poor LVO was associated with reduced systolic and mean velocities of SMA and DAo and increased incidence of absent end diastolic flow.

Conclusions: LVO up-regulation in the first 3 days of life may be vital to improve systemic perfusion in preterm neonates. Failure of LVO up-regulation is associated with reduced superior mesenteric and descending aorta perfusion. The presence of a PDA in the setting of a poor LVO exacerbates this phenomenon.

\begin{tabular}{|c|c|c|c|c|}
\hline & $\begin{array}{c}\text { Good LVO } \\
\Gamma=6\end{array}$ & $\begin{array}{l}\text { Poor LWO } \\
\Gamma=8\end{array}$ & \begin{tabular}{|c|}
$P$ \\
value \\
\end{tabular} \\
\hline \multicolumn{2}{|c|}{$\begin{array}{l}\text { LVO dfferential D1 to D3 } \\
\text { [mls/kg/min] }\end{array}$} & $+78(43 \%)$ & $-30(-12 \%)$ & 0.004 \\
\hline \multicolumn{2}{|c|}{ SV differertial D1 to $D B$ (mls) } & $+0.35(22 \%)$ & $-0.23(-142)$ & 006 \\
\hline \multirow{3}{*}{ SMA } & Systdic velocity $(\mathrm{m} / \mathrm{s})$ & 055 & 0.35 & 0.004 \\
\hline & Mean velocity $(\mathrm{m} / \mathrm{s})$ & 0,33 & 0.23 & 002 \\
\hline & QEDF & $1 / 6(16 \%)$ & $3 / 8(37 \%)$ & NA \\
\hline \multirow[t]{3}{*}{ D\%o } & Systdic velocity $(\mathrm{m} / \mathrm{s})$ & 126 & 0.73 & 007 \\
\hline & Mean vel ocity $(\mathrm{m} / \mathrm{s})$ & 0.70 & 0.42 & 0.1 \\
\hline & QEDF & $0 / 6(0 \%)$ & $4 / 8(50 \%)$ & NA \\
\hline \multicolumn{2}{|c|}{ Total Parerteral Nurition (dayw) } & $14.4(+42.12)$ & $22.1(+45.10)$ & 003 \\
\hline
\end{tabular}

\section{DIURESIS FOLLOWING INDOMETHACIN TREATMENT IN PRETERM INFANTS WITH PERSISTENT DUCTUS ARTERIOSUS IS DEPENDENT ON FLUID INTAKE BEFORE TREATMENT}

\author{
C M Lameijer, M van Stuijvenberg, A F Bos
}

Department of Pediatrics, Division of Neonatology, University Medical Center, Groningen, The Netherlands

Background and Aims: Indomethacin treatment leads to a transient decline in diuresis in many preterm infants. Administration of cyclo-oxygenase inhibitors to hypovolaemic patients bears a significant risk of renal failure. The severity of side-effects following indomethacin treatment in preterm infants might be influenced by fluid management. The aim of this study was to determine the relationship between fluid intake before and diuresis following indomethacin treatment for patent ductus arteriosus (PDA).

Methods: We retrospectively studied all preterm infants born in 2004 and admitted to the NICU, University Medical Centre Groningen, who were treated with indomethacin for PDA. Fluid intake and diuresis were determined on a daily basis, from 2 days before indomethacin treatment up to 5 days after treatment.

Results: 24 preterm infants were included, gestational age median $28 \mathrm{w}$ (range: $25-31 \mathrm{w}$ ), birth weight median $993 \mathrm{~g}$ (range: 675-1840 g). Following indomethacin treatment, diuresis decreased in all infants to a minimum of (median) $48 \mathrm{ml} / \mathrm{kg} / 24 \mathrm{~h}$ (range 13-98 ml/ $\mathrm{kg} / 24 \mathrm{~h}$ ) on day 1 (range 0-4 days). A lower fluid intake before treatment was associated with a stronger decline in diuresis on day 1 to 4 following treatment (Spearman's $\mathrm{r}=0.545, \mathrm{p}=0.011$ for fluid intake 2 days before and $\mathrm{r}=0.670, \mathrm{p}<0.001$ for fluid intake 1 day before treatment). Gestational age, postnatal day of treatment with indomethacin and success of ductal closure did not influence the severity of the decline in diuresis.
Conclusions: A higher fluid intake before indomethacin treatment might reduce the severity of the decline in diuresis after treatment.

\section{PERTUSSIS REQUIRING INTENSIVE CARE}

\author{
J Surridge ${ }^{1}$, E R Segedin ${ }^{1}$, C C Grant ${ }^{2,3}$ \\ ${ }^{1}$ Paediatric Intensive Care Unit, Auckland District \\ Health Board, Auckland, New Zealand, ${ }^{2}$ Department of \\ General Paediatrics, Auckland District Health Board, \\ Auckland, New Zealand, ${ }^{3}$ Department of Paediatrics, \\ University of Auckland, Auckland, New Zealand
}

Background and Aims: Severe pertussis in young infants remains difficult to treat. Pertussis is prevalent in New Zealand and thus many infants are hospitalised with severe pertussis. The study aims were to describe children with pertussis who required intensive care.

Methods: An audit in Auckland, New Zealand, of all pertussis admissions to the national paediatric intensive care unit (PICU) 1991-2003.

Results: 72 children were admitted to PICU, 97\% $<12$ months old. The annual number increased with time $(\mathrm{p}=0.04) .40$ children $(56 \%)$ had coughed for $<8$ days before admission. Apnoea or paroxysmal cough were present in $33(83 \%)$ of these 40 . Of the 72 children $19 \%$ were readmitted to PICU. Those readmitted presented with more atypical disease, had a shorter first admission but longer total PICU admission (9 versus 5 days, $\mathrm{p}=0.009) . \quad 35 \quad(49 \%)$ children received assisted ventilation. 4 children died. 9 of 58 children from Auckland either died (3), or had subsequent respiratory or neurodevelopmental problems (6). There was an increased risk (RR, 95\% CI) of death or disability associated with an elevated lymphocyte count (5.26, $1.48-12.85)$, seizures or encephalopathy $(5.38,1.54-$ $8.44)$, shock $(6.80,2.22-8.95)$, abnormal neurological signs $(8.82,3.10-14.56)$, readmission to PICU (4.31, 1.36-8.41), intubation/ventilation (14.43, 3.64-31.24), and receiving inotropes $(14.33,1.73-16.41)$ or nitric oxide (8.80, 3.50-10.98).

Conclusions: Apnoea and paroxysmal cough are key symptoms in those with shorter cough duration. Death or disability are frequent. Clinical factors define children at increased risk of these poor outcomes. Early discharge from PICU is associated with an increased risk of readmission and poor outcome.

\section{AUTOLOGUES FIBRIN GLUE USE AFTER TONSILLECTOMY}

N Cekic ${ }^{1}$, K Georgievski $^{1}$, R Filipovski $^{1}$, O Zografski ${ }^{2}$, K Cekic Dimovska ${ }^{3}$

${ }^{1}$ ENT Unit, Military Hospital, Skopje, Macedonia, ${ }^{2}$ ENT Unit, MC Veles, Skopje, Macedonia, ${ }^{3}$ PO Pinokio,

Skopje, Macedonia

Background and Aims: The aim of the study is to evaluate the effects of autologues fibrin glue use after tonsillectomy.

Methods: Two groups of patients underwent tonsillectomy in endotracheal anesthesia. All patients were previously tested on coagulation status/disorders. In all 22 patients of the first group, immediately after 
tonsillectomy, tonsillar fossae were filled up with autologues fibrin glue with $4 \%$ Lidocaine. Three of the patients had some coagulation disorders (extended protrombine time or extended partial trombine time). In 20 patients of second group, mono or bipolar electrocutter to stop bleeding, was used.

Results: Wong-Baker pain scale was used for postoperative pain prediction after operation and during first four postoperative days, in regular time periods. Bleeding within 24 hours after operation, were considered as early postoperative bleeding. Postoperative pains in patients of the first group last 2-3 days and after that time were very week, while in second group pains last 7 to 10 days. There were no postoperative bleeding in both groups.

Conclusions: These results suggest that the use of autologues fibrin glue with lidocaine applied in tonsillar fossae after tonsillectomy reduce postoperative pains and period to normal nourishment. Haemostatic effect of autologues fibrin glue has shown as perfect, without early and postoperative bleeding even in patients with coagulation disorders.

\section{SURFACTANT IN AN INFANT WITH ASPIRATION SYNDROME}

\author{
M Bisceglia $^{1}$, M Piccirillo ${ }^{2}$, F Tancioni ${ }^{2}$, M Verre $^{2}$ \\ ${ }^{I}$ Neonatal Intensive Care Unit, Crotone Hospital, \\ Crotone, Italy, ${ }^{2}$ Intensive Care Unit, Hospital S. \\ Giovanni di Dio, Crotone, Italy
}

Background and Aims: After impressive results in the treatment of respiratory failure in preterm infants, naturl surfactant has been proposed in lung pathologies involving suspected surfactant deficiency: bronchiolitis, pneumonia, aspiration and chest trauma, which can lead to adult respiratory distress syndrome (ARDS).

Methods: A.D., an 18 months-old infant, had febrile seizure and loss of conscience. At the admission he was connected to the ventilator limiting tindal volume of less than $8 \mathrm{ml} / \mathrm{kg}$; fraction of inspired oxygen (FiO2) of 0.6 ; peak inspiratory pressure (PIP) of less then $36 \mathrm{~mm}$ $\mathrm{mmHg}$, ventilation rate of $36 \mathrm{~b} / \mathrm{min}$. The chest $\mathrm{x}$ ray was characterized by aspiration syndrome and pneumotorax. The severe respiratory distress and the ventilatory parameters led us to consider the use of surfactant. We supply surfactant by intratracheal instillation at the dose of $0.5 \mathrm{ml} / \mathrm{kg}$ (CUROSURF).

Results: It was observed a rapid and persistent improvement after the somministration of surfactant during the first 4 hours. In fact, after 4 hours we could modify ventilatory parameters using $\mathrm{FiO} 2$ values near the 0.21 and using CPAP ventilation. Even the $\mathrm{X}$ ray was clearly improved. The patient was extubated and he was discharged three days after.

Conclusions: Surfactant dysfunction probably plays a role in the pathophysiology of severe paediatric ARDS triggered by pneumonia, or aspiration syndrome etc., as it was found that surfactant instillation rapidly improved gas exchange in many cases of infants and children as described in literature. Larger randomized controlled studies are necessary to evaluate the effects of surfactant treatment on morbidity and mortality.

\section{INCONTINENTIO PIGMENTI WITH GLAUCOMA, RETINAL ABLATION AND RETROBULBAR MASS IN A SIX YEAR OLD GIRL (CASE REPORT)}

G Jovanov ${ }^{1}$, K Damovska ${ }^{2}$, V Tasic ${ }^{2}$, A Jancevska ${ }^{2}$, Z S Gucev ${ }^{2}$

${ }^{1}$ Health Center Skopje, Skopje, Macedonia, ${ }^{2}$ Medical Faculty Skopje, Skopje, Macedonia

Background and Aims: Description of patient with incontinentio pigmenti and eye complications (glaucoma, retinal ablation and retrobulbar mass).

Methods: Six year old girl with incontinentio pigmenti, neonatal diagnosed and eye complications. The diagnoses was performed by clinical signs and laboratory methods dermal biopsy, EKG, X-rays of chest, ultrasound, MRI and genetic analyses).

Results: A 6 year girl was first observed neonatal. Blisters and erythema in linear fashion were present. Dermal histology showed acantotic Malpighi structures, intracellular edema and vesicles filled with eosinofils. The perivesicular epiderm was filled with eosinophil cells, as well as the upper and middle dermis. At the age of 6 year she has normal stature ( 75 percentile on the growth curve), bird facies and long and hyperextensive fingers. Rare teeth were also noted. The hair is thin and moderately sparse. Two supernumerary nipples are present under the normal ones. Additionally she was found to have mitral valve prolapse (EKG, X-rays of the chest, ultrasound). Left eye glaucoma (treated with antiglaucomatous drugs) was found. Retinal ablatia was also present on the same glaucomatous eye. Retrobulbar tumorous mass with features of connective tissue was found on ultrasound and MRI. The family history was uneventful. Genetic diagnosis for the eventual IP2 type (NEMO gene) is pending.

Conclusions: The association between IP and glaucoma, retinal ablation and retrobulbar mass is of interest because of the combination of these rare conditions.

\section{FATAL STROKE AND MULTIORGAN FAILURE AS SUDDEN ONSET OF A MITOCHONDRIAL DISEASE IN A YOUNG INFANT}

M Benedetti, M Soffiati, P Santuz, C Ghizzi, M Bolognani, P Bonetti, F Zaglia, S Marzini, P Biban

Paediatric and Neonatal Intensive Care Unit, Major City Hospital, Azienda Ospedaliera di Verona, Verona, Italy

Background and Aims: MELAS (mitochondrial myopathy, lactic acidosis and stroke-like episodes) is a fairly common metabolic syndrome characterised by various clinical manifestations. The majority of patients with MELAS are adults younger than 40 years. A relatively small number of diagnoses have been confirmed on the basis of muscle biopsy in childhood, while diagnosis is rarely performed in infancy. We describe a young infant with an abrupt and fatal onset of MELAS.

Methods: The subject was a 4-month female infant, born at term by consanguineous parents. The neonatal period was uneventful. Family history revealed a sister 
who died at 2 years of age with MELAS and respiratory failure. Muscle biopsy showed multiple enzymatic deficits of respiratory chain complexes (I, II, III, IV and citrate synthase).

Results: The patient was referred to our Unit for a stroke-like episode with loss of conscience, circulatory failure, seizures and apnoea. On day 1, CT scan and cerebral MRI revealed severe bilateral parieto-temporooccipital ischemic lesions. Main laboratory findings included lactic acidosis, hypoglicemia, AST/ALT elevation and progressive renal failure. No signs of sepsis were found. Despite aggressive treatment, the patient died on day 7 , due to progressive multiple organ failure. Muscle biopsy showed the same multiple deficits of respiratory chain complexes found in the patient's sister.

Conclusions: Stroke and fatal multiorgan failure in infants may be a sudden presentation of a mitochondrial disease. MELAS should be suspected in any infant with these symptoms. Once the diagnosis is confirmed, aggressive treatment must be attempted and genetic counselling to parents provided.

\section{HAEMOPHAGOCYTIC LYMPHOHISTIOCYTOSIS AND PAEDIATRIC INTENSIVE CARE ADMISSIONS}

\section{O Kapellou, P Lister}

\section{PICU, Great Ormond Street Hospital NHS Trust, London, UK}

Background and Aims: Haemophagocytic Lymphohistiocytosis (HLH) is a rare cause of Multi Organ Dysfunction Syndrome (MODS). Our data of patients with HLH admitted to intensive care from January 1993 to January 2001 showed poor outcome, despite treatment, with 15 out of 16 children dying due to multiorgan failure. Recently an overall survival of $58.5 \%$ was reported after haematopoietic stem cell transplantation. We wanted to review outcomes over the last 5 years and compare to the earlier period.

Methods: Retrospective review of the medical records of children admitted to PICU with HLH from February 2001 to March 2006.

Results: We identified 14 patients with 19 admission episodes. They all had failure of 2 or more organ systems on admission. 5 patients died during their admission. All the death episodes had 4 or more systems failing on admission in PICU. 3 of the 5 newly diagnosed patients died. 3 of the deaths were during the first PICU admission. 3 had Bone Marrow transplant and survived their admission.

Conclusions: There seems to be an improvement in the episode outcome of the PICU admissions with MODS due to HLH.

\section{UTILITY OF OESOPHAGEAL DOPPLER MONITORING IN FLUID MANAGEMENT IN PAEDIATRIC SEPTIC SHOCK}

R Chhabra, P Maheshwari, A Desai, S Nadel, D P Inwald
Background and Aims: To determine the utility of oesophageal Doppler monitoring in the assessment and management of fluid status in paediatric septic shock.

Methods: 8 patients with inotrope-dependent septic shock were studied, with a median age of 12 months (range 6 months-2 years) and weight of $10 \mathrm{~kg}(5-12 \mathrm{~kg})$. A KDP72 oesophageal Doppler probe (Deltex Medical, UK) was inserted. Stroke volume and cardiac output were calculated from aortic blood flow velocity using nomograms based on age, weight and height. Flow time corrected for heart rate (FTc), which provides an index of preload, was also measured. The patients received 16 boluses of $4.5 \%$ albumin solution $(10 \mathrm{mls} / \mathrm{kg})$ prescribed on the basis of decreased FTc, indicative of hypovolaemia. Heart rate (HR, bpm), mean arterial blood pressure (MAP, $\mathrm{mmHg}$ ), lactate $(\mathrm{mmol} / \mathrm{l})$, cardiac index $(\mathrm{CI}, 1 / \mathrm{min} / \mathrm{m} 2)$ and FTc $(\mathrm{ms})$ were recorded before and after the fluid bolus. Inotropic support remained unchanged between paired measurements.

Results: Administration of fluid was associated with significant increases in FTc from $307(+7)$ to $332(+10)$, $\mathrm{p}=0.0008$ (mean $+95 \%$ confidence limits, paired t-test), CI from $3.17(+0.31)$ to $3.55(+0.33), \mathrm{p}=0.01$ and MAP from $58(+6)$ to $62(+5), \mathrm{p}=0.02$. There were significant decreases in HR from $163(+9)$ to $158(+8), \mathrm{p}=0.02$ and lactate from $1.6(+0.3)$ to $1.4(+0.3), \mathrm{p}=0.003$. Increase in FTc following fluid correlated with increase in CI $(\mathrm{p}=0.02)$.

Conclusions: These findings support the use of the oesophageal Doppler monitoring in the management of fluid status in septic shock in children.

\section{NATIONAL PAEDIATRIC INTENSIVE CARE ACTIVITY (UK) 1998 - 2003 AS RECORDED BY THE HOSPITAL EPISODE STATISTICS DATABASE}

\section{H Livingston, S M Whiteley}

Paediatric Intensive Care Unit, St James's University Hospital, Leeds, UK

Background and Aims: The hospital episode statistics database 1 (HES) provides information on all inpatients admitted to NHS hospitals in England from 1989 onwards. We examined the data held in relation to PICU admissions, to identify trends in activity and PICU outcome.

Methods: A search strategy was devised to identify the number of admissions to general paediatric intensive care units for the period 1998-2003. The data was stratified for age, mode of original admission to both hospital and PICU (acute/elective), source of admission to PICU and mortality.

Results: The HES query returned large quantities of useful data. Key findings are shown below.

Conclusions: The hospital episode statistics database provides useful information describing the activity of paediatric critical care facilities throughout England. This data will provide a useful comparison to the prospective data set being collected by the UK Paediatric Intensive Care Audit Network (PICANET). 1 Department Of Health, Hospital Episode Statistics: London, UK.

PICU, St Mary's Hospital, London, UK 


\begin{tabular}{|c|c|c|c|c|c|c|c|c|}
\hline \multirow{2}{*}{\multicolumn{2}{|c|}{$\begin{array}{l}\text { Table } 1 \text { PICU admission } \\
\text { rate }\end{array}$}} & \multicolumn{7}{|c|}{ Year } \\
\hline & & $\begin{array}{l}1998- \\
1999\end{array}$ & 1999-2 & 2000 & & 001 & $\begin{array}{l}2001- \\
2002\end{array}$ & $\begin{array}{l}2002- \\
2003\end{array}$ \\
\hline \multicolumn{2}{|c|}{$\begin{array}{l}\text { Total Paedlatric Hospital } \\
\text { Admissions }\end{array}$} & 1021132 & \multicolumn{2}{|c|}{1001604} & \multicolumn{2}{|c|}{977123} & 987927 & 981347 \\
\hline \multicolumn{2}{|c|}{ Total PICU Admissions } & 6540 & \multicolumn{2}{|c|}{6808} & \multicolumn{2}{|c|}{8426} & 10375 & 11201 \\
\hline \multicolumn{2}{|c|}{$\begin{array}{l}\text { PICU Admission Rate from } \\
\text { Hospital Inpatients }\end{array}$} & $0.64 \%$ & \multicolumn{2}{|c|}{$0.68 \%$} & \multicolumn{2}{|c|}{$0.86 \%$} & $1.05 \%$ & $1.14 \%$ \\
\hline \multirow{2}{*}{\multicolumn{2}{|c|}{$\begin{array}{l}\text { Table } 2 \text { Overall Mortality } \\
\text { for PICU admission }\end{array}$}} & \multicolumn{7}{|c|}{ Age Group } \\
\hline & & $0-28 d$ & $29-362 d$ & $1-5 y$ & & 6-10yт & $11-15 y \mathrm{r}$ & $16-19 \mathrm{yr}$ \\
\hline \multirow{3}{*}{$\begin{array}{c}\text { Planned } \\
\text { PICU } \\
\text { Admissions }\end{array}$} & Survived & 880 & 3274 & 305 & & 1381 & 2164 & 2807 \\
\hline & Died & 43 & 78 & 48 & & 20 & 24 & 47 \\
\hline & $\%$ Mortality & 4.66 & 2.33 & 1.5 & & 1.43 & 1.10 & 1.65 \\
\hline \multirow{3}{*}{$\begin{array}{l}\text { Unplanned } \\
\text { PICU } \\
\text { Admissions }\end{array}$} & Survived & 2129 & 5494 & 588 & & 2748 & 4125 & 6010 \\
\hline & Died & 176 & 355 & 332 & & 150 & 259 & 471 \\
\hline & $\%$ Mortality & 7.64 & 6.07 & 5.3 & & 5.18 & 5.91 & 7.27 \\
\hline
\end{tabular}

\section{DIAGNOSTIC ROLE OF PROINFLAMATORY CYTOKINES, C-REACTIVE PROTEIN AND PROCALCITONIN IN PEDIATRIC NEUTROPENIC FEVER PATIENTS}

\author{
I Devrim ${ }^{1}$, G Seçmeer ${ }^{1}$, T Kutluk ${ }^{2}$, M Ceyhan ${ }^{1}$, \\ A Kara ${ }^{1}, \mathrm{H}_{\text {Tezer }}{ }^{1}$, A K Uludağ $\breve{~}^{3}$, A B Cengiz ${ }^{1}$ \\ ${ }^{1}$ Pediatric Infectious Disease Unit, ${ }^{2}$ Pediatric Oncology \\ Unit, ${ }^{3}$ Department of Biostatics, Hacettepe University, \\ Faculty of Medicine, Ankara, Turkey
}

Background and Aims: A reliable highly spesific and sensitive marker which could be helpful in neutropenic fever is needed. This study was planned to evaluate the diagnostic value of C-reactive protein (CRP), interleukin-6 (IL-6), tumour necrosis factor- $\alpha$ (TNF- $\alpha$ ), interleukin-1 (IL-1), procalcitonin (PCT), erythrocyte sedimentation rate (ESR) in pediatric neutropenic fever patients with documented infection and no documented infections (FUO).

Methods: We have evaluated the diagnostic value of the parameters in determining the infection and optimum time to collect samples in febrile episodes of 20 different neutropenic patients.

Results: When serum levels of all parameters at the $0,8,24$ and 48th hours of administration at hospital were compared in documented infections and FUO, we found no statistically significant difference in both group and sequential analysis did not show any difference in both group. There is no significant difference in all parameters in all durations found in the patients with short $(<7$ days) and long ( $\geq 7$ days) duration of neutropenia. The PCT levels in all time period were found to be stastistically significantly higher in patients with fever lasting more than 3 days when compared to the patients with fever less than 3 days.

Conclusions: All of the reactants failed to differentiate the patients with FUO and documented infections or estimate the duration of neutropenia, severity of the disease and duration of fever. Only PCT levels were found to be useful in prediction of duration of fever. For this reason, multicentral large studies with should be done to determine the exact place of PCT in neutropenic fever.

\section{LATE ONSET SEPSIS IN VLBW INFANTS: ROLE OF CAROTENOIDS}

D van Zoeren-Grobben ${ }^{1}$, A Vogelsang ${ }^{1}$, R A Van Lingen ${ }^{1}$, J Slootstra ${ }^{2}$, A Schaafsma ${ }^{3}$

${ }^{I}$ Department of Neonatology, Isala Clinics, Zwolle, The Netherlands, ${ }^{2}$ Department of Clinical Chemistry, Isala
Clinics, Zwolle, The Netherlands, ${ }^{3}$ Friesland Foods, Leeuwarden, The Netherlands

Background and Aims: In vitro studies have shown that carotenoids are antioxidants and immune modulators. VLBW infants may be deficient in carotenoids since carotenoids are not present in total parenteral nutrition (TPN), the main feeding source of these infants in the first week of life. We studied the role of carotenoids as antioxidants and have shown that low beta-carotene intake and plasma levels are associated with an increased incidence of BPD. In this study we evaluated the role of carotenoids as immune modulators and studied whether carotenoid levels are related to the occurrence of late onset sepsis (LOS).

Methods: Plasma concentrations of $\alpha$ - and $\beta$-carotene, lycopene, lutein, vitamin $A$ and the vitamin E/cholesterol ratio were studied at days 1, 3, 7 and 21 in a group of 109 preterm infants: 76 control, 33 with LOS. LOS was defined as clinical or culture proven sepsis $>3$ days of age.

Results: In the LOS group gestational age did not differ, birth weight was lower and TPN duration was longer (all $\mathrm{p}<0.001$ ); concentrations of vitamin A (day 7, $\mathrm{p}<0.001$ ) and $\beta$-carotene (day 3, $\mathrm{p}<0.004$; day 7, $\mathrm{p}<0.001)$ were lower. Concentrations of lycopene, lutein, $\alpha$-carotene and vitamin $E /$ cholesterol ratios did not differ between groups.

Conclusions: Low concentrations of $\beta$-carotene and vitamin A are associated with an increased risk of LOS and supplementation in TPN may be necessary. Since other carotenoids did not differ between groups, we speculate that lower concentrations of $\beta$-carotene and vitamin A in LOS infants cannot only be due to longer TPN duration.

\section{BIOELECTRICAL IMPEDANCE ANALYSIS AND SERUM LEPTIN IN HEALTHY INFANTS IN THE FIRST YEAR OF LIFE: RELATION TO THE KIND OF EARLY FEEDING}

F Savino, S A Liguori, M F Fissore, E C Grassino,
C Guidi, E Palumeri, R Oggero, R Miniero

Department of Pediatrics, "Regina Margherita" Children's Hospital, Torino, Italy

Background and Aims: Bioelectrical impedance analysis (BIA) estimates body composition through the measurement of resistance (R), reactance (Xc) and phase angle (PA). $\mathrm{R}$ is high in adipose tissue, $\mathrm{Xc}$ and PA are indicators of body cellular mass. Leptin, mainly synthesized by the adipose tissue, is also produced by mammary epithelial cells and secreted into breast milk. We studied the relationships between serum leptin concentration and BIA measurements in infants in the first year of life according to the kind of early feeding.

Methods: We studied 90 healthy term infants aged 0-12 months, 48 exclusively breast fed (BF) for at least 4 months and 42 formula fed (FF). Serum leptin concentration was determined by RIA test; BIA was performed by a single-frequency, phase-sensitive impedance analyser.

Results: In FF infants aged 0-4 months we observed a negative correlation between leptin and $\mathrm{Xc}(\mathrm{n}=23 ; \mathrm{r}=-$ $0.421 ; \mathrm{P}=0.045)$ and leptin and $\mathrm{PA}(\mathrm{r}=-0.521 ; \mathrm{P}=0.011)$. 
In $\mathrm{BF}$ infants aged 8-12 months a negative correlation between leptin and $\mathrm{R}(\mathrm{n}=20 ; \mathrm{r}=-0.521 ; \mathrm{P}=0.018)$ emerged.

Conclusions: Diet-related differences in serum leptin concentration (1) and the different correlations observed in $\mathrm{BF}$ and $\mathrm{FF}$ infants between serum leptin and BIA parameters support a possible influence of early feeding on body composition later in life. We might speculate that only in FF infants serum leptin could be an indicator of fat mass, because $\mathrm{BF}$ ones receive an exogenous source of leptin from breast milk. References: (1) Savino F et al. Ghrelin, leptin and IGF-I levels in breast-fed and formula-fed infants in the first years of life. Acta Paediatr 2005;94(5):531-7.

\section{TH1|TH2 LYMPHOCYTES RESPONSE DURING THE COURSE OF LUNG INFECTION IN CF CHILDREN}

\section{A Jelenina ${ }^{1}$, A V Orlov ${ }^{2}$, T Bulgakova ${ }^{1}$, T S Ses ${ }^{2}$

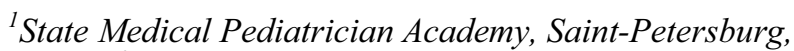 Russia, ${ }^{2}$ Institute of Pulmonology, Saint-Petersburg, Russia}

Background and Aims: Cystic Fibrosis (CF) - is characterized by early airway inflammation and recurrent respiratory infections. The aim of study was to evaluate the relationships between lung infections and production of main cytocines of Th1 and Th2 lymphocytes- IFN- $\gamma$ and IL-4 in CF children with obstructive syndrome.

Methods: Levels of IL-4 and IFN- $\gamma$ and total IgE in serum were detected by ELISA method.

Results: Frequency of S.aureus infection $(71,4 \%)$ was higher than Ps. aeruginosa infection $(28,6 \%, \mathrm{p}<0,05)$ in children with moderate $\mathrm{CF}$. Pseudomonas aeruginosa was predominant pathogen in $\mathrm{CF}$ children with severe obstructive syndrome $(100 \%)$. The total $\operatorname{IgE}$ concentration in serum of these patients was 4-5 times higher than in the healthy children. Opposite the normal level of IgE was in $35 \%$ of CF patients with S.aureus. Significant increasing of IL-4 in serum in children with severe obstructive syndrome compared with moderate and healthy children was shown. In the same time the level of IFN- $\gamma$ was higher in moderate obstructive lung syndrome (Table N1).

Conclusions: These date, whilst confirming the importance of Th1-type CD4 cells and IFN- $\gamma$ in moderate course of $\mathrm{CF}$, also suggest that the presence of Th2 lymphocytes may contribute to tissue damage associated with Ps.aeruginosa chronic lung infection.

\begin{tabular}{|c|c|c|}
\hline Patients & IL-4 (pg\ml) & INF- $\boldsymbol{\gamma}$ (pg\ml) \\
\hline I-moderate $(\mathrm{n}=14)$ & $81,64 \pm 4,62$ & $170,90 \pm 20,07$ \\
\hline II-severe $(\mathrm{n}=18)$ & $131,20 \pm 14,04$ & $87,89 \pm 0,26$ \\
\hline Healthy $(\mathrm{n}=20)$ & $32,8 \pm 2,6$ & $28,40 \pm 2,90$ \\
\hline & I-II $\mathrm{p}<0,01$ & I-II $\mathrm{p}<0,001$ \\
\hline
\end{tabular}

\section{SHOULD WE ARREST SPONTANEOUS PUBERTY IN TURNER'S SYNDROME TO OPTIMIZE GROWTH?}

M Toumba $^{1}$, I Bacopoulou ${ }^{1}$, S C Savva ${ }^{2}$, T Apsiotou ${ }^{1}$, T Georgiou ${ }^{1}$, S Stavrou $^{1}$, N Skordis ${ }^{1}$
${ }^{1}$ Pediatric Endocrine Unit, Makarios Hospital, Nicosia, Cyprus, ${ }^{2}$ Research and Education Institute of Child Health, Nicosia, Cyprus

Background and Aims: The combined treatment with gonadotrophin-releasing hormone analogues $(\mathrm{GnRH})$ and growth hormone $(\mathrm{GH})$ has been beneficial in increasing final height in girls with precocious or early puberty with or without GH deficiency and in girls with ISS. The hope that interrupting puberty might increase adult height has led to the thought of using combined treatment in girls with Turner syndrome (TS) and spontaneous puberty (SP).

Methods: Three girls with TS and SP were involved. They started treatment with GH at the age of diagnosis (4.5, 10.0, 10.0 years). All of them entered puberty spontaneously at the age of $10.9,11.0$ and 10.2 years respectively. After the confirmation of their pubertal onset with LHRH stimulation test, GnRH analogue treatment was added. Auxological and pubertal development were evaluated at 3-6 months intervals. Bone age (BA) was also determined using the Greulich and Pyle method. The special CDC TS-charts were used.

Results: Just before staring GH treatment, the mean height was $0.63 \pm 0.92$ SDS whereas just before adding $\mathrm{GnRH}$ analogue was $1.11 \pm 0.46$ SDS $(\mathrm{p}=0.18)$ ( $\triangle \mathrm{SDS}: 0.48$ ). After a mean duration of $22.50 \pm 2.12$ months of combined treatment the mean height was $2.11 \pm 0.85$ SDS $(p=0.10)(\Delta$ SDS:1.00). BA was not significantly advanced.

Conclusions: Combined treatment with $\mathrm{GnRH}$ analogue and GH seems to improve height in girls with TS and SP probably more rather than GH treatment alone. There were only 3 patients and definite conclusions will arise after the patients reach their final heights. However we should always consider the economical and ethical cost for such therapies.

\section{EVALUATION OF TEN STEPS FOR SUCCESSFUL BREAST-FEEDING IN VALIASR HOSPITAL IN KURMSHAHR IRAN 2004}

S Nouhjah ${ }^{1}$, M Mojadam ${ }^{2}$

${ }^{l}$ Department of Public Health, School of Health Medical Sciences, University of Joundishapoor, Ahwaz, Iran

${ }^{2}$ Province Health Center, Ahwaz, Iran

Background and Aims: The baby friendly hospital intiative is considered one of the most successful international efforts ever performed to protect, promote and support breast feeding. Objective of this study was detrmine the level of implementation to each of ten steps and to analyze the relationship between the implementation for each of ten steps and selected demographic characteristics.

Methods: A descriptive survey was conducted in Khuramshahr (a city in Khuzestan, Southwestern of Iran) in 2004. 376 women who attendies to health centers for receiving health care were participated in this study. They had done delivery in Valiasr hospital (baby friendly hospital) during last year. An 27itemsquestionnaire was fulled by trained questioners. Observation and questionnaire were instruments for data collection . 
Results: The average breast feeding initiation rate was $95.9 \%$ in the hospital three of ten steps were implemented with a low adherence rate (steps: 6,7,10). Three steps were implemented with a moderate adherence rate (steps $: 3,4,5$ ) The highest implementation rate observed in step of $6(95.9 \%)$ and the lowest rate was related to implementation of step of $8(66.5 \%)$. Two way ANOVA revealed that women who had a Cesarean Section experienced a significant difference in the initiation and continue to breastfeeding $(\mathrm{p}<0.001)$. Conclusions: The implantation of ten steps overall is relatively good, but maternity services must increase their efforts to consistently implement the ten steps, to become more baby friendly and to more fully establish a breast feeding culture within their institutions. Key words: Baby friendly hospital, ten steps to successful breastfeeding.

\section{THE DIFFERENCES IN SOMATOMETRIC PARAMETERS AMONG CHILDREN LIVING IN URBAN/RURAL AREAS AND GYPSY CHILDREN}

\section{Vlacha, A Filias \\ Department of Pediatrics, University Hospital of Rion, Patras, Greece}

Background and Aims: The obesity epidemic is a growing concern in the developing countries. The aim of the study was to compare the somatometric parameters of children living in urban/rural areas and Gypsy children.

Methods: A total of 343 children were included in the study; Group A: 208 city children, Group B: 99 children from rural areas and Group C: 36 Gypsy children. The age, sex, weight, height, and the body mass index (BMI) were recorded.

Results: The mean (sd) of BMIs were; Group A: BMI=19.44 (4.09), Group B: BMI=19.55 (3.55), Group C: $\mathrm{BMI}=18.15$ (2.6). No statistical differences were reported among the 3 groups. $\mathrm{BMI}<18$ and $\mathrm{BMI}>25$ was documented in $43 \%$ and $9.6 \%$ of city children, $40 \%$ and $7 \%$ of children from rural areas and $58 \%$ and $0 \%$ of Gypsy children, respectively. Importantly, a positive linear relationship was reported between BMI and age in children from Group A $(\mathrm{R} 2=0.218, \mathrm{p}<0.001)$ and from Group B $(\mathrm{R} 2=0.184, \mathrm{p}<0.0001)$. Moreover, the differences in BMI were more prominent in children older than 10 years in Group A $(p<0.001)$ and Group B $(p<0.001)$ Those differences were not seen in Gypsy children.

Conclusions: There were no differences in BMI among children living in urban, rural areas and Gypsy children. About half of the children had BMI $<18$. One out of 10 of city children were overweight. None of the Gypsy children were overweight. The higher prevalence of overweight was particularly large in older children.

\section{NON-CYSTIC FIBROSIS BRONCHIECTASIS IN CHILDHOOD: REPORT OF TWO CASES}

P Korovessi $^{1}$, I Papadea ${ }^{1}$, T Poukoulidou ${ }^{1}$, A Mihalopoulou $^{2}$, M Tibisranis $^{2}$, E Papadakis $^{1}$
${ }^{1}$ Department of Paediatrics, Thriasion General Hospital, Athens, Greece, ${ }^{2}$ Department of Radiology, Thriasion General Hospital, Athens, Greece

Background and Aims: Bronchiectasis is a morphological term used to describe abnormal irreversibly dilated and often thick walled bronchi. However, the precise relationship between the clinical features, radiological, and histological findings in children is unknown. Chest High Resolution Computed Tomography, has greatly improved our ability to recognise this condition and has now replaced bronchography as the 'gold standard' imaging modality for diagnosing bronchiectasis.

Methods: We present two boys, 6 and a 3 years old of age, admitted to our department with lower lobe consolidation and digital clubbing. Both children were treated several times with antibiotics for radiologically diagnosed pneumonias with the symptoms started before their first year of life. They have also received treatment for asthma on many occasions.

Results: In view of persistence of chest radiographic abnormalities we performed a chest HRCT that revealed bronchiectatic changes at the right middle lobe and thickening of bronchial walls in the right and left lower lobes in both children. The patients were investigated to determine the aetiology of bronchiectasis, with bronchoscopy, measurement of serum immunoglobulins (Igs), IgG subclasses, specific antibody responses to tetanus toxoid, and to the capsular polysaccharides of Haemophilus influenzae type b (Hib) and Streptococcus pneumoniae. Bacterial culture of bronchoalveolar lavage (BAL) fluid and Sweat tests were also performed. Both patients received a 3 weeks course of oral antibiotics and regular chest physiotherapy

Conclusions: Bronchiectasis should be suspected in children with recurrent bronchitis or pneumonia, and when despite appropriate therapy, pulmonary infiltrates or atelectasis persist 12 weeks beyond the index illness.

\section{NUTRITION AND PREVALENCE OF CONSTIPATION IN LESS THAN 2 YEARS OF INFANT}

\section{A Kazemi}

Department of Pediatric Gastroenterology, Alzahra Hospital, Isfahan, Iran

Background and Aims: Constipation is one of the common gastrointestinal problems in infant. This research has been done in order to determine constipation prevalence as well as the relationship between the age and kind of nutrition with constipation prevalence in infants

Methods: A cross-sectional study dine on 350 infants aged 1-24 months referred to 3 teaching pediatric climatic in Isfahan during 4 month in 2004. The bowel habits less than two times per week or having hard and dried stool is that meant by constipation. To compare constipation prevalence and stool consistency in different groups a Chi square test was used, and to compare the frequency of bowel habit an ANOVA test was used.

Results: 350 infants under the study showed the constipation prevalence in different groups as follows: 
76 breast - fed infants aged 1-6 months (5.2\% constipation), 44 formula - fed infants aged 1-6 months (11.4\% constipation), 19 both breast and formula - fed infants aged 1-6 months (10.6\% constipation), 119 breast infants aged 6-24 months ( $7.6 \%$ constipation), 58 formula - fed infants aged 6-24 months (19\% constipation), 34 both breast and formula - fed infants aged 6-24 months (11.8\% constipation).

Conclusions: Considering the obtained results for different aged groups. It can be concluded that constipation prevalence in formulae -fed infants in more than breast fed infants. Constipation prevalence in two groups doesn't show any significant difference. The average frequency of bowel habit in breast - fed infants is more than formula - fed.

\section{OBESITAS, IS THERE MORE?}

V Rena ${ }^{1}$, M Hegedus-Jungvirth ${ }^{2}$, D Vulic $^{3}$, A Persoglia $^{4}$, Z Konjarik ${ }^{5}$, I Francula ${ }^{6}$, S Zahirovic ${ }^{1}$

${ }^{1}$ Private Paediatric Practice, Rijeka, Croatia ${ }^{2}$ Department of Paediatrics, County Hospital Medjimurje, Cakovec, Croatia, ${ }^{3}$ Private Paediatric Practice, Split, Croatia, ${ }^{4}$ Private Paediatric Practice, Sisak, Croatia, ${ }^{5}$ Private Paediatric Practice, Djakovo, Croatia, ${ }^{6}$ Clinic of Paediatrics, Rijeka Clinical Hospital Centre, Rijeka, Croatia

Background and Aims: Obesity is growing problem among children in developed countries as in transitional countries as Croatia today. The cause of obesity is not simple and there is more than one condition causing final problem. The aim of this study is to found out how cultural, geographical and religious habits, social status and family life style influence the child obesity.

Methods: We investigated social and psychological status and eating disorders in obese children in five counties in Croatia (three in continental and two in costal part of country). In five paediatric practice in primary care, body mass index were calculated for children from two to 16 years old, during one month period. Children belonging above $85 \%$ (nearly 300 ) were included in study. A questionnaire consisted of several parts investigate social and psychological status and eating disorders, including child's habits and activity at home, kindergarten and school, and was filled up by paediatrician and clinical psychologist with active participation of parents.

Results: The results indicate importance and influence of family life style and social and psychological identity and nutrition in kindergarten and school on obesity prevalence. The results will be of great importance for guidelines for prevention of obesity among children and education of parents, nursing, teachers and children from early age to adolescence.

Conclusions: It is of great importance for obese children to be aware of their physical appearance, their status in families, schools or kindergartens and with proper education try to pass over difficulties and to raise the quality of future life.

\section{GROWTH IN TWO GROWTH HORMONE TREATED CHILDREN WITH RUSSEL-SILVER SYNDROME}

\author{
A Jancevska $^{1}$, G Jovanov ${ }^{2}$, V Tasic ${ }^{1}$, Z S Gucev ${ }^{1}$ \\ ${ }^{1}$ Pediatric Clinic, Medical Faculty Skopje, Skopje, \\ Macedonia, ${ }^{2}$ Health Center Skopje, Skopje, Macedonia
}

Background and Aims: Two children with short stature, triangular face, prominent forehead, clinodactuly, subcutaneous fat tissue hypoplasia were diagnosed as Russel-Silver syndrome. Normal intelligence was assessed in both cases. Moderate hemihypertrophy was noted in both children. In addition, the girl had a $2 \mathrm{~cm}$ shorter leg.

Methods: The girl was born small for gestational age (birth weight $1700 \mathrm{~g}$ and length $40 \mathrm{~cm}$ ), while the boy was a term born child with weight and height within normal limits. The boys bone age (BA) was significantly retarded (at age of 11 years, BA was 5), the girls BA was 3 months at the age of two. The tests for pituitary reserve were normal in both of them.

Results: Children's height at the beginning of the growth hormone treatment was significantly lower for age and sex (-3.5 SDS for the girl and -4 SDS for the boy). The first year height gain for the boy was $13 \mathrm{~cm}$, and his final height is 158 centimeters after five years of treatment. It is of note that the parents voluntarily discontinued the $\mathrm{GH}$ treatment and that this is the probably one of the reasons that he did not reach the target height of $169 \mathrm{~cm}$. The first year gain for the girl was $10 \mathrm{~cm}$. Initially well bellow the third percentile on the growth chart she reached the 3rd percentille in the course of treatment. She is still GH treated and is growing on the third percentile.

Conclusions: Both children responded favorably on the GH treatment.

\section{DEVELOPMENT OF A SIMPLE NUTRITIONAL SCREENING TOOL FOR ILL CHILDREN}

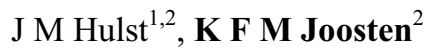 \\ ${ }^{1}$ Department of Pediatrics, HAGA Hospital, Juliana \\ Children's Hospital, The Hague, The Netherlands \\ ${ }^{2}$ Department of Pediatrics, Erasmus MC, Sophia \\ Children's Hospital, Rotterdam, The Netherlands
}

Background and Aims: Although hospitalized children are at risk of malnutrition, routine nutritional screening is impeded by lack of a simple nutritional screening tool. The aim of the study was to assess the feasibility of performing a nutritional risk screening instrument, and to relate the nutritional risk score to anthropometric parameters.

Methods: Cross-sectional pilot study at medium care units of two pediatric hospitals, in all admitted children aged $>14$ days. Measurements of weight, length and mid upper arm circumference were performed. The screening instrument consisted of 6 items: (1) Subjective clinical assessment (2) Underlying pathology (3) Diarrhea, vomiting, loss of fluids or dysphagia (4) Decreased food intake (5) Weight loss or poor weight increase (6) Poor length growth.

Results: A total of 108 children were included (median age of 5.1 years and median hospital stay 3 days). Items 
$1-5$ of the screening instrument were successfully scored in $87-100 \%$ of children, but item 6 could only be answered in $64 \%$. Total scores including items $1-5$ were obtained and nutritional risk was scored as none in $25 \%$, moderate in $57 \%$ and high in $18 \%$ of children. The median SD-scores for anthropometric measurements were significantly different between the risk groups, with the high risk group having the lowest SD-scores.

Conclusions: Standard nutritional risk screening using a simple questionnaire was possible in the majority of children and the overall score was related to anthropometric parameters. Awareness for malnutrition can be increased by using a nutritional screening tool, but further prospective investigation is needed.

\section{CORRELATIONS OF C-REACTIVE PROTEIN WITH THE METABOLIC RISK FACTORS IN CHILDREN AND ADOLESCENTS}

E Stefanaki ${ }^{1}$, E Thalassinos ${ }^{2}$, S Stefanaki ${ }^{1}$, P Neonaki ${ }^{1}$, M Mavrokosta $^{1}$, C Lydakis $^{2}$, A Tsilimigaki-Christaki ${ }^{1}$

${ }^{1}$ Second Pediatric Department, Venizelio General Hospital, Heraklio, Crete, Greece, ${ }^{2}$ Antihypertensive Unit, Venizelio General Hospital, Heraklio, Crete, Greece

Background and Aims: Our purpose was to investigate the relationship between the risk factors for metabolic syndrome and the plasma C-reactive protein (CRP) concentration as measured by high-sensitive CRP assay in children and adolescents.

Methods: We studied 74 children and adolescents. The ages ranged from 6,5 to 17 years old. Body mass index, triglycerides, high-density lipoprotein cholesterol, fasting glucose, insulin, systolic and diastolic blood pressures, and CRP were measured. Blood pressure was measured three times while seated and it was averaged for analysis. The degree of insulin resistance was determined with the use of homeostasis model assessment (HOMA) and it was calculated as the product of fasting plasma insulin level $(\mathrm{mu} / \mathrm{ml})$ and the fasting plasma glucose level (mmoles/lt) divided by 22,5 .

Results: There were statistically significant positive correlations for CRP with body mass index (Spearman's, $\mathrm{r}=0,592, \mathrm{p}<0,01)$, triglycerides $(\mathrm{r}=0,244, \mathrm{p}<0,05)$, diastolic blood pressure $(\mathrm{r}=0,351, \mathrm{p}<0,01)$ and homeostasis model assessment $(\mathrm{r}=0,464, \mathrm{p}<0,01)$. High-density lipoprotein cholesterol showed a significant negative correlation with CRP $(r=-0,366$, $\mathrm{p}<0,01$ ). No correlation was found with systolic blood pressure and with fasting glucose.

Conclusions: These results indicate that CRP, a marker of inflammation that underlies atherosclerosis, is associated with almost all metabolic syndrome risk factors in children and adolescents.

\section{THE METABOLIC SYNDROME IN CHILDREN AND ADOLESCENTS}

E Stefanaki ${ }^{1}, \mathrm{~S}$ Stefanaki ${ }^{1}, \mathrm{E}$ Thalassinos ${ }^{2}, \mathrm{P} \mathrm{Neonaki}^{1}$, M Mavrokosta $^{1}$, C Lydakis $^{2}$, A Tsilimigaki-Christaki ${ }^{1}$
${ }^{1}$ Second Pediatric Department, Venizelio General Hospital, Heraklio, Crete, Greece, ${ }^{2}$ Antihypertensive Unit, Venizelio General Hospital, Heraklio, Crete, Greece

Background and Aims: The metabolic syndrome in adults is correlated with higher cardiovascular risk. Recently, several studies discuss the presence of metabolic syndrome in children and adolescents. Our purpose was to calculate of the frequency of metabolic syndrome in three groups of children and adolescents according Body Mass Index (BMI). 1) BMI $>$ 95th percentile (obese), 2) 95th $<$ BMI $>85$ th (overweight) and 3) BMI $<85$ th percentile.

Methods: We studied 106 obese, 30 overweight children and 36 with BMI $<$ 85th percentile who were examined in the Pediatric Obesity Unit in our hospital. The ages ranged from 6,5 to 17 years old. Blood samples were received from all children after 12 hours night fasting and glucose, triglycerides and HDL were calculated. Blood pressure was also measured. The children of our study were considered to have metabolic syndrome if they completed 4 out of 5 of the following criteria according age and sex: BMI $>85$ th percentile, systolic pressure or/and diastolic pressure $>95$ th percentile, triglycerides $>$ 95th percentile, HDL $<5$ th percentile and fasting glucose $>110 \mathrm{mg} / \mathrm{dl}$.

Results: 11 out of $106(10,36 \%)$ of the obese children completed the criteria for metabolic syndrome. The youngest child was a boy 6,5 years old. Only 2 out of the $30(6,6 \%)$ overweight children also completed the necessary conditions for metabolic syndrome. In the group with BMI $<85$ th percentile there was not found any child with metabolic syndrome.

Conclusions: The relatively high frequency of metabolic syndrome in obese children should alert us in maximizing our efforts towards prevention and treatment of obesity.

\section{PAEDIATRIC HYPONATRAEMIA: INCIDENCE, AETIOLOGY AND MANAGEMENT}

A M Mc Morrow, N P Corrigan

Department of Paediatric, Altnagelvin Area Hospital, Londonderry, UK

Background and Aims: Recent publicity has highlighted the potentially catastrophic results of paediatric hyponatraemia. Little data exists however on the overall incidence and course of uncomplicated hyponatraemia in general paediatric practice.

Methods: All children admitted to Altnagelvin Hospital over a 21-month period with hyponatraemia $(<133)$ were identified using our laboratory database. Newborns and infants in NICU were excluded. Retrospective chart review enabled data collection regarding incidence, aetiology and management.

Results: 6,276 children were admitted to Altnagelvin Hospital over the study period. 153 patients $(2.4 \%)$ had at least one documented episode of hyponatraemia (medical $68 \%$ vs surgical $32 \%$ ). Of 105 medical children, the most common diagnoses were gastroenteritis $(25 \%)$, viral infections $(25 \%)$ and lower respiratory tract infection $(21 \%)$. Of 21 general surgical children, acute appendicitis accounted for $50 \%$. The 
incidence of hyponatraemia complicating acute appendicitis was $11 \%$. The primary pathophysiological trigger for the hyponatraemia was attributable to SIADH in $66 \%$ of children overall. Hyponatraemia was a presenting feature in $88 \%$ of children. Intravenous fluid therapy was prescribed for $61 \%$ of children. $0.45 \%$ saline / dextrose (64\%) and isotonic saline solutions $(27 \%)$ comprised the majority of prescribed fluids with no children receiving solution 18 . Fluid volumes were given as maintenance in $60 \%$, restricted in $31 \%$ and potentially generous in $7 \%$. No serious complications of hyponatraemia were identified.

Conclusions: Hyponatraemia is a common complication of acute illness in both medical and surgical children, with SIADH as the primary mechanism. Very hypotonic intravenous solutions are no longer being prescribed, in keeping with regional guidelines.

\section{THE TOLERANCE OF ENTERAL NUTRITION IN CHILDREN WITH ACUTE PANCREATITIS}

M Kostrzewska, E Toporowska-Kowalska, J Kudzin, K Wąsowska-Królikowska

Department of Children's Allergology, Gastroenterology and Nutrition, Poland

Background and Aims: Enteral nutrition (EN) is a preferable alimentation form for children with acute pancreatitis (AP). The subject of the study was evaluation EN tolerance in AP patients hospitalized in Children's Allergology, Gastroenterology and Nutrition Department of Medical University in Łódź, in 2005-2006.

Methods: Retrospective analysis involved the course of EN: duration, amount of calories, change of body weight and clinical tolerance of EN in 13 patients aged from 4,5 to 18 years (average 10,6 $\pm 3,96$ ), with 16 episodes observed. Low-fat, half-elementary diet was applied by nasojejunal catheter, using pomp.

Results: 13 episodes AP were mild and 3 severe (2 pseudocysts and 1 rupture of pancreas, all treated by drainage). AP etiology: abdominal trauma $(n=4)$, hereditary AP $(n=1,4$ episodes $)$, pancreas divisum $(n=1)$, cholelithiasis $(n=1)$, infectious AP $(n=2)$, post valproic acid $(n=1)$, idiopatic $(n=3)$. EN time: 5 to 46

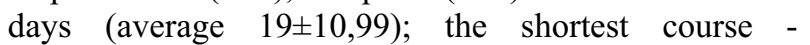
hereditary AP (average $8 \pm 2,58$ ), the longest - in posttraumatic AP (average 28,5 $\pm 12,28$ ). By EN we ensured the supply of $42,6 \pm 13,28 \mathrm{kcal} / \mathrm{kg}$ per day on average, reaching rise of body weight $900 \pm 678,23 \mathrm{~g}$ $(2,68 \pm 2,02 \%)$ in 6 children; the initial weight -2 and decrease - 8 patients (average 600 $\pm 538,52 \mathrm{~g}$; $1,37 \pm 1,02 \%$ ). Undesirable effects (nausea, diarrhoea, vomitus) were observed in 6 patients (37\%): 3 (19\%) were passing, $3(19 \%)$ - needed modification of nutritional therapy (2 - lower dose of EN, 1 - TPN).

Conclusions: EN is well tolerated by children with mild AP. The patients with posttraumatic AP, developing complications, may cause worse EN tolerance.

\section{CONGENITAL CHLORIDE DIARRHEA: A REVIEW OF 12 ARABIAN CHILDREN}

${ }^{1}$ Department of Pediatrics, Faculty of Medicine, ElMansoura University, El-Mansoura, Egypt

${ }^{2}$ Department of Pediatrics, Faculty of Medicine, Benha University, Tukh, Kaliobia, Egypt

Background and Aims: Congenital chloride diarrhea (CCD), a rare autosomal recessive disorder, is characterized by sustained watery diarrhea (due to defect of active Chloride/HCO3 exchange in ileum and colon) with high fecal chloride. The estimated incidence of CCD was 1 in 14000 in Kuwait reported in 1989. Aim of our study was to spotlight the common presentation of infants with CCD which appears not to be an uncommon disorder in Kuwait for early management and prevention of complications.

Methods: : Reviewing the inpatient database of Pediatric Department of Al-Adan Hospital, Kuwait for patients who were admitted as chronic diarrhea in the first year of life, 12 patients were diagnosed as CCD. The diagnosis of CCD was based on antenatal and early infantile presentation and confirmed by laboratory finding of excess chloride loss in stool and low serum chloride level.

Results: All 12 patients were born to consanguineous parents, had antenatal history of IUGR, polyhydramnios and distended hypoechoic fetal bowel and presented with abdominal distension, hypotonia and muscle wasting. Whereas, 10 patients $(90 \%)$ were delivered prematurely, and 9 patients $(75 \%)$ had absence of normal meconium at birth. Our patients showed significant decrease in serum sodium, potassium, chloride and urine chloride compared with the average for age and had significant increase in serum bicarbonates, plasma renin activity, serum aldosterone and stool chloride.

Conclusions: High index of suspicious and awareness should be considered for early diagnosis of CCD in this population especially in the presence of consanguineous marriage and the characteristic features in antenatal ultrasound for early investigations and appropriate management.

\section{OUT OF HOSPITAL CARDIAC ARREST IN A PEDIATRIC POPULATION: AN EPIDEMIOLOGICAL ANALYSIS}

\author{
G van Berlaer, V Van Gorp, S Hachimi-Idrissi
}

Department of Pediatric Critical Care, Academical Hospital of the 'Vrije Universiteit Brussel', Brussels, Belgium

Background and Aims: Resuscitation for pre-hospital cardio-pulmonary arrest (PCPA) in children has a very poor outcome. We believe that training Emergency Medical Service personnel in pediatric life support skills improves survival rate and neurological outcome. Our aim is to describe a large pediatric population who developed PCPA, and to outline the influence of precardiopulmonary cerebral resuscitation (CPCR) factors on survival rate.

Methods: From 1991 till 2005, clinical records of all patients younger than 16 years with PCPA were reviewed. All data were prospectively registered using a uniform standard registry, and adjusted to the Utstein style report. The studied pre-CPCR factors were 
restricted to age, site and cause of PCPA, bystander CPCR (lay or skilled), time to intubation and administration of drugs, initial cardiac rhythm and respiratory pattern on arrival of the mobile intensive care unit team.

Results: Out of all data of registered pediatric patients with PCPA, we present their median age, sites and causes of PCPA, the number of dead on arrival, the number of attempted CPCR and achievement of restoration of spontaneous circulation (ROSC). We compare the influence of different initial cardiac rhythms and respiratory patterns on the percentage of ROSC and regaining of consciousness. We also compare results of CPCR performed by lay bystanders or by EMS-personnel as a witness, skilled in pediatric life support, on survival rate and neurological outcome.

Conclusions: Rapid intervention and resuscitation by EMS-bystanders skilled in pediatric life support increases survival rate and neurological outcome in children. For this reason CPCR training, appropriate equipment and rapid intervention are mandatory.

\section{BCR/ABL FUSION IN A CHILD WITH ACUTE MYELOIC LEUCAEMIA}

Z Gucev $^{1}$, S Koceva ${ }^{1}$, O Muratovska ${ }^{1}$, S Glamocanin ${ }^{1}$, G Jovanov $^{2}$, V Vankovska ${ }^{1}$, L Tasevska ${ }^{1}$, B Taseva ${ }^{1}$, A Jancevska ${ }^{1}$

${ }^{1}$ Pediatric Clinic, Medical Faculty Skopje, Skopje, Macedonia, ${ }^{2}$ Health Center Skopje, Skopje, Macedonia

Background and Aims: Head to tail fusion of ABL protooncogene from chromosome 9 to breakpoint cluster region $(\mathrm{BCR})$ on chromosome 22 generates a hybrid $\mathrm{BCR} / \mathrm{ABL}$ gene. This hybrid is found in adult and in childhood myelogenous leucemia. In addition, the same fusion is found in $2-10 \%$ of childhood lymphoblastic leucemias. Since this BCR/ABL fusion is an ominous prognostic factor (conferring drug resistance) its early determination is important for the choice of the intensity of the treatment protocol.

Methods: We analyzed 52 children with acute leucaemias for the BCR/ABL fusion. DNA was isolated and RTPCR was performed with specific primers.

Results: Among 42 children with acute lymphoblastic leucaemias and 6 children with acute myeloic leucemia no BCR/ABL fusion was found. One out of four children with chronic myelogenous leucemia was BCR/ABL positive (25\%). Hyperleucocitosis and skin haemathomas were initial signs. Cytological and cytochemical analysis confirmed a chronic myelogenous leucemia. A chemotherapy was started and bone marrow transplantation was performed.

Conclusions: Further monitoring of the patient for $\mathrm{BCR}-\mathrm{ABL}$ fusion is in progress.

\section{CLINICAL COURSE AND TREATMENT EFFICACY IN CHILDREN WITH ATOPIC DERMATITIS}

N Kavlashvili, M Kherkheulidze, N Adamia

State Medical University, Tbilisi, Georgia
Background and Aims: Recently prevalence of allergic disorders and among them atopic dermatitis is quite high (10-20\% according data of different countries) and have increasing tendency. So, atopic dermatitis remains one of the serious problems of pediatricians. The aim of our study was to assess clinical course of atopic dermatitis in children using SCORAD index.

Methods: We studied 32 patients aged 1-6 year, who admitted to M. Guramishvili Pediatric clinic in 2005-2006 year. All patients fulfilled the criteria of Hannifin and Rajka at least 3 major and 3 minor criteria for diagnosis of atopic dermatitis. The SCORAD index was used to evaluate severity of disease. Were assessed objective signs - spread of lesions, intensity (erythema, edema, oozing, excoriation, lichenification, xerosis) and subjective signs (pruritus and sleeping disorders).

Results: The mean SCORAD index before treatment was 45,9. Treatment was conducted by Advantan (Methylprednisolole aceponate - 0,15) - corticosteroid for topical use. Advantan was used once a day on damaged skin. On third-forth days of treatment was founded improvement of clinical (SCORAD index decreases) and subjective signs. After 7 days of treatment in $70 \%$ of patients and after 10 days in rest $30 \%$ was observed clinical remission of disease (The mean SCORAD index after treatment was 5.6).

Conclusions: So, we can conclude that SCORAD index is effective and easy method for evaluation of treatment efficacy and clinical course of atopic dermatitis in children.

\section{TUBULAR AND GLOMRULAR FUNCTION IN MAJOR TALASSEMIA}

\section{S Sadeghi Bojd}

Department of Pediatrics, Zahedan Medical Sciences, Zahedan, Iran

Background and Aims: Thalassemia is a heterogenous groups of disease and are the most common genetic disorder on a worldwide basin. The severity correlated with the degree of anemia, hyper transfusion and iron chelating therapy This study is designed to define renal tubular and glumerolar abnormalities with beta thalassemia and to correlate the renal finding with clinical parameters.

Methods: One hundred sixty six patients (mean age 13.34), mean weight (31.78) were studied. The patients were 96 male $(57.8 \%)$ and 70 female (42.8\%). 24 hours urine secretion was collected and was examined for level of $\mathrm{Na}, \mathrm{K}$, Creatinin, $\mathrm{Mg}$, Uric acid, Beta 2 Microglobulin, Protein, $\mathrm{Ca}$ and Phospharose.

Results: $78.3 \%$ of patients had normal GFR and $21.7 \%$ had abnormal GFR. The mean of tubular parameters such as protein, phospharose, Uric acid, Na, Beta 2 Microglobulin, were higher than normal level. Significantly higher levels of Uric acid, P, Na, Beta 2 MG, were found compared with normal level.

Conclusions: Correlation was not found between serum ferritin and urine parameters. $(p<0.01)$. We do not know that the degree of renal dysfunction correlated to response to therapy (Iron chelation), frequent transfusion and/or splenectomy, or not? Further study in particular long term follow up in thalassemic patients 
after efficient iron chelation and hypertransfusion is needed to clarify our observation.

\section{ALL THAT GLITTERS IS NOT GOLD; ALL THAT PEES RED IS NOT HAEMATURIA}

\author{
H F Ayyash ${ }^{1}$, N Seneviratne ${ }^{1}$, A Godhamgaoknat ${ }^{1}$, \\ P Ohadike ${ }^{1}$, J Joseph $^{2}$ \\ ${ }^{I}$ Department of Paediatrics, Doncaster Royal Infirmary, \\ Doncaster, South Yorkshire, UK, ${ }^{2}$ Department of \\ Haematology, Doncaster Royal Infirmary, Doncaster, \\ South Yorkshire, UK
}

Background and Aims: Paroxysmal Cold Hemoglobinuria $(\mathrm{PCH})$ is characterised by a sudden onset of hemoglobinuria either spontaneously or after exposure to cold. The authors illustrate some of the difficulties in making the diagnosis of this very rare condition in childhood and discuss the benefits of early communication between the on call Paediatrician and Haematologist.

Methods: We report on the clinical and laboratory findings of a 3 years old boy who presented with coryzal symptoms, cough, fever and 'blackcurrant juice' coloured urine which was initially thought to be haematuria. Two weeks prior to this episode he has had chicken pox. Examination showed pallor, congested tonsils, suprapubic tenderness, ankle oedema and periorbital swelling.

Results: Blood investigations showed haemoglobin 6.4gr/dL, normal U/E's and LFT's but haemolysed potassium and bilirubin. Urinalysis showed $3+$ of blood and red cell casts but no red cells. Direct Coombs and Donath-Landsteiner Tests were positive. The patient received blood transfusion due to drop of his $\mathrm{Hb}$ to 5.3 $\mathrm{gr} / \mathrm{dL}$ and deterioration of some of his vital signs.

Conclusions: $\mathrm{PCH}$ pose a challenge for Paediatricians as it is very rare disease and mimics other conditions. It should be considered in every patient with sudden drop in hemoglobin and hemoglobinuria.

\section{HENOCH-SCHONLEIN PURPURA. OUR EXPERIENCES}

\section{P Zlatkovska}

Department of Pediatrics, Opsta Bolnica, Stip, Macedonia

Background and Aims: HSP is generally hypersensitive vasculitis on smaller blood vessels, with unknown ethyopathogenesis. Children are most common susceptible group. It is characterized by triad of symptoms: on the skin, joints and gastrointestinal track. Renal involvement is also frequent, while CNS, testicals and lungs are less frequent attacked. To present experience with children treated for HSP in our institution. Treatment, analysis and monitoring.

Methods: A retrospective study on 4 patients treated on HSP in 2003-2005. All four of them were children. Three girls and one boy, with average age of 5 years, corresponding to literature.

Results: On girls, signs typical for HSP were developed. On male patient the first signs developed on testicals. At the latter phase, the rash developed on typical locations. Arthromialgies were present on all of the patients. GIT symptoms were identified through colicky and vomiting. Kidney disorder developed on one of the patient, accompanied with long term rash, hematuria and proteinuria. Peak of the disease lasted for 2 weeks. One of the patients recurred, with equal severity of the disease (rash, gastrointestinal pain and HS nephrite). The treatment was untypical: rest, antiinflammatory drugs, vitamins, and in cases of infection therapy with antibiotics was applied. In cases of severe abdominal pain and kidney involvement, corticosteroids were applied as well. Soon after their admission, disease signs disappeared.

Conclusions: HSP has a self-limiting condition, but needs close follow-up of the patients. If there is no kidney disorder, as a result to HSP, generally this disease has excellent prognosis.

\section{SEVERE CHILDHOOD DERMATOMYOSITIS COMPLICATED WITH PANCREATITIS}

E G Martinez Iriart, D Bottaro, A Pizzo, G Ortellao, A Awad

Department of Medicine, Children Hospital 'Dr Ricardo Gutierrez', Buenos Aires, Argentina

Background and Aims: Dermatomyositis (DM) is an idiopathic rheumatologic disease with characteristic cutaneous and muscular findings. DM is a systemic disorder that frequently affects the joints, esophagus, lungs, and less commonly, the heart. Pancreatitis and hepatitis are extremely rare and are associated to severe forms of the disease.

Methods: We report a 3-years-old male, previously healthy, who developed malar rash, cutaneous ulcers on axillary area, generalized lymphadenopathy, poliarthritis, hepatomegaly, poliarthritis, myalgias and muscular weakness. Laboratory: Anemia. Functional hepatic and muscular laboratory: Elevated levels of serum hepatic enzymes, aldolasa, CPK and LDH. FAN, ANA, ASMA, JO1, LCM, MI2: Negative. Virals and parasitic serology: Negatives. PPD: Negative. IgG, IgM, IgA, C4 elevated. C3: Normal electromyography: Inflammatory myopathy. Deglutition tests: Oronasal reflux with active disease who develops abdominal pain. Ocular fundus examination: Normal. Diagnostic of severe form of DM was done and successive treatments with intravenous gammaglobulin, corticosteroids, methothrexate and cyclophosphamide were carried out. During the clinical course he developed pancreatitis. It required intensive care. The patient improved and he reinitiated primary DM treatment.

Results: Pancreatitis, if it appears, demands us to diagnose infectious and toxic (drug-induced) etiology. Hepatitis and pancreatitis, excluded infectious and toxic etiology, must be considered as several forms of DM. We wish to alert physicians that pancreatitis may develop in childhood dermatomyositis. It should be considered as a differential diagnosis in the child findings.

Conclusions: Hepatitis and pancreatitis, although rare, are reported in pediatric patients and must be consider a severe form of the disease and requires intensive and aggressive treatments. 


\section{JUVENILE SYSTEMIC LUPUS ERYTHEMATOSUS (SLE) ASSOCIATED WITH PHOTOSENSITIVITY INDUCED BY HYDROXYCLHOROQUINE}

D Bottaro, E G Martinez Iriart, A Pizzo, G Ortellao, A Awad

\section{Department of Medicine, Children Hospital 'Dr Ricardo Gutierrez', Buenos Aires, Argentina}

Background and Aims: Systemic lupus erythematosus (SLE) is an autoimmune disease that causes disseminated tisular inflammation. Hydroxyclhoroquine is an effective antilupus drug with phototoxic side effects.

Methods: We report a case of juvenile SLE with photosensivity associated to hydroxycloroquine. We present a female adolescent who developed in the last year neumonitis, poliserositis, miocarditis, malar erythema, arterial hypertension and pancytopaenia. Antinuclear antibodies (ANA) and anti-DNA antibodies were positives. She was treated with corticosteroids and hydroxycloroquine.12 months later, she presented progressive photosensivity leading to generalizated erythrodermia. Treatment with local and oral hydrocortisone and antibiotics was unsuccessful. Skin biopsy revealed Interphase dermatitis. Suspecting drug sensivity hydroxycloroquine was interrupted and the patient improved.

Results: Hydroxycloroquin has effective response in the treatment of SLE with muscular and skin involvement. But hydroxycloroquine photosensitivity also occurred. Physicians must consider it as differential diagnosis of re-activated SLE.

Conclusions: 1) Differential diagnoses of a patient with active SLE and photosensivity is often difficult. 2) Skin biopsy can be used to diagnose this entity 3 ) We must consider hydroxycloroquin photosensivity in a patient with poor response to standard therapy.

\section{THALASEMIA MAJOR - A PROBLEM IN THE STRUMICA REGION, REPUBLIC OF MACEDONIA}

M Gligorova $^{1}$, K Kuzmanovska ${ }^{1}$, T Deleva ${ }^{2}, \mathrm{~V} \mathrm{Tasic}^{3}$, M Nikoloski ${ }^{4}$, S Nikoloska ${ }^{5}$

${ }^{1}$ Department of Paediatric, PHI General Hospital, Strumica, Macedonia, ${ }^{2}$ Department of Haemodialysis, PHI General Hospital, Strumica, Macedonia

${ }^{3}$ Paediatric Clinic, Clinical Center, Skopje, Macedonia

${ }^{4}$ Department of Paediatric Surgery, Special Hospital for

Surgical Diseases St. Naum Ohridski, Skopje,

Macedonia, ${ }^{5}$ ORL Clinic, Clinical Center, Skopje,

Macedonia

Background and Aims: Thalasemia major - a problem in the Strumica region, Republic of Macedonia, in the 1990-2003 period. Thalasemia, also known as Mediterranean anaemia, are quantitative disorders in the normal globulin chain synthesis, building blocks of haemoglobin. The thalasemia is encountered around the Mediterranean, which is also the region the city of Strumica and its county belongs to.
Methods: A review of clinical histories of patients treated of the aforementioned disease at the hospital in Strumica during 1990-2003.

Results: In this period we had 6 children aged 6-18 years. All but one (the oldest) underwent splenectomy, and afterwards received a supstitution of washed Eryhtrocytes once a month, as well as Desferal parenterally. Each child was hospitalized ten times a year. Out of total number of hospitalizations, in this period, these patients account for 40 days. Genetic examination performed in 4 children (oldest in their families). Thanks to the preventive pregnancy screening, only one additional case was included in year 1997. The oldest patient treated from Thalasemia major, passed away in January 2004, at the age of 18; the diagnosis was Myocardiopathia chronica decompensate.

Conclusions: Thalasemia major is a real problem in our region and we must always think about this disease.

\section{ERYTHEMA NODOSUM - CASE REPORT OF FOUR DIFFERENT ETIOLOGIES}

C Dâmaso, P Ferreira, C Constantino, A Casimiro, F Cunha

Serviço de Pediatria, Hospital Reynaldo dos Santos, Vila Franca de Xira, Portugal

Background and Aims: Erythema nodosum (EN) is an acute, nodular, erythematous eruption, usually limited to the extensor aspects of lower legs. EN is presumed to be a hypersensitivity reaction and may occur in association with several systemic diseases, drug therapies and may be idiopathic. The authors describe the clinical presentation, investigation pathways, clinical course and treatment outcomes of four patients with EN.

Methods: Review of four patient's files followed in our Paediatric Department.

Results: Four patients with EN clinical diagnosis were studied (1 boy-3 girls), aged $7-12$ years, who had a 2 year follow-up after initial diagnosis. Two patients required admission to the hospital and two were followed exclusively in the outpatient clinic. All started with the typical clinical picture, with fever and painful, red nodules on the legs. None had history of drug use, known exposure to tuberculosis or diarrhea. Blood tests showed increased acute reactants; Tuberculin Skin Test (Mantoux) was negative as were product cultures. Upon extensive investigation based on this same clinical presentation, the etiology of three cases was determined: case 1 - post- Streptococcal pharyngitis; case 2 systemic lupus erythematosus (SLE); case 3 - acute Mycoplasma pneumoniae infection. In the fourth case, after exhaustion of investigation pathways, an idiopathic EN was assumed. Treatment directed to the underlying etiology was ensued and all four cases had a good clinical outcome.

Conclusions: EN is a hypersensitivity reaction with many etiologies. Although usually a self limited condition, it can be the first manifestation of an underlying chronic disease. 
INTRACRANIAL HEMORRHAGE IN A NEWBORN WITH HEMOPHILIA

S Abtahi ${ }^{1}$, M Khalili ${ }^{2}$, M R Khazaee ${ }^{3}$

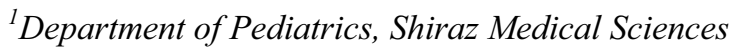
University, Namazi Hospital, Shiraz, Fars, Iran

${ }^{2}$ Department of Pediatrics, Zahedan Medical Sciences

University, Ali-Ebne-Abitaleb Hospital, Zahedan,

Sistanobaloochestan, Iran, ${ }^{3}$ Department of Pediatrics, Mashhad Azad University, 22 Bahman Hospital, Mashhad, Khorasan, Iran

Background and Aims: Intracranial hemorrhage (ICH) in the newborn is an uncommon presenting manifestation of hemophilia, occurring with an incidence of $1-4 \%$, however, it has dramatic consequences. Apart from its high mortality rate, over $50 \%$ of survivors are left with psychoneurological sequelae, especially seizures and motor impairment.

Methods: A male full-term infant with birth weight of 4 $\mathrm{kg}$ was born into a family without history of hemophilia. He was born with vaginal delivery, soon after birth, the child became irritable and difficult to feed. He was found to be hypoactive with full anterior fontanelle and cyanosis, the patient's head circumference (HC) was 37 $\mathrm{cm}$, heart rate was $170 / \mathrm{min}$, respiratory rate was $66 / \mathrm{min}$, and $\mathrm{BP}$ was $90 / 60 \mathrm{~mm} \mathrm{Hg}$. He was immediately transferred to NICU for close observation, but he deteriorated and became semiconscious, with shallow breathing.

Results: Arterial blood gas analysis showed respiratory acidosis that was managed with assisted ventilation. ICH was highly suspected and confirmed by CT scan of the head. The patient was screened for hemophilia on the second day after birth. PTT was raised (120 seconds, control 34 seconds) and factor VIII was $1 \mathrm{u} / \mathrm{ml}$.

Conclusions: Appropriate doses of cryoprecipitate were infused every 12 hours to raise factor VIII level to more than 50 units $/ \mathrm{ml}$. Infusion of cryoprecipitate was continued for 16 days. A few days later, the patient regained consciousness, was crying for feeds and suckling well, and was moving all the limbs, though with slight increase in the tone. He was, extubated from the ventilator.

\section{REVERSIBLE MODERATE THROMBOPENIA IN A 11 YEAR OLD CHILD TREATED WITH VALPROATE}

N Boutrid, H Rahmoune, H Allas

\section{Mansourah Pediatric Hospital, Constantine, Algeria}

Background and Aims: It is well known that therapy with valproate may induce a thrombocytopenia. In this case report, a 11-year-old child having symptomatic epilepsy - Petit Mal - was under Valproate since age 8 years.

Methods: Regular controls of liver enzymes and blood counts were ruled, and a thrombopenia was discovered, without any clinical feature, after the girl received Valproate $(750 \mathrm{mg} /$ day $)$ for 2 years. Blood count showed moderate thrombocytopenia of 89 GIGA/L (normal: 150-450 GIGA/L), with normal coagulation parameters.
Results: Simple survey, with weekly clinical controls and periodic platelet counts, noted spontaneous remission, and the thrombocyte count normalized within 4 months.

Conclusions: No screening methods preview presence of a severe or fatal Valproate therapy complication. Therefore clinical and biological controls are indicated for patients under valproate.

\section{CLINICAL AND RADIOLOGIC INVESTIGATION OF 36 CHILDREN WITH FANCONI ANEMIA}

S Ansari, N Ahanchi, T Sadedel, M Hashemi

Department of Hematology, Iran University, Ali Asghar Children's Hospital, Tehran, Iran

Background and Aims: Fanconi anemia is the most form of inherited aplastic anemia and characterized by progressive bone marrow failure, congenital anomalies and cancer susceptibility. Common anomalies are skeletal abnormalities, skin pigmentation disorder, short stature, head abnormalities, kidney and gonad disorders respectively. Complication of fanconi anemia including leukemia, liver tumors in 5\% and other cancers occurs in $5 \%$ of them. In this study, in addition of clinical and radiologic investigation of children with fanconi anemia, survival, prognostic factors and complication of disease were also determined.

Methods: This retrospective analytic cross-sectional study was carried out on 36 patients with fanconi anemia who were diagnosed in Ali Asghar Children's Hospital from 1985-2002. Data was analyzed by Spss software.

Results: Skeletal abnormalities was most common congenital anomalies and other anomalies were skin pigmentation disorder, short stature, kidney disorder, microcephaly and male genital disorder respectively. 5 and 10 years survival rates of fanconi anemia patients were $80 \%, 71 \%$ respectively. In bivariate analysis, the age at diagnosis correlated with survival rate significantly $(\mathrm{p}=0.006)$ and patients with age less than 10 years at diagnosis had more survival rate than others. In our study, prevalence of kidney disorder as a congenital anomalies is more than other investigation and in contrast, frequency of short stature, microcephaly, skin pigmentation and male genitalia disorder are less than others.

Conclusions: Complication of fanconi anemia including leukemia, because of defective DNA repair in approximately $10 \%$ of patients, liver tumors in 5\%. One of considerable result of this research is absence of neoplastic.

\section{ATYPICAL HOENOCH-SCHONLEIN VASCULITIS}

S D Todorovska ${ }^{1}$, M I Trenceva ${ }^{2}$, S S Smugreska ${ }^{2}$, K Cekic-Dimovska ${ }^{3}$, R Hadzi-Manceva ${ }^{4}$, V B Tasic ${ }^{5}$

${ }^{l}$ Pediatric Service, PZU Pantelejmon, Skopje,

Macedonia, ${ }^{2}$ Pediatric Service, Health Center Skopje, Skopje, Macedonia, ${ }^{3}$ Pediatric Office, PZU Pinokio, Skopje, Macedonia, ${ }^{4}$ Pediatric Service, Remedica Hospital, Skopje, Macedonia, ${ }^{5}$ Department of Pediatric 
Nephrology, Children's Hospital Skopje, Skopje, Macedonia

Background and Aims: Hoenoch Schonlein vasculitis may present with very atypical features, particularly in very young infants. In this work we present a 3 year old female who initially presented with very unusual localization of angioedema in lumbar region.

Methods: A descriptive case, standard clinical laboratory and serologic investigations.

Results: A three year old female presented with pains in ankles and knees. The joints were swollen and tender. There was a sudden appearance of a soft tissue formation in the lumbar region. Laboratory revealed increased ASTO titer (600 U/l) suggesting diagnosis of rheumatic fever. The treatment with oral penicillin $\mathrm{V}$ was started. The throat swab was negative. Two days latter a purpuric rash developed over lower extremities, characteristic for Hoenoch Schonelin purpura. Analysis of the urine did not reveal renal affection. There were no abdominal symptoms. Angioedema in the lumbar region resolved spontaneously.

Conclusions: This young female presented very atypical features of Hoenoch Schonlein vasculitis: arthritis preceding appearance of purpura, mimicking rheumatic fever. Although angioedema may be seen in Hoenoch Schonlein patients, lumbar localization is very unusual.

\section{PREVALENCE AND CAUSES OF IRON DEFICIENCY ANAEMIA IN INFANTS AGED 9 TO 12 MONTHS IN ESTONIA} N Vendt ${ }^{1}$, H Grünberg $^{1,2}$, S Leedo $^{3}$, V Tillmann
T Talvik

${ }^{1}$ Department of Paediatrics, University of Tartu, Tartu, Estonia, ${ }^{2}$ Children's Clinic, Tartu University Hospital, Tartu, Estonia, ${ }^{3}$ United Laboratory, Tartu University Hospital, Tartu, Estonia

Background and Aims: Iron deficiency anaemia (IDA) in children is common nutritional problem all over the world. The aim of our study was to investigate the prevalence and associated background factors of iron deficiency (ID) and iron deficiency anaemia (IDA) in infants aged 9 to 12 months in Estonia.

Methods: Every second child aged 9-12 months was randomly selected from primary medical centres in 7 counties from all over Estonia. A questionnaire concerning eating habits and lifestyle was sent to their parents. Thirty six percent $(n=195)$ of contacted families agreed to participate in the study. Haemoglobin $(\mathrm{Hb})$, mean corpuscular volume (MCV), serum ferritin, soluble transferrin receptors (TfR) and C-reactive protein (CRP) were measured in 171 infants. Anaemia was defined as $\mathrm{Hb}<105 \mathrm{~g} / \mathrm{l}$, and iron deficiency (ID) as ferritin $<12 \mu \mathrm{g} / \mathrm{L}$ and MCV $<74 \mathrm{fl}$.

Results: The prevalence of ID was $14.0 \%$ and IDA 9.4\%. Birthweight (BW) less than $3000 \mathrm{~g}$ was the main associated background factor for ID $(\mathrm{OR}=9.4$; $\mathrm{p}<0.0005$ ). Infants fed with breast-milk and solid food had lower ferritin concentration than infants fed with formula and solid food $[18.5 \mu \mathrm{g} / \mathrm{L}$ (95\% CI 14.0-23.0) vs. $32.8 \mu \mathrm{g} / \mathrm{L}(26.6-39)(\mathrm{p}<0.005)]$.
Conclusions: IDA was common in infants aged 9-12 months in Estonia. The main associated background factor for ID was BW less than $3000 \mathrm{~g}$.

\section{PASSIVELY ACQUIRED ANTI-CAPSULAR PNEUMOCCOCAL POLYSACCHARIDE ANTIBODIES IN INFANTS BORN TO HIV- INFECTED IMMUNIZED MOTHERS}

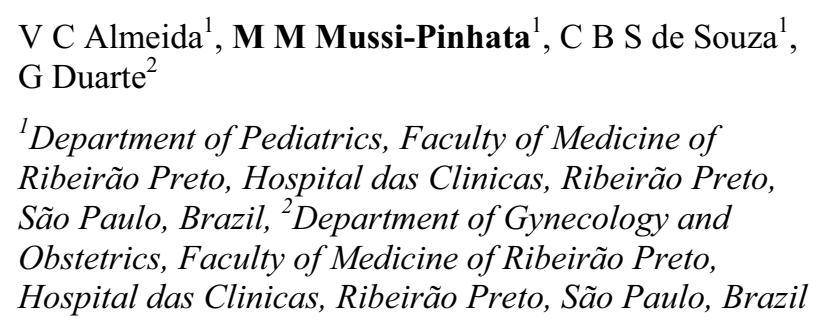

Background and Aims: Maternal immunization may protect young infants by provision of passive immunity. Pneumococcal polysaccharide vaccine (PSV) has been shown to elicit protective levels of antibodies in normal pregnant women and their infants. We evaluated immunogenicity of PSV vaccine in HIV-infected $(\mathrm{HIV}+)$ pregnant women and the antibody kinetics from birth to 6 months of age.

Methods: Serotype-specific antibodies to 6 capsular pneumococcal polysaccharides (CPs) $(1,3,5,6 \mathrm{~B}, 9 \mathrm{~V}$ and 14) were quantified before PSV immunization (gestational age of 32-34 wks) and at delivery in 44 $(\mathrm{HIV}+)$ pregnant women and in their infants at birth, 1 , 2, 3 and 6 months. All mothers had prevaccination (PRV) CD4+ counts > 200/mm3, 97.7\% were asymptomatic, and $97.8 \%$ received antiretroviral prophylaxis. Anti-CPs antibodies were measured with an ELISA.

Results: Overall, PRV antibodies $>1.3 \mathrm{ug} / \mathrm{ml}$ were found in 48 to $82 \%$ of the women. Significant increases in postvaccination antibody concentrations were found for all serotypes except CP 3. At delivery, higher antibody levels were detected in mothers $(1.3-9.2 \mathrm{ug} / \mathrm{ml})$ than in neonates $(0.7-4.4 \mathrm{ug} / \mathrm{ml})$. Placental transfer ratios (newborn/mother) ranged from $47-59 \%$. Protective levels of antibodies were estimated for CPs 5 , $6 \mathrm{~B}, 9 \mathrm{~V}$ and 14 in neonates, and for CPs $5,6 \mathrm{~B}$ and 14 in 1 month infants. Except for S14, lower than protective levels were estimated for $5 / 6$ serotypes in infants $>=2$ months.

Conclusions: Although (HIV+) mothers respond to most CPs present in PSV, maternal vaccination is not adequate to maintain protective levels of anti-CPs antibodies in infants due to the limitations of placental transport. Funded by FAPESP, Brazil.

\section{DOPPLER ASSESSMENT OF THE MIDDLE CEREBRAL ARTERIES BY TWO DIFFERENT APPROACHES}

S J Moltu, E Schumacher, T Stiris

Division of Neonatology, Ullevaal University Hospital, Oslo, Norway

Background and Aims: Cerebral ultrasound is a well established method to assess brain integrity in the preterm infant. The major cerebral lesions in these 
infants are of circulatory origin and pulsed and color Doppler evaluation has become an important tool for studying cerebral hemodynamics. To aim for minimal handling of the premature infants, we wanted to investigate whether measuring blood flow velocity toward or away from the transducer probe gave equal blood flow velocity (BFV) estimates within the same vessel, thus enabling us to measure both middle cerebral arteries (MCA's) from one side only.

Methods: Using an $8.5 \mathrm{~Hz}$ probe $\mathrm{BFV}$ was measured in the right and left MCA by placing the probe over the respective temporal template. In addition to the flow estimates of the ipsilateral vessel, we also obtained the negative BFV estimate of the contra lateral vessel (flow away from the probe). The same procedure was repeated from the opposite side and ultimately the two separate estimates were compared. The order of left and right was chosen randomly.

Results: The intraclass correlation coefficient was used for comparison between the two methods of measurements. There was a strong concordance between the flow velocity estimates with an Inter-Item Correlation matrix of 0.831 .

Conclusions: Our results depicts that measuring blood flow velocity toward or away from the transducer probe gives equal estimates, hence allowing measurements of the right and left MCA from just one side, and thereby avoiding having to turn the head from one side to another in the often unstable premature infant.

\section{IMMUNOLOGICAL FEATURES OF HUMAN MILK IN DIFFERENT ETHNIA}

L Ciardelli $^{1}$, F Garofoli ${ }^{1}$, M A Avanzini ${ }^{1}$, A De Silvestri ${ }^{2}$, A Serra ${ }^{3}$, A Gasparoni $^{4}$, M Stronati ${ }^{3}$

${ }^{I}$ Research Laboratories, Neonatal Immunology and Paediatric Oncohematology, IRCCS Policlinico San Matteo, Pavia, Italy, ${ }^{2}$ Biometric Unit, IRCCS Policlinico San Matteo, Pavia, Italy, ${ }^{3}$ Neonatal Intensive Care Unit, IRCCS Policlinico San Matteo, Pavia, Italy, ${ }^{4}$ Neonatal Intensive Care Unit, Spedali Civili, Brescia, Italy

Background and Aims: To evaluate immunological secretory memory, we studied production of s-IgA specific for E. coli (Anti-Ec-s-IgA) in mothers' milk of different ethnia previously exposed to poor conditions, but living in Italy in adequate conditions. Moreover, we explored cytokines levels (IL-6, IL-8, IL-10). 112 colostrums samples were included into the study: 24 from European Eastern Countries (EEC), 24 from Asia, 24 from Africa, 40 from Italy.

Methods: Anti-Ec-s-IgA and cytokines were quantified by ELISA technique. Statistic analysis was performed with respect to Italians. Univariate analysis was performed for Anti-Ec- s-IgA and cytokines. Multiple Regression was performed when residence period, home returns and cytokines concentration were considered explication variables (EV).

Results: Foreign milk presented higher levels of AntiEc-s-IgA than Italians, for Asian women the difference was significant $(\mathrm{p}=0.007)$. Asian and African's milk $(\mathrm{p}=0.031, \quad \mathrm{p}=0.015)$ reported significant higher concentration when data were corrected for EV. A positive correlation between Anti-Ec-s-IgA and IL-8 was always observed $(\mathrm{p}=0.01)$. Milk from EEC revealed the highest IL-8 levels $(\mathrm{p}=0.026)$. Milk from Asians presented IL-6 greatest concentration $(\mathrm{p}=0.004)$. Africans reported IL-10 littlest concentration ( $\mathrm{p}=0.046)$. Conclusions: High levels of Anti-Ec-s-IgA in foreign milk may due to mothers' previous exposition. Since all mothers in Italy were in adequate conditions, this could be the expression of a secretory immunologic memory. The highest amounts of IL- 8 in EEC milk and of IL-6 in Asian milk may promote defense mechanisms useful where infections are severe. Opposite IL-10, a downregulator of pro-inflammatory cytokine, is less useful in those countries where stimulatory mediators are required to defeat environmental infections.

\section{RIGHT ILIAC FOSSA GROANS - REMEMBER WORMS!}

\section{E Jamalullail, L Reed, C Chianakwalam}

Department of Surgery, William Harvey Hospital, Ashford, UK

Background and Aims: Enterobius vermicularis (pinworm) infestation is a recognized but often forgotten cause of acute right iliac fossa pain especially in children. It is a ubiquitous parasite of man transmitted faeco-orally that resides primarily in the caecum. The aim of this study was to evaluate the incidence and clinico-pathological findings in children undergoing appendicectomy.

Methods: A retrospective review of all appendicectomies in patients below the age of 21 between January 2004 and July 2005. Data on clinical presentation and pathological findings were recorded.

Results: Of a total of 369 appendicectomies, 18 (4.9\%) had Enterobius vermicularis on histology. There were 12 females and 6 males with a mean age of 13 years. Their mean duration of symptoms was 2.4 days, temperature $37.1 \mathrm{o} \mathrm{C}$ and WBC 8.7 X 109/L. There was no histological evidence of acute inflammation in 16 appendices and in the remaining 2 the inflammation was graded as only very mild.

Conclusions: Enterobius vermicularis is a significant cause of right iliac fossa pain in children and should be considered in the differential diagnosis of acute appendicitis. It is not associated with acute inflammatory changes in the appendix.

\section{LEMIERRE'S SYNDROME WITH BENIGN PROGNOSIS: A CASE REPORT}

R Fernandes, J Caiado, F Prata, A Mouzinho, P Valente

Infectious Diseases Unit, Department of Paediatrics, Santa Maria's Hospital, Lisbon, Portugal

Background and Aims: Lemierre's syndrome is a rare form of septicaemia caused by the anaerobic gramnegative bacterium Fusobacterium Necrophorum. The characteristic clinical picture follows oropharyngeal infections and includes septic thrombophlebitis of the internal jugular vein. It may be complicated by multisystem septic embolism and a high mortality, but less severe cases have also been described. The aim of 
this report is to stress the need for a high index of suspicion to prompt diagnosis, and to discuss current therapeutic options.

Methods: Case-report.

Results: We present a case of a previously healthy eleven-year old girl with a transient episode of mild sore throat followed a week later by fever, a prominent cervical abscess and elevated laboratory inflammatory markers. Early surgical debridement was performed and the patient received amoxicillin-clavulanate with metronidazol for eight days and improved considerably. Fusobacterium Necrophorum was isolated from the drained purulent material and a right vein jugular vein thrombosis was confirmed by neck ultrasonography. The antibiotic therapy was maintained for three weeks with clindamycin, along with short-duration (two weeks) anticoagulation therapy with enoxaparin. No systemic complications were identified and there were no sequelae at follow-up.

Conclusions: The benign nature of this case, after adequate management, highlights the role of both imaging and microbiology techniques in diagnosis. Appropriate therapy includes adequate antibiotic treatment and early surgical intervention, if required. The role of anticoagulation remains controversial.

\section{PROGNOSTIC VALUE OF PROCALCITONIN AND CRP IN PEDIATRIC NEUTROPENIC FEVER}

I Devrim ${ }^{1}$, G Seçmeer ${ }^{1}$, M Ceyhan ${ }^{1}$, A Kara ${ }^{1}$, H Tezer ${ }^{1}$, A K Uludag ${ }^{2}$, A B Cengiz ${ }^{1}$

${ }^{\text {I} P e d i a t r i c ~ I n f e c t i o u s ~ D i s e a s e ~ U n i t, ~ H a c e t t e p e ~}$ University, Faculty of Medicine, Ankara, Turkey

${ }^{2}$ Department of Biostatics, Hacettepe University, Faculty of Medicine, Ankara, Turkey

Background and Aims: In clinical practice in neutropenic fever patients with no microbiologically and clinically defined infection, the decision for the clinicians when to stop antibiotic therapy or the risk of underestimating an occult infection is the main problem. A reliable highly specific and sensitive marker which could be helpful in early prediction of infection in neutropenic fever is needed in the era of antibiotic resistance.

Methods: We have evaluated the diagnostic value of Procalcitonin (PCT) compared with C-reactive protein (CRP) and erythrocyte sedimentation rate (ESR) in predicting the infection in neutropenic fever patients and durational change for 48 (at $0,8,24,48$ hour) hours of these parameters in the presence of defined infection in 60 febrile episodes of 49 patients.

Results: PCT and CRP levels were significantly higher in neutropenic fever patients than afebrile cancer patients $(\mathrm{p}<0.001)$ throughout the study period, but ESR levels did not show any significant difference $(p>0.05)$. In sequential analysis of the patients without documented infections, the median of PCT concentrations show tendency to fall after the 8th hour of onset of fever, while in patients with documented infection those normalized after the 48th hour. Sequential analysis of median PCT levels were found to decrease in 48 hours period in patients with fever lasting in 72 hours while in patients with prolonged fever PCT sustained its level in overall period.

Conclusions: In conclusion, our study suggests PCT when measured periodically is more useful diagnostic inflammatory parameter in pediatric neutropenic fever patients than CRP in both estimating the duration and origin of fever and deciding the antimicrobial choice.

\section{BACTERIAL MENINGITIS IN A PORTUGUESE PAEDIATRIC HOSPITAL}

M Ferreira, C Mendes, P Janeiro, M Conde, T Aguiar, M J Brito

Department of Paediatric, Hospital Fernando Fonseca, Amadora, Portugal

Background and Aims: Bacterial meningitis is a serious infection in pediatric age, associated with high risk of complications and sequelae. Objective: To characterize the etiology and evolution of bacterial meningitis and evaluate risk factors associated with poor prognosis.

Methods: Retrospective study, between July 1996 and December 2005, of all children admitted with bacterial meningitis. Analysed demographic, clinical, laboratory and evolution data. SPSS 13.0 for Windows was used for statistical analysis.

Results: Total of 107 cases were observed; $49,5 \%$ less than 2 years old and $43,4 \%$ had chronic disease. All children were immunized against Haemophilus influenzae, 4,1\% had pneumococcal vaccine and 3,1\% meningocococal $\mathrm{C}$ vaccine. Ethiologic diagnosis made in $65(60,7 \%)$ cases; Neisseria meningitidis (32), Streptococus pneumoniae (22), Haemophilus influenzae (3), Streptococcus agalactae (4) and others (5). Complications occurred in $25 \%$ cases: seizures (14), coma (8), cranial nerve paralysis (6), cerebral stroke (6), motor disabilities (4), hydrocephalus (4) and others (11). Sequelae occurred in $14 \%$ cases: hearing loss (8), mental disabilities (7), motor disabilities (5), epilepsy (5) and others (5). One child died. complications were associated with Streptococcus pneumoniae $(58,3 \% \mathrm{vs} 15,9 \%, \quad \mathrm{p}=0,006, \quad \operatorname{Exp}(\mathrm{B}) \quad 21,472), \quad \mathrm{CSF}$ glucose $<30 \mathrm{mg} / \mathrm{dL} \quad(68,2 \% \mathrm{vs} 28,6 \%, \quad \mathrm{p}=0,011, \operatorname{Exp}(\mathrm{B})$ $8,900)$ and sepsis $(44,4 \% v s 20,3 \% ; p=0,014, \operatorname{Exp}(B)$ $10,813)$. Risk factors for sequelae where Streptococcus pneumoniae $(69,2$ vs $21,8 \%, p=0,009, \operatorname{Exp}(B) 11,127)$ and CSF glucose $<30 \mathrm{mg} / \mathrm{dL}(83,3 \% \mathrm{vs} 31 \%, \mathrm{p}=0,047$, $\operatorname{Exp}(B) 10,468)$.

Conclusions: Bacterial meningitis is an important cause of morbility in pediatric age. Specific immunizations and other intervention strategies can modified in the future the epidemiology of this disease.

\section{ANTIBIOTICS EXPENDITURE IN A PAEDIATRIC HOSPITAL WITH A REFERRAL MATERNITY}

\section{Ventura, M T Neto, L Ribeiro, R M Barros \\ Committee for Hospital Infection Control, Hospital Dona Estefania, Lisbon, Portugal}

Background and Aims: The correct use of antibiotics is the most effective way to prevent bacterial resistance. 
The knowledge and control of its prescription should be a constant concern of the Hospital's Committee for Infection Control. Hospital Dona Estefania is a referral paediatric hospital with a high risk maternity with obstetrical clinics. Our aim was to quantify the expenditure on intravenous antibiotics in a tertiary paediatric hospital with obstetrical clinics.

Methods: The source of data were the Pharmacy's and the Institute for Informatics and Financial Management files. For each intravenous antibiotic the expenditure in $\mathrm{g}$, costs in Euros and per $\mathrm{g}$ were evaluated for the years of 2003-2004.

Results: Penicillin's were the main group of antibiotics prescribed ( $43 \%$ of the total of prescriptions, $32 \%$ of the total of expenditure) followed by the group of second generation cephalosporins $(20 \% ; \quad 27 \%)$, aminoglycosides $(13 \% ; 3 \%)$ and third generation cephalosporins $(9 \% ; 16 \%)$. Amongst penicillin's the most prescribed were benzylpenicillins, ampicillin and amoxycillin/clavulanic acid while amongst first and second generation cephalosporins it was cefoxitin $(70 \%)$. Cefazolin, the antibiotic advised for surgical prophylaxis, accounted for $9 \%$. Amongst aminoglycosides, gentamicin was the most frequently used $(90 \%)$ while for third generation cephalosporins it was ceftriaxone, followed by cefotaxime and ceftazidime The expenditure with intravenous antibiotics accounted for $62 \%$ of the total expenses with antibiotics, $32 \%$ with anti infectious agents and $6.1 \%$ of the total therapeutic charge.

Conclusions: Intravenous antibiotics use in Dona Estefania hospital seems very reasonable. Prescriptions follow ancient teachings with preference for old antibiotics but surgical prophylaxis has to be improved.

\section{MORBIDITY OF PARAINFLUENZA 3 OUTBREAK IN A NEONATAL UNIT- KKH EXPERIENCE}

\author{
W Y Teo ${ }^{1}$, M Z Han ${ }^{2}$, S Bhavani², V S Rajadurai ${ }^{2}$ \\ ${ }^{I}$ Department of Paediatric Medicine, \\ ${ }^{2}$ Department of Neonatology, Kandang Kerbau \\ Women's \& Children's Hospital, Singapore
}

Background and Aims: Parainfluenza type 3 virus (PIV-3) is an important nosocomial pathogen causing pneumonia and bronchiolitis in infants. We report an outbreak of PIV-3 respiratory infection in the Department of Neonatology of KKH in June 2005. This is the second PIV-3 outbreak in our unit after the first in December 1994.

Methods: Retrospective review of the clinical characteristics and outcome of 7 infants tested positive for PIV-3 on nasopharyngeal aspirate in June 2005.

Results: 7 cases were infected with PIV-3 during this outbreak. Mean birthweight of affected infants was $1370 \mathrm{~g}$ (range $740 \mathrm{~g}-2585 \mathrm{~g}$ ) and mean gestational age was 29 weeks (range 24-35 weeks). Apnoeas and bradycardias were the significant symptoms in 3 infants. 5 infants had progressive respiratory distress while 2 infants had flu-like illness. 5 infants required ventilatory support. There were no deaths. The index case was an infant with chronic lung disease who was on oxygen supplementation and subsequently required ventilatory support with nasal CPAP +6 with increasing oxygen requirements. She continues to have severe post-viral hyper-reactivity and is still hospitalised. Early identification with strict cohorting of infected cases was implemented. Contact tracing and screening of contact cases was done. Hand hygiene precautions were reinforced.

Conclusions: PIV-3 respiratory infections in infants can present with non-specific symptoms, leading to significant morbidity. Early recognition of symptoms and detection is necessary to limit extent of infection, institute ventilatory support promptly and avoid unnecessary investigations and treatment. Though infrequent, nosocomial respiratory infection is a significant cause of morbidity in infants, especially in those with underlying pulmonary pathology.

\section{A COMPARATIVE ANALYSIS OF THE REPORTS OF ACCOMPLISHMENT OF THE PROGRAMME FOR INFECTION CONTROL IN 1999 AND 2005 - DEPARTMENT OF NEONATOLOGY}

\section{Dzarlieva}

Department of Neonatology, Hospital, Bitola, Macedonia

Background and Aims: The Epidemiology Service does four visits on average over one year. Each one of those visits contains from 15 to 20 smear samples, based on variation of requirements. To compare the microbiological analysis of the smears collected in 1999 with the ones collected in 2005.

Methods: The material used were the reports of nosocomial infections of the Epidemiology Service in these two different years.

Results: In 1999, from the total number of 482 smear samples collected from around the hospital, 58 (12.0\%) were positive, 13 of which $(23.6 \%)$ were from a smaller collection of 55 samples taken at the Department of Neonatology. As a figure form the overall hospital collection, these 13 represent $22.4 \%$ of the entirety of positive ones. The sample contained 24 smears from objects of general use, 7 obtained from noses, 12 from skin and 12 from eyes. In 2005, from the total number of 397 smear samples, 114 (28.7\%) were diagnosed positive. From the collected 37 samples at The Department of Neonatology, 12 were positive, which makes up $10.5 \%$ from the entire number of smears accumulated from the whole hospital. The sample of positive ones consisted of 23 smear samples from objects of general use, 7 from noses, 4 from eyes, 2 from skin and 1 from a breast.

Conclusions: The reduction from $22.4 \%$ in 1999 to $10.5 \%$ in 2005 of positive isolations can be perceived very evidently.

\section{NON TYPABLE HAEMOPHILUS INFLUENZAE MENINGITIS FOLLOWING FACIAL TRAUMA IN A CHILD WITH INTERCURRENT CONJUCTIVITIS AND UPPER RESPIRATORY TRACT INFECTION}

I M Velissariou, E Latoufi 
Paediatric Intensive Care Unit, Athens Medical Centre, Athens, Greece

Background and Aims: Meningitis due to CSF leakage is a complication of skull base trauma. There is, however, no clear consensus regarding the use of antibiotic prophylaxis following such trauma.

Methods: A 3 year old girl was hospitalized for acute meningitis, occurring 24 hours after an orbito-maxillar trauma.

Results: The girl was previously healthy and vaccinated for Streptococcus pneumoniae and Haemophilus influenzae. She was involved in a car crash, causing her an injury of the left eye and maxilla. Initial cranial-facial X-ray was unremarkable. Fever and vomiting began 12 hours after the injury, followed by nuchal rigidity and impaired consciousness. In addition to a left lower eyelid haematoma, a purulent conjunctivitis and nasopharyngeal congestion were noticed. CSF contained 6000 cells $/ \mathrm{mm} 3$ and culture yielded $140 \mathrm{CFU} / \mathrm{ml}$ of non typable Haemophilus influenzae beta-lactamase positive. Nasopharyngeal aspirate culture performed after one dose of ceftriaxone yielded Streptococcus pneumoniae, Branhamella catarrhalis and Staphylococcus aureus. Computed tomography documented a medial wall fracture of the orbit. Bacteriological eradication was sustained 48 hours after the beginning of ceftriaxone, which was continued for 14 days intravenously. Amoxicillin-clavulanate and rifampicin were then administered orally for 14 days for prophylaxis, with no relapse during this period.

Conclusions: In skull base trauma of young children, CSF leakage should be investigated thoroughly and antibiotic prophylaxis for meningitis may be considered. Purulent conjunctivitis and nasopharyngeal congestion could indicate a rapid onset of this complication.

\section{ANALYSIS ON LOW RESPIRATORY TRACT INFECTION CAUSED BY HAEMOPHILUS INFLUENZAE IN 216 CHILDREN}

\author{
C Z Hua ${ }^{1}, \mathrm{H} \mathrm{M} \mathrm{Yu}^{2}$, S Q Shang ${ }^{2}$ \\ ${ }^{l}$ Department of Pediatrics, Hangzhou Teachers College, \\ Hangzhou No.2 Hospital, Hangzhou, China \\ ${ }^{2}$ Children's Hospital, Zhejiang University, School of \\ Medicine, Zhejiang, China
}

Background and Aims: To investigate the phenotypes of Haemophilus influenzae isolated from low respiratory tract infection children and the manifestations of these cases.

Methods: Sputum specimens for bacterial cultures, were collected in all low respiratory tract infection children during August 2001 to July 2002, and clinical materials were collected in cases whose etiology were identified as Haemophilus influenzae. Serotypes were performed with slide agglutination method, and the sensitivities of 216 strains of Haemophilus influenzae against 13 antibiotics were determined in vitro by disc diffusion methods.

Results: Of all 216 strains isolated from low respiratory tract infection children, 82 strains (38.0\%) were typable and $89.0 \%$ of them belonged to type $\mathrm{d}$, followed by type c $(6.1 \%), f(2.4 \%), b(1.2 \%)$ and a $(1.2 \%)$, while 134 strains $(62.0 \%)$ were nontypable. Higher incidence of typable Haemophilus influenzae was found in male $(61 / 142,43.0 \%)$ than that in female $(21 / 74,28.4 \%)$ and the difference was significant $\mathrm{P}<0.05$. Thirty-nine isolates $(18.1 \%)$ were $\beta$-lactamase-positive and 10 strains could not grow on medium in antibiotics sensitivity test. Of all 206 isolates tested successfully, $83.5 \%$ were susceptible to ampicillin, and the sensitivity rates to cefaclor, ceftriaxone, cefotaxime and clarithromycin were as high as $98.5 \%, 99.5 \%, 99.5 \%$ and $91.7 \%$, respectively. All strains were sensitive to amoxicillin/clavulanic acid, ampicillin/sulbactan and imipenem.

Conclusions: Both nontypable and type $d$ are major cause in low respiratory tract infection in children due to Haemophilus influenzae, and ampicillin or other $\beta$ lactam actibiotics are still the choices for treatment of infections with Haemophilus influenzae.

\section{IL-6, CRP AND ELASTASE IN PRETERM INFANTS}

\author{
A Sofijanova ${ }^{1}, \mathrm{~K}_{\text {Piperkova }}{ }^{2}$, A Sajkovski ${ }^{1}$, \\ D Fidanovski ${ }^{1}$, A Hristovski ${ }^{1}$, L Koic $^{1}$ \\ ${ }^{I}$ Department of Intensive Care Unit, Pediatric Clinic, \\ Skopje, Macedonia, ${ }^{2}$ Department of Neonatology, \\ Pediatric Clinic, Skopje, Macedonia
}

Background and Aims: Il-6 is early and sensitive marker, CRP is late and specific, but elastase is a direct measure for the granulocytes. The aim is to assess correlation between Il-6, elastase and CRP in preterm infants as early markers.

Methods: 18 infected premature infants with proven sepsis were analized at the NICU with BW in average $2.74 \mathrm{~kg}$. and GA $35 \pm 5.2$. There were 3 blood samples: 1) at the admission before given antibiotics 2) after 4872h. 3) after 5-7 days. The group was analysed and compared for a range of perinatal and neonatal factors and analysed using ANOVA for conttinuous variables and contingency tables for categorical variables.

Results: The results revealed that the concentration of Il-6 is statistically significant in infants with bacterial infection on first day of life compared with control. The severity of the disease correlates with the risk for death in the first 24-48h. A significant correlation between Il-6 and CRP showed that higher Il- 6 and lower CRP in the first analysis and if higher the concentration of CRP and Il-6 the infection is more severe. All infants with sepsis showed statistically higher values of elastase after $24 \mathrm{~h}$.

Conclusions: Careful monitoring of the dynamics of CRP and Il-6 are useful in administrating antibiotics. Il6 is early and sensitive marker and CRP is late and specific, but the combination of both is the most secure marker. So if both are negative after $48 \mathrm{~h}$. the treatment with antibiotics should be cut off. Elastase is a good marker for the follow up.

\section{BOTULISM: THE IMPORTANCE OF THE INITIAL TREATMENT WITH THE ANTITOXIN BOTULINUM}

M B Sampaio ${ }^{1}$, H Antunes ${ }^{1}$, A Antunes ${ }^{1}$, C Castro $^{1}$, S Figueiredo ${ }^{1}$, R Maré ${ }^{2}$ 
${ }^{I}$ Department of Pediatrics, São Marcos Hospital, Braga, Portugal, ${ }^{2}$ Department of Neurology, São Marcos Hospital, Braga, Portugal

Background and Aims: Botulism is a rare condition, caused by a neurotoxin produced from Clostridium botulinum.

Results: A 12 years old adolescent was brought to the Emergency Room with blurred vision and diplopia initiated in the previously $12 \mathrm{~h}$. He also mentioned constipation in the formerly 2 days and paresthesias in the earlier 4 days. One week sooner, he had abdominal pain and diarrhea that last 1 day. He mentioned the ingestion of commercially produced mushrooms and fast-food 1 day before. It was only found dilated pupils. The routine analysis and the cerebral TC didn't show any alteration. At that time foodborne botulism was thought. In the next $12 \mathrm{~h}$ he developed ptosis and extraocular muscle weakness. Serum and stools specimens were collected. It was administrated trivalent antitoxin. Five days after admission he as initiated weakness and diminished tendons reflexes of the upper extremities. The lumbar puncture performed was normal. The electromyography revealed an incremental increase in amplitude to rapid repetitive stimulation. Since then the clinical features suffered a favourable evolution. Nine days after admission it was confirmed the diagnosis by the identification of the $\mathrm{B}$ toxin. $\mathrm{He}$ was released home. Six months later is physical exam was normal.

Conclusions: In the reported cases in our country the antitoxin administration was not performed but the evolution were less favourable than in our case. It's essential to make survey's in our country to question legitimise of the antitoxin administration, one that it was only found B and E type, and not the lethally A type.

\section{BACTERIAL INFECTION IN INFANTS WITH BRONCHIOLITIS ADMITTED AT PEDIATRIC CRITICAL CARE UNIT (PICU)}

Z Martinez de Compañon, E Esteban, J Lozano, I Jordan, J Caritg, M Balaguer, S Gala, F J Cambra

Pediatric Critical Care Unit, Hospital Sant Joan de Deu, Barcelona, Spain

Background and Aims: Bacterial infection in bronchiolitis is usual although is more frequent and serious in PICU patients. To characterize bacterial infections in infants with bronchiolitis at PICU.

Methods: Prospective, observational study. Data from infants with bronchiolitis admitted at PICU from 2004 to 2005 was collected and compared with 2002-2003 period. CDC definitions for nosocomial infection were used.

Results: Were enrolled 72 patients, 45 were male $(62.5 \%)$. Mean age was 1.93 (DS \pm 2.69$)$ months and 23.6\% were expremature. Wood Downes-modified score gravity at admission was 10.6 (DS \pm 2.8 ). Respiratory Syncitial virus was positive in $52.8 \%$. Antibiotherapy was indicated in 40 cases $(55.6 \%)$ related to clinical infection suspicion $(7.5 \%)$, chest-ray alterations $(41.6 \%)$ analytic suspicion $(43.9 \%)$ and other causes $(7 \%)$. Final diagnostics were respiratory tract infection in 30 cases $(41.6 \%)$, urinary tract infections in
$4(5.6 \%)$ and 6 sepsis $(8.3 \%)$. There was isolated some bacteria in $35 \%$ from antibiotic treated infants. In tracheal sample were 5 Haemophilus influenzae, 3 streptococcues pneumoniae and 1 moraxella catarralis; in urine culture 2 E.coli, 1 Enterobacter cloacae and 1 candida sp; and a Streptococcus pneumoniae from blood isolation. Fourteen infants required ionotropics related to bacterial infection. The PICU stay was significative longer in infants with infectious complication (mean 4.2 days versus 6.4) $\mathrm{p}=0.04$. Any infected patients become exitus. All data was similar to 2002-03 years, despite antibiotherapy: indicated in $88.3 \%$ patients, $\mathrm{p}=0.03$.

Conclusions: Respiratory tract infection was the most frequent bacterial infection in bronchiolitis. It results in a higher morbidity and longer stay at PICU.

\section{ASSOCIATION OF KAWASAKI DISEASE WITH PRIMARY CYTOMEGALOVIRUS INFECTION} B E Guc ${ }^{1}$, S V Yildirim ${ }^{2}$, N Cengiz ${ }^{3}$, E Cekinmez $^{1}$,
Y Uslu $^{3}$

${ }^{1}$ Department of Pediatrics, Baskent University, Adana

Teaching and Medical Research Center, Adana, Turkey

${ }^{2}$ Department of Pediatric Cardiology, Baskent

University, Adana Teaching and Medical Research

Center, Adana, Turkey, ${ }^{3}$ Department of Pediatric

Nephrology, Baskent University, Adana Teaching and

Medical Research Center, Adana, Turkey

Background and Aims: Kawasaki disease is an acute systemic vasculitis that occurs predominantly in children younger than 5 years of age. The cause of Kawasaki disease remains unknown. On the basis of clinical and epidemiologic features, Kawasaki disease is widely believed to have an infectious cause.

Methods: Case: A 9-month-old female infant was admitted to our hospital for the treatment of fever of unknown origin. She had erythema of the oral and pharyngeal mucosa, nonexudative conjunctivitis, and extreme irritability. Her leukocyte was $23.100 / \mathrm{mm}^{3}$; hemoglobin level was $10.1 \mathrm{~g} / \mathrm{dl}$; C-reactive protein level was $146 \mathrm{mg} / \mathrm{L}$, and ESR was $116 \mathrm{~mm} / \mathrm{h}$. No bacterial or fungal agent grew on cultures of blood or urine samples. The results of serologic tests for CMV IgM and IgG were positive. CMV DNA was identified by polymerase chain reaction in a blood sample. Echocardiogram showed a slight dilatation of the coronary arteries and pericardial effusion. The diagnosis was Kawasaki disease.

Conclusions: Our case report suggests that patients with Kawasaki Disease should be tested for CMV infection, and the results of that observation imply something more than coincidence.

\section{REALIZATION OF CALENDAR OF VACCINATIONS IN PUBLIC AND NOT-PUBLIC PRACTICES}

A Nitsch-Osuch, E Gyrczuk, A Wardyn

Department of Family Medicine, Warsaw, Poland

Background and Aims: Polish calendar of vaccinations consists of: obligatory (paid by government) and recommended (paid by patients) vaccinations (including 
for example acellular pertussis, Haemophilus influenzae, varicella, hepatitis A vaccinations).

Methods: The aim of our study was to find out differences in realization of the calendar of vaccinations in public family practices (PFP) and non-public family practices (NFP).

Results: We compared the realization of calendar of vaccinations in one public and one non-public family practice in 2005. We estimated number of dosages of recommended vaccinations (including acellular pertussis and Haemophilus influenzae vaccinations) in public and non-public family practice. There were 287 and 143 children aged 0-24 months respectively in NPF and PFP. In the non-public family practice 838/842 (99\%) dosages of DTPa were administrated while in public family practice only 67/384 (17,5\%) dosages of DTPa were given (the rest were the whole cell pertussis vaccine - DTPw). In a NPF all children were vaccinated against Haemophilus influenzae while in a PFP - 19/143 (19\%) children.

Conclusions: The recommend vaccinations are more often prescribed in non-public family practice. It may be a result of: better financial status of patients who belong to nonpublic family practice, better contact between doctors and patents (patients) in non-public practices (doctors have more time for visits so they are able to explain and encourage parents to use recommended vaccines). The aim of public family practices should be more often usage of recommended vaccines among their patients.

\section{ATYPICAL KAWASAKI PRESENTING AS PNEUMONIA. A CASE REPORT}

P Korovessi $^{1}$, M Lariou ${ }^{1}$, M Psalti ${ }^{1}$, A Natsiopoulou ${ }^{2}$, M Tibisranis $^{2}$, E Papadakis ${ }^{1}$

${ }^{I}$ Department of Pediatrics, Thriasion General Hospital, Athens, Greece, ${ }^{2}$ Department of Radiology, Thriasion General Hospital, Athens, Greece

Background and Aims: Atypical KD is defined as a fever of more than five days duration in the presence of less than four of the five classical clinical criteria. Here we report one child with marked pulmonary symptoms in the course of Kawasaki disease who was initially diagnosed with pneumonia.

Methods: A 2 year old, previously healthy boy, was hospitalised with prolonged fever and 'unresolving pneumonia'. He developed a temperature up to $40 \mathrm{C}$ and was initially diagnosed as having bullous myringitis; he was treated with Amoxycillin. On the fifth day of fever he was referred to the emergency department for further evaluation. The only positive physical examination findings were mild oropharyngeal erythema, small submandibular lymph nodes, and left bullous myringitis. There were no respiratory symptoms or signs.

Results: Initial laboratory results showed leucocytosis with mildly elevated ESR and CRP. Chest radiographs revealed consolidation in the upper part of the right lobe. He was treated with IV Ceftriaxone for 3 days but the fever persisted, although the XR findings were resolved. His 8 th day of the illness he developed conjunctivitis and cheilitis and was treated with IVIG as an atypical Kawasaki. The fever subsided after $24 \mathrm{hrs}$ and on the 10th day marked thrombocytosis was noted. There were no Coronary Arteries Aneurysms (CAA) on echocardiograms performed on 2nd and 6th week of illness.

Conclusions: In summary, we have shown that atypical $\mathrm{KD}$ can present as prolonged fever with lobar pneumonia. Early clinical suspicion and therapy can reduce the risk of developing CAA .

\section{A CASE OF MILIARY T.B. WITH HYDROCEPHALLY}

\section{S Emadoleslami}

Department of Pediatrics, Isfahan University of Medical Sciences, Isfahan, Iran

Background and Aims: Tuberculosis is a contagious infectious disease that is still common in Iran. It may appear in different patterns. Its symptoms are depends on host immunity, age and disease severity. Miliary T.B and meningitis usually occurs in early years of life. Pulmonary symptoms, hepatosplenomegally, generalized lymphadenopathy are seen in military form Methods: It is a case report article.

Results: We report a 5 year old girl with miliary T.B and meningitis without hepatosplenomegally. She was referred to pediatrics emergency room with fever and productive cough. She had been sick since 6 months ago. She complained from fever, lymph node enlargement and diaphoresis. This patient had been maltreated as a case of asthma due to dyspnea, productive cough and fever. She has also has been treated with so many antibiotics. Even laryngoscopy was done purposelessly. Unfortunately these drugs had postponed the patient's diagnosis and treatment.

Conclusions: T.B evaluation is suggested in children with chronic cough, fever and weight loss and especially brain C.T scan may help us to evaluate military T.B cases who are suffering from meningitis too.

\section{KAWASAKI DISEASE IN INFANTS - CASE REPORTS}

S Tomé, A Sequeira, C Novais, M Figueiredo, R Cardoso, S Carvalho, F Carvalho, P Fonseca, $\mathrm{P}$ Teixeira

Paediatric Service, Hospital São João de Deus, Vila Nova de Famalicão, Portugal

Background and Aims: Kawasaki Disease (KD) is a syndrome of unknown etiology, defined by well established clinical features (Classical Kawasaki Disease). In the last years, it has been recognised forms of the disease that do not full fill sufficient clinical criteria (Incomplete Kawasaki Disease) most in infants who have higher risk of cardiovascular disease. The presence of additional laboratory or ecographic criteria, establish the diagnosis in this cases and the need of treatment

Methods: Revision the clinical cases of incomplete Kawasaki Disease by medical registrations.

Results: We had two cases on infants during 2005. A six months old boy admitted with 3 days of fever and macular rash. On fifth day of disease began bilateral 
conjunctival injection, fissured lips and skin descamation. He presented leucocitosis, elevated Creactive protein and erythrosedimentation rate, increase platelet count and sterile pyuria. A two months old girl admitted with fever, irritability and diffuse maculopapular rash with perineal descamation for 24 hours. Extremities changes with desquamation on the 9th day. She had elevated C-reactive protein and transaminases, anemia, increased platelet count, low albumin and pyuria. Both infants had normal ecocardiographic studies and began treatment with immunoglobulin and salicilate, with good clinical response.

Conclusions: Early treatment of $\mathrm{KD}$ is important for minimise the risk of cardiovascular disease and have a better outcome. It is important to recognise incomplete forms of the disease or occurrence in infancy to establish early treatment.

\section{DELAYS IN ANTIBIOTIC ADMINISTRATION IN MENINGOCOCCAL DISEASE}

\author{
H M Lala ${ }^{1}$, G D Mills ${ }^{1}$, K Barratt ${ }^{2}$, J Bonning ${ }^{3}$, \\ D Martin $^{4}$, N E Manikkam
}

${ }^{I}$ Department of Infectious Diseases, Waikato District Health Board, Hamilton, New Zealand, ${ }^{2}$ Department of Molecular Biology, Waikato District Health Board, Hamilton, New Zealand, ${ }^{3}$ Emergency Department, Waikato District Health Board, Hamilton, New Zealand ${ }^{4}$ Environmental Science Research, Kenepuru Science Centre, Porirua, New Zealand, ${ }^{5}$ Department of Paediatrics, Waikato District Health Board, Hamilton, New Zealand

Background and Aims: Meningococcal disease (MCD) is responsible for a large number of deaths among children. This study assessed the intervals between symptom onset, clinical assessment and antibiotic administration.

Methods: A retrospective review of hospital records and laboratory data of confirmed and presumed MCD deaths in children aged 0-18 in New Zealand during the serogroup B epidemic 1991-2004.

Results: Clinical records were available on 82 of 135 (61\%) fatal cases. Confirmed MCD was present in $79 \%$. Clinical diagnosis from the records suggested $52 \%$ with septicaemia alone, $41 \%$ with mixed disease, and 5\% with meningitis alone. The median age was 2 years (range $0-18$ ). 59\% (48) of the cohort had one or more consultations with a doctor prior to hospital presentation yet only $38 \%$ (18) received antibiotics prior to hospital presentation. Only $60 \%$ of patients received antibiotics within 2 hours of their first medical consultation. Death occurred rapidly within the cohort with $76 \%$ dying within 24 hours of hospital presentation.

Conclusions: Deaths from MCD in our cohort occurred rapidly, which is common to all MCD death cohorts. Although early antibiotic administration has been shown to save lives, many of those who died did not receive antibiotics at the earliest opportunity. A delay of more than two hours was seen in $40 \%$ of the cohort. The availability of improved diagnostic markers is urgently required to ensure antibiotics are administered at the earliest possible opportunity.

\section{INFECTIOUS DISEASES AND UNEXPECTED DEATH OF CHILDREN - PROBLEMS, REASONS AND PROGNOSIS}

\section{N P Skorodumova}

Chair of Children Disease and Children Infections, Donetsk State Medical University, Donetsk, Ukraine

Background and Aims: Infections play the main role among the reasons of unexpected death of children.

Methods: We have analyzed 80 cases of unexpected death of children of the first year, which took place in out of hospital departments. In $88,7 \%$ of children the death took place on the background of non-diagnostic infections diseases (meningococcous infection, encephalitis, leptomeningitis, generalized viral, viralbacterial infection).

Results: The main reasons of death in group of neuroinfectious were: infectious-toxic shock, edema of brain end lungs, acute adrenal deficiency, syndrome of disseminated intravascular coagulation. Death of $68,7 \%$ of children took place on the background of the acute respiratory viral infection because of reason of edema and swelling of brain, acute violation of microcirculation, cardiopulmonary deficiency. Gealin membranes were discovered in $8 \%$ of children. Children of 6-12 months died more often, men $(52,1 \% 0$, with low weight in birth, with the indexes of Apgar scale low than 6. As a rule, $(95,4 \%)$ children died at night during sleeping, more often in autumn-winter season, of the year. $54,7 \%$ of mothers were before 20 years old, $32,1 \%$ were exposure of much artificial abortion, in $38 \%$ of women the pregnancy developed with toxicosis, $39,1 \%$ smoked during pregnancy, $31 \%$ of women drenched, $24,1 \%$ took different medicines.

Conclusions: The totality of enumerated negative factors determine the prognosis of development of unexpected death in children and dictate the necessity of detachment of them in «risk group» the elaboration of schema of clinical examination.

\section{BLOODSTREAM AND BRONCHOPULMONARY FUNGAL INFECTIONS IN THE PICU}

\author{
I Gotsi ${ }^{1}$, G Arsenis ${ }^{2}$, A Velegraki ${ }^{2}$, G Paphitou ${ }^{1}$, \\ P Kalabalicis $^{1}$, T Hatzis ${ }^{1}$ \\ ${ }^{I}$ PICU, Agia Sophia Children Hospital, Athens, Greece \\ ${ }^{2}$ Mycology Laboratory, Microbiology Department, \\ Medical School, University of Athens, Athens, Greece
}

Background and Aims: To determine the epidemiology and antifungal susceptibility of blood and lung fungal infections, in PICU patients.

Methods: Retrospective study. All patients admitted to the PICU over a 6year period (2000-2006) with blood and/or Bal cultures positive for fungal infection. The Minimal Inhibitory Concentrations (MICs) were determined.

Results: 66 patients (35 girls, 31 boys), mean age 4 years. 5 of them $(7,8 \%)$ had a blood positive infection: isolates were $60 \%$ Candida ( $20 \%$ C.Albicans, $20 \%$ C.Tropicalis, $20 \%$ C.Mananis) and $40 \%$ Aspergillus Flavus. In 61 children $(92,4 \%)$ with $\mathrm{Bal}$ positive infections: $67 \%$ had Candida, 26\% Aspergillus, $7 \%$ both. Candida group: 9\% C.Albicans, 17\% C.Tropicalis, 15\% 
C.Parapsilosis, 5\% C.Quilliermondii, 2\% C.Glabrata, 2\% C.Krusei. Aspergillus group: 50\% A.Fumigatus, 31\%A.Flavus, $7 \%$ A.Restrictum, 6\% A.Penicillioides, $6 \%$ A.Terreus. In $44 \%$ cultures were $(+)$ on admission, in $56 \%$ became $(+)$ after a mean of 16 days and sterile after a mean of 17 days. E-test susceptibility for Candida: AmphotericineB $>$ Ketoconazole $>$ Intraconazole $>$ 5Fluorocytocine $>$ Voriconazole $>\mathrm{F}$ luconazole $>$ Caspofungine and for Aspergillus:AmphotericineB > Voriconazole > Ketoconazole > Caspofungine >Intraconazole > 5Fluorocytocine.

Conclusions: Blood fungal infections mortality was $80 \%$ compared to Bal (Candida 24\%, Aspergillus 37\%). C.Albicans is the commonest pathogen with a shift toward non Albicans spp during the last 3 years. AmphotericineB remains the drug of choice for Candida and Aspergillus.

\section{OUTBREAK OF METHICILLIN RESISTANT STAPHYLOCOCCUS AUREUS (MRSA) SEPTICAEMIA IN THE PAEDIATRIC INTENSIVE CARE UNIT (PICU) OF A DEVELOPING COUNTRY}

\author{
A Deep $^{1}, \mathrm{R}$ G Ghildiyal ${ }^{1}, \mathrm{~S}$ Kandian ${ }^{2}$ \\ ${ }^{I}$ Department of Pediatrics, T.N Medical College, \\ Mumbai, Maharashtra, India, ${ }^{2}$ Department of \\ Microbiology, T.N Medical College, Mumbai, \\ Maharashtra, India
}

Background and Aims: The emergence of MRSA in a critical care setting in a developing country has both health related concerns as well as serious financial implications.

Methods: A one year prospective study was undertaken to study the microbiological profile of nosocomial infections in PICU from April 2000 to March 2001. In September 2000, MRSA was isolated from the patients and the environmental samples were sent for phage typing. During the outbreak, an MRSA case was defined as any patient in the PICU with MRSA colonization or clinical disease. Before sending for phage typing, the Staph aureus cultures were subcultured. All bacterial isolates were subjected to antibiotic sensitivity testing.

Results: 6 patients had MRSA isolated from their blood. All these patients had copious secretions requiring frequent suctioning. The duration of stay in PICU before MRSA was isolated ranged from 5 days to 22 days. All MRSA isolates were highly drug resistant being sensitive only to vancomycin and rifampicin. Phage typing of MRSA isolated from these patients as well as those from portable suction pump revealed non-typable MRSA, thus tracing the sources of outbreak to the portable suction pump. The PICU remained closed for 2 wks until two sets of surveillance cultures were negative.

Conclusions: This outbreak demonstrated that lack of adherence to infection control guidelines in a intensive care setup can lead to grave consequences. It also demonstrates the role of extensive surveillance (both environmental and personnel) in the event of an outbreak of nosocomial infection in an intensive care unit.

\section{A DEDICATED PAEDIATRIC RETRIEVAL TEAM CAN SIGNIFICANTLY IMPROVE THE MANAGEMENT OF PERSISTENT PULMONARY HYPERTENSION OF THE NEWBORN}

A Deep, A Desai, A Petros, D Lutman, M Montgomery

The Children's Acute Transport Service, London, UK

Background and Aims: The Children's Acute Transport Service (CATS) transports over 1200 critically ill children/neonates $(>85 \%$ ventilated $)$ annually. We aimed to audit outborn cases of persistent pulmonary hypertension of the newborn (PPHN) referred for ECMO.

Methods: Case note audit of 135 consecutive cases (November 2001-August 2005).

Results: Two did not require transport. Of the remaining 133 (male:female 1.7:1) the commonest aetiology was meconium aspiration (48\%). Median (+ interquartile range) gestational age was 38 (34-40) weeks, age at referral 19.2 (9-31) hours, and weight 3.2 (2.8-3.62) kg. Stabilisation time was 153.5 (125-195) minutes, $111(83 \%)$ received 20ppm nitric oxide, and two died with the CATS team present. Twenty (15\%) underwent ECMO. Calculated risk of mortality deduced from PIM2 scores was higher for neonates referred aged $<12$ cf $>12$ hours ( $\mathrm{n}=48$ vs 42 : Risk of mortality -29.2 vs. 21.1 respectively, $\mathrm{p}=0.02$ ), and $>24 \mathrm{cf}<24$ hours $(\mathrm{n}=35$ vs 90, Risk of mortality-22.5 vs 25.15 respectively, $\mathrm{p}=0.04)$. Stabilization times were 153.5 (110-195), 152.5 (130-182.5), 150 (130-185) and 157.5 $(127.5-180) \min$ for $<12, \quad>12, \quad<24$, and $>24$ respectively.

Conclusions: (1) Risk of mortality calculated from PIM2 scores was inversely related to post-natal age, consistent with worse disease severity or lack of time for initial dynamic pulmonary vascular resistance to relax. (2) Stabilisation times were independent of PIM2. (3) Despite outborn neonates referred for ECMO being likely to represent more severe PPHN, mortality was only $1.5 \%$ : an experienced specialist service can safely transfer the majority of even severe PPHN cases.

\section{ASPIRATION PNEUMONIA PREVENTION IN INFANTS, USING ACE INHIBITORS}

\author{
P Avram, M Craiu, A V Cochino
}

Institute for Mother and Child Care, Bucharest, Romania

Background and Aims: In adults studies, protective respiratory outcome was documented for ACEinhibitors treatment. 1. ACE inhibitors play a role in setting the threshold for the cough and swallowing reflex in humans. 2. No data is available for pediatric population.

Methods: Six months case control prospective study in 6 infants with severe neurologic impairment (birth asphyxia). They were treated by nasogastric (NG) tube, with captopril $(0.7-1 \mathrm{mg} / \mathrm{kg} /$ day). Control group: 14 infants, admitted in the same unit. All patients had cardiovascular assessment (exclusion criteria included congenital heart disease and hypotension).

Results: In the captopril group, 2 episodes of aspiration pneumonia ( 2 cases/3 patient-year); were documented 
(clinically and radiologically) in control group were 20 such episodes (20/7 patient-year). Four of the episodes from the control group and none from the captopril group were severe and prolonged, eventually receiving mechanical ventilation. None of the children died.

Conclusions: 1. Captopril has proven efficiency in preventing aspiration pneumonia in neurological compromized patients $(\mathrm{OR}=0.23$ for pneumonia in the captopril group). 2. There were no secondary effects related to captopril at the used dosage. 3. Further studies are required to document these findings. Bibliography: 1. Teramoto S, Yamamoto H, Yamaguchi Y, Ouchi Y, Matsuse T. A novel diagnostic test for the risk of aspiration pneumonia in the elderly. Chest 2004;125:801-8022. Teramoto S, Ishii T, Yamamoto H, Yamaguchi Y, Ouchi Y. Nasogastric tube feeding is a cause of aspiration pneumonia in ventilated patients. Eur Respir J 2006; 27:436-437.

\section{ONE YEAR FOLLOW-UP OF VARICELLA VACCINE IN HEALTHY CHILDREN IN A POLISH VILLAGE}

\author{
T Jackowska ${ }^{1}$, J Dziadek ${ }^{2}$, R Wasilewski ${ }^{3}$ \\ ${ }^{I}$ Department of Pediatric Hematology and Oncology, \\ Medical University, Warsaw, Poland, ${ }^{2}$ Family Practice, \\ Gruta, Poland, ${ }^{3}$ Department of Laboratory Diagnostics \\ and Pediatric Clinical Immunology, Warsaw, Poland
}

Background and Aims: The rate of varicella and the persistence of varicella antibody after a one dose regimen of varicella virus vaccine in healthy children, in a Polish village, were compared after 46 days and after 13 months.

Methods: 106 healthy children (5 9girls, 47 boys), with no history of varicella, were vaccinated with VARILRIX ${ }^{\circledR}$ (GSK) between March and April 2004. The subjects were aged 9 months to 12 years (median47 months). After 46 days (group-VE1) and 13 months (group-VE2), the concentration of IgG-antibody against VZV was measured using the ELISA IgGTestkit.

Results: No local or general symptoms were observed after vaccination. Two vaccinated children with borderline values (9-11VE) developed a mild form of varicella one year after completing the vaccination regimen. 46 days after vaccination the seroconversion rate was $103(97.2 \%)$ of 106 . The seroconversion rate was $83(78.3 \%)$ of 106 patients 13 months after vaccination. The GMT in group VE1 was 19.4 (95\%CI$9.5,23.1)$ and $15.0(95 \%$ CI-15.5,23.1) in group VE2 respectively and was significantly lower after one year $(\mathrm{p}<0.001)$. In both groups no significant statistical differences were noted between antibody levels in girls (VE1-19.0; VE2-15.1) and boys (VE1-19.8;VE2-14.9). In children under 7 years of age $(\mathrm{N}=58)$ and in older children $(\mathrm{N}=48)$ there were no significant statistical differences between antibody levels both after 46 days and 13 months (VE1-17.9;VE2-14.6 and VE1-21.3; VE2-15.5 respectively).

Conclusions: Varicella vaccine was $98.1 \%$ effective in preventing varicella. After one year, antibody titers were negative in $21.7 \%$ children. Booster vaccinations or a two dose regimen of varicella vaccine might be considered to prevent breakthrough infection.

\section{MEASLES OUTBREAK - ATHENS, GREECE 2005-2006: DATA FROM THE HOSPITALIZATION OF CHILDREN}

D Dimitriou ${ }^{1}, \mathrm{~S}$ Leka $^{1}, \mathrm{~N}$ Tsilifis ${ }^{1}, \mathrm{M}$ Stamatiou ${ }^{1}$, G Mostrou $^{1}$, M Giannaki ${ }^{2}$, M Theodoridou ${ }^{1}$

${ }^{l}$ Department of Infectious Diseases A, Pediatric Clinic, University of Athens, 'Aghia Sofia Childrens Hospital', Athens, Greece, ${ }^{2}$ Microbiological Laboratory, 'Aghia Sofia Childrens Hospital', Athens, Greece

Background and Aims: Recording of clinicalepidemiological data from children with measles from 9/2005 until 7/2006.

Methods: 112 children, 1:1 male-female ratio, aged 7 months -12 years old (mean age 3yo), verified by Elisa and indirect immunofluorescence.

Results: Population distribution: 50\% Roma, 25\% Greek, 25\% children of immigrants. $97 \%$ were not vaccinated, $3 \%$ received one dose MMR. $22 \%$ were $<24$ months old, $65.5 \% 2-5$ yo, $12.5 \%$ over 6 yo. Mean duration of hospitalization was 4.5 days. Serological confirmation was established in 4 cases $(3.5 \%)$ that clinical data was insufficient. In $41 \%$ the manifestations were atypical. Complications rose in $91 \%, 72 \%$ as upper respiratory infections, $3 \%$ as GI complications, $3 \%$ as CNS involvement of which two children were admitted in the ICU. The outcome was excellent in all cases. Source of infection was family $31 \%$ and $12.5 \%$ Outpatient-clinic visit, compatible with the incubation time.

Conclusions: 1. The clinical outcome was excellent. 2. The Roma population has a significant number of children that are not vaccinated. 3. The atypical clinical manifestations may be contributed at the small age of the children $(<15$ m.o.) and possibly at the virulence of measles virus. 4 . The outbreak is a critical alert for the intensification of measles vaccination in Greece.

\section{TRANSPORT SYSTEM IN PORTUGUESE CENTER REGION: 15 YEARS EXPERIENCE}

\section{Fonseca, E Santos, J C Peixoto, J F Neves}

Unidade de Cuidados Intensivos, Hospital Pediátrico de Coimbra, Coimbra, Portugal

Background and Aims: Neonatal transfer is a subsystem of the National Medical Emergency Institute, born as part of the Perinatal Regionalisation Net from the National Health Department in 1990. This subsystem is organised in 3 regions: North, Center and South. Center Region transfer was extended to children up to 14 , since 2004. The authors aim to describe the neonatal and paediatric transport model of Portuguese Center Region and present the quantity and nature of the transfers.

Methods: To describe the neonatal and paediatric transport model, of Portuguese Center Region and present transfers' data during 1991-2005.

Results: Regionalisation Center Net comprehends 3 tertiary care hospitals (Polyvalent Intensive Care Unit in Hospital Pediátrico de Coimbra, headquarters of coordination of the transport service and 2 NICU in Maternities) and 8 secondary care hospitals that cover an area of $23.672 \mathrm{Km} 2$ and a population of 16.900 live 
birth/year and 261.000 children up to 14 years. The team includes an intensive care senior paediatrician and nurse available by a single phone number $24 \mathrm{~h} /$ day. There were 2997 transfers (not transported 51: dead prior to team arrival - 31; without indication - 20). They were 2480 newborns $(<1000 \mathrm{~g}$ : 8,9\%, 1000-1499g: 17,4\%, 15002499g: 25,7\%), 443 infants and 36 children. Boys $60 \%$. The main diagnoses were: prematurity, cardiorespiratory problems and malformations. Medical procedures: mechanical ventilation - $47,8 \%$, inotropics $25,8 \%$; central catheterization - 41,7\%, surfactant administration - 7\%. Destination: NICU/PICU - 48,4\%, surgery-14,8\%, secondary care hospitals - 21,1\%, complementary exams - 15,7\%.

Conclusions: Throughout 15 years, our transfer system, as a part of Regionalisation, proved its effectiveness and efficiency, and with the same team it was possible to extend it to children up to 14 years.

\section{PAEDIATRIC CRITICAL CARE TRANSPORT REVIEW: A NOVEL APPROACH}

\author{
J W Davis ${ }^{1}$, R H Taylor ${ }^{2}$, C B Imrie ${ }^{1}$, B A Bell ${ }^{3}$ \\ ${ }^{I}$ Department of Paediatrics, Altnagelvin Area Hospital, \\ Derry, Co. Londonderry, Northern Ireland \\ ${ }^{2}$ Paediatric Intensive Care, Royal Belfast Hospital for \\ Sick Children, Belfast, Co. Antrim, Northern Ireland \\ ${ }^{3}$ Department of Paediatrics, Craigavon Area Hospital, \\ Portadown, Co. Armagh, Northern Ireland
}

Background and Aims: Sick or injured children may initially present to a unit, which provides adequate resuscitation or stabilisation but is unable to offer intensive care management. Such children must be transported to another hospital or department. At present there is no regional paediatric retrieval service in Northern Ireland. Our aim was to review the transfer of critically ill children from a non-tertiary care hospital to the regional paediatric intensive care unit (PICU) in the Royal Belfast Hospital for Sick Children, Northern Ireland.

Methods: A forum was created using teleconferencing facilities to present, discuss and critically evaluate recently transferred cases. These cases were appraised by expert review and involved the multidisciplinary team involved in paediatric transport.

Results: Teleconferencing sessions have allowed multidisciplinary learning and identified areas for practice development; including documentation, clinical guidelines, fluid/airway management and drug administration. An inter-departmental group has been established to progress these developments locally.

Conclusions: This review is facilitating non-tertiary units to develop 'best practice', training and multidisciplinary care-pathways in paediatric transport. This model has been extended to create a network involving a three-way teleconference between two nontertiary units and the regional PICU. We have found that teleconferencing is popular with all staff incorporated into an education program and permits sharing of knowledge in a no-blame format with a greater understanding of each other's capabilities and limitations. This model may also benefit regions that already have an established transport service, including education and retention of skills for trainees who are no longer involved in paediatric transfer.

\section{INTRAOSSEOUS NEEDLE IN PAEDIATRIC CRITICAL CARE TRANSPORT SETTING}

\author{
A Bansal, P Ramnarayan, M Montogmery, \\ B Rossouw
}

\section{Children's Acute Transport Services, London, UK}

Background and Aims: To describe the frequency, efficacy and complications of intraosseous needle (IO) use for venous access during intensive care transport of critically ill children. Setting: Paediatric critical care transports performed by regionalized retrieval service.

Methods: Retrospective chart review was performed from Nov 2001-Nov 2005. All children who were referred for retrieval and required IO access during resuscitation at hospital or during transport were included. SPSS 10.0 was used for data analysis.

Results: 144 IO were placed in 114 children (54 males and 60 females; $1.7 \%$ of all retrievals in that period) during resuscitation and/or transport. Majority of children $(100 / 114,87.7 \%)$ were transported to an intensive care unit after successful resuscitation; 14 children died at the referring institution with the retrieval team. Mean age was $12.8 \pm 21.2$ month (range: 1 days -180 months) and mean weight was $8.0 \pm 5.3$ $\mathrm{kg}$. IO's were placed by referring paediatricians on retrieval team's advice in $64.9 \%$ children and by the retrieval team in $25.4 \%$. An average of 1.3 attempts were made (maximum 5 attempts), with a first attempt success rate of $82.5 \%$. The IO was maintained for $4.2 \pm$ 1.5 hours (range 2-9 hours). The main admitting diagnoses included sepsis $(23.7 \%)$, cardiac $(21.9 \%)$ and respiratory distress $(19.3 \%)$. IO placement was mainly for difficult intravenous access (55.8\%), cardiopulmonary arrest (25.4\%) and shock (18.4\%). Complications were seen in $16.7 \%$ cases, mainly displacement and local infiltration.

Conclusions: Intraosseous needle constitutes an important means of venous access for initial resuscitation and subsequent transport in critically ill children.

\section{VACCINEPROFILAX WITH POLIO VACCINE ON PRE-SCHOOL AND SCHOOL CHILDREN IN THE MUNICIPALITY OF DEMIR KAPIJA}

\author{
K Bakieva-Bojceva, Z Rajcanovska ${ }^{1}$, B Filipova \\ Health Centre Negotino, Faculty of Medicine Skopje, \\ Negotino, Macedonia
}

Background and Aims: In the past the contagious diseases have been taking many human lives or have left difficult consequences on the human organisms with permanent impairment. The source of contamination is an ill person contaminated with this disease. The provoker is the polio virus. The aim of the work is to present the percentage of primovaccine and revaccine coverage on pre-school and school children as well as the morbidity and the epidemiology in the Medicine Centre in Demir Kapija. 
Methods: For the production of these materials there are used vaccination cards and contagious diseases evidence books for the period 1995- 2005.

Results: Results are shown numerically, tabularly.

Conclusions: The successfully carried out vaccineprofilax $98.8 \%$ is a method for extermination (eradication) of polio in our country. We hope it will remain like that in the next millennium, too.

\begin{tabular}{|l|c|cc}
\hline & planned & done & $\%$ \\
\hline Prim ovaccine 1,2,3 & 849 & 839 & 98.8 \\
\hline 1 Revaccine & 303 & 302 & 99.6 \\
\hline 2 Revaccine & 403 & 403 & 100 \\
\hline 3 Revaccine & 607 & 607 & 100 \\
\hline
\end{tabular}

\section{AN IMPORTED CASE OF CHOLERA}

A Vasconcelos, E Almeida, H Almeida, M Brito, M Machado

Department of Paediatrics, Hospital Fernando Fonseca, Amadora, Portugal

Background and Aims: Even though cholera no longer poses a threat to developed countries it is still able to be imported from areas where it remains a devastating cause of death. One should know when to suspect it, effective methods for isolation of the Vibrio cholerae, how to provide prompt treatment and management of the contacts in order to avoid a major public-health disaster.

Methods: Clinical case presentation.

Results: An eleven-year old girl was admitted to the emergency unit in hypovolemic shock. She had arrived in Portugal from Guinea-Bissau just the previous day. Vomits and diarrhoea started 6 hours before admission. Signs of severe dehydration were present along with metabolic acidosis (pH:7.09, HCO3:15.3, EB:-14.6), hyponatraemia $(\mathrm{Na}+: 129 \mathrm{mmol} / \mathrm{L})$ and acute renal failure (creatinine: $2.3 \mathrm{mg} / \mathrm{dL}$ ). It was then observed profuse painless diarrhoea with watery stools resembling 'rice water' and facing this clinical picture cholera was suspected. Fluid replacement was immediately started along with doxycycline. In the first 24 hours she had lost up to a total of 11 litres of stools. The local health authority was reported and management of contacts proceeded. On the 5th day diarrhoea and clinical complications were solved. After two attempts, diagnosis was finally confirmed by isolation of Vibrio cholerae - serogroup O1 Inaba when alkaline peptone water was used.

Conclusions: In Portugal throughout many decades there were no reports of cholera, what made this case a special challenge in diagnosis. Nowadays, one should never underestimate the risk of importing highly contagious disease and the possibility of an outbreak if proper measures and diagnosis is delayed.

\section{MORTALITY RATES OF A DEDICATED PAEDIATRIC RETRIEVAL SERVICE}

A R Desai, A Deep, D Lutman, A Petros, M Montgomery
The Children's Acute Transport Service, London, UK

Background and Aims: The Children's Acute Transport Service (CATS) is a dedicated transport service for critically ill children and neonates requiring Intensive care in North London. We reviewed data for patients over a one year period to determine the mortality rate from point of referral.

Methods: A retrospective review was performed of all patients referred who died from December 2003 November 2004. Data extracted included death before CATS mobilisation, death in presence of the CATS team, death in presence of other teams, and death on PICU within 6 hours of arrival. Calculated risk of mortality (from PIM2) was reported.

Results: A total of 1658 patients were referred. The mortality rate of patients referred was $7 \%$. No patients died on transport. 20 deaths occurred prior to CATS mobilisation, 9 in the presence of the CATS team at the referring unit and 2 when other teams were present. 19 deaths occurred in the PICU after transfer by CATS. The calculated risk of mortality (from PIM2) of these 19 patients was 89.7 (IQR 78.6-94.9).

Conclusions: Our data support the view that a dedicated retrieval service can successfully transfer extremely sick children without mortality during transfer. Earlier referral may enable a reduction in the number of deaths at the referring institution.

\section{NOSOCOMIAL INFECTION SURVEILLANCE IN A PEDIATRIC INTENSIVE CARE UNIT (PICU)}

C Fernandez ${ }^{1}$, I Aguilar ${ }^{1}$, I Jordan ${ }^{1}$, E Esteban ${ }^{1}$, M Urrea $^{2}$, S Gala $^{1}$, A Palomeque $^{1}$

${ }^{1}$ Pediatric Critical Care Unit, Hospital Sant Joan de Deu, Barcelona, Spain, ${ }^{2}$ Department of Epidemiology, Hospital Sant Joan de Deu, Barcelona, Spain

Background and Aims: Hospital-acquired infections are usual in PICU. It results in a higher morbidity and mortality. Deaths attributable to nosocomial infection in PICU are estimated in $11 \%$. To describe the epidemiology of nosocomial infections in our PICU.

Methods: Prospective and observational study was performed in our unit from October to December 2005. CDC definitions for nosocomial infections were used.

Results: Medical data from 134 patients was analyzed. Were exposed to central venous catheter (CVC) 107 patients, to ventiloteraphy 67 and to urological catheter 110. Mean exposition days was $7.4 \pm 7.1,4.1 \pm 4.0$ and $5.0 \pm 4.8$ respectively with a mean stay in PICU of $5.3 \pm$ 4.5 days. Twenty patients suffered nosocomial infection, with 22 episodes (15\% from patients exposed and $10 \%$ of total admissions). Infection rate was 6,0 infections/100 patients-days stay and 11,0 infections/ 100 admissions. The infection site was: urinary tract in 10 cases $(45.5 \%), 3$ primary bloodstream infection $(13.6 \%)$, pneumonia and respiratory tract infections in 6 (27.6\%), septicemia related to CVC in 2 patients and one case of bronchiolitis. The more frequent germs isolated were enterobacterias (E coli and Enterobacter sp): 7 cases in the urinary cultures, 3 in blood sample and one in bronchoalveolar lavage. Length of exposition to extern devices and stay in PICU (13.6 versus 3.9 
days) were significantly longer in patients with nosocomial infections, $\mathrm{p} 0.02$.

Conclusions: Tract urinary and respiratory infections were the most common nosocomial sites of infection. Surveillance and prevention strategies are essential in care units.

\section{IMPROVING TRANSPORT MEDICINE: THE IMPACT OF REFLECTIVE PRACTICE}

\section{T Vince \\ Childrens Acute Transport Service, London, UK}

Background and Aims: Reflective practice is a useful skill for all doctors to help maximise the learning benefit of their experiences. This is imperative in critical care transport where trainees work in foreign, exposed environments. Consequently, critical examination of practice is essential. This study evaluates the effect of reflective practice on a group of paediatric critical care trainees during an attachment to a specialised retrieval service.

Methods: Trainees already maintained a mandatory logbook of their retrievals. After receiving instruction and on-going support, trainees recorded retrievals as episodes of reflection over an 8-month. The impact on trainees was gauged using semi-structured questionnaires and interviews. These techniques also explored factors that facilitated or hindered reflective practice.

Results: Despite intensity of work and time constraints, trainees were able to reflect on their practice. They perceived benefits both in clinical management skills and professional development, particularly in areas not covered by conventional training. There was broad agreement on organisational factors (time, service requirements and training) as facilitators of reflection and both organisational (time and extra work) and emotional factors (fear of criticism, fear of competency assessment) as barriers. Trainees were reluctant to document reflections and preferred oral group sessions.

Conclusions: This study confirms that reflective practice is beneficial. It is well suited to transport medicine and may be used to augment current paediatric training. However, some doctors will always find the process unacceptable and implementing mandatory reflection is likely to be counterproductive. Finding suitable alternatives is challenging.

\section{EFFICACY OF CIPROFLOXACIN IN AN EXPERIMENTAL MODEL OF ESCHERICHIA COLI CHORIOAMNIONITIS IN RABBITS}

\author{
E Launay $^{1}$, N Joram ${ }^{1}$, C Jacqueline ${ }^{2}$, J Caillon ${ }^{2}$, \\ J C Roze ${ }^{1}$, C Gras-Le Guen ${ }^{1}$ \\ ${ }^{1}$ Pediatric Intensive Care, CHU, Nantes, France \\ ${ }^{2}$ UPRES EA 3826, School of Medicine, Nantes, France
}

Background and Aims: Chorioamnionitis is a purulent infection causing prematurity, neonatal sepsis and death. Usual treatment ( $\beta$-lactams and aminoglycosides) is not optimal with the persistence of foetal bacteremia. So we studied the efficacy of ciprofloxacin, active in pus and not used in this indication, in a rabbit model of chorioamnionitis.

Methods: $12 \mathrm{~h}$ after the intrauterine inoculation of $\mathrm{E}$. coli, 6 pregnant rabbits were treated with ciprofloxacin $(30 \mathrm{mg} / \mathrm{kg}$ IV), 6 with ciprofloxacin + gentamicin $(10 \mathrm{mg} / \mathrm{kg} \mathrm{IM})$ and 4 others were untreated (controls). At 12,36 and $60 \mathrm{~h}$ after inoculation, caesarian was performed. The therapeutic efficacy was evaluated by the fotal survival, maternal and foetal blood cultures and tissular and amniotic liquid cultures.

Results: $60 \mathrm{~h}$ after inoculation, none of the untreated fetus survived, the survival rate was significantly increased in the ciprofloxacin and the ciprofloxacin + gentamicin groups $(46,7 \%$ and $71,2 \%$ with $\mathrm{p}=0,02$ and $\mathrm{p}=0,0003$ vs untreated respectively). Ciprofloxacin produced a dramatic reduction of fetal bacteremia and the sterilisation of maternal blood culture after $48 \mathrm{~h}$. The association with gentamicin allowed the sterilization of all the maternal and survival fœtal blood cultures as soon as $24 \mathrm{~h}$ of treatment. The quantitative cultures of amniotic liquid, lung, brain, liver and placenta were significantly reduced after a $24 \mathrm{~h}$-treatment in the two treated groups ( $\mathrm{p}<0,001 \mathrm{vs}$ controls).

Conclusions: Ciprofloxacin appears as an efficient treatment of acute chorioamnionitis with better survival rates and decreased quantitative blood cultures. The addition of gentamicin improves the in vivo activity with faster and greater bactericidal effect.

\section{INSULIN RESISTANCE IN CHILDREN WITH MENINGOCOCCAL SEPSIS: DOES IT OCCUR?}

\section{J J Verhoeven, A C S Hokken-Koelega, M den Brinker, K F M Joosten}

Department of Pediatric Intensive Care, Erasmus MC Sophia Children's Hospital, Rotterdam, The Netherlands

Background and Aims: Hyperglycemia (glucose $>8$ $\mathrm{mmol} / \mathrm{l}$ ) in critically ill children with various diseases is associated with increased mortality. It is not known whether insulin therapy will be beneficial and which children should be treated. The objective was to investigate the occurrence of insulin resistance by analyzing blood glucose levels in relation with insulin levels in children with meningococcal sepsis.

Methods: Prospective observational study. In all children with meningococcal sepsis on the pediatric intensive care between October 1997 and May 2004 blood levels of glucose and insulin were analyzed on admission and related with glucose intake and corticosteroid use.

Results: 78 Children were included (59\% male), mean age 5,3 years (1 month-16 years), mean PRISM score 21. 10 children did not survive (13\%). Hyperglycemia was found in $38 \%$. Non-survivors had a significant lower blood glucose level compared to survivors (5 vs 8 $\mathrm{mmol} / \mathrm{l})$. High insulin glucose ratios were found both in the group with low glucose $(<4 \mathrm{mmol} / \mathrm{l})$ and high glucose $(>11 \mathrm{mmol} / \mathrm{l})$.

Conclusions: Hyperglycemia occurred in $40 \%$ of the patients. In the group with hyperglycemia $>11 \mathrm{mmol} / 1$ the highest insulin-glucose ratios were seen, suggesting insulin resistance. Hyperglycemia $>11 \mathrm{mmol} / \mathrm{l}$ was 
correlated with higher age, higher glucose intake and corticosteroid use.

\begin{tabular}{|c|c|c|c|c|c|c|c|}
\hline $\begin{array}{l}\text { ghucose on } \\
\text { admissiom } \\
\text { (mmol/) }\end{array}$ & $\begin{array}{l}\text { patients } \\
(\%))\end{array}$ & $\begin{array}{l}\text { age } \\
\text { (yrs) }\end{array}$ & $\begin{array}{l}\text { survival } \\
(\%)\end{array}$ & $\begin{array}{l}\text { ghocose } \\
\text { intake } \\
\text { (mg/kg/min) }\end{array}$ & $\begin{array}{l}\text { insulin } \\
\text { (pmoll) }\end{array}$ & $\begin{array}{l}\text { invulin/ } \\
\text { ghroose ratio } \\
\text { (pmol/nmol) }\end{array}$ & $\begin{array}{l}\text { steroid } \\
\text { use } \\
(\%)\end{array}$ \\
\hline 84 & 9 & 1,1 & 33 & 1,6 & 57 & 21 & 0 \\
\hline 48 & 53 & 4,5 & 88 & 2,7 & 81 & 14 & 5 \\
\hline $8,1-11$ & 25 & 7,4 & 100 & 32 & $1 \pi 0$ & 18 & 6 \\
\hline$>11$ & 13 & 7,1 & 89 & 5,0 & 320 & 23 & 33 \\
\hline
\end{tabular}

\section{EFFECT OF BETAMETHASONE ON CEREBRAL BLOOD FLOW VELOCITIES IN PRETERM NEONATES WITH SEVERE BRONCHOPULMONARY DYSPLASIA}

G Cambonie, O Pidoux, R Mesnage, C Milesi, M Badr, P Plan, E Sabatier, B Veit, F Montoya, J C Picaud

\section{Neonatal Intensive Care Unit, Arnaud de Villeneuve Hospital, University Hospital of Montpellier, France}

Background and Aims: In fetal sheep, betamethasone administration in late gestation exerts a major vasoconstrictor effect on cerebral blood flow. In human preterm infants, postnatal corticosteroids are associated with an increased risk of neurodevelopmental delay, but little is known about the haemodynamic involvement in the occurrence of cerebral injury. We aimed to assess the effects on cerebral blood flow velocities (CBFV) of betamethasone $(\mathrm{BM})$ given to premature infants for the treatment of exceptionally severe bronchopulmonary dysplasia (ESBPD).

Methods: We prospectively measured $\mathrm{CBFV}$ in subjects treated with BM for ESBPD and lifethreatening conditions. BM dosage was $0.5-0.5-0.5-0.3-$ $0.3-0.1 \mathrm{mg} / \mathrm{kg} / \mathrm{d}$. After standardization of the procedure, $\mathrm{CBFV}$ were recorded in the anterior cerebral (ACA) and the lenticulostriate arteries (LSA) before and 2 hours after each dose of BM. Results expressed as mean (SEM) and analyzed using repeated-measure ANOVAs.

Results: Ten neonates [BW:737(46)g, GA:25.5 (0.3) weeks] were studied at 34(3) days of life. In the ACA and LSA, both systolic (S) and diastolic (D) velocities significantly decreased during BM treatment. The resistance index (RI) increased in the ACA over the treatment period [baseline: $0.78(0.01), \mathrm{d} 1: 0.83(0.02)^{*}$, d3: $0.83(0.01)^{*}$, d5: $0.89(0.01)^{*}$, d8: $0.81(0.01)$, $\mathrm{p}<0.05]$, whereas the increase in LSA was significant only at day3.

Conclusions: Our results support the hypothesis of a vasoconstrictor effect of betamethasone in premature infants with ESBPD. This effect may be implicated in the occurrence of brain injury and later neurodevelopmental delay in this specific population.

\section{DELAYED NEURAL CONDUCTION IN THE AUDITORY BRAINSTEM IN PRETERM INFANTS WITH CHRONIC LUNG DISEASE}

\section{Z D Jiang, D M Brosi, A R Wilkinson}

Department of Pediatrics, University of Oxford, John

Radcliffe Hospital, Oxford, UK
Background and Aims: Neonatal chronic lung disease (CLD) is known to affect growth and development of the immature brain. However, it is not known whether infants with neonatal CLD are at risk of auditory deficits. This study aims to detect functional abnormality in the auditory brainstem at term in preterm infants with CLD.

Methods: We recruited 25 preterm infants who suffered neonatal CLD, with gestational age 23-30 wks. None had any concomitant other major perinatal problems. At 37-42 wks postconceptional age, brainstem auditory evoked response (BAER) was recorded with clicks of 21-91/s.

Results: Compared to those recorded in normal term controls, BAER wave V latency and I-V and III-V interpeak intervals in the CLD infants increased significantly (ANOVA $\mathrm{p}<0.01-0.001$ ). The latencies of waves I and III did not differ significantly from the controls. However, no abnormalities were found in BAER wave amplitudes. These BAER findings were seen at all click rates $21-91 / \mathrm{s}$, although the increase in III-V interval tended to be more significant at higher rates compared to those at lower rates. Click ratedependent changes in BAER variables in the CLD infants were generally similar to the term controls, although slightly different.

Conclusions: BAER components, mainly reflecting central auditory function, in the preterm infants with CLD increased significantly. The increase in wave $\mathrm{V}$ latency and I-V interval is due to the increase in III-V interval. These results suggest that there is delayed neural conduction in the more central portion of the auditory brainstem following neonatal CLD.

\section{PILOT STUDY USING NIRO-200 IN VERY LOW BIRTH WEIGHT (VLBW) INFANTS TO ASSESS CEREBRAL AUTOREGULATION}

A C Doolan, A R Twomey

National Maternity Hospital, Dublin, Ireland

Background and Aims: Near Infrared Spectroscopy (NIRO) uses light spectroscopy, to provide us with information about oxygenation within the tissues, measuring changes in the concentration of oxygenated haemoglobin (Hb02), deoxygenated haemoglobin $(\mathrm{HHb})$, total haemoglobin $(\mathrm{HbT})$, tissue oxygenation index (TOI) and tissue haemoglobin index (THI), thus giving us information about brain oxygenation, cerebral blood volume and cerebral blood flow.

Methods: The aim was to observe if infants $<1.5 \mathrm{~kg}$ demonstrated evidence of cerebral autoregulation in response to position changes. A total of 12 VLBW babies were observed on Day 1, 2 and 3. The NIRO-200 (Hamamatsu) was used to record $\mathrm{Hb} 02, \mathrm{HHb}, \mathrm{HbT}$, TOI and THI. The changes in these variables in response to the tilt and changes in vital signs were recorded. Infants with a CRIB score of $6+$ were considered separately to those who were assigned a score of 5 or less.

Results: Of the 12 babies included, 8 were male and 4 were female with an average gestation of $29+4$ and weight of $1.08 \mathrm{~kg}$. There were 8 babies in the group with CRIB score of 5 or less, with 4 babies in the more unwell group. Statistically significant differences were 
seen in the two group's response to tilt. Stable babies had an increase in $\mathrm{HbO} 2$ and $\mathrm{HbT}$ and an increase in BP. Sicker babies tended to drop their $\mathrm{HbO} 2, \mathrm{HbT}$ and THI and BP.

Conclusions: The purpose of our study was to observe the effect of change in position in VLBW infants. Our study would suggest that more unstable babies show impaired cerebral autoregulation.

\section{ULTRASOUND DIAGNOSIS OF HYPOXIC- ISCHEMIC ENCEPHALOPATHY IN NEWBORNS}

\section{B A Burov}

\section{Tashkent Medical Institute of Postgraduate Education, Tashkent, Uzbekistan}

Background and Aims: Hypoxic-ischemic damages of the brain in newborns is the significant current problem of neonatology. The lesions of the nervous system account for $50 \%$ in the structure of the pediatric disability. The diseases of nervous system are related to perinatal factors in $70-80 \%$.

Methods: Neurological symptoms do not always reflect the character and severity degree of the central nervous system damage, and so ultrasound diagnosis has the important role in the identification of these pathologies. Neurosonography is one of the current methods of ultrasound diagnosis of encephalopathy.

Results: Investigation was carried out on 110 premature newborns with encephalopathy. There were studied data of medical history, risk factors of the period of gestation. There was noted high frequency of preterm deliveries. Among the neurological symptoms the symptom of Grefe, strained look, pathological increase in muscular tone, clonic-tonic convulsions are prevailed. Neurosonography of $38(34,55 \%)$ infants revealed isolated peri-intraventricular hemorrhages of the $1 \mathrm{st}$ degree and hemorrhages of the second degree were found in $12(10,91 \%)$. Peri-intraventricular hemorrhages of the 3 degree were found in 27 patients (24,55\%).

Conclusions: Thus, neurosonography in the complex investigation of newborns with hypoxic-ischemic encephalopathy appeared to be highly effective reliable methods of early diagnosis of cerebral pathology which may be recommended to perform from the 3rd day of life. This method allows to identify predominantly changes of hemorrhagic character (peri- and intraventricular) hemorrhages in the brain of different severity.

\section{OUTCOME OF INFANTS EXCLUDED FROM A HYPOTHERMIA TRIAL BY AMPLITUDE-EEG (aEEG)}

\section{J D Barks ${ }^{1}$, D V Azzopardi², S M Donn ${ }^{1}$}

${ }^{1}$ Department of Pediatrics, University of Michigan, Ann Arbor, MI, USA, ${ }^{2}$ Department of Paediatrics, Imperial College, London, UK

Background and Aims: Infants with hypoxic-ischemic encephalopathy (HIE) were excluded from a recent hypothermia trial if an analog aEEG (Lectromed) tracing failed to meet criteria for moderate-to-severe abnormality. Expertly interpreted aEEG is predictive of outcome of HIE; yet, its prognostic utility by novice or infrequent users is uncertain.

Methods: Cases were ascertained from the recruitment log from a single study center. 25 infants $>=36$ wks with birth depression and moderate-to-severe HIE in the first $5.5 \mathrm{~h}$ of life were logged over 2 years. 12 were randomized, 1 was ineligible because of equipment malfunction, and 12 did not meet aEEG criteria.

Results: Of the 12 excluded infants, 3 were lost to followup and 1 died neonatally with severe HIE. Of 8 with 3-6 yrs followup; 50\% had spastic or athetoid $\mathrm{CP}$, microcephaly and developmental delay, one had ADHD, and three were normal.

Conclusions: There was a high burden of death or severe disability in infants excluded based on criteria intended to enhance specificity. Possible explanations include (i) the cause of birth depression and CP might not have been acute perinatal HI; or (ii) the aEEG step may have excluded patients that might have benefited. A limitation of the Lectromed aEEG was inability to evaluate raw EEG. With new monitors, and raw EEG review capability, the role of "aEEG" in patient selection may need to be re-evaluated. We speculate that the new digital EEG monitors might play a greater role in enhancing sensitivity of ascertainment of eligible infants than in excluding candidates from a safe and effective therapeutic intervention.

\section{GLUTATHIONE FRACTIONAL SYNTHESIS RATES IN NEONATES WITH PERINATAL ASPHYXIA}

K de Groof ${ }^{1}$, F W J Te Braake ${ }^{1}$, H Schierbeek ${ }^{2}$, J B Van Goudoever ${ }^{1}$

${ }^{I}$ Department of Pediatrics, Division of Neonatology, Erasmus MC - Sophia Children's Hospital, Rotterdam, The Netherlands, ${ }^{2}$ Department of Pediatrics, Division of Neonatology, Mass Spectrometry Laboratory, Erasmus MC - Sophia Children's Hospital, Rotterdam, The Netherlands

Background and Aims: Perinatal asphyxia is one of the major causes of neonatal death. During hypoxia, reactive oxygen species are formed by reperfusion and by resuscitation with $100 \%$ oxygen. Reactive oxygen species cause brain injury and organ failure. Glutathione (GSH) is the major intracellular antioxidant. It protects cells against the toxic effects of these reactive oxygen species. The fractional synthesis rate (FSR) of glutathione in healthy adults is $65 \pm 13 \%$ /day [Lyons, 2000]. Our aim is to quantify the FSR of glutathione in term asphyxiated infants, shortly after birth.

Methods: Neonates with a gestational age $>35$ weeks, umbilical $\mathrm{pH}<7.10$ and 5 min Apgar score $<7$ were included. To quantify glutathione synthesis, we infused [1-13C]glycine between 48 and 96 hours after the hypoxic event. Blood was sampled after 4, 5 and 6 hours. Free [1-13C]glycine enrichment was measured by gas chromatography - isotope ratio mass spectrometry (IRMS) and was used as a precursor for glutathione synthesis. Labelled glycine incorporation in glutathione was measured by liquid chromatography - IRMS.

Results: The four infants were measured on postnatal day 3 and reached a plateau within 4 hours. See table. 
Conclusions: Neonates ( $>35$ weeks) with asphyxia have a low FSR of glutathione as compared to healthy adults.

\begin{tabular}{|l|c|c|c|c|c|c|c|}
\hline $\mathrm{n}$ & $\begin{array}{c}\text { Gestational } \\
\text { age(wk) }\end{array}$ & $\begin{array}{c}\text { Umbilical } \\
\mathrm{pH}\end{array}$ & $\begin{array}{c}\text { Apgar } \\
\text { score } \\
5 \mathrm{~min}\end{array}$ & $\begin{array}{c}\text { Non-protein } \\
\text { intake } \\
(\mathrm{kcal} / \mathrm{kg} / \mathrm{d})\end{array}$ & $\begin{array}{c}\text { Protein } \\
\text { intake } \\
(\mathrm{g} / \mathrm{kg} / \mathrm{d})\end{array}$ & $\begin{array}{c}\text { Glycine } \\
\text { Enrichment } \\
(\mathrm{MPE})\end{array}$ & $\begin{array}{c}\mathrm{FSR} \\
\mathrm{GSH} \\
(\% / \text { day })\end{array}$ \\
\hline 1 & $38^{2} / \gamma$ & 6.79 & 4 & 51 & 2.25 & 3.37 & 50 \\
\hline 2 & $41^{0} / \gamma$ & 7.09 & 5 & 34 & 1.50 & 3.66 & 49 \\
\hline 3 & $352 / 7$ & 6.94 & 6 & 57 & 1.75 & 2.89 & 58 \\
\hline 4 & $38^{2} / \gamma$ & n.a. & 4 & 50 & 0.75 & 3.90 & 55 \\
\hline mean & $38^{3} / \gamma$ & 6.94 & 5 & 48 & 1.56 & 3.46 & 53 \\
\hline
\end{tabular}

\section{BREATHING XENON WHILE COLD IS COOL}

C E Hobbs ${ }^{1}$, J J Dingley ${ }^{1,2}$, A M Tucker ${ }^{1}$, M Thoresen ${ }^{1}$

${ }^{I}$ Department of Child Health, CSSB, University of

Bristol, Bristol, UK, ${ }^{2}$ Department of Anaesthetics,

Morriston Hospital, Swansea, UK

Background and Aims: Hypothermia (HT), administered after hypoxia-ischaemia (HI) in newborn infants, is neuroprotective. However, the long-term efficacy is uncertain and the number to treat is 6 . Experimental models show that the inert gas Xenon (Xe) protects the brain after HI. Preliminary work combining the two treatments to enhance neuroprotection shows great promise. Xe has a high density and viscosity, and at high concentration $(70 \%)$ can cause respiratory depression. Moderate $\mathrm{Xe}$ concentration $(50 \%)$ does not depress respiration at normothermia (NT) but the effect of the combination on respiration in neonates is unknown. Thus, our aim was to examine the respiratory effects of $50 \% \mathrm{Xe}$ and $\mathrm{HT}$ after $\mathrm{HI}$ in the neonatal rat.

Methods: 29, 7-day-old (P7) Wistar rats underwent ligation of the left common carotid artery, followed by 90 minutes of hypoxia ( $8 \%$ oxygen) at NT. Pups were then randomised to receive $5 \mathrm{~h}$ of: Air/NT, Air/HT, 50\% $\mathrm{Xe} / \mathrm{NT}$ or $50 \% \mathrm{Xe} / \mathrm{HT}$. Sampling of blood gases was performed immediately after the treatment period.

Results: Measured respiratory outcomes (pH, pCO2 and lactate) and $\mathrm{HCO} 3$ were not significantly affected by $5 \mathrm{~h}$ of $50 \%$ Xenon or HT alone, nor the combination, after HI. pO2 was significantly higher in the $50 \% \mathrm{Xe} / \mathrm{NT}$ group than the Air/HT group, apparently reflecting the variability of this measure in mixed arterial-venous samples.

Conclusions: The combination of $50 \%$ Xenon and hypothermia for 5 hours following HI does not depress respiration in the neonatal rat. For further clinical trials we would suggest that $50 \%$ Xenon is a safe and effective concentration to use in combination with hypothermia.

\section{IS VIGINTIPHOBIA REMERGING? \\ BIOCHEMICAL MARKERS OF \\ NEUROTOXICITY DUE TO \\ HYPERBILIRUBINEMIA IN NEONATES}

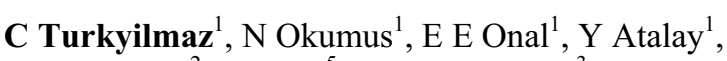
A Serdaroglu ${ }^{2}, \mathrm{G} \mathrm{Deda}^{5}$, Y K Kemaloglu ${ }^{3}, \mathrm{H} \mathrm{Pasaoglu}^{4}$, $\mathrm{S} \mathrm{Elbeg}^{4}, \mathrm{~A} \mathrm{Cansu}^{2}$, B Gunduz ${ }^{3}$

${ }^{1}$ Department of Neonatology, Gazi University Medical Faculty, Ankara, Turkey, ${ }^{2}$ Department of Pediatric Neurology, Gazi University Medical Faculty, Ankara, Turkey, ${ }^{3}$ Department of Otorhinolaryngology, Gazi University Medical Faculty, Ankara, Turkey
${ }^{4}$ Department of Medical Biochemistry, Gazi University Medical Faculty, Ankara, Turkey, ${ }^{5}$ Department of Pediatric Neurology, Ankara University Medical Faculty, Ankara, Turkey

Background and Aims: The aim of this study is to investigate the relationship between serum levels of S-100 and Tau protein and bilirubin encephalopathy findings in neonates.

Methods: Sixty one jaundiced and 28 healthy term newborns were included. Serum levels of S-100 and Tau protein were analysed by ELISA. Neurological examination, Electroencephalogram (EEG), otoacustic emission (OAE), brainstem auditory evoked response (BAER) were done in infants on admission and at the age of three months.

Results: Mean serum levels of Tau and S-100 protein $(183.6 \pm 9.6 \mathrm{pg} / \mathrm{mL}, 151.1 \pm 18.2 \mathrm{ng} / \mathrm{L})$ in jaundiced infant was higher than in controls $(245.5 \pm 64.6 \mathrm{pg} / \mathrm{mL}$, $979.6 \pm 986.7 \mathrm{ng} / \mathrm{L}) \quad(\mathrm{p}<0.000, \quad \mathrm{p}<0.000)$ respectively. There were significant positive correlations between total bilirubin concentrations and serum Tau protein $(\mathrm{r}=0.825, \quad \mathrm{p}=0.000)$ and serum $\mathrm{S}-100$ protein concentrations $(\mathrm{r}=0.794, \mathrm{p}=0.000)$. In jaundice group, 8 cases had EEG abnormalities, 9 cases had BAER abnormalities and 6 cases failed OAE, while no abnormality were detected in controls. In infants whose bilirubin levels higher than $20 \mathrm{mg} / \mathrm{dl}, 10$ were hypotonic and 22 had poor sucking on admission, but there are no clinical abnormalities in jaundiced infants whose bilirubin levels lower than $20 \mathrm{mg} / \mathrm{dl}$. In the age of three months-old, all previous abnormalities were disappeared.

Conclusions: Serum Tau and S-100 proteins can be used as markers of bilirubin induced neurotoxicity in neonates. Vigintiphobia could be a reality.

\section{PERINATAL CHANGES OF NEUROTROPHINS IN INTRAUTERINE GROWTH RESTRICTED AND APPROPRIATE FOR GESTATIONAL AGE TERM OFFSPRING AND THEIR MOTHERS}

K E Nikolaou, A Malamitsi-Puchner, E Economou, M Boutsikou, T Boutsikou, K P Puchner,

M Kyriakakou, D Hassiakos

2nd Department Obstetrics and Gynecology, Athens University, Medical School, Athens, Greece

Background and Aims: Intrauterine growth restricted (IUGR) are fetuses with estimated weight $<10$ th customized centile, displaying signs of chronic malnutrition and hypoxia, possibly leading to brain sparing effect. Neurotrophins, [Nerve Growth Factor (NGF), Brain Derived Neurotrophic Factor (BDNF), Neurotrophin-3 (NT-3), Neurotrophin-4 (NT- 4)] are important for pre- and postnatal brain development. This study investigated circulating levels of NGF, BDNF, NT-3 and NT-4 in IUGR and appropriate for gestational age (AGA) term fetuses and neonates and their mothers. Methods: BDNF, NGF, NT-4, NT-3 were determined by enzyme immunoassays in the plasma of 60 mothers (MS) and their 30 IUGR and 30 AGA fetuses (UC) and neonates on postnatal day 1 (N1) and 4 (N4). 
Results: No statistically significant differences were observed between IUGR and AGA MS, UC, N1 and N4 of BDNF, NT-3 and NT-4 levels. NGF levels were significantly higher in AGA than IUGR MS $(p=0.007)$, UC $(p=0.01), N 1(p=0.043)$ and N4 $(p=0.003)$ and positively correlated with the infants' centiles and birthweights. IUGR and AGA maternal neurotrophin levels were higher than respective fetal and neonatal levels. Neurotrophin levels showed no correlation with gender or delivery mode in both groups.

Conclusions: The brain sparing effect, caused by fetal hypoxia, could be responsible for similar BDNF, NT-3 and NT-4 levels in IUGR and AGA fullterm fetuses and neonates. NGF levels positively correlated with infants' customized centile and birthweight, thus they were higher in AGA. Elevated maternal neurotrophin levels could reflect nervous system maturity. No differentiation in neurotrophin levels among genders might imply their similar neurodevelopment.

\section{EFFECT OF hGH ON BRAIN ANTIOXIDANT SYSTEM AND LIPID PEROXIDATION OF HYPOXIC-ISCHEMIC NEWBORN RATS}

\author{
H Yildizdas ${ }^{1}$, M Satar ${ }^{1}$, N Canacankatan ${ }^{2}$, E Tutak ${ }^{1}$, \\ $\mathrm{N} \mathrm{Narli}^{1}$, Y Sertdemir ${ }^{3}$ \\ ${ }^{I}$ Department of NICU, Çukurova University Faculty of \\ Medicine, Adana, Turkey, ${ }^{2}$ Department of Biochemistry, \\ Çukurova University Faculty of Medicine, Adana, \\ Turkey, ${ }^{3}$ Department of Biostatistics, Çukurova \\ University Faculty of Medicine, Adana, Turkey
}

Background and Aims: We investigated the effect of hGH on newborn rat brain superoxide dismutase (SOD), glutathione (GSH) and malondialdehyde (MDA) levels in hypoxic-ischemic newborn rats.

Methods: Forty-eight 7 days old newborn rats were randomised to healthy (n:15), hypoxic ischemic (H-I, $\mathrm{n}: 18)$ and $\mathrm{GH}$ administered hypoxic ischemic (GH-HI, $\mathrm{n}: 15)$ group. Permanent, left common carotid ligation was performed in the hypoxic ischemic groups. In the GH-HI group, $50 \mathrm{mg} / \mathrm{kg}$ s.c. hGH was administered just before carotid artery ligation. After 2 hours of hypoxemia rats were decapitated. Right and left cerebral hemispheres (CHs) and cerebellum-brain stem (C-BS) were separated.

Results: GSH levels of each region were not statistically different from each other in and between the groups. SOD levels were higher in CBSs compared to CHs ( $<<0,01)$. CHs and C-BS MDA levels were similar in the control and H-I groups but MDA levels of both CHs in GH-HI group were significantly higher than the levels of H-I group. Left CH of GH-HI group was also higher compared to left $\mathrm{CH}$ of the control group $(\mathrm{p}=0$, 045) while there was no difference between right $\mathrm{CHs}$. In GH-HI group left $\mathrm{CH}$ MDA level was higher than CBS $(p=0,03)$. MDA levels of the C-BSs did not differ between groups $(\mathrm{p}>0,05)$.

Conclusions: In conclusion, although we have not evaluated the effect of GH histopathologically, increased lipid peroxidation especially in the hypoxicischemic (left) hemisphere of the GH treated rats might suggest that GH treatment may be harmful in hypoxic ischemic encephalopathy.
NEONATAL BRAIN INJURY - HYPOXIA PLUS INFECTION. WHICH IS THE OUTCOME?

M Craiu, A V Cochino, P Avram

Institute for Mother and Child Care, Bucharest, Romania

Background and Aims: As stated in literature, the association between hypoxia and infection is a worsening factor of neonatal brain injury. We performed a 2 years study, to determine the value of the risk increment in this concern.

Methods: This is a retrospective case control study. The data were gathered from clinical files of 2004-2005. The infected group: 11 newborns with documented infection and hypoxic/ischemic brain damage. The control group: 27 newborns with perinatal brain damage. We have evaluated the risk factor distribution, especially the infection, and the severity of neurologic impairment, as documented by clinical and transfontanelar echographic (TFE) evaluation.

Results: No significant Apgar scores differences were found between the two groups (mean Apgar scores at 1 minute: 5.75 in infected, versus 5.5 in control group). The mean number of clinical signs of neurologic impairment was 3.63/patient as compared to 2.44/patient, in the control group. Cerebral edema and dilated lateral ventriculi, as evaluated by TFE, were more frequent in the infected group. There were $18.51 \%$ patients with a normal TFE in the control group but none in the infected group.

Conclusions: 1 . Infection presence worsens the clinical and TFE aspect of newborns with hypoxic brain injury (clinical and imagistic proves). 2. Other important risk factors were: birth at home, lack of pregnancy followup, maternal infection during gestation and prolonged ruptured membranes. 3. Gestation follow-up, economic development (related to hospital versus home birth) and infection control might improve neurologic outcome of newborns.

\section{LEPTIN RECEPTOR EXPRESSION IN THE BRAIN OF NEWBORN RATS DECREASES FOLLOWING HYPEROXIA AND INCREASES AFTER INJURY CAUSED BY PHORBOLESTER}

C Peiser, P Koehne, M Dzietko, O Polley, M Obladen, U Felderhoff-Müser

Department of Neonatology, Charité Virchow-Campus, Berlin, Germany

Background and Aims: Leptin is a pleitropic proteohormone and cytokine, which acts by binding at the long isoform of the leptin receptor (OB- $\mathrm{Rb}$ ). Next to metabolic and immunomodulating functions, leptin also promotes angiogenesis, takes part in brain development, and plays a role in neuroprotection. The aim of this study was to investigate OB-Rb expression in the brain of newborn rats in relation to neurodegeneration, either induced by hyperoxic or inflammatory brain injury, both important causes of neonatral neurodegeneration.

Methods: $6 \mathrm{~d}$ old Wistar rat pups were either subjected to $80 \%$ oxygen or treated with phorbolester by injecting $500 \mu \mathrm{g}$ intraperitoneally. After $0,2,6,12$ and $24 \mathrm{~h}$ of exposition animals ( $\mathrm{n}=5$ per group) were sacrificed and 
the brain cortices were processed for molecular experiments on mRNA (quantitative RT-PCR) and protein (Western blot) levels using OB-Rb specific primers and antibodies.

Results: In association with hyperoxia a decrease in $\mathrm{OB}-\mathrm{Rb}$ mRNA and protein expression down to about $40 \%$ of the basal level of the control animals was noted. In case of phorbolester treatment we have seen an increase of $\mathrm{OB}-\mathrm{Rb}$ expression up to about $170 \%$ compared to the controls. The effects had their maximum / minimum after 6-12 $\mathrm{h}$ on the transcriptional and after $24 \mathrm{~h}$ on the translational level.

Conclusions: Exposure to hyperoxia or treatment with phorbolester induce statistically significant timedependent changes in OB-Rb expression, indicating the influence of the leptin system in neuronal injury to the immature brain caused by hyperoxic or inflammatory brain injury.

\section{LATERAL VENTRICULAR SIZE IN EXTREMELY PREMATURE INFANTS: 3D MRI CONFIRMS 2D ULTRASOUND MEASUREMENTS}

S Horsch ${ }^{1}$, J Bengtsson ${ }^{2}$, A Nordell ${ }^{2}, \mathrm{Z} \mathrm{Nagy}^{2}$, B Hallberg ${ }^{1}$, H Lagercrantz $^{1}$, U Ådén ${ }^{2}$, B Nordell ${ }^{2}$, M Blennow ${ }^{1}$

${ }^{1}$ Department of Neonatology, Karolinska University Hospital, Stockholm, Sweden, ${ }^{2}$ Department of Hospital Physics and MR Centrum, Karolinska University

Hospital, Stockholm, Sweden

Background and Aims: Cerebral ventriculomegaly at term equivalent age is an predictor of adverse outcome in preterms. It can easily be detected with cranial ultrasound (US), whereas 3D MRI provides a valid assessment of ventricle volumes. Previous studies have found limited correlations between 2D ventricular US measurements and 3D MRI volumes; however CSF volumes were not subdivided into extracerebral and ventricular CSF.

Methods: Paired cranial MRI and US scans in a population based cohort of 43 ELGA infants were obtained. Infants were scanned at term equivalent age on a 1.5T MRI system. To segment the lateral ventricles, an image processing software was developed using a semiautomatic Active Contour model. Cranial US was performed in the coronal and parasagittal planes through the anterior fontanelle. Five different ventricular dimensions were measured on each side.

Results: Correlations ( $\mathrm{r} 2$ ) between MRI volumes of US measurements of the frontal horns (coronal short axis: right 0.91 , left 0.82 , long axis right 0.74 , left 0.67 ; sagittal frontal horn height right 0.88 , left 0.70 ) and ventricular midbody (right 0.83 , left 0.80 ) were consistently good to excellent. Highest correlation was achieved by combining the coronal frontal horn measurements to a frontal horn product (=short axis*long axis; right 0.95 , left 0.92 ). The thalamusoccipital horn distance showed the poorest correlation (right 0.53 , left 0.37 ).

Conclusions: We have been able to show that 2D ultrasound measurements of the frontal horns and midbody have a very good correlation to the total ventricular volume measured by MRI. This study underlines the reliability and value of neonatal cranial ultrasound.

\section{THE EFFECT OF CHANGES IN CEREBRAL PERFUSION PRESSURE (CPP) ON AMPLITUDE INTEGRATED EEG (AEEG) IN A NEONATAL PIGLET MODEL OF INTRAVENTRICULAR HAEMORRHAGE}

\author{
K Aquilina $^{1,2}$, A M Tucker ${ }^{1}$, C E Hobbs ${ }^{1}$, M Thoresen ${ }^{1}$ \\ ${ }^{l}$ Clinical Science at South Bristol, St Michael's Hospital, \\ Bristol, UK, ${ }^{2}$ Department of Neurosurgery, Frenchay \\ Hospital, Bristol, UK
}

Background and Aims: aEEG is an important tool in the evaluation of neonatal brain activity and prediction of outcome. Changes in aEEG related to changes in CPP were studied.

Methods: Five piglets $(<24$ hours old $)$ were anaesthetised. Four mls of high hematocrit blood were injected into the lateral ventricles over two hours (1), under continuous intracranial pressure (ICP), invasive mean arterial pressure (MAP) and aEEG monitoring. Post-injection ultrasonography confirmed the intraventricular location of the injected blood.

Results: The median of the maximal ICP was $25 \mathrm{mmHg}$ at 80-100 minutes after the commencement of injection. The median of the lowest CPP was $18 \mathrm{mmHg}$ (range $8-40 \mathrm{mmHg}$ ) and occurred at the time of maximal ICP. Maximal drop in aEEG baseline occurred during the period of lowest CPP in four piglets. Recovery occurred within 15 minutes of return to baseline CPP (Figure 1).

Conclusions: Reduction in aEEG upper and lower voltage margin correlates with reduced CPP in this model 1). aEEG may be useful as a physiological marker of poor brain perfusion.

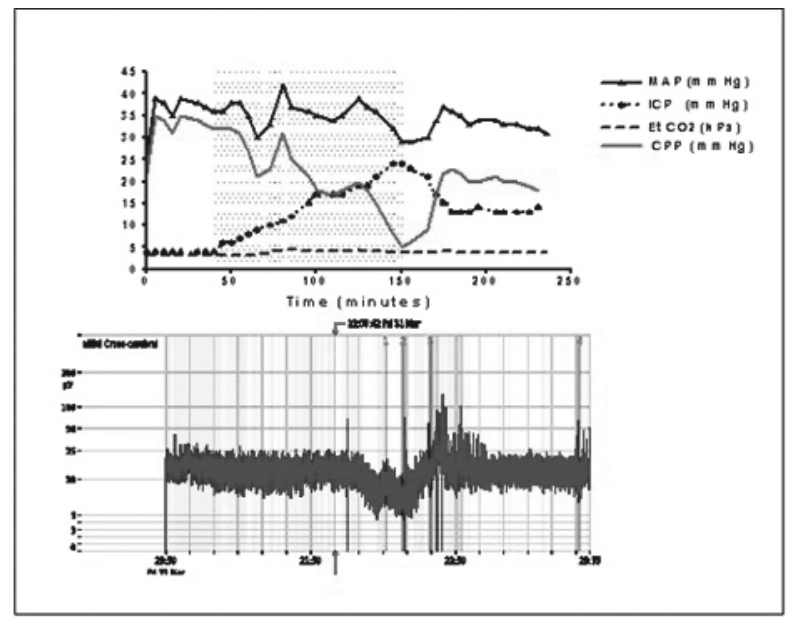

Figure 1. Data from invasive monitoring correlated with aEEG changes during a typical $\mathrm{TVH}$ injection procedure in a neonatal piglet. aEEG and CPP are closely correlation.

Reference

1. Thoresen M, Hobbs C, Aquilina K, Cherian S, Whitel aw A. A long-term survival, piglet model of posthemorrhagic ventricul ar dilatation. Paediatric Research, April, 2006 (abstract). 


\section{THE EFFECT OF INTRAVENTRICULAR HEMORRHAGE ON SIMULTANEOUSLY RECORDED TWO-CHANNEL AEEG IN NEWBORN RAT PUPS}

\section{A M Tucker, C E Hobbs, K Aquilina, M Thoresen}

\section{Dept of Child Health (CSSB), University of Bristol, UK}

Background and Aims: Intraventricular hemorrhage (IVH) is associated with a high incidence of disability or mortality, particularly in cases involving posthaemorrhagic ventricular dilatation (hydrocephalus). Amplitude-integrated EEG (aEEG) allows continuous monitoring of cerebral activity and is a strong predictor of neurodevelopmental outcome. Analysis of simultaneously-recorded aEEG during and after IVH may elucidate the effect of IVH on brain function, including the occurrence of seizures.

Methods: Using our IVH model (Cherian, 2003), 80- $\mu \mathrm{L}$ of citrated rat blood was infused into alternate lateral ventricles of 12, anaesthetized Wistar rat pups on postnatal day (P) 7 and P8. Continuous, two-channel aEEG (BRM2, BrainZ Instruments Ltd, NZ) was recorded from each hemisphere; before (baseline), during and after the infusion of blood, until the needle was removed. The aEEG recording was made on both days of the blood injection (P7 and P8).

Results: During infusion, there was a dramatic depression in both upper and lower voltage margins and a shrinking of overall trace bandwidth representing a nearly isoelectric trace pattern bilaterally. Changes in aEEG background activity were delayed by 3 minutes from the beginning of blood infusion. The effect of IVH on aEEG was more profound on the first day of infusion than on the second.

Conclusions: This study confirms that IVH has a significant effect on overall aEEG background activity, notably upper and lower voltage margins and trace bandwidth, in the newborn rat.

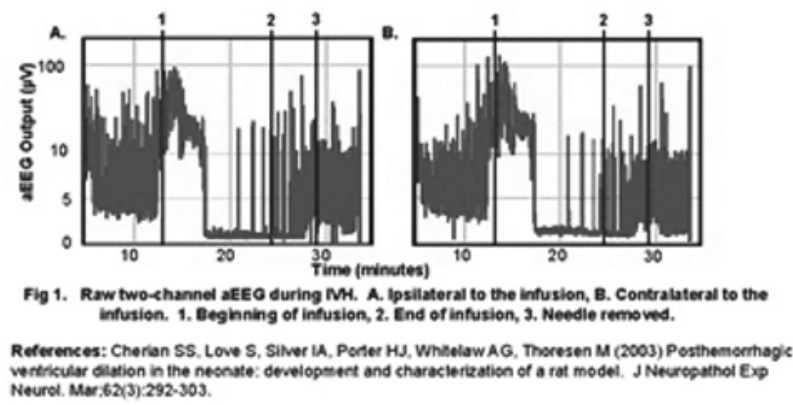

\section{EFFECT OF CHRONIC FETAL HYPOXIA ON CHICKEN EMBRYO SURFACTANT MATURATION}

J V Been, B Zoer, L J Zimmermann, N Kloosterboer, C G Kessels, E Villamor, J F van Iwaarden

Department of Neonatology, Maastricht University Hospital, Maastricht, The Netherlands

Background and Aims: The processes of pulmonary growth and maturation are critical in preparing the fetus for postnatal life. Previous studies suggest a modulatory effect of prenatal hypoxia on lung development. We hypothesise that normal surfactant development is altered by chronic prenatal hypoxia in the chicken fetus.
Methods: White Leghorn fertilised eggs were incubated at either room air or $15 \%$ hypoxia from day 6 of the 21 day incubation period. Normoxic and hypoxic chick embryos were sacrificed at either day $16(\mathrm{n}=15$ for each group) or day 19 ( $\mathrm{n}=15$ for each group). Lungs were excised and total protein was measured in the homogenate. After butanol extraction, total phospholipids (PL) and disaturated phospholipids (DSPL) were measured. Surfactant protein B (SP-B) was measured by ELISA.

Results: The main results are summarised in the table. Chronic fetal hypoxia was associated with lower birth weight. Lung PL and DSPL, mainly derived from surfactant, were significantly decreased at day 19. Lung SP-B content was lower after hypoxia, however no effect was seen after correction for either total protein or lung weight.

Conclusions: Chronic fetal hypoxia was associated with a decrease in whole lung surfactant content in the chick embryo on day 19. Further research is aimed at investigating the underlying mechanisms and determining the effect of variation in exposure time and severity of hypoxia.

\begin{tabular}{l|cc|cc} 
& \multicolumn{2}{|c|}{ day 16} & \multicolumn{2}{c}{ day 19} \\
& normoxia & hypoxia & normoxia & hypoxia \\
embryonic wt ( $\mathrm{g}$ ) & $17.0 \pm 1.0$ & $16.3 \pm 0.9$ & $29.9 \pm 2.3$ & $25.8 \pm 1.2^{* *}$ \\
lung wt (mg) & $80 \pm 14$ & $74 \pm 13$ & $137 \pm 18$ & $123 \pm 22$ \\
lung wt (\%o embryo wt) & $4.7 \pm 0.8$ & $4.6 \pm 0.8$ & $4.6 \pm 0.5$ & $4.8 \pm 0.9$ \\
total protein (mg/g lung) & $28.5 \pm 4.6$ & $30.0 \pm 3.8$ & $34.0 \pm 2.9$ & $35.7 \pm 8.3$ \\
total PL (mg/g lung) & $7.3 \pm 2.0$ & $6.2 \pm 0.9$ & $8.2 \pm 0.8$ & $7.0 \pm 0.7^{* *}$ \\
total DSPL (mg/glung) & $0.8 \pm 0.1$ & $0.8 \pm 0.1$ & $2.7 \pm 0.5$ & $2.2 \pm 0.4^{* *}$ \\
total SP-B (ug/lung) & $0.54 \pm 0.11$ & $0.47 \pm 0.06$ & $5.58 \pm 1.10$ & $4.66 \pm 0.71^{*}$ \\
& & & $* \mathrm{p}<0.05 ; * * 0<0.01$
\end{tabular}

\section{MATERNAL PULSE OXIMETRY PERFUSION INDEX AND ADVERSE NEONATAL RESPIRATORY OUTCOME AFTER ELECTIVE CAESAREAN DELIVERY}

\author{
C De Felice ${ }^{1}$, L Leoni ${ }^{2}$, E Tommasini ${ }^{2}$, O Pede ${ }^{2}$, \\ G Tonni ${ }^{3}$, A Del Vecchio ${ }^{4}$, G Ladisa $^{4}$, G Latini ${ }^{4,5}$ \\ ${ }^{1}$ Neonatal Intensive Care Unit, Azienda Ospedaliera \\ Universitaria Senese, Policlinico "Le Scotte", Siena, \\ Italy, ${ }^{2}$ Department of Intensive Care Unit, Azienda \\ Ospedaliera Universitaria Senese, Policlinico "Le \\ Scotte”, Siena, Italy, ${ }^{3}$ Division of Obstetrics, Guastalla \\ Hospital, Reggio Emilia, Italy, ${ }^{4}$ Division of \\ Neonatology, Perrino Hospital, Brindisi, Italy \\ ${ }^{5}$ Clinical Physiology Institute, National Research \\ Council of Italy (IFC-CNR), Lecce, Italy
}

Background and Aims: No reliable maternal predictors of adverse neonatal outcome at ELCS are known to date.

Methods: The value of maternal pulse oximetry [perfusion index (PI), pulse rate (PR), and oximetry (SpO2)] monitoring during the elective cesarean section (ELCS) in identifying newborns with adverse respiratory outcome was assessed in a prospective casecontrol study. ELCS was sub-divided into 9 phases. Analysis of pulse oximetry-derived signals, systolic (sys-BP), diastolic (dia-BP) and differential (diff-BP) blood pressure were recorded during the ELCS in 44 healthy mothers as a function of neonatal morbidity. 
Maternal arterial and newborn cord blood gas analysis and placental histology were performed.

Results: Significant variations in PI $(\mathrm{p}<0,0001)$, PR $(\mathrm{p}<0,0001), \mathrm{SpO} 2(\mathrm{p}<0,0001)$, sys BP $(\mathrm{p}<0,001)$ and dia BP $(p<0,001)$ were observed. $13.6 \%$ of newborns showed early postnatal complications and increased illness severity scores $(p<0.0001)$. In $4 / 6$ cases a subclinical chorioamnionitis was evidenced. Their mothers showed significantly decreased PI $(p<0.0001)$, and $\mathrm{SpO} 2(\mathrm{p}<0.0001)$, increased PR $(\mathrm{p}<0.0001)$, with increased sys-BP $(p=0.0039)$ and $\operatorname{diaBP}(\mathrm{p}=0.0331)$ as compared to controls. A maternal PI $\leq 2.15$ during the pre-anesthesia phase of the ELCS showed N\% 94.9\% sensitivity (95\% CI: $82.6-99.2)$ and $70.2 \%$ specificity (95\% CI: 60.4-78.8) in identifying cases from controls $(\mathrm{AUC}=0.881$, SE: $0.028 ; 95 \%$ CI: 0.816-0.929; $\mathrm{p}$ $<0.0001)$.

Conclusions: Our findings indicate that a decreased PI value occurring in the pre-anesthesiology phase of the ELCS is a maternal predictor of increased neonatal morbidity, and is significantly related to subclinical placental inflammatory disease. These observations suggest the feasibility of a noninvasive pulse-oximeter prenatal screening of high risk newborns in ELCS.

\section{PULMONARY ENDOSTATIN PERINATALLY AND IN LUNG INJURY OF THE NEWBORN INFANT}

\author{
J Janér ${ }^{1}$, S Andersson ${ }^{1}$, C Haglund ${ }^{2}$, P Lassus ${ }^{3}$ \\ ${ }^{1}$ Hospital for Children and Adolescents, University of \\ Helsinki, Helsinki, Finland, ${ }^{2}$ Department of Surgery, \\ University of Helsinki, Helsinki, Finland, ${ }^{3}$ Department \\ of Plastic Surgery, University of Helsinki, Helsinki, \\ Finland
}

Background and Aims: Endostatin (ES) is a potent angiogenesis inhibitor. Angiogenesis is central for the development of the human lung. The role of ES in the development of the human lung and connection to chronic lung disease remains unclear. We set out to study the role of ES in the developing human lung, and in acute and chronic lung injury in the preterm infant.

Methods: 9 fetuses, 14 control neonates without primary lung disease, 14 preterm infants with respiratory distress syndrome, and 8 infants with bronchopulmonary dysplasia were included in the immunohistochemistry study. Tracheal aspirate fluid samples of intubated very low birth weight infants during postnatal weeks 1-5 were analyzed with ELISA.

Results: Endothelial cell staining was positive for ES in all 45 samples. Staining of epithelial cells (cuboidal, bronchiolar and alveolar) was seen mostly in fetuses as well as late RDS and BPD cases. Staining in alveolar macrophages was most abundant in late RDS and BPD cases. ES was expressed consistently in tracheal aspirate fluid, being highest during the first postnatal day. Higher ES concentrations correlated with parameters reflecting lower lung maturity.

Conclusions: The pattern of pulmonary ES protein expression in immunohistochemistry and consistent ES protein appearance in tracheal aspirate fluid in human preterm infants indicate a role in the physiological development of the lung. An increase in ES expression postnatally could indicate a disruption in the normal development of the lung and contribute to chronic lung injury.

\section{ALGORITHM FOR ECMO (EXTRACORPORAL MEMBRANE OXYGENATION) IN CONGENITAL DIAPHRAGMATIC HERNIA (CDH) - CHANGES OVER 15 YEARS}

S T Hien ${ }^{1}$, F Loersch ${ }^{1}$, J Sartoris ${ }^{1}$, S Demirakca ${ }^{1}$, V Varnholt ${ }^{1}$, S Loff $^{2}$, T Schaible $^{1}$

${ }^{I}$ Neonatal Intensive Care Unit, Pediatric Hospital, University of Mannheim, Mannheim, Germany ${ }^{2}$ Pediatric Surgery Unit, University of Mannheim, Mannheim, Germany

Background and Aims: ECMO is a lifesaving intervention, its role on $\mathrm{CDH}$ survival is still controversial. We examined the relationship between ECMO and its effect on CDH survival in our NICU over 15 years (1991-2005).

Methods: In 15 years, our NICU received 280 patients with $\mathrm{CDH}$. For evaluation and interpretation of different management protocols, this period was divided into 3 periods: The unit received 38 patients with $\mathrm{CDH}$ (19 ECMO) in 1991-1995, 58 (29) in 1996-2000 and 184 (88) in 2001-2005.

Results: Antenatal Diagnosis was known in 11 cases $(29 \%)$ in the first and $133(72 \%)$ in the last period. Number of patients requiring ECMO decreased during these periods $(55 \%, 50 \%, 48 \%)$. Concerning the last hundred patients, rate of ECMO felt to $35 \%$. Survival after ECMO changed from $34 \%$ to $76 \%$ to $64 \%$. Overall mortality in $\mathrm{CDH}$ was reduced from $50 \%$ to $24 \%$ to $20 \%$. Most patients who died in last 5 years didn't get surgery because of evidence of poor survival according to selection criteria and management protocol.

Conclusions: ECMO may be used to overwhelm associated pulmonary hypertension in $\mathrm{CDH}$ and seems to have an impact on improved survival. The study underlines importance of referral to specialized units to develop experience and to standardize treatment algorithms even for patients with poor prognosis. Although $50 \%$ of $\mathrm{CDH}$ patients required ECMO during the different time periods, overall-mortality and mortality under ECMO was reduced dramatically over time. Improvement of selection-criteria to estimate perinatale risk and further outcome analysis is needed to optimize the use of ECMO in CDH.

\section{DIAGNOSTIC CRITERIA AND MOLECULAR MECHANISMS OF ABCA3 DEFICIENCY}

O Danhaive ${ }^{1,2}$, D Peca $^{2}, \mathrm{~S}$ Petrini ${ }^{3}, \mathrm{R}$ Boldrini ${ }^{4}$, $\mathrm{J} \mathrm{Rechichi}^{1}$, F Savignoni ${ }^{1}$, A Gallo ${ }^{5}, \mathrm{C} \mathrm{Corchia}^{1}$

${ }^{I}$ Division of Neonatology, Bambino Gesù Children's Hospital, Rome, Italy, ${ }^{2}$ Laboratory of Neonatal Biology, Bambino Gesù Children's Hospital, Rome, Italy, ${ }^{3}$ Division of Molecular Medicine, Bambino Gesù Children's Hospital, Rome, Italy, ${ }^{4}$ Division of Clinical Pathology, Bambino Gesù Children's Hospital, Rome, Italy, ${ }^{5}$ Laboratory of RNA Editing, Bambino Gesù Children's Hospital, Rome, Italy 
Background and Aims: Mutations in the $\mathrm{ABCA} 3$ gene are associated with neonatal and pediatric lung disease. However, clinical and histological phenotypes vary widely. Our aim was to identify diagnostic criteria for ABCA3 deficiency.

Methods: 6 neonates with progressive unexplained respiratory failure underwent lung biopsy. Standard staining and immunohistochemestry for $\mathrm{SpB}$, proSpB, proSpC were performed on formalin-fixed/paraffinembedded sections. Electron microscopy was done with lead citrate/uranyl acetate contrast and after immunogold staining. $\mathrm{ABCA} 3, \mathrm{SpB}$ and $\mathrm{SpC}$ coding exons were sequenced on PCR-amplified genomic DNA from patients' leukocytes; when possible, gene transcription (mRNA) was measured by real-time RTPCR on lung tissue and protein expression by immunofluorescence on frozen lung sections.

Results: None carried $\mathrm{SpB} / \mathrm{SpC}$ mutations. Case 1 carried a homozygote frameshift $\mathrm{ABCA} 3$ mutation, and had no ABCA3 expression. Cases 2, 3 and 4 carried double, bi-allelic heterozygote $\mathrm{ABCA} 3$ mutations. ABCA3 expression was normal on case 2, present but mislocated on case 3 and markedly decreased on case 4 , where mRNA was also reduced. In case 5 , in whom a single $\mathrm{ABCA} 3$ mutation was found, both $\mathrm{ABCA} 3$ mRNA and protein were increased; proSpB was normal, but mature $\mathrm{SpB}$ was decreased. In case 6 , despite no ABCA3 mutations could be found and mRNA was twofold increased, there was a marked decrease in protein expression. In all patients, lamellar bodies were smaller than normal, almost completely deficient in pseudomyelin structures (intracellular surfactant), most containing a dense core.

Conclusions: Although mutational pattern and molecular mechanisms are various, including trancription abortion (1) or decrease (4), translational (6), trafficking (3) and/or functional $(2,5)$ defects, ABCA3 is characterized by typical lamellar body alterations.

\section{A RISK INDEX TO PREDICT THE RDS OCCURRENCE IN NEONATES FROM MOTHERS WITH INTRAHEPATIC CHOLESTASIS OF PREGNANCY}

\author{
D De Luca ${ }^{1}, \mathrm{M}_{\text {Marras }}{ }^{1}, \mathrm{G}_{\text {Barbato }}{ }^{1}$, T Bernardini ${ }^{2}$, \\ C Romagnoli ${ }^{1}$, E Zecca ${ }^{1}$
}

${ }^{l}$ NICU, Division of Neonatology, Department of Pediatrics, Catholic University of The Sacred Heart, Rome, Italy, ${ }^{2}$ Department of Obstetrics and Gynaecology, Catholic University of The Sacred Heart, Rome, Italy

Background and Aims: Intrahepatic cholestasis of pregnancy (ICP) has an incidence ranging from $0.1 \%$ to $1.5 \%$ of pregnancies in Europe. We recently demonstrated that ICP is significantly associated with the occurrence of RDS in the newborn but we failed to find a direct correlation between maternal or neonatal BA levels and the RDS occurrence. We looked for a scoring system able to predict the occurrence of neonatal RDS in infants from ICP mothers.

Methods: We reviewed our database about 70 pregnancies complicated by ICP from 2000 to 2003 and we elaborated a risk index which includes factors significantly related to the RDS occurrence in the multivariate analysis: exposure time to abnormal maternal BA levels (ET), maternal BA levels at delivery and gestational age $(\mathrm{GA})$. RDS risk index $=\mathrm{BA}(\mu \mathrm{mol} / \mathrm{l})$ $\mathrm{x}$ ET (days) / GA (days). The risk index was then prospectively verified in 30 neonates born from ICP pregnancies in 2004-2005.

Results: ROC analysis showed 9 to be the cut-off value with the highest sensitivity and specificity (AUC $=0.75$; $\mathrm{p}=0.004)$. With a mean RDS incidence of $28 \%$ in newborns from ICP pregnancies, having a positive score increases this risk to $62 \%$ while a negative score decreases it to $5 \%$ in the validation cohort (sensitivity $80 \%$; specificity $87.5 \%$ ).

Conclusions: Perinatal management of ICP pregnancies is still questioned because mechanisms of foetal morbidity are unknown and perinatal complications are not easily predicted. Our risk index is useful to identify those infants deserving special nursery surveillance for RDS occurrence.

\section{HIGHER IL-10 IN CORD BLOOD OF PREMATURE INFANTS WITH RDS BUT NOT BPD}

J Y Kim ${ }^{1}$, J Y Park ${ }^{1}$, S J Cho ${ }^{1}$, Y J Kim², E A Park ${ }^{1}$

${ }^{1}$ Department of Pediatrics, Ewha Womans University College of Medicine, Seoul, South Korea, ${ }^{2}$ Department of Obstetrics, Ewha Womans University College of Medicine, Seoul, South Korea

Background and Aims: RDS and BPD are characterized by inflammation, destruction and repair in the premature lung. We hypothesized that the cord blood levels of anti-inflammatory IL-10, proinflammatory IL-12, and the balance between the two cytokines related to Th1 response and IFN-gamma production would be useful in predicting the occurrence of RDS and BPD in premature babies.

Methods: Cord blood samples from 56 premature infants born at Ewha Womans University Mok Dong Hospital from January 2003 to June 2005 were studied after prior parental consent at mid pregnancy. Cord blood of infants with maternal history of chorioamnionitis, maternal infections, and major congenital anomalies were excluded. The cord blood were stored in -70 and IL-10 and IL-12 levels were measured with ELISA. A retrospective study of the medical records was performed for clinical data.

Results: The mean gestational age of the infants was $30.5 \pm 2.5$ weeks. Thirty- two infants $(57.1 \%)$ were diagnosed with RDS and 18 infants (32.1\%) were diagnosed with BPD. Higher IL-10 levels were demonstrated in the RDS group. $(3.03 \pm 4.79 \mathrm{pg} / \mathrm{ml}$ vs. $1.02 \pm 0.90 \mathrm{pg} / \mathrm{ml}: \mathrm{p}<0.05)$. No significant difference were found in IL-12 and IL-12/IL-10 ratios in the RDS and non-RDS group. IL-10, IL-12 and IL-12/IL-10 ratio did not differ in the BPD and non-BPD group.

Conclusions: High IL-10 levels in premature infants with RDS suggests that inflammation may be an important component in the pathogenesis of RDS. Whereas the pathogenesis of BPD involves more 
complicated postnatal factors and cannot be explained solely via cord blood cytokine levels.

\section{CLINICAL SIGNIFICANCE OF PLASMA B-TYPE NATRIURETIC PEPTIDE IN RESPIRATORY DISTRESS SYNDROME OF THE NEONATE}

\author{
G Rocha $^{1}$, F Clemente ${ }^{2}$, T Rodrigues ${ }^{3}$, H Guimarães ${ }^{4}$ \\ ${ }^{I}$ Division of Neonatology, Department of Pediatrics, \\ Hospital de São João, Porto, Portugal, ${ }^{2}$ Division of \\ Neonatology, Department of Pediatrics, Hospital de São \\ João, Porto, Portugal, ${ }^{3}$ Department of Obstetrics and \\ Gynecology, Department of Epidemiology, Faculty of \\ Medicine of Porto University, Porto, Portugal, ${ }^{4}$ Division \\ of Neonatology, Department of Pediatrics, Hospital de \\ São João e Faculty of Medicine of Porto University, \\ Porto, Portugal
}

Background and Aims: Plasma brain-type natriuretic peptide (BNP) is secret by cardiac ventricular myocytes in response to pressure or volume overload. The aim of this study was to determine the relationship between the severity of respiratory distress syndrome (RDS) and plasma levels of NT pro-BNP.

Methods: Plasma levels of NT pro-BNP were measured in premature neonates $(<34$ wks gestation), at 24 hours (day 1), 7, 14 and 21 days of life and in 18 term neonates without RDS and congenital heart disease at 24 hours of life. Levels of NT pro-BNP were compared between premature neonates with and without RDS and term neonates.

Results: 45 premature neonates, F25 / M20, GA 30 (2533) wks, BW 1290 (500-2220) g. RDS occurred in 21 (47\%) neonates. Comparative analysis revealed a significant increase in plasma level of NT pro-BNP at 24 hours $(\mathrm{p}=0,001)$ and day $7(\mathrm{p}=0,015)$ of life in patients with RDS. Plasmatic levels of NT pro-BNP at 24 hours of age were significantly increased in patients with severe RDS than in patients with mild RDS $(p<0,001)$ or moderate RDS ( $<<0,002)$, after adjustment for $\mathrm{GA}$ and $\mathrm{BW}$.

Conclusions: Our data demonstrate that plasma levels of NT pro-BNP are directly related to increasing severity of RDS in neonates, and decrease during the neonatal period, suggesting a close relation to the functional impairment of pulmonary hemodynamic changes associated with RDS.

\section{CONGENITAL CYSTIC ADENOMATOID MALFORMATION OF THE LUNG - EXPERIENCE OF FIVE CENTERS}

G Rocha ${ }^{1}$, P C Fernandes ${ }^{2}$, E Proença ${ }^{3}$, C Quintas ${ }^{4}$, T Martins $^{5}$, I Azevedo ${ }^{6}$, H Guimarães ${ }^{7}$

${ }^{I}$ Division of Neonatology, Department of Pediatrics, Hospital de São João, Porto, Portugal, ${ }^{2}$ Service of Neonatal and Pediatric Intensive Care, Hospital Geral de Santo António, Porto, Portugal, ${ }^{3}$ Neonatal Intensive Care Unit, Maternidade Júlio Dinis, Porto, Portugal ${ }^{4}$ Neonatal Intensive Care Unit, Service of Pediatrics, Centro Hospitalar de Vila Nova de Gaia, Porto, Portugal, ${ }^{5}$ Neonatal Intensive Care Unit, Service of Pediatrics, Hospital Pedro Hispâno, Matosinhos, Porto,
Portugal, ${ }^{6}$ Unit of Pulmonology, Department of Pediatrics, Hospital de São João, Faculdade de Medicina da Universidade do Porto, Porto, Portugal ${ }^{7}$ Division of Neonatology, Department of Pediatrics, Hospital de São João, Faculdade de Medicina da Universidade do Porto, Porto, Portugal

Background and Aims: The clinical spectrum of congenital cystic adenomatoid malformation of the lung (CCAML) ranges from severe respiratory distress to asymptomatic lesions. The objective of this study was to review our experience with CCAML.

Methods: A retrospective chart review in five tertiary medical centers in the north of Portugal, between 1999 and 2005.

Results: 15 neonates with CCAML, 9F/6M, BW 3100 (645-3975) g, GA 38 (24-40) wks. Antenatal diagnosis was performed in $11(73 \%)$ cases, at a median age of 22 (19-30) wks. Spontaneous in utero regression of the lesion was observed in 2 cases. Antenatal intervention was performed in 1 fetus (pleurocentesis and thoracoamniotic shunting) with impending hydrops. The lesion was right sided in $6(40 \%)$, left sided in $8(53 \%)$ and bilateral in $1(7 \%)$ case. Six neonates became symptomatic during the neonatal period (respiratory distress) and 1 after the neonatal period (spontaneous pneumothorax). Seven patients underwent thoracotomy and appropriate excisional surgery. Histological examination showed definitive features of CCAML (type $\mathrm{I}=4$; type $\mathrm{II}=1$; type $\mathrm{III}=2$ ). Two neonates deceased (a 24 wks GA with a type III CCAML; a 38 wks GA neonate with a type I CCAML). Eight (53\%) patients are asymptomatic and did not undergo surgery. Conclusions: Antenatally diagnosed CCAML has a good prognosis in the absence of severe fetal distress; treatment of asymptomatic CCAML is controversial; surgery may be advocated because of the low morbidity and the prevention of late complications, as malignancy.

\section{SYSTEMIC HEMODYNAMIC EFFECTS OF THE ASSOCIATION OF INHALED NITRIC OXIDE AND INTRAVENOUS PROSTACYCLIN IN ISOLATED DIAPHRAGMATIC HERNIA}

\section{T Gaillot ${ }^{1,2}$, A Beuchée ${ }^{1,2}$, A Sauret ${ }^{1}$, P Pladys ${ }^{1,2}$, P Bétrémieux ${ }^{1}$ \\ ${ }^{1}$ Rennes Teaching Hospital, Rennes, France, ${ }^{2}$ University of Rennes 1, Rennes, France}

Background and Aims: Inhaled nitric oxide (iNO) is used to treat persistent pulmonary hypertension of newborn (PPHN). The use of intravenous prostacyclin (IVP), another pulmonary vasodilatator, is limited because of systemic hypotensive effects. Our objective was to investigate the hemodynamic dose-related side effects of IVP in prenatally diagnosed isolated congenital diaphragmatic hernia (PDICDH) presenting PPHN despite iNO.

Methods: Data from 16 consecutive neonates with PDICDH admitted between December 1999 and September 2004 were analyzed retrospectively. iNO (20 ppm) was started with mechanical ventilation. Intravenous prostacyclin was secondary infused in case of PPHN despite iNO, suspected from pre and postductal peripheral oxygen saturation difference and 
confirmed by US scan. IVP was adapted from the repeated PPNH evaluation. Systemic hypotension was treated with dopamine or noradrenaline. IVP doses were recorded during the rise of doses. Concomitantly, blood lactate levels, dopamine and noradrenalin doses, hourly trends in blood pressure and urine output were recorded. Statistical analysis used ANOVA tests. Results are presented as median [min-max].

Results: Two babies died at day 1. IVP was started at $2[2-6]$ nanog $/ \mathrm{Kg} / \mathrm{min}$ and increased to 23[4-41] nanog $/ \mathrm{Kg} / \mathrm{min}$ at 29 [7-34] hours. Nine babies received vasoconstrictor therapy (dopamine, $15[10-40] \mathrm{ug} / \mathrm{kg} / \mathrm{min}$ and/or noradrenalin, $0.10[0.06-0.25] \mathrm{ug} / \mathrm{kg} / \mathrm{min}$ ). Low urine output was observed during the first hours. Statistical analysis did not shown any effect of IVP on MAP or urine output. Low lactate levels $(1.8[1.4 ; 2.4])$ suggest the absence of associated shock.

Conclusions: In our experience, the use of IVP in PDICDH presenting despite iNO is safe. Hypotension is frequent but easily controlled with vasoconstrictor therapy.

\section{CAPILLARY PCO2 AS A METHOD OF MONITORING INFANTS WITH CHRONIC LUNG DISEASE (CLD) ON SUPPLEMENTARY HOME OXYGEN}

\author{
K Reeve $^{1}$, S McKenzie ${ }^{2}$, A Shah ${ }^{3}$, S Fang ${ }^{1}$ \\ ${ }^{I}$ Department of Neonatalogy, Homerton University \\ Hospital, London, UK, ${ }^{2}$ Department of Paediatric \\ Respiratory Medicine, Royal London Hospital, London, \\ UK, ${ }^{3}$ Department of Paediatrics, North Middlesex \\ University Hospital, London, UK
}

Background and Aims: The discharge criteria and weaning protocols of CLD babies going home on oxygen vary between neonatal units. If pCO2 is raised, pO2 will be reduced and there will be an increased risk of desaturation. Infants with CLD at Homerton are discharged home with oxygen if their $\mathrm{pCO} 2$ is $>6.0 \mathrm{kPa}$. The aim is to inform a hypothesis that monitoring of capillary pCO2 is an efficient and reliable method to identify CLD infants who need oxygen at home.

Methods: This is a retrospective study of all infants discharged on home oxygen from 2000 to 2004. Infants were followed up at home and capillary blood gases collected at 1 or 2 monthly intervals at clinic visits.

Results: Results are expressed as median (range). 55 infants born at a gestational age of 26 (23-32) weeks and birth weight 900 (618-1530) g were discharged on home oxygen. The pC02 and weight at discharge were $7.2(5-$ $11.4) \mathrm{kPa}$ and 3.3 (2.2-6.1) $\mathrm{kg}$ respectively. The length of hospital stay was 105 (50-222) days. The corrected gestational age when stopping oxygen therapy completely was 56 (45-104) weeks. 20 infants (36\%) had 39 hospital admissions with respiratory illnesses in the year following discharge.

Conclusions: $\mathrm{PCO} 2$ is inversely proportional to $\mathrm{pO} 2$ (alveolar-air equation). Because measurement of pCO2 is less time consuming than 24 hour saturation monitoring it confers a potential financial benefit. Further studies are required to determine how different methods used in assessing infants' oxygen requirements compare in terms of clinical and economic factors.

\section{DO 27 WEEKERS BENEFIT FROM PROPHYLACTIC SURFACTANT? A COMPARISON OF TWO TREATMENT POLICIES}

N Yousef ${ }^{1}$, V Champion $^{1}$, J Patkai $^{2}$, G Dassieu $^{1}$, J H Jarreau ${ }^{2}$

${ }^{I}$ Neonatal Intensive Care Unit, Centre Hospitalier Intercommunal de Creteil, Creteil, France, ${ }^{2}$ Neonatal Intensive Care Unit, Centre Hospitalier Cochin-PortRoyal, Paris, France

Background and Aims: Current recommendations for prophylactic surfactant use for prevention of RDS in ELBW infants do not include recommendations on threshold GAThis study aims to determine the impact of prophylactic versus curative surfactant administration in a population of inborn ELBW infants at $27 \mathrm{wk}$ GA.

Methods: Bi-centric retrospective study from 2 NICUs with comparable recruitment, but different surfactant use policies (prophylactic surfactant $<28$ wk GA for centre $1(\mathrm{C} 1)$ and $<27 \mathrm{wk}$ GA for centre 2 (C2).), comparing results for ELBW for 27 wk GA. ELBW for 26 wk and 28 wk GA were included to assess bias due to inter-hospital variations.

Results: 290 infants were included. Significant differences between $\mathrm{C} 1$ versus $\mathrm{C} 2$ include: medical treatment of PDA $(58 \%$ vs $31 \%, \mathrm{p}<0.01)$, post natal steroids $(27 \%$ vs $0 \%, \mathrm{p}<0.01)$, pulmonary haemorrhage $(2 \%$ vs $10 \%, p<0.01)$, nosocomial septicaemia ( $8 \%$ vs $17 \%, \mathrm{p}<0.05)$, BPD36wk-free survival $(80 \%$ vs $57 \%$, $\mathrm{p}=0.01)$ and length of hospital stay $(82+/-23 \mathrm{~d}$ vs $89+/-$ $28 \mathrm{~d}, \mathrm{p}<0.05)$. There was no significant difference in cerebral ultrasound scan lesions or mortality rates. In the 27wk GA subgroup, intubation rates were comparable $(100 \%$ vs $93 \%, p=0.19)$, but surfactant use was different $(100 \%$ vs $66 \%, \mathrm{p}<0,05)$. RDS was less severe in $\mathrm{C} 1$ at 27wk GA, (but not at 26wk and 28wk GA) (oxygenation index $>10: 8 \%$ vs $24 \%, \mathrm{p}=0,07$; HFV as rescue: $6 \%$ vs $27 \%, \mathrm{p}<0,02)$. BPD 36wk-free survival rate was higher in $\mathrm{C} 1$ ( $83 \%$ vs $59 \%, \mathrm{p}<0.05)$.

Conclusions: Prophylactic surfactant use for $27 \mathrm{wk} \mathrm{GA}$ ELBW does not lead to excessive intubation rates and might reduce the severity of the initial respiratory disease.

\section{SURVIVAL RATE AND PREVALENCE OF BRONCHOPULMONARY DYSPLASIA IN ELBW INFANTS: THE BRINDISI EXPERIENCE}

\author{
G Latini $^{1,2}$, A Del Vecchio ${ }^{1}$, G Presta ${ }^{3}$, G Ladisa ${ }^{1}$, \\ C De Felice ${ }^{4}$ \\ ${ }^{1}$ Division of Neonatology, Perrino Hospital, Brindisi, \\ Italy, ${ }^{2}$ Clinical Physiology Institute (IFC-CNR), \\ National Research Council of Italy, Lecce Section, Italy \\ ${ }^{3}$ Neonatal Intensive Care Unit, Azienda Ospedaliera \\ Cardinal Panico, Tricase, Italy, ${ }^{4}$ Neonatal Intensive \\ Care Unit, Azienda Ospedaliera Universitaria Senese, \\ Siena, Italy
}

Background and Aims: Bronchopulmonary dysplasia (BPD) continues to be one of the most common longterm complications of preterm birth. BPD is currently infrequent in infants with birth weight greater than $1200 \mathrm{~g}$ or $>30$ weeks' gestation against a reported BPD 
prevalence of about 30\% among extremely low birth weight (ELBW) infants, as an increasing number of them survive. Although a new form is emerging, BPD is very often preceded by the use of mechanical ventilation early in life. A very low prevalence of BPD in our neonatal intensive care unit (NICU), commonly using a minimal handling ventilatory approach, has recently been reported. The aim of this study was to compare survival rate and in our ELBW infants population between two different time periods (period 2: last 42 months vs. period 1: July 1, 1986 -June 30, 2002).

Methods: Frequencies of oxygen-dependency $\geq 28$ days (BPD 28-d) and/or oxygen-dependency $\geq 36$ weeks postmenstrual age plus oxygen-dependency $\geq 28$ days (BPD $36 \mathrm{wk}$ ) and survival rates for the selected time periods were obtained.

Results: Survival rate in the latter period was $80.6 \%$ $(50 / 62)$ vs. $45 \%(72 / 160)$ of the previous period $(\mathrm{p}=$ $0.0007)$. The frequency of BPD $28-\mathrm{d}$ was $34 \%(17 / 50)$ as compared to $25 \%(18 / 72)(\mathrm{p}=0.3803)$. Likewise, the frequency of BPD $36 \mathrm{wk}$ was $8 \%(4 / 50)$ vs. $1 / 72$ $(1.38 \%)(\mathrm{P}=0.1577)$.

Conclusions: These findings strongly suggest that an increased survival rate among ELBW infants population does not necessarily negatively reflect on the incidence of BPD in a NICU commonly using a minimal handling approach.

\section{THE CORRELATION BETWEEN THE SEVERITY OF RESPIRATORY DISTRESS SYNDROME AND TRACHEAL PROCALCITONIN LEVELS IN PREMATURE BABIES}

\author{
N Narli ${ }^{1}$, F Mahsereci $^{1}, \mathrm{~T}$ İnal $^{2}, \mathrm{~K}_{\text {Özcan }}{ }^{1}$, \\ F Mahsereci ${ }^{1}$, H Yapıcıoğlu ${ }^{1}$, M Satar ${ }^{1}$ \\ ${ }^{1}$ Div of Neonatology, ${ }^{2}$ Dept of Biochemistry, Çukurova \\ University Faculty of Medicine, Adana, Turkey
}

Background and Aims: We aimed to study the correlation between the severity of Respiratory Distress Syndrome (RDS) and tracheal procalcitonin levels in premature babies.

Methods: 60 premature babies with RDS with a mean gestational age of 29,5 $\pm 1,8$ (26-32) weeks and mean birth weight of $1017,8 \pm 168,3(650-1300) \mathrm{g}$ were included in the study. Tracheal aspirates were collected on days $1,3,5,7,14,21$ and 28 if the babies were still on ventilator, and procalcitonin levels were studied.

Results: Procalcitonin levels were highest on day 5 in all patients. Although there was a positive correlation between Oxygen index (OI) and alveolar-arterial O2 (AaDO2) levels and procalcitonin; procalcitonin levels were not statistically different in patients who developed chronic lung disease.

Conclusions: Correlation between $\mathrm{OI} / \mathrm{AaDO} 2$ and procalcitonin may suggest procalcitonin level monitorization in babies with RDS.

\section{USE OF SILDENAFIL IN THREE CASES OF NEONATAL PULMONARY HYPERTENSION}

G Cavallaro, C Bottura, E Cerini, L Andaloro, R Astolfi, G Compagnoni
Neonatal Intensive Care Unit, 'Carlo Poma' Hospital, Mantua, Italy

Background and Aims: The neonatal pulmonary hypertension (PPHN) (1,9\% alive births) is characterized by persistent high pulmonary vascular resistances. Therapy can be based on the use of selective vasodilatators like epoprostenol and inhaled nitric oxide (iNO), expensive, not so easy to use and toxic. Sildenafil, vasodilatator developed for the treatment of the erectile dysfunction, has been recently proposed as alternative or associative therapy, because of its efficacy, safety, low cost, easiness to use.

Methods: Study show the clinical history of three newborns (> 33 weeks G.A.) with acute respiratory distress syndrome (RDS) caused by early onset sepsis and pulmonary hypertension, efficacy treated with Sildenafil.

Results: All cases developed RDS. They started with mechanical conventional ventilation and surfactant therapy without any improvement. Echocardiography showed pulmonary hypertension, right-left shunt in the ductus arteriosus and foramen ovale, for which they start high frequency oscillatory ventilation (HFOV) and therapy with iNO, without clinical improvement. Then they started oral Sildenafil $(2 \mathrm{mg} / \mathrm{Kg} / 6 \mathrm{~h})$ and in the first hour after, a significant and progressive improvement in oxygenation, with reduction of $\mathrm{AaDO} 2$ was observed. The therapy was maintained for 36 - $48 \mathrm{~h}$, with definite resolution.

Conclusions: These cases demonstrate the beneficial pulmonary vasodilatator effect of sildenafil in PPHN after the conventional treatment failure outlines the absence of rebound effect after iNO suspension. Such preliminary data suggest the opportunity of a multicentric, randomized trial in order to demonstrate the real effectiveness and the absence of adverse effects of the sildenafil in the treatment of the neonatale pulmonary hypertension.

\section{ORAL SILDENAFIL (VIAGRA) TREATMENT OF PULMONARY HYPERTENSION IN A PRETERM NEONATE WITH SEVERE IUGR}

K Bohlin, M Bartocci, M Blennow, B Sahlgren

Karolinska Institutet, Division of Pediatrics, Karolinska University Hospital, Stockholm, Sweden

Background and Aims: The phosphodiesterase inhibitor sildenafil is a selective pulmonary vasodilator and a promising new treatment option in pulmonary hypertension. In neonates, sildenafil has mainly been used for refractory cases in addition to inhaled nitric oxide (iNO). Placental insufficiency with intrauterine growth restriction (IUGR) may impair lung development and sildenafil has experimentally been shown to promote alveolar growth and lung angiogenesis.

Methods: We report a case of severe pulmonary hypertension in a preterm infant with pronounced IUGR, gestational age 28 weeks, birth weight $450 \mathrm{~g}$.

Results: The patient presented with only mild respiratory symptoms, but deteriorated on day of life 2 with signs of pulmonary hemorrhage and required mechanical ventilation for 4 days. Echocardiography at 
that time revealed only moderately elevated pulmonary resistance. Over the first 3 weeks of life, pulmonary artery pressure increased until reaching supra-systemic levels of $100 \mathrm{mmHg}$ with signs of right ventricular failure. Despite the severe pulmonary hypertension, the infant was not in respiratory failure and iNO treatment would have required placing the infant on mechanical ventilation. Inhaled iloprost showed only transient effect. Hence, oral sildenafil was administered $(4 \mathrm{mg} / \mathrm{kg} / \mathrm{d})$. There was a rapid treatment response with decreasing pulmonary artery pressure over the first week. Treatment was continued for three months and resulted in a complete recovery and no evidence of rebound pulmonary hypertension.

Conclusions: Oral sildenafil as single therapy was effective in a preterm infant with pulmonary hypertension believed to be mainly due to structural changes in the pulmonary vessels secondary to extreme IUGR.

\section{NEONATAL PERSISTENT PULMONARY HYPERTENSION REFRACTORY TO INHALED NITRIC OXIDE TREATED WITH MILRINONE: PRESENTATION OF A CASE}

C Tzialla, R M Cerbo, G F Perotti, T Figar, C Viganò, M Stronati

\section{Neonatal Intensive Care Unit, IRCCS Policlinico San Matteo, Pavia, Italy}

Background and Aims: Current therapy of persistent pulmonary hypertension of the newborn (PPHN) consists of optimal lung inflation, hemodynamic support and selective vasodilatation with inhaled nitric oxide (NO). However, not all infants will respond. Several animal studies have demonstrated that Milrinone, a phosphodiesterase III inhibitor routinely used to prevent post-operative cardiac failure in infants dilates the pulmonary vessels. We present a case of PPHN treated with a combination of $\mathrm{NO}$ and Milrinone.

Methods: A 38- week-gestation female infant (birth weight: $3680 \mathrm{~g}$.) was born by elective caesarean section. Respiratory failure was developed shortly after birth. Echocardiogram confirmed PPHN: estimated right ventricle systolic pressure of $64 \mathrm{mmHg}$ and bidirectional flow through a large PFO and PDA. Fever, neutropenia, increased C-reactive protein were also developed. Despite full ventilation she remained hypoxemic and NO was administered at maximum dose of $26 \mathrm{ppm}$, but the initial good response did not last long. Milrinone i.v. was started at $0,25 \mathrm{mcg} / \mathrm{kg} / \mathrm{min}$, then increased at $0,75 \mathrm{mcg} / \mathrm{kg} / \mathrm{min}$. Milrinone and NO were discontinued 8 days later and the infant was extubated.

Results: After Milrinone administration, oxygenation improved within few hours, she developed systemic hypotension refractory to dopamine $(15 \mathrm{\gamma} / \mathrm{Kg} / \mathrm{min})$ but responsive to adrenaline $(0,1 \mathrm{mcg} / \mathrm{kg} / \mathrm{min})$. Seizures were developed prior to Milrinone infusion but head ultrasound does not demonstrate signs of cerebral hemorrhage.

Conclusions: PPHN was secondary to sepsis and there was no sustained response to NO. The use of Milrinone has determinate the complete resolution, suggesting, according to the recent data in literature, that may be an adjunctive treatment in the management of PPHN.

\section{CONGENITAL IDIOPATHIC CHYLOTHORAX: REPORT OF TWO CASES}

\author{
A Nika, N Iacovidou, S Mouskou, \\ $\mathrm{H}$ Dellagrammaticas
}

\section{Neonatal Department, Aglaia Kyriakou Childrens Hospital, Athens, Greece}

Background and Aims: Chylothorax is the accumulation of chyle in the pleural space. The congenital idiopathic form is rare, being more common in males.

Methods: Case 1Bilateral chylothorax was diagnosed in a male fetus and pleuroamniotic tubes were inserted at 31 weeks and maintained until birth at $32+2$ weeks gestation. After birth the pleural effusion resolved on the left side within 48 hours and the chest tube was removed. However reappearance of the infusion of increasing volume on the right side required multiple insertions of chest tubes. The course was further complicated by systemic fungal infection requiring removal of the two drains on the right side both of which grew Candida albicans. Repeated thoracocenteses were subsequently performed and a trial of the somatostatin analogue, ocreotide was finally associated with successful resolution of the pleural effusion.

Results: Case 2 Fetal hydrops was diagnosed in a male infant at 32 weeks and in utero effusion punction was performed. Following premature labour the infant was born by caesarean section at 34 weeks gestation. At birth bilateral thoracic drains were inserted. He received parenteral nutrition for 10 days followed by gradual introduction of an MCT diet. He had an uncomplicated hospital stay being extubated on day 5 with complete resolution of the effusions and drain removal on day 10 .

Conclusions: We report two cases of congenital idiopathic chylothorax diagnosed antenatally. Parents were unrelated and caryotype was normal on both cases. Postnatal management was conservative and both made full recovery.

\section{TRACHEAL BRONCHUS SUPPLYING THE RIGHT AND MIDDLE LOBE: A CASE REPORT IN A PREMATURE NEONATE}

A Nika, N Iacovidou, A Alexaki, H Dellagrammaticas

Neonatal Department, Aglaia Kyriakou Childrens Hospital, Athens, Greece

Background and Aims: Tracheal bronchus encompasses a variety of congenital anomalies diagnosed with increasing frequency as a result of refinements of diagnosing imaging and endoscopy. It is a recognized cause of respiratory morbidity in children and association with other structural airway anomalies, cardiac defects, Down's and VACTERL syndrome have been described. We report an apparently unique case of Rt tracheal bronchus supplying the whole right upper and right middle lobe in a premature neonate and its association with vascular anomalies and dysplastic changes in the liver. 
Methods: His hospital course was complicated by long term ventilation following persistent Rt lobe atelectasis and consequently almost massive collapse of the Rt lung. Bronchoscopy and airway imaging confirmed the presence of tracheal stenosis and tracheal bronchus. The infant had a stormy course and died at the age of 4 months.

Results: Autopsy studies revealed an anomalous bronchus arising at an angle of 90 degrees below the stenotic trachea and bifurcating in the Rt upper and Rt middle lobe bronchus. Associated malformations included vascular anomalies, a VSD and histologic changes in the liver resembling haepatoblastoma.

Conclusions: Tracheal bronchus can interfere with routine care of children in NICU and it is important for neonatologists to consider early the potential for tracheal bronchus to cause or be associated with pulmonary problems in complicated cases.

\section{THE EFFECT OF CENTRALISATION ON MORTALITY OF INFANTS BORN VERY PREMATURELY IN FINLAND}

L Rautava $^{1}$, L Lehtonen ${ }^{1}$, M Peltola $^{2}$, E Korvenranta ${ }^{1}$, H Korvenranta $^{3}$, M Linna ${ }^{2}$, M Hallman ${ }^{4}$, S Andersson ${ }^{5}$, M Gissler ${ }^{2}$, J Leipälä ${ }^{6}$, O Tammela ${ }^{7}$, U Häkkinen ${ }^{2}$, For the PERFECT Preterm Infant Study Group ${ }^{8}$

${ }^{1}$ Department of Pediatrics, Turku University Hospital, Turku, Finland, ${ }^{2}$ National Research and Development Centre for Welfare and Health (STAKES), Centre for

Health Economics (CHESS), Helsinki, Finland

${ }^{3}$ Turku University Hospital, Turku, Finland

${ }^{4}$ Department of Pediatrics, Oulu University Hospital, Oulu, Finland, ${ }^{5}$ Department of Pediatrics, Hospital for Children and Adolescents, Helsinki, Finland

${ }^{6}$ Department of Pediatrics, Kuopio University Hospital, Kuopio, Finland, ${ }^{7}$ Department of Pediatrics, Tampere University Hospital, Tampere, Finland, ${ }^{8}$ Finland

Background and Aims: The rapid development of neonatal intensive care may widen the performance differences between centres. We need up-to-date comparison of outcomes on prematurely born infants treated in different hospitals. Our aim was to test the hypothesis that the level of the delivery hospital affects one-year mortality of very preterm infants in Finland. Methods: This Medical Birth Register study included all Finnish delivery hospitals with very preterm births in 2000 through to 2003 . There were 2291 infants born very prematurely (gestational age below 32 weeks or birth weight 1500 grams or less) in 14 level II (central) or 5 level III (university) hospitals during the study period. The main outcome measure was one-year mortality.

Results: The adjusted total one-year mortality and oneyear mortality of live born infants were increased in level II hospitals compared to level III hospitals, OR 3.8 (95\% CI 2.8 to 5.2 ) and OR 2.1 (95\% CI 1.3 to 3.3 ), respectively. The total one-year mortality was increased in very preterm infants born outside office hours, OR 1.8 (CI 1.3 to 2.3 ). Theoretically, if all the very preterm infants would have been born in level III hospitals, 69 out of the 170 infants who died would have survived.
Calculating from the data of live born infants only, 18 out of 45 would have survived.

Conclusions: More efficient centralisation of very preterm deliveries would increase one-year survival of very preterm infants in Finland.

\section{IMPACT OF RESUSCITATION OF LIKELY NON-VIABLE INFANTS: A COST-UTILITY ANALYSIS IN THE UNITED STATES}

J C Partridge ${ }^{1}$, A B Caughey ${ }^{2}$, M D Sendowski ${ }^{1}$, E A Drey ${ }^{2}, S^{2}$ Little ${ }^{2}$, A M Martinez ${ }^{1}$

${ }^{I}$ Department of Pediatrics, University of California San Francisco, San Francisco, CA, USA, ${ }^{2}$ Department of

Obstetrics and Gynecology, University of California San Francisco, San Francisco, CA, USA

Background and Aims: Under current practices of selective resuscitation in the United States, most infants born at 20-24 weeks gestation are not resuscitated. Recently-issued guidelines on enforcement of federal laws defining "born-alive" infants may affect physician resuscitation practices. Under these guidelines, withholding of "appropriate medical care" could be construed as a violation of Child Abuse Prevention Treatment Act or the Emergency Medical Treatment and Labor Act. We wished to examine the theoretical effects of enforcement of this federal legislation by comparing routine resuscitation were this legislation enforced to current selective resuscitation practices.

Methods: We designed a decision analysis model comparing current individualized resuscitation practices to the enforced resuscitating of all potentially-viable infants (20-24 wks GA) whether born after preterm delivery (PTD) or elective termination of pregnancy (TAb). We used a theoretical US-population cohort with primary outcomes as maternal quality-adjusted life years (QALYs), numbers of moderately/severely disabled survivors, and costs.

Results: When all 20-24 wk GA infants are resuscitated, maternal QALYs fell from 23.76 to 23.71 per woman for PTD and from 26.11 to 26.06 per woman for TAb. Costs per pregnancy increased from US\$10,562 to US $\$ 21,402$ for PTD and from US\$1080 to US\$1870 for $\mathrm{TAb}$. In addition, mandated resuscitation would save 49 moderately/severely disabled infants, but only 30 unaffected survivors. These findings were persistently true with sensitivity analyses.

Conclusions: Enforced resuscitation of all neonates 2024 wks GA increases perinatal care costs and worsens maternal outcomes. Compared to selective individualized resuscitation, mandated resuscitation of live-born immature infants would not improve health care costs nor quality of life.

\section{EFFECTS OF ORAL AND VAGINAL INDOMETHACIN ON NO, CYCLIC GMP AND CYCLIC AMP IN A RABBIT MODEL FOR PRETERM BIRTH}

K D Beharry ${ }^{1}$, W Fortson ${ }^{2}$, H D Modanlou ${ }^{1}$

${ }^{1}$ Department of Pediatrics, University of California Irvine, Irvine, CA, USA, ${ }^{2}$ Department of Obstetrics and 
Gynecology, Miller Children's Hospital, Long Beach, CA, USA

Background and Aims: NO maintains uterine quiescence and promotes cervical ripening via cGMP and cAMP. Suppression of cAMP in amniotic fluid is associated with fetal distress. The effects of oral and vaginal indomethacin on NO, cGMP and cAMP were examined in a rabbit model for preterm birth.

Methods: Pregnant rabbits ( $\mathrm{n}=6$ /group) were induced with RU486 prior to administration of oral indomethacin $(\mathrm{OI}, 20 \mathrm{mg})$; oral vehicle $(\mathrm{OV})$; vaginal indomethacin (VI, $20 \mathrm{mg}$ in cocoa butter); or cocoa butter only (placebo, VP) once daily for 2 days. Uterus, cervix, fetal membranes and amniotic fluid were analyzed for $\mathrm{NO}$ metabolites (NOx), cGMP and cAMP levels.

Results: VI delayed delivery $>72$ hours in $100 \%$ of the rabbits. VI significantly increased NOx levels in the uterus and fetal membranes ( $\mathrm{p}<0.01$ vs VP), cGMP in the fetal membranes ( $p<0.01$ vs OV and OI), and cAMP in the amniotic fluid ( $<<0.05$ vs VP). OI increased NOx in the amniotic fluid $(\mathrm{p}<0.05$ vs OV), cGMP in the cervix $(p<0.05$ vs OV), and cAMP in the uterus $(p<0.05$ vs OV and VI). Conversely, OI suppressed cAMP in the amniotic fluid ( $\mathrm{p}<0.01$ vs. OV and VI).

Conclusions: The effects of VI on NOx, cGMP and cAMP suggest a mechanism in part, for its tocolytic effects. Suppression of amniotic fluid cAMP with OI may represent elevated vasoconstrictor prostaglandins and fetal distress. We propose that VI is as effective as oral for prolonging gestation and may be considered as an alternate method for tocolysis.

\section{INCREASED SERUM MONO-ETHYLHEXYL PHTHALATE CONCENTRATIONS IN WOMEN WITH MISCARRIAGES}

\section{G Latini $^{1.3}$, S Luisi ${ }^{3}$, C De Felice ${ }^{4}$, A Del Vecchio ${ }^{1}$, F Ruggeri $^{5}$, P Mazzeo $^{5}$, F Petraglia ${ }^{3}$ \\ ${ }^{1}$ Division of Neonatology, Perrino Hospital, Brindisi, Italy, ${ }^{2}$ Clinical Physiology Institute (IFC-CNR) National Research Council of Italy, Lecce Section, Italy ${ }^{3}$ Department of Pediatrics, Obstetrics, and Reproductive Medicine, University of Siena, Italy, ${ }^{4}$ Neonatal Intensive Care Unit, Azienda Ospedaliera Universitaria Senese, Siena, Italy \\ ${ }^{5}$ Department of Chemistry, Chemical Engineering and Materials, University of L'Aquila, L'Aquila, Italy}

Background and Aims: Di-(2-ethylhexyl)-phthalate (DEHP), the most commonly used plasticiser in flexible polyvinylchloride (PVC) formulations, is a widespread ubiquitous environmental contaminant. To date, little information exists on the potential role of exposure to DEHP and its primary active metabolite, monoethylhexyl phthalate (MEHP), on early pregnancy development. The aim of the present study was to evaluate the concentration in maternal circulation of DEHP and/or MEHP in women with high risk of spontaneous miscarriage.

Methods: Three groups of pregnant women were enrolled: Threatened miscarriage $(\mathrm{n}=17)$, complete miscarriage $(n=20)$ and healthy pregnancy $(n=20)$. Serum DEHP and MEHP concentrations were measured by High Performance Liquid Chromatography (HPLC).
Results: Detectable serum MEHP was found in 95\%, $82.3 \%$, and $65 \%$ of the samples from miscarriages, threatened miscarriage and control subjects, respectively, while serum DEHP was detectable in $100 \%, 100 \%$ and $95 \%$, respectively. Detectable serum MEHP and DEHP were found in $95 \%, 82.3 \%$ in patients with miscarriage and threatened miscarriage, respectively, as compared to $60 \%$ of the control subjects. Patients with miscarriages showed significantly increased serum MEHP concentrations (median: $0.445 \mu \mathrm{g} / \mathrm{ml}$ [0.37-0.50], range:0-1.1), as compared to controls $(0.32 \mu \mathrm{g} / \mathrm{ml}$ [0-0.39], range: $0-1.55 ; \mathrm{p}=0.0066$ ), while a non-significant trend was observed for patients with threatened miscarriage. In the presence of serum DEHP and MEHP, patients with miscarriage showed a relative risk of 1.58 folds $(95 \%$ C.I.:1.09 -2.29), as compared to the control women. In contrast, no significant differences were observed for serum DEHP concentrations.

Conclusions: These findings indicate that human phthalate exposure is significantly associated with fetal loss.

\section{LONG-TERM OUTCOME OF VERY LOW BIRTH WEIGHT INFANTS (VLBWI) BORN TO MOTHERS WITH DIABETES}

Y Kono, J Mishina, H Hara, T Takamura, I Sakuma, S Kusuda, H Nishida

\section{Maternal and Perinatal Center, Tokyo Women's Medical University, Tokyo, Japan}

Background and Aims: The long-term outcome of VLBWI that could be complicated by maternal diabetes mellitus (DM) is not known. We investigated influence of maternal DM on the outcome of VLBWI admitted in TWMU Hospital from 1984 to 2003.

Methods: Mortality and neurological impairment (after following for more than $18 \mathrm{mo}$ ) rates were compared in singleton VLBWI $(\mathrm{BW}<1500 \mathrm{~g})$ born to mothers with DM (IDMs, $n=30$, 3 with gestational DM) and without DM (controls, $n=421$, excluding cases with maternal complications before pregnancy). Numbers of infants for long-term follow-up were 18 vs 332 (IDMs vs controls).

Results: There were no differences between IDMs and controls in the mean BW (1075 vs $1033 \mathrm{~g}$ ) or GA (29.8 vs $29.0 \mathrm{wks}$ ). SGA rate was slightly higher in IDMs (47 vs $37 \%$ ). There was no significant difference in longterm mortality rate (10 vs $8.3 \%)$. No significant difference was found in rates of cerebral palsy ( 0 vs $11 \%)$, visual impairment ( 0 vs $1.5 \%$ ), and hearing impairment ( 0 vs $1.2 \%$ ). Mental retardation was found more often in IDMs, but not significantly (16.7 vs $8.1 \%$ ). Cognitive tests at 6 and 9 yrs showed lower tendency in PIQ in IDMs. Overall prevalence categorized as normal in the follow-up was $67 \%$ in IDMs and $72 \%$ in controls.

Conclusions: There was no significant increase in mortality and major impairment rates in VLBWI born to DM mothers compared to controls. Since prematurity seems to affect the outcome of the infants, appropriate control of diabetic mothers preventing premature delivery is important. 


\section{SHORT TERM FOLLOW UP OF CHILDREN FROM PREGNANCIES OBTAINED THROUGH IN VITRO FERTILIZATION}

\author{
B Marinescu ${ }^{1}$, A I Toma ${ }^{2}$, L Dracea ${ }^{1}$, A Vultur ${ }^{1}$ \\ ${ }^{I}$ Department of Human Assisted Reproduction, 'Panait \\ Sarbu' Hospital, Bucharest, Romania, ${ }^{2}$ Department of \\ Neonatology, 'Panait Sarbu' Hospital, Bucharest, \\ Romania
}

Background and Aims: To follow the somatic and neurobehavioral outcome at 2 years of age for children from pregnancies obtained after in vitro fertilization (IVF).

Methods: There were formed 2 groups: One from children born after IVF procedures and one control group from children born after normal pregnancies, all born at term. They were followed at 21-24 months by Bayley Infant Neurodevelopmental Screener (BINS). There were noted: The total score, the risk group, the scores obtained at different items, weight and head circumference. For comparisons between the 2 samples there was used the Chi Square test (statistically significant for $\mathrm{p}<0.05$ ).

Results: There were 30 children in the IVF group and 30 in the control group. There were no significant differences regarding birth weight $(p<0.24)$, gestational age (p,0.97). The BINS scores were all in the low risk zone in both groups. There was no difference from the point of view of the weight percentile (IVF/control) 56 $(+28.1) / 54(+20.24)(\mathrm{p}<0.43)$, head circumference: 56 $(+23.57) / 61(+30.2)(\mathrm{p}<0.54)$. If there are compared in the IVF sample the singletons with the twins $(22 / 8$ cases) there are statistically significant differences between the 2 groups (singletons/twins) total score: $11,18(+1.77) / 10.5(+0.5)(\mathrm{p}<0.03605)$, weight percentile: $66.36(+25.69) / 27.5(+2.88)(\mathrm{p}<0.01501)$, head circumference percentile $65(+21.33) / 33.75$ $(+11.87)(\mathrm{p}<0.04990)$.

Conclusions: The children born at term after IVF are not different from the other children born at term regarding the neurobehavioral and somatic development. In the group of IVF children, the twins have a greater risk for abnormalities in the BINS score and somatic growth than the singletons.

\section{HOME OXYGENOTHERAPY IN CHILDREN WITH BPD: 10 YEARS' EXPERIENCE AT NEONATAL TERTIARY CENTRE IN PLZEN}

\section{J Dort, E Dortova \\ Department of Neonatology, Charles University Hospital, Plzen, Czech Republic}

Background and Aims: To evaluate the contribution of home oxygenotherapy (OT) to the treatment of infants with bronchopulmonary dysplasia (BPD). BPD occurs in a significant proportion of very and extremely low birth weight infants in the Czech Republic as in other developed countries. OT is an important therapeutic tool of their treatment.

Methods: A retrospective cohort study of infants with BPD on home OT. Authors describe the way oxygenotherapy is conducted at home conditions under supervision of skilled Neonatologist.
Results: Since 1996 a group of 21 children received home OT. Mean gestational age was 25.8 wks, mean birth weight 733 grams, average length of home OT was 173 days. 3 infants died during home OT, 2 of them had the new form of BPD.

Conclusions: Main advantages of home OT are: Considerably shorter hospital stay thus eliminating nosocomial infection risk, psychomotor development and mother - child bonding is better at home, and costs are lower. The mobility when using liquid oxygen container is excellent. No serious complications occurred.

\section{OUTCOMES IN MODERATELY PREMATURE INFANTS IN THE NICU}

I Rod, T Farstad

Department of Neonatology, Akershus University Hospital, Nordbyhagen, Norway

Background and Aims: Newborns 30- 34 weeks gestational age (wGA) make up to $20-25 \%$ of all admission to NICU. The aim of present study was to describe the NICU and post discharge course of this group.

Methods: Hospital based prospective study done by chart review and post discharge charts from hospital and outpatient clinic.

Results: During the five years period a cohort of 182 infants born with $30-34 \mathrm{w} \mathrm{GA}$ ( $2.2 \%$ of all inborn) were identified. 90/182 (49\%) were delivered by caesarean section, $92(51 \%)$ were male, $32 \pm 2 \mathrm{wGA}$ (mean $\pm \mathrm{SD}$ ), BW $1761 \pm 410$ g. $91 \%$ were AGA, 2,7\% were SGA and $4,9 \%$ were LGA. Four needed resuscitation with intubations at delivery room. $1 / 3$ were suspected to have sepsis and treated with antibiotics, $70 \%$ needed phototherapy. 12/182 were surfactant treated, $10 \%$ needed mechanical ventilation and 82/182 (45\%) needed NCPAP support. 1/3 were given oxygen for $6.3 \pm 4.6$ days. There was a tendency toward male gender in association with longer need for ventilatory support. Four infants had IVH and to had seizures. Length of stay (LOS) was $33.3 \pm 16.3$ days however 16 (8.8\%) infants stay longer at NICU than 40 wGA. 54 infants were later hospitalised.

Conclusions: Moderately premature infants experience significant morbidity during and after NICU stay.

\begin{tabular}{|l|l|}
\hline Post discharge morbidity & \\
\hline Neurological problems & $6 \%$ \\
\hline Respiratory symptoms & $17 \%$ \\
\hline Infections & $12 \%$ \\
\hline Laryngological problems & $9 \%$ \\
\hline Nutritional problems & $5 \%$ \\
\hline Cumulative morbidity & $32 \%$ \\
\hline
\end{tabular}

\section{EARLY HOME SENDING FOR PREMATURES}

\section{A Kyhnæ}

\section{NICU, Hvidovre Hospital, Hvidovre, Denmark}

Background and Aims: At Hvidovre Hospital we are offering the families the possibility for Early Home Sending (EHS). This means that the parents can take their infant home when he/she no longer needs surveillance, are after $34 \mathrm{GA}$ but still needs feeding help. It gives the parents opportunity to re-establish a normal family life with their infant in well known 
surroundings. Being at EHS means that the family are at home with their infant, getting a visit from a NICU nurse once a week, and goes to the NICU for at control also once a week.

Methods: The parents are being introduced to the EHS in the beginning of the admitting to the NICU, and about 2 weeks before expected EHS we start preparing the parents in going home with their infant. They learn how to tube feed their infant, and how to place the feeding tube. Both parents learn first aid to infants. We have a conversation with the parents regarding their expectations, plans and practical things.

Results: By offering the parents this opportunity for the last period of the infants admittance, we strengthen their development of parents competence, and give them the opportunity to be with their infant day and night.

Conclusions: The attachment between the parents and the infant is strengthening. Better possibilities to establish the lactation. Often the mother gets more milk to her infant, because she can practice skin to skin the whole day.

\section{EARLY OXIDATIVE STRESS IN DOWN'S SYNDROME PREGNANCIES}

\section{S Perrone, M Longini, G Centini, L De Marco, A Brogna, A Plantulli, B Marzocchi, G Buonocore \\ Department of Pediatrics, Obstetrics and Reproductive Medicine, University of Siena, Siena, Italy}

Background and Aims: Individuals with Down syndrome (DS) have signs of fetal growth restriction (FGR) and possible brain damage prior to birth. Increased oxidative stress (OS) in brains of fetuses with DS has been proposed on the basis of evidence of impaired metabolism of oxygen free radicals and enhanced lipid peroxidation in vitro. We recently demonstrated that isoprostanes (IPs), a new marker of free radical-catalyzed lipid peroxidation, are a reliable marker of early OS in amniotic fluid of pregnancies with FGR. We test the hypothesis that oxidative stress occurs early in DS pregnancies.

Methods: Concentrations of IPs were measured by colorimetric enzyme immunoassay in amniotic fluid samples from 56 normal (group I), 23 growth restricted (group II) and 10 DS (group III) fetuses, diagnosed by karyotype analysis of amniotic cells.

Results: Concentrations of IPs were highest in group III (595.15; 542.96-631.64 pg/ml, median; 95\% CI), followed by group II $(155 ; 130.57-172.23 \mathrm{pg} / \mathrm{ml})$ and group I $(67 ; 49.82-98.38 ; \mathrm{p}<0.0001)$. No overlap of values was observed between normal and DS gestations. Concentrations over $400 \mathrm{pg} / \mathrm{ml}$ were only found in pregnancies with DS fetuses.

Conclusions: IP concentrations in amniotic fluid of pregnancies with DS fetuses was greater than in pregnancies with FGR. OS occurs even more in pregnancies with DS fetuses due to an imbalance between oxidant generation and antioxidant capacity. We believe that this study will add important information on the role of OS in the DS. Prenatal antioxidant therapy, if effective, could represent a possible therapeutic strategy to improve the quality of life in the DS population.

\section{POTENTIAL USE OF AUTOLOGOUS CORD BLOOD ERYTHROCYTES FOR EARLY TRANSFUSION NEEDS OF PREMATURE INFANTS}

\author{
J S von Lindern ${ }^{1}$, A Brand ${ }^{2}$, M Jansen ${ }^{1}$, F J Walther ${ }^{1}$

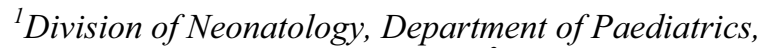 \\ LUMC, Leiden, The Netherlands, ${ }^{2}$ Sanquin Blood Bank, \\ Leiden, The Netherlands
}

Background and Aims: This prospective study investigated whether the odds of receiving a red blood cell transfusion in premature infants can be predicted at birth and for whom of these infants harvesting of cord blood for autologous transfusion within 30 days after birth would be worthwhile. Methods: Characteristics were evaluated from 288 premature infants with a gestational age between 24 and 36 weeks who were admitted to our neonatal centre. In 144 (63\%) of these infants cord blood collection was attempted and the early transfusion needs could be compared with the amount of cord blood available for transfusion. Results: Sixty-nine of 114 (61\%) inborn infants with a gestational age of less than 32 weeks received one or more erythrocyte transfusions. Apgar score at 1 minute of less than 6 and gestational age of less than 32 weeks were independently associated with the chance of receiving a transfusion. In $46 \%$ at least $15 \mathrm{ml}$ of cord blood per $\mathrm{kg}$ of birth weight was collected in $41 \%$ this would have been sufficient to cover their early transfusion needs. Conclusions: The decision to collect cord blood for postnatal transfusion can be made just after labour. The collection of cord blood is most effective and efficient for premature infants between 29 and 31 weeks of gestation. For infants less than 29 weeks of gestation, the technical aspects of umbilical cord blood collection need improvement. This pilot study requires a prospective clinical study to evaluate the proportion of premature infants that can be fully or substantially supported with autologous cord blood.

\section{CORRELATION OF RED CELL VOLUME WITH HEMATOCRIT IN VLBW INFANTS}

J S Mercer ${ }^{1}$, D Erickson-Owens ${ }^{1}$, P McMillan ${ }^{2,3}$, $\mathrm{O}$ Silbert ${ }^{2}, \mathrm{~W} \mathrm{Oh}^{2,} \mathrm{H} \mathrm{Rabe}^{3}$

${ }^{1}$ University of Rhode Island, Kingston, RI, USA

${ }^{2}$ Brown Medical School, Providence, RI, USA

${ }^{3}$ Rhode Island Hospital Core Research Laboratory, Providence, RI, USA, 3 Department of Neonatology, Brighton and Sussex University Hospitals, Brighton, United Kingdom

Background and Aims: The aim of this pilot study was to assess red cell volume (RCV) and to correlate RCV with hematocrit (Hct) in VLBW infants. Evidence suggests that the current practice of Hct to predict RCV is not always an accurate measure of available cells for oxygen delivery to the tissues.

Methods: Eleven Infants (24 - 29.6 weeks gestation, birth weight $830-1,250 \mathrm{gm}$ and were transfused between 7 and 27 days of life) were enrolled in the study with parental permission. Before and after transfusion, $0.2 \mathrm{~mL}$ of blood was withdrawn. Using flow cytometry, the percentage of red blood cells (RBCs) containing 
fetal hemoglobin $(\mathrm{HgF})$ were estimated by staining with a fluorescent antibody to the gamma chains of $\mathrm{HgF}$ after glutaraldehyde fixation and Triton permeabilization of the RBCs in the pre- and post-transfusion samples. The difference between $\mathrm{HgF}$ and $\mathrm{HgA}$ in the post-transfusion sample allows for estimates of the pre-transfusion RCV. Prior validation of this method in preterm infants using the biotin method showed a correlation of 0.99 .

Results: The mean RCV was $21 \pm 4.3, \mathrm{~mL} / \mathrm{kg}$ (SD). The pre-transfusion hematocrit was $30.4+4.2 \%$. The correlation between the pre-transfusion Hct and RCV was 0.7 .

Conclusions: Hematocrit appears to overestimate RCV by $30 \%$. This flow cytometry technique may serve as a useful method for the estimate of RCV in VLBW infants.

\section{USE OF SERIAL BIOCHEMICAL MARKERS IN PRETERM NEONATES TO PREDICT PDA AND SEPSIS}

\author{
A De ${ }^{1}$, D Housley ${ }^{2}$, I Ossuetta ${ }^{1}$, N Smith ${ }^{2}$, \\ D Freedman $^{2}$ \\ ${ }^{I}$ Department of Neonatology, Luton and Dunstable \\ Hospital, Luton, UK, ${ }^{2}$ Department of Chemical \\ Pathology, Luton and Dunstable Hospital, Luton, UK
}

Background and Aims: Patent ductus arteriosus (PDA) and sepsis can both present with non-specific signs like apnea and bradycardia in preterm neonates. Biochemical markers like B-type natriuretic peptide (BNP) is known to have positive correlation with hemodynamically significant PDA, but debate about the threshold value continues. Procalcitonin (PCT) and high-sensitive creactive protein (hsCRP) are sensitive markers of sepsis, but little data on their physiological values exists in preterms. Our study aims at 1) finding the physiological range of BNP, hsCRP and PCT during the first 8 weeks in neonates born at 24-32 weeks gestation and 2) whether serial measurement of BNP, hsCRP and PCT can support or exclude the diagnoses of PDA and sepsis in this group.

Methods: Serial measurement of BNP, hsCRP and PCT are done weekly for first 8 weeks in preterm neonates of 24-32 weeks gestation. Echocardiography done to diagnose / follow up PDA. Blood cultures taken as clinically indicated.

Results: Examples of diagnostic performance (cut-off; specificity; sensitivity; PPV; NPV; LR(+); LR(-)): BNP (110 ng/ml: 96\%; 56\%; 83\%; 86\%; 14; 0.46); hsCRP $(0.5 \mathrm{mg} / \mathrm{L}: 56 \% ; 79 \% ; 61 \% ; 80 \% ; 1.8 ; 2.7)$; PCT $(0.5$ ng/ml: $50 \% ; 88 \% ; 80 \% ; 70 \% ; 4.2 ; 1.8)$. Further details, ROC analysis, performance at various concentrations and analysis of false positive / negative results will be presented.

Conclusions: BNP, hsCRP and PCT in preterms are variable. However, serial monitoring of BNP can confirm persistence or closure of ductus arteriosus. In preterms hsCRP is a better marker of sepsis than CRP. Procalcitonin adds little to hsCRP to diagnose sepsis in this group.
PILOT STUDY USING NIRO-200 TO OBSERVE TISSUE OXYGENATION INDEX IN THE FIRST THREE DAYS OF LIFE IN VERY LOW BIRTH WEIGHT INFANTS

A C Doolan, A R Twomey

National Maternity Hospital, Dublin, Ireland

Background and Aims: Near Infrared Spectroscopy (NIRO) uses light spectroscopy, to provide us with information about oxygenation within the tissues (in this application in the brain). The aims were to measure the Tissue Oxygenation Index (TOI) of Very Low Birth Weight babies (VLBW) over the first three days of life and to determine if the TOI was different in sicker infants.

Methods: A total of 12 VLBW babies were observed on Day 1, 2 and 3. These babies were assigned a CRIB score. The NIRO-200 (Hamamatsu) was used to record the TOI. The average TOI was calculated for each baby. The TOI in babies with a CRIB score of 6 and above was then compared to babies with a CRIB score of 5 and below.

Results: Of the 12 babies included, 8 were male and 4 were female, with an average gestational age of $29+4$ (standard deviation 2.3, CI 1.3) and weight of $1.08 \mathrm{~kg}$ (CI 0.16). The average TOI on Day 1,2 and 3 was $73.07 \%$ (CI 3.63), $70.05 \%$ (CI 4.45) and $75.94 \%$ (4.2) respectively. Infants with a CRIB score of 5 or below had a TOI of $76 \%, 74 \%$ and $79 \%$ in the first three days of life, while more unwell infants had a TOI of $67 \%$ (p 0.007), 63\% (p 0.01) and 68\% (p 0.017).

Conclusions: In this group the TOI remained stable in the first days of life. However infants who were more unwell had significantly lower TOI despite no difference in the oxygen saturations or blood pressure.

\section{THE ROLE OF COMPLEMENT AND CD14 IN MECONIUM-INDUCED CYTOKINE PRODUCTION IN HUMAN WHOLE BLOOD}

\author{
B Salvesen $^{1,2}$, M Fung $^{3}$, O D Saugstad ${ }^{2}, \mathrm{~T} \mathrm{E} \mathrm{Mollnes}^{1}$ \\ ${ }^{1}$ Institute of Immunology, University of Oslo and \\ Rikshospitalet University Hospital, Oslo, Norway \\ ${ }^{2}$ Department of Pediatric Research, University of Oslo \\ and Rikshospitalet University Hospital, Oslo, Norway \\ ${ }^{3}$ Tanox Inc., Houston, TX, USA
}

Background and Aims: Meconium aspiration syndrome (MAS), an important cause of respiratory distress in term newborns, has a complex and not well defined pathophysiology. We have previously shown that meconium is a potent activator of the complement system in vitro. MAS is associated with a systemic inflammatory response reflected by cytokine production in vivo in a newborn pig model. We investigated whether complement contributed to the inflammatory reaction in MAS and whether CD14-dependent signalling was involved in meconium-induced cytokine production.

Methods: Human whole blood and cord blood collected from different donors were incubated with meconium for 4 hours in the prescence or abscence of anti-CD14 and/or monoclonal antibodies blocking complement (anti-factor D and anti-C2). Proinflammatory cytokines 
and the terminal complement complex (TCC) were measured.

Results: Complement inhibition completely blocked meconium-induced TCC formation whereas anti-CD14 had no effect on TCC. IL- 6 and IL-1 beta production was markedly reduced by CD14 inhibition but only slightly by blocking complement. TNF alpha production was markedly inhibited ( $>50 \%)$ by blocking either complement or CD14. Interestingly, the combined inhibition of CD14 and complement completely abolished the production of all three cytokines.

Conclusions: Meconium-induced cytokine production is mediated by both complement and CD14. Combined inhibition of these two effector mechanisms may be an interesting therapeutic approach to reduce the inflammatory reaction in MAS.

\section{MICROPARTICLES AS A INDEX OF PLATELETS ACTIVATION IN NEWBORNS}

A Wasiluk ${ }^{1}$, M Mantur ${ }^{2}, \mathrm{H} \mathrm{Kemona}^{2}$, M Szczepanski ${ }^{1}$, A Butkiewicz ${ }^{2}, \mathrm{~J} \mathrm{Warda}^{1}$

${ }^{1}$ Department of Neonatology, Medical University of Bialystok, Bialystok, Poland, ${ }^{2}$ Department of Clinical

Laboratory Diagnostics, Medical University of Bialystok, Bialystok, Poland

Background and Aims: Microparticles are procoagulant vesicles shed from an activated platelet membrane. They provide a catalytic phospolipid surface for assembly of factors $\mathrm{Xa}, \mathrm{Va}$, and $\mathrm{Ca}+2$ (prothrombinase complex), accelerating blood coagulation. Therefore they play main hemostatic function. We have tested whether there is or not activation of blood platelets in healthy term newborns in umbilical artery expressed by the increase the percentage of microparticles and whether gender affect or not?

Methods: 55 healthy term newborns, 25 girls and 30 boys were introduced to this studies. Microparticles was tested in umbilical artery. Microparticles positively labelled with CD61 were calculated in flow cytometer.

Results: The percentage of microparticles in healthy term newborns was 4,32. Our attention has been directed whether gender affect or not the tested parameter. In female newborns on resting platelets we have noted the percentage of microparticles 4,67 and in male 4,02. One can pay attention for big differences between minimal and maximal values in both genders. The percentage of microparticles in adults is 0,76 .

Conclusions: Our findings demonstrate 5-fold increase of the percentage of microparticles in healthy term newborns in comparison to adults. The activation of platelets measured as percentage of microparticles is higher in female newborns than male newborns. Acknowledgment: This work was supported by a grant P-3-31484 from Medical University, Bialystok, Poland.

\section{TORTICOLLIS IN NEWBORN}

\section{O Mojsova}

Department of Paediatrics, Hospital Veles, Veles, Macedonia
Background and Aims: Presentation of torticolis in newborns during a period of three years conducted in the obstetrics ward, the pediatric ward and the Physical Therapy Department of the Public Health Centre in Veles.

Methods: Presentation of a retrospective study in the period of three years (2003, 2004 and 2005) representing fact about newborns in urban and rural areas according to their sex and nationality and their condition at the age of 7 days 15 days 1 month and 6 months respectively.

Results: Table 1 presents cases of torticollis among the newborns in the above mentioned years. Table 2 shows sex place of living (town or village) and the nationality of newborns. Table 3 shows age groups: Up to the age of 7 days, 14 days, 1 month, 3 months, 6 months and over 6 months.

Conclusions: Complete cooperation between neonatologists pediatricians orthopedic specialists and gynecologists is of utmost importance for early detection and prevention of this illness. More details about this cooperation will be specified in the presentation of this study which is accompanied by pictures displaying the most common characteristics of this illness.

\section{DELAY IN MEASURING OFC IN PRETERM INFANTS}

\author{
E Chakkarapani ${ }^{1}$, D Rogahn ${ }^{2}$
}

${ }^{1}$ Department of Neonatology, Liverpool Womens

Hospital, Liverpool, UK, ${ }^{2}$ Department of Paediatrics, Countess of Chester Hospital, Chester, UK

Background and Aims: Poor head growth in the postnatal period after preterm birth correlates strongly with minor motor deficits. Abnormal individual head circumference measurements may indicate neurological abnormalities or inadequate brain growth. The late intrauterine and early postnatal periods in human development are characterised by high growth velocity, particularly of the brain. To investigate if Occipitofrontal circumference[OFC] is measured at birth in Preterm infants ( $<33$ weeks gestation).

Methods: 50 Preterm infants born less than 33 weeks gestation and admitted to SCBU from 2004 - 2005 were studied. Infants transferred from other units were excluded. Data collected: Gestation, birth weight, if birth OFC was measured/not, day OFC measured, ventilated or Cpap used on day 1. Method: Retrospective analysis of case notes and growth chart.

Results: Mean [s.d] of gestation and birth weight were 29.15 weeks [1.9] and 1319 grams [356.45] respectively. $14[28 \%]$ infants had their birth OFC measured and $36[72 \%]$ did not have their birth OFC measured. Gestational age [G.A] and birth weight [B.W] were negatively correlated with $\mathrm{OFC}$ measurement day. [Pearson correlation for [G.A] $-0.60, p<0.0001] \&$ B.W: $[-0.55, \mathrm{p}<0.0001]$. There is no significant difference in ventilation [Fisher's exact test $\mathrm{p}=0.2$ ] or cpap on day 1 [Fisher's exact test, $\mathrm{p}=0.3$ ] between the babies whose OFC was and wasn't measured at birth.

Conclusions: OFC measurement is delayed in preterm and very low birth weight infants. This group needs 
monitoring of head growth for future neurodevelopment monitoring. Every available opportunity needs to be used for measuring OFC early.

\section{VARIABILITY OF CRIB ON PREDICTING NEONATAL OUTCOMES ACROSS TEN EUROPEAN COHORTS OF VERY PRETERM BABIES. RESULTS FROM THE MOSAIC PROJECT}

D Di Lallo ${ }^{1}$, M R G Carrapato ${ }^{2}$, R Maier ${ }^{3}$, D Milligan $^{4}$, P Pezzotti ${ }^{2}, \mathrm{~J}_{\text {Zeitlin }}{ }^{5}$, E Draper ${ }^{6}$

${ }^{1}$ Agency for Public Health, Rome, Italy, ${ }^{2}$ Hospital de São Sebastião, Porto, Portugal, ${ }^{3}$ Phillips University, Marburg, Germany, ${ }^{4}$ Royal Victoria Infirmary, Newcastle upon Tyne, UK, ${ }^{5}$ INSERM, U149, Paris, France, ${ }^{6}$ University of Leicester, Leicester, UK

Background and Aims: The Clinical Risk Index for Babies (CRIB) was derived and validated almost a decade ago. This analysis describes the predictive ability of CRIB for in-hospital mortality and morbidity in a more recent European cohort of very preterm babies (VPBs).

Methods: The study population consisted of 4,989 VPBs (22-31 wks of gestational age) born in ten European regions during 2003 and admitted to neonatal care. Perinatal information, including CRIB variables and health outcomes until final discharge or death were collected. 4156 babies had complete data for CRIB score $(83.3 \%$ of entire cohort). To evaluate the performance of CRIB score we chose as neonatal outcome in-hospital mortality and in-hospital mortality or morbidity (intraventricular haemorrhage of grade IIIIV and/or periventricular leukomalacia and/or bronchopulmonary dysplasia). To examine the discrimination of the CRIB score, we estimated the Area Under the Receiver Operating Characteristic (AUROC) with $95 \%$ confidence intervals $(95 \% \mathrm{CIs})$.

Results: The median CRIB in the cohort was 2. The estimated AUROC for in-hospital mortality was 0.87 (95\% CIs 0.86-0.88), ranging from 0.77 (95\% CIs $0.74-$ 0.80 ) for France to 0.93 (95\% CIs 0.91-0.96) for Poland. The estimated AUROC for in-hospital mortality/morbidity were always lower compared to estimates for in-hospital mortality $(0.81 ; 95 \%$ CIs 0.80 0.82), ranging from 0.70 (95\% CIs $0.67-0.73$ ) for France to 0.89 (95\% CIs $0.85-0.92$ ) for Poland.

Conclusions: The CRIB performed well in all regions in predicting mortality, but less well for predicting a composite variable combining mortality and morbidity. Further analysis should compare the validity of the CRIB score with other neonatal severity variables.

\section{VERY LOW BIRTH WEIGHT CHILDREN AT FIVE YEARS OF AGE}

C Nascimento, L Mendes, F Torgal, R Gouveia

Department of Child Development, Hospital de Santa Maria, University of Lisbon, Lisbon, Portugal

Background and Aims: To assess psycho-motor development and neuro-behavioural impairment in very low birth weight children (VLBW) followed at the Child
Development Department of the University Hospital of Lisbon.

Methods: The A. analysed 68 clinical records of VLBW children followed at the Child Development Department, between January 1992 and June 2000, for obstetrical and perinatal data. The Griffiths Mental Developmental Scale was used to assess psycho-motor development.

Results: Of the 68 cases, $18 \%$ had a birth weight below $750 \mathrm{gr}, 22 \%$ between $750 \mathrm{gr}$ and $1000 \mathrm{gr}, 32 \%$ between 1000 gr and 1250 gr and $28 \%$ between 1250 gr and 1500 gr. Only $4 \%$ of children were born at term, and $47 \%$ had a gestational age below 29 weeks. Maternal disease was found in $28 \%$ of cases and obstetrical complications occurred in $72 \%$. Foetal disease was found in $16 \%$ of cases. The main perinatal problems were: respiratory distress, septicaemia, high bilirrubin levels, intraventricular haemorrhage and anaemia. At 5 years of age $22 \%$ of children had mental retardation. More cases of mental retardation were found in children with birth weight below 1000 gr. Most children did not have major visual or hearing impairment. Cerebral palsy (all forms) was found in $29 \%$ of cases and autism spectrum disorder in 3\%. Hyperactive and inattentive behaviour was found in $20 \%$.

Conclusions: VLBW children are at a greater risk of developing psycho-motor and neuro-behavioural impairment. It is therefore important that they be followed at a Child Development Clinic, in order to get appropriate intervention and to improve prognosis.

\section{CAN THE CRITERIA FOR SCREENING OF RETINOPATHY OF PREMATURITY (ROP) BE SAFELY REDUCED?}

J U M Termote ${ }^{1}$, C S P M Uiterwaal ${ }^{2}$, $M$ van der Heide-Jalving ${ }^{1}$, M J van Schooneveld ${ }^{3}$

${ }^{I}$ Department of Neonatology, Wilhelmina Children's Hospital, University Medical Centre, Utrecht, The Netherlands, ${ }^{2} J u l i u s$ Centre for Health Sciences and Primary Care, University Medical Centre, Utrecht, The Netherlands, ${ }^{3}$ Department of Ophthalmology, University Medical Centre, Utrecht, The Netherlands

Background and Aims: To evaluate whether a birth weight $(\mathrm{BW})<1250 \mathrm{~g}$ and/or gestational age $(\mathrm{GA})<30$ weeks could provide a safe screening guideline for identification of all infants at risk for threshold ROP in the central Netherlands.

Methods: Data of 521 infants admitted between 2000 and 2005 and completely screened for ROP according to the Dutch national guideline (BW $<1500 \mathrm{~g}$ and/or GA $<32$ weeks or preterms treated $\geq 3$ days with $\mathrm{FiO} 2 \geq$ $0.4)$ were retrospectively studied. Incidence and severity of ROP were analysed as well as risk factors for ROP. Statistics: logistic regression.

Results: Incidence of ROP was $24.9 \%$. Incidence of ROP stage 1 was $17.5 \%$, ROP stage $26.1 \%$ and ROP stage 3,1.3\%. Incidence of infants treated for threshold ROP was $0.8 \%$. Mean GA of infants with ROP stage 3 was $26.9 \pm 0.9$ weeks (range 25.6-28.1 weeks), mean $\mathrm{BW} 830 \pm 214 \mathrm{~g}$ (range 580-1270 g). Independent risk factors for ROP were gestational age, postnatal steroids and patent ductus arteriosus. Using the guideline of GA 
$<30$ weeks and/or BW < 1250 g no infants with ROP stage 3 would have been missed, $21.3 \%$ of the infants of the study group could have been excluded from screening, $6.7 \%$ of the infants with ROP stage 1 would have been missed.

Conclusions: Although GA is a significant risk factor for ROP in our study group, the screening guideline for ROP can be safely lowered to GA $<30$ weeks and/or $\mathrm{BW}<1250 \mathrm{~g}$ in the central Netherlands.

\section{CARE-ASSISTANTS ON THE NICU}

\section{Y Hoekstra, H M Kolenbrander-Buurman}

\section{Erasmus MC-Sophia, Rotterdam, The Netherlands}

Background and Aims: Since 2003 we work in our hospital, the Erasmus MC-Sophia in Rotterdam, on the implementation of the NIDCAP (Newborn Individualized Developmental Care and Assessment Program). This involves a lot of changes in the care of the infant and the involvement of the parents. The care will change from task oriented to patient oriented care.

Methods: To reduce the stress of the infant we wanted to take care with two persons. We prefer to do this with one of the parents. Our NICU is a large unit with a lot of very premature and very sick infants. We often work with less staff. Parents could not always be there because of different reasons (possibilities to stay at the bedside, region and culture). Since two years we work in the Erasmus MC-Sophia with care-assistants. These people (with different background), have had a special individual training. During the care they give support to the baby with containment and consulates the infants. Also for other tasks they could be asked for.

Results: Giving continue support and containment during and after the care reduces the stress of the infant. The baby is more relaxed, has less changes in vital functions and recovers faster after the care giving.

Conclusions: From our experience we can say that the care-assistants have a very important task in supporting the infants, but only when the parents are not able to be there.

\section{TWIN-TWIN TRANSFUSION SYNDROME: A REVIEW}

J Campos, F Duque, P Lapa, R Henriques

Department of Obstetrics and Neonatology, Coimbra's University Hospital, Coimbra, Portugal

Background and Aims: To determine the incidence, complications, management and outcome of infants with twin-twin transfusion syndrome over a period of five years.

Methods: Retrospective review of all infants with twintwin transfusion syndrome born in our department during a period of five years (January 2001 to December 2005). Twin-twin transfusion syndrome was diagnosed in monochorionic twins if one had olygoamnios and was pale and the other had hydramnios and was plethoric with a haemoglobin difference $>5 \mathrm{~g} / \mathrm{dl}$ and/or birth weight differences $>15 \%$.

Results: Sixteen $(2,75 \%)$ of the 582 twin pairs had twin-twin transfusion syndrome. Decompression amniocentesis was performed in 4 cases $(50 \%)$ and 2 (25\%) performed laser of the placental anastomosis. Of the 16 infants, $14(88 \%)$ were girls and $2(12 \%)$ were boys. Their mean birth weight was $1055 \mathrm{~g}$ (range 490 2290 ) and gestation was 28,8 weeks (range 25-36). The mean intrapair haemoglobin difference was $7,2 \mathrm{~g} / \mathrm{dl}$ and the mean intrapair birth weight difference was $20 \%$. Seven (44\%) infants died in utero (five donors and two recipients) and the rest nine (56\%) were admitted to our neonatal unit. Of those admitted, 8 had respiratory distress, 2 had hypotension, 4 had anemia and 5 had hyperbilirrubinemia. Transfontanelar ultrasound revealed one intraventricular hemorrhage (III-IV) which evolution was to hydrocephaly. There was one neonatal death.

Conclusions: Although the incidence of twin-twin transfusion syndrome is low $(2,75 \%)$, it is an important phenomenon in view of its association with morbidity and mortality in the affected twins. Prompt recognition and management of the haemodynamic and haematological problems of infants with this syndrome is essential for a better outcome.

\section{LOW PREPREGNANT BODY MASS INDEX AND BREASTFEEDING PRACTICES OF HEALTHY TERM INFANTS}

G Banderali $^{1}$, I Giulini Neri ${ }^{1}$, V Carmine ${ }^{1}$, G Radaelli ${ }^{2}$, C Agostoni ${ }^{1}$, M Giovannini ${ }^{1}$

${ }^{I}$ Department of Paediatrics, San Paolo Hospital, University of Milan, Milan, Italy, ${ }^{2}$ Unit of Medical Statistics, San Paolo Hospital, University of Milan, Milan, Italy

Background and Aims: The relationship of maternal underweight with breastfeeding has been scantily investigated. The aim of this survey was to examine the relationship of low versus normal prepregnant body mass index (BMI) with breastfeeding practices of healthy infants.

Methods: This prospective observational survey recruited 1272 mother-infant pairs among births occurred at the San Paolo Hospital in Milan, Italy. Infants with birthweight $<2500 \mathrm{~g}$ and/or gestational age $<37$ weeks, and overweight or obese women (BMI $>26$ $\mathrm{kg} / \mathrm{m} 2$ according to the Institute of Medicine) were excluded. Prepregnant BMI was calculated from selfreported weight before pregnancy and height measured at the hospital. Underweight was defined as BMI $<19.8$ $\mathrm{kg} / \mathrm{m} 2$. Women were interviewed via telephone through 12 months postdelivery about breastfeeding. Breastfeeding was classified according to the World Health Organization.

Results: The participation rate was $100 \%$ and $88.9 \%$ at baseline and after 12 months, respectively. A total of $410(32.4 \%)$ mothers was underweight. Initiation of breastfeeding was 97\%. High vs. low maternal education level (odds ratio $[\mathrm{OR}]=1.41$ ), primiparous status $(\mathrm{OR}=1.35)$, caesarian section $(\mathrm{OR}=0.74)$, and birthweight of the infant ( $£$ vs. $>4000 \mathrm{~g}, \mathrm{OR}=1.89$ ) were associated with low BMI. At multiple analyses, no significant difference was found between underweight and normal weight women for initiation or duration of breastfeeding (mean adjusted difference, 95\% 
confidence interval $[95 \% \mathrm{CI}], 0.4[-0.1 ; 0.9]$ months) or exclusive breastfeeding $(0.1[-0.1 ; 0.3]$ months $)$.

Conclusions: Underweight versus normal weight mothers of healthy term infants may be not at increased risk for not initiating or shorter breastfeeding.

\section{HIGH PREVALENCE OF COHEN AMONGST IRISH TRAVELLERS}
A M Murphy ${ }^{1}$, O S Flanagan ${ }^{2}$, K P Dunne ${ }^{2}$, S A Lynch ${ }^{1}$
${ }^{I}$ The National Centre for Medical Genetics, Our Lady's Hospital for Sick Children, Dublin, ${ }^{2}$ Department of Paediatrics, University College Hospital Galway, Galway, Ireland

Background and Aims: Cohen syndrome is a rare autosomal recessive disorder, prevalent in the Finnish and Jewish populations. Mutations in $\mathrm{COH} 1$, a large gene on chromosome 8 produce a complex phenotype which evolves with age. The features are a characteristic facies, non- progressive mental retardation and motor delay, microcephaly, neutropenia and recurrent minor infections in the first 6 years of life, long tapering fingers and toes, prominent central incisors, truncal obesity from mid childhood, a warm and socialable personality and a progressive chorioretinal dystrophy which causes almost total blindness by adulthood. We report our recent finding of 5 cases from three different families from the highly consanguineous Irish Traveller community. We are aware of a sixth case in a UK bases Irish Traveller child.

Methods: Clinical assessment, molecular genetic testing, detailed family pedigrees, estimation of prevalence using Traveller population data from the 2002 Government of Ireland census.

Results: All cases have an identical homozygote c. $4471 \mathrm{G}>\mathrm{T}$ mutation in the $\mathrm{COH} 1$ gene. We estimate a minimum prevalence of Cohen syndrome of 8 per 1000 Irish Traveller children in the County Galway region of the West of Ireland and 0.5 per 1000 in the Republic of Ireland.

Conclusions: A diagnosis of Cohen Syndrome should be considered in any child from this population who presents with developmental delay and microcephaly. Because the mutation in this ethnic group is now known molecular confirmation is easy and pre natal testing is possible.

\section{UNIVERSAL NEONATAL HEARING SCREENING: OUR EXPERIENCE ON 18,774 NEWBORNS}

\author{
B De Capua ${ }^{1}$, D Costantini $^{1}$, C Martufi $^{2}$, C De Felice ${ }^{3}$ \\ ${ }^{1}$ Dipartimento Cefalico e Scheletrico, U.O.S. Audiologia \\ Clinica, ${ }^{2}$ Dipartimento Cefalico e Scheletrico, U.O.C. \\ Otorinolaringoiatria, ${ }^{3}$ D.A.I. Materno-Infantile, U.O.C. \\ Terapia Intensiva Neonatale, A.O.U.S., Siena, Italy
}

Background and Aims: Hearing loss (HL) is likely to be the most common congenital abnormality in humans. As early detection and intervention is critical to prevent the adverse consequences of a delayed diagnosis on speech, language and cognitive development. As 33$50 \%$ of all congenital HLs cannot be detected in a selective hearing risk, use of universal neonatal hearing screening (UNHS) programs is expanding. Aims: We tested the value of an UNHS protocol, based on a Transient Evoked Otoacoustic Emissions (TEOAEs) two-stage strategy.

Methods: TEOAEs (292 DP Echoport OAE Analyzer) were combined to diagnostic ABR evaluation (Amplaid MK12) in newborns at high audiologic risk according to the Joint Committee on Infant Hearing. A total of 18,774 infants were screened (April 1, 1998 - March 27, 2006). Accuracy of the UNHS strategy in predicting congenital HL was evaluated by Receiver Operating Characteristic (ROC) curve analysis.

Results: Prevalence for all HLs in the neonatal period was $1.81 / 1000$ 1.b. $(34 / 18,774)$, with bilateral HL in 1.44/1000 1.b. (27/18,774) [low-risk rate: 0.46/1,000 1.b $(8 / 17,476)$; high risk infants rate: $14.63 / 1,000$ 1.b (19/1298)]. All HL infants were diagnosed $<3$ and received intervention $<6$ months' age. ROC curves results showed $100 \%$ sensitivity (95\% C.I.: $89.0-100)$ and $99.3 \%$ specificity (95\% C.I.: 99.2-99.4) of the TEOAEs two-stage strategy in detecting congenital HLs [area under the ROC curve: 0.997 (95\% C.I.: 0.9950.997)].

Conclusions: (1) The epidemiology of congenital HLs widely justifies UNHS; and (2) a two-stage TEOAEsbased screening for congenital HL is feasible, minimally invasive and accurate in the early detection of congenital HL.

\section{HISTOLOGIC CHORIOAMNIONITIS AND OTITIS MEDIA WITH EFFUSION IN PRETERM INFANTS DURING THE FIRST YEAR OF LIFE}

C De Felice ${ }^{1}$, B De Capua ${ }^{2}$, D Costantini' ${ }^{2}$, C Martufi $^{3}$, P Toti ${ }^{4}$, G Latini ${ }^{5,6}$

${ }^{I}$ Neonatal Intensive Care Unit, D.A.I. Materno-Infantile, Azienda Ospedaliera Universitaria Senese (AOUS), Siena, Italy, ${ }^{2}$ U.O.S. Audiologia Clinica, AOUS, Siena, Italy, ${ }^{3}$ U.O.C. Otorinolaringoiatria, Dipartimento Cefalico e Scheletrico, AOUS, Siena, Italy, ${ }^{4}$ Department of Human Pathology and Oncology, University of Siena, Siena, Italy, ${ }^{5}$ Division of Neonatology, Perrino Hospital, Brindisi, Italy, ${ }^{6}$ Clinical Physiology Institute, National Research Council of Italy (IFC-CNR), Lecce Section, Italy

Background and Aims: Otitis media with effusion (OME) has the highest incidence in children younger than 2 years, and is a leading cause of acquired hearing loss. Cumulating evidence indicates that histological chorioamnionitis (HCA) is a often subclinical cause of preterm birth and neonatal morbidity, linked to a fetal inflammatory response syndrome. Here, we tested the hypothesis of an association between OME during the first year of life and HCA.

Methods: A total of 243, randomly selected, infants born $<37$ weeks' gestation (gestational age of $31.9 \pm 3.3$ weeks; birth weight: $1725 \pm 660 \mathrm{~g}$ ) were examined during the first year of life for the presence of OME [diagnostic criteria: (1) Type B tympanogram; (2) ipsilateral absence of transient evoked otoacoustic emissions responses; and (3) ipsilaterally increased threshold at auditory brain responses]. Placental 
histology was performed for all cases and HCA was defined as $\geq 10$ polymorphonuclear leukocytes /field in 10 nonadjacent 400 -power fields in membranes and/or placental chorionic plate.

Results: $17.28 \%$ (42/243) prevalence of OME, [15.22\% $(37 / 243)=$ bilateral $\quad$ OME] and $23.45 \% \quad(57 / 243)$ prevalence of HCA were observed. OME was detected at a mean age of $6 \pm 3.6$ months (range: $1-12$ ) in $85.96 \%$ (49/57) of infants born with HCA vs. $10.21 \%(19 / 186)$ of those without HCA $(p<0.0001)$. OME was significantly related to HCA (adjusted O.R.: 56.22, 95\% CI: 25.84-122.33, $\mathrm{p}<0.0001)$ and small for gestation birth (adjusted O.R.: 6.95, 95\% CI: 3.09-15.65, $\mathrm{p}<0.0001)$.

Conclusions: HCA is a major risk factor for OME in preterm infants during the first year of life.

\section{MORTALITY, MORBIDITY AND LENGTH OF HOSPITALIZATION IN PREMATURE INFANTS IN THE SHAHIDBEHESHTI HOSPITAL IN KASHAN, IRAN 2001-2002}

\author{
Z Sadat $^{1}$, Z Mossayebi $^{2}$, A Movahhedian ${ }^{2}$, M Barati $^{3}$ \\ ${ }^{1}$ Nursing and Midwifery Faculty, ${ }^{2}$ Department of \\ Pediatrics, ${ }^{3}$ Kashan University of Medical Science, \\ Kashan, Iran
}

Background and Aims: Due to important etiology of mortality in neonatal unit is premature birth and its complication are varied in others society, the present study was performed in order to determined mortality, morbidity and length of hospitalization in premature infants in Shahid Beheshti Hospital in Kashan.

Methods: The records of all premature neonates that admitted into the Neonatal Unit of the University Teaching Hospital Shahid Beheshti from October 2001 to October 2002 were retrospectively analyzed in order to determine the outcome and mortality of premature new borns.

Results: There were 408 neonates admissions in during study that 112 of which was premature. Of the 112 enrolled subjects three records was incomplete and excluded from study. Of 109 children 59 (54.1) were male and 50 subject (45.9) were female. Mean birth weight was $1866 \pm 391 \mathrm{gr}$ and mean gestational age was 33.6 weeks. Common morbidity was sepsis $(80.7 \%)$, neonatal jaundice $(49.5 \%)$, anemia $(52.2 \%)$, respiratory distress syndrome $(40.3 \%)$, hypocalcaemia $(33 \%)$ and hypoglycemia $(25.6 \%)$, asphyxia (16.5\%), pneumothorax (5.6\%), DIC (13.7\%), meconium aspiration $(1.8 \%)$ and convulsion was $(5.5 \%)$. The average length of stay in hospital of the surviving children was 15.7 days. Mortality in premature birth was $28.4 \%$.

Conclusions: It is inconsiderable that $27.5 \%$ of neonates that admitted in unit neonates are premature also mortality and morbidity in this children is high.

\section{EMERGENCY MANAGEMENT OF CARDIAC TAMPONADE AND BILATERAL PLEURAL EFFUSION IN A VLBW INFANT}

C Haass ${ }^{1}$, A Tempera $^{1}$, E Sorrentino ${ }^{1}, \mathrm{C}_{\text {Consigli }}{ }^{1}$, D De Paola ${ }^{2}$, G Calcagni ${ }^{1}$, M Finocchi ${ }^{1}$
${ }^{1}$ Neonatal Intensive Care Unit, Fatebenefratelli S. Pietro Hospital, Rome, Italy, ${ }^{2}$ Department of Cardiology, Fatebenefratelli S. Pietro Hospital, Rome, Italy

Background and Aims: Life-threatening extravasation complications are occasionally associated with peripherally inserted central catheters (PICCs) placement. Uncommonly, severe complications may occur even after several days of correct PICC function.

Methods: Illustrative case.

Results: A 630 g premature newborn (25 weeks gestation) was electively intubated at birth and underwent HFOV for 2 days due to neonatal RDS. On the 3rd day of life a 27 G PICC was insterted, with radiological control. On the 29th day, while on CPAP, HFOV was reinstituted due to apneic episodes. Thereafter, an increasing respiratory distress and hemodynamic compromise rapidly developed. Chest Xray revealed a bilateral pleural effusion with increasing cardiomegaly ; 2DEcho showed a 'swinging heart' with marked pericardial effusion. The diastolic volume resulted reduced, thereby impairing LV outflow. Suddenly, the infant became severely hypotensive and bradycardic. A rescue emergency pericardiocentesis [6 $\mathrm{mL}$ ] avoided the impending cardiogenic shock. A right chest tube was inserted draining $26 \mathrm{~mL}$ of milky fluid. Recovered pleural and pericardial effusion fluid analysis was similar to the intravenous fluids given through PICC. Subsequently, oxygenation markedly improved and ventilator settings could be greatly reduced; inotropes were no longer required. Chest $\mathrm{X}$ rays confirmed the lung clearing.

Conclusions: Cardiac tamponade represents an emergency situation leading to a fatal outcome without early diagnosis/timely intervention. Pericardial effusion and cardiac tamponade have been reported in $1-3 \%$ of neonates with PICC $(30-50 \%$ mortality $)$ The coexistence of acute pericardial and bilateral pleural effusions from parenteral fluid extravasation remains a very uncommon finding.

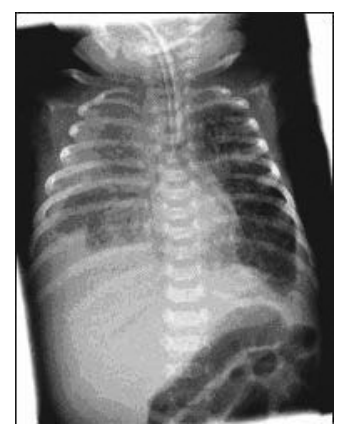

\section{COMMON REASONS FOR TRANSPORT OF NEWBORN INFANTS TO INTENSIVE CARE}

J T Guleva, M K Zip, V V Delovska-Stojkova, B T Mihajlovik, T B Pensovska

\section{SHGO Cair, Skopje, Macedonia}

Background and Aims: The aim of this article is to present the most common raesons for transport of the newborns who urgently need to be treated adequately at the department of the intensive care (NICU). The basic 
goal is to identify and reduce the reasons that directly contributed to it.

Methods: Database of the department of the newborn infants had been used and analysis of gestation age and clinical diagnoses of infants vas done. The analysed material covers period from January 2001 to December 2005.

Results: Three hundred and two newborns $(1,65 \%)$ out of 18288 live born infants have been transported during 2001- 2005. One hundred eighty nine of them $(62,58 \%)$, have been transported to the Clinic for Children' $\mathrm{s}$ diseases at NICU. The most common diagnosis were: premature with RDSy 115 (60,85\%), aspiration syndrom $29(15,34 \%)$, perinatal asphyxia was diagnosed in 19 $(10,05 \%), 13(6,88 \%)$ showed sings of HIE (hypoxicischemic encephalophaty) and congenital malformation was diagnosis in13 (6,88\%) newborns. Sixty-one $(32,27 \%)$ of the newborns were below 33 gestational ege and $90(47,62 \%)$ were below 37 gestational week (GW).

Conclusions: These carefully made analyses confirm that priority for transport to NICU, have those diagnosed with Praematurus and RDSy, and newborns with gestation age below $33 \mathrm{GW}$. On the whole, all these analyses and results clearly state the idea of urgent improvement of prenatal protection of the pregnant women, regular follow up of pregnancy and immediate transfer to the adequate clinic.

\section{THE PROBIOTIC E. COLI NISSLE 1917 STOPS ACUTE DIARRHOEA IN INFANTS AND TODDLERS}

\author{
J Henker $^{1}$, B M Blokhin ${ }^{2}$, Y K Bolbot ${ }^{3}$, \\ V G Maydannik ${ }^{4}$, C Wolff ${ }^{5}$, J Schulze ${ }^{5}$ \\ ${ }^{1}$ Universitätsklinikum Carl Gustav Carus Der TU \\ Dresden, Dresden, Germany, ${ }^{2}$ Russian State Medical \\ University, Moscow, Russia, ${ }^{3}$ Dnepropetrovsk State \\ Medical Academy, Dnepropetrovsk, Ukraine, ${ }^{4}$ National \\ Medical University O.O. Bogomolets, Kyiv, Ukraine \\ ${ }^{5}$ Ardeypharm, Herdecke, Germany
}

Background and Aims: In most cases acute diarrhoea will be self-limiting. For children health risks may arise when diarrhoea lasts longer than 3 days. The purpose of the present trial was to normalize the stool frequency of infants and toddlers suffering from acute diarrhoea by administering the probiotic E. coli Nissle 1917 (EcN) quicker than with placebo.

Methods: In total, 113 children (aged 2 to 47 months) with $>3$ watery or loose stools in 24 hours were randomized to either verum $(\mathrm{EcN}$ suspension with $10 \mathrm{E} 8$ viable $\mathrm{EcN}$ per $\mathrm{ml}, \mathrm{n}=55)$ or placebo $(\mathrm{n}=58)$ in a confirmative, double-blind clinical trial. Depending on the age of patients $1-3 \mathrm{ml}$ per day were administered orally.

Results: As a reason for the diarrhoea viral infection was stated in $31 \%$, bacterial infection in $11.5 \%$, and unspecific infection in the rest of the children. Onset of the response to treatment (reduction of stool frequency to $\leq 3$ watery or loose stools in 24 hours over a period of at least 2 consecutive days) in the EcN group was more rapid (median of 2.5 days) than in the placebo group (median of 4.8 days), a significant difference of 2.3 days
( $p=0.0007$, Kaplan-Meier analysis). The number of patients showing response was significantly higher $(\mathrm{p}=0.0003)$ in the $\mathrm{EcN}(94.5 \%)$ than in the placebo group (67.2\%).

Conclusions: EcN showed significant superiority compared to placebo in the treatment of acute diarrhoea in infants and toddlers. This concerns the time to response as well as the response rate.

\section{INTESTINAL PARASITIC INFECTIONS AND ANTHROPOMETRIC INDICATORS OF CHILDREN GROWTH: A LARGE POPULATION- BASED SURVEY}

J Nematian, A Gholamrezanezhad, E Nematian

Department of Mycology and Parasitology, Faculty of Medicine, Azad University of Tehran, Tehran, Iran

Background and Aims: School children carry the heaviest burden of morbidity of intestinal parasitic infections. There are numerous data suggestive of their adverse effect on children growth. In the present study, the association between previously undiagnosed intestinal parasitic infections and children growth was assessed cross-sectionally.

Methods: 19213 elementary school children were selected randomly. The physical growth of these children was investigated by taking anthropometric measurements which included body weight and standing height and Z-scores were calculated using New Anthro 1.02 software (CDC/WHO, 1999). Coproparasitoscopic examination was also done with three different methods. The association between intestinal parasitic infection and growth was analyzed with multivariable models adjusted for the influence of age and polyparasitism.

Results: The prevalence of intestinal parasitic infection was $18.4 \%$. The prevalence of stunting $(3.8 \% \mathrm{Vs}$. $2.8 \%)$, wasting $(22.7 \%$ Vs. $20.4 \%)$ and also the combination of stunting-wasting $(3.7 \%$ Vs. $2.8 \%)$ was significantly higher in those children involved by parasitic infections as compared with uninfected children (all $\mathrm{P}<0.01$ ); Only Giardia lamblia infection and Enterobius vermicularis infections were significantly associated with reductions in height for age (stunting) and weight for height (wasting). There was not such reduction in infections by Ascaris lumbricodes, Trichiuris trichiura, Hookworms, Blastocystis hominis, Hymenolepis nana, and Entamoeba coli.

Conclusions: The data confirmed the high prevalence of previously undiagnosed intestinal parasitic infections and highlighted important interactions between nutrition and parasites. These data strongly support the need for school health programmes aimed at reducing the prevalence of infection in schoolchildren (particularly Giardiasis and Enterobiasis), with potentially beneficial effects on educational outcomes. 
EFFECTS OF NUTRITIONAL AND PSYCHOSUPPORTIVE INTERVENTIONS ON THE COURSE OF INFANTILE COLIC - FIRST RESULTS OF A PROSPECTIVE CLINICAL STUDY

\author{
K Sargsyan, K Burmucic, M Dunitz-Scheer, \\ A C Hauer
}

\section{University Hospital for Children and Adolescent Health, Graz, Austria}

Background and Aims: Infantile colic is a syndrome characterized by paroxysmal and inconsolable crying without identifiable cause in healthy infant. The aim is the evaluation of a nutritional (lactose reduced partially hydrolysed study formula vs. standard formula) and a psychosupportive intervention in "colicky" babies.

Methods: Inclusion criteria are "Wessels's" criteria, exclusion criteria are potential organic causes for a colicky state. Study infants are randomly assigned to following groups: 1) Nutritional intervention group (study formula) 2) Nutritional control group 1 (standard formula) 3) psychosupportive intervention group ( $3 \times 45$ min. psychotherapy) 4) Nutritional plus psychosupportive intervention group 5) Nutritional observational group (breastfed babies). Infants of group 3 and 4 are seen by the recruiting paediatrician and the psychotherapist on 3 occasions (4-6 weeks); those of group 1,2, and 5 have only 2 assessment. At recruitment questionnaires are filled in and videotaping of typical feeding scene is performed. Pain scales are used to check continuously colic intensity and quantity.

Results: Our results indicate that infants of groups 1, 3 and 4 had an improved state after 2 weeks. Their"colicky" state was reduced by $50.3 \%, 51.1 \%$, $69.5 \%$ resp.

Conclusions: We conclude that dietary intervention study formula should be combined with psychosupportive intervention to ameliorate the colicky state in these infants. Higher numbers of patients are required to confirm our observations so far. This work was supported by Nutricia Research Foundation.

\section{PREVALENCE OF CELIAC DISEASE IN CHILDREN AND ADOLESCENTS WITH DIABETES MELLITUS TYPE I IN SOUTH- EASTERN POLAND: THE EFFECTIVENESS OF 5-YEAR SCREENING PROGRAMME}

\author{
B Korczowski $^{1,2}$, L Lisowicz ${ }^{1}$, R Jakubiec ${ }^{1}$, B Surdej ${ }^{1}$ \\ ${ }^{l}$ Department of Pediatrics, Regional Hospital No 2, \\ Rzeszów, Poland, ${ }^{2}$ Institute of Physiotherapy, University \\ of Rzeszów, Rzeszów, Poland
}

Background and Aims: The prevalence of celiac disease among children with type I diabetes mellitus exceeds the prevalence of the condition in the general population. To investigate the prevalence of celiac disease in the large cohort of children and adolescents with type I diabetes mellitus was the aim of the study. Methods: 765 children from the Podkarpatian Region with diabetes type I diagnosed in years 1990-2005 were enrolled into the study. Prospective serologic screening was performed in 355 children with diabetes diagnosed during 5 consecutive years between January 2000 and
December 2005. Among 410 children with diabetes diagnosed in the period of 10 years - between January 1990 and December 1999, serologic tests for celiac disease were performed exclusively in children with clinical symptoms of malabsorption syndrome. Intestinal biopsy was performed in all children with positive results of serologic tests.

Results: Celiac disease was diagnosed in 8 out of 355 children $(2,25 \%)$ screened in the years $2000-2005$ and in 3 out of 410 children with diabetes mellitus type I diagnosed in the years 1990-1999.

Conclusions: The prevalence of celiac disease in children and adolescents with diabetes type I in southeastern Poland is relatively low. Screening for celiac disease in type I diabetes, although costly, was an effective method to detect silent form of the disease.

\section{LONG TERM GASTRO-ESOPHAGEAL REFLUX FOLLOWING CONGENITAL DIAPHRAGMATIC HERNIA}

M G Peetsold ${ }^{1}$, H A Heij ${ }^{2}$, C M Kneepkens ${ }^{1}$, $\mathrm{R} J ~ G e m k e^{1}$

${ }^{I}$ Department of Pediatrics, VU University Medical Center, Amsterdam, The Netherlands, ${ }^{2}$ Department of Pediatric Surgery, VU University Medical Center, Amsterdam, The Netherlands

Background and Aims: Gastro-esophageal reflux (GER) is a recognized complication of congenital diaphragmatic hernia $(\mathrm{CDH})$, yet its mechanism has not been clarified. In the first years after $\mathrm{CDH}$ repair the incidence of GER varies from 20-72\%. Long term follow up studies are scarce while most are based on small numbers of patients. The goal of this study was to determine the incidence of GER after CDH using a standardized symptom checklist for increased risk of GER in $\mathrm{CDH}$ survivors and to identify predictive variables for GER following $\mathrm{CDH}$.

Methods: During a period of 18 years, 70 of 159 patients (44\%) with high-risk $\mathrm{CDH}$ survived after surgery. 55 of the 70 survivors $(79 \%)$ (mean age 12.1 years \pm 3.4 ; range $6-18$ years) completed a standardized questionnaire (C. Manterola, 2002). Patient who had a score indicating increased risk of GER were referred for gastroscopy and $\mathrm{pH}$-metry.

Results: GER in the first years after repair was proven in 23 of 55 patients $(42 \%)$. Nine of them underwent a surgical antireflux-procedure within 2 years after $\mathrm{CDH}$ repair. At the time of follow-up 10 of 55 patients (18\%) had symptoms indicating an increased risk of GER. In 5 GER was confirmed. All responded well to proton pump inhibitors. There were no perinatal or postnatal risk factors associated with long term GER.

Conclusions: $\mathrm{CDH}$ survivors are at risk for (subclinical) GER, although no predictive variables could be identified. Long-term follow-up for all $\mathrm{CDH}$ patients focusing on GER is justified to prevent adverse consequences, such as chronic esophagitis and Barrett's esophagus. 
CELIAC DISEASE IN CHILDREN. ITS RELATION WITH HEART INVOLVEMENT

\section{P Sciacca, C Mattia, M Spina, G Distefano \\ Department of Pediatrics, University of Catania, Catania, Italy}

Background and Aims: Coeliac disease (CD), is a permanent gluten intolerance occurring in genetically susceptible subjects. Recent report have correlated cases of dilated cardiomyopathy with CD in adults. For this reason we started a systematic cardiac evaluation of children with CD.

Methods: We studied 40 children with $\mathrm{CD}$ diagnosis (TTGa dosing). Medium age 5,35 y; medium weight $15,5 \mathrm{Kg}$. We performed an echocardiography when CD diagnosis was confirmed by biopsy and a second Echo2d 12 months after beginning of gluten free diet (GFD). Control group was of 20 healthy children.

Results: In the 1 Echo2d we observed 8 (20\%) children with mild heart involvement: slight mitral valve regurgitation (MVr) in 5; slight aortic valve regurgitation in 2 ; pulmonary and tricuspid valve regurgitation in 1 and impaired ejection fraction (EF) $(0,58)$ in 1 . In the 2 Echo2d all valve regurgitations had regressed except 1 case of MVr. The patient with impaired $\mathrm{EF}$ had significant improvement from 0,58 to 0,78 . In these 8 patients medium $\mathrm{EF}$ value was of $0,61 \pm$ 0,025 in the 1 examination and $0,72 \pm 0,066$ after 1 year of GFD $(\mathrm{p}<0,001)$. TTGa medium values passed from $381 \pm 45,72$ to $15,20 \pm 2,62 \mathrm{UI} / 1$.

Conclusions: Our preliminary data show that, as in adults, children with $\mathrm{CD}$ are at risk of reversible heart involvement. The pathogenetic mechanisms may be linked to chronic malabsorption or to an associated autoimmune process. Recently has been demonstrated an upregulation of transglutaminase II mRNA in rat models of heart failure.

\section{SCREENING FOR LIVER DISEASE IN PATIENTS WITH CYSTIC FIBROSIS}

\author{
S Fustik $^{1}$, T Jakovska ${ }^{1}$, M Trajkovska $^{2}$, T Josifovska ${ }^{3}$, \\ $\mathrm{S}$ Kocova $^{4}$, G D Efremov ${ }^{4}$ \\ ${ }^{l}$ CF Centre, Department for Pediatrics, Clinical Centre, \\ Skopje, Macedonia, ${ }^{2}$ Department for Gastroenterology \\ and Hepatology, Clinical Centre, Skopje, Macedonia \\ ${ }^{3}$ Institute for Nuclear Medicine, Skopje, Macedonia \\ ${ }^{4}$ Research Center for Genetic Engineering and \\ Biotechnology, Macedonian Academy of Science and \\ Arts, Skopje, Macedonia
}

Background and Aims: The prevalence of liver disease (LD) associated with cystic fibrosis (CF) is increasing as the population of patients with CF lives longer, and this may have a negative prognostic impact. The aim of study was to analyze the results from screening program for diagnosis of $\mathrm{LD}$ in $\mathrm{CF}$.

Methods: All patients older than 3 years $(n=52)$, regularly followed at the $\mathrm{CF}$ Centre in Skopje were screening for LD through clinical, biochemical, echo graphic, and hepatobiliary scintigraphic assessment. LD was defined by the finding of hepatomegaly and/or splenomegaly; significant and persistent increased of at least two serum liver enzyme levels; suggestive ultrasonographic abnormalities (score $>4$ ); and morphologic or functional scintigraphic abnormalities.

Results: According to our predefined criteria, 18 patients $(34.6 \%)$ were classified as having LD, three of them with portal hypertension. Clinical presentations of LD had 6 (33.3\%), abnormal liver blood test results 12 (66.6\%), ultrasound abnormalities 18 (100\%), and scintigraphic abnormalities 13 (72.2\%) of the patients with LD. Prevalence of LD increased with age (average $13.2 \pm 5.3$ years in the LD group vs. $9.9 \pm 5.6$ years in the no LD group). A male predominance was found in the group with LD (72\%). Pancreatic insufficiency was present in all patients with LD. Genetic analysis show higher frequency of $\triangle \mathrm{F} 508$ mutation in $\mathrm{LD}$ group $(77.8 \%)$ vs. no LD group (66.2\%). All patients with LD had severe mutations: $\triangle F 508$, G542X, N1303K, CFTRdel.21Kb, 1811+1G $\rightarrow$ C, and $\mathrm{Y} 1092 \mathrm{X}$.

Conclusions: Hepatic screening enable early identification of patients with LD and early therapeutic intervention for prevention of liver complications.

\section{TOLERANCE AND ADEQUACY OF FEEDING DURING PHARMACOLOGICALLY INDUCED PENTOBARBITAL COMA IN CRITICALLY ILL ICU PATIENTS}

\author{
S Naghib ${ }^{1,2}$, K Hofmann ${ }^{1}$, M de Hoog ${ }^{1}$, D Tibboel ${ }^{2}$, \\ K F M Joosten ${ }^{1}$ \\ ${ }^{I}$ Department of Pediatrics, Erasmus MC, Sophia \\ Children's Hospital, Rotterdam, The Netherlands \\ ${ }^{2}$ Department of Pediatric Surgery, Erasmus MC, Sophia \\ Children's Hospital, Rotterdam, The Netherlands
}

Background and Aims: In adults difficulties in nutritional support following pentobarbital coma are repeatedly shown. In contrast no data are available in children. We determined the tolerance and adequacy of feeding in children receiving continuous pentobarbital infusion during pharmacological induced coma in a retrospective data analysis in 2003-2005.

Methods: Children were eligible for the study if they received at least 48 hours continuous pentobarbital as treatment modality for refractory seizures. Desired intake and the type of formula was guided by a nutritional protocol and adjusted to the child's condition by the attending intensivist. Actual energy intake was recorded, compared to measured energy expenditure (MEE) in 5 patients and compared with calculated resting energy expenditure (REE) in 3 patients.

Results: Eight children were eligible. The mean age was 4 years (range $0-13$ ). During 94 pentobarbital coma days a total of 63 records of feeding days were recorded. Seven patients were successfully fed on a duodenal tube. Enteral feeding was given in $87 \%$ of the children. The MEE of the 5 measured children was $25 \%$ lower in comparison to calculated REE.

Conclusions: This study shows that children requiring continuous pentobarbital during pharmacological coma tolerated enteral feeding well but have a decreased energy expenditure and a considerable risk of overfeeding. 
BLOOD LEAD LEVELS OF MATERNAL-CORD PAIRS IN INTRAUTERINE GROWTH RETARD AND NORMAL TERM NEONATES

R Iranpour ${ }^{1}$, A A Besharati ${ }^{1}$, F Naseri ${ }^{1}$, M Hashemipour ${ }^{1}$, M Balali ${ }^{2}$, R Kelishadi ${ }^{3}$

${ }^{I}$ Department of Pediatrics, Isfahan University of Medical Sciences, Al-Zahra Hospital, Isfahan, Iran ${ }^{2}$ Department of Toxicology, Mashad University of Medical Sciences, Emam-Reza Hospital, Mashad, Iran ${ }^{3}$ Department of Preventive Pediatric Cardiology, Isfahan Cardiovascular Research Center, Isfahan University of Medical Sciences, Isfahan, Iran

Background and Aims: As birth weight predicts infant outcome, growth and development, determining the risk for intrauterine growth retardation (IUGR) is important in prenatal care. In this study we aimed to compare the blood lead levels of maternal-cord pairs, in normal and IUGR term neonates in Isfahan city.

Methods: From April 2005 to December 2005, blood lead levels were measured in umbilical cord and maternal venous blood samples in 32 mother-infant pairs with IUGR full term neonates and 34 motherinfant pairs with normal full term neonates. Blood-lead levels were analyzed by atomic absorption spectrometry. Results: The mean lead concentration in neonates of IUGR and normal groups were not significantly different $(107.47+/-16.75$ vs. $113.08+/-19.08 \mu \mathrm{g} / \mathrm{L}$, respectively $\mathrm{p}=0.2$ ). The mean lead concentration in mothers of IUGR group was lower than normal groups, but this difference was not significant $(124.56+/-19.71$ vs.135.26 +/- $26.91 \mu \mathrm{g} / \mathrm{L}$, respectively, $\mathrm{p}=0.07$ ). Maternal lead levels were strongly related with cord blood in both IUGR and normal groups ( $\mathrm{r}=0.8$, and $\mathrm{p}<0.0001)$. Maternal and cord blood lead levels were not correlated with birth weight of newborns in neither group. Overall, $65.6 \%$ of IUGR neonates and $76.5 \%$ of normal neonates were above the critical level defined for lead poisoning as $>100 \mu \mathrm{g} / \mathrm{L}$ by the centers for disease control.

Conclusions: Our results indicate that whole blood lead was not related to the birth weight; in addition, there were remarkable lead burdens on both the mothers and their neonates in this industrial area.

\section{CYSTIC FIBROSIS ASSOCIATED HEPATOBILIARY DISEASE: IS IT INFLUENCED BY GENOTYPE?}

\author{
I M Popa ${ }^{1}$, I Popa ${ }^{1}, \mathrm{~L} \mathrm{Pop}^{1}, \mathrm{Z} \mathrm{Popa}^{2}$, L Tamas ${ }^{3}$ \\ ${ }^{1}$ Pediatrics IInd Department, UMF Timisoara, \\ Timisoara, Romania, ${ }^{2}$ Cystic Fibrosis Centre, \\ Timisoara, Romania, ${ }^{3}$ Department of Molecular \\ Genetics, Department of Biochemistry, ' V. Babes', \\ Timisoara, Romania
}

Background and Aims: Cystic fibrosis (CF) associated hepatobiliary disease include many expressions like: liver disease (LD), neonatal cholestasis, cholelithiasis, microgallbladder, common bile duct stenosis. LD is frecquently associated with severe genotype. The aim of the study was to evaluate the genotype impact on $\mathrm{CF}$ associated hepatobiliary disease.
Methods: Lot study included patients with typical CF, $\mathrm{n}=85$ patients $(0-18$ years old $)$, followed up by clinical assessment, biochemical markers, ultrasound examinations and in some cases liver biopsy. All were genetically tested for the most common $29 \mathrm{CF}$ mutations.

Results: Hepatobiliary disease was diagnosed in 36 patients $(42,35 \%)$; in 56 children younger then 10 years, only $11,76 \%$ had liver disease, 2 associated neonatal cholestasis (F508 homozygous). 30,59\% of children (older than 10 years) had hepatobiliary expression; in 8 children of patients, multilobular cirrhosis was diagnosed, four of them confirmed by biopsy. Genotype structure of 85 patients revealed 32 F508 homozygous genotypes, 22 - F508/X, 5 non - F508/X and 26 unknown $(\mathrm{X} / \mathrm{X})$, the frequency of F508 allele being $50,58 \%$. Severe liver disease developed in 7 patients, 4 children with F508 homozygous genotype. Among 32 F508 homozygous genotypes, only $15,29 \%$ had a hepatobiliary expression.

Conclusions: We could not establish a specific correlation between HD expressions and a certain mutation. The heterogeneous phenotypes in CF patients having the same genotype suggest that other environmental and/or genetic factors are implicated.

\section{APPENDECTOMY WITH ONE TROCAR THROUGH THE UMBILICUS: RESULTS OF OUR SERIES AND A COST APPROXIMATION}
A Perez-Martinez ${ }^{1}$, M A Martínez-Bermejo ${ }^{1}$, J Conde-Cortes ${ }^{1}$, C Goñi-Orayen ${ }^{2}$, J Pisón-Chacón ${ }^{1}$, L Bento-Bravo ${ }^{1}$
${ }^{1}$ Department of Pediatric Surgery, Virgen del Camino Hospital, Pamplona, Navarra, Spain, ${ }^{2}$ Department of Pediatrics, Virgen del Camino Hospital, Pamplona, Navarra, Spain

Background and Aims: We perform the appendectomy through a single periumbilical incision. Using a double channel laparoscope we locate and exteriorize the appendix and then proceed to remove it in the standard fashion. We studied this practice to determine if this technique makes it possible to make use of the advantages of laparoscopic surgery and of open surgery, decreasing costs without increasing the rate of complications.

Methods: The study group was made up of transumbilical appendectomies (TA) for acute, uncomplicated appendicitis carried out in 2004 and 2005. The control group consisted of a group of open appendectomies (OA) performed for acute, uncomplicated appendicitis in our department in 2002 and 2003. Both study groups were comparable.

Results: We performed a total of 208 TA of which 162 were due to uncomplicated appendicitis. The mean hospital stay was 2.84 days (versus 4.83 days with OA) (significant difference). Infectious complications presented in $4.29 \%$ (versus $1.75 \%$ in the OA group) (this difference was not significant). Postoperative pain and need for analgesia were less in the TA group than in the OA (difference did not reach significance). The savings resulting from the transumbilical technique represents some 90,561.97€ / year compared with the 
cost that laparoscopic appendectomy would incur, and some $42,232.37 €$ / year versus traditional, open surgery. Conclusions: Once the learning curve is overcome, we trust that the percentage of infectious complications will decline sharply. This technique is highly beneficial because it improves the postoperative recovery with unbeatable esthetic results. The decreased costs should serve to promote its use.

\section{ISOLATED INTESTINAL PERFORATION OF THE NEWBORN, PERIVENTRICULAR WHITE MATTER DAMAGE AND INFLAMMATION - IS THERE A LINK?}

\author{
S Kout $^{1}$, F Kieffer ${ }^{1}$, K Husseini ${ }^{2}$, M Mokhtari ${ }^{2}$, \\ C Dupont $^{2,3}$, J F Magny ${ }^{1}$, A Lapillonne ${ }^{2,3}$ \\ ${ }^{1}$ Institut de Puericulture, Paris, France, ${ }^{2}$ Saint-Vincent \\ de Paul Hospital, Paris, France, ${ }^{3}$ Rene Descartes Paris \\ 5 University, Paris, France
}

Background and Aims: To determine prognostic factors associated with isolated intestinal perforation of the newborn.

Methods: Data of newborns with intestinal perforation, but without NEC and intestinal malformation, were collected retrospectively from in-patient medical record of 2 neonatal units over a 5-year period. Periventricular white matter damage (WMD) was considered present on cranial US if cystic periventricular leukomalacia, echodensities persisting for more than $14 \mathrm{~d}$ without cyst formation, periventricular parenchymal hemorrhagic involvement or ventricular dilatation associated with transient periventricular echodensities was detected.

Results: Among the 30 infants (mean $\mathrm{GA} \pm \mathrm{SD}=$ $29 \pm 3.9$ wks; birth weight $=1126 \pm 571 \mathrm{~g}$ ) included, 13 (43\%) died and WMD was present in 9 cases $(31 \%)$. Materno-fetal infection, antenatal use of cortisteroid, GA, birth weight, $\mathrm{pH}$ at birth, postnatal age, positive blood culture, or CRP at time of intestinal perforation were not significantly associated with death or WMD. In contrast, white blood cell (WBC) count was significantly higher in infants who had WMD $(33 \pm 18$ vs. $9 \pm 8 \times 109 / \mathrm{L}, \mathrm{p}=0.004)$ and tended to be higher in infants who died $(24 \pm 20$ vs. $12 \pm 12 \times 109 / \mathrm{L}, \mathrm{p}=0.07)$. Infants with a WBC count above $20 \times 109 / \mathrm{L}$ had a significant risk of death $(\mathrm{OR}=9 ; 95 \% \mathrm{CI}=1-63)$ and WMD $(\mathrm{OR}=19 ; 95 \% \mathrm{CI}=2-170)$.

Conclusions: WBC count at time of intestinal perforation is a strong predictor of death or WMD. This suggests that the cascade of events that lead to adverse outcomes of newborns with isolated intestinal perforation is mediated by a profound inflammatory response.

\section{RECURRENT ABDOMINAL PAIN IN PEDIATRICS: A POTENTIAL PRECURSOR OF FUNCTIONAL GASTROINTESTINAL DISORDERS IN ADOLESCENTS AND YOUNG ADULTS?}

M T Fernández ${ }^{1}, \mathrm{~J}$ M Marugan ${ }^{1}, \mathrm{~L}_{\text {Regueras }}{ }^{1}$,
M C Torres ${ }^{2}, \mathrm{M}$ C de Fuentes ${ }^{1}$
${ }^{1}$ Hospital de León, León, Spain, ${ }^{2}$ CAP Jose Aguado,
León, Spain
Background and Aims: To determine if functional recurrent abdominal pain in pediatric age (RAF), can predict gastrointestinal or extra digestive functional disorders.

Methods: Retrospective longitudinal design of cases and controls. Symptoms-based diagnostic criteria (Roma II) were used to classify the patients with RAF between 1992-1998. The average time since discharge was 7,76 $+1,4$ years. The control group (matched for age and sex with the case group) were patients from a primary care center who didn't have RAF. When former consent was obtained, we realized a telephone interview that evaluated the presence of pain, its characteristics, symptoms associated and repercussion in life quality. The study was approved by our Ethic Committee. Statistic comparison with Fischer test.

Results: We studied 116 patients (58 cases/58 controls), the average of age was $16,2+3,16$ in both $(62 \%$ women). From the RAF patients $25(43,1 \%)$ continued with abdominal pain $(66 \%$ women) and $22(37,9 \%)$ from the control group ( $77 \%$ women) $(p>0,05)$, although $10 / 25$ in the cases group consider that pain is a important limitation for their quality of life, and only $3 / 22$ in the control group $(p=0,056)$. Another symptoms compatible with functional gastrointestinal disorders were presented in 15/25 cases and 10/22 controls. Another symptoms were, respectively cases/controls: migraine 18/8 $(p<0,05)$, constipation $9 / 11(p>0,05)$ and maladaptative behavioral responses 17/14 ( $>0,05)$.

Conclusions: There is a elevated prevalence of RAF and other functional disorders in adolescents and young adults as many cases as controls, although cases patients with pediatric RAF have more migraine prevalence, with significance statistics.

\section{DIENCEPHALIC CACHEXIA OF INFANCY: RUSSELL'S SYNDROME}

\author{
A M Murphy ${ }^{1}$, B Drumm², C Brenner ${ }^{3}$, S A Lynch ${ }^{1}$ \\ ${ }^{I}$ The National Centre for Medical Genetics, Our Lady's \\ Hospital for Sick Children, Dublin, Ireland, ${ }^{2}$ The \\ Children's Research Centre, Our Lady's Hospital for \\ Sick Children, Dublin, Ireland, ${ }^{3}$ Department of \\ Radiology, Our Lady's Hospital for Sick Children, \\ Dublin, Ireland
}

Background and Aims: The diencephalon is the most rostal part of the brain stem and includes the thalamus, hypothalamus, pituitary and pineal glands and the structures surrounding the third ventricle. Lesions (usually neoplasms) of this region produce a complex of symptoms and signs thought to be related to hypothalamic dysfunction although the exact mechanisms behind the complex phenotype remain unexplained. This particular form of failure to thrive occurs in the setting of elevated growth hormone levels. The condition first came to medical attention at the annual general meeting of the British Paediatric Association in 1951 when Russell described the typical features. In descending order of frequency these are emaciation, increased alertness, hyperkinesis, vomiting, euphoria, nystagmus, irritability, hydrocephalous and optic atrophy. We report a female infant who presented at 16 months of age with severe generalised lipoatrophy, 
constipation, a normal developmental profile and a subtle rotatory nystagmus which was initially overlooked.

Methods: Clinical assessment, clinical photographs and brain imaging at diagnosis and at follow up post chemotherapy one year later.

Results: The tumor has shrunk, constipation has resolved. Weight gain is restored and normal development continues.

Conclusions: Diencephalic syndrome should be considered as a diagnosis in any child with infancy onset failure to thrive despite adequate caloric intake and normal absorptive function of the small bowel and an inappropriate euphoric mood with or without neurological signs. Long term survival is possible if control can be achieved.

\section{THE RELATIONSHIP BETWEEN 'CHILD FEEDING' BEHAVIOUR OF MOTHERS AND F.T.T IN THEIR CHILDREN}

\section{A Amini \\ Department of Pediatrics, Medicine Faculty, Isfahan University, Isfahan, Iran}

Background and Aims: Some children with F.T.T have no organic diseases and their F.T.T may be due to mother-infant relationship during child feeding. The major goal of this study is to find that which elements in maternal 'child feeding' behaviour could lead to F.T.T.

Methods: To do this study we chose 50 children with F.T.T and without any organic diseases and 100 healthy children as control group. All children were between 230 months. The study was done in four health care centers in Isfahan city. At first, all children had complete physical examination and evaluation of growth cards. The behavior and reactions of each mother to her child's feeding habits was checked by interview with mothers and a standard questionnaire sheet. The data was evaluated by Mann-Whitney formula and calculation of P-values. The P-value $<0.05$ was accepted as valuable difference between 2 groups.

Results: The factors that have no relationship with F.T.T were: age of mothers, educational status of mothers, preparing special foods for children and having snack between meals. Effective factors in prevention of F.T.T were: Doesn't be worry when the child refuses to eat. Doesn't control the child behavior when he/she spills the food. oRestarting spoon feeding again and again, when child refuses to feed with spoon. Conclusions: Not only amount of food is important in prevention of F.T.T but also behavior of mother versus feeding habits of child has important role in prevention of F.T.T. By knowing this fact and reminding it to mothers we can decrease incidence of F.T.T in children without any extra costs.

\section{SOME FALLS IN PREMATURE BABY'S NUTRITION MANAGEMENT DURING THE NON-INVASIVE RESPIRATORY SUPPORT}

S B Jancevska ${ }^{1}$, Z S Gucev $^{2}$, E L Petkovska ${ }^{1}$
${ }^{1}$ Clinic of Gynecology and Obstetrics, Clinical Center Skopje, Skopje, Macedonia, ${ }^{2}$ Pediatric Clinic, Clinical Center Skopje, Skopje, Macedonia

Background and Aims: Premature babies with respiratory distress syndrome (RDSy) on continuous positive airway pressure (CPAP) therapy can be feed. We confirmed relation between wrong nutrition management steps and premature infant's disturbing during the CPAP-nasal prongs therapy.

Methods: We analyzed one group of 25 premature babies from 27 to 31 gestation weeks. All babies were with respiratory distress syndrome problems and had non-invasive mechanical support. CPAP-NP type therapy gives opportunities to feed babies.

Results: The most frequently falls in a baby's feed preparing and realization are:

The baby is not in a supine position with the head elevated about 30 degrees.

The baby has a pain by nasal prongs wrong position,

The frequently or out of suction nasal cavities, mouth and pharynx,

Do not pass an oro/naso-gastric tube and aspirate the stomach air and other contents,

Do not remove the tube father aspiration,

Do not feed the baby by gravidity but by pressure,

Do not remove the feeding tube the stomach.

Every of this falls produces bay's uncomfortable, an exiting, a crying and movements. Vomits was presented in quarter of group. An aspiration was confirmed in 2 babies.

Conclusions: Small changes in nutrition management produce big problems. So, CPAP-NP advantages transform to CPAP-NP disadvantages.

\section{ANOMALIES OF GASTROINTESTINAL TRACT /GIT/ AND STRUCTURE OF MORBIDITY OF THE NEWBORNS}

R Rudanovic, M Rudanovic, D Dakic, L Globarevic, M Perosevic, D Milidrag, M Rudanovic

Department of Neonatology, Children's Clinic, Podgorica, Serbia and Montenegro

Background and Aims: Importance of early detection of (echography, amniocentosis, genetic counselling and prenatal therapy and laboratory diagnosis) anomalies of Git and anomalies of diaphragm - impact onto adequate treatment and final outcome.

Methods: Prospectus monitoring for four year period (2001 - 2005) of the frequency of the above mentioned anomalies at newborn children cured in Neonatology Center.

Results: Results for the above mentioned period: total 2980 newborn children were under the cure. Total 50\% of those children with the anomalies had joint anomalies, out of which $25 \%$ jeopardized life of patients and required urgent surgery intervention. According to our data, $21 \%$ out of total number of all anomalies refers to Git anomalies. Half of those are anorectal anomalies, congenital anomalies, anomalies of heart and big blood arteries make $20 \%$, urinary tract anomalies $10 \%$, CNS anomalies $4 \%$, while remaining $45 \%$ refers to other anomalies (respiratory tract, chromosomopathies, etc). Esolagus atresia in sample amounts to $5,7 \%$, diaphragm 
hernia $0,5 \%$, i.e. 1 in 1000 babies born alive or 1 in 1000 babies born dead. In total sample, prematurus were 1,8 temporarius (hypotrophicus 4,4\%).

Conclusions: Survival in the group of children with diaphragmal hernia is $96 \%$, specifically in the group of children with highest percentage of prenatally determined diagnosis. All the children were at Mechanical ventilation and surgical treatment was made within 24 hours upon reception.

\section{MOLECULAR ADSORBENT RECIRCULATING SYSTEM (MARS) IN CHILDREN WITH CHOLESTASIS AND INTRACTABLE PRURITUS}

J Campos ${ }^{1}$, F Duque ${ }^{1}$, C Veiga ${ }^{2}$, A Dinis ${ }^{1}$, L Carvalho $^{1}$, I Gonçalves ${ }^{2}, \mathrm{~J}$ F Neves ${ }^{1}$

${ }^{I}$ Paediatric Intensive Care Unit, Coimbra's Paediatric

Hospital, Coimbra, Portugal

${ }^{2}$ Gastroenterology/Hepatology Unit, Coimbra's

Paediatric Hospital, Coimbra, Portugal

Background and Aims: Pruritus related to cholestasis, is a serious and difficult clinical problem to manage, and when intractable, represents an indication for liver transplantation. Its etiology is unknown, most therapeutic modalities are empirical, and none are universally satisfactory. The Molecular Adsorbents Recirculating System (MARS) is a novel dialysis technology which efficiently removes albumin bound substances (including bile salts), with promising preliminary results in the management of cholestatic disease. Our aim was to evaluate the usefulness of MARS in relieving cholestasis associated resistant pruritus in a child with Allagile Syndrome.

Methods: A 9 year old boy with Allagile syndrome with intractable pruritus, associated with a complex congenital heart disease and moderate renal dysfunction, was studied. He had received standard and second line anti-pruritic medication without symptomatic relief, being send to our institution for evaluation of the possibility of liver transplantation. In spite of his clinical condition, liver transplant was contra-indicated and he was proposed for realization of albumin dialysis (MARS).

Results: He underwent two sessions of MARS with seven hours each. The procedure was well tolerated and resulted in a relief of pruritus as well as in a decline of plasma bilirubin (from $93 \mathrm{umol} / 1$ to $70,5 \mathrm{umol} / 1$ ). Despite of the increased serum bile acid levels there was a change in their plasma profile. After one month, the child showed sustained improvement of pruritus and a further decline of bilirubin levels.

Conclusions: In our case, MARS revealed to be a safe and effective tool in the management of cholestatic pruritus resistant to conventional therapy.

\section{INDICATION FOR SURGERY OF NEONATAL HYDRONEPHROSIS CAN PREDICT THE OUTCOME OF RENAL FUNCTION}

J Langer $^{1,3}$, J Sedlacek ${ }^{2,3}$, R Kocvara ${ }^{2,3}$, J Dvoracek $^{2,3}$, Z Dite ${ }^{2,3}, \mathrm{~J} \mathrm{Zeman}^{1}$
${ }^{I}$ Department of Paediatrics, Faculty Hospital, Charles University, Prague, Czech Republic, ${ }^{2}$ Department of Urology, Faculty Hospital, Charles University, Prague, Czech Republic, ${ }^{3}$ Department of Urology, Postgraduate Medical School, Prague, Czech Republic

Background and Aims: Management of neonatal hydronephrosis is a clinical challenge. In our study we evaluated the outcome of renal function in small children with neonatal hydronephrosis after surgically repair according to indication criteria for surgery.

Methods: Neonatal hydronephrosis was diagnosed in 38 children. In 28 asymptomatic neonates diagnosed by ultrasound, the indication for surgery was low renal function at the first diuretic radionuclide renography in 11 patients (Group A), progressive pelvicalyceal dilatation in 11 (Group B) and progressive decline in split renal function in 6 (Group C). In another 10 symptomatic neonates or infants, surgery was indicated because of urosepsis (group D).

Results: The renal function before and after surgery was $28 \%$ and $32 \%$ in Group A, $47 \%$ and $46 \%$ in Group B, $40 \%$ and $47 \%$ in Group C and $48 \%$ and $44 \%$ in group D, respectively. A decrease of renal function after surgery was observed only in two patients from the Group A ( $23 \%$ to $17 \%$ and $24 \%$ to $14 \%$ ). In the Group C, all patients regained the level of their original renal function with no irreversible deterioration.

Conclusions: Increase in renal function after surgery is hardly to be expected in children with primarily hypofunctional kidney and hydronephrosis. Renal function was stable in operated children because of progressive dilatation. Preoperative deterioration of renal function was reversible in all patients. Symptomatic patients with urosepsis had normal renal function before and after surgery. Supported by IGA-MZ CR, 8320-4.

\section{G349A, C881T, G1223A AND A3330G NPHS1 MUTATION IN CHILDREN WITH DIFFUSE MESANGIAL HYPERCELLULARITY}

A Brodkiewicz ${ }^{1}$, A Bińczak-Kuleta ${ }^{2}$, G Adler $^{2}$, E Szychot $^{2}$, J Peregud-Pogorzelski ${ }^{1}$, W Jarmużek ${ }^{3}$, M Litwin ${ }^{3}$, R Grenda ${ }^{3}$, I Kostro ${ }^{4}$,

M Pańczyk-Tomaszewska ${ }^{4}$, M Roszkowska-Blaim ${ }^{4}$, A Ciechanowicz ${ }^{2}$

${ }^{1} 1$ st Department of Pediatrics, Pomeranian Medical University, Szczecin, Poland, ${ }^{2}$ Department of Pathobiochemistry and Molecular Biology, Pomeranian Medical University, Szczecin, Poland, ${ }^{3}$ Department of Nephrology and Transplantology, Medical University, Warsaw, Poland, ${ }^{4}$ Department of Paediatrics and Nephrology, Medical University, Warsaw, Poland

Background and Aims: Mutations in the NPHS1 gene encoding nephrin, major protein of slit-diaphragm, are responsible for the development of an autosomal recessive nephrotic syndrome of the Finnish type (CNF). Therefore, it raises the question whether the NPHS1 mutations might play a role in the pathogenesis of proteinuria in other renal diseases. The aim of our study was to evaluate the association between G349A, C881T, G1223A and A3330G NPHS1 mutation and both the predisposition to mesangial proliferation and 
prognosis in children with idiopathic nephrotic syndrome (INS) with histologic manifestation of diffuse mesangial hypercellurarity (DMH).

Methods: The study group consisted of 56 children with DMH and 105 healthy children as controls. The C881T NPHS1 mutation was investigated using PCR-RFLP technique. G349A, G1223A and A3330G NPHS1 mutation were investigated using PCR-OLA technique.

Results: No significant difference in genotype frequency has been observed between both groups. In addition, no significant differences with respect to various genotypes in the $\mathrm{DMH}$ group were noted for frequency of primary steroid-resistance as well as for gender, age of INS onset, INS recurrences, creatinine and cholesterol concentrations in serum, frequency of increased systolic and diastolic arterial blood pressure.

Conclusions: The results of our preliminary study suggest the lack of association between the G349A, C881T, G1223A and A3330G NPHS1 mutation and both the predisposition to DMH and prognosis in Polish INS children with histologic manifestation of diffuse mesangial hypercellularity.

\section{URINARY TRACT INFECTION IN A PEDIATRIC EMERGENCY DEPARTMENT: LABORATORY AND CLINICAL PRESENTATION}

N Manakou, A Gianissi, S Liosi, O Gkika, P Anastasopoulou, G Z Zantopoulos, Z Patrona

Department of Paediatrics, General Hospital of Korinthos, Korinthos, Greece

Background and Aims: The identification of Urinary Tract Infection (UTI) in the emergency department which leads to early diagnosis and adequate treatment is imperative for the prevention of chronic renal damage. Our aim was to describe the laboratory and clinical features of urinary tract infections in childhood in an emergency department.

Methods: By reviewing medical charts for patients admitted to the pediatric emergency department of our hospital between January 2004 and December 2005, we identified and enrolled patients 14 years of age or less in which defended UTI that was confirmed by positive urine culture.

Results: 336 patients ranging from 4 weeks to 14 years of age (2-13years on the average) made up our sample. Urine colony count and culture were done on the suspected cases. UTI was 1.38 times more frequent in girls. $33.03 \%$ of children with UTI were hospitalized. $21.7 \%$ of the patients were found in urinalysis to have 5 white blood cells or fewer per high power field and $12.3 \%$ had positive nitrite reaction. Escherichia coli was the most common organism isolated $(78.0 \%)$, followed by Klebsiella (11.8\%), Proteus (6.1\%), Staphylococcus (3.1\%) and Pseudomonas (1\%). The most common clinical presentations were fever $(87 \%)$, abdominal or suprapubic pain (22\%), dysuria (14\%), failure to thrive $(13 \%)$, malodorous urine or hematuria $(8 \%)$, febrile convulsions $(4.5 \%) .92 \%$ of the patients did not have another potential source for their fever.

Conclusions: UTI is prevalent in young children, particularly females, without a definite source of fever. Specific clinical signs and symptoms of UTI are uncommon. Therefore, a UTI should be considered in all children with a fever in whom other sources have been excluded. Urinalysis provides presumptive evidence of infection, whereas urine culture is definitive.

\section{TRAMADOL DISPOSITION TO SIMULTANEOUSLY ASSESS IN VIVO CYP2D6 AND CYP3A4 ONTOGENY IN NEONATAL LIFE}

\author{
K Allegaert ${ }^{1}$, J de Hoon $^{2}$, R Verbesselt ${ }^{2}$, V Cossey ${ }^{1}$, \\ G Naulaers ${ }^{1}, J$ van den Anker ${ }^{3}$ \\ ${ }^{I}$ Neonatal Intensive Care Unit, University Hospital \\ Gasthuisberg, Leuven, Belgium, ${ }^{2}$ Center for Clinical \\ Pharmacology, University Hospital Gasthuisberg, \\ Leuven, Belgium, ${ }^{3}$ Departments of Paediatrics, \\ Pharmacology and Physiology, George Washington \\ University School of Medicine and Health Sciences, \\ Washington DC, USA
}

Background and Aims: To document maturational changes of in vivo CYP2D6 and CYP3A4 activity in the first months of life.

Methods: The relative contribution of tramadol, O-demethyl tramadol (M1, CYP2D6) and N-demethyl tramadol (M2, CYP3A4) to overall renal tramadol elimination was calculated during continuous intravenous administration of tramadol. The $[\log \mathrm{M} / \mathrm{M} 1$ / $\log \mathrm{M} / \mathrm{M} 2]$ ratio was calculated to compare CYP2D6 versus CYP3A4 ontogeny and correlations with the postmenstrual age were investigated.

Results: Of the total amount tramadol administered, $34.5 \%$ (SD 6.1) was retrieved in the urine as parent compound or metabolite in 25 neonates and young infants (PMA 25-53 weeks). This retrieval primarily consisted of tramadol 79\% (SD 18), M1 contributed $10 \%$ (SD 17) and M2 3\% (SD 3.4). The mean [log $\mathrm{M} / \mathrm{M} 1 / \log \mathrm{M} / \mathrm{M} 2]$ ratio was 0.65 (SD 0.54) and a significant inverse correlation between $[\log \mathrm{M} / \mathrm{M} 1 / \log$ $\mathrm{M} / \mathrm{M} 2]$ ratio and PMA $(\mathrm{r} 2=-0.26,95 \% \mathrm{CI}$ for $\mathrm{r}=-0.76$ to -0.13 ) was observed.

Conclusions: PMA is the most important variable observed in the maturational changes of both in vivo CYP2D6 and CYP3A4 activity [1] Compared to developmental changes in CYP2D6 activity, CYP3A4 activity is relatively delayed in the first months of life [2]. However, the overall weak correlations reflect that PMA only in part explains the interindividual variability observed. [1] Allegaert K et al. Eur J Clin Pharmacol 2005;61:837-42[2] Allegaert $\mathrm{K}$ et al. Int $\mathrm{J}$ Clin Pharmacol Toxicol (in press).

\section{URINARY INFECTIONS IN CHILDREN - ETHIOLOGY AND TREATMENT}

I Timovski, V Angelovska, J Markov, V Timovski, S Naunova

Department of Pediatrics, Military Hospital, Skopje, Macedonia

Background and Aims: Urinary infections are frequent problem in the pediatric practice. During every unclear febrile condition we should consider the possibility of a urinary infection. Aim: On time diagnosing and 
treatment of the urinary infections for on time prevention of damaging the kidney function.

Methods: Processed data of 630 children age $0-18$ years in a period of 2 years. Analytical and descriptive methods were used.

Results: Urinoculture is taken from 259 children and 74 children were positive. Echerichia coli was found in 34 children (45,94\%), E.Coli haemolytica with 15 children $(20,7 \%)$, Proteus mirabilis is isolated with 11 children $(14,8 \%)$ and in some cases Klebsiela and Pseudomonas aeruginosa were isolated. Recidivicious infections were found with 17 children $(22,9 \%)$, out of which 3 children were diagnosed with VUR. The children were treated with Cefixime, Amoxiciline+Clavulonic acid and Trimetoprim + Sulfonamid depending from the antibiogram. With all the children we reached negativization of the urinocultures and the lab results while the children with VUR are under regular nephrologic control of the pediatric clinics.

Conclusions: 1. Every febrile condition deserves routine analyses of the urine, with on time diagnosing. 2. Every on time diagnosing and treatment successfully prevent the kidney damage.

\section{THE ROLE OF ACUTE PERITONEAL DIALYSIS IN PAEDIATRIC INTENSIVE CARE UNIT}

M Novak ${ }^{1}$, Z Puretic $^{2}$, J Slavicek ${ }^{2}$, M Cvitkovic ${ }^{1}$, $\mathrm{S}_{\text {Galic }}{ }^{1}, \mathrm{~V}$ Benjak ${ }^{1}$, B Filipovic ${ }^{1}$

${ }^{1}$ PICU, Department of Paediatrics, University Hospital Center Zagreb, Zagreb, Croatia, ${ }^{2}$ Department of

Dialysis, University Hospital Center Zagreb, Zagreb, Croatia

Background and Aims: The role of acute peritoneal dialysis (APD) in the treatment of critically ill children was assessed.

Methods: The records of children in whom APD was performed because of acute renal failure (ARF) in our PICU from 1997 to 2003 period were reviewed.

Results: Of 42 APD treated children, 10 had known preexisting renal disease, and 32 did not. Causes of ARF in 32 children without known preexisting renal disease included open-heart surgery for congenital heart disease repair (12 children), hemolytic-uremic syndrome (3 children), newborn sepsis with consequent MOSF (12 neonates), and miscellaneous (5 patients). Mean age of 32 children without known underlying renal disease was $30.91 \pm 39.353$ months (range 9 hours to $150 \mathrm{mo}$ ), and mean body weight (BW) was $12,272 \pm 12.075 \mathrm{~kg}$ (range from 1.97 to $55 \mathrm{~kg}$ ). Mean age of 10 children with known renal disease was $110 \pm 52.398$ months (range 24 to $192 \mathrm{mo}$ ), and mean $\mathrm{BW}$ was $23.25 \pm 13.875 \mathrm{~kg}$ (range 8 to $56 \mathrm{~kg}$ ). Total mortality rate was $45.23 \%$ (19 of 42 children died), but all children with previously known renal disease and all children with HUS survived.

Conclusions: PD remains a suitable modality choice for the treatment of children with ARF in the era of continuous renal replacement therapies, mainly in the youngest infants. Further studies are needed to better define the role of each modality in the pediatric ARF management.

\section{ULTRASOUND SCREENING FOR URINARY TRACT MALFORMATIONS IN FAMILY RELATIVES}

V Tasic ${ }^{1}$, S Zeynel $^{2}$, N Ristoska-Bojkovska ${ }^{1}$, Z Gucev $^{1}$

${ }^{1}$ Children's Hospital, Clinical Center, Skopje, Macedonia, ${ }^{2}$ Medical School, University St. Cyrilus and Methodius, Skopje, Macedonia

Background and Aims: There is growing evidence that urinary tract malformation (UTM) have genetic basis. The aim of this study was to evaluate prevalence of UTM in families of index children with established diagnosis of UTM.

Methods: One hundred and nineteen families agreed to participate in this study. The diagnosis of UTM in index patients was established with ultrasound, radionuclide and X-ray studies. Available family relatives were investigated non-invasively with ultrasound scanning. If serious abnormality was detected by US, they were referred to their physician for further work up.

Results: There were 300 relatives (111 mothers, 103 fathers, 38 male siblings and 48 female siblings). In 42 families at least one relative was found to have UTM $(35.3 \%)$. Among 300 relatives there were 50 with UTM $(16.7 \%)$. The prevalence of UTM in parents [35 out of $214(16.3 \%)$ ] did not statistically differ from that in siblings [15 out of $86(17.4 \%)], \mathrm{P}>0.05$. The most common UTM's among family relatives were: duplex anomalies, simple cysts and hypoplastic kidneys. One parent had terminal uremia. Serious UTM in relatives were considered if there was need for surgical/conservative treatment or strict follow up (16 out of 50).

Conclusions: Ultrasound scanning is useful noninvasive screening tool for detection of UTM among family relatives of index children with diagnosed UTM. The true prevalence of UTM in relatives is most likely higher than $16.7 \%$ since invasive studies were not performed due to ethical limitations.

\section{EVALUATION AND ACCURACY OF CYSTATIN C TO ESTIMATE GLOMERULAR FILTRATION AFTER CARDIAC SURGERY}

J C López-Menchero ${ }^{1}$, A Alcaraz ${ }^{1}$, C Romero ${ }^{2}$, $\mathrm{M} \mathrm{D} \mathrm{Morales}^{3}$, A Luque ${ }^{3}$, P Blanco ${ }^{2}$, M J Santiago ${ }^{1}$, G Brochet ${ }^{4}$

${ }^{I}$ Pediatric Intensive Care Unit, Hospital General Universitario Gregorio Marañón, Madrid, Spain ${ }^{2}$ Department of Clinical Chemistry, Hospital General Universitario Gregorio Marañón, Madrid, Spain ${ }^{3}$ Department of Pediatric Nephrology, Hospital General Universitario Gregorio Marañón, Madrid, Spain ${ }^{4}$ Department of Pediatric Cardiac Surgery, Hospital General Universitario Gregorio Marañón, Madrid, Spain

Background and Aims: Assessment of renal function by glomerular filtration rate (GFR) is essential in the evaluation of the critically ill pediatric patient. The objectives of this study were: to compare plasma Cystatin C (CysC), Schwartz's Formula (CcrFS) and Creatinine clearance (Ccr24) for the estimation of GFR 
and to asses the efficacy of $\mathrm{CysC}$ in quantifying significant changes in renal function.

Methods: Prospective observational study in 64 consecutive children admitted to the pediatric intensive care unit (PICU) after cardiac surgery. $\mathrm{Cr}$, CysC, and $24 \mathrm{~h}$ urinary creatinine were determined on the second and fourth day after admission. Cr was measured by Jaffé's method and $\mathrm{CysC}$ by an automated inmunonephelometric assay (Dade Behring). GFR was estimated by Schwartz's formula, by Ccr 24 and by a equation based on CysC: $(\mathrm{FGcysc}=4.32+80.35 / \mathrm{CysC}$ ). Diuresis, furosemide administration or necessity of continuous hemofiltration (HF) were also recorded. Renal impairment was defined as 50\% decrease in GFR assessed by CcrFS. Correlation between different methods was evaluated by Spearman rank correlation and sensitivity by ROC curve analysis.

Results: FGcysc was significantly correlated with $\mathrm{CcrFs}$ $(\mathrm{r}=0.82)$ and $\mathrm{CCr} 24 \quad(\mathrm{r}=0.77)$. CysC $(\mathrm{AUC}=0.90)$ showed similar sensitivity to $\mathrm{CCrFS}(\mathrm{AUC}=0.84)$ and to $\mathrm{CCr} 24(0.84)$ in the detection of a decrease of GFR $>50 \%$. But CysC (AUC $=0.90)$ sensitivity to predict the necessity of HF was better than the exhibited by $\mathrm{CCr} 24$ $(\mathrm{AUC}=0.75)$ and $\mathrm{CCrFs}(\mathrm{AUC}=0.70)$.

Conclusions: $\mathrm{CysC}$ and $\mathrm{FGcysc}$ are closely correlated with GFR estimated by both CCrFS and $\mathrm{CCr} 24$ in critically ill patients. CysC proved to be a sensitive and specific biomarker to detect renal dysfunction after cardiac surgery.

\section{NEWLY DIAGNOSED UREA CYCLE DEFECT IN FIFTEEN YEAR OLD ENCEPHALOPATHIC PATIENT}

N Mettauer Lenz ${ }^{1}$, M Peters ${ }^{1}$, C Pierce ${ }^{1}$, P Clayton ${ }^{2}$, H Mundy $^{2}$

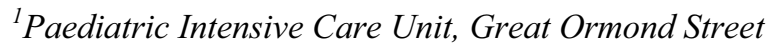
Hospital, London, UK, ${ }^{2}$ Department of Metabolic Medicine, Great Ormond Street Hospital, London, UK

Background and Aims: We report of a fifteen year old patient with developmental delay, auto-selective vegetarian diet and seizures in his past history who was admitted with the diagnosis of status epilepticus. Subsequently he was diagnosed to have hyperammonaemic encephalopathy due to an urea cycle defect.

Methods: A 15 year old boy was referred due to progressive lethargy and prolonged seizures after having been pyrexial for one week. He was treated with anticonvulsants and started on antimicrobial treatment for meningitis cover. His CT Head showed no abnormalities. His past medical history revealed global development delay and seizures. He had a dairy and egg allergy diagnosed and is currently living on an autoselective vegetarian diet.

Results: 24 hours after stopping sedation the patient remained comatosed with abnormal posturing and abnormal tone. An EEG showed severe non-specific encephalopathy. A metabolic screen revealed a hyperammonaemia which together with his history of protein avoidance and developmental delay suggested a urea cycle defect. Treatment consisted of haemofiltration, nitrogen scavengers and arginine supplementation. Neurologically the patient improved to a pre-admission mental state within the next week. Plasma amino acids showed elevated citrulline and argininoaccinic acid which prompted the diagnosis of argininosuccinic aciduria. This diagnosis was confirmed by enzyme analysis.

Conclusions: Metabolic disorders should be included in the diffential diagnosis of encephalopathic patients regardless of the patient's age. This case demonstrates how important a good history is, as this patient showed multiple signs and symptoms indicating a urea cycle defect. Early recognition and treatment might have avoided neurological handicap.

\section{TRANSIENT SEVERE METASTASTIC CALCIFICATION IN ACUTE RENAL FAILURE}

H Krymko ${ }^{1}$, H Shalev ${ }^{1}$, S Agronovich ${ }^{2}$, D Landau ${ }^{1}$

${ }^{1}$ Department of Pediatrics, Soroka Medical Center, Ben Gurion University, Beer Sheva, Israel, ${ }^{2}$ Department of Nuclear Medicine, Soroka Medical Center, Ben Gurion University, Beer Sheva, Israel

Background and Aims: Metastatic calcification (MC) (or calcific uremic arteriolopathy) is the most common extra-osseous calcification in end stage renal disease. Ulceration and necrosis of involved areas may carry a high mortality rate. $\mathrm{MC}$ has been rarely described as a complication of short-term acute renal failure and the therapeutic role of sodium thiosulfate has been seldom reported.

Methods: A 10-month-old male developed acute renal failure due to atypical hemolytic uremic syndrome and needed acute peritoneal dialysis, using regular $\mathrm{Ca}(3.5$ meq/L) dialysate. Intravenous $\mathrm{Ca}$ was given for hypocalcemia and depressed myocardial function. High serum $\mathrm{Ca}-\mathrm{P}$ products values $(>70 \mathrm{mg} 2 / \mathrm{dl} 2)$ were controlled with diet, enteral calcium salts and $1-\alpha-(\mathrm{OH})$ D3.

Results: On hospital day 10, a painful and swollen left arm was found. Radiographies and bone scan revealed diffuse calcification and MDP uptake in the muscles around the humerus and axilla. Dialysis solution was switched to low-calcium and later stopped, as well as enteral calcium salts. Intravenous sodium thiosulfate $(100 \mathrm{mg} / \mathrm{kg} / \mathrm{day})$ was given for 12 days. Improvement in arm movement and resolution of local pain were seen after 6 weeks. X- ray and bone scan revealed resolution of MC.

Conclusions: MC, a known complication of prolonged ESRD, is herein described for the first time in the setup of acute renal failure. Sodium thiosulfate (in addition to the withdrawal of enteral calcium and vitamin D) was associated with the resolution of MC. The exact duration of this treatment in acute renal failure is unclear.

\section{SUCCESSFUL MANAGEMENT OF POST- DIARRHEA HEMOLYTIC UREMIC SYNDROME BY CONTINUOUS VENO-VENOUS HEMODIAFILTRATION: ABOUT 3 CASES}

J Papadopoulos ${ }^{1}$, C Heymans ${ }^{2}$, W Marion ${ }^{1}$, B Debont ${ }^{1}$ 
${ }^{1}$ Department of Pediatric Intensive Care, Hopital de Jolimont, Haine St Paul, Belgium, ${ }^{2}$ Department of Nephrology, Hopital de Jolimont, Haine St Paul, Belgium

Background and Aims: Hemolytic Uremic Syndrome (HUS) is the most common cause of acute renal failure (ARF) in young children. Anuric post-diarrhea HUS has a good outcome when treated aggressively i.e. with conventional renal replacement therapy (RRT). Continuous Veno-Venous HemoDiaFiltration (CVVHDF) is an alternative RRT to peritoneal dialysis and we describe 3 cases treated successfully with CVVHDF.

Methods: Cases series.

Results: At 11 months, 21/2 years and $41 / 2$ years children have been admitted in our pediatric intensive care unit with typical sporadic post-diarrhea HUS. All three developed, 5 to 15 days after an acute gastro-enteritis, an ARF with moderate hypertension, uremia, hemolytic anemia with schizocytosis, thrombopenia and anuria despite medical treatment (normal saline loading and RBC transfusion, with continuous IV diuretic therapy). We didn't find any causal microbial agent for these patients. All three benefited of CVVHDF by a double lumen catheter with heparin anticoagulation despite of low platelets account. Hypertension, fluid overload and metabolic disorders were corrected in the first 24 hours, for a mean duration of CRRT of 53 hours, without any complication. All three patients recuperated a normal renal function without hypertension and one had a residual proteinuria.

Conclusions: Continuous veno-venous hemodiafiltration is a safe technique for renal replacement therapy in post-diarrhea acquired microangiopathy. We prefer CVVHDF to peritoneal dialysis because it is a more effective therapy for the management of hydroelectrolytic balance and for the optimal control of intakes (total fluids, proteins and calories).

\section{DIAGNOSIS AND MANAGEMENT OF URINARY TRACT INFECTION (UTI) IN OUR MEDIUM DURING THE FIRST YEAR OF LIFE}

I Ledesma, L M Rodríguez, S Lapeña, B Herrero, M C De Fuentes, M T Fernández, R Álvarez

\section{Servicio de Pediatria, Hospital de León, León, Spain}

Background and Aims: To know the frequency with which UTI is diagnosed in our medium during the first year of life, the method used for its diagnosis and the treatment and imaging studies recommended.

Methods: The parents of 472 children born in our hospital were surveyed to find out whether the children had been diagnosed with UTI in their first year. If the answer was positive, they were questioned about the age of the child at diagnosis, the use of uroculture, the method to collect urine samples, whether they needed to be hospitalized and the form of treatment, and what imaging studies were practiced.

Results: 24 children had been diagnosed with UTI during their first year. 5 of those had been diagnosed during their first month. All had been diagnosed through uroculture and the method used to collect urine samples was transurethral catheterization in 15 cases and collecting bag in the remaining. 16 children were hospitalized and the treatment was exclusively oral in 9 cases. Ultrasonography was used with 23 of the 24 patients, and cystourethrography wasn't performed in $1 / 3$ of the children. Scintigraphy was carried out in 18 of the 24 cases.

Conclusions: There's a very high incidence of UTI in our medium during the first year. However, in more than a third of the cases, the diagnosis is not reached through reliable samples. The percentage of children hospitalized and with parenteral treatment is high, although, $1 / 3$ of the patients didn't undergo some of the studies recommended for infants with UTI.

\section{ORNITHINE TRANSCARBAMYLASE DEFICIENCY (OTC) IN GIRLS: LONG-TERM OUTCOME FROM CHILDHOOD TO ADOLESCENSE}

D Esteban, M Eril, A Rodriguez-Palmero, G Pintos, C Rodrigo

Hospital Universitari Germans Trias I Pujol, Badalona, Barcelona, Spain

Background and Aims: Ornithine transcarbamylase (OTC) deficiency, is a chronic X-linked disease with hyperammonemic outbreaks featuring seizures and encephalopathy.

Methods: Four female cases are reported herein.

Results: 1. A full-term female with hyperammonemic coma (580 micromol/L) requiring peritoneal dialysis. Several outbreaks with common infections despite early treatment. At 16-year-old, she was admitted because of generalized seizure with moderate hyperammonemia. Nowadays, she is 18-year-old and she has important mental retardation. 2. A 23-month-old child with Reyelike syndrome (ammonia: $300 \mathrm{micromol} / \mathrm{L}$ ) requiring $\mathrm{K}$ vitamin. Increased levels of urine orotic, glutammine and alanine. Clinical improvement with citruline, sodium benzoate, carnitine and hypoproteic diet. Acute relapses occurred during upper airway infections. After eleven years follow-up, she shows moderate mental retardation and behavioral problems. 3. A 3-year-old girl with hyperammonemic coma (568 micromol/L). Previous history of restlessness, hypotonia and monthly vomiting since 18 months. Plasmatic levels of glutamine and urine orotate were increased. Hyperammonemic outbursts during upper airway infections occurred until she underwent tonsilectomy. Since then she has held a normal mental development and good schooling. 4. A 3year-old infant was admitted because of vomiting, headache, ataxy and lethargy. Hyperammonemia of 539 micromol/L. Increased ammonia and urine orotate after protein overload. Relapses occurred during catabolic illnesses. Nowadays her IQ is on the lower level of the normal range.

Conclusions: OTC deficiency usually has a more benign course in women. Diagnosis and early treatment with limited protein intake and ammonia removers (phenilbutirate, sodium benzoate), along with a strict follow-up, imply an increase of the survival. Nevertheless, eventual relapses can limit neuropsychological development. 


\section{HYPERCALCUIRIA AND URINARY TRACT INFECTION}

\section{S Sadeghi Bojd}

Department of Pediatrics, Ali Ebne Abitaleb Hospital, Zahedan Medical Sciences, Zahedan, Iran

Background and Aims: Urinary tract infection is one of the most common and most important of infection complaints in children. Idiopathic hypercalciuria is a common metabolic abnormality in children of all ages. There is evidence of an association of idiopathic hypercalciuria with nephrolithiasis hematuria and osteoporosis. Hypercalciuria is believed to be the cause of variety of urinary tract complaints in children such as UTI. The aim of this study to evaluate the Zahedan. Prevalence of hypercalciuria in children with UTI in children hospital in Iran.

Methods: We studied seventy children (3 month - 15 years) with urinary tract infection one month after recovery and negative urine Culture and compared with control group (normal children) without UTI in the same range of age for hypercalciuria.

Results: In 70 patients with urinary tract $(\mathrm{F} / \mathrm{M}=1)$ $34.2 \%$ had idiopathic hypercalciuria and in control group $(48.5 \%$ female- $51.9 \%$ male $) \quad 14.2 \%$ had hypercalciuria. Idiopathic hypercalciuria were the most common in 1- 5 years old group and females. The patients with hypercalciuria often had recurrent UTI and upper urinary tract infection.

Conclusions: We conclude that hypercalciuria may be important contributing factor in urinary tract infection and almost often it ameliorate after treatment. The severe inflammation of the kidney may explain this complication ca oxalate crystals may damage uroepithial and it suspiteble the patient for urinary tract infection.

\section{PREDICTION OF VESICOURETERAL REFLUX IN INFANTS AFTER FIRST FEBRILE URINARY TRACT INFECTION}

\author{
B Orive ${ }^{1}, \mathrm{~J}_{\text {Elorz }}{ }^{2}$, A Rodriguez ${ }^{1}$ \\ ${ }^{I}$ Department of Pediatrics, Hospital Txagorritxu, \\ Vitoria, Spain, ${ }^{2}$ Department of Pediatrics, Hospital \\ Basurto, Bilbao, Spain
}

Background and Aims: Vesicoureteral reflux is a common anomaly in children with urinary tract infection. The presence of reflux, especially high grade reflux, is the most important risk of definitive renal scarring. Controversy remains if now days, with the routine use of prenatal echography, renal ultrasound has additional diagnostic value in the study of uncomplicated urinary tract infection. We analysed clinical, biochemical and imaging parameters associated with vesicoureteral reflux to determine the adjusted value of renal ultrasound.

Methods: Prospective study of 383 infants with first episode of febrile ITU. There were analysed as predictor variables of vesicoureteral reflux: Age, sex, days of fever after therapy, leucocytosis (>15000), CRP (> 20 $\mathrm{mg} / \mathrm{l}$ ), and ultrasonography. Variables that result significant in univariate analysis with $\mathrm{p}<0,15$ were introduced in a logistic regression. Adjusted OR of significant variables with $\mathrm{p}<0,1$ were used in a Receiver Operating Characteristic Curve Analysis

Results: None had obstructive uropathy. In univariant analysis only age more than a year, days of fever after therapy and abnormal ultrasound were significantly associated with vecoureteral reflux, $p<0,01, p 0,001$ and $\mathrm{p}<0,001$ respectively. In the logistic regression all of them continued significant, adjusted OR (CI 95\%) 1,78 $(1,04-3,04), 1,72(1,06-2,83)$ and 3,23 $(1,9-5,47)$. Area under curve was $0,693(0,634-0,753)$. The model was poorly predictive: Sensitivity $26 \%$, specificity $91 \%$, LR $+3,02$, LR- 0,81 , probability post positive test $53 \%$ and probability post negative test $28 \%$.

Conclusions: Predictive value for vesicoureteral reflux of clinical variables and renal ultrasound are low, cystouretrography is obligatory in the study of a infant with first urinary tract infection.

\section{DOES SURGERY IMPROVE RENAL FUNCTION IN PELVIURETERIC JUNCTION OBSTRUCTION?}

B Orive ${ }^{1}, \mathrm{~J} \mathrm{Elorz}^{2}, \mathrm{~J}$ Jiménez ${ }^{3}$, A Villanueva ${ }^{3}$

${ }^{l}$ Department of Pediatrics, Hospital Txagorritxu, Vitoria, Spain, ${ }^{2}$ Department of Pediatrics, Hospital Basurto, Bilbao, Spain, ${ }^{3}$ Department of Pediatric Surgery, Hospital Donostia, San Sebastian, Spain

Background and Aims: Ureteropelvic junction obstruction is the most common urinary tract obstruction in children. The aim of the present study was to evaluate the renal function before and after Anderson-Hynes pyeloplasty in children diagnosed of ureteropelvic junction obstruction.

Methods: We retrospectively reviewed two groups of patients with unilateral ureteropelvic obstuction divided by mode of presentation. Group 1: 31 children diagnosed by prenatal ultrasound. Group 2: 11 patients with symptomatic hydronephrosis. All prenatal diagnosis had obstuctive pattern on 99 TC Mercaptoacetyltriglycine Scintigraphy normalized after pyeloplasty. Descriptive statistic is expressed as median and interquartile range. Age for surgery was : 0,83 years $(0,38-1,46)$ and 8,45 years $(6,71-11,83)$ (median and interquartile) respectively. The paired Wilcoxon and Spearman rank test for nonparametric data were used for statistical analysis.

Results: There was statistical differences between renal function before and after pyeloplasty: Prenatal $40 \%$ $(33 \%-47 \%), 45 \% \quad(36 \%-50 \%) \quad \mathrm{p}<0,05$. Symptomatic $40 \% \quad(36 \%-46 \%), \quad 45 \% \quad(40 \%-47 \%) \quad \mathrm{p}<0,01$. No correlation was found between age of surgery and improvement in renal function $\mathrm{p} 0,87$. A negative correlation (correlation coefficient $-0,77, p<0,01$ ) was detected among renal function prior surgery and improvement after on symptomatic group. Prenatal group didn't show a significant correlation.

Conclusions: Our follow up of obstruction prenatally diagnosed permitted the observation of this children in a critical interval, avoiding permanent loss of renal function and averting unnecessary surgical procedures. We didn't observe any correlation between age of surgery and improvement of renal function, that in fact showed little amelioration, suggesting that renal injury 
is probably present in utero. The symptomatic group evidenced amelioration in less severe affected patients.

\section{DOES RENAL ULTRASONOGRAPHY PREDICT VESICOURETERAL REFLUX IN INFANTS WITH FEBRILE URINARY TRACT INFECTION?}

\author{
B Orive ${ }^{1}, \mathrm{~J}_{\text {Elorz }}{ }^{2}$, A Rodriguez ${ }^{1}$, I Hualde ${ }^{1}$ \\ ${ }^{I}$ Department of Pediatrics, Hospital Txagorritxu, \\ Vitoria, Spain, ${ }^{2}$ Department of Pediatrics, Hospital \\ Basurto, Bilbao, Spain
}

Background and Aims: Guidelines from the American Academy of Pediatrics recommend renal ultrasound and voiding cystouretrography for young children after first urinary tract infection. The non-invasive nature, the lack of radiation, and the low cost of renal ultrasound have made it an ideal tool for the initial screening investigation in children with febrile urinary tract infection. We evaluate the sensitivity and predictive value of renal ultrasound for vesicoureteral reflux in the diagnostic workup of urinary tract infections within the first 24 months of life.

Methods: Prospective study of 383 infants hospitalized with first febrile urinary tract infection. All of them had a normal prenatal ultrasound. $141(36,8 \%)$ were males and $242(63,2 \%)$ were females. $291(76 \%)$ were younger than a year. Ultrasonography was performed at hospitalization and cystography was carried out four weeks later. Analysis of frequencies was done with the chi square test. Kendall's Tau-b was used as a measure of ordinal association. Sensitivity, specificity and predictive values for vesicoureteral reflux were calculated.

Results: 124 infants $(27,2 \%)$ had vesicoureteral reflux. $91(23,8 \%)$ had small reflux (1-3) and $13(3,4 \%)$ had high grade (4-5). Ultrasonography was abnormal in 86 $(22,5 \%)$. Pathological ultrasound was more frequent in the reflux group $(p<0,001)$. Whereas, there was a mild ordinal association between grade of reflux and ultrasound result; Kendall's Tau-b 0,269 $(\mathrm{p}<0,001)$. Sensitivity and specificity were $39 \%$ and $84 \%$ respectively (PPV 48\%, PNV 79\%, LR -0,72 and Posttest negative probability $21 \%$ ).

Conclusions: Renal ultrasound is not a reliable procedure to predict vesicoureteral reflux in infants.

\section{NEPHROLITIASIS IN A PATIENT WITH CYSTIC FIBROSIS}

B Orive $^{1}, \mathrm{~J}$ Elorz ${ }^{2}, \mathrm{C}$ Vázquez ${ }^{2}$, A Mari ${ }^{1}$

${ }^{I}$ Department of Pediatrics, Hospital Txagorritxu, Vitoria, Spain, ${ }^{2}$ Department of Pediatric Pulmonology, Hospital Cruces, Bilbao, Spain

Background and Aims: Cystic fibrosis (CF) is caused by mutations in the $\mathrm{CF}$ transmembrane conductance regulator (CFTR) gene. Defects in the CFTR gene cause abnormal chloride conductance across the apical membrane of epithelial cells. It results in progressive lung disease and also affects different organs. With longer life expectancy other complications of $\mathrm{CF}$ have become more apparent.
Methods: We present a patient with $\mathrm{CF}$ and symptomatic nephrolithiasis

Results: Several stones were evident in both kidneys. Hyperoxaluria and Hypocitraturia were evidenced in the twenty four urine sample, $141 \mathrm{mg} / 24 \mathrm{~h} / 1,73 \mathrm{~m} 2$ and $206 \mathrm{mg} / 24 \mathrm{~h} / 1,73 \mathrm{~m} 2$ (177 mg citrate/gr creatinine), respectively.

Conclusions: Nephrolithiasis should be present in the differential diagnosis of a patient with CF who has abdominal pain and urinary excretion of oxalate and citrate should be investigated.

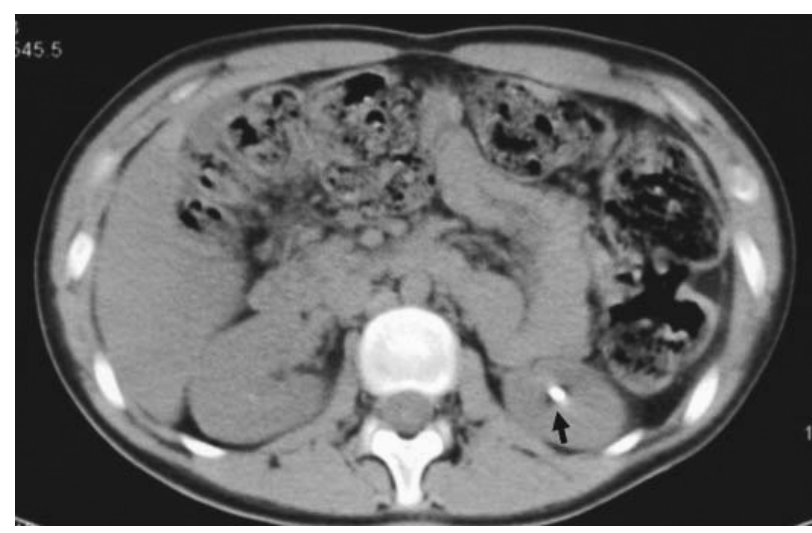

CPR AND UNCONTROLLED TIDAL VOLUMES (TV) IN THE DELIVERY ROOM (DR) INCREASED RISK FOR BRONCHOPULMONARY DYSPLASIA (BPD) IN VERY PRETERM INFANTS $<1250 G$ (VLBW)?

A Castillo, R Deulofeut, A Sola

Department of Neonatal-Perinatal Medicine, Emory University, Atlanta, GA, USA

Background and Aims: BPD is multi-factorial. It is unknown if VLBW likely to be exposed to brief periods of "uncontrolled" ventilation, like those requiring "full" DR-cardiopulmonary resuscitation (DR-CPR), are at a higher risk for BPD. The objective was to determine if DR-CPR is associated with BPD in VLBW.

Methods: Analysis of a prospectively collected database of all VLBW infants admitted to two Emory University NICU's from 1/00-12/04 that survived to $\geq 36$ weeks post-conceptional age (PCA). Exclusion criteria were major congenital malformations. DR-CPR was defined as intubation and manual ventilation with chest compression and/or epinephrine use in the DR. The primary outcome variable was BPD, defined as the need for oxygen at 36 weeks PCA. Statistical analysis included bivariate analyses and multivariate logistic regression.

Results: 443 infants met enrollment criteria and 57 $(13 \%)$ were exposed to DR-CPR. Gestational age [26.7 $\pm 2.1 \mathrm{vs} .27 .3 \pm 2.3 ; \mathrm{p}=0.498]$, were similar between DR-CPR and non DR-CPR groups. The proportion of infants with BPD (61\%vs.38\%:p=0.001) was significantly higher in the infants treated with DR-CPR than in the infants in the non DR-CPR group[OR=2.7 $(95 \% \mathrm{CI}=01.51-4.73)]$ and than the intubated infants without DR-CPR (61\%vs.45\%:p=0.019)[OR=1.92 (95\%CI=1.07-3.43)] Additionally, these infants who were intubated but did not receive DR-CPR had higher 
rate of $\mathrm{BPD}(45 \%$ vs. $16 \% ; \mathrm{p}=0.000)$ than those who were not intubated $[\mathrm{OR}=4.34(95 \% \mathrm{CI}=2.45-7.68)]$.

Conclusions: DR-CPR is associated with a higher risk for BPD in VLBW infants. This lends support to the hypothesis that a brief period of artificial poorly controlled delivery of TV in the DR may be sufficient to produce significant lung injury and predispose to BPD.

\section{THE ROUTINE USE OF NASAL CONTINUOUS POSITIVE AIRWAY PRESSURE AS PRIMARY SUPPORT IN RESPIRATORY DISTRESS SYNDROME MAY RESULT IN A SIGNIFICANT DELAY IN THE ADMINISTRATION OF SYNTHETIC SURFACTANT}

\section{J Dewhurst, L Phillips, D Roghan \\ Department of Paediatrics, Countess of Chester Hospital NHS Trust, Chester, Cheshire, UK}

Background and Aims: In infants judged to be at risk of developing RDS, early prophylactic administration of synthetic surfactant has been demonstrated to improve clinical outcome. Increasingly NCPAP is being used in such infants for respiratory support. It was our unit's policy that babies who commenced NCPAP did not receive prophylactic surfactant. The aims of this study were to identify factors that could predict which babies would 'fail' on NCPAP support and need subsequent surfactant administration and ventilation.

Methods: Retrospective study of 70 case notes picked at random, of babies born between May 03 to May 05 at less than 33 completed weeks gestation, were reviewed and data collected on their early respiratory course. This data was entered into Microsoft Excel and analysed. Results: Sixteen (23\%) babies received NCPAP as primary support for RDS, with $6(38 \%)$ requiring subsequent ventilation. Birth weight differed between the CPAP successes and failures (1519g vs 1283g, p value $=0.1)$. All babies over $1400 \mathrm{~g}$ succeeded on CPAP. $60 \%$ of babies under $1400 \mathrm{~g}$ required subsequent ventilation with surfactant administration delayed by on average 9.8 hours. There was no difference in sex, gerstation, Apgar score or complication rates between the two groups.

Conclusions: Using NCPAP as primary support for respiratory distress syndrome in infants less than $1400 \mathrm{~g}$ should be undertaken with caution as it may result in a delay of surfactant administration. Further, larger studies are required to identify factors that predict cpap success or failure.

\section{PREDICTIVE VALUE OF DIFFERENT PARAMETERS FOR OPTIMAL ENDOTRACHEAL TUBE POSITION IN NEONATES}

\section{F A M van den Dungen, M L J Brouwer, F Meulink, K P Bach, J I M L Verbeke, R M van Elburg \\ Department of Pediatrics, Subdivision of Neonatology, VU University Medical Center, Amsterdam, The Netherlands}

Background and Aims: Simple parameters to estimate the optimal endotracheal tube (ETT) position are warranted as chest radiography is considered the gold standard for ETT position. To estimate the optimal ETT position in AGA and SGA neonates, we studied both retrospectively and prospectively the predictive value of birthweight, weight at intubation, and birth length. In addition, we studied only prospectively sternum length, foot length and nose-tragus distance.

Methods: Neonates who were nasotracheally intubated were studied retrospectively and prospectively. Values of birthweight, weight at intubation, birth length, sternum length, foot length and nose-tragus distance were measured. After correction for neutral head position, optimal ETT position in chest radiograph was defined as the position of the tip between $5 \mathrm{~mm}$ above tracheal bifurcation and the level of the first thoracic vertebra. Only the first intubation in each patient was used for statistical analysis.

Results: In the retrospective and prospective study 28 and 42 neonates were included respectively (gestational age 26-42 weeks, birthweight 609-4680 grams). Birthweight correlated best with optimal ETT position $(\mathrm{r} 2=0,82)$. The best intra-class correlation was tubedepth $(\mathrm{mm})=11,71 *$ birth weight $(\mathrm{kg})+70,78(\mathrm{r} 2=0,90)$. Predictive values for optimal ETT position in AGA (AGA + SGA) infants were birthweight 73\% (69\%), weight at intubation $62 \%(57 \%)$, birth length $69 \%$ (61\%), foot length $63 \%(66 \%)$, sternum length $66 \%$ (60\%), and nose-tragus distance 60\% (63\%).

Conclusions: Birthweight is the best predictor for optimal ETT position in nasotracheally intubated neonates. However, chest radiography remains necessary to check for the optimal position.

\section{BRONCHOPULMONARY DYSPLASIA AND VENTILATION MODE}

T Farstad, M Olsen

Department of Neonatology, Akershus University Hospital, Norbyhagen, Norway

Background and Aims: Major risk factors in Bronchopulmonary Dysplasia (BPD) are prematurity, barotrauma, oxygen therapy, and perinatal infections. The aim of this study was to investigate how mechanical ventilation and early nasal CPAP (NCPAP) correlate with incidence of BPD.

Methods: Infants with Birth Weight $(\mathrm{BW})<1500 \mathrm{~g}$ and Gestation Age (GA) < 34 weeks were identified from prospective NICU records. Data were analyzed to predict risk factors associated with occurrence of BPD. Diagnosis was based on radiological findings and oxygen dependency at postconceptional age of 36 weeks.

Results: Mean (SD) BW was 1020 (242)g and GA 27(2) wks for the whole group. $18 \%$ of infants fulfilled criteria for BPD. 28\% received only NCPAP, $68 \%$ were intubated and received surfactant within one hour after birth.

Conclusions: There seems to be a correlation between need for mechanical ventilation for longer than 5 days and BPD. NCPAP for less than 14 days seems to protect against BPD However, early surfactant treatment is not associated with lower BPD incidence. 


\begin{tabular}{|l|l|l|l|}
\hline & Non- $\mathrm{BPD} \mathrm{n}=114$ & $\mathrm{BPD} \mathrm{n}=26$ & $\mathrm{P}$ \\
\hline Male/Female & $59 / 55$ & $18 / 8$ & $\mathrm{NS}$ \\
\hline Birth Weight (g) & $853 \pm 207$ & $1059 \pm 233$ & $\mathrm{NS}$ \\
\hline Gestation Age (wks) & $27.2 \pm 2.1$ & $28.1 \pm 1.9$ & $\mathrm{NS}$ \\
\hline Surfactant treatment (\%) & $70(61)$ & $22(85)$ & $<0.05$ \\
\hline None mechanical ventilation (\%) & $40(35)$ & $4(15)$ & $<0.05$ \\
\hline Mechanical ventilation 1-5 days (\%) & $46(63.8)$ & $7(31.8)$ & $<0.05$ \\
\hline NCPAP $\leq 14$ days (\%) & $32(29)$ & $2(8)$ & $<0.05$ \\
\hline
\end{tabular}

\section{HEALTH CARE ASSOCIATED INFECTION IN PAEDIATRIC PATIENTS ON EXTRACORPOREAL LIFE SUPPORT: IMPACT OF MULTIDISCIPLINARY SURVEILLANCE}

\author{
K L Brown ${ }^{1}$, D A Ridout ${ }^{2}$, M Shaw ${ }^{1}$, I Dodkins ${ }^{1}$, \\ M O'Callaghan ${ }^{1}$, L Smith ${ }^{1}$, A P Goldman ${ }^{1}$, \\ J C Hartley ${ }^{1}$
}

${ }^{1}$ Great Ormond Street Hospital for Sick Children, London, UK, ${ }^{2}$ The Centre for Paediatric Epidemiology and Biostatistics, Institute of Child Health, London, UK

Background and Aims: Objective was to describe the use of a multi-disciplinary approach to sepsis surveillance and evaluate impact on outcome.

Methods: 215 children supported with ECMO January 1999 - December 2004 were included. A multidisciplinary team met monthly to evaluate cases of sepsis and update unit policies. Changes in practice were made at the end of 2001 in order to tackle a high rate of sepsis: 1) re-education, 2) introduction of electively preprimed ECMO circuits and 3) preference for neck rather than chest cannulation in cardiac patients. Prophylactic antibiotics were used for 24 hours for procedures.

Results: Over the study period, 39 children had 47 septic episodes, with a rate of 24.9 per 1000 ECMO days. Multiple logistic regression analyses indicated that infection was associated with duration of support (OR $1.24,95 \%$ CI $1.15,1.35$ per day) and case-type: closed versus open chest was protective in cardiac patients (OR $0.0895 \%$ CI $0.01,0.50$ ). Infection increased the odds of death by $2.01(95 \%$ CI $1.00,4.05)$ but this effect was less important than case-type and ECMO days. After policy changes, there was reduced sepsis in respiratory patients: neonates 28.0 to 6.6 and paediatrics 42.4 to 16.9 episodes per 1000 ECMO days. Despite policy changes, sepsis remained a problem in cardiac patients with open sternum: 65.1 per 1000 ECMO days.

Conclusions: ECMO support is high-risk for nosocomial infection, especially cardiac patients with open sternum for whom antibiotic prophylaxis is justified. Multi-disciplinary surveillance offers an excellent approach for quality improvement in this challenging field.

\section{MIDAZOLAM REQUIREMENTS ARE AGE DEPENDANT IN A PEDIATRIC INTENSIVE CARE UNIT}

D H de Gast-Bakker ${ }^{1}, S$ D van der Werff $^{1}$, R Sibarani-Ponsen ${ }^{1}$, E L Swart ${ }^{2}$, F B Plötz ${ }^{1}$

${ }^{I}$ Department of Pediatric Intensive Care, VU Medical Center, Amsterdam, The Netherlands

${ }^{2}$ Department of Pharmacology, VU Medical Center,

Amsterdam, The Netherlands
Background and Aims: Midazolam is one of the most widely used sedative agents in the pediatric intensive care unit (PICU). We tested the hypothesis that midazolam requirements during prolonged mechanical ventilation in critically ill children is age dependent.

Methods: Chart records of all children (28 days - 18 year) who were admitted to the PICU between January 1st 2002 and January 1st 2005 were retrospectively analyzed. Inclusion criteria were controlled mechanical ventilation for at least five days and initial sedation with midazolam. The total daily dosage per kilogram body weight of midazolam and other sedatives were calculated during the first five days. Statistical analysis was carried out by means of SPSS 11 .

Results: A total of 1186 children were admitted, of which fifty-eight children fulfilled the inclusion criteria. The children were divided into three age groups: 28 days - 1 year $(n=28), 1-4$ years $(n=16)$, older than 4 years $(n=14)$. Within two days after start of mechanical ventilation the children age 1-4 years received the maximum midazolam dosage $(0.3 \mathrm{mg} / \mathrm{kg} / \mathrm{hr})$. In addition, the total daily dosage of midazolam was higher at all days compared to the other two age groups. At day $5,14.3 \%$ of children 28 days -1 year and $7.1 \%$ of children older than 4 years could be adequately sedated with only midazolam, in contrast none of the children between 1-4 years.

Conclusions: Children between 1-4 years required more midazolam as compared to children who are younger or older and also required several sedative agents in order to achieve adequate sedation.

\section{CONGENITAL DIAPHRAGMATIC HERNIA - A 15 YEAR SURVEY}

E Santos, C Veiga, J Peixoto, L Carvalho, J F Neves

Paediatric Intensive Care Unit, Coimbra's Paediatric Hospital, Coimbra, Portugal

Background and Aims: Congenital Diaphragmatic Hernia (CDH) is a condition with a significant morbidity and mortality, with new treatment strategies assayed in the last two decades. Coimbra's Paediatric Intensive Care Unit (PICU) is the referral neonatal surgical center of Portugal's central region since the last 25 years. The authors report on the incidence of $\mathrm{CDH}$ in the region and the effect of delayed surgery on mortality.

Methods: A retrospective analysis of demographic data and prognostic risk factors were made including all the newborn with $\mathrm{CDH}$ admitted to PICU during the last 15 years. The newborns were submitted to a similar protocol management except for high frequency oscillatory ventilation (HFO) $(>1995)$ and inhaled nitric oxide (iNO) (> 1998).

Results: Sixty neonates had a diagnosis of $\mathrm{CDH}$, with an incidence of 2.2 cases/ 10000 life births. Forty one of them were submitted to conventional ventilatory support only, and 18 required HFO ventilation. Eight required in addition iNO. Seven newborns died before surgery ( 6 in the first 24 hours of life). Surgery was delayed in 33 of the 53 patients and no deaths occurred in this group. In the remaining 20 newborns, 5 died $(25 \% ; \mathrm{p}=0,005)$. The survival rate at discharge was $80 \%$. 
Conclusions: The low incidence of $\mathrm{CDH}$ in our region, might be underestimated by hidden mortality before admission in PICU. No mortality was observed in patients submitted to delayed surgery. The majority of deaths occurred in the first 24 hours, meaning the failure of achieving adequate pulmonary hypertension control.

\section{TWO RARE PRESENTATIONS OF CONGENITAL DIAPHRAGMATIC HERNIA}

A Fernandes ${ }^{1}, \mathrm{~S}$ Castro ${ }^{1}, \mathrm{C}$ Didelet ${ }^{1}, \mathrm{~A}$ Clington $\mathrm{Jr}^{2}$, H Sá Couto ${ }^{3}$, A Amador ${ }^{1}$, J Oliveira Santos ${ }^{4}$

${ }^{1}$ Pediatric Respiratory Diseases Outpatient Department, Nossa Senhora do Rosário Hospital, Barreiro, Portugal ${ }^{2}$ Departament of Neonatology, Dona Estefânia Pediatric Hospital, Lisbon, Portugal, ${ }^{3}$ Departament of Surgery, Dona Estefânia Pediatric Hospital, Lisbon, Portugal ${ }^{4}$ Pneumology Unit, Dona Estefânia Pediatric Hospital, Lisbon, Portugal

Background and Aims: Congenital diaphragmatic hernia $(\mathrm{CDH})$ is the result of incomplete fusion or hypoplasy of one or more of the embryonic components of diaphragm, with an estimated incidence of 1/2000 to $1 / 5000$ births. Morgagni hernia is the least common type of $\mathrm{CDH}$ and most are diagnosed late because patients can be asymptomatic or present with non-specific respiratory or gastrointestinal symptoms. Bochdaleck hernia is the most common type of $\mathrm{CDH}$ and, unlike Morgagni's, most are dignosed either antenatally or will present with respiratory distress in the first few hours of life. However, some present beyond the neonatal period, posing considerable diagnostic challenges. Chest radiograph is the first and most important diagnostic tool.

Methods: Two cases are reported of late presenting $\mathrm{CDH}$. The first is a Morgagni hernia detected in the chest radiograph of a four month old healthy female infant with first episode of respiratory distress syndrome and confirmed with toracoabdominal ultrasound and computed tomogram. The second is a Bochdaleck hernia diagnosed in a six month old (cronologic) preterm male infant in an abdominal ultrasound for gastroesophageal reflux research, during etiologic investigation of persistent respiratory distress syndrome.

Results: In both cases, the defect was repaired promptly after diagnosis, with full recovery of respiratory function and no recurrence in the follow-up.

Conclusions: We dicuss the diagnostic importance of chest radiograph in the first episode of respiratory distress that doesn't improve with appropriate theraputic measures, as the diagnostic possibility of respiratory congenital anatomic anomaly must always be considered in this instance.

\section{THREE DIFFERENT CLINICAL PRESENTATIONS OF THE SAME ASPIRATED MATERIAL}

A R Fernandes ${ }^{1}$, O Sedelnikova ${ }^{1}$, A J Amador ${ }^{1}$, J Oliveira Santos ${ }^{2}$

${ }^{I}$ Department of Pediatric Respiratory Diseases

Outpatient, Nossa Senhora do Rosário Hospital,
Barreiro, Portugal, ${ }^{2}$ Pneumology Unit, Dona Estefânia Pediatric Hospital, Lisbon, Portugal

Background and Aims: Foreign body aspiration is a serious situation which may result into respiratory distress, pulmonary lesion or even death. More than two thirds of foreign body aspiration cases occur among children under 3 years old and organic materials are the most commonly aspirated. Diagnosis is frequently difficult and delayed, as in about half of the cases the chocking episode is missed, there is a wide range of clinical presentation, which frequently mimics other illnesses, and none of the imaging methods employed in such cases are diagnostic. Bronchoscopy is frequently necessary for diagnosis and treatment. When diagnosis is delayed, complications of a retained foreign body such as unresolving pneumonia, lung abscess, recurrent haemoptysis and bronchiectasis may occur.

Methods: The authors present three cases of foreign body aspiration (sunflower seeds) in patients at different ages and with different clinical presentations; all had a delay in diagnosis ranging from days to months.

Results: All cases were diagnosed and treated with bronchoscopy, with immediate recovery from respiratory symptoms.

Conclusions: These cases demonstrate that a high index of suspicion and a careful history in a child with persistent/recurrent respiratory symptoms, along with bronchoscopy, are the cornerstones of diagnosis and treatment of foreign body in the airway.

\section{TRACHEO-ESOPHAGEAL FISTULA: IS CHRONIC PULMONARY DISEASE POSSIBLE?}

\section{Z Sarevska, M Maneva, L Stojanovska}

Institute for Respiratory Diseases in Children-Kozle, Skopje, Macedonia

Background and Aims: Tracheo-esophageal fistula (TEF) is congenital anomaly of two types: With and without atresio oesophagi. Late sequels are possible like bronchiectasis as result of recurrent respiratory infections or aspiration.

Methods: We would like to describe case of a boy who was born with atresio oesophagy and TEF, which were corrected with surgery, the second day after birth. After that, he had a great number of hospitalizations because of pneumonias e aspirationem, strydor, and cough. During the first year, oesophagus balloon dilatation was made. In this period, bronchoscopy exploration showed bronchomalatio, as a result of surgery. Because of recurrent symptoms, many investigations were made when a boy was 5 years old.

Results: Lab tests results (SE, blood count, immunoglobulins, sweet test, and other) were in regular values. Chest X-ray: bilateral patchy infiltrates, hyperinflation. Bronchoscopic findings: Tracheomalatio in middle part of trachea (surgery side); generally white mucus and like casts in bronchus of middle lobe. Perfusion scan: Left-hypoperfusio of posterior and latero-basal segments at the lower lobe; right subsegmental changes in postero-basal segment at the lower lobe. HRCT: Proximal part of trachea with membranous part thickness $(3-4 \mathrm{~cm})$, thickness of the bronchi wall for a middle and upper lobe. 
Conclusions: In our case, at this moment, chronic pulmonary disease is not evident. Our patient likes healthy. We proved only tracheomalatio, probably transitory, as a surgery sequel, some changes in perfusion and bronchi walls. Further follow-up is necessary.

\section{EVENTRATION OF THE DIAPHRAGM - CASE REPORT}

\section{Dervishov}

\section{Children Hospital, Veles, Macedonia}

Background and Aims: Relaxation and atrophy of the diaphragm is also called event ration. In that situation diaphragm is having high position, causing prolapse of organs in thorax cavity, compression on lungs and dislocation mediastinal organs on the opposite site. Goal: To present 6 months old baby hospitalised on the Children's Department in Medical centre in Veles.

Methods: Heteroanamnesis, clinical examination, RTG of lungs, ultrasonography and CTM of diaphragm, laboratory analysis

Results: Six months old boy conscious, febrile with tachydyspnea. On auscultation crude vesicular breathing and tiny and middle wet bronchitis snores with prolonged expiration.

Conclusions: Examinations RTG of lungs: High position of the right part of diaphragm (picture 1). Ultrasonography: Eventration of diaphragm in her medial and frontal part with normal density. In elevated part is noted liver tissue (picture 2). KTM of diaphragm: Mediastinum is normal. Scintigraphy: From 14th to 19th is detected liver issue on the right side like result of eventrated (hypoplastic) diaphragm (picture 3). Laboratory analysis. After treatment with antibiotics, i.v. and inhalator corticosteroids, oxygen, the patient is sent in Clinical centre on Surgery department. Conclusion: Eventration of diaphragm is rear disease with incidence of $10 \%$ of all congenital diaphragm disorders. The treatment of choice is surgical.

\section{RECURRENT SPASMODIC CROUP AND ALLERGY}

S Galbova, M Caparoska, O Zafirovski, L Petrusevska, B Mladenovic

Institute for Respiratory Diseases in Children, KozleSkopje, Macedonia

Background and Aims: The causes of recurrent spasmodic croup are unclear. The acute onset of the attacks without signs of acute infection, the short duration (few hours or less) and usually excellent respond to a symptomatic therapy, show that allergy may play an important role. The aim of this study is to determinate the probably role of allergy as etiological factor of recurrent spasmodic croup.

Methods: A group of 75 children with anamnesis of recurrent croup episodes who were performed to skin prick tests was analyzed. The evidence of family and personal atopy was explored. The most frequent allergens were determined.
Results: 49 children $(65,3 \%)$ from this group had positive skin prick test. 29 of them had another allergy comorbidity (asthma, dermatitis allergica, rhinitis allergica). 11 children from the 26 with negative prick skin tests had positive anamnesis for personal or/and family allergy: 6 dermatitis atopica, 2 asthma, 5 recidivate bronchoobstructive episodes, 7 positive anamnesis of asthma. Only in 11 children $(21,3 \%)$ there was no evidence for allergy. The most frequent positive allergens were: pollens 18 children, Dermatofagoides pteronnysimmus 16 children and etc.

Conclusions: It appears that allergy is an important factor in etiology of recurrent spasmodic croup in children.

\section{VENTILATORY STRATEGIES IN BELGIAN NICUS}

I Van Herreweghe, G Delanghe, E Chevalier, R Ciardelli, D Haumont

\section{Neonatal Intensive Care Unit, Saint-Pierre University Hospital, Brussels, Belgium}

Background and Aims: In 2004 all Belgian NICU admissions were registered in a national database. One fifth of the babies was hospitalised for respiratory distress syndrome (RDS). Evidence based guidelines on ventilatory strategies and surfactant administration are well established. The aim of this study was to compare among the different NICU centres the application of these strategies and the pulmonary outcome in Very Low Birth-weight Infants (VLBWI).

Methods: Records of 840 VLBWI were analysed. Duration of endotracheal ventilation and CPAP, surfactant administration, chronic lung disease (CLD) at 36 weeks, presence of retinopathy of prematurity (ROP) (grade 3 or $>$ ) and steroid use were recorded.

Results: 538 infants (68\% of all VLBWI) had diagnosis of RDS. Of the 495 intubated infants $80 \%$ were treated with surfactant (inter-centre range $56 \%$ to $100 \%$ ). The mean gestational age in this group was significantly lower $(27.6+/-1.9$ weeks versus $28.4+/$ - 1.8 weeks; $\mathrm{p}<0.0001)$. Below 28 weeks $83 \%$ were treated. CPAP use varied among centres (median CPAP ventilation days: range 1.0 to 43.0 days). Steroids were used in $35 \%$ of the infants (range $0 \%$ to $64 \%$ ). CLD occurred in $11.5 \%$ of the babies (range $0 \%$ to $42 \%$ ) receiving steroids in $50 \%$ (range 0 to $100 \%$ ). ROP was found in $5 \%$ of the survivors and was related to gestational age. Only 7 of 17 centres reported ROP.

Conclusions: In Belgian NICUs there are large intercentre variations in medical strategies for RDS. Existing evidence based guidelines were not systematically applied. Establishing national guidelines on surfactant use and ventilation seems necessary.

\section{INDOOR ECOLOGY AND RISK FACTORS FOR BRONCHIAL ASTHMA IN CHILDREN}

R L Karseladze ${ }^{1}$, L D Jorjoliani ${ }^{2}$, N A Katamadze ${ }^{3}$, M K Tskhakaia ${ }^{2}$

${ }^{I}$ Department of Paediatrics, Iv. Javakhishvili Tbilisi

State University, Tbilisi, Georgia, ${ }^{2}$ Department of 
Allergology and Clinical Immunology, Institute of Paediatrics, Tbilisi, Georgia, ${ }^{3}$ Primary Health Care Department, Batumi, Georgia

Background and Aims: Problem statement: High morbidity of Bronchial asthma; High rate of temporary or chronicle disability, caused by allergic diseases.

Methods: Problem statement: High morbidity of Bronchial asthma; High rate of temporary or chronicle disability, caused by allergic diseases; Sometimes doctors are unable to manage well bronchial asthma (BA), because of neglected early diagnostic.

Results: Study findings confirm causal connection (with high relative risk) and significant probability of the disease (with high attributive risk), with 'major risk factors': allergic diseases and atopic dermatitis in relatives and with 'minor risk factors': allergic rhinitis. Relatively weak risk factors (with low relative risk), but with high prevalence in child population in Batumi were found: respiratory infections and bottle-feeding. According to finding data indoor ecological factors (dust collectors, humidity, mould, active cigarette smoking, house heating and number of family members) have significant influence on sensibility occurrence frequency to house dust mite, mould fungi and other everyday allergens and bronchial asthma formation in children.

Conclusions: Revealing individual and population risk phenomenology of bronchial asthma in children population in Batumi, on the basis of that the most of risk factors can be monitored, will promote asthma prevention and population health care, which is more rational and cost-effective.

\section{DETERMINANTS OF PROLONGED ASTHMA HOSPITALIZATION IN INNER-CITY CHILDREN}

\section{P O Ozuah, M Reznik}

\section{Children's Hospital at Montefiore, Albert Einstein College of Medicine, Bronx, NY, USA}

Background and Aims: Identifying the factors associated with prolonged asthma hospitalizations for this population may help to decrease length of stay and reduce hospital costs. Objective: To identify determinants of prolonged hospitalization in inner-city minority children with asthma.

Methods: Retrospective cohort study between 1/1/98$10 / 1 / 04$. Long-stay group defined as children hospitalized with asthma for more than 3 days. Shortstay group were hospitalized 3 days or less. Bivariate analyses (t-test, chi-square test) and logistic regression were used.

Results: Of 479 subjects, $60 \%$ were Hispanic, 36\% African American, 64\% male and the mean age was 6.6 years (SD 5.3). Mean length of stay was 3.1 days (SD 1.7). $30 \%$ of subjects were in the long-stay group; and were older ( 7.6 years vs 6.1 years, $p=.006$ ), had greater mean number of lifetime asthma admissions (8.2 vs 3.8, $\mathrm{p}=.002$ ) and were more likely to receive a pulmonary consultation during the index hospitalization (OR 4.2, 95\% CI 2.6-6.9). Long-stay group were also more likely to be admitted to the ICU (OR 2.6, 95\% CI 1.6-4.2) and to require oxygen supplementation (OR $3.2,95 \% \mathrm{CI}$ 2.1-4.9) during the index hospitalization. Using logistic regression, oxygen requirement, multiple asthma admissions, and pulmonary consultation during the index admission were independent predictors of prolonged hospitalization. We found no significant group differences on exposure to environmental triggers, mean number of days with worsening asthma symptoms and home management prior to admission.

Conclusions: Our findings suggest that inner-city children with more severe asthma have longer hospital stay.

\section{EFFECT OF VOLUME GUARANTEE COMBINED WITH SYNCHRONIZED INTERMITTENT MANDATORY VENTILATION VS SYNCHRONIZED INTERMITTENT MANDATORY VENTILATION IN THE EXTREME PREMATURE}

M D Salvia, J Figueras, X Miracle, J M Rodríguez-Miguélez, X Carbonell

Servei de Neonatologia, ICGON, Agrupació Sanitària H. Clínic - H. Sant Joan de Déu, Universitat de Barcelona, Barcelona, Spain

Background and Aims: To compare the effect of synchronized intermittent mandatory ventilation (SIMV) vs. synchronized intermittent mandatory ventilation with volume-guarantee (SIMV + VG) on tidal volume (VT), peak inspiratory pressure (PIP), mean airway pressure (MAP), oxygen saturation ( $\mathrm{SpO} 2)$ in the extreme premature.

Methods: A total of 58 VLBW were randomised to receive SIMV or SIMV+VG from 30 min after the first surfactant dose until extubation or failure of ventilation mode or dead.

Results: There were no differences in demographic variables in the two groups. In the first 48 hours airway pressure was higher in SIMV (PIP 18.7 \pm 2.9 vs $14.9 \pm 1.9$, $\mathrm{p}<0.0001 /$ MAP $7.7 \pm 1.7$ vs $5.3 \pm 0.91, \mathrm{p}<0.001)$, VT vas lower and less variable during SIMV+VG $(6.2 \pm 1.1$ vs $4.7 \pm 0.9, \mathrm{p}<0.0001)$ for similar $\mathrm{CO} 2$ concentration (45.8 \pm 6.6 in SIMV vs $48.6 \pm 6.1$ in SIMV+VG, NS). In SIMV group there were three episodes hypocarbia vs none in de $\mathrm{SIMV}+\mathrm{VG}$ group. There was lower $\mathrm{FiO} 2$ $(27.7 \pm 4.5$ vs $25.1 \pm 3.6, \mathrm{p} 0,03)$ for similar $\mathrm{SpO} 2$ during SIMV+VG.

Conclusions: SIMV+VG appears to be more effective and less dangerous than SIMV. Less pressure is needed to obtain similar blood gases. Lower and more stable VT are recorded, with less episodes of hypocarbia.

\section{LUNG TISSUE MECHANICS PREDICT LUNG HYPOPLASIA IN A RABBIT MODEL FOR DIAPHRAGMATIC HERNIA}

A W Flemmer ${ }^{1}$, J C Jani ${ }^{2,4}$, F Bergmann ${ }^{3}$, O J Münsterer ${ }^{3}$, D Gallot ${ }^{2}$, K Hajek ${ }^{1}$, J Deprest ${ }^{2,4}$

${ }^{l}$ Department of Neonatology, University Children's Hospital, Center of Perinatology Grosshadern, LMUniversity Munich, Munich, Germany, ${ }^{2}$ Center for Surgical Technologies, Faculty of Medicine, Katholieke Universiteit Leuven, Leuven, Belgium, ${ }^{3}$ University Hospital for Pediatric Surgery, LM-University Munich, 
Munich, Germany, ${ }^{4}$ Department of Obstetrics and Gynaecology, University Hospital Gasthuisberg, Leuven, Belgium

Background and Aims: Several animal models have been proposed to study the pathophysiology of congenital diaphragmatic hernia (DH). Surgical induction of $\mathrm{DH}$ in fetal rabbits during the pseudoglandular phase has been shown to induce severe pulmonary hypoplasia, but functional studies in this model are scarce. We aimed to measure neonatal pulmonary impedance and related it to the severity of lung hypoplasia.

Methods: DH was surgically created in rabbits at 23 days of gestation. Following cesarean delivery at term (31 days) pups were subjected to measurement of total lung capacity (TLC), lung to body weight ratio (LBWR) and lung impedance by forced oscillation technique. Airway resistance (Raw), elastance (HL), tissue damping (GL) and hysteresivity (GL/HL) were calculated from impedance data.

Results: $12 \mathrm{DH}$ fetuses and 15 controls were available for final analysis. LBWR and TLC were significantly lower in the $\mathrm{DH}$ group compared to gestational and age matched controls $(p<0.001)$. Raw, HL, GL and hysteresivity were significantly increased in DH fetuses. Group differences in tissue mechanics were best described by hysteresivity, indicating a dominant contribution of lung tissue mechanics to DH-induced lung hypoplasia.

Conclusions: We successfully introduced lung impedance measurement by forced oscillation technique in neonatal rabbits. Following surgical induction of $\mathrm{DH}$ in the pseudoglandular phase, they have, next to morphological evidence of pulmonary hypoplasia, changes in lung mechanics. Our results for lung tissue mechanics support the concept of delayed pulmonary tissue modeling. We propose to use functional studies in future experiments when evaluating prenatal interventions aimed at reversing pulmonary hypoplasia.

\section{JOUBERT SYNDROME: A REVIEW OF 8 CASES}

A F El-Hassanien ${ }^{1}$, H A Al-Ghiaty ${ }^{2}, \mathrm{~S} \mathrm{Mohamed}^{3}$

${ }^{l}$ Department of Pediatrics, Faculty of Medicine, ElMansoura University, El-Mansoura, Egypt

${ }^{2}$ Department of Pediatrics, Faculty of Medicine, Benha

University, Tukh, Kaliobia, Egypt, ${ }^{3}$ Department of

Opthalmology, Faculty of Medicine, Benha University,

Tukh, Kaliobia, Egypt

Background and Aims: Joubert Syndrome (JS) is a rare genetic brain malformation characterized by absence or underdevelopment of cerebellar vermis. The common features of JS in infants include hyperpnea, nystigmus, mental retardation, and ataxia. The purpose of study is to increase awareness for the clinical and neuroimaging findings of Joubert syndrome for the correct diagnosis of this rare congenital malformation.

Methods: Eight patients with Joubert syndrome were identified from 3 hospital pediatric departments databases covering a 5-year period. Criteria for diagnosis included absent or markedly hypoplastic cerebellar vermis, abnormal eye movements, and developmental delay. Radiological scans reports, clinical notes, biochemical results and opthalmological evaluation were available for all the 8 patients. 6 cases were clinically reviewed to assess visual electrophysiology and 2 patients had died.

Results: Cerebellar vermis hypoplasia/aplasia and apnea were present in all patients, polydactly in 1 of 8 , renal problems with cysts in 3 patients and 4 of 6 had abnormal electroretinograms (ERGs). Blood investigations of organic acids, amino acids and verylong-chain fatty acid, were normal in the 7 patients tested.

Conclusions: JS is a rare genetic brain malformation with association of retinal dystrophy and renal abnormalities. The retinal dystrophy may be progressive. The prognosis of patients depends mainly on the degree of brain malformation.

\section{THE TIME TO PEE STUDY: A RANDOMISED CONTROLLED TRIAL OF A VIBRATING BLADDER STIMULATOR IN CHILDREN}

\author{
P Davies $^{1}$, R Greenwood ${ }^{2}$, J Benger ${ }^{3}$ \\ ${ }^{I}$ Children's Emergency Department, Bristol Royal \\ Hospital for Children, Bristol, UK, ${ }^{2}$ Research and \\ Development Support Unit, United Bristol Healthcare \\ Trust, Bristol, UK, ${ }^{3}$ Faculty of Health and Social Care, \\ University of The West of England, Bristol, UK
}

Background and Aims: To evaluate a commercially available vibrating bladder stimulator for use in reducing the time spent waiting for children to produce a urine sample in acute paediatrics.

Methods: All non-continent children needing a urine sample attending a children's emergency department were included. Exclusions were neuro-anatomical abnormalities and those who were too unwell. After informed consent, children were randomised either to an "advice" group, given an advice sheet of traditional methods for urine stimulation, or a "device" group, where they were offered the use of a bladder stimulator. Both were administered by the parents. Ethics committee approval was granted.

Results: 110 patients consented, from which we had 97 datasets (48 advice, 49 device). 11 advice and 15 device children left the department without a sample being obtained (not significant). Mean waiting time was 1 hour and 2 minutes. $61 \%$ in the advice group waited for over one hour compared to $46 \%$ in the device group $(\mathrm{p}=0.15)$. and a mean reduction in waiting times by 18 minutes $(\mathrm{p}=0.2)$. Kaplan-Meyer plot identified a population of children who may benefit from bladder stimulation.

Conclusions: There is a trend towards reduced waiting times when the bladder is vibrated by a device compared to traditional methods. However, we still had a significant waiting time even with use of the stimulator. The device is noisy and can frighten small children. Although the use of this cheap and easily available device may reduce waiting times, the search for a reliable urinary stimulator in children continues. 
THE NEW STEROID-FREE ASCANCOL OINTMENT SAFE FOR CHILDREN WITH ATOPIC DERMATITIS

N F Mgeladze ${ }^{1}$, R L Karseladze ${ }^{1}$, I D Dadeshidze ${ }^{2}$, E G Karseladze ${ }^{1}$

${ }^{I}$ Department of Pediatrics, Iv. Javakhishvili State University of Tbilisi, Tbilisi, Georgia, ${ }^{2}$ I.G Kutateladze Institute of Pharmacochemistry Georgian Academy of Sciences, Tbilisi, Georgia

Background and Aims: Atopic dermatitis - common inflammatory skin disease, with an high prevalence in children and infants. The aim of this study was to investigate clinical efficacy of the new steroid-free Ascancol - bentonite gel in patients with atopic dermatitis.

Methods: The study was designed as open-controlled, 80 children aged 36 month to 14 years with Atopic Dermatitis were randomized 1:1 to a Ascancol-based regimen $(n=40)$ or a conventional treatment (hormonal, symptomatic indifferent ointments) regimen $(n=40)$. All patients underwent a 3-9 months (catamnesis) run-in period, to assess their baseline conditions. The effectiveness of the treatment is evaluated by determining the index SCORAD until and after the treatment.

Results: The results shows that in patients with severe atopic dermatitis there were no significant differences among the clinical features, basal symptoms or atopic status between the children with administration of Ascancol and OSMF for external use. Ascancol was effective in $66,7 \%$ of cases (significant improvement in $46,7 \%$, improvement in-26, $7 \%$ ); in cases of intertrigo significant improvement in $75 \%$, improvement in $25 \%$ of patients; in cases of Eczema - significant improvement in $48 \%$, improvement in $25 \%$ of patients; ill health-in $13 \%$ of patients with eczema (dry) and neurodermitis.

Conclusions: Generalizing results according to SCORAD index, in AD monotherapy with Ascancol and with OSMF, according to comparison data of drying and antipruritic effects, Ascancol is preferential than OSMF for external use, especially in early childhood.

\section{HEALTH PROMOTION OF THE INTERNATIONALLY ADOPTED AND IMMIGRATED CHILDREN. AN EXPERIENCE FROM FERRARA, ITALY}

M Bergamini ${ }^{1}$, G Gabutti ${ }^{1}$, M Cova ${ }^{2}$, G Giovannini ${ }^{3}$, R Carfora $^{1}$, K Cervato $^{1}$, F Gigli $^{1}$, F Pizzo ${ }^{1}$, R Rizzello ${ }^{1}$, $\mathrm{P}_{\text {Gregorio }}{ }^{1}$

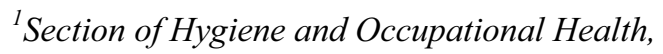
Department of Clinical and Experimental Medicine, University of Ferrara, Ferrara, Italy, ${ }^{2}$ Public Health Service, Local Health Care Unit of Ferrara, EmiliaRomagna Region, Italy, ${ }^{3}$ Health Children Service, Local Health Care Unit of Ferrara, Emilia-Romagna Region, Italy

Background and Aims: In Italy, the enlarged practice of international adoptions and increasing immigration of children highlights the need to adequate Italian public health services. In particular, international adopted children represent a group with unique health care needs. Data from published studies suggest that the risk of serious infections in adoptees is low, although evidence-based medicine on which to base screening protocols for this segment of population is lacking.

Methods: All health and vaccination records of some child immigrated or internationally adopted in Ferrara, Italy, were reviewed. If a reliable immunization record is presented, one need not repeat the vaccines or check titers. In absence of documentation, routine serological screening tests for diphtheria, tetanus, poliomyelitis and hepatitis B have been performed. A tuberculin skin test was performed on someone, and a chest radiograph had been obtained for any individual with a positive PPD. In addition, special screening tests (e.g., for malaria, hepatitis C, MMR) were indicated by paediatricians on the basis of specific child's age and/or acute medical needs.

Results: This study started one year ago. Of 94 immigrants notified in the same period, $72(76,5 \%)$ were sent referral letters. Most of the others lacked valid addresses. Of the $44(46.8 \%)$ who were examined, 26 $(59 \%)$ were serologically screened, 15 (34\%) were recommended vaccination, and 14 (31.8\%) completed it. Conclusions: Indicators based on measurements of health in foreign or adopted children are important for identifying problems and priorities, changes over time, and newly emergent issues in a newly segment of Italian population.

\section{TONSILLOADENOIDECTOMIA - YES OR NO}

S Stankovska-Kceva ${ }^{1}$, A Jancevska ${ }^{2}$, S Dzekova ${ }^{1}$, L Veljanovska $^{1}$

${ }^{1}$ ENT Department, Health Center Skopje, Skopje, Macedonia, ${ }^{2}$ Pediatric Clinic, Medical Faculty Skopje, Skopje, Macedonia

Background and Aims: Short resume of different approaches about Tonsilloadenoidectomia who have specialist for children daisies and ENT specialist during chronicle infected Waldeyerov lymphatic ring considering children.

Methods: The diagnosis of the children was made with ENT examination, hemogram, microbiological finding nose and throat received antybiogram, RTG after Hurthz. Twenty children between age of 3 and 6 year have been analyzed during the period of 2 years.

Results: Following problems relating our examination of this children were: Angina catharalis, Angina Lacunaris, Otitis media acuta, supp, Sinusitis maxillaris billateralis, Anemia, difficulties in breathing and problems during growing. According to the obtain antybiograms most often proved evokers are: Straphyloccocus Beta haemiliticus, pyogenus, Haemophylus infl, Staphylococus aureus. Fourteen of twenty children have been expose to surgery and their health problems were improved and the condition of other six children remain the same.

Conclusions: According to opinion of ENT specialist Tonsilloadenoidectomia should be used to all children with chronicle infected Waldeyerov lymphatic in order to prevent possible complications. 
CLINICAL AND IMMUNOLOGICAL CHARACTERISTICS OF NONBACTERIAL ACUTE RESPIRATORY TRACT INFECTIONS IN CHILDREN

\author{
K Stavric, S Peova, K Mironska, L Kareva
}

Department of Immunology, University Children's Hospital Skopje, Skopje, Macedonia

Background and Aims: Present the clinical and immunological characteristics of acute nonbacterial respiratory tract infections in preschool children.

Methods: 38 children with bronchopneumonia at age of 6 months to 7 years, hospitalized at Department of Immunology, were investigated. Pneumoslide $M$ (Vircell) - immunflurescent test for detection of specific IgM and $\operatorname{IgG}$ antibodies was performed.

Results: 20 children (57\%) had positive result for more than one infection. The most frequent was Mycoplasma pneumonia infection - 17 children (48.6\%), from which $12 \mathrm{had}$ co-infection. The frequency of the other present infections was as follows: Adenovirus and Influenza B infection in 12 children (31.5\%), Parainfluenza in 10 children (28.6\%) and RSV in 9 children (25.7\%). Laboratory tests of children with Mycoplasma infection showed: $\mathrm{CRP}=34 \pm 33 \mathrm{~g} / \mathrm{l}$, ESR $35.7 \pm 36 \mathrm{~mm}$ and normal level of immunoglobulin. Children with Adenovirus showed increased level of inflammatory markers $(\mathrm{ESR}=52 \pm 25 \mathrm{~mm}, \mathrm{CRP}=57 \pm 66 \mathrm{~g} / \mathrm{l}), \mathrm{IgM}$ $(1.28 \pm 0.7 \mathrm{~g} / \mathrm{l})$ and a lower level of $\operatorname{IgA}(0.79 \pm 0.6 \mathrm{~g} / \mathrm{l})$. Urticaria or exanthematous rash were connected with Mycoplasma pneumonia infection (35\%), RSV (33\%) and $25 \%$ with adenovirus and influenza B infection. Children with isolated viral infection had significantly lower level of ESR, IgA and IgM $(\mathrm{p}<0.05)$ compared with children with more infections.

Conclusions: Nonbacterial infections are common in preschool children with acute respiratory tract infection. The children are competent to produce specific antibodies, which is stronger if there is more than one infection. The most frequent is Mycoplasma pneumonia infection with most common clinical sign of urticaria or exanthematous rash.

\section{CLINICAL AND EPIDEMIOLOGICAL CHARACTERISTICS OF CHILDREN HOSPITALIZED FOR COMMUNITY-ACQUIRED PNEUMONIA IN A RURAL AREA}

\begin{abstract}
A Giannisi, N Manakou, O Gkika, P Anastasopoulou, G Z Zantopoulos, S Liosi, C Rossas, I Anastasopoulos, K Gantas, Z Patrona
\end{abstract}

Department of Paediatrics, General Hospital of Korinthos, Korinthos, Greece

Background and Aims: Community-acquired Pneumonia (CAP) is one of the most common infections in the pediatric age group and one of the leading diagnoses that result in overnight hospital admission for children. The aim of the study was to evaluate the clinical and epidemiological characteristics of children hospitalized for pneumonia in our geographic area.

Methods: We retrospectively reviewed the medical records of 37 children hospitalized for pneumonia confirmed by radiological images, total leucocyte count
$(>15.000 / \mathrm{ml})$ and C-reactive protein value $(>80 \mathrm{mg} / \mathrm{l})$. Age, sex, symptoms on admission, diagnosis, seasonal variation and outcome were evaluated. All patients were followed-up for 15 to 30 days after diagnosis to ascertain outcome.

Results: Between February 2005 and January 2006, 37 children aged 3 months to 14 years old with pneumonia were enrolled. The mean age was $5.6 \pm 1.2$ years. There were $18(49 \%)$ boys and $19(51 \%)$ girls. A total of $8 \%$ were aged less than 6 months. The individual background was unremarkable in $27(73 \%)$ children. The main symptoms were fever (86\%), cough $(22.3 \%)$, general malaise $(14.9 \%)$, respiratory distress $(9.2 \%)$, thoracic pain $(3.3 \%)$, and headache $(1.2 \%)$. During the winter months, there was up to a $50 \%$ increase in the number of cases compared with the summer months. Outcome was favorable in all children.

Conclusions: CAP in hospitalized children is most frequent in previously healthy children, especially aged 2 to 6 years old, with fever nonspecific signs and symptoms that are similar to those of other infections and respiratory processes. Recommendations for diagnosing and treating CAP may be advisable.

\section{DEVELOPING INFECTION CONTROL STRATEGY: RISK FACTOR ANALYSIS IN TERTIARY REFERRAL UNIT FROM ROMANIA}

P Avram, M Craiu, A V Cochino

Institute for Mother and Child Care, Bucharest, Romania

Background and Aims: Before 1989, in Romania, nosocomial infections were exceptionally reported. To improve infection control in our ICU, we conducted a case series study.

Methods: Two years (2004-2005) retrospective study in 17 infants, admitted in ICU. Control group: 46 infants, admitted in the same period, for documented noninfectious causes. We studied comparative risk factors (sex, socials, gestationals, birth related, invasive procedures, septic work-up, antibiotic treatment).

Results: Identified pathogens: Klebsiella spp. (47.05\%), E.coli (29.4\%), Staphylococcus spp. (17.64\%), Pseudomonas aeruginosa (11.76\%), enterococcus (5.88\%), resistant Streptococcus pneumoniae $(5.88 \%)$. Risk factors (table). Five infants expired - 2 from the infected group (infection-related death $\mathrm{OR}=2.87$ ).

Conclusions: 1. Most pathogens were gram negative rods $(76.47 \%)$, Klebsiella dominating $(47.05 \%)$. 2. Multiresistant strains frequency was higher in 2004; similar findings regarding concomitant multiple bacterial infection. 3. Home delivery was the most important risk factor for severe neonatal infection. 4. Empiric treatment with antibiotics before referral increases the chance of severe bacterial infection $(\mathrm{OR}=1.9)$. 5. Prematurity was not a significant risk factor; probably related to advanced care provided. Neither were invasive procedures (MV, central lines). 6. In 2005 were admitted 854 more patients than in 2004; still, the relative reduction of severe infection frequency was $20.17 \%$. 


\begin{tabular}{|l|c|c|c|}
\hline \multicolumn{1}{|c|}{ Risk factor } & "Infection" group & Control & Odds ratio \\
\hline Sex ratio F:M & $1,42: 1$ & $0,43: 1$ & 1.85 \\
\hline No follow-up gestation & $41.17 \%$ & $17.39 \%$ & 2.24 \\
\hline Home delivery & $11.76 \%$ & $2.17 \%$ & 6 \\
\hline Gestational age $<37 \mathrm{w}$ & $47.05 \%$ & $60.86 \%$ & 0.57 \\
\hline Birth weight<1500 & $41.17 \%$ & $39.13 \%$ & 1.12 \\
\hline Invasive procedures & $11.76 \%$ & $19.56 \%$ & 0.51 \\
\hline Maternal infection during gestation & $23.52 \%$ & $6.52 \%$ & 4.41 \\
\hline Previous antibiotic treatment & $76.47 \%$ & $63 \%$ & 1.9 \\
\hline Prolonged ruptured membranes (>8h) & $47.05 \%$ & $23.91 \%$ & 2.82 \\
\hline
\end{tabular}

\section{CONGENITAL ANOMALIES AND GENETIC DISEASES IN CHILDREN LESS THAN 15 YEARS IN AN ISRAELI ARAB TOWN}

\section{Jaber}

Neurology Clinic, Schneider Children's Medical Center of Israel, Petah Tikva, Israel, The Bridge to Peace Community Pediatrics Center, Taibe, Israel, Clalit Health Services, Israel

Background and Aims: The aim of this study was to establish the profile of genetic disorders and congenital malformation in children less than 15 years old in an Israeli Arab town.

Methods: We studied 7200 children in an Arab town in the center of Israel, over a six-year period. The study was partly conducted prospectively and partly retrospectively. The prospective part, which comprised all the children born after January 1998, and the retrospective part was concerned with children who were born prior to January 1998 by examining the medical records of the children. The cut off age for these children was the 15th birthday. The genetic disorder and major congenital malformations were recorded.

Results: 514 (71.4/1000) had genetic disorders or congenital malformations. $39(7.6 \%)$ had multiple malformations and $430(83.7 \%)$ had involvement of single system. The most common system with isolated single malformation was cardiovascular 109 (21.2\%) and in other systems it was in the CNS, Genitourinary, Ophthalmological, Dermatological, Gastrointestinal, Musculoskeletal, Hematological/Immunological and Ear and Nose: 85 (18\%), 85 (18\%), 44 (8.6\%), 29 (5.6\%), $27(5.27 \%), 22(4.3 \%), 14(2.7 \%)$ and $8(1.55 \%)$ respectively. Twelve $(1.6 \%)$ of the multiple anomalies were syndromes and twenty seven children had abnormalities which could not be classified. The prevalence of defined and suspected autosomal recessive disorders in the population was 1:90. Conclusions: Each village and town in this high risk community has its own genetic and congenital malformations. Suitable comprehensive plan for prevention is warranted.

\section{THE ROLE OF PEDIATRICIANS IN PROPHYLAXIS OF DENTAL CARIES}

\section{A Nitsch-Osuch, E Gyrczuk, A Wardyn \\ Department of Family Medicine, Medical University of Warsaw, Warsaw, Poland}

Background and Aims: Dental caries (DC) is an infectious and preventable disease of a great social impact. In Polish pre-school children DC is present in more than $90 \%$. The aim of a pediatrician should be to prevent DC by education of children and their parents regarding hygienic and nutritional habits and fluoride supplementation. Our task has been to learn parents knowledge concerning prophylactic procedures connected with DC in their preschool children.

Methods: We obtained fulfilled surveys with questions about prevention of DC from 98 parents of children attending to one of public day care facilities in Warsaw.

Results: Most of parents (76\%) self estimated their knowledge about prophylaxis of DC as being 'not enough'. Only in $38 \%$ parents the main source of information about DC was medical staff $(61 \%$ learnt some information from popular newspapers). The mean age of first visit of a child to a dentist was 3,6 years. In parents opinion pediatrician was not oriented for prophylaxis of dental caries ( $88 \%$ opinions). Only 56\% of them were provided by pediatricians with some peaces of advice regarding oral hygiene, fluoride supplementation or nutritional habits - playing the role in prophylaxis of DC.

Conclusions: Our results show that pediatricians are not active enough in a field of DC prophylaxis. Parents are not aware enough how to prevent DC in their children. We should encourage pediatricians to educate their patients in a field of oral hygiene, nutritional habits, safe fluoride supplementation.

\section{BREASTFEEDING THE PREMATURE INFANT}

\section{R Maastrup}

\section{NICU, Rigshospitalet, University Hospital, Copenhagen, Denmark}

Background and Aims: A great number of the premature infants are not able to suck at birth due to their immaturity. This challenges the nurse at her professional efforts to create frames and expectation for breastfeeding, and to give support and counselling to the mother for a successfully breastfeeding before discharge.

Methods: A breastfeeding policy is developed: Already the first day the mother will be informed about the importance of breastfeeding for the premature infant and encouraged in expressing her milk. Written material is distributed to support oral information. The breastfeeding process, "The Milky-Way" is a guideline in transitioning the premature infant from tube-feeding to exclusively breastfeeding. The first step on "The Milky Way" is skin-to-skin contact. This is easy as in Denmark we mostly use nasal-CPAP rather than ventilator treatment when possible. As soon as the infant is stabile with nasal-CPAP it will be put to the mother's breast to smell and taste and suck for pleasure. This will help the infant to be able to suck sooner when strong enough. The transitioning process will often take time for the premature infant, from days to weeks. During this period the parents and nurse will make daily evaluation of the infant's maturity and abilities as for the plan of breastfeeding.

Results: All mothers start expressing milk. All infants receive skin-to-skin contact, and all infants suck for pleasure. $67 \%$ of the infants are exclusive breastfed at discharge and $15 \%$ partial breastfed. 
Conclusions: Breastfeeding is science for the nurse but art for the mother.

\section{DEVELOPMENT AND APPLIANCE OF EDUCATIONAL MATERIAL TO PREPARE CHILDREN AND PARENTS TO VENOPUNCTURE}

\section{L G Pedreira, M A M Pettengill, A R Bezerra, V Guarise \\ Department of Nursing, Federal University of São Paulo, São Paulo, São Paulo, Brazil}

Background and Aims: Many children and their parents consider venopuncture as one of the most traumatic event during illness and hospitalization because of their fear of needles and pain. There are evidences relating parent's anxiety during venopuncture and children's stress. Considering that both children and their parents should be prepared for this situation, it is essential to elaborate strategies and instruments, in order to meet their needs, such as the development of educational and instructional material regarding venopuncture. The aim of this paper is to describe the material's construction and its appliance in clinical setting.

Methods: A participant methodological framework was performed in a pediatric unit of a public teaching hospital in São Paulo city, Brazil. In order to be acquainted with the scientific evidences, a literature review was carried out. After that, a field phase was conducted, applying qualitative contend analysis to understand the child and parents' needs of information allied to venopunction, in their perspective, as well in the professionals. Data were compared and organized in a creative book format. Finally, three specialists and the subjects validated the material.

Results: The book has been systematically applied with children and their parents before the venopuncture procedure. They reveal their satisfaction with the preparation for the procedure with the educational material, acknowledging its importance.

Conclusions: The evaluation disclose that it is a very significant instrument to be used in nursing practice with children and their parents because attend their needs of information and strengthen their capacity to participate in the care, which contributes to minimize their fear and anxiety.

\section{LEADERSHIP IN NEONATAL NURSING: TAKING IT GLOBAL}

\section{D Philips, W Moulsdale, M DeLand}

Neonatal Intensive Care Unit, Sunnybrook and Womens Health Sciences Centre, Toronto, ON, Canada

Background and Aims: Canadian Neonatal Network and The Shanghai Children's Hospital of Fudan University launched, in October 2005, the People's Republic of China Neonatal Nurses Training Program. This global partnership is aimed at improving the quality and efficacy of neonatal care in China, and provides a unique opportunity for nurses to participate in an evidence-based clinical collaboration. Specific objectives of the program include, establishing a centre dedicated to training neonatal nurses, as well as establishing a national standard for neonatal nursing education in China.

Methods: Planning an international training program requires a focused and committed management team. Essential elements include developing the content and methods for its' delivery, as well as strategizing logistical details, from immigration requirements, to the preparation and printing of course materials. This dynamic process is ongoing and must include evaluation, and creative problem solving at every stage of program development.

Results: Benefits of our successful international exchange of knowledge included, increased crosscultural awareness, personal and professional development and potential for international research collaboration.

Conclusions: The value of mentorship in adult learning is increasingly recognized. This global initiative places an emphasis on mentorship, while encouraging leadership and helping to establish excellence in clinical practice in order to advance neonatal nursing worldwide. The collaboration recognizes, realizes, and enhances the impact of nursing, while developing a new avenue for professional enrichment. Developing exciting new ways to utilize knowledge and experience leads to increased job satisfaction, staff retention, and morale. The ultimate goal of targeted international academic exchange however is improved patient and family outcomes.

\section{PRACTICE EDUCATOR ON THE PEDIATRIC INTENSIVE CARE UNIT: A MUCH-NEEDED PROFESSIONAL!}

\author{
R F W Van Den Berg, A P Verest, I van 't Wout,
} $M$ van Dijk

\section{Department of Pediatric Surgery, Erasmus MC-Sophia Children's Hospital, Rotterdam, The Netherlands}

Background and Aims: There is a clear role for the practice educator in the pediatric intensive care environment considering the high nursing shortages and turnover of patients. Supervising adults requires skills such as teaching at the learner's level, helping learners to use their knowledge and skills, and adapting supervision to individual needs. This study evaluates the work of two practice educators in pediatric intensive care.

Methods: The two practice educators of the Pediatric Surgical Intensive Care Unit of the Erasmus MC-Sophia Children's Hospital retrospectively classified focus of their supervision during the previous two years broadly as follows: Knowledge/skills, psychological and supportive. Thirty-eight nurse students were asked to provide a strength/weakness analysis and information on prior education and working experience.

Results: From October 2003 to October 2005 two practice educators supervised a total of 38 students. Median age of the 34 female and 4 male students was 29 years (range 21 to 41 years). They were training for general pediatric nurse $(\mathrm{n}=10)$, high care nurse $(\mathrm{n}=16)$, or pediatric intensive care nurse $(\mathrm{n}=12)$. Supervision focused on knowledge/skills in 16 (42.1\%), supportive 
and/or psychological in $5(13.1 \%)$, and on all three or two areas including knowledge/skills in 17 students (44.8\%). Six students struggled with their student role. Strengths and weaknesses mentioned were giving/receiving feedback, perfectionist, connect theory and practice.

Conclusions: Focus and intensity of supervision in pediatric intensive care should be geared to a student's work experience, educational level, personality and life experience, and require flexible and highly individualized approach from the practice educator.

\section{CLINICAL SUPERVISION IN PRACTICE}

\section{M Thruston}

\section{Paediatric Intensive Care Unit, Evelina Childrens' \\ Hospital, Guys' and St Thomas' NHS Foundation Trust, London, UK}

Background and Aims: Clinical Supervision is an effective process in reducing stress and burnout that staff working in critical care areas can experience. Clinical Supervision has been defined as 'A formal process of professional support and learning which enables individual practitioners to develop knowledge and competence, assume responsibility for their own practice, and enhance consumer protection and safety of care in complex clinical situations. It is central to the process of learning and to the expansion of the scope of practice and should be seen as the means for encouraging self-assessment and analytic and reflective skills' (Department of Health, (DOH) 1994).

Methods: Supervisory models are useful to provide structure and direction to clinical supervision sessions. Guided reflection offers an effective way of structuring clinical supervision and is central to the process. Many methods for reflection have been identified and different models have been designed.

Results: Benefits of Clinical Supervision have been shown in recruitment and retention and continuing professional development. Participants of supervision have reported increased competence, confidence, increased job satisfaction and accountability, feeling valued and supported, reduced stress, reduced sickness, professional development, improved teamwork, and healthier work environment.

Conclusions: Clinical supervision requires organisational endorsement and commitment and stakeholders want evidence of its effectiveness. Clinical supervision is supported both at national and local level. Guys' and St Thomas' NHS Foundation Trust endorses clinical supervision in its' 'Nursing and Midwifery Strategy' and actively encourages staff to take part in it. Clinical supervision is an essential component of the clinical governance framework to support and safeguard quality practice.

\section{NURSING INDICATORS IC NEONATOLOGY}

\section{A T A M Claassen}

Department of Neonatology, Emma Children's Hospital, Academic Medical Center, Amsterdam, The Netherlands
Background and Aims: To test the 'List of requirements of the Nursing Care within the Neonatology Department' (1997). The current testing methods are time consuming and labour-intensive. To develop a set of indicators derived from the norms in the list of requirements. With these indicators the nurses should be able to monitor and direct the delivered care by themselves, and receive continuous feedback of the quality-level of nursing practice.

Methods: The project consists of a development, realization and after-care phase. In the development phase the indicators are developed stepwise. Based on set criteria selections were made. An indicatordevelopment-form describes the method of measurement, the applied criteria and the method of collecting data. During the realization phase the developed indicators will be measured supported by an instruction-measurement-form, which enables every nurse to perform the test (once a month, takes about 3 hours). Results are published monthly on a poster. In the after-care phase, the results are evaluated. When the results are repeatedly below the norm for a specific indicator, an improvement project is started.

Results: Since the beginning of the project (2003), a total of 20 indicators are tested, 3 improvement projects are finalized and 2 new projects are started. The improvement projects are coordinated by nurses. Overall these results lead to critical contemplation and reflection in our department. Employees feel responsible for the results, and thus for delivering high-quality-care.

Conclusions: In our Neonatology department we showed with a small set of indicators important aspects of nursing care can be measured.

\section{A RANDOMIZED STUDY OF ORAL CARE INFLUENCE ON DURATION OF MECHANICAL VENTILATION AND LENGTH OF STAY IN A PICU}

D M Kusahara ${ }^{1}$, M A S Peterlini ${ }^{1}$, W B Carvalho ${ }^{2}$, A R Ruiz ${ }^{2}$, S R Pereira ${ }^{1}$, M L G Pedreira ${ }^{1}$

${ }^{l}$ Department of Nursing, Federal University of São Paulo, São Paulo, Brazil, ${ }^{2}$ Pediatric Intensive Care Unit, Hospital São Paulo, São Paulo, Brazil

Background and Aims: Oral care has been described as capable to prevent VAP, reducing duration of mechanical ventilation, length of stay (LOS) and use of systemic antibiotics in adult patients. The aim of this study was to compare the duration of mechanical ventilation and LOS of children in a PICU, according oral care intervention.

Methods: Prospective randomized study realized in a PICU in the city of São Paulo, Brazil. The research was approved by the ethics committee. The sample was composed of 56 children, 29 (51.8\%) in Control GroupCG (mechanical intervention) and $27(48.2 \%)$ in Experimental Group-EG (0.12\% chlorhexidine); 47 $(83.9 \%)$ children received mechanical ventilation. Complementary variables of children's characteristics and intensive care were collected. Significance level (p) was set at $5 \%$.

Results: There was no significant influence of oral care intervention on duration of mechanical ventilation (CG 
122.6 \pm 120.0 ; EG $147.4 \pm 157.4$ hours; $p=0.670$ ) and

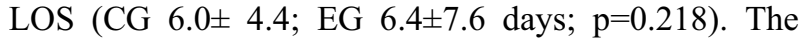
results demonstrated no significant differences between the distribution of demographic characteristics of the sample in the groups: age $(\mathrm{p}=0.854)$, gender $(0.945)$, skin color $(0.983)$, nutritional status $(0.531)$, clinical conditions of mouth $(0.414)$, teeth $(0.343)$, presence of infections $(0.222)$ at admission, tracheal intubation status (0.716), and use of enteral tubes (0.712).

Conclusions: There was no significant influence of the studied oral care interventions on the duration of mechanical ventilation and PICU's LOS. Acknowledgments: Dr. Pedreira acknowledges support for research by the grant 04/13361-2 from FAPESP Fundacao de Amparo - Pesquisa do Estado de Sao Paulo.

\section{NASAL CONTINUOUS POSITIVE AIRWAY PRESSURE IN SEVERE ACUTE BRONCHIOLITIS: EVALUATION OF TIME CONSUMPTION FOR NURSES}

C Veyssiere, H Guerrini, A Louis, S Essouri, D Devictor

\section{Pediatric Intensive Care Unit, CHU Kremlin Bicêtre,} Kremlin Bicêtre, France

Background and Aims: Nasal continuous positive airway pressure (NCPAP) in severe acute bronchiolitis is now more largely use. The assistance time spent by nurses in relation to ventilatory support either NCPAP or tracheal ventilation was retrospectively studied.

Methods: 71 infants were included during the 2 last epidemia (2004-2005 and 2005-2006), 42 received NCPAP and 29 were intubated. Quantification of nurse's act (suctioning practices, comfort and relation care) was noted for each infant during the first 24 hours of stay.

Results: General characteristics were similar for the 2 populations with a mean age of $4.9 \pm 2.3$ weeks and a mean weight of $4 \pm 0.8$ kilos. Requirement of suctioning practices per day is significantly decreased in the NCPAP group $(12 \pm 2$ versus $31 \pm 4, p<0.01)$. Need of comfort care (massage and installation) per day is significantly decreased in the NCPAP group compared to the intubation group $(6.6 \pm 1.4$ versus $12 \pm 1.4$, $\mathrm{p}<0.01$ ). No sedation is required during NCPAP allowing a better infant-parent relation and setting in the arms of parents. Length of respiratory support and stay were reduce in the NCPAP group $(\mathrm{p}<0.002)$.

Conclusions: This study seems to favour a low time spent by nurses in relation to ventilatory time during NCPAP for severe acute bronchiolitis allowing them to spent more time for parents-infant relation.

\section{MEDIATION IN PAEDIATRIC INTENSIVE CARE - THE WAY FORWARD?}

\author{
B J Teuten ${ }^{1}$, C M Pierce ${ }^{2}$ \\ ${ }^{l}$ Medical Directorate, Great Ormond Street Hospital for \\ Children NHS Trust, London, UK, ${ }^{2}$ Paediatric and \\ Neonatal Intensive Care Unit, Great Ormond Street \\ Hospital for Children NHS Trust, London, UK
}

Background and Aims: Conflict between health professionals and families within the intensive care setting is increasing. A new avenue for resolution of potential conflicts within our unit (PICU) came via the Patient Advice and Liaison Service (PALS) in 2002.

Methods: We performed a review of formal complaints to our hospital and later to PALS between 2001-2004. Point of contact and reason for contact and outcome were reviewed.

Results: Between 2001-4 our hospital received 542 formal complaints. Referrals to PALS were 470 for the period 2002-4 of which 32 were in respect of intensive care, 9 (3.54\%) in 2001-2 dropping to 3 in 2002-3 $(1.67 \%)$ and 2 in 2003-4 after the arrival of PALS. Content of complaints about PICU altered significantly after arrival of PALS.

Conclusions: A liaison service can become an intergral part of intensive care working alongside the medical and nursing staff to improve communication and family satisfaction. Cases involving end of life care and treatment decisions may on occasion cause significant conflict for staff and families. An approach based on impartial mediation meets the needs of the family and staff and can prove significantly successful in the deescalation and resolution of conflicts and the subsequent reduction of complaints received.
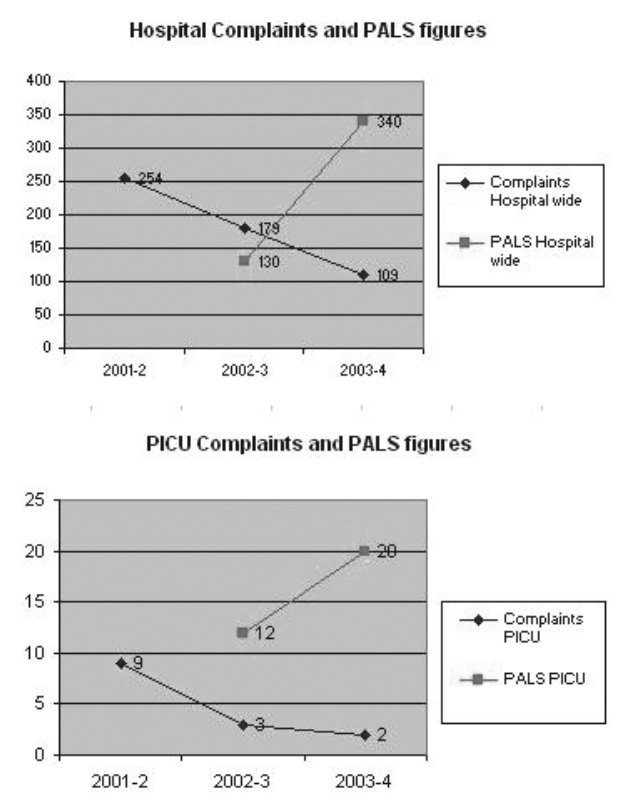

\section{TOWARDS DEBRIEFING ON THE PICU!}

O Stoffels, J Reuselaars, R J Houmes, A van den Bos, I van 't Wout, D Tibboel, M van Dijk

Pediatric Surgical Intensive Care Unit, Erasmus MCSophia, Rotterdam, The Netherlands

Background and Aims: Critical Incident Stress Debriefing (CISD), was developed in 1983 to manage stress for workers in high risk workplaces such as the emergency room. CISD sessions help participants: (1) to verbalize distress; (2) to form appropriate concepts about stress reactions before false interpretations of the experience are formed; and (3) to return to routine functioning. Debriefing has not yet gained ground in pediatric intensive care despite high incidences of distressing and acute situations. To determine the need 
for debriefing, we asked our PICU caregivers to assess stressful incidents in which they had been involved.

Methods: Caregivers completed a six-item questionnaire after watching videotaped material of incidents in which they had participated, such as resuscitation during ECMO and care of a patient with neurotrauma. The questionnaire included closed questions about communication, safety and negative emotions afterwards. Total score could range from 0 (no impact) to 18 (serious impact of incident).

Results: Eighteen questionnaires were returned from twelve nurses and six physicians. Thirteen respondents (72\%) agreed that there had been stress/turmoil on the ward. Ten respondents (61\%) judged division of roles as unclear. Seven reported ineffective communication $(39 \%)$, and three unsafety $(17 \%)$. Median total score was 6.5 for physicians and 7.5 for nurses.

Conclusions: This questionnaire could be valuable to evaluate the impact of incidents in the PICU environment, both on nurses and physicians. More data are required to confirm these findings and to determine elements (severity of illness or collaboration in the team) of greatest impact.

\section{EVELINA CHILDRENS HOSPITAL (ECH): A NEW PAEDIATRIC INTENSIVE CARE (PICU)}

\section{C A Mangani, T J Fox}

\section{PICU, Forest Ward, Evelina Childrens Hospital, London, UK}

Background and Aims: Guy's \& St Thomas PICU have been based at Guy's site for the past 20 years. Finally on the 17th October 2005 they moved into a new location to the new children's hospital at the St Thomas site which is the beginning of a new era for PICU.

Methods: The aim of this presentation is to concentrate on the planning involved in moving an entire department from one hospital to another and to show the benefits PICU have gained since moving from a 12 funded bed unit to a 20 bedded unit which is now all located on the same floor (previously on two floors) and in close proximity to cardiac theatres and others departments. The move was performed in one day with in-house South Thames Retrieval Service.

Results: Advantages state of the art medical equipment (pendants, monitoring) PICU located on the same floor. In close proximity to Cardiac Theatres and recovery. South Thames Retrieval Service operating within PICU. Effective communication and control of medical equipment (wireless).

Conclusions: The patients and the PICU can only benefit from having a new unit which is in close proximity to other essential departments with a unit which has been specifically designed to make life easier.

\section{IMPLEMENTATION OF A PATIENT DATA MANAGEMENT SYSTEM IN THE NICU: CAN THE PICU-TEMPLATE BE CONVERTED INTO THE NICU?}

S De Reus ${ }^{1}$, B A H Versluis ${ }^{2,3}$
${ }^{I}$ NICU, Erasmus MC, Sophia Children's Hospital, Rotterdam, The Netherlands, ${ }^{2}$ Directorate Information, Department of Management and Maintenance Information Systems, Erasmus MC, Rotterdam, The Netherlands, ${ }^{3}$ PICU/NICU, Erasmus MC, Sophia Children's Hospital, Rotterdam, The Netherlands

Background and Aims: The Neonatal Intensive Care Unit (NICU) was the last intensive care unit of the Erasmus MC - Sophia Children's Hospital, which had to implement the Patient Data Management System (PDMS). The aim is to work with a PDMS-template that covers the complete nursing record and part of the medical record of the patients in the NICU.

Methods: In the development of a PDMS-template for the NICU, the PDMS-template of the PICU was reviewed for its usage and compatibility of the NICU care processes. The advantage of using the PICUtemplate might save time and the administrative differences between the NICU and the PICU might be reduced to a better cooperation between healthcare professionals of both units. A group of 2 physicians, 1 nurse practitioner and 17 nurses were trained in the PDMS programme. The professionals placed the data of 30 neonates in the PICU-template and concluded that many specific items could not be placed in this template. Results: The data for the neonatal population missing in the PICU-template were for example: Gestational age, weights, accuracy of calculations and treatments. The professionals advised to make a separate NICU-template and a list of recommendations was established based upon the experiences of the 30 neonatal cases. Based on these recommendations, the system administrator made a specific NICU-template.

Conclusions: It is not possible to convert the current PICU-template of the PDMS into a NICU-template. The PICU-template can be used as a basic template to develop a specific NICU-template.

\section{IMPORTANCE OF PREVENTIVE CHILD CARE FROM THE VIEW OF MOTHERS}

\author{
N Dede Çinar ${ }^{1}$, T M Filiz ${ }^{2}$, S Sahin ${ }^{1}$, P Topsever ${ }^{2}$ \\ ${ }^{1}$ School of Health Sciences, Sakarya University, \\ Sakarya, Turkey, ${ }^{2}$ Faculty of Medicine, Department of \\ Family Medicine, Kocaeli University, Kocaeli, Turkey
}

Background and Aims: Appropriate feeding, immunization and periodic health controls have an impact on pediatric health care. The aim of this study was to assess protective behaviour of families with children between the age of 0-16 years concerning immunization, nutrition and periodic health care.

Methods: Inclusion criteria for this cross- sectional, descriptive study were, having child/ children between the age of 0-16 years, sharing the same domicile with them, giving informed consent to participate in the study. The study group $(\mathrm{n}=368)$ was randomly selected among mothers fulfilling the above criteria. Data were collected during one year between February 2003-2005. For socio-demographic inquiry a semistructured questionnaire consisting of 19 questions was used. After test-retest procedures these 10 items likert scale $(1=$ low importance, $10=$ utmost importance) questions were used 
to assess mothers' attitudes towards their offsprings' immunization, nutrition, and periodic health care.

Results: The scores for immunization $(9.4 \pm 1.2)$ was leading, nutrition $(8.5 \pm 1.4)$ and periodic health care (8.2 \pm 1.6$)$ were found relatively less important by the mothers. The attributed importance to nutrition and periodic health care was significantly increasing with higher education levels $(\mathrm{r}=0.34, \mathrm{p}<0.001 ; \mathrm{r}=0.24$, $\mathrm{p}<0.001$, respectively) while there was no correlation with immunization and mothers' education level $(\mathrm{r}=0.1$, $\mathrm{p}=0.06$ ).

Conclusions: In spite of globalization children are still dying due to malnutrition and preventable diseases in the world. Families should be educated in terms of child care emphasizing the importance of nutrition, immunization and periodic health care. This study suggests that childhood vaccines are well known and appreciated by mothers of all levels of education.

\section{A COMPARISON OF GROWTH IN PRETERM INFANTS FED TWO DIFFERENT TYPES OF INFANT FORMULA PROTEIN}

\author{
K N Florendo ${ }^{1}$, A VanZwol ${ }^{2}$, B Bellflower ${ }^{1}, \mathrm{R} \mathrm{J} \mathrm{Cooke}^{1}$ \\ ${ }^{l}$ Division of Neonatology, University of Tennessee, \\ Memphis, TN, USA, ${ }^{2}$ Division of Neonatology, VU \\ University Medical School, Amsterdam, The Netherlands
}

Background and Aims: Feeding dies suggest that feeding tolerance is better in preterm infants fed formulas containing hydrolyzed whey proteins. However, concern also exists about the biologic value of such formula. We speculated that tolerance, and, therefore, growth would be better in preterm infants fed hydrolyzed whey-based formula when compared to a standard preterm infant formula.

Methods: In a single-center, double-blind randomized controlled trial, infants weighing $<$ or $=1750 \mathrm{~g}$ at birth, $<$ or $=34$ weeks gestation, and tolerating $150 \mathrm{cc} / \mathrm{k} /$ day enterally for $>24$ hours were randomized to receive standard premature infant formula (Group A) or a premature infant formula containing $100 \%$ hydrolyzed whey protein (Group B) for 3 weeks. Anthropometry and blood chemistries (BUN, total protein, albumin) were determined at the beginning of the study and weekly thereafter. At the end of the study, plasma amino acids, fatty acids, transferrin, and retinol binding protein were also determined. Data were analyzed on an intention-totreat basis and a per protocol basis. According to data distribution, a difference in weight gain of $3 \mathrm{~g} / \mathrm{d}$ would be significant. Primary outcome was weight gain; secondary outcomes were feeding tolerance and gains in length and head circumference.

Results: Of the 80 infants enrolled, 74 completed the study; 2 were never started due to age, 2 were lost to follow-up, and 2 were lost due to sepsis, 1 of whom had NEC. Two infants (1 from each group)were dropped from the per protocol data due to lack of compliance but were included in the intention-to-treat analysis. No significant difference was found between treatment groups for demographics, protocol compliance, or gains in weight $(0.9 \mathrm{~g} / \mathrm{d}$ difference, $\mathrm{p} 0.54)$ length $(0.434 \mathrm{~mm} / \mathrm{wk}$ difference, $\mathrm{p} 0.51)$, or head circumference $(0.328 \mathrm{~mm} / \mathrm{wk}$, $\mathrm{p}$ 0.44). There were 2 episodes of feeding intolerance, 1 in each group. Two adverse events (1 NEC, Group A; 1 GBS sepsis, Group B) were also equally distributed.

Conclusions: Preterm infants fed hydrolyzed whey protein-based formula have equivalent growth compared to preterm infants fed standard non-hydrolyzed wheycasein based formula.

\section{EFFECT OF INTRAVENOUS IBUPROFEN ON BILIRUBIN BINDING IN PRETERM INFANTS: INTERIM DATA}

\section{Desfrere}

France

Abstract not available at the time of printing. 